

\section{Nebraska Statewide Wind Integration Study}

\section{April 2008 - January 2010}

EnerNex Corporation

Knoxville, Tennessee

Ventyx

Atlanta, Georgia

Nebraska Power Association

Lincoln, Nebraska

NREL Technical Monitor: Brian Parsons

Prepared under Subcontract No. AAM-9-89030-01

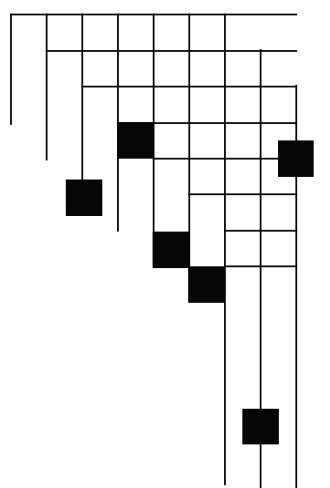




\section{NOTICE}

This report was prepared as an account of work sponsored by an agency of the United States government. Neither the United States government nor any agency thereof, nor any of their employees, makes any warranty, express or implied, or assumes any legal liability or responsibility for the accuracy, completeness, or usefulness of any information, apparatus, product, or process disclosed, or represents that its use would not infringe privately owned rights. Reference herein to any specific commercial product, process, or service by trade name, trademark, manufacturer, or otherwise does not necessarily constitute or imply its endorsement, recommendation, or favoring by the United States government or any agency thereof. The views and opinions of authors expressed herein do not necessarily state or reflect those of the United States government or any agency thereof.

Available electronically at http://www.osti.gov/bridge

Available for a processing fee to U.S. Department of Energy and its contractors, in paper, from:

U.S. Department of Energy

Office of Scientific and Technical Information

P.O. Box 62

Oak Ridge, TN 37831-0062

phone: 865.576 .8401

fax: 865.576 .5728

email: mailto:reports@adonis.osti.gov

Available for sale to the public, in paper, from:

U.S. Department of Commerce

National Technical Information Service

5285 Port Royal Road

Springfield, VA 22161

phone: 800.553 .6847

fax: 703.605.6900

email: orders@ntis.fedworld.gov

online ordering: http://www.ntis.gov/ordering.htm

This publication received minimal editorial review at NREL 


\section{CONTENTS}

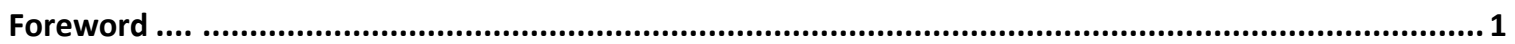

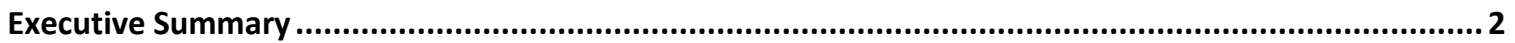

Section 1Introduction - Project Scope, Objectives, and Deliverables .............................................31

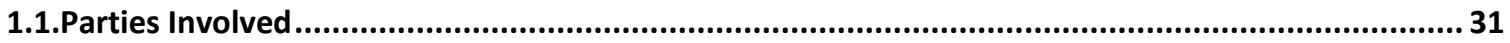

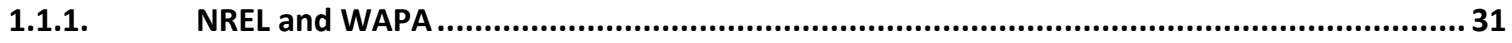

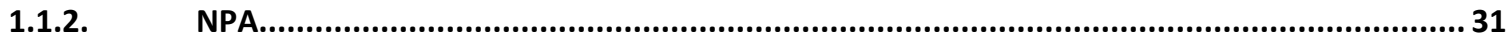

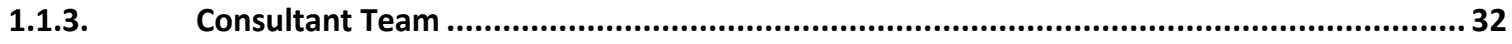

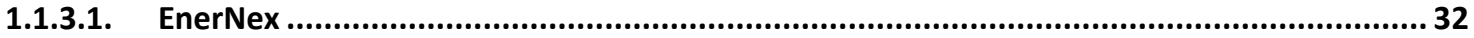

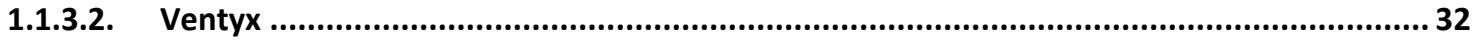

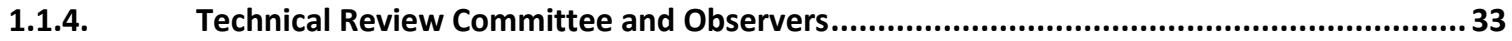

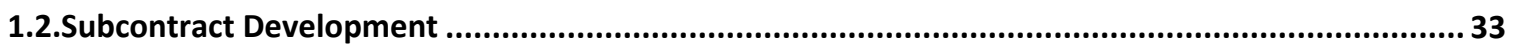

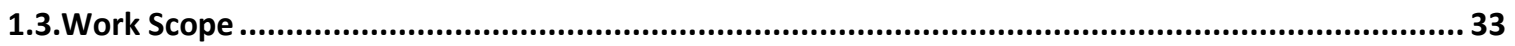

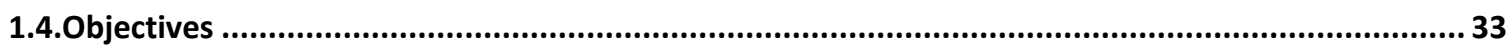

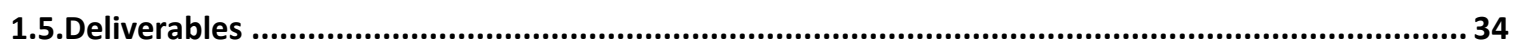

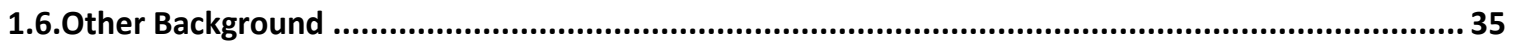

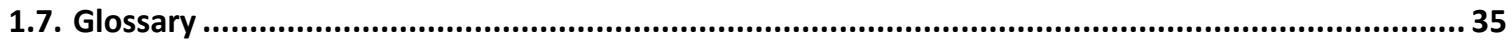

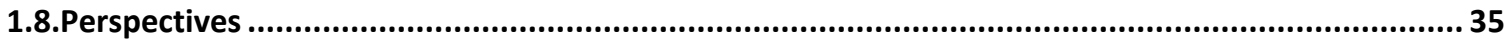

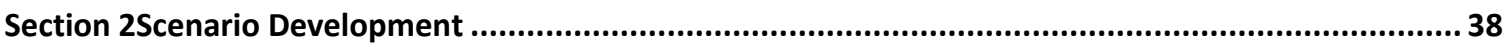

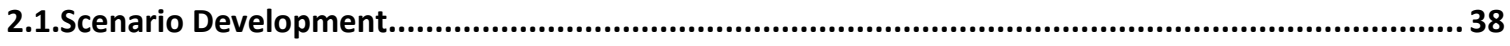

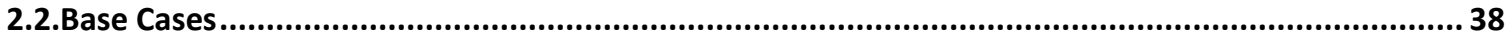

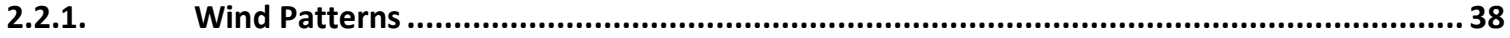

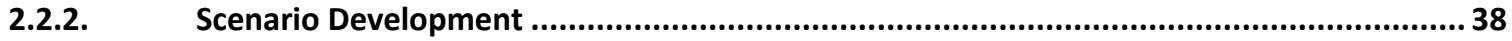

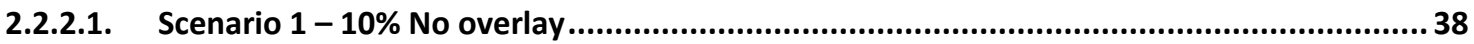

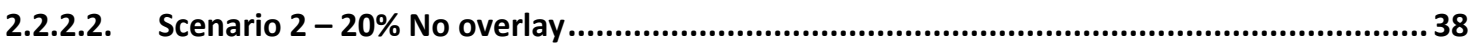

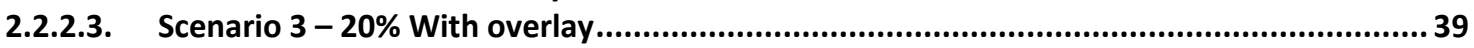

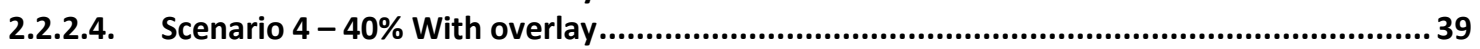

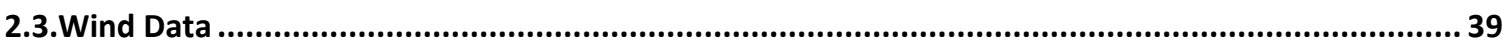

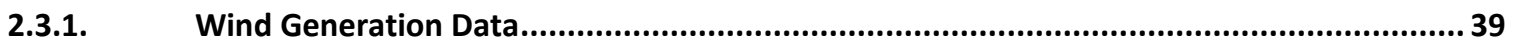

2.3.2. Selection Criteria .................................................................................. 40

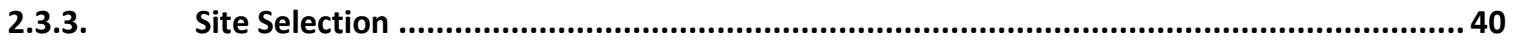

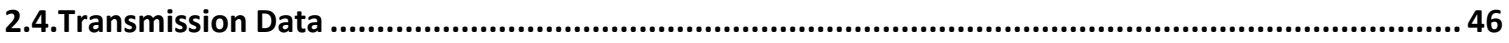




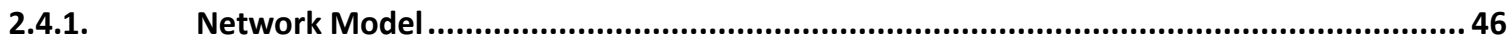

2.4.2. SPP - Transmission Model (No Overlay Cases) ..........................................................46

2.4.3. SPP -Transmission Model - EHV Overlay .............................................................47

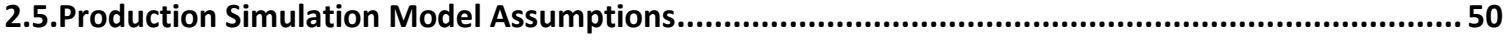

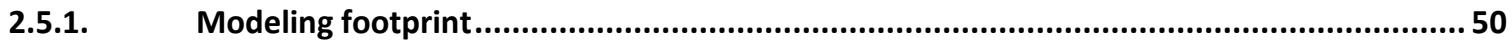

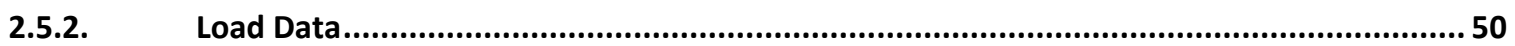

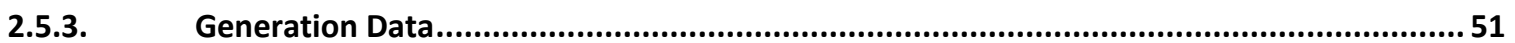

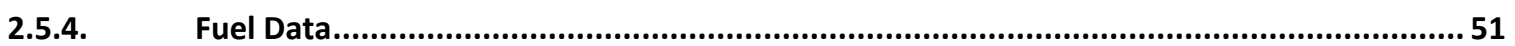

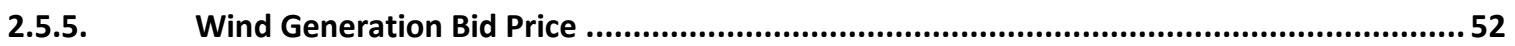

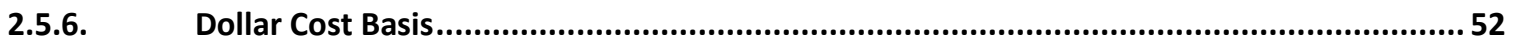

Section 3Power System Regulation and Balancing with substantial Wind Generation.........................53

3.1.Power System Operation and Control ............................................................................5 53

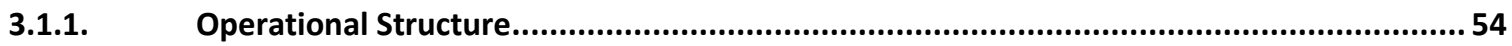

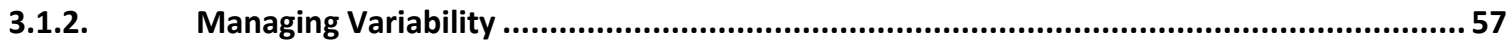

3.1.3. Measuring Control Performance ...................................................................59

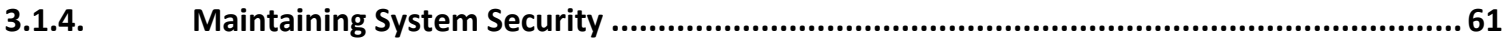

3.2.Effects of Wind Generation on Power System Control ...........................................................62

3.3.Modeling and Analysis for Assessing Wind Integration Impacts .............................................64

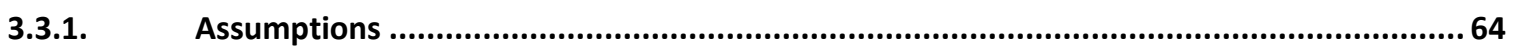

3.3.2. Mapping Reserve Requirements for Production Simulations .........................................65

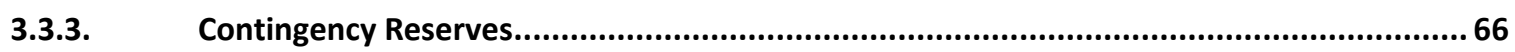

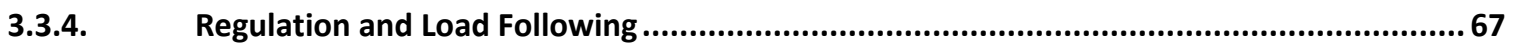

3.4.Regulating Reserve Results for Study Scenarios....................................................................76

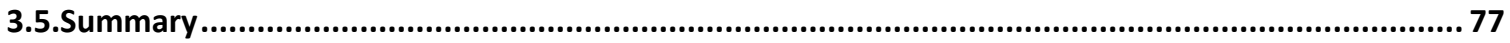

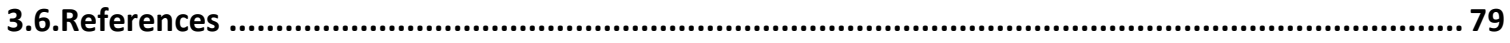

Section 4Assessing Impacts on Power System Operations .............................................................8 80

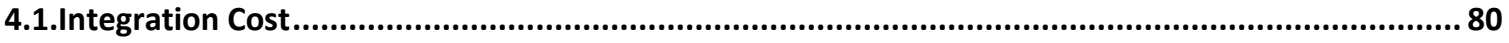

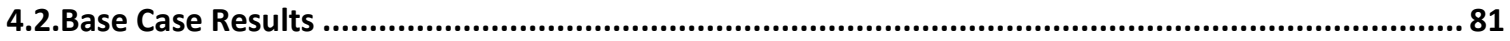

4.2.1. Integration Effects on Thermal Generators ...........................................................82

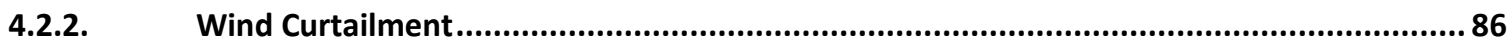

4.2.3. Transmission System Usage and LMP...........................................................88

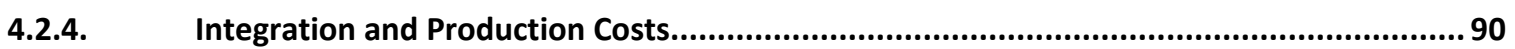

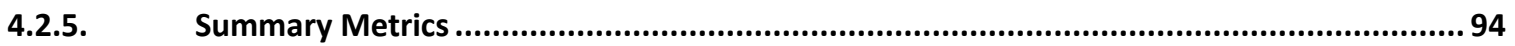

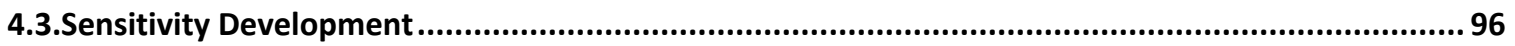




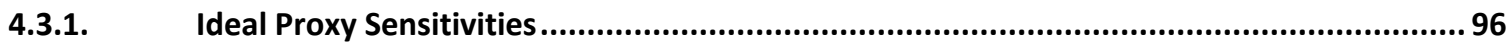

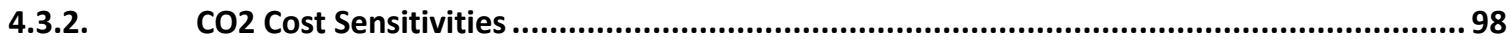

4.3.3. Market sensitivity \#1 - Nebraska standalone market..........................................................99

4.3.4. Market sensitivity \#2 - Hurdle rate raised from $\$ 5 / \mathrm{MWh}$ to $\$ 20 / \mathrm{MWh} \ldots \ldots . . . . . . . . . . . . . . . . . .100$

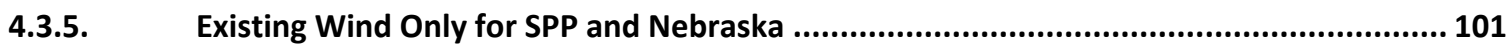

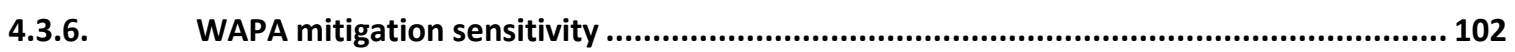

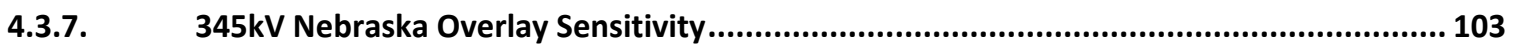

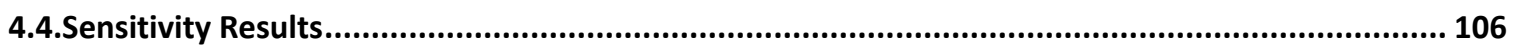

4.4.1. Sensitivity - Nebraska Standalone Market.................................................................. 109

4.4.2. Market Sensitivity \# 2- Tariff/Hurdle Rate @ \$20/MWh ............................................... 111

4.4.3. Sensitivity - Existing Wind .............................................................................................. 113

4.4.4. Sensitivity - Proxy Resource Comparison ................................................................. 115

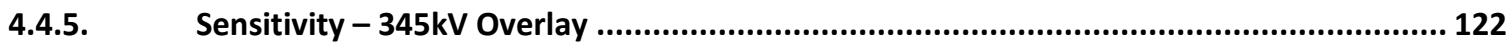

4.4.6. Sensitivity - WAPA Mitigation .......................................................................................... 125

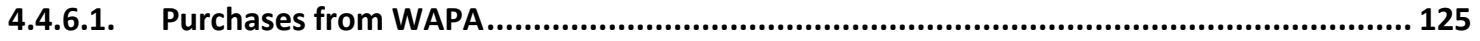

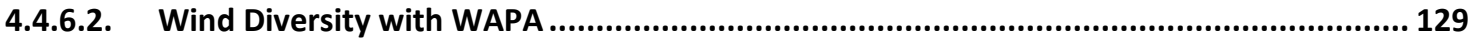

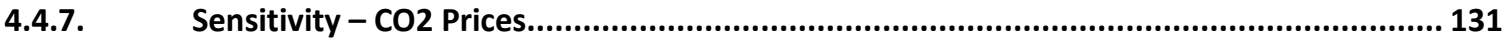

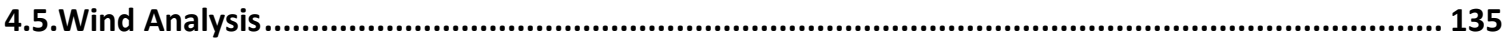

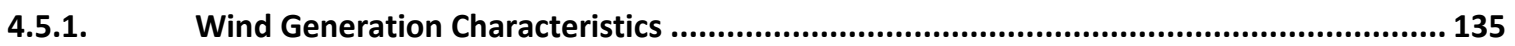

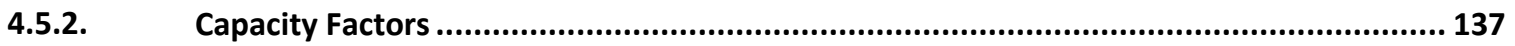

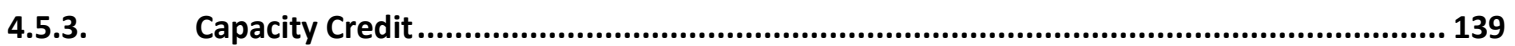

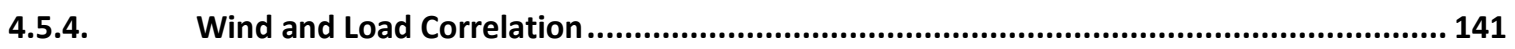

4.5.5. Mean Absolute Error of Wind Forecast ...................................................................... 142

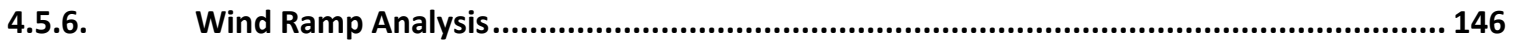

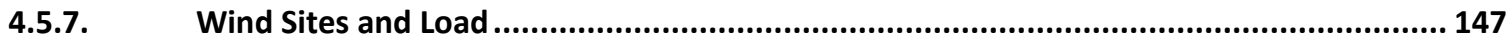

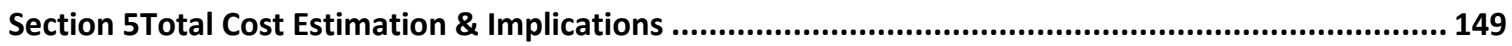

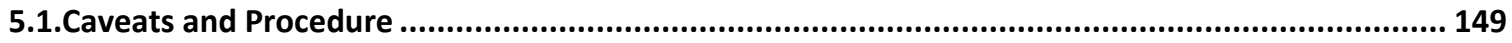

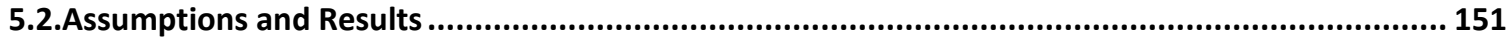

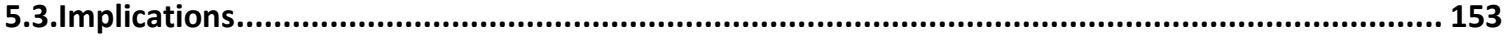

Section 6Western Nebraska Wind - Western Interconnection: a Brief Review.................................... 157

6.1.Executive Summary - Western Interconnection ...................................................................... 157

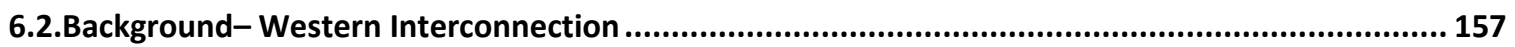

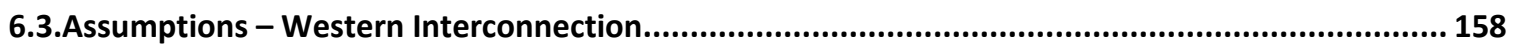

6.3.1. NPA asked WAPA to perform two primary studies:................................................... 158 
6.3.2. Other Assumptions - Western Interconnection ............................................................ 159

6.4.Study Conclusions- Western Interconnection: .............................................................................. 160

6.4.1. Cost Estimate for Upgrades (Cost estimates given are in 2008 dollars) ...........................160

6.4.1.1. 2012 Base Case (for $300 \mathrm{MW}$ injection) ......................................................................... 160

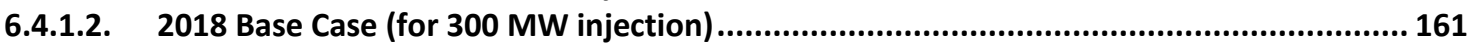

6.4.2. Wind Integration for Western Nebraska ........................................................................ 161

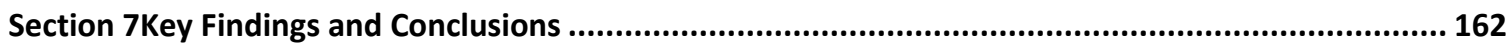

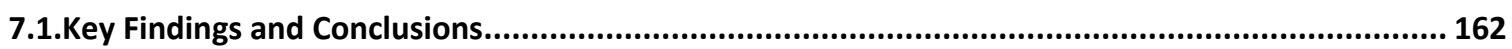

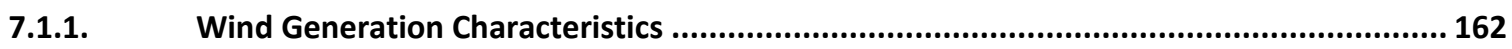

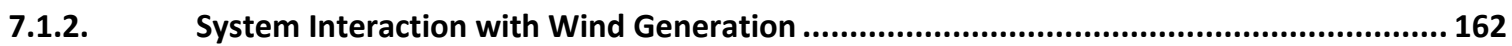

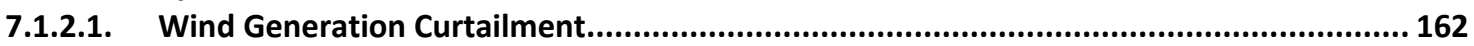

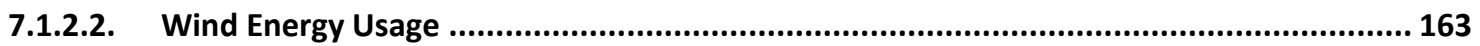

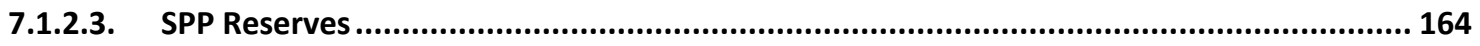

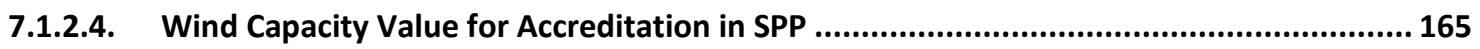

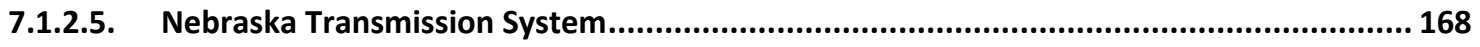

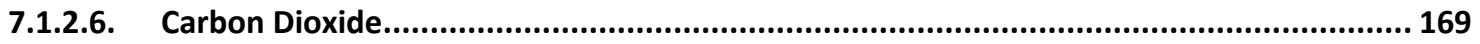

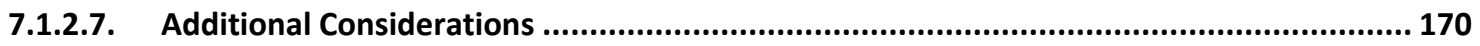

7.1.2.8. Total Costs (annual amounts in 2018 and assuming REC price of zero)........................... 170

7.1.3. WAPA Interface for Integration ...................................................................................... 171

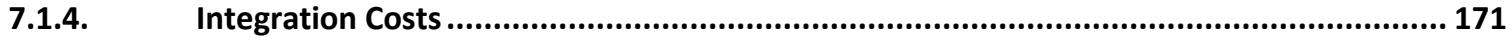

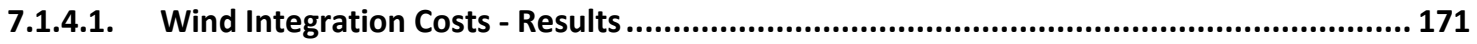

7.1.4.2. Wind Integration Costs - Methodology and Proxy Selection ......................................... 176

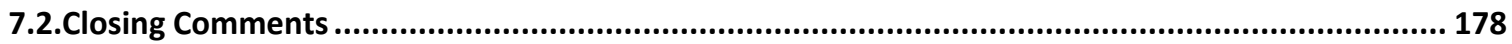

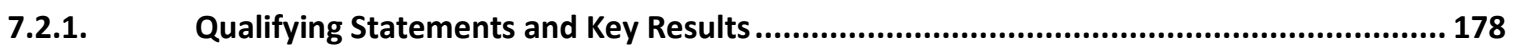

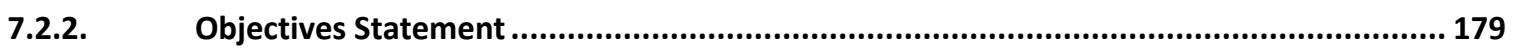

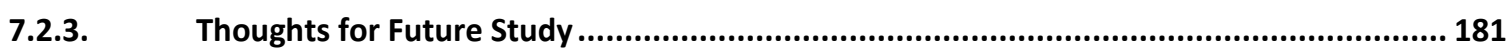

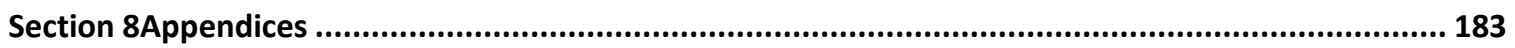

8.1.Technical Review Committee for the Nebraska Statewide Wind Integration Study ........................ 183

8.2.Additional Wind Generation Characteristics Scenario Information ................................................ 185

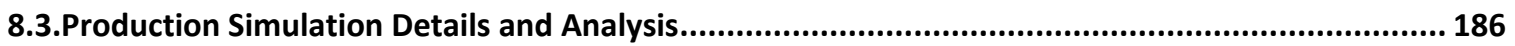

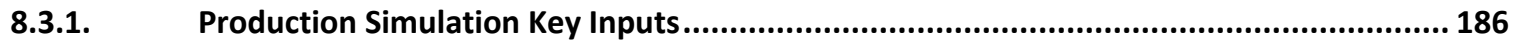

8.3.2. Production Simulation Generation Results..................................................................... 189

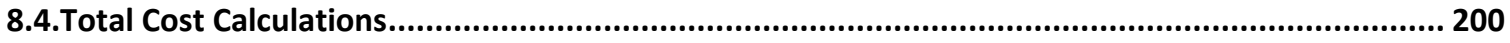

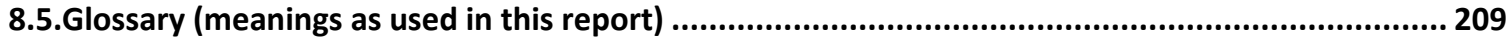




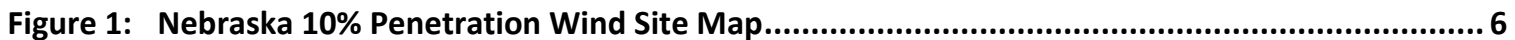

Figure 2: Nebraska Wind Sites Added to 10\% Penetration Sites to Achieve 20\% Penetration ................ 7

Figure 3: Nebraska Wind Sites Added to 20\% Penetration Sites to Achieve 40\% Penetration ................ 7

Figure 4: Conceptual Extra High Voltage (EHV) Overlay with Base Case Upgrades .................................9

Figure 5: SPP Wind Generation Curtailment by Scenario (note: no curtailment in NPA) ..................... 15

Figure 6: Nebraska Exports by Scenario................................................................................................... 16

Figure 7: Monthly NPA Wind Capacity Value Estimates by Scenario ..................................................... 20

Figure 8: Monthly SPP (non-NPA) Wind Capacity Value Estimates by Scenario..................................... 20

Figure 9: Monthly SPP with NPA Wind Capacity Value Estimates by Penetration ................................. 20

Figure 10: Total SPP Cost Estimates (inc Neb) in \$2018 millions - Cap \& Trade Reg with 117.7 M s-ton

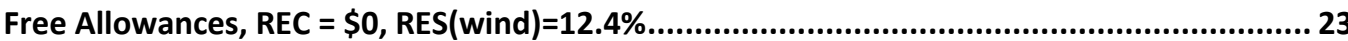

Figure 11: Change in Nebraska Generation with Incremental Reserve Requirements and Additional Uncertainty due to Wind Generation ("actual" case)

Figure 12: SPP (including Nebraska) Normalized Wind Integration Costs (shaped proxy) by Scenario and Wind Year for 2018 Conditions shown both in 2018\$ (top) and 2009\$ (bottom)

Figure 13: SPP (including Nebraska) Integration Costs Using Different Proxy Resources for 2018 Conditions shown both in 2018\$ (top) and 2009\$ (bottom)

Figure 14: Representation of all possible NPA and SPP wind plants from NREL meso-scale database. ... 39

Figure 15: Nebraska 10\% Penetration Wind Siting Map........................................................................... 42

Figure 16: Nebraska Wind Sites Added to 10\% Penetration Sites to Achieve 20\% Penetration ...............43

Figure 17: Nebraska Wind Sites Added to 20\% Penetration Sites to Achieve 40\% Penetration ............... 44

Figure 18: SPP w/o Nebraska 40\% Wind Siting Map ................................................................................ 45

Figure 19: EWITS Reference case less Nebraska sites................................................................................. 46

Figure 20: Base Transmission Upgrades Used in all Scenarios. .......................................................... 47

Figure 21: EHV Conceptual Overlay for NPA Study with Base Case Upgrades ..................................... 48

Figure 22: SPP EHV Overlay with Nebraska 345 kV Sensitivity with Base Case Upgrades ....................... 49

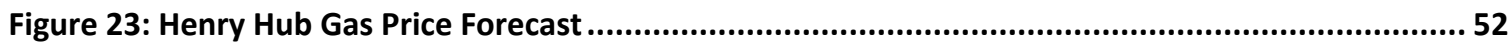

Figure 24: NERC Reliability Regions and Balancing Authorities as of January 2005 (top) and August 2007

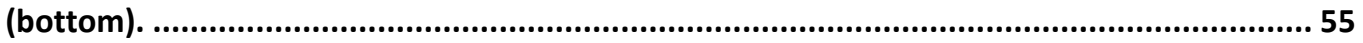

Figure 25: U.S. Regional Transmission Organizations (RTOs) .................................................................. 56

Figure 26: Depiction of Regulation and Load Following Characteristics of Demand. ..............................58 
Figure 27: NERC CPS2 equations.

Figure 28: Normalized 10-minute Variability for Five Different Groups of Wind Generation. The $500 \mathrm{MW}$ Scenario is Part of the 5000, which is Part of the 15000.

Figure 29: Illustration of Short-Term (next 10-minute interval) Forecasts of Load and Wind Generation.69

Figure 30: Errors in SPP Short-Term Forecasts of Load and Wind Generation; Load Error is assumed to be

Zero in the Mathematical Procedure.

Figure 31: Illustration of short-term (ten minute ahead) wind generation forecast errors as a function of average hourly production 71

Figure 32: Standard Deviation of 1-Hour Persistence Forecast Error for Nebraska 40\% Wind Penetration72

Figure 33: Distributions of hourly regulating reserve requirements for SPP and Nebraska $-40 \%$ wind Penetration, for load only (ideal wind generation) and load net of wind generation.

Figure 34: Comparison of SPP (including Nebraska) Actual and Ideal Generation by Type 83

Figure 35: Comparison of Nebraska Actual and Ideal Generation by Type........................................ 83

Figure 36: SPP Generation by Fuel and Unit Type .......................................................... 84

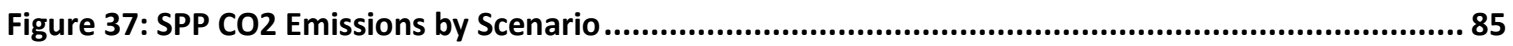

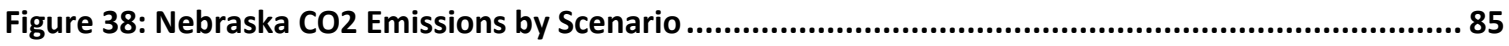

Figure 39: Wind Curtailment (TWh) in non-Nebraska SPP ....................................................... 86

Figure 40: Wind Curtailment (\% of annual expected wind energy) in non-Nebraska SPP .....................87

Figure 41: SPP (Including Nebraska) Annual Average Generation Weighted LMP ................................90

Figure 42: SPP (including Nebraska) Adjusted Production Costs...................................................... 91

Figure 43: SPP (including Nebraska) Wind Integration Costs (shaped proxy) in Million\$...................... 91

Figure 44: SPP (including Nebraska) Wind Integration Costs (shaped proxy) Normalized to Wind Energy for 2018 Conditions shown both in 2018\$ (top) and 2009\$ (bottom) ................................. 92

Figure 45: Summary of Base Case Metrics(All dollars are 2018\$) ................................................ 95

Figure 46: Comparison of Block vs. Shaped Proxy Types ........................................................97

Figure 47: Comparison of Moving Average vs. Shaped Proxy Types .............................................. 98

Figure 48: Representation of separate Nebraska Market............................................................. 100

Figure 49: Representation of Hurdle Rates in PROMOD IV .................................................... 101

Figure 50: Comparison of Load and 'Load Net Wind' (Jan 1-7, 2018) ......................................... 103

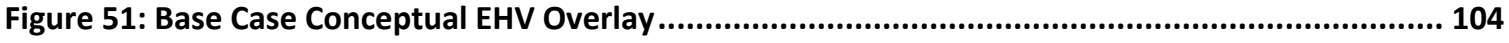

Figure 52: Conceptual EHV Overlay with 345 kV in Nebraska................................................... 105

Figure 53: Conceptual 345 kV Overlay in Nebraska............................................................ 105

Figure 54: Summary of Sensitivity Case Metrics (All dollars are 2018\$) .................................... 108

Figure 55: Thermal Generation Change from Nebraska Market Case to Base Case ........................... 110

Figure 56: Comparison of Exports in Base Case and Nebraska Market Case ...................................... 111 
Figure 57: Exports Comparison between Base Case and \$20 Tariff Case

Figure 58: Thermal Generation Changes between Base Case and \$20 Tariff Case

Figure 59: Comparison of Thermal Generation in Nebraska Market Case and Existing Wind Case ....... 114

Figure 60: Exports Comparison of Nebraska Market Case and Existing Wind Case ............................ 114

Figure 61: Thermal Generation Comparison between Shaped Proxy and flat Block Proxy Cases.......... 117

Figure 62: Comparison of Normalized Wind Integration Costs for SPP (including Nebraska) for Different Proxy Resource Sensitivities for Different 10\% Cases for 2018 Conditions for both 2018\$ (top) and 2009\$ (bottom)

Figure 63: Comparison of Adjusted Production Costs for SPP (including Nebraska) for Different 10\% Cases in 2018\$

Figure 64: Comparison of Normalized Integration Costs for SPP (including Nebraska) with Alternate Proxies at $10 \%$ and $20 \%$ Wind for 2018 conditions shown in both $2018 \$$ (top) and $2009 \$$ (bottom)

Figure 65: Flow Durations for Nebraska 345 kV Overlay (20\%)................................................... 122

Figure 66: Flow Durations for Nebraska 345 kV Overlay (40\%)................................................... 123

Figure 67: Comparison of CO2 Emissions for 345 kV Overlay Sensitivity........................................ 124

Figure 68: Comparison of Exports for 345 kV Overlay Sensitivity ............................................... 125

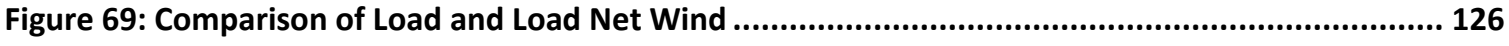

Figure 70: Comparison of WAPA Firm Purchase Schedules .................................................... 127

Figure 71: Comparison of Load Following Methods ................................................................. 127

Figure 72: WAPA Firm Purchase Duration Curve - 2018 annual .................................................... 128

Figure 73: Annual Thermal Generation Change in Nebraska for WAPA Sensitivity (using Pure Load

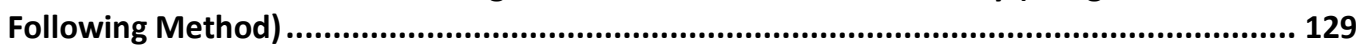

Figure 74: Nebraska MAE of Actual and Day Ahead Forecast .................................................. 130

Figure 75: WAPA MAE of Actual and Day Ahead Forecast................................................. 130

Figure 76: Nebraska and WAPA MAE of Actual and Day Ahead Forecast......................................... 131

Figure 77: CO2 Emissions Comparison for different Penalty Levels ............................................ 132

Figure 78: Thermal Generation Comparison between CO2 Reduction Case and Base Case ................. 133

Figure 79: Comparison of Pumped Storage Output with Different CO2 Penalties ............................... 134

Figure 80: SPP On and Off Peak Annual Average LMP .......................................................... 135

Figure 81: Nebraska Wind Site capacity credit................................................................. 141

Figure 82: Correlation of Hourly Plant Output between Nebraska Wind Sites ................................... 142

Figure 83: Nebraska Day Ahead Forecast Mean Absolute Error .................................................. 144

Figure 84: Nebraska 1 Hour Persistence Forecast Mean Absolute Error .................................... 145

Figure 85: Nebraska Load Duration Curve for Load and Load net Wind .......................................... 147

Figure 86: One Year of Nebraska Load and 40\% Wind Penetration ............................................... 148 
Figure 87: Additional Revenue Increase \%.

Figure 88: Total SPP Cost Estimates (including Nebraska) in \$2018 millions - Cap \& Trade Reg with $117.7 \mathrm{M}$ s-ton Free Allowances, $\mathrm{REC}=\$ 0, \mathrm{RES}($ wind $)=12.4 \%$.

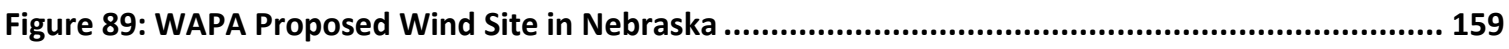

Figure 90: Nebraska exports by Scenario and Wind Year ......................................................... 164

Figure 91: Aggregated Wind Site Capacity Value in Nebraska .................................................. 167

Figure 92: Aggregated Wind Site Capacity Value in SPP (without Nebraska) .................................... 167

Figure 93: Monthly SPP with NPA Wind Capacity Value Estimates by Penetration ............................. 168

Figure 94: Change in Nebraska Generation with Incremental Reserve Requirements and Additional Uncertainty due to Wind Generation ("actual" case).

Figure 95: SPP (including Nebraska) Normalized Wind Integration Costs by Scenario and Wind Year for 2018 Conditions shown in 2018\$ (top) and 2009\$ (bottom) .

Figure 96: SPP (including Nebraska) of Integration Costs using Different Proxy Resources for 2018 Conditions in 2018\$ (top) and 2009\$ (bottom)

Figure 97: Total SPP Cost Estimates (inc Neb) in \$2018 millions - Cap \& Trade Reg with 117.7M s-ton Free Allowances, $\operatorname{REC}=\$ 0, \operatorname{RES}($ wind) $=12.4 \%$

Figure 98: Total SPP Cost Estimates (inc Neb) in \$2018 millions - Cap \& Trade Reg with 117.7M s-ton Free Allowances, $\mathrm{REC}=\$ 15, \mathrm{RES}($ wind) $=12.4 \%$.

Figure 99: Total SPP Cost Estimates (inc Neb) in \$2018 millions - Cap \& Trade Reg with 117.7M s-ton Free Allowances, $\mathrm{REC}=\$ 0, \mathrm{RES}($ wind $)=12.4 \%$

Figure 100:Total SPP Cost Estimates (inc Neb) in \$2018 millions - Tax Regulation - No Free Allowances, $\operatorname{REC}=\$ 15, \operatorname{RES}($ wind $)=12.4 \%$

Figure 101:Total SPP Cost Estimates (inc Neb) in \$2018 millions - Tax Regulation - No Free Allowances, REC $=\$ 0, \operatorname{RES}($ wind $)=12.4 \%$

Figure 102: Additional Revenue Increase \% 


\section{TABLES}

Table 1: Characteristics of Wind Penetration for Study..................................................................... 5

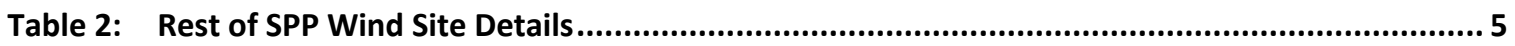

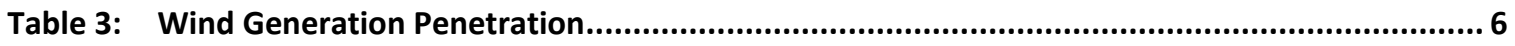

Table 4: Detailed Results for Base Scenarios - all dollars are 2018\$.................................................... 13

Table 5: Regulating Reserve Requirements for Study Scenarios ................................................... 17

Table 6: Regulating Reserve Requirements for NPA as a Single Balancing Authority and SPP with and

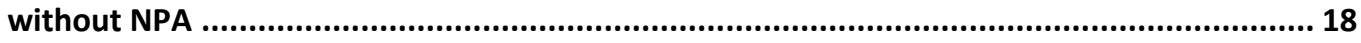

Table 7: Estimated Capacity Accreditation per SPP Methodology for Wind Generation Sites in

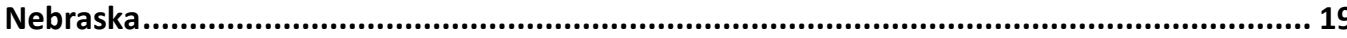

Table 8: Monthly Capacity Values for Aggregated Nebraska Wind Generation Scenarios by

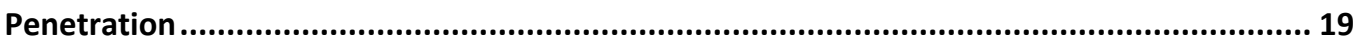

Table 9: Number of Nebraska Branches at or Near Limits ............................................................. 21

Table 10: Shaped and Sub-period Block Proxy Analysis at 10\% and 20\% Wind Penetration .................. 28

Table 11: Nebraska Selected Wind Generation Penetration Details ................................................... 41

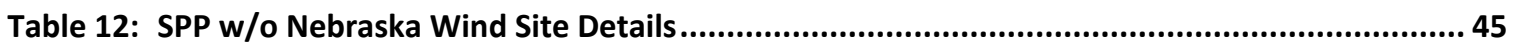

Table 13: Nebraska and SPP Coincident Peak and Energy .................................................................. 51

Table 14: Regional Wind Penetrations (2018) Outside of SPP ............................................................... 51

Table 15: Excerpts from NERC Glossary of Terms Related to Operating Reserves ................................ 57

Table 16: 2009 CPS2 Bounds for some Eastern Interconnection Balancing Authority Areas ...................60

Table 17: Mapping of Reserve Components in categories for production simulations ............................66

Table 18: Contingency Reserve Requirements by Operation Region and Scenario for 2018...................67

Table 19: Summary of Reserve Methodology for Study Scenarios ........................................................ 73

Table 20: Example Application of Reserve Methodology to Hourly Data (SPP including Nebraska)....... 74

Table 21: Adjustment of spinning reserve for reduction in wind generation (SPP including Nebraska) 75

Table 22: Regulating Reserve Requirements for the SPP Including Nebraska Load and Load Net Wind. 77

Table 23: Regulating Reserve Requirements for Nebraska Only and SPP without Nebraska for $10 \% 2006$ Profile

Table 24: Nebraska Dump Energy as a Percentage of Wind Energy..................................................... 88

Table 25: Numbers of Constrained Hours for Flowgates in or near Nebraska ......................................... 88

Table 26: Number of Nebraska transmission branches near thermal limits.......................................... 89

Table 27: Integration Costs (shaped proxy) in Millions for SPP and Modeled Footprint......................... 93

Table 28: Changes in Integration Cost (shaped proxy) With Wind Penetration Increase ........................ 94

Table 29: Normalized Wind Integration Costs (shaped proxy) for SPP and Modeled Footprint .............99 
Table 30: Contingency Reserve Requirements for Nebraska and SPP

Table 31: Wind Energy Comparison between Existing and 10\% Cases ......................................... 102

Table 32: Separate Nebraska Market Wind Curtailment Comparison ............................................ 110

Table 33: Comparison of Costs (2018\$) in Base Case and Nebraska Market Case ............................ 111

Table 34: SPP including NPA Integration Costs (2018\$) - Shaped Proxy ........................................ 113

Table 35: Wind Energy Comparisons of Existing Wind and 10\% Wind Nebraska Standalone Market

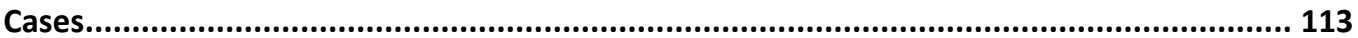

Table 36: Cost Comparison (2018\$) of Existing Wind Case and Nebraska Market Case - Daily Block Proxy

Table 37: Comparison of Costs (2018\$) between Daily Block Proxy Sensitivity and 10\% Base Case (Shaped Proxy)...

Table 38: Integration Costs in Millions using different proxies for SPP and Modeled Footprint .... 121

Table 39: Native and Implied Integration Costs for SPP using Daily and Sub-period Block Proxies ...... 121

Table 40: Costs Comparisons (2018\$) for Overlay Sensitivities (Shaped Proxy) ............................... 123

Table 41: Comparison of Load and WAPA Firm Purchase................................................. 126

Table 42: Cost Results for Nebraska for WAPA Sensitivity using Load Following with Historical Mins. 128

Table 43: Cost Results for Nebraska for WAPA Sensitivity using Pure Load Following ....................... 128

Table 44: Nebraska Capacity Factor Comparison between Base Case and CO2 Reduction Case .......... 133

Table 45: Nebraska Wind Site Characteristics..................................................................... 136

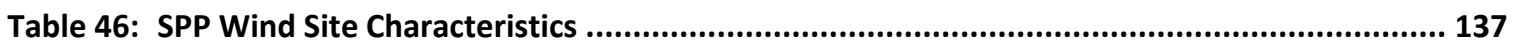

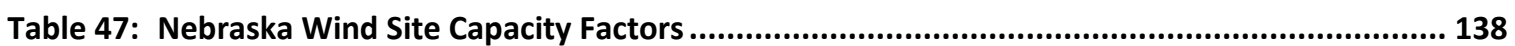

Table 48: Rest of SPP Wind Site Capacity Factors ................................................................ 139

Table 49: Correlation between Nebraska Wind Sites................................................................. 141

Table 50: Maximum Hourly Up-Ramp for Nebraska with 20\% Wind Penetration ............................ 146

Table 51: Maximum Hourly Down-Ramp for Nebraska with 20\% Wind Penetration .......................... 146

Table 52: NPA Load and $20 \%$ Wind Penetration Summary..................................................... 148

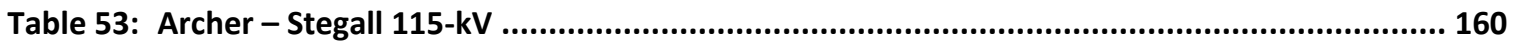

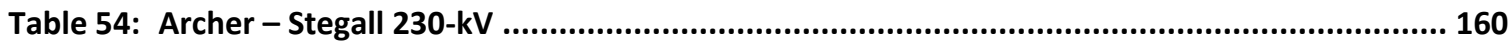

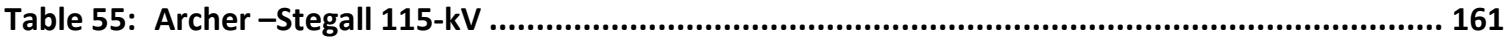

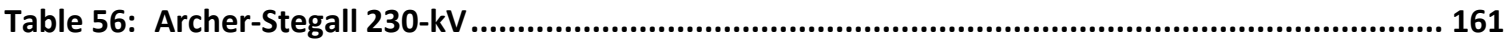

Table 57: Wind Generation Scenarios("SPP" here is rest of SPP).............................................. 162

Table 58: Regulating Reserves for SPP Load and Load net Wind .............................................. 165

Table 59: Regulating Reserves with Load and Load net Wind for Nebraska only and SPP without

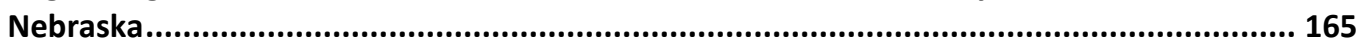

Table 60: Nebraska Monthly Wind Site Capacity Values .......................................................... 166 
Table 61: Monthly Capacity Values for Aggregated Nebraska Wind Generation Scenarios by Penetration.

Table 62: Number of Nebraska Branches at or near limits ......................................................... 168

Table 63: Proxy Integration Costs for 2018 and 2009 Dollars ..................................................... 179

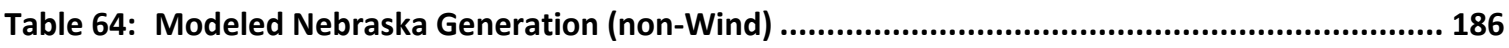

Table 65: Nebraska Wind Site Mapping to Bus Number ......................................................... 188

Table 66: Modeled Flow Gates ........................................................................................ 188

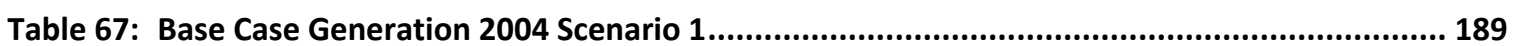

Table 68: Base Case Generation 2004 Scenario 2 ................................................................. 189

Table 69: Base Case Generation 2004 Scenario 3....................................................................... 189

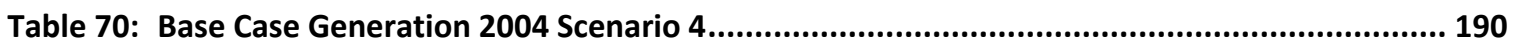

Table 71: Base Case Generation 2005 Scenario 1..................................................................... 190

Table 72: Base Case Generation 2005 Scenario 2................................................................. 190

Table 73: Base Case Generation 2005 Scenario 3............................................................... 191

Table 74: Base Case Generation 2005 Scenario 4..................................................................... 191

Table 75: Base Case Generation 2006 Scenario 1....................................................................... 191

Table 76: Base Case Generation 2006 Scenario 2.................................................................. 192

Table 77: Base Case Generation 2006 Scenario 3................................................................ 192

Table 78: Base Case Generation 2006 Scenario 4................................................................ 192

Table 79: Sensitivity Case Generation 2006 Nebraska 345 kV 20\% Penetration............................... 193

Table 80: Sensitivity Case Generation 2006 Nebraska 345 kV 40\% Penetration................................ 193

Table 81: Sensitivity Case Generation 2006 Tariff = \$20/MWh 20\% Penetration ............................ 193

Table 82: Sensitivity Case Generation 2006 CO2 = \$0/MWh 20\% Penetration................................. 194

Table 83: Sensitivity Case Generation 2006 CO2 = \$50 20\% Penetration ........................................ 194

Table 84: Sensitivity Case Generation 2006 CO2 = \$120 20\% Penetration ..................................... 194

Table 85: Sensitivity Case Generation 2006 Nebraska Market ................................................... 195

Table 86: Sensitivity Case Generation 2006 Daily block Proxy 10\% Penetration .............................. 195

Table 87: Sensitivity Case Generation 2006 5-Hr MA 10\% Penetration............................................ 195

Table 88: Sensitivity Case Generation 2006 Sub-Period Block 10\% Penetration ............................. 196

Table 89: Sensitivity Case Generation 2006 13-Hr MA 10\% Penetration....................................... 196

Table 90: Sensitivity Case Generation 2006 WAPA Sensitivity .................................................. 196

Table 91: Sensitivity Case Generation 2006 WAPA II - Pure ........................................................ 197

Table 92: Sensitivity Case Generation 2006 Existing Wind ...................................................... 197

Table 93: Sensitivity Case Generation 20065 Hour MA 20\% ..................................................... 197 
Table 94: Sensitivity Case Generation 2006 Daily Block Wind 20\%............................................... 198

Table 95: Sensitivity Case Generation 2006 Sub-period Block Wind 20\%...................................... 198

Table 96: Sensitivity Case Generation 200613 Hour Moving Average 20\% ................................... 198

Table 97: Nebraska Non-monitored Branches with number of hours of potential overloads (2006 load and wind profiles) ....................................................................................... 199

Table 98: Additional Revenue Requirements (CO2 Cap \& Trade Reg, REC=\$15............................ 204

Table 99: Additional Revenue Requirements (CO2 Cap \& Trade Reg, REC=\$0................................ 205

Table 100:Additional Revenue Requirements (CO2 TAX Reg, REC=\$15) ........................................206

Table 101:Additional Revenue Requirements (CO2 TAX Reg, REC=\$0) ........................................ 207 


\section{FOREWORD}

This work is a result of the U.S. Department of Energy's (DOE) Wind Program and the National Renewable Energy Laboratory's (NREL) interest in advancing the understanding of wind integration impacts in the Western Area Power Administration's (WAPA) service area and among consumer-owned electric power cooperatives. The DOE through NREL provided costshare support to the Nebraska Power Association (NPA) utilities to help carry out the program objectives of this wind integration study.

NREL's expectation was that the integration study should incorporate the best practices for performing such a study, including the assessment of wind's impact in the different time scales (regulation, load following, and unit commitment/scheduling) using high-quality wind speed and/or wind power data.

The NPA is deeply appreciative of this learning opportunity afforded by the financial and technical support of NREL as provided to this study. The study process and results will be an aid in preparing for the rapidly developing wind future of Nebraska and its participation in regional markets. Further, the NPA is appreciative of the dedicated and talented work of the consultant team of EnerNex Corporation and Ventyx, Inc. Representatives of NREL, the consultants, and the rest of the Technical Review Committee (TRC) are listed in Appendix Section 8.1. Finally, the NPA thanks all members of the TRC and the observers for their steady attention, cooperative efforts and creative thinking. The NPA does not make any representations regarding use of the information beyond the context presented in this report.

WAPA offers this statement: "Western appreciated the opportunity to participate in this study. The results and conclusions offer insight as related to potential impacts/benefits resulting from the integration of large quantities of wind generation. Further, Western concurs with the statements contained in Section 7.2.1, in that Western does not make any representations regarding use of the information beyond the context presented in this report."

The National Renewable Energy Laboratory and the Nebraska Power Association do not make any representations regarding use of the information in this report beyond the context presented in this report. 


\section{EXECUTIVE SUMMARY}

\section{Introduction}

Wind generation is a source of electric energy that, in sufficient quantity, can cause concern for the operation of the bulk power system because of the variability and uncertainty of the wind. Most short-term planning and operational functions supporting the electric power grid are centered on supply resources that have capacity attributes - i.e. they can be dispatched (controlled up and down) to a specified power level for a specified period of time. The need to maintain very high levels of system reliability reinforces the preference in operations toward resources having capacity attributes. Wind generation usually has relatively low capacity value at system peak.

The characteristics of wind generation increase the variability of the net demand in the balancing area and the uncertainty about the net demand over operational planning horizons e.g. next hour, next 24 hours beginning at midnight, etc. Managing a system with significant wind generation requires conventional resources to potentially "work a little harder". Increased costs are a consequence of the additional duties performed by conventional generators.

Over the past decade, many "wind integration" studies have been performed to quantify the operational impacts of wind generation.

Wind generation resources in Nebraska will play an increasingly important role in the environmental and energy security solutions for the state and the nation. In a reference document entitled "Renewable Energy Background and Outlook for Nebraska Electricity Consumers ${ }^{1 "}$, the Nebraska Power Association utilities recognized the growing need for a specific study of wind generation in the state.

This Nebraska Statewide Wind Integration Study provides that initial study.

\section{Study Background}

The Nebraska Power Association (NPA) (www.nepower.org) is a voluntary organization that represents all segments of the Nebraska power industry: municipalities, public power districts, public power and irrigation districts, and cooperatives engaged in generation, transmission, and/or distribution of electric energy in the nation's only totally public power state.

The organization represents approximately $28,248 \mathrm{GWh}$ of annual retail load (2007 value - DOE) served by approximately $9,000 \mathrm{MW}$ of generation capacity (including capacity associated with out-of-state purchases and sales). Six Nebraska utilities own and operate or purchase the output from a total of $152 \mathrm{MW}$-nameplate of wind generation projects in five separate locations spread across the state. During 2009 power purchase agreements were completed for two new

${ }^{1}$ dated December 28, 2007, downloadable from www.nepower.org/NPA\%20Report.pdf 
wind generation plants totaling $102 \mathrm{MW}$. Negotiations are underway to add more wind generation and still more wind additions are planned for later on according to the state utility integrated resource plans.

In April of 2008, NPA submitted a proposal to and received an award from the U.S. Department of Energy through the National Renewable Energy Laboratory for funding to conduct a statewide wind integration study.

Nebraska electric utilities, large and small, are represented directly or indirectly by their power supplier in this study of wind integration effects to the public power system in Nebraska.

Qualified and interested regional and national entities were invited to participate as observers or as active members of the Technical Review Committee. This Nebraska Statewide Wind Integration Study (Study) will help Nebraska implement wind energy in an intelligent manner, while maintaining Nebraska's success in operating a reliable and economical $\left(5^{\text {th }}\right.$ lowest state in 2007 at 6.28 cents per kWh) electrical system and achieving the diversity of a significant statewide renewable portfolio.

Of significant interest to NPA was the opportunity to work directly with the U.S. DOE's Wind Program, NREL, and WAPA not only because of their expertise, but also as an opportunity to foster regional solutions to wind integration issues.

Three balancing areas are fully represented: Nebraska Public Power District, Omaha Public Power District, and the Lincoln Electric System. Additionally all of the Nebraska utilities, small, medium, and large, are participating in the study through their NPA representation.

\section{Study Objectives}

Objectives of the work to be done were established by NREL and supplemented by NPA as:

- $\quad$ Evaluate the impact of wind energy on system operations using synchronized wind and load data and state of the art analysis techniques.

- Wind energy penetration of at least $10 \%$, based on wind energy to total energy sales should be evaluated. Additional scenarios at higher penetrations are encouraged.

- $\quad$ Evaluation should be a combination of statistical analysis and production simulation.

- $\quad$ Evaluate the extent to which the Federal hydroelectric system can be used to help with cost-effective wind integration that is consistent with reliable system operation.

- $\quad$ Evaluate potential mitigation approaches that may include innovative markets, institutional arrangements within or between Balancing Areas, or physical response on an economic basis.

- $\quad$ Produce meaningful and supported results in coordination with a Technical Review Committee (TRC), which should include stakeholders and experts in wind integration analysis. 
- $\quad$ Nebraska utilities see this NREL/WAPA grant program as a real opportunity to advance statewide wind development giving consideration not only to the integration impacts but also to making progress in finding relevant solutions in a multi-stakeholder process.

- $\quad$ Quantify, using valid evaluation techniques, the expected wind integration costs, both sub-hourly and hourly and longer for Nebraska wind generation development.

- Involve outside experts and interested parties in order to advance team building for future Nebraska wind generation development and increase the level of understanding among state leaders concerning wind integration issues for Nebraska.

- $\quad$ Build a data base and utility expertise for ongoing improved in-house modeling of wind generation impacts for future studies.

\section{Assumptions, Data, and Methodology}

\section{Wind Generation Scenarios}

Wind generation penetration levels equivalent to $10 \%, 20 \%$, and $40 \%$ of Nebraska retail electric energy sales in 2018 were defined as the targets for the study.

Using a variety of criteria, individual plants were selected from the NREL Meso-Scale database ${ }^{2}$. This database was created with funding from the Department of Energy through NREL and consists of production profiles for wind plants east of the Rocky Mountains. The resolution of the profiles is ten minutes for the entirety of historical years 2004, 2005, and 2006. The database contains profiles for $580,000 \mathrm{MW}$ of wind generation.

The "sites" selected corresponded to general areas where wind generation facilities already existed, or where good wind resources have been identified. An attempt was made to include a diversity of wind resource regions in the state. It must be made clear that the site selection process is not intended to indicate where the best resources or project potential exist, but rather to develop representative scenarios for study of their impact on the operation of the NPA system.

Characteristics of the aggregate wind generation in the defined scenarios are given in Table 1 as average values for 2004-2006. The existing wind generation facilities in Nebraska are considered to be included in and modeled as part of these data.

${ }^{2}$ http://www.nrel.gov/wind/integrationdatasets/eastern/data.html 
Table 1: Characteristics of Wind Penetration for Study

\begin{tabular}{|l|r|r|r|}
\hline NPA & $10 \%$ Pen & $20 \%$ Pen & 40\% Pen \\
\hline Energy (GWh) & 4,523 & 8,855 & 16,831 \\
Capacity (MW) & 1,249 & 2,488 & 4,727 \\
Capacity Factor & 0.41 & 0.41 & 0.41 \\
Average Site Size (MW) & 250 & 311 & 430 \\
\hline
\end{tabular}

Figure 1 through Figure 3 provide specific locations (close to center of each dark circle in the figure) of the production profiles (wind sites) selected from the NREL database for the $10 \%$, $20 \%$, and $40 \%$ penetrations.

Wind generation in areas adjacent to Nebraska can influence system operations within Nebraska. This is especially true with modern energy markets, where generating units are committed to operation and dispatched based on price signals. These signals are a function of load, transmission, and "stack" of available resources. With marginal cost nearly equal to zero, wind generation is a "price taker", which has an effect of lowering locational pricing. If transmission allows, prices are depressed across a wide region due to the delivery of wind energy to the grid. With transmission congestion, prices may only be depressed on a local or sub-regional basis.

Therefore, it is important that some consideration be made for likely wind development outside of Nebraska, so as not to skew the study results. It was assumed for the study that wind generation penetration in the rest of SPP would be equivalent percentage-wise with that in Nebraska. Table 2 provides details of the assumed wind generation in the rest of SPP for each scenario as average values for 2004-2006.

Table 2: $\quad$ Rest of SPP Wind Site Details

\begin{tabular}{|l|r|r|r|}
\hline Rest of SPP & $10 \%$ Pen & $20 \%$ Pen & $40 \%$ Pen \\
\hline Number of Sites & 7 & 18 & 33 \\
Energy (GWH) & 22,901 & 45,807 & 91,548 \\
Capacity (MW) & 6,256 & 12,596 & 25,431 \\
CF & 0.42 & 0.41 & 0.41 \\
Average Site Size (MW) & 894 & 700 & 771 \\
\hline
\end{tabular}

SPP (including NPA) has interconnections to other regional entities, including MISO/MAPP and WAPA. Still other areas farther east were also modeled. To account for wind generation in these areas, and not dramatically increase the scope of this study, the reference wind generation scenario developed for the Eastern Interconnection Wind Integration and Transmission Study (EWITS) was employed as the wind scenario for these regions. This reference case represents wind penetration that achieves current renewable portfolio standards. The installed capacity of wind modeled in these regions is around 50,000 MW as seen in Table 3 and held constant for each of the study scenarios, representing a wind penetration outside SPP of approximately $6 \%$. It is understood that holding external penetrations constant will encourage more exports out of SPP for the $20 \%$ and $40 \%$ SPP penetration levels than if the penetrations in the external areas were increasing along with those inside SPP. 
Table 3: $\quad$ Wind Generation Penetration

\begin{tabular}{|l|r|r|r|r|r|r|r|r|r|}
\hline & \multicolumn{3}{|c|}{$10 \%$ Penetration } & \multicolumn{2}{c|}{$20 \%$ Penetration } & \multicolumn{2}{c|}{$40 \%$ Penetration } \\
\cline { 2 - 9 } \multicolumn{1}{c|}{ Region } & \multicolumn{1}{c|}{$\begin{array}{c}\text { Pame } \\
\text { Plate }\end{array}$} & \multicolumn{1}{c|}{ CF } & $\begin{array}{c}\text { Reference } \\
\text { Energy }\end{array}$ & $\begin{array}{c}\text { Name } \\
\text { Plate }\end{array}$ & \multicolumn{1}{c|}{ CF } & $\begin{array}{c}\text { Reference } \\
\text { Energy }\end{array}$ & $\begin{array}{c}\text { Name } \\
\text { Plate }\end{array}$ & \multicolumn{1}{c|}{ CF } & $\begin{array}{c}\text { Reference } \\
\text { Energy }\end{array}$ \\
\hline NPA & 1,249 & $41 \%$ & 4,523 & 2,488 & $41 \%$ & 8,855 & 4,727 & $41 \%$ & 16,831 \\
SPP w/o NPA & 6,256 & $42 \%$ & 22,901 & 12,596 & $41 \%$ & 45,807 & 25,431 & $41 \%$ & 91,548 \\
PJM & 25,807 & $36 \%$ & 81,460 & 25,807 & $36 \%$ & 81,460 & 25,807 & $36 \%$ & 81,460 \\
MISO/MAPP & 19,547 & $36 \%$ & 61,700 & 19,547 & $36 \%$ & 61,700 & 19,547 & $36 \%$ & 61,700 \\
SERC & 3,615 & $36 \%$ & 11,410 & 3,615 & $36 \%$ & 11,410 & 3,615 & $36 \%$ & 11,410 \\
TVA & 1,397 & $36 \%$ & 4,410 & 1,397 & $36 \%$ & 4,410 & 1,397 & $36 \%$ & 4,410 \\
\hline Total & 57,871 & $37 \%$ & 186,405 & 65,450 & $37 \%$ & 213,642 & 80,524 & $38 \%$ & 267,359 \\
\hline
\end{tabular}

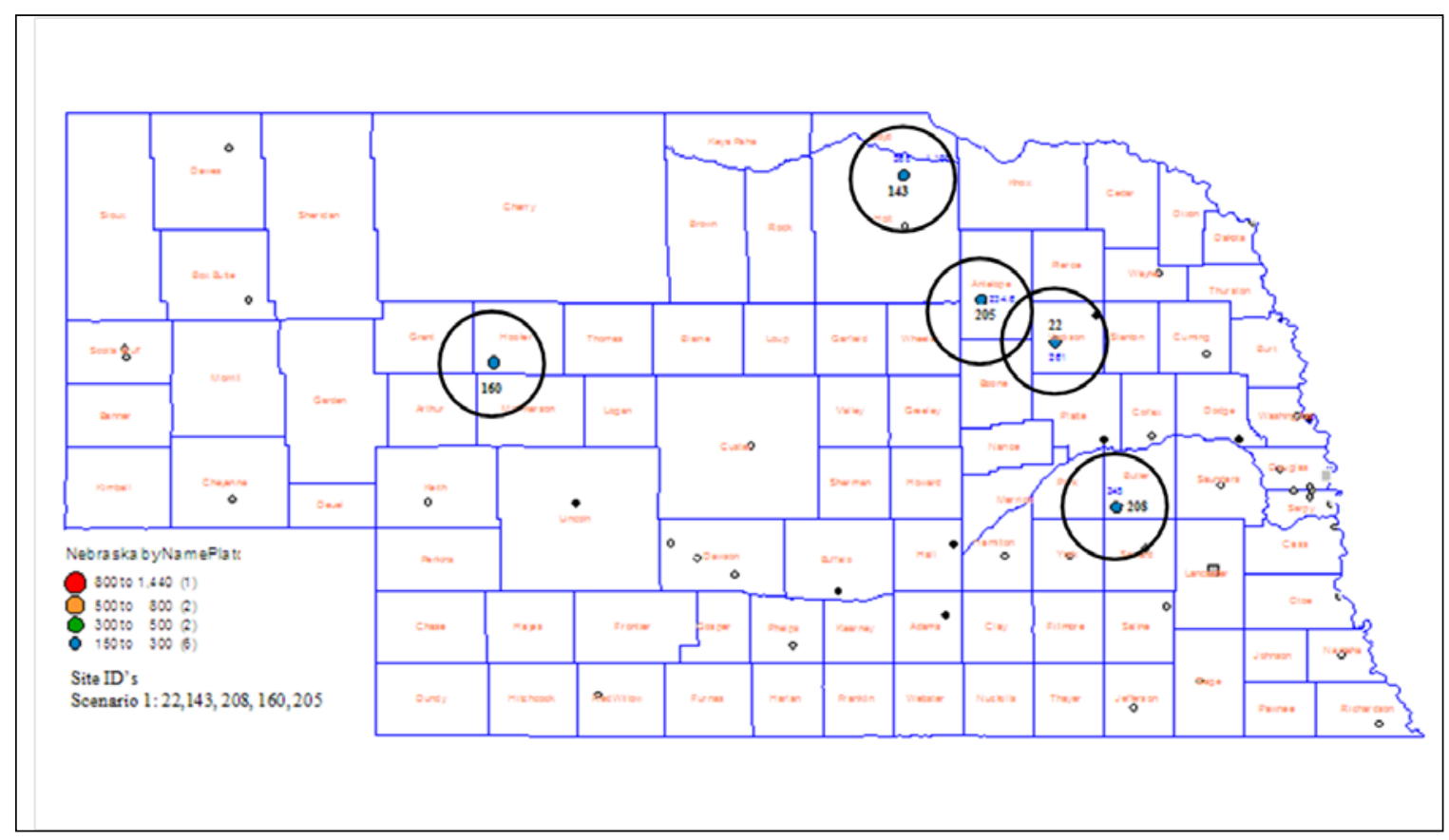

Figure 1: Nebraska 10\% Penetration Wind Site Map 


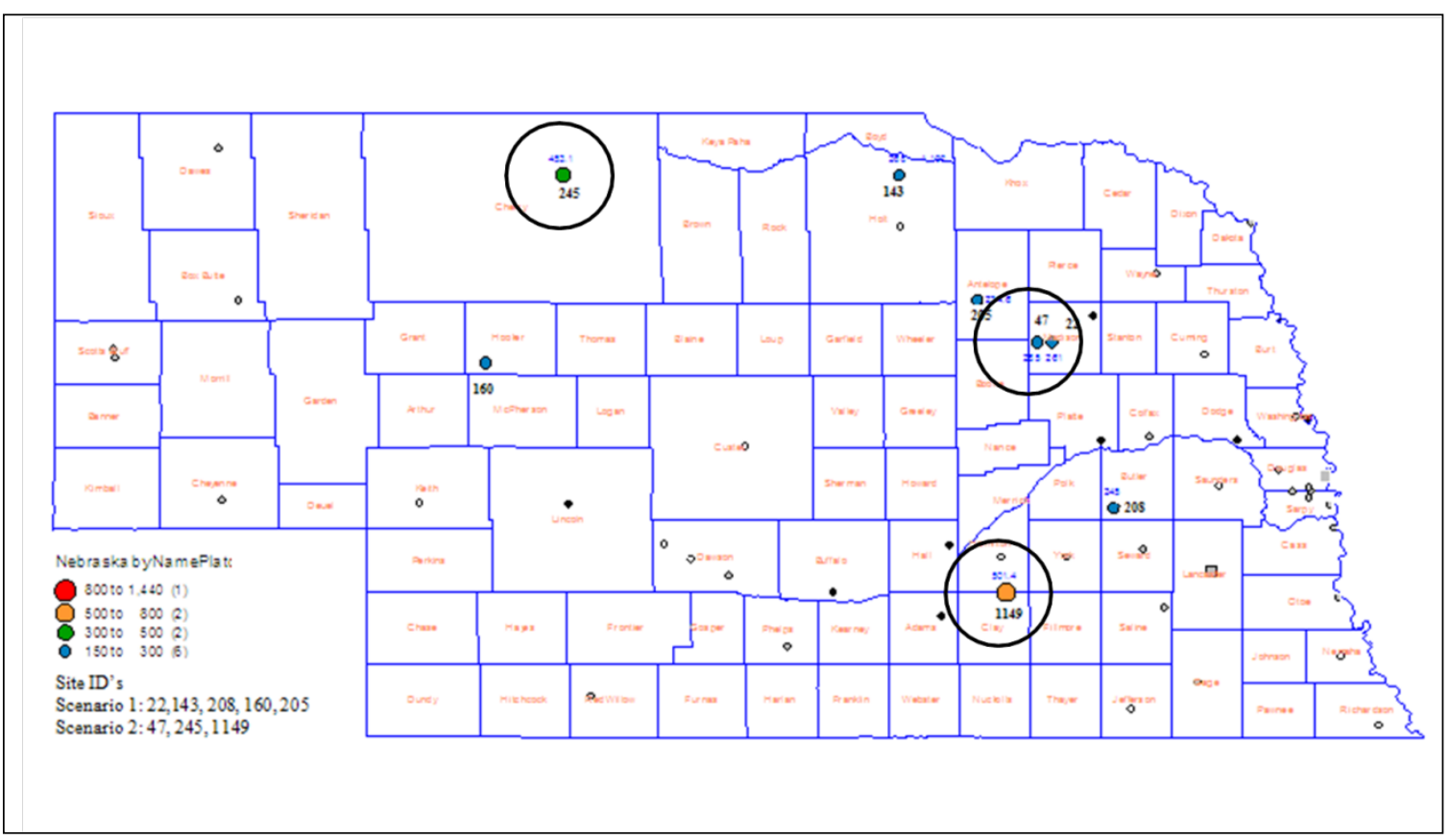

Figure 2: $\quad$ Nebraska Wind Sites Added to $10 \%$ Penetration Sites to Achieve $20 \%$ Penetration

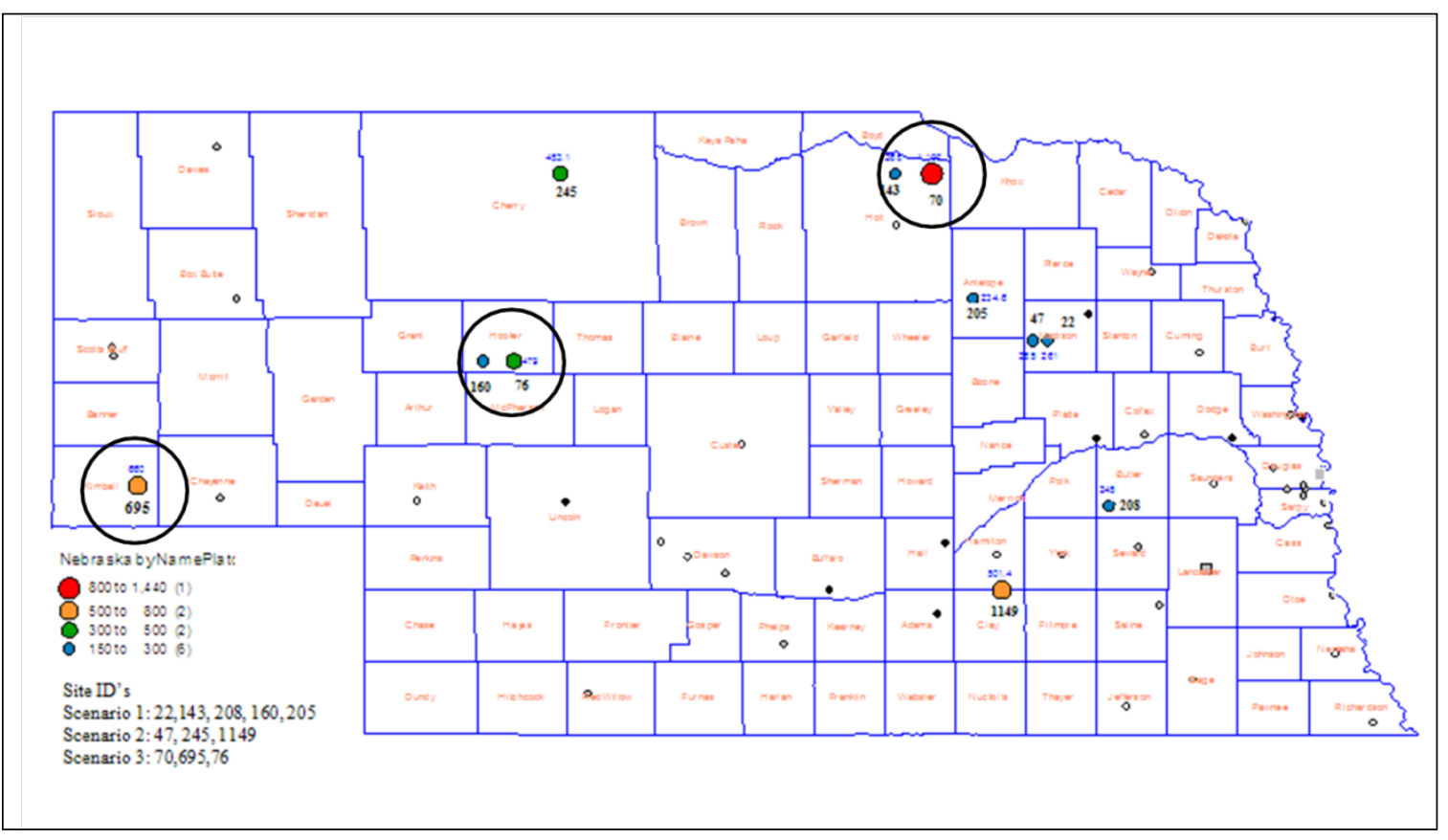

Figure 3: Nebraska Wind Sites Added to 20\% Penetration Sites to Achieve $40 \%$ Penetration 


\section{NPA and SPP Power System in 2018}

An Eastern Interconnection power flow case for 2018 was the starting point for developing the detailed model to be used in this study. The power flow case had previously been developed and then used for the JCSP study and was also the starting point for the DOE/NREL Eastern Interconnection Wind Integration and Transmission Study (EWITS).

The basic model data includes generating units, the transmission network, hourly bus loads, and wind generation profiles as described above. Completing the model from this relatively straightforward foundation requires that many additional assumptions be made. These are detailed in the report.

NPA loads in the model were adjusted to match monthly peak demand and energy projected for 2018. Care was taken to retain the shape and pattern characteristics from historical years 2004 to 2006 to preserve correlation to the wind profile data for those same historical years.

Generating unit data in the base model was also audited and updated based on inputs from NPA members.

Finally, because NPA utilities became participants in the SPP market at the onset of this study, the modeling was structured to reflect this operational reality (the SPP day ahead and ancillary services markets modeled are not in place today but are expected to operate in 2018). In the simulations described later, NPA generating units were committed and dispatched along with all other units in the SPP operating area. Hourly transactions of energy, both within the SPP market footprint and with external areas such as MISO, SERC, and TVA, were made on an economic basis. A "hurdle rate" between regions of $\$ 5 / \mathrm{MWh}$ was included in the interchange decision to reflect additional costs that needed to be overcome for interchange to take place between buyer and seller. Unit shares, whether ownership or participation transactions, were modeled for Nebraska purchases from out-of-state units and for Nebraska sales to out-of-state utilities.

\section{Transmission Assumptions}

The base model for the study contains a detailed representation of the transmission system, representing explicitly all buses at or above $115 \mathrm{kV}$. Planned transmission additions through at least 2013 are part of the power flow case from which the study model was derived. However, for the higher wind penetrations considered in the study, it was thought that transmission capacity could be an important issue and potentially lead to significant curtailment of wind generation without an adequate transmission build out.

The $10 \%$ wind penetration case (Scenario 1 ) was run with existing and currently planned/committed transmission facilities, as was an initial case at $20 \%$ penetration in NPA and the rest of SPP (Scenario 2). In addition, some localized transmission constraints were removed from the model without actually identifying specific transmission facilities necessary in operation to do so. A third case, (Scenario 3) again at $20 \%$ wind generation penetration, incorporated a substantial regional transmission expansion that has been under study at SPP for some time. With the addition of NPA utilities to SPP, the $765 \mathrm{kV}$ EHV overlay that constitutes the core of this plan was modified to loop through Nebraska as seen in Figure 4 below.

The EHV overlay was also initially used for the $40 \%$ wind penetration case (Scenario 4 ). 


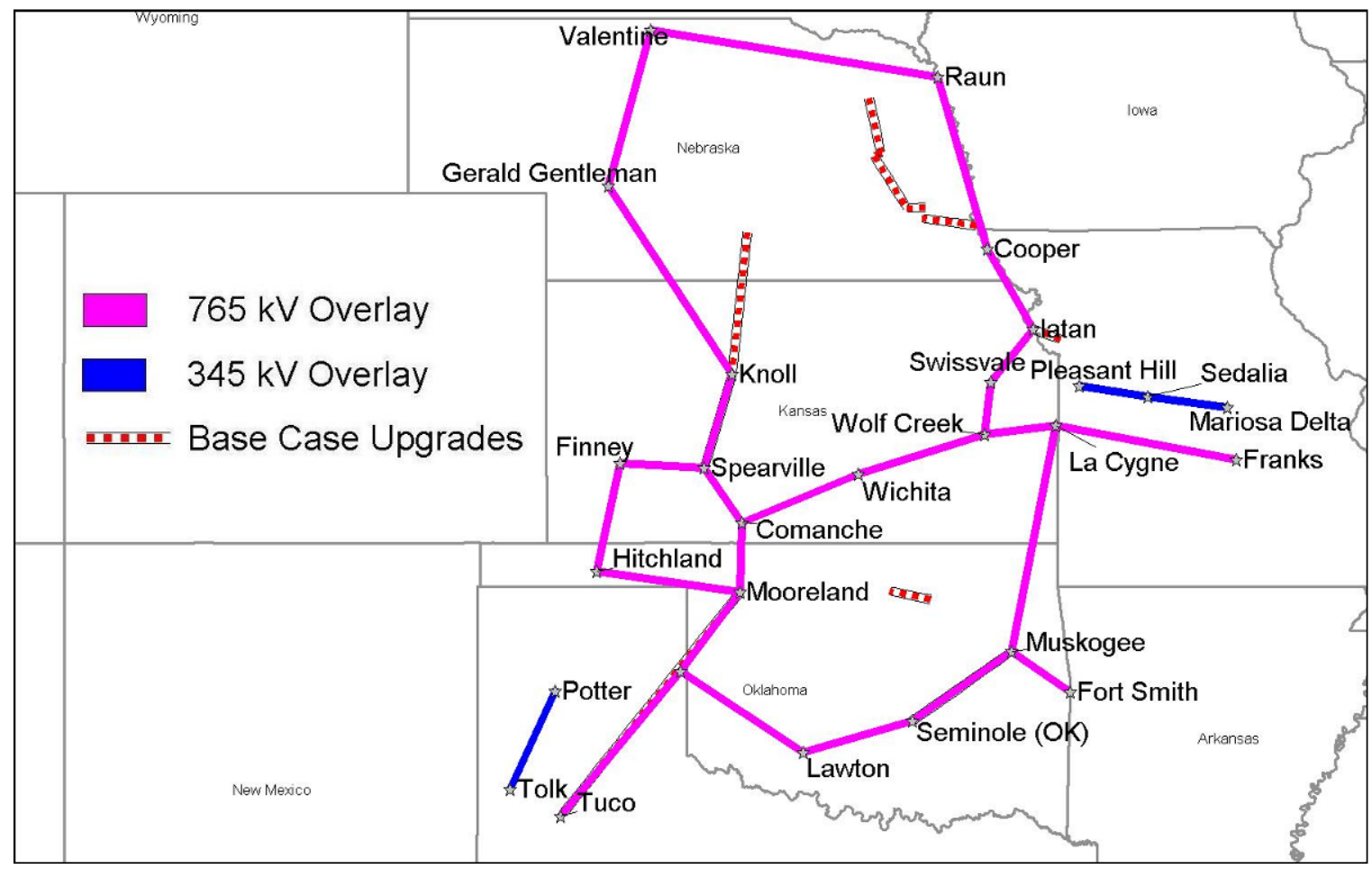

Figure 4: $\quad$ Base Case Conceptual EHV Overlay

\section{Methodology}

Wind generation impacts on power systems operations are assessed through chronological production simulations. This is the preferred method for analyzing wind integration impacts, and has been used in nearly all of the major integration studies to date.

In the simulations, units are committed and dispatched to serve load at each bus while honoring transmission constraints and recognizing the security needs of the system, and at the same time adjusting generation to accommodate the delivery of wind energy to the system. Three full years with unique 8760 hour load and wind profile data representing the study year of 2018 are simulated. A nodal model is employed, where all transmission in the study area is represented explicitly, along with all generating units and loads at bulk delivery points.

With annual production simulations as the basic tool, a large number of cases are run to determine how increasing amounts of wind generation affect the utilization of other generating resources and the transmission system, consumption of fuel, and market prices for electric energy. In addition to analysis of the four base scenarios, a significant number of additional production simulation cases were run to explore how key variables or assumptions would affect the results.

A key goal of a wind integration study is to determine integration costs associated with wind generation. The additional variability and uncertainty attributable to wind generation increase the requirement for regulating reserves in real-time operations (above that already required for regulating load), and also introduce some additional inefficiency in the day ahead energy market due to increased errors in the aggregate forecast of load and wind generation. These general 
effects of wind generation are captured in the production simulations by a) optimizing the deployment of generating units (i.e. unit commitment) to forecasts of hourly wind and load for the next day rather than the "actual" quantities per the profiles mentioned above; and b) increasing the hourly reserve constraint (or requirement) consistent with the amount of wind generation during the hour. Additionally the shape or variability of the wind can be considered as an integration cost.

Integration costs are driven by changes in the use of the thermal generation fleet in response to the wind. Typically, the "actual" wind case simulation (including the effects of forecast error, incremental reserves and wind variability) will result in higher production costs than the "ideal" wind case (no forecast error, incremental wind reserves or wind variability). This is explained by the increased use of higher cost resources (combined cycles versus coal) in response to the wind. Given the make-up of Nebraska's thermal generation fleet, increased reserve requirements typically cause coal units to back down to carry reserves. This results in higher cost resources, particularly combined cycle units, being brought on to serve the energy previously generated by the coal units. These higher cost resources also respond to the faster ramps and forecast errors associated with wind.

The intent of these simulations is to mimic as closely as possible the assumed operational structure for SPP in the study year. It is assumed that the SPP energy markets in 2018 consist of day-ahead and real-time (or sub-hourly) energy markets as well as an ancillary services market. These markets are not all operational in SPP today.

Simulation outputs include the hourly operational profile of each generating unit in the model and hourly flows on all transmission lines in the model. Locational marginal prices (LMPs) and production costs are also key metrics.

\section{Dollar Cost Basis}

Throughout the report dollars are given in 2018 nominal values unless otherwise noted.

Current cost numbers were escalated to 2018 using various rates depending on the particular

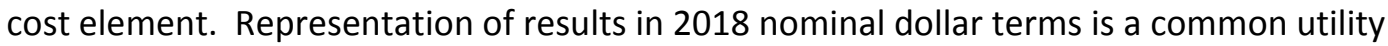
procedure and provides for the resulting (estimated) future relationship between such cost elements. However, for the purposes of potential comparison to other studies, normalized wind integration cost results are presented in 2009 dollar terms by deescalating the 2018 results to 2009 at $2.5 \%$ per year. Additionally the cost value given in the Western Nebraska Wind Western Interconnection is presented on a 2008 dollar basis.

\section{Costs and Implications}

A simplified methodology was developed to facilitate calculation of the total costs associated with each of the scenarios studied, as well as certain sensitivity cases. Because of the necessary simplifications, the total costs are useful as a relative metric by which the cases can be compared. They do not represent a full accounting of all the details associated with each of the scenarios, and are very dependent on the assumptions established for this study. 
The cost estimation procedure considers all of SPP including Nebraska, because of the sharedreserve modeling, and can be thought of in a step-wise manner as follows:

- A reference 2018 total cost is estimated with no $\mathrm{CO} 2$ regulation (cost) or federal Renewable Energy Standard or significant REC pricing.

- $\quad$ An incremental wind purchase cost is estimated for the increment of wind generation that is added above the existing amount now installed in the SPP.

- $\quad$ An incremental transmission cost is estimated for the increment of transmission line and substation facilities that will be needed as wind generation is added.

- A cost of $\mathrm{CO} 2$ emissions is included at various levels depending on the scenario description. The cap-and trade estimates are patterned after the proposed Waxman-Markey bill. Alternative total cost results are presented for both capand-trade and tax regulation scenarios.

- Essentially, the total cost estimate for a case then becomes:

the reference cost

plus incremental wind cost

plus incremental transmission cost

minus the delta on PROMOD adjusted production cost (to convert from tax to cap-and trade, if done for that case).

The list of assumptions used in these calculations is detailed in the report. Implications of this costing analysis are listed in the "Results, Findings, and Conclusions" section of this Executive Summary.

\section{Western Nebraska Wind - Western Interconnection}

The very western-most part of the state of Nebraska is part of the Western Interconnection in the U.S.; the remainder of the state operates as part of the Eastern Interconnection. This artifact of history and the distribution of population across the lower 48 states have the effect of dramatically limiting the transfer of electric energy from eastern Nebraska to western Nebraska. There are several connections between the grids via back-to-back AC-DC-AC ties, of which two are located in Nebraska. Transfer capability over these ties, however, is quite limited.

Western Nebraska is a very low load area, meaning that large amounts of wind generation will require transmission capability to "export" power out of the area to load centers. Going either to the east or to the west, there is limited transmission capability due to commitments already in place for other resources. Consequently, it was not practical to incorporate those areas (and potential wind generation) operating in the Western Interconnection directly into the analysis.

Given that the likely load center for wind in far western Nebraska would actually be in the Western Interconnection, a separate transmission evaluation was conducted to assess challenges for development of wind generation in this portion of the state. 
In collaboration with the WAPA Rocky Mountain Region, the following topics pertaining to wind in the western portion of Nebraska in the Western Interconnection were addressed:

- Identification of how much wind generation could be injected into the western Nebraska transmission system without requiring transmission system upgrades.

- Determination of the transmission system upgrades required to add $300 \mathrm{MW}$ of wind generation in western Nebraska in the Western Interconnection.

The focus for this part of the study was also nearer-term than the larger analytical effort, and looked at the 2012 time frame before considering 2018.

The analysis determined that the existing transmission network could connect and provide delivery for about $50 \mathrm{MW}$ of wind generation. Approximately $\$ 16$ million (2008\$) in transmission improvements would be necessary to interconnect $300 \mathrm{MW}$.

In the 2018 time frame, additional transmission improvements would also be required to connect $300 \mathrm{MW}$ of wind generation, although the details of the necessary upgrades differ from what was determined for the earlier year.

Wind integration costs, as computed for the eastern regions of the state and for the SPP market footprint, were not addressed in this component of the complete study.

\section{Results, Findings, and Conclusions}

Some details of the production simulations for the base scenarios are found in Table 4. The large volume of numerical results precludes a substantive discussion in this Executive Summary of the production simulation cases run and analyzed as part of this study effort. In this section, a review of the key study findings and conclusions, along with supporting tables, charts, and graphs from the body of the report is provided.

For brevity in referencing, four scenarios are numbered:

- $\quad$ Scenario 1: 10\% wind penetration in Nebraska (Figure 1) and the rest of SPP without the addition of an EHV transmission overlay.

- $\quad$ Scenario 2: 20\% wind penetration in Nebraska (Figure 1 and Figure 2) and the rest of SPP without the addition of an EHV transmission overlay.

- $\quad$ Scenario 3: 20\% wind penetration in Nebraska (Figure 1 and Figure 2) and the rest of SPP with the addition of a $765 \mathrm{kV}$ transmission overlay.

- $\quad$ Scenario 4: 40\% wind penetration in Nebraska (Figure 1, Figure 2, and Figure 3) and the rest of SPP with the addition of a $765 \mathrm{kV}$ transmission overlay.

Within each of the scenarios, the wind and load patterns for all three historical years (2004, 2005 , and 2006) were also modeled as separate cases. Further, localized transmission additions necessary to handle the wind generation injections were not specifically identified but were implicitly assumed to be present as described in the Transmission Assumptions section. 
Table 4: $\quad$ Detailed Results for Base Scenarios - all dollars are $2018 \$$

\begin{tabular}{|c|c|c|c|c|c|c|c|c|c|c|c|c|}
\hline & \multicolumn{3}{|c|}{ Scenario 1} & \multicolumn{3}{|c|}{ Scenario 2} & \multicolumn{3}{|c|}{ Scenario 3} & \multicolumn{3}{|c|}{ Scenario 4} \\
\hline & 2004 & 2005 & 2006 & 2004 & 2005 & 2006 & 2004 & 2005 & 2006 & 2004 & 2005 & 2006 \\
\hline Load (TWh) - SPP & 265 & 265 & 265 & 265 & 265 & 265 & 265 & 265 & 265 & 265 & 265 & 265 \\
\hline Actual Wind (TWh) - SPP & 25.75 & 27.76 & 27.52 & 49.69 & 51.63 & 52.95 & 52.39 & 54.79 & 56.32 & 100.31 & 104.20 & 107.23 \\
\hline Ideal Wind (TWh) - SPP & 25.68 & 27.15 & 27.45 & 49.61 & 51.51 & 52.86 & 52.38 & 54.78 & 56.31 & 100.27 & 104.14 & 107.18 \\
\hline All Other Gen/Resources (TWh) - SPP & 250.72 & 249.41 & 250.05 & 237.34 & 236.55 & 236.09 & 237.17 & 236.29 & 235.83 & 214.41 & 212.45 & 211.00 \\
\hline Actual APC (\$M) - SPP & 14,815 & 14,685 & 14,655 & 13,162 & 13,013 & 12,924 & 12,772 & 12,579 & 12,462 & 9,759 & 9,488 & 9,334 \\
\hline Ideal APC (\$M) - SPP & 14,770 & 14,639 & 14,607 & 13,079 & 12,922 & 12,828 & 12,683 & 12,480 & 12,355 & 9,542 & 9,283 & 9,101 \\
\hline Delta APC (Actual minus Ideal) - SPP & 45 & 45 & 48 & 82 & 90 & 96 & 89 & 100 & 107 & 217 & 204 & 233 \\
\hline Integration Costs (\$/MWh) - SPP & 1.75 & 1.66 & 1.74 & 1.65 & 1.75 & 1.81 & 1.70 & 1.82 & 1.90 & 2.16 & 1.96 & 2.18 \\
\hline Wind Curtailment (\%) - Nebraska & $0 \%$ & $0 \%$ & $0 \%$ & $0 \%$ & $0 \%$ & $0 \%$ & $0 \%$ & $0 \%$ & $0 \%$ & $0 \%$ & $0 \%$ & $0 \%$ \\
\hline Wind Curtailment (\%) - Rest of SPP & $2 \%$ & $2 \%$ & $3 \%$ & $6 \%$ & $7 \%$ & $7 \%$ & $0 \%$ & $0 \%$ & $0 \%$ & $5 \%$ & $5 \%$ & $5 \%$ \\
\hline Exports (TWh) & 11.48 & 12.24 & 12.59 & 21.24 & 22.40 & 23.09 & 24.53 & 26.09 & 27.13 & 49.69 & 51.37 & 52.85 \\
\hline CO2 Emissions (Million Short Tons) & 203.07 & 202.83 & 202.75 & 193.22 & 192.93 & 192.46 & 194.53 & 194.22 & 193.59 & 175.64 & 174.14 & 172.58 \\
\hline SPP Wind Reserve (Max MW) & 884 & 853 & 852 & 1,538 & 1,540 & 1,542 & 1,538 & 1,540 & 1,542 & 3,003 & 3,041 & 3,034 \\
\hline SPP Wind Reserve (Avg MW) & 513 & 505 & 499 & 1,021 & 1,007 & 996 & 1,021 & 1,007 & 996 & 2,094 & 2,058 & 2,042 \\
\hline
\end{tabular}

Notes on Base Scenario Results:

- $\quad$ Reserves noted as "wind reserves" are the incremental regulating reserves associated with the additions of wind generation.

- $\quad \mathrm{APC}=$ Adjusted Production Cost

- $\quad$ SPP includes NPA unless otherwise noted

- Scenario1: 10\% Wind Penetration

- $\quad$ Scenario2: 20\% Wind Penetration

- $\quad$ Scenario3: 20\% Wind Penetration w EHV Overlay

- $\quad$ Scenario4: 40\% Wind Penetration w EHV Overlay

- Integration costs are based on shaped proxy resource. 


\section{Wind Generation Curtailment}

Assuming certain localized transmission line additions are made and Knoll-Axtell $345 \mathrm{kV}$ line is built, there was no significant wind generation curtailment in Nebraska in any case with or without the overlay. That is, all scheduled wind generation was accommodated by redispatching other generation and exporting excess wind energy, all within modeled transmission limits. As described in the Transmission Assumptions section, lines nearby to the wind injection points were allowed to overload for study simplicity rather than determine all the specific additions needed. Further the $40 \%$ case was only run with the overlay in place.

There was also no significant violation of Nebraska thermal unit minimums (i.e. dump energy) in any of the cases.

- $\quad$ With the wind dispatch price of $-\$ 40 / M W h$ (assuming $\$ 15 / M W h$ Renewable Energy Credit, REC and $\$ 25 \mathrm{MWh}$ Production Tax Credit, PTC) wind has priority over other resources. I.e., other resources re-dispatch or even curtail before wind does.

- $\quad$ Access to large export markets was a key factor in accommodating the wind generation additions.

Although 20 major flow gates were monitored in Nebraska, it's possible that some local congestion was not captured in the analysis which could lead to curtailment.

In the rest of SPP for the base cases, wind curtailment was found to be:

- $2 \%$ at the $10 \%$ penetration without the overlay.

- $\quad 7 \%$ at the $20 \%$ penetration without the overlay.

- $\quad 0 \%$ at the $20 \%$ penetration with the overlay.

- $5 \%$ at the $40 \%$ penetration with the overlay.

These results are summarized in Figure 5. 


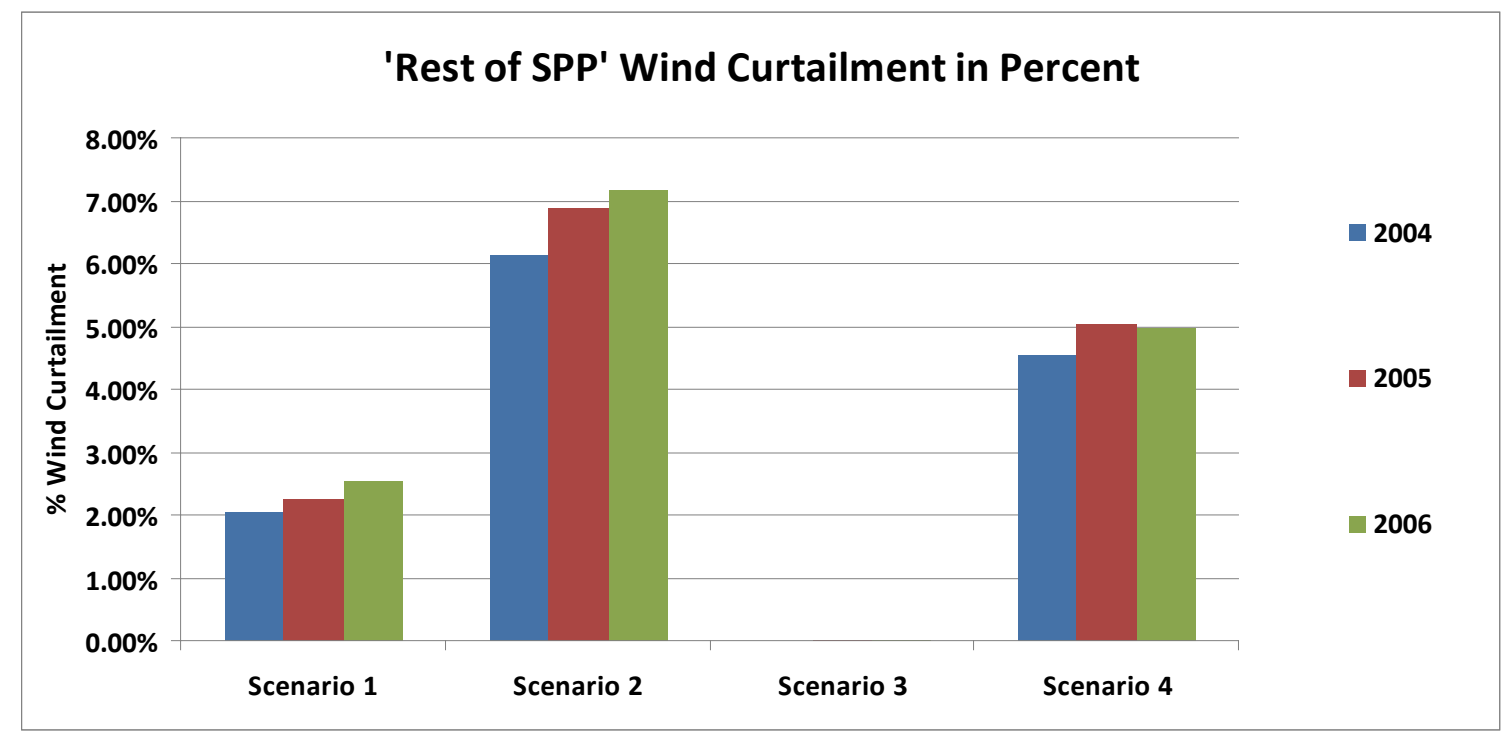

Figure 5: $\quad$ SPP Wind Generation Curtailment by Scenario (note: no curtailment in NPA)

\section{Wind Energy Usage}

Consistently as wind penetration increased, the study results had SPP (including Nebraska) increasing exports by approximately half of the amount of the wind generation increases. Specifically for Nebraska only:

- $\quad$ From $10 \%$ wind penetration to $20 \%$ wind penetration Nebraska wind energy increases by 4.35 TWh and exports increase by $3.20 \mathrm{TWh}(74 \%)$ by using averages of 3 wind years for base cases for scenarios 1 and 2, see Figure 6 .

- $\quad$ From $20 \%$ wind penetration to $40 \%$ wind penetration (with the EHV overlay) Nebraska wind energy increases by 8.12 TWh and exports increase by $4.74 \mathrm{TWh}$ $(58 \%)$ by using averages of 3 wind years for base cases for scenarios 3 and 4 , see Figure 6.

Although it is impossible to say exactly from which sources energy is exported, the increased wind penetrations in Nebraska / SPP result in increased energy exports. Note that in areas external to SPP the wind penetrations were held constant at a weighted $6 \%$ level across all of the SPP variations in penetration. As such the external system had greater flexibility at the higher penetration scenarios to accept imports of wind than if it had the same higher penetrations as in SPP/NPA. 


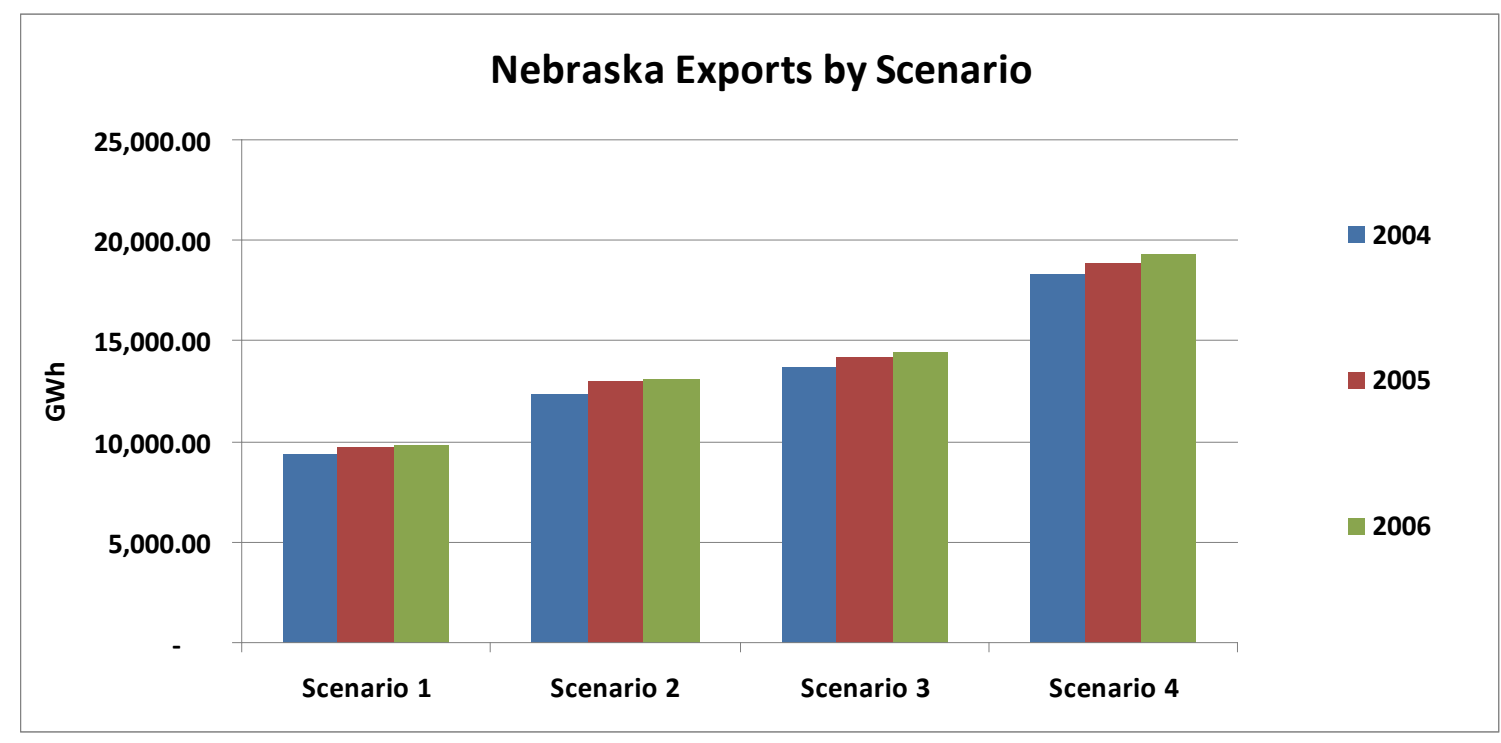

Figure 6: Nebraska Exports by Scenario

\section{Incremental Reserves}

Wind generation has a substantial impact on the requirements for regulating reserves (an amount of spinning reserve responsive to Automatic Generation Control (AGC) that is sufficient to provide normal regulating margin) relative to what the load by itself requires. For the SPP market, $10 \%$ wind energy in NPA and SPP will increase the average hourly requirement by around $500 \mathrm{MW}$. At 20\%, the incremental amount is about $1000 \mathrm{MW}$, and rises to about an additional $2000 \mathrm{MW}$ at $40 \%$ penetration, all being relatively linear.

There is cost associated with carrying these additional spinning reserves, which have been captured in the production simulations. It should also be noted that the incremental hourly amounts are small when wind generation is low and, obviously the largest at high levels of wind production. Under these conditions, however, conventional generation will be "unloaded" and freed up to contribute to the reserve needs.

The calculation of the increased regulating reserve requirement was done outside of and prior to the production simulations. The additional amounts of reserves are estimated by employing some statistical and mathematical analysis of higher resolution (10 minute intervals or less) wind and load data.

Results of these computations for the base scenarios are shown in Table 5. Because the requirements vary hourly with both wind and load, the average and maximum values for each year of the wind and load profile data are shown. At $10 \%$ wind penetration in SPP, including NPA, the average value of the hourly regulating reserve more than doubled. At $40 \%$ wind penetration, regulating reserves requirements increase by over 7 times. 
Table 5: $\quad$ Regulating Reserve Requirements for Study Scenarios

\begin{tabular}{|c|c|c|c|c|c|c|c|}
\hline \multirow{2}{*}{$\begin{array}{c}\text { SPP with Nebraska } \\
10 \% \text { Penetraton }\end{array}$} & \multicolumn{2}{|c|}{ Load Only } & \multicolumn{2}{|c|}{ Load and Wind } & \multicolumn{2}{|c|}{$\begin{array}{l}\text { Delta Load and } \\
\text { Wind - Load }\end{array}$} & \multirow{2}{*}{$\begin{array}{c}\text { Wind } \\
\text { Nameplate } \\
\text { MW }\end{array}$} \\
\hline & Max & Average & Max & Average & $\operatorname{Max}$ & Average & \\
\hline 2004 profile & 533 & 301 & 1142 & 814 & 884 & 513 & 7,505 \\
\hline 2005 profile & 526 & 301 & 1146 & 806 & 853 & 505 & 7,505 \\
\hline 2006 profile & 528 & 295 & 1152 & 794 & 852 & 499 & 7,505 \\
\hline \multicolumn{8}{|l|}{ 20\% Penetration } \\
\hline 2004 profile & 533 & 301 & 1796 & 1322 & 1538 & 1021 & 15,084 \\
\hline 2005 profile & 526 & 301 & 1802 & 1308 & 1540 & 1007 & 15,084 \\
\hline 2006 profile & 528 & 295 & 1800 & 1291 & 1542 & 996 & 15,084 \\
\hline \multicolumn{8}{|l|}{ 40\% Penetration } \\
\hline 2004 profile & 533 & 301 & 3261 & 2395 & 3003 & 2094 & 30,158 \\
\hline 2005 profile & 526 & 301 & 3261 & 2359 & 3041 & 2058 & 30,158 \\
\hline 2006 profile & 528 & 295 & 3257 & 2337 & 3034 & 2042 & 30,158 \\
\hline
\end{tabular}

*Delta Max colum represents the coincidental maximum delta of Load Only and Load and Wind

The increase in regulating reserves to manage the additional variability and short-term uncertainty attributable to wind generation is one of the major operating impacts. There is cost associated with the increased requirement, and potentially control performance degradation if adequate reserve capacity cannot be found among units in the operating area. Both of these issues are evaluated directly in the production simulations.

Table 6 illustrates one of the benefits of larger operating areas for managing wind generation. If NPA were operated as a single balancing area, not part of the SPP market, it would be responsible for providing regulating reserves for the wind generation in Nebraska. For the $10 \%$ case, the average hourly regulating reserve requirement for load and wind in the NPA balancing area on its own would be around $150 \mathrm{MW}$. This is in contrast with a $750 \mathrm{MW}$ requirement for the rest of the SPP market footprint. The value of aggregation can be even more clearly seen by comparing the SPP requirements from the two tables: Adding Nebraska - and its wind generation for the $10 \%$ case - to the SPP operating area (and ancillary services market) increases the regulating requirement by about $50 \mathrm{MW}(794 \mathrm{MW})$, which is less than the sum of the requirement for SPP and Nebraska operating separately (742 MW + $148 \mathrm{MW}=890 \mathrm{MW}$ ). With more wind generation, the aggregation effect is even more pronounced. 
Table 6: Regulating Reserve Requirements for NPA as a Single Balancing Authority and SPP with and without NPA

\begin{tabular}{|l|r|r|r|r|r|r|r|}
\hline \multirow{2}{*}{$\begin{array}{c}\text { 10\% Penetration } \\
\text { 2006 profile }\end{array}$} & \multicolumn{2}{|c|}{ Load Only } & \multicolumn{2}{|c|}{ Load and Wind } & \multicolumn{2}{c|}{$\begin{array}{c}\text { Delta Load and } \\
\text { Wind - Load }\end{array}$} & $\begin{array}{c}\text { Wind } \\
\text { Nameplate }\end{array}$ \\
\cline { 2 - 8 } & \multicolumn{1}{|c|}{ Max } & Average & Max & Average & Max & Average & MW \\
\hline SPP with Nebraska & 528 & 295 & 1152 & 794 & 852 & 499 & 7,505 \\
SPP w/o Nebraska: A & 460 & 256 & 1111 & 742 & 821 & 486 & 6,256 \\
Nebraska alone: B & 75 & 45 & 216 & 148 & 177 & 118 & 1,249 \\
\hline Total A + B & 535 & 301 & 1327 & 890 & 998 & 604 & 7,505 \\
\hline
\end{tabular}

"Delta Max Column represents the coincidental maximum delta of Load Only and Load and Wind

\section{Wind Capacity Value for Accreditation in SPP}

The SPP criteria for calculating monthly capacity value were used for these findings, because that is the criteria now applicable to Nebraska utilities. It is noted that it is not based on an Effective Load Carrying Capacity methodology as many criteria are. Three years of Nebraska hourly wind data were correlated with corresponding 3 years of Nebraska hourly load.

The monthly capacity value for individual wind sites studied in Nebraska ranged from a $0.06 \%$ minimum to a $16.5 \%$ maximum, Table 7 . It is noteworthy that the two most common months in which Nebraska has its annual peak (July and August) are unfortunately the same two months in which the capacity values are the lowest.

When all the Nebraska wind sites were aggregated, the lowest monthly capacity values were observed in the summer months June, July and August, while the highest values were observed in April, May, September and October, as shown for $40 \%$ penetration in Table 8 and in Figure 7. The aggregated minimum increased to $6.55 \%$ while the maximum remained at $16.5 \%$, Table 8 , $40 \%$.

For SPP (without Nebraska) the monthly capacity values for individual wind sites ranged in value from a $0.03 \%$ minimum to a maximum of $16.55 \%$. The lowest values were observed in December and January.

When the SPP (without Nebraska) wind sites were aggregated the monthly capacity value minimum and maximum increased to $4.99 \%$ and $22.4 \%$ respectively, as shown in Figure 8.

Aggregating all wind sites of Nebraska and SPP improved the monthly capacity values by increasing the minimum to $10.79 \%$ and maximum to $30.35 \%$, as shown in Figure 9 . This demonstrates the benefit of diversity across broader regions with increased wind capacity, assuming sufficient transmission exists. Although the data for November in these two figures stands out as atypical, nothing could be found in the NREL data that was obviously inappropriate.

It is noted however, that the SPP accreditation criteria is currently applied to individual wind units only, rather than to an aggregation of plants.

Methods of calculating capacity credit for wind vary across the country and produce different results. A standardized method of calculating the capacity credit of wind is under investigation by FERC. 
Table 7: Estimated Capacity Accreditation per SPP Methodology for Wind Generation Sites in Nebraska

\begin{tabular}{|l|r|r|r|r|r|r|r|r|r|r|r|}
\hline \multirow{2}{*}{ Nameplate } & \multicolumn{4}{|c|}{ NPA - 10\% Wind Sites } & \multicolumn{3}{|c|}{ NPA 20\% Wind Sites } & \multicolumn{2}{|c|}{ NPA 40\% Wind Sites } \\
\cline { 2 - 10 } & 261 & 268 & 240 & 235 & 245 & 285 & 453 & 501 & 1100 & 479 & 660 \\
\hline Site ID & 22 & 143 & 160 & 205 & 208 & 47 & 245 & 1149 & 70 & 76 & 695 \\
\hline Jan & $2.79 \%$ & $1.19 \%$ & $1.82 \%$ & $2.24 \%$ & $3.59 \%$ & $2.66 \%$ & $2.81 \%$ & $2.25 \%$ & $1.63 \%$ & $2.30 \%$ & $4.49 \%$ \\
Feb & $6.60 \%$ & $2.79 \%$ & $4.23 \%$ & $4.06 \%$ & $10.85 \%$ & $6.26 \%$ & $2.52 \%$ & $10.16 \%$ & $4.36 \%$ & $4.77 \%$ & $6.45 \%$ \\
Mar & $5.27 \%$ & $2.22 \%$ & $2.98 \%$ & $4.24 \%$ & $3.90 \%$ & $4.64 \%$ & $4.01 \%$ & $2.95 \%$ & $3.79 \%$ & $3.57 \%$ & $4.95 \%$ \\
Apr & $6.46 \%$ & $8.25 \%$ & $8.42 \%$ & $4.69 \%$ & $3.04 \%$ & $5.46 \%$ & $6.38 \%$ & $0.86 \%$ & $8.12 \%$ & $8.73 \%$ & $3.08 \%$ \\
May & $13.52 \%$ & $8.35 \%$ & $5.35 \%$ & $9.26 \%$ & $16.53 \%$ & $12.09 \%$ & $4.55 \%$ & $14.57 \%$ & $11.77 \%$ & $4.11 \%$ & $4.39 \%$ \\
Jun & $4.45 \%$ & $1.86 \%$ & $1.98 \%$ & $0.63 \%$ & $6.22 \%$ & $5.41 \%$ & $2.80 \%$ & $8.19 \%$ & $1.58 \%$ & $2.11 \%$ & $3.22 \%$ \\
Jul & $1.54 \%$ & $1.17 \%$ & $1.08 \%$ & $0.06 \%$ & $1.96 \%$ & $0.54 \%$ & $0.39 \%$ & $1.26 \%$ & $1.37 \%$ & $1.51 \%$ & $2.41 \%$ \\
Aug & $3.07 \%$ & $0.81 \%$ & $2.22 \%$ & $0.34 \%$ & $1.94 \%$ & $3.29 \%$ & $1.39 \%$ & $4.11 \%$ & $1.19 \%$ & $1.86 \%$ & $3.35 \%$ \\
Sep & $10.40 \%$ & $6.79 \%$ & $4.05 \%$ & $5.35 \%$ & $12.66 \%$ & $10.19 \%$ & $3.14 \%$ & $12.53 \%$ & $9.25 \%$ & $4.02 \%$ & $3.13 \%$ \\
Oct & $7.92 \%$ & $10.23 \%$ & $1.47 \%$ & $5.21 \%$ & $8.36 \%$ & $7.35 \%$ & $2.32 \%$ & $5.47 \%$ & $10.23 \%$ & $1.63 \%$ & $5.37 \%$ \\
Nov & $2.10 \%$ & $0.86 \%$ & $2.43 \%$ & $1.20 \%$ & $0.98 \%$ & $1.64 \%$ & $5.57 \%$ & $0.53 \%$ & $1.77 \%$ & $4.33 \%$ & $6.23 \%$ \\
Dec & $3.94 \%$ & $3.59 \%$ & $3.76 \%$ & $3.87 \%$ & $2.73 \%$ & $4.07 \%$ & $4.34 \%$ & $1.74 \%$ & $3.55 \%$ & $2.80 \%$ & $3.31 \%$ \\
\hline
\end{tabular}

Table 8: $\quad$ Monthly Capacity Values for Aggregated Nebraska Wind Generation Scenarios by Penetration

\begin{tabular}{|l|r|r|r|}
\hline \multirow{2}{*}{ Nameplate } & \multicolumn{3}{|c|}{ All Nebraska Sites } \\
\cline { 2 - 4 } & $10 \%$ & $20 \%$ & $40 \%$ \\
\cline { 2 - 4 } Jan & 1249 & 2488 & 4727 \\
Feb & $6.18 \%$ & $7.96 \%$ & $9.02 \%$ \\
Mar & $7.73 \%$ & $10.30 \%$ & $12.74 \%$ \\
Apr & $7.29 \%$ & $7.71 \%$ & $11.18 \%$ \\
May & $11.19 \%$ & $14.29 \%$ & $15.65 \%$ \\
Jun & $13.41 \%$ & $16.39 \%$ & $16.56 \%$ \\
Jul & $7.75 \%$ & $9.34 \%$ & $8.91 \%$ \\
Aug & $2.94 \%$ & $4.75 \%$ & $6.55 \%$ \\
Sep & $4.98 \%$ & $8.83 \%$ & $7.56 \%$ \\
Oct & $10.61 \%$ & $11.20 \%$ & $13.78 \%$ \\
Nov & $8.60 \%$ & $11.34 \%$ & $13.05 \%$ \\
Dec & $5.52 \%$ & $7.19 \%$ & $10.50 \%$ \\
\hline
\end{tabular}




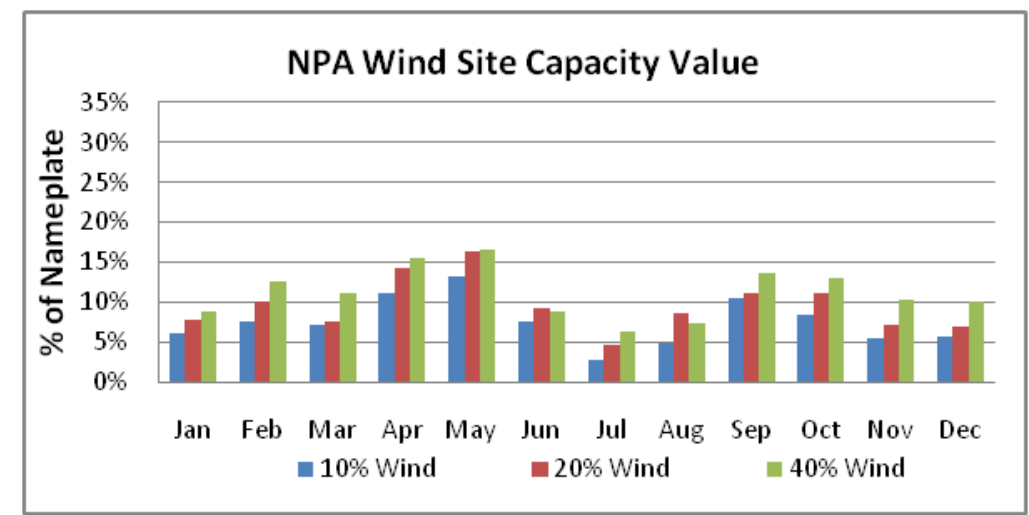

Figure 7: $\quad$ Monthly NPA Wind Capacity Value Estimates by Scenario

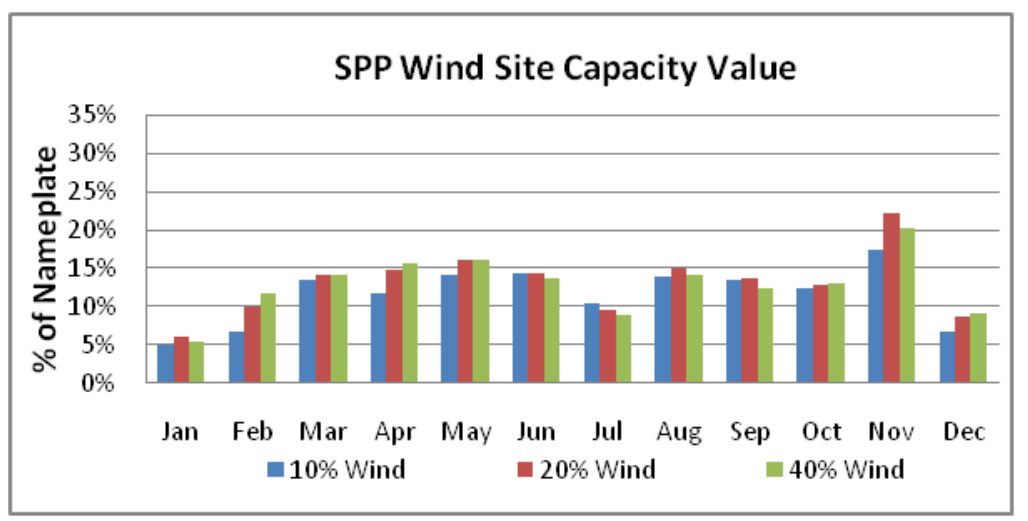

Figure 8: Monthly SPP (non-NPA) Wind Capacity Value Estimates by Scenario

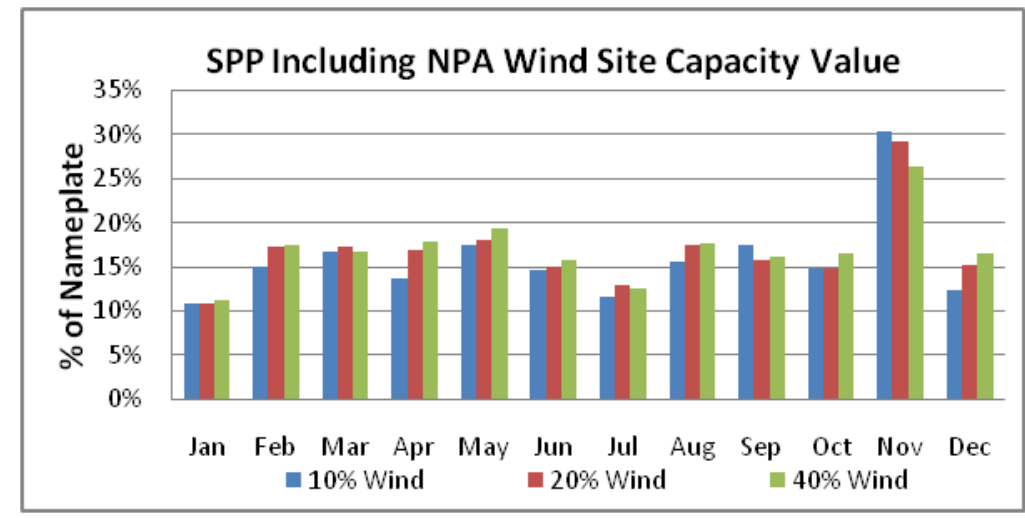

Figure 9: Monthly SPP with NPA Wind Capacity Value Estimates by Penetration

\section{Nebraska Transmission System}

New transmission will be required to bring any significant new wind resources online. The transmission system will be stressed with increased wind penetration. The transmission system expansion must be designed with wind expansion in mind to minimize those stresses.

- $\quad$ Twenty flowgates involving Nebraska were modeled and fifteen of them were constrained somewhat and ranging from one hour to 4,970 hours out of the 
year depending on the scenario and flowgate. Nine flowgates had no constrained hours.

- $\quad$ Table 9 shows the number of non-monitored branches (not flowgates) in Nebraska that approached or exceeded their thermal limits by scenario using the 2006 wind/load patterns. These branches are $115 \mathrm{kV}-345 \mathrm{kV}$ lines that are allowed in the model to exceed limits recognizing that other transmission will need to be designed and installed to relieve these overloads.

- $\quad$ Specifically, the transmission system shows increased usage and risk of overloads as wind penetration increases, but those overloads decrease with the addition of transmission designed to deliver the wind energy (i.e., the $765 \mathrm{kV}$ overlay) as shown in Table 9. That is, from Scenarios 1 to 2 and from 3 to 4 , the exceedance numbers increase with penetration increases without adding transmission. Conversely from Scenario 2 to 3 the numbers decrease with the addition of the overlay.

Table 9: $\quad$ Number of Nebraska Branches at or Near Limits

\begin{tabular}{|l|r|r|r|r|}
\hline & Scenario 1 & Scenario 2 & Scenario 3 & Scenario 4 \\
\hline Violations of Thermal Limit & 6 & 15 & 10 & 23 \\
\hline $\begin{array}{l}\text { Over 90\% of Thermal Limit (excludes } \\
\text { violations) }\end{array}$ & 6 & 11 & 4 & 2 \\
\hline $\begin{array}{l}\text { Over 80\% of Thermal Limit (excludes } \\
\text { violations and 90\% violations) }\end{array}$ & 15 & 15 & 11 & 10 \\
\hline $\begin{array}{l}\text { Total Non-monitored Branches over } \\
80 \% \text { of Thermal Limit }\end{array}$ & 27 & 41 & 25 & 35 \\
\hline
\end{tabular}

\section{Carbon Dioxide}

Increased wind penetrations result in lower $\mathrm{CO} 2$ emissions, as shown in Table 4.

- $\quad$ Nebraska CO2 emissions decrease by about $8 \%$ between the $10 \%$ wind case and the $40 \%$ wind case (using averages of 3 wind years).

- $\quad$ The rest of SPP decreases CO2 emissions by about $15 \%$ between the same cases (using averages of 3 wind years).

- Increased exports due to wind will offset emissions outside of SPP.

A pure price penalty on $\mathrm{CO} 2$ is not very effective at reducing $\mathrm{CO} 2$ emissions in SPP, including Nebraska, (given the consistent expansion fleet modeled for all penetration levels) until the penalty gets very large (using 2006, scenario 3 and its variations).

- $\quad \mathrm{A} \$ 25$ / short ton penalty reduced SPP CO2 emissions by about $2 \%$ from the no penalty case.

- $\quad \mathrm{A} \$ 50 /$ short ton penalty reduced SPP CO2 emissions by about $6 \%$ from the no penalty case. 
- $\quad$ A $\$ 120$ / short ton penalty reduced SPP CO2 emission by about $19 \%$ from the no penalty case.

Increased price penalties on $\mathrm{CO} 2$ result in decreased wind integration costs as more gas resources are committed due to $\mathrm{CO} 2$ penalties in both the ideal and actual cases that diminish the cost difference between coal and gas.

Significant carbon reductions through dispatch penalties or emissions caps result in huge increases in the use of natural gas for electricity.

- $\quad$ Combined cycle use in Nebraska increases by $138 \%$ in the $\mathrm{CO} 2$ reduction case (\$120/short ton) when compared to the base case (\$25/short ton), increasing CC capacity factors to over $80 \%$.

\section{Other Considerations}

There would be some additional costs associated with managing wind generation not captured in these production simulations:

- $\quad$ Additional maintenance and forced outages and de-rates

- Degradation of heat rate from ramping, cycling and range of operation

- Increase in emission rate per MWh production due to increased ramping that is not fully embodied in historical data that is used to determine modeled emission rates

- $\quad$ Other potential factors

Production simulation results could be used to make estimates for some of these factors, but that was not attempted in this study.

\section{Total Costs (annual amounts in $\mathbf{2 0 1 8}$ and assuming REC price of zero)}

Regarding the assumption that REC price is modeled as zero. In most cases, two incentives for wind generation are being modeled: a production tax credit (PTC) and a carbon dioxide emission price. It is judged as not likely that three incentives will exist in 2018. It is acknowledged that the PTC is extended through 2012, but it is not certain that it would remain in 2018 after a federal Renewable Energy Standard is adopted.

The primary results of the total cost estimation process for SPP including Nebraska are shown in Figure 10, which shows in bar chart and tabular form four cost categories for fifteen primary cases run on the 2006 wind and load patterns: incremental wind generation cost (cost above existing wind), incremental transmission, $\mathrm{CO} 2$ prices, and other costs (production and other fixed costs). For this set of cases shown, the total cost estimates for 2018 range from \$23.7 billion to $\$ 28.5$ billion, a $20 \%$ increase over the reference case to the $\mathrm{CO} 2$ reduction case identified as $\$ 50 /$ short ton ( $\$ 120$ in the dispatch). If the CO2 regulation is a tax instead, the corresponding case is accordingly higher yet in cost. 


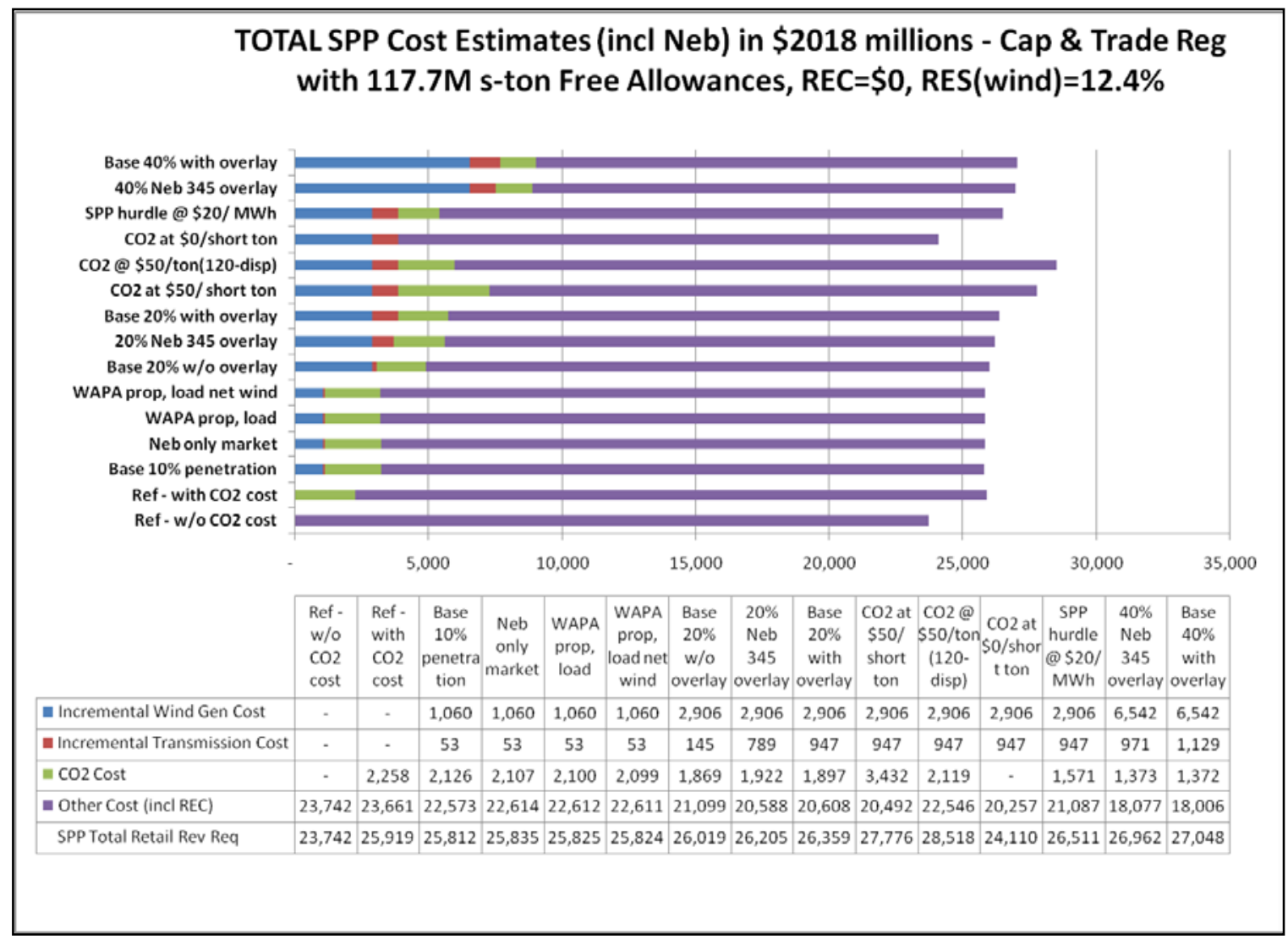

Figure 10: Total SPP Cost Estimates (inc Neb) in \$2018 millions - Cap \& Trade Reg with 117.7 M ston Free Allowances, REC $=\$ 0$, RES (wind) $=12.4 \%$

The following observations and conclusions assume a Renewable Energy Credit (REC) price of \$0:

- $\quad \$ 25 /$ short ton $\mathrm{CO} 2$ price for cap-and-trade adds approximately $9 \%$ (or $\$ 2.2$ billion) to the total cost reference, which is estimated at $\$ 23.7$ billion for existing wind and no $\mathrm{CO} 2$ cost in 2018. Under a tax regulation, that increase becomes approximately $22 \%$.

- $\quad$ Each $10 \%$ penetration (7,540 MW) adds approximately $1.7 \%$ to the total cost reference cost when the $\mathrm{CO} 2$ cap-and-trade price is $\$ 25 /$ short ton (comparing $10 \%$ and $40 \%$ bases).

- $\quad$ Each 1\% increase in revenue requirement equates to about $\$ 237$ million per year.

- $\quad$ Only the CO2 reduction case ('120/50') has emissions that approach the cap by substituting gas for coal generation.

- $\quad$ At the $10 \%$ penetration level, operating Nebraska as a standalone market (rather than as a part of the SPP market) raises the SPP-wide cost (including Nebraska) by $\$ 22$ million annually for either $\mathrm{CO} 2$ regulation with price at 
$\$ 25 /$ short ton; however, this is not considered to be a comprehensive market analysis.

- Under cap-and-trade, there is a bigger jump in cost (9.5\%) from $\$ 0$ to $\$ 25 /$ ton than from $\$ 25$ to $\$ 50 /$ ton (6.0\%). Likely due to the loss of 13.5 TWh exports in going from $\$ 0$ to $\$ 25 /$ ton.

- $\quad$ Assuming the same case dispatches for the $20 \%$ penetration case, and the same $\mathrm{CO} 2$ prices, the total costs for SPP under a tax regulation for $\mathrm{CO} 2$ (rather than cap-and-trade), are approximately $\$ 3$ billion higher at $\$ 25 /$ short ton tax for $\mathrm{CO} 2$ emissions and $\$ 6$ billion higher at $\$ 50 /$ short ton tax.

\section{WAPA Interface for Integration}

Scheduling WAPA firm power to Nebraska utilities proportional to load-net-wind vs. proportional to load may save Nebraska on the order of $\$ 1$ million, subject to Missouri River Basin management priorities.

\section{Integration Costs}

Wind Integration Costs - Results

Wind integration costs are production cost increases due to wind forecast error, wind regulating reserves and wind shape variability. The costs are typically normalized to wind energy by dividing production cost delta by total wind energy (see Methodology section for more background).

Costs to integrate wind increase with wind energy penetrations as more natural gas fired resources are used to respond to wind forecast error and displacement of coal energy backed down for reserves as shown in Figure 11. SPP integration costs increase from an average of $\$ 46$ million at $10 \%$ wind to an average of $\$ 218$ million at $40 \%$ wind penetration using the shaped proxy.

Normalized Integration Costs (as initially defined with shaped proxy ${ }^{3}$ resource) are relatively small and in a narrow range of \$1.65 - \$2.18/MWh in 2018\$ (or \$1.32 - \$1.75/MWh in 2009\$) for the multiple penetration levels and years modeled for the base case, as shown in Figure 12.

The base penetration scenarios show a consistent increase in use of gas fired resources (especially combined cycle plants) to deal with wind forecast error and increased (and variable) reserve requirements when comparing actual wind to ideal wind runs. In accommodating wind reserves and forecast error, the Nebraska combined cycle usage increases by $18 \%$, when comparing the actual case to the ideal case at $40 \%$ wind penetration.

The initial definition of integration costs (with shaped proxy) is only capturing effects of incremental reserves for wind and wind forecast error and is not capturing shape variability costs.

\footnotetext{
${ }^{3}$ Shaped Proxy - a proxy wind resource that uses the actual delivered shape of the wind but is perfectly known (no forecast error) and requires no additional regulating reserves.
} 


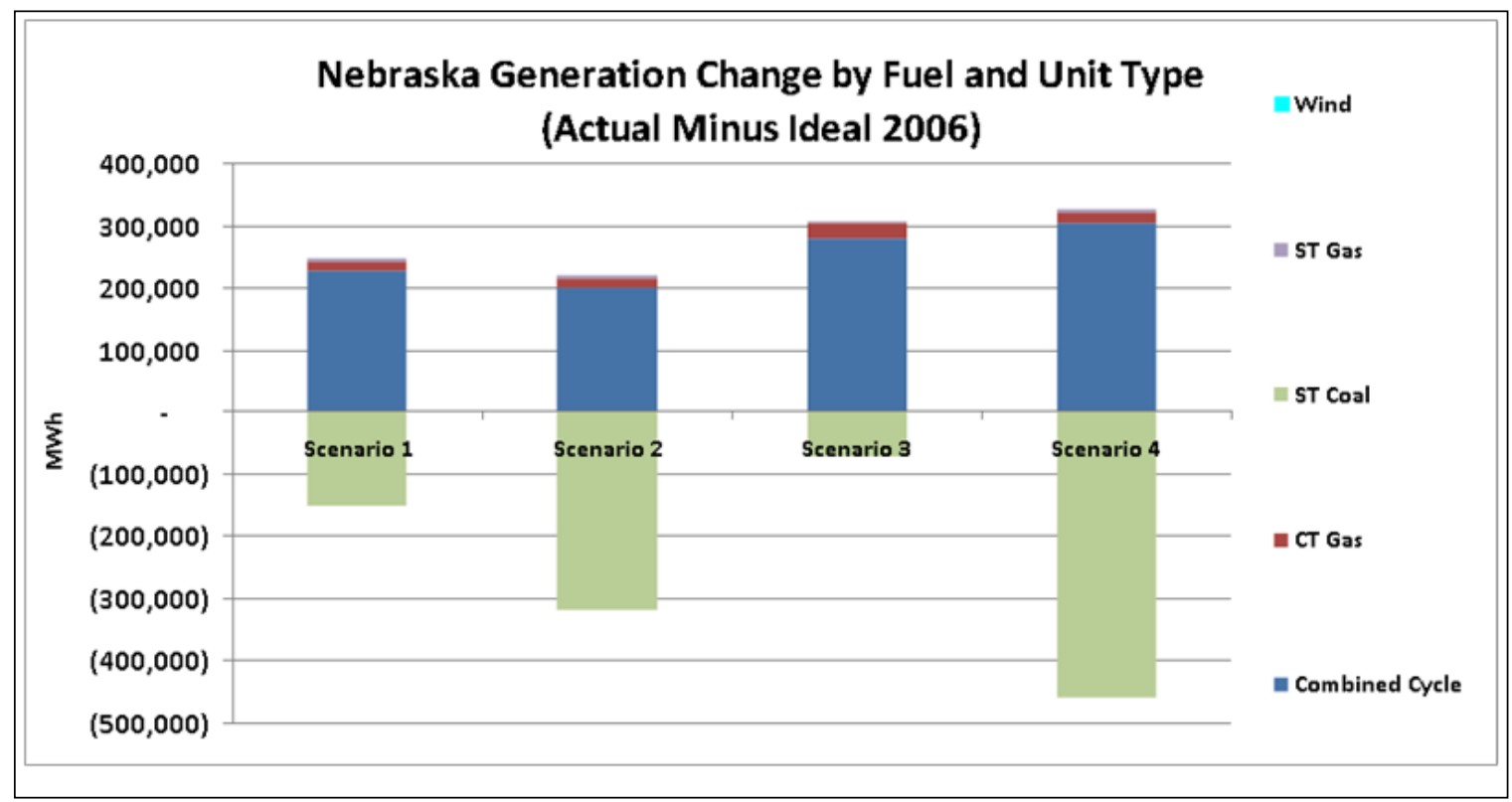

Figure 11: Change in Nebraska Generation with Incremental Reserve Requirements and Additional Uncertainty due to Wind Generation ("actual" case) 

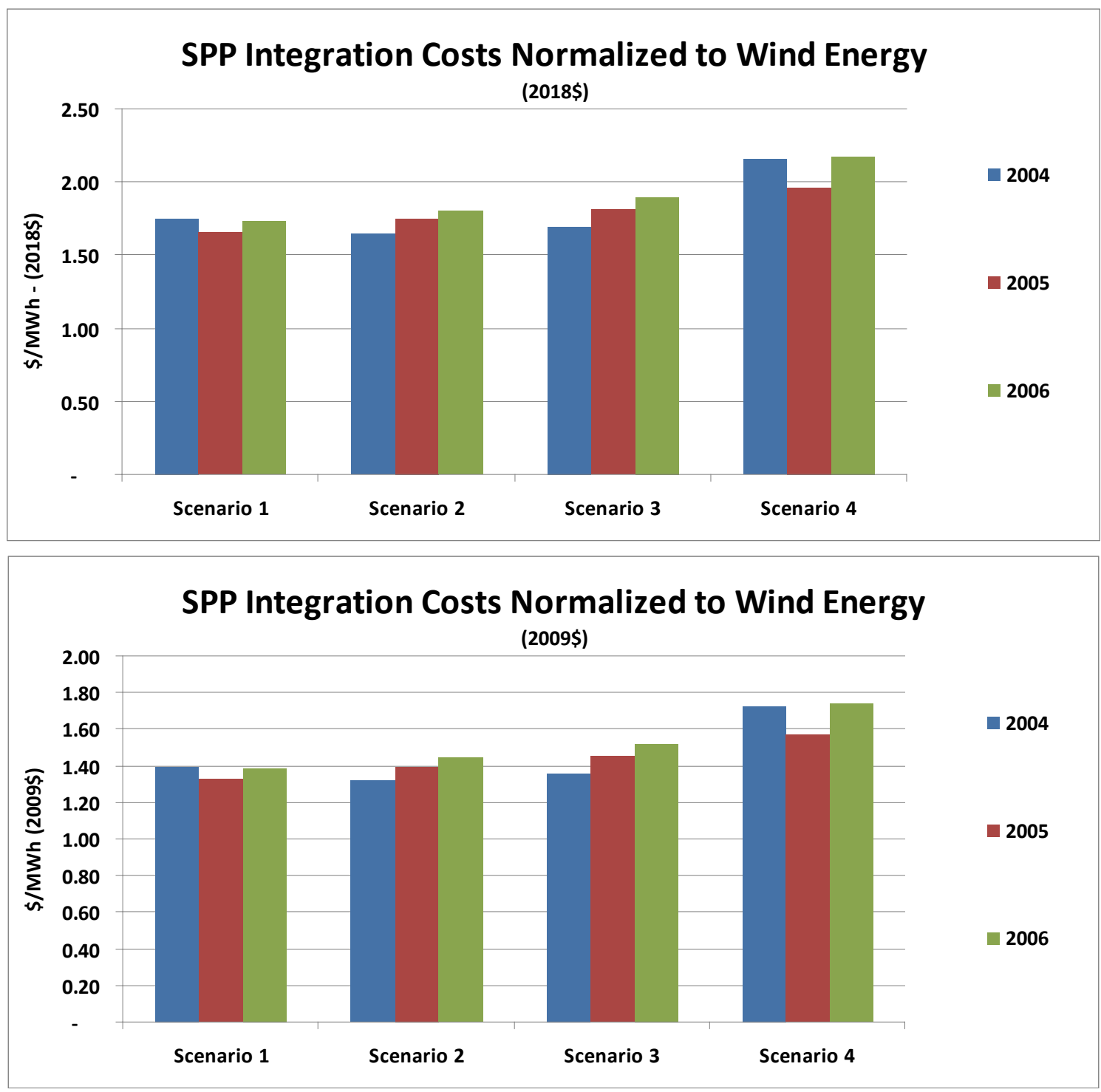

Figure 12: $\quad$ SPP (including Nebraska) Normalized Wind Integration Costs (shaped proxy) by Scenario and Wind Year for 2018 Conditions shown both in 2018\$ (top) and 2009\$ (bottom) Other proxy resources were tested to help measure effects of hour to hour wind variability on the generation fleet. Sensitivity cases show that a moving average or block (sub-period or flat) for proxy captures more integration costs, called herein shape or variability costs, see Figure $13^{4}$.

${ }^{4}$ Flat block proxy consists of a 24 hour constant block of energy equal to the amount of energy of the actual wind profile for the same day. Sub period block proxy distributes the daily energy during on peak and off peak hours

Moving average proxy is a profile that represents a periodic hourly average of energy, ( 5 and 13 hour averages were used) 
- $\quad$ Proxy resource sensitivity results show $\$ 4.01 / \mathrm{MWh}$ (or $\$ 3.21 / \mathrm{MWh}$ in $2009 \$$ ) for $10 \%$ penetration with daily block proxy and $\$ 2.40 / \mathrm{MWh}$ (or $\$ 1.92 / \mathrm{MWh}$ in 2009\$) for a sub-period block proxy.

- At $20 \%$ wind penetration those integration cost results increase to $\$ 5.36 / \mathrm{MWh}$ (or $\$ 4.29 / \mathrm{MWh}$ in $2009 \$$ ) for the daily block proxy and $\$ 3.88 / \mathrm{MWh}$ (\$3.11/MWh in 2009\$) with the sub-period block proxy.

- $\quad$ Non-normalized integration costs using the daily block proxy for SPP are \$110 million at $10 \%$ wind and $\$ 66$ million using the sub-period block proxy; these values increase to $\$ 286$ million and \$206 million respectively for $20 \%$ wind penetration.

- $\quad$ These increasing differentials for alternative proxies as wind penetration increases may indicate that integration costs associated with wind variability are increasing with the amount of wind although the other costs (reserves and uncertainty as measured with the shaped proxy) are remaining relatively flat as wind penetration increases.

- $\quad$ Analysis indicates that the modeled system outside of SPP is absorbing some of the costs of wind variability represented in the block proxies (daily and subperiod) and that total SPP integration costs could be as high as $\$ 5.41 / \mathrm{MWh}$ (2009\$) at $10 \%$ wind penetration and $\$ 9.26 / \mathrm{MWh}(2009 \$)$ at $20 \%$ wind penetration when considering those exported costs (using the sub-period block proxy), Table 10. 

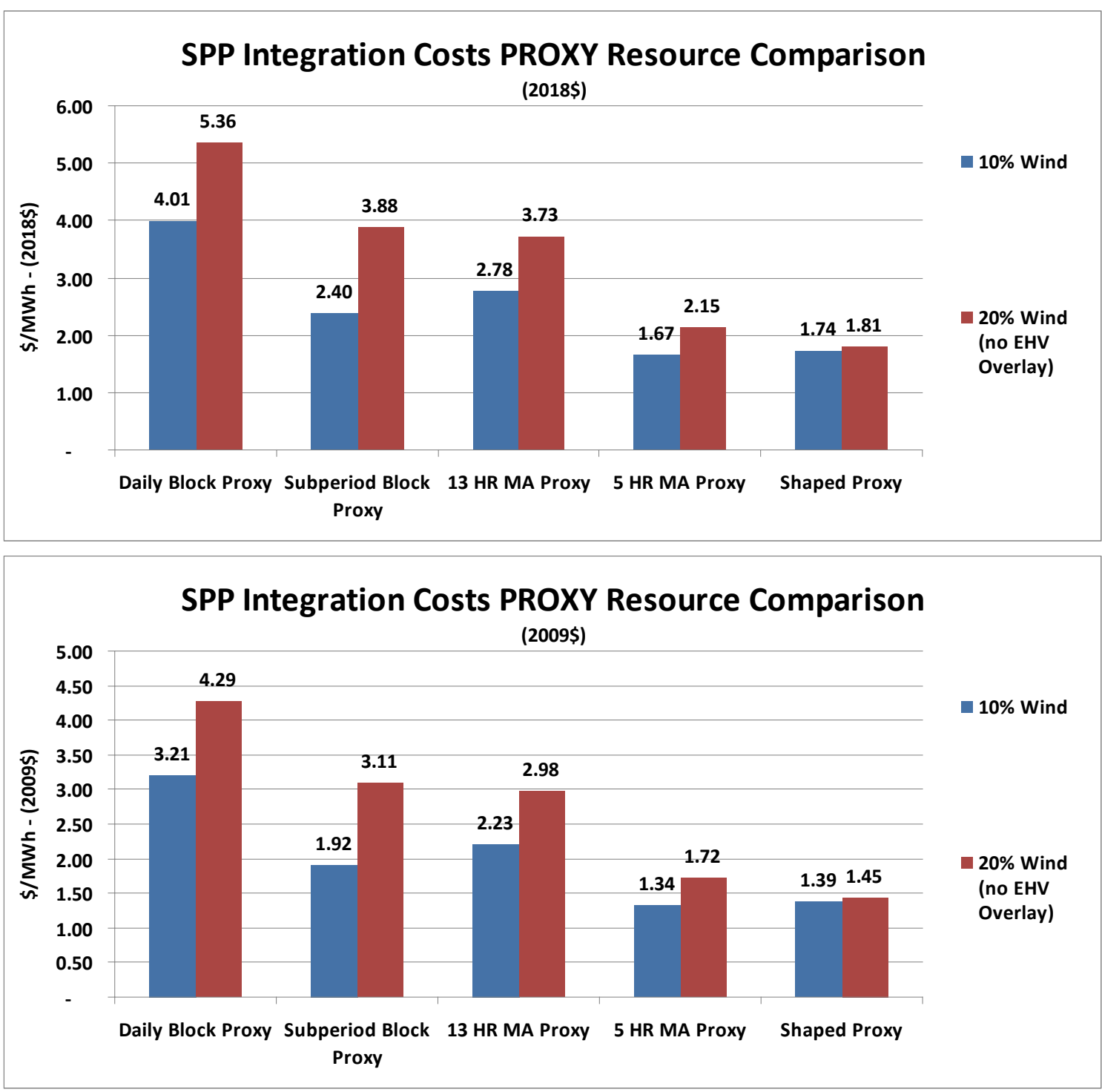

Figure 13: SPP (including Nebraska) Integration Costs Using Different Proxy Resources for 2018 Conditions shown both in 2018 (top) and 2009\$ (bottom)

Table 10: Shaped and Sub-period Block Proxy Analysis at 10\% and $20 \%$ Wind Penetration

\begin{tabular}{|c|c|c|c|c|}
\hline \multirow[t]{2}{*}{ Proxy Type } & \multicolumn{2}{|c|}{$2018 \$ / M W h$} & \multicolumn{2}{|c|}{$2009 \$ / M W h$} \\
\hline & $\begin{array}{l}10 \% \text { Wind } \\
\text { Penetration }\end{array}$ & $\begin{array}{l}\text { 20\% Wind } \\
\text { Penetration }\end{array}$ & $\begin{array}{c}10 \% \text { Wind } \\
\text { Penetration }\end{array}$ & $\begin{array}{l}\text { 20\% Wind } \\
\text { Penetration }\end{array}$ \\
\hline Shaped Proxy & 1.74 & 1.81 & 1.39 & 1.45 \\
\hline $\begin{array}{l}\text { Sub-period Block Proxy -Native } \\
\text { Sub-Period Block Proxy - Implied }\end{array}$ & 2.4 & 3.88 & 1.92 & 3.11 \\
\hline (including "exported" shape costs) & 6.76 & 11.56 & 5.41 & 9.26 \\
\hline
\end{tabular}


As discussed throughout the report, there are a number of cost considerations involved with incorporating wind other than the wind integration costs labeled as such in this report, such as:

- Wind generation and transmission facilities installation and operation and maintenance.

- $\quad$ Effects on heat rates, emission rates, and outage frequency and duration from increased thermal unit ramping. The magnitude of these effects at high penetration rates is unknown, because utilities generally don't have such operating experience yet.

- $\quad$ Feasibility and pricing of highly variable exports associated with high wind penetrations.

- $\quad$ Effects from external areas increasing their wind penetrations along with SPP and associated reduction of external system's ability to absorb SPP wind variations.

- Lack of "shape" costs from the "shape proxy" methodology (as contained in the "daily block" proxy).

- Apparent need to consider the portion of the shape costs that get "exported" and do not show up in the calculated integration cost for the originating wind area.

\section{Wind Integration Costs - Methodology and Proxy Resource Selection}

The wind integration cost evaluation involves comparing the wind resource complete with its variability and uncertainty to an "ideal" proxy, or reference, resource by performing two simulations. The resulting adjusted production cost difference is the integration cost that can be normalized by dividing this result by the amount of wind generation being integrated. A key element in the determination of integration costs is the definition of the proxy resource. A second issue is whether or not the modeling approach addresses all the integration costs.

\section{Conclusions}

- On the first issue the report does not definitively conclude what is the single best proxy resource for establishing wind integration costs for all conditions, but indicates the sub-period block best minimizes the concerns associated with other proxies - there is minimal energy shift from off peak to on peak periods (comparing to the daily block), but shape costs are captured (comparing to the shaped proxy).

- On the second issue, the report indicates that when including shape (or variability) costs by use of the block or moving average proxies, it is important to examine whether and what amount of these shape costs are being "exported" to external areas. And lastly that there are likely additional wind integration costs that are not yet identified by conventional modeling.

\section{Closing Comments}

In closing, a qualifying statement, a statement concerning how the study objectives were accomplished, and thoughts for future study are presented. 


\section{Qualifying Statement}

The results, findings, and conclusions presented here relate to the scenarios defined for the calendar year 2018. Critical to these scenarios are the extensive assumptions made regarding wind generation, load, fuel prices, energy market and operational structure, and certain aspects of policy regarding carbon. While the results paint a reasonably detailed picture regarding wind integration challenges and opportunities for NPA for the scenarios studied, care must be taken in extrapolating their meaning beyond the context in which they are presented in this report.

\section{Objectives Statement}

The ten study objectives listed earlier were fully accomplished as described in the study report. Some key components were use of NREL's wind data base, wind penetration from $10 \%$ to $40 \%$, statistical and PROMOD analyses, WAPA purchase scheduled on load net wind, SPP future market, large and active TRC, active utility and stakeholder participation in Nebraska, various approaches to integration cost evaluation, use of technical experts, and transfer of data to the utilities.

\section{Thoughts for Future Study}

Detailed thoughts are offered in the study report on what might be important and productive areas to investigate further if the opportunity should arise. In summary, these areas relate to:

- $\quad$ Additional work on integration cost concerning proxy resource definition, influence of interchange, influence of wind penetration in external areas, and influence of hurdle rate.

- More hourly examination of hourly specifics for generators and interchange, high wind/low load and low wind/high load periods, and extreme ramping situations.

- $\quad$ Transmission design, land use studies, and export studies.

- $\quad$ More wind capacity valuations, outside the current SPP criteria.

- $\quad$ More completely involve operational and transmission personnel. 


\section{Section 1}

\section{InTRODUCTION - PROJECt SCOPE, OBjeCtIVES, AND DeLIVERABLes}

\subsection{PARTIES InVolved \\ 1.1.1. NREL and WAPA}

The National Renewable Energy Laboratory (NREL, www.nrel.gov) is a national laboratory of the U.S. Department of Energy (DOE), Office of Energy Efficiency and Renewable Energy, and is operated by the Alliance for Sustainable Energy, LLC. This study began on October 23, 2008 and is an outcome of NREL's Request for Proposal (RFP) RAM-8-89030. Federal funding of $\$ 500,000$ for this study was matched by an equal contribution of cash and in-kind labor provided by the participating NPA utilities.

The NPA utilities are customers of the Western Area Power Administration (WAPA, www.wapa.gov), one of four power marketing administrations within the DOE. WAPA's role is to market and transmit electricity from multi-use water projects having 57 power plants that have an installed capacity of 10,395 megawatts.

\subsubsection{NPA}

The Nebraska Power Association (NPA) (www.nepower.org) is a voluntary organization that represents all segments of the Nebraska power industry: municipalities, public power districts, public power and irrigation districts, and cooperatives engaged in generation, transmission, or distribution of electric energy in the nation's only totally public power state.

Three balancing areas are fully represented: Nebraska Public Power District, Omaha Public Power District, and the Lincoln Electric System. Additionally all of the Nebraska utilities, small, medium, and large, are participating in the study through their NPA representation.

As such NPA represents approximately $28,248 \mathrm{GWh}^{5}$ of annual retail load served by approximately 9,000 MW of generation capacity (including capacity associated with out-of-state purchases and sales). Six Nebraska utilities own and operate or purchase the output from a total of $152 \mathrm{MW}$-nameplate of wind generation projects in five separate locations spread across the state.

\footnotetext{
${ }^{5}$ Energy Information Administration, DOE/EIE-0348(01)/2, Date of Data: 2007
} 
During 2009 power purchase agreements were completed for two new wind generation plants totaling $102 \mathrm{MW}$. Negotiations are underway to add more wind generation and still more wind additions are planned according to the state utility integrated resource plans.

For this project the NPA contributed in excess of $\$ 500,000$ in the form of direct project funding, direct expenses, and utility in-kind labor cost. The NPA provided oversight to the consultants through separate contracts with each firm.

\subsubsection{Consultant Team}

\subsubsection{EnerNex}

EnerNex Corporation is an electric power engineering and consulting firm specializing in the application and integration of new electric power technologies. EnerNex provides engineering and consulting services, along with software development/customization and market/technology analysis, for energy producers, distributors, users and research organizations.

The EnerNex project team consists of Robert Zavadil, Vice President and Principal Consultant, Jack King, Consulting Engineer and Tom Mousseau, Senior Consultant.

EnerNex has extensive experience with wind turbine technology, wind plant design and operating issues and technical questions associated with transmission grid interconnection and power system operation. EnerNex also provides technical and operating support to the Utility Wind Integration Group, the leading resource for technical knowledge about integration of wind generation. In addition, EnerNex is/was involved in and helped establish several industry working groups such as UWIG, NRECA Task force, IEEE Power Engineering Society Wind Power Coordinating Committee, and the IEEE PES Power Systems Dynamics Committee. EnerNex has been recognized on several occasions for work in wind technologies; these honors include 2005 AWEA Technical Achievement Award.

\subsubsection{Ventyx}

Ventyx is a leading energy consulting, data intelligence and analytics simulation software firm. The consulting practice, Ventyx Advisors, offers subscription advisory services and strategic consulting services in power market and transmission analysis, asset valuation and project finance, energy portfolio risk analysis, resource planning and rate studies, and fuels and environmental policy analysis. Ventyx is currently supporting 13 of the top 15 wind developers and 7 of the top 8 wind turbine manufacturing companies with strategic decision support, project-specific analysis and software/data intelligence solutions. Ventyx also performs substantial market-related transmission and wind analysis in SPP, PJM, MISO, MAPP, CAISO, the broader WECC and ERCOT. Ventyx is the developer of the PROMOD IV security constrained economic dispatch simulation model.

Formed from the 2007 merger of Global Energy Decisions and New Energy Associates, Ventyx Advisors brings more than 30 years of experience to our clients. These clients include over 900 worldwide and over 430 US clients: federal and regional agencies, state regulators, integrated utilities, independent transmission companies, small and large wind developers, merchant generators, consulting firms, and financial hedge firms, among many others. 
The Ventyx project team consists of Gary Moland, Vice President and Rick Hunt, Principal Consultant.

\subsubsection{Technical Review Committee and Observers}

A Technical Review Committee (TRC) consisting of technical experts, interested parties, consultants and utility personnel was formed and met four times in Omaha and three times as webinars hosted by Ventyx. The TRC and the observers provided very helpful guidance to the NPA and the consultants. The thirty-seven TRC members and thirty-one observers are listed in Appendix 8.1.

\subsection{Subcontract DeVelopment}

NPA responded to NREL RFP RAM-8-89030 of April 2, 2008 titled "Wind Integration Study: Cost Share Program" with a study proposal on April 28, 2008 titled "NPA Proposal for a Nebraska Statewide Wind Integration Study". At the request of NREL a follow-up re-scope proposal was submitted on August 26, 2008 with the same title. On October 23, 2008 the Alliance for Sustainable Energy, LLC, the management and operating contractor for NREL executed a subcontract with NPA for subcontract number AAM-9-89030-01 for the NPA to do the year-long study outlined in the August 26 proposal.

\subsection{WORK SCOPE}

NREL described the work to be done as three tasks:

Task 1 - Model/Characterize the Wind Power Resources

Task 2 - Ascertain Wind Integration Impacts

Task 3 - Identify Issues and Potential Solutions

NPA detailed the three NREL-identified tasks into a 25-task list of Study Plan Details that was an integral part of the NPA proposal and incorporated into the subcontract.

\subsection{OBJECTIVES}

Objectives of the work to be done were established by NREL and supplemented by NPA as:

- $\quad$ Evaluate the impact of wind energy on system operations using synchronized wind and load data and state of the art analysis techniques.

- Wind energy penetration of at least $10 \%$, based on wind energy to total energy sales should be evaluated. Additional scenarios at higher penetrations are encouraged.

- Evaluation should be a combination of statistical analysis and production simulation.

- $\quad$ Evaluate the extent to which the Federal hydroelectric system can be used to help with cost-effective wind integration that is consistent with reliable system operation. 
- $\quad$ Evaluate potential mitigation approaches that may include innovative markets, institutional arrangements within or between Balancing Areas, or physical response on an economic basis.

- $\quad$ Produce meaningful and supported results in coordination with a Technical Review Committee (TRC), which should include stakeholders and experts in wind integration analysis.

- $\quad$ Nebraska utilities see this NREL/WAPA grant program as a real opportunity to advance statewide wind development giving consideration not only to the integration impacts but making progress in finding relevant solutions in a multistakeholder process.

- $\quad$ To quantify, using valid evaluation techniques, the expected wind integration costs, both sub-hourly and hourly and longer for Nebraska wind generation development.

- $\quad$ To involve outside experts and interested parties in order to advance team building for future Nebraska wind generation development and increase the level of understanding among state leaders concerning wind integration issues for Nebraska.

- $\quad$ Builds data base and utility expertise for ongoing improved in-house modeling of wind generation impacts for future studies.

\subsection{DeLIVERABLeS}

Deliverables of the work to be done were established by NREL and supplemented by NPA as:

Hold four TRC meetings that correspond to the 3 tasks above, and a final TRC meeting near the end of the project. The final meeting should be held sufficiently in advance of the end of the project so that TRC concerns or questions can be addressed in the final report. Deliver a meeting summary following each meeting.

Prepare and deliver at least one presentation at a Utility Wind Integration Group workshop, and at least two presentations at appropriate public power forums. Deliver summary reports after the workshop and two presentations at public power forums.

Produce a detailed final report that clearly explains the data, modeling, analysis, assumptions, and results. The report will provide details of the hourly production simulation analysis, and the sub-hourly statistical analysis, along with recommendations for system or institutional improvements that can help improve the system ability to absorb wind economically and reliably.

A detailed list of Expected Results and Usage, as well as Key Outcomes and Deliverables, was also specified in the NPA proposal. 


\subsection{OTHER BACKGROUND}

The NPA describes some of its work in the renewable area on its website at www.nepower.org. In particular, note:

- Meeting documentation (agendas and summaries) for this study.

- $\quad$ "Renewable Energy Background and Outlook for Nebraska Electricity Consumers: A Reference Document" by the Nebraska Power Association December 28, 2007.

- $\quad$ Six white papers provided to the Natural Resources Committee of the Nebraska Legislature in support of their Interim Study Resolution, LR 83. The purpose of LR 83 is to study issues relating to expanded development of wind energy in Nebraska, while preserving the ability of the state's unique public power system to continue serving the state with low-cost, reliable electricity.

EnerNex and Ventyx have provided consulting services and authored reports and papers for many other studies of this type including the Eastern Wind Integration and Transmission Study (EWITS), another current project sponsored by NREL. For further information on their work see their websites of www.enernex.com and www.ventyx.com.

At the time this report was finalized, summary presentations had been made to a Large Public Power Council - Chief Operating Officers (LPPC/COO) meeting on September 30, 2009 in Omaha, Nebraska, the lowa/Nebraska Conference: Renewable Energy Day - Wind Focus on October 6, 2009 in Omaha, Nebraska, the Utility Wind Integration Group's Fall Technical Conference on October 9, 2009 in Cedar Rapids, lowa, and the Nebraska Wind Working Group meeting on November 9-10, 2009 in Kearney, Nebraska.

\subsection{GLOSSARY}

A glossary of terms is provided as an Appendix Section 8.5 that provides a central place for looking up various terms used in this study report.

\subsection{Perspectives}

This section briefly discusses this NPA-NREL study in relation to other initiatives and studies that may have a relationship to this general topic of Nebraska wind integration.

Nebraska Legislative Resolution (LR) 83: The Natural Resources Committee of the Nebraska Legislature is conducting an interim study relating to expanded development of wind energy in Nebraska, while preserving the ability of the state's unique public power system to continue serving the state with low-cost, reliable electricity. The NPA is actively involved in all the LR 83 study subcommittees. The LR83 study considers the DOE 20\% scenario whereby Nebraska would host 7,800 MW of Nebraska wind generation capacity by year 2030. This NPA-NREL wind integration study's highest penetration level of $40 \%$ measured by energy results in 4,727 MW in Nebraska in year 2018, which would be about $60 \%$ of the way to the LR 83 wind goal in about $45 \%$ of the elapsed time to 2030 . In either year 2018 or 2030 , these referenced penetrations represent very aggressive schedules for installing wind generation and would require significant 
external market and investment interest. It is expected that the results of this NPA-NREL study will be helpful to the conduct of the LR83 legislative study.

Federal Renewable Energy Standard (RES) possibility - The Waxman-Markey HR 2454 bill was incorporated into this study as a potential regulation that would require an estimated $12.4 \%$ renewable energy, as described in Section 5.2 of this report. As such, the $10 \%$ penetration scenario studied herein would not satisfy such an RES, unless other non-wind renewable made up the rest of the standard requirement, but the $20 \%$ and $40 \%$ scenarios easily would.

NPA Utility Goals - Some of the NPA utilities have included wind generation additions in their integrated resource plans for the future in recognition of their utility goals to reach $10 \%$ renewable penetrations by year 2020 .

Governor's "Top 10" Statement - Nebraska Governor Dave Heineman made a statement in fall 2008 , without giving particulars, about a potential Nebraska goal to be in the top 10 for wind generation installations. One NPA interpretation of such a goal would be installing an estimated $2,184 \mathrm{MW}$ by 2018 . This would amount to an $18 \%$ penetration in 2018 , and be very similar to the $20 \%$ scenario used in this study.

Nebraska Renaissance Group (NRG) State Population Growth Recommendation - An NRG economic study in summer 2009 recommended that the state strive for a population of two million by year 2020 . This would amount to a $0.96 \%$ per year growth from the 1.78 million population estimate for 2008 . This is approximately double the population growth rate during the last decade. Applying the possibility that loads would grow accordingly (and keeping the same per person usage), this would mean for example that a studied $20 \%$ penetration scenario in 2018 would actually be approximately 19\% penetration in 2018 because of the slightly higher loads.

Interim 2009 Nebraska Energy Plan - A couple excerpts:

"POLICY STATEMENT: Support a balanced integration of resources so as to provide Nebraskans with affordable, reliable, and environmentally sustainable electricity."

"...Nebraska is committed to increasing the amount of electricity generated by wind in the state. The Governor, the Nebraska Energy Office, and other state offices and representatives are exploring ways in which Nebraska's abundant wind resources could be harnessed both to meet demand in Nebraska, and to export to other states. Exportation of electricity could provide Nebraska with revenue sources which can be used to avoid rate increases, reduce tax rates, and to re-invest in yet more wind generation and transmission capacity, thereby creating more jobs. Finally, Nebraska's exported wind energy can benefit other states as well by providing them with an additional source for meeting their renewable energy goals and mandates."

Nebraska Wind Working Group - Its purpose is "to educate and update Nebraskans with the current information on wind energy markets, technologies, economics, policies, prospects, and issues". Its activities include "periodic meetings; targeted workshops such as wind integration for utilities, policy options for legislators and regulators; and a state-wide wind conference every 12-18 months." NPA utilities regularly participate in these activities. The U.S. Department of Energy funds the operations of the Nebraska Wind Working Group under the Wind Powering America program and the operation of the Group is facilitated by the Nebraska Energy Office. 
Distributed Wind Generation - The NPA utilities recognize that wind generation that is installed on the distribution (low voltage) system can have certain system benefits like minimizing transmission requirements. Further, that others like the Minnesota utilities have extensively studied such scenarios for the Minnesota Department of Commerce. This NPA-NREL study does not delve into such distributed wind generation scenarios largely due to the intent to represent the larger penetrations of $10 \%$ to $40 \%$.

Southwest Power Pool (SPP) Wind Integration Task Force Study - SPP is well along in the process of completing this study, which has similar penetrations and similar wind generation sitings to the NPA-NREL study, but is focused more on transmission system performance and uses a shorter term model than does this NPA-NREL study.

Eastern Wind Integration and Transmission Study (EWITS) - The EWITS study of the whole Eastern Interconnection was started and completed during about the same time as was this NPA-NREL study. Further both EnerNex and Ventyx were also involved in the EWITS study. While there are quite a few similarities between the two studies, EWITS was focused later in time (year 2024) and contained higher levels of penetration in that $20 \%$ and $30 \%$ penetrations were applied to the whole interconnection which put the Midwest penetrations much higher than those studied in the NPA-NREL study. For example, in its most extreme case, EWITS modeled 25,000 MW of wind generation compared to the largest Nebraska amount studied here of 4,727 MW. Also the EWITS study had a greater focus on transmission analysis and design.

Other Initiatives and Groups - The NPA recognizes that there are a number of other important initiatives and groups formed and forming in Nebraska and elsewhere that have an interest in wind generation development in Nebraska. There are many interests to balance in this arena and we have not attempted to identify them all but rather list some that have come up in discussion throughout the study. 


\section{Section 2}

\section{SCENARIO DEVELOPMENT}

\subsection{SCENARIO DeVELOPMENT}

This section identifies the general source of data used in the study along with a general description of any adjustments that have been made for the purposes of this study. Key data for this study includes wind siting and profile data for varying penetration levels, production simulation data including generation, load forecasts and fuel forecasts and transmission data including underlying network model, constraint set, upgrades and overlay assumptions. Data is considered in three basic pieces: Nebraska, rest of SPP, and the rest of the modeling footprint. Ventyx and EnerNex developed an initial view of all data pieces and provided this to NPA for review and adjustments of the assumptions as appropriate.

\subsection{BASE CASES}

\subsubsection{Wind Patterns}

The NREL meso-scale wind database developed by AWS Truewind, is the source for all wind generation data in this study. The database focuses on meso-scale data as a whole and includes 34 states in the Eastern Interconnection. There are 1325 separate wind plants with a total installed capacity of $580 \mathrm{GW}$ which are aggregations of nearby data points corresponding to a 2 $\mathrm{km}$ grid. For the Nebraska study the NREL database provided significant data for modeling of wind generation. This analysis included sub hourly, 10-minute resolution, and hourly data with day ahead forecast and actual production data for the years 2004, 2005 and 2006. Annual wind patterns were used for each wind site.

\subsubsection{Scenario Development}

The wind penetration development for SPP including Nebraska consisted of four base scenarios (cases). Separate production simulations were performed using 2004, 2005 and 2006 load and wind profile data making a total of 12 cases for examination.

\subsubsection{Scenario $1-10 \%$ No overlay}

This scenario consisted of a $10 \%$ wind penetration for Nebraska and SPP with transmission capability as described in Section 2.4 .

\subsubsection{Scenario $2-20 \%$ No overlay}

This scenario consisted of a $20 \%$ wind penetration for Nebraska and SPP with transmission capability as described in Section 2.4. 


\subsubsection{Scenario $3-20 \%$ With overlay}

This scenario consisted of a $20 \%$ wind penetration for Nebraska and SPP with transmission overlay consisting primarily of $765 \mathrm{kV}$ additions with some also being at $345 \mathrm{kV}$, see Section 2.4 .

\subsubsection{Scenario $4-40 \%$ With overlay}

This scenario consisted of a $40 \%$ wind penetration for Nebraska and SPP with transmission overlay consisting primarily of $765 \mathrm{kV}$ additions with some also being at $345 \mathrm{kV}$, see Section 2.4 .

\subsection{WIND Data}

\subsubsection{Wind Generation Data}

Nebraska and rest of SPP wind sites were selected from the NREL meso-scale database. This was the same source of data used for the selection of wind sites in the EWITS study. Figure 14 provides an overview of all wind locations within the NREL database for the Nebraska and SPP regions.

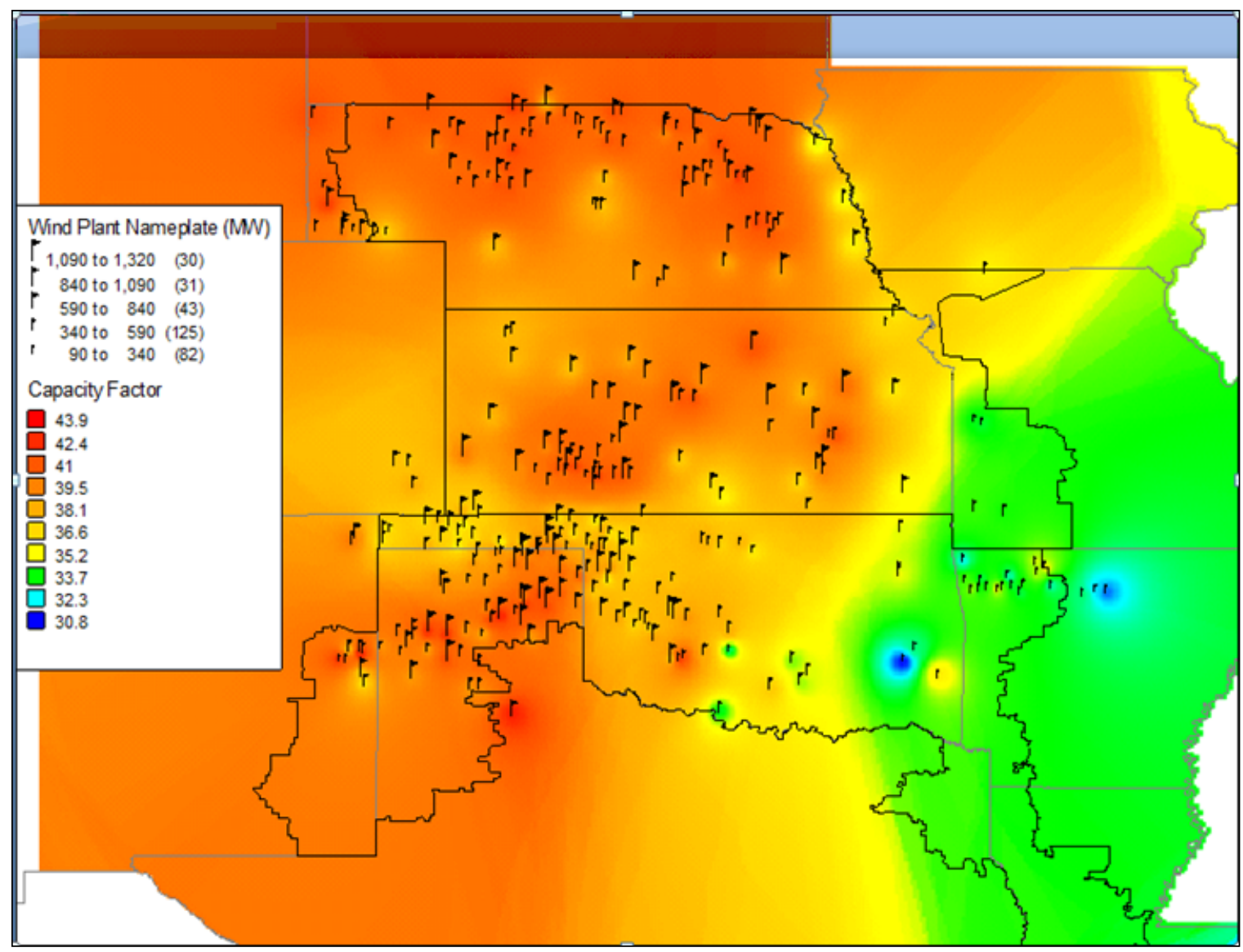

Figure 14: Representation of all possible NPA and SPP wind plants from NREL meso-scale database. 
Deliberating with the NPA project team, the wind scenarios for the study were established with regard to a target energy penetration: Three penetration scenarios corresponding to $10 \%, 20 \%$, and $40 \%$ of NPA electric energy demand. The precise installed capacity to achieve these energy targets depends on the aggregate capacity factor and size of the selected plants from the NREL database.

\subsubsection{Selection Criteria}

NPA calculated an estimate for the amount of wind generation energy necessary to provide $1 \%$ penetration for Nebraska (energy basis) as being $405 \mathrm{GWh}$ in year 2018. This estimate conservatively included not only a $1 \%$ percentage of the retail load (as a typical Renewable Portfolio Standard would be defined) but also a $1 \%$ percentage of the transmission and distribution losses from the generation bus point to the retail meter bus point. That is, in this calculation losses of $7.4 \%$ were assumed; therefore of the $405 \mathrm{GWh}$ generated $375 \mathrm{GWh}$ was for retail load and $30 \mathrm{GWh}$ for the losses.

EnerNex developed the wind energy targets by multiplying the $405 \mathrm{GWh}$ by 10,20 , and 40 to establish the energy targets for the penetration scenarios. The established wind plants in Nebraska on the Eastern Interconnection (Ainsworth, Elkhorn Ridge, Lincoln, and Valley) constitute a portion of these wind generation amounts, although specific data is not used for these known sites. Rather they can each be considered as part of the nearest selected site. EnerNex used the NREL data base to fully achieve the target energies in the three penetration scenarios. The Kimball wind plant is connected to the Western Interconnection.

The TRC-approved site selection criteria involved consideration of the high capacity factor locations while also factoring in good geographical diversity (primarily for the purpose of achieving some degree of non-coincidence in the aggregated wind resource for reliability purposes). The TRC also gave attention to environmental and transmission access considerations.

One un-anticipated issue in the site selection process was that the NREL site data involve necessarily large capability wind sites. So to match or exceed the energy targets, there was typically some excess of wind generation beyond the targeted amount. This approach was taken rather than taking a partial site to exactly match the target amount, as this could somewhat distort the sub hourly data characteristics that assume a certain aggregated site size.

\subsubsection{Site Selection}

It is important to recognize that the results of the site selection process are only a sample set of sites and the sites selected are not to be interpreted as implying that transmission requirements or environmental factors are fully considered, or that these are the best or even the most likely development sites.

In the site selection process, six options were examined. The following characteristics in Table 11 summarize the sixth option, which was chosen for modeling purposes in this study, totaling 4,727 MW nameplate capacity with an average site size of $430 \mathrm{MW}$ for the full $40 \%$ penetration scenario.

This study does not claim that the wind generation must or even will likely be constructed according to the siting modeled. In this respect we have tried to coordinate the siting for the 
areas outside Nebraska with other studies, and in Nebraska we strived to show a dispersed wind development over the fairly large area of good wind resources in order to see the effects of geographic diversity on the wind profiles.

Table 11: Nebraska Selected Wind Generation Penetration Details

\begin{tabular}{|r|r|r|r|}
\hline Nebraska 3 Yr Average & $10 \%$ Pen & $20 \%$ Pen & $40 \%$ Pen \\
\hline Target (GWH) & 4,052 & 8,104 & 16,208 \\
\hline Selected (GWH) & 4,523 & 8,856 & 16,831 \\
\hline \% of Target & $112 \%$ & $109 \%$ & $104 \%$ \\
\hline Nameplate Capacity (MW) & 1,249 & 2,488 & 4,727 \\
\hline Capacity Factor & 0.41 & 0.41 & 0.41 \\
\hline Number of Sites & 5 & 8 & 11 \\
\hline Average Site Size (MW) & 250 & 311 & 430 \\
\hline
\end{tabular}

Figure 15, Figure 16, and Figure 17 show the wind sitings for each of the three penetration levels for Nebraska. 


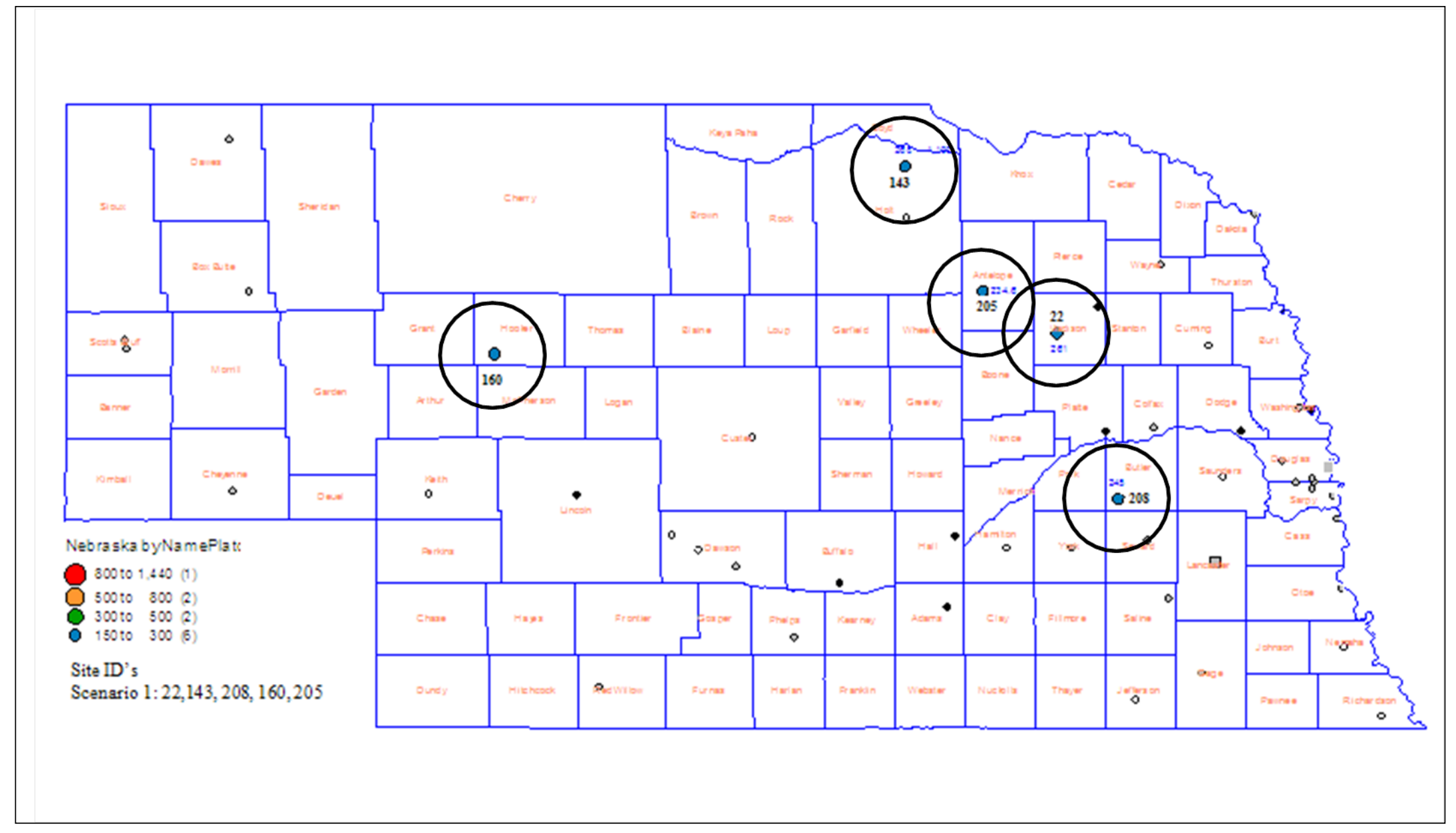

Figure 15: Nebraska 10\% Penetration Wind Siting Map 


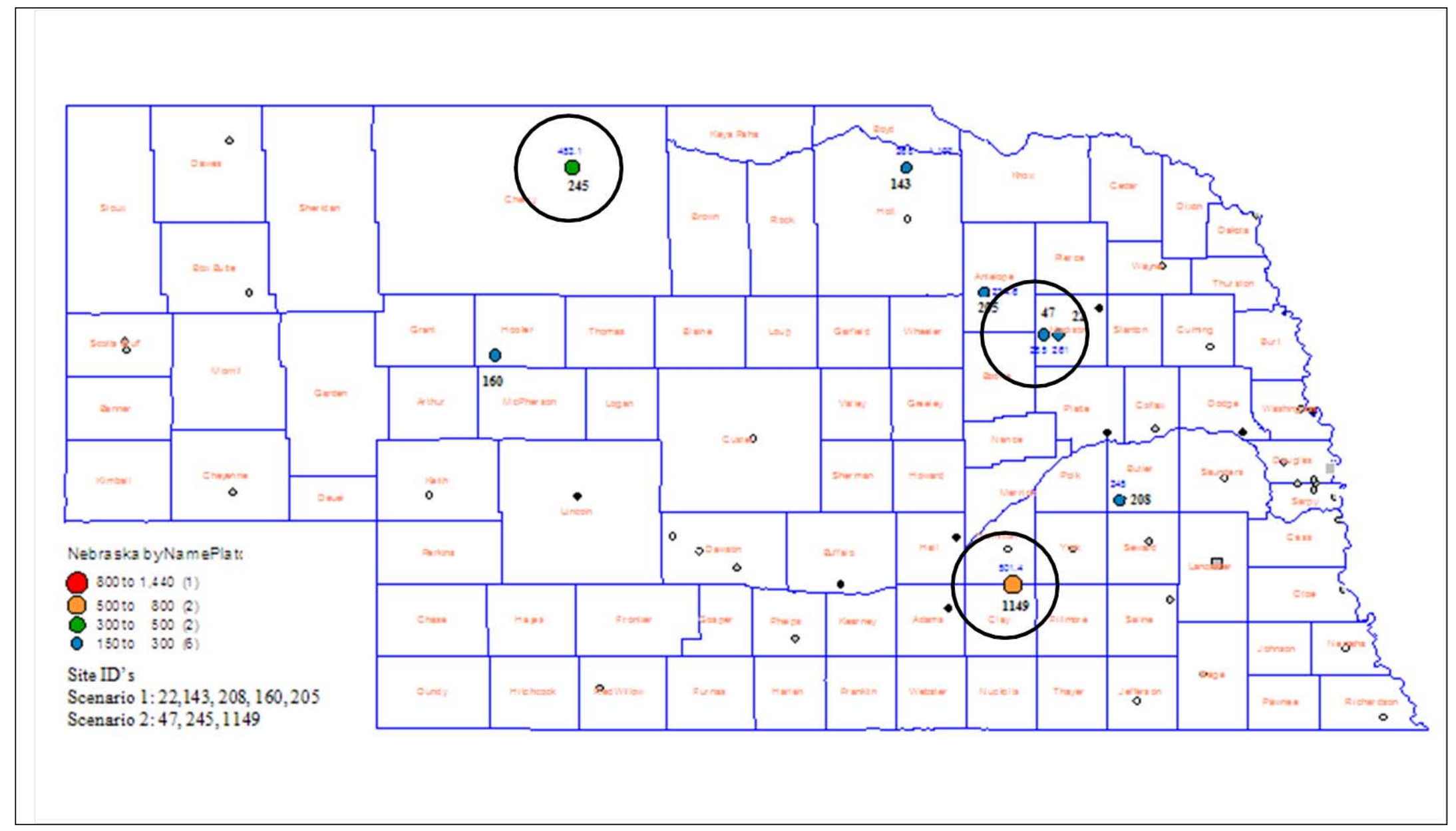

Figure 16: Nebraska Wind Sites Added to 10\% Penetration Sites to Achieve 20\% Penetration 


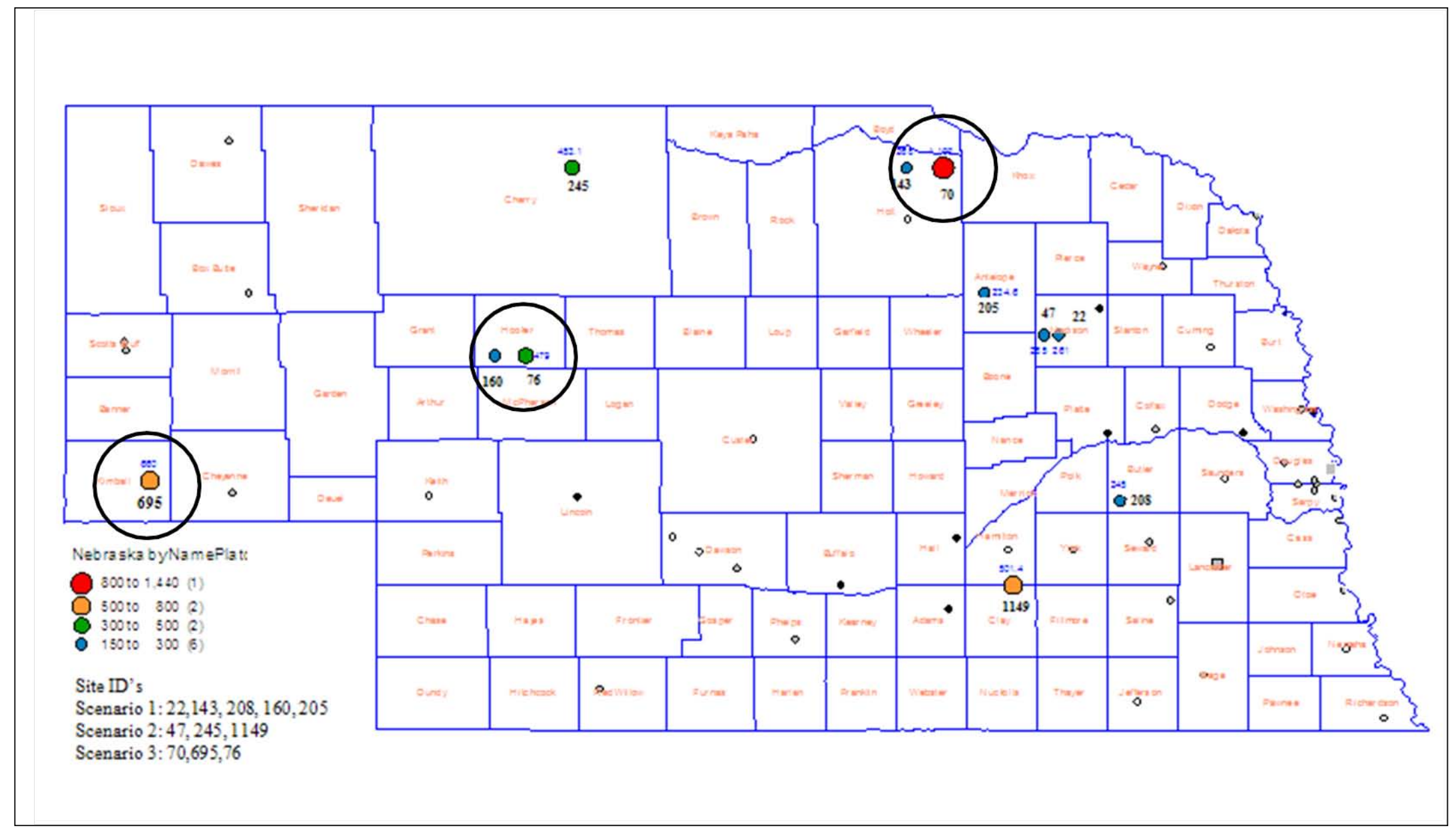

Figure 17: Nebraska Wind Sites Added to 20\% Penetration Sites to Achieve 40\% Penetration 
In a separate activity EnerNex worked with SPP to identify a representative selection of wind sites for each penetration level. In Figure 18 the $40 \%$ penetration scenario shows all wind sites being modeled in the rest of SPP. Table 12 provides a summary of the aggregated wind sites for each penetration level.

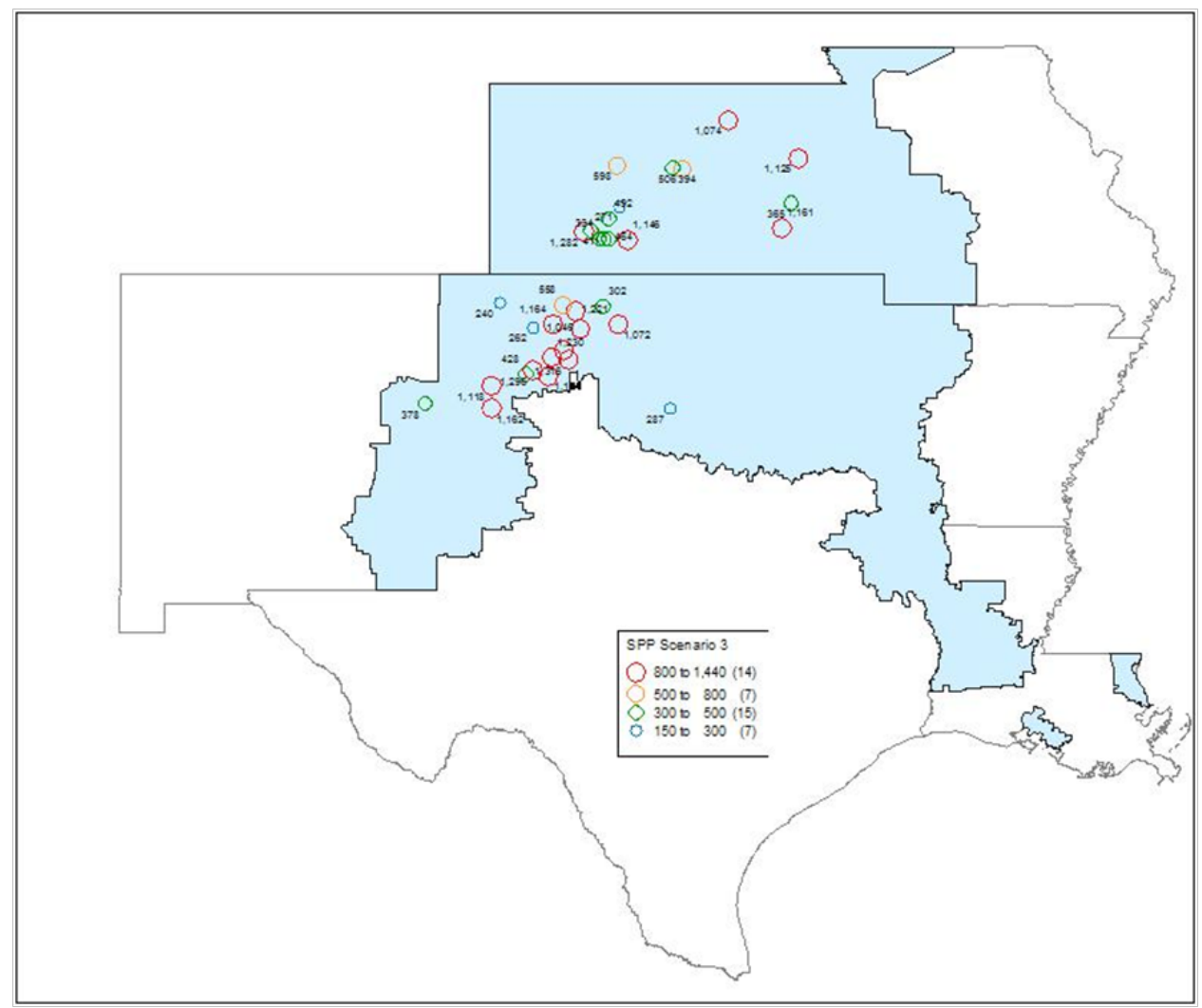

Figure 18: SPP w/o Nebraska $40 \%$ Wind Siting Map

Table 12: SPP w/o Nebraska Wind Site Details

\begin{tabular}{|r|r|r|r|}
\hline Rest of SPP & \multicolumn{1}{|c|}{$10 \%$ Pen } & \multicolumn{1}{|c|}{$20 \%$ Pen } & \multicolumn{1}{c|}{$40 \%$ Pen } \\
\hline Target (GWH) & 22,321 & 44,641 & 89,283 \\
\hline Selected (GWH) & 22,901 & 45,807 & 91,548 \\
\hline \% of Target & $103 \%$ & $103 \%$ & $103 \%$ \\
\hline Nameplate Capacity (MW) & 6,256 & 12,596 & 25,431 \\
\hline Capacity Factor & 0.41 & 0.41 & 0.40 \\
\hline Number of Sites & 7 & 18 & 33 \\
\hline Average Site Size (MW) & 894 & 700 & 771 \\
\hline
\end{tabular}

Figure 19 shows wind sitings for the rest of the system outside of SPP and Nebraska which remained constant throughout the study. It should be noted that the sitings shown in the EWITS map that are in the SPP control area were replaced with the SPP agreed sites. 


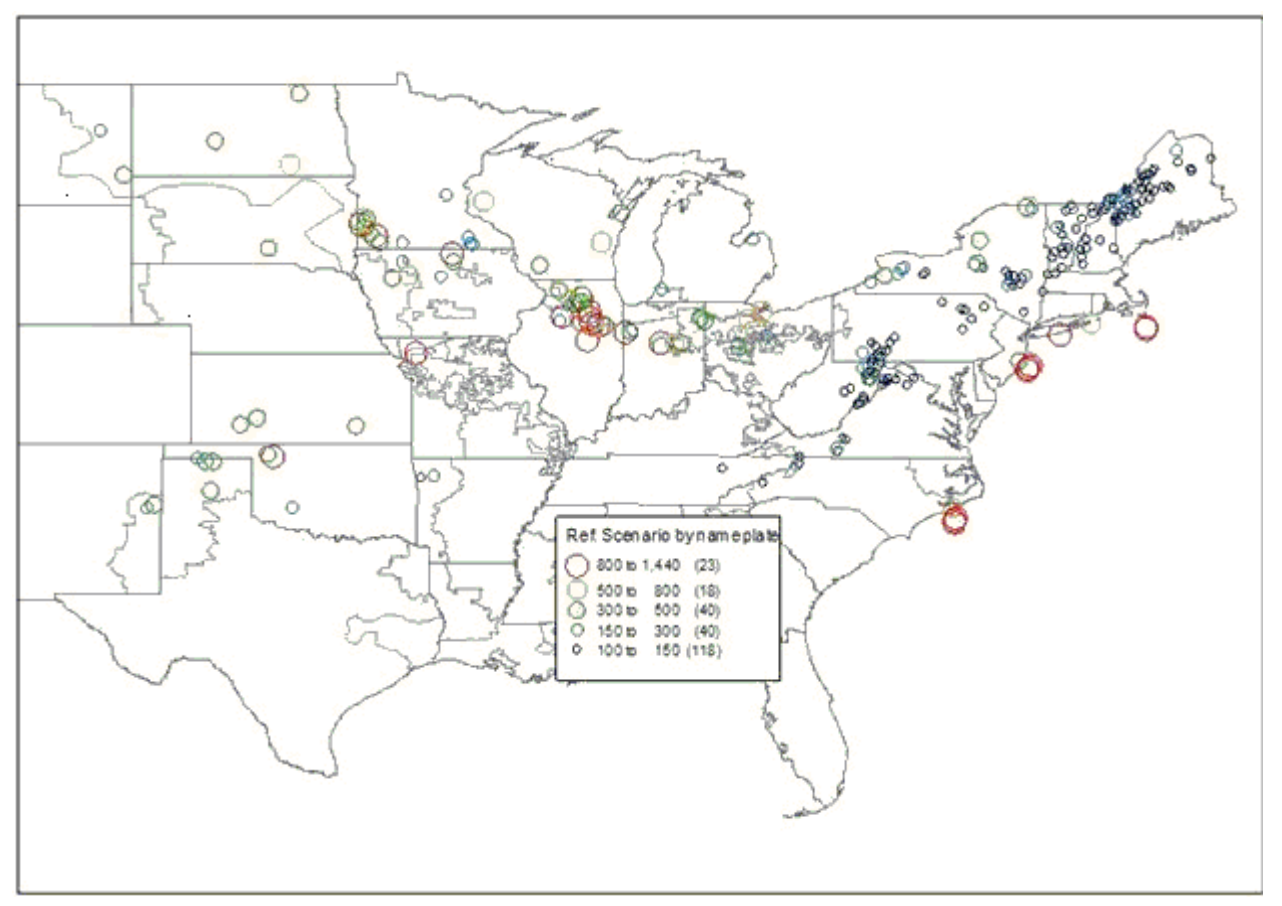

Figure 19: EWITS Reference case less Nebraska sites

\subsection{TRANSMISSION DATA}

\subsubsection{Network Model}

The Nebraska Wind study is focused on conditions in 2018 and thus uses a 2018 representation of transmission system topology, loads and generation. The network model used is from the Eastern Interconnection Wind Study (EWITS) reference case and is the basis for transmission topology representation in the study. This power flow has its origins in the ERAG MMWG 2007 Series 2018 summer peak power flow model and was subsequently modified for the Joint Coordination System Planning (JCSP) process. In addition to that powerflow representation, the EWITS Reference case transmission expansion is included here in order to remain consistent with wind penetration assumptions in areas outside of SPP. Finally the power flow was modified to include SPP balanced portfolio projects and other known Nebraska transmission upgrades. These transmission topology assumptions are combined with constraint representations based on NERC/MISO/SPP books of flow gates and reviewed by NPA staff for Nebraska specific constraints. A list of flowgates modeled in Nebraska is shown in Table 66 of the Appendix Section 8.3.1. DC Tie schedules in western Nebraska were set to zero.

\subsection{2. $\quad$ SPP - Transmission Model (No Overlay Cases)}

As mentioned above particular focus was paid to transmission upgrades in SPP that will be in place by 2018. Specifically, the model was adjusted to include the Balanced Portfolio 3E "Adjusted" transmission projects endorsed by the SPP Markets and Operations Policy Committee on April 15, 2009. A map of these projects is shown on page 5 of the SPP Balanced Portfolio Report (6/23/2009) available at http://www.spp.org/publications/2009 Balanced 
Portfolio - Final Approved Report.pdf. In addition, other lines recently under construction or recently placed in service in Nebraska were also added to this model. The base transmission upgrades (of $345 \mathrm{kV}$ or higher) used in all scenarios are shown in Figure 20 below.

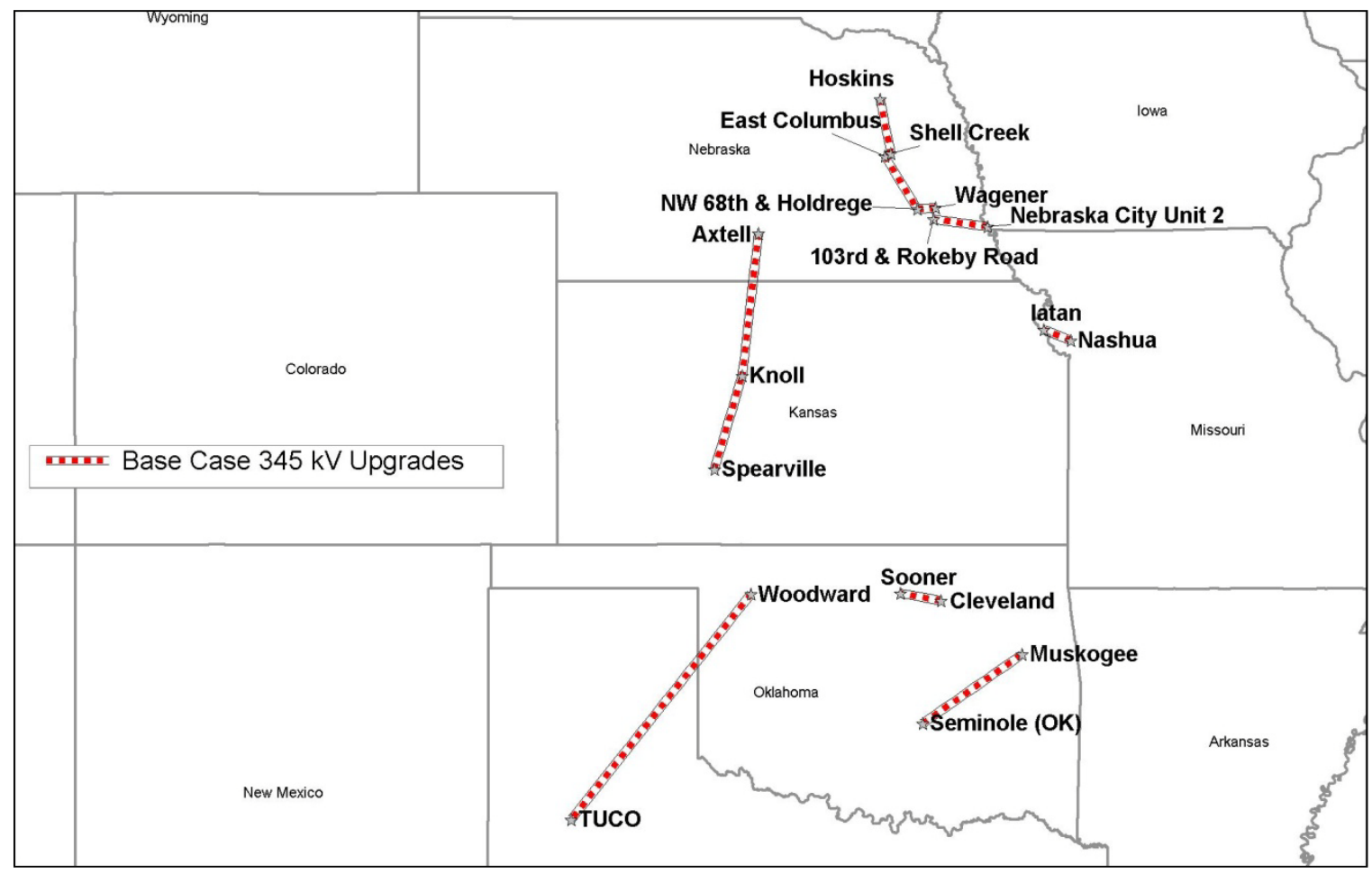

Figure 20: Base Transmission Upgrades Used in all Scenarios.

\subsection{3. $\quad$ SPP -Transmission Model - EHV Overlay}

As described previously, Scenarios $3(20 \%)$ and 4 (40\%) include an assumption of a conceptual EHV overlay for SPP in order to simulate transmission needed to deliver large amounts of wind. This overlay primarily consists of $765 \mathrm{kV}$ lines and is shown as Figure 14 in the DRAFT 2008 SPP EHV Overlay Report (12/26-2008) available at http://www.spp.org/publications/2008\%20SPP\%20\%20EHV\%200verlay\%20Report DRAFT 12 2608 FINAL.pdf

Figure 14 in the SPP Overlay Report is a plan studied for 2,000 MW of wind generation located in Nebraska and 13,500 MW located in the rest of SPP (as reference, in our 2018 model these MW levels would represent about $17 \%$ in Nebraska and $21 \%$ in the rest of SPP). The actual $765 \mathrm{kV}$ Overlay used in the model had a few modifications from the SPP Report's Figure 14 plan, especially in relocating some of the terminals of the Nebraska loop and in making some connections east from the network in the rest of SPP as shown in the overlay Figure 21 of this report. 


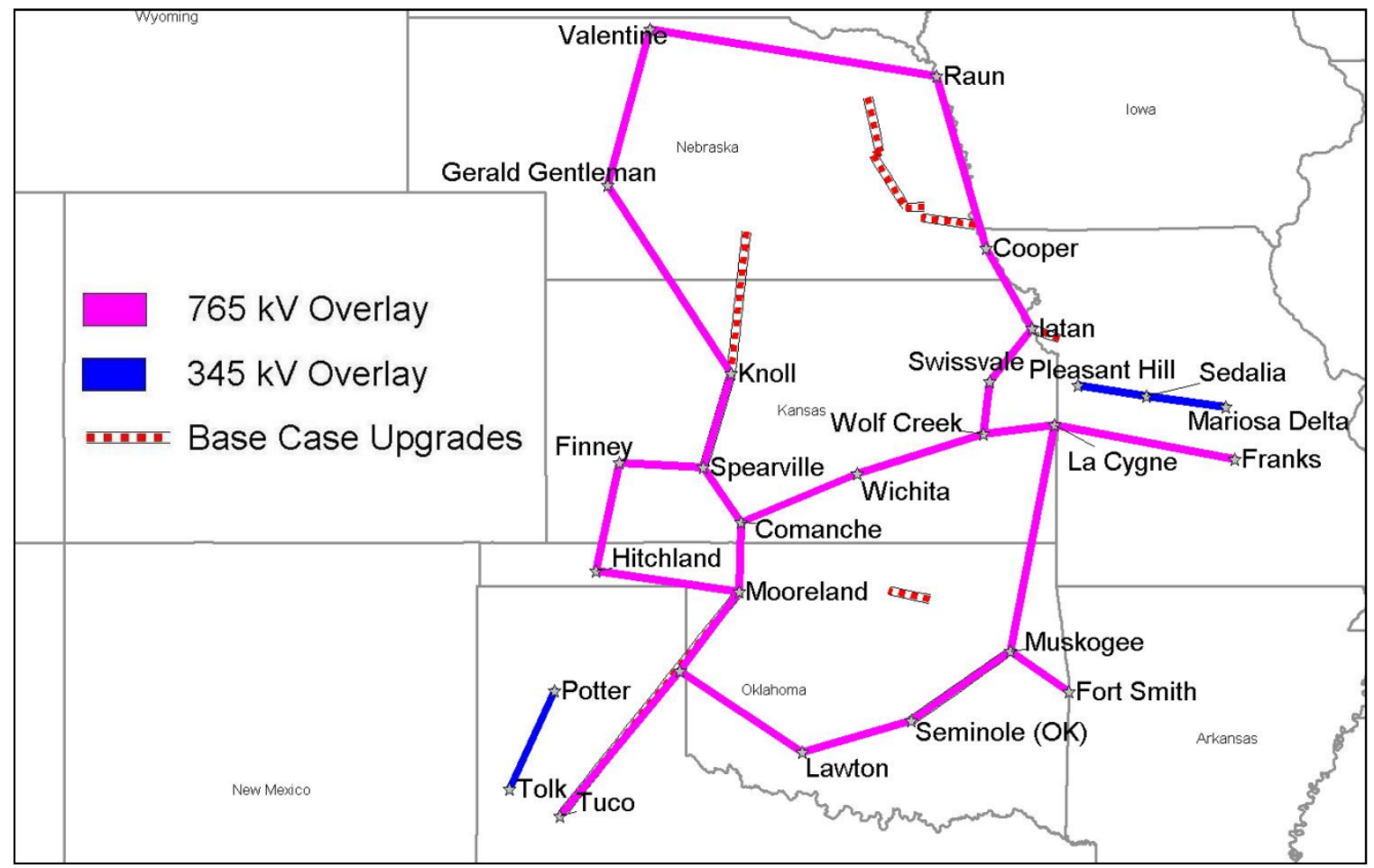

Figure 21: EHV Conceptual Overlay for NPA Study with Base Case Upgrades

Two overlay sensitivity cases were run where the Nebraska $765 \mathrm{kV}$ loop was replaced with $345 \mathrm{kV}$ additions as shown in the Nebraska only 345kV Overlay Figure 22 of this report (the overlay in the rest of SPP remained the same). These cases were run to investigate transmission and generation dispatch efficiencies related to the two voltage levels and associated capacity for the Nebraska transmission overlay. 


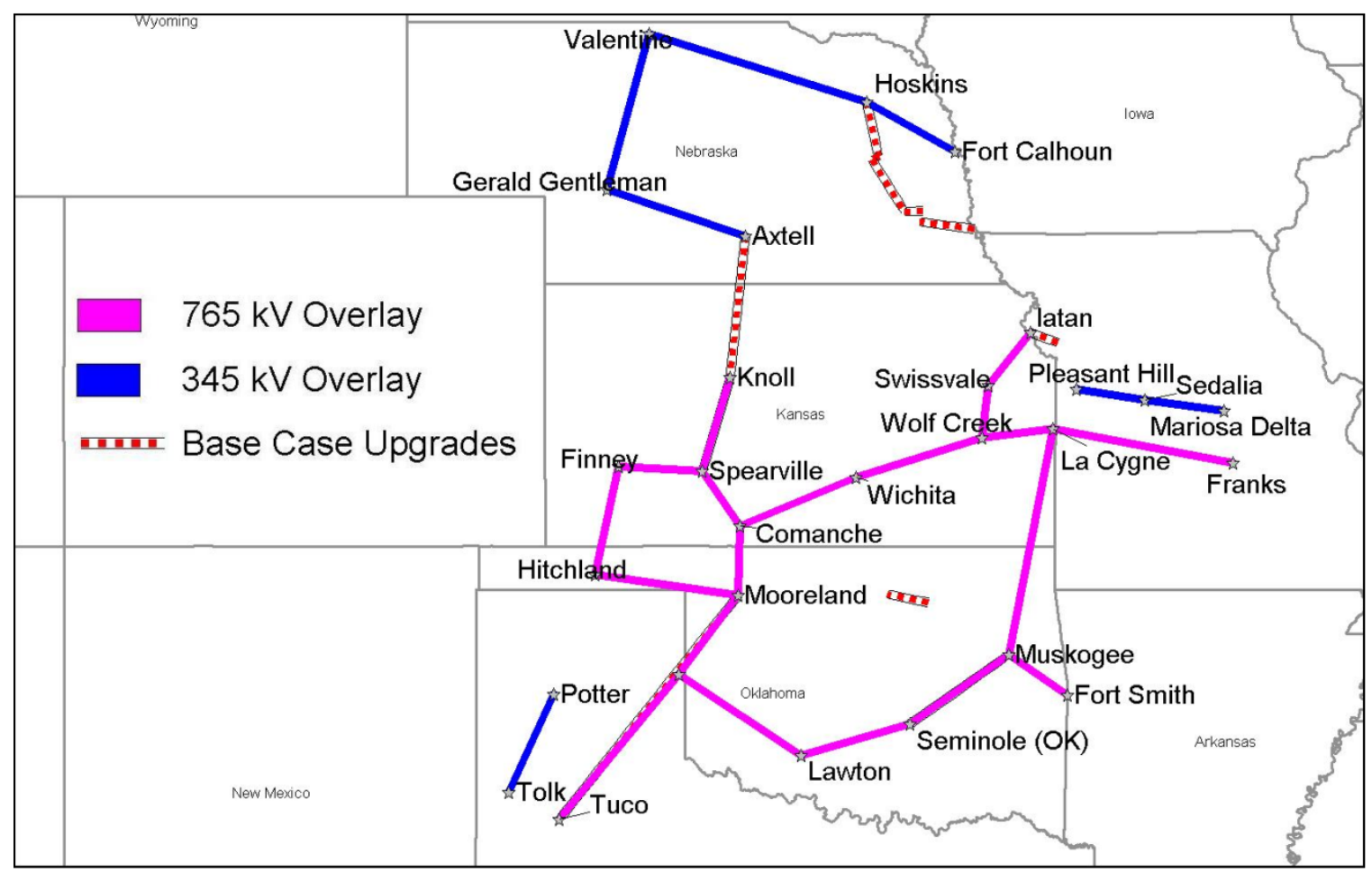

Figure 22: SPP EHV Overlay with Nebraska 345 kV Sensitivity with Base Case Upgrades

At best the comparative performance and cost results for the with and without overlay cases might be useful as a proxy for "what if" type comparisons. Because we were not able to do indepth transmission analysis the transmission-related results have several drawbacks that need to be recognized:

1. Alternative configurations were not studied for either the with- or withoutoverlay cases, except for the limited look at a 345kV alternative for Nebraska.

2. Localized transmission requirements for wind generation injections were not identified; instead limits on facilities localized to the injections were not enforced, but rather the power was allowed to enter the network.

3. Backup/collector $115 \mathrm{kV}-345 \mathrm{kV}$ elements required were not identified so the cost estimates are very approximate.

4. Some of the elements of the overlay are already under consideration for nearterm construction as part of the Priority Projects transmission study process scheduled to be completed early 2010. If some of these projects are approved then they should analytically be a part of the without overlay cases if this study were to be done over. In other words transmission planning is very dynamic at the moment in SPP. 
Considering all these drawbacks, we still present our results on a proxy "what if" basis to show general relationships between transmission capability, wind generation levels, and effects on other generation in the system, both from performance and cost bases.

Performance of the transmission system is evaluated by PROMOD primarily through the calculation of Adjusted Production Cost which can be lowered by virtue of a flexible, highcapacity transmission network that improves deliverability of the lowest cost resources to the loads. As modeled in PROMOD in this study, Adjusted Production Costs are area or regional production costs adjusted to account for purchase and sale energy, by subtracting revenue from sales or adding costs of purchases to the native production costs. Sales are priced at generation weighted LMP for the region and purchases are priced at load weighted LMP for the region. PROMOD monitors flowgates in order to limit to realistic levels places in the network where congestion is likely. Once at a limit, PROMOD would then re-dispatch to next lower cost units that can be delivered through the network.

Section 5.1 describes how the cost of the transmission system requirements were estimated as well as the wind generation and other costs to determine an estimated total cost for each case starting with the PROMOD calculated adjusted production costs.

\subsection{Production Simulation Model Assumptions}

\subsubsection{Modeling footprint}

Although the study focus is on Nebraska and SPP, production simulations were performed using a representation of most of the Eastern Interconnection. This insures a full market for interchange, especially an outlet for wind generation, and minimizes any 'seams issues' near the focus area. The modeled footprint includes:

- SPP - (includes Nebraska)

- MISO - For the purposes of this study Includes WAPA and other MAPP areas

- Saskatchewan \& Manitoba

- All PJM

- All SERC

- TVA - includes E.ON (LGE), Big River, East Kentucky Power Cooperative In the PROMOD runs for this study, except where specified, Nebraska is treated as part of SPP with interchange limited only by transmission capabilities. This means that the resources within the SPP energy market including Nebraska were dispatched as a single control area. Interchange with the rest of the interconnection is limited additionally by a $\$ 5 / \mathrm{MWh}$ tariff (or hurdle rate) in order to simulate market inefficiencies.

\subsubsection{Load Data}

Load forecasts for 2018 are developed from a combination of sources. Forecasts of peak and energy data by operating company are combined with historical hourly load patterns to create an hourly (8760) load shape for the future year being modeled. For this study, historical load shapes from 2004, 2005 and 2006 filed information were combined with the peak and energy forecasts for 2018. Nebraska load forecast data was provided directly by NPA; the remainder of the regional data was developed from filed data and used as provided in Ventyx's Simulation 
Ready Data release from February of 2009. The historical hourly profiles are adjusted to the forecasted peak and energy using an algorithm that maintains the peak and valley hours and attempts to keep essential shape characteristics intact. Table 13 shows the modeled peak (coincident) and energy for 2018.

Table 13: Nebraska and SPP Coincident Peak and Energy

\begin{tabular}{|l|r|r|}
\hline 2018 (2006 Load Shape) & Peak (MW) & Energy (GWh) \\
\hline Nebraska Coincident Peak & 7,489 & 39,405 \\
Rest of SPP Coincident Peak & 46,403 & 225,134 \\
\hline
\end{tabular}

\subsubsection{Generation Data}

Generation data for production simulations includes the generation fleet list and key operating characteristics of those generators including capacity, heat rates, emissions rates, fuel used, start-up costs, O\&M costs, ramp rates, run times, down times and nodal location (bus). These data are all developed from publicly filed data that are then organized into Ventyx's Simulation Ready Data (vintage February 2009) for use in production modeling with PROMOD IV. Nebraska generator lists and characteristics were then reviewed and adjusted where appropriate by NPA staff for this study. See the appendix Section 8.3.1 for a list of Nebraska generation and associated capacity used in the study.

The selected Nebraska wind generation described in Section 2.3.3 was interconnected in the PROMOD model to the transmission system described in Section 2.4 at injection points. These injection points are described in Table 65 of appendix Section 8.3.1.

In the rest of the Eastern Interconnection, in addition to the load and generation data described above, the EWITS Reference Case was used for wind siting and expansion generation resources and held constant for all the NPA cases. This assumption put wind penetration in these areas outside SPP averaging at approximately the 6\% level, although areas nearby to SPP had higher wind penetrations. This represents a view of wind expansion incorporating current Renewable Portfolio Standards. See Table 14 below for penetration levels by energy using 2006 load and wind profiles.

Table 14: Regional Wind Penetrations (2018) Outside of SPP

\begin{tabular}{|l|r|r|r|}
\hline Region & Load (TWh) & Wind (TWh) & Penetration (Energy) \\
\hline PJM & $1,119.82$ & 81.46 & $7.3 \%$ \\
\hline MISO/MAPP & 742.87 & 61.70 & $8.3 \%$ \\
\hline SERC & 502.81 & 11.41 & $2.3 \%$ \\
\hline TVA & 276.77 & 4.41 & $1.6 \%$ \\
\hline Total & $2,642.27$ & 158.97 & $6.0 \%$ \\
\hline
\end{tabular}

\subsubsection{Fuel Data}

Fuel forecasts for generators were developed by the Ventyx Advisors fuel team and vetted by the Nebraska wind study project team and the TRC. To forecast future burner-tip gas prices, Ventyx incorporates a fundamental gas forecasting model into the forecast methodology for medium- to long-term analysis. The model is a general equilibrium model of gas supply and 
demand in a competitive environment for the North American natural gas industry. The Henry Hub gas price forecast through 2018 is shown in Figure 23.

In addition there was a $\$ 25 /$ short ton $\mathrm{CO} 2$ emission charge used in the study.

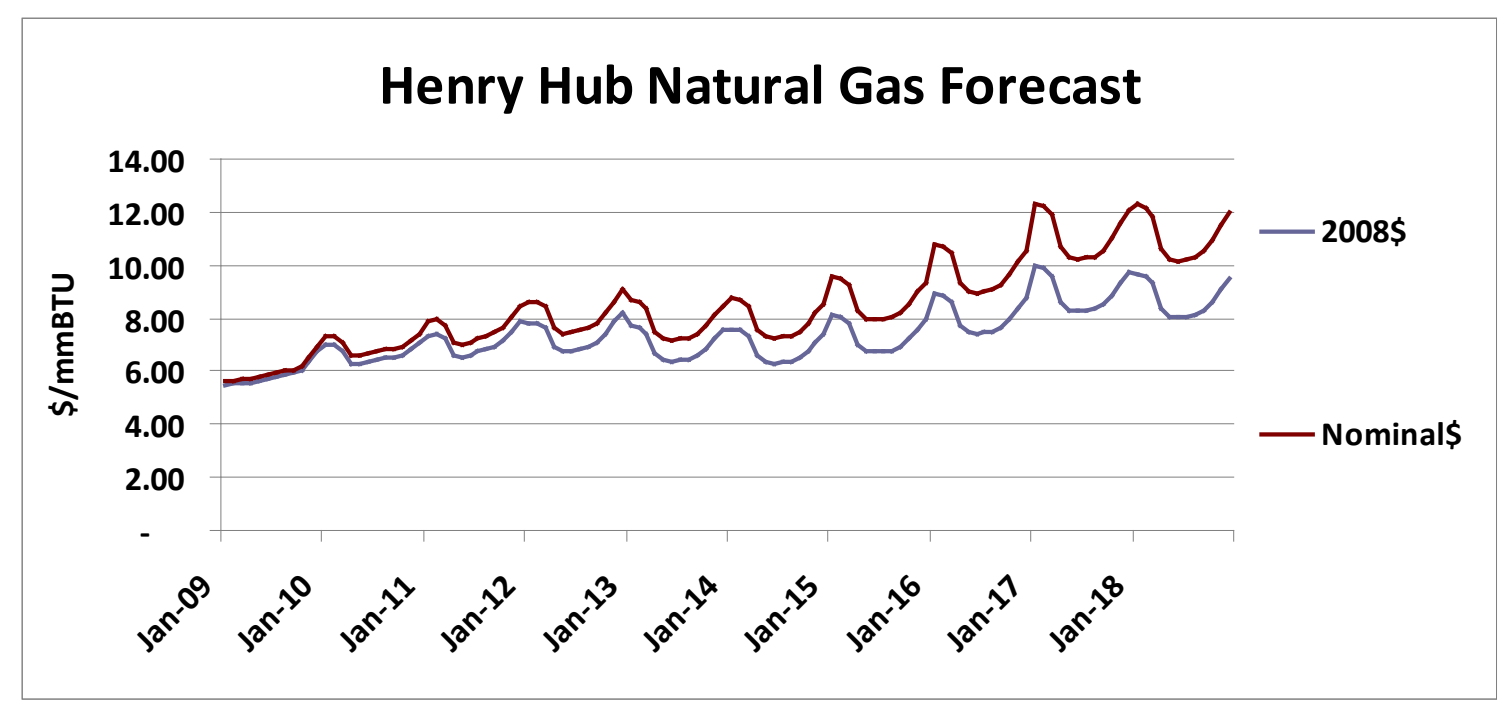

Figure 23: Henry Hub Gas Price Forecast

\subsubsection{Wind Generation Bid Price}

The modeling approach for wind generation in this study was to locate the generation at injection buses and treat the wind generation as a dispatchable resource having a bid price of \$-40/MWh (assuming \$15/MWh Renewable Energy Credit, REC, and \$25/MWh Production Tax Credit, PTC) when it goes through the unit commitment and dispatch simulation process described in Section 3.3 and Section 4.2. This negative bid-in price causes the wind generation to be taken before any other generation as long as all transmission and other constraints are satisfied. If the locational marginal price (LMP) at that bus is below that bid price (due to transmission congestion or minimum generation constraints), then PROMOD IV will curtail an amount of wind that results in the LMP rising above that threshold.

Note that this bid price is not reflected as a cost in adjusted production cost. In adjusted production cost wind is priced at $\$ 0 / \mathrm{MWh}$; wind costs are considered in the total costs section.

\subsubsection{Dollar Cost Basis}

Throughout the report dollars are given in 2018 nominal values unless otherwise noted.

Current cost numbers were escalated to 2018 using various rates depending on the particular cost element. Representation of results in 2018 nominal dollar terms is a common utility procedure and provides for the resulting (estimated) future relationship between such cost elements. However, for the purposes of potential comparison to other studies, normalized wind integration cost results are presented in 2009 dollar terms by deescalating the 2018 results to 2009 at 2.5\% per year. Additionally the cost value given in Section 6 is presented on a 2008 dollar basis. 


\section{Section 3}

\section{POWER SySTEM Regulation AND BALANCING WITH SUBSTANTIAL Wind Generation}

Matching the supply of electric energy to the electric demand, over time frames ranging from seconds to decades, is a fundamental building block for maintaining high bulk power system reliability. The additional variability and uncertainty introduced by wind generation makes the general task incrementally more challenging.

\subsection{POWER SyStem Operation ANd CONTROL}

Power system operation is near the "real-time" end of the spectrum of the operating horizon referred to above. There are a number of functions which need to be performed to maintain the reliability of the system in day-to-day operations. These functions were traditionally performed by individual utility "control areas" and now may be performed by one or several NERC-approved entities in a Balancing Authority. These reliability functions can be categorized by different names and sometimes broken down into more components depending on the context. These functions or "ancillary services" include:

1. Scheduling (unit commitment), system control, and dispatch

2. Reactive supply and voltage control from generation

3. Energy imbalance

4. Regulation and frequency response

5. Operating reserve - spinning

6. Operating reserve - supplemental (e.g. non-spinning)

7. Generator imbalance

As a result of the U.S. Energy Policy Act of 2005, reliability standards are now mandatory, and the North American Electric Reliability Corporation (NERC) is the federally-mandated Electric Reliability Organization (ERO). In the NERC Functional Model, the term for the actions above is "Reliability-Related Services", and include the range of services other than the supply of energy for load that are physically provided by generators, transmitters, and loads in order to maintain reliability. 


\subsubsection{Operational Structure}

A Balancing Authority (BA) operates within metered boundaries that define a Balancing Authority Area (BAA). Every element of the Bulk Power System - generator, transmission facility, end-use customer - is in one and only one BAA.

The four synchronous interconnections in the U.S. each are comprised of one or more BAAs (ERCOT and Quebec are single BAA's). The original BAAs except for the three "tight" power pools in the Northeast (New England, New York and PJM) were individual electric utility control areas. With restructuring of the electric power industry over the previous two decades and the emergence of wholesale energy markets, the number of both BAAs and BAs has been reduced (Figure 24). Further consolidation is expected over the coming years. The Midwest ISO and SPP RTO are examples. BAs that are part of the Midwest ISO (shown as MISO RTO on Figure 25) energy market, located in the MRO, MAIN, SERC, and RFC Regional Reliability Organizations were consolidated under a single BAA with the start-up of the MISO ancillary services market. The SPP RTO began market operations with an Energy Imbalance Service and is transitioning to other offerings that may eventually supplant traditional individual balancing authority functions within its market footprint.

In this study, the subset of Reliability-related Services that involve the control of generation to meet demand, facilitate the delivery of wind energy, and maintain the security of the bulk power system are of primary interest. Further, in this chapter the focus is on the control of generation in real time in response to the changes in wind generation and load. The generation capacity assigned to serve these roles is generally known as "reserves", and specific categories of reserves are designated to fulfill specific functions.

The terminology for reserves is not rigidly defined, and varies by region and country. For example, common definitions for operating reserve categories used in the UCTE in Europe are different that those in the U.S. Even within the U.S., variations in operational practice have lead to reserve definitions that are not uniform across the country.

Relevant definitions from the NERC Glossary of Terms are presented in Table 15. It can be seen that the definitions are somewhat overlapping - operating reserve is comprised of regulating reserve and contingency reserve - and not completely consistent or precise; "operating reserve - spinning" does not seem to include regulating reserve, while the general category of operating reserve does. Mapping each of these terms to the Reliability-related Services (enumerated above) in the NERC Functional Model is also not straightforward.

For purposes of this study, the categories of operating reserve to be specifically evaluated consist of the following:

- $\quad$ Regulating Reserve - generation responsive to automatic generation control that is adjusted to support the frequency of the interconnection and compensate for errors in short-term forecasts of balancing area demand.

- $\quad$ Contingency Reserve - the unloaded capacity carried to guard against major system disruptions, such as the sudden loss of a large generating unit or major transmission facility. 
- $\quad$ Contingency Reserve (Spinning) - that portion of the contingency reserves that is synchronized to the system and fully available serve load within the time specified by the NERC Disturbance Control Standard (DCS).

- $\quad$ Contingency Reserve (Supplemental) - that portion of the contingency reserve consisting of generation that is either synchronized to the system or capable of being synchronized to the system within a specified window of time that is fully available to serve load within the time specified by the NERC DCS.

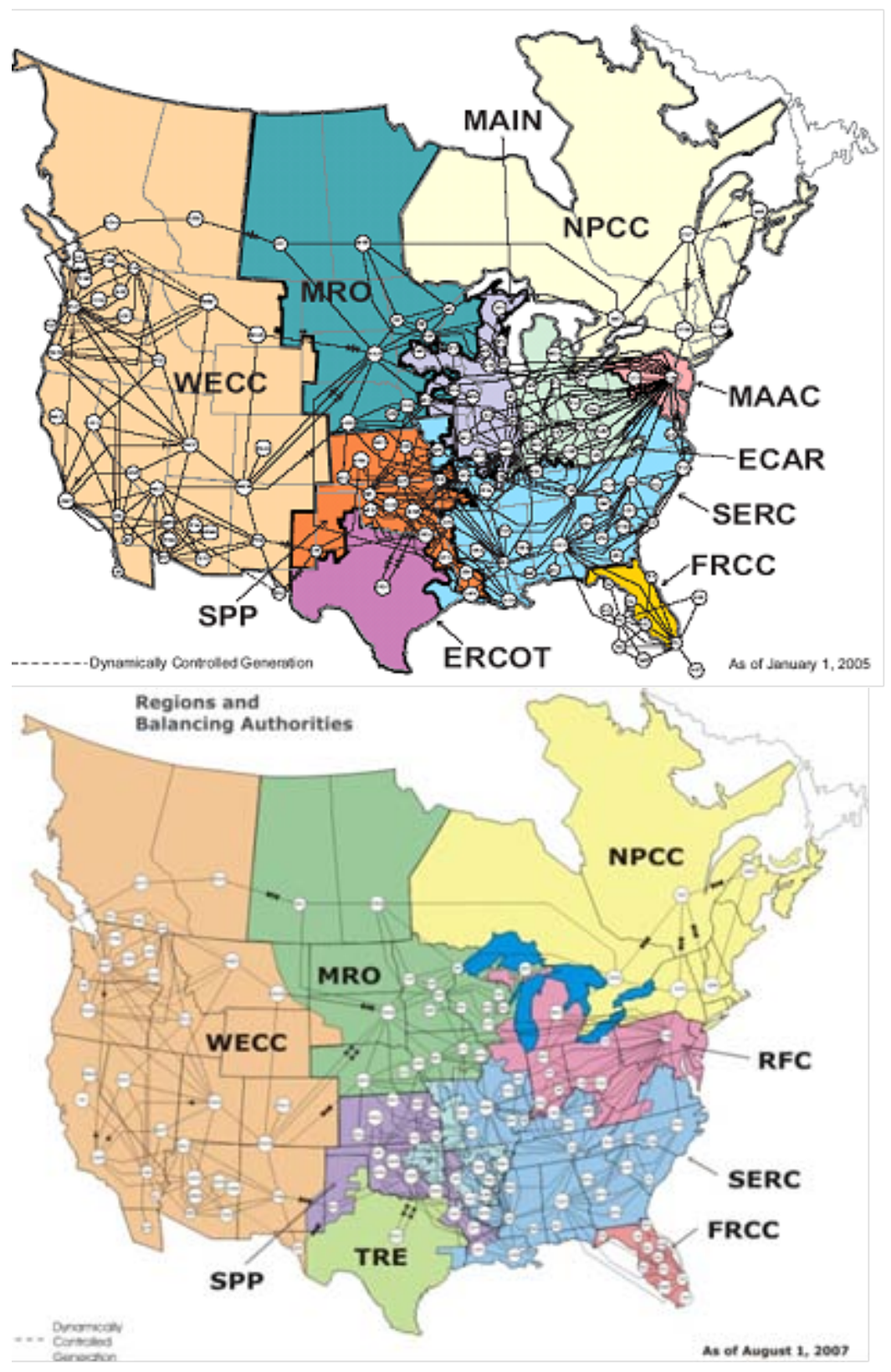

Figure 24: NERC Reliability Regions and Balancing Authorities as of January 2005 (top) and August 2007 (bottom). 


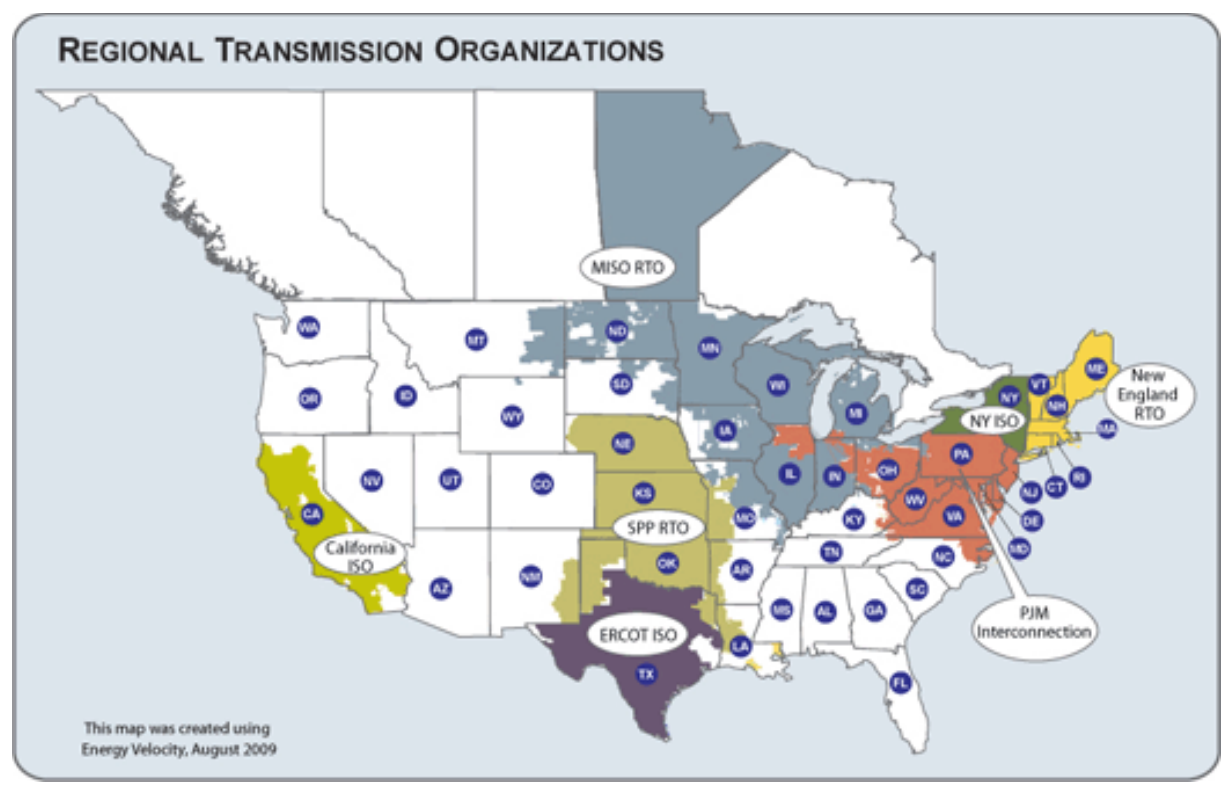

Figure 25: U.S. Regional Transmission Organizations (RTOs) 
Table 15: Excerpts from NERC Glossary of Terms Related to Operating Reserves

\begin{tabular}{|c|c|}
\hline Term & Definition \\
\hline Ancillary Service & $\begin{array}{l}\text { Those services that are necessary to support the transmission of capacity and energy } \\
\text { from resources to loads while maintaining reliable operation of the Transmission } \\
\text { Service Provider's transmission system in accordance with good utility practice. } \\
\text { (From FERC order } 888-A \text {.) }\end{array}$ \\
\hline Contingency Reserve & $\begin{array}{l}\text { The provision of capacity deployed by the Balancing Authority to meet the } \\
\text { Disturbance Control Standard (DCS) and other NERC and Regional Reliability } \\
\text { Organization contingency requirements. }\end{array}$ \\
\hline Operating Reserve & $\begin{array}{l}\text { That capability above firm system demand required to provide for regulation, load } \\
\text { forecasting error, equipment forced and scheduled outages and local area } \\
\text { protection. It consists of spinning and non-spinning reserve. }\end{array}$ \\
\hline $\begin{array}{l}\text { Operating Reserve - } \\
\text { Spinning }\end{array}$ & $\begin{array}{l}\text { The portion of Operating Reserve consisting of: } \\
\text { - Generation synchronized to the system and fully available to serve load } \\
\text { within the Disturbance Recovery Period following the contingency event; } \\
\text { or } \\
\text { - Load fully removable from the system within the Disturbance Recovery } \\
\text { Period following the contingency event. }\end{array}$ \\
\hline $\begin{array}{l}\text { Operating Reserve - } \\
\text { Supplemental }\end{array}$ & $\begin{array}{l}\text { The portion of Operating Reserve consisting of: } \\
\text { - Generation (synchronized or capable of being synchronized to the system) } \\
\text { that is fully available to serve load within the Disturbance Recovery Period } \\
\text { following the contingency event; or } \\
\text { - Load fully removable from the system within the Disturbance Recovery } \\
\text { Period following the contingency event. }\end{array}$ \\
\hline Regulating Reserve & $\begin{array}{l}\text { An amount of reserve responsive to Automatic Generation Control, which is } \\
\text { sufficient to provide normal regulating margin. }\end{array}$ \\
\hline Spinning Reserve & Unloaded generation that is synchronized and ready to serve additional demand. \\
\hline
\end{tabular}

\subsubsection{Managing Variability}

Each BAA must assist the larger interconnection with maintaining frequency at the target level (usually $60 \mathrm{~Hz}$ ) and maintain scheduled energy flows to BAAs with which it is interconnected. Balancing real power supply with real power demand is the means by which frequency is maintained. Regulation and load following are mechanisms for achieving this control under normal operating conditions. Figure 26 illustrates the load characteristics that drive the demand for these services. Variations in the aggregate electric demand are continuous, and can be roughly separated into two components:

- $\quad$ Fast variations that are nearly random in nature, consequences of a great number (millions) of individual decisions or actions such as the flipping of light switches.

- $\quad$ Slower trends that are relatively predictable, such as the rising load in the morning and the falling load through the evening into nighttime. 


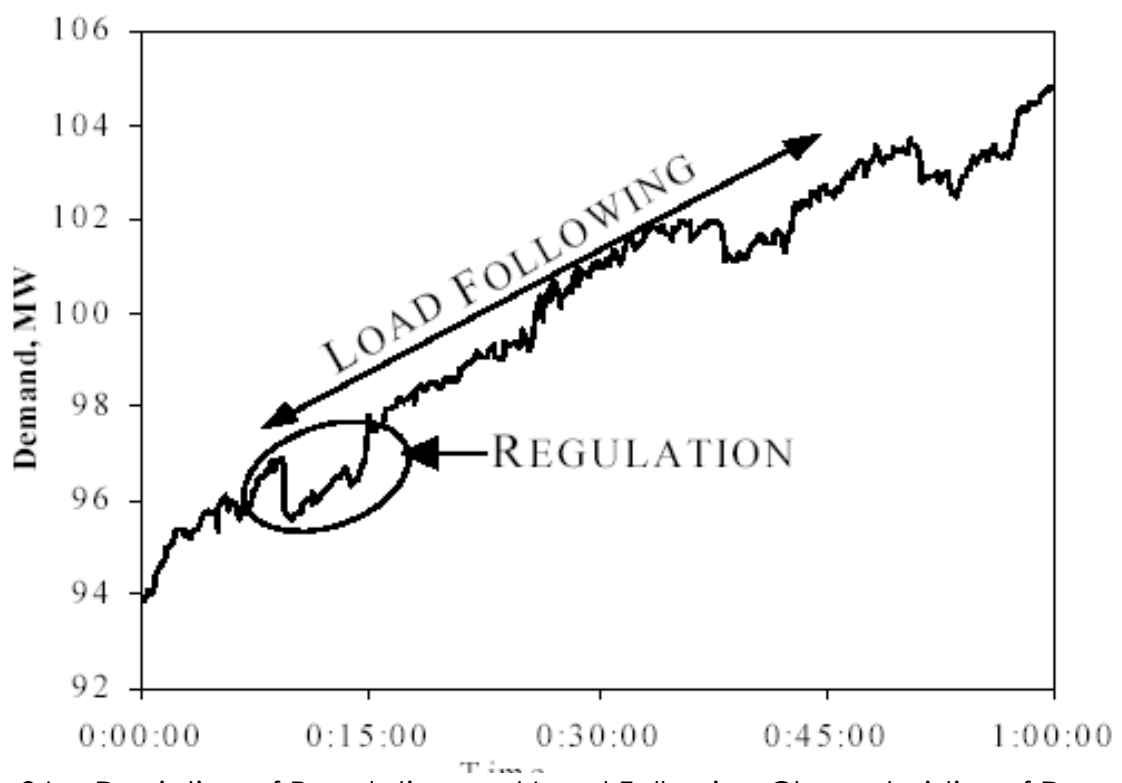

Figure 26: Depiction of Regulation and Load Following Characteristics of Demand.

Generation units on regulation duty are adjusted to compensate for random or sudden changes in demand. These adjustments take place automatically through AGC and occur, depending on the characteristics of the balancing area, over periods of tens of seconds to a minute.

Regulation movements are required both up and down, and the amount of net energy over a period is small as the movements tend to cancel each other. To provide regulation, therefore, a generating unit must reserve capacity and operate below its maximum (to reserve room for upward movement) and above its minimum (for downward movement). In addition, only generating units that meet the BA's requirements for providing regulation and frequency service can participate in the regulation market.

The term "load following" does not appear in the NERC Glossary of Terms, but is generally taken to mean the adjustment of generation over periods of several minutes to hours to compensate for changes in demand. Generation movement is in response to economic dispatch commands from the balancing area energy management system. In real-time or sub-hourly energy markets, clearing points are determined from short-term forecasts of demand, and generating units participating in that market are instructed to move to the forecasted clearing point. Subhourly market intervals of as short as five minutes are in use today, with the clearing points established two or three intervals prior.

Sub-hourly markets are dispatched economically, meaning that the least costly units available (i.e., participating in the sub-hourly market) that satisfy system security constraints are called upon to follow the forecasted change in demand. Providing regulation service requires a commitment on the part of generators to leave capacity both up and down and to allow their units to be moved automatically by the market operator. Consequently, analysis of current market operation reveals that regulation can be quite expensive [1]. Conversely, load following obtained via sub-hourly markets is not. While prices within the hour can vary dramatically, on average prices in sub-hourly markets track day-ahead energy prices quite closely. This has 
important consequences for the methodology used to calculate incremental operating reserve requirements with large amounts of wind generation.

\subsubsection{Measuring Control Performance}

A running evaluation of control performance is kept for each Balancing Authority Area. The primary measure of control performance is Area Control Error, or ACE. The equation for a BAA's ACE has interchange and frequency error terms, and is written as:

$$
\mathrm{ACE}=\left(\mathrm{NI}_{\mathrm{A}}-\mathrm{NI}_{\mathrm{S}}\right)-10 ß\left(\mathrm{~F}_{\mathrm{A}}-\mathrm{F}_{\mathrm{S}}\right)-\mathrm{I}_{\mathrm{ME}}
$$

Where

$$
\begin{aligned}
& \mathrm{NI}_{\mathrm{A}}=\text { the sum of the actual interchange with other balancing areas } \\
& \mathrm{NI}_{\mathrm{S}}=\text { the total scheduled interchange with other balancing areas } \\
& \beta=\quad \text { the balancing area frequency bias, reflecting the fact that load will change with } \\
& \text { frequency } \\
& \mathrm{F}_{\mathrm{A}}=\text { the actual frequency of the interconnection } \\
& \mathrm{F}_{\mathrm{S}}=\text { the scheduled frequency of the interconnection; this is usually } 60 \mathrm{~Hz} \text {, although } \\
& \text { there are times when the scheduled frequency is slightly above or below the } \\
& \mathrm{I}_{\mathrm{ME}}=\text { mominal value to affect what is known as "time error correction" }
\end{aligned}
$$

ACE is computed automatically by the balancing area EMS every few seconds. The adequacy of generation adjustments by the balancing area operators and the EMS are gauged by two metrics that use ACE as an input. The first metric, Control Performance Standard 1 (CPS1), uses ACE values averaged over a 1 minute period. It is a measure of how the BA is helping to support and manage the frequency of the entire interconnection. If the interconnection frequency is low, it signifies that there is more demand than generation (the "machine" is slowing down"). If a particular balancing area has a negative ACE, it is contributing to this frequency depression. Conversely, if ACE were positive during that period, over-generation in the balancing area is helping to restore the interconnect frequency.

The CPS1 "score" for balancing authorities is based on performance over a rolling 12-month period. This score must be greater than $100 \%$, which is an artifact of the equations used to compute the compliance factor. Maintaining adequate capacity on automatic generation control is a major factor in complying with CPS1.

The second metric is Control Performance Standard 2 (CPS2). It utilizes the average ACE over a ten minute period. Over each period, the ten-minute average ACE for a balancing area must be within specific bounds, known as $L_{10}$. These bounds are unique for each balancing area and are based generally on system size. 2009 CPS2 bounds for BAAs in the Eastern Interconnection relevant to this study are shown in Table 16. 
Table 16: 2009 CPS2 Bounds for some Eastern Interconnection Balancing Authority Areas

\begin{tabular}{|c|c|c|c|c|c|c|c|}
\hline & & $\begin{array}{c}\text { Est. Peak } \\
\text { Demand (MW) }\end{array}$ & $\begin{array}{l}\text { Freq. Bias } \\
(\mathrm{MW} / .1 \mathrm{~Hz})\end{array}$ & $\begin{array}{c}\text { Bias/Load } \\
(\%)\end{array}$ & $\begin{array}{c}\text { Bias/Total } \\
\text { Bias (\%) }\end{array}$ & $\begin{array}{l}\mathrm{L} 10 \\
(\mathrm{MW})\end{array}$ & $\begin{array}{c}\text { Variable } \\
\text { Bias? }\end{array}$ \\
\hline SPP & & 44,518 & 483 & 1.08 & 7.42 & & \\
\hline Board of Public Utilities & $\mathrm{KACY}$ & 507 & -7 & 1.38 & 0.11 & 20.08 & \\
\hline Central and Southwest & csws & 10,230 & -102.3 & 1.00 & 1.57 & 76.75 & \\
\hline City of Independence Missouri & $\mathrm{NDN}$ & 329 & -3.3 & 1.00 & 0.05 & 13.78 & \\
\hline City of Lafayette & LAFA & 478 & -5 & 1.05 & 0.08 & 16.97 & \\
\hline Cleco Corporation, Inc. & CIEC & 2,128 & -26 & 1.22 & 0.40 & 38.69 & \\
\hline Empire District Electric Co., The & $\mathrm{EDE}$ & 1,179 & -11.8 & 1.00 & 0.18 & 26.07 & \\
\hline Grand River Dam Authority & GRDA & 998 & -10 & 1.00 & 0.15 & 24.00 & \\
\hline Kansas City Power \& Light, Co & $\mathrm{KCPL}$ & 3,618 & -37 & 1.02 & 0.57 & 46.16 & \\
\hline $\begin{array}{l}\text { Louisiann Energy \& Power } \\
\text { Atrhority }\end{array}$ & LEPA & 221 & -3 & 1.36 & 0.05 & 13.14 & \\
\hline $\begin{array}{l}\text { Missouri Public Service (Aquila } \\
\text { Networks - MPS) }\end{array}$ & MPS & 1,995 & -20 & 1.00 & 0.31 & 33.94 & \\
\hline Oklahoma Gas and Electric & OKGE & 6,570 & -73 & 1.11 & 1.12 & 64.83 & \\
\hline $\begin{array}{l}\text { Southwestem Power } \\
\text { Administration }\end{array}$ & SPA & 1,660 & -27 & 1.63 & 0.41 & 39.43 & \\
\hline $\begin{array}{l}\text { Southwestem Public Service } \\
\text { Coupany }\end{array}$ & SPS & 5,646 & -60 & 1.06 & 0.92 & 58.78 & \\
\hline $\begin{array}{l}\text { Sunflower Electric Power } \\
\text { Corporation }\end{array}$ & SECI & 1,086 & -11 & 1.01 & 0.17 & 25.17 & \\
\hline $\begin{array}{l}\text { Westem Farmers Electric } \\
\text { Cooperative }\end{array}$ & WFEC & 1,463 & -14.6 & 1.00 & 0.22 & 28.99 & \\
\hline $\begin{array}{l}\text { Westem Resources dba Westar } \\
\text { Energy }\end{array}$ & $\mathrm{WR}$ & 6,410 & -71.9 & 1.12 & 1.10 & 64.34 & \\
\hline Dairyland Power Coopentive & $\mathrm{DPC}$ & 924 & -9.2 & 1.00 & 0.14 & 23.02 & \\
\hline Lincoln Electric System & LES & 774 & -7.7 & 0.99 & 0.12 & 21.06 & \\
\hline MHEB, Transwission Services & MHEB & 4,487 & 44.9 & 1.00 & 0.69 & 50.85 & \\
\hline MidAmerican Energy Coumany & MEC & 5,136 & -75 & 1.46 & 1.15 & 65.72 & \\
\hline Mascatine Power and Water & MPW & 145 & -1.5 & 1.03 & 0.02 & 9.29 & \\
\hline Nebnaksa Public Power District & NPPD & 3,253 & -45 & 1.38 & 0.69 & 50.90 & \\
\hline Omaha Public Power District & OPPD & 2,859 & -28.6 & 1.00 & 0.44 & 40.58 & \\
\hline SaskPower Grid Control Centre & SPC & 3,246 & -41 & 1.26 & 0.63 & 48.59 & \\
\hline $\begin{array}{l}\text { Westem Area Power } \\
\text { Administration, Upper Great } \\
\text { ra.i... Th.. }\end{array}$ & WAUE & 3,400 & -45 & 1.32 & 0.69 & 50.90 & \\
\hline
\end{tabular}

Source: http://www.nerc.com/docs/oc/rs/CPS2Bounds 2009.9b.pdf

The CPS2 metric is tabulated monthly. To comply with CPS2 requirements, $90 \%$ or more of the ten- minute average ACE values must be within the designated $L_{10}$ bounds for the BA. Minimum performance allows 14.4 violations per day. Most BAs maintain CPS2 scores in the mid $90 \%$ range.

$$
\begin{aligned}
& A V G_{10-\text { minute }}(A C E) \leq L_{10} \\
& \text { CPS2 }=\left[1-\frac{\text { Violations }_{\text {month }}}{\left(\text { Total Periods }_{\text {month }}-\text { Unavailabe Periods }_{\text {month }}\right)}\right] * 100
\end{aligned}
$$

Figure 27: NERC CPS2 equations 
Balancing area compliance with NERC performance standards is defined as a combination of CPS1 and CPS2 scores:

- In compliance: CPS1 $>100 \%$, and CPS2 $>90 \%$

- Out of compliance: CPS1 < $100 \%$, or CPS2 < $90 \%$

Compliance is based solely on control performance relative to the required scores for the two metrics; there is no direct specification of required reserve amounts for each operating area. It is up to each operating area to establish policies and practices to comply with the NERC standards.

Field trials of a new Reliability-Based Control standard (NERC draft standard BAL-007-1) are currently underway. If adopted, two new performance metrics - CPM (Control Performance Measure) and BAAL (Balancing Authority ACE Limit) - would replace CPS1 and CPS2. The new metrics are designed to improve interconnection frequency support, reduce short-term frequency deviations due to ramping associated with transaction schedules, and provide for timely transmission congestion relief. How the new standards affect the challenge of managing significant wind generation in a balancing area has not yet been studied quantitatively.

\subsubsection{Maintaining System Security}

Achieving high reliability requires that the bulk power system be operated in a way that it can withstand the loss of major elements without cascading failure or tripping of additional elements, and be able to resume normal operation within a specified period of time. The Operating Reserve elements of the Reliability-Related Services listed previously are intended for preservation of bulk power system security.

"Contingency reserve" is the traditional name for the spare generating capacity that can be called upon in system emergencies. The spinning portion of the contingency reserve is synchronized with the grid and ready to respond immediately; offline-capacity that can be called upon, started, and synchronized with a defined period of time (10 minutes or 30 minutes) makes up the non-spinning or supplemental contingency reserve.

Unlike reserves for regulation, which are for supporting normal system operations within applicable reliability criteria, contingency reserves which are spinning are not dispatched continuously by AGC in response to ACE And held in reserves for system emergencies. They are also unidirectional, in that the ability to move upward - serve more load - is that attribute being counted as contingency reserve.

Currently, the basis for the required contingency reserves varies across the interconnection. The need is usually defined by the magnitude of the single or two largest loss-of-source events which could result from a single contingency. For example, in an operating region where the largest plant is a $900 \mathrm{MW}$ nuclear unit, enough additional generation must be available to cover the sudden loss of this large unit, assuming it normally operates at its rated output. In many reliability regions, a substantial portion of this additional generation must be synchronized with the grid, i.e. spinning. The required fraction of contingency reserves that must be spinning is in many, but not all, cases around $50 \%$ of total contingency reserves.

Immediately upon loss of the large generator described above, system frequency would begin to decline, as the amount of load now exceeds the available supply. As frequency declines 
however, governors on all generating units whether they are regulating units, units participating in the energy market or operating reserve units would detect the abnormal low frequency, and if the deviation is large enough or exceeds a defined deadband, increase the mechanical power inputs to the generators.

The system operator would use the operating reserves to replace the loss of generation. NERC's Disturbance Control Standard (DCS) requires BA's to rebalance their system within 15 minutes of a major disturbances and to restore the contingency reserves that were deployed at the event inception within 105 minutes.

\section{2. $\quad$ Effects of Wind Generation On Power System Control}

Actions to support frequency and maintain scheduled interchanges in a BAA are driven by the variety of errors in the generation and load balance. Therefore, it is the effects of wind generation variability and uncertainty on the net variability and uncertainty of the BAA aggregate demand that defines how a given amount of wind generation affects power system control. Measurable impacts would be manifested in increased requirements for regulation capacity and load following capability. Wind plants typically do not impact contingency reserve requirements since the individual generators are relatively small.

Previous integration studies have shown that the net variability concept is extremely important, and effects of aggregation and diversity are very powerful. With load alone, it is demonstrated in practice that the normalized variability of larger aggregations of load - i.e. larger BAAs - is much less than for smaller areas. The same phenomenon is observed with wind generation due to spatial and geographic diversity effects. As the number of turbines and the area over which they are installed grow, the aggregate variability declines. When these aggregations increase to span multiple Balancing Authorities, realizing any potential benefit of these aggregations may require consideration of the impacts on current operating protocols.

The effects of diversity on the variability of wind generation are illustrated in Figure 28 using actual wind profile data for the Eastern Interconnection. The curves represent the changes in wind generation over a 10-minute interval; the value plotted is the standard deviation of all incremental changes over three years of data for hourly production levels (in per-unit) corresponding to the value on the horizontal axis. The curves illustrate that more variability can be expected when the wind generation is in the mid-range of the aggregate nameplate production. Secondly, and also of great interest for this study, the per-unit variability declines significantly as more wind is aggregated. 


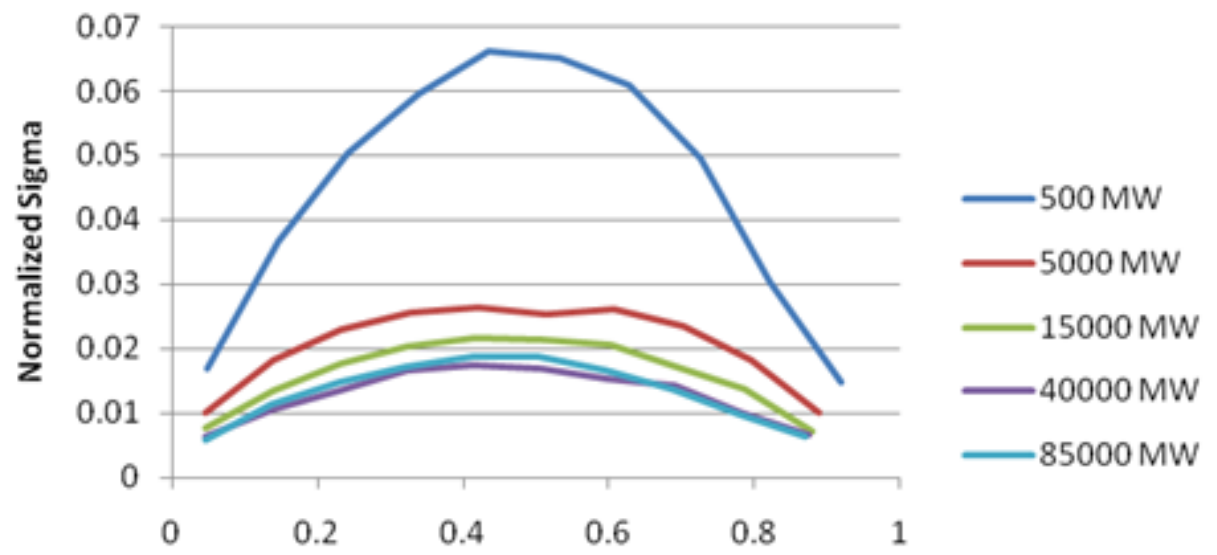

Production Level as Percent of Nameplate

Figure 28: Normalized 10-minute Variability for Five Different Groups of Wind Generation. The 500 MW Scenario is Part of the 5000, which is Part of the 15000

The magnitude of the effects on the variability of the BA net load will depend on the amount of wind generation relative to load, the variability of load alone, and the amount of diversity that characterizes the aggregate wind generation.

Changes in wind generation over other time frames must also be factored into operational practices. Large drops in wind energy production may be of the magnitude of the contingency for which operating reserves are carried, but there is a significant difference in the event duration. The nuclear unit described earlier may be lost in an instant, providing $900 \mathrm{MW}$ one minute and off-line the next. Large reductions in aggregate wind generation do not occur suddenly, but may instead evolve over a one- to several- hour duration. This is due to the large number of individual turbines, the large geographic area over which they are installed, and the time it takes for major meteorological phenomena such as fronts to propagate.

Smaller, but more frequent changes in wind generation over periods of one to four hours are also important operationally. On these time scales, the uncertainty regarding how much wind generation will be available takes on more importance than variability. Because of the short lead time, replacement capacity for forecast wind generation that does not materialize in this timeframe must be found from units already committed, from regulating reserves until economic replacement energy can be committed, units with quick-start capability if insufficient regulating reserves are available, or from a neighboring BA. Consequently, the expected error in wind generation forecasts over these horizons could play a role in the policy and practice for operating reserves. A centralized wind production forecast will assist balancing authorities in mitigating the impact of changes in wind generation; however, a level of operating reserves may still be required to address the remaining errors. 


\subsection{MODELING AND ANALYSIS FOR ASSESSING WIND INTEGRATION IMPACTS}

The analysis of wind generation impacts on power system regulation and balancing for this study had two primary objectives:

- With wind generation and load profile data as a starting point, use engineering judgment and technical knowledge of power system operation and control to develop a methodology for estimating how wind generation in the study scenarios would be managed in real-time operations, and

- Develop a process for mapping these requirements to the chronological production simulations that will be used to assess overall wind integration impacts.

The second bullet is very important to the overall analytical methodology employed in this study. The within-the-hour impacts of varying load and wind generation are accounted for, approximately at least, in the production simulations by setting constraints on the unit commitment and economic dispatch algorithms. In each hour, specified amounts of reserves must be set aside, not used to serve load.

Impacts of changing load and wind generation are more explicitly considered in the production simulation at increments of one hour or more. Additional constraints are defined for each generating unit in terms of the amount that output can be changed over a single hour, maximum and minimum output, start-up and shut down times, and minimum run and minimum down times. The unit commitment and economic dispatch steps must observe these constraints on each unit. Consequently, situations of specific interest for wind integration, such as minimum load and minimum generation periods, are being evaluated in the production simulation program. Violations of constraints are report, or appear as dump energy or load that is not served.

\subsubsection{Assumptions}

There is a trend in the U.S. electric power industry towards larger effective operating pools, either through energy markets or inter-area operating agreements. Previous wind integration studies have concluded that larger operating areas are an effective means for managing wind integration, because they take natural advantage of geographic diversity of load and wind and aggregate a larger set of discrete generating units to compensate for load variations and provide frequent economic dispatch of units with movement capability to follow slower variations in balancing area demand.

For the study horizon of 2018, it was assumed that the interface with SPP including Nebraska will contain four major operating areas corresponding to the current boundaries of the following entities:

- PJM

- MISO

- tVA

- SERC 
It was further assumed that by 2018 all of the operating areas will have a uniform structure in terms of market products, unit dispatch, and real-time operations and fulfill the functions of a Balancing Authority. This structure consists of:

- A day-ahead energy market followed by a security-constrained unit commitment (SCUC) later on the day prior to the operating day.

- A real-time energy market, cleared at frequent intervals during the operating hour. Each real-time market clearing point is based on short-term forecasts of load and wind generation. To line up with the data available for the study, the clearing interval was defined to be 10 minutes, with the market clearing point based on information available at the previous 10-minute interval.

- An ancillary services market, where a large pool of resources competes to provide the defined regulating and operating reserves.

The areas modeled in this study currently operate according to these assumptions in varying degrees. Although the progress in consolidation and advancement of markets in the direction of the study assumptions is significant, the operation of the entire study footprint by these assumptions by 2018 is not a foregone conclusion. Additionally, it is assumed that reserves can be shared across the entire operating area; transmission congestion internal to a region does not create sub-regions with reserve requirements that must be met locally, which will need further investigation.

There is a general recognition that renewable wind, in the current operating and markets constructs, would face very significant barriers to realizing these levels of penetration. Instead, this analysis looks at wind impacts in a possible "future world" operating and market construct that may be able to accommodate high levels of wind. This study also recognizes that considerable work remains to realize this operating scenario.

Existing practice was used as a starting point for determining the amount of regulating reserve required for load alone. A value of $1 \%$ of the hourly load was assumed, although that fraction of the forecast daily peak load for each hour of the day would have been somewhat more reflective of current practice and policies, $1 \%$ of hourly load is a reasonable working assumption.

\subsubsection{Mapping Reserve Requirements for Production Simulations}

The methodology used in this study for assessing the impacts and cost of integrating wind energy into a utility balancing area is based on chronological simulations of scheduling and realtime operations. Production costing and other optimization tools are used to conduct these simulations. In most cases, the "time-step" for these simulations is in one-hour increments. Consequently, many details of real-time operation cannot be simulated explicitly. Generation capacity that is used by operators to manage the system in real-time - i.e. the units on AGC utilized by the EMS for both fast response to ACE and that which is frequently economically redispatched to follow changes in balancing area demand - is assigned to one or more reserve categories defined in the various programs.

At this level of granularity, the reserve requirements for the system are constraints on the optimization and dispatch. Supply resources are designated by their ability to contribute to system requirements in one or more reserve categories. In the course of the optimization or 
dispatch, the solution algorithm must honor system reserve needs, and therefore is not able to use some capacity to meet load or fulfill transactions.

The reserve requirements with wind generation for the study operating areas are computed on a technical basis from the functional considerations for system reliability and security. Utilizing them in the production simulations necessitates the translation of the various components into the reserve categories considered by the simulation tools. For the large scale production simulations in this study, only two types of reserves can be considered: Spinning, and NonSpinning (Synchronized and Non-Synchronized may be clearer terms here, as "spinning" is generally associated with a specific ancillary service; however, since the simulation tools actually uses those terms, they are retained here). .

Table 17 shows the mapping for the reserve types discussed here. Regulating reserve by definition must be spinning, as it must compensate continuously for changes in balancing area demand to assist in controlling the frequency of the interconnection. In the mapping, regulation is divided into two components, which are assumed to be additive. And, per the NERC glossary definition, regulation is also carried to cover errors in demand forecasts, which is assigned to a second category of regulation, for reasons that will be explained later in the section.

Contingency reserve, per the Reliability-Related Services definition, consists of both spinning and non-spinning portions, and is computed for the scenarios as described in the next section.

Table 17: Mapping of Reserve Components in categories for production simulations

\begin{tabular}{lcc}
\hline $\begin{array}{l}\text { Reserve } \\
\text { Component }\end{array}$ & Spinning & Non-Spinning \\
\hline $\begin{array}{l}\text { Regulation } \\
\text { (variability) }\end{array}$ & $100 \%$ & $0 \%$ \\
$\begin{array}{l}\text { Regulation } \\
\text { (forecast error)_ }\end{array}$ & $100 \%$ & $0 \%$ \\
Contingency & $50 \%$ & $50 \%$ \\
\hline
\end{tabular}

\subsubsection{Contingency Reserves}

Since sufficient contingency reserves are maintained to respond to largest generator within a Balancing Authority or as part of a Reserve Sharing Group, it is conceivable that the existing contingency reserves are sufficient to maintain the same level of reliability at varying levels of wind penetration. Contingency reserves would need to be increased if it is determined that the total output of a Wind Plant or multiple Wind Plants within a location are larger than the current contingency and have the potential to trip off-line within a few minutes.

In this study, it is assumed that the spinning and non-spinning (supplemental) contingency reserves are not influenced by the amount of wind generation in the operating area, but rather a function of conventional equipment and the network as is the current practice.

The operating regions defined for this study do not exactly conform to the existing reliability regions and reserve sharing groups. Consequently, it is necessary to define requirements for contingency reserves on some other basis for the study. Adapting existing practice, the total contingency reserve requirement was defined to be 1.5 times the single largest hazard (SLH) in 
each balancing area if no information was available from current practice with at least half required to be spinning.

Assumed contingency reserves requirements for each operating region and scenario are shown in Table 18. The total operating reserve is split 50/50 between spinning and non-spinning (supplemental) except in MISO and TVA.

Table 18: Contingency Reserve Requirements by Operation Region and Scenario for 2018 Total Operating Reserve Requirement

\begin{tabular}{lcc}
\multicolumn{1}{c}{ Region } & $\begin{array}{c}\text { Total Operating Reserve Requirement } \\
-\end{array}$ & $\begin{array}{c}\text { Spinning/Supplemental split } \\
\text { (\%) }\end{array}$ \\
\hline $\begin{array}{l}\text { Nebraska } \\
\text { alone }\end{array}$ & $132 \mathrm{MW}$ & $50 / 50$ \\
$\begin{array}{l}\text { SPP with or } \\
\text { without }\end{array}$ & \\
Nebraska & $1539 \mathrm{MW}$ & $50 / 50$ \\
MISO & $2271 \mathrm{MW}$ & $100 / 0$ \\
\hline TVA & $1750 \mathrm{MW}$ & $23 / 77$ \\
SERC (partial) & $1140 \mathrm{MW}$ & $50 / 50$ \\
\hline
\end{tabular}

\subsubsection{Regulation and Load Following}

The approach for calculating the incremental regulation and load following capacity required to maintain control performance in each of the BAAs defined for the study was based on a number of observations from current market operations and experience from previous studies.

First, the minute-to-minute variability of wind generation, relative to that of the aggregate load, is very small. While the NREL mesoscale data has inadequate resolution for quantifying the fast variations in the study scenarios, measurement data collected by NREL ${ }^{[1]}$ and others has provided an empirical basis for analyzing such variations.

The measurement data in [1] shows that the standard deviation of the minute-to-minute variability - faster than that which can be dealt with by the sub-hourly energy market or subhourly scheduling - is about $1 \mathrm{MW}$ for a $100 \mathrm{MW}$ wind plant, based on separating the fastest variations from longer-term trends using a 20 minute rolling average window. (Note: as the results will show, the details of this process - i.e. resolution of the data, width of the averaging window - are not critical to the results and conclusions).

Minute-to-minute variability is also uncorrelated between individual wind plants and between wind and load. Considering a BAA with 100,000 MW of load and 60,000 MW of wind generation, the impact of wind generation on the fast variations of the net BAA demand can be estimated:

- Assume that the $15,100 \mathrm{MW}$ of wind generation is comprised of $100 \mathrm{MW}$ plants (to use the variability characteristics from above). If each of the $100 \mathrm{MW}$ plants exhibits a minute-to-minute variability of $1 \mathrm{MW}$ (as measured by the standard deviation of these variations), and they are uncorrelated with similar variations from other wind plants in 
the sample, the standard deviation of the variability for all 15,100 MW would be the square root of the sum of the squares of the individual $100 \mathrm{MW}$ plant deviations, shown in the equation below:

$$
\sigma_{\text {aggregate_wind }}=\sqrt{\left(\left(\frac{15,100}{100}\right) \cdot(1 M W)^{2}\right)}=12.3 \mathrm{MW}
$$

- Assume that the $1 \%$ regulation amount carried for load alone (See Section 3.3.1; 50,000 $\mathrm{MW}$ of load in this example) is three times ${ }^{6}$ the standard deviation of the load variability on this same time scale:

$$
\sigma_{\text {load }}=\frac{50,000 M W \cdot 1 \%}{3}=166.7 \mathrm{MW}
$$

- The standard deviation of the load net of wind generation, which is a basis for the regulating reserve, can be computed assuming that the fast variations from load are not correlated with those from the aggregate wind generation:

$$
\sigma_{\text {Total }}=\sqrt{\sigma_{\text {load }}^{2}+\sigma_{\text {aggregate_wind }}^{2}}=167.1 \mathrm{MW}
$$

As the calculation shows, the effect of the fast variations in aggregate wind production is negligible.

Considering the uniform structure assumed for the operating areas in the study, it becomes apparent that wind variability would likely have larger impacts on time scales associated with the sub-hourly markets and economic dispatch of generating resources.

Sub-hourly market clearing points are based on short term forecasts of demand. In an existing 5-minute energy market, for example, the clearing point is based on projections of demand made 15 to 20 minutes prior to the interval. Participating units are instructed to move to cover the projected change in load; any difference between the forecast load and the actual load for the interval (assuming that all generating units follow dispatch instructions precisely) will effectively "spill over" into the regulation bin.

Very short term aggregate forecasts of large amounts of load can be quite accurate. For wind generation, the variations over these same time periods are less so. Errors in the short-term forecast of wind generation will therefore increase the requirement for regulation.

\footnotetext{
${ }^{6}$ A multiple of 3 times the standard deviation encapsulates almost $99.9 \%$ of all samples in a normal distribution. There is precedent in the U.S. electric utility industry for using a multiplier of 3, although there are instances of higher multiples that can be found. The multiplier assumed here is thought to be more appropriate for the very large Balancing Areas defined for the 2018 scenario. In smaller balancing areas, multipliers of up to 5 are used.
} 
The 10-minute resolution wind generation profile data for each scenario is used to estimate this impact on regulation. Using a persistence forecast where the average production for the last several intervals (six intervals in this case) is to the forecast for the next ten-minute interval, the expected error in this simple short-term wind generation forecast can be easily calculated and characterized. Persistence performs reasonably well as a forecast technique for limited horizons, on average. Other techniques may be better for predicting significant ramps, but over all of the intervals in a year may not out-perform simple persistence. The objective here was to employ a simple, yet reasonable, approximation to a more sophisticated approach that would be used in practice.

Figure 29 illustrates the short term forecast errors for load and wind generation with data from one of the scenarios and operating regions. Here it is assumed that the sub-hourly market operates on 10-minute intervals (to match the resolution of data available for this study), and the load forecast is generated one interval prior. A simple regression-extrapolation technique performs very well for forecasting load; this is likely due to the smoothness of the variations. In reality, more sophisticated techniques are used, and can account for the expected load shape and other factors which would further improve performance near peak intervals.

The persistence forecast for wind generation performs reasonably well, but the variations at 10minute intervals for even this large amount of wind generation exhibit more volatility than is observed in the aggregate load. Consequently, the errors in wind generation forecasts dominate the net error, as can be seen in Figure 30.

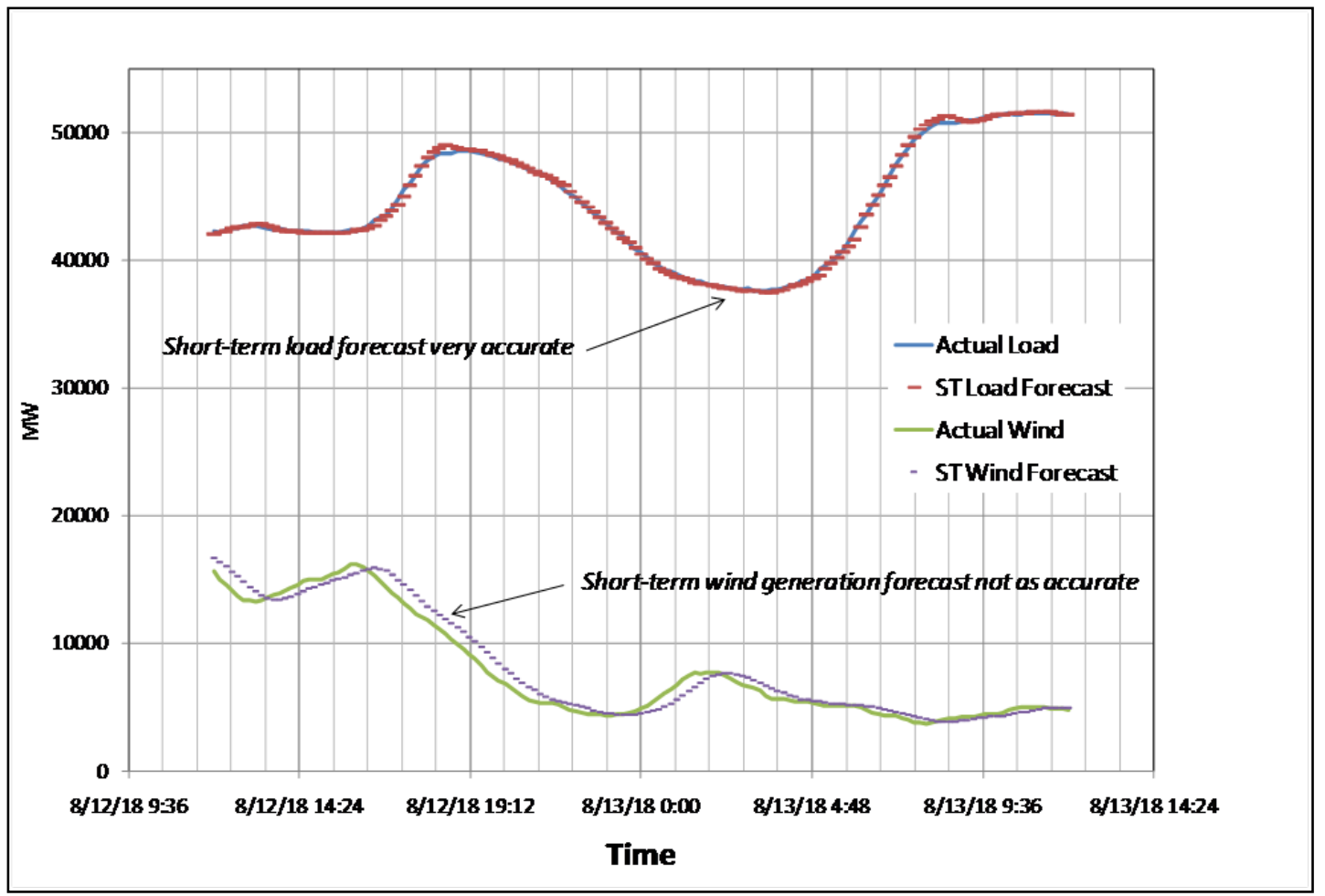

Figure 29: Illustration of Short-Term (next 10-minute interval) Forecasts of Load and Wind Generation. 


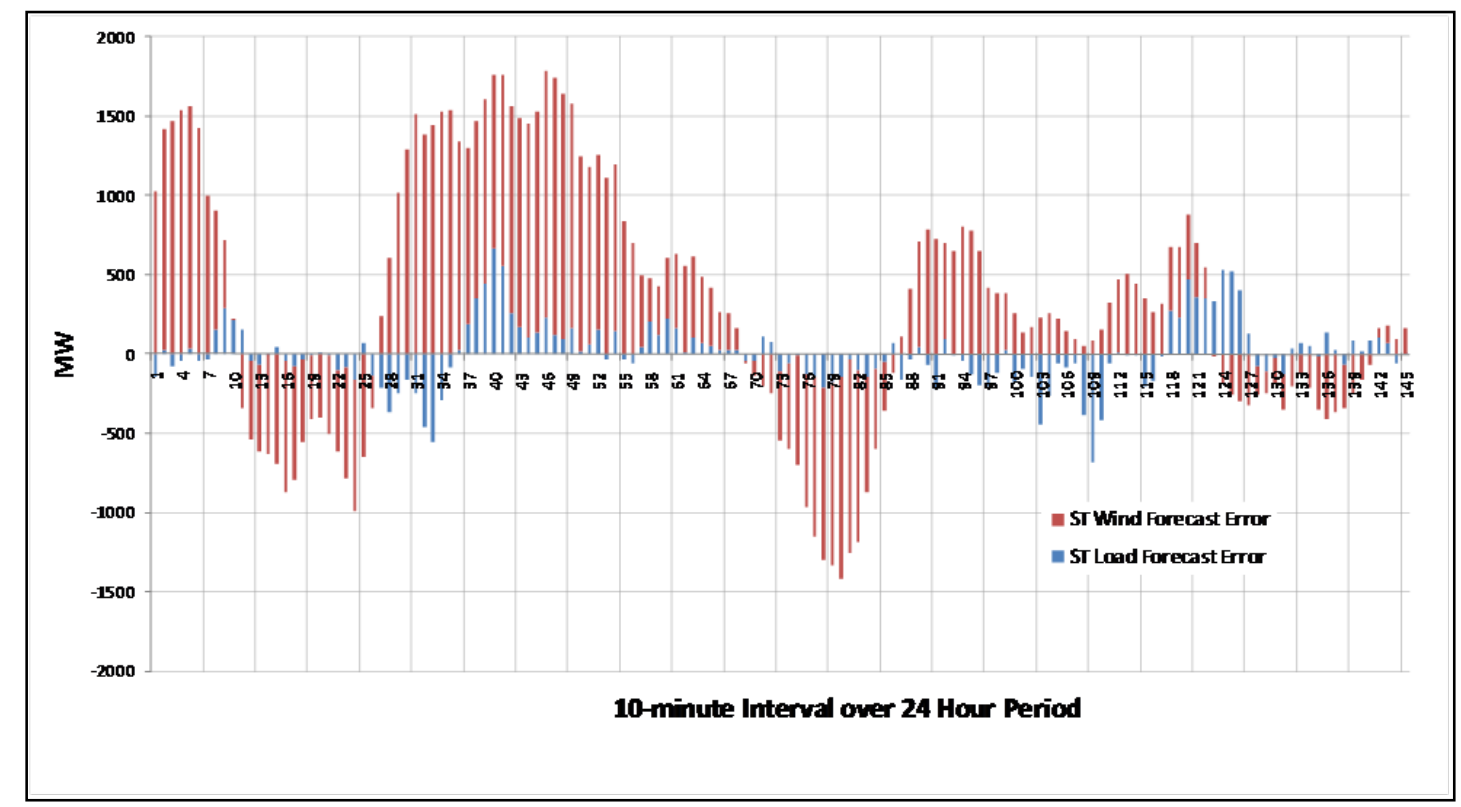

Figure 30: Errors in SPP Short-Term Forecasts of Load and Wind Generation; Load Error is assumed to be Zero in the Mathematical Procedure.

The high-resolution data available for the study allows the expected errors in short-term wind generation forecasts to be statistically characterized. The errors for each interval forecast are sorted into deciles based on the average hourly production at the time of the forecast. The errors in each of the deciles appear to be normally distributed, so the standard deviation is calculated and used as a measure of the expected forecast error.

Figure 31 shows the result for one of the scenarios and operating regions. The maximum expected error occurs in the mid-range of the aggregate production, which is expected as would be where the largest number of turbines is operating on the steep part of their power curves. For low levels of production, the error is small because the output is small; at higher production levels, the error also declines, as it is in this region that many turbines are operating above rated wind speed, where fluctuating wind speed does not translate into varying energy production.

The empirical expected error characteristic can be approximated with a quadratic expression as shown on the figure. The input to this expression is the average hourly production, with the output being the standard deviation of the expected error in the short term wind generation forecast for the current level of wind production.

Fast variations in load are almost certainly uncorrelated with the short-term forecast errors for wind generation. Therefore, the regulation requirements for load alone and short-term wind generation forecast errors do not add arithmetically. To account for this, the individual requirements will be combined as a root of the sum of the squares.

In summary, for regulating reserves with no wind generation, the amount of regulation capacity carried is equal to $1 \%$ of the hourly load. The total spinning reserve carried forward to the production simulations is the regulation amount plus the spinning part of the contingency reserve defined earlier, Table 17 and Table 18: 


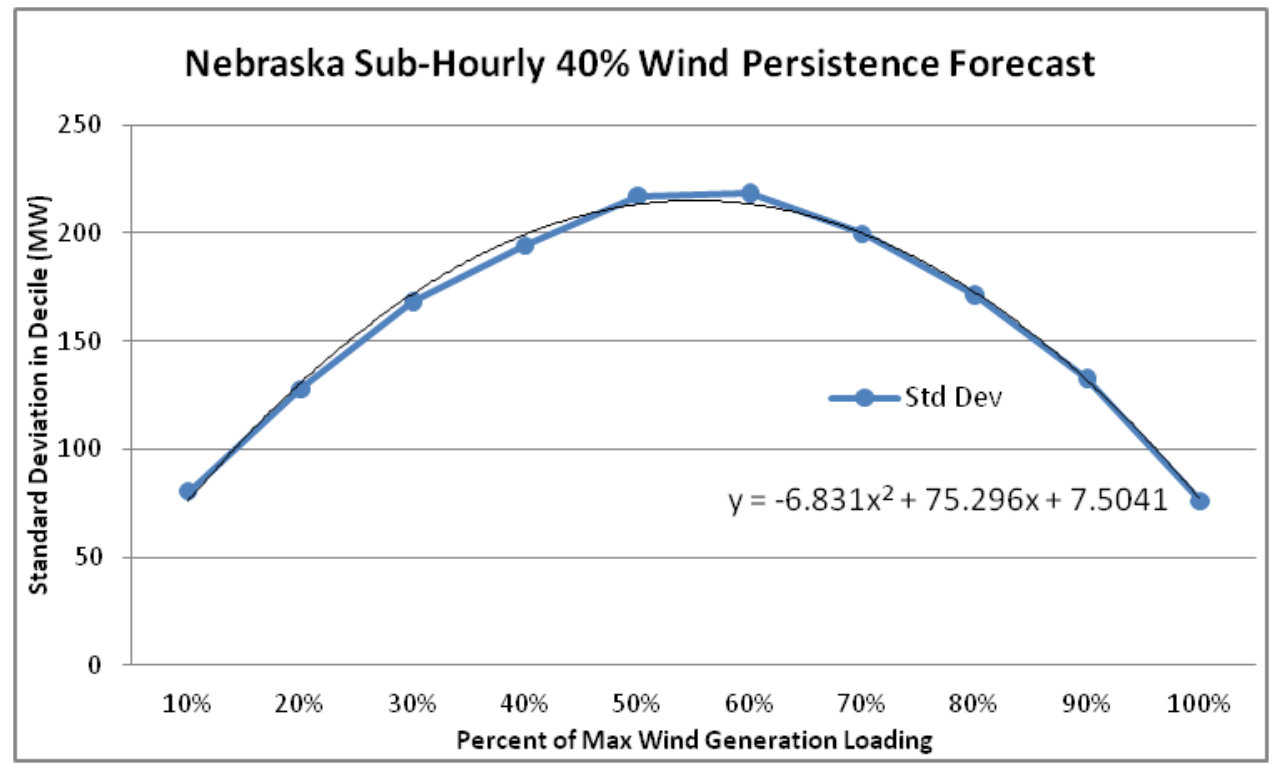

Figure 31: Illustration of short-term (ten minute ahead) wind generation forecast errors as a function of average hourly production

Spinning Reserve $($ Load Only $)=1 \% \cdot($ Hourly Load $)+$ Spinning Contingency Reserve

With wind generation, the regulation reserve is augmented to account for the short term wind generation forecast errors using statistical characterizations like the one shown in Figure 31:

Regulation Requirement (with wind) $=3 * \sqrt{\left(\frac{\text { Load Regulation }}{3}\right)^{2}+\operatorname{Variability}(\text { Hourly Wind })^{2}}$

Where:

$\sigma_{\text {ST }}$ (Hourly Wind $)=\quad$ the function described in Figure 31 for the specific operating area and wind generation scenario.

The amount of regulation capacity is taken to be three times the standard deviation of the combined variability of load and wind, which accounts for the division of load regulation by three and the multiple of three on the radical in the equation. Again, a multiplier of 3 was selected because of the large size of the operating areas in this study.

As described previously, movements of generators to follow trends in load are assumed to come from the sub-hourly energy market. Economic dispatch in the production simulation honors individual unit ramp rates on an hourly basis, and, as discussed previously, Section 3.1.2, the average price for energy in the sub-hourly market is assumed not to diverge from the day-ahead price. Therefore, the movements of generation to follow trends in the aggregate load will be reasonably captured in the production simulations. This, of course, is based on an additional assumption that a significant increase in demand for such capabilities would not increase the price. 
Uncertainty in the amount of wind generation to be delivered in the next hour also has impact on the reserve picture. Using a procedure similar to that employed to characterize the very short term forecast errors, the expected hour-ahead error for wind generation in each operating area and scenario can be characterized as shown in Figure 32. The expected next-hour forecast errors (using a simple persistence forecast) exhibit similar characteristics as the very short-term forecasts; the highest errors occur when the aggregate wind production is in the mid-range of capability, and decline for both lower and higher production levels.

Reductions in next-hour wind generation output - which, given the persistence forecast assumption is equivalent to the forecast being more than what actually is delivered - could possibly be covered by quick-start (non-spinning) generation. The assumption used in this study was that some additional spinning reserve would be held to cover next-hour forecast errors that are expected on a frequent basis, once or more per day. The amount of additional spinning reserve was set at one standard deviation of the expected error. Additional supplemental or non-spinning reserve was also allocated to cover the larger, but less frequent forecast errors. An amount equivalent to twice the standard deviation of the expected next-hour wind generation forecast error was used here.

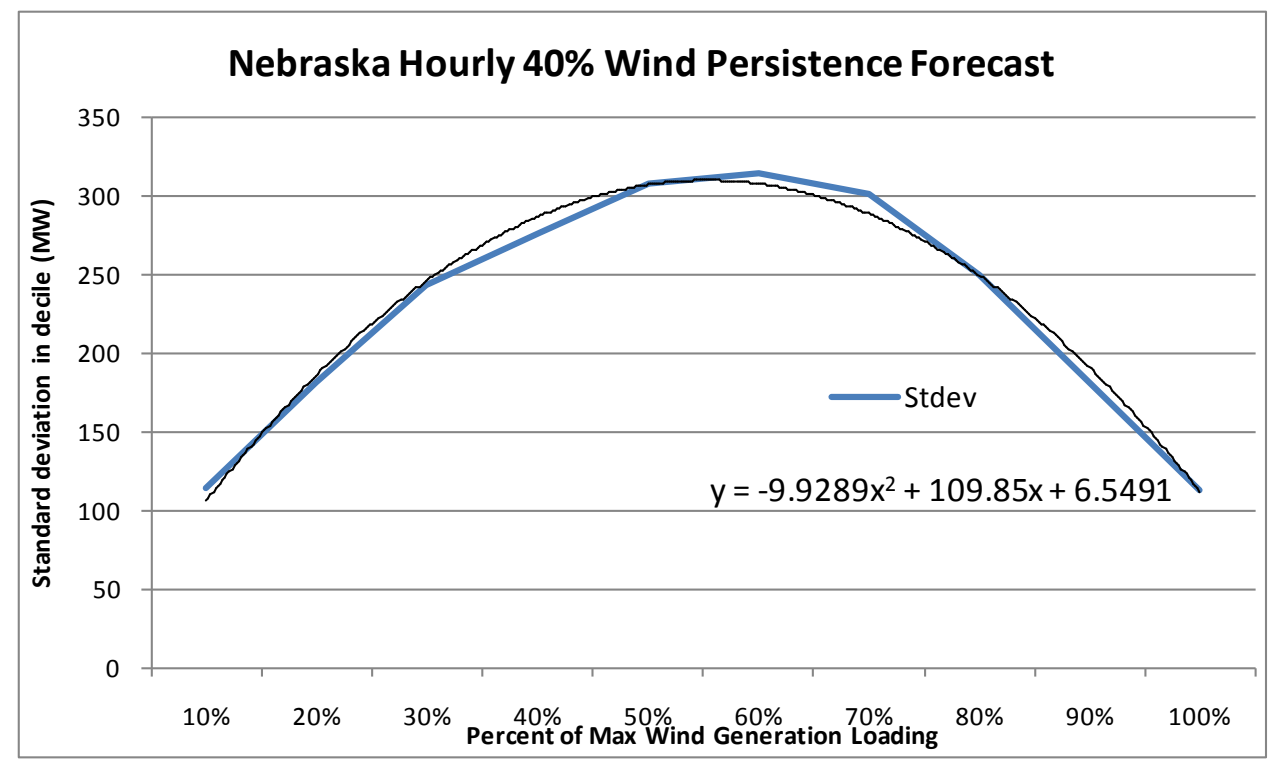

Figure 32: Standard Deviation of 1-Hour Persistence Forecast Error for Nebraska $40 \%$ Wind Penetration

Table 19 summarizes the elements of the spinning and non-spinning reserves used in the production simulations. Since hourly wind and load are inputs to certain of the components, the result is an hourly profile rather than a single number. By using the statistical characterizations of short-term and next-hour wind generation forecast error, aspects of the specific wind generation scenarios are embedded within the determination of reserves. 
Table 19: Summary of Reserve Methodology for Study Scenarios

\begin{tabular}{lcc}
\hline $\begin{array}{l}\text { Reserve } \\
\text { Component }\end{array}$ & $\begin{array}{c}\text { Spinning } \\
(\mathrm{MW})\end{array}$ & $\begin{array}{c}\text { Non-Spinning } \\
\text { (MW) }\end{array}$ \\
$\begin{array}{l}\text { Regulation } \\
\text { (variability and } \\
\text { short-term wind } \\
\text { forecast error) }\end{array}$ & $3 \cdot \sqrt{\left(\frac{1 \% \cdot \text { HourlyLoad }}{3}\right)^{2}+\sigma_{S T}(\text { HourlyWind })^{2}}$ & 0 \\
$\begin{array}{l}\text { Regulation } \\
\text { (next-hour wind } \\
\text { forecast error) }\end{array}$ & $1 \cdot \sigma_{\text {NextHourError } \text { (PreviousHourWind) }}$ & 0 \\
$\begin{array}{l}\text { Additional } \\
\text { Reserve }\end{array}$ & $\begin{array}{c}\text { 2.(Regulation for next hour } \\
\text { wind forecast error) }\end{array}$ \\
$\begin{array}{l}\text { Contingency } 1.5 \times \text { SLH } \\
\text { (or designated fraction) }\end{array}$ & $\begin{array}{c}50 \% \text { of } 1.5 \times \text { SLH } \\
\text { (or designated fraction) }\end{array}$ \\
\hline $\begin{array}{l}\text { Total (used in } \\
\text { production } \\
\text { simulations) }\end{array}$ & Sum of above & Sum of above \\
\hline
\end{tabular}

Load forecast errors, both very short-term and for the next hour or hours, has similar impacts on the regulating and load following reserves. Some of these errors are actually considered in the assumption of $1 \%$ regulation for load. With sufficient data and information on load behavior and forecast accuracy, the process used here to assess requirements with wind generation could be applied to determine the regulation and load following requirements for load.

Finally, Table 20 provides an example of the calculations used to determine the hourly regulating and spinning reserve requirement for each operating area. Hourly load (column 1) and wind generation (column 2) are the key inputs, along with the equations from Figure 31 and Figure 32. These equations are developed for each operating area for each scenario from the high-resolution and hourly production data.

The regulation amount for load alone is assumed to be $1 \%$ of the hourly load (column 3 ). The standard deviation of the short-term wind generation forecast error is calculated using the appropriate equation and the hourly average wind production (column 4). The regulation for load net of wind generation is then computed by statistically combining the load regulation (assuming that it represents three times the standard deviation of load) with the standard deviation of the short-term wind generation forecast error (column 5).

The spinning portion of the contingency reserve (column 6) is constant for each hour. In column 7 , the expected error of the forecast wind generation for the hour is computed by using the appropriate equation and the previous hour's wind generation. The total spinning reserve requirement for the hour (column 8 ) is then the sum of total regulation (column 5 ), the spinning portion of the contingency reserve (column 6), and the additional regulating reserve that was set aside the previous hour to cover expected reductions in wind generation (column 7). 
Table 20: Example Application of Reserve Methodology to Hourly Data (SPP including Nebraska)

\begin{tabular}{|c|c|c|c|c|c|c|c|c|}
\hline & (1) & $(2)$ & (3) & (4) & (5) & (6) & $(7)$ & (8) \\
\hline Hour & $\begin{array}{l}\text { Actual } \\
\text { Load } \\
(\mathrm{MW})\end{array}$ & $\begin{array}{l}\text { Actual } \\
\text { Wind } \\
(\mathrm{MW})\end{array}$ & $\begin{array}{l}\text { Regulation } \\
\text { for Load } \\
(\mathrm{MW})\end{array}$ & $\sigma_{\mathrm{ST}}(\mathrm{MW})$ & $\begin{array}{l}\text { Total } \\
\text { Regulation } \\
\text { (MW) }\end{array}$ & $\begin{array}{l}\text { Contingency } \\
\text { Reserve - } \\
\text { Spinning }\end{array}$ & $\begin{array}{l}\sigma_{\text {NextHourError }} \\
(\mathrm{MW})\end{array}$ & $\begin{array}{l}\text { Total } \\
\text { Spinning } \\
\text { Reserve }\end{array}$ \\
\hline 1 & 28321 & 22329 & 283 & 163 & 565 & 1539 & 251 & 2355 \\
\hline 2 & 27666 & 22079 & 277 & 166 & 571 & 1539 & 236 & 2346 \\
\hline 3 & 27380 & 20903 & 274 & 181 & 608 & 1539 & 241 & 2388 \\
\hline 4 & 27286 & 19776 & 273 & 193 & 639 & 1539 & 262 & 2440 \\
\hline 5 & 27370 & 17413 & 274 & 209 & 684 & 1539 & 278 & 2502 \\
\hline 6 & 27588 & 16330 & 276 & 213 & 696 & 1539 & 302 & 2537 \\
\hline 7 & 28207 & 16586 & 282 & 212 & 696 & 1539 & 308 & 2543 \\
\hline 8 & 29051 & 14801 & 291 & 215 & 707 & 1539 & 307 & 2553 \\
\hline 9 & 29844 & 13914 & 298 & 214 & 708 & 1539 & 310 & 2558 \\
\hline 10 & 30380 & 14012 & 304 & 214 & 711 & 1539 & 309 & 2559 \\
\hline 11 & 30227 & 11781 & 302 & 206 & 688 & 1539 & 309 & 2536 \\
\hline 12 & 29529 & 9912 & 295 & 192 & 647 & 1539 & 297 & 2483 \\
\hline 13 & 28759 & 9959 & 288 & 192 & 645 & 1539 & 276 & 2460 \\
\hline 14 & 28192 & 10180 & 282 & 194 & 647 & 1539 & 277 & 2463 \\
\hline 15 & 27496 & 10641 & 275 & 198 & 655 & 1539 & 280 & 2474 \\
\hline 16 & 27028 & 12692 & 270 & 211 & 687 & 1539 & 285 & 2511 \\
\hline 17 & 26947 & 12962 & 269 & 212 & 689 & 1539 & 304 & 2532 \\
\hline
\end{tabular}

Notes on Table 20 :

Equation for Column (4) is from Figure 31 and uses current hour wind generation from Column (2):

$$
\sigma_{S T}(\text { HourlyWind })=-6.381 \cdot(\text { HourlyWind })^{2}+76.296 \cdot(\text { HourlyWind })+7.504
$$

Column (5) value is computed from Column (3) and Column (4) using:

$$
3 \cdot \sqrt{\left(\frac{1 \% \cdot \text { HourlyLoad }}{3}\right)^{2}+\sigma_{S T}(\text { HourlyWind })^{2}}
$$

Equation for Column (7) is from Figure 32 and uses wind generation from previous hour:

$$
\sigma_{\text {NextHourError }}=-9.93 \cdot\left(\text { Wind }_{H-1}\right)^{2}+109.85 \cdot\left(\text { Wind }_{H-1}\right)+6.55
$$

The total spinning reserve contains a component that is allocated specifically to be used if wind generation is less than was forecast in the previous hour. To avoid double counting of these reserves, the profile is adjusted to deploy this capacity in the production simulation. This is accomplished by reducing the hourly spinning reserve constraint by the amount of the reduction in wind generation from the previous hour, up to the amount that was held. This is illustrated in Table 21. In Hour 3, $241 \mathrm{MW}$ of extra spinning reserve was being carried to cover hourly wind generation forecast error (column 3). Wind generation declined by $1176 \mathrm{MW}$ from the previous hour (column 5). All of the $241 \mathrm{MW}$ was deployed to cover this drop, so the total spinning reserve constraint for that hour in the production simulation is reduced by that amount, from $2388 \mathrm{MW}$ to $2147 \mathrm{MW}$. 
As can be seen in Hour 2, if wind generation increases from the previous hour, there is no adjustment.

If it were desired to cover a larger number of the reductions in wind generation output with regulation versus non-spinning (quick-start) generation, the amount of regulating reserve would increase. In this example, an amount equivalent to one standard deviation of the next-hour persistence forecast error was held; increasing the amount to two standard deviations, which would be adequate to cover about $90 \%$ of the reductions in next-hour wind generation output, would double this component of the overall spinning reserve. It would also result in more spinning reserve that is not actually dispatched to cover forecast errors, and thus the cost.

The "cost" of releasing the spinning reserves is tabulated by the production simulation program; generation capacity that would have otherwise be unloaded will be dispatch to cover the loss in wind, and associated production costs accumulated.

Table 21: Adjustment of spinning reserve for reduction in wind generation (SPP including Nebraska)

\begin{tabular}{|c|c|c|c|c|c|c|c|}
\hline & (1) & (2) & (3) & (4) & (5) & (6) & (7) \\
\hline Hour & $\begin{array}{l}\text { Actual } \\
\text { Load } \\
(\mathrm{MW})\end{array}$ & $\begin{array}{l}\text { Actual } \\
\text { Wind } \\
\text { (MW) }\end{array}$ & $\begin{array}{c}\sigma_{\text {NextHourErr }} \\
\text { or }(\mathrm{MW})\end{array}$ & \begin{tabular}{|c|} 
Total \\
Spinning \\
Reserve \\
(MW)
\end{tabular} & $\begin{array}{c}\text { Change in } \\
\text { Wind } \\
\text { Generation } \\
\text { (MW) }\end{array}$ & $\begin{array}{l}\text { Adjustment } \\
\text { (MW) }\end{array}$ & $\begin{array}{c}\text { Adjusted } \\
\text { Spinning } \\
\text { Reserve } \\
\text { (MW) }\end{array}$ \\
\hline 1 & 28,321 & 22,329 & 251 & 2,355 & 764 & 0 & 2,355 \\
\hline 2 & 27,666 & 22,079 & 236 & 2,346 & -249 & 236 & 2,110 \\
\hline 3 & 27,380 & 20,903 & 241 & 2,388 & $-1,176$ & 241 & 2,147 \\
\hline 4 & 27,286 & 19,776 & 262 & 2,440 & $-1,128$ & 262 & 2,178 \\
\hline 5 & 27,370 & 17,413 & 278 & 2,502 & $-2,363$ & 278 & 2,223 \\
\hline 6 & 27,588 & 16,330 & 302 & 2,537 & $-1,083$ & 302 & 2,235 \\
\hline 7 & 28,207 & 16,586 & 308 & 2,543 & 256 & 0 & 2,543 \\
\hline 8 & 29,051 & 14,801 & 307 & 2,553 & $-1,785$ & 307 & 2,246 \\
\hline 9 & 29,844 & 13,914 & 310 & 2,558 & -886 & 310 & 2,247 \\
\hline 10 & 30,380 & 14,012 & 309 & 2,559 & 98 & 0 & 2,559 \\
\hline 11 & 30,227 & 11,781 & 309 & 2,536 & $-2,231$ & 309 & 2,227 \\
\hline 12 & 29,529 & 9,912 & 297 & 2,483 & $-1,869$ & 297 & 2,186 \\
\hline 13 & 28,759 & 9,959 & 276 & 2,460 & 47 & 0 & 2,460 \\
\hline 14 & 28,192 & 10,180 & 277 & 2,463 & 221 & 0 & 2,463 \\
\hline 15 & 27,496 & 10,641 & 280 & 2,474 & 460 & 0 & 2,474 \\
\hline 16 & 27,028 & 12,692 & 285 & 2,511 & 2,051 & 0 & 2,511 \\
\hline 17 & 26,947 & 12,962 & 304 & 2,532 & 270 & 0 & 2,532 \\
\hline
\end{tabular}

The resulting 8760-hour profiles for each year and scenario are input to the production simulation program as operating area requirements, which constrain the algorithms for optimization and economic dispatch. 


\subsection{Regulating Reserve ResUlts fOR StUdy ScEnarios}

Statistics of the regulation portion of the spinning reserves for each operating region and wind generation scenario are documented in Table 22 through Table 23. This amount includes the additional spinning operating reserve for covering next-hour wind generation deficits from the hour-ahead forecast. The tables list the maximum and average values of an 8760 -hour profile. A more detailed view of the SPP with Nebraska $40 \%$ wind penetration requirements showing distributions of the regulating requirement for load only and load net wind is shown in Figure 33. The blue bars on the histogram show the percent of hours of regulating reserve for serving load while the red bars shows the percent of hours of regulating reserve value serving both load and wind. Connecting the information in Figure 33 to that in Table 22, the maximum and average values of 3257 and 2337 for 2006 patterns at $40 \%$ penetration are the same data represented as red hourly statistical data in Figure 33.

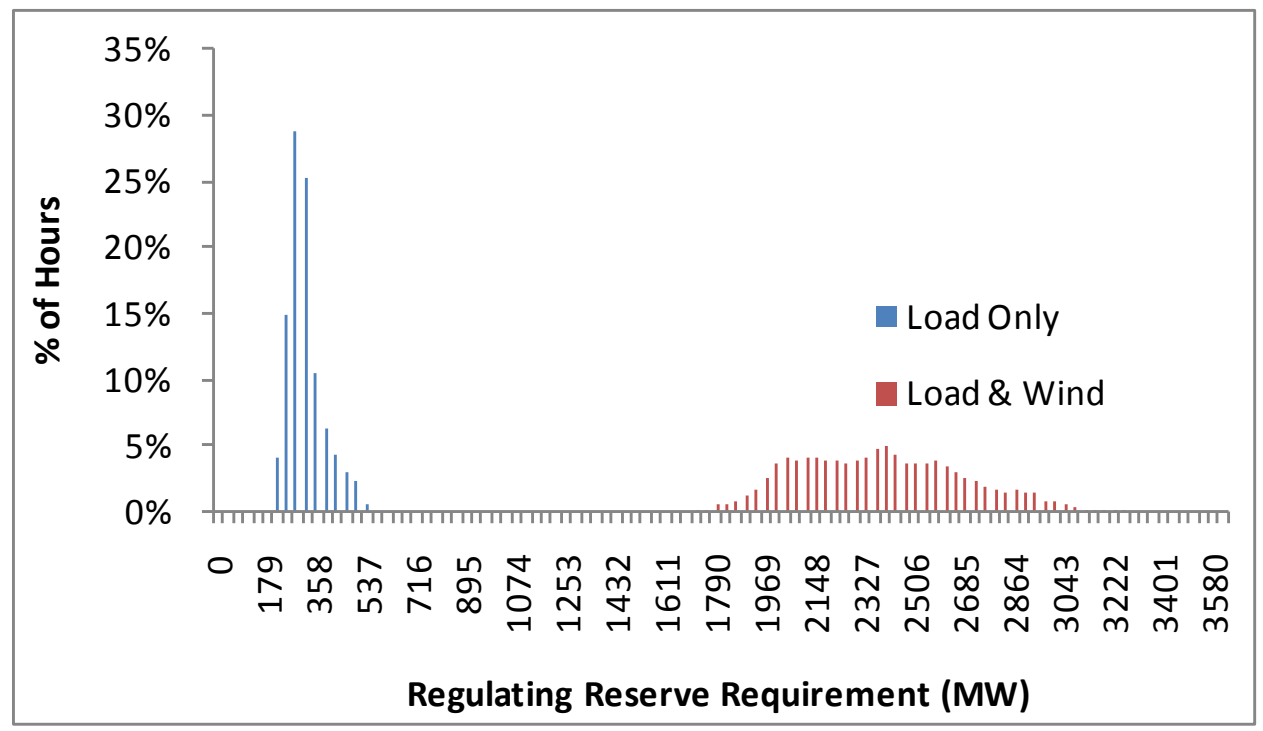

Figure 33: Distributions of hourly regulating reserve requirements for SPP and Nebraska - $40 \%$ wind Penetration, for load only (ideal wind generation) and load net of wind generation. 
Table 22: Regulating Reserve Requirements for the SPP Including Nebraska Load and Load Net Wind

\begin{tabular}{|c|c|c|c|c|c|c|c|}
\hline \multirow{2}{*}{$\begin{array}{l}\text { SPP with Nebraska } \\
10 \% \text { Penetraton }\end{array}$} & \multicolumn{2}{|c|}{ Load Only } & \multicolumn{2}{|c|}{ Load and Wind } & \multicolumn{2}{|c|}{$\begin{array}{l}\text { Delta Load and } \\
\text { Wind - Load }\end{array}$} & \multirow{2}{*}{$\begin{array}{c}\text { Wind } \\
\text { Nameplate } \\
\text { MW }\end{array}$} \\
\hline & Max & Average & Max & Average & $\operatorname{Max}$ & Average & \\
\hline 2004 profile & 533 & 301 & 1142 & 814 & 884 & 513 & 7,505 \\
\hline 2005 profile & 526 & 301 & 1146 & 806 & 853 & 505 & 7,505 \\
\hline 2006 profile & 528 & 295 & 1152 & 794 & 852 & 499 & 7,505 \\
\hline \multicolumn{8}{|l|}{$20 \%$ Penetration } \\
\hline 2004 profile & 533 & 301 & 1796 & 1322 & 1538 & 1021 & 15,084 \\
\hline 2005 profile & 526 & 301 & 1802 & 1308 & 1540 & 1007 & 15,084 \\
\hline 2006 profile & 528 & 295 & 1800 & 1291 & 1542 & 996 & 15,084 \\
\hline \multicolumn{8}{|l|}{ 40\% Penetration } \\
\hline 2004 profile & 533 & 301 & 3261 & 2395 & 3003 & 2094 & 30,158 \\
\hline 2005 profile & 526 & 301 & 3261 & 2359 & 3041 & 2058 & 30,158 \\
\hline 2006 profile & 528 & 295 & 3257 & 2337 & 3034 & 2042 & 30,158 \\
\hline
\end{tabular}

*Delta Max colum represents the coincidental maximum delta of Load Only and Load and Wind

Table 23: Regulating Reserve Requirements for Nebraska Only and SPP without Nebraska for $10 \%$ 2006 Profile

\begin{tabular}{|l|r|r|r|r|r|r|r|}
\hline \multirow{2}{*}{$\begin{array}{l}\text { 10\% Penetration } \\
\text { 2006 profile }\end{array}$} & \multicolumn{2}{|c|}{ Load Only } & \multicolumn{2}{c|}{ Load and Wind } & \multicolumn{2}{c|}{$\begin{array}{c}\text { Delta Load and } \\
\text { Wind - Load }\end{array}$} & $\begin{array}{c}\text { Wind } \\
\text { Nameplate }\end{array}$ \\
\cline { 2 - 7 } & Max & Average & Max & Average & Max & Average & MW \\
\hline SPP with Nebraska & 528 & 295 & 1152 & 794 & 852 & 499 & 7,505 \\
SPP w/o Nebraska: A & 460 & 256 & 1111 & 742 & 821 & 486 & 6,256 \\
\cline { 2 - 4 } Nebraska alone: B & 75 & 45 & 216 & 148 & 177 & 118 & 1,249 \\
\hline Total A + B & 535 & 301 & 1327 & 890 & 998 & 604 & 7,505 \\
\hline
\end{tabular}

"Delta Max Column represents the coincidental maximum delta of Load Only and Load and Wind

\subsection{SUMMARY}

As mentioned above, the spinning reserves profiles for each operating region are treated as constraints by the algorithms within the production simulation program. Generation must be committed and dispatched to meet load at minimum costs while honoring all constraints, of which the hourly spinning reserve requirement is one.

The reserve constraints have an impact only when they are binding on either the unit commitment or economic dispatch. By the tables above, the regulating reserve requirements appear to be very significant (re: SPP), it should be remembered that they are highest when wind generation is moderate to high. If generation mix doesn't change except for the introduction of renewable wind, heavy penetration of wind generation frees up conventional generation to provide the required regulating reserves to support frequency and balance generation with demand. The decreased revenues of the fleet of intermediate generation and 
market structures, however, could affect the availability of these services in the market, as discussed further in the balance of this summary.

There are costs associated with carrying significant spinning reserve for wind generation, however. If additional conventional generation has to be committed simply to meet the spinning reserve requirement, the reserve constraint is binding and additional operating costs will be incurred. Even without a change in commitment, units may not be loaded to their maximums, and thereby not operate as efficiently.

The reserve costs that can be extracted from production simulations reflect the less efficient dispatch and opportunity costs. However, there are some additional operational costs associated with regulation duty that are not captured. In current markets, regulation is a relatively expensive service compared to provision of spinning operating reserve.

The assumptions defined earlier are critical to the results presented here, and merit some additional discussion. First, while the philosophy behind the view of short-term forecast errors in wind generation as a contributor to needs for incremental regulation is sound, the persistence forecast technique is acknowledged to be rudimentary and likely not what would be implemented in practice. Improvements in short-term forecasts would reduce the impact on regulation requirements. The persistence assumption employed here likely leads to conservative estimates of regulation requirements.

Second, high penetrations of wind generation and the increased requirements for regulation and flexibility means that providing those services would have more value. Moving up the supply curve for those services may reach into units that are much less efficient at providing them, further increasing the cost. Finally, there are questions about the depth of the resource stack for flexibility, which could potentially be another limitation. Alternatively, loads and storage are beginning to supply regulation in at least three ISOs. Responsive load and storage may significantly increase the supply of regulation by 2018.

Third, if large amounts of wind energy displace conventional units and significantly reduce capacity factors, additional questions are raised about compensation in lieu of energy sales for those units and keeping them economically viable to provide the flexibility that the system requires.

Finally, the importance of the assumptions regarding the structure for operations in this 2018 scenario must be reiterated. Functional sub-hourly markets provide the most economic means to compensate for short-term changes in load and wind generation that can be forecasted. Very large balancing areas with adequate transmission take maximum advantage of diversity in both load and wind generation. By contrast, the Western Interconnection, with the exception of California, is comprised of smaller, less tightly interconnected balancing areas. Even modest penetrations of wind generation, much smaller than considered in this study, can have very significant operational and cost impacts due to the additional requirements they bring for regulation and balancing.

The penetrations of wind generation considered in this study are well beyond what experience can speak to definitively; further analysis is certainly warranted. The knowledge gained from operating experience around the country and world as wind generation penetrations continue 
to grow will provide an increasingly better foundation for technical insights into this important challenge.

\subsection{REFERENCES}

[1] Kirby, B., Milligan, M, Gramlich, R., Goggin, M. "Impact of Electric Industry Structure on High Wind Penetration Potential" NREL Technical Report NREL/TP-550-46723, July, 2009.

[2] Wan, Y., et.al. "Short-Term Power Fluctuations of Large Wind Power Plants" NREL Technical Report NREL/CP-500-30747, January, 2002. 


\section{Section 4}

\section{AsSessing IMPACTS ON POWER SYSTEM Operations}

The analytical methods used in this study build off those established in the prior integration studies conducted over the past ten years. A chronological data set of wind generation and load data is the critical input for the study. Load and wind data must be temporally synchronized since meteorology has influence on load patterns and is obviously a critical factor for wind energy production.

Development of the wind generation profile data from the NREL meso-scale data was described in Section 2.2.1.

The consensus approach for assessing wind integration impacts is to simulate the scheduling and operation of the power system with wind generation over an extended period of time. If the hourly load and wind data that drive the simulation extend over a sufficient period of time, the range of conditions evaluated can be considered statistically valid - i.e. all combinations of wind and load and their respective variability and uncertainty characteristics are represented in the input data. This prevents a focus on only those severe events, like major wind ramps, that would be expected to occur infrequently (e.g., once per year or less).

\subsection{INTEGRATION COST}

The additional variability and uncertainty introduced by wind generation will increase the duty on other conventional units for supporting the frequency of the interconnection and balancing total generation with load.

The basic process for assessing the impacts of wind generation on power system operations involves simulations of system operation. A chronological production simulation computer program is the tool of choice. The simulations are set up and run in a way to mimic as closely as possible the actual scheduling and operation of the power system. Therefore in the part of that process where decisions are being made regarding which generating units to run to meet anticipated loads over the coming day or days, a forecast of wind generation is used. The dispatching of committed generating units to meet hourly loads represents, roughly, the realtime operation of the system. Here, additional capacity is held back to represent the incremental regulating and other reserves that would be required to manage the variability of load and wind generation combined and to insure the security of the system.

Production costs incurred over the simulation will then reflect the impacts of the total variability and uncertainty of load net of wind generation. To determine the cost of the wind generation variability and uncertainty by themselves, an additional step is required. With significant wind 
generation, the conventional generation "stack" will change, as marginal units are displaced by what is usually considered to be a "must take" wind energy resource. Consequently, the costs related to uncertainty in the optimization process and the requirements for carrying additional reserves will be different than a case with no wind because of this displacement. In recognition of this factor, previous studies have utilized the concept of a "proxy resource" to represent the energy provided by wind generation but in a way that affects scheduling and real-time operations as little as possible, i.e. it neither helps nor hurts the scheduling and dispatch of other conventional resources and therefore is close to cost-neutral operationally.

One fictitious energy resource that meets this definition consists of a daily flat block of energy equivalent to the energy provided in the actual wind profile for that same day. Despite much discussion and debate but little consensus regarding alternatives, this type of proxy resource has been used in many of the previous integration studies. As the amount of wind generation increases relative to load and conventional resources, this daily flat block of energy can introduce some artifacts, as the ramp between one daily level and the next can become large at high wind penetrations. This daily flat block proxy resource was used for a few sensitivity cases primarily to relate to past studies.

In consideration of the potential problems with the fictitious resource described above, a second logical candidate for the proxy resources is a profile that matches the actual hourly shape of the delivered wind energy and is called herein "shaped proxy". As with other proxy resources, this resource requires no additional regulating or operating reserves to manage the system in real-time. This shaped proxy resource was used for most of the cases.

"Intermediate" proxy resource types were also investigated as sensitivity cases to try to identify methodologies thought to be most useful. These types included subperiod (on-peak/off-peak) block and moving average proxies as explained in Section 4.3.1.

Integration costs are determined by comparing the case where wind generation introduces additional uncertainty into the commitment process and requires additional reserves in the economic dispatch steps to the case with the proxy resource, where only load carries uncertainty and exhibits variability.

While there has been much focus on the costs of integrating wind generation in previous studies, it must be remembered that those costs are only one piece of the larger set of wind generation costs and benefits.

\subsection{Base CASe Results}

For purposes of this report the 'Base Case' includes four scenarios simulated for three different wind / load years as shown below.

- Scenario $1-10 \%$ Wind Penetration in Nebraska and Rest of SPP

- $\quad$ Scenario $2-20 \%$ Wind Penetration in Nebraska and Rest of SPP

- $\quad$ Scenario 3-20\% Wind Penetration plus EHV Overlay for Nebraska and SPP

- Scenario 4-40\% Wind Penetration in Nebraska and SPP (includes EHV Overlay from Scenario 3)

- $\quad$ Simulations are performed using wind and load for 2004, 2005 and 2006. 
- Wind penetrations in other areas averages $6 \%$ for all scenarios as described in Table 14.

For each scenario there are two PROMOD simulations in order to determine integration costs of wind generation.

- $\quad$ Actual Wind Case - case with effects of wind forecast errors and incremental reserves for wind, and

- $\quad$ Ideal Wind Case - case using the 'Proxy Resource' where the wind forecast is perfect and no incremental reserves for wind are required.

For the four scenarios and three wind years comprising the base case, results are shown together in order to facilitate observations about trends between the years and wind penetration levels. Results are presented for either SPP including Nebraska, SPP excluding Nebraska or Nebraska only and are indicated as such.

A key goal of a wind integration study is to determine integration costs associated with wind. Those integration costs are driven by changes in the use of the thermal generation fleet in response to the wind. Typically, the actual wind case simulation (including the effects of forecast error, incremental reserves and wind variability) will result in higher production costs than the ideal wind case (no forecast error, incremental wind reserves or wind variability). This is explained by the increased use of higher cost resources (combined cycles versus coal) in response to the wind. Increased reserve requirements and need for response to variability can cause coal units to back down and combined cycle generation to increase. This results in higher cost resources, particularly combined cycle units, to be brought on to serve the energy displaced on the coal units. These higher cost resources also respond to the faster ramps and forecast errors associated with wind.

\subsubsection{Integration Effects on Thermal Generators}

Prior to looking at costs associated with those changes, it is useful to look at the changes themselves. Figure 34 and Figure 35 show the changes associated with generation types for SPP (including Nebraska) and Nebraska respectively for the 2006 load and wind year. These are the differences in generation between the actual case and the ideal case as described above. Note, the biggest changes are to the Coal fired Steam Turbines and the Combined Cycle Units. Also, notice that the differences increase most with scenario number which is basically the direction of wind penetration increase. The notable exception to that is Scenario 3 for Nebraska. In that scenario, the addition of the transmission overlay allows for substantial exports and thus less change in the usage of the coal plants, while still showing an increase in the use of combined cycle for wind response.

There are some small differences in the wind generation between the actual and ideal cases, especially in the scenarios without the overlay. These differences (actual wind ranges from $0 \%$ to $0.28 \%$ higher than ideal wind gen in 2006) can be explained by different congestion patterns due to different commitment and dispatch levels driven by the wind forecast error and incremental reserve requirements. In other words, the wind generation in the actual case can be greater than that in the ideal case if there is transmission congestion that is relieved by the thermal units running lower to provide for the incremental reserves modeled in the actual case 
or additional committed units providing counter flows to congestion. These differences related to the base cases that used the shaped proxy described in Section 4.1. All such differences resulted from actual wind generation being slightly higher than ideal wind generation results. If the wind generations were equalized (and incremental value, or lower APC, be assigned to the ideal case), then the integration cost would slightly increase accordingly. Such small adjustments were not made in the study results. The opposite relationship is true for the nonshaped proxy resources as noted in Section 4.4.4.

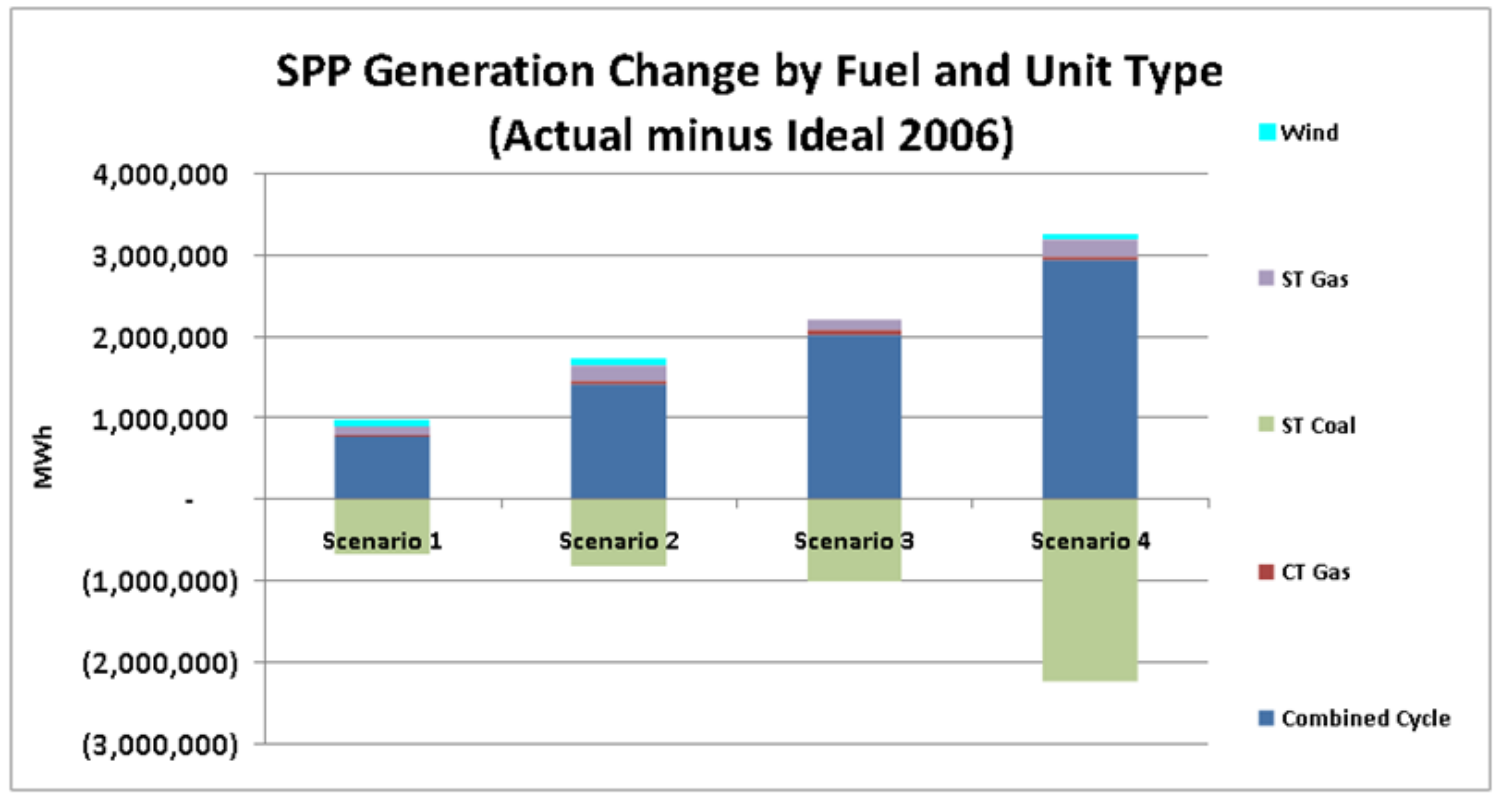

Figure 34: Comparison of SPP (including Nebraska) Actual and Ideal Generation by Type

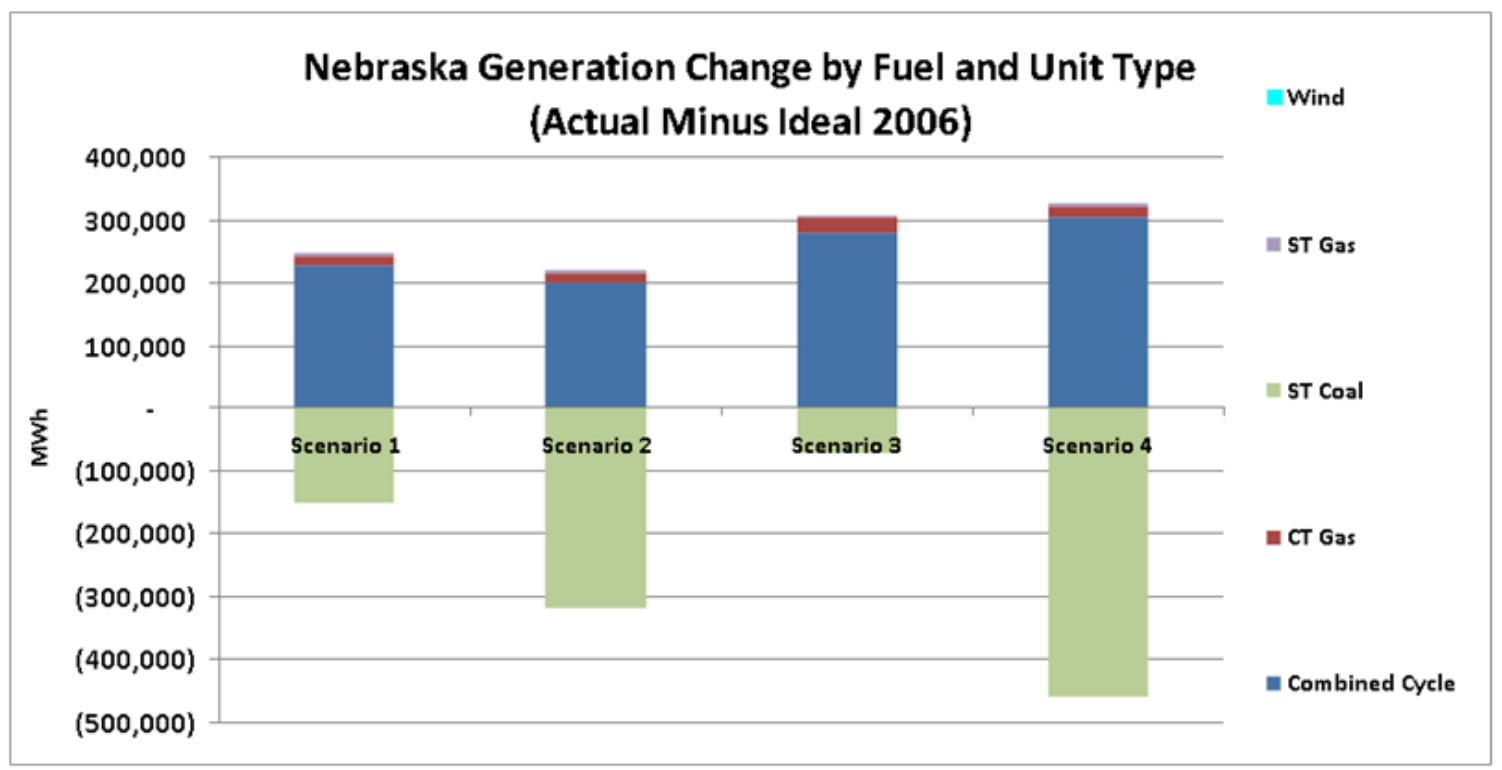

Figure 35: Comparison of Nebraska Actual and Ideal Generation by Type 
In addition to the changes to generation between the ideal and actual cases (i.e., integration effects), it is useful to observe the changes to generation usage as wind energy penetration increases. Figure 36 shows the major fuel / unit types and their associated energy production at each penetration level for the 2006 load and wind patterns. Note the decrease in coal fired steam turbine output and in combined cycle output as wind penetration increases. There is a slight increase in coal usage in Scenario 3 when the EHV overlay allows for more exports; there is also an increase in wind output for that scenario as the EHV overlay reduces wind curtailment caused by transmission congestion. Beginning with Scenario $2(20 \%)$ wind becomes the second largest energy producer in SPP behind only coal fired steam turbines.

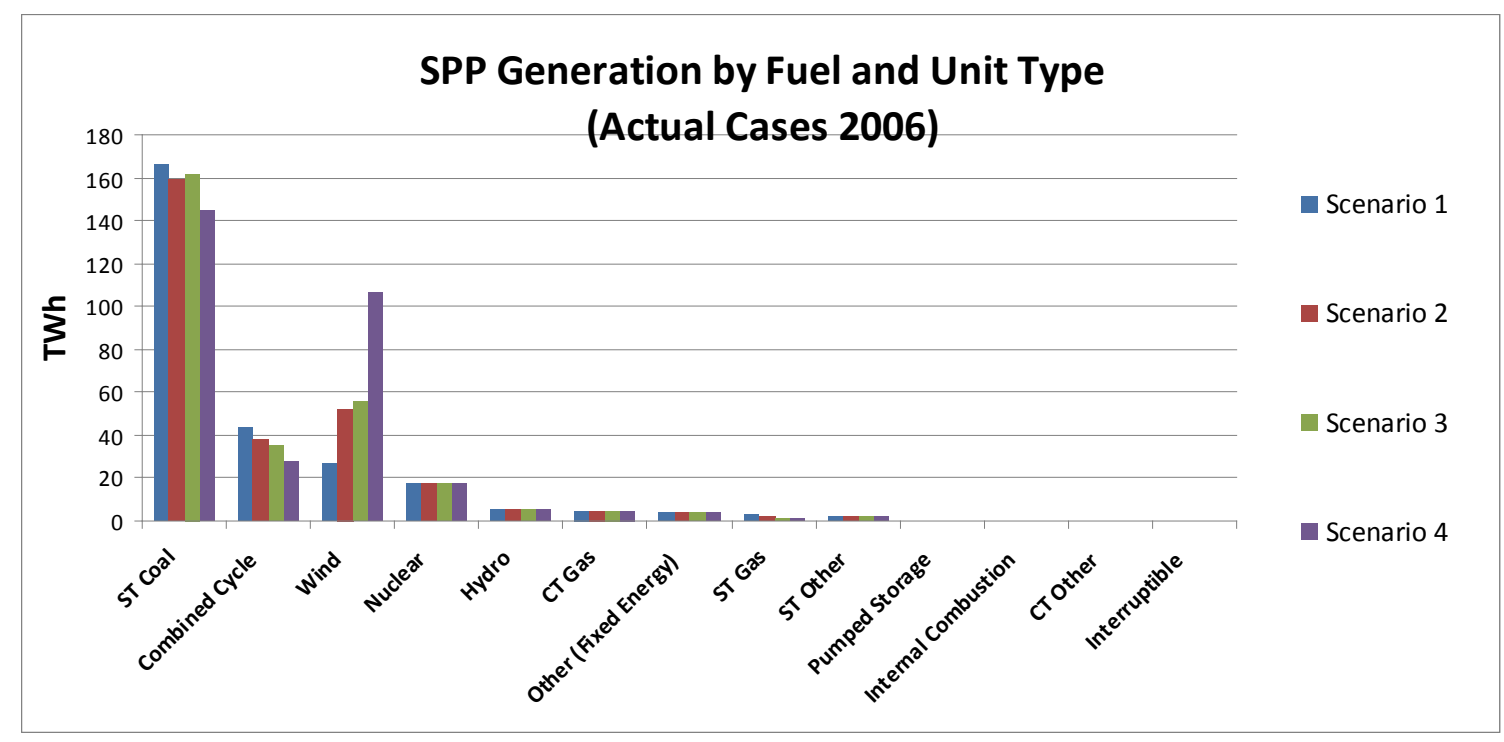

Figure 36: SPP Generation by Fuel and Unit Type

The change in energy output from thermal generation as wind energy increase warrants looking at the effect on emissions, particularly $\mathrm{CO} 2$, associated with those energy output decreases. CO2 emissions for SPP (including Nebraska) are shown below in Figure 37. Consistent with the output decreases from coal plants and combined cycle plants, the production of $\mathrm{CO} 2$ in the region decreases substantially as wind penetration increases. There is an average decrease in CO2 emissions of about $14 \%$ across the three study years from the $10 \%$ cases to the $40 \%$ cases. The slight upward tick with the addition of the transmission overlay is visible here as well. 


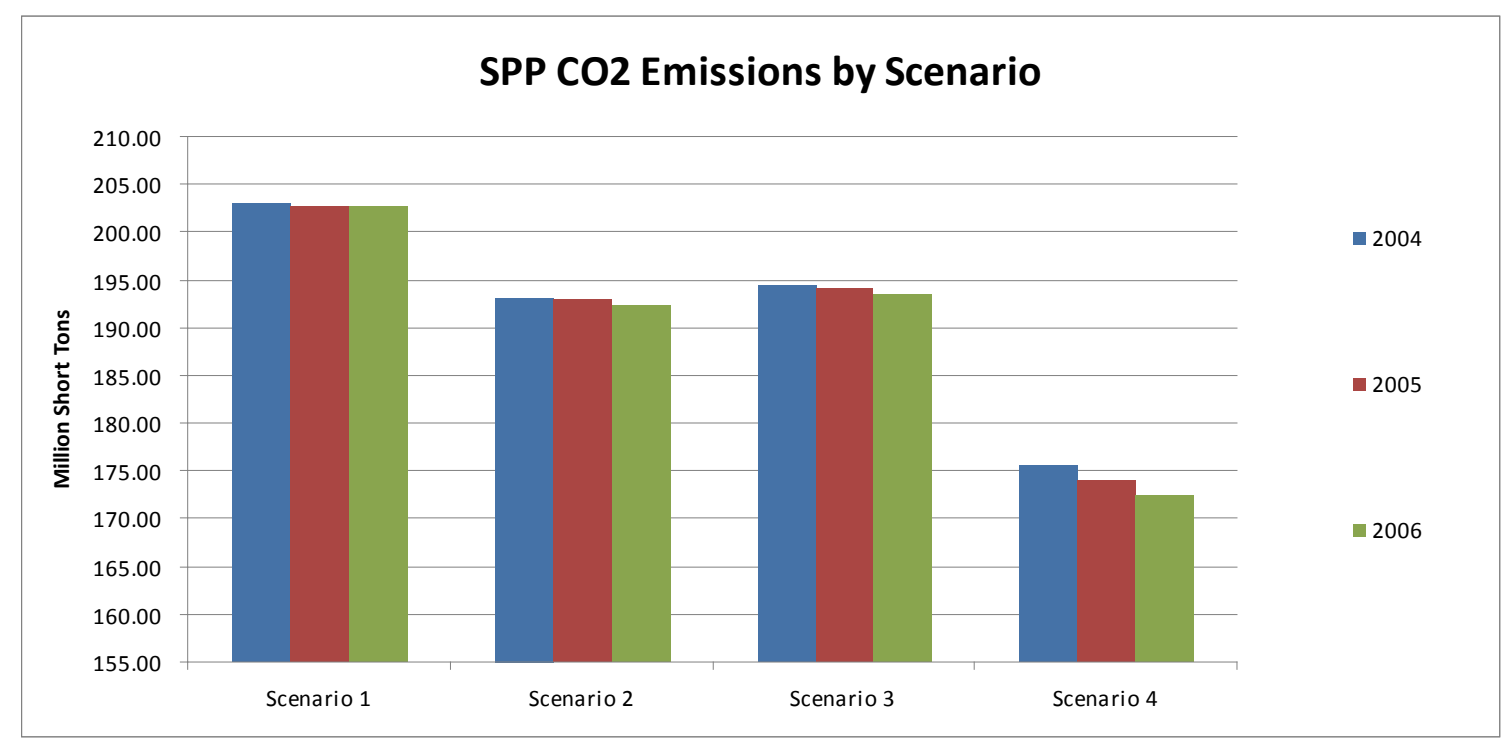

Figure 37: SPP CO2 Emissions by Scenario

As shown in Figure 38, the CO2 emissions pattern for Nebraska is similar to the whole of SPP in that $\mathrm{CO} 2$ production decreases with the addition of more wind energy; however, there is a much bigger increase in $\mathrm{CO} 2$ production with the addition of the EHV transmission. This is due to a bigger increase in exports and the use of coal generation with the EHV overlay in Nebraska.

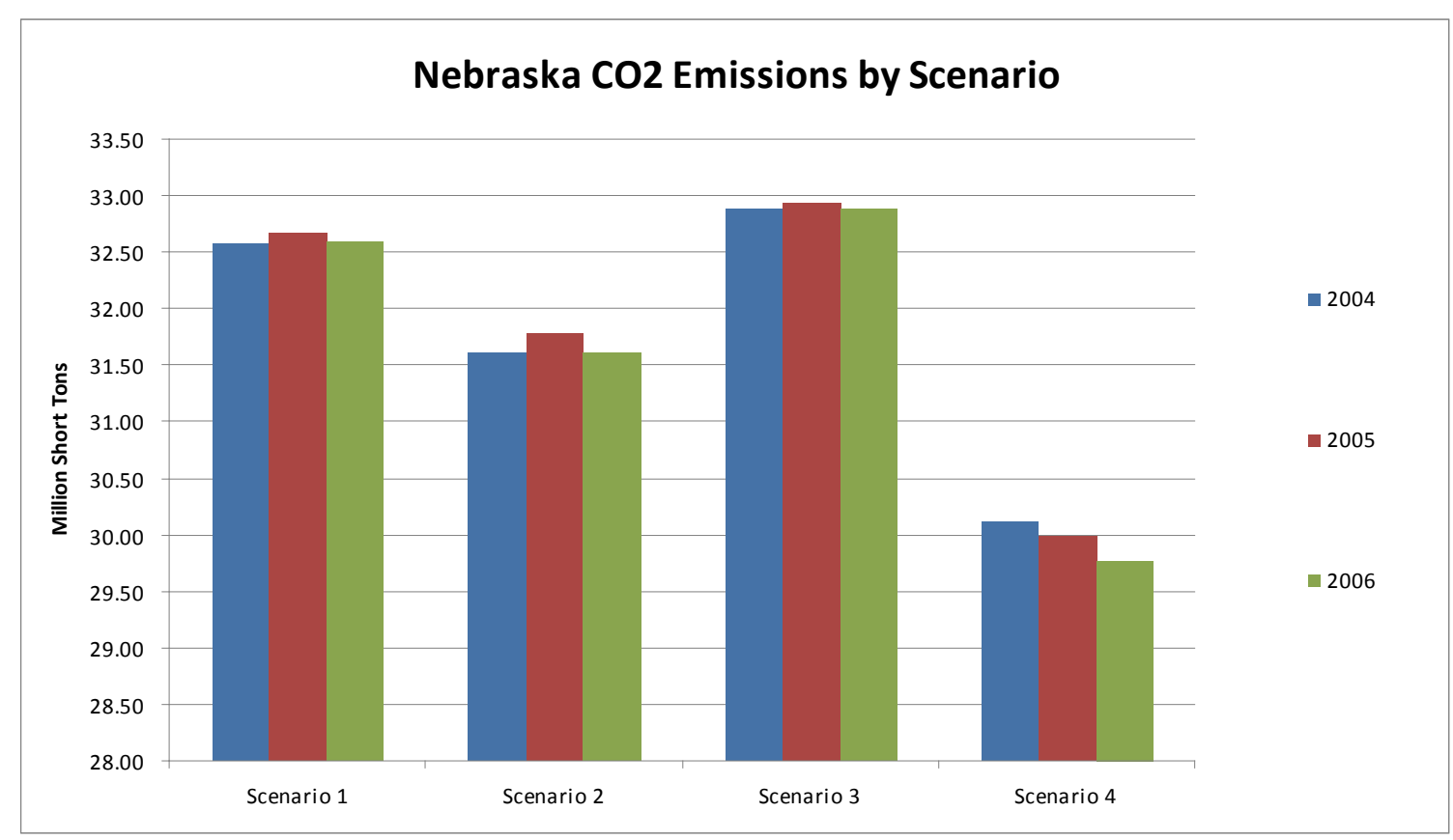

Figure 38: Nebraska CO2 Emissions by Scenario 


\subsubsection{Wind Curtailment}

In addition to effects of the wind on the thermal generation fleet, effects on the wind from the fleet and transmission system can be analyzed. When the production simulations are performed, a set amount of wind is requested at each injection point (bus). If the locational marginal price (LMP) at that bus is below a certain threshold, then PROMOD IV will curtail an amount of wind that results in the LMP rising above that threshold. That threshold is the bid-in price of wind which is negative $\$ 40 / \mathrm{MWh}$, as discussed in Section 2.5.5. Wind curtailment, although directly driven by LMP, is a function of transmission congestion that results in LMPs below the curtailment threshold and possibly minimum generation limits. This also drives down LMP. Curtailment values for 'Rest of SPP' are shown here both as percentages and MWh values in Figure 39 and Figure 40. There is no curtailment occurring in Nebraska in any of the cases and thus no chart is shown.

Curtailment is typically a local phenomenon driven primarily by local congestion and to a lesser extent minimum generation (which itself can be driven by congestion), so the lack of wind curtailment in Nebraska is an indicator that those problems are not occurring in a manner that affects wind injections. More specifically, curtailment in the model is determined by extremely low LMPs (below the $-\$ 40 / \mathrm{MWh}$ wind bid price) and the lack of curtailment indicates that these low LMPs are not occurring at the Nebraska wind injection sites. This is a reasonable outcome given the siting of the wind in this study (at higher voltage buses making the assumption that the wind interconnection will be designed for full delivery) and the addition of the EHV overlay at the higher penetrations. The curtailment in the non-Nebraska portions of SPP is primarily occurring in the Texas panhandle and is an indicator that more transmission development is required to deliver any energy that is sited as in this study.

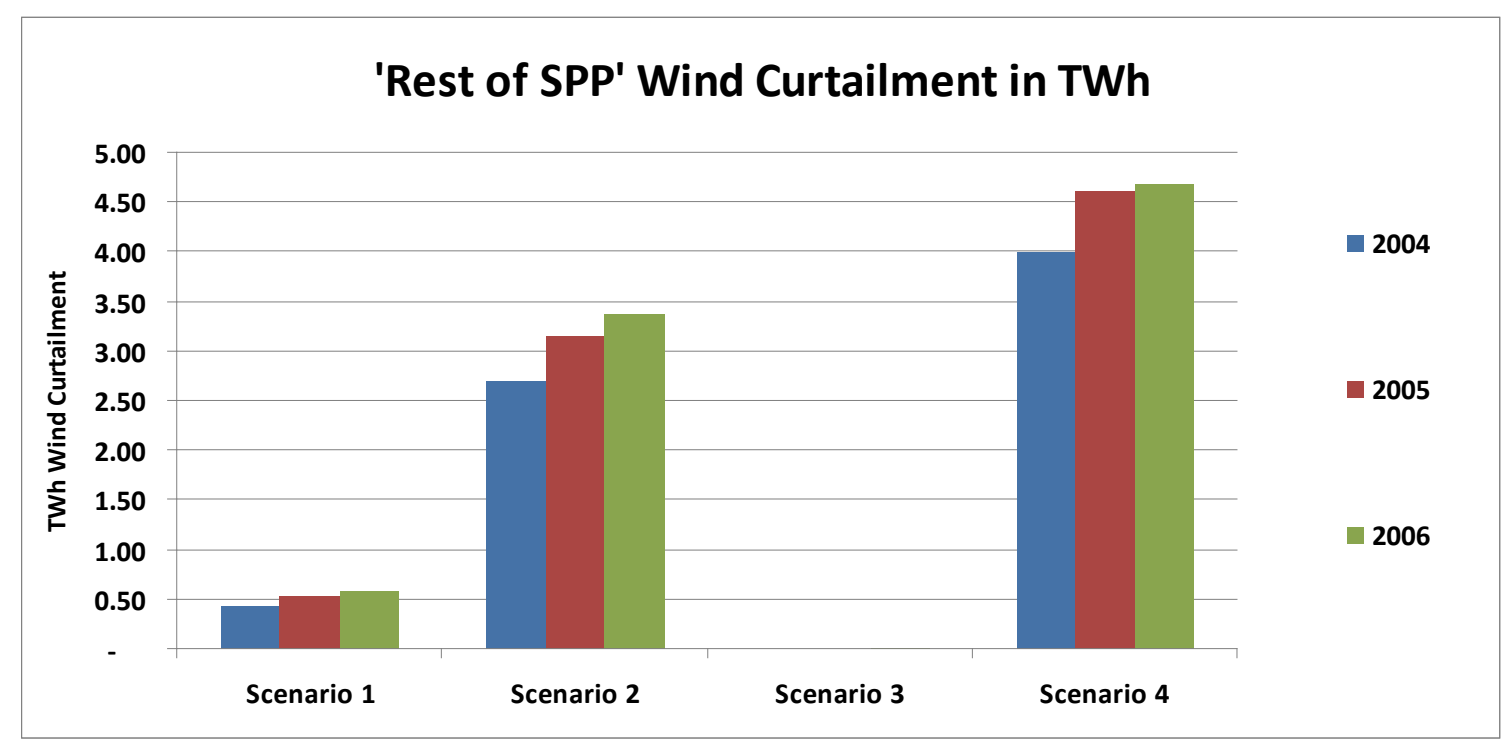

Figure 39: Wind Curtailment (TWh) in non-Nebraska SPP 


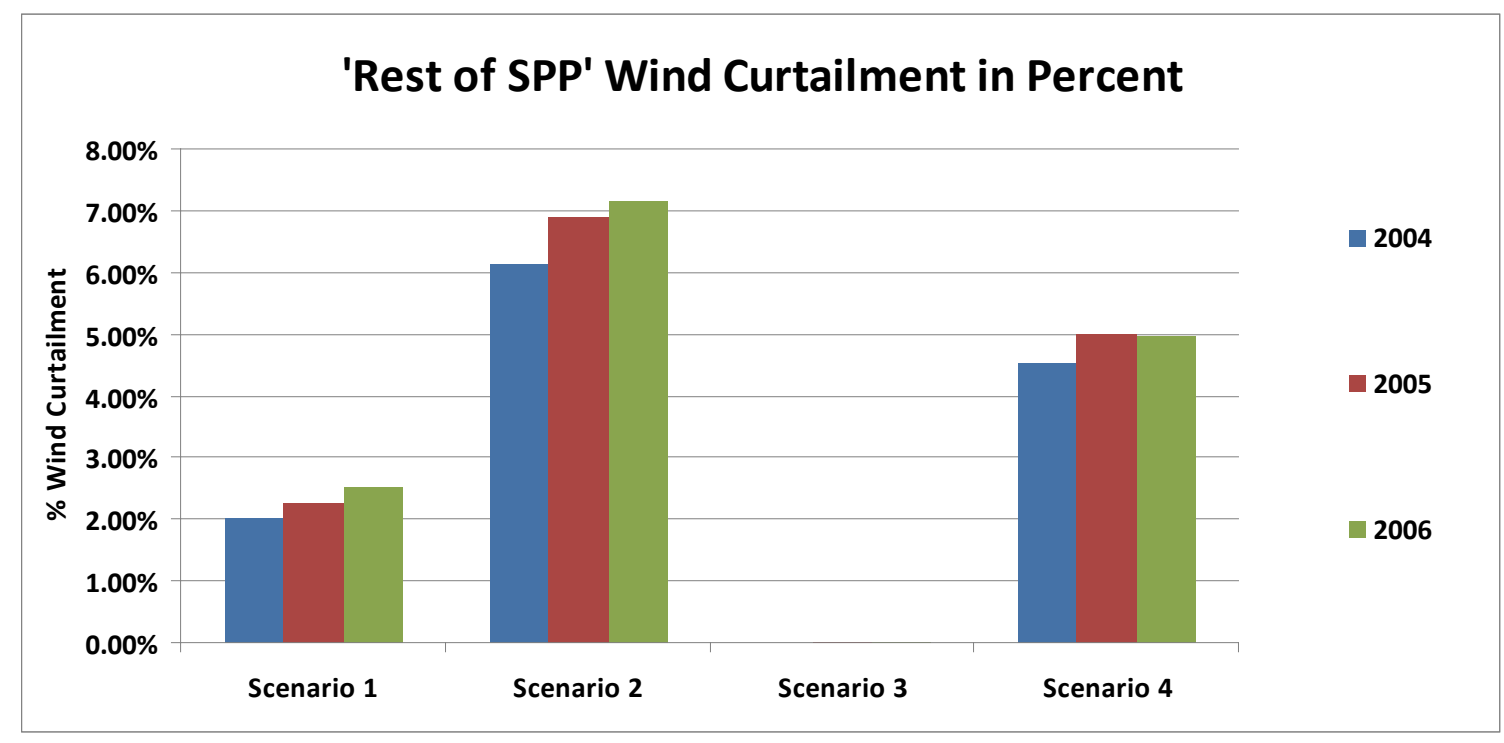

Figure 40: Wind Curtailment (\% of annual expected wind energy) in non-Nebraska SPP

In all scenarios, for all years, wind assigned to Nebraska experienced no curtailment and the wind in the rest of SPP experienced curtailment that increased with penetration level and decreased with the addition of EHV transmission overlay. Although this has been discussed earlier in the report, it is worth noting again that transmission congestion (and thus wind curtailment) is driven by the modeled flowgates in the PROMOD IV network model. Those constraints are apparently not causing congestion that results in wind curtailment in Nebraska, but that does not mean that no curtailment will ever occur even at these penetration levels. Congestion causing curtailment is often a local issue; this study has assumed that these wind injections will be interconnected in such a manner as to allow deliverability to the high voltage network.

Another consideration in the curtailment discussion is that of minimum generation levels and dump energy. Because wind is bid in at $-\$ 40 / \mathrm{MWh}$, the wind resources are the last to have their output levels affected. Thermal generation will be re-dispatched first and possibly attempt to violate minimum output levels to accommodate wind. When a thermal generator produces energy that can't be used by the system it is known in PROMOD as dump energy. More specifically, dump energy is the total amount of energy produced by generators in an area that could not be used by the system whether for native load, charging of storage or export. This energy may be unusable due to unit minimum segments exceeding load requirements, unit ramp constraints preventing desired unit movement or transmission congestion preventing energy deliverability; all of these issues could occur more frequently with the presence of wind energy. Thus it is useful in the curtailment analysis to also view dump energy results, which could be considered as possible wind curtailment. For Nebraska, we also see minimal dump energy for the simulations indicating that Nebraska is able to accommodate or export additional wind energy without violating unit minimums or ramping constraints and that transmission congestion is not preventing energy delivery. Dump energy as a percent of wind energy is presented in the Table 24 below. 
Table 24: Nebraska Dump Energy as a Percentage of Wind Energy

\begin{tabular}{|r|r|r|r|r|}
\hline & Scenario 1 & Scenario 2 & Scenario 3 & Scenario 4 \\
\hline 2004 & $0.16 \%$ & $0.09 \%$ & $0.00 \%$ & $0.12 \%$ \\
\hline 2005 & $0.01 \%$ & $0.00 \%$ & $0.00 \%$ & $0.00 \%$ \\
\hline 2006 & $0.10 \%$ & $0.05 \%$ & $0.00 \%$ & $0.05 \%$ \\
\hline
\end{tabular}

In PROMOD simulations discussions of dump energy are often paired with discussions on emergency energy. Although not related to wind curtailment, emergency energy in PROMOD (also known as unserved energy) is an effective metric for validating model performance.

Emergency energy is simply an artificial resource used to balance generation to load when no more modeled generation is available and is an indication of a potential for the inability to serve load. In these base case simulations there was almost no emergency energy called on, with a maximum (out of all twelve base cases) of $108 \mathrm{MWh}$ or $0.00004 \%$ of load for SPP in the 2006 based Scenario 4.

\subsubsection{Transmission System Usage and LMP}

The lack of curtailment in Nebraska is somewhat misleading about the stress the transmission grid may be experiencing. There are other indicators which show the impact of the additional wind resources on the Nebraska transmission grid. Table 25 shows the number of hours in 2006 that modeled flowgates involving Nebraska branches are constrained (at their limits). In some cases the flowgates show an increase in constrained hours as the wind penetration increases (from Scenario 1 to 2 and from Scenario 3 to 4). Some even show a decrease in the amount of hours constrained as wind penetration increases indicating that the wind is providing counter flow that reduces the strain on the flowgate. All of the 15 constrained flowgates (out of the 20 Nebraska flowgates modeled) show a significant decrease in constrained hours with the addition of the EHV overlay in Scenario 3. The complete list of Nebraska flowgates is listed in Table 66 of appendix Section 8.3.1.

Table 25: Numbers of Constrained Hours for Flowgates in or near Nebraska

\begin{tabular}{|c|c|c|c|c|}
\hline Flowgate Name & Scenario 1 & Scenario 2 & Scenario 3 & Scenario 4 \\
\hline Kelly-Tecumseh 161 kV flo Cooper - St.Joe 345 kV & 102 & 39 & 0 & 0 \\
\hline Gill-Clearwater 138 flo Red Willow - Mingo 345 kV & 2725 & 4025 & 574 & 4975 \\
\hline St. Joe-Midway 161kV flo Fairport-Cooper/St. Joe 345kV & 123 & 246 & 81 & 371 \\
\hline Council Bluffs-Avoca 161kV flo Council Bluffs-Madison County 345kV & 28 & 99 & 15 & 100 \\
\hline Council Bluffs - River Bend 161 kV FLO Cooper - St.Joe 345 kV & 86 & 118 & 0 & 0 \\
\hline Gentleman to Red Willow 345 kV & 812 & 596 & 0 & 0 \\
\hline Sheldon-20th \& Pioneer 115kV flo Wagener-Mark Moore 345kV & 0 & 0 & 0 & 2 \\
\hline Red Willow to Mingo $345 \mathrm{kV}$ & 0 & 3 & 0 & 0 \\
\hline Nebraska City-Cooper 345kV & 30 & 20 & 7 & 2 \\
\hline S1226-Tekamah 161kV flo S3451-Raun 345kV & 11 & 22 & 0 & 0 \\
\hline 70th \& Bluff Xfmr flo Sub 3454-Wagener 345kV & 6 & 67 & 1 & 240 \\
\hline COOPER_S Interface & 34 & 131 & 0 & 0 \\
\hline FTCAL_S Interface & 697 & 603 & 88 & 255 \\
\hline GGS Interface & 51 & 44 & 0 & 0 \\
\hline GRIS_LNC Interface & 323 & 1230 & 0 & 96 \\
\hline
\end{tabular}

*flo = "for the loss of" 
Additionally, Table 26 shows the number of non-monitored branches in Nebraska that are approaching their limits by scenario (using the 2006 load and wind patterns). Note the increased number of branches approaching their limits as the wind penetration increases. Also note the significant decrease in that same number when the EHV overlay is added in Scenario 3. These are all branches that were not forced to honor their limits in the PROMOD IV simulation (i.e., not in the flowgates shown above) and are thus indicative of potential overloads or needs for re-dispatch that were not captured in the PROMOD IV results. The specific numbers of hours for the specific branches relating to Table 26 data can be found in Table 97 of the appendix Section 8.3.2.

Table 26: Number of Nebraska transmission branches near thermal limits

\begin{tabular}{|l|r|r|r|r|}
\hline & Scenario 1 & Scenario 2 & Scenario 3 & Scenario 4 \\
\hline Violations of Thermal Limit & 6 & 15 & 10 & 23 \\
\hline $\begin{array}{l}\text { Over 90\% of Thermal Limit (excludes } \\
\text { violations) }\end{array}$ & 6 & 11 & 4 & 2 \\
\hline $\begin{array}{l}\text { Over 80\% of Thermal Limit (excludes } \\
\text { violations and 90\% violations) }\end{array}$ & 15 & 15 & 11 & 10 \\
\hline $\begin{array}{l}\text { Total Non-monitored Branches over } \\
80 \% \text { of Thermal Limit }\end{array}$ & 27 & 41 & 25 & 35 \\
\hline
\end{tabular}

Locational Marginal Price is an effective indicator of both fleet usage and congestion. Figure 41 shows the average generation weighted LMP for SPP for all scenarios and all years. There is a consistent trend towards decreased LMP as wind penetration increases. It is interesting to consider decreased LMP in an environment where there is increased use of more expensive gas generation as shown above. As less expensive resources are being backed down to a) carry reserves, b) accommodate the wind and c) accommodate the faster responding units, it stands to reason that the marginal resource would be a less expensive, backed down unit leading to decreased LMPs with increased wind penetration. It is also interesting to note the small increase of LMPs between the $20 \%$ penetration cases with and without the transmission overlay (Scenarios 2 and 3 ) indicating that although the overlay is not having a big effect on LMPs, the increased export capability is resulting in some slight equalization of higher prices from the east with the lower LMPs in SPP. 


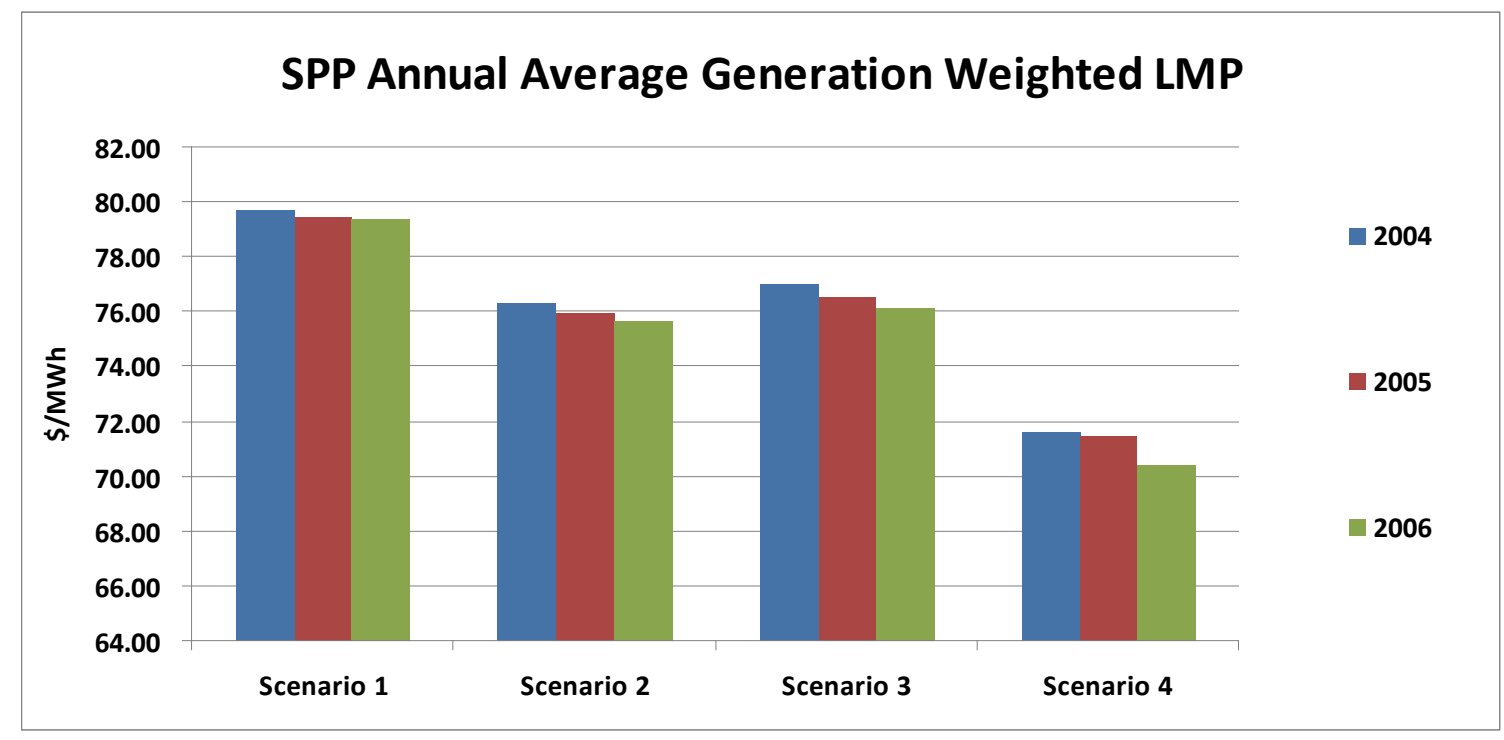

Figure 41: SPP (Including Nebraska) Annual Average Generation Weighted LMP

\subsubsection{Integration and Production Costs}

The changes in the fleet usage as reflected in the data above result in changes in the production cost for a given scenario. The difference in those production costs (between the actual wind case and the ideal wind case) are the integration costs and can be described in terms of dollars or dollars per megawatt hour of wind. It is important to note that this calculation is being performed using Adjusted Production Cost in order to account for interchange between areas and the cost differences of that interchange in different wind cases. As modeled in PROMOD in this study, Adjusted Production Costs are area or regional production costs adjusted to account for purchase and sale energy. It is also worth pointing out that these adjusted production costs do not include the cost of the wind energy - it is the cost associated with the thermal units. Adjusted productions costs for SPP, including Nebraska, are shown in Figure 42, followed by Integration Costs in dollars in Figure 43. The production costs consist of fuel and operations and maintenance costs, but do not include the installation cost or the fixed and variable O\&M costs of wind. Such costs are estimated and included in the Section 5 discussion on total cost, which also incorporates transmission and distribution costs.

In this section relating to base case runs, all integration costs were determined using the shaped proxy resource. Results for alternate proxy resource runs are included in Section 4.4 Sensitivity Results. 


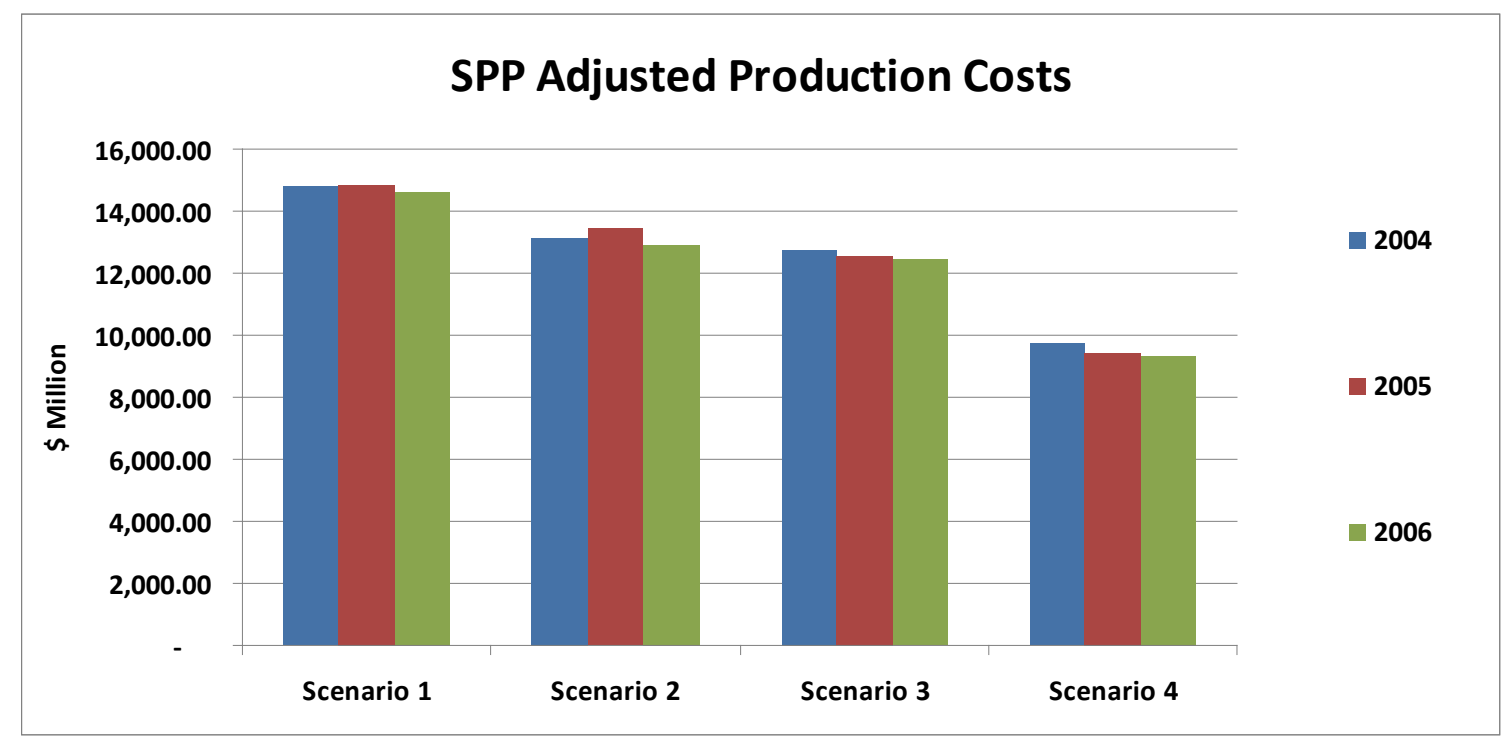

Figure 42: SPP (including Nebraska) Adjusted Production Costs

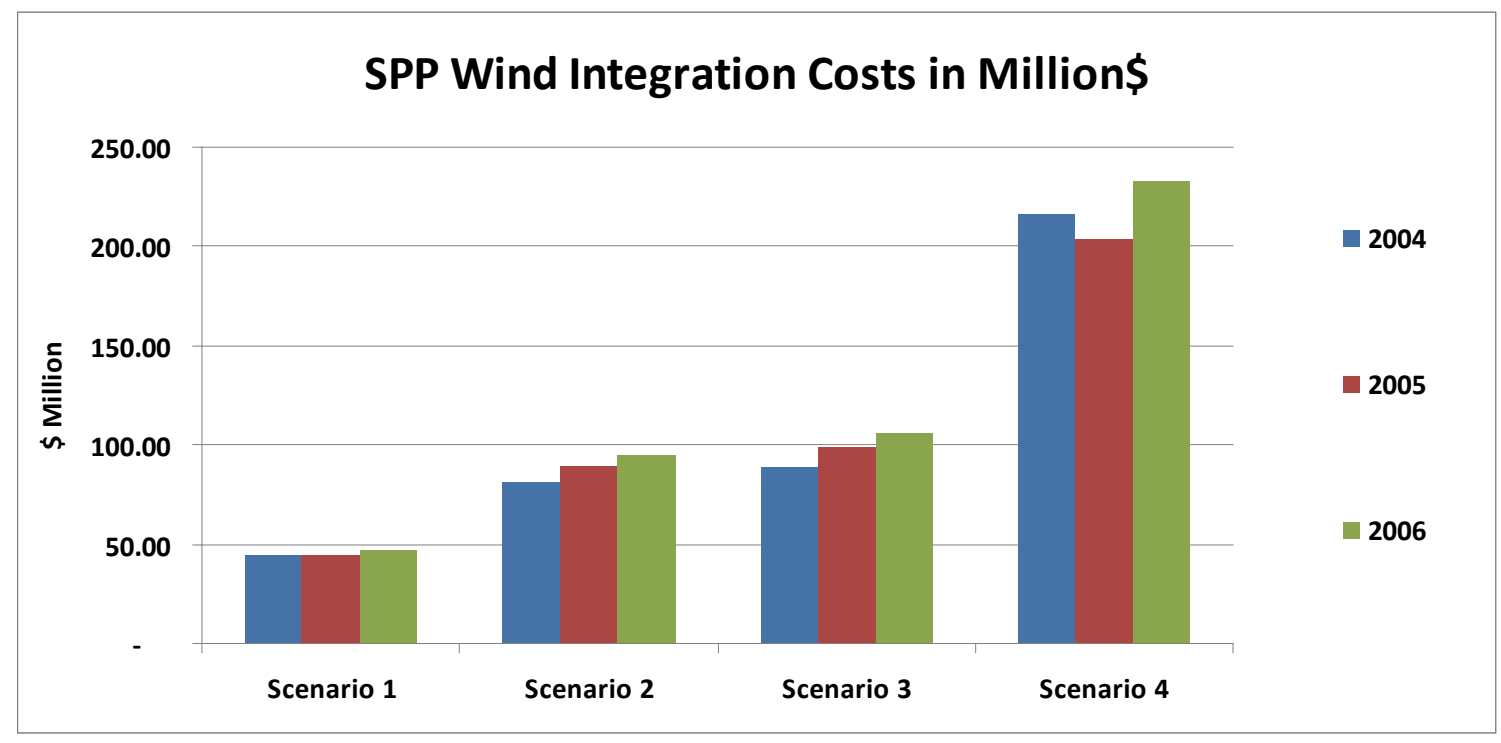

Figure 43: SPP (including Nebraska) Wind Integration Costs (shaped proxy) in Million\$

Adjusted Production Costs for the actual cases are decreasing with increased wind penetration because the wind (having no modeled production cost) is displacing thermal generation that has a cost associated with it. (This is not an indication of total portfolio costs; see Section 5 for a discussion of total costs including wind costs.) However, the adjusted production costs difference between the actual wind case and the ideal wind case increases as the addition of wind results in the use of more expensive gas resources in order to meet increased reserve requirements and cover wind forecast errors. Those increasing differences are roughly proportional to the increase in wind amounts as shown by the flatness of the normalized 
integration costs in 2018 dollars in Figure 44. These integration cost results use a "shaped proxy resource" in the ideal case, and is reflective of integration costs due to regulating reserves for wind and wind forecast errors.

Investigations into other proxy resource definitions are discussed in the Sensitivity Results Section 4.4.4. Such alternate proxies include additional integration costs called shape (or variation) costs herein. Section 4.4.4 also contains results from an examination of the degree to which these shape costs are "exported" to areas outside of SPP. Lastly, regarding these shape costs especially, it is noted that if the external regions had been modeled with higher penetrations, the computed integration costs may have been higher because the ability of these external systems to "help" integrate the SPP wind would have been reduced.
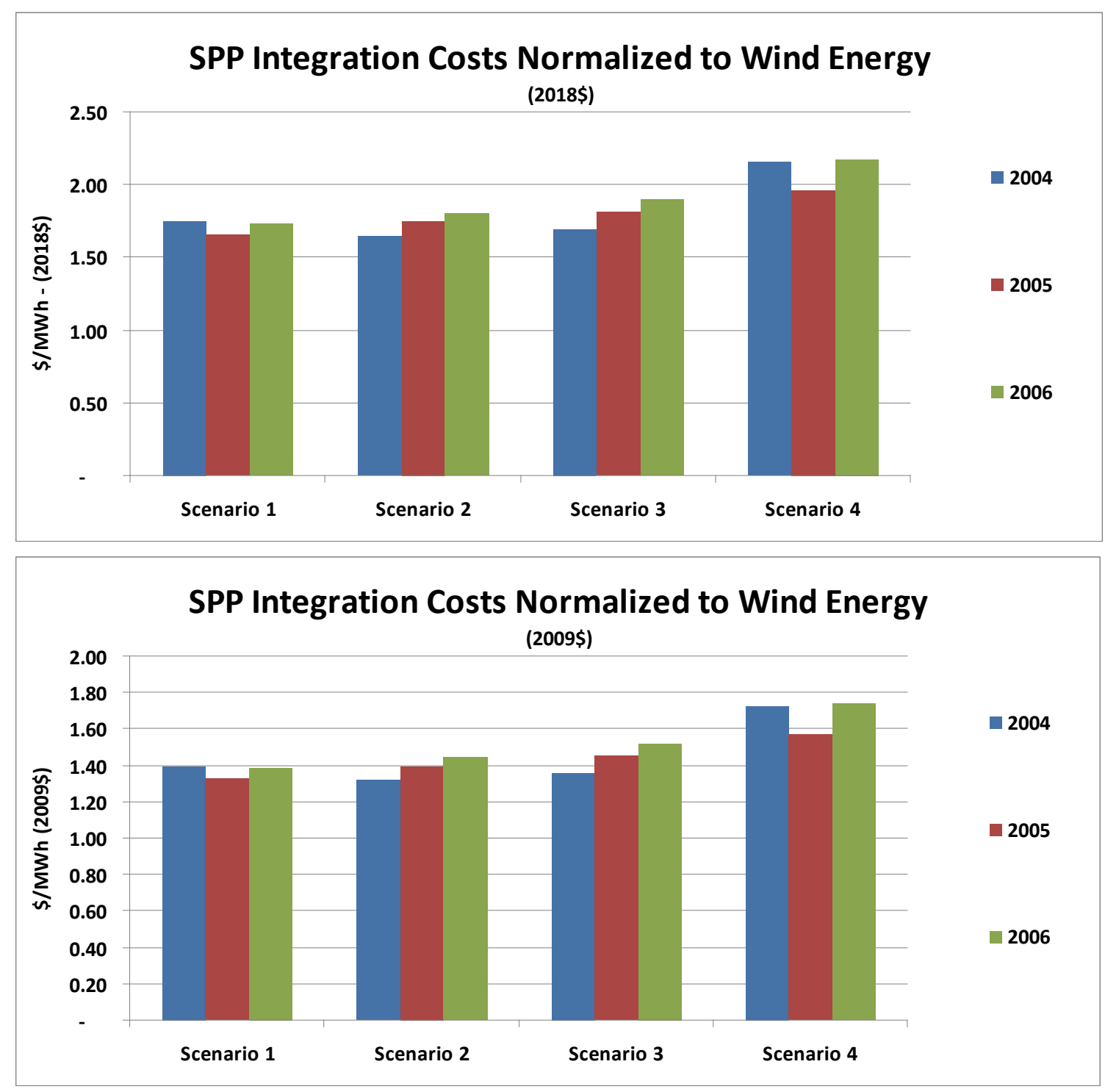

Figure 44: SPP (including Nebraska) Wind Integration Costs (shaped proxy) Normalized to Wind Energy for 2018 Conditions shown both in $2018 \$$ (top) and $2009 \$$ (bottom) 
There is a very slight increase in integration costs from Scenario 2 (20\% Wind) to Scenario 3 (20\% Wind with EHV Overlay). Although adjusted production costs are decreasing with the addition of the transmission overlay, the difference between the actual and ideal cases is increasing. This increasing difference is consistent with an increase in the amount of dispatched wind from Scenario 2 to Scenario 3. In other words, addition of the EHV overlay is resulting in decreased wind curtailment and that additional wind energy has an integration cost. Those incremental changes are relatively small however. Specifically, as a result of adding the overlay in Scenario 3 the 2006 pattern wind generation increases 3.37 TWh (curtailment is eliminated) and the difference between actual and ideal adjusted production costs increases by $\$ 11$ Million which results in an increase of 9 cents / MWh in SPP normalized integration costs.

Through the course of this study the project team and Technical Review Committee have discussed the potential effects of calculating regional integration costs in the contexts of a larger study footprint. Basically the team was interested in whether or not integration costs may be being exported from SPP to the rest of the country or more generically from high wind penetration areas to lower penetrations areas. Given that the regional (SPP) wind penetration is increasing and a substantial amount of energy is being exported to the rest of the footprint, it seems logical that there may also be some integration costs exported along with that energy.

To investigate that possibility, integration costs for the full footprint can be calculated and compared to the regional integration costs that have already been presented. To perform this analysis full footprint integration costs (non-normalized) for the 4 base case scenarios using the 2006 load and wind profiles were developed. To clarify, these are not integration costs for the rest of the country excluding SPP; they are for the whole footprint inclusive of SPP. It should also be noted that when calculating integration costs at the footprint level, there is no need for an adjusted production cost calculation. The adjustment is not necessary because all interchange is accounted for in the total production cost.

Below in Table 27 are integration costs in \$Million (2018) for both SPP and the footprint by scenario. The first point of interest here is the relatively consistent delta between the two ranging from $\$ 78$ to $\$ 87$ Million. These are the integration costs associated with the non-SPP portion of the footprint. By remaining somewhat flat rather than showing a consistent pattern of increase with increased SPP wind penetration, these results indicate that integration costs are not being exported from SPP to the rest of the footprint but are being captured within SPP.

Table 27: Integration Costs (shaped proxy) in Millions for SPP and Modeled Footprint

\begin{tabular}{|c|r|r|r|r|r|r|}
\hline & \multicolumn{3}{|c|}{ 2018 Dollars } & \multicolumn{3}{c|}{ 2009 Dollars } \\
\cline { 2 - 7 } \$ Million & \multicolumn{1}{|c|}{ SPP } & Footprint & Delta & SPP & Footprint & Delta \\
\cline { 2 - 7 } Scenario 1 & 47.82 & 135.36 & 87.54 & 38.29 & 108.38 & 70.09 \\
Scenario 2 & 95.85 & 174.07 & 78.22 & 76.75 & 139.38 & 62.63 \\
Scenario 3 & 106.94 & 186.36 & 79.42 & 85.63 & 149.22 & 63.59 \\
Scenario 4 & 233.42 & 316.49 & 83.07 & 186.90 & 253.42 & 66.51 \\
\hline
\end{tabular}

Next, here is a comparison of the change from scenario to scenario in the Integration costs, Table 28. This is Scenario 2 results (from the above table) minus Scenario 1 results (from the above table) and so on. The point of interest to here is that the changes are roughly the same for SPP or the total footprint indicating that the integration cost increases captured in the SPP 
calculation are all of the integration costs increases occurring in the footprint. Especially look at the last line comparing Scenario 4 to Scenario 1 and showing a very small difference in increase for SPP or the footprint.

Table 28: Changes in Integration Cost (shaped proxy) With Wind Penetration Increase

\begin{tabular}{|l|r|r|r|r|}
\hline \multirow{2}{*}{ \$Million } & \multicolumn{2}{|c|}{2018 Dollars } & \multicolumn{2}{c|}{2009 Dollars } \\
\cline { 2 - 5 } & \multicolumn{1}{|c|}{ SPP } & Footprint & SPP & Footprint \\
\hline $10 \%$ to $20 \%$ & 48.03 & 38.70 & 38.46 & 30.99 \\
$20 \%$ to $20 \%$ w Overlay & 11.09 & 12.29 & 8.88 & 9.84 \\
$20 \%$ to $40 \%$ (both w Overlay) & 126.48 & 130.14 & 101.27 & 104.20 \\
$10 \%$ to $40 \%$ & 185.60 & 181.13 & 148.61 & 145.03 \\
\hline
\end{tabular}

Finally, here are normalized integration costs for the footprint in Table 29. These are less insightful but, like the above data, there is a similar pattern of increases by scenario rather than values indicating that the rest of the footprint is carrying an increasing burden.

Table 29: Normalized Wind Integration Costs (shaped proxy) for SPP and Modeled Footprint

\begin{tabular}{|l|r|r|r|r|}
\hline \multirow{2}{*}{ \$/MWh } & \multicolumn{2}{|c|}{ 2018 Dollars } & \multicolumn{2}{c|}{ 2009 Dollars } \\
\cline { 2 - 5 } & SPP & Footprint & SPP & Footprint \\
\hline Scenario 1 & 1.74 & 0.95 & 1.39 & 0.76 \\
Scenario 2 & 1.81 & 1.00 & 1.45 & 0.80 \\
Scenario 3 & 1.90 & 1.05 & 1.52 & 0.84 \\
Scenario 4 & 2.18 & 1.19 & 1.75 & 0.95 \\
\hline
\end{tabular}

Given these results it appears that this data doesn't support the theory that integration costs based on shaped proxy are being exported to the rest of the footprint. In fact, it supports the opposite. It is critical to point out though that these costs are associated with incremental regulating reserves and wind forecast error since these were done with the shaped proxy. Investigations into costs of the wind variability being exported are presented in Section 4.4.4.

\subsubsection{Summary Metrics}

Figure 45 is a summary of key numerical results for the base case simulations for all scenarios and all years. Some of the charts above were limited to 2006 in order to avoid overloading the reader; however, a review of the table below should confirm that the trends presented above are consistent regardless of wind year. 


\begin{tabular}{|c|c|c|c|c|c|c|c|c|c|c|c|c|}
\hline & \multicolumn{3}{|c|}{ Scenario 1} & \multicolumn{3}{|c|}{ Scenario 2} & \multicolumn{3}{|c|}{ Scenario 3} & \multicolumn{3}{|c|}{ Scenario 4} \\
\hline & 2004 & 2005 & 2006 & 2004 & 2005 & 2006 & 2004 & 2005 & 2006 & 2004 & 2005 & 2006 \\
\hline Load (TWh) - SPP & 265 & 265 & 265 & 265 & 265 & 265 & 265 & 265 & 265 & 265 & 265 & 265 \\
\hline Actual Wind (TWh) - SPP & 25.75 & 27.76 & 27.52 & 49.69 & 51.63 & 52.95 & 52.39 & 54.79 & 56.32 & 100.31 & 104.20 & 107.23 \\
\hline Ideal Wind (TWh) - SPP & 25.68 & 27.15 & 27.45 & 49.61 & 51.51 & 52.86 & 52.38 & 54.78 & 56.31 & 100.27 & 104.14 & 107.18 \\
\hline All Other Gen/Resources (TWh) - SPP & 250.72 & 249.41 & 250.05 & 237.34 & 236.55 & 236.09 & 237.17 & 236.29 & 235.83 & 214.41 & 212.45 & 211.00 \\
\hline Actual APC (\$M) - SPP & 14,815 & 14,685 & 14,655 & 13,162 & 13,013 & 12,924 & 12,772 & 12,579 & 12,462 & 9,759 & 9,488 & 9,334 \\
\hline Ideal APC (\$M) - SPP & 14,770 & 14,639 & 14,607 & 13,079 & 12,922 & 12,828 & 12,683 & 12,480 & 12,355 & 9,542 & 9,283 & 9,101 \\
\hline Delta APC (Actual minus Ideal) - SPP & 45 & 45 & 48 & 82 & 90 & 96 & 89 & 100 & 107 & 217 & 204 & 233 \\
\hline Integration Costs (\$/MWh) - SPP & 1.75 & 1.66 & 1.74 & 1.65 & 1.75 & 1.81 & 1.70 & 1.82 & 1.90 & 2.16 & 1.96 & 2.18 \\
\hline Wind Curtailment (\%) - Nebraska & $0 \%$ & $0 \%$ & $0 \%$ & $0 \%$ & $0 \%$ & $0 \%$ & $0 \%$ & $0 \%$ & $0 \%$ & $0 \%$ & $0 \%$ & $0 \%$ \\
\hline Wind Curtailment (\%) - Rest of SPP & $2 \%$ & $2 \%$ & $3 \%$ & $6 \%$ & $7 \%$ & $7 \%$ & $0 \%$ & $0 \%$ & $0 \%$ & $5 \%$ & $5 \%$ & $5 \%$ \\
\hline Exports (TWh) & 11.48 & 12.24 & 12.59 & 21.24 & 22.40 & 23.09 & 24.53 & 26.09 & 27.13 & 49.69 & 51.37 & 52.85 \\
\hline CO2 Emissions (Million Short Tons) & 203.07 & 202.83 & 202.75 & 193.22 & 192.93 & 192.46 & 194.53 & 194.22 & 193.59 & 175.64 & 174.14 & 172.58 \\
\hline SPP Wind Reserve (Max MW) & 884 & 853 & 852 & 1,538 & 1,540 & 1,542 & 1,538 & 1,540 & 1,542 & 3,003 & 3,041 & 3,034 \\
\hline SPP Wind Reserve (Avg MW) & 513 & 505 & 499 & 1,021 & 1,007 & 996 & 1,021 & 1,007 & 996 & 2,094 & 2,058 & 2,042 \\
\hline \multicolumn{13}{|l|}{ Nebraska Generation (TWh) } \\
\hline Nebraska Total Gen & 48.86 & 49.14 & 49.25 & 51.83 & 52.45 & 52.57 & 53.15 & 53.65 & 53.91 & 57.75 & 58.37 & 58.81 \\
\hline Nebraska Combined Cycle & 3.24 & 3.15 & 3.16 & 2.84 & 2.73 & 2.71 & 3.08 & 2.95 & 2.97 & 2.18 & 2.06 & 1.97 \\
\hline Neb Comb Cycle Cap Factor & $40 \%$ & $39 \%$ & $39 \%$ & $35 \%$ & $34 \%$ & $34 \%$ & $38 \%$ & $37 \%$ & $37 \%$ & $27 \%$ & $26 \%$ & $24 \%$ \\
\hline Neb CT Gas & 0.10 & 0.08 & 0.09 & 0.09 & 0.07 & 0.07 & 0.06 & 0.06 & 0.06 & 0.04 & 0.04 & 0.03 \\
\hline Neb ST Coal & 28.87 & 29.00 & 28.91 & 28.13 & 28.35 & 28.20 & 29.24 & 29.34 & 29.28 & 26.98 & 26.92 & 26.74 \\
\hline Neb ST Coal Cap Factor & $79 \%$ & $80 \%$ & $79 \%$ & $77 \%$ & $78 \%$ & $77 \%$ & $80 \%$ & $80 \%$ & $80 \%$ & $74 \%$ & $74 \%$ & $73 \%$ \\
\hline Neb Wind Actual & 4.30 & 4.56 & 4.75 & 8.44 & 8.96 & 9.26 & 8.44 & 8.96 & 9.26 & 16.23 & 17.03 & 17.75 \\
\hline \multicolumn{13}{|l|}{ SPP Generation (TWh) } \\
\hline Rest of SPP Total Gen & 227.62 & 228.02 & 228.32 & 235.20 & 235.73 & 236.46 & 236.41 & 237.42 & 238.24 & 256.97 & 258.28 & 259.42 \\
\hline Rest of SPP Comb Cycle & 41.19 & 40.56 & 40.72 & 35.72 & 35.10 & 35.18 & 32.83 & 32.10 & 32.39 & 26.58 & 25.83 & 26.15 \\
\hline SPP CC Cap Factor & $42 \%$ & $41 \%$ & $41 \%$ & $36 \%$ & $36 \%$ & $36 \%$ & $33 \%$ & $33 \%$ & $33 \%$ & $27 \%$ & $26 \%$ & $27 \%$ \\
\hline Rest of SPP CT Gas & 5.44 & 5.38 & 5.38 & 5.18 & 5.14 & 5.12 & 5.19 & 5.15 & 5.13 & 5.03 & 5.03 & 4.98 \\
\hline Rest of SPP ST Coal & 137.54 & 137.49 & 137.45 & 131.73 & 131.55 & 131.23 & 133.41 & 133.40 & 132.80 & 121.00 & 119.95 & 118.77 \\
\hline Rest of SPP ST Coal Cap Factor & $76 \%$ & $76 \%$ & $76 \%$ & $73 \%$ & $72 \%$ & $72 \%$ & $74 \%$ & $74 \%$ & $73 \%$ & $67 \%$ & $66 \%$ & $65 \%$ \\
\hline Rest of SPP Wind Actual & 21.45 & 22.67 & 22.77 & 41.26 & 42.66 & 43.68 & 43.95 & 45.82 & 47.05 & 84.08 & 87.17 & 89.48 \\
\hline \multicolumn{13}{|l|}{ Interchange } \\
\hline Nebraska Net Position (Exports) & 9.45 & 9.74 & 9.85 & 12.42 & 13.05 & 13.17 & 13.75 & 14.25 & 14.50 & 18.35 & 18.96 & 19.40 \\
\hline SPP Incl Neb Net Position (Exports) & 11.48 & 12.24 & 12.59 & 21.24 & 22.40 & 23.09 & 24.53 & 26.09 & 27.13 & 49.69 & 51.37 & 52.85 \\
\hline \multicolumn{13}{|l|}{ Carbon } \\
\hline Nebraska CO2 Emissions (MT) & 32.59 & 32.68 & 32.59 & 31.62 & 31.78 & 31.61 & 32.89 & 32.94 & 32.88 & 30.12 & 30.00 & 29.78 \\
\hline Rest of SPP CO2 Emissions (MT) & 170.49 & 170.15 & 170.16 & 161.61 & 161.15 & 160.84 & 161.64 & 161.28 & 160.70 & 145.52 & 144.13 & 142.80 \\
\hline Nebraska CO2 Emissions (M\$) & 814.67 & 816.98 & 814.86 & 790.39 & 794.52 & 790.27 & 822.20 & 823.46 & 822.12 & 753.07 & 750.01 & 744.54 \\
\hline Rest of SPP CO2 Emissions (M\$) & $4,262.18$ & $4,253.84$ & $4,253.98$ & $4,040.20$ & $4,028.71$ & $4,021.11$ & $4,041.09$ & $4,031.93$ & $4,017.54$ & $3,637.89$ & $3,603.36$ & $3,570.03$ \\
\hline
\end{tabular}

Figure 45: Summary of Base Case Metrics(All dollars are 2018\$) 
Notes on Base Case summary results metrics (Figure 45)

- $\quad$ Reserves noted as "wind reserves" are the incremental regulating reserves associated with the additions of wind generation (i.e., the difference between load and wind vs. load alone), as in Table 22.

- $\quad \mathrm{APC}=$ Adjusted Production Cost

- $\quad$ SPP includes NPA unless otherwise noted

- $\quad$ Scenario1: 10\% Wind Penetration

- $\quad$ Scenario2: 20\% Wind Penetration

- $\quad$ Scenario3: 20\% Wind Penetration w EHV Overlay

- $\quad$ Scenario4: 40\% Wind Penetration w EHV Overlay

- $\quad$ Thermal unit capacity factors calculated using maximum annual (Winter) Capacity

- $\quad$ Load regulating reserve values are shown in tables Table 58 and Table 59.

- Integration costs are based on shaped proxy resource.

- $\quad$ More detailed production simulation generation results can be found in appendix Section 8.3.2.

\subsection{Sensitivity DeVELOPMENT}

This section describes how the sensitivities were developed and in Section 4.4 the results of the sensitivities are presented.

"Sensitivity" PROMOD cases provide a useful method for testing an assumption and its effects on results. Typically one assumption is changed (sensitivity case) from another case (base case) in order to examine the effect of that change on some result. Several sensitivity cases were studied in the Nebraska wind study in order to explore in more detail the effects of market structure, methodology, transmission system, mitigation methods and more on wind integration costs and specifically these study results. In the Nebraska wind study, all of the sensitivity cases were run using the $\mathbf{2 0 0 6}$ wind generation and load patterns. Depending on the nature of the sensitivity, mostly the $10 \%$ (Scenario 1) case and the $20 \%$ with overlay (Scenario 3 ) were used as the base cases for the sensitivity. In addition, an "existing wind" case was run, non-shaped proxy resource runs were made with Scenario 2 (as well as with 1), and two transmission sensitivities were run with a transmission overlay modification to Scenarios 3 and 4 . In some cases the sensitivities were tested against other already developed sensitivities.

\subsubsection{Ideal Proxy Sensitivities}

Most previous wind integration studies have been performed using an ideal wind resource (the proxy resource) represented as an average of daily wind energy, but in this study all the base case simulations were performed using a 'shaped' proxy due to concerns about large ramps between daily averages at higher penetration levels. The shaped proxy is simply the actual shape of the scheduled wind energy with no forecast error or incremental reserve requirement. 
The purpose of these non-shaped proxy sensitivities was to include some shape (or variability) costs in the integration cost evaluation and to relate this study to previous study techniques (daily block proxy). Some other proxy approaches were considered in order to investigate a potentially more useful proxy for high penetration wind integration studies by minimizing large transitions found in the data, yet keeping a more steady proxy than the shaped proxy.

Proxy sensitivity cases notes:

- $\quad$ Run against Scenarios 1 and 2 (shaped proxy, 10\% and 20\%-no overlay, SPPwide market, $\mathrm{CO} 2$ at $\$ 25 /$ ton)

- Daily block - traditional daily average energy

- $\quad$ Sub-period block - on and off peak average energy by day, with 2 hour ramp between sub-periods. (Where on-peak is considered to be Hour ending 7 through hour ending 22, and the other hours are off peak).

- 13-hr moving average - average of the current hour $+/-6$ hours energy values for a given hour

- $\quad 5$-hour moving average - average of the current hour +/- 2 hours energy values for a given hour

- $\quad$ The proxy variations are only applied to Nebraska and the rest of the SPP while the rest of the Eastern Interconnection remains at the shaped proxy assumption.

Sample weeks for each proxy resource type are shown in Figure 46 and Figure 47 and compared to the actual shape of the wind. These samples are for a single week (168 hours) for a single bus.

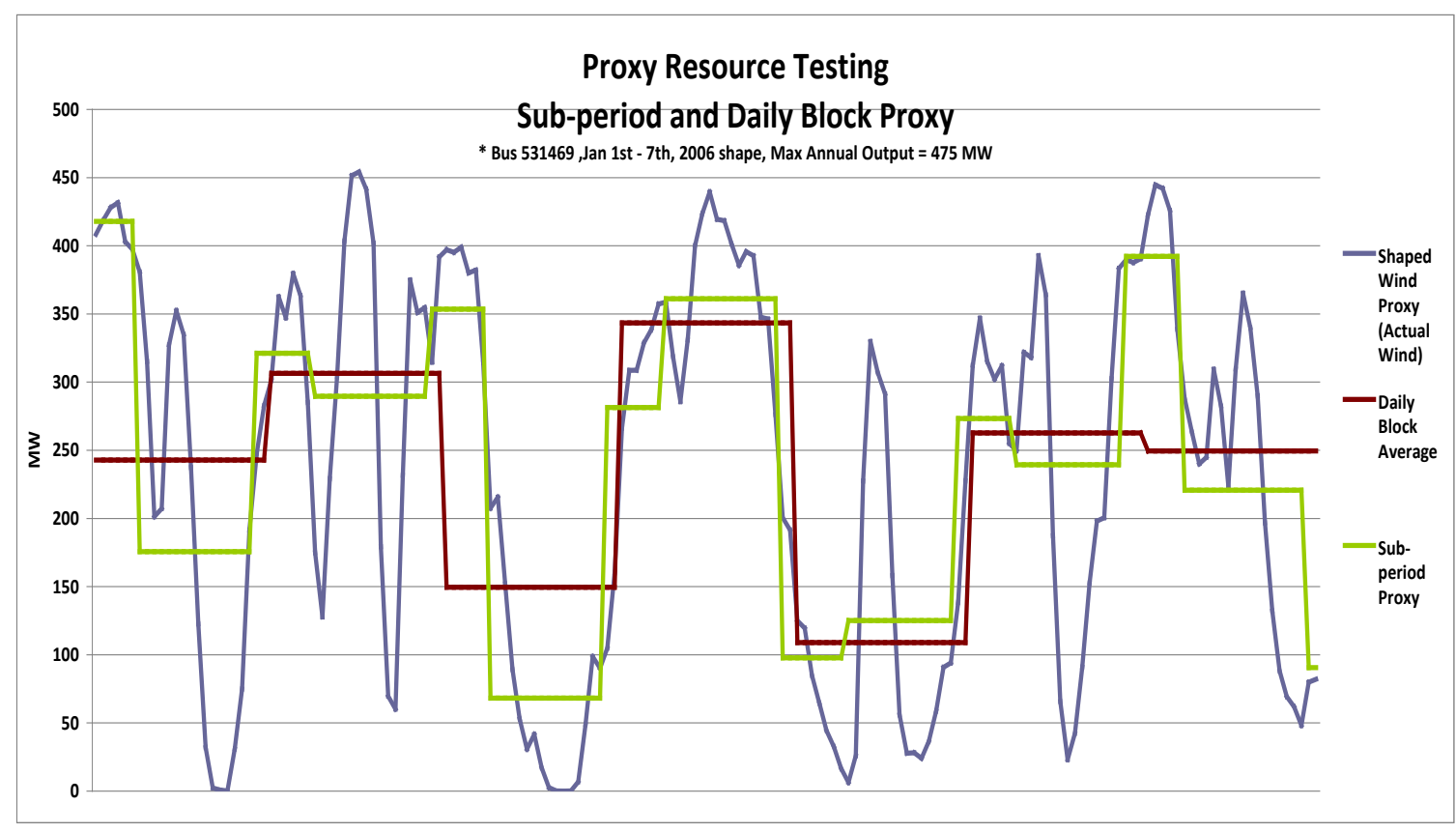

Figure 46: Comparison of Block vs. Shaped Proxy Types 


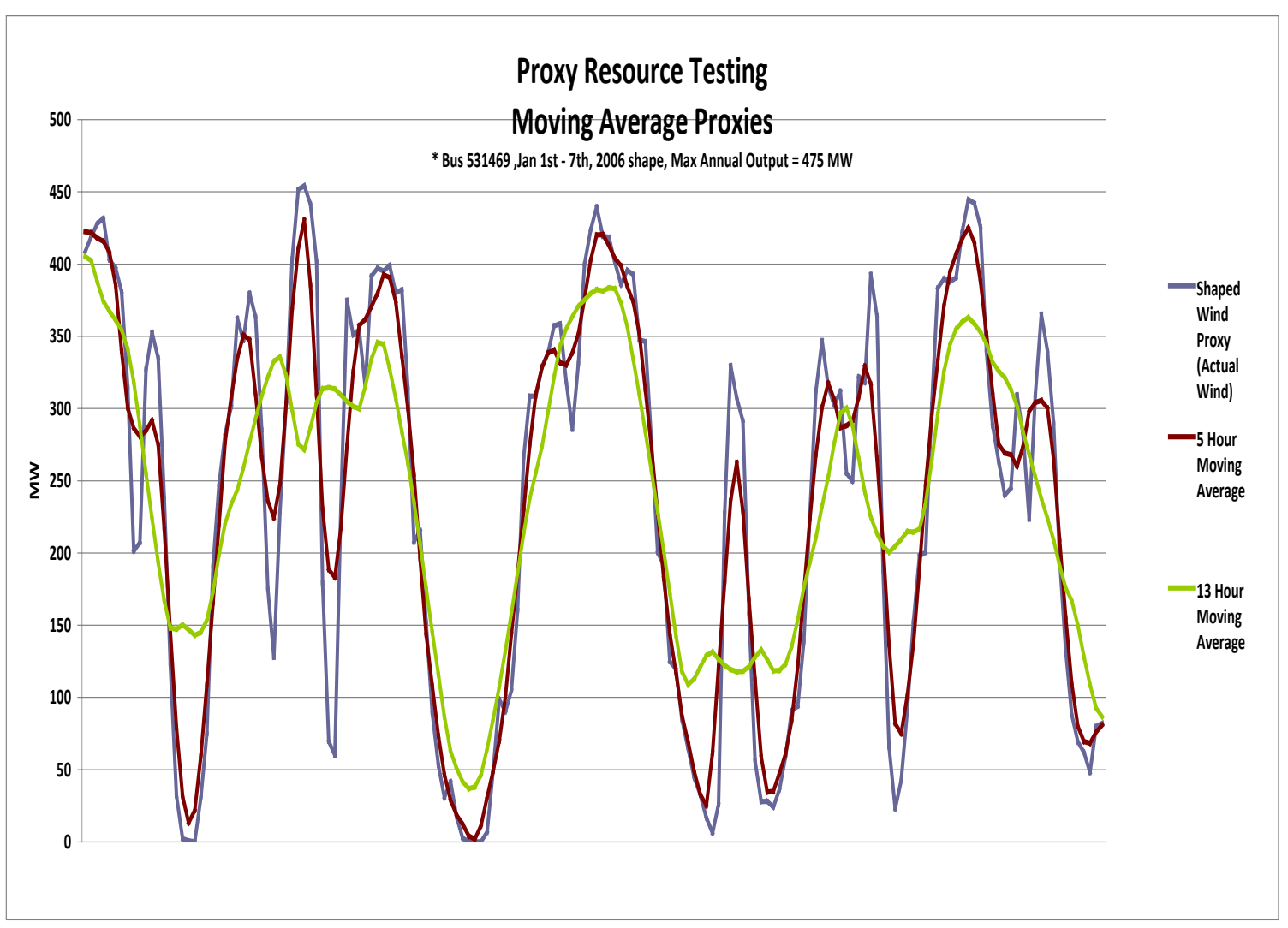

Figure 47: Comparison of Moving Average vs. Shaped Proxy Types

\subsubsection{CO2 Cost Sensitivities}

Given the current uncertainty as to what form future carbon legislation may take, if any, the study team undertook several 'CO2 cost sensitivities'. The possibilities for tax versus cap and trade and for varying levels of emissions caps are substantial. Towards the goal of capturing a range of those possibilities, there are three sensitivities on the dispatch signal for $\mathrm{CO} 2$. This is in addition to the base case assumption of $\$ 25$ / Ton (Short Tons) of CO2. PROMOD includes this price along with fuel costs, other emissions penalties and thermal variable $O \& M$, (wind variable O\&M is not part of the APC calculation) in a given unit's commitment and dispatch decisions.

CO2 sensitivity notes:

- $\quad$ Run against a base of scenario 3 (shaped proxy, 20\%-with overlay, SPP-wide market, $\mathrm{CO} 2$ at $\$ 25 /$ short ton)

- $\quad$ No penalty - $\$ 0 /$ ton - established a baseline 'no penalty' view, given the other assumptions including study year (2018), generation and transmission expansion and wind penetration (20\%)

- $\quad$ High Penalty - $\$ 50 /$ ton - doubled the CO2 penalty of the base case

- $\quad$ CO2 Reduction Case - $\$ 120 /$ ton - this penalty was derived from some analysis done to determine a point at which gas-fired combined cycle resources would 
displace coal. The concept was to force a reduction in $\mathrm{CO} 2$ through resource switching, not a prediction of a future price or tax on $\mathrm{CO} 2$ emissions.

- $\quad$ These values were applied universally across the entire model.

The $\mathrm{CO} 2$ reduction sensitivity was designed to result in a roughly $20 \%$ reduction in $\mathrm{CO} 2$ emissions (from current) in Nebraska. This was done by determining the crossover or breakeven point of $\mathrm{CO} 2$ pricing at which gas fired combined cycles would become cheaper than coal fired steam turbines. This analysis considers the forecast costs of both fuels (natural gas and coal), representative full load average heat rates for both plant types and representative $\mathrm{CO} 2$ emissions rates for each plant type. The analysis determined a breakeven cost (2018 dollars) of $105 \$$ /Short Ton. For simulation purposes that value was increased to $120 \$$ /short ton to account for variability in fleet heat rates and emissions rates and to insure maximum $\mathrm{CO} 2$ reduction.

\subsubsection{Market sensitivity \#1 - Nebraska standalone market}

Although the Nebraska entities have already joined SPP, the interest in the wind integration effects on a smaller market as compared to the full regional market of SPP to see more closely operation in today's market structure were studied. Today there is no SPP-wide day-ahead market or ancillary service market in SPP and there is no guarantee in 2018 that such markets will materialize although they are being planned. To investigate that issue a sensitivity was developed that treated Nebraska as a smaller coordinated market separated from SPP. It is important to note the distinction between this treatment of a coordinated Nebraska and historical operations that did not involve joint dispatch of the three separate balancing areas in Nebraska.

Nebraska Standalone sensitivity notes:

- $\quad$ Run against a base of Daily Block proxy sensitivity with $10 \%$ wind penetration (i.e., this is 2 step changes from Scenario 1 - change to the daily block proxy and change to the Nebraska only market.)

- $\quad$ All of the rest of the SPP remains as a single market with coordination of commitment and dispatch among those remaining entities.

- $\quad$ Nebraska operates alone, but coordination within Nebraska (joint dispatch) exists.

In order to properly simulate Nebraska operations separated from the rest of SPP all reserves assumptions had to be addressed as the base case simulations include reserve sharing for the whole of SPP including Nebraska. This includes splitting out the regulation for wind as well as establishing independent contingency reserve assumptions.

Contingency reserves for SPP as a whole are $1539 \mathrm{MW}$ and remain that way even with Nebraska split out. Nebraska reserves for this sensitivity were developed based on 2007 Midwest Reserve Contingency Sharing Group values as outlined in an August 25, 2006 letter to FERC from Gregory A. Troxwell. A single Nebraska contingency reserve total of $132 \mathrm{MW}$ was created from the values reported for NPPD, OPPD, LES, MEAN and City of Hastings. Total contingency reserves for the sensitivity and base case are shown in Table 30. 
Table 30: $\quad$ Contingency Reserve Requirements for Nebraska and SPP

Contingency Reserve Requirements

\begin{tabular}{|l|c|c|}
\hline & Single Market (Base Case) & Split Market (Sensitivity) \\
\hline Nebraska & NA & $132 \mathrm{MW}$ \\
\hline SPP & $1539 \mathrm{MW}$ & $1539 \mathrm{MW}$ \\
\hline
\end{tabular}

Figure 48: Represents the changes made to reserves, hurdle rates and market structure in the Nebraska standalone market sensitivity.

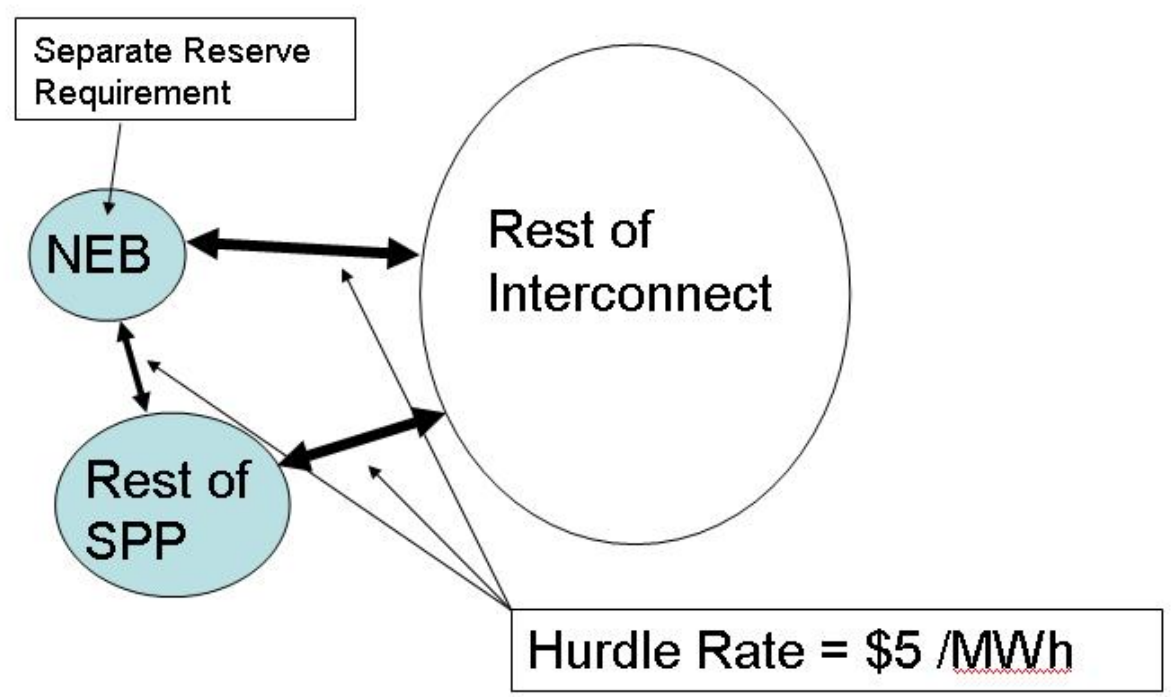

Figure 48: Representation of separate Nebraska Market

\subsubsection{Market sensitivity \#2 - Hurdle rate raised from $\$ \mathbf{5} / \mathbf{M W h}$ to $\mathbf{\$ 2 0} \mathbf{M W h}$}

During commitment and dispatch simulations PROMOD uses a 'hurdle rate' or 'tariff' to determine whether or not interchange should occur between regions. If LMPs in those regions have a bigger differential than the hurdle rate, interchange can occur. If not, no interchange occurs. The hurdle rate is intended to simulate the effects of uncoordinated markets and inefficiencies in interchange between those markets. The base hurdle rate assumption for this study is $\$ 5 / \mathrm{MWh}$ and is a typical assumption for modeling interchange between regions. In an attempt to investigate whether or not interchange with outside regions is dampening wind integration effects this sensitivity was developed using a $\$ 20 / \mathrm{MWh}$ hurdle rate. Thus, only very large LMP differences (over $\$ 20 / \mathrm{MWh}$ ) will result in energy being exchanged between SPP and an outside region.

Hurdle rate sensitivity notes:

- $\quad$ Run against Scenario 3 base case $-20 \%$ with overlay.

- $\quad$ Run with the shaped proxy resource. 
- Hurdle rate change was between SPP (including Nebraska) and the rest of the regional interconnections.

Figure 49 represents the changes to hurdle rate between SPP and the rest of the interconnection in the hurdle rate sensitivity.

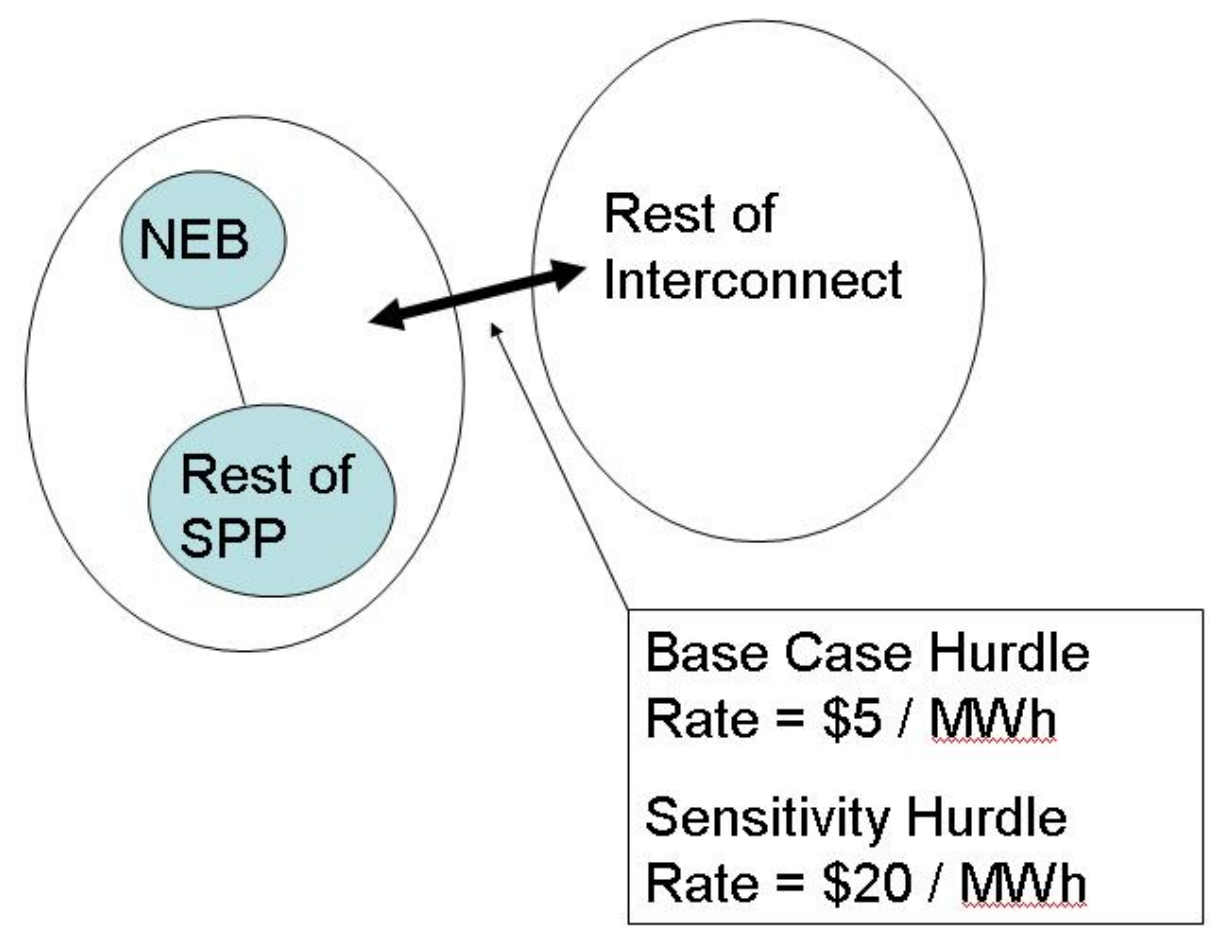

Figure 49: Representation of Hurdle Rates in PROMOD IV

\subsection{5. $\quad$ Existing Wind Only for SPP and Nebraska}

The existing wind sensitivity was developed in order to establish a reference case of integration effects given current (2009) minimal wind penetration levels. The Nebraska standalone market was used to more closely represent today's operations without SPP-wide day-ahead market and ancillary services market. The Nebraska existing wind scenario has $140 \mathrm{MW}$ represented primarily Ainsworth and Elkhorn Ridge (approximately $1 \%$ penetration). The rest of SPP is based on a 3,000 MW value published on a Q\&A webpage on the SPP website at this time, http://www.spp.org/publications/SPP Wind Integration QA.pdf (approximately 5\% penetration). Incremental reserves for wind were adjusted to account for these lower penetration values, as shown later in Figure 54; however, contingency reserves remained at the level for the $10 \%$ - Nebraska standalone case listed in Section 4.3.3.

The existing wind sensitivity was

- $\quad$ Run with Nebraska standalone market and Daily Block proxy sensitivity (i.e., 3 changes from base case Scenario 1 - change to daily block proxy, then change to 
Nebraska only market, then change to existing wind penetration; in other words it is one change from Market Sensitivity \#1).

Table 31 compares scheduled wind energy in the existing wind sensitivity to scheduled wind energy in the $10 \%$ base case.

Table 31: Wind Energy Comparison between Existing and 10\% Cases

\begin{tabular}{|l|r|r|}
\hline & Existing Wind & $10 \%$ Case (2006) \\
\cline { 2 - 3 } & Energy (GWh) & Energy (GWh) \\
\hline Nebraska & 492 & 4,749 \\
\hline Rest of SPP & 11,204 & 23,368 \\
\hline
\end{tabular}

\subsubsection{WAPA mitigation sensitivity}

One of the purposes of this study is to investigate potential methods of mitigating wind integration effects. One often discussed mitigation method is the use of flexible hydro resources to respond to the hourly variability in the wind that would normally be covered by more expensive thermal (usually gas) resources. The Nebraska entities have allocations from WAPA that could theoretically be useful for that mitigation. This sensitivity was designed with that in mind.

WAPA mitigation sensitivity notes:

- $\quad$ Run using the Nebraska only sensitivity (Market sensitivity \#1, with $10 \%$ wind penetration) as the base setup. The Nebraska only market was selected to focus the results on the destination of the WAPA energy.

- $\quad$ Does not involve the ideal case or proxy resource (Actual wind cases with two different hydro schedules are compared).

- $\quad$ Modeling changes affect only the Nebraska firm purchases from WAPA. Peaking purchases are not involved in the changes as they are a very small portion of the energy purchased from WAPA and have different contractual limitations.

- $\quad$ The sensitivity does not involve changing the hydro facilities generation patterns, just Nebraska's purchase patterns. WAPA generation pattern is set at the year 2000 pattern, a normal generation year identified by WAPA in their "Wind and Hydropower Feasibility Study" done in 2008.

The methodology for the WAPA mitigation sensitivity was relatively simple. In one case the WAPA firm purchases were scheduled in a load following pattern against the Nebraska entities' composite native load. This scheduling is done prior to any thermal unit commitment or dispatch and thus provides a 'net load' pattern to schedule those resources against. In the second case, the same load following algorithm was applied, but in this 'test case' the energy was scheduled to the load net of hourly wind. That results in a different 'net load' to perform unit commitment and dispatch on.

The load following algorithm applied for the scheduling method honors a weekly energy amount as well as a weekly maximum (capacity) amount. Hourly energy is scheduled proportional to the 
load such that the maximum amount is scheduled in the hour of the peak and all other hours purchase amounts are proportionally lower than that. There is no consideration for time of day or day of week, so that the schedules to load net of wind can have maximum adjustment within the energy and capacity constraints.

Figure 50, shows a sample week of Nebraska load and load net of wind along with the total hourly wind production for the week.

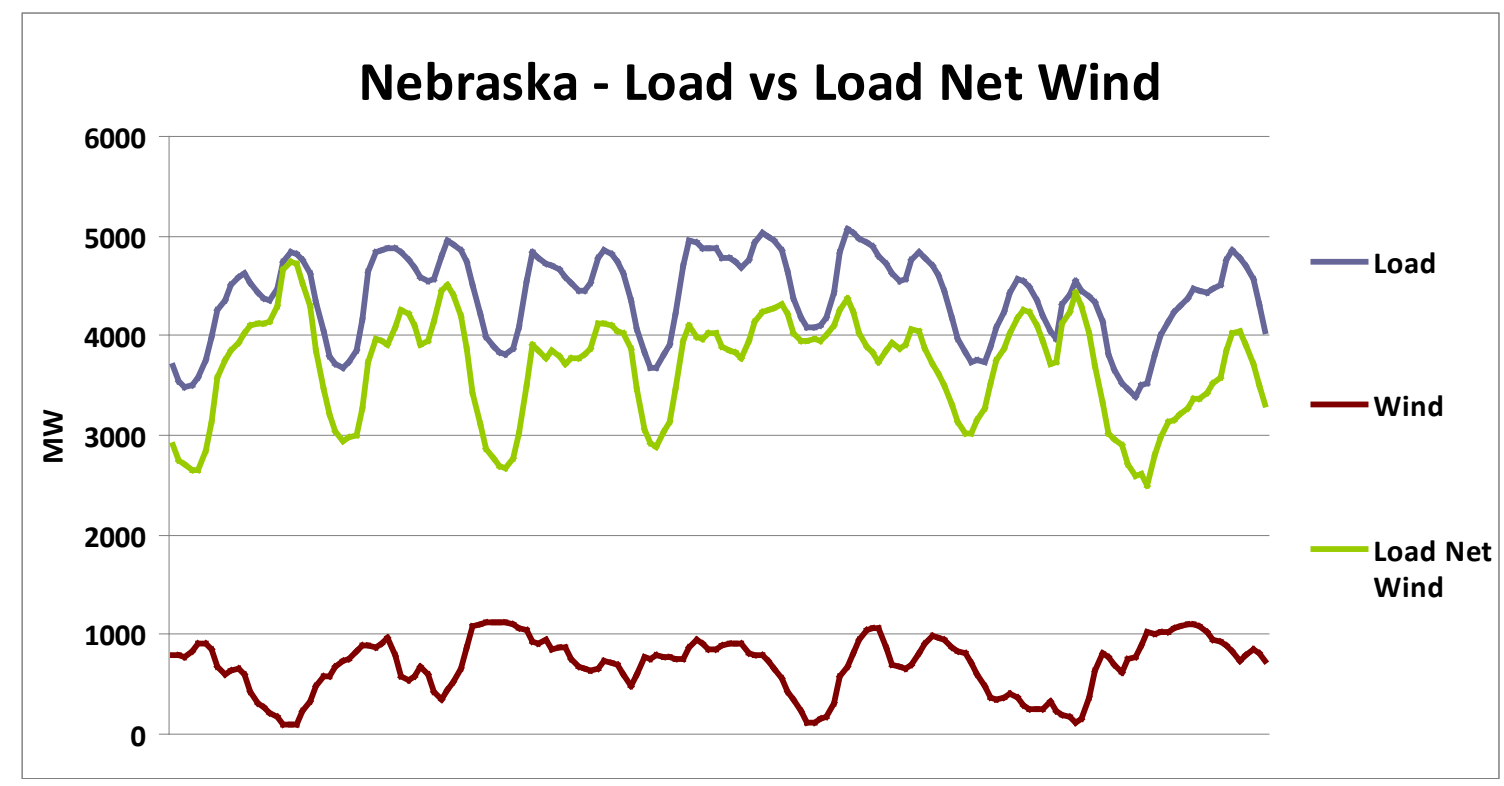

Figure 50: Comparison of Load and 'Load Net Wind' (Jan 1-7, 2018)

\subsubsection{5kV Nebraska Overlay Sensitivity}

As described earlier, base case Scenarios 3 ( $20 \%$ wind) and 4 ( $40 \%$ wind) utilize a conceptual extra high voltage transmission overlay for all of SPP including Nebraska. That overlay as modeled consists of $765 \mathrm{kV}$ branches throughout SPP. To further test possible refinement of this conceptual overlay, the Nebraska $345 \mathrm{kV}$ sensitivity simulates the $20 \%$ and $40 \%$ wind penetration levels with a $345 \mathrm{kV}$ overlay in Nebraska that interconnects with the $765 \mathrm{kV}$ overlay in the rest of SPP. This $345 \mathrm{kV}$ overlay mostly just replaces the $765 \mathrm{kV}$ overlay in Nebraska; however, there are some topology differences as well.

Nebraska 345 kV overlay notes:

- $\quad$ Run against Scenarios 3 (20\%) and $4(40 \%)$ base cases.

- $\quad$ Run with the shaped proxy resource.

- $\quad$ Assumes a $345 \mathrm{kV}, 3000 \mathrm{amp}$ design as is recommended by current SPP practices.

- The overlay is only changed in Nebraska and connections into Nebraska. The rest of SPP uses the same EHV overlay assumptions as the base case. 
Figure 51, Figure 52 and Figure 53 show the base case EHV overlay topology assumptions, the sensitivity EHV topology assumptions and a closer view of the Nebraska $345 \mathrm{kV}$ EHV sensitivity topology, respectively.

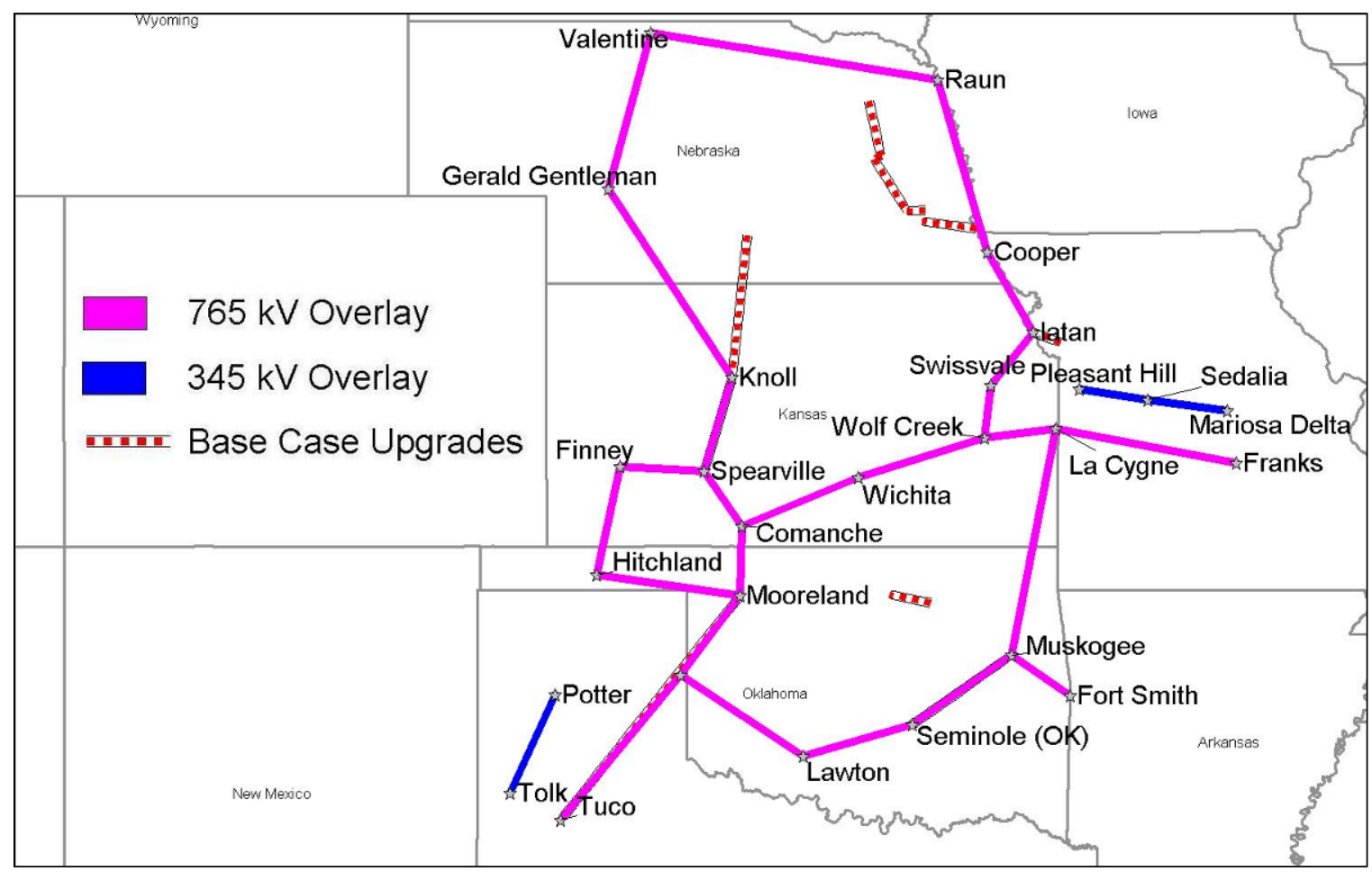

Figure 51: Base Case Conceptual EHV Overlay 


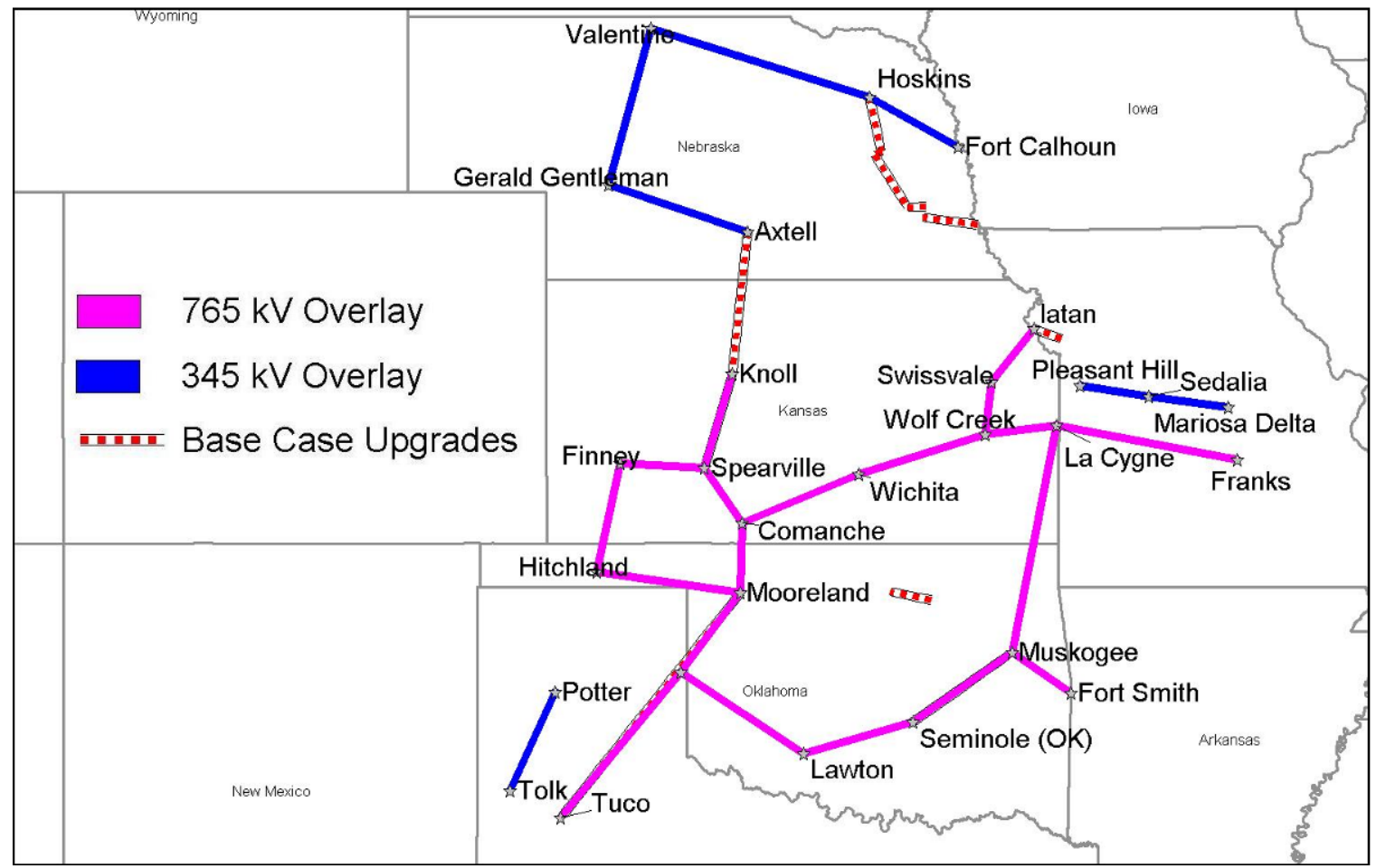

Figure 52: Conceptual EHV Overlay with 345 kV in Nebraska

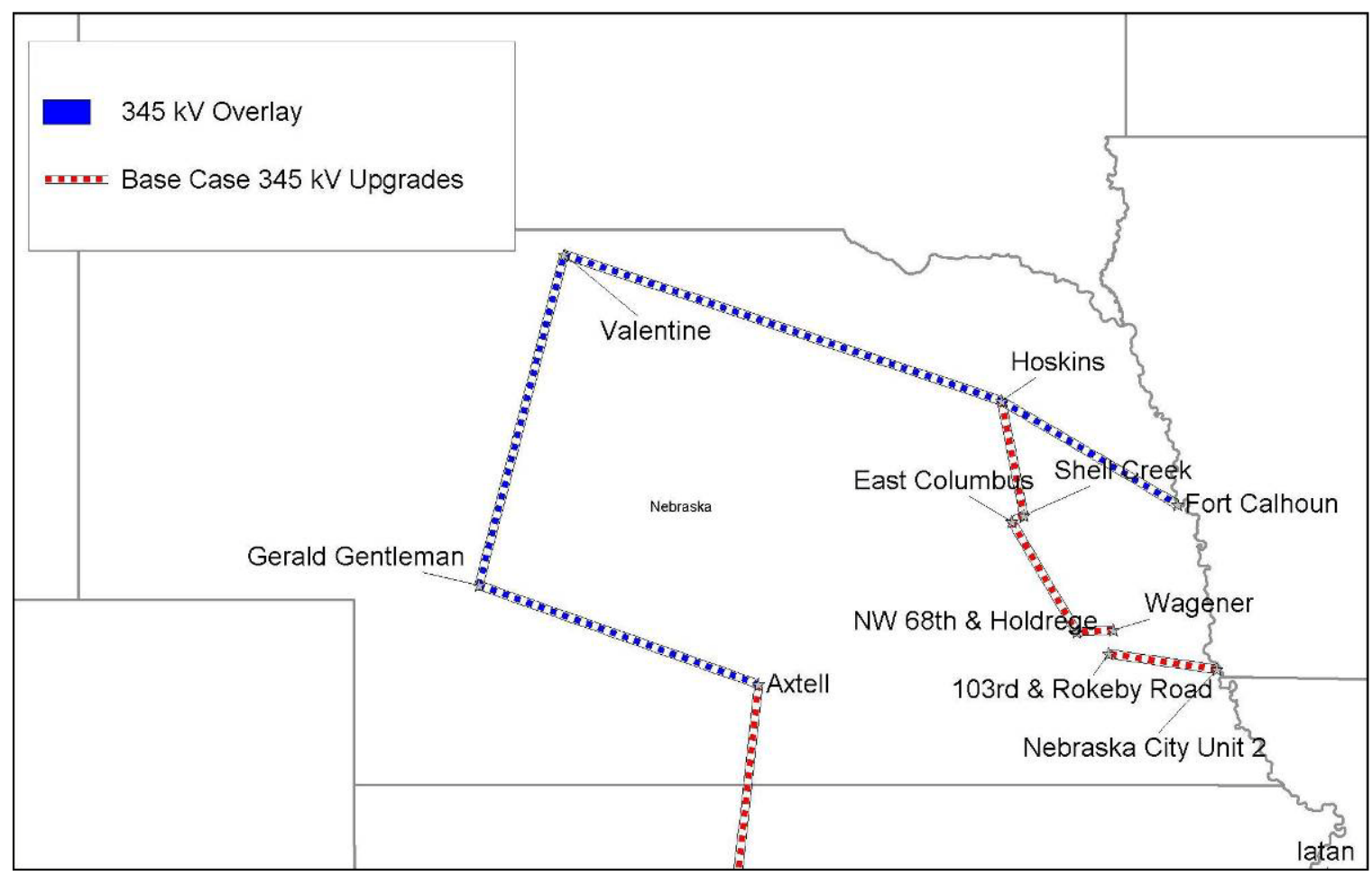

Figure 53: Conceptual 345 kV Overlay in Nebraska 


\subsection{SENSITIVITY RESULTS}

A series of sensitivities were performed as stress or investigative cases to compare to the base cases. Most were performed using the 2006 load and wind year either using the $10 \%$ wind assumptions (Scenario 1) or $20 \%$ with EHV overlay (Scenario 3). In addition, an "existing wind" case was run, non-shaped proxy resource runs were made with Scenario 2 (as well as with 1), and two transmission sensitivities were run with a transmission overlay modification to Scenarios 3 and 4 . The specifics for each sensitivity's setup, assumptions and results are described in the following sections. In each of the sensitivity sections below, key metrics deemed relevant to the scenario have been reported and discussed. A more comprehensive slate of metrics is available for all of the sensitivity simulations in Figure 54. 


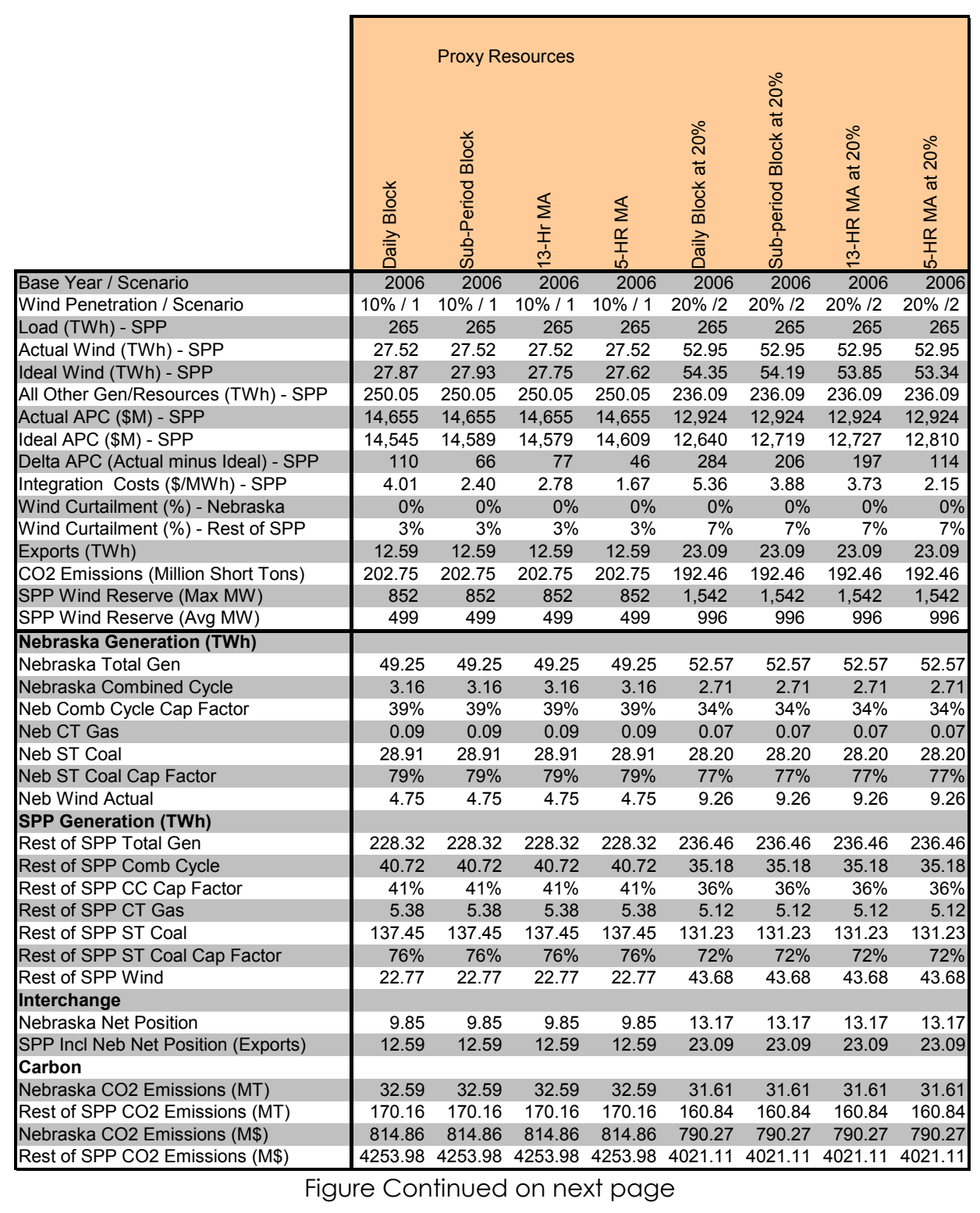




\begin{tabular}{|c|c|c|c|c|c|c|c|c|c|c|}
\hline & \multicolumn{5}{|c|}{ Market Sensitivities } & \multicolumn{3}{|c|}{ CO2 Sensitivities } & \multicolumn{2}{|c|}{$\begin{array}{l}\text { Transmission } \\
\text { Sensitivities }\end{array}$} \\
\hline & 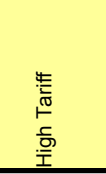 & 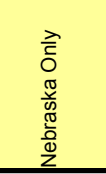 & 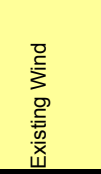 & 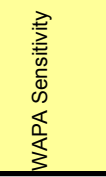 & 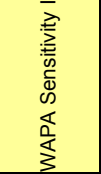 & \begin{tabular}{l}
\multicolumn{1}{c}{} \\
$\stackrel{\circ}{\circ}$ \\
$\varnothing$
\end{tabular} & 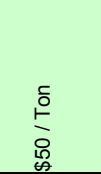 & 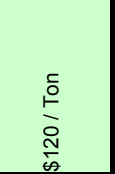 & 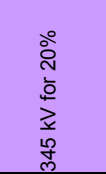 & 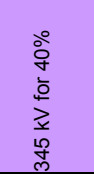 \\
\hline Base Year / Scenario & 2006 & 2006 & 2006 & 2006 & 2006 & 2006 & 2006 & 2006 & 2006 & 2006 \\
\hline Wind Penetration / Scenario & $20 \% / 3$ & $10 \% / 1$ & NA & $10 \% / 1$ & $10 \% / 1$ & $20 \% / 3$ & $20 \% / 3$ & $20 \% / 3$ & $20 \% / 3$ & $40 \% / 4$ \\
\hline $\begin{array}{l}\text { Load (TWh) - SPP } \\
\text { Letratio }\end{array}$ & 265 & 265 & 265 & 265 & 265 & 265 & 265 & 265 & 265 & 265 \\
\hline Actual Wind (TWh) - SPP & 56.32 & 27.54 & 11.70 & 27.53 & 27.52 & 56.31 & 56.31 & 56.32 & 56.31 & 107.23 \\
\hline Ideal Wind (TWh) - SPP & 56.30 & 27.87 & 11.70 & 27.53 & 27.53 & 56.30 & 56.32 & 56.32 & 56.30 & 106.87 \\
\hline All Other Gen/Resources (TWh) - SPP & 219.21 & 249.15 & 257.57 & 248.44 & 248.44 & 236.50 & 236.29 & 236.75 & 237.28 & 182.31 \\
\hline Actual APC (\$M) - SPP & 12,615 & 1,599 & 1,895 & 1,601 & 1,600 & 7,271 & 16,822 & 27,276 & 12,467 & 9,406 \\
\hline Ideal APC (\$M) - SPP & 12,511 & 1,574 & 1,880 & 1,601 & 1,599 & 7,121 & 16,732 & 27,246 & 12,359 & 9,169 \\
\hline Delta APC (Actual minus Ideal) - SPP & 103 & 25 & 15 & 0.2 & 0.9 & 149 & 90 & 30 & 108 & 238 \\
\hline Integration Costs (\$/MWh) - SPP & 1.83 & 5.27 & 30.08 & NA & NA & 2.65 & 1.60 & 0.54 & 1.92 & 2.22 \\
\hline Wind Curtailment (\%) - Nebraska & $0 \%$ & $0 \%$ & $0 \%$ & $0 \%$ & $0 \%$ & $0 \%$ & $0 \%$ & $0 \%$ & $0 \%$ & $0 \%$ \\
\hline Wind Curtailment (\%) - Rest of SPP & $0 \%$ & $2 \%$ & $0 \%$ & $3 \%$ & $3 \%$ & $0 \%$ & $0 \%$ & $0 \%$ & $0 \%$ & $5 \%$ \\
\hline Exports (TWh) & 10.52 & 11.70 & 4.30 & 11.10 & 11.11 & 40.56 & 27.83 & 28.45 & 28.56 & 52.74 \\
\hline CO2 Emissions (Million Short Tons) & 180.53 & 201.97 & 208.02 & 201.65 & 201.68 & 197.95 & 186.33 & 160.08 & 194.59 & 172.61 \\
\hline SPP Wind Reserve (Max MW) & 1,542 & 177 & 64 & 177 & 177 & 1,542 & 1,542 & 1,542 & 1,542 & 3,034 \\
\hline SPP Wind Reserve (Avg MW) & 996 & 118 & 28 & 118 & 118 & 996 & 996 & 996 & 996 & 2,042 \\
\hline \multicolumn{11}{|l|}{ Nebraska Generation (TWh) } \\
\hline Nebraska Total Gen & 52.07 & 47.23 & 44.59 & 47.05 & 47.08 & 53.93 & 54.46 & 51.58 & 53.52 & 56.57 \\
\hline Nebraska Combined Cycle & 2.26 & 2.29 & 2.95 & 2.16 & 2.17 & 2.56 & 4.43 & 7.07 & 2.95 & 2.13 \\
\hline Neb Comb Cycle Cap Factor & $28 \%$ & $28 \%$ & $37 \%$ & $27 \%$ & $27 \%$ & $32 \%$ & $55 \%$ & $88 \%$ & $37 \%$ & $26 \%$ \\
\hline Neb CT Gas & 0.06 & 0.07 & 0.08 & 0.06 & 0.06 & 0.06 & 0.06 & 0.13 & 0.08 & 0.05 \\
\hline Neb ST Coal & 28.16 & 27.77 & 28.70 & 27.73 & 27.74 & 29.72 & 28.38 & 22.74 & 28.89 & 26.45 \\
\hline Neb ST Coal Cap Factor & $77 \%$ & $76 \%$ & $79 \%$ & $76 \%$ & $76 \%$ & $82 \%$ & $78 \%$ & $62 \%$ & $79 \%$ & $73 \%$ \\
\hline Neb Wind Actual & 9.26 & 4.75 & 0.49 & 4.75 & 4.75 & 9.26 & 9.26 & 9.26 & 9.26 & 17.75 \\
\hline \multicolumn{11}{|l|}{ SPP Generation (TWh) } \\
\hline Rest of SPP Total Gen & 223.46 & 229.46 & 224.68 & 228.91 & 228.90 & 238.88 & 238.13 & 241.49 & 240.06 & 232.96 \\
\hline Rest of SPP Comb Cycle & 26.47 & 41.27 & 44.44 & 40.88 & 40.84 & 28.33 & 40.17 & $\begin{array}{r}73.88 \\
73.48\end{array}$ & 33.14 & 26.65 \\
\hline Rest of SPP CC Cap Factor & $27 \%$ & $42 \%$ & $45 \%$ & $41 \%$ & $41 \%$ & $29 \%$ & $41 \%$ & $75 \%$ & $34 \%$ & $27 \%$ \\
\hline Rest of SPP CT Gas & 5.11 & 5.10 & 5.10 & 5.35 & 5.35 & 5.06 & 5.48 & 7.94 & 5.08 & 4.94 \\
\hline Rest of SPP ST Coal & 123.99 & 137.96 & 140.82 & 138.02 & 138.04 & 137.23 & 124.62 & 90.04 & 133.91 & 118.84 \\
\hline Rest of SPP ST Coal Cap Factor & $68 \%$ & $76 \%$ & $78 \%$ & $76 \%$ & $76 \%$ & $76 \%$ & $69 \%$ & $50 \%$ & $74 \%$ & $65 \%$ \\
\hline Rest of SPP Wind & 47.05 & 22.79 & 11.20 & 22.78 & 22.78 & 47.05 & 47.05 & 47.06 & 47.04 & 89.17 \\
\hline \multicolumn{11}{|l|}{ Interchange } \\
\hline Nebraska Net Position & 12.66 & 7.82 & 5.19 & 7.71 & 7.74 & 16.36 & 15.05 & 12.18 & 14.10 & 19.25 \\
\hline SPP Incl Neb Net Positi & 10.52 & 11.70 & 4.30 & 11.10 & 11.11 & 40.56 & 27.83 & 28.45 & 28.56 & 52.74 \\
\hline \multicolumn{11}{|l|}{ Carbon } \\
\hline Nebraska CO2 Emissions (MT) & 31.39 & 31.01 & 32.30 & 30.90 & 30.92 & 33.18 & 32.50 & 27.72 & 32.48 & 29.56 \\
\hline Rest of SPP CO2 Emissions (MT) & 149.14 & 170.96 & 175.72 & 170.75 & 170.76 & 164.76 & 153.83 & 132.36 & 160.70 & 142.80 \\
\hline Nebraska CO2 Emissions (M\$) & 784.65 & 775.18 & 807.58 & 772.45 & 772.96 & 0 & $1,624.9$ & $3,326.52$ & 812.06 & 739.07 \\
\hline Rest of SPP CO2 Emissions (M\$) & $3,728.49$ & $4,274.00$ & $4,392.92$ & $4,268.81$ & $4,269.01$ & 0 & $7,691.6$ & $15,883.51$ & $4,017.54$ & $3,570.03$ \\
\hline
\end{tabular}

Figure 54: Summary of Sensitivity Case Metrics (All dollars are 2018\$) 
Notes on sensitivity results table Figure 54:

- $\quad$ Reserves noted as "wind reserves" are the incremental regulating reserves associated with the additions of wind generation (i.e., the difference between load and wind vs. load alone, as in Table 22.

- $\quad$ For proxy resource sensitivities, the proxies used are indicated. For CO2, transmission and high tariff sensitivities, the shaped proxy was used. For Nebraska only and existing wind sensitivities, the daily block proxy was used. For the WAPA sensitivities, no proxy was needed.

- $\quad$ For proxy resource cases there are two actual cases: one for all the $10 \%,(2006)$ cases and one for all the $20 \%$-w/o overlay, (2006) cases.

- $\quad$ For WAPA Sensitivities the actual case $=$ schedule purchase to load, ideal case $=$ schedule purchases to load net wind.

- In WAPA Sensitivity I, historical minimum purchases are used as a constraint, but such minimums are not used as a constraint in WAPA Sensitivity II.

- In the WAPA sensitivities, Nebraska sensitivity and Existing Wind Sensitivity, APC is reported for Nebraska alone, not all of SPP.

- $\quad$ Capacity factors calculated using annual maximum (Winter) capacity.

- $\quad$ Unless otherwise indicated SPP = SPP including Nebraska.

- $\quad$ See Sections 4.3.3, 4.3.5, and 4.3.6 for more detailed description of setup, including reserve requirements for Nebraska standalone and existing wind sensitivities and WAPA scheduling.

- $\quad$ More detailed production simulation generation results can be found in appendix Section 8.3.2.

\subsubsection{Sensitivity - Nebraska Standalone Market}

Nebraska joined the Southwest Power Pool after the start of this study. As such, most of the simulations in this study treat the Nebraska entities as members of the SPP market with reserve sharing, no hurdle rates between SPP members and coordinated commitment and dispatch. However, for four sensitivities, Nebraska was treated as a standalone entity with its own market, operations and reserve requirements. Those sensitivities are the Nebraska Standalone sensitivity (Section 4.3.3), the existing wind sensitivity (Section 4.3.5), and the two WAPA (including shape costs) sensitivities (Section 4.3.6). In order to attempt to more completely capture the integration costs, the Nebraska sensitivity was performed using the daily block proxy in the ideal case and is thus compared to the sensitivity using $10 \%$ wind penetration with daily block proxy (SPP market), where in both cases the daily block is used in Nebraska and the rest of SPP and the shaped proxy in the rest of the footprint.

Consistent with other cases there was no wind curtailment in Nebraska in the Nebraska Standalone sensitivity (Table 32). Curtailment also remains approximately the same in the rest of SPP as is shown in Table 32. Curtailment is typically a local phenomenon driven primarily by local congestion and to a lesser extent minimum generation, so it is not unexpected that a 
change in market structure would have little effect on curtailment. More specifically, curtailment in the model is determined by extremely low LMPs (below the \$-40/MWh wind bid price) and the lack of curtailment indicates that these low LMPs are not occurring at the Nebraska wind injection sites. There were no changes to the input flowgate set that would create any different congestion patterns. Specifically however, there was a substantial decrease in Nebraska generation (see Figure 45 and Figure 55 show 49.25 TWh for Nebraska in SPP market compared to 47.23 TWh for the Nebraska only case), which would make curtailment even less likely due to lower use of the Nebraska transmission system.

Table 32: Separate Nebraska Market Wind Curtailment Comparison

\begin{tabular}{|l|r|r|r|r|}
\hline \multirow{2}{*}{ Wind Curtailment } & \multicolumn{2}{|c|}{ Ideal } & \multicolumn{2}{c|}{ Actual } \\
\cline { 2 - 5 } & Nebraska & SPP* & Nebraska & SPP* \\
\hline Nebraska Market & $0 \%$ & $1.04 \%$ & $0 \%$ & $2.47 \%$ \\
\hline Basecase (Single Market) & $0 \%$ & $1.08 \%$ & $0 \%$ & $2.54 \%$ \\
\hline
\end{tabular}

*Non-Nebraska portion of SPP

When Nebraska is modeled as an independent entity, local generation decreases for Nebraska, and increases for rest of SPP. This is likely due to lesser coordination of the markets, the $\$ 5 / \mathrm{MWh}$ hurdle rate between Nebraska and the rest of SPP and the associated decrease in exports. Figure 55 shows the change in generation of three major categories of generation between the Nebraska market case and the Base Case (both using actual wind). Note that while the rest of SPP is increasing generation by about 1.1 TWh, local Nebraska generation is decreasing by about 2.0 TWh, indicating that much of that Nebraska energy was being exported beyond SPP in addition to serving coordinated operations.

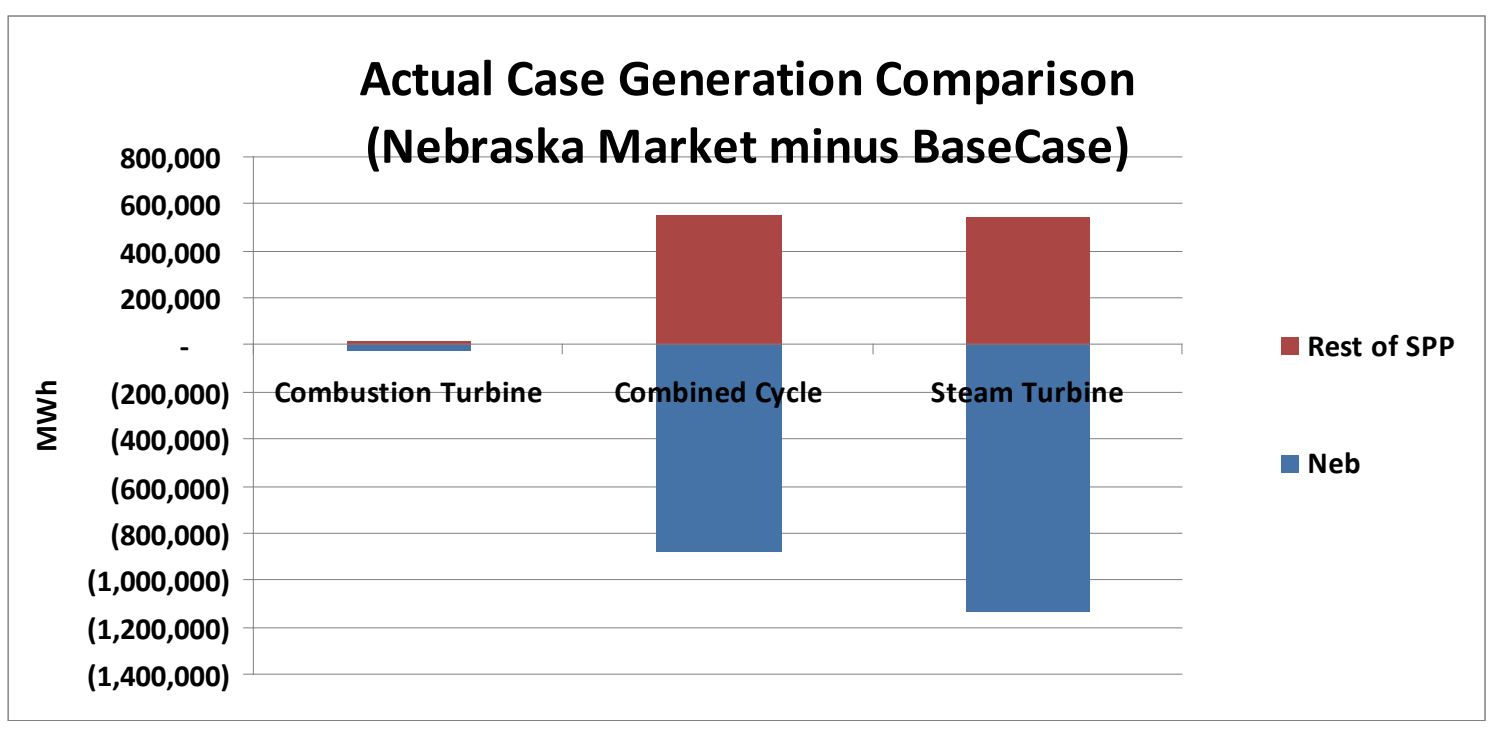

Figure 55: Thermal Generation Change from Nebraska Market Case to Base Case

Consistent with generation decrease, Nebraska exports decrease by about $20 \%$ in the Nebraska Market sensitivity as shown in Figure 56. 


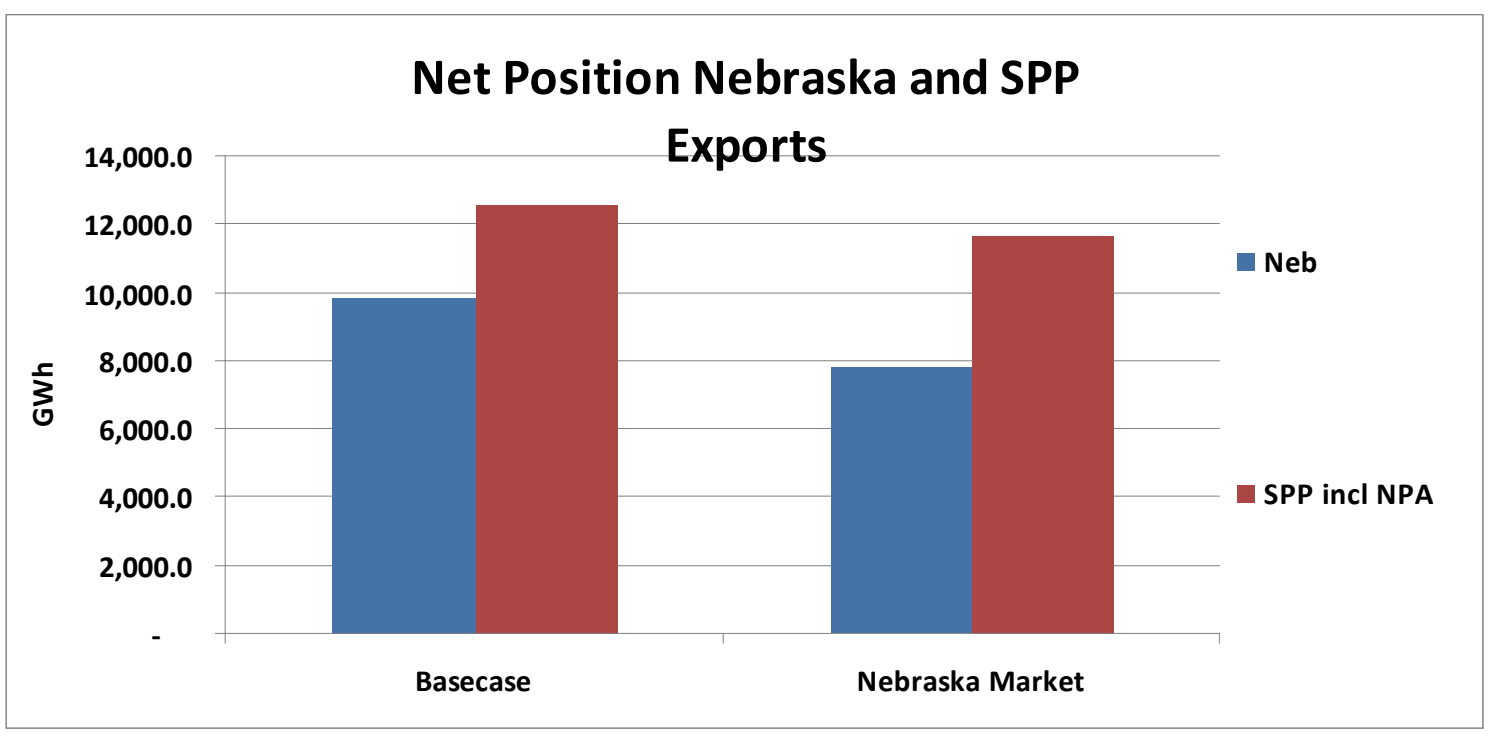

Figure 56: Comparison of Exports in Base Case and Nebraska Market Case

As discussed earlier, base case integration costs have been calculated at the SPP regional level rather than at a sub-regional Nebraska level due to the assumption of coordinated reserves and unit operations. For the Nebraska sensitivity, those integration costs have been calculated for the sub-regions of Nebraska and the rest of SPP. Although that difference makes integration costs difficult to compare, it does appear that the integration costs are higher for the smaller area (i.e. with Nebraska modeled as a separate entity). This is due to a smaller fleet and less load to absorb wind variability, reserves and forecast errors. Put another way, Nebraska's membership in SPP serves as a benefit to integrating wind. It should be noted that APCs for the two components are not additive to get an SPP total due to interchange pricing between components. Integration and production costs comparisons are shown in Table 33.

Table 33: Comparison of Costs (2018\$) in Base Case and Nebraska Market Case

\begin{tabular}{|l|c|l|r|r|r|r|}
\hline & $\begin{array}{l}\text { Actual APC } \\
(\mathrm{M} \$)\end{array}$ & $\begin{array}{l}\text { Ideal APC } \\
(\mathrm{M} \$)\end{array}$ & Delta $(\mathrm{M} \$)$ & $\begin{array}{l}\text { Wind } \\
(\mathrm{TWh})\end{array}$ & $\begin{array}{l}\text { Integration } \\
\text { Costs (\$/MWh) }\end{array}$ \\
\hline Nebraska & $1,599.03$ & $1,574.01$ & 25.02 & 4.75 & 5.27 \\
\hline Rest of SPP & $13,398.17$ & $13,329.66$ & 68.51 & 22.79 & 3.01 \\
\hline & & & & & \\
\hline All SPP One Market* & $14,655.28$ & $14,544.90$ & 110.38 & 27.52 & 4.01 \\
\hline
\end{tabular}

*Scenario1 with Daily Block Wind Proxy

\subsubsection{Market Sensitivity \# 2- Tariff/Hurdle Rate @ $\quad$ \$20/MWh}

A second market sensitivity was performed with the intent of investigating the effects of interchange on integration costs. This was performed by increasing the hurdle rate between SPP and outside entities. The hurdle rate (also called a tariff) is used to determine whether or not interchange can occur between areas. The hurdle rate is simply the size of LMP differential 
required for interchange to occur - in the base case that hurdle rate was $\$ 5 / \mathrm{MWh}$, but in this sensitivity that value was increased to $\$ 20 / \mathrm{MWh}$.

For the tariff/hurdle rate at $\$ 20 / \mathrm{MWh}$ sensitivity, exports decrease by a small amount in Nebraska but the exports for SPP decrease by over half from the base $\$ 5 / \mathrm{MWh}$ scenario. Interchange between Nebraska and the rest of SPP is not governed by a tariff, but all of SPP to the outside markets is limited by that tariff and it is obviously having a significant effect. Nebraska and SPP exports are shown in Figure 57.

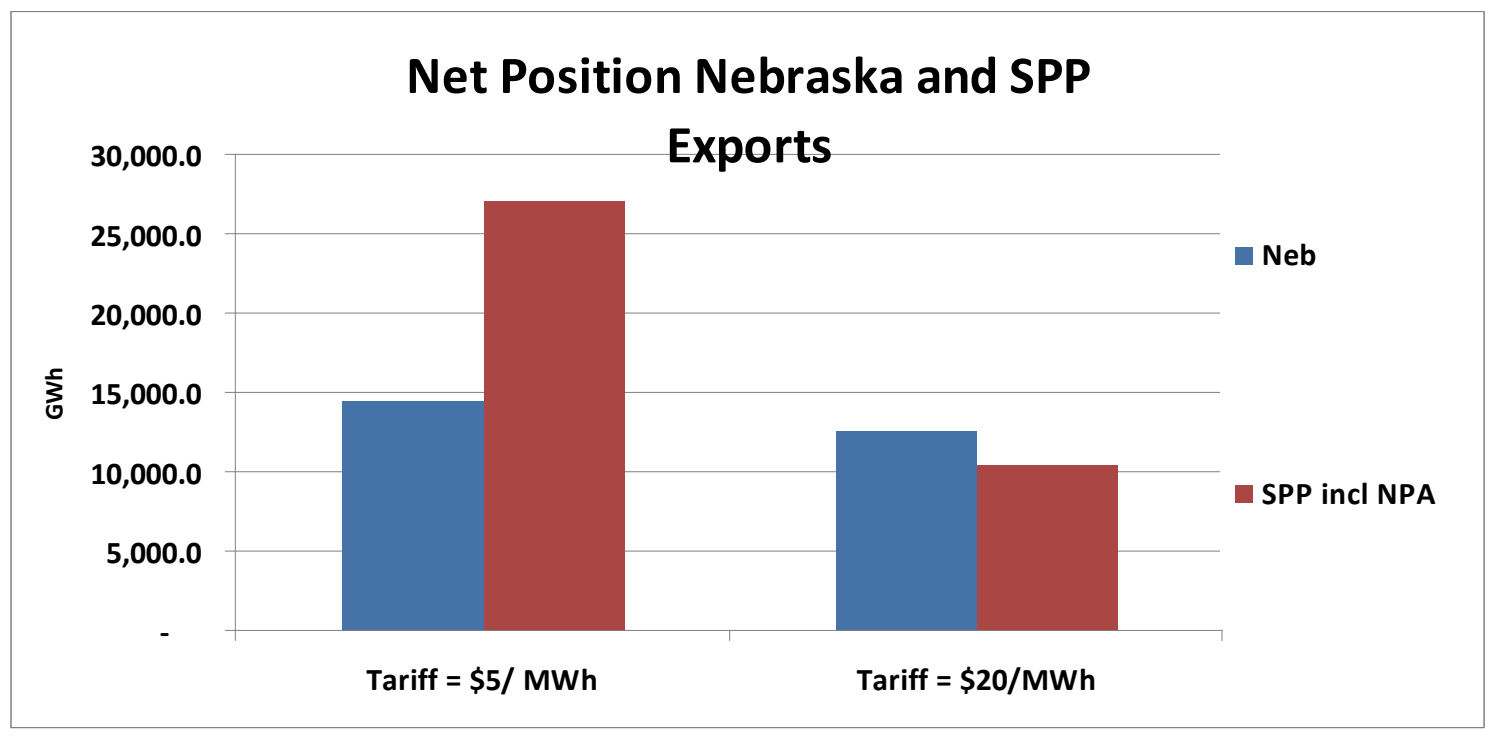

Figure 57: Exports Comparison between Base Case and \$20 Tariff Case

Consistent with those decreased imports is decreased use of local thermal resources as shown in Figure 58.

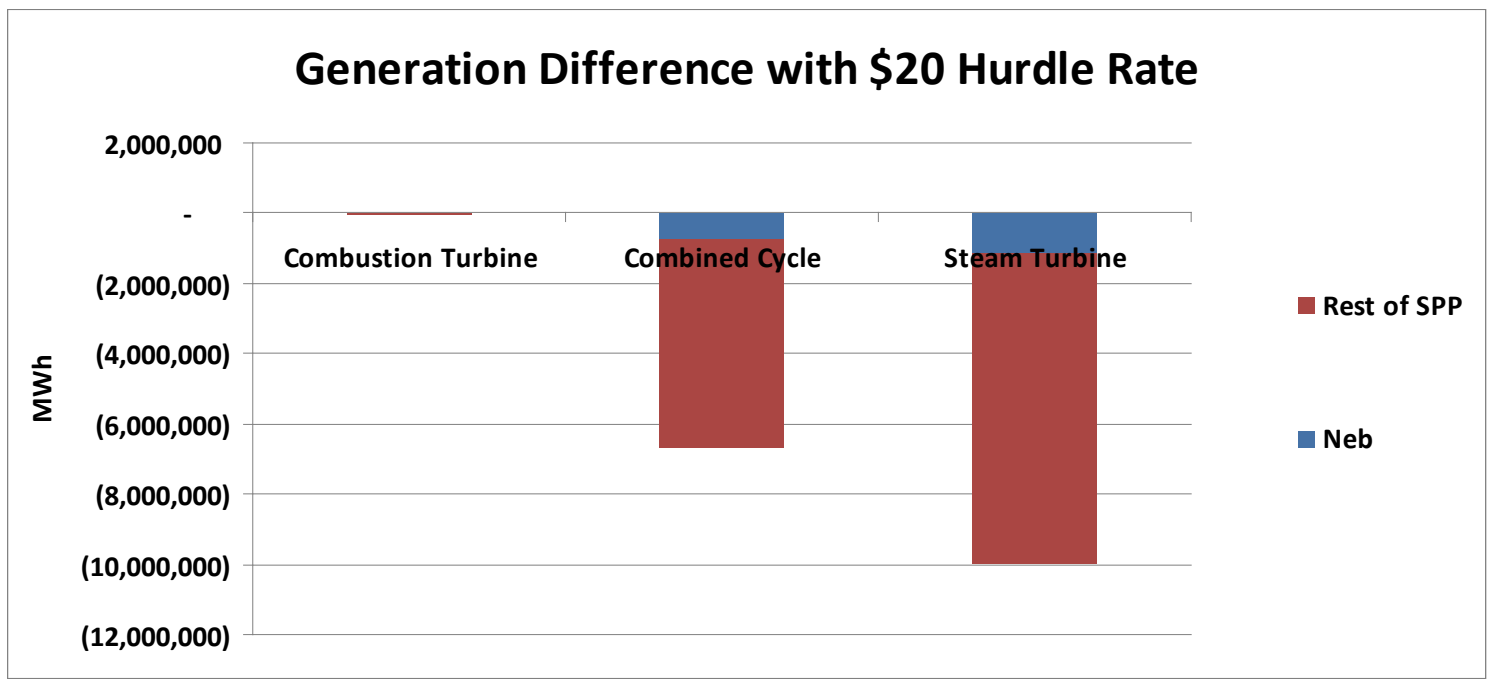

Figure 58: Thermal Generation Changes between Base Case and $\$ 20$ Tariff Case 
Despite this large change in exports and local generation, there is minimal effect on integration costs (using shaped proxy) as shown below in Table 34. There is about a $1 \%$ increase in Adjusted Production Costs for all of SPP, but a very slight decrease in wind integration costs in both dollars and $\$ / M W h$, as a result of the higher tariff. The minimal change in integration costs is an interesting finding given that there has been some concern about the effect of the large modeling footprint on specific regional results when based on the shaped proxy. This is an indicator that exporting energy is not the same thing as exporting integration costs. When considering the components of integration costs this is a reasonable finding. When using the shaped proxy resource, there are two components of integration costs: costs of incremental regulating reserves and costs of wind forecast error (there is no variability cost associated with using the shaped proxy). It is a modeling certainty that reserves are maintained by the region they are assigned to; that leaves wind forecast error as the only cost that could be exported. The results of this sensitivity indicate that that is not happening either; at least not at a cost less than $\$ 20 / M W h$.

Table 34: SPP including NPA Integration Costs (2018\$) - Shaped Proxy

\begin{tabular}{|l|l|l|r|l|l|r|}
\hline SPP (Including NPA) & $\begin{array}{l}\text { Actual APC } \\
\text { (M\$) }\end{array}$ & $\begin{array}{l}\text { Ideal APC } \\
\text { (M\$) }\end{array}$ & Delta (M\$) & $\begin{array}{l}\text { Wind } \\
\text { (TWh) }\end{array}$ & $\begin{array}{l}\text { Integration } \\
\text { Costs (\$/MWh) }\end{array}$ \\
\hline$\$ 20$ Tariff & $12,614.58$ & $12,511.43$ & 103.16 & 56.32 & 1.83 \\
\hline$\$ 5$ Tariff* & $12,462.26$ & $12,355.32$ & 106.94 & 56.32 & 1.90 \\
\hline
\end{tabular}

*Scenario3 Basecase

\subsubsection{Sensitivity - Existing Wind}

The existing wind sensitivity was developed in order to simulate wind integration effects given current (2009) wind penetration levels. The wind energy amounts (before curtailment) for the existing wind sensitivity and the $10 \%$ stand alone market case and other results are shown in Table 35.

Table 35: Wind Energy Comparisons of Existing Wind and 10\% Wind Nebraska Standalone Market Cases

\begin{tabular}{|l|c|c|}
\hline & Existing Wind & $10 \%$ Nebraska Standalone \\
\hline NEBRASKA & & 4,749 \\
\hline Wind Energy - GWh & 500 & $\mathrm{NA}$ \\
\hline Contingency Reserves - MW & 132 & 31.01 \\
\hline Emissions - millions of short tons & 32.3 & 118 \\
\hline Avg Incremental Wind Reserves - MW & 28 & $0 \%$ \\
\hline Curtailment - \% & $0 \%$ & 7.82 \\
\hline Exports - TWh & 5.19 & \\
\hline REST OF SPP & & 23,368 \\
\hline Wind Energy - GWh & 11,433 & 1,539 \\
\hline Contingency Reserves - MW & 1,539 & 170.96 \\
\hline Emissions - millions of short tons & 175.72 & \\
\hline
\end{tabular}


The rest of the fleet generates more with less wind injections as shown in Figure 59. To make up for the loss of 4.2 TWh of Nebraska wind generation moving from the $10 \%$ Nebraska only case to the existing wind case, the Nebraska combined cycle and steam turbine units generate 1.6 TWh (Figure 59) more and the Nebraska exports are reduced 2.6 TWh (Figure 60).

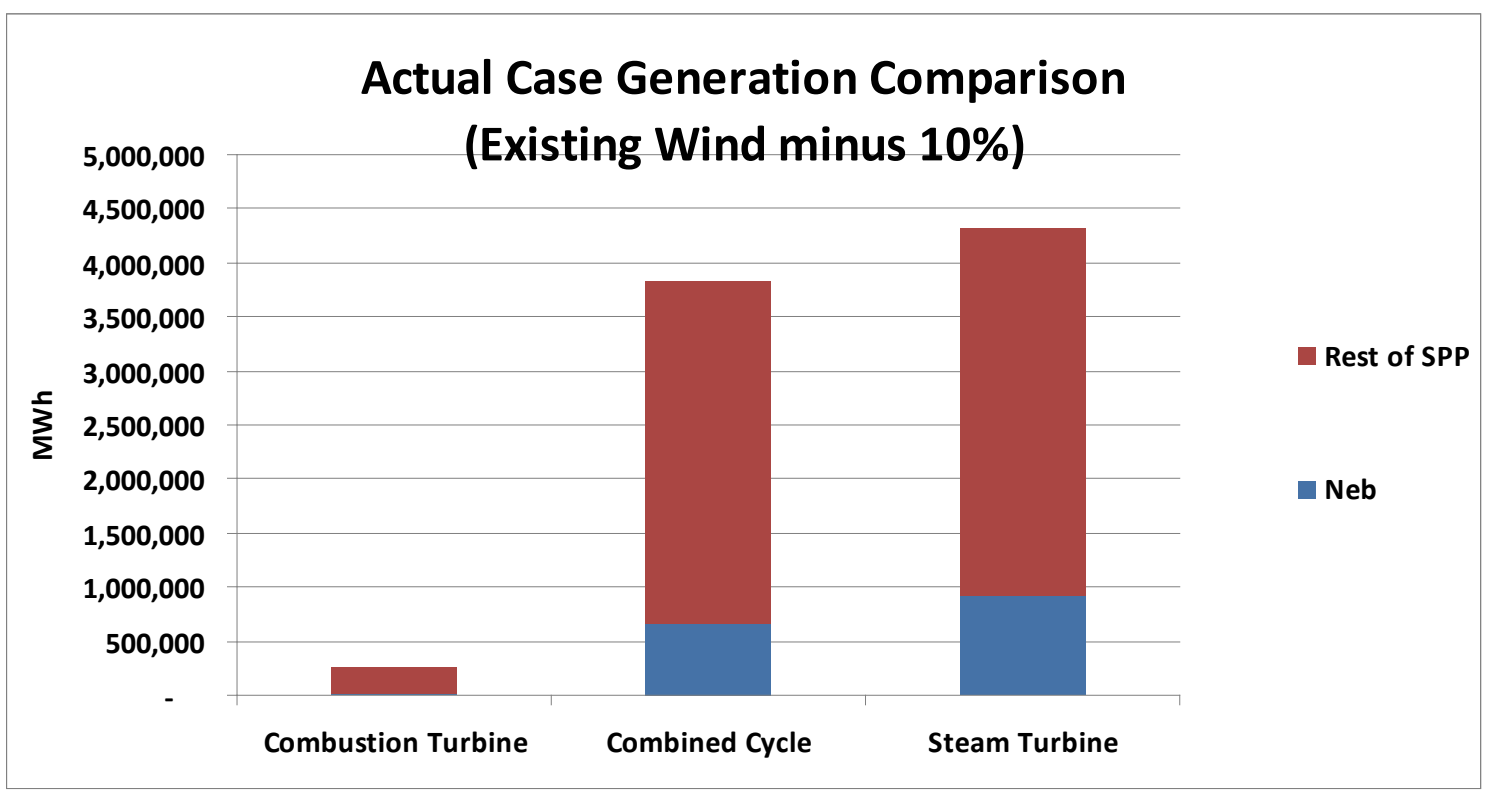

Figure 59: Comparison of Thermal Generation in Nebraska Market Case and Existing Wind Case

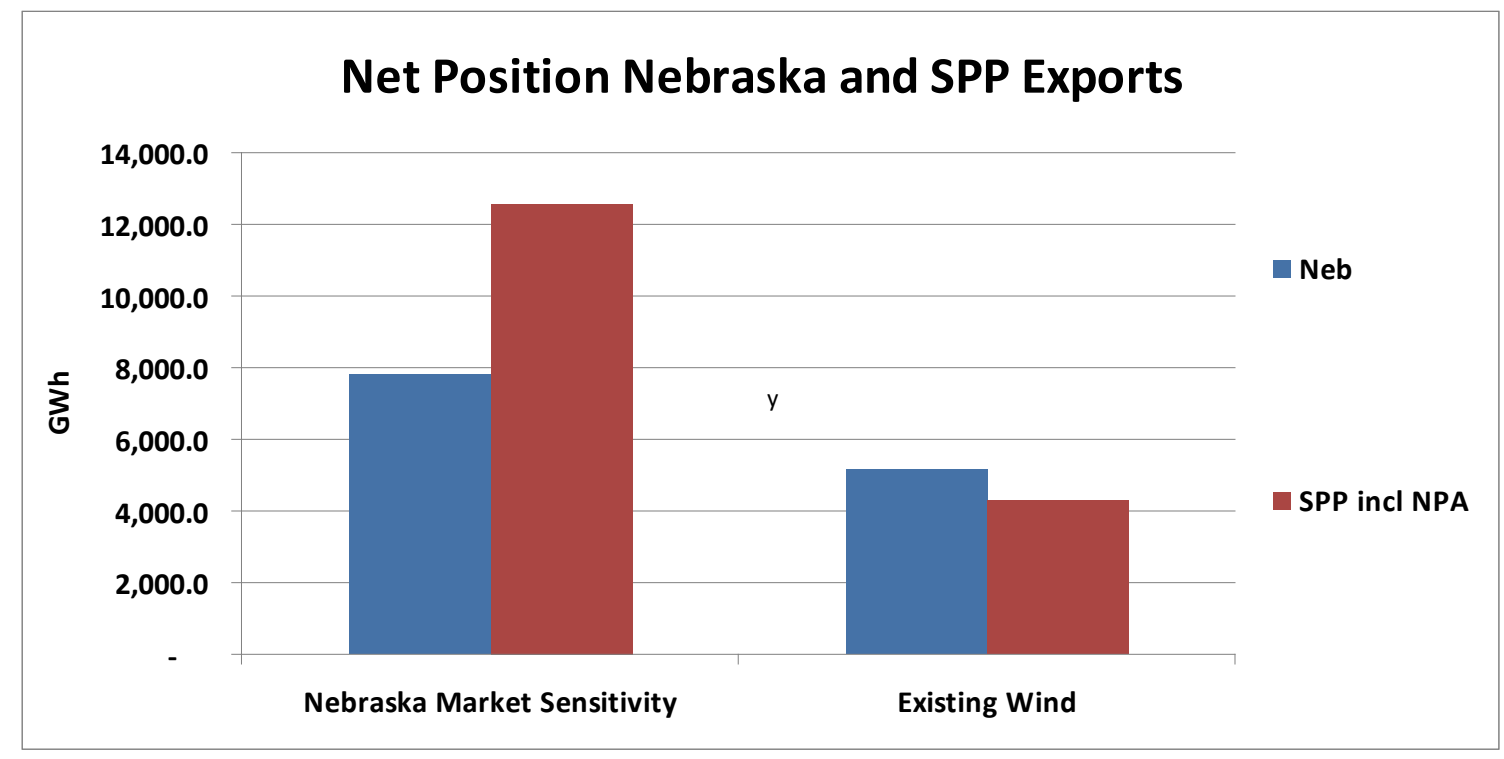

Figure 60: Exports Comparison of Nebraska Market Case and Existing Wind Case

The integration costs exhibit a phenomenon seen in some other studies: regions with very low penetration levels show very high integration costs in $\$ / M W h$ terms. Although Nebraska's APC changes by only $\$ 15$ million from ideal to actual, as shown in Table 36 , the small wind energy 
value in the denominator results in high integration costs. The rest of SPP integration costs (based on daily block proxy) in $\$ / M W h$ terms decrease very slightly from the $10 \%$ scenario to the existing scenario. In this sensitivity, the Nebraska wind penetration level is inconsistent with levels of surrounding areas: Nebraska $\sim 1 \%$, the rest of SPP $5 \%$.

The resulting $\$ 30.08 / \mathrm{MWh}$ in normalized integration costs should not be cause for alarm. Other studies with low penetration regions bordered by higher penetration regions have shown similar effects. Although difficult to analyze, this is believed to be driven by 3 factors:

- Non-linear effects of the costs of integrating the first few megawatts into a system - it is possible that a few unit commitment changes required to accommodate this small amount of wind might be able to accommodate even more wind, but the system cannot make less changes and still accommodate this $1 \%$.

- The simple math of a small denominator - the non-normalized costs of integration for Nebraska at $1 \%$ is a relatively small $\$ 15$ million or slightly under $1 \%$ of APC. However, dividing this by the small wind energy value results in a higher normalized integration cost.

- Absorption of shape-based integration costs from surrounding regions - although Nebraska is at $1 \%$ penetration, the rest of SPP is around $5 \%$ penetration and Western MISO to the north is near $17 \%$ wind penetration. In instances of large enough LMP differentials, integration costs could be transferred from those areas to Nebraska. The results from Market Sensitivity \#2 indicate that this may not be occurring; however, the use of the daily block proxy (instead of shaped proxy) in the existing wind sensitivity could provide another integration cost (wind variability) to be shifted between regions. This was found to be the case as discussed in Section 4.4.4.

Table 36: Cost Comparison (2018\$) of Existing Wind Case and Nebraska Market Case - Daily Block Proxy

\begin{tabular}{|l|c|c|r|r|r|r|}
\hline Regions & $\begin{array}{l}\text { Actual APC } \\
\text { (M\$) }\end{array}$ & $\begin{array}{l}\text { Ideal APC } \\
(\mathrm{M} \$)\end{array}$ & Delta (M\$) & $\begin{array}{l}\text { Wind } \\
(\mathrm{TWh})\end{array}$ & $\begin{array}{l}\text { Integration } \\
\text { Costs (\$/MWh) }\end{array}$ \\
\hline Nebraska Existing & $1,895.21$ & $1,880.41$ & 14.80 & 0.49 & 30.08 \\
\hline Nebraska 10\% & $1,599.03$ & $1,574.01$ & 25.02 & 4.75 & 5.27 \\
\hline Rest of SPP Existing & $14,245.41$ & $14,214.02$ & 31.39 & 11.20 & 2.80 \\
\hline Rest of SPP 10\% & $13,398.17$ & $13,329.66$ & 68.51 & 22.79 & 3.01 \\
\hline
\end{tabular}

\subsubsection{Sensitivity - Proxy Resource Comparison}

Determining the proper proxy resource to use for a wind integration study has been, and continues to be, an ongoing discussion. Most studies to date have used the 'daily block' proxy for the ideal case, although some recent studies, including this one, have used the 'shaped' proxy for the ideal case. The daily block proxy is the daily ( 24 hour) average of a wind plants energy output for the day. The shaped proxy is the actual shape of the delivered wind without consideration for wind forecast error or incremental reserves required by the wind. The move away from the daily block was driven by observation of very large single hour ramps in the daily 
block when working with higher penetration wind regions. There have also been concerns that the daily block average is resulting in wind energy being shifted from low cost to high cost (or vice versa) periods and thus artificially influencing integration costs.

With that background and those concerns in mind, a series of simulations using different proxy resources for the ideal wind case were performed. Initially these simulations were performed for a $10 \%$ wind penetration scenario and the 2006 load and wind shapes and then repeated at the $20 \%$ penetration. The proxy shapes tested are shown in Section 4.3.1.

Initially, only the daily block wind proxy was tested and it resulted in integration costs more than double what the shaped wind proxy had shown. Results for that run are compared to the base case results in Table 37. Integration costs (at 10\%) using daily block proxy resource are $\$ 4.01$ /MWh, with \$2.27 attributable to the difference between using the shaped wind and the flat block wind.

Table 37: Comparison of Costs (2018\$) between Daily Block Proxy Sensitivity and 10\% Base Case (Shaped Proxy)

\begin{tabular}{|l|l|l|r|l|l|}
\hline SPP (Including NPA) & $\begin{array}{l}\text { Actual APC } \\
(\mathrm{M} \$ \text { ) }\end{array}$ & $\begin{array}{l}\text { Ideal APC } \\
(\mathrm{M} \$)\end{array}$ & Delta (M\$) & $\begin{array}{l}\text { Wind } \\
(\mathrm{TWh})\end{array}$ & $\begin{array}{l}\text { Integration } \\
\text { Costs (\$/MWh) }\end{array}$ \\
\hline Daily Block Proxy & $14,655.28$ & $14,544.90$ & 110.38 & 27.52 & 4.01 \\
\hline Shaped Proxy* & $14,890.61$ & $14,842.78$ & 47.82 & 27.52 & 1.74 \\
\hline Shape Cost & \multicolumn{3}{|l}{} & & 2.27 \\
\hline
\end{tabular}

\section{* Scenario1 BaseCase}

The differences in integration cost between the two proxies can be explained by the different resources used to respond to the wind variability and forecast errors. The shaped proxy run uses much more combined cycle and less steam turbine than the daily block proxy case Figure 61. The increased use of the more expensive combined cycle pushes the Shaped Proxy case closer in price to that of the actual wind case, thus decreasing integration costs when comparing actual wind to shaped proxy. 


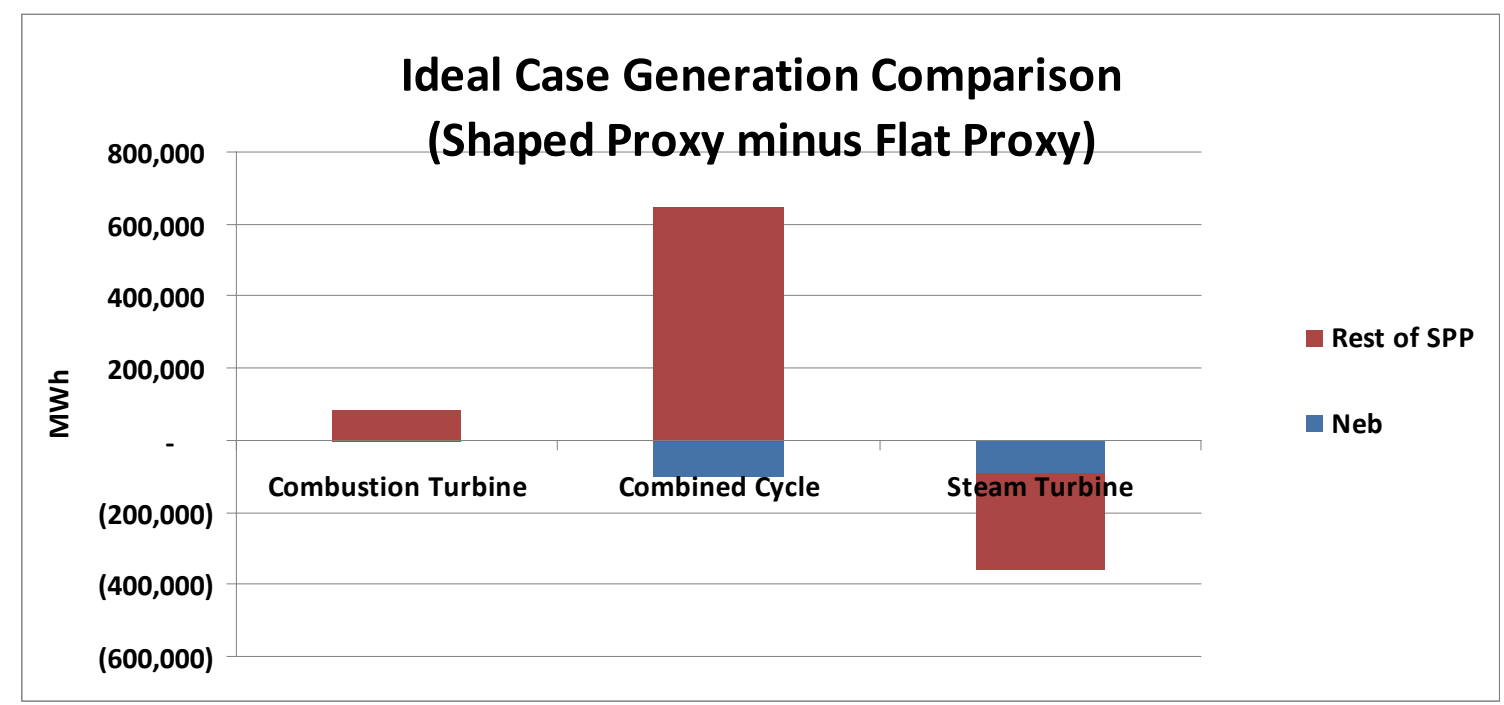

Figure 61: Thermal Generation Comparison between Shaped Proxy and flat Block Proxy Cases

The results from the study of the daily block proxy led to queries into other possible proxies and the previously mentioned concerns about energy and cost shifting. The results of performing those several simulations at $10 \%$ wind are shown in Figure 62 and Figure 63 along with the previously run daily block and shaped proxies. The other proxies involved a sub-period proxy with two blocks per day (on-peak and off-peak) instead of one, and then two moving average proxies for 13 -hour and 5-hour moving averages. The new tested proxies give integration costs across a range between that of flat block proxy and the base case (shaped) proxy. 

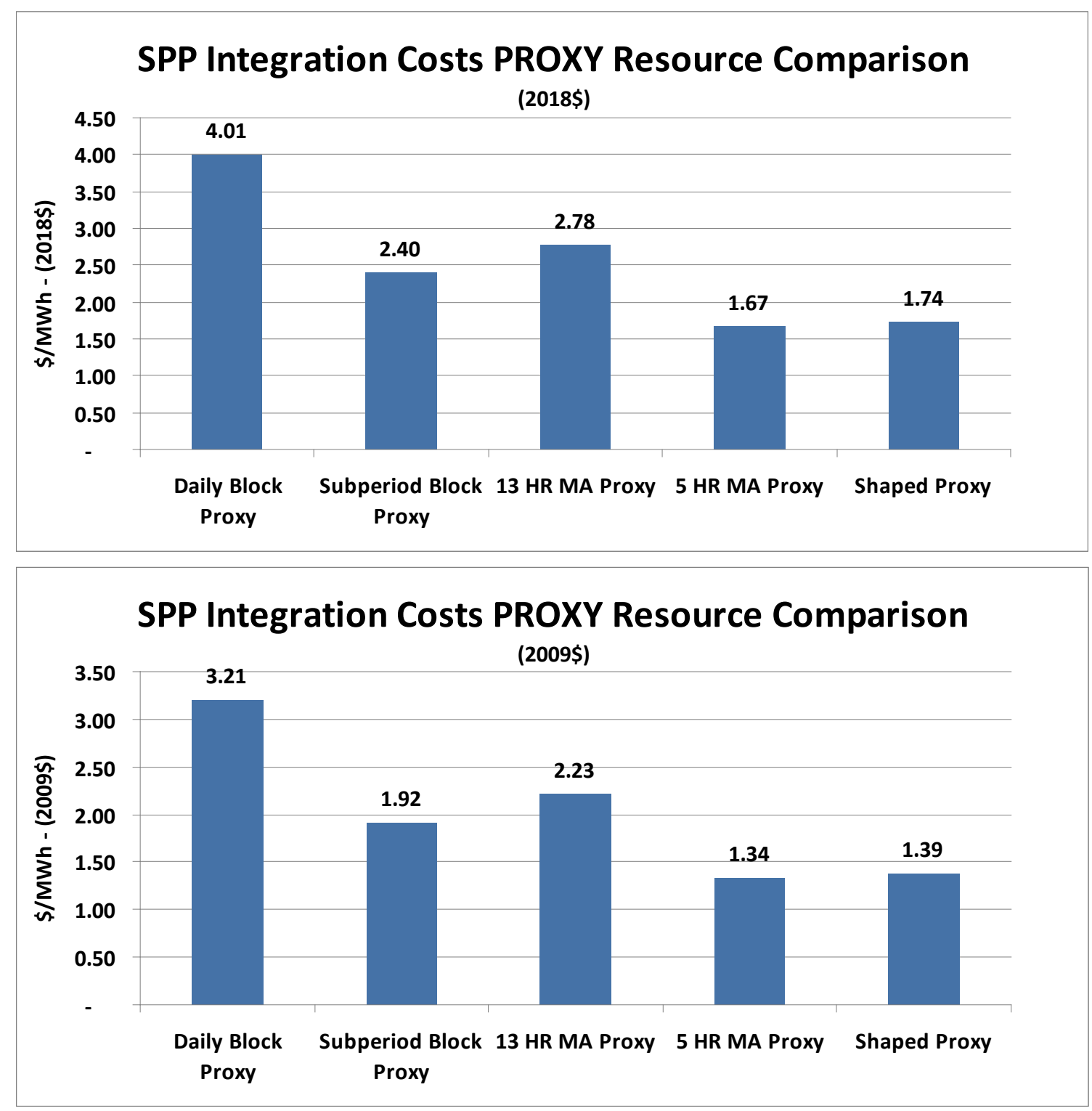

Figure 62: Comparison of Normalized Wind Integration Costs for SPP (including Nebraska) for Different Proxy Resource Sensitivities for Different 10\% Cases for 2018 Conditions for both $2018 \$$ (top) and $2009 \$$ (bottom) 


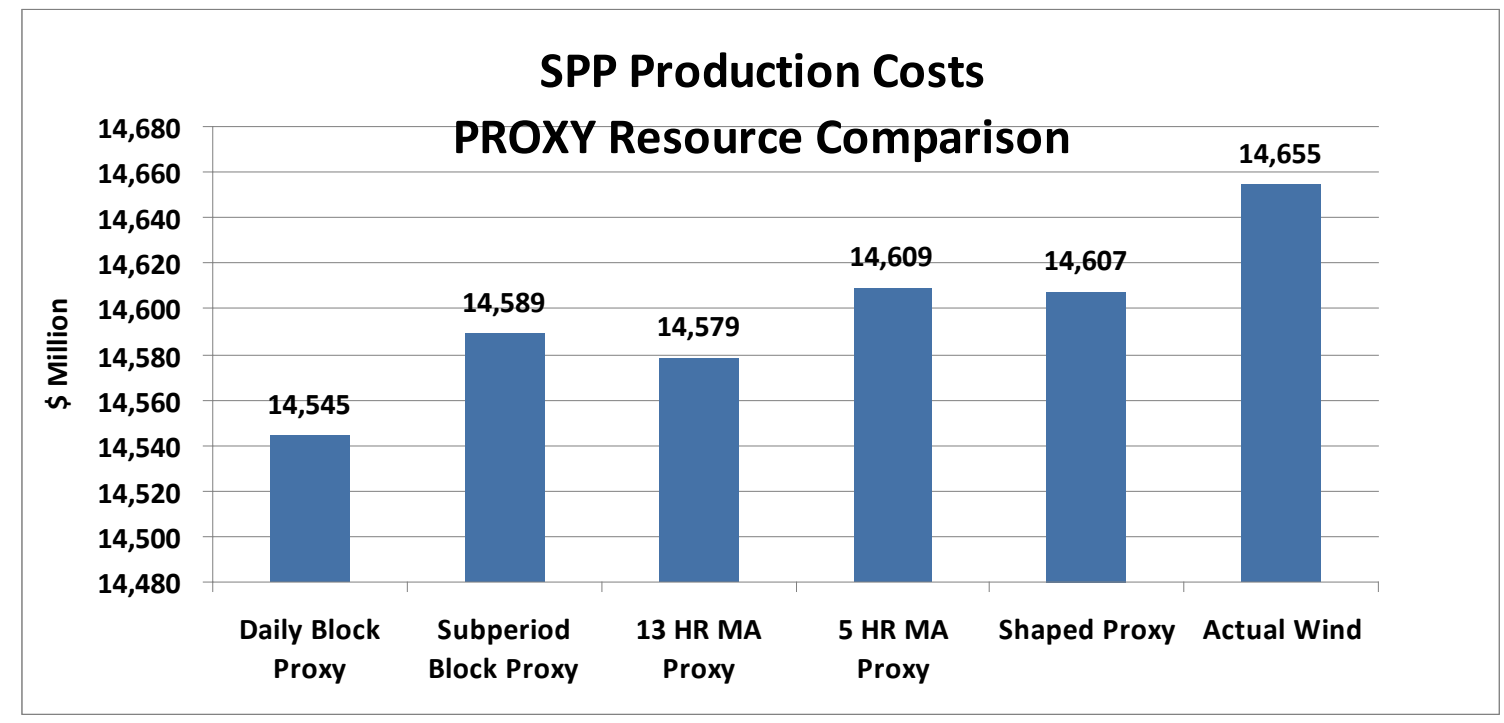

Figure 63: Comparison of Adjusted Production Costs for SPP (including Nebraska) for Different 10\% Cases in $2018 \$$

Subsequent to the interesting results of the $10 \%$ proxy tests, a simulation using the same proxies were performed for the $20 \%$ wind penetration case (Scenario 2 ). Those results are shown below in Figure 64 alongside the $10 \%$ results. Consistent with most of the results of the $10 \%$ simulations, the use of the other proxy resources in the ideal case results in normalized integration costs higher than those using the shaped proxy (as was done in the base cases). The exception is that the use of the 5 -hour moving average proxy in the $20 \%$ case results in increased normalized integration costs relative to the base case, while the same proxy in the $10 \%$ case results in slightly lower integration costs relative to the $10 \%$ base case. The daily block and subperiod block proxies show a bigger differential in integration costs than they did in the $10 \%$ cases indicating that costs associated with the shape or variability of the wind may be increasing with penetration.

The proxy resource is a fictional reference point for establishing costs to integrate wind and each definition of the proxy has certain shortcomings that must be recognized when considering results of analysis using them. These results indicate that the shaped proxy is not capturing all of the integration costs and that another proxy may be more useful, specifically at capturing costs of wind variability which are not captured by the use of the shaped proxy. However, there is also concern that the traditionally used daily flat block proxy shifts too much energy from low cost hours to higher cost hours and thus overstates integration costs. The sub-period block proxy appears to be a reasonable solution to those two issues, but should be carefully studied in order to ascertain what its shortcomings might be. 

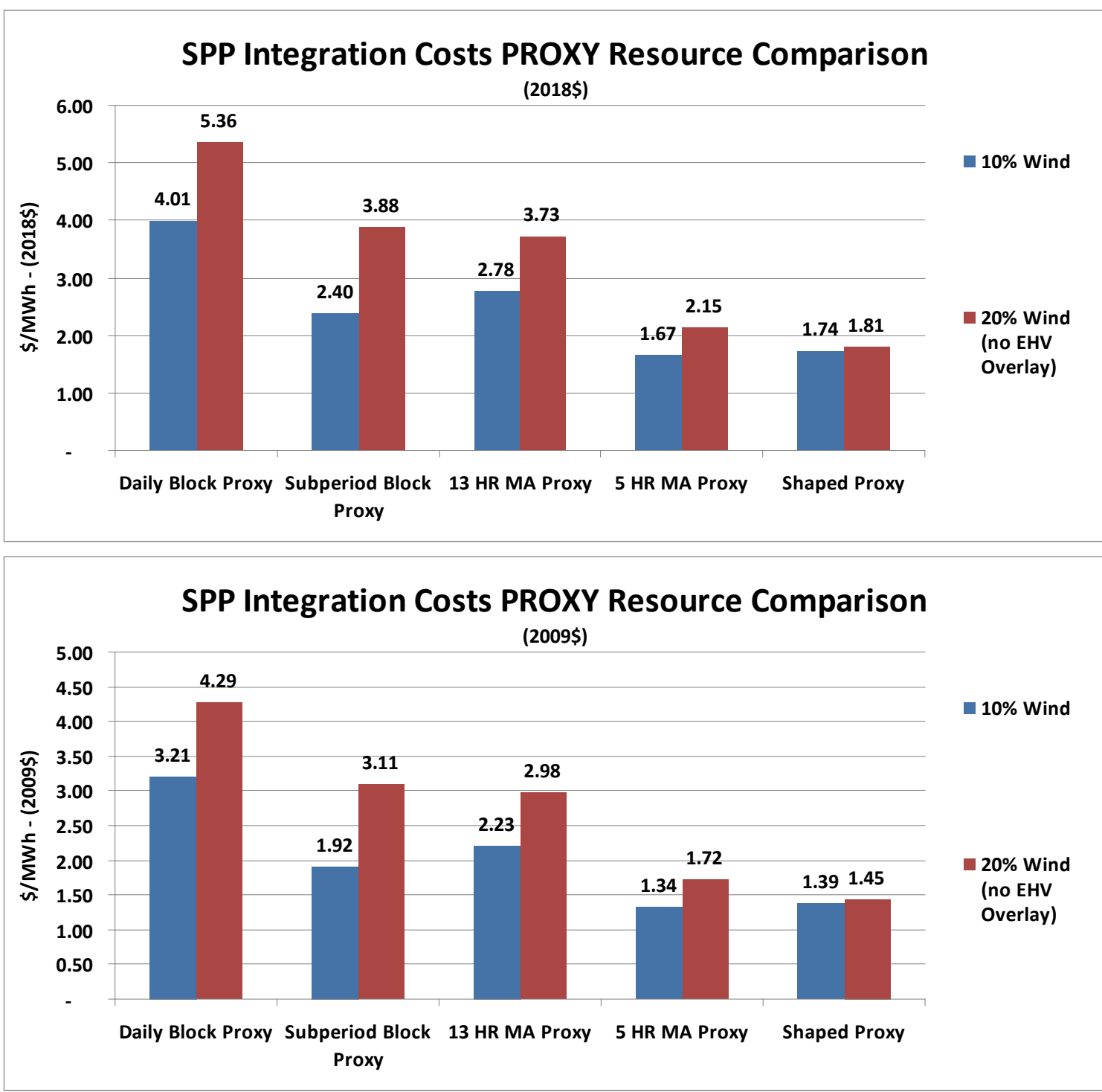

Figure 64: Comparison of Normalized Integration Costs for SPP (including Nebraska) with Alternate Proxies at $10 \%$ and $20 \%$ Wind for 2018 conditions shown in both $2018 \$$ (top) and $2009 \$$ (bottom)

As noted in Section 4.2 for base case results with the shaped proxy, wind generation is typically slightly higher for the actual wind case than for the ideal wind case, which would result in a slightly higher integration cost if an adjustment to equalize generations were to be made. The opposite situation appears for the other proxy definitions where as shown in Figure 54, ideal wind generation exceeds that for actual wind in the non-shaped proxy resource runs. As noted before, these adjustments are not made in the results Section 4.4.

In Section 4.2.4 results were presented analyzing the question of exported integration costs based on shaped proxy. Given the increased integration costs shown here when using some other proxy resources, it is useful to again investigate that question in the context of these proxy resources. In other words are shape/variability costs being exported? Table 38 below shows 
the integration costs using the daily block and sub-period block proxies at $10 \%$ and $20 \%$ wind penetration.

Table 38: Integration Costs in Millions using different proxies for SPP and Modeled Footprint

\begin{tabular}{|l|r|r|r|r|r|r|}
\hline \multirow{2}{*}{ \$ Million } & \multicolumn{3}{|c|}{ 2018 Dollars } & \multicolumn{3}{c|}{ 2009 Dollars } \\
\cline { 2 - 7 } & SPP & Footprint & Delta & SPP & Footprint & Delta \\
\cline { 2 - 7 } & 110.38 & 380.61 & 270.23 & 88.38 & 304.76 & 216.37 \\
Scenario 1 (Daily Block) & 283.80 & 789.44 & 505.64 & 227.24 & 632.11 & 404.87 \\
Scenario 2 (Daily Block) & 66.03 & 265.45 & 199.42 & 52.87 & 212.55 & 159.68 \\
Scenario 3 (Subperiod Block) & 205.66 & 694.94 & 489.28 & 164.67 & 556.44 & 391.77 \\
Scenario 4 (Subperiod Block) & &
\end{tabular}

It should first be noted that in the proxy resource sensitivities, the only proxies that were changed were the Nebraska and SPP wind plants. The rest of the footprint consistently used the shaped proxy. Based on that, the rest of the footprint integration costs should remain consistent with the values reported above in Table 27 or around 78 to 87 million. However, as is shown in Table 38 above, that is not the case. In fact, the integration costs being carried by the rest of the footprint are much bigger than the values reported in Table 27 indicating that some of shape or variability costs are being exported from SPP to surrounding regions.

Assigning those variability costs back to SPP results in the calculation of an implied integration cost for SPP (using these other proxy resources) that are a good bit higher than previously reported, Table 39. (This is done by subtracting the Delta in Table 27 from the Delta in Table 38 and then adding that to the integration costs calculated in the original proxy resource runs then normalizing that total to wind energy in SPP).

Table 39: Native and Implied Integration Costs for SPP using Daily and Sub-period Block Proxies

\begin{tabular}{|l|r|r|r|r|}
\hline \multirow{2}{*}{ \$/MWh } & \multicolumn{2}{|c|}{ 2018 Dollars } & \multicolumn{2}{c|}{ 2009 Dollars } \\
\cline { 2 - 5 } & Native & Implied & Native & Implied \\
\hline Scenario 1 (Daily Block) & 4.01 & 10.65 & 3.21 & 8.53 \\
Scenario 2 (Daily Block) & 5.36 & 13.43 & 4.29 & 10.75 \\
Scenario 1 (Subperiod Block) & 2.4 & 6.76 & 1.92 & 5.41 \\
Sceanrio 2 (Subperiod Block) & 3.88 & 11.56 & 3.11 & 9.26 \\
\hline
\end{tabular}

These results along with the results for the base cases explain a difference between the $\$ 20 / \mathrm{MWh}$ tariff sensitivity (presented in Section 4.4.2) and the existing wind sensitivity (presented in Section 4.4.3). The $\$ 20 / M W h$ case seems to support the theory that no integration costs are being exported - it is run with the shaped proxy. The existing wind case appears to show Nebraska carrying an excess of integration costs - it is run with the daily block proxy, so perhaps Nebraska is importing integration costs from the rest of SPP in that case.

Lastly, regarding these shape costs especially, it is noted that if the external regions had been modeled with higher penetrations, the computed integration costs may have been higher because the ability of these external systems to "help" integrate the SPP wind would have been reduced. 


\subsubsection{Sensitivity - $\mathbf{3 4 5 k V}$ Overlay}

Scenarios 3 and 4 of the base case were developed using a 765 kV EHV overlay for all of SPP including Nebraska. These sensitivities sought to test the possibility of utilizing a lower voltage overlay in Nebraska and thus a $345 \mathrm{kV}$ overlay was laid out for Nebraska and used for $20 \%$ and $40 \%$ wind penetration scenarios. It is described in detail in Section 2.4.3. Comparing the lower voltage overlay included checking for changes in production cost and wind integration costs as well as ability to deliver the wind energy to load. This exercise is an abbreviated transmission evaluation, similar to the comparison of the complete $765 \mathrm{kV}$ overlay case that was done at a $20 \%$ wind penetration.

$345 \mathrm{kV}$ power flows on the $345 \mathrm{kV}$ overlay branches are all within their $1793 \mathrm{MW}$ (3000 amp) design limits. Flow duration curves for the Nebraska $345 \mathrm{kV}$ overlay are shown below for both the $20 \%$ (Figure 65 ) and the $40 \%$ (Figure 66) cases.

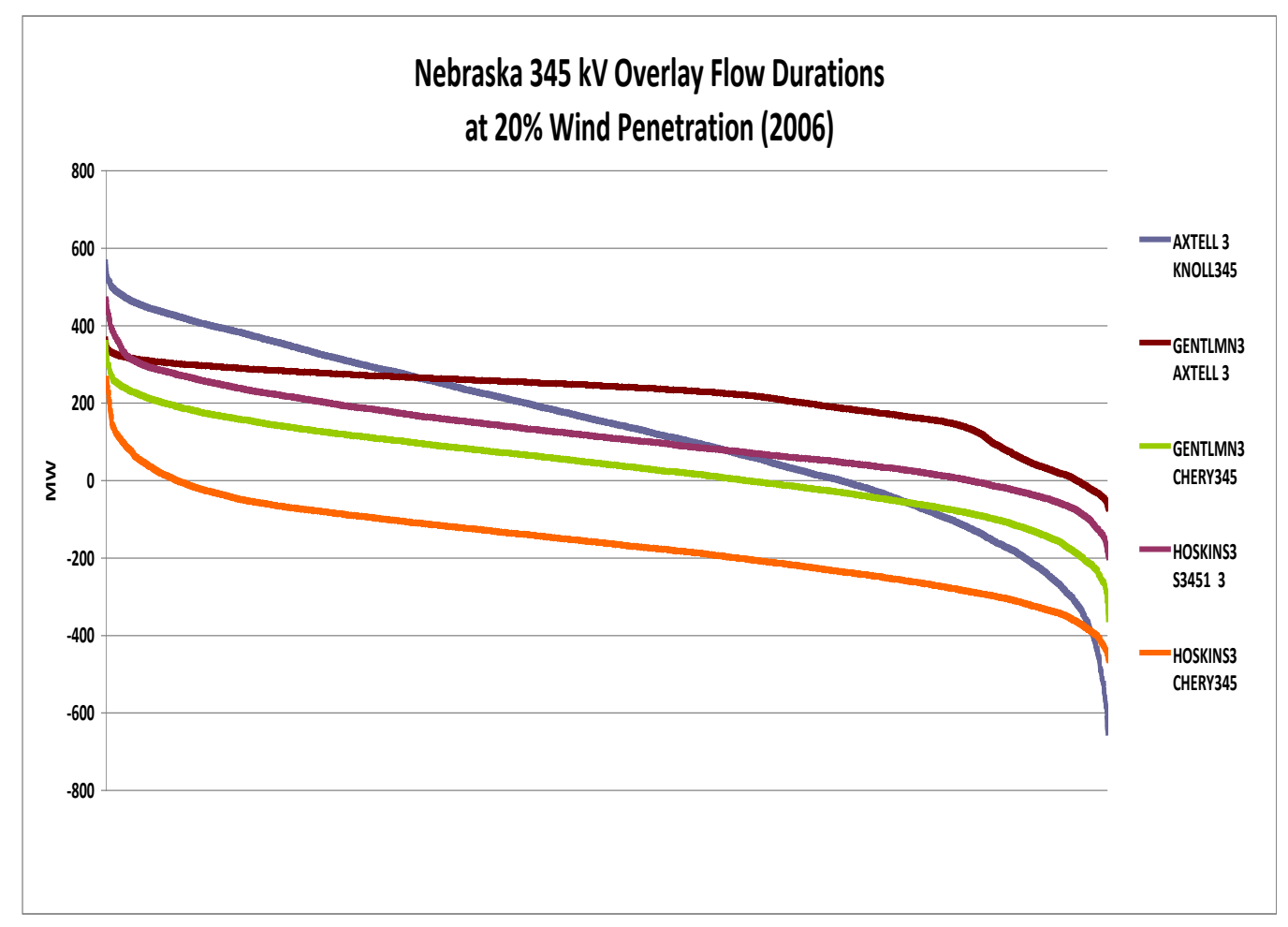

Figure 65: Flow Durations for Nebraska 345 kV Overlay (20\%) 


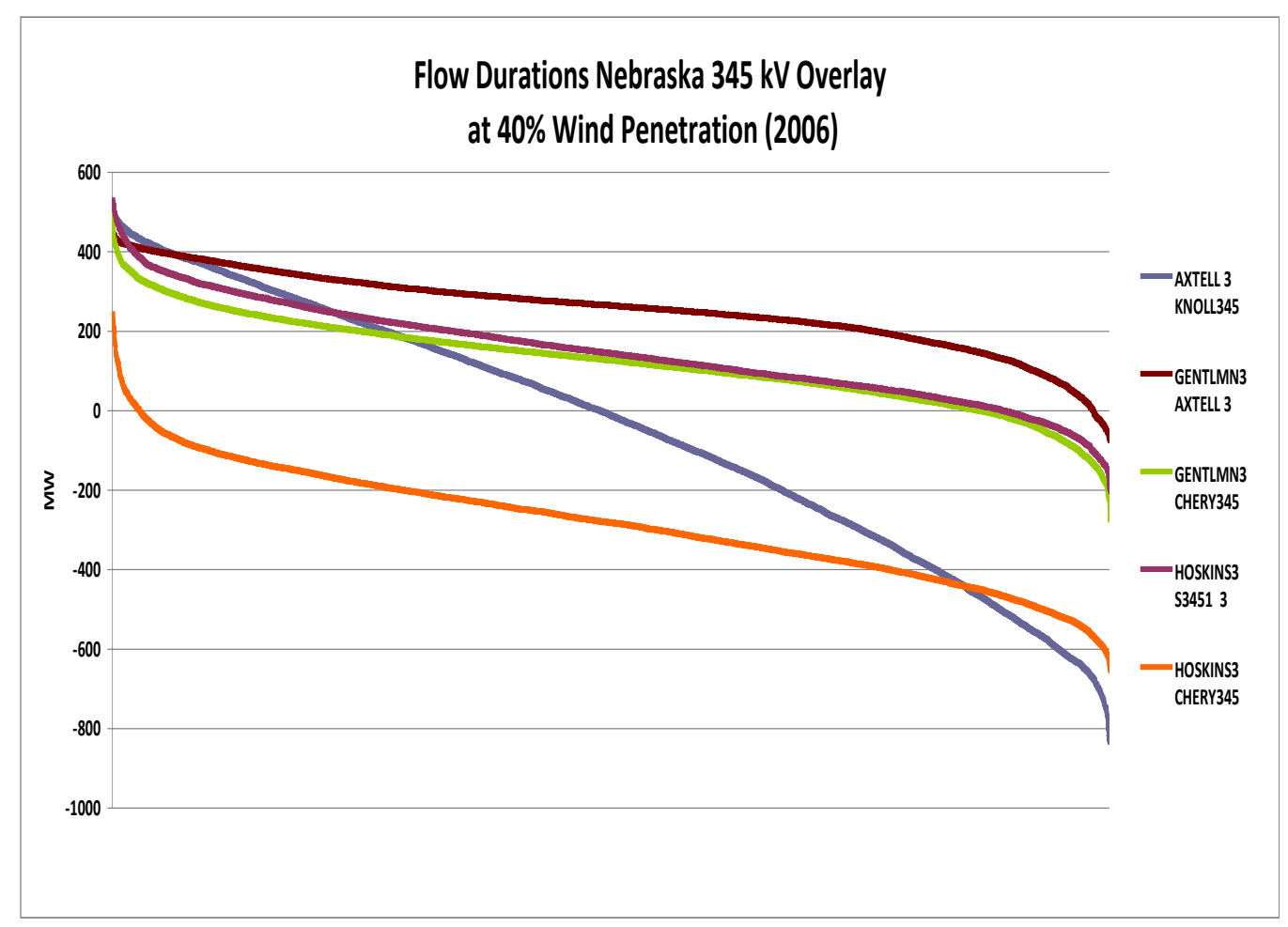

Figure 66: Flow Durations for Nebraska 345 kV Overlay (40\%)

Despite the absence of any violations of the overlay when using the $345 \mathrm{kV}$ design, there are some differences in production costs and integration costs. These are driven by higher impedances of the $345 \mathrm{kV}$ branches (i.e. less energy carried) and by a slightly different topology for the $345 \mathrm{kV}$ overlay. These results are using the shaped proxy shown in Table 40, where the $765 \mathrm{kV}$ overlay improves integration cost over the 345kV Nebraska overlay by $\$ 0.02 / \mathrm{MWh}$ at $20 \%$ and $\$ 0.04 / \mathrm{MWh}$ at $40 \%$ while the APC is decreased by $\$ 4.4$ million at $20 \%$ and $\$ 72.0$ million at $40 \%$ for year 2018.

Table 40: Costs Comparisons (2018\$) for Overlay Sensitivities (Shaped Proxy)

\begin{tabular}{|l|l|l|r|r|r|r|}
\hline SPP (Including NPA) & $\begin{array}{l}\text { Actual APC } \\
\text { (M\$) }\end{array}$ & $\begin{array}{l}\text { Ideal APC } \\
\text { (M\$) }\end{array}$ & Delta (M\$) & $\begin{array}{l}\text { Wind } \\
(\text { TWh) }\end{array}$ & $\begin{array}{l}\text { Integration } \\
\text { Costs (\$/MWh) }\end{array}$ \\
\hline 345 kV Overlay - 20\% & $12,466.66$ & $12,358.70$ & 107.96 & 56.31 & 1.92 \\
\hline 765 kV Overlay* - 20\% & $12,462.26$ & $12,355.32$ & 106.94 & 56.32 & 1.90 \\
\hline & & & & & 2.22 \\
\hline 345 kV Overlay - 40\% & $9,406.10$ & $9,168.53$ & 237.57 & 107.23 & 2.18 \\
\hline 765 kV Overlay* - 40\% & $9,334.14$ & $9,100.72$ & 233.42 & 107.23 & \\
\hline
\end{tabular}

*Basecase

As mentioned in the base case results (Section 4.2) the decrease in APC for the $40 \%$ penetration ( $\$ 3.1$ billion or about $25 \%$ ) from the $20 \%$ penetration case is a result of the "no-cost" 
representation of the added wind generation and transmission in PROMOD. Total costs are discussed in Section 5.

There are relatively small changes in exports and $\mathrm{CO} 2$ emissions with the use of the $345 \mathrm{kV}$ overlay in Nebraska as shown in Figure 67 and Figure 68. Using relevant data from Figure 45 and Figure 54 , the $40 \%$ penetration case shows the least amount of change in exports, as Nebraska exports decrease by $0.77 \%$ and SPP (total) exports decrease by $0.21 \%$. However, the $20 \%$ case shows a slightly larger $2.8 \%$ decrease in Nebraska exports and a $5 \%$ increase in exports in SPP total. These differences are likely driven by the somewhat different topology of the 345 $k \mathrm{~V}$ overlay and that topology's effect on exports.

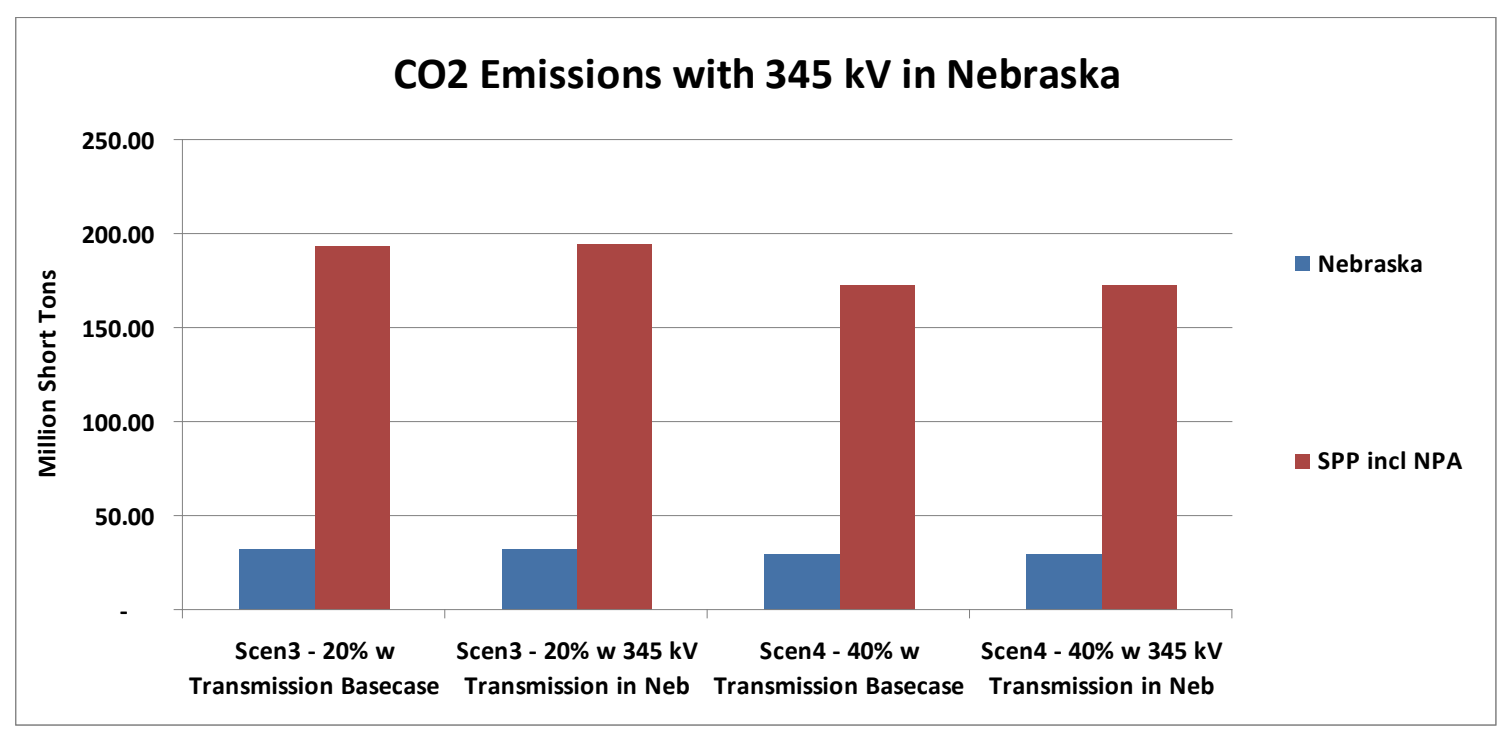

Figure 67: Comparison of CO2 Emissions for 345 kV Overlay Sensitivity 


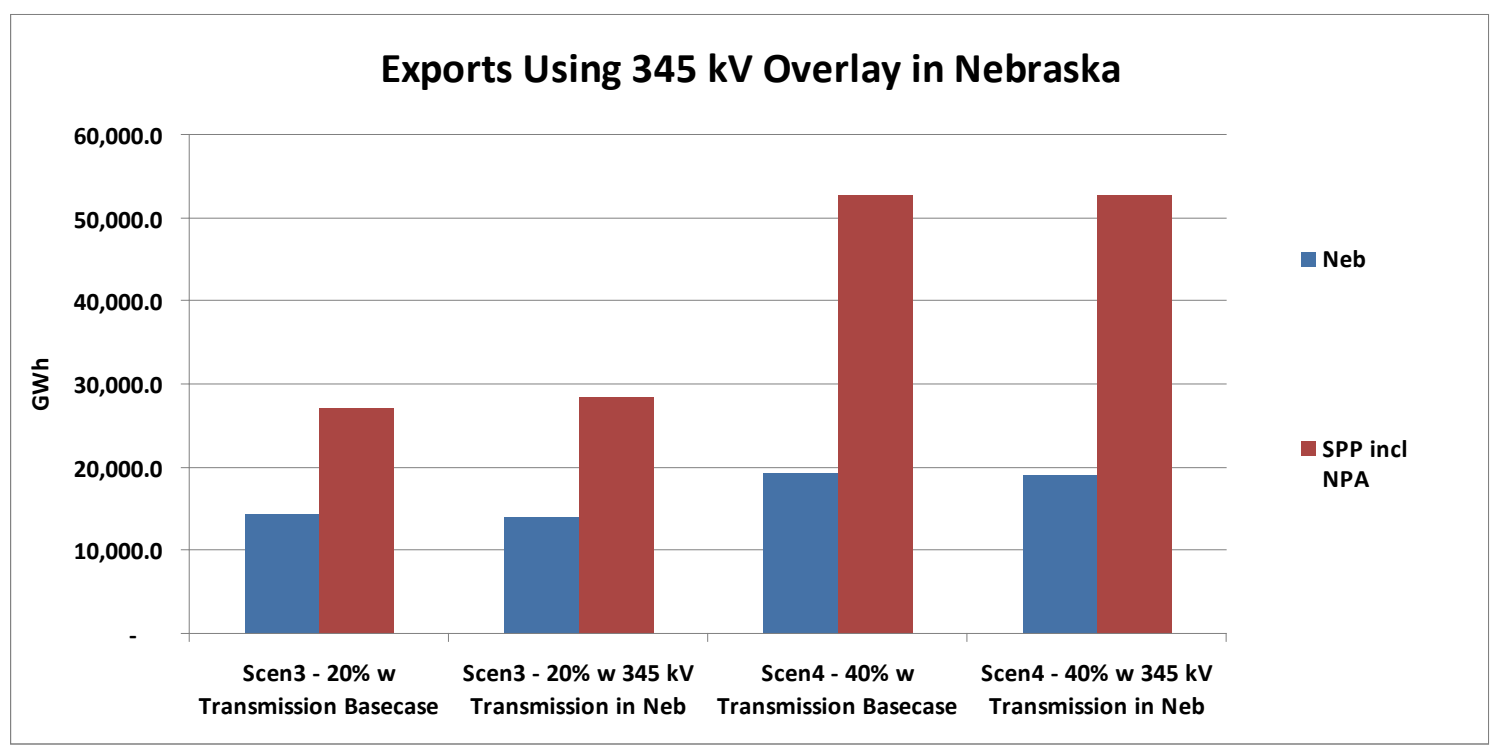

Figure 68: Comparison of Exports for $345 \mathrm{kV}$ Overlay Sensitivity

\subsubsection{Sensitivity - WAPA Mitigation}

\subsubsection{Purchases from WAPA}

Integration costs are driven by increased use of more expensive resources to respond to variability, reserve requirements and forecast error. If another resource with no variable costs could be used to respond to these needs then integration costs could be reduced. In the case of Nebraska, purchases from WAPA (federal hydropower) might meet these criteria. As such, this sensitivity investigates the ability to mitigate integration effects and costs with purchased energy from WAPA.

For the WAPA Mitigation sensitivity, production costs for Nebraska entities were compared between cases using WAPA Firm Purchases scheduled against load versus those scheduled against load net wind. Previous (base case and other sensitivity) simulations constrained purchases using monthly energy and capacity; these cases were built using historical weekly energy, maximum and minimum purchases and schedules were fixed. The purpose of this change is that scheduling against wind or load net of wind with only weekly flexibility is more likely to be a realistic representation of the firm purchase constraints. For the mitigation runs, hourly schedules for the WAPA firm purchases were developed using two load following methodologies where energy scheduled is proportional to the load (given energy and capacity constraints): (a) load following with historical minimums and (b) pure load following. The sensitivity only involved the firm purchases, and did not include the WAPA peaking transactions. This sensitivity used 10\% penetration, 2006 load and wind patterns, and Nebraska only market case - the proxy resource was not involved. The WAPA hydro schedule was a 2000 pattern - a typical generation year. In other words, the hydro plants outputs were not changed for these simulations, only the purchases from WAPA.

It is first worth noting, the amount of energy that Nebraska entities purchase from WAPA on an annual basis relative to the total load in the simulation year 2018 is fixed. As shown in Table 41 
the 1.6 TWh is about 4\% of annual load energy in Nebraska. Although not shown in the table, it is also about $34 \%$ of the Nebraska wind energy in the $10 \%$ penetration cases.

Table 41: Comparison of Load and WAPA Firm Purchase

\begin{tabular}{|c|c|c|c|}
\hline & Nebraska Load & $\begin{array}{l}\text { Firm Purchase from } \\
\text { WAPA }\end{array}$ & $\begin{array}{l}\text { WAPA Purchase } \\
\text { as \% of Load }\end{array}$ \\
\hline Total (MWh) & $39,405,013$ & $1,641,236$ & $4.17 \%$ \\
\hline $\operatorname{Min}(\mathrm{MW})$ & 2,475 & 60 & $2.44 \%$ \\
\hline $\operatorname{Max}(\mathrm{MW})$ & 7,489 & 361 & $4.82 \%$ \\
\hline
\end{tabular}

Figure 69 shows the total Nebraska load for a sample week as well as the load net of wind for that same week. The comparison for this sensitivity will be the results of scheduling the WAPA purchase against the green as compared to the blue lines.

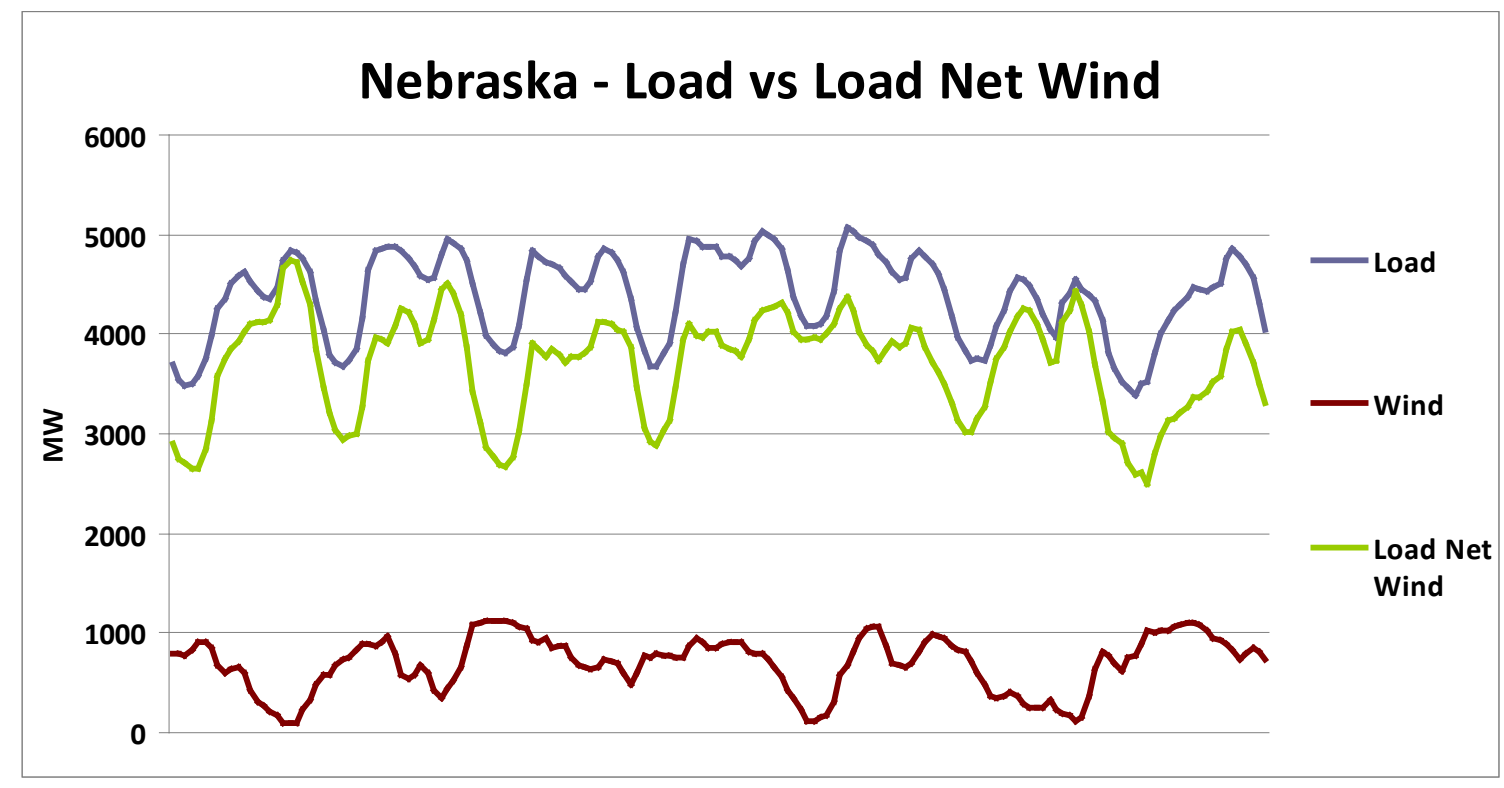

Figure 69: Comparison of Load and Load Net Wind

In setting up the sensitivity and scheduling methodology there was some question as to whether or not historical minimum purchases from WAPA should be considered as a constraint in the scheduling methodology. Analysis of historical data indicated that it may be an artificial constraint rather than a pure load following methodology. In order to remove that question, schedules with and without historical minimums honored were created.

The first set of tests used the schedules with historical minimum purchases honored as a constraint in the scheduling methodology. Figure 70 shows the difference between scheduling the WAPA purchase to load and load net wind. Note that since the purchase has a maximum take and a fixed energy, the schedules are not radically different - the peak amount is the same and the weekly total is the same, only hourly values have changed. 


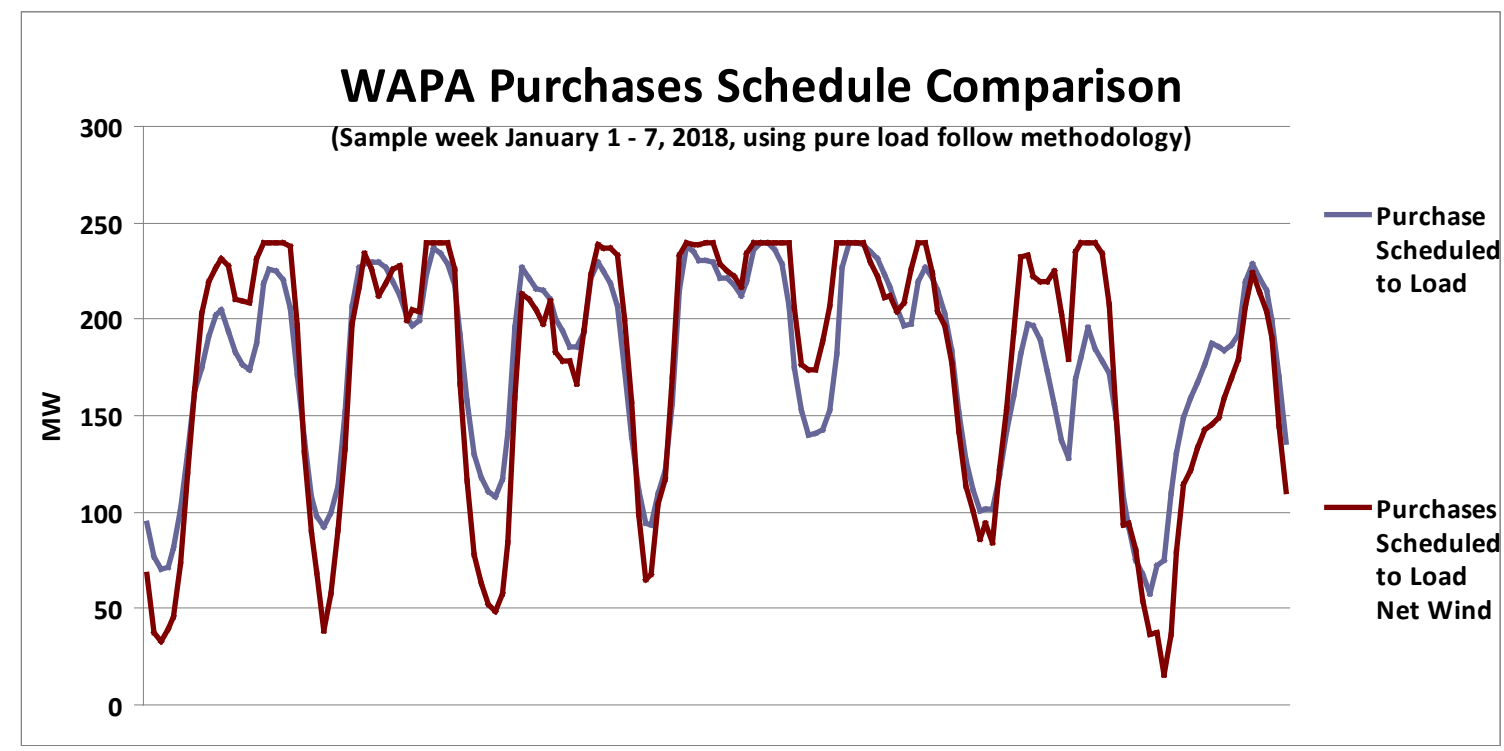

Figure 70: Comparison of WAPA Firm Purchase Schedules

As a second set of tests and when not honoring historical minimum schedules, more energy is available in the higher load shoulder hours. Figure 71 demonstrates this by comparing the 'Pure Load Following' and the 'Load Following with Mins' Methods.

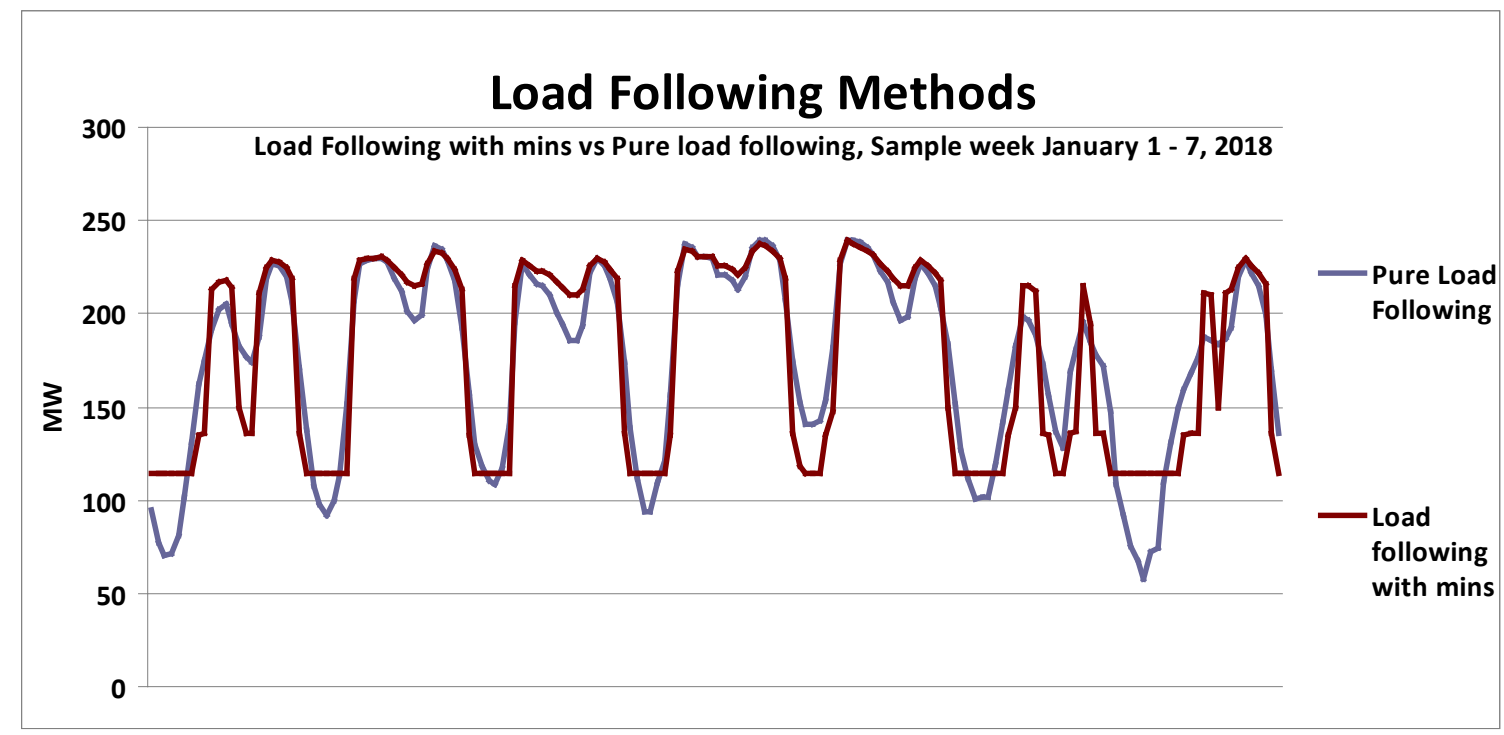

Figure 71: Comparison of Load Following Methods

The net result of the new schedule (to load net of wind with historical minimums honored) was that 186,000 MWh (about 10\%) of the WAPA firm purchase shifted over the year. There were 7700 hours affected (88\%). The maximum changes were $227 \mathrm{MW}$ decrease and $202 \mathrm{MW}$ increase. Those same statistics for pure load following (i.e. historical minimums not honored) are 195,000 MWh (about 12\%) of energy shifted, affecting 8400 hours (96\%) with maximum increase of $120 \mathrm{MW}$ and decrease of $104 \mathrm{MW}$. Figure 72 is a duration curve of the WAPA Firm 
purchases against load with the same hour values when scheduled against load net of wind without historical minimums honored. The direction of the energy change from one scheduling method to another is evenly spread between increases and decreases.

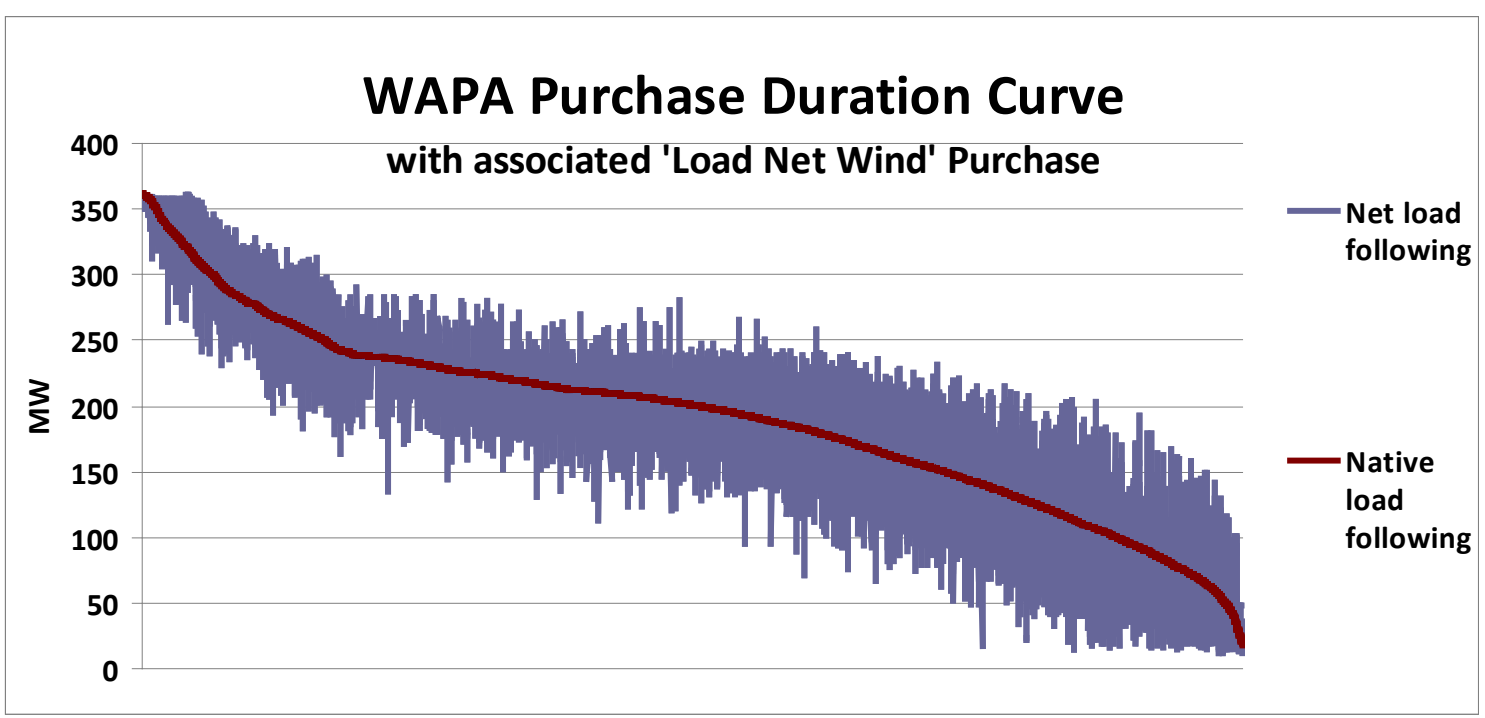

Figure 72: WAPA Firm Purchase Duration Curve - 2018 annual

The results were compared using Nebraska (only) production costs and adjusted production costs in Table 42 and Table 43. The 'pure' load following method shows more improvement to Nebraska production costs: a $\$ 910,000$ benefit (Table 43) to scheduling the purchases to 'load net wind'. However, the improvement is small: $0.06 \%$ decrease in Nebraska adjusted production costs. Looking at this result another way, for the approximate $12 \%$ of WAPA Firm Purchase energy that is shifted, each MWh shifted carries an increased value of about $\$ 5 / \mathrm{MWh}$ under the 'pure' load following analysis. $(\$ 910,000 / 195,000 \mathrm{MWh}=\$ 4.67 / \mathrm{MWh})$.

"Basecase" means following load, "TestCase" means following load net wind.

Table 42: Cost Results for Nebraska for WAPA Sensitivity using Load Following with Historical Mins.

\begin{tabular}{|l|r|r|r|}
\hline With Historical Mins & WAPA Basecase & WAPA TestCase & Delta \\
\hline Total Production Costs (M\$) & $2,161.15$ & $2,161.15$ & $(0.01)$ \\
\hline Net interchange (M\$) & $(523.69)$ & $(523.86)$ & 0.17 \\
\hline APC (M\$) & $1,637.46$ & $1,637.29$ & 0.16 \\
\hline
\end{tabular}

Table 43: Cost Results for Nebraska for WAPA Sensitivity using Pure Load Following

\begin{tabular}{|l|r|r|r|}
\hline Pure Load Following & WAPA Basecase & WAPA TestCase & Delta \\
\hline Total Production Costs (M\$) & $2,162.85$ & $2,162.53$ & 0.32 \\
\hline net interchange (M\$) & $(526.61)$ & $(527.19)$ & 0.59 \\
\hline APC (M\$) & $1,636.24$ & $1,635.33$ & 0.91 \\
\hline
\end{tabular}


Consistent with other results, it is useful here to look at the effect on the thermal fleet of generators. Figure 73 shows that there is very little change in the generation of any of the combustion turbine, combined cycle or steam turbines categories in Nebraska - well under half a percent change.

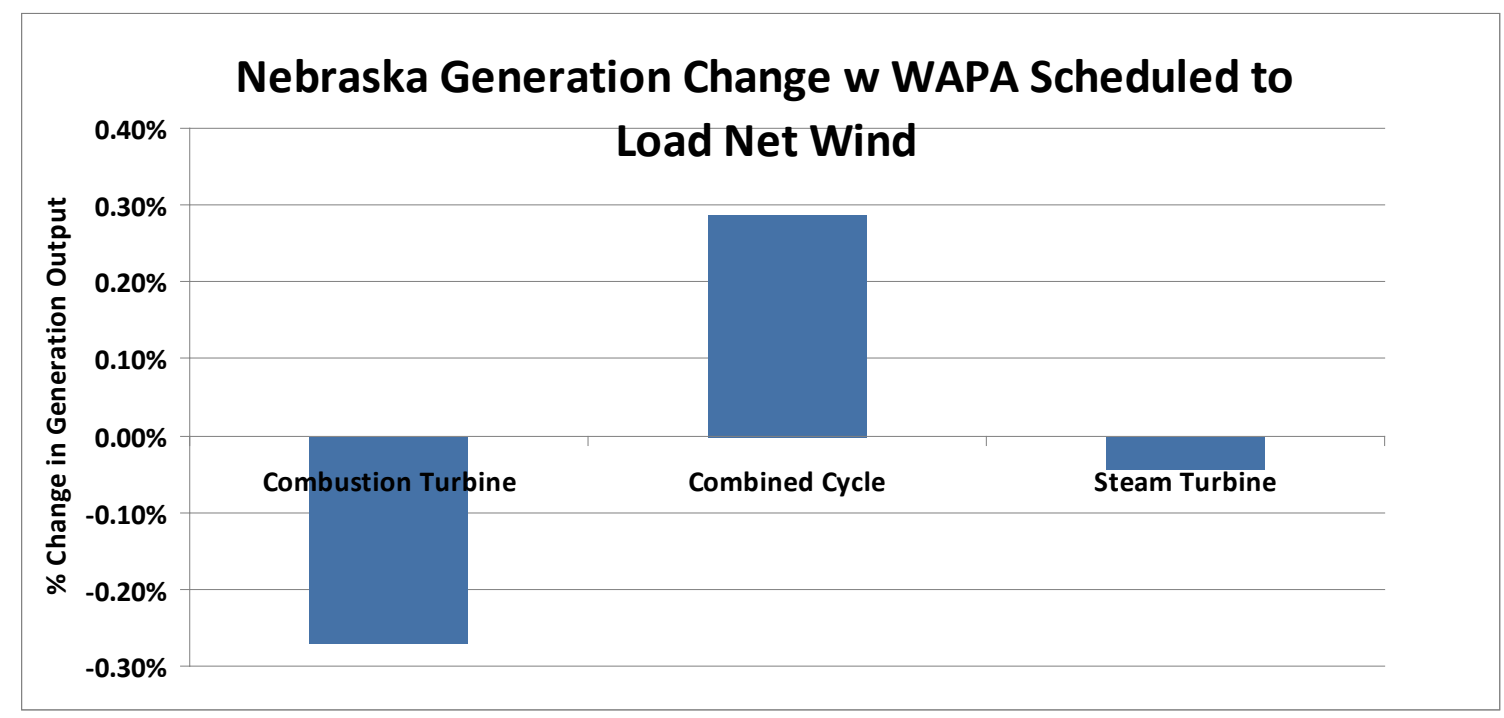

Figure 73: Annual Thermal Generation Change in Nebraska for WAPA Sensitivity (using Pure Load Following Method)

\subsubsection{Wind Diversity with WAPA}

In addition to the sensitivities that focused on the purchases from WAPA, analysis was performed on the wind generation forecast. The forecast error for wind generation was calculated by finding the difference between the hourly values of the day ahead wind generation forecast and the actual wind generation. The Mean Absolute Error (MAE) is a statistical way of determining how close a forecast is to its actual value. For the $10 \%$ penetration scenario it was found that the MAE of the day-ahead wind generation forecast for Nebraska and WAPA combined could be reduced to $10.9 \%$ compared to individual values of $11.4 \%$ for Nebraska and 12.3\% for WAPA, see Figure 74, Figure 75 and Figure 76. This indicates some potential diversity benefits from coordination of wind resources. 


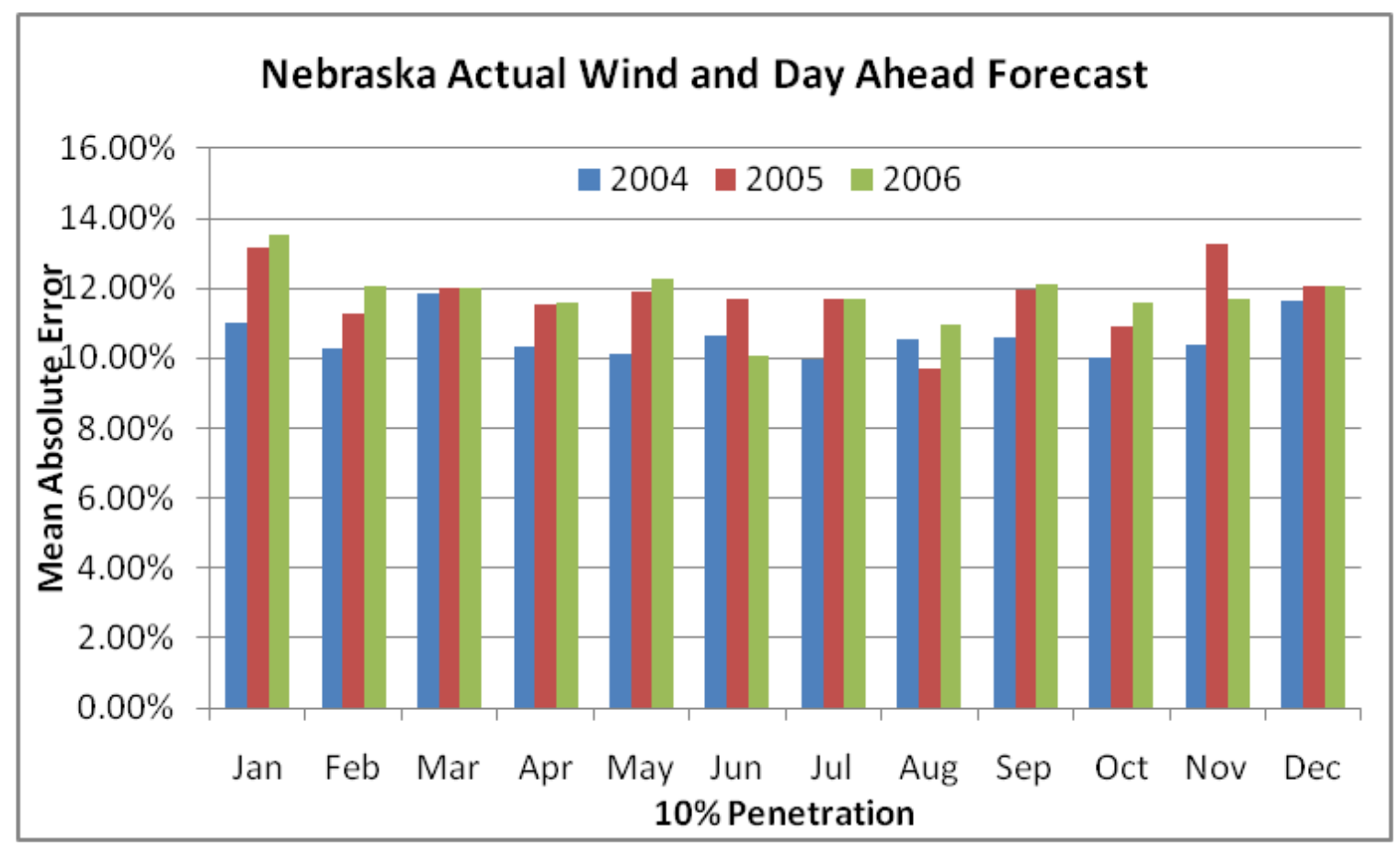

Figure 74: Nebraska MAE of Actual and Day Ahead Forecast

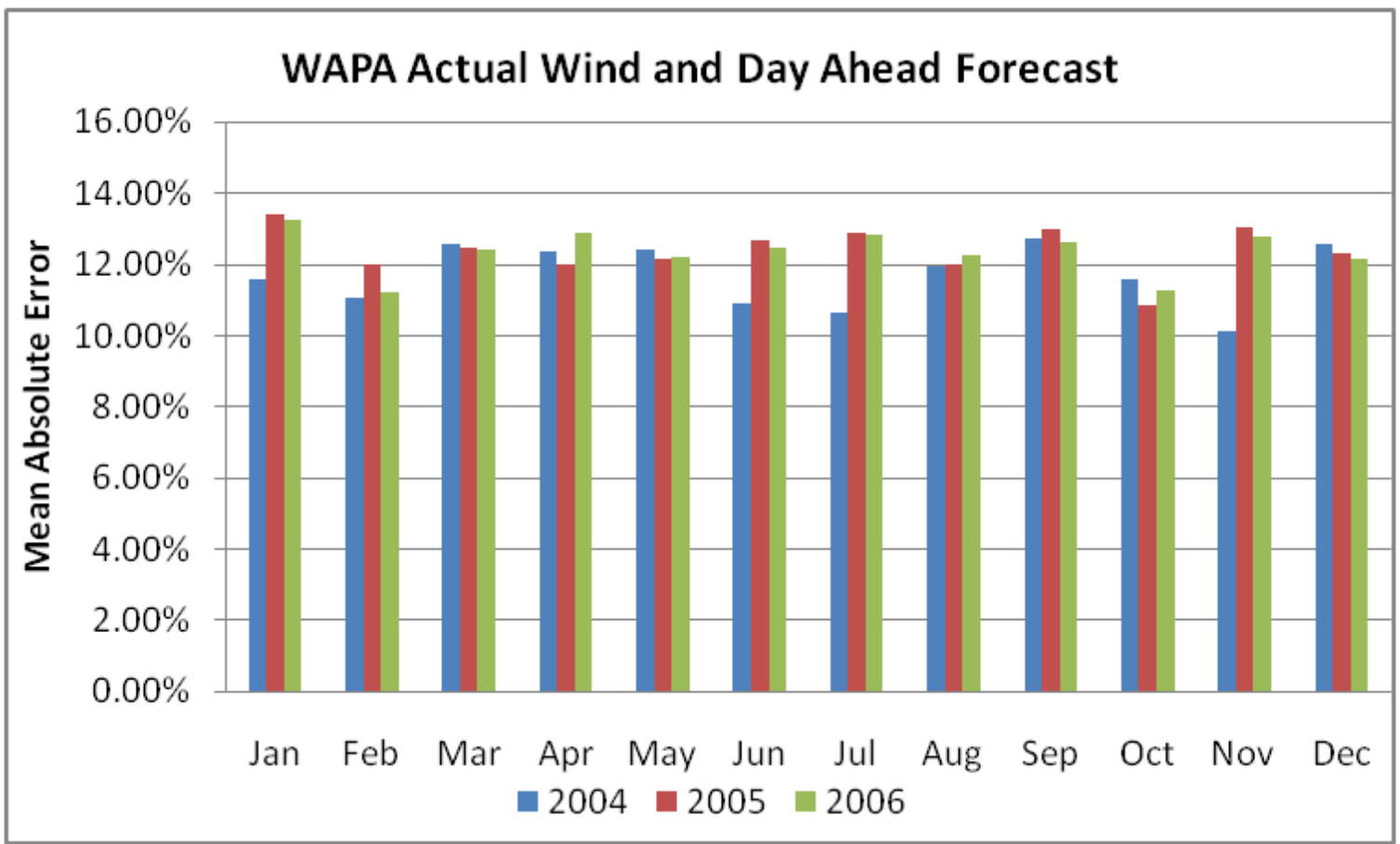

Figure 75: WAPA MAE of Actual and Day Ahead Forecast 


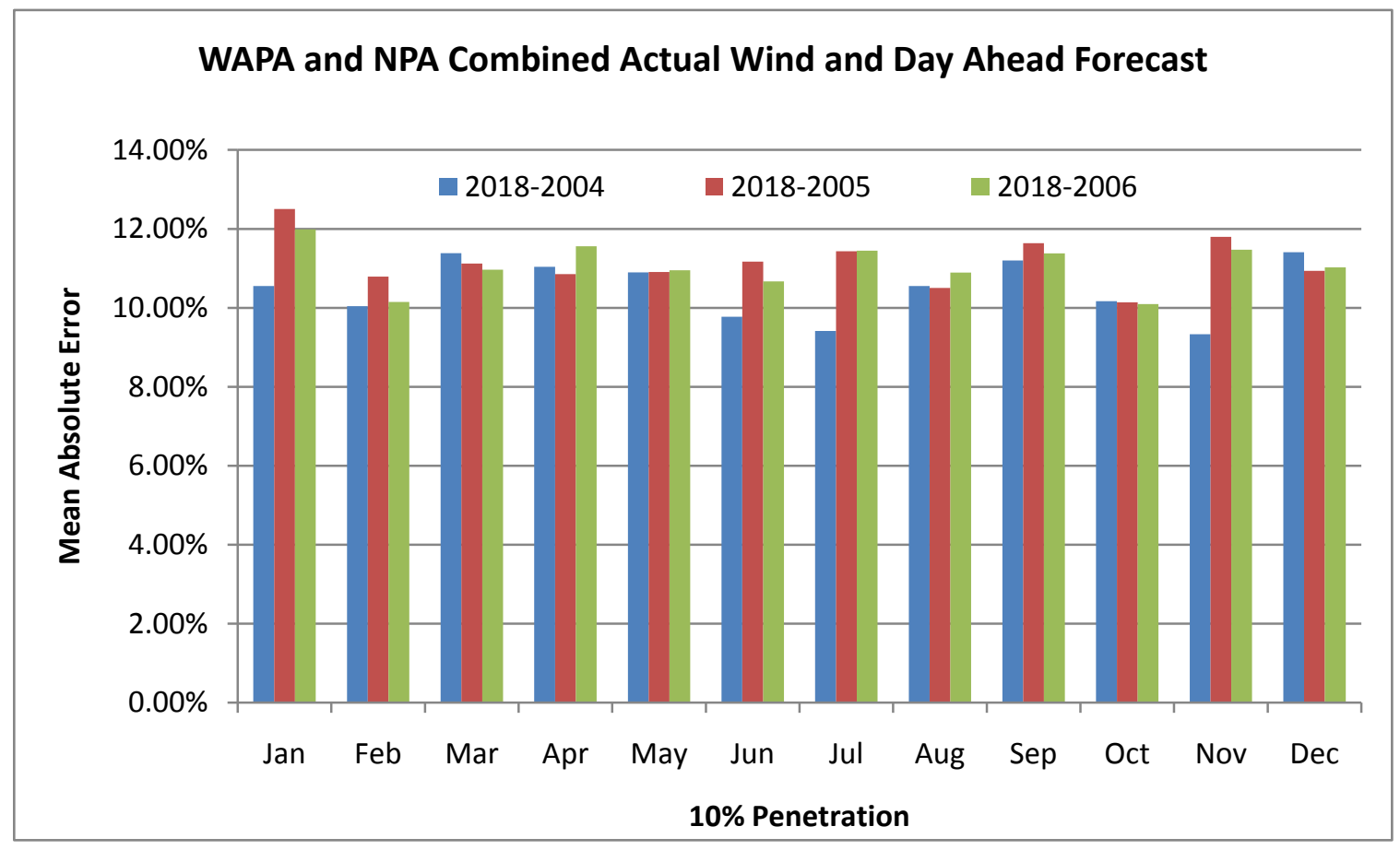

Figure 76: Nebraska and WAPA MAE of Actual and Day Ahead Forecast

\subsubsection{Sensitivity - CO2 Prices}

There is still uncertainty as to what form future carbon legislation may take, if any. The possibilities for tax versus cap and trade and for varying levels of emissions caps are substantial. Towards the goal of capturing a range of those possibilities, this study includes three sensitivities on the dispatch signal for $\mathrm{CO} 2$. This is in addition to the base case assumption of \$25 / Ton (Short Tons) of CO2. Those sensitivities are:

- $\quad \mathrm{CO} 2$ priced at $\$ 0 /$ ton - no price penalty on $\mathrm{CO} 2$

- $\quad \mathrm{CO} 2$ prices at $\$ 50 /$ ton - double the penalty of the base case

- $\quad$ CO2 priced at $\$ 120$ / ton - the 'CO2 reduction' case. This is not a prediction of future $\mathrm{CO} 2$ prices, but rather a dispatch signal based on an analysis of the dispatch switching point between coal and natural gas in order to force the use of natural gas fired generation over that of coal fired generation.

The results indicate very little change in total $\mathrm{CO} 2$ emissions when using $\$ 25 /$ ton or $\$ 50$ / ton as compared to no penalty. However, the $\mathrm{CO} 2$ reduction sensitivity resulted in an approximate $19 \%$ reduction in $\mathrm{CO} 2$ emissions using $\$ 120 /$ ton (down from 2018, $\$ 0 /$ ton). This indicates noneconomic operations are required in order to try and achieve an emission cap (see the 120/50 bullet discussion in Section 5.3); this is not a forecast of emissions price. The resulting 160 million tons of $\mathrm{CO} 2$ emission (for SPP including Nebraska) in the $\mathrm{CO} 2$ reduction case are slightly higher than the expected cap of 156 million tons under the Waxman-Markey bill, as described in Section 5.3. In the long term, it is expected that the price differential between carbon sources with $\mathrm{CO} 2$ pricing and low carbon sources will converge or reverse in order for the reductions to be market driven. It is also worth noting that this level of emission occurred at $20 \%$ wind 
penetration. $\mathrm{CO} 2$ emissions for each $\mathrm{CO} 2$ price sensitivity as well as the basecase are shown in Figure 77.

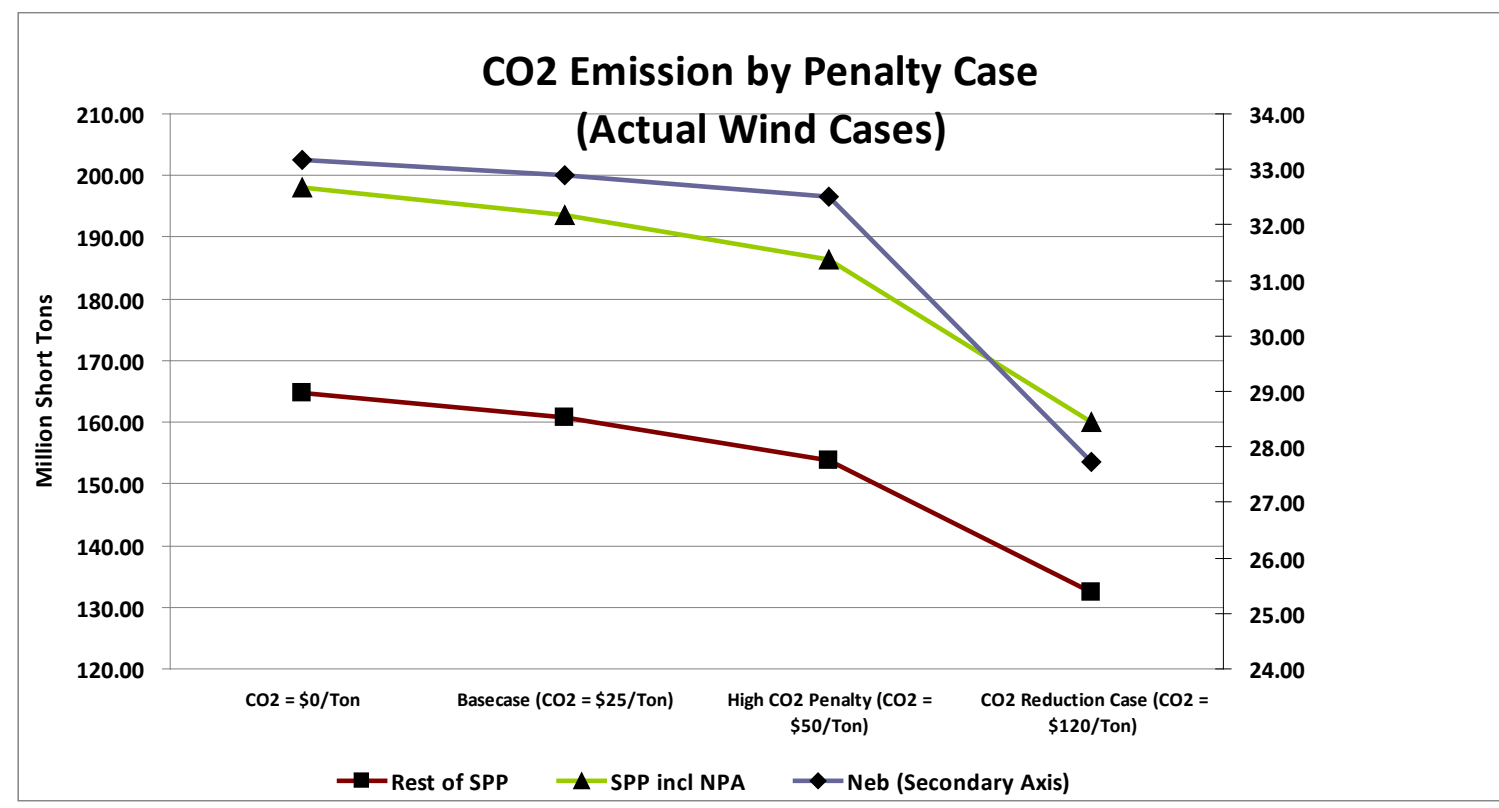

Figure 77: CO2 Emissions Comparison for different Penalty Levels

Figure 45 shows that the reduction in SPP's (including Nebraska) CO2 emissions from $10 \%$ to $40 \%$ wind penetrations is 30 million short tons as compared to the 33 million short tons reduction achieved by raising the base $\mathrm{CO} 2$ price of $\$ 25 /$ short ton to $\$ 120 /$ short ton.

Comparing the generation changes between the $\mathrm{CO} 2$ reduction case ( $\$ 120 /$ ton) and the base case ( $\$ 25 /$ ton) shows the expected change of a large increase in combined cycle usage with a large decrease in steam turbine (primarily coal) usage (Figure 78). It is important to note the scale of these changes with respect to some other changes observed. The over 40 million $\mathrm{MWh}$ increase in gas usage and decrease in coal usage are substantially larger than the generation changes observed in any of the other cases, including when comparing the change from $10 \%$ wind to $40 \%$ wind (where the thermal reductions are $18 \mathrm{TWh}$ for coal and $15 \mathrm{TWh}$ for gas) or comparing any actual to ideal cases. In the $\mathrm{CO} 2$ reduction case, combined cycle generation increased $(+138 \%$ in Nebraska, $+128 \%$ in rest of SPP) while coal shows substantial reduction ($22 \%$ in Nebraska, $-30 \%$ in rest of SPP). Additionally, combustion turbine usage increases as well: by $163 \%$ in Nebraska and by $61 \%$ in the rest of SPP. 


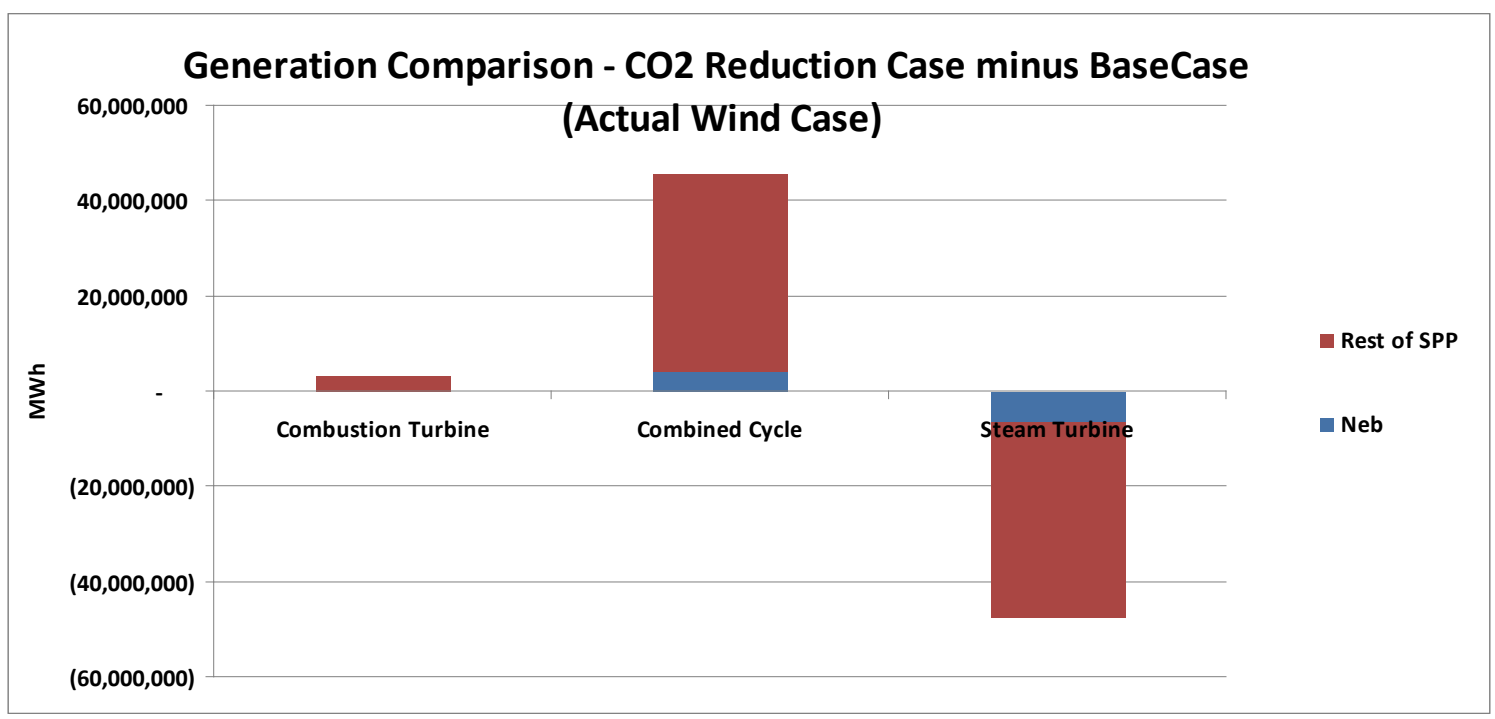

Figure 78: Thermal Generation Comparison between CO2 Reduction Case and Base Case

It's also worth noting the change in capacity factors for two critical generation types in Nebraska - combined cycle and coal fired steam turbines. Table 44 shows that the combined cycle capacity factor increased to over $80 \%$ (from a range of $27 \%$ to $44 \%$ ) between the base case and the $\mathrm{CO} 2$ reduction case and that ST Coal units decreased from about $80 \%$ to a range of $58 \%$ to $67 \%$.

Table 44: Nebraska Capacity Factor Comparison between Base Case and CO2 Reduction Case

\begin{tabular}{|l|c|c|c|c|}
\hline \multirow{2}{*}{$\%$} & \multicolumn{2}{|c|}{ Combined Cycles } & \multicolumn{2}{c|}{ ST Coal } \\
\cline { 2 - 5 } & BaseCase & CO2 @ \$120 & BaseCase & CO2 @ \$120 \\
\hline LES & 27 & 80 & 84 & 58 \\
\hline NPPD & 28 & 82 & 82 & 67 \\
\hline OPPD & 44 & 94 & 78 & 59 \\
\hline
\end{tabular}

An interesting side effect of the substantial increase in $\mathrm{CO} 2$ penalties is the dampening of the split between on-peak and off-peak LMPs. This decreasing split results in uneconomic conditions for the operation of existing pumped storage generation facilities in SPP. Figure 79 shows the decrease in pumped storage usage as $\mathrm{CO} 2$ costs are increased. The effect is dramatic even at lower $\mathrm{CO} 2$ prices.

This is a significant finding in that pumped storage capability (hydro or compressed air) is one of the alternatives that can help mitigate wind generation (pumping when winds are high and generating when winds are low). Mitigation will continue to be a benefit from pumped storage but its other benefits from "transferring" energy from off-peak periods to on-peak periods will be diminished as prices for coal generation and gas generation come together under $\mathrm{CO} 2$ regulation. 


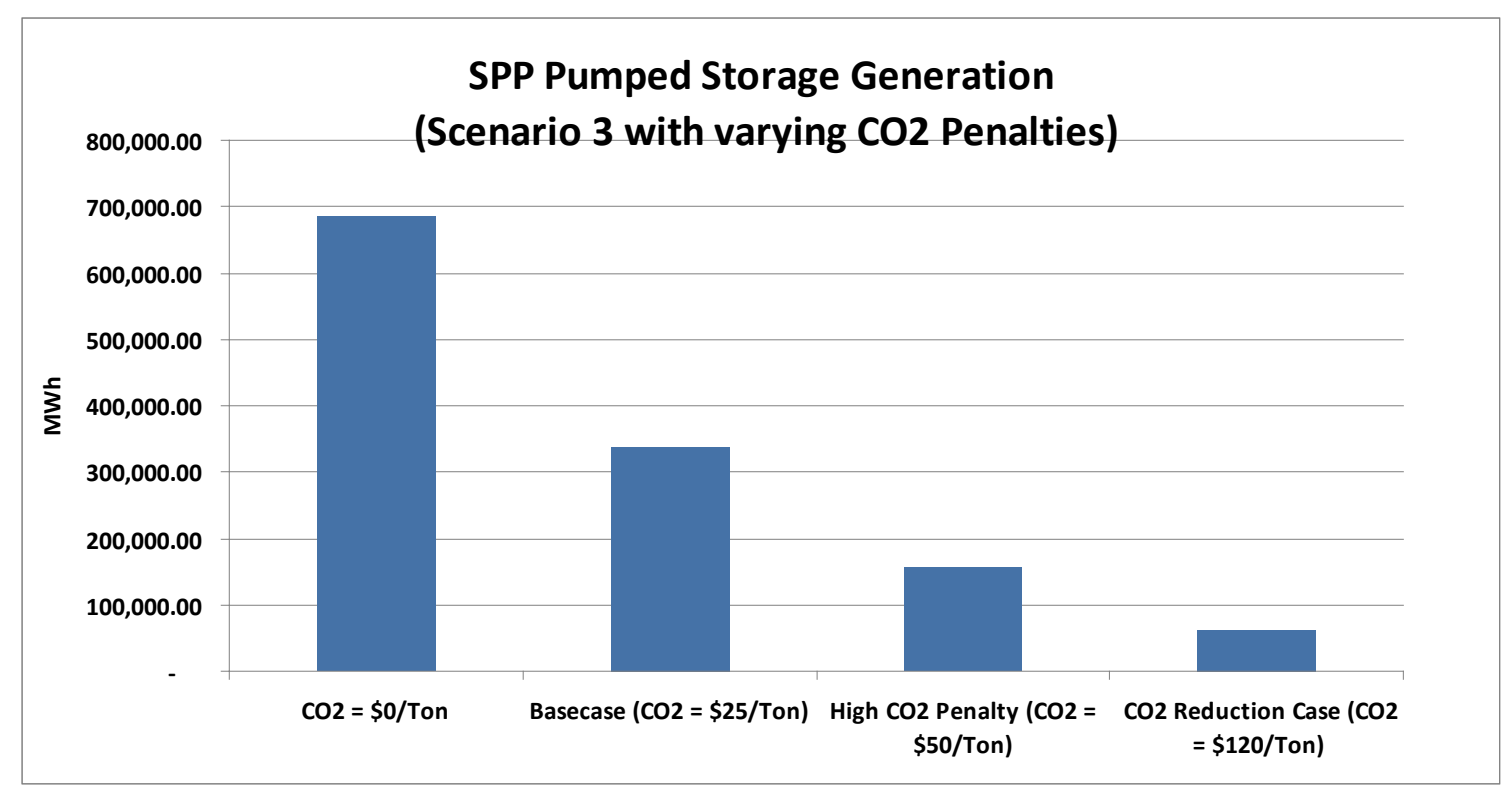

Figure 79: Comparison of Pumped Storage Output with Different CO2 Penalties

Some of this reduction in pumped storage usage may be a function of PROMOD modeling techniques. PROMOD will only allow pumped storage units to refill during off peak (night and weekend) hours, so if lower priced hours occurred during the day PROMOD would not be able to take advantage of that and pump. However, it's not likely that this is a significant driver of the results being shown. First, note that the wind penetration is consistent at $20 \%$ for each of the $\mathrm{CO} 2$ sensitivity cases and that the wind patterns are exactly the same. So, any peak switching that is occurring is taking place in all of the sensitivities. Second, one of the purposes of the $\mathrm{CO} 2$ sensitivities (especially the $\mathrm{CO} 2$ reduction case) was to create a penalty that resulted in gas units and coal units switching places in the economic stack. Thus, it stands to reason that LMPs would converge between off peak periods where coal is often on the margin and on peak periods where gas units are often the marginal resource. Figure 80 below shows both the increasing LMPs as $\mathrm{CO} 2$ penalties increase and the generally decreasing differential between on peak and off peak LMPs with the same $\mathrm{CO} 2$ increase. 


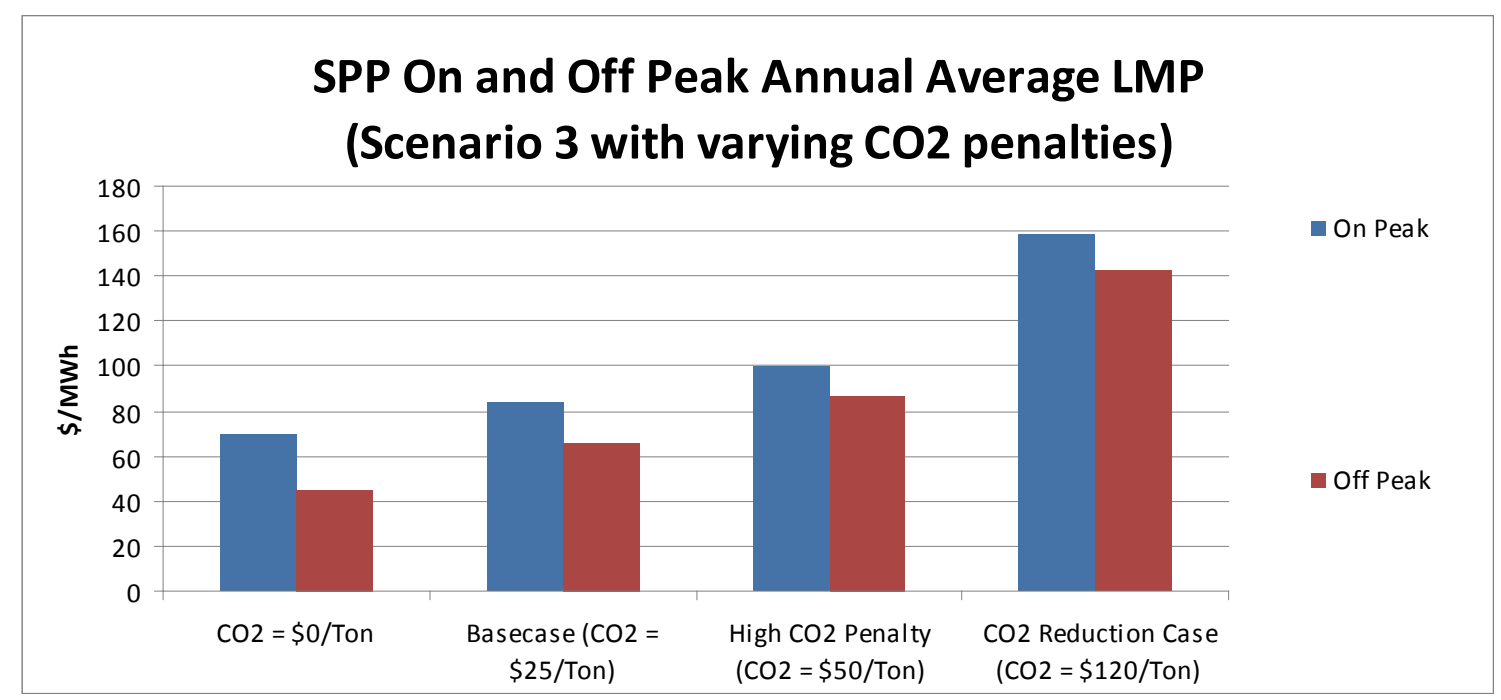

Figure 80: SPP On and Off Peak Annual Average LMP

The decreasing use of the pumped storage generation with the increase in $\mathrm{CO} 2$ penalties indicates that the differential in LMP is not enough to overcome the inherent inefficiency in pumped storage and thus its operation becomes uneconomic. It may indicate that a future with high penalties on $\mathrm{CO} 2$ could call for new operating procedures for storage plants that have some objective other than economics determining their operations.

\subsection{WIND ANALYSIS}

The NREL wind site database provides forecast and actual hourly data representations for each of the selected wind sites. From this data several analyses were performed to find relationships between wind pattern and load. It was possible to obtain trend information for individual sites or as an aggregate. Wind is intermittent with varying patterns throughout time. To obtain a better understanding of these patterns several analyses of the wind data were performed for the NPA and the SPP sites. In addition to the charts and figures presented here the document referenced in appendix Section 8.2 contains additional information, charts and figures.

\subsubsection{Wind Generation Characteristics}

As discussed in Section 2.3.1 the wind generation site production was obtained from the NREL database. The sites in the NREL data were necessarily large, given their original purpose (EWITS study). Therefore for the Nebraska smaller penetrations it was not possible to get exactly the nominal penetration amount; especially at $10 \%$ (hence actually $11.2 \%$ was modeled in Nebraska).

Additionally there was no evidence found in the NREL database for sites that show loss of wind generation occurrences due to icing, cold temperatures, hot temperatures, or high wind trips.

There were a total of 11 wind sites selected for Nebraska, five sites for the $10 \%$ penetration scenario and 3 additional sites each for the $20 \%$ and $40 \%$ penetration scenarios. Individual site resources characteristics are shown in Table 45 . The $10 \%$ scenario sited $1249 \mathrm{MW}$ of nameplate generation that provided an average $4.5 \mathrm{TWh}$ annual energy. The next $10 \%$ 
penetration of wind siting consists of $1239 \mathrm{MW}$ of nameplate generation providing an additional average energy of $4.4 \mathrm{TWh}$. To obtain $40 \%$ wind penetration an additional 2,239 MW of nameplate capacity was added along with the additional average energy of $8.1 \mathrm{TWh}$.

Table 45: Nebraska Wind Site Characteristics

\begin{tabular}{|c|c|c|c|c|c|c|}
\hline Nebraska Sites & \multicolumn{6}{|c|}{ Initial 10\% Penetration } \\
\hline SitelD & 22 & 143 & 160 & 205 & 208 & Total \\
\hline NamePlate & 261 & 268 & 240 & 235 & 245 & 1,249 \\
\hline $2004 \mathrm{MWH}$ & 954,524 & 928,527 & 836,829 & 785,406 & 831,805 & $4,337,092$ \\
\hline $2005 \mathrm{MWH}$ & 991,648 & 966,194 & 864,056 & 842,712 & 885,040 & $4,549,651$ \\
\hline $2006 \mathrm{MWH}$ & $1,045,368$ & $1,001,097$ & 902,692 & 881,119 & 920,530 & $4,750,806$ \\
\hline & \multicolumn{4}{|c|}{ Add for $20 \%$ penetration } & & \\
\hline SitelD & 47 & 245 & 1149 & Total & & \\
\hline NamePlate & 285 & 453 & 501 & 1,239 & & \\
\hline $2004 \mathrm{MWH}$ & 997,720 & $1,541,920$ & $1,622,240$ & $4,161,879$ & & \\
\hline $2005 \mathrm{MWH}$ & $1,054,278$ & $1,643,371$ & $1,699,189$ & $4,396,838$ & & \\
\hline $2006 \mathrm{MWH}$ & $1,116,852$ & $1,665,893$ & $1,732,331$ & $4,515,075$ & & \\
\hline
\end{tabular}

\begin{tabular}{|c|r|r|r|r|}
\hline \multicolumn{5}{|c|}{ Add for 40\% Penetration } \\
\hline SitelD & \multicolumn{1}{|c|}{70} & $\mathbf{7 6}$ & \multicolumn{1}{c|}{ 695 } & \multicolumn{1}{c|}{ Total } \\
\hline NamePlate & 1,100 & 479 & 660 & 2,239 \\
\hline $2004 \mathrm{MWH}$ & $3,951,551$ & $1,726,076$ & $2,184,190$ & $7,861,817$ \\
\hline $2005 \mathrm{MWH}$ & $4,046,332$ & $1,774,646$ & $2,226,755$ & $8,047,732$ \\
\hline $2006 \mathrm{MWH}$ & $4,201,907$ & $1,845,977$ & $2,432,432$ & $8,480,316$ \\
\hline
\end{tabular}

Note - Other summary tables in this report may have slightly different totals due to averaging and rounding

Similarly, Table 46 shows the wind site characteristics selected for SPP. There were a total of 33 wind sites identified with 7 sites having total nameplate capacity of $6,256 \mathrm{MW}$ and an average annual energy of $22.9 \mathrm{TWh}$ for the first $10 \%$ penetration. The next $10 \%$ of wind generation added 11 more wind sites with nameplate capacity of 6,340MW and an additional average annual energy of 22.9 TWh. To reach the $40 \%$ penetration level an additional 15 wind sites were selected having total nameplate capacity of $12,835 \mathrm{MW}$ and average annual energy of 45.8 TWh. For the $40 \%$ wind penetration case the total nameplate capacity of wind sites was 25,431 MW providing an average annual energy of 91.6 TWh. 
Table 46: SPP Wind Site Characteristics

\begin{tabular}{|c|c|c|c|c|c|c|c|c|}
\hline SPP Sites & \multicolumn{7}{|c|}{ Initial 10\% Penetration } & \multirow[b]{2}{*}{ Total } \\
\hline SitelD & 34 & 56 & 62 & 81 & 439 & 441 & 2299 & \\
\hline Nameplate MW & 1,296 & 1,230 & 1,282 & 1,118 & 598 & 492 & 240 & 6,256 \\
\hline $2004 \mathrm{MWH}$ & $4,734,368$ & $4,367,627$ & $4,507,747$ & $4,019,125$ & $2,041,887$ & $1,670,794$ & 703,078 & $22,044,626$ \\
\hline $2005 \mathrm{MWH}$ & $4,901,477$ & $4,558,024$ & $4,871,654$ & $4,141,099$ & $2,149,001$ & $1,777,090$ & 745,579 & $23,143,924$ \\
\hline $2006 \mathrm{MWH}$ & $5,038,118$ & $4,717,498$ & $4,796,483$ & $4,274,121$ & $2,103,165$ & $1,740,228$ & 777,946 & $23,447,559$ \\
\hline
\end{tabular}

\begin{tabular}{|c|c|c|r|r|r|r|}
\hline \multicolumn{7}{|c|}{ Add for 20\% Penetration } \\
\hline SitelD & $\mathbf{7}$ & $\mathbf{1 4}$ & $\mathbf{5 3}$ & $\mathbf{9 2}$ & $\mathbf{1 0 0}$ & $\mathbf{1 3 7}$ \\
\hline Nameplate MW & 378 & $\mathbf{2 8 7}$ & $\mathbf{4 2 8}$ & 1197 & $\mathbf{4 6 4}$ & 1162 \\
\hline $2004 \mathrm{MWH}$ & $1,415,067$ & $1,019,600$ & $1,502,370$ & $4,190,173$ & $1,658,685$ & $4,111,422$ \\
\hline $2005 \mathrm{MWH}$ & $1,454,043$ & 987,966 & $1,585,122$ & $4,345,059$ & $1,727,583$ & $4,125,998$ \\
\hline $2006 \mathrm{MWH}$ & $1,502,521$ & $1,112,854$ & $1,628,825$ & $4,519,685$ & $1,734,220$ & $4,404,074$ \\
\hline \multicolumn{7}{|c|}{} \\
\hline SitelD & $\mathbf{2 3 9}$ & $\mathbf{2 9 5}$ & $\mathbf{4 7 3}$ & $\mathbf{4 7 6}$ & $\mathbf{4 9 6}$ & Total \\
\hline Nameplate MW & 334 & 365 & 262 & 1161 & 302 & 6,340 \\
\hline 2004 MWH & $1,132,819$ & $1,316,290$ & 853,398 & $4,039,288$ & 986,616 & $22,225,729$ \\
\hline $2005 \mathrm{MWH}$ & $1,212,663$ & $1,269,530$ & 895,231 & $3,920,673$ & $1,051,553$ & $22,575,421$ \\
\hline $2006 \mathrm{MWH}$ & $1,210,538$ & $1,364,604$ & 937,731 & $4,270,328$ & $1,088,020$ & $23,773,401$ \\
\hline
\end{tabular}

\begin{tabular}{|c|c|c|c|c|c|c|c|c|}
\hline & \multicolumn{8}{|c|}{ Add for $40 \%$ Penetration } \\
\hline SitelD & 30 & 93 & 105 & 136 & 173 & 189 & 193 & 194 \\
\hline Nameplate & 1,316 & 1,104 & 411 & 1,146 & 1,046 & 427 & 1,221 & 1,164 \\
\hline 2004 Total & $4,745,206$ & $3,768,133$ & $1,444,560$ & $3,992,502$ & $3,562,118$ & $1,504,947$ & $4,154,147$ & $3,978,644$ \\
\hline 2005 Total & $4,907,959$ & $3,955,734$ & $1,545,801$ & $4,169,422$ & $3,699,833$ & $1,557,084$ & $4,319,122$ & $4,089,286$ \\
\hline 2006 Total & $5,146,879$ & $4,137,703$ & $1,520,865$ & $4,216,489$ & $3,862,329$ & $1,559,144$ & $4,476,646$ & $4,256,330$ \\
\hline SitelD & 212 & 240 & 299 & 365 & 389 & 443 & 632 & Total \\
\hline Nameplate & 506 & 1,074 & 394 & 558 & 271 & 1,125 & 1,072 & 12,835 \\
\hline 2004 Total & $1,794,236$ & $3,781,703$ & $1,411,818$ & $1,869,555$ & 931,619 & $3,925,669$ & $3,568,644$ & $44,433,498$ \\
\hline 2005 Total & $1,858,988$ & $3,863,049$ & $1,436,729$ & $1,955,653$ & 981,523 & $3,898,434$ & $3,624,855$ & $45,863,472$ \\
\hline 2006 Total & $1,839,087$ & $3,966,842$ & $1,410,246$ & $2,004,181$ & 959,170 & $4,075,532$ & $3,817,722$ & $47,249,164$ \\
\hline
\end{tabular}

Note - Other summary tables in this report may have slightly different totals due to averaging and rounding

For the rest of the Interconnection, Figure 19 shows the general locations and sites modeled. Table 3, Table 14 and Table 18 show some numerical data on how the regions external to SPP were modeled.

\subsubsection{Capacity Factors}

Wind sites selected for NPA were intentionally located in areas with high wind characteristics thus providing an increased probability of achieving high capacity factors. Table 47 presents the annual capacity factors for each of the site id's selected for Nebraska and the overall average. For the SPP wind sites Table 48 provides site capacity factors for each of the reference years and the average for all three years.

It is important to note that these capacity factors have not been reduced by NPA (nor NREL, it is believed) to account for high or low temperature generation cutouts, high wind or icing cutouts, turbine malfunction or maintenance, or wind generation curtailment as a result of transmission congestion. 
Table 47: Nebraska Wind Site Capacity Factors

\begin{tabular}{|r|r|r|r|r|}
\hline $\begin{array}{c}\text { Nebraska } \\
\text { SitelD }\end{array}$ & 2004 CF & 2005 CF & 2006 CF & 3 Year CF \\
\hline 22 & 0.42 & 0.43 & 0.42 & 0.42 \\
\hline 143 & 0.39 & 0.41 & 0.43 & 0.41 \\
\hline 160 & 0.40 & 0.41 & 0.43 & 0.41 \\
\hline 205 & 0.38 & 0.41 & 0.43 & 0.41 \\
\hline 208 & 0.39 & 0.41 & 0.43 & 0.41 \\
\hline 47 & 0.40 & 0.42 & 0.41 & 0.41 \\
\hline 245 & 0.39 & 0.41 & 0.42 & 0.41 \\
\hline 1149 & 0.37 & 0.39 & 0.39 & 0.38 \\
\hline 70 & 0.41 & 0.42 & 0.40 & 0.41 \\
\hline 76 & 0.41 & 0.42 & 0.40 & 0.41 \\
\hline 695 & 0.38 & 0.39 & 0.42 & 0.39 \\
\hline
\end{tabular}


Table 48: Rest of SPP Wind Site Capacity Factors

\begin{tabular}{|l|r|r|r|r|}
\hline $\begin{array}{l}\text { SPP Site } \\
\text { ID }\end{array}$ & 2004 CF & 2005 CF & \multicolumn{1}{l|}{2006 CF } & 3 Year CF \\
\hline 34 & 0.42 & 0.43 & 0.44 & 0.43 \\
\hline 56 & 0.40 & 0.42 & 0.44 & 0.42 \\
\hline 62 & 0.40 & 0.43 & 0.43 & 0.42 \\
\hline 81 & 0.41 & 0.42 & 0.44 & 0.42 \\
\hline 439 & 0.39 & 0.41 & 0.40 & 0.40 \\
\hline 441 & 0.39 & 0.41 & 0.40 & 0.40 \\
\hline 2299 & 0.33 & 0.35 & 0.37 & 0.35 \\
\hline 7 & 0.43 & 0.44 & 0.45 & 0.44 \\
\hline 14 & 0.40 & 0.39 & 0.44 & 0.41 \\
\hline 53 & 0.40 & 0.42 & 0.43 & 0.42 \\
\hline 92 & 0.40 & 0.41 & 0.43 & 0.41 \\
\hline 100 & 0.41 & 0.43 & 0.43 & 0.42 \\
\hline 137 & 0.40 & 0.41 & 0.43 & 0.41 \\
\hline 239 & 0.39 & 0.41 & 0.41 & 0.40 \\
\hline 295 & 0.41 & 0.40 & 0.43 & 0.41 \\
\hline 473 & 0.37 & 0.39 & 0.41 & 0.39 \\
\hline 476 & 0.40 & 0.39 & 0.42 & 0.40 \\
\hline 496 & 0.37 & 0.40 & 0.41 & 0.39 \\
\hline 30 & 0.41 & 0.43 & 0.45 & 0.43 \\
\hline 93 & 0.39 & 0.41 & 0.43 & 0.41 \\
\hline 105 & 0.40 & 0.43 & 0.42 & 0.42 \\
\hline 136 & 0.40 & 0.42 & 0.42 & 0.41 \\
\hline 173 & 0.39 & 0.40 & 0.42 & 0.40 \\
\hline 189 & 0.40 & 0.42 & 0.42 & 0.41 \\
\hline 193 & 0.39 & 0.40 & 0.42 & 0.40 \\
\hline 194 & 0.39 & 0.40 & 0.42 & 0.40 \\
\hline 212 & 0.40 & 0.42 & 0.41 & 0.41 \\
\hline 240 & 0.40 & 0.41 & 0.42 & 0.41 \\
\hline 299 & 0.41 & 0.42 & 0.41 & 0.41 \\
\hline 365 & 0.38 & 0.40 & 0.41 & 0.40 \\
\hline 389 & 0.39 & 0.41 & 0.40 & 0.40 \\
\hline 443 & 0.40 & 0.40 & 0.41 & 0.40 \\
\hline 632 & 0.38 & 0.39 & 0.41 & 0.39 \\
\hline
\end{tabular}

\subsubsection{Capacity Credit}

Because of the intermittent nature of wind power plants, including that wind generation output is typically low at system peak times; capacity credit for wind generators is typically low. Using the SPP criteria, which is the criterion applicable to Nebraska utilities, the capacity credit for wind sites in Nebraska were calculated. Capacity credit is a monthly calculation. For the 
purpose of analysis an annual credit is presented as being those values from the month of system peak demand. A brief summary of the criteria used is described below ${ }^{7}$. It should be noted that only 3 years of wind data was used in this analysis.

- $\quad$ Assemble up to the most recent ten years, with a minimum of the most recent five years, of hourly net power output (MW) data, measured at the system interconnection point. Values may be calculated from wind data, if measured MW values are not yet available. Wind data correlated with a reference tower beyond fifty miles is subject to Generation Working Group approval. For calculated values, at least one year must be based on site specific wind data.

- $\quad$ Select the hourly net power output values occurring during the top $10 \%$ of load hours for the SPP Load Serving Member for each month of each year for the evaluation period (e.g., 72 hours for a typical 30 day month and 360 hours for a 5 year period).

- $\quad$ Select the hourly net power output value that can be expected from the plant $85 \%$ of the time or greater. For example, for a 5 year period with the 360 hourly net power output values ranked from highest to lowest, the capacity of the wind plant will be the MW value in the $306^{\text {th }}$ data point.

- $\quad$ A seasonal or annual net capability may be determined by selecting the appropriate monthly MW values corresponding to the Load Serving Member's peak load month of the season of interest.

Figure 81 shows the capacity credit for a sample of the 5 selected Nebraska wind sites identified for the $10 \%$ penetration scenario. The chart shows the capacity credit, as a percentage of nameplate capacity, on a monthly basis for each site. It should be noted that the annual capacity credit is biased toward the lower capacity credit value by the nature of the calculation criteria. It can also be observed how the tendency of wind to be lower in the summer months is reflected in the lower capacity credit. Additional charts of capacity credit can be found in the document referenced in appendix Section 8.2.

Methods of calculating capacity credit for wind vary across the country and produce different results. A standardized method of calculating the capacity credit of wind is under investigation by FERC.

${ }^{7}$ From SPP Criteria, revision 7/28/2009 pages $12-6$ and $12-7$ 


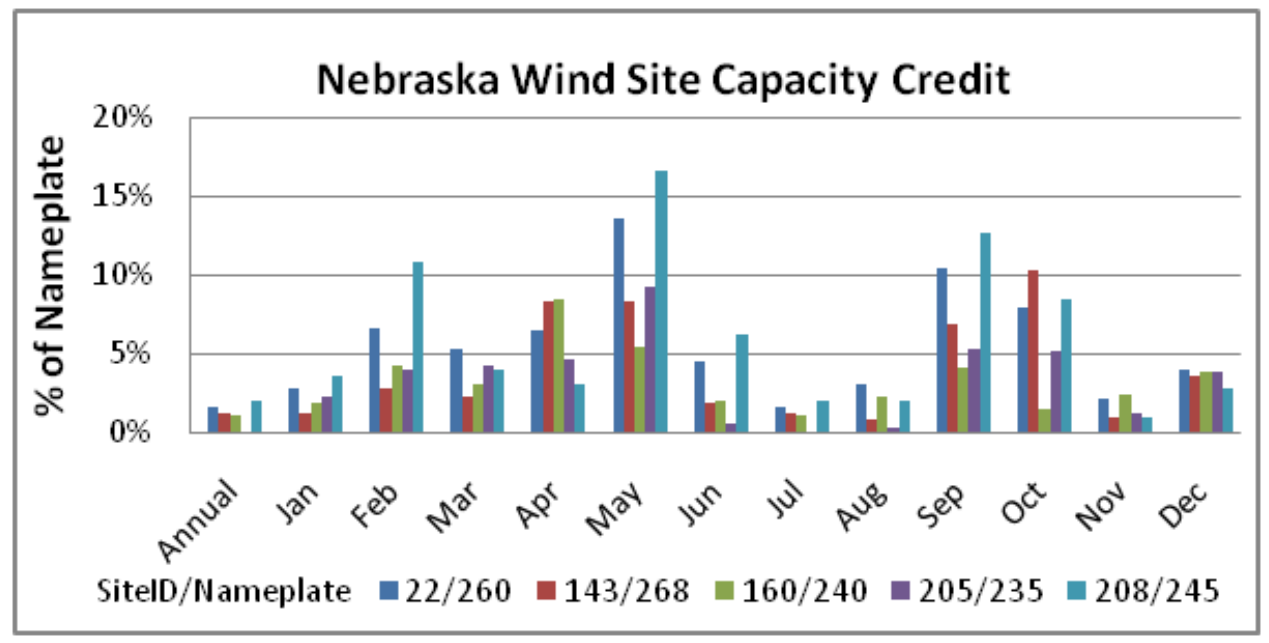

Figure 81: Nebraska Wind Site capacity credit

\subsubsection{Wind and Load Correlation}

One of the criteria for selecting the Nebraska wind sites was geographic diversity. Looking at the selection of sites on the map shown in Figure 15, Figure 16, and Figure 17, one can see that some sites have closer proximity which intuitively would indicate a high level of correlation with generator output. On the other hand sites with distant proximity would be expected to have lower or close to zero correlation. Wind site output for Nebraska was correlated to other Nebraska wind sites for all three years of actual wind data. The correlation between Nebraska sites can be seen on Table 49. Looking at this table the suggested ranking of the wind site correlation would be:

- Zero to 2 no or negligible correlation (Zero pairs)

- $\quad .2$ to .4 low degree of correlation $\quad$ (12 pairs)

- $\quad .4$ to .6 moderate degree of correlation (15 pairs)

- $\quad .6$ to .8 marked degree of correlation (14 pairs)

- .8 to 1.0 high correlation (14 pairs)

Table 49: Correlation between Nebraska Wind Sites

\begin{tabular}{|c|c|c|c|c|c|c|c|c|c|c|c|}
\hline \multicolumn{12}{|c|}{ Correlation of hourly wind data between wind sites for actual wind data in years 2004 to 2006} \\
\hline \multirow[b]{2}{*}{ Site ID } & \multicolumn{5}{|c|}{$10 \%$ sites } & \multicolumn{3}{|c|}{$20 \%$ sites } & \multicolumn{3}{|c|}{$40 \%$ sites } \\
\hline & 22 & 143 & 160 & 205 & 208 & 47 & 245 & 1149 & 70 & 76 & 695 \\
\hline 22 & 1 & 0.77 & 0.47 & 0.93 & 0.81 & 0.99 & 0.49 & 0.78 & 0.82 & 0.49 & 0.24 \\
\hline 143 & 0.77 & 1 & 0.61 & 0.83 & 0.58 & 0.75 & 0.69 & 0.58 & 0.97 & 0.64 & 0.32 \\
\hline 160 & 0.47 & 0.61 & 1 & 0.55 & 0.35 & 0.46 & 0.83 & 0.39 & 0.60 & 0.98 & 0.57 \\
\hline 205 & 0.93 & 0.83 & 0.55 & 1 & 0.74 & 0.92 & 0.57 & 0.73 & 0.88 & 0.57 & 0.28 \\
\hline 208 & 0.81 & 0.58 & 0.35 & 0.74 & 1 & 0.82 & 0.34 & 0.90 & 0.63 & 0.36 & 0.21 \\
\hline 47 & 0.99 & 0.75 & 0.46 & 0.92 & 0.82 & 1 & 0.48 & 0.79 & 0.81 & 0.48 & 0.23 \\
\hline 245 & 0.49 & 0.69 & 0.83 & 0.57 & 0.34 & 0.48 & 1 & 0.37 & 0.67 & 0.83 & 0.51 \\
\hline 1149 & 0.78 & 0.58 & 0.39 & 0.73 & 0.90 & 0.79 & 0.37 & 1 & 0.62 & 0.41 & 0.22 \\
\hline 70 & 0.82 & 0.97 & 0.60 & 0.88 & 0.63 & 0.81 & 0.67 & 0.62 & 1 & 0.62 & 0.30 \\
\hline 76 & 0.49 & 0.64 & 0.98 & 0.57 & 0.36 & 0.48 & 0.83 & 0.41 & 0.62 & 1 & 0.54 \\
\hline 695 & 0.24 & 0.32 & 0.57 & 0.28 & 0.21 & 0.23 & 0.51 & 0.22 & 0.30 & 0.54 & 1 \\
\hline
\end{tabular}


Figure 82 graphically shows the generation output correlation between the Nebraska wind sites. The wind sites are identified along the $x$ axis. Site 22 has high correlation higher than $70 \%$ with 6 of the other ten wind sites. Each subsequent wind site moving to the right has one less site to compare since its correlation with each of the sites listed to the left has already been charted to the left. Note site 695 is the only site located in the southwestern part of the state and subsequently has lower correlation with the rest of the sites.

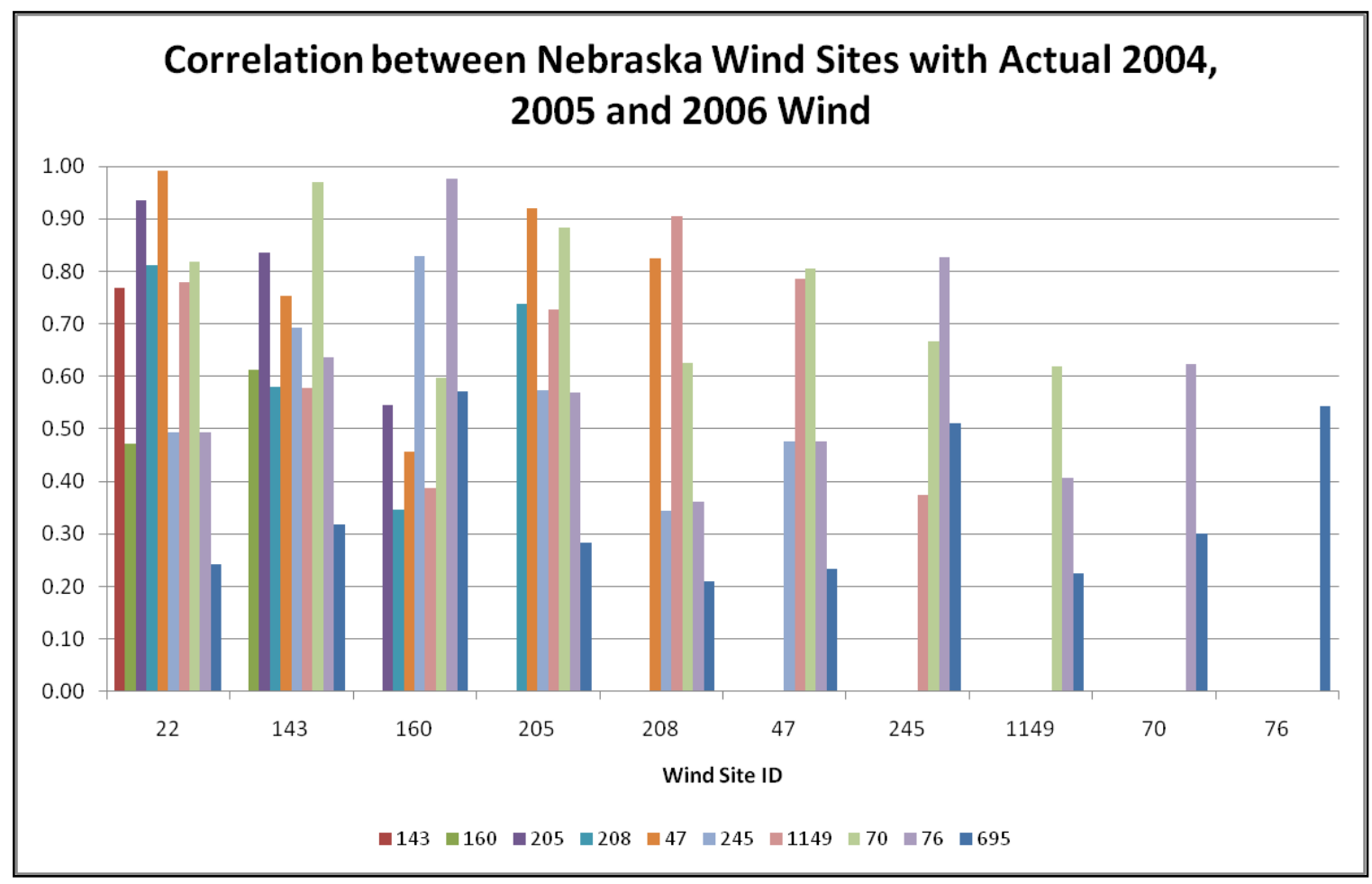

Figure 82: Correlation of Hourly Plant Output between Nebraska Wind Sites

\subsubsection{Mean Absolute Error of Wind Forecast}

The mean absolute error of the Nebraska day-ahead wind forecast was calculated for each penetration level for the Nebraska aggregated wind sites. Wind variability can have a large impact on dispatch decisions. Wind forecasts that turn out to be too high can cause an under commitment of resources and conversely low wind forecasts can result in over committing resources from an economic standpoint. Subsequently the accuracy of the wind forecast influences the costs of operations.

Two different analyses were performed. The first examined the accuracy of the day ahead forecast by comparing the forecast provided 18 hours before the next day's wind and compared this to the actual wind for the next day. It was found in this analysis that the mean absolute error for the day ahead forecast using all three study years and all three scenarios was about $11 \%$. Individual years and months show there are times when the error fluctuates between $9 \%$ and $14 \%$. In Figure 83 it can be observed looking at the annual bars that the mean absolute error decreases as wind penetration increases, although only slightly because much of this reduction has already been accomplished in the first $10 \%$ penetration. This error reduction 
results from the occasional offsetting of errors. Also the more windy months have somewhat greater mean absolute error values than less windy months for the day-ahead forecast.

When performing intra hour and near term dispatch and commitment decisions the persistence forecast can have the greatest accuracy. The second analysis examined the error of the persistence forecast using the actual wind in the current hour to predict the wind forecast for the next hour. This analysis showed a monthly variation from $3 \%$ to $6 \%$. It was also observed in Figure 84 that as wind penetration increased the accuracy of the persistence forecast increased (i.e., errors decreased). However, opposite to the day-ahead forecast, the mean absolute error of the persistence forecast has somewhat greater values in the less windy months (summer) than the more windy months. 


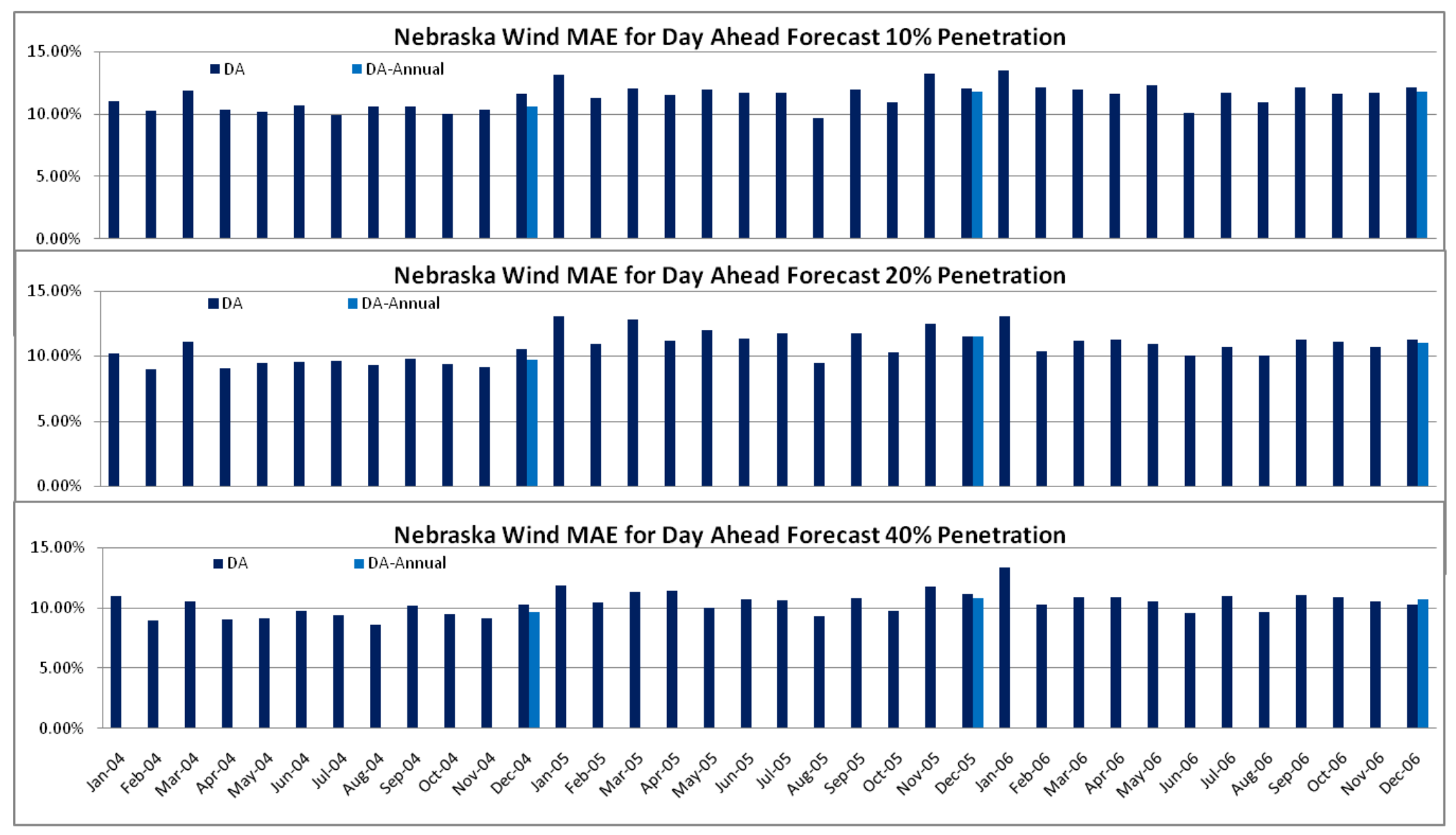

Figure 83: Nebraska Day Ahead Forecast Mean Absolute Error 


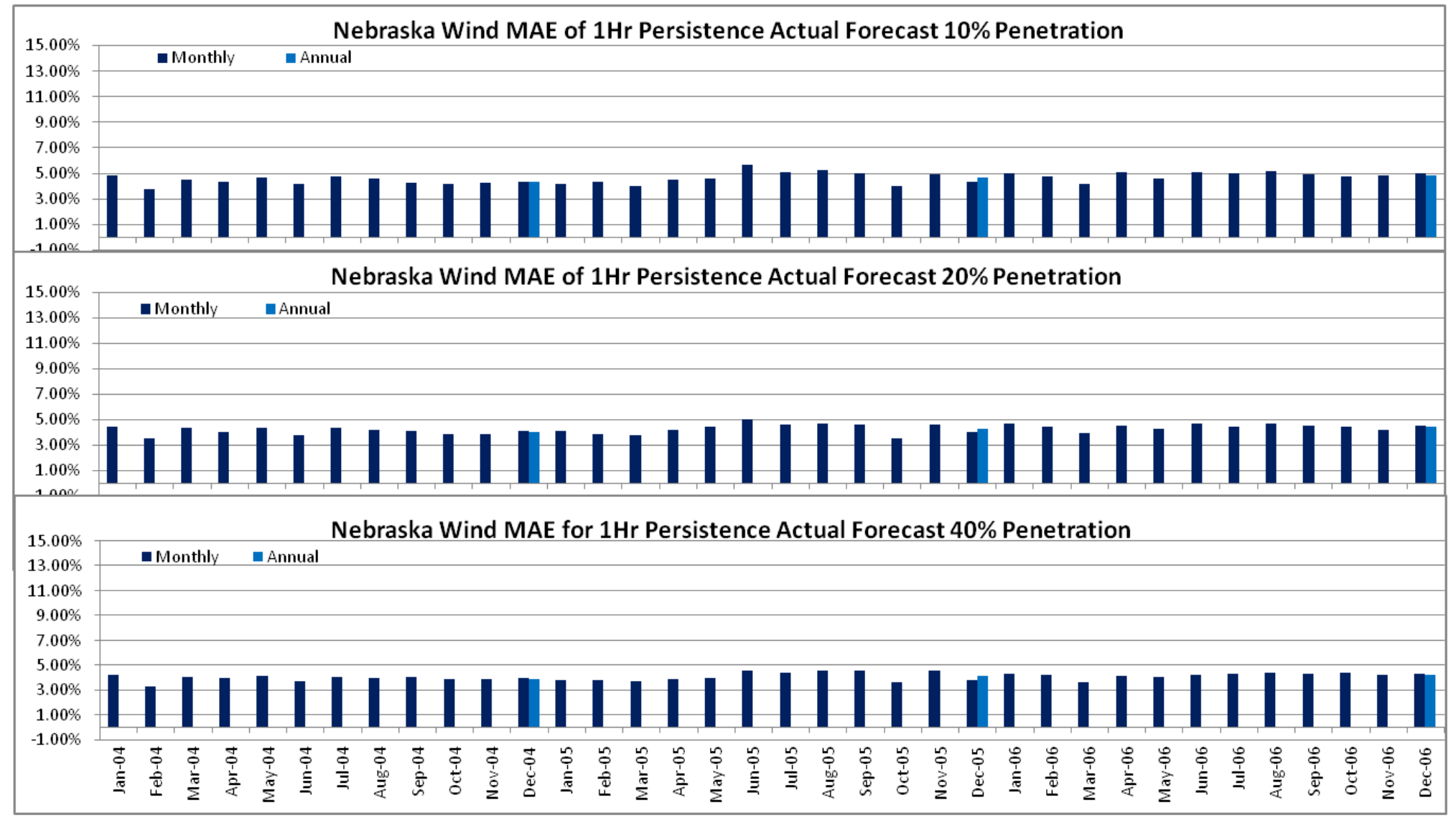

Figure 84: Nebraska 1 Hour Persistence Forecast Mean Absolute Error 


\subsubsection{Wind Ramp Analysis}

Additionally wind analysis was performed to study the impact of wind on morning ramp-up and evening ramp-down periods. The Nebraska load was examined and evaluated with wind effects for morning ramp-up in hours ending 5 to 11 and for night ramp-down from hour ending 21 to hour ending 3. During these hours operators tend to have to ramp generation to serve increasing or decreasing load. The 2018 Nebraska loads using the 2006 profile was examined with a $20 \%$ wind penetration. The maximum seasonal hourly changes are shown in Table 50 and Table 51. Additional ramp-up and ramp-down tables can be found in the document referenced in appendix Section 8.2 .

Table 50: Maximum Hourly Up-Ramp for Nebraska with 20\% Wind Penetration

\begin{tabular}{|c|c|c|c|c|c|c|c|c|c|c|c|c|c|c|}
\hline & \multicolumn{14}{|c|}{ Nebraska Maximum Hourly Ramp-up MW Change 2018 - 2006 Profile 20\% Wind } \\
\hline & \multicolumn{2}{|c|}{$\mathrm{Hr} 5$} & \multicolumn{2}{|c|}{$\mathrm{Hr} 6$} & \multicolumn{2}{|c|}{$\mathrm{Hr} 7$} & \multicolumn{2}{|c|}{$\mathrm{Hr} 8$} & \multicolumn{2}{|c|}{\begin{tabular}{|l|}
$\mathrm{Hr} 9$ \\
\end{tabular}} & \multicolumn{2}{|c|}{\begin{tabular}{|l|l|}
$\mathrm{Hr} 10$ \\
\end{tabular}} & \multicolumn{2}{|c|}{$\operatorname{Hr} 11$} \\
\hline & Load & $\begin{array}{l}\text { Load } \\
\text { Net } \\
\text { Wind }\end{array}$ & Load & $\begin{array}{l}\text { Load } \\
\text { Net } \\
\text { Wind }\end{array}$ & Load & $\begin{array}{l}\text { Load } \\
\text { Net } \\
\text { Wind }\end{array}$ & Load & $\begin{array}{l}\text { Load } \\
\text { Net } \\
\text { Wind }\end{array}$ & Load & $\begin{array}{l}\text { Load } \\
\text { Net } \\
\text { Wind }\end{array}$ & Load & $\begin{array}{l}\text { Load } \\
\text { Net } \\
\text { Wind }\end{array}$ & Load & $\begin{array}{l}\text { Load } \\
\text { Net } \\
\text { Wind }\end{array}$ \\
\hline Winter & 161 & 434 & 395 & 651 & 643 & 866 & 511 & 1113 & 196 & 398 & 195 & 473 & 113 & 322 \\
\hline Spring & 134 & 400 & 314 & 610 & 592 & 676 & 397 & 674 & 367 & 728 & 329 & 442 & 275 & 522 \\
\hline Summer & 87 & 344 & 405 & 434 & 632 & 741 & 436 & 886 & 474 & 801 & 502 & 696 & 442 & 941 \\
\hline Fall & 146 & 440 & 334 & 472 & 586 & 738 & 407 & 661 & 352 & 523 & 393 & 674 & 328 & 583 \\
\hline Year & 161 & 440 & 405 & 651 & 643 & 866 & 511 & 1113 & 474 & 801 & 502 & 696 & 442 & 941 \\
\hline
\end{tabular}

Table 51: Maximum Hourly Down-Ramp for Nebraska with 20\% Wind Penetration

\begin{tabular}{|c|c|c|c|c|c|c|c|c|c|c|c|c|c|c|}
\hline & \multicolumn{14}{|c|}{ NPA Maximum Hourly Ramp-down MW Change 2018 - 2006 Profile 20\% Wind } \\
\hline & \multicolumn{2}{|c|}{$\mathrm{Hr} 21$} & \multicolumn{2}{|c|}{$\operatorname{Hr} 22$} & \multicolumn{2}{|c|}{$\mathrm{Hr} 23$} & \multicolumn{2}{|c|}{\begin{tabular}{|l|}
$\mathrm{Hr} 24$ \\
\end{tabular}} & \multicolumn{2}{|c|}{\begin{tabular}{|l|}
$\mathrm{Hr} 1$ \\
\end{tabular}} & \multicolumn{2}{|c|}{\begin{tabular}{|l|l|}
$\mathrm{Hr} 2$ \\
\end{tabular}} & \multicolumn{2}{|c|}{$\mathrm{Hr} 3$} \\
\hline & Load & \begin{tabular}{|l} 
Load \\
Net \\
Wind
\end{tabular} & Load & $\begin{array}{l}\text { Load } \\
\text { Net } \\
\text { Wind }\end{array}$ & Load & $\begin{array}{l}\text { Load } \\
\text { Net } \\
\text { Wind }\end{array}$ & Load & $\begin{array}{l}\text { Load } \\
\text { Net } \\
\text { Wind }\end{array}$ & Load & $\begin{array}{l}\text { Load } \\
\text { Net } \\
\text { Wind }\end{array}$ & Load & $\begin{array}{l}\text { Load } \\
\text { Net } \\
\text { Wind }\end{array}$ & Load & $\begin{array}{l}\text { Load } \\
\text { Net } \\
\text { Wind } \\
\end{array}$ \\
\hline Winter & -460 & -551 & -250 & -674 & -420 & -927 & -481 & -900 & -522 & -652 & -186 & -455 & -158 & -319 \\
\hline Spring & -220 & -604 & -231 & -671 & -518 & -757 & -540 & -639 & -667 & -808 & -242 & -807 & -219 & -462 \\
\hline Summer & -302 & -706 & -212 & -878 & -502 & -843 & -620 & -913 & -488 & -799 & -383 & -547 & -288 & -436 \\
\hline Fall & -147 & -622 & -309 & -698 & -561 & -1008 & -508 & -948 & -718 & -777 & -308 & -464 & -186 & -561 \\
\hline Year & -460 & -706 & -309 & -878 & -561 & -1008 & -620 & -948 & -718 & -808 & -383 & -807 & -288 & -561 \\
\hline
\end{tabular}

Wind and load ramps can either counterbalance or compound the generation ramp requirement, depending on their relative directions of movement. At $20 \%$ penetration the maximum hourly generation ramp up requirement increases by $470 \mathrm{MW}$ due to the wind (from $643 \mathrm{MW}$ for load and 1,113 MW for load net wind), and changes timing. The maximum hourly ramp down requirement is a larger negative value by $-290 \mathrm{MW}$ due to the wind (from -718 MW for load and -1,008 MW for load net wind), and changes seasons.

In the production cost simulation results the integration costs associated with these hourly ramps would mostly not be captured in the base case comparison between the actual case and the shaped proxy ideal case. Since the shaped proxy case contains those same hourly ramps there is little wind ramping difference (except where forecast errors may change the ramp) 
between the actual and ideal case and thus no integration cost differential for that particular aspect. However, the daily block proxy or sub-period proxy will capture these effects to some extent in that the steam units were modeled in PROMOD with heat rate curves that vary with the unit's hourly output levels. See Section 4.4.4 for the results of sensitivities with those proxy cases.

\subsubsection{Wind Sites and Load}

To show the relationships between wind and load, analysis was performed on the aggregated wind sites by season for each scenario. Figure 85 shows load duration curves for Nebraska Load and the Nebraska Load net Wind for each wind penetration levels for the 2006 wind pattern. Note, the fixed line on the chart depicts the annual minimum load, the point where the blue line representing the 2006 load profile intersects at the far right. The minimum is one of 8760 hours and used in this chart as a visual aid to show the number of hours when the load net wind is less than the annual minimum. The chart provides an indication of an impact of the wind generation on operations, but it does not indicate the number of hours that load net wind is less than load. Figure 85 does show however that wind generation for $40 \%$ penetration exceeds Nebraska load approximately 600 hours of the year or $7 \%$ of the time (time below the zero line).

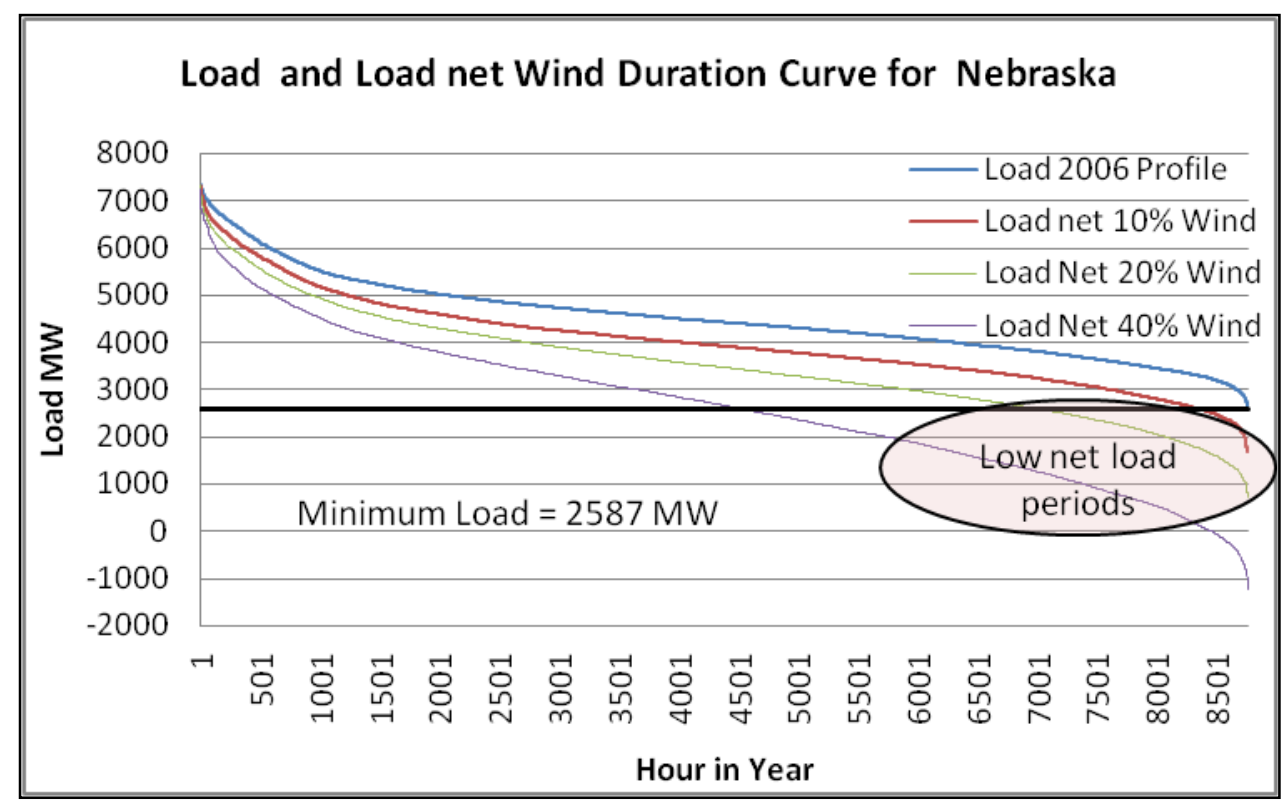

Figure 85: Nebraska Load Duration Curve for Load and Load net Wind

The relationship of wind and load for the $40 \%$ wind penetration case, Figure 86, shows the 2006 wind pattern's tendency to diminish in July and August while load in these months increases. It can also be observed that at other times during the year available wind can exceed load as was also shown in Figure 85. During such situations, PROMOD would first export energy if markets permit, reduce thermal generation as needed, and finally curtail wind generation if necessary. 


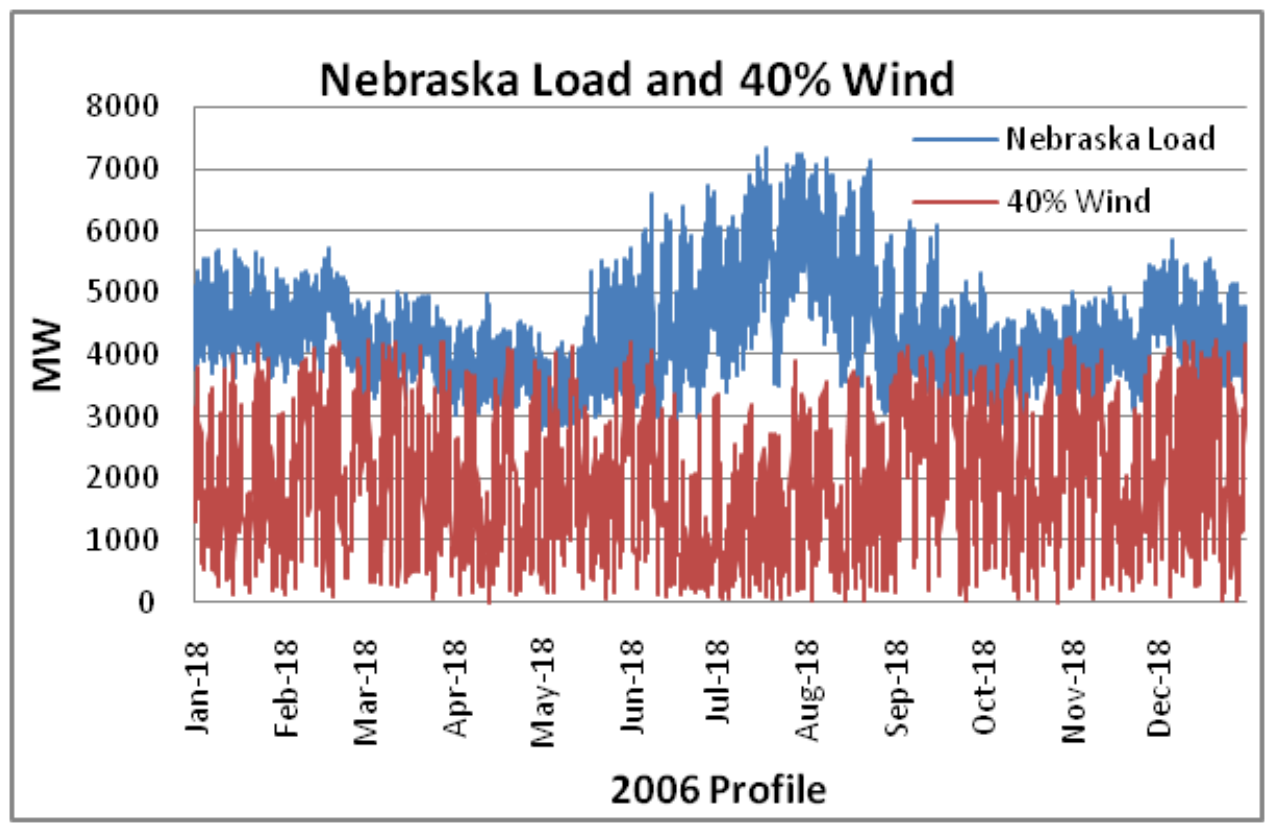

Figure 86: One Year of Nebraska Load and $40 \%$ Wind Penetration

Table 52 shows key data for each of the yearly profiles with $20 \%$ wind penetration for Nebraska. The impact of a $20 \%$ wind penetration on load is observed by examining the load net wind values. For this penetration the peak load net wind value twice remains in July but on a different day of the week and once shifts to August. However there is more impact on minimum load. The period for the annual minimum changes from May, in the 2004 and 2006 profile to September-October and from June in the 2005 profile to September.

Table 52: NPA Load and $20 \%$ Wind Penetration Summary

\begin{tabular}{|c|c|c|c|c|c|}
\hline \multirow[b]{2}{*}{ Load } & \multicolumn{5}{|c|}{ NPA Load 2018} \\
\hline & TWh & Peak MW & Date & Min MW & Date \\
\hline 2004 Profile & \multicolumn{2}{|c|}{$\begin{array}{ll}39.402 & 7,553\end{array}$} & $7 / 17 / 1815: 00$ & 2,441 & $5 / 27 / 183: 00$ \\
\hline 2005 Profile & \multicolumn{2}{|l|}{39.402} & $7 / 20 / 1815: 00$ & 2,654 & $6 / 10 / 187: 00$ \\
\hline \multirow[t]{2}{*}{2006 Profile } & 39.405 & 7,489 & $7 / 18 / 1815: 00$ & 2,475 & $5 / 6 / 184: 00$ \\
\hline & \multicolumn{5}{|c|}{ NPA 20\% Wind Penetration } \\
\hline Wind & TWh & Peak MW & Date & Min MW & Date \\
\hline 2004 Profile & 8.499 & 2,231 & $9 / 19 / 1818: 00$ & 0 & $10 / 26 / 1815: 00$ \\
\hline 2005 Profile & 8.946 & 2,322 & $11 / 27 / 1821: 00$ & 1 & $10 / 11 / 1812: 00$ \\
\hline \multirow[t]{2}{*}{2006 Profile } & 9.266 & 2,313 & $10 / 7 / 180: 00$ & 2 & $10 / 2 / 1815: 00$ \\
\hline & \multicolumn{5}{|c|}{ Load net Wind } \\
\hline Load net Wind & TWh & Peak MW & Date & Min MW & Date \\
\hline 2004 Profile & 30.903 & 7,490 & $7 / 18 / 1817: 00$ & 652 & $9 / 30 / 186: 00$ \\
\hline 2005 Profile & 30.456 & 7,315 & $8 / 1 / 1817: 00$ & 691 & $9 / 28 / 1815: 00$ \\
\hline 2006 Profile & 30.136 & 7,070 & $7 / 27 / 18$ 19:00 & 400 & $10 / 8 / 182: 00$ \\
\hline
\end{tabular}




\section{Section 5}

\section{TOTAL COST Estimation \& IMPLICATIONS}

In this section a general view of costs is presented that estimates the other costs that are not a part of the statistical and production analyses. For example, the cost of the wind generation, associated transmission, and other related costs. In addition adjustments are estimated to reflect $\mathrm{CO} 2$ regulation being a cap-and-trade system (PROMOD models $\mathrm{CO} 2$ cost as a tax) and Renewable Energy Credit (REC) pricing is considered. In this way, cases can be more completely compared to attempt to see more of the bigger picture.

Throughout the report dollars are given in 2018 nominal values unless otherwise noted.

\subsection{Caveats and Procedure}

As the methodology for total cost estimation is described here, it will become clear that the process to derive projected 2018 costs involves broad aggregations of utility expenses and therefore the results should only be used for indications and not be considered conclusive. Results depend directly on the assumptions that are made. This study has many assumptions for some large and very uncertain variables, such as fuel cost, $\mathrm{CO} 2$ regulation and pricing, wind generation and transmission costs, etc.

Not only are there uncertainty issues, but the total cost results depend on aspects that are not fully represented or analyzed. For example, the transmission overlay represented here has not been studied in depth as study scope and resources did not provide for such, so estimates were made. As particular clarification, the no-overlay case is not just the existing transmission system, but rather:

- $\quad$ some additional lines that do not exist today are modeled in it, and

- $\quad$ transmission limits in the area of wind injections were relaxed rather than doing the transmission planning work to determine what is required to accept the injections.

What has been done is to include some estimated costs for local transmission needs for both the no-overlay and overlay cases without determining those lines specifically, and include costs as well as for any specific lines, including the overlay lines. The transmission overlay is somewhat coordinated with other studies, but is considered only as a sample plan. Bottom line, the transmission line estimations are not to be concluded as necessary or sufficient, but perhaps as a start to such a look.

The wind generation siting is also a sample plan that is designed to study dispersed wind sites as well as having good energy production capability, while being somewhat coordinated with other studies. 
The cost estimation procedure considers all of SPP including Nebraska, because of the sharedreserve modeling, and can be thought of in a step-wise manner as follows:

- $\quad$ A reference 2018 total cost is estimated by escalating an assumption for 2007 state-specific revenues from data reported to the US DOE. For some states in SPP, the fraction of the statewide total needed to be estimated. This reference revenue requirement is assumed to include all existing and new generation, transmission, and distribution costs to serve load with existing wind generation, but with no $\mathrm{CO} 2$ regulation (cost) or federal Renewable Energy Standard or significant REC pricing.

- $\quad$ An incremental wind purchase cost is estimated for the increment of wind generation that is added above the existing amount now installed in the SPP.

- $\quad$ An incremental transmission cost is estimated for the increment of transmission line and substation facilities that will be needed as wind generation is added. This is intended to include local transmission development to handle the wind injections and to provide for the overlay as identified in the scenario description. As noted above, some new lines are already assumed to be present in all cases, even without these "incremental" lines.

- A cost of $\mathrm{CO} 2$ emissions is included at various pricing levels depending on the scenario description. These are already included in the PROMOD-calculated production costs as a tax (including a cost for each $\mathrm{CO} 2$ ton emitted). Then for a cap-and-trade scenario, the $\mathrm{CO} 2$ costs included by PROMOD are backed out of the production cost for the estimated free allowances. The cap-and trade estimates are patterned after the proposed Waxman-Markey bill. Alternative total cost results are presented for both cap-and-trade and tax regulation scenarios.

- $\quad$ A price received/paid for Renewable Energy Credits (RECs) that are in excess/short under a federal Renewable Energy Standard (RES) contained within the Waxman-Markey bill is used to estimate REC impacts. Alternative total cost results are presented for both a zero REC price and a non-zero REC price given the uncertainties involved.

- $\quad$ Essentially, the total cost estimate for a case then becomes:

the reference cost

plus incremental wind cost

plus incremental transmission cost

minus the delta on PROMOD adjusted production cost (to convert from tax to cap-and trade, if done for that case)

plus/minus the cost/value to purchase/sell RECs that are short/in excess of the RES requirement, if the REC price is non-zero. 


\section{2. $\quad$ ASSUMPTIONS AND RESULTS}

It is recognized that many of the assumptions that were taken for calculating total cost have large impacts and yet are quite uncertain. Here is a list of key assumptions that were used:

- $\quad$ Projected system conditions and 2018 dollars as described in Section 2.5.6.

- $\quad$ Existing wind generation in SPP including Nebraska for the reference case is 3,140 MW, a $4.3 \%$ penetration.

- Incremental wind generation can be purchased on site for $\$ 50 / \mathrm{MWh}$ in 2009, which is then escalated to 2018 at $3 \%$ per year.

- Incremental local transmission cost is $5 \%$ of the incremental wind generation cost.

- $\quad$ The "base overlay" consists of 230 miles of $345 \mathrm{kV}$ line at $\$ 1.10$ million per mile in 2008 plus 2,930 miles of $765 \mathrm{kV}$ line at $\$ 2.17$ million in 2008 , both escalated at $3 \% / \mathrm{yr}$ to 2018.

- $\quad$ The "Neb $345 \mathrm{kV}$ Overlay" consists of 840 miles of $345 \mathrm{kV}$ line at $\$ 1.10$ million per mile in 2008 plus 2,020 miles of $765 \mathrm{kV}$ line at $\$ 2.17$ million in 2008 , both escalated at $3 \% / y r$ to 2018.

- $\quad$ Substation costs are $12 \%$ in addition to line costs above.

- Annual carrying charge rate is $8 \%$.

- $\quad$ Adjusted production costs are taken from PROMOD runs for 2006 wind/load patterns applied to year 2018 (these PROMOD results include the CO2 price on all emitted tons, i.e., treating $\mathrm{CO} 2$ price as a tax).

- Depending on the case, the $\mathrm{CO} 2$ price assumption is set at $\$ 0 /$ short ton, $\$ 25 /$ short ton, $\$ 50 /$ short ton, or "120/50" (meaning that PROMOD calculates using $\$ 120 /$ short ton, but in post processing that price is adjusted down to $\$ 50)$.

- $\quad$ CO2 cost is converted (reduced) to a cap-and-trade program using APPA's summer 2009 interpretation of the Waxman-Markey HR2454 bill as applied by NPA to SPP historical emission characteristics, which yielded 117.70 million short tons of free allowances for year 2018. For the tax scenario no adjustment is generally necessary.

- In two situations, adjustments to $\mathrm{CO} 2$ cost were made to change the $\mathrm{CO} 2$ price while keeping the same dispatch in determining (1) the delta between existing wind $\mathrm{w} /$ and $\mathrm{w} / \mathrm{o} \mathrm{CO} 2$ cost and (2) for the $\mathrm{CO} 2$ reduction case where PROMOD dispatched at $\$ 120 /$ ton but total cost is figured at $\$ 50 /$ ton. For these situations, the average price premium achieved for net exports was assumed at $75 \%$ of the cost of typical coal emission rate of one ton per MWh.

- $\quad$ There is a "good case" to be made that the relevant REC price for this study should be zero because:

- $\quad$ The model already has two big incentives for renewable generation in (a) the approximate $\$ 25 / \mathrm{MWh}$ production tax credit assumed for 2018 , 
as part of the wind generation cost and in (b) the base assumption of $\$ 25 /$ short ton of $\mathrm{CO} 2$ cost.

- It may be that as a result of instituting a federal RES, the production tax credit would be removed by 2018 .

- $\quad$ This thinking would say that either a REC market price, or a production tax credit exists once the $\mathrm{CO} 2$ regulation is set up, but not both.

- $\quad$ For completeness, total cost estimates are also provided for the assumption that a REC price of $\$ 15 / \mathrm{MWh}$ is present along with the other two incentives of production tax credit and $\mathrm{CO} 2$ pricing. The Renewable Energy Standard estimated for 2018 is $12.4 \%$ derived from the assumption that, under WaxmanMarkey, the RES goes from 6\% in 2012 to $20 \%$ in 2020, that it is linear between the two dates, and that $25 \%$ of the requirement is covered with efficiency and the rest is all renewable wind generation.

Appendix 8.4 contains the total cost estimate results for the fifteen primary cases and displays the results in three different forms (four bar charts with tables, four tables, and a single bar chart of percentage comparisons to the reference) for the four combinations of:

- $\quad$ CO2 Cap-and-Trade, REC $=\$ 15 / \mathrm{MWh}$

- $\quad$ CO2 Cap-and-Trade, REC $=\$ 0 / M W h$

- $\quad$ CO2 Tax, REC $=\$ 15 / \mathrm{MWh}$

- $\quad \mathrm{CO} 2 \mathrm{Tax}, \mathrm{REC}=\$ 0 / \mathrm{MWh}$

The bars on the first four bar charts and the data in the tables at the bottom of the charts are the same information. For each case, the four cost categories of Incremental wind generation; incremental transmission, CO2 Cost, and Other (including REC) are displayed. Separating out the REC component on the graph does not work well because for some cases it is a positive value and some a negative value. However, the REC value that is included into the other category does show up as a separate column on the other tables.

The primary purpose of the next four tables is to show the additional revenue requirement (above the reference amount) and then to translate this into percentage increases from the reference. Other columns on the table display penetration percentage, REC cost, CO2 price, CO2 emission tonnage, and SPP exports in millions of MWh (or TWh).

Finally the last bar chart in Appendix 8.4,also shown here as Figure 87, displays the percentage increase in total cost for all the fifteen primary cases in all the four combinations for $\mathrm{CO} 2$ regulation and REC pricing. These cases (bars) are sorted first by wind penetration level, then by $\mathrm{CO} 2$ price, then by percentage cost increase. Note that for the assumptions used and the cases estimated, the total cost increases above that for the reference case range up to $45 \%$. This result is for the case with $20 \%$ penetration (with overlay), $\mathrm{CO} 2$ regulation as a tax of $\$ 50 /$ short ton with coal generation reduced by running more gas generation (by dispatching with $\mathrm{CO} 2$ at $\$ 120 /$ short ton), and REC price set to zero. 


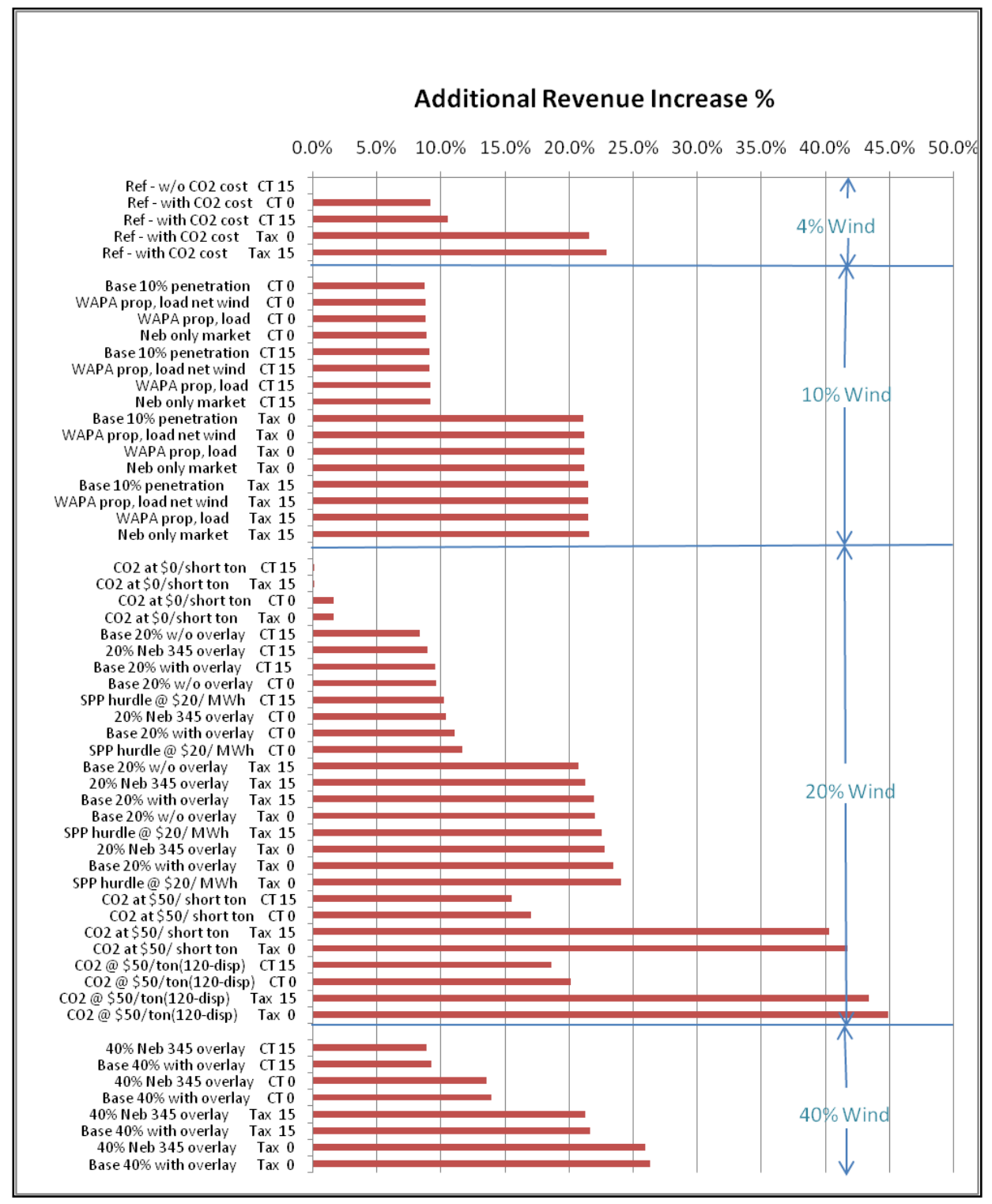

Figure 87: Additional Revenue Increase \%

\subsection{IMPLICATIONS}

The primary results of the total cost estimation process for SPP including Nebraska are shown in Figure 88, which shows in bar chart and tabular form four cost categories for fifteen primary cases run on the 2006 wind and load patterns: incremental wind generation cost (cost above existing wind), incremental transmission, $\mathrm{CO} 2$ prices, and other costs (production and other fixed costs). 


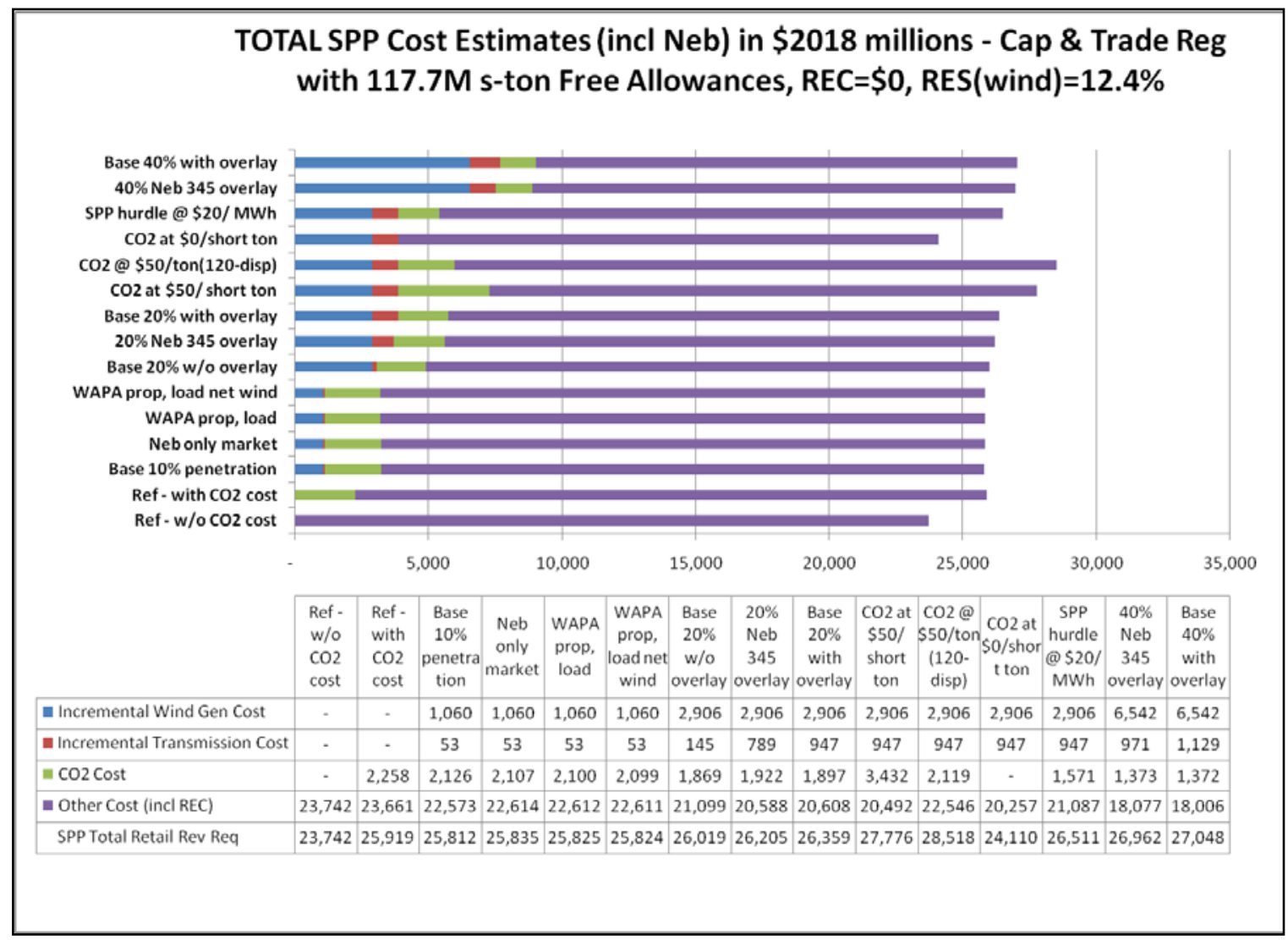

Figure 88: Total SPP Cost Estimates (including Nebraska) in \$2018 millions - Cap \& Trade Reg with 117.7M s-ton Free Allowances, REC $=\$ 0, \operatorname{RES}($ wind) $=12.4 \%$

Examining the full set of charts and tables of Total Cost Estimates (annual amounts in 2018\$) in Appendix 8.4 some implications can be observed, albeit recognizing all the caveats noted above:

- $\quad \$ 25 /$ short ton $\mathrm{CO} 2$ price for cap-and-trade adds approximately $9 \%$ (or $\$ 2.2$ billion) to the total cost reference which is estimated at $\$ 23.7$ billion for existing wind and no CO2 cost or REC pricing in 2018 (Table 99). Under a tax regulation, that increase becomes approximately $22 \%$ (Table 101).

- $\quad$ Each $10 \%$ penetration (7,540 MW) adds approximately $1.7 \%$ to the total cost reference when the $\mathrm{CO} 2$ cap-and-trade price is $\$ 25 /$ short ton, and the REC price is zero (comparing $10 \%$ and $40 \%$ bases, Table $99[(13.9 \%-8.7 \%) / 3]$ ). In this case if the REC price is $\$ 15 / \mathrm{MWh}$ the cost increase from a $10 \%$ penetration increase is only about $0.1 \%$, Table $98[(9.22 \%-9.05 \%) / 3]$.

- $\quad$ Each $1 \%$ increase in revenue requirement equates to about $\$ 237$ million per year (Table 99; 23,742*1\%).

- It appears that fossil exports will need to be reduced to keep CO2 emissions under the rough estimate cap of 156.6 million short tons. The case with the lowest CO2 emissions (160.1 million short tons) is slightly above the cap but SPP 
is still making 28.5 TWh of exports, of which some of the fossil-based export production could be reduced (Table 99).

- $\quad$ Only the CO2 reduction case ('120/50') has emissions that approach the cap by substituting gas for coal generation. Note the $\$ 1.4 \mathrm{~B}$ increase going from $\$ 25$ to $\$ 50 /$ short ton $[(4,035-2,617) / 1000)$, then an additional \$0.7B from the switch to gas $[(4,777-4,035) / 1000]$ (while exports are relatively steady at 27-28 TWh). However, this case is always the most costly. (Table 99)

- $\quad$ This bullet discusses whether the investment in wind generation and transmission yields equal or more benefits from reduced $\mathrm{CO} 2$ and other costs (production) in the first year for selected cases, assuming the $\mathrm{CO} 2$ price is \$25/short ton.

- If the REC price is zero, the first year benefit-to-cost ratio is 1.10 comparing $10 \%$ wind to existing wind and is 0.81 comparing $40 \%$ wind to $10 \%$ (Figure 88 or Figure 99 ), determined as follows:

$$
\begin{aligned}
& 1.10=[(2,177-2,126)+(23,742-22,573)] /[(1,060-0)+(53-0)] \\
& 0.81=[(2,126-1,372)+(22,573-18,006)] /[(6,542-1,060)+(1,129-53)]
\end{aligned}
$$

- If the REC price is $\$ 15 / \mathrm{MWh}$, the first year benefit-to-cost ratio is 1.31 comparing $10 \%$ wind to existing wind and is 0.99 comparing $40 \%$ wind to $10 \%$ (Figure 98), determined as follows:

$$
\begin{aligned}
& 1.31=[(2,177-2,126)+(24,057-22,651)] /[(1,060-0)+(53-0)] \\
& 0.99=[(2,126-1,372)+(22,651-16,888)] /[(6,542-1,060)+(1,129-53)] .
\end{aligned}
$$

- $\quad$ These $\mathrm{B} / \mathrm{C}$ estimates apply whether the $\mathrm{CO} 2$ regulation is cap-and-trade or a tax (using the same method applied to Figure 100 and Figure 101).

- The 345 kV Nebraska overlay appears to save \$153 - \$154 million annually over the $765 \mathrm{kV}$ overlay at $20 \%$ wind penetration $(2,264-2110)$ and $\$ 86$ million annually at $40 \%$ penetration $(2,189-2,103)$, regardless of the REC price. These estimates apply whether the $\mathrm{CO} 2$ cost of $\$ 25 /$ short ton is regulated as cap-andtrade or as a tax. (Table 98 - Table 101) As a reminder, neither transmission overlay plan is optimized. For example, the full $765 \mathrm{kV}$ (base) overlay has 300 miles more transmission than does the $345 \mathrm{kV}$ Nebraska overlay plan. Estimating the study costs associated with 300 miles of line using the assumptions in Section 5.2, yields $\$ 40$ million annually to add to the $345 \mathrm{kV}$ plan or $\$ 78$ million annually to subtract from the $765 \mathrm{kV}$ plan, depending on which equivalent is considered most appropriate. Either way such an adjustment would lower the estimated "savings" associated with the $345 \mathrm{kV}$ overlay.

The $765 \mathrm{kV}$ overlay initially studied for the $20 \%$ and $40 \%$ penetration level might not be required in Nebraska for these penetrations, and a $3000 \mathrm{amp} 345 \mathrm{kV}$ system may be satisfactory. More complete analytical work on system design and economics, including range of future scenarios, is certainly indicated for both options before reaching conclusions on the exact transmission upgrades required. For example, even higher wind penetrations for Nebraska are being 
modeled in some external studies. These decisions need to consider many additional factors such as dynamic system performance, land use issues, habitat fragmentation, and corridor fatigue.

- If the REC price is zero in the $20 \%$ wind penetration scenario, the $765 \mathrm{kV}$ overlay appears to add a net $\$ 340$ million cost annually $(2,617.5-2,277.6)$ (compared to the no-overlay case) even though it allows 4.0 TWh of additional SPP exports. However, if the REC price is $\$ 15 / \mathrm{MWh}$, then the added net cost from the $765 \mathrm{kV}$ overlay is reduced to $\$ 289$ million annually $(2,263.7-1,974.4)$. These estimates apply whether the CO2 cost of $\$ 25 /$ short ton is regulated as cap-and-trade or as a tax. (Table 98 - Table 101) As noted above, if one were to equivalence the mileages, these added costs would each be reduced by $\$ 40-\$ 78$ million annually.

- However, this analysis should not be considered as a complete transmission analysis from either the operating or economic perspectives.

- $\quad$ At the $10 \%$ penetration level, operating Nebraska as a standalone market (rather than as a part of the SPP market) raises the SPP-wide cost by $\$ 22$ million $(2,171-2,149)$ for either REC price or either $\mathrm{CO} 2$ regulation with price at $\$ 25 /$ short ton; however, this is not considered to be a comprehensive market analysis. (Table 98 - Table 101)

- With REC price at zero and under cap-and-trade, there is a bigger jump in cost $(9.5 \%)(=11.02 \%$ - $1.55 \%)$ from $\$ 0$ to $\$ 25 /$ ton than from $\$ 25 /$ ton to $\$ 50 /$ ton $(6.0 \%)(=17.0 \%-11.0 \%$. Likely due to the loss of $13.5 \mathrm{TWh}$ exports in going from $\$ 0$ to $\$ 25 /$ ton. (Table 99)

- $\quad$ Assuming the same case dispatches for the $20 \%$ penetration case, and the same $\mathrm{CO} 2$ prices, the total costs for SPP under a tax regulation for $\mathrm{CO} 2$ (rather than cap-and-trade), are approximately $\$ 3$ billion higher [Table 99 and Table 101; $(5,560-2,617) / 1000]$ at $\$ 25 /$ short ton tax for CO2 emissions and $\$ 6$ billion higher [Table 99 and Table 101; $(9,920-4,035) / 1000]$ at $\$ 50 /$ short ton tax. This is true regardless of the REC price.

In conclusion, Section 5 includes many detailed examinations that are supplementary findings that sometimes involve billions of dollars between comparative cases rather than the millions between an actual wind case and an ideal wind case that defines integration costs. Both views are important. In integration cost only the adjusted production cost and wind generation values are used. As part of the total cost, the installation and operating cost of facilities for wind generation and transmission are also examined.

Even under a cap-and-trade regulation for $\mathrm{CO} 2$, where a considerable number of emission allowances are evaluated as free, the remaining allowances cost more than $20 \%$ wind energy if the $\mathrm{CO} 2$ price gets to $\$ 50 /$ short ton as shown in Figure 88 . The highest cost adder for any of the cases modeled is $45 \%$ as shown in Figure 87. 


\section{Section 6}

\section{Western Nebraska Wind - Western INTERCONNECTION: A BRIEF REVIEW}

\subsection{EXECUTIVE SUMMARY - WESTERN INTERCONNECTION}

Nebraska is separated electrically into the Eastern and Western Interconnections, with nearly all being in the Eastern Interconnection. The Nebraska Power Association (NPA) - National Renewable Energy Laboratory (NREL) Study Technical Review Committee (TRC) concluded that the wind integration study should focus on that portion of the state of Nebraska that lies in the Eastern Interconnection. The TRC developed a secondary requirement that the report include a technical analysis of transmission availability on the Western Area Power Administration (WAPA) transmission system in the part of Nebraska in the Western Interconnection.

The TRC identified a site in western Nebraska and asked WAPA to study the feasibility of injecting up to $300 \mathrm{MW}$ of wind capacity into the WAPA system. WAPA determined that the best place to interconnect wind would be to either its Archer-Stegall 115-kV Transmission Line or its Archer-Stegall 230-kV Transmission Line. WAPA performed abbreviated power flow studies and did not perform stability or interconnection facility type studies. The results provide a general sense of the potential capability of the system in that area in the years 2012 and 2018.

WAPA concluded that in 2012, it would be possible to interconnect up to $50 \mathrm{MW}$ of wind generation without requiring significant system upgrades, although the connecting line and substation would be required. To interconnect $300 \mathrm{MW}$ of wind generation, upgrades ranging between $\$ 6$ and $\$ 16$ million would be required depending on the configuration.

By 2018, WAPA estimates that there will be no available capacity without system upgrades. To inject $300 \mathrm{MW}$ of wind in 2018 would require additional upgrades of $\$ 6$ to $\$ 10$ million, plus interconnection costs. Parties interested in pursuing an interconnection in western Nebraska would need to conduct more detailed studies to refine the cost of an interconnection.

Integration costs in western Nebraska were not analyzed by the TRC or WAPA in this report. However, there are reference materials available that provide indicative costs for wind integration in western Nebraska.

\subsection{BACKGROUND- WESTERN INTERCONNECTION}

The electric grid in the United States and Canada is electrically isolated into three distinct interconnections: The Eastern Interconnection, the Western Interconnection and the Electric Reliability Council of Texas. The only connections between these grids are back-to-back AC-DCAC ties. There are two such ties located in western Nebraska: the David A. Hamil Tie, in Stegall 
and the Virginia Smith Converter Station in Sidney. Tri-State Generation and Transmission Association owns the Hamil Tie and WAPA own the DC tie in Sidney.

The electrical separation of the grid is essential to maintain system reliability. The electrical characteristics of the Eastern and Western Interconnections are slightly different resulting, in the 1970s and early 1980s, in system instability that caused transmission lines to instantaneously overload and go out of service. Back-to-back AC-DC-AC ties were installed as measures to allow power to flow between the interconnections without jeopardizing system reliability. The DC Ties have limited capability to move power freely between interconnections.

This separation has an additional drawback that affects integration of renewable resources onto the grid. DC ties are rarely, if ever, allowed to instantaneously change output levels in response to changing conditions. They are operated at set transfer levels for an entire hour. That means that resources in one interconnection cannot effectively support resources in the other interconnection.

Western Nebraska is a very low load area, meaning that large amounts of wind generation will require transmission capability to "export" power out of the area to load centers. Going either to the east or to the west, there is limited transmission capability due to commitments already in place for other resources.

As a result of these limitations on system configuration, it was not practical to perform a statewide integration study that included the Western Interconnection. An integration study for resources in the Western Interconnection in Nebraska would require the involvement of utilities in Colorado and Wyoming. The portion of western Nebraska in the Western Interconnection is part of a Balancing Area, or Balancing Authority, commonly referred to as Western Area Colorado-Missouri (WACM). WACM is operated by WAPA. A Balancing Area matches generation to load within a given area bounded by an agreed-to set of metering. WACM provides this service in the Western Interconnection for large portion of Wyoming and Colorado and western Nebraska. Public Service Company of Colorado and PacifiCorp are other major Balancing Areas adjacent to WACM.

These hurdles incorporating western Nebraska directly into this study are further described below in Section 6.4.2. As an alternative to performing an integration study for western Nebraska, the TRC asked WAPA to perform a transmission study in western Nebraska. This study was completed by WAPA's Rocky Mountain Region.

\subsection{ASSUMPTIONS - WESTERN INTERCONNECTION}

\subsection{1. $\quad$ NPA asked WAPA to perform two primary studies:}

- $\quad$ Identify how much Nebraska wind generation could be injected into the Western Interconnection transmission system without requiring transmission system upgrades.

- $\quad$ Determine what transmission system upgrades would be required to add 300 MW of wind generation in western Nebraska in the Western Interconnection.

The rationale for choosing the minimum level of input without upgrades was to provide a threshold level for adding wind generation to the system. The $300 \mathrm{MW}$ level was determined by 
looking at various scenarios for delivering wind energy to load. One possible scenario is to export wind into the Eastern Interconnection across the two DC ties. The maximum capability of the ties is approximately $300 \mathrm{MW}$. (This actual scenario was not studied by WAPA, because WAPA does not have adequate transmission facilities in the Eastern Interconnection and because the TRC anticipated that the upgrade costs would be significantly higher than can be justified to move the power to the east.)

The TRC identified a site in western Nebraska that was initially identified as a prime location for wind development. This site was used by WAPA in its analysis. (See Figure 89, site identified as "Proposed Wind Site".) The final NPA report identifies a $660 \mathrm{MW}$ site that is approximately 40 miles to the southeast (east of Kimball) that is included in the $40 \%$ penetration scenario and would require new transmission lines from there to Sidney to connect to the Eastern Interconnection.

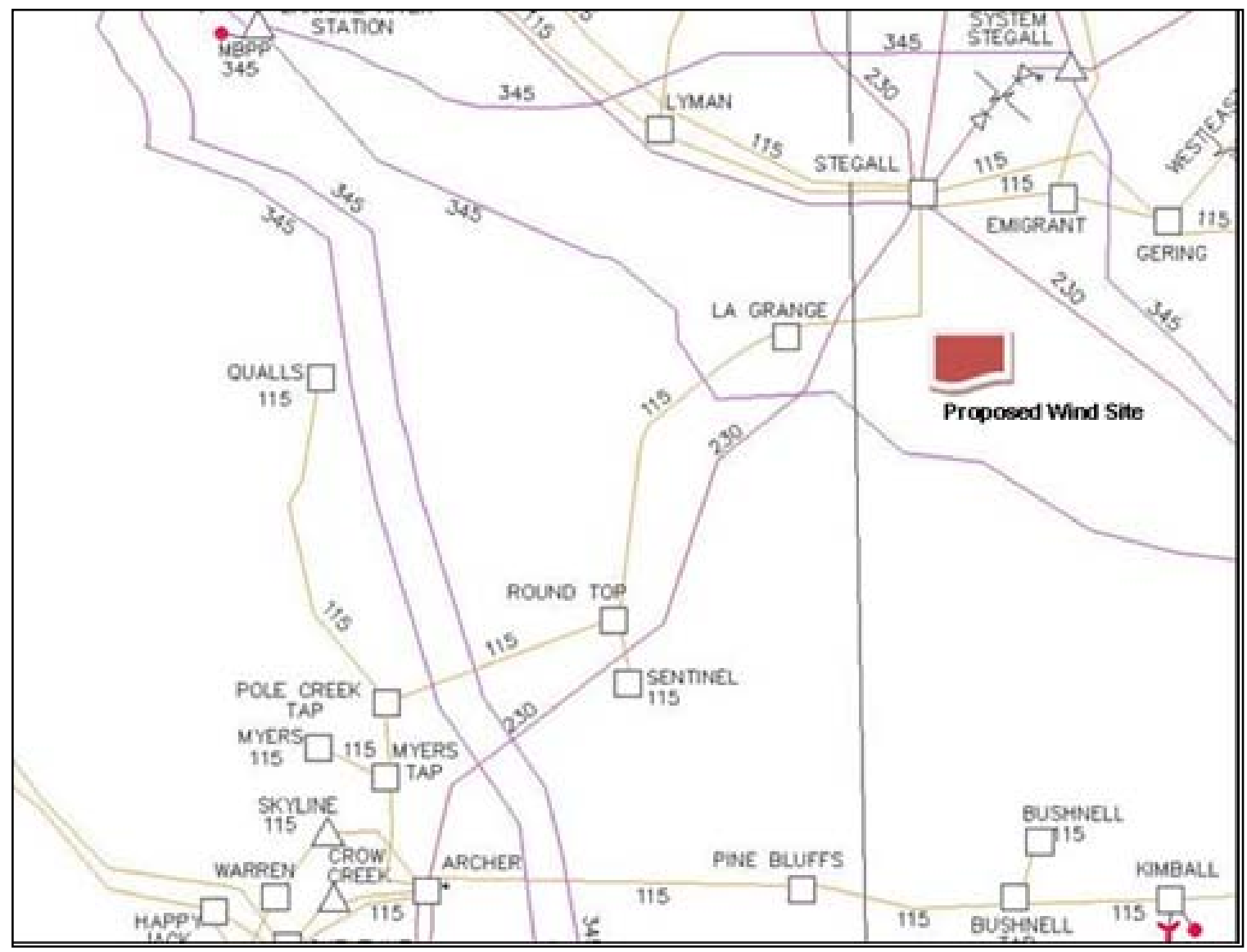

Figure 89: WAPA Proposed Wind Site in Nebraska

\subsubsection{Other Assumptions - Western Interconnection}

- $\quad$ Study years are 2012 and 2018

- $\quad$ To minimize extensive transmission upgrades in Colorado, generation in Wyoming was reduced to accommodate the addition of wind generation in 
Nebraska. (Exports from western Nebraska and Wyoming into Colorado are already limited and would result in extensive system upgrades.)

- $\quad$ The following transmission additions were assumed in the 2018 case:

- $\quad$ Archer - Laramie River 345-kV transmission line

- $\quad$ Archer - Story 345-kV transmission line

- $\quad$ Addition of PacifiCorp's Windstar 230-kV Substation

- $\quad$ Addition of Miracle Mile - Windstar 230-kV transmission line

- $\quad$ Addition of Dave Johnston - Windstar 230-kV transmission line.

- $\quad$ WAPA would evaluate interconnecting the proposed wind generation with either WAPA's Archer-Stegall 115-kV line or WAPA's Archer -Stegall 230-kV line.

\subsection{STUDY CONCLUSIONS- WESTERN INTERCONNECTION:}

In 2012, based upon initial power flow studies, it may be possible to interconnect $50 \mathrm{MW}$ of wind generation onto WAPA's Archer-Stegall 115-kV transmission line without any additional upgrades, provided the in-service date is 2012 . Injection of the full $300 \mathrm{MW}$ will require upgrades as outlined below.

In 2018, based upon initial power flow studies, it is not possible to interconnect additional wind generation without significant upgrades as described below.

The results displayed here are very preliminary. More detailed analysis of system stability and other factors, such as location of buyer, type and size of wind turbines, and other potential interconnection requests in WAPA's queue, will be required to determine actual system upgrades and construction costs.

\subsubsection{Cost Estimate for Upgrades (Cost estimates given are in 2008 dollars)}

Further case specific analysis will be required to determine final upgrade costs).

\subsubsection{2012 Base Case (for $300 \mathrm{MW}$ injection)}

Table 53: $\quad$ Archer - Stegall 115-kV

\begin{tabular}{|l|l|l|l|l|}
\hline Line Segment & Conductor Type & Distance (Miles) & Cost per mile & Total Cost \\
\hline Archer-Stegall 115-kV & 954 ACSR & 61 & $\$ 97,934$ & $\$ 5,973,976$ \\
\hline & & & Total Cost & $\$ 5,973,976$ \\
\hline
\end{tabular}

Table 54: Archer-Stegall 230-kV

\begin{tabular}{|l|l|l|l|l|}
\hline Overloaded Element & Equipment Type & $\begin{array}{l}\text { Distance } \\
\text { (Miles) }\end{array}$ & Cost per mile & Total Cost \\
\hline Archer-Stegall 230-kV & 1272 ACSS & 40 & $\$ 329,070$ & $\$ 13,162,824$ \\
\hline
\end{tabular}




\begin{tabular}{|l|l|l|l|l|}
\hline Archer 115/230 XFMR & 250 MVA & & & $\$ 2,962,440$ \\
\hline & & & Total Cost & $\$ 16,125,264$ \\
\hline
\end{tabular}

6.4.1.2. 2018 Base Case (for 300 MW injection)

Table 55: Archer-Stegall 115-kV

\begin{tabular}{|l|l|l|l|l|}
\hline Overloaded Element & Conductor Type & Distance (Miles) & Cost per mile & Total Cost \\
\hline Archer-Stegall 115-kV & 795 ACSR & 61 & $\$ 95,304$ & $\$ 5,836,453$ \\
\hline & & & Total Cost & $\$ 5,836,453$ \\
\hline
\end{tabular}

Table 56: Archer-Stegall 230-kV

\begin{tabular}{|l|l|l|l|l|}
\hline Line Segment & Equipment Type & Distance (Miles) & Cost per mile & Total Cost \\
\hline Sidney-Sterling 115-kV & 477 ACSR & 39.24 & $\$ 168,000$ & $\$ 6,592,320$ \\
\hline Sidney 115/230 XFMR & $200 \mathrm{MVA}$ & & & $\$ 2,424,000$ \\
\hline Capacitor at Ft. Morgan & $30 \mathrm{MVAR}$ & & & $\$ 356,918$ \\
\hline Capacitor at Sidney & $15 \mathrm{MVAR}$ & & & $\$ 178,459$ \\
\hline Capacitor at Laramie & $15 \mathrm{MVAR}$ & & & $\$ 178,459$ \\
\hline & & & Total Cost & $\$ 9,730,153$ \\
\hline
\end{tabular}

\subsubsection{Wind Integration for Western Nebraska}

Wind integration costs were not addressed in the WAPA transmission study. As studied, the wind generation analyzed in the transmission study lies within WAPA's WACM balancing area. WAPA has not performed a detailed wind integration study within the WACM boundaries. One of the primary reasons that it has not performed such a study is that, unlike most other balancing areas, there is not a single primary generation owner or load serving entity. Any wind integration study would require extensive collaboration among a wide range of utilities and might result in cost shifts among those companies. Wind generators interconnecting within WACM will be required to pay ancillary service charges under WAPA's Open Access Transmission Tariff.

Excel Energy's subsidiary, Public Service Company of Colorado (PSCo), operates a balancing area that is interconnected with WACM. In July 2009, PSCo completed a wind integration study in its area. This study attempted to quantify three categories of integration costs: regulation, system operations (opportunity costs, higher production costs due to less-than optimal operations, etc), and gas supply. The study did not quantify any additional integration costs associated with curtailment of wind generation, electricity trading inefficiencies introduced by wind uncertainty, or increased O\&M costs at existing thermal units that may be called upon more often to ramp output over a broader range with shorter notice. This study can be found at:

http://www.xcelenergy.com/SiteCollectionDocuments/docs/CRPWindIntegrationStudy.pdf 


\section{Section 7}

\section{Key Findings AND CONCLUSIONS}

\subsection{Key Findings ANd Conclusions}

\subsubsection{Wind Generation Characteristics}

As mentioned in Section 2.2 the four base scenarios include three wind penetrations. A summary of the wind aggregated nameplate, capacity factor and reference energy for each wind penetration percentage is provided in Table 57. The reference energy column is the average of the 3 historical years. The total amounts of wind modeled in PROMOD ranged from $58 \mathrm{GW}$ for the $10 \%$ scenario to $81 \mathrm{GW}$ for the $40 \%$ scenario, with Nebraska and SPP being the only regions in which wind penetration was varied. Outside SPP/NPA the wind penetration was held constant at $6 \%$.

Table 57: Wind Generation Scenarios("SPP" here is rest of SPP)

\begin{tabular}{|l|r|r|r|r|r|r|r|r|r|}
\hline \multirow{2}{*}{ Region } & \multicolumn{3}{|c|}{$10 \%$ Penetration } & \multicolumn{2}{c|}{$20 \%$ Penetration } & \multicolumn{2}{c|}{$40 \%$ Penetration } \\
\cline { 2 - 9 } & $\begin{array}{c}\text { Name } \\
\text { Plate }\end{array}$ & \multicolumn{1}{c|}{ CF } & $\begin{array}{c}\text { Reference } \\
\text { Energy }\end{array}$ & $\begin{array}{c}\text { Name } \\
\text { Plate }\end{array}$ & CF & $\begin{array}{c}\text { Reference } \\
\text { Energy }\end{array}$ & $\begin{array}{c}\text { Name } \\
\text { Plate }\end{array}$ & \multicolumn{1}{c|}{$\begin{array}{c}\text { CF } \\
\text { Energy }\end{array}$} \\
\hline NPA & 1,249 & $41 \%$ & 4,523 & 2,488 & $41 \%$ & 8,855 & 4,727 & $41 \%$ & 16,831 \\
SPP & 6,256 & $42 \%$ & 22,901 & 12,596 & $41 \%$ & 45,807 & 25,431 & $41 \%$ & 91,548 \\
PJM & 25,807 & $36 \%$ & 81,460 & 25,807 & $36 \%$ & 81,460 & 25,807 & $36 \%$ & 81,460 \\
MISO/MAPP & 19,547 & $36 \%$ & 61,700 & 19,547 & $36 \%$ & 61,700 & 19,547 & $36 \%$ & 61,700 \\
SERC & 3,615 & $36 \%$ & 11,410 & 3,615 & $36 \%$ & 11,410 & 3,615 & $36 \%$ & 11,410 \\
TVA & 1,397 & $36 \%$ & 4,410 & 1,397 & $36 \%$ & 4,410 & 1,397 & $36 \%$ & 4,410 \\
\hline Total & 57,871 & $37 \%$ & 186,405 & 65,450 & $37 \%$ & 213,642 & 80,524 & $38 \%$ & 267,359 \\
\hline
\end{tabular}

\subsubsection{System Interaction with Wind Generation}

\subsubsection{Wind Generation Curtailment}

- $\quad$ Assuming certain localized transmission line additions are made and Knoll-Axtell $345 \mathrm{kV}$ line is built, there was no significant wind generation curtailment in Nebraska in any case with or without the overlay. That is, all scheduled wind generation was accommodated by re-dispatching other generation and exporting excess wind energy, all within modeled transmission limits. As described in the Section 2.4.3, lines nearby to the wind injection points were allowed to overload for study simplicity rather than determine all of the specific additions needed. Further the $40 \%$ case was only run with the overlay in place.

- $\quad$ There was also no significant violation of Nebraska thermal unit minimums (i.e. dump energy) in any of the cases. 
- $\quad$ With the wind dispatch price of $-\$ 40 / \mathrm{MWh}$ wind has priority over other resources. I.e., other resources re-dispatch or even curtail before wind does.

- $\quad$ Access to large export markets was a key factor in accommodating the wind generation additions.

- $\quad$ Although 20 major flow gates were monitored in Nebraska, it's possible that some local congestion was not captured in the analysis which could lead to curtailment.

- In the rest of SPP for the base cases, wind curtailment was found to be:

- $2 \%$ at the $10 \%$ penetration without the overlay

- $\quad 7 \%$ at the $20 \%$ penetration without the overlay

- $0 \%$ at the $20 \%$ penetration with the overlay

- $5 \%$ at the $40 \%$ penetration with the overlay

\subsubsection{Wind Energy Usage}

Consistently as wind penetration increased, the study results had SPP (including Nebraska) increasing exports by approximately half of the amount of the wind generation increases (41\% from Scenario 1 to 2 and 51\% from Scenario 3 to 4, using data from Figure 45). Figure 90 shows the Nebraska exports by scenario and wind/load pattern year.

- $\quad$ From $10 \%$ wind penetration to $20 \%$ wind penetration Nebraska wind energy increases by 4.35 TWh and exports increase by 3.20 TWh $(74 \%$ using averages of 3 wind years for base cases for Scenarios 1 and 2; see Figure 90).

- $\quad$ From $20 \%$ wind penetration to $40 \%$ wind penetration (with the EHV overlay) Nebraska wind energy increases by 8.12 TWh and exports increase by 4.74 TWh (58\% using averages of 3 wind years for base cases for Scenarios 3 and 4; see Figure 90).

- $\quad$ Although it is impossible to say exactly from which sources energy is exported, the increased wind penetrations in Nebraska / SPP result in increased energy exports. Note that in areas external to SPP the wind penetrations were held constant at a weighted $6 \%$ level across all of the SPP variations in penetration. As such the external system had greater flexibility at higher penetrations to accept imports of wind than if it had the same higher penetrations as in SPP/NPA. 


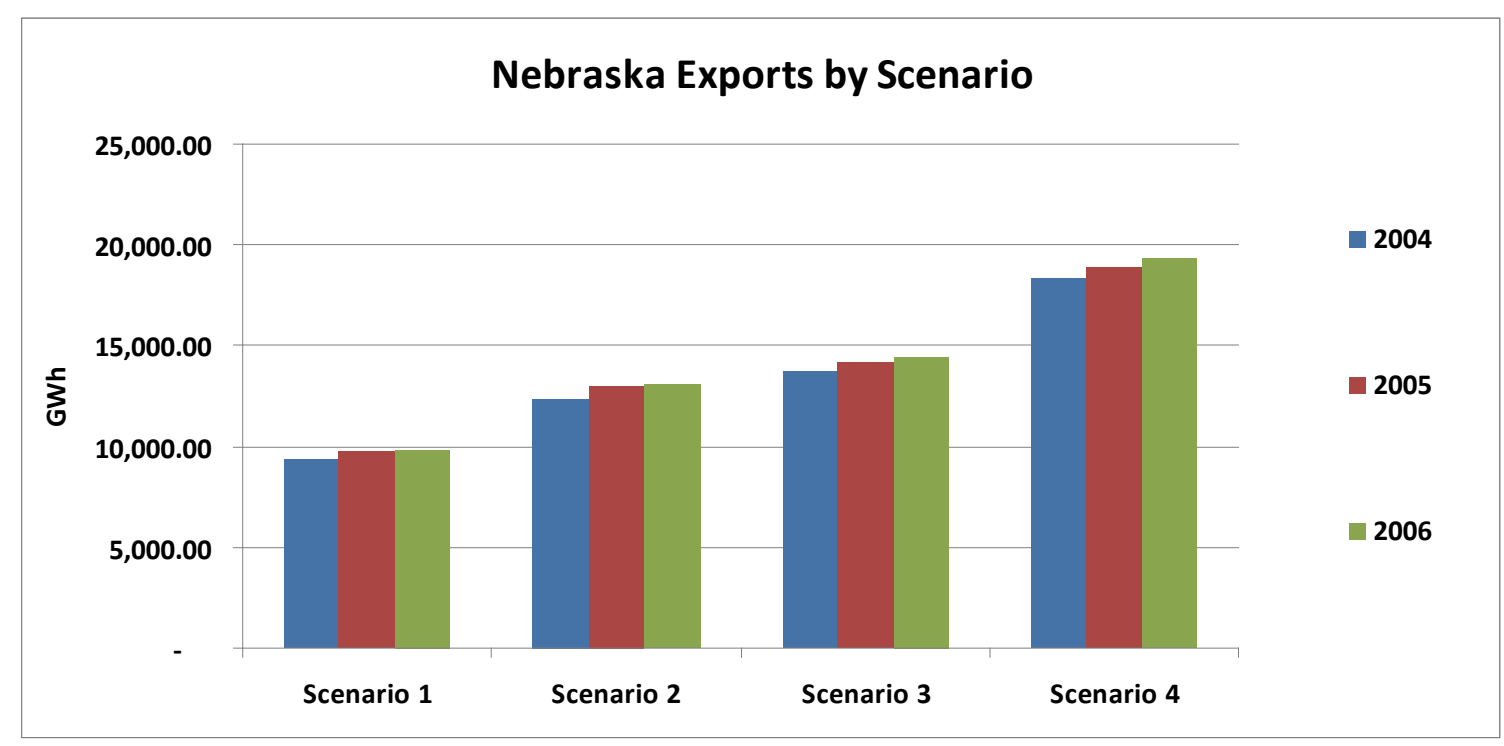

Figure 90: Nebraska exports by Scenario and Wind Year

\subsubsection{SPP Reserves}

- $\quad$ The effect of wind on total regulating reserves increases non-linearly as wind penetration increases and nearly linearly in incremental reserve requirement. Table 58 shows that for the 2006 profile with $10 \%$ wind penetration the average regulating reserves increase by $169 \%$. For the same year profile with $20 \%$ wind penetration the average regulating reserves increase $338 \%$ and the $40 \%$ wind penetration increases average regulating by $692 \%$.

- $\quad$ But for example, the incremental reserve requirements (amounts that reserves needed for load and wind exceed reserves needed for load alone) increase fairly linearly:

- $\quad$ From $10 \%$ to $20 \%$ penetrations, the average incremental reserve increase is $497 \mathrm{MW}=(1,291-295)-(794-295)$.

- $\quad$ From $10 \%$ to $40 \%$ penetrations, the average incremental reserve increase is $1,543 \mathrm{MW}=(2,337-295)-(794-295)$, which is approximately three times the $10 \%$ to $20 \%$ incremental reserve delta and three times the wind generation delta. That is, incremental reserve requirements increase approximately linearly with wind penetration.

- $\quad$ Pooling the SPP and Nebraska wind and dispatch reduce overall regulating reserves when compared to individual operations, Table 59. For the 2006 profile with $10 \%$ wind penetration Nebraska alone the average regulating reserve increases by $190 \%$ and the $10 \%$ wind penetration for SPP without Nebraska wind penetration increases average regulating reserves by $229 \%$. In other words for the 2006 profile with $10 \%$ wind penetration Nebraska independent of SPP has an average regulating reserve requirement of $148 \mathrm{MW}$. SPP without Nebraska has an average regulating reserve requirement of 742 MW. Combining the regulating reserve requirement for Nebraska alone with 
the rest of SPP results in a greater regulating reserve than when Nebraska and SPP are combined (148 MW + $742 \mathrm{MW}>794 \mathrm{MW}$ ).

Table 58: $\quad$ Regulating Reserves for SPP Load and Load net Wind

\begin{tabular}{|c|c|c|c|c|c|c|c|}
\hline \multirow{2}{*}{$\begin{array}{l}\text { SPP with Nebraska } \\
10 \% \text { Penetraton }\end{array}$} & \multicolumn{2}{|c|}{ Load Only } & \multicolumn{2}{|c|}{ Load and Wind } & \multicolumn{2}{|c|}{$\begin{array}{l}\text { Delta Load and } \\
\text { Wind - Load }\end{array}$} & \multirow{2}{*}{$\begin{array}{c}\begin{array}{c}\text { Wind } \\
\text { Nameplate }\end{array} \\
\mathrm{MW}\end{array}$} \\
\hline & Max & Average & Max & Average & $\operatorname{Max}$ & Average & \\
\hline 2004 profile & 533 & 301 & 1142 & 814 & 884 & 513 & 7,505 \\
\hline 2005 profile & 526 & 301 & 1146 & 806 & 853 & 505 & 7,505 \\
\hline 2006 profile & 528 & 295 & 1152 & 794 & 852 & 499 & 7,505 \\
\hline \multicolumn{8}{|l|}{$20 \%$ Penetration } \\
\hline 2004 profile & 533 & 301 & 1796 & 1322 & 1538 & 1021 & 15,084 \\
\hline 2005 profile & 526 & 301 & 1802 & 1308 & 1540 & 1007 & 15,084 \\
\hline 2006 profile & 528 & 295 & 1800 & 1291 & 1542 & 996 & 15,084 \\
\hline \multicolumn{8}{|l|}{$40 \%$ Penetration } \\
\hline 2004 profile & 533 & 301 & 3261 & 2395 & 3003 & 2094 & 30,158 \\
\hline 2005 profile & 526 & 301 & 3261 & 2359 & 3041 & 2058 & 30,158 \\
\hline 2006 profile & 528 & 295 & 3257 & 2337 & 3034 & 2042 & 30,158 \\
\hline
\end{tabular}

"Delta Max colum represents the coincidental maximum delta of Load Only and Load and Wind

Table 59: Regulating Reserves with Load and Load net Wind for Nebraska only and SPP without Nebraska

\begin{tabular}{|l|r|r|r|r|r|r|r|}
\hline \multirow{2}{*}{$\begin{array}{c}\text { 10\% Penetration } \\
\text { 2006 profile }\end{array}$} & \multicolumn{2}{|c|}{ Load Only } & \multicolumn{2}{c|}{ Load and Wind } & \multicolumn{2}{c|}{$\begin{array}{c}\text { Delta Load and } \\
\text { Wind - Load }\end{array}$} & $\begin{array}{c}\text { Wind } \\
\text { Nameplate }\end{array}$ \\
\cline { 2 - 8 } & \multicolumn{1}{|c|}{ Max } & Average & \multicolumn{1}{c|}{ Max } & Average & Max & Average & \multicolumn{1}{c|}{ MW } \\
\hline SPP with Nebraska & 528 & 295 & 1152 & 794 & 852 & 499 & 7,505 \\
SPP w/o Nebraska: A & 460 & 256 & 1111 & 742 & 821 & 486 & 6,256 \\
Nebraska alone: B & 75 & 45 & 216 & 148 & 177 & 118 & 1,249 \\
\hline Total A + B & 535 & 301 & 1327 & 890 & 998 & 604 & 7,505 \\
\hline
\end{tabular}

"Delta Max Column represents the coincidental maximum delta of Load Only and Load and Wind

\subsubsection{Wind Capacity Value for Accreditation in SPP}

- $\quad$ The SPP criterion, which is the criteria applicable to Nebraska utilities, for calculating monthly capacity value, was used for these findings. It is not based on an Effective Load Carrying Capacity methodology as many criteria are. Three years of Nebraska hourly wind data were correlated with corresponding 3 years of Nebraska hourly load.

- $\quad$ The monthly capacity value for individual wind sites studied in Nebraska ranged from a $0.06 \%$ minimum to a $16.5 \%$ maximum as shown in Table 60 . It is noteworthy that the two most common months in which Nebraska has its annual peak (July and August) are unfortunately the same two months in which the capacity values are the lowest. When all Nebraska wind sites were 
aggregated, the lowest monthly capacity values were observed in the summer months June, July and August, while the highest values were observed in April, May, September and October, as shown for $40 \%$ penetration in Table 61 and Figure 91 . The aggregate minimum increased to $6.55 \%$ while the maximum remained at $16.5 \%$, Table $8,40 \%$.

Table 60: Nebraska Monthly Wind Site Capacity Values

\begin{tabular}{|l|r|r|r|r|r|r|r|r|r|r|r|}
\hline \multirow{2}{*}{ Nameplate } & \multicolumn{5}{|c|}{ NPA - 10\% Wind Sites } & \multicolumn{3}{|c|}{ NPA 20\% Wind Sites } & \multicolumn{2}{|c|}{ NPA 40\% Wind Sites } \\
\cline { 2 - 11 } & 261 & 268 & 240 & 235 & 245 & 285 & 453 & 501 & 1100 & 479 & 660 \\
\hline Site ID & 22 & 143 & 160 & 205 & 208 & 47 & 245 & 1149 & 70 & 76 & 695 \\
\hline Jan & $2.79 \%$ & $1.19 \%$ & $1.82 \%$ & $2.24 \%$ & $3.59 \%$ & $2.66 \%$ & $2.81 \%$ & $2.25 \%$ & $1.63 \%$ & $2.30 \%$ & $4.49 \%$ \\
Feb & $6.60 \%$ & $2.79 \%$ & $4.23 \%$ & $4.06 \%$ & $10.85 \%$ & $6.26 \%$ & $2.52 \%$ & $10.16 \%$ & $4.36 \%$ & $4.77 \%$ & $6.45 \%$ \\
Mar & $5.27 \%$ & $2.22 \%$ & $2.98 \%$ & $4.24 \%$ & $3.90 \%$ & $4.64 \%$ & $4.01 \%$ & $2.95 \%$ & $3.79 \%$ & $3.57 \%$ & $4.95 \%$ \\
Apr & $6.46 \%$ & $8.25 \%$ & $8.42 \%$ & $4.69 \%$ & $3.04 \%$ & $5.46 \%$ & $6.38 \%$ & $0.86 \%$ & $8.12 \%$ & $8.73 \%$ & $3.08 \%$ \\
May & $13.52 \%$ & $8.35 \%$ & $5.35 \%$ & $9.26 \%$ & $16.53 \%$ & $12.09 \%$ & $4.55 \%$ & $14.57 \%$ & $11.77 \%$ & $4.11 \%$ & $4.39 \%$ \\
Jun & $4.45 \%$ & $1.86 \%$ & $1.98 \%$ & $0.63 \%$ & $6.22 \%$ & $5.41 \%$ & $2.80 \%$ & $8.19 \%$ & $1.58 \%$ & $2.11 \%$ & $3.22 \%$ \\
Jul & $1.54 \%$ & $1.17 \%$ & $1.08 \%$ & $0.06 \%$ & $1.96 \%$ & $0.54 \%$ & $0.39 \%$ & $1.26 \%$ & $1.37 \%$ & $1.51 \%$ & $2.41 \%$ \\
Aug & $3.07 \%$ & $0.81 \%$ & $2.22 \%$ & $0.34 \%$ & $1.94 \%$ & $3.29 \%$ & $1.39 \%$ & $4.11 \%$ & $1.19 \%$ & $1.86 \%$ & $3.35 \%$ \\
Sep & $10.40 \%$ & $6.79 \%$ & $4.05 \%$ & $5.35 \%$ & $12.66 \%$ & $10.19 \%$ & $3.14 \%$ & $12.53 \%$ & $9.25 \%$ & $4.02 \%$ & $3.13 \%$ \\
Oct & $7.92 \%$ & $10.23 \%$ & $1.47 \%$ & $5.21 \%$ & $8.36 \%$ & $7.35 \%$ & $2.32 \%$ & $5.47 \%$ & $10.23 \%$ & $1.63 \%$ & $5.37 \%$ \\
Nov & $2.10 \%$ & $0.86 \%$ & $2.43 \%$ & $1.20 \%$ & $0.98 \%$ & $1.64 \%$ & $5.57 \%$ & $0.53 \%$ & $1.77 \%$ & $4.33 \%$ & $6.23 \%$ \\
Dec & $3.94 \%$ & $3.59 \%$ & $3.76 \%$ & $3.87 \%$ & $2.73 \%$ & $4.07 \%$ & $4.34 \%$ & $1.74 \%$ & $3.55 \%$ & $2.80 \%$ & $3.31 \%$ \\
\hline
\end{tabular}

Table 61: Monthly Capacity Values for Aggregated Nebraska Wind Generation Scenarios by Penetration

\begin{tabular}{|l|r|r|r|}
\hline \multirow{2}{*}{ Nameplate } & \multicolumn{3}{|c|}{ All Sites } \\
\cline { 2 - 4 } & $10 \%$ & $20 \%$ & $40 \%$ \\
\cline { 2 - 4 } Jan & 1249 & 2488 & 4727 \\
Feb & $7.73 \%$ & $7.96 \%$ & $9.02 \%$ \\
Mar & $7.29 \%$ & $7.71 \%$ & $11.18 \%$ \\
Apr & $11.19 \%$ & $14.29 \%$ & $15.65 \%$ \\
May & $13.41 \%$ & $16.39 \%$ & $16.56 \%$ \\
Jun & $7.75 \%$ & $9.34 \%$ & $8.91 \%$ \\
Jul & $2.94 \%$ & $4.75 \%$ & $6.55 \%$ \\
Aug & $4.98 \%$ & $8.83 \%$ & $7.56 \%$ \\
Sep & $10.61 \%$ & $11.20 \%$ & $13.78 \%$ \\
Oct & $8.60 \%$ & $11.34 \%$ & $13.05 \%$ \\
Nov & $5.52 \%$ & $7.19 \%$ & $10.50 \%$ \\
Dec & $5.80 \%$ & $7.05 \%$ & $10.23 \%$ \\
\hline
\end{tabular}




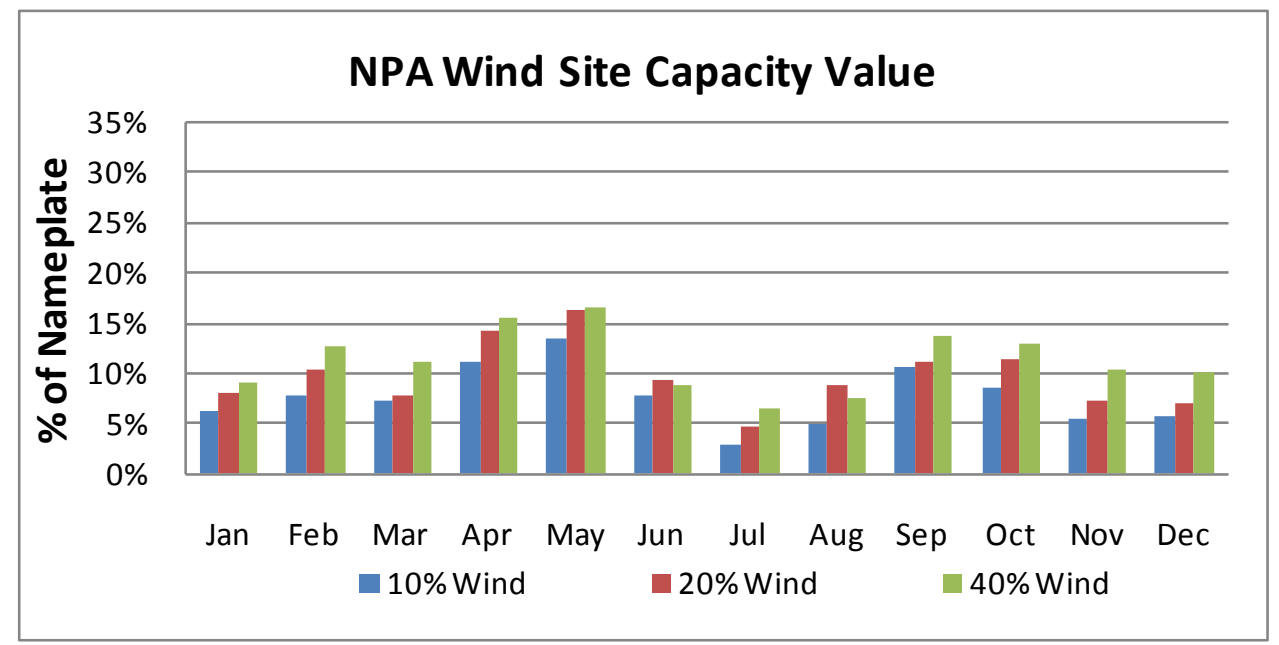

Figure 91: Aggregated Wind Site Capacity Value in Nebraska

- $\quad$ For SPP (without Nebraska) the monthly capacity values for individual wind sites ranged in value from a $0.03 \%$ minimum to a maximum of $16.55 \%$. The lowest values were observed in December and January.

- When the SPP (without Nebraska) wind sites were aggregated the monthly capacity value minimum and maximum increased to $4.99 \%$ and $22.4 \%$ respectively as shown in Figure 92.

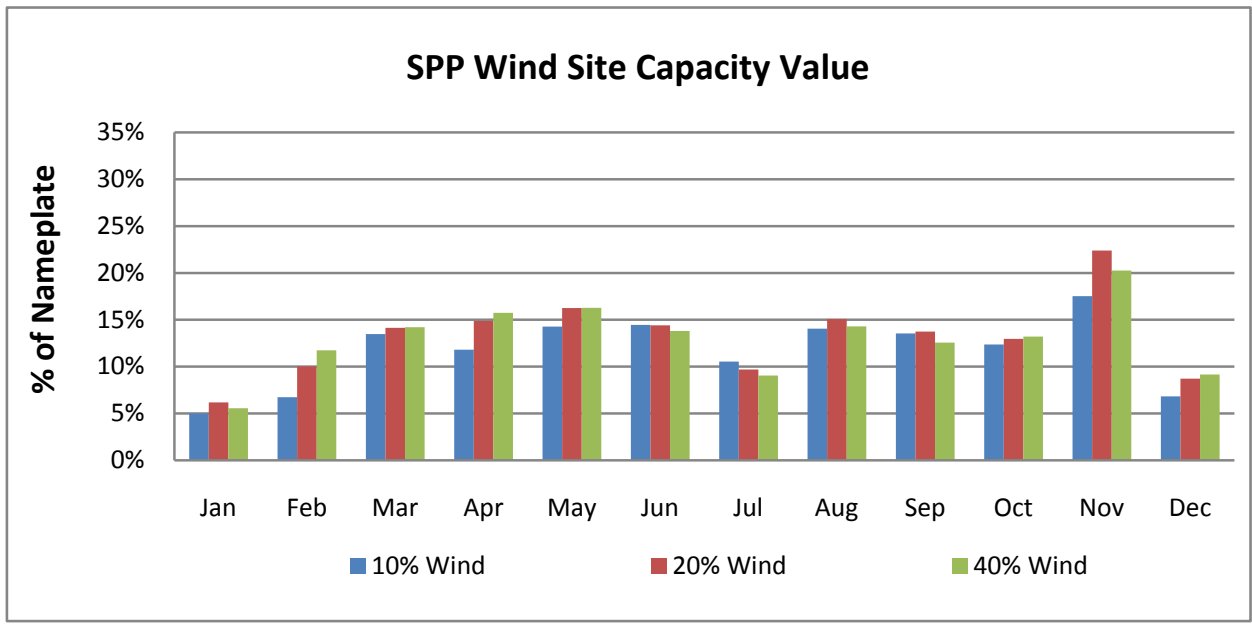

Figure 92: Aggregated Wind Site Capacity Value in SPP (without Nebraska)

- $\quad$ Aggregating all wind sites of Nebraska and SPP improved the monthly capacity values by increasing the minimum to $10.79 \%$ and maximum to $30.35 \%$, as shown in Figure 93 . This demonstrates the benefit of diversity across broader regions with increased wind capacity, assuming sufficient transmission exists. Although the data for November in this figure stands out as atypical, nothing could be found in the NREL data that was obviously inappropriate. 
- It is noted however, that the SPP accreditation criteria is currently applied to individual wind plants only rather than to an aggregation of plants.

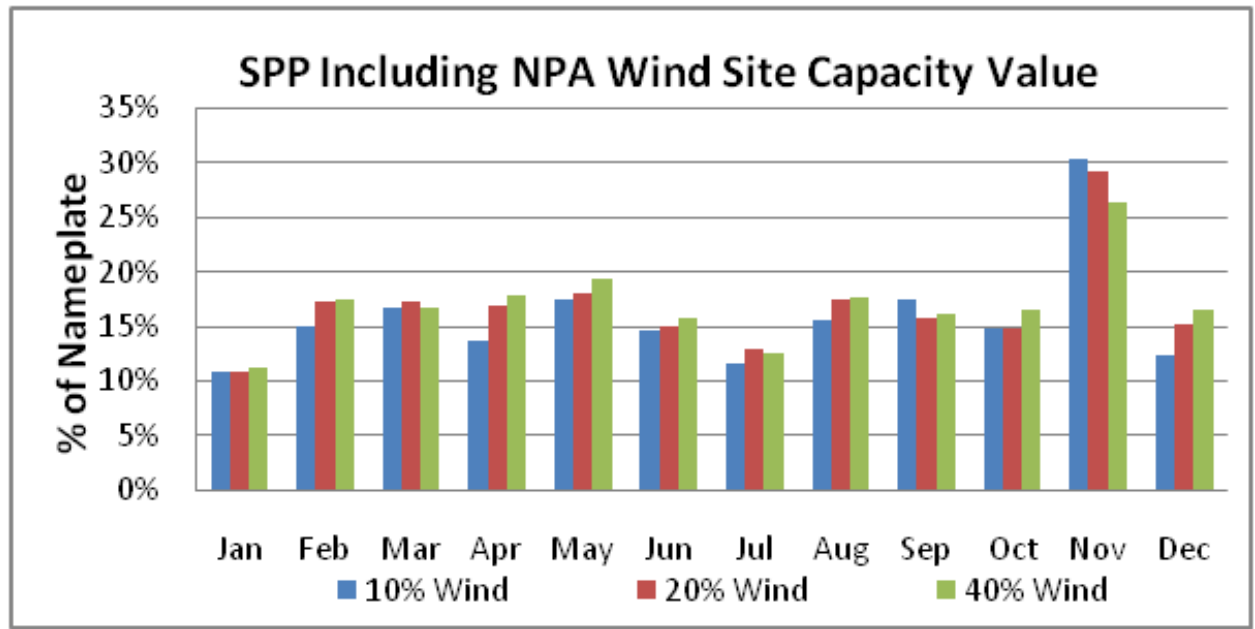

Figure 93: Monthly SPP with NPA Wind Capacity Value Estimates by Penetration

\subsubsection{Nebraska Transmission System}

- $\quad$ New transmission will be required to bring any significant new wind resources online. The transmission system will be stressed with increased wind penetration. The transmission system expansion must be designed with wind expansion in mind to minimize those stresses.

- $\quad$ Twenty flowgates involving Nebraska were modeled and fifteen of them were constrained somewhat and ranging from one hour to 4,970 hours out of the year depending on the scenario and flowgate. Nine flowgates had no constrained hours.

- $\quad$ Table 62 shows the number of non-monitored branches (not flowgates) in Nebraska that approached or exceeded their thermal limits by scenario using the 2006 wind/load patterns. These branches are 115kV-345kV lines that are allowed in the model to exceed limits recognizing that other transmission will need to be designed and installed to relieve these overloads.

Table 62: Number of Nebraska Branches at or near limits

\begin{tabular}{|l|r|r|r|r|}
\hline & Scenario 1 & Scenario 2 & Scenario 3 & Scenario 4 \\
\hline Violations of Thermal Limit & 6 & 15 & 10 & 23 \\
\hline $\begin{array}{l}\text { Over 90\% of Thermal Limit (excludes } \\
\text { violations) }\end{array}$ & 6 & 11 & 4 & 2 \\
\hline $\begin{array}{l}\text { Over 80\% of Thermal Limit (excludes } \\
\text { violations and 90\% violations) }\end{array}$ & 15 & 15 & 11 & 10 \\
\hline $\begin{array}{l}\text { Total Non-monitored Branches over } \\
80 \% \text { of Thermal Limit }\end{array}$ & 27 & 41 & 25 & 35 \\
\hline
\end{tabular}


- $\quad$ Specifically, the transmission system shows increased usage and risk of overloads as wind penetration increases, but those overloads decrease with the addition of transmission designed to deliver the wind energy (i.e., the $765 \mathrm{kV}$ overlay) as shown in Table 68. That is, from Scenarios 1 to 2 and from 3 to 4 , the exceedance numbers increase with penetration increases without adding transmission. Conversely from Scenario 2 to 3 the numbers decrease with the addition of the overlay.

\subsubsection{6. $\quad$ Carbon Dioxide}

- Increased wind penetrations result in lower $\mathrm{CO} 2$ emissions.

- $\quad$ Nebraska CO2 emissions decrease by about $8 \%$ between the $10 \%$ wind case and the $40 \%$ wind case (using averages of 3 wind years).

- The rest of SPP decreases CO2 emissions by about $15 \%$ between the same cases (using averages of 3 wind years).

- Increased exports due to wind will offset emissions outside of SPP.

- A pure price penalty on $\mathrm{CO} 2$ is not very effective at reducing $\mathrm{CO} 2$ emissions in SPP including Nebraska (given a constant expansion fleet modeled for all penetration levels) until the penalty gets very large (using 2006, Scenario 3 and its variations).

- $\quad$ A $\$ 25 /$ short ton penalty reduced SPP CO2 emissions by about $2 \%$ from the no penalty case.

- $\quad A \$ 50 /$ short ton penalty reduced SPP $\mathrm{CO} 2$ emissions by about $6 \%$ from the no penalty case.

- A $\$ 120 /$ short ton penalty reduced SPP CO2 emission by about $19 \%$ from the no penalty case.

- Increased penalties on $\mathrm{CO} 2$ result in decreased wind integration costs as more gas resources are committed due to $\mathrm{CO} 2$ penalties in both the ideal and actual cases diminishing the cost difference between coal and gas.

- $\quad$ Significant carbon reductions through dispatch penalties or emissions caps result in huge increases in the use of natural gas for electricity.

- $\quad$ Combined cycle use in Nebraska increases by $138 \%$ in the $\mathrm{CO} 2$ reduction case ( $\$ 120 /$ short ton) when compared to the base case $(\$ 25 /$ short ton), increasing CC capacity factors to over $80 \%$.

- $\quad$ As $\mathrm{CO} 2$ cost increased, the split between on-peak and off-peak LMPs dampened. This decreasing split results in uneconomic conditions for the operation of existing pumped storage generation facilities in SPP. Pumped storage capability (hydro or compressed air) is one of the alternatives that can help mitigate wind generation (pumping when winds are high and generating when winds are low). Mitigation will continue to be a benefit from pumped storage but its other benefits from "transferring" energy from off-peak periods to on-peak periods 
will be diminished as prices for coal generation and gas generation come together under $\mathrm{CO} 2$ regulation.

\subsubsection{Additional Considerations}

- There would be some additional costs associated with managing wind generation not captured in these production simulations:

- $\quad$ Additional maintenance and forced outages and derates

- Degradation of heat rate from ramping, cycling and range of operation

- Increase in emission rate per MWh production due to increased ramping that is not fully embodied in historical data that is used to determine modeled emission rates

- $\quad$ Other potential factors

- $\quad$ Production simulation results could be used to make estimates for some of these factors, but that was not attempted in this study.

\subsubsection{Total Costs (annual amounts in 2018 and assuming REC price of zero)}

- $\quad$ Regarding the assumption that REC price is modeled as zero. In most cases, two incentives for wind generation are being modeled: a production tax credit (PTC) and a carbon dioxide emission price. It is judged as not likely that three incentives will exist in 2018. It is acknowledged that the PTC is extended through 2012, but it is not certain that it would remain in 2018 after a federal Renewable Energy Standard is adopted.

- $\quad$ The primary results of the total cost estimation process for SPP including Nebraska are shown in Figure 95 which shows in bar chart and tabular form four cost categories for fifteen primary cases run on the 2006 wind and load patterns: incremental wind generation cost (cost above existing wind), incremental transmission, $\mathrm{CO} 2$ prices, and other costs (production and other fixed costs).

- $\quad \$ 25 /$ short ton $\mathrm{CO} 2$ price for cap-and-trade adds approximately $9 \%$ (or $\$ 2.2$ billion) to total cost reference, which is estimated at $\$ 23.7$ billion for existing wind and no $\mathrm{CO} 2$ cost in 2018. Under a tax regulation, that increase becomes approximately $22 \%$.

- $\quad$ Each $10 \%$ penetration (7,540 MW) adds approximately $1.7 \%$ to the total cost reference when CO2 cap-and-trade price is at $\$ 25 /$ short ton (comparing $10 \%$ and $40 \%$ base cases).

- $\quad$ Only the $\mathrm{CO} 2$ reduction case has emissions that approach the cap estimated for SPP under the Waxman-Markey proposal studied here by substituting gas for coal generation.

- $\quad$ At the $10 \%$ penetration level, operating Nebraska as a standalone market (rather than as a part of the SPP market) raises the SPP-wide cost (including 
Nebraska) by $\$ 22$ million annually for either $\mathrm{CO} 2$ regulation with price at $\$ 25 /$ short ton; however, this is not considered to be a comprehensive market analysis.

- Under cap-and-trade, there is a bigger jump in cost (9.5\%) from $\$ 0$ to $\$ 25 /$ ton than from $\$ 25$ to $\$ 50 /$ ton (6.0\%). Likely due to the loss of 13.5 TWh exports in going from $\$ 0$ to $\$ 25 /$ ton.

- $\quad$ This bullet discusses whether the investment in wind generation and transmission yields equal or more benefits from reduced $\mathrm{CO} 2$ and other costs (production) in the first year for selected cases, assuming the $\mathrm{CO} 2$ price is $\$ 25 /$ short ton.

- $\quad$ The first year benefit-to-cost ratio is 1.10 comparing $10 \%$ wind to existing wind and is 0.81 comparing $40 \%$ wind to $10 \%$.

- $\quad$ These $\mathrm{B} / \mathrm{C}$ estimates apply whether the $\mathrm{CO} 2$ regulation is cap-and-trade or a tax.

- $\quad$ At the $20 \%$ wind penetration the annual expense of the overlay does not appear to be totally recovered in year 2018; however, this study is not considered to be a complete transmission analysis from either the operating or economic perspectives.

- $\quad$ Compared to cap and trade, a $\mathrm{CO} 2$ tax is estimated to add $\$ 3$ billion at $\$ 25 /$ short ton price and $\$ 6$ billion at $\$ 50 /$ ton, at the $20 \%$ penetration.

- $\quad$ Additional results are calculated in Section 5, including for a non-zero REC price of $\$ 15 / \mathrm{MWh}$ making wind penetrations more favorable but it is quite uncertain that the federal production tax credit will be continued in the case of a federal RES and a carbon regulation (so a third incentive of REC pricing is not included in these Section 7 key findings).

\subsubsection{WAPA Interface for Integration}

- $\quad$ Scheduling WAPA firm power to Nebraska utilities proportional to load-net-wind vs. proportional to load may save Nebraska on the order of $\$ 1$ million, subject to Missouri River Basin management priorities.

- $\quad$ For the $10 \%$ penetration scenario it was found that the Mean Absolute Error of the day-ahead wind generation forecast could be reduced to $10.9 \%$ for a combined forecast compared to individual values of $11.4 \%$ for Nebraska and $12.3 \%$ for WAPA. This indicates some potential diversity benefits from coordination of wind resources.

\subsubsection{Integration Costs}

\subsubsection{Wind Integration Costs - Results}

Wind integration costs are production cost increases due to wind forecast error, wind regulating reserves and wind shape variability. The costs are typically normalized to wind energy by 
dividing production cost delta by total wind energy (see Section 3, Section 4.1, and Section 4.2.4 for more background).

- $\quad$ Costs to integrate wind increase with wind energy penetrations as more natural gas fired resources are used to respond to wind forecast error and displacement of coal energy backed down for reserves, as shown in Figure 94.

- $\quad$ SPP integration costs (based on shaped proxy) increase from an average of $\$ 46$ million at $10 \%$ wind to an average of $\$ 218$ million at $40 \%$ wind penetration using the shaped proxy as shown in Figure 45.

- $\quad$ Normalized Integration Costs (as initially defined with shaped proxy resource) are relatively small and in a narrow range of $\$ 1.65 / \mathrm{MWh}-\$ 2.18 / \mathrm{MWh}$ in $2018 \$$ (or $\$ 1.32-\$ 1.75 / \mathrm{MWh}$ in $2009 \$$ ) for the multiple penetration levels and years modeled for the base case as shown in Figure 95.

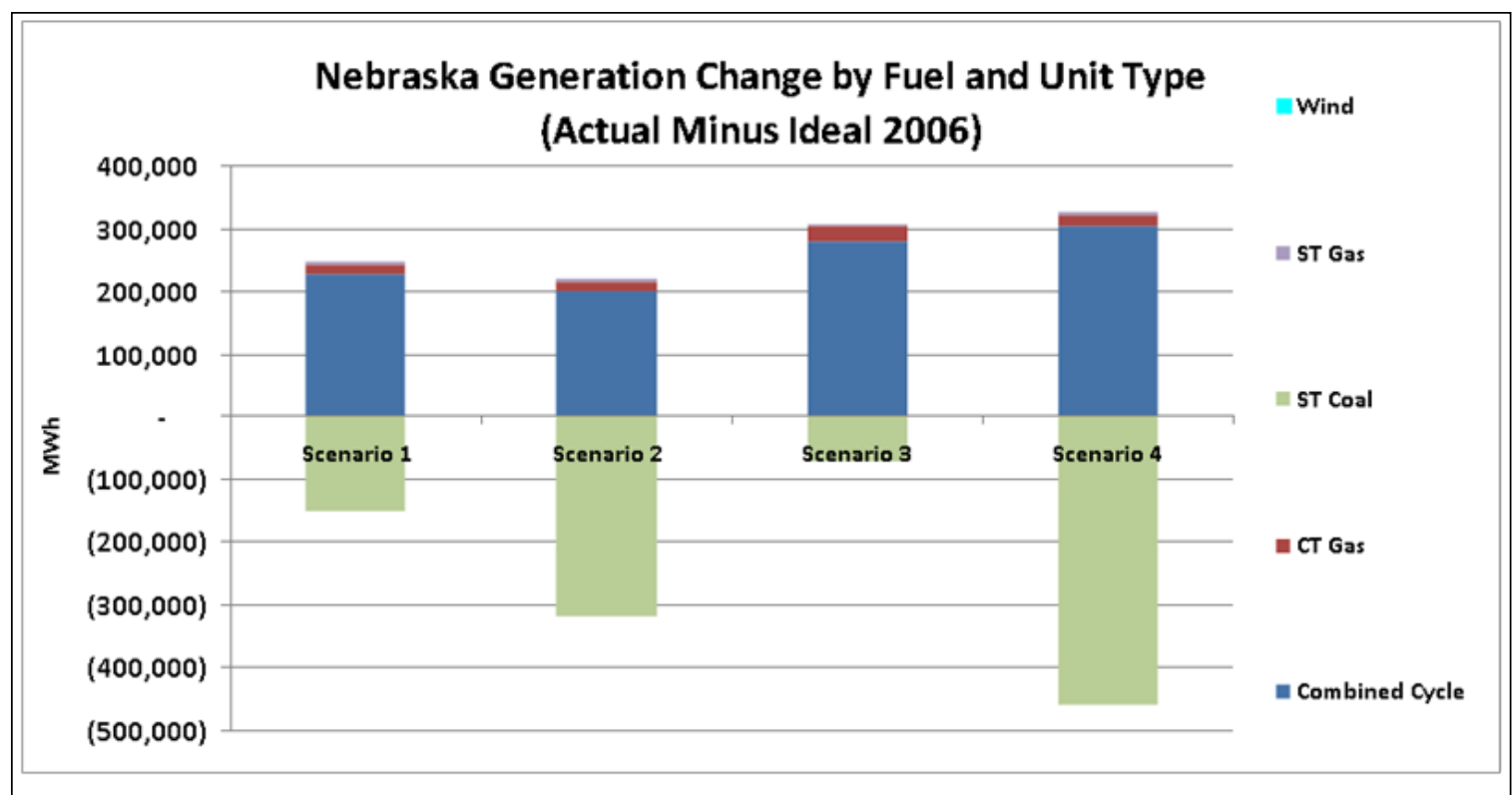

Figure 94: Change in Nebraska Generation with Incremental Reserve Requirements and Additional Uncertainty due to Wind Generation ("actual" case) 

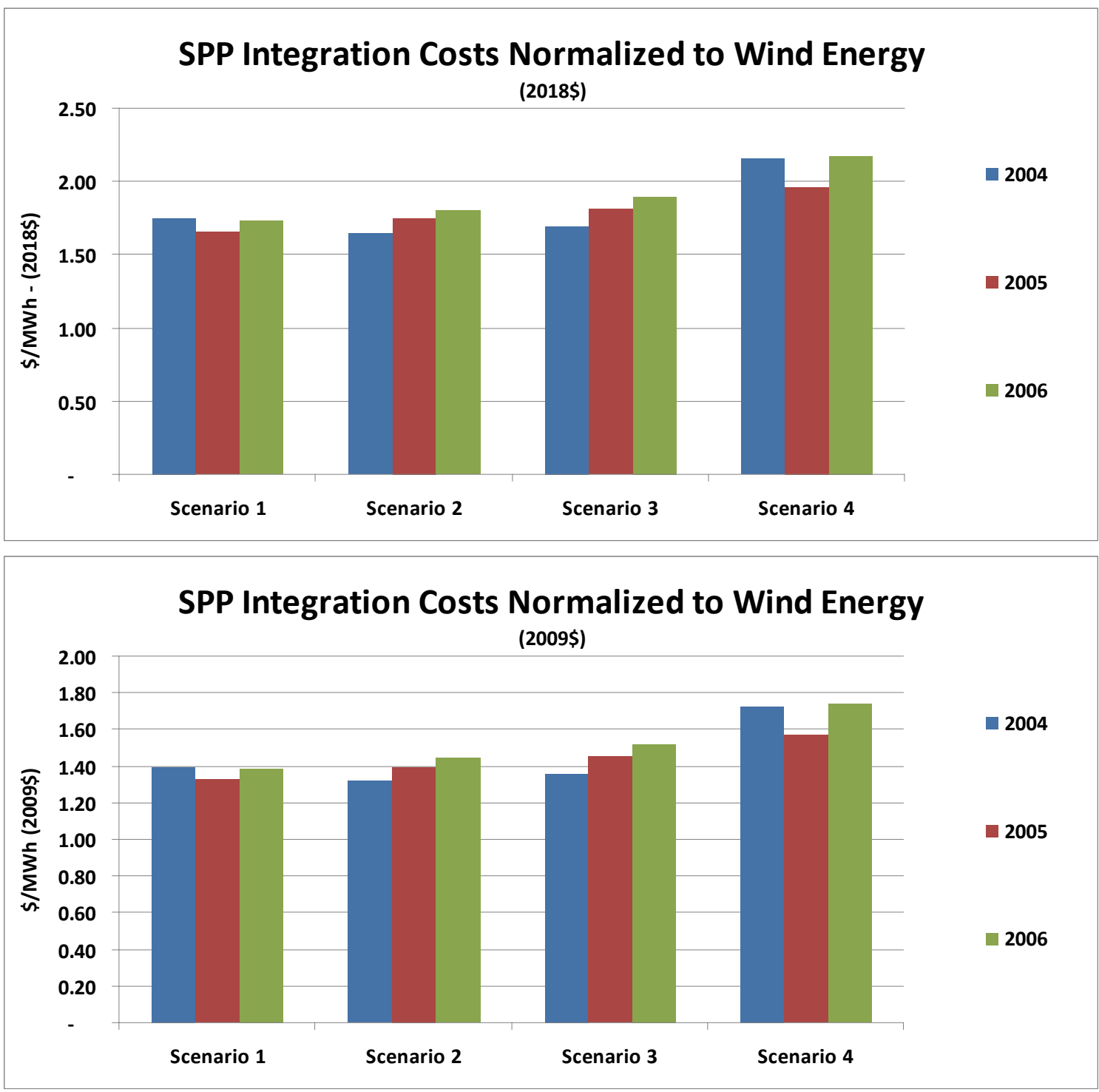

Figure 95: SPP (including Nebraska) Normalized Wind Integration Costs by Scenario and Wind Year for 2018 Conditions shown in 2018 (top) and 2009\$ (bottom)

- $\quad$ The base penetration scenarios show a consistent increase in use of gas fired resources (especially combined cycle plants) to deal with wind forecast error and increased (and variable) reserve requirements when comparing actual wind to ideal wind runs.

- In accommodating wind reserves and forecast error, the Nebraska combined cycle usage increases by $18 \%$, when comparing the actual case to the ideal case at $40 \%$ wind penetration.

- $\quad$ The initial definition of integration costs (with shaped proxy) is only capturing effects of incremental reserves for wind variability and wind forecast error and is not capturing shape variability costs. 
- $\quad$ Other proxy resources were tested to help measure effects of hour to hour wind variability on the generation fleet. Sensitivity cases show that a moving average or block (sub-period or flat) for proxy captures more integration costs, called herein shape or variability costs, see Figure 96.

- $\quad$ Proxy resource sensitivity results show $\$ 4.01 / \mathrm{MWh}$ (or $\$ 3.21 / \mathrm{MWh}$ in $2009 \$$ ) for $10 \%$ penetration with daily block proxy and $\$ 2.40 / \mathrm{MWh}$ (or 1.92/MWh in 2009\$) for a sub-period block proxy.

- $\quad$ At $20 \%$ wind penetration those integration cost results increase to $\$ 5.36 / \mathrm{MWh}$ (or $\$ 4.29 / \mathrm{MWh}$ in $2009 \$$ ) for the daily block proxy and $\$ 3.88 / \mathrm{MWh}$ (or $\$ 3.11 / \mathrm{MWh}$ in $2009 \$$ ) with the sub-period block proxy.

- $\quad$ Non-normalized integration costs using the daily block proxy for SPP are $\$ 110$ million at $10 \%$ wind and $\$ 66$ million using the sub-period block proxy; these values increase to $\$ 286$ million and \$206 million respectively for $20 \%$ wind penetration.

- $\quad$ These increasing differentials for alternative proxies as wind penetration increases may indicate that integration costs associated with wind variability are increasing with the amount of wind although the other costs (reserves and uncertainty as measured with the shaped proxy) are remaining relatively flat as wind penetration increases. 

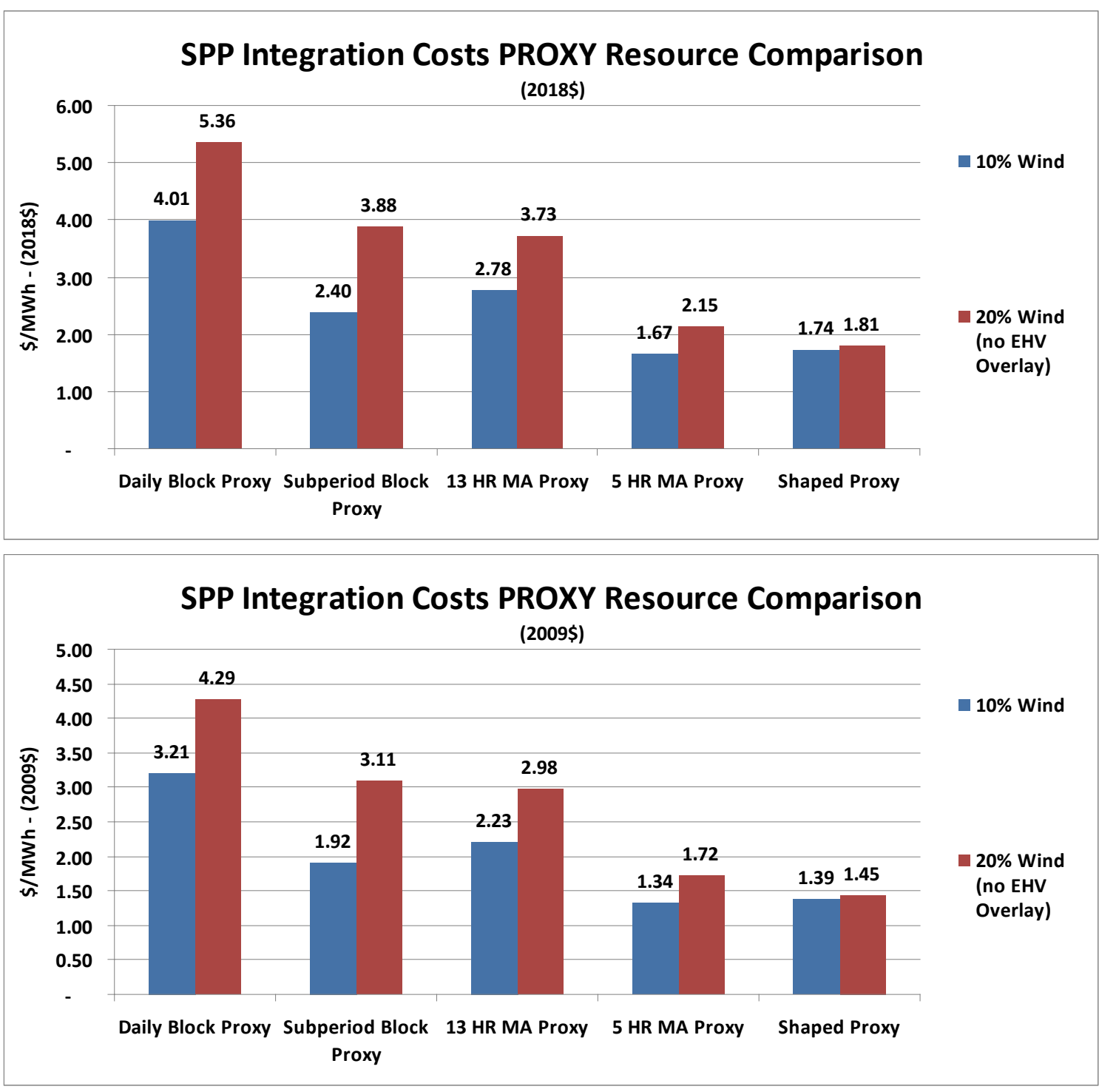

Figure 96: SPP (including Nebraska) of Integration Costs using Different Proxy Resources for 2018 Conditions in 2018 (top) and $2009 \$$ (bottom)

- $\quad$ Analysis indicates that the modeled system outside of SPP is absorbing some of the costs of wind variability represented in the block proxies (daily and subperiod) and that total SPP integration costs could be as high as $\$ 5.41 / \mathrm{MWh}$ (2009\$) at $10 \%$ wind penetration and $\$ 9.26 / \mathrm{MWh}(2009 \$)$ at $20 \%$ wind penetration when considering those exported costs (using the sub-period block proxy), see Section 4.4.4.

- $\quad$ Lastly, regarding these shape costs especially, it is noted that if the external regions had been modeled with higher penetrations, the computed integration costs may have been higher because these external systems would have had less capability to "help" integrate the shape of the SPP wind generation. 
As discussed throughout the report, there are a number of cost considerations involved with incorporating wind other than the wind integration costs that use the shaped proxy, such as:

- Wind generation and transmission facilities installation and operation and maintenance.

- $\quad$ Effects on heat rates, emission rates, and outage frequency and duration from increased thermal unit ramping. The concern is that these effects could be significant, especially at higher penetration rates because utilities generally don't have such operating experience yet.

- $\quad$ Feasibility and pricing of highly variable exports associated with high wind penetrations.

- $\quad$ Effects from external areas increasing their wind penetrations along with SPP and associated reduction of external system's ability to absorb SPP wind variations.

- Lack of "shape" costs from the "shape proxy" methodology (as contained in the "daily block" proxy).

- Apparent need to consider the portion of the shape costs that get "exported" and do not show up in the calculated integration cost for the originating wind area.

\subsubsection{Wind Integration Costs - Methodology and Proxy Selection}

The wind integration cost evaluation involves comparing the wind resource complete with its variability and uncertainty to an "ideal" proxy, or reference, resource by performing two simulations. The resulting adjusted production cost difference is the integration cost that can be normalized by dividing this result by the amount of wind generation being integrated. A key element in the determination of integration costs is the definition of the proxy resource. A second issue is whether or not the modeling approach addresses all the integration costs.

Conclusions -

- $\quad$ On the first issue the report does not definitively conclude what is the single best proxy resource for establishing wind integration costs for all conditions, but indicates the sub-period block best minimizes the concerns associated with other proxies - there is minimal energy shift from off peak to on peak periods (comparing to the daily block), but shape costs are captured (comparing to the shaped proxy).

- $\quad$ On the second issue, the report indicates that when including shape (or variability) costs by use of the block or moving average proxies, it is important to examine whether and what amount of these shape costs are being "exported" to external areas. And lastly that there are likely additional wind integration costs that are not yet identified by conventional modeling.

The following represents the current assumptions of the report and associated rationales. 
- $\quad$ Many previous studies coupled the daily block proxy resource with fixed interchange schedules. However, the associated issues with this approach are:

- $\quad$ The proxy resource having more on-peak (and less off-peak) energy than the actual wind resource, assuming wind energy is typically biased to the off-peak period,

- Wind generation will affect interchange rather than leaving it fixed, and

- $\quad$ At high wind penetrations, the daily transition from one daily block to another creates unreasonably large ramps.

- $\quad$ All the other proxy resources examined (sub-period block, moving average or shaped) are assumed to address the concern about the value difference from energy shifting from off-peak to on-peak discussed in the first sub bullet above.

- $\quad$ Modeling interchange between markets with typical hurdle rates applied to LMPs allows the wind generation to affect interchange. However, results show that the shape portion of integration costs can be "exported" and can be significant. For the purposes of calculating integration costs that consider and include such "exported" costs, the integration costs for the whole footprint can be used to impute such implied costs. Or it may be that more refined representation of external market response to the increased volatility of interchange under high wind penetration would work. For example, identifying an ancillary service premium for externally absorbing the wind variations might be appropriate.

- $\quad$ Daily transition ramps might be at least partially mitigated by the subperiod block proxy, more so by the moving average proxies, and totally by the shaped proxy.

- $\quad$ The shaped proxy clearly only evaluates the cost effects from incremental reserve requirement and wind forecast errors, but includes no consideration of shape costs.

- For the time being, at present penetration levels, the report proposes the sub period block proxy to be the most appropriate proxy for the integration cost elements that PROMOD was set up to evaluate, as it does result in costs for shape, incremental reserves, and forecast error without introducing energy shifting concerns. Further, some consideration is believed appropriate for "exported" integration costs as described. Lastly, the report recognizes that there are other potential wind integration costs that exist as a result of market questions noted above and unmodeled factors as noted in Section 7.1.4.1.

- $\quad$ For very high wind penetrations, such as $40 \%$, it may be that a good share of the wind generation will be dynamically scheduled out of the area anyway, resulting that wind integration cost is not borne by the local utilities, but by the receiving utility. 


\subsection{Closing Comments}

In closing, a qualifying statement with key results, a statement concerning how the study objectives were accomplished, and thoughts for future study are presented.

\subsubsection{Qualifying Statements and Key Results}

The results, findings, and conclusions presented here relate to the scenarios defined for the calendar year 2018. Critical to these scenarios are the extensive assumptions made regarding wind generation, load, fuel prices, energy market and operational structure, and certain aspects of policy regarding carbon. While the results paint a reasonably detailed picture regarding wind integration challenges and opportunities for NPA for the scenarios studied, care must be taken in extrapolating their meaning beyond the context in which they are presented in this report.

Figure 97 represents typical sample total cost results found in the study for all these major variables and interactions, showing $\mathrm{CO} 2$ cost effects, transmission and generation cost effects, and other remaining costs.

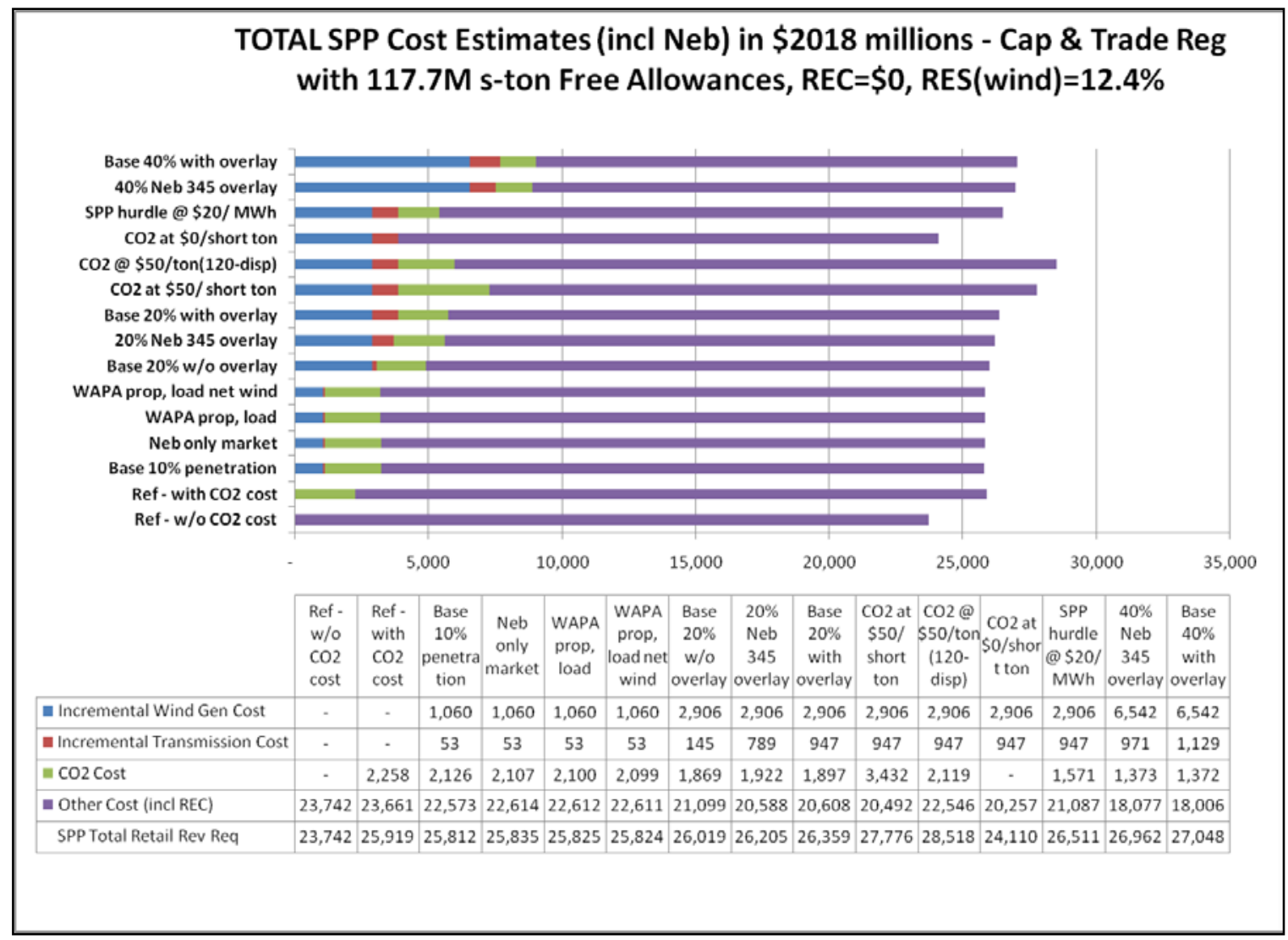

Figure 97: Total SPP Cost Estimates (inc Neb) in $\$ 2018$ millions - Cap \& Trade Reg with 117.7M s-ton Free Allowances, REC $=\$ 0$, RES (wind) $=12.4 \%$

Table 63 compares the integration costs for SPP including Nebraska for the two primary proxies for two penetrations for three perspectives in the two dollar-time-frames. 
Table 63: Proxy Integration Costs for 2018 and 2009 Dollars

\begin{tabular}{|l|r|r|r|r|}
\hline \multirow{2}{*}{ Proxy Type } & \multicolumn{2}{|c|}{2018 \$MWh } & \multicolumn{2}{c|}{2009 /MWh } \\
\cline { 2 - 5 } & $\begin{array}{c}\text { 10\% Wind } \\
\text { Penetration }\end{array}$ & $\begin{array}{c}20 \% \text { Wind } \\
\text { Penetration }\end{array}$ & $\begin{array}{c}10 \% \text { Wind } \\
\text { Penetration }\end{array}$ & $\begin{array}{c}\text { 20\% Wind } \\
\text { Penetration }\end{array}$ \\
\hline Shaped Proxy & 1.74 & 1.81 & 1.39 & 1.45 \\
Sub-period Block Proxy -Native & 2.4 & 3.88 & 1.92 & 3.11 \\
Sub-Period Block Proxy - Implied & & & & \\
(including "exported" shape costs) & 6.76 & 11.56 & 5.41 & 9.26 \\
\hline
\end{tabular}

\subsubsection{Objectives Statement}

The ten study objectives listed in Section 1.4 were accomplished in the following ways:

- $\quad$ The NREL wind generation and forecast data files and historical load data for Nebraska and much of the Eastern Interconnection for 2004-2006 were synchronized into the PROMOD production model software running base and sensitivity cases to evaluate system characteristics such as: wind integration costs, production costs, total system costs, wind generation curtailment, dump energy, emergency energy, flowgate performance, $\mathrm{CO} 2$ emissions, $\mathrm{CO} 2$ emission cost, interchange, unit production, ramping, proxy resource variations, market structure influence, WAPA interaction sensitivity, transmission overlay influence, capacity credit, and more. Many of these results are identified for Nebraska, but also for SPP as a whole.

- $\quad$ Four wind penetration scenarios were examined: $10 \%$ without transmission overlay, $20 \%$ with and without overlay, and $40 \%$ with overlay.

- Statistical analysis determined reserve components necessary as an input for the PROMOD production simulation runs.

- $\quad$ Two variations on scheduling WAPA firm power were evaluated to see what level of benefits might exist, fully recognizing that other water priorities may not allow such changes. A statistical evaluation was also performed on the reduction of wind forecast error potentially achievable if the wind generation forecasts in the WAPA and Nebraska areas were to be coordinated.

- $\quad$ The base assumption was a future SPP coordinated day-ahead and ancillary services market, which was then compared to a Nebraska standalone market to see some of the market aggregation benefits.

With regard to a pumped storage mitigation, the study produced a not-soencouraging finding that shows declining pumped storage economics as $\mathrm{CO} 2$ regulation tends to equalize on- and off-peak pricing. 
The study also suggests that there will likely be a shift from coal to gas generation in order to meet $\mathrm{CO} 2$ regulations, which might provide some opportunity for coal generation to partially mitigate wind variability (lower coal generation when the wind generation is high, and vice- versa).

Gas generation is shown as very helpful to accommodate wind generation variability.

- A Technical Review Committee consisting of 37 members was assembled including stakeholders, consultants, and utility members. The TRC met four times in person and three times by webinar. Additionally, 33 observers participated at various levels and received the same communications as did the TRC. The TRC and observers provided considerable guidance and support to the direction and accomplishments of the study.

- Nebraska involvement through this study is described as: 36 technical utility personnel committing over 4,700 hours of labor as TRC members or observers or in providing other technical support, 12 non-utility stakeholders representing 10 Nebraska interests on the TRC or as observers, three presentations to date in Nebraska on the study results, active and detailed participation in the direction of the study and development of presentations and final report, and associated discussions and updates to utility boards and managements. These study activities have advanced statewide wind development through better understanding and communication of the associated challenges and opportunities. This study also serves as a localized reference and direction for future work in the dynamic area of interest.

- Wind integration costs are developed using traditional and newer methodologies that consider sub-hourly and hourly and longer time periods for Nebraska as part of an SPP future market and also in a Nebraska standalone market. Results are also developed for the shape portion of integration costs that get "exported" to external systems.

- $\quad$ Experts and interested parties, nationally and locally, were included through the TRC participation described above and two well-qualified consultant teams, EnerNex and Ventyx, were employed for the technical and study management work.

- The statistical and production model information are available to the utilities, as well as the study process itself, are expected to yield improved utility modeling for future wind studies. 


\subsubsection{Thoughts for Future Study}

The study uncovered several areas of interest for further study:

- $\quad$ Determine how much the integration costs would increase if the complete footprint had consistently increasing penetration (e.g., $20 \%$ throughout examine the EWITS results to see if those results shed any light on this question).

- Do more hourly investigation on interactions between generators, wind and export.

- Do further study on the "exporting" of integration costs.

- $\quad$ Determine if the "mixture" of daily block proxy for SPP/NPA and shaped proxy in the rest of the system have a significant effect over daily block for all.

- $\quad$ Determine a best proxy resource for high wind penetrations in multiple operating areas with large footprints and developed markets.

- In the very critical operating periods of high wind/low load and low wind/high load, determine what the specific stresses on the system are.

- $\quad$ For the extreme ramping situations determine what the specific stresses on the system are.

- Examine the ramping "duty" that is being "exported" to see if these "volatile" interchanges that provide integration "service" are being priced adequately in the current modeling processes. And determine if a new ancillary service needs to be identified for model studies like this.

- $\quad$ Determine what the transmission system design for Nebraska wind development should be. Determine what is needed in the rest of SPP to relieve the wind curtailment. Also make land use studies and export studies.

- $\quad$ Compare findings with other studies such as SPP Wind Integration Study and EWITS.

- $\quad$ Evaluate reserve categorization, reserve sharing policies, and anything that relates to better integration of wind generation.

- $\quad$ Pursue wind capacity valuations in aggregate vs. individual plant valuations for accreditation as possible future criteria.

- Look at existing units in more detail to identify best capabilities and use, including limiting reserves to fewer units to provide regulation.

- Involve more people from operations in more in depth work for their benefit, but mainly to get more real-world modeling input.

- $\quad$ Consider running Nebraska in a more isolated manner to better tie back to previous studies. 
- Determine the value of short term storage that could handle the large shortterm reversals noted in the wind patterns (Idea being that could lesser cost for shorter term storage provide payback when longer term storage may not be able to, by mitigating the most costly fluctuations).

- $\quad$ Develop a method for adjusting integration cost results that involves equalizing the wind energy in the ideal and actual wind cases, or more likely, adjusting for the difference in wind energy value contributions. 


\section{Section 8 APPENDICES}

\subsection{Technical ReView Committee for the Nebraska STATEWIDE WIND INTEGRATION STUDY}

--led by the NPA subcontracting with the National Renewable Energy Laboratory

(NREL)

\section{Nebraska Utility Members:}

Clint Johannes - Nebraska Electric G\&T Cooperative, Inc. (Chair of the TRC and the NPA Joint Planning Subcommittee)

Paul Malone, Dave Rich, and Doug Kallesen - Nebraska Public Power District

David Ried, Marc Nichols, and Jon Iverson - Omaha Public Power District

Bruce Merrill - Lincoln Electric System

Billy Cutsor - NMPP Energy and Municipal Energy Agency of Nebraska

Dave Mazour and Ron Steinbach - Tri-State G\&T Association, Inc.

\section{Consultants:}

Bob Zavadil, Tom Mousseau, and Jack King - EnerNex Corporation (Zavadil - Study Project Manager)

Gary Moland, Rick Hunt, and Brenton Meese - Ventyx Energy, LLC (Moland - Lead)

\section{Technical Experts and Stakeholders:}

Michael Milligan - National Renewable Energy Laboratory / Transmission and Grid Integration Group

Matt Schuerger and Ed DeMeo - representing National Renewable Energy Laboratory

Charlie Smith - Utility Wind Integration Group

Laverne Kyriss and Mike Radecki - Western Area Power Administration

Jay Caspary - Southwest Power Pool staff

Sohrab Asgarpoor - University of Nebraska - Lincoln (Electrical Engineering)

Neil Moseman, Bruce Hauschild, and Sarah Hurt - Nebraska Department of Energy / Nebraska Energy Office

John Hansen - Nebraska Wind Working Group

Tim Texel - Nebraska Power Review Board

Michael Goggin - American Wind Energy Association

Steve Eveans - representing Community-Based Energy Development (C-BED) 
Five tribes were invited and copied on TRC correspondence: Tribal Council of the Winnebago Tribe of Nebraska; Ponca Tribe of Nebraska; Omaha Tribe of Nebraska \& lowa; Santee Sioux Tribe of Nebraska; and Oglala Sioux Tribe - Pine Ridge, South Dakota

\section{NREL Technical Monitor:}

Brian Parsons - National Renewable Energy Laboratory

\section{Observers to the TRC:}

\section{Subcontract Official:}

Neil Wikstrom - National Renewable Energy Laboratory

\section{Technical Experts and Stakeholders:}

Joe Sullivan and Mark Ahlstrom - Wind Logics, Inc.

John Cochnar - U. S. Fish and Wildlife Service

Tim McCoy - Nebraska Game and Parks Commission

Bill Doan - U.S. Army Corps of Engineers

Patrick Dalseth - Midwest Wind Energy, LLC

Dave Dingman - Nebraska Energy Office

Laurie Lage - Office of Nebraska Senator Chris Langemeier, Chair, Natural Resources Committee, Nebraska Legislature.

\section{Utilities (other NPA Joint Planning Subcommittee members, other Southwest Power Pool} staff, etc.):

Bruce Pontow - Nebraska Electric G\&T Cooperative, Inc.

Randy Lindstrom, Jim Fehr, Jon Sunneberg, Rocky Plettner, Ron Thompson, Tim Owens, Mike

Matheson, Joe Citta, Frank Thompson, Brian Wilcox, and John Richards - Nebraska

Public Power District

Jim Spiers, Mike Stortz, and Gerald Brooks - Tri-State G\&T Association, Inc.

Travis Burdett - City of Grand Island, Utilities Department

Don Cox - Hastings Utilities

Max VanSkiver - South Central Public Power District

Ron Ziola - Loup Power District

Eric Hixson - Central Nebraska Public Power \& Irrigation District

Charles Hendrix, Jason Smith, and Juliano Freitas - Southwest Power Pool staff 


\subsection{Addional Wind Generation Characteristics Scenario INFORMATION}

Because of size, the data provided in this section is contained in a separate document that has two parts. 


\section{Nebraska Statewide Wind Integration Study}

\section{Part 1 of Report Section 8.2}




\section{Contents}

Section $8 \quad$ Appendix...................................................................................................

Section 8.2 Additional Wind and System Generation Characteristics and Scenario Information 1

Section 8.2.1 Characterization of NREL Mesoscale Data $\quad 2$

Section 8.2.2 Wind Site Selection and Analysis $\quad 4$

Section 8.2.2.1 Wind Scenario Development by Region ......................................................4

Section 8.2.2.2 Installed Capacity by Scenario ................................................................. 14

Section 8.2.2.3 Annual Energy \& Capacity Factors................................................................. 14

Section 8.2.2.4 Wind Generation Capacity Factors (monthly and accumulative) .......................15

Section 8.2.2.5 Nebraska Wind Diversity ........................................................................... 18

Section 8.2.2.6 Wind Variability Histograms ....................................................................... 19

Section 8.2.2.7 Variability and Uncertainty - Wind ........................................................ 23

Section 8.2.2.8 Wind Capacity Value Estimates.............................................................25

Section 8.2.3 Wind and Load Characteristics $\quad 35$

Section 8.2.3.1 Hourly Characteristics by Year/Scenario/Region ............................................35

Section 8.2.3.2 Histogram Charts for Hourly Loads................................................................ 45

Section 8.2.3.3 Average Seasonal Load and Wind Production ............................................... 46

Section 8.2.3.4 Load, Wind and Load net Wind .................................................................54

Section 8.2.3.5 Nebraska Load/Wind Hourly Correlation .......................................................58

Section 8.2.3.6 NPA and WAPA Load and Wind Forecast Analysis .......................................63

Section 8.2.4 Operational Considerations $\quad 67$

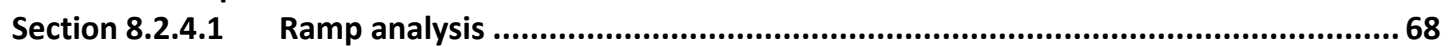

Section 8.2.4.2 Weekly Load, Resource and Wind Charts (Separate Document): .......................68 
Figure 1: Scenario Nameplate Capacity summary by Region $\quad 6$

Figure 2: Average Annual Energy by Scenario by Region 6

Figure 3: $\quad$ Wind Siting for the EWITS Reference Case - All sites 7

Figure 4: $\quad$ MISO West EWITS Reference case $\quad 8$

Figure 5: $\quad$ TVA Site ID's for all Penetrations 9

Figure 6: NPA Site ID's for 10\% Penetration 10

Figure 7: NPA Site ID's for 20\% Penetration $\quad 11$

Figure 8: Nebraska Site ID's for 40\% Penetration 12

Figure 9: SPP Site ID's for 10\% Penetration 13

Figure 10: SPP Site ID's for 20\% Penetration 13

Figure 11: SPP Site ID's for 40\% Penetration $\quad 14$

Figure 12: NPA Aggregated Capacity Factor for Wind Generation 16

Figure 13: NPA 10\% Penetration Wind Capacity Factors during Highest Load Hours 17

Figure 14: NPA 20\% Penetration Wind Capacity Factors during Highest Load Hours 17

Figure 15: NPA 40\% Penetration Wind Capacity Factors during Highest Load Hours 18

Figure 16: Nebraska wind site wind correlation $\quad 19$

Figure 17: Histogram of NPA Hourly Wind Variability - 10\% Penetration 20

Figure 18: Histogram of NPA Hourly Wind Variability as Percent of Nameplate - 10\% Penetration 20

Figure 19: Histogram of NPA Hourly Wind Variability - 20\% Penetration 21

Figure 20: Histogram of NPA Hourly Wind Variability as Percent of Nameplate - 20\% Penetration 21

Figure 21: Histogram of NPA Hourly Wind Variability - 40\% Penetration 21

Figure 22: Histogram of NPA Hourly Wind Variability as Percent of Nameplate - 40\% Penetration 21

Figure 23: Histogram of SPP Hourly Wind Variability - 10\% Penetration 22

Figure 24: Histogram of SPP Hourly Wind Variability as Percent of Nameplate - 10\% Penetration 22

Figure 25: Histogram of SPP Hourly Wind Variability - 20\% Penetration 22

Figure 26: Histogram of SPP Hourly Wind Variability as Percent of Nameplate - 20\% Penetration 23

Figure 27: Histogram of SPP Hourly Wind Variability - 40\% Penetration 23

Figure 28: Histogram of SPP Hourly Wind Variability as Percent of Nameplate - 40\% Penetration 23

Figure 29: Nebraska Persistence Hourly Wind Forecast Error 40\% Penetration 24 
Figure 30: Nebraska Persistence Sub Hourly Wind Forecast Error 40\% Penetration

Figure 31: Forecast error SPP 40\% Penetration

Figure 32: NPA Wind Site Capacity Values - 10\% Penetration

Figure 33: NPA Wind Site Capacity Values - 20\% Penetration

Figure 34: NPA Wind Site Capacity Values - 40\% Penetration

Figure 35: NPA Aggregated Wind Site Capacity Values by Penetration 30

Figure 36: SPP Wind Sites 1-4 Capacity Values - 10\% Penetration 31

Figure 37: SPP Wind Sites 5-7 Capacity Values - 10\% Penetration 31

Figure 38: SPP Wind Sites 1-4 Capacity Values - 20\% Penetration 31

Figure 39: SPP Wind Sites 5-8 Capacity Values - 20\% Penetration 32

Figure 40: SPP Wind Sites 9-11 Capacity Values - 20\% Penetration 32

Figure 41: SPP Wind Sites 1-4 Capacity Values - 40\% Penetration 33

Figure 42: SPP Wind Sites 5-8 Capacity Values - 40\% Penetration 33

Figure 43: SPP Wind Sites 9-12 Capacity Values - 40\% Penetration 34

Figure 44: SPP Wind Sites 13-15 Capacity Values - 40\% Penetration 34

Figure 45: NPA + SPP Wind Site Capacity Value by Penetration 35

Figure 46: NPA 2004 Load and Wind Analysis - 10\% Penetration 36

Figure 47: NPA 2004 Load and Wind Analysis - 20\% Penetration 36

Figure 48: NPA 2004 Load and Wind Analysis - 40\% Penetration 37

Figure 49: NPA 2005 Load and Wind Analysis - 10\% Penetration 37

Figure 50: NPA 2005 Load and Wind Analysis - 20\% Penetration 38

Figure 51: NPA 2005 Load and Wind Analysis - 40\% Penetration 38

Figure 52: NPA 2006 Load and Wind Analysis - 10\% Penetration 39

Figure 53: NPA 2006 Load and Wind Analysis - 20\% Penetration 39

Figure 54: NPA 2006 Load and Wind Analysis - 40\% Penetration 40

Figure 55: SPP 2004 Load and Wind Analysis - 10\% Penetration 41

Figure 56: SPP 2004 Load and Wind Analysis - 20\% Penetration 41

Figure 57: SPP 2004 Load and Wind Analysis - 40\% Penetration 42

Figure 58: SPP 2005 Load and Wind Analysis - 10\% Penetration 42

Figure 59: SPP 2005 Load and Wind Analysis - 20\% Penetration 43

Figure 60: SPP 2005 Load and Wind Analysis - 40\% Penetration 43

Figure 61: SPP 2006 Load and Wind Analysis - 10\% Penetration 44

Figure 62: SPP 2006 Load and Wind Analysis - 20\% Penetration 44

Figure 63: SPP 2006 Load and Wind Analysis - 40\% Penetration 45 
Figure 64: Histogram of Average NPA 2018 Load 45

Figure 65: Histogram of Average SPP 2018 Load Including NPA 46

Figure 66: Histogram of Average SPP - NPA 2018 Load Excluding NPA 46

Figure 67: NPA - 24-Hour Average Seasonal Load and Wind 10\% Penetration 47

Figure 68: NPA - 24-Hour Average Seasonal Load and Wind 20\% Penetration 47

Figure 69: NPA - 24-Hour Average Seasonal Load and Wind 40\% Penetration 48

Figure 70: NPA - 24 Hour Average Seasonal Wind 10\% Penetration 48

Figure 71: NPA - 24 Hour Average Seasonal Wind 20\% Penetration 49

Figure 72: NPA - 24 Hour Average Seasonal Wind 40\% Penetration 49

Figure 73: NPA - 24 Hour Average Monthly Wind 10\% Penetration 50

Figure 74: NPA - 24 Hour Average Monthly Wind 20\% Penetration 50

Figure 75: NPA - 24 Hour Average Monthly Wind 40\% Penetration 51

Figure 76: SPP - 24 Hour Average Seasonal Load and Wind 10\% Penetration 51

Figure 77: SPP - 24 Hour Average Seasonal Load and Wind 20\% Penetration 52

Figure 78: SPP - 24 Hour Average Seasonal Load and Wind 40\% Penetration 52

Figure 79: SPP - 24 Hour Average Seasonal Wind 10\% Penetration 53

Figure 80: SPP - 24 Hour Average Seasonal Wind 20\% Penetration 53

Figure 81: SPP - 24 Hour Average Seasonal Wind 40\% Penetration 54

Figure 82: Time Series Plot for April Day of Load, Wind, and Load net Wind 54

Figure 83: Generation and Transaction to accommodate load and wind variations and Ramp Requirements for April 2, 2018 for 2006 profile 56

Figure 84: Time Series Plot for April Week of Load, Wind, and Load net Wind 57

Figure 85: Load Duration for NPA load and Load net Wind 57

Figure 86: NPA-Annual Summary Correlation of Load and Wind 58

Figure 87: NPA 2004 Load and Wind Correlation by Month $\quad 58$

Figure 88: NPA 2005 Load and Wind Correlation by Monty $\quad 59$

Figure 89: NPA 2006 Load and Wind Correlation by Month 59

Figure 90: SPP Load and Wind Correlation by Year $\quad 60$

Figure 91: SPP 2004 Load and Wind Correlation by Month 60

Figure 92: SPP 2005 Load and Wind Correlation by Month 61

Figure 93: SPP 2006 Load and Wind Correlation by Month 61

Figure 94: SPP with NPA Load and Wind Correlation by Year 61

Figure 95: SPP with NPA 2004 Load and Wind Correlation by Month 62

Figure 96: SPP with NPA 2005 Load and Wind Correlation by Month 62 
Figure 97: SPP and NPA 2006 Load and Wind Correlation by Month

Figure 98: Nebraska Actual Wind and Day Ahead Forecast MAE for 10\% Penetration 63

Figure 99: Nebraska Actual Wind and Day Ahead Forecast MAE for 20\% Penetration 63

Figure 100: Nebraska Actual Wind and Day Ahead Forecast MAE for 40\% Penetration 64

Figure 101: MAE of Nebraska Load with Persistence Forecast 64

Figure 102: WAPA Actual Wind and Day Ahead Forecast MAE 65

Figure 103: WAPA Load with 1 hr. Persistence Forecast MAE 65

Figure 104: Nebraska and WAPA Actual Wind and Day Ahead Forecast MAE for 10\% Penetration 66

Figure 105: Nebraska and WAPA Actual Wind and Day Ahead Forecast MAE for 20\% Penetration 66

Figure 106: Nebraska and WAPA Actual Wind and Day Ahead Forecast MAE for 40\% Penetration 67

Figure 107: Nebraska and WAPA Load with $1 \mathrm{hr}$ Persistence Forecast MAE 67

Figure 108: 2004 Profile Monthly Average Hourly MW Change for Hours 5 to 1169

Figure 109: 2004 Profile Monthly Maximum Hourly MW Change for Hours 5 to 1169

Figure 110: 2004 Profile Monthly Minimum Hourly MW Change for Hours 5 to 1170

Figure 111: 2004 Profile Monthly 98 Percentile Hourly MW Change for Hours 5 to 11

Figure 112: 2005 Profile Monthly Average Hourly MW Change for Hours 5 to 11

Figure 113: 2005 Profile Monthly Maximum Hourly MW Change for Hours 5 to 11

Figure 114: 2005 Profile Monthly Minimum Hourly MW Change for Hours 5 to 11

Figure 115: 2005 Profile Monthly 98 Percentile Hourly MW Change for Hours 5 to 11

Figure 116: 2006 Profile Monthly Average Hourly MW Change for Hours 5 to 11

Figure 117: 2006 Profile Monthly Maximum Hourly MW Change for Hours 5 to 11

Figure 118: 2006 Profile Monthly Minimum Hourly MW Change for Hours 5 to 11

Figure 119: 2006 Profile Monthly 98 Percentile Hourly MW Change for Hours 5 to 11

Figure 120: 2004 Profile Seasonal Maximum Hourly MW Change for Hours 5 to 1175

Figure 121: 2004 Profile Seasonal Minimum Hourly MW Change for Hours 5 to 11

Figure 122: 2004 Profile Seasonal 98 Percentile Hourly MW Change for Hours 5 to 11

Figure 123: 2005 Profile Seasonal Maximum Hourly MW Change for Hours 5 to 1176

Figure 124: 2005 Profile Seasonal Minimum Hourly MW Change for Hours 5 to 1176

Figure 125: 2005 Profile Seasonal 98 Percentile Hourly MW Change for Hours 5 to 11

Figure 126: 2006 Profile Seasonal Maximum Hourly MW Change for Hours 5 to 11

Figure 127: 2006 Profile Seasonal Minimum Hourly MW Change for Hours 5 to 11

Figure 128: 2006 Profile Seasonal 98 Percentile Hourly MW Change for Hours 5 to 11

Figure 129: 2004 Profile Monthly Average Hourly MW Change for Hours 21 to 3

Figure 130: 2004 Profile Monthly Maximum Hourly MW Change for Hours 21 to 3 
Figure 131: 2004 Profile Monthly Minimum Hourly MW Change for Hours 21 to 3

Figure 132: 2004 Profile Monthly 98 Percentile Hourly MW Change for Hours 21 to 3

Figure 133: 2005 Profile Monthly Average Hourly MW Change for Hours 21 to 3

Figure 134: 2005 Profile Monthly Maximum Hourly MW Change for Hours 21 to 3

Figure 135: 2005 Profile Monthly Minimum Hourly MW Change for Hours 21 to 3

Figure 136: 2005 Profile Monthly 98 Percentile Hourly MW Change for Hours 21 to 3

Figure 137: 2006 Profile Monthly Average Hourly MW Change for Hours 21 to 3

Figure 138: 2006 Profile Monthly Maximum Hourly MW Change for Hours 21 to 3

Figure 139: 2006 Profile Monthly Minimum Hourly MW Change for Hours 21 to 3

Figure 140: 2006 Profile Monthly 98 Percentile Hourly MW Change for Hours 21 to 3

Figure 141: 2004 Profile Season Maximum Hourly MW Change for Hours 21 to 3

Figure 142: 2004 Profile Season Minimum Hourly MW Change for Hours 21 to 3

Figure 143: 2004 Profile Season 98 Percentile Hourly MW Change for Hours 21 to 3

Figure 144: 2005 Profile Season Maximum Hourly MW Change for Hours 21 to 3

Figure 145: 2005 Profile Season Minimum Hourly MW Change for Hours 21 to 3

Figure 146: 2005 Profile Season 98 Percentile Hourly MW Change for Hours 21 to 3

Figure 147: 2006 Profile Season Maximum Hourly MW Change for Hours 21 to 3

Figure 148: 2006 Profile Season Minimum Hourly MW Change for Hours 21 to 3

Figure 149: 2006 Profile Season 98 Percentile Hourly MW Change for Hours 21 to 3

Figure 150: Contour plot of Nebraska Load Net Wind Hourly Changes with 20\% Penetration for 2006 profile. 
Table 1: $\quad$ Counts of Plants by Size $\quad 2$

Table 2: Wind Plants by State and Size 3

Table 3: $\quad$ Installed Capacity by State and Size of Plant 4

Table 4: $\quad$ Nebraska Selected Wind Generation Penetration Details 5

Table 5: $\quad$ SPP w/o Nebraska Selected Wind Generation Penetration Details 5

Table 6: $\quad$ Summary of Capacity, Capacity Factor and Energy for each Scenario by Region 6

Table 7: $\quad$ Nebraska Site Details $\quad 9$

Table 8: $\quad$ Wind Generation Capacity Assumptions by Region $\quad 14$

Table 9: Annual Wind Energy (GWh) by Region - 10\% Penetration 15

Table 10: Annual Wind Energy (GWh) by Region - 20\% Penetration 15

Table 11: Annual Wind Energy (GWh) by Region - 40\% Penetration 15

Table 12: Aggregate Capacity Factor by Region - 10\% Penetration 15

Table 13: Aggregate Capacity Factor by Region - 20\% Penetration 15

Table 14: Aggregate Capacity Factor by Region - 40\% Penetration 15

Table 15: Monthly Capacity Factors for NPA wind 16

Table 16: Correlation between NPA wind sites $\quad 19$

\begin{tabular}{lr} 
Table 17: & NPA Peak Load \\
\hline
\end{tabular}

\begin{tabular}{ll} 
Table 18: & SPP Peak Load \\
\hline
\end{tabular}

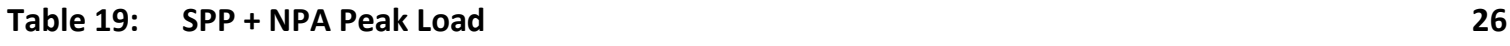

Table 20: $\quad$ NPA Wind Site Capacity Values - 10\% Penetration 27

Table 21: $\quad$ NPA Wind Site Capacity Values - 20\% Penetration 28

Table 22: NPA Wind site Capacity Values - 40\% Penetration 29

Table 23: $\quad$ NPA Aggregated Wind Site Capacity Values by Penetration 30

Table 24: $\quad$ Sample Day of Hourly Data (April 2, 2018) for Nebraska Load and Wind with 2006 profile 55 


\section{Section $8 \quad$ APPENDIX}

\section{Section 8.2 Additional Wind and System Generation Characteristics and Scenario Information}

This document is a part of the work performed by EnerNex for the Nebraska Statewide Wind Integration Study and is the appendix to this study final report. Ventyx provided the output results from PROMOD runs used to create the charts provided in Section 8.2.4.2 (Part 2 of Section 8.2).

The objective of this section of the appendix is to document the wind profile data from the mesoscale database developed for National Renewable Energy Laboratory (NREL) by AWS Truewind as it is used in the Nebraska Statewide Wind Integration Study. Load profile data obtained from NPA is also reported, including its correlation to wind generation. The focus is on the data for Nebraska and the rest of the Southwest Power Pool (unless otherwise noted herein "SPP" is used to refer to the non-Nebraska portion of the Southwest Power Pool, even though most Nebraska utilities are members of SPP). Data used in the model is also reported for other portions of the Eastern Interconnection of the U.S. power grid.

Because of the very significant volume of data, for both the database as a whole and the scenarios defined and developed for use in the study, the documentation is necessarily in the form of summary charts, graphs, and tables that depict relevant characteristics of the time series data.

The analyses conducted conform to the structure defined in the project scope, which consists of the following five items for the data included in this appendix, Section 8.2

- Group wind sites into regions.

- Conduct statistical analysis with spatial and temporal slices, to examine resource correlation across the region and wind/load correlation over time.

- Examine the energy production value of wind sites.

- Develop three scenarios with 10\%, 20\% and $40 \%$ wind energy penetration in the SPP portion of the study footprint based on these analyses, and with a goal of low cost of energy and low integration costs.

- Conduct statistical analysis on these three scenarios to examine the feasibility of integrating these levels of resources into the individual balancing areas, or authorities.

The raw data used in this work can be accessed and downloaded from NREL's website at http://www.nrel.gov/wind/integrationdatasets/eastern/data.html. Before downloading, it is suggested that one review the FAQ webpage referenced there for direction.

Section 8.2.1 of this appendix focuses on the mesoscale data as a whole. The database for 34 states in the Eastern Interconnection contains 1,325 separate "plants" - which are aggregations of nearby data points corresponding to a $2 \mathrm{~km}$ grid - and a total installed capacity of $580 \mathrm{GW}$, see Table 1, Table 2, and Table 3.

Section 8.2.2 of the report documents the process used to define the study scenarios being evaluated, as well as several charts and tables of the site-specific and aggregated wind 
generation characteristics in terms of capacity and energy, capacity factor, capacity value (in SPP), diversity/correlation, variability, and uncertainty .

Section 8.2.3 provides:

- charts and tables showing relationships between NPA and SPP loads and wind site generation for the selected sites.

- $\quad$ NPA and WAPA wind diversification for combined forecast reduction.

Section 8.2.4 displays weekly details for the year's time for each of the NPA and for the rest of SPP in how wind generation reduces the net load and how the remainder of the generation system responds to the wind generation characteristics as determined by PROMOD.

\section{Section 8.2.1 Characterization of NREL Mesoscale Data}

The NREL mesoscale wind database for the Eastern Interconnection, developed by AWS Truewind, is the source for all wind generation data in this study. The data is available for selective download at http://www.nrel.gov/wind/integrationdatasets/eastern/data.html.

Characteristics of the NREL mesoscale database, which represents $580 \mathrm{GW}$ of total nameplate capacity, are presented in this section, along with characteristics of wind generation profiles by the operating footprints defined for this study.

Table 1: Counts of Plants by Size

\begin{tabular}{|rc|}
\hline Plant Size (MW) & $\begin{array}{c}\text { Number of Plants } \\
\text { in Database }\end{array}$ \\
\hline $0-150$ & 265 \\
\hline $150-250$ & 155 \\
\hline $250-350$ & 214 \\
\hline $350-450$ & 194 \\
\hline $450-550$ & 146 \\
\hline $550-650$ & 95 \\
\hline $650-750$ & 52 \\
\hline $750-850$ & 38 \\
\hline $850-950$ & 11 \\
\hline $950-1050$ & 57 \\
\hline $1050-1150$ & 54 \\
\hline $1150-1250$ & 29 \\
\hline $1250-1350$ & 12 \\
\hline $1350-1450$ & 325 \\
\hline Total & \\
\hline
\end{tabular}


Table 2: Wind Plants by State and Size

\begin{tabular}{|c|c|c|c|c|c|c|c|c|c|c|c|c|c|c|}
\hline State & $0-150$ & $\begin{array}{c}150- \\
250 \\
\end{array}$ & $\begin{array}{c}250- \\
350 \\
\end{array}$ & $\begin{array}{c}350- \\
450 \\
\end{array}$ & $\begin{array}{c}450- \\
550 \\
\end{array}$ & $\begin{array}{c}550- \\
650 \\
\end{array}$ & $\begin{array}{c}650- \\
750 \\
\end{array}$ & $\begin{array}{c}750- \\
850 \\
\end{array}$ & $\begin{array}{c}850- \\
950 \\
\end{array}$ & $\begin{array}{l}950- \\
1050 \\
\end{array}$ & $\begin{array}{c}1050- \\
1150\end{array}$ & $\begin{array}{c}1150- \\
1250 \\
\end{array}$ & $\begin{array}{c}1250- \\
1350\end{array}$ & \begin{tabular}{|c|}
$1350-$ \\
1450 \\
\end{tabular} \\
\hline Arkansas & 11 & 6 & 2 & & & & & & & 1 & & & & \\
\hline Colorado & & & 2 & 3 & 1 & & 1 & 1 & & & & & & \\
\hline Connecticut & 6 & 2 & & & & & & & & & & & & \\
\hline Delaware & 6 & & 1 & & & & & & & & & & & \\
\hline Illinois & & 5 & 19 & 23 & 6 & 5 & 2 & 5 & 2 & 6 & 4 & 1 & 1 & \\
\hline Indiana & & 5 & 17 & 12 & 9 & 6 & 3 & 1 & & 3 & 4 & 1 & & \\
\hline lowa & & 7 & 13 & 17 & 17 & 13 & 6 & 6 & 1 & 4 & 1 & 2 & 4 & 1 \\
\hline Kansas & & & 6 & 12 & 10 & 10 & 5 & 3 & 1 & 1 & 11 & 5 & 2 & \\
\hline Kentucky & 3 & & 1 & 1 & 1 & & & & & & & & & \\
\hline Maine & 37 & 4 & & & & & & & & & 1 & & & \\
\hline Maryland & 7 & 2 & & & & & & & & & & & & \\
\hline Massachusetts & 18 & 1 & & & & & & & & & & & & \\
\hline Michigan & 9 & 13 & 12 & 5 & 5 & 1 & 3 & 1 & 1 & 3 & 1 & 2 & & 1 \\
\hline Minnesota & 1 & 9 & 33 & 22 & 22 & 13 & & 4 & 1 & 4 & 7 & 4 & 1 & \\
\hline Missouri & & 1 & 4 & 4 & 2 & 5 & & & 1 & 1 & 1 & & & \\
\hline Montana & 2 & 2 & 2 & 2 & 1 & & & & 1 & 1 & & & & 1 \\
\hline Nebraska & & 8 & 16 & 17 & 13 & 9 & 8 & 3 & & 10 & 4 & 1 & & \\
\hline New & 20 & 1 & & & & & & & & & & & & \\
\hline New Jersey & 5 & 2 & & 1 & & & & & & & & & & \\
\hline New Mexico & 2 & 5 & 4 & 3 & 5 & & 2 & & 1 & 1 & 1 & & & \\
\hline New York & 25 & 26 & 5 & 5 & 1 & & 2 & 1 & & & 1 & & & \\
\hline North Carolina & 6 & 2 & & 1 & 1 & & & & & & & & & \\
\hline North Dakota & & 6 & 13 & 10 & 10 & 6 & 3 & 2 & & 5 & 3 & 2 & & \\
\hline Ohio & & 4 & 9 & 4 & 4 & 2 & 6 & 1 & & 1 & 2 & 1 & & \\
\hline Oklahoma & 4 & 9 & 14 & 21 & 11 & 7 & & 5 & & 5 & 4 & 1 & 1 & \\
\hline Pennsylvania & 48 & 7 & 1 & & & & & & & & & & & \\
\hline Rhode Island & 4 & 3 & & & & & & & & & & & & \\
\hline South Dakota & 2 & 9 & 14 & 18 & 13 & 13 & 6 & 2 & 2 & 5 & 4 & 2 & 1 & \\
\hline Tennessee & 7 & 1 & & & & & & & & & & & & \\
\hline Texas & & & 11 & 8 & 6 & 4 & 3 & 1 & 1 & 2 & 4 & 6 & 2 & \\
\hline Vermont & 14 & 3 & & & & & & & & & & & & \\
\hline Virginia & 13 & 1 & 2 & & & & & & & & & & & \\
\hline West Virginia & 15 & 2 & & 1 & & & & & & & & & & \\
\hline Wisconsin & & 8 & 14 & 4 & 8 & 1 & 2 & 1 & & 4 & 1 & 1 & & \\
\hline
\end{tabular}


Table 3:Installed Capacity by State and Size of Plant

\begin{tabular}{|c|c|c|c|c|c|c|c|c|c|c|c|c|c|c|c|}
\hline State & $\begin{array}{l}0- \\
150\end{array}$ & $\begin{array}{l}150 \\
- \\
250\end{array}$ & $\begin{array}{l}250 \\
- \\
350\end{array}$ & $\begin{array}{l}350 \\
- \\
450\end{array}$ & $\begin{array}{l}450- \\
550\end{array}$ & $\begin{array}{l}550 \\
- \\
650\end{array}$ & $\begin{array}{l}650 \\
- \\
750\end{array}$ & $\begin{array}{l}750 \\
- \\
850\end{array}$ & $\begin{array}{l}850 \\
- \\
950\end{array}$ & $\begin{array}{l}950- \\
1050\end{array}$ & $\begin{array}{l}1050 \\
- \\
1150\end{array}$ & $\begin{array}{l}1150 \\
- \\
1250\end{array}$ & $\begin{array}{l}1250 \\
- \\
1350\end{array}$ & $\begin{array}{l}1350 \\
- \\
1450\end{array}$ & $\begin{array}{l}\text { Total } \\
\text { - GW }\end{array}$ \\
\hline Arkansas & 1342 & 1101 & 557 & & & & & & & 1049 & & & & & 4 \\
\hline Colorado & & & 541 & 1191 & 456 & & 732 & 840 & & & & & & & 3 \\
\hline Connecticut & 685 & 346 & & & & & & & & & & & & & 1 \\
\hline Delaware & 688 & & 330 & & & & & & & & & & & & 1 \\
\hline Illinois & & 1162 & 5776 & 9076 & 3021 & 2854 & 1357 & 3979 & 1747 & 6164 & 4370 & 1234 & 1291 & & 42 \\
\hline Indiana & & 1135 & 4963 & 4878 & 4564 & 3663 & 2181 & 823 & & 3104 & 4456 & 1199 & & & 31 \\
\hline lowa & & 1595 & 3989 & 6895 & 8474 & 7798 & 4081 & 4762 & 919 & 4024 & 1107 & 2414 & 5083 & 1435 & 53 \\
\hline Kansas & & & 1778 & 4936 & 4997 & 5918 & 3441 & 2418 & 906 & 1011 & 12153 & 5930 & 2581 & & 46 \\
\hline Kentucky & 300 & & 264 & 381 & 545 & & & & & & & & & & 1 \\
\hline Maine & 4026 & 753 & & & & & & & & & 1084 & & & & 6 \\
\hline Maryland & 769 & 345 & & & & & & & & & & & & & 1 \\
\hline Massachusetts & 1998 & 168 & & & & & & & & & & & & & 2 \\
\hline Michigan & 1029 & 2671 & 3508 & 1992 & 2470 & 578 & 2107 & 797 & 896 & 3085 & 1082 & 2369 & & 1361 & 24 \\
\hline Minnesota & 147 & 2036 & 9839 & 8973 & 10774 & 7726 & & 3209 & 880 & 4075 & 7762 & 4777 & 1281 & & 61 \\
\hline Missouri & & 245 & 1316 & 1658 & 950 & 2907 & & & 878 & 1038 & 1147 & & & & 10 \\
\hline Montana & 269 & 463 & 598 & 772 & 497 & & & & 850 & 1025 & & & & 1357 & 6 \\
\hline Nebraska & & 1875 & 4708 & 6792 & 6289 & 5279 & 5509 & 2273 & & 10209 & 4366 & 1171 & & & 48 \\
\hline New & 2188 & 183 & & & & & & & & & & & & & 2 \\
\hline New Jersey & 548 & 357 & & 423 & & & & & & & & & & & 1 \\
\hline New Mexico & 203 & 1076 & 1161 & 1207 & 2396 & & 1418 & & 897 & 1038 & 1128 & & & & 11 \\
\hline New York & 2756 & 4992 & 1373 & 1934 & 516 & & 1377 & 825 & & & 1086 & & & & 15 \\
\hline North Carolina & 642 & 386 & & 425 & 546 & & & & & & & & & & 2 \\
\hline North Dakota & & 1267 & 4016 & 4035 & 4879 & 3500 & 2141 & 1570 & & 5121 & 3222 & 2388 & & & 32 \\
\hline Ohio & & 822 & 2715 & 1540 & 1892 & 1194 & 4098 & 795 & & 969 & 2212 & 1207 & & & 17 \\
\hline Oklahoma & 400 & 1927 & 4179 & 8295 & 5336 & 4222 & & 4016 & & 5062 & 4361 & 1163 & 1291 & & 40 \\
\hline Pennsylvania & 5517 & 1176 & 294 & & & & & & & & & & & & 7 \\
\hline Rhode Island & 462 & 578 & & & & & & & & & & & & & 1 \\
\hline South Dakota & 271 & 1847 & 4312 & 7279 & 6376 & 7708 & 4247 & 1529 & 1772 & 5047 & 4480 & 2374 & 1304 & & 49 \\
\hline Tennessee & 730 & 156 & & & & & & & & & & & & & 1 \\
\hline Texas & & & 3317 & 3142 & 2874 & 2440 & 2176 & 789 & 890 & 2046 & 4413 & 7196 & 2613 & & 32 \\
\hline Vermont & 1537 & 482 & & & & & & & & & & & & & 2 \\
\hline Virginia & 1340 & 197 & 561 & & & & & & & & & & & & 2 \\
\hline West Virginia & 1543 & 430 & & 403 & & & & & & & & & & & 2 \\
\hline Wisconsin & & 1611 & 4245 & 1597 & 3940 & 560 & 1397 & 753 & & 4035 & 1125 & 1230 & & & 20 \\
\hline Total GW & 29 & 31 & 64 & 78 & 72 & 56 & 36 & 29 & 11 & 58 & 60 & 35 & 15 & 42 & 580 \\
\hline
\end{tabular}

As shown in Table 3 approximately $8 \%$ (or $48 \mathrm{GW}$ ) of the wind plant capability identified in this 34-state database resides in Nebraska. It also shows that the potential installations listed for Nebraska are about ten times the largest penetration scenario in this study.

\section{Section 8.2.2 Wind Site Selection and Analysis}

\section{Section 8.2.2.1 Wind Scenario Development by Region}

Wind generation siting for the Nebraska Statewide Wind Integration Study were selected from the wind resources identified in the NREL mesoscale database. The selection criteria were based upon plant capability, energy, location dispersion and ability to meet the energy criteria for each scenario. 
Wind selection was made for Nebraska labeled as the Nebraska Power Association (NPA) and the rest of the Southwest Power Pool (SPP). It was determined at the study's Technical Review Committee (TRC) meeting in March that the wind siting identified in the reference case for the Eastern Wind Integration and Transmission Study (EWITS) would be used. This provides a constant reference point with this interconnection and focuses the wind penetration impact on NPA, and SPP. The Western Area Power Administration (WAPA) and the Dakotas (MAPP) are represented in the EWITS reference case and held constant. The Tennessee Valley Authority (TVA) wind sites also remain constant for each scenario.

The energy levels for wind selection were based upon the estimated system energy for each region in year 2018. NPA calculated the wind generation energy necessary to provide an estimated $1 \%$ penetration for Nebraska in year 2018. The amount, $405 \mathrm{GWh}$ included consideration of both retail load and transmission and distribution losses from the generation bus to the retail meter bus. Losses of $7.4 \%$ were assumed which would make the $405 \mathrm{GWh}$ generated equivalent to $375 \mathrm{GWh}$ to serve retail load and with the balance of $30 \mathrm{GWh}$ for losses. The energy targets for NPA were established by multiplying the $405 \mathrm{GWh}$ by 10, 20 and 40 for each respective scenario Table 4, Figure 1, and Figure 2.

The energy targets for SPP were derived from publicly available data filed by the utilities with FERC and adjusted to year 2018 assuming typical growth rate. For these regions wind penetration levels of $10 \%, 20 \%$ and $40 \%$ of energy were calculated Table 5, Figure 1 and Figure 2. Wind sites from the mesoscale database were selected to closely achieve meeting these energy levels.

Table 4: $\quad$ Nebraska Selected Wind Generation Penetration Details

\begin{tabular}{|l|r|r|r|}
\hline \multicolumn{1}{|c|}{ Nebraska } & \multicolumn{1}{c|}{ 10\% Pen } & 20\% Pen & \multicolumn{1}{c|}{ 40\% Pen } \\
\hline Target (GWH) & 4,052 & 8,104 & 16,208 \\
\hline Selected (GWH) & 4,523 & 8,856 & 16,831 \\
\hline$\%$ of Target & $112 \%$ & $109 \%$ & $104 \%$ \\
Nameplate (MW) & 1,249 & 2,488 & 4,727 \\
\hline Capacity Factor & 0.41 & 0.41 & 0.41 \\
\hline Number of Sites & 5 & 8 & 11 \\
\hline Average Site Size & 250 & 311 & 430 \\
\hline
\end{tabular}

Table 5: $\quad$ SPP w/o Nebraska Selected Wind Generation Penetration Details

\begin{tabular}{|l|r|r|r|}
\hline \multicolumn{1}{|c|}{ SPP } & 10\% Pen & 20\% Pen & 40\% Pen \\
\hline Target (GWH) & 22,321 & 44,641 & 89,283 \\
\hline Selected (GWH) & 22,901 & 45,807 & 91,548 \\
\hline \% of Target & $103 \%$ & $103 \%$ & $103 \%$ \\
Nameplate (MW) & 6,256 & 12,596 & 25,431 \\
\hline Capacity Factor & 0.41 & 0.41 & 0.40 \\
\hline Number of Sites & 7 & 18 & 33 \\
\hline Average Site Size (MW) & 894 & 700 & 771 \\
\hline
\end{tabular}




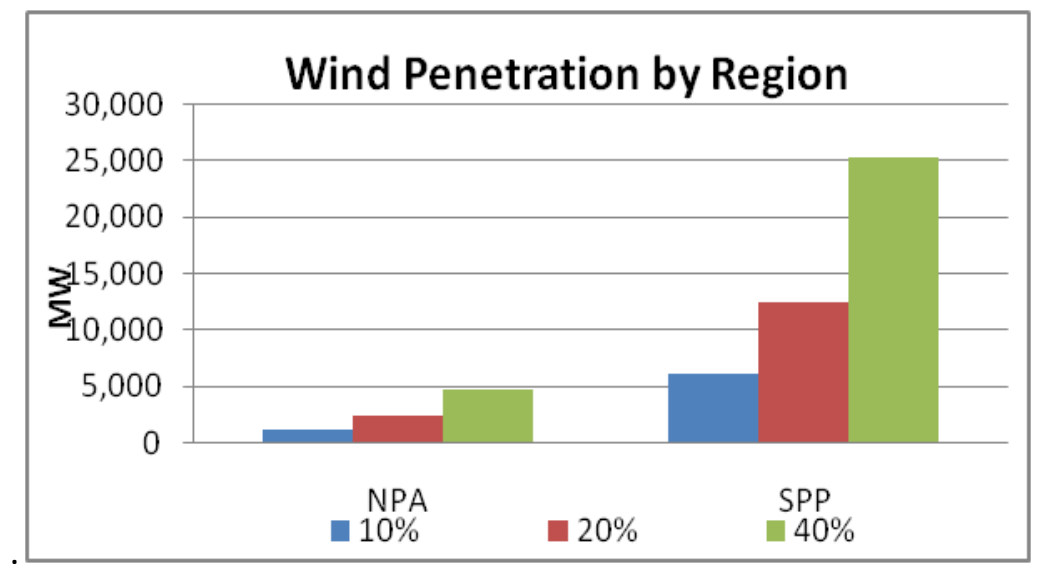

Figure 1: Scenario Nameplate Capacity summary by Region

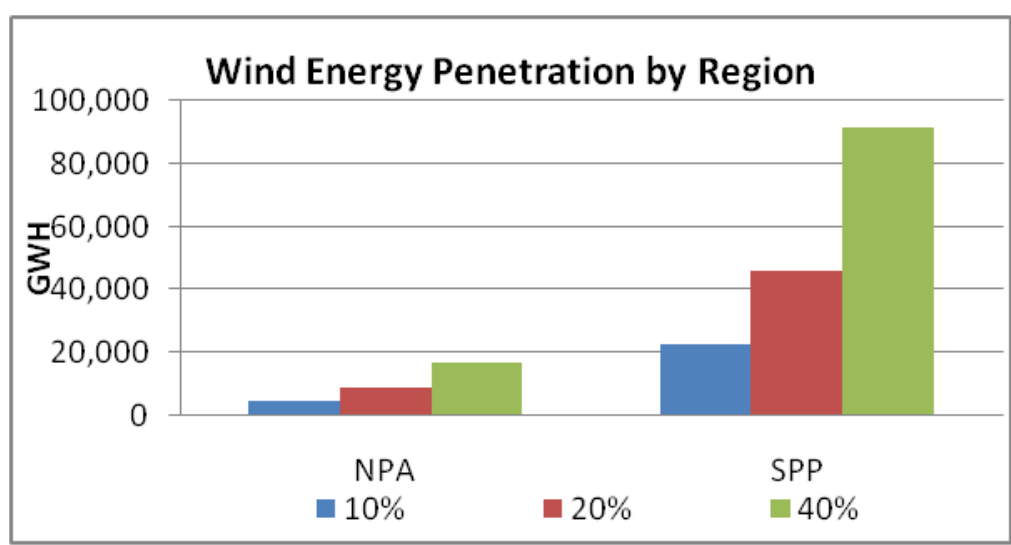

Figure 2: Average Annual Energy by Scenario by Region

Table 6: $\quad$ Summary of Capacity, Capacity Factor and Energy for each Scenario by Region

\begin{tabular}{|l|r|r|r|r|r|r|r|r|r|}
\hline & \multicolumn{3}{|c|}{$10 \%$ Penetration } & \multicolumn{2}{c|}{$20 \%$ Penetration } & \multicolumn{2}{c|}{$40 \%$ Penetration } \\
\cline { 2 - 9 } \multicolumn{1}{|c}{ Region } & $\begin{array}{c}\text { Name } \\
\text { Plate }\end{array}$ & \multicolumn{1}{c|}{ CF } & $\begin{array}{c}\text { Reference } \\
\text { Energy }\end{array}$ & $\begin{array}{c}\text { Name } \\
\text { Plate }\end{array}$ & CF & $\begin{array}{c}\text { Reference } \\
\text { Energy }\end{array}$ & $\begin{array}{c}\text { Name } \\
\text { Plate }\end{array}$ & $\begin{array}{c}\text { Reference } \\
\text { Energy }\end{array}$ \\
\hline NPA & 1,249 & $41 \%$ & 4,523 & 2,488 & $41 \%$ & 8,855 & 4,727 & $41 \%$ & 16,831 \\
SPP & 6,256 & $42 \%$ & 22,901 & 12,596 & $41 \%$ & 45,807 & 25,431 & $41 \%$ & 91,548 \\
PJM & 25,807 & $36 \%$ & 81,460 & 25,807 & $36 \%$ & 81,460 & 25,807 & $36 \%$ & 81,460 \\
MISO/MAPP & 19,547 & $36 \%$ & 61,700 & 19,547 & $36 \%$ & 61,700 & 19,547 & $36 \%$ & 61,700 \\
SERC & 3,615 & $36 \%$ & 11,410 & 3,615 & $36 \%$ & 11,410 & 3,615 & $36 \%$ & 11,410 \\
TVA & 1,397 & $36 \%$ & 4,410 & 1,397 & $36 \%$ & 4,410 & 1,397 & $36 \%$ & 4,410 \\
\hline Total & 57,871 & $37 \%$ & 186,405 & 65,450 & $37 \%$ & 213,642 & 80,524 & $38 \%$ & 267,359 \\
\hline
\end{tabular}

The following figures provide visual locations of wind siting utilized in the study. The sitings identified for MISO include the MAPP and WAPA regions. These sitings along with the TVA sitings are constant for each scenario. Wind penetrations are varied only in the NPA and SPP areas. 
The map in Figure 3 shows the Wind Siting for the EWITS Reference Case. Figure 4 is a sub set of the EWITS showing West MISO which includes MAPP and WAPA areas. TVA sitings are shown in Figure 5. Sitings for Nebraska are shown in Figure 6 - Figure 8 and details are listed in Table 7. The Reference Energy column in Table 7is the average annual energy for three years of hourly data. The SPP siting, not including Nebraska, are shown in Figure 9 - Figure 11.

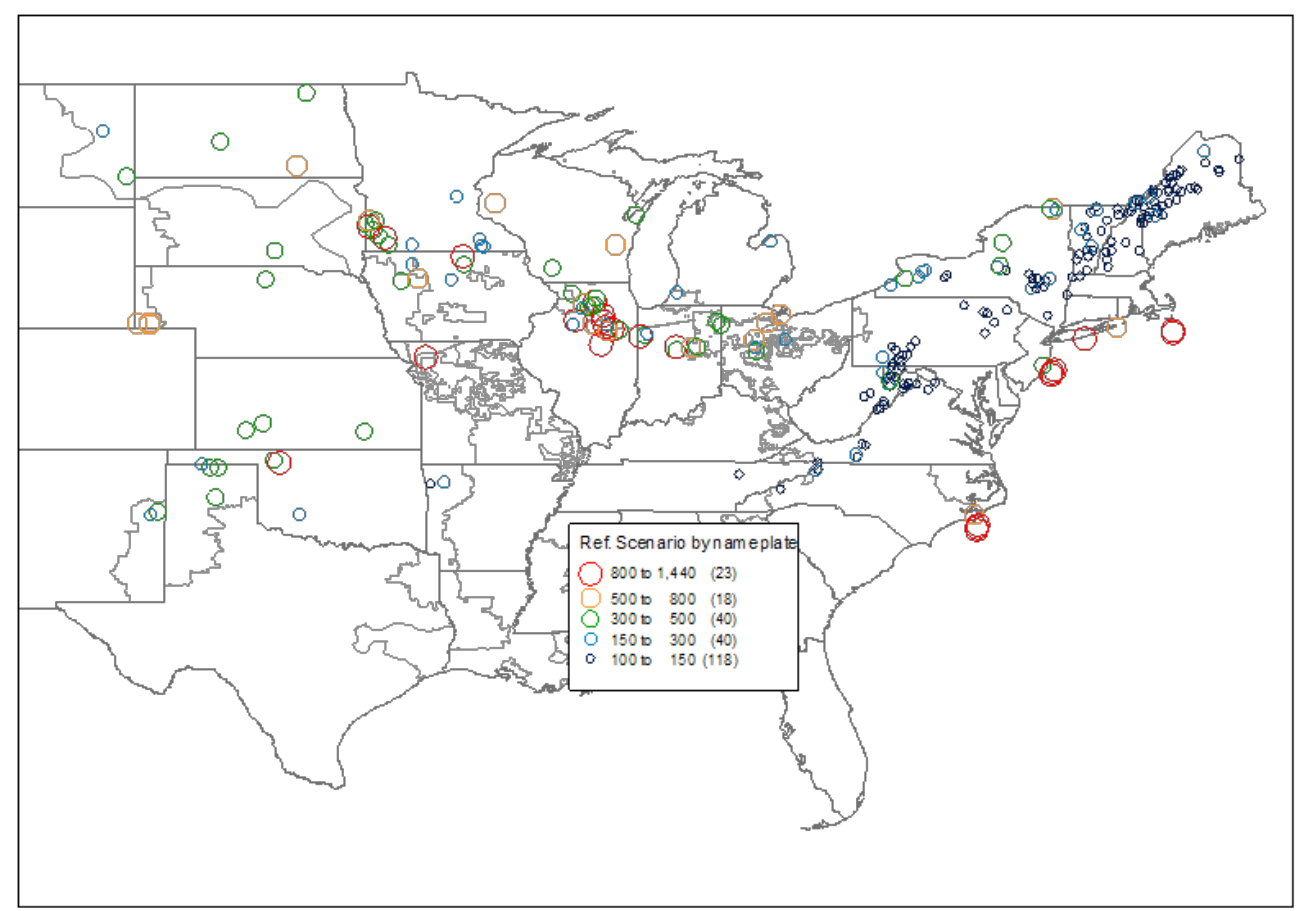

Figure 3: Wind Siting for the EWITS Reference Case - All sites 


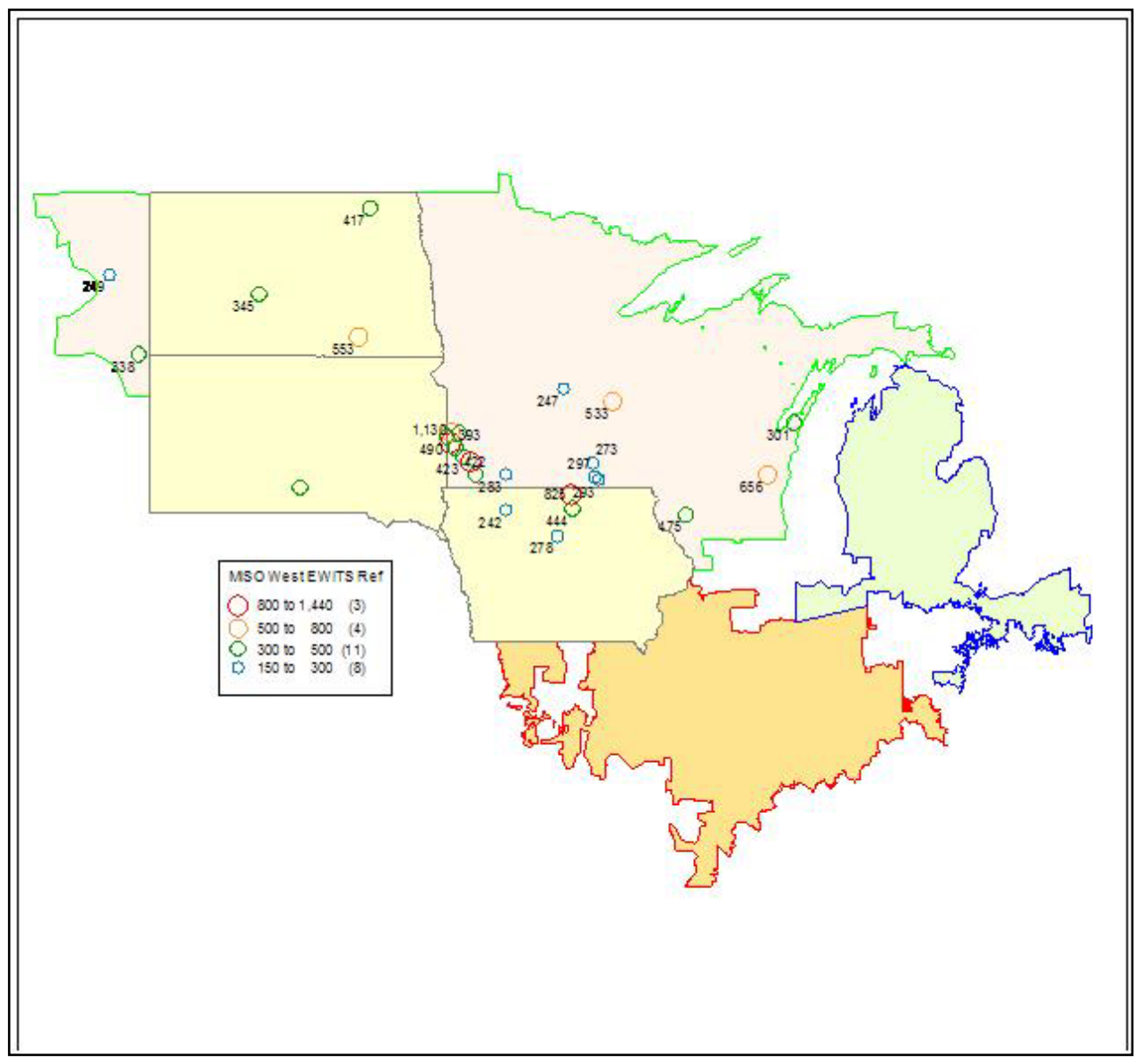

Figure 4: MISO West EWITS Reference case 


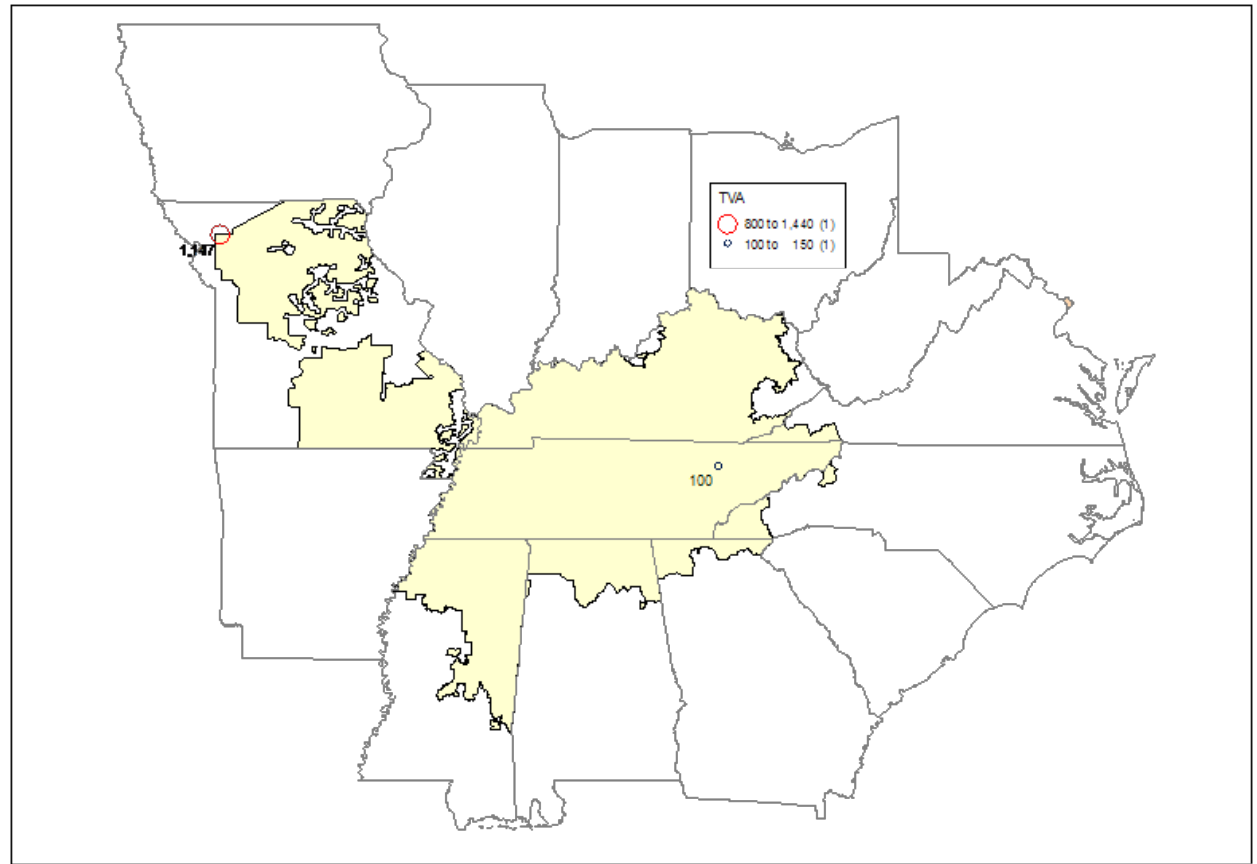

Figure 5: TVA Site ID's for all Penetrations

Table 7: $\quad$ Nebraska Site Details

\begin{tabular}{|r|r|r|r|r|r|r|r|}
\hline $\begin{array}{c}\text { Penetration } \\
\text { Level }\end{array}$ & Site ID & Lat & \multicolumn{1}{c|}{ Lon } & \multicolumn{1}{c|}{$\begin{array}{c}\text { Name } \\
\text { plate }\end{array}$} & $\begin{array}{c}\text { Capacity } \\
\text { Factor }\end{array}$ & $\begin{array}{c}\text { Reference } \\
\text { Energy }\end{array}$ & GWH ID \\
\hline $10 \%$ & 22 & 41.92 & -97.77 & 261 & 0.42 & 971 & 640226 \\
\hline $10 \%$ & 143 & 42.66 & -98.66 & 268 & 0.41 & 966 & 652509 \\
$10 \%$ & 208 & 41.05 & -97.29 & 245 & 0.41 & 880 & 640271 \\
$10 \%$ & 160 & 41.84 & -101.27 & 240 & 0.41 & 869 & 659247 \\
$10 \%$ & 205 & 42.07 & -98.16 & 235 & 0.41 & 837 & 640227 \\
\hline $20 \%$ & 47 & 41.92 & -97.68 & 285 & 0.41 & 1027 & 640226 \\
\hline $20 \%$ & 245 & 42.66 & -100.80 & 453 & 0.41 & 1619 & 640392 \\
\hline $20 \%$ & 1149 & 40.66 & -97.97 & 501 & 0.38 & 1686 & 640271 \\
\hline $40 \%$ & 70 & 42.65 & -98.42 & 1100 & 0.41 & 3958 & 652509 \\
\hline $40 \%$ & 695 & 41.19 & -103.54 & 660 & 0.39 & 2283 & 659133 \\
\hline $40 \%$ & 76 & 41.77 & -101.11 & 479 & 0.41 & 1734 & 640183 \\
\hline
\end{tabular}




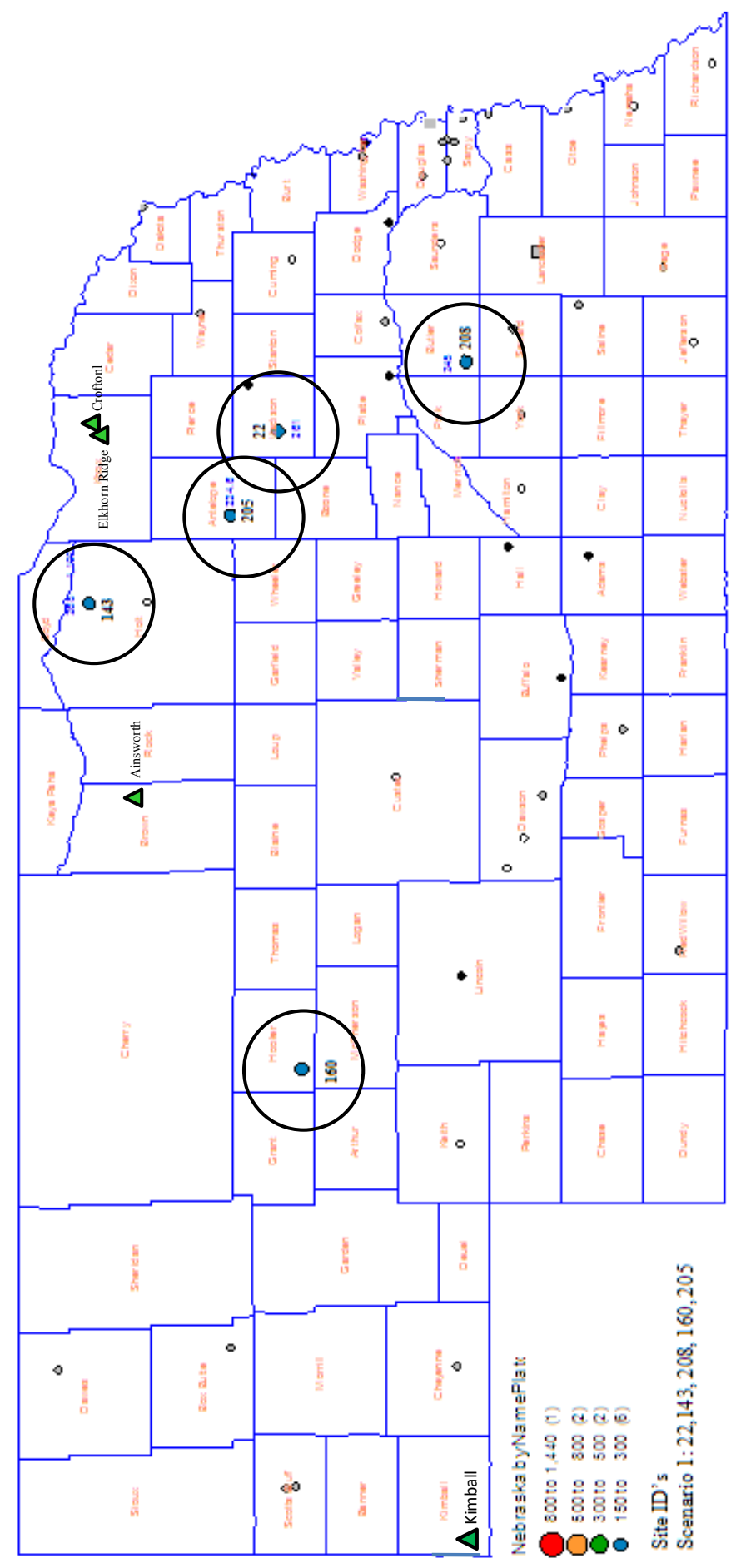

Figure 6: NPA Site ID's for 10\% Penetration 
Figure 7 identifies with circles the Nebraska wind sites that are added to the sites in the $10 \%$ penetration scenario make the $20 \%$ penetration scenario.

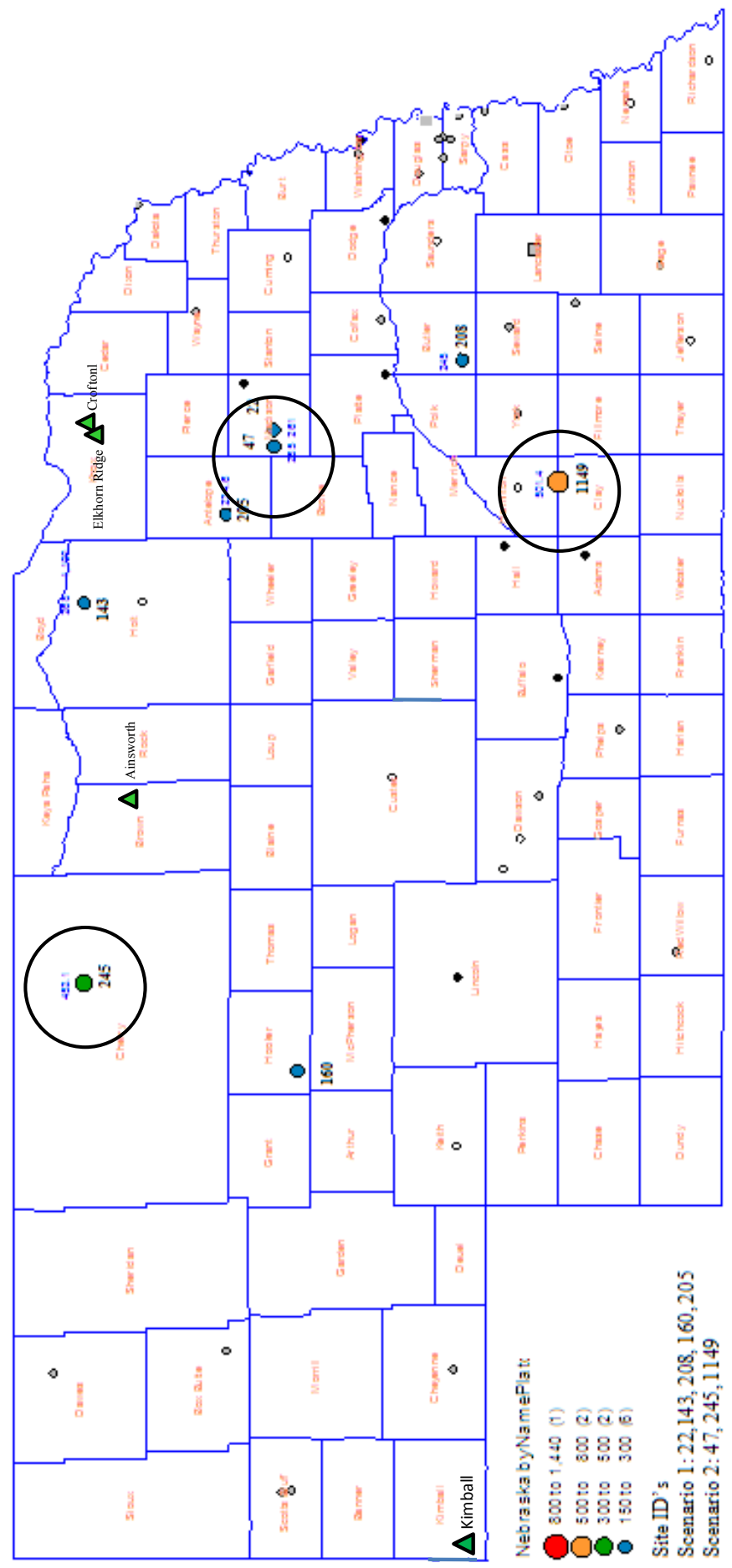

Figure 7: NPA Site ID's for 20\% Penetration 
Figure 8 identifies with circles the Nebraska wind sites that are added to the sites in the $20 \%$ penetration scenario to make the $40 \%$ penetration scenario.

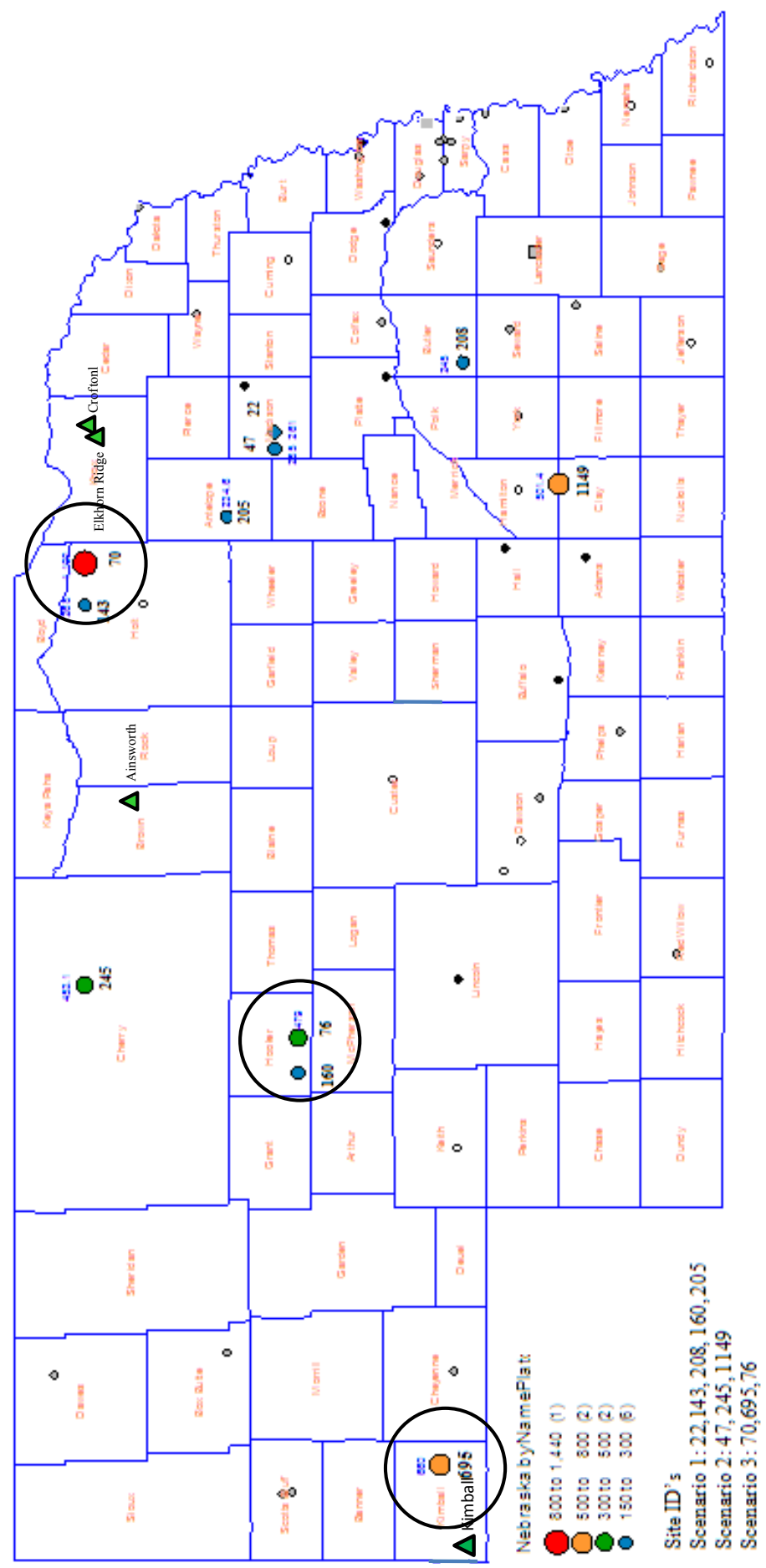

Figure 8: Nebraska Site ID's for $40 \%$ Penetration 


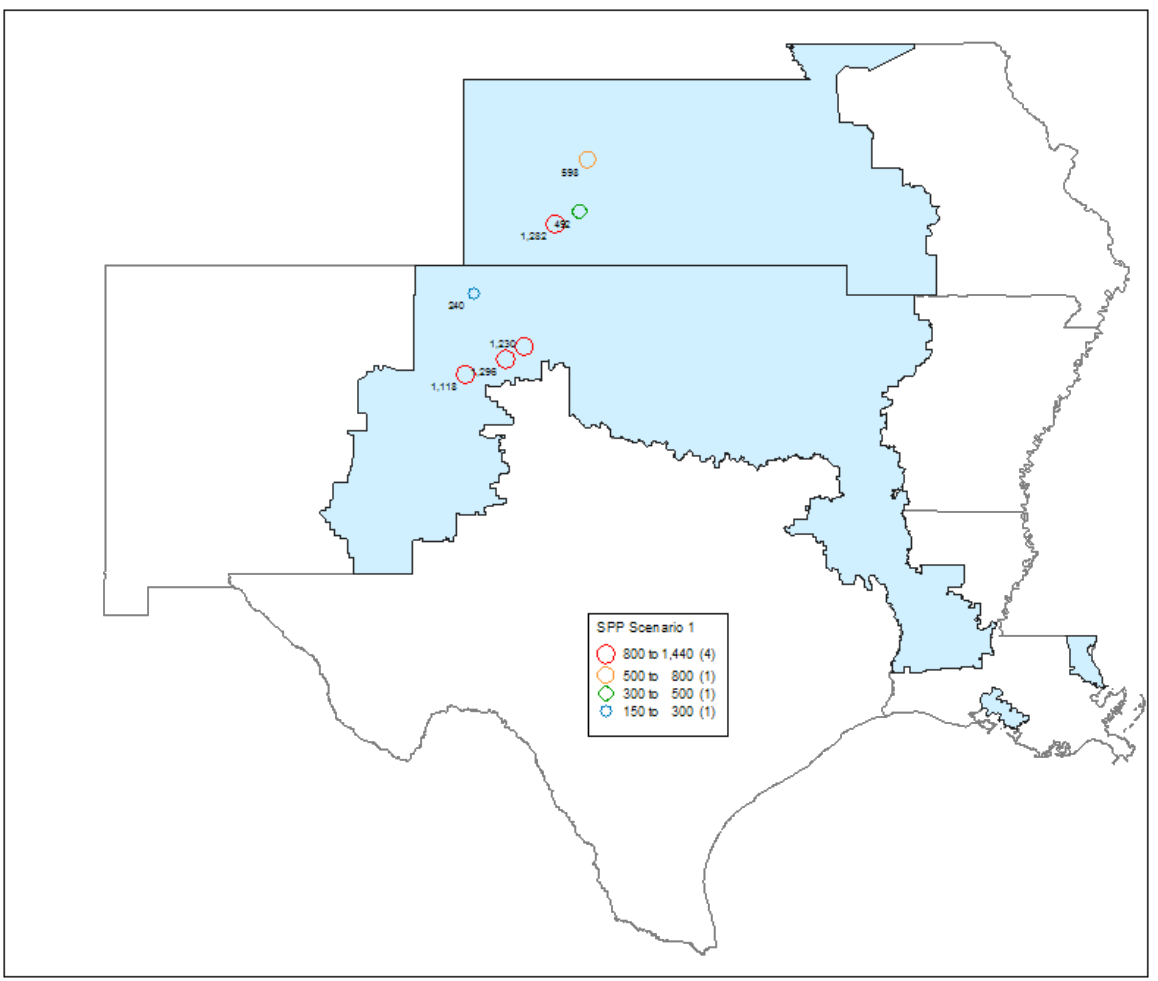

Figure 9: SPP Site ID's for 10\% Penetration

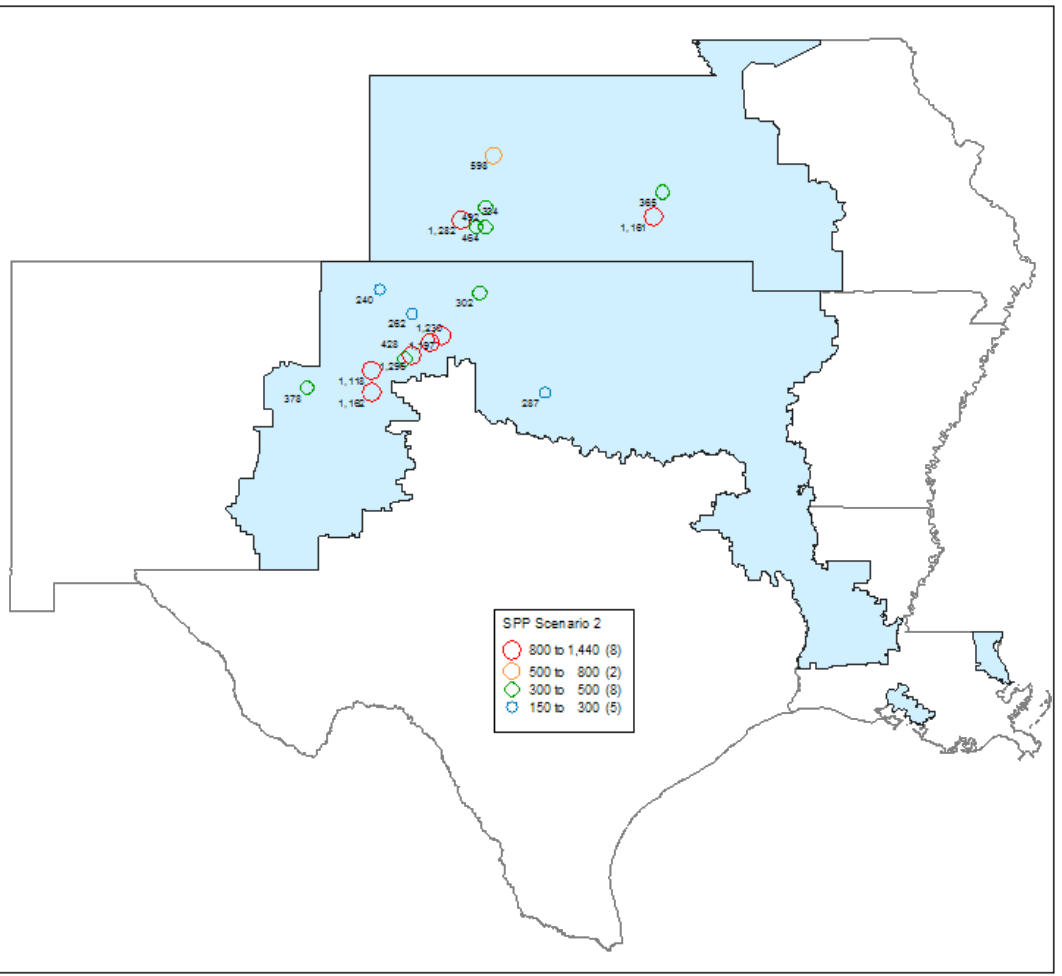

Figure 10: SPP Site ID's for 20\% Penetration 


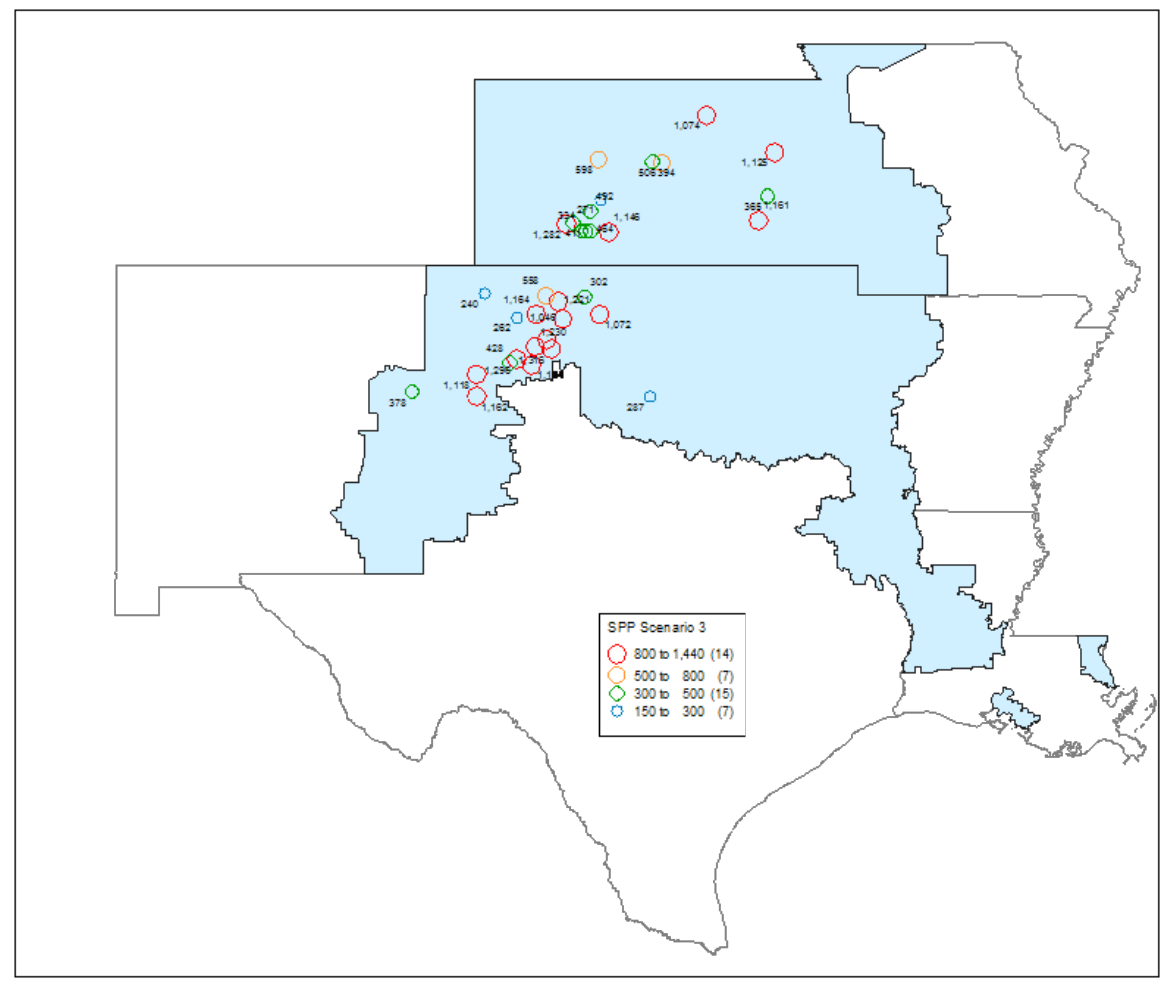

Figure 11: SPP Site ID's for 40\% Penetration

\section{Section 8.2.2.2 Installed Capacity by Scenario}

Table 8 shows a summary of the nameplate values for the aggregated wind sites modeled in the study for NPA, the rest of SPP, and for the rest of the system.

Table 8: Wind Generation Capacity Assumptions by Region

\begin{tabular}{|l|r|r|r|}
\hline \multicolumn{1}{|c|}{ Region } & $\begin{array}{r}10 \% \text { Penetration } \\
\text { Nameplate (MW) }\end{array}$ & $\begin{array}{l}\text { 20\% Penetration } \\
\text { Nameplate (MW) }\end{array}$ & $\begin{array}{l}\text { 40\% Penetration } \\
\text { Nameplate (MW) }\end{array}$ \\
\hline NPA & 1,249 & 2,488 & 4,727 \\
SPP & 6,256 & 12,596 & 25,431 \\
Rest of System & 50,366 & 50,366 & 50,366 \\
\hline Total & 57,871 & 65,450 & 80,524 \\
\hline
\end{tabular}

\section{Section 8.2.2.3 Annual Energy \& Capacity Factors}

Table 9, Table 10, and Table 11 show summaries of the aggregate wind energy from the selected sites for NPA and the rest of SPP for three historical years 2004-2006. Table 12, Table 13, and Table 14 convert the data in Table 8 - Table 11 into annual capacity factor values. 
Table 9: $\quad$ Annual Wind Energy (GWh) by Region - 10\% Penetration

\begin{tabular}{|l|r|r|r|}
\hline \multicolumn{4}{|c|}{ Annual Energy by Region - 10\% } \\
\hline Region & 2004 & 2005 & 2006 \\
\hline NPA & 4,326 & 4,549 & 4,736 \\
SPP & 22,045 & 23,144 & 23,448 \\
\hline
\end{tabular}

Table 10: Annual Wind Energy (GWh) by Region - 20\% Penetration

\begin{tabular}{|l|r|r|r|}
\hline \multicolumn{4}{|c|}{ Annual Energy by Region - 20\% } \\
\hline Region & 2004 & 2005 & 2006 \\
\hline NPA & 8,478 & 8,945 & 9,235 \\
\hline SPP & 44,270 & 45,719 & 47,221 \\
\hline
\end{tabular}

Table 11: Annual Wind Energy (GWh) by Region - 40\% Penetration

\begin{tabular}{|l|r|r|r|}
\hline \multicolumn{4}{|c|}{ Annual Energy by Region -40\% } \\
\hline Region & 2004 & 2005 & 2006 \\
\hline NPA & 16,317 & 16,992 & 17,696 \\
\hline SPP & 88,704 & 91,583 & 94,470 \\
\hline
\end{tabular}

Table 12: Aggregate Capacity Factor by Region - 10\% Penetration

\begin{tabular}{|l|r|r|r|}
\hline \multicolumn{4}{|c|}{ Aggregate Capacity Factor by Region $10 \%$} \\
\hline Region & 2004 & 2005 & 2006 \\
\hline NPA & $39.5 \%$ & $41.6 \%$ & $43.3 \%$ \\
\hline SPP & $40.2 \%$ & $42.2 \%$ & $42.8 \%$ \\
\hline
\end{tabular}

Table 13: Aggregate Capacity Factor by Region - 20\% Penetration

\begin{tabular}{|l|r|r|r|}
\hline \multicolumn{3}{|c|}{ Aggregate Capacity Factor by Region $20 \%$} \\
\hline Region & 2004 & 2005 & 2006 \\
\hline NPA & $38.9 \%$ & $41.0 \%$ & $42.4 \%$ \\
\hline SPP & $40.1 \%$ & $41.4 \%$ & $42.8 \%$ \\
\hline
\end{tabular}

Table 14: Aggregate Capacity Factor by Region - 40\% Penetration

\begin{tabular}{|l|r|r|r|}
\hline \multicolumn{3}{|c|}{ Aggregate Capacity Factor by Region $40 \%$} \\
\hline Region & 2004 & 2005 & 2006 \\
\hline NPA & $39.4 \%$ & $41.0 \%$ & $42.7 \%$ \\
\hline SPP & $39.8 \%$ & $41.1 \%$ & $42.4 \%$ \\
\hline
\end{tabular}

Section 8.2.2.4

Wind Generation Capacity Factors (monthly and accumulative)

Figure 12 shows the aggregated wind capacity factor for NPA penetrations. The generally high capacity factor can be attributed to the wind site diversity. Observe not only the monthly variations but also from year to year (2006 is the highest overall). 


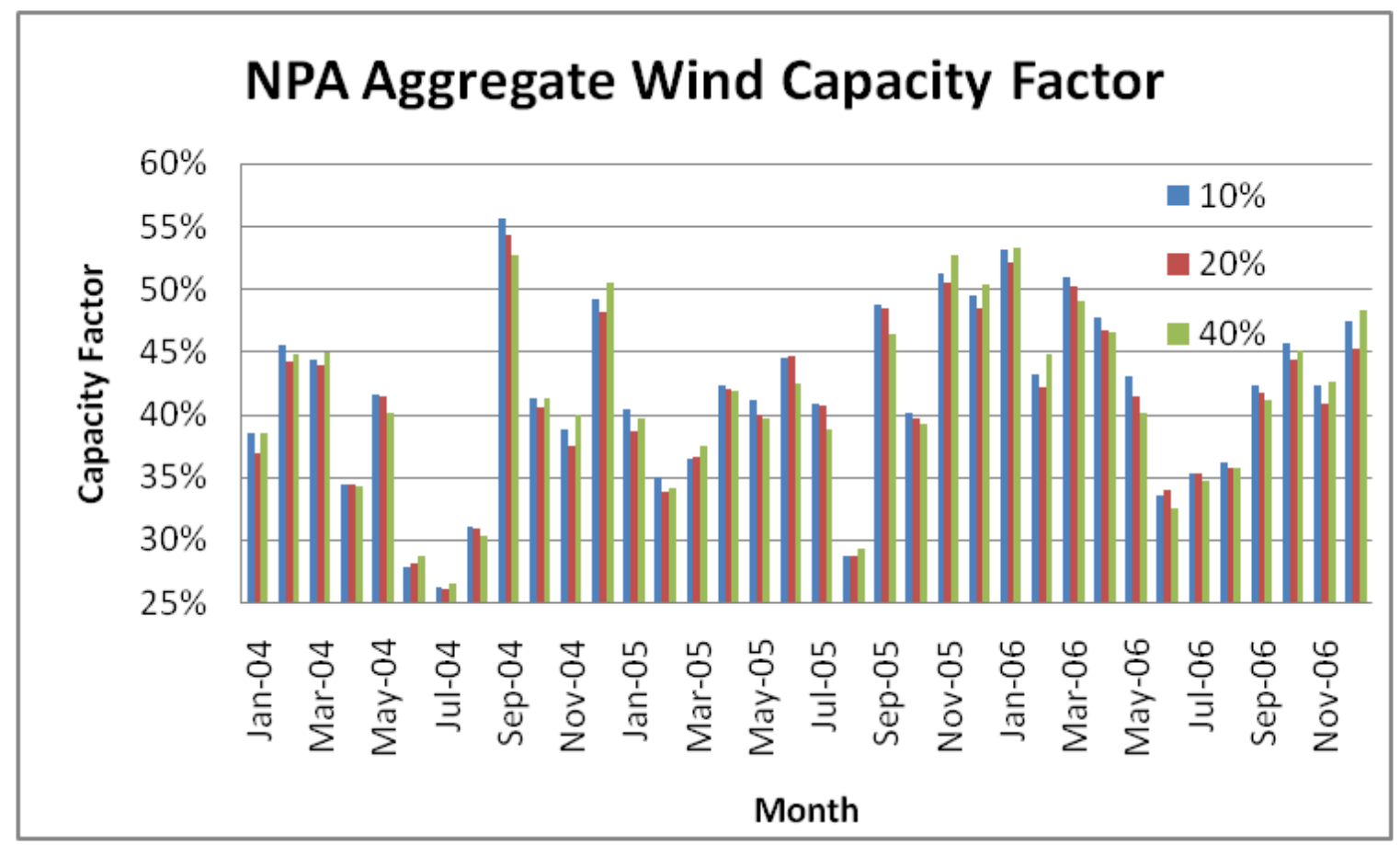

Figure 12: NPA Aggregated Capacity Factor for Wind Generation

Table 15: Monthly Capacity Factors for NPA wind

\begin{tabular}{|c|c|c|c|c|c|c|c|c|c|c|c|c|}
\hline $\begin{array}{c}\text { NPA } \\
\text { Aggregate } \\
\text { Wind }\end{array}$ & Jan & Feb & Mar & Apr & May & Jun & Jul & Aug & Sep & Oct & Nov & Dec \\
\hline $10 \%$ CF 2004 & $39 \%$ & $46 \%$ & $44 \%$ & $34 \%$ & $42 \%$ & $28 \%$ & $26 \%$ & $31 \%$ & $56 \%$ & $41 \%$ & $39 \%$ & $49 \%$ \\
\hline $10 \%$ CF 2005 & $40 \%$ & $35 \%$ & $36 \%$ & $42 \%$ & $41 \%$ & $45 \%$ & $41 \%$ & $29 \%$ & $49 \%$ & $40 \%$ & $51 \%$ & $49 \%$ \\
\hline $10 \%$ CF 2006 & $53 \%$ & $43 \%$ & $51 \%$ & $48 \%$ & $43 \%$ & $34 \%$ & $35 \%$ & $36 \%$ & $42 \%$ & $46 \%$ & $42 \%$ & $47 \%$ \\
\hline $20 \%$ CF 2004 & $37 \%$ & $44 \%$ & $44 \%$ & $34 \%$ & $42 \%$ & $28 \%$ & $26 \%$ & $31 \%$ & $54 \%$ & $41 \%$ & $38 \%$ & $48 \%$ \\
\hline $20 \%$ CF 2005 & $39 \%$ & $34 \%$ & $37 \%$ & $42 \%$ & $40 \%$ & $45 \%$ & $41 \%$ & $29 \%$ & $48 \%$ & $40 \%$ & $51 \%$ & $49 \%$ \\
\hline $20 \%$ CF 2006 & $52 \%$ & $42 \%$ & $50 \%$ & $47 \%$ & $42 \%$ & $34 \%$ & $35 \%$ & $36 \%$ & $42 \%$ & $44 \%$ & $41 \%$ & $45 \%$ \\
\hline $40 \%$ CF 2004 & $39 \%$ & $45 \%$ & $45 \%$ & $34 \%$ & $40 \%$ & $29 \%$ & $27 \%$ & $30 \%$ & $53 \%$ & $41 \%$ & $40 \%$ & $51 \%$ \\
\hline $40 \%$ CF 2005 & $40 \%$ & $34 \%$ & $38 \%$ & $42 \%$ & $40 \%$ & $42 \%$ & $39 \%$ & $29 \%$ & $46 \%$ & $39 \%$ & $53 \%$ & $50 \%$ \\
\hline $40 \%$ CF 2006 & $53 \%$ & $45 \%$ & $49 \%$ & $47 \%$ & $40 \%$ & $33 \%$ & $35 \%$ & $36 \%$ & $41 \%$ & $45 \%$ & $43 \%$ & $48 \%$ \\
\hline
\end{tabular}

Figure 13, Figure 14, and Figure 15 examine, in an accumulative fashion, the capacity factors for the 1,000 highest load hours for each of the historical years and each of the three penetrations. That is, the first data point at the left side is the aggregated capacity factor of the highest load hour (the July peak hour). Then the next data point is the average of the aggregated capacity factors for the two hours with the highest loads for the year, and so on. Note that the aggregated averages after 1,000 hours center around 30\% capacity factor thereby indicating that the wind is reduced during the higher load hours, as the capacity factors over all hours is approximately $40 \%$, or more. 


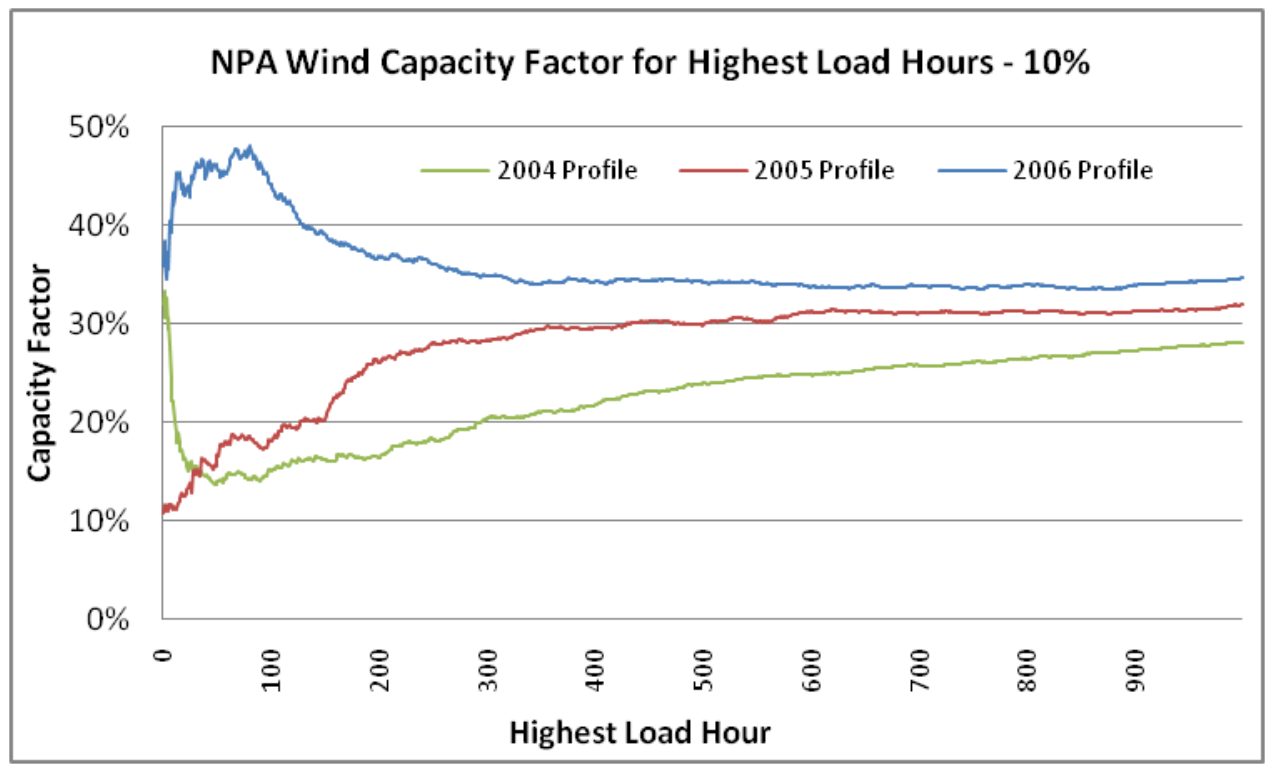

Figure 13: NPA 10\% Penetration Wind Capacity Factors during Highest Load Hours

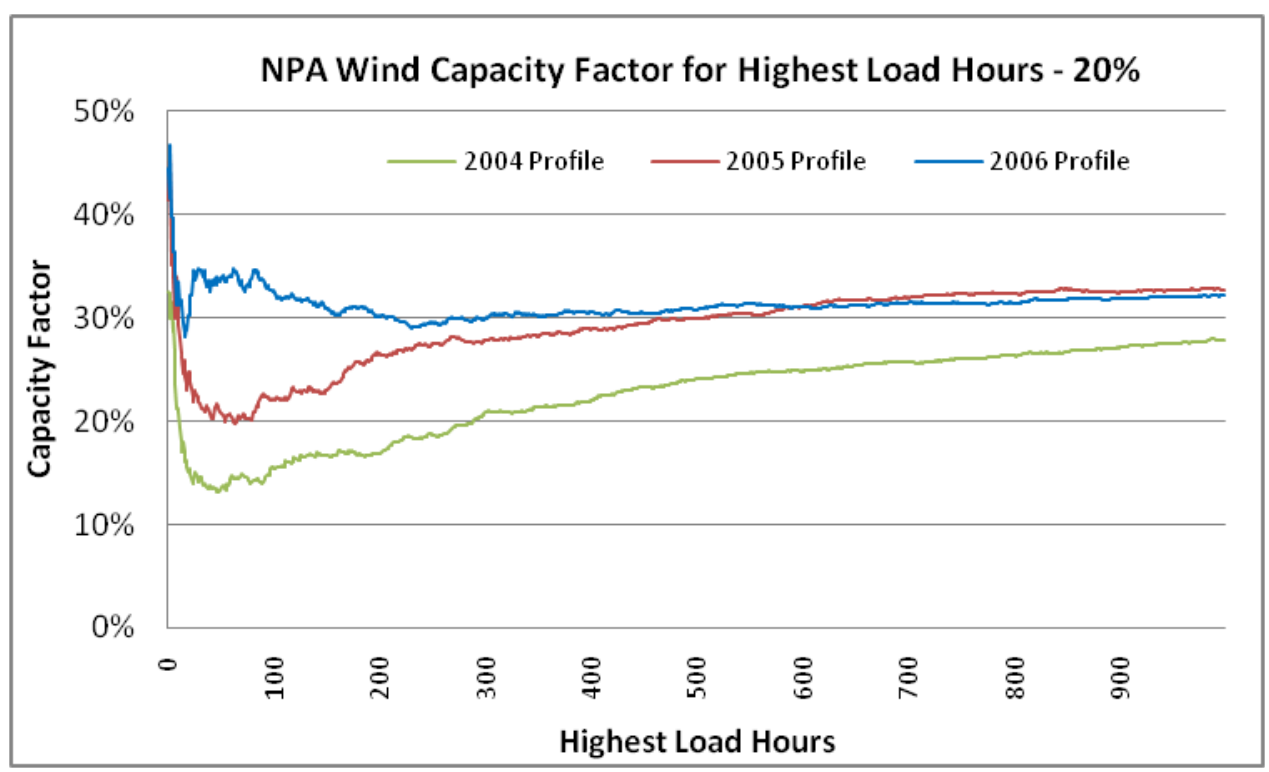

Figure 14: NPA 20\% Penetration Wind Capacity Factors during Highest Load Hours 


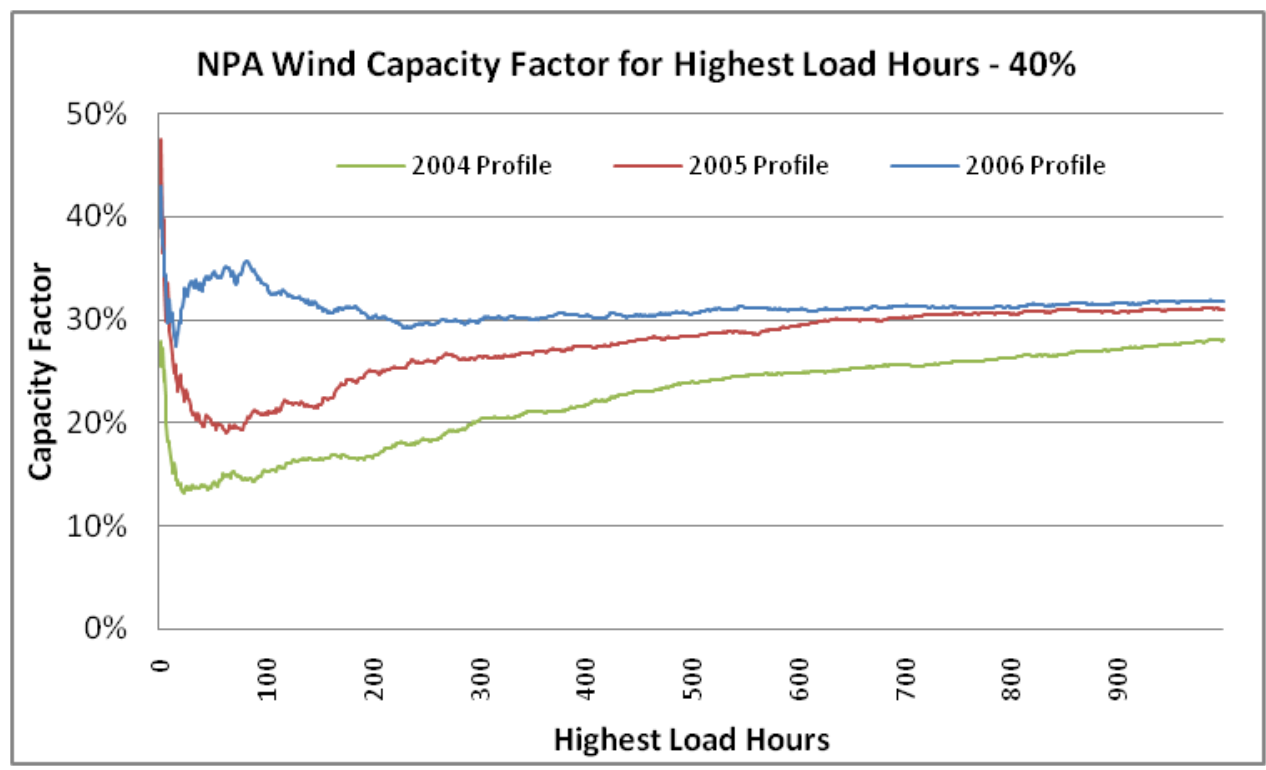

Figure 15: NPA 40\% Penetration Wind Capacity Factors during Highest Load Hours

\section{Section 8.2.2.5 Nebraska Wind Diversity}

Wind diversity for sites selected could be quantified by calculating the correlation between the hourly wind generation of each plant in the scenarios. A correlation value of 1 is a perfect correlation between sites meaning as one site increases so does the other. A correlation of 0 indicates no correlation. A correlation of -1 indicates a negative correlation meaning as one site increases in generation the other site decreases by the same amount. Values in between these limits shows where the data correlates to these limits.

Table 16 presents a matrix of correlations between wind sites. The correlation values for the 55 pairings of the eleven Nebraska wind sites might be quantified in the following way:

- Zero to .20 negligible correlation or high wind diversity (zero pairs)

- .20 to .40 low degree of correlation or marked degree of wind diversity (12 pairs)

- .40 to .60 moderate degree of correlation or moderate degree of wind diversity (15 pairs)

- .60 to .80 marked degree of correlation or low degree of wind diversity (14 pairs)

- .80 to 1.00 high correlation or low degree of wind diversity (14 pairs) 
Table 16: Correlation between NPA wind sites

\begin{tabular}{|c|c|c|c|c|c|c|c|c|c|c|c|}
\hline \multicolumn{12}{|c|}{ Correlation of hourly wind data between wind sites for actual wind data in years 2004 to 2006} \\
\hline \multirow[b]{2}{*}{ Site ID } & \multicolumn{5}{|c|}{\begin{tabular}{|c|}
$10 \%$ sites \\
\end{tabular}} & \multicolumn{3}{|c|}{$20 \%$ sites } & \multicolumn{3}{|c|}{$40 \%$ sites } \\
\hline & 22 & 143 & 160 & 205 & 208 & 47 & 245 & 1149 & 70 & 76 & 695 \\
\hline 22 & 1 & 0.77 & 0.47 & 0.93 & 0.81 & 0.99 & 0.49 & 0.78 & 0.82 & 0.49 & 0.24 \\
\hline 143 & 0.77 & 1 & 0.61 & 0.83 & 0.58 & 0.75 & 0.69 & 0.58 & 0.97 & 0.64 & 0.32 \\
\hline 160 & 0.47 & 0.61 & 1 & 0.55 & 0.35 & 0.46 & 0.83 & 0.39 & 0.60 & 0.98 & 0.57 \\
\hline 205 & 0.93 & 0.83 & 0.55 & 1 & 0.74 & 0.92 & 0.57 & 0.73 & 0.88 & 0.57 & 0.28 \\
\hline 208 & 0.81 & 0.58 & 0.35 & 0.74 & 1 & 0.82 & 0.34 & 0.90 & 0.63 & 0.36 & 0.21 \\
\hline 47 & 0.99 & 0.75 & 0.46 & 0.92 & 0.82 & 1 & 0.48 & 0.79 & 0.81 & 0.48 & 0.23 \\
\hline 245 & 0.49 & 0.69 & 0.83 & 0.57 & 0.34 & 0.48 & 1 & 0.37 & 0.67 & 0.83 & 0.51 \\
\hline 1149 & 0.78 & 0.58 & 0.39 & 0.73 & 0.90 & 0.79 & 0.37 & 1 & 0.62 & 0.41 & 0.22 \\
\hline 70 & 0.82 & 0.97 & 0.60 & 0.88 & 0.63 & 0.81 & 0.67 & 0.62 & 1 & 0.62 & 0.30 \\
\hline 76 & 0.49 & 0.64 & 0.98 & 0.57 & 0.36 & 0.48 & 0.83 & 0.41 & 0.62 & 1 & 0.54 \\
\hline 695 & 0.24 & 0.32 & 0.57 & 0.28 & 0.21 & 0.23 & 0.51 & 0.22 & 0.30 & 0.54 & 1 \\
\hline
\end{tabular}

Figure 16 shows graphically the correlations in Table 16. From this chart SitelD 695 shows a marked degree of wind diversity (low correlation) when compared to wind sites 22, 143, 205, 208, 47, 1149 and 70.

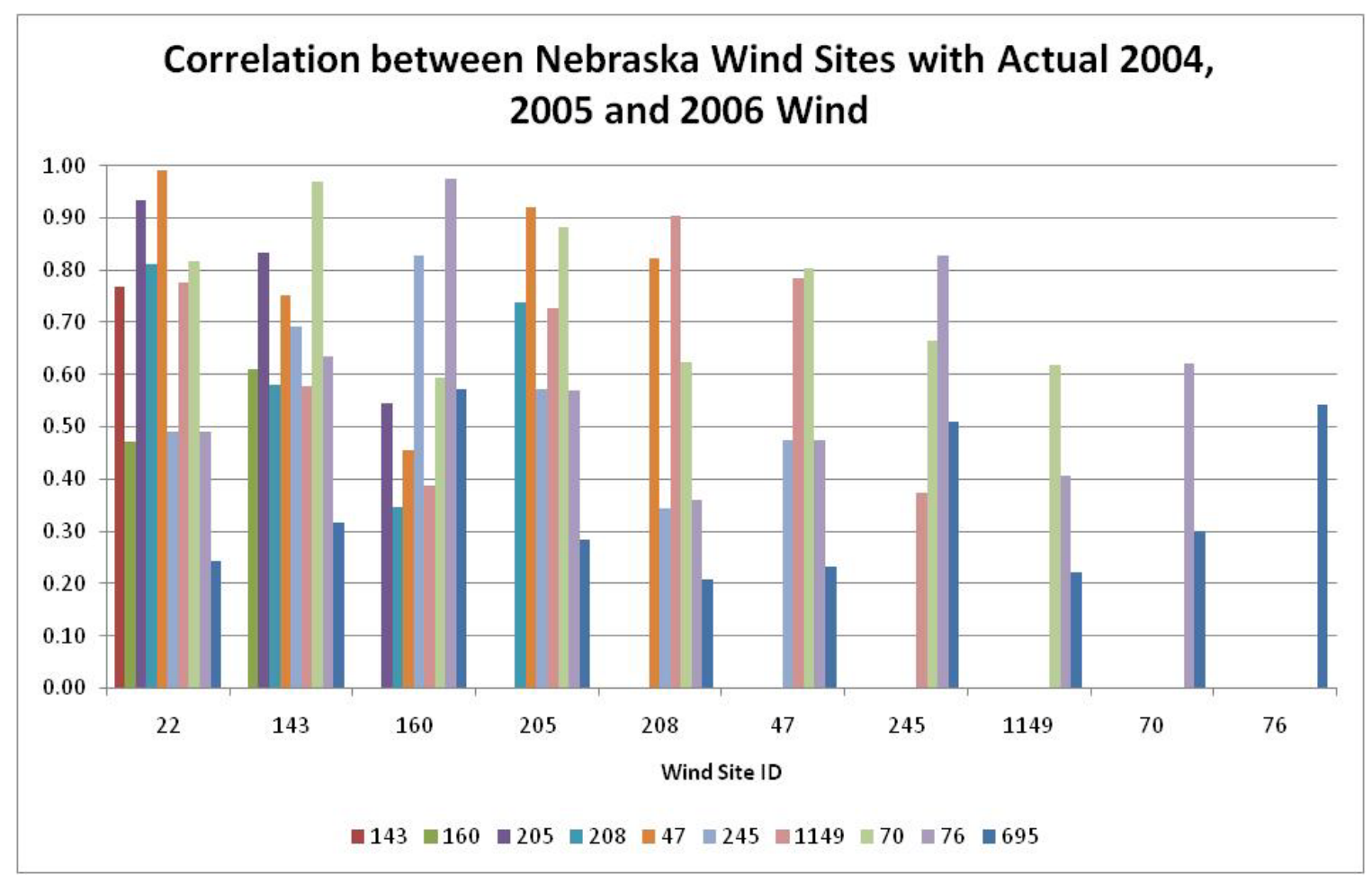

Figure 16: Nebraska wind site wind correlation

\section{Section 8.2.2.6 Wind Variability Histograms}

The following twelve histograms Figure 17 - Figure 28 depict the change in wind variability from one hour to the next. For the 3 years of hourly wind data (26,280 data points) the maximum and minimum changes were identified. The range of change was divided into deciles. The histograms show the count when the MW changes by the decile amount. For example in Figure 17 there are about 4,000 hours when there is a downward change in MW between -144MW and 
$-58 \mathrm{MW}$ whereas there are over 10,000 hours when the change between hour is between -58 $\mathrm{MW}$ and $28 \mathrm{MW}$. For this example the minimums change (i.e., largest negative) is $-402 \mathrm{MW}$, and the maximum is $458 \mathrm{MW}$, yielding deciles that each span $86 \mathrm{MW}$. A corresponding histogram shows the change as a $\%$ of nameplate capacity.

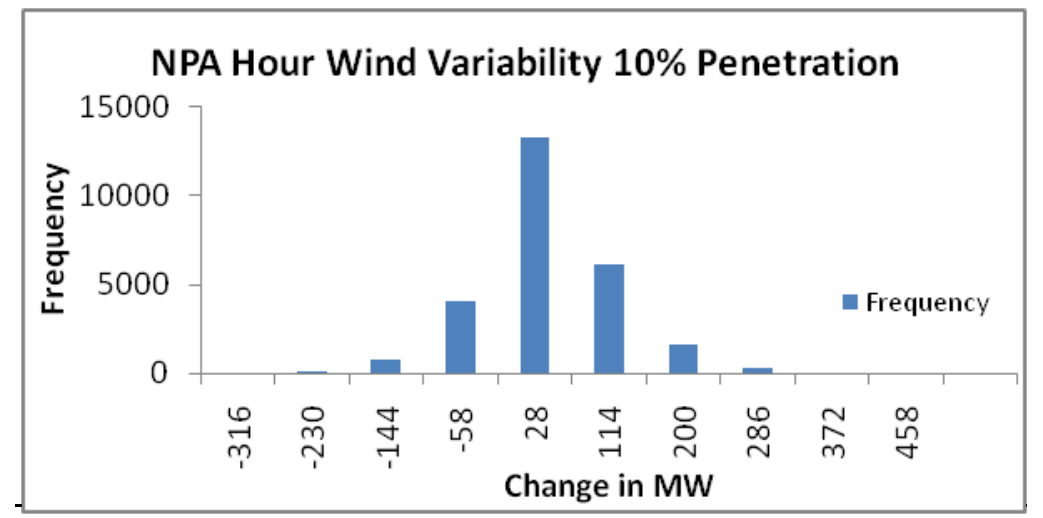

Figure 17: Histogram of NPA Hourly Wind Variability - 10\% Penetration

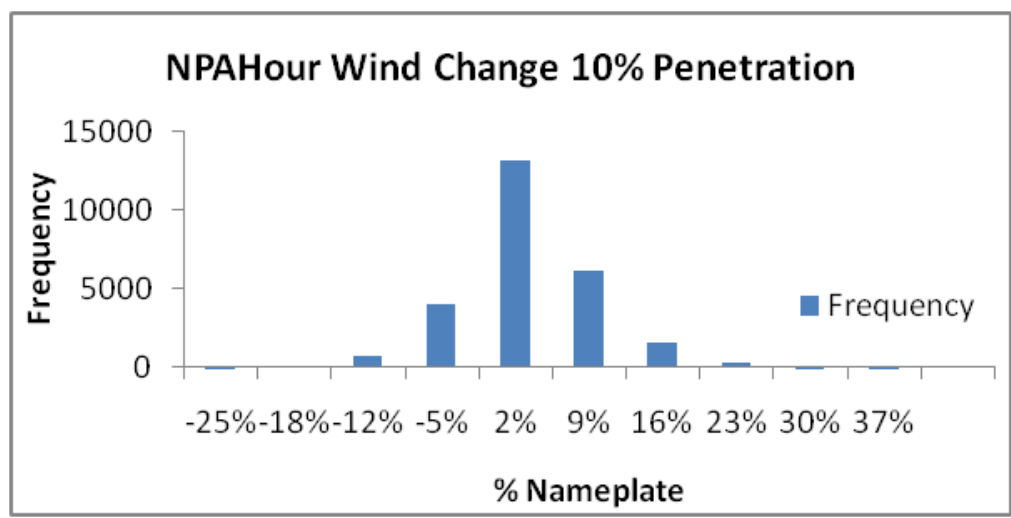

Figure 18: Histogram of NPA Hourly Wind Variability as Percent of Nameplate - $10 \%$ Penetration

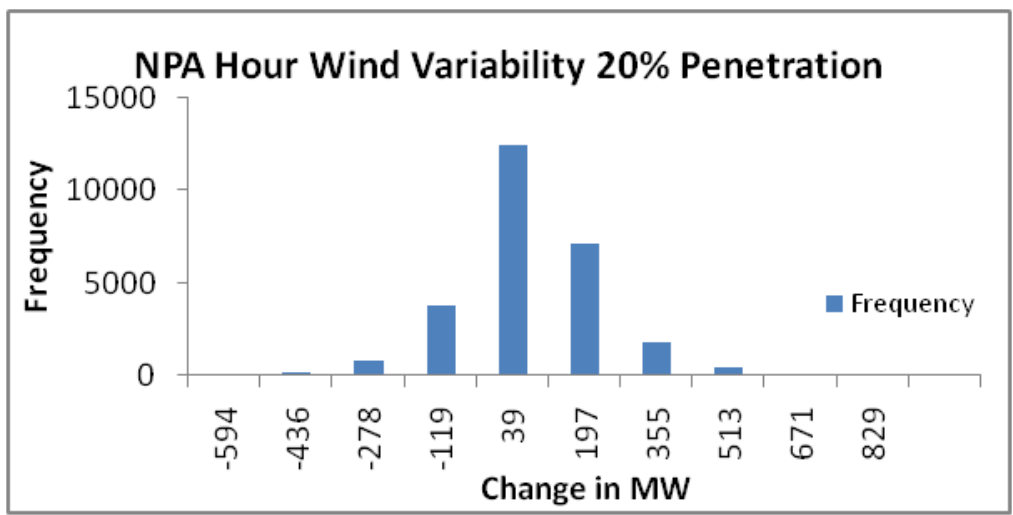


Figure 19: Histogram of NPA Hourly Wind Variability - $20 \%$ Penetration

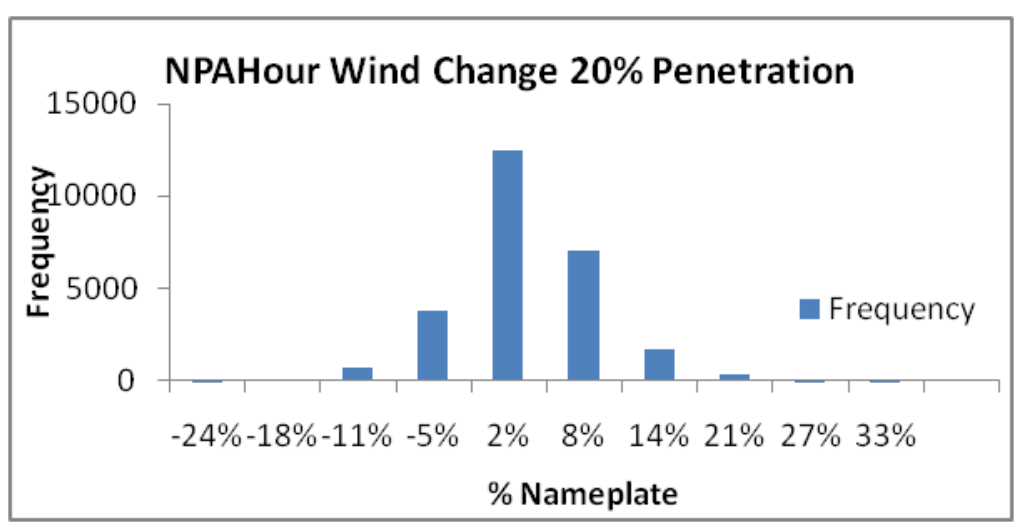

Figure 20: Histogram of NPA Hourly Wind Variability as Percent of Nameplate - $20 \%$ Penetration

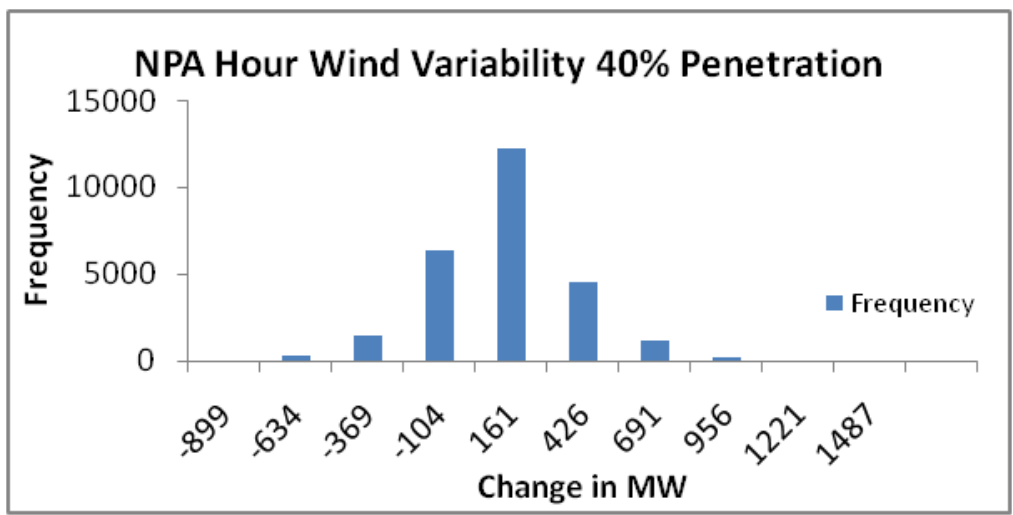

Figure 21: Histogram of NPA Hourly Wind Variability - 40\% Penetration

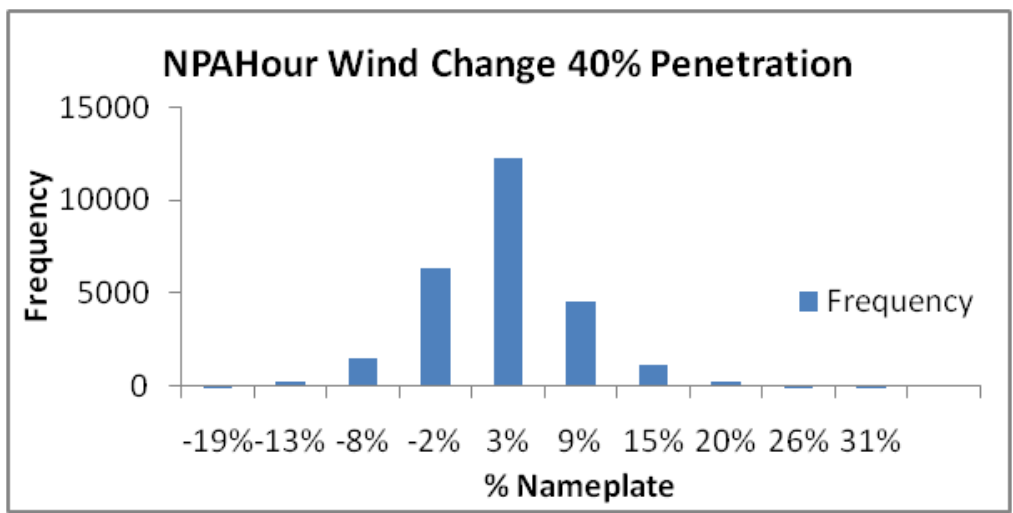

Figure 22: Histogram of NPA Hourly Wind Variability as Percent of Nameplate - $40 \%$ Penetration 


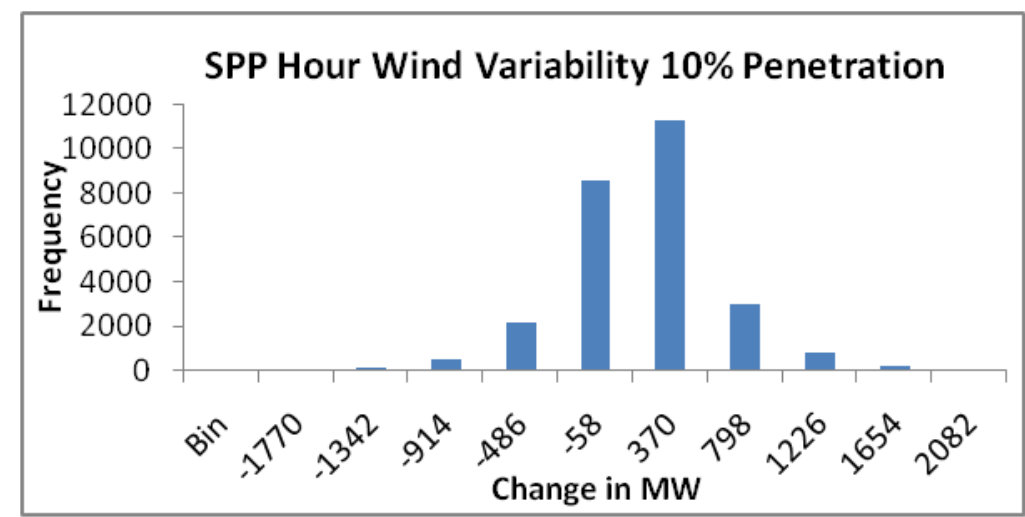

Figure 23: $\quad$ Histogram of SPP Hourly Wind Variability - 10\% Penetration

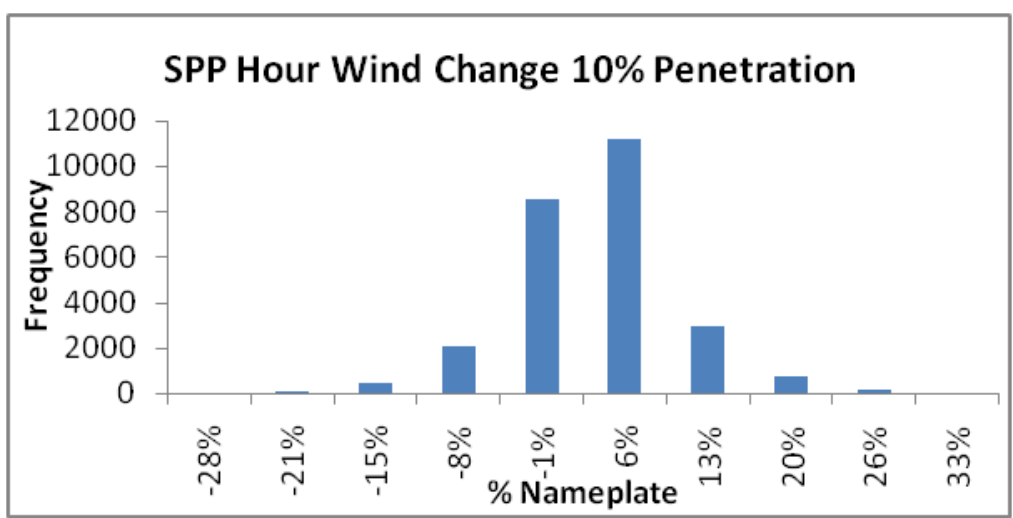

Figure 24: Histogram of SPP Hourly Wind Variability as Percent of Nameplate - 10\% Penetration

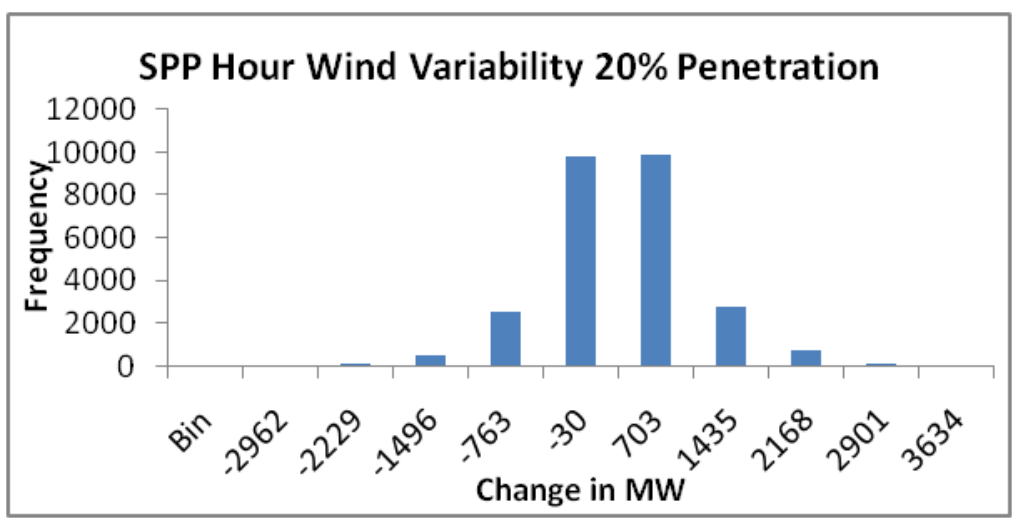

Figure 25: Histogram of SPP Hourly Wind Variability - 20\% Penetration 


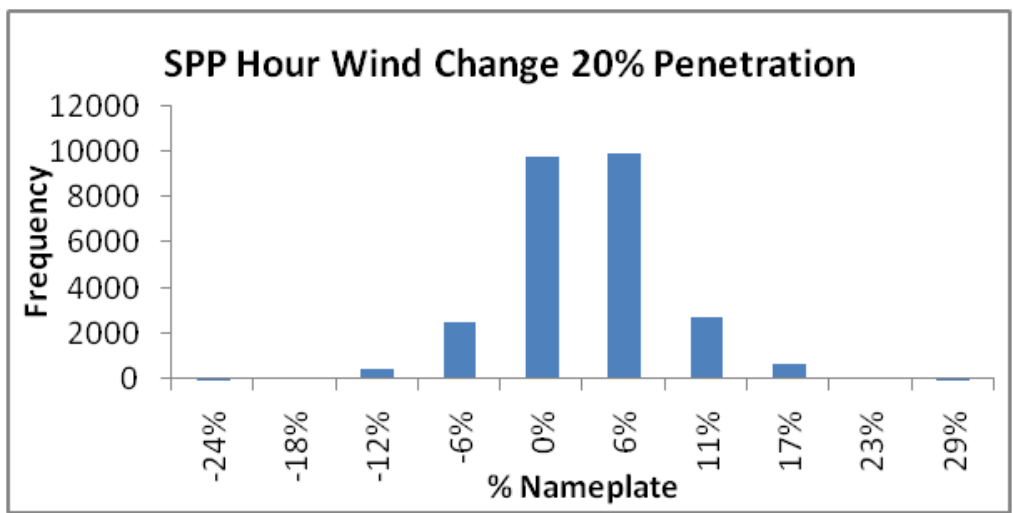

Figure 26: Histogram of SPP Hourly Wind Variability as Percent of Nameplate - $20 \%$ Penetration

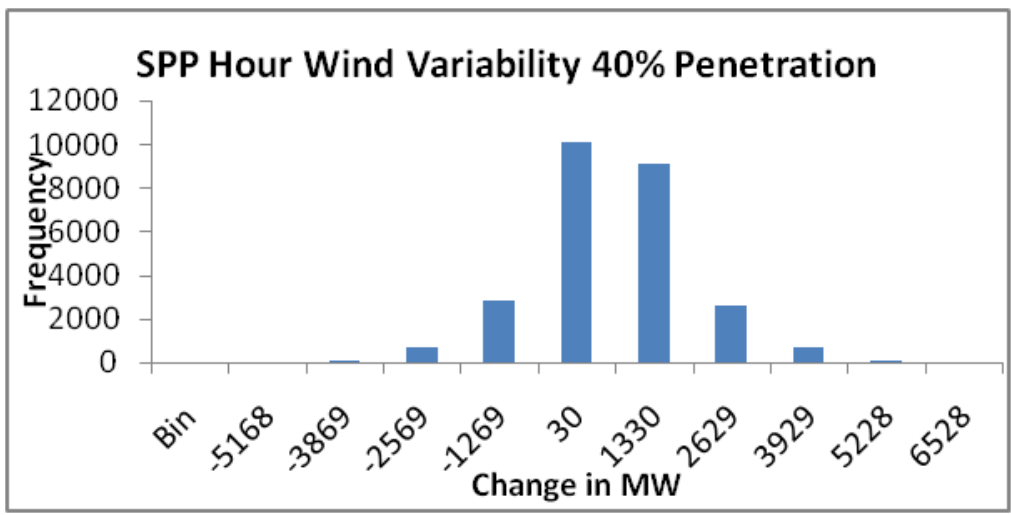

Figure 27: Histogram of SPP Hourly Wind Variability - 40\% Penetration

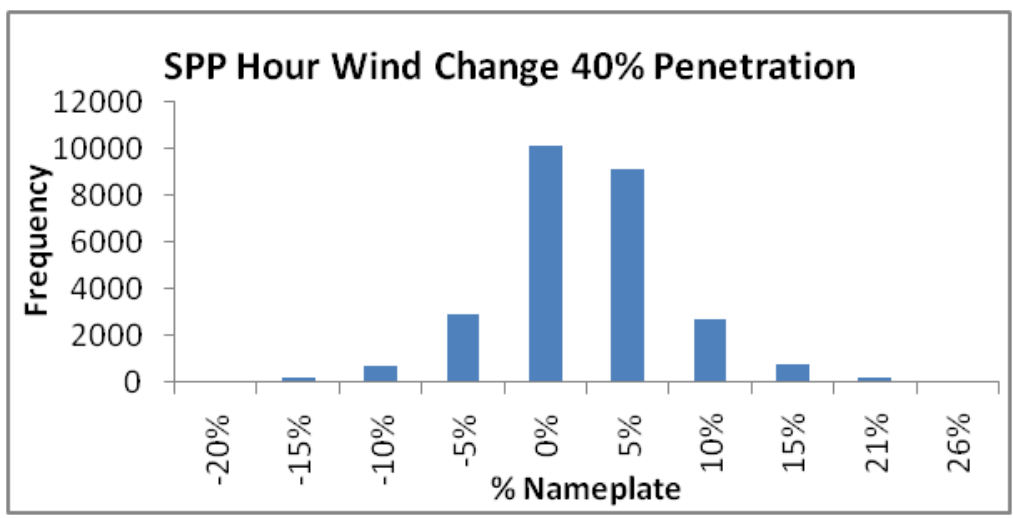

Figure 28: Histogram of SPP Hourly Wind Variability as Percent of Nameplate - $40 \%$ Penetration

\section{Section 8.2.2.7 Variability and Uncertainty - Wind}

Figure 29, Figure 30, and Figure 31 show plots of an analysis of short term forecast errors. Figure 29 uses a one hour persistence forecast of actual wind generation for the aggregated wind in the $40 \%$ penetration case. The expected next-hour forecast errors are calculated along 
with sigma. The generation is sorted low to high with respective forecast error. The sigma of each deciles forecast error are plotted on these charts. Figure 30 uses sub hourly (10 minute) generation data. Note the reduction in sigma from greater than 100 in the 1 hour persistence forecast for low and high range wind generation to less than 100 in the sub-hourly persistence forecast. The mid range operation of wind generation has similar reduction from greater than 300 to less than 250.

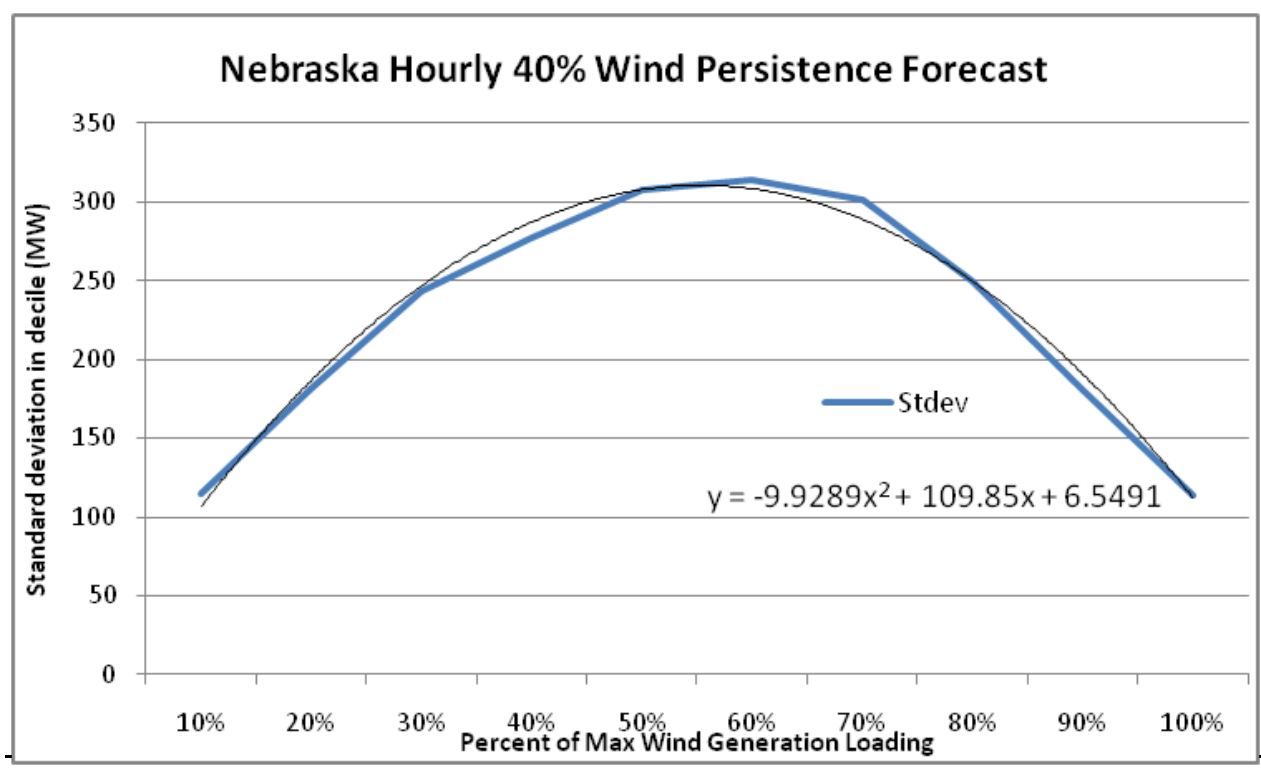

Figure 29: Nebraska Persistence Hourly Wind Forecast Error 40\% Penetration

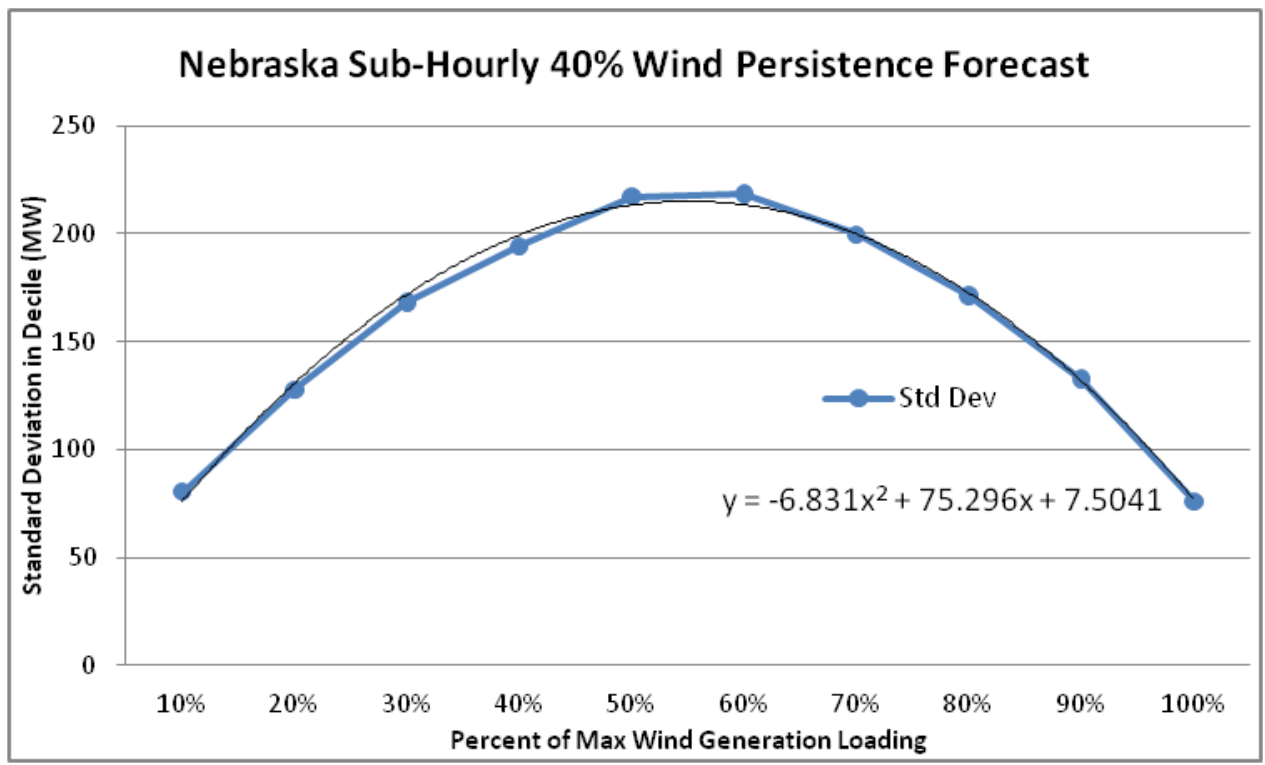

Figure 30: Nebraska Persistence Sub Hourly Wind Forecast Error 40\% Penetration 


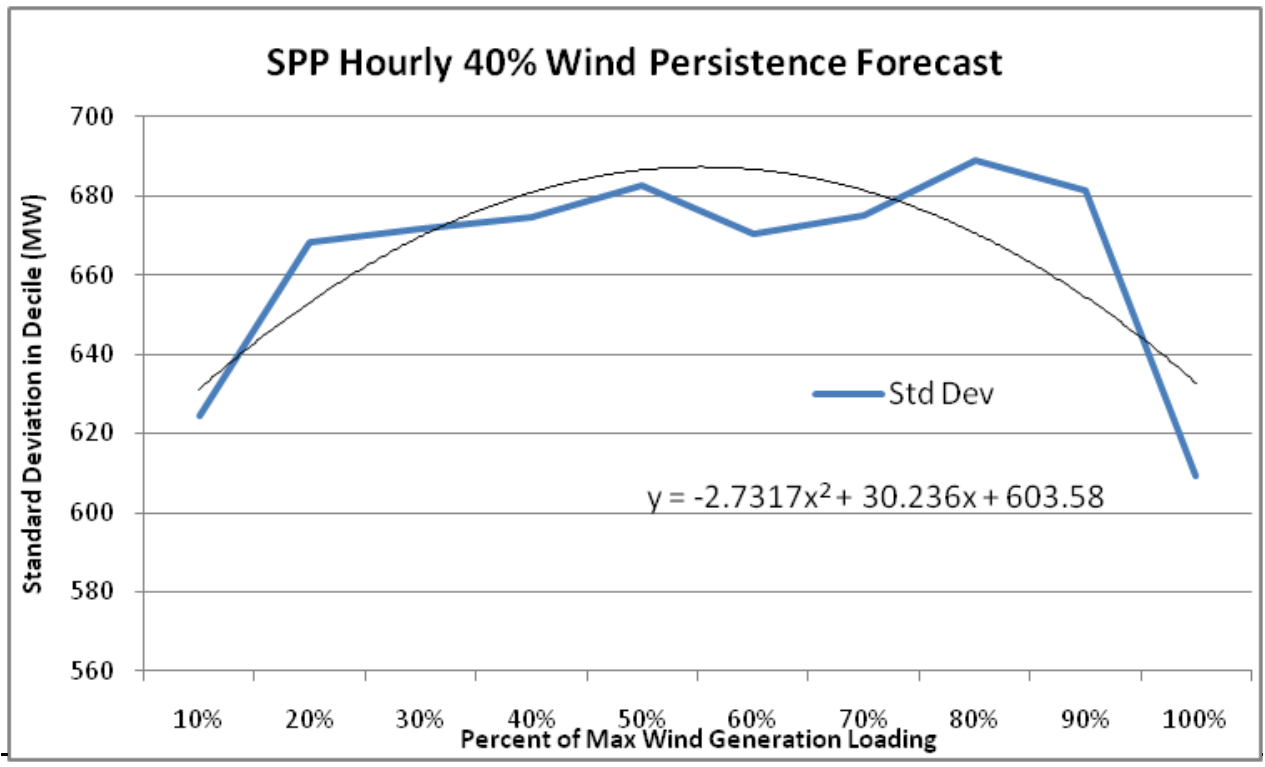

Figure 31: Forecast error SPP 40\% Penetration

\section{Section 8.2.2.8 Wind Capacity Value Estimates}

The following Capacity Value estimates are calculated using rules outlined in the SPP Wind Accreditation Criteria that can be found on pages 12-6 and 12-7 of their document. SPP Criteria with Appendices can be accessed at: http://www.spp.org/section.asp?group=215\&pagelD=27 (currently dated 2009/07/28).

These criteria can be summarized as follows:

- Calculations result in twelve unique monthly values.

- Normally 10 years of data is used - here data is only available for the years 2004-2006.

- Select the hourly wind generation values during the top $10 \%$ of load hours for each month of each of the three years (e.g., 72 hours from June 2004, 72 hours from June 2005, and 72 hours from June 2006).

- Select the hourly wind generation value that can be expected from the plant $85 \%$ of the time or greater (e.g., the $184^{\text {th }}$ value from the 216 values for June ranked highest to lowest).

- A seasonal or annual net capability may be determined by selecting the appropriate monthly MW values corresponding to the Load Serving Member's peak load month of the season of interest.

For the calculation of seasonal or annual net capability NPA's peak load month is July for each reference year in the study, see Table 17. 
Table 17: NPA Peak Load

\begin{tabular}{|r|r|c|}
\hline \multicolumn{3}{|c|}{ NPA Load } \\
\hline Ref. Year & Peak MW & Date \\
\hline 2004 & 7553 & $7 / 17 / 1815: 00$ \\
2005 & 7461 & $7 / 20 / 1815: 00$ \\
2006 & 7489 & $7 / 18 / 1815: 00$ \\
\hline
\end{tabular}

Table 18: SPP Peak Load

\begin{tabular}{|r|r|r|}
\hline \multicolumn{3}{|c|}{ SPP w/o NPA } \\
\hline Ref Year & Peak MW & \multicolumn{1}{c|}{ Date } \\
\hline 2004 & 46403 & $8 / 23 / 1815: 00$ \\
2005 & 46023 & $8 / 1 / 1815: 00$ \\
2006 & 46005 & $8 / 8 / 1815: 00$ \\
\hline
\end{tabular}

Table 19: SPP + NPA Peak Load

\begin{tabular}{|r|r|r|}
\hline \multicolumn{3}{|c|}{ SPP with NPA } \\
\hline Ref Year & Peak MW & Date \\
\hline 2004 & 53607 & $8 / 23 / 1815: 00$ \\
2005 & 53352 & $8 / 1 / 1815: 00$ \\
2006 & 53387 & $7 / 18 / 1815: 00$ \\
\hline
\end{tabular}

Capacity Value estimates were calculated for each SiteID identified in each scenario for NPA and SPP, see Figure 32 - Figure 45 and Table 20 - Table 23. The Capacity Value was also calculated for each scenario after aggregating the sites. Capacity Values were calculated separately by scenario for NPA and SPP. A separate Capacity Value for SPP + NPA is also provided. The load profiles used for identifying the top $10 \%$ load hours were the aggregate NPA and SPP profiles, as appropriate.

The charts shown in Figure 32 through Figure 34 display the capacity value for NPA sites as a percentage of each sites nameplate capacity. Figure 35 shows the NPA aggregate capacity value of all wind by scenario, although capacity value by aggregation is not a part of the SPP criteria this exercise is for information purposes only.

Key observations noted using the SPP criteria are:

- Very low summer values that trend with the generally negative correlation between wind and load, see Figure 90. The peak hours over the year tend to be in the summer months of July and August at which time wind is low.

The effects of wind diversity can be seen with the wide variety in capacity values such as:

- In the $10 \%$ scenario for November, the capacity values for NPA are relatively low (typically less than $5 \%$ whereas the values for SPP are typically greater than $10 \%$ ), Table 20 - Table 22 and Figure 36 - Figure 44.

- The site-specific charts (Figure 32- Figure 34 and Table 20 - Table 22) show considerable variation in capacity value from site to site. For example in the $40 \%$ scenario for May, 
the capacity value varies from $4 \%$ for site 76 to $12 \%$ for site 70 while the reverse relationship appears in several other months.

- However, Figure 35 for NPA shows the diversity benefit in capacity value terms that as more wind generation is added (moving from $10 \%$ to $40 \%$ penetration), there is nearly always more capacity value in normalized percentage terms.

- Wind site diversity contributes to the wide variety of capacity values for different sites, e.g., site 208 for NPA has values less than $5 \%$ in many months but some of the highest values, greater than $10 \%$ in February, May and September when compared to other sites in this scenario.

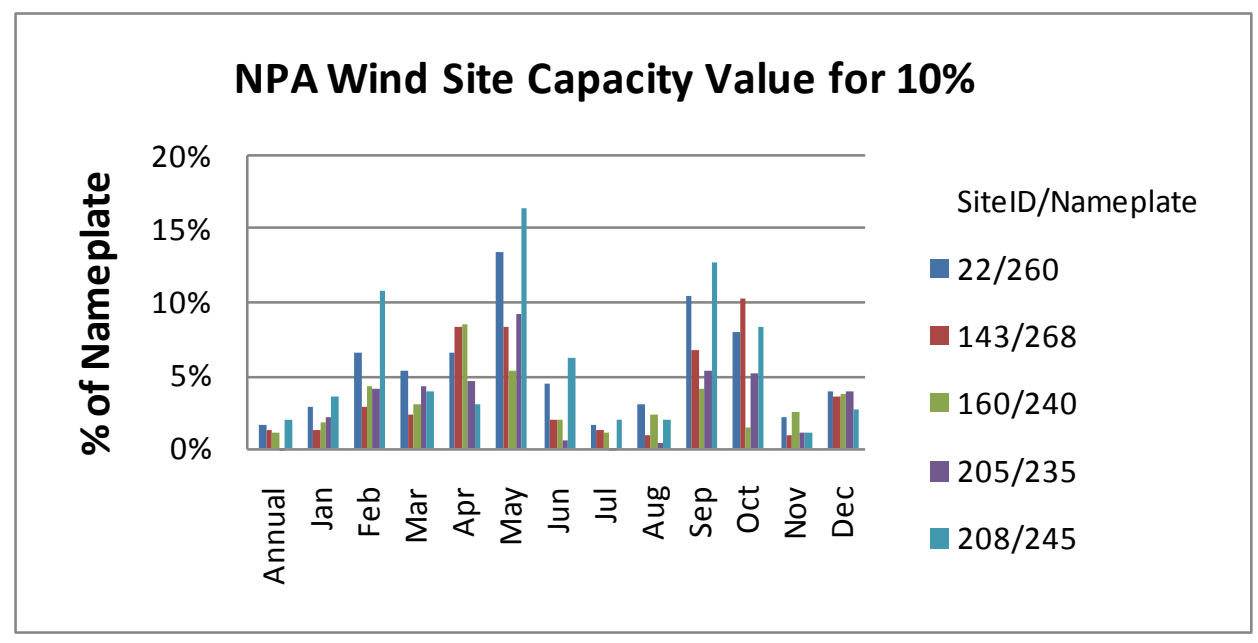

Figure 32: NPA Wind Site Capacity Values - 10\% Penetration

Table 20: NPA Wind Site Capacity Values - 10\% Penetration

\begin{tabular}{|l|r|r|r|r|r|}
\hline \multirow{2}{*}{$\begin{array}{c}\text { SitelD/ } \\
\text { Nameplate }\end{array}$} & \multicolumn{5}{|c|}{ NPA - 10\% Penetration } \\
\cline { 2 - 6 } & $22 / 260$ & $143 / 268$ & $160 / 240$ & $205 / 235$ & $208 / 245$ \\
\hline Annual & $1.54 \%$ & $1.17 \%$ & $1.08 \%$ & $0.06 \%$ & $1.96 \%$ \\
Jan & $2.79 \%$ & $1.19 \%$ & $1.82 \%$ & $2.24 \%$ & $3.59 \%$ \\
Feb & $6.60 \%$ & $2.79 \%$ & $4.23 \%$ & $4.06 \%$ & $10.85 \%$ \\
Mar & $5.27 \%$ & $2.22 \%$ & $2.98 \%$ & $4.24 \%$ & $3.90 \%$ \\
Apr & $6.46 \%$ & $8.25 \%$ & $8.42 \%$ & $4.69 \%$ & $3.04 \%$ \\
May & $13.52 \%$ & $8.35 \%$ & $5.35 \%$ & $9.26 \%$ & $16.53 \%$ \\
Jun & $4.45 \%$ & $1.86 \%$ & $1.98 \%$ & $0.63 \%$ & $6.22 \%$ \\
Jul & $1.54 \%$ & $1.17 \%$ & $1.08 \%$ & $0.06 \%$ & $1.96 \%$ \\
Aug & $3.07 \%$ & $0.81 \%$ & $2.22 \%$ & $0.34 \%$ & $1.94 \%$ \\
Sep & $10.40 \%$ & $6.79 \%$ & $4.05 \%$ & $5.35 \%$ & $12.66 \%$ \\
Oct & $7.92 \%$ & $10.23 \%$ & $1.47 \%$ & $5.21 \%$ & $8.36 \%$ \\
Nov & $2.10 \%$ & $0.86 \%$ & $2.43 \%$ & $1.20 \%$ & $0.98 \%$ \\
Dec & $3.94 \%$ & $3.59 \%$ & $3.76 \%$ & $3.87 \%$ & $2.73 \%$ \\
\hline
\end{tabular}




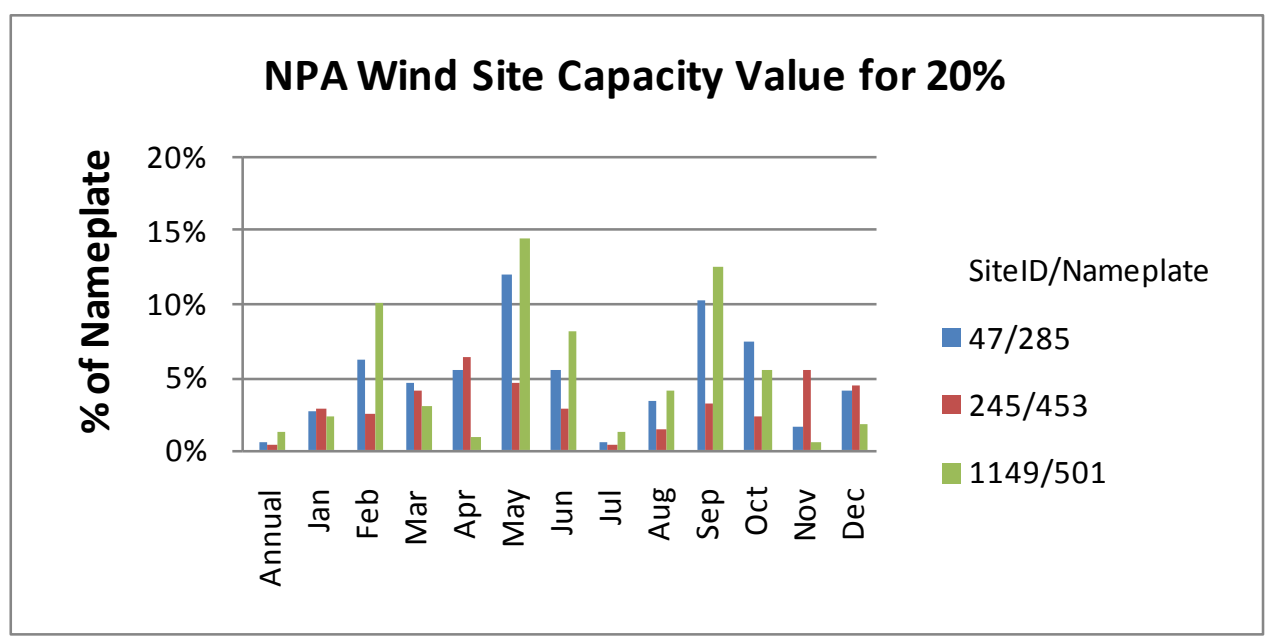

Figure 33: NPA Wind Site Capacity Values - 20\% Penetration

Table 21: NPA Wind Site Capacity Values - 20\% Penetration

\begin{tabular}{|l|r|r|r|}
\hline \multirow{2}{*}{ SitelD/ } & \multicolumn{3}{|c|}{ NPA 20\% Penetration } \\
\cline { 2 - 4 } Nameplate & $47 / 285$ & $245 / 453$ & $1149 / 501$ \\
\hline Annual & $0.54 \%$ & $0.39 \%$ & $1.26 \%$ \\
Jan & $2.66 \%$ & $2.81 \%$ & $2.25 \%$ \\
Feb & $6.26 \%$ & $2.52 \%$ & $10.16 \%$ \\
Mar & $4.64 \%$ & $4.01 \%$ & $2.95 \%$ \\
Apr & $5.46 \%$ & $6.38 \%$ & $0.86 \%$ \\
May & $12.09 \%$ & $4.55 \%$ & $14.57 \%$ \\
Jun & $5.41 \%$ & $2.80 \%$ & $8.19 \%$ \\
Jul & $0.54 \%$ & $0.39 \%$ & $1.26 \%$ \\
Aug & $3.29 \%$ & $1.39 \%$ & $4.11 \%$ \\
Sep & $10.19 \%$ & $3.14 \%$ & $12.53 \%$ \\
Oct & $7.35 \%$ & $2.32 \%$ & $5.47 \%$ \\
Nov & $1.64 \%$ & $5.57 \%$ & $0.53 \%$ \\
Dec & $4.07 \%$ & $4.34 \%$ & $1.74 \%$ \\
\hline
\end{tabular}




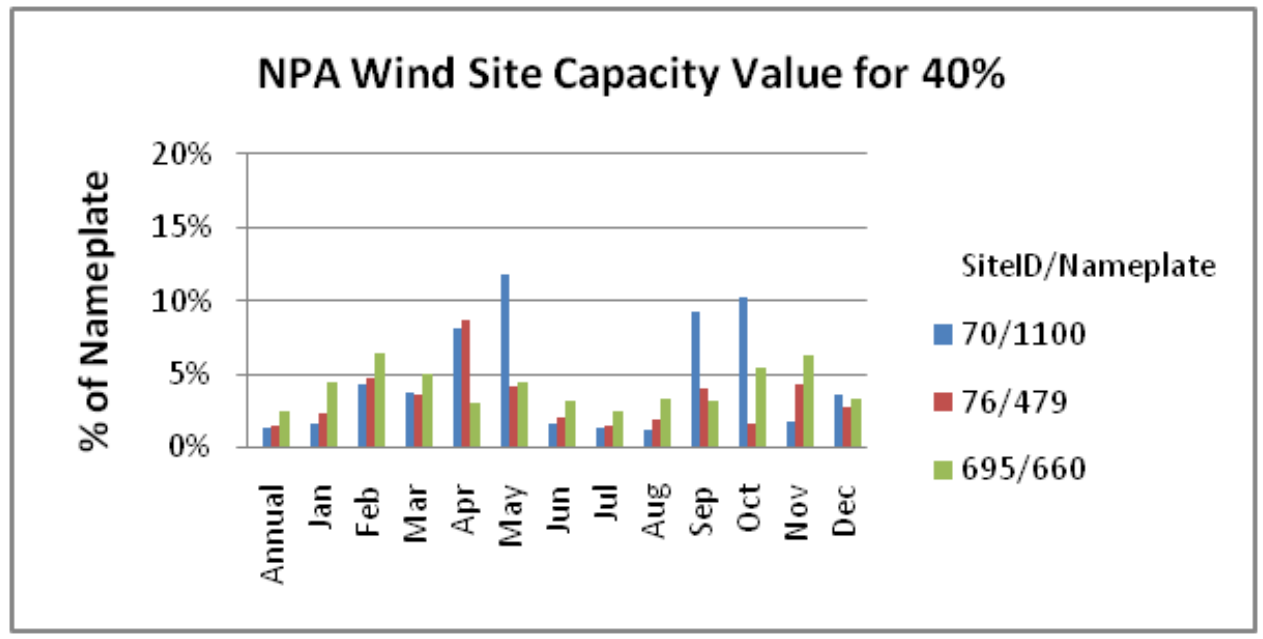

Figure 34: NPA Wind Site Capacity Values - 40\% Penetration

Table 22: NPA Wind site Capacity Values - 40\% Penetration

\begin{tabular}{|l|r|r|r|}
\hline \multirow{1}{*}{$\begin{array}{c}\text { SitelD/ } \\
\text { Nameplate }\end{array}$} & \multicolumn{3}{|c|}{ NPA 40\% Penetration } \\
\cline { 2 - 4 } & $70 / 1100$ & $76 / 479$ & $695 / 660$ \\
\hline Annual & $1.37 \%$ & $1.51 \%$ & $2.41 \%$ \\
Jan & $1.63 \%$ & $2.30 \%$ & $4.49 \%$ \\
Feb & $4.36 \%$ & $4.77 \%$ & $6.45 \%$ \\
Mar & $3.79 \%$ & $3.57 \%$ & $4.95 \%$ \\
Apr & $8.12 \%$ & $8.73 \%$ & $3.08 \%$ \\
May & $11.77 \%$ & $4.11 \%$ & $4.39 \%$ \\
Jun & $1.58 \%$ & $2.11 \%$ & $3.22 \%$ \\
Jul & $1.37 \%$ & $1.51 \%$ & $2.41 \%$ \\
Aug & $1.19 \%$ & $1.86 \%$ & $3.35 \%$ \\
Sep & $9.25 \%$ & $4.02 \%$ & $3.13 \%$ \\
Oct & $10.23 \%$ & $1.63 \%$ & $5.37 \%$ \\
Nov & $1.77 \%$ & $4.33 \%$ & $6.23 \%$ \\
Dec & $3.55 \%$ & $2.80 \%$ & $3.31 \%$ \\
\hline
\end{tabular}




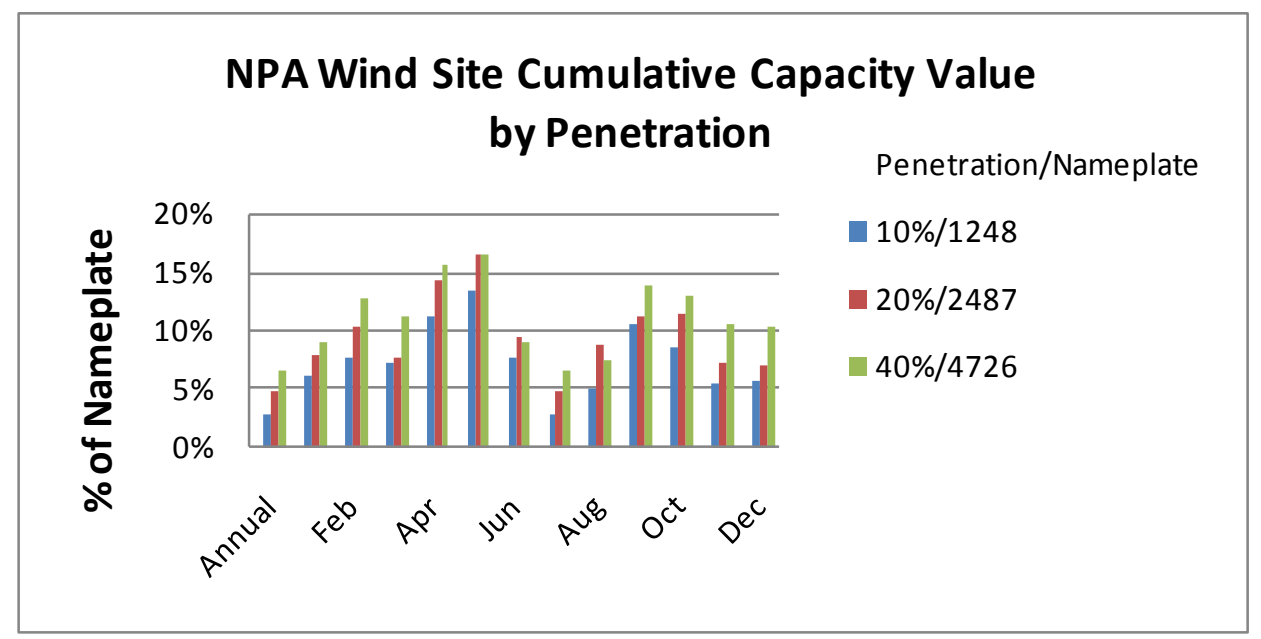

Figure 35: NPA Aggregated Wind Site Capacity Values by Penetration

Table 23: NPA Aggregated Wind Site Capacity Values by Penetration

\begin{tabular}{|l|r|r|r|}
\hline \multirow{2}{*}{ Nameplate } & $10 \%$ & $20 \%$ & $40 \%$ \\
\cline { 2 - 4 } & 1249 & 2488 & 4727 \\
\hline Annual & $2.94 \%$ & $4.75 \%$ & $6.55 \%$ \\
Jan & $6.18 \%$ & $7.96 \%$ & $9.02 \%$ \\
Feb & $7.73 \%$ & $10.30 \%$ & $12.74 \%$ \\
Mar & $7.29 \%$ & $7.71 \%$ & $11.18 \%$ \\
Apr & $11.19 \%$ & $14.29 \%$ & $15.65 \%$ \\
May & $13.41 \%$ & $16.39 \%$ & $16.56 \%$ \\
Jun & $7.75 \%$ & $9.34 \%$ & $8.91 \%$ \\
Jul & $2.94 \%$ & $4.75 \%$ & $6.55 \%$ \\
Aug & $4.98 \%$ & $8.83 \%$ & $7.56 \%$ \\
Sep & $10.61 \%$ & $11.20 \%$ & $13.78 \%$ \\
Oct & $8.60 \%$ & $11.34 \%$ & $13.05 \%$ \\
Nov & $5.52 \%$ & $7.19 \%$ & $10.50 \%$ \\
Dec & $5.80 \%$ & $7.05 \%$ & $10.23 \%$ \\
\hline
\end{tabular}

Charts in Figure 36 through Figure 44 show capacity value for each site ID in the SPP scenarios. Figure 45 shows the SPP aggregated capacity value for each scenario. 


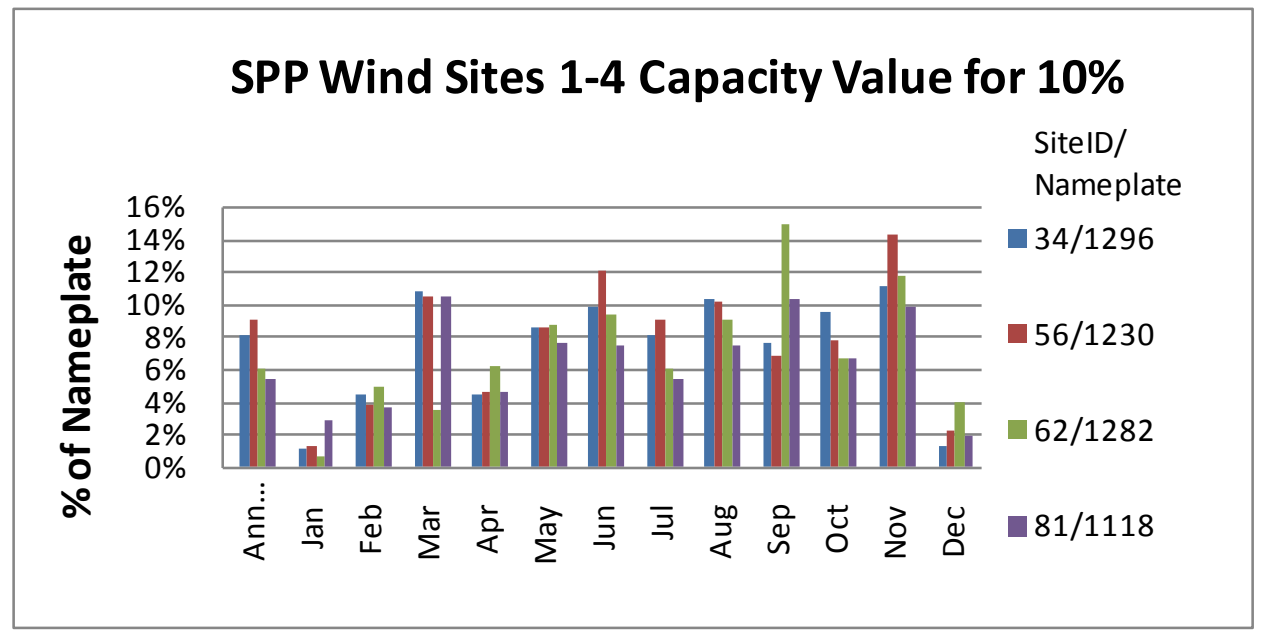

Figure 36: SPP Wind Sites 1-4 Capacity Values - 10\% Penetration

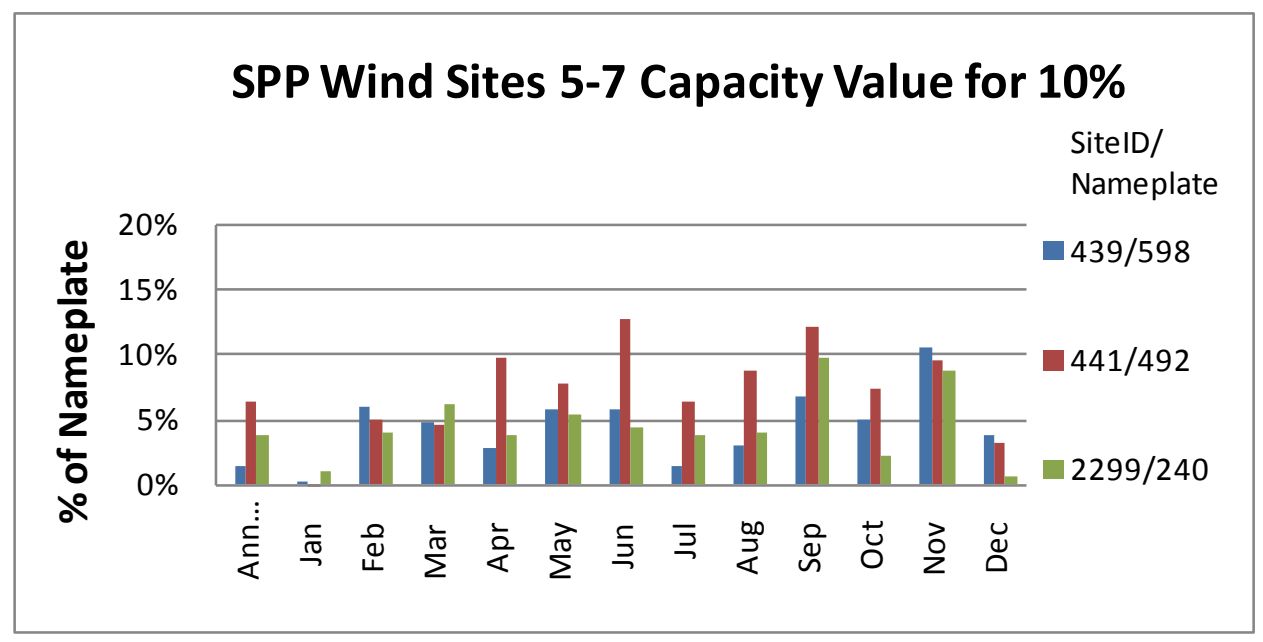

Figure 37: SPP Wind Sites 5-7 Capacity Values - 10\% Penetration

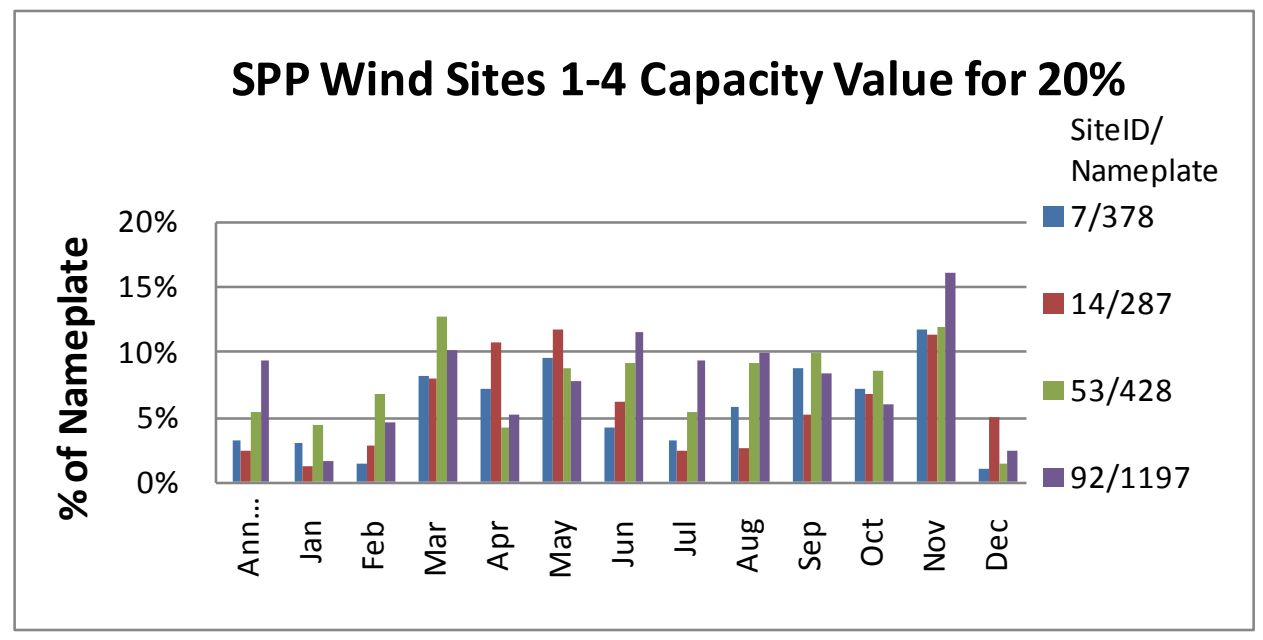

Figure 38: SPP Wind Sites 1-4 Capacity Values - 20\% Penetration 


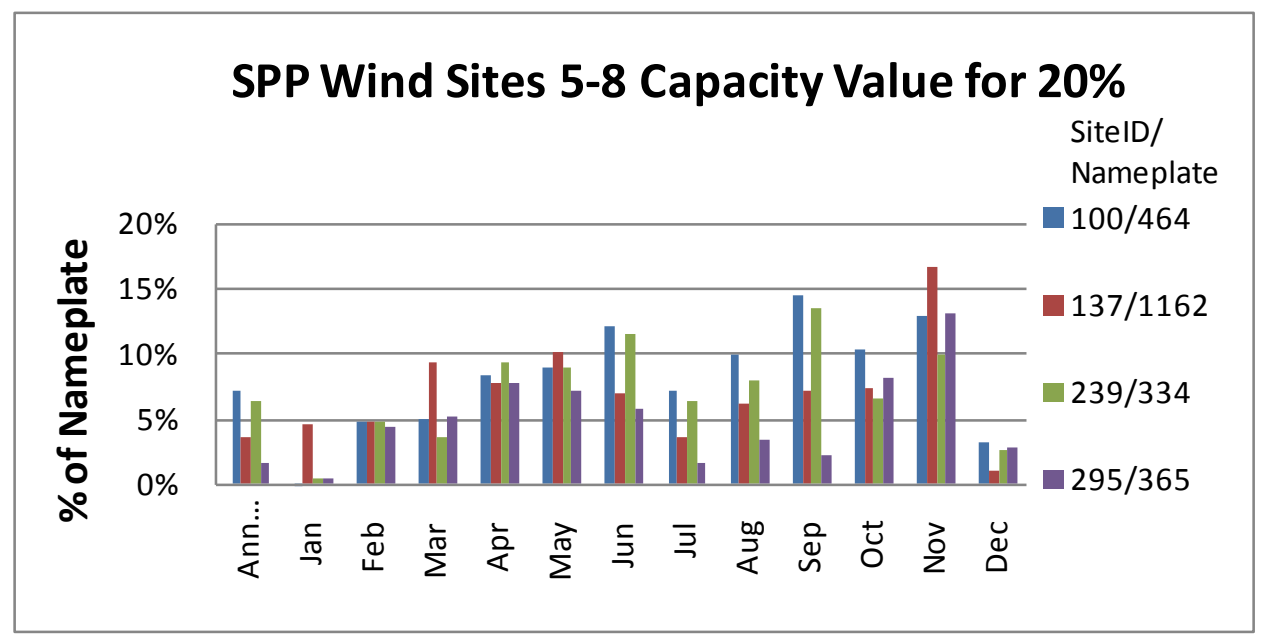

Figure 39: SPP Wind Sites 5-8 Capacity Values - 20\% Penetration

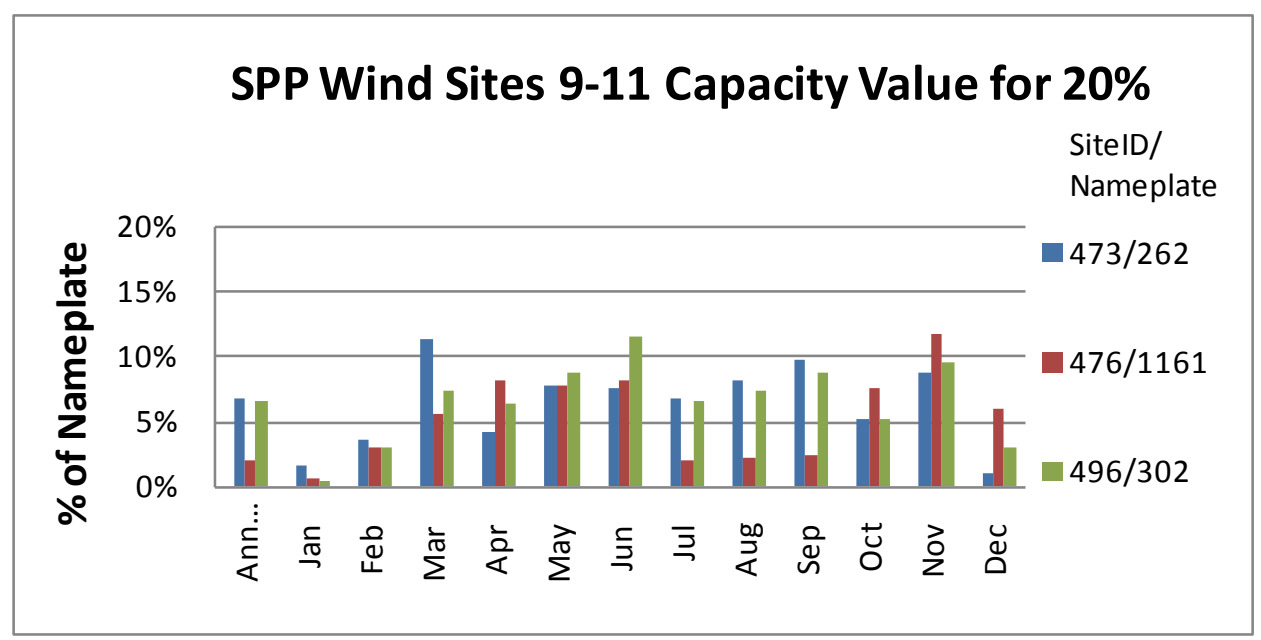

Figure 40: SPP Wind Sites 9-11 Capacity Values - 20\% Penetration 


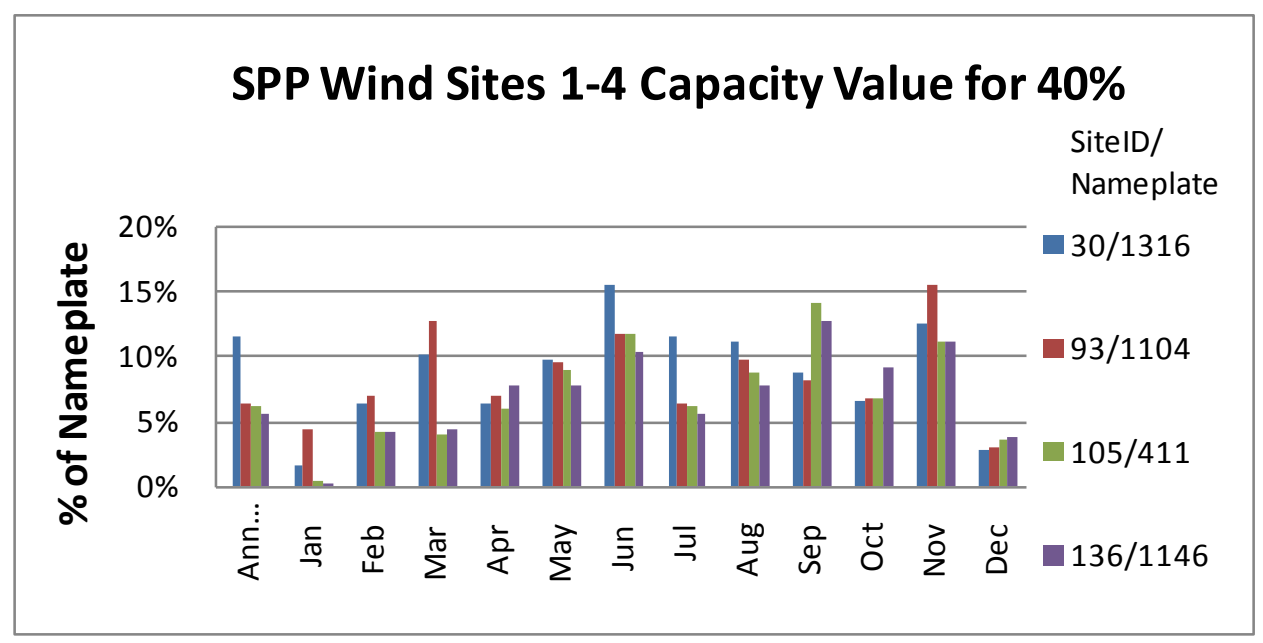

Figure 41: SPP Wind Sites 1-4 Capacity Values - 40\% Penetration

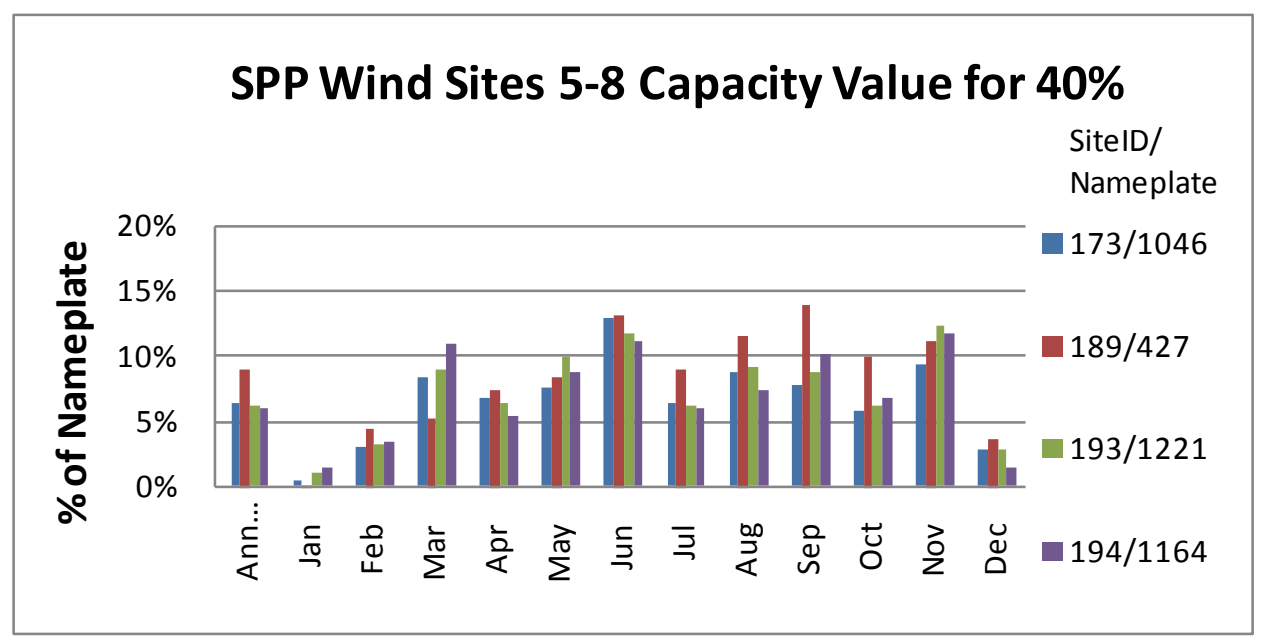

Figure 42: SPP Wind Sites 5-8 Capacity Values - 40\% Penetration 


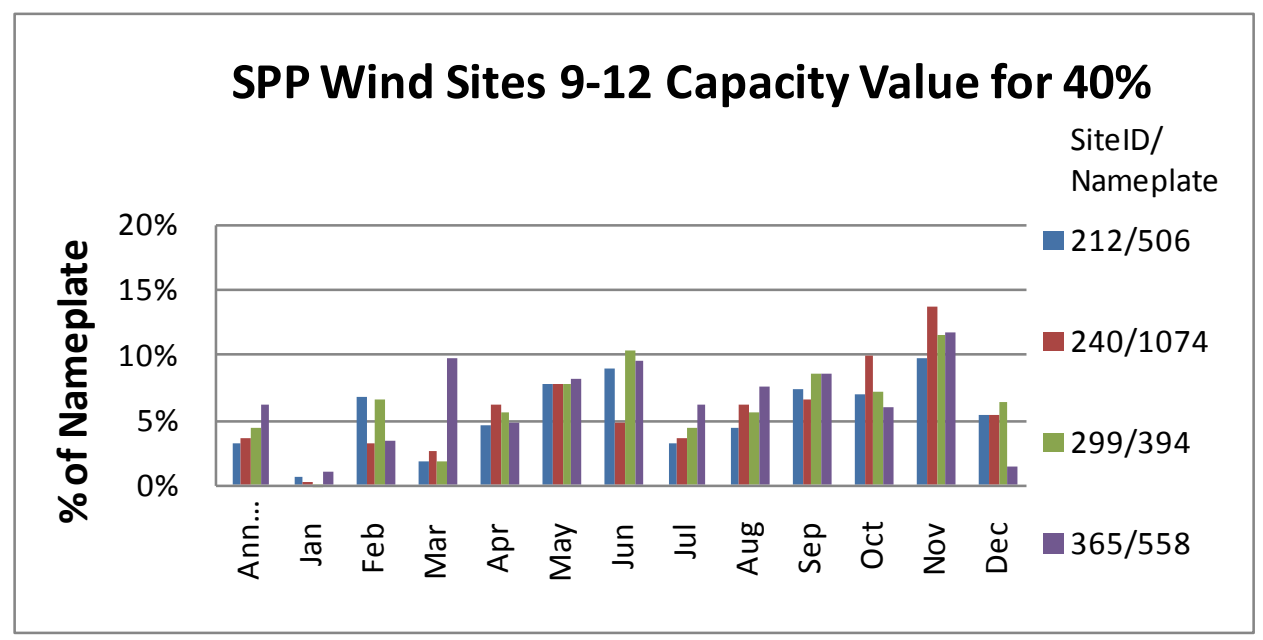

Figure 43: SPP Wind Sites 9-12 Capacity Values - 40\% Penetration

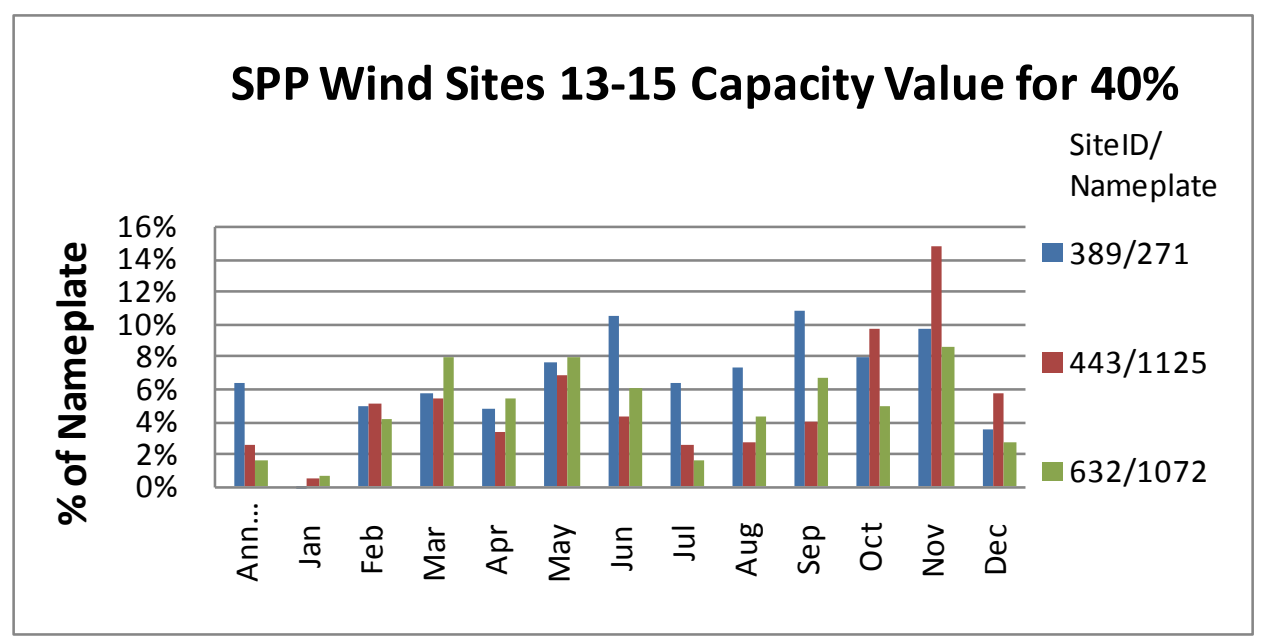

Figure 44: SPP Wind Sites 13-15 Capacity Values - 40\% Penetration 


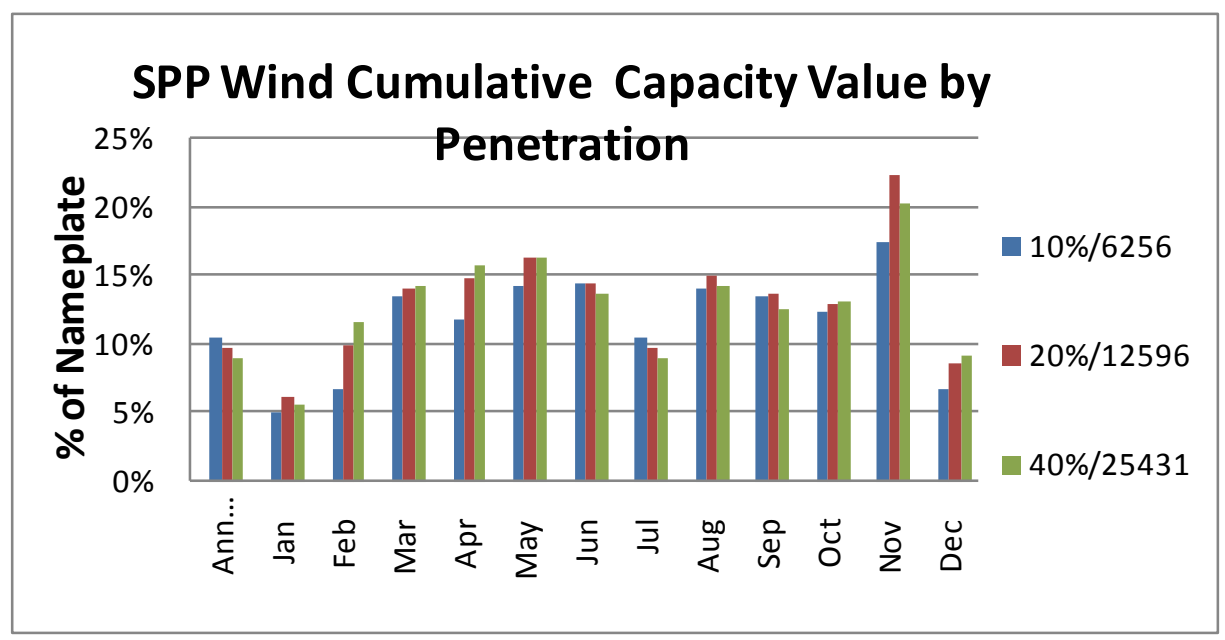

Figure 45: NPA + SPP Wind Site Capacity Value by Penetration

\section{Section 8.2.3 Wind and Load Characteristics}

NPA provided hourly load data for 2004, 2005 and 2006. For each year's data loads were escalated to 2018. Load data for the remaining regions were taken from the EWITS study and escalated to 2018. As stated previously, the wind data came from the NREL database for the Eastern Interconnection.

\section{Section 8.2.3.1 Hourly Characteristics by Year/Scenario/Region}

The next several pages show charts that display wind and load for different regions. Figure 46 through Figure 54 show the relationship of each year of NPA load with corresponding year of wind data. Figure 55 to Figure 63 shows SPP load with SPP wind.

All SPP Load and Wind data shown in the charts do not include Nebraska unless specifically noted.

At $40 \%$ penetrations there are hours when wind generation exceeds system load. 


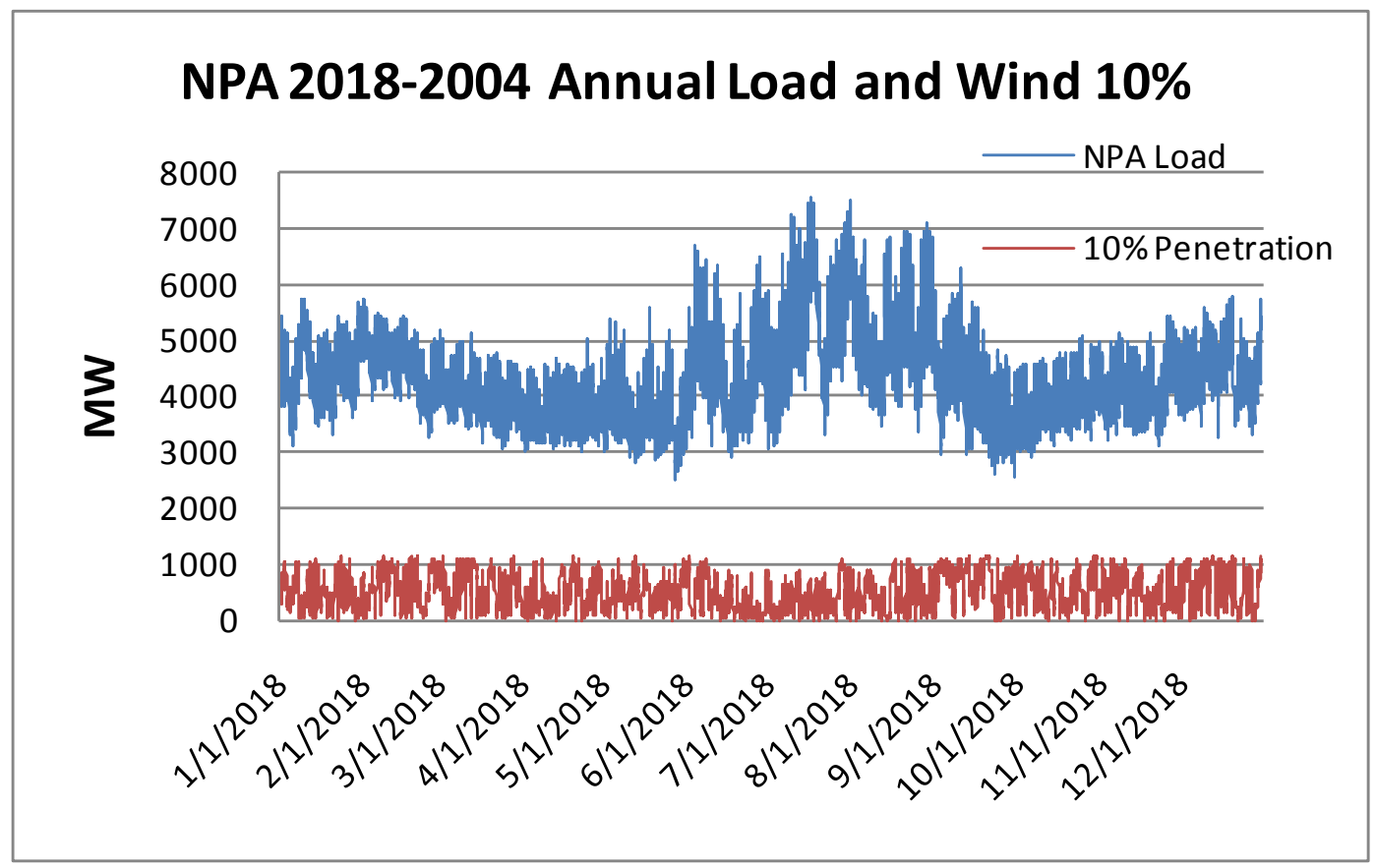

Figure 46: NPA 2004 Load and Wind Analysis - 10\% Penetration

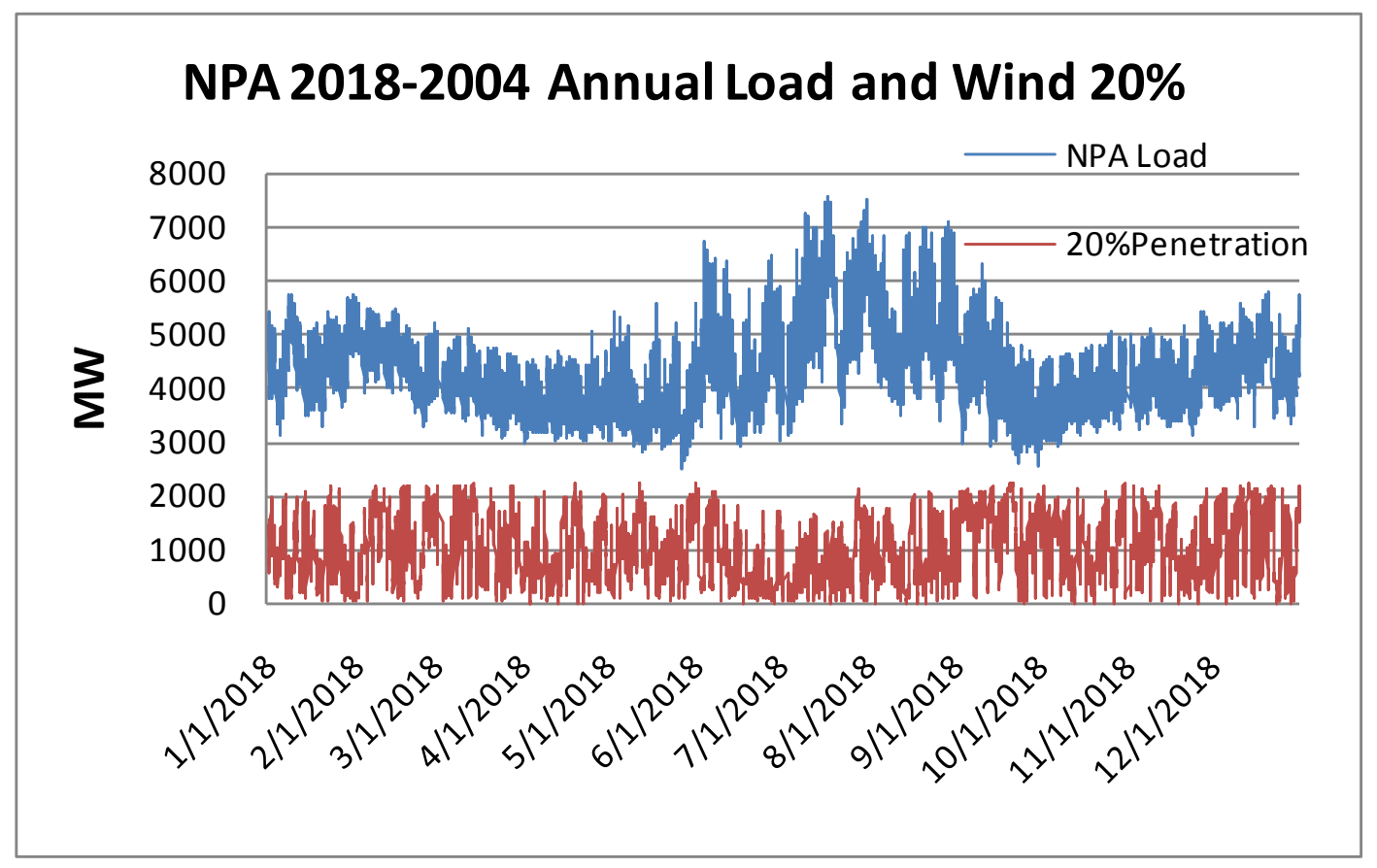

Figure 47: NPA 2004 Load and Wind Analysis - 20\% Penetration 


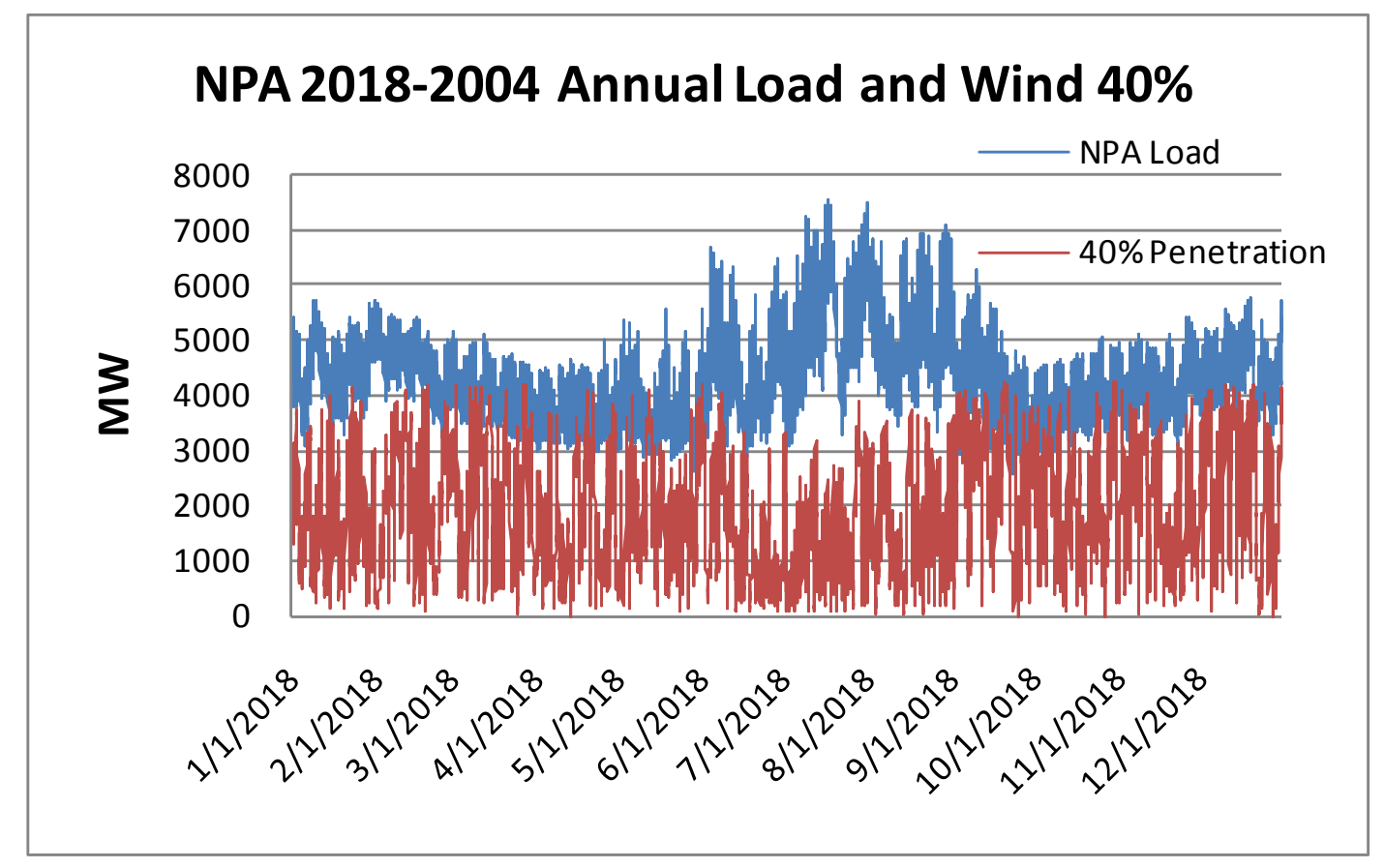

Figure 48: NPA 2004 Load and Wind Analysis - 40\% Penetration

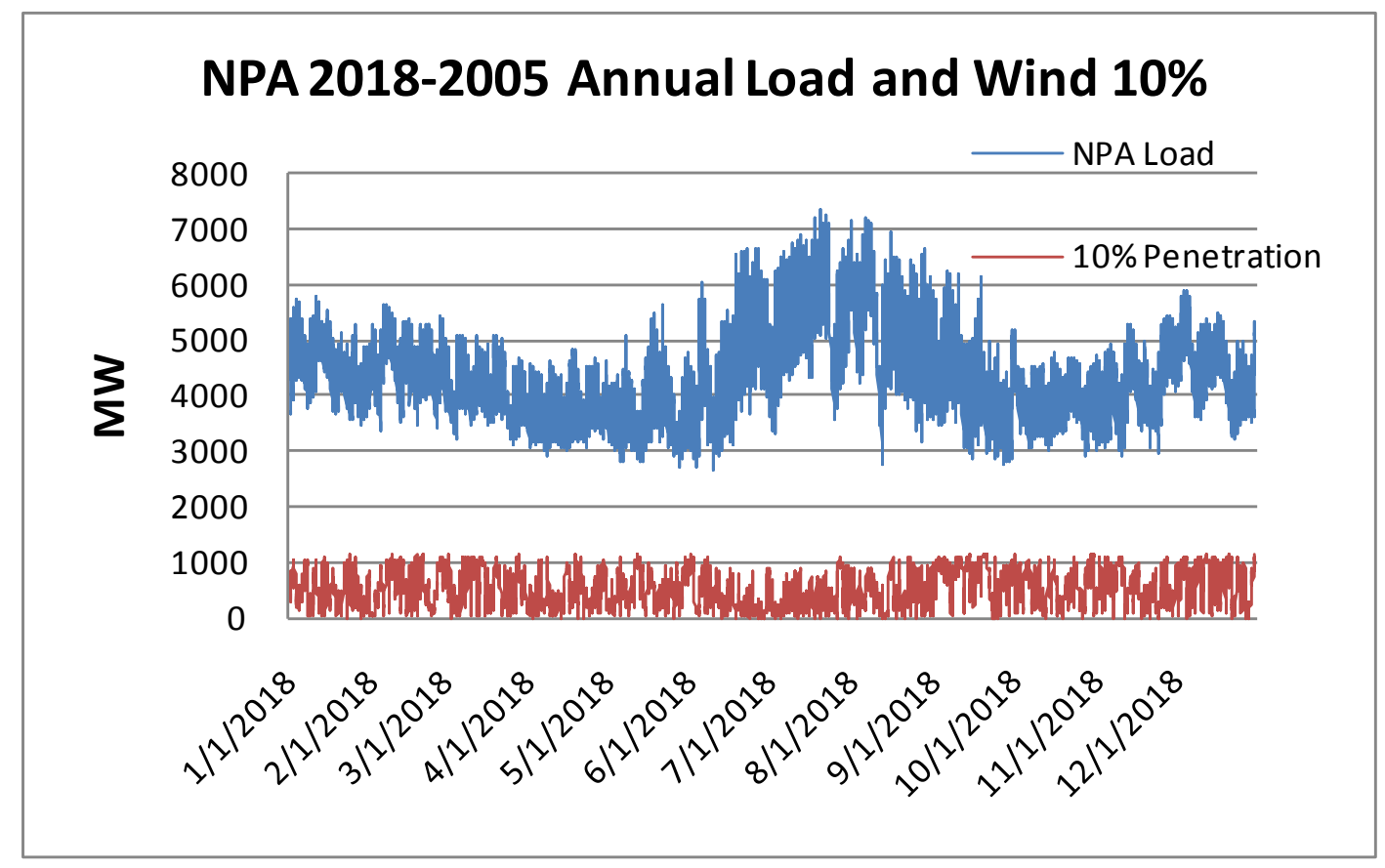

Figure 49: NPA 2005 Load and Wind Analysis - 10\% Penetration 


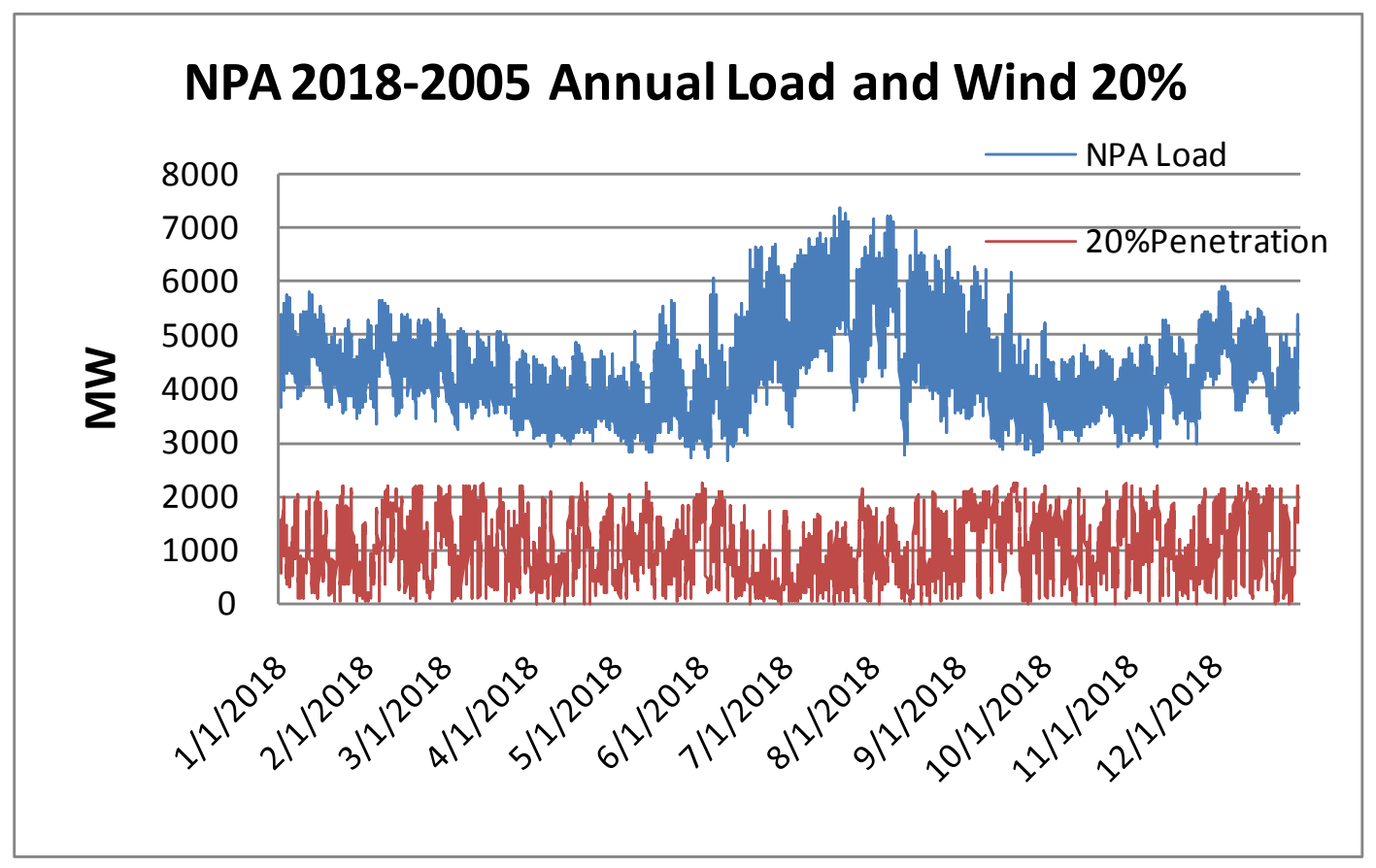

Figure 50: NPA 2005 Load and Wind Analysis - 20\% Penetration

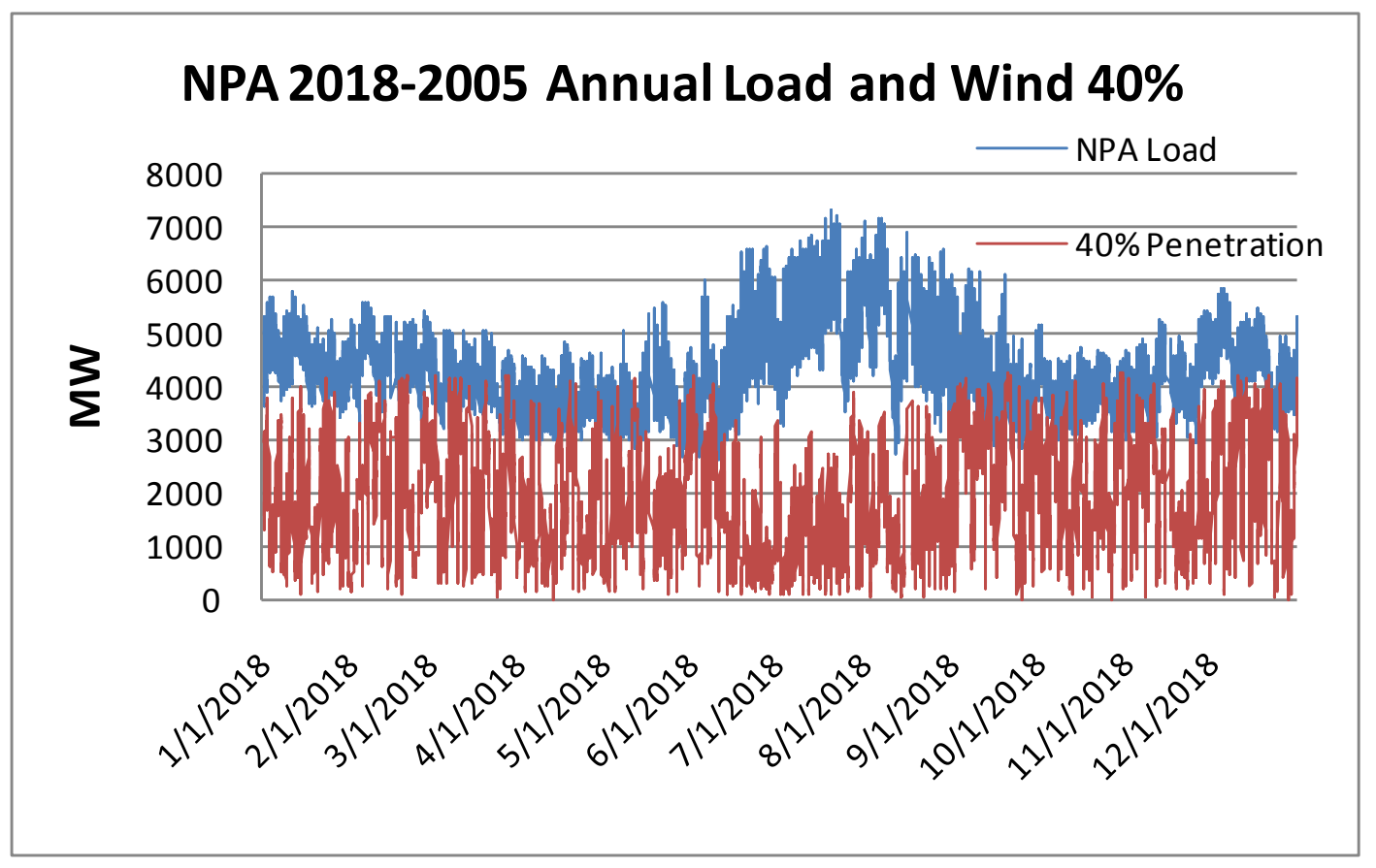

Figure 51: NPA 2005 Load and Wind Analysis - 40\% Penetration 


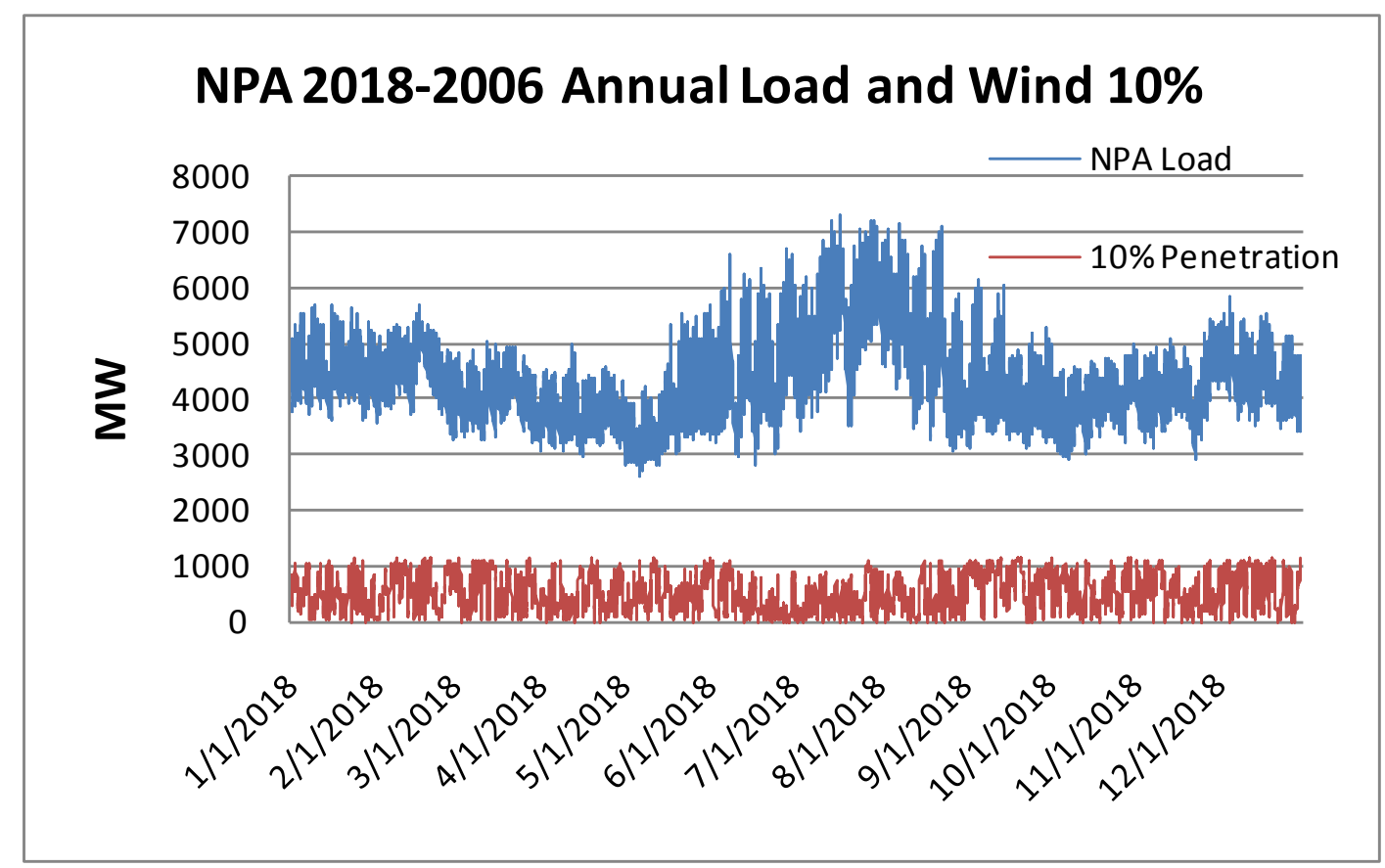

Figure 52: NPA 2006 Load and Wind Analysis - 10\% Penetration

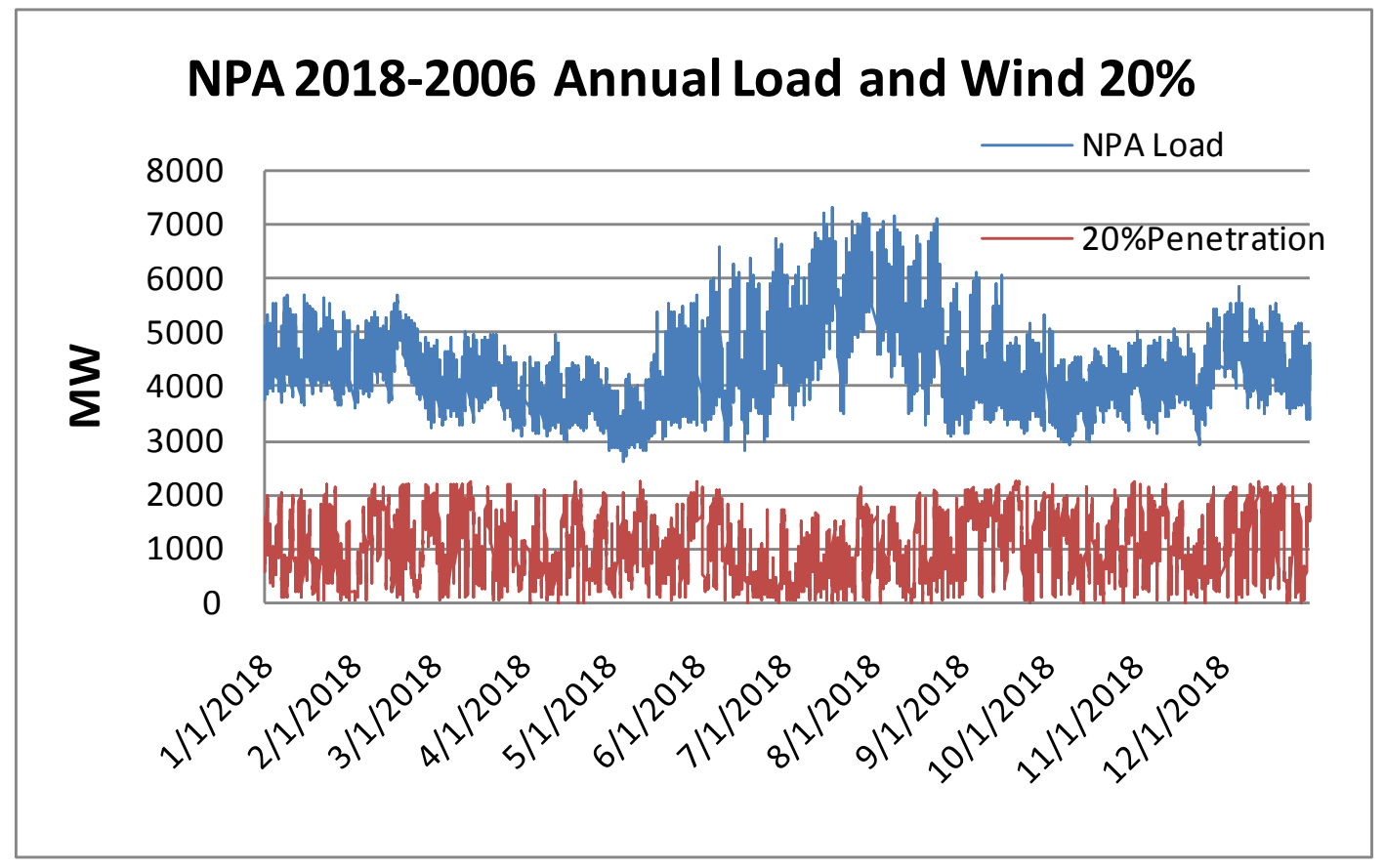

Figure 53: NPA 2006 Load and Wind Analysis - 20\% Penetration 


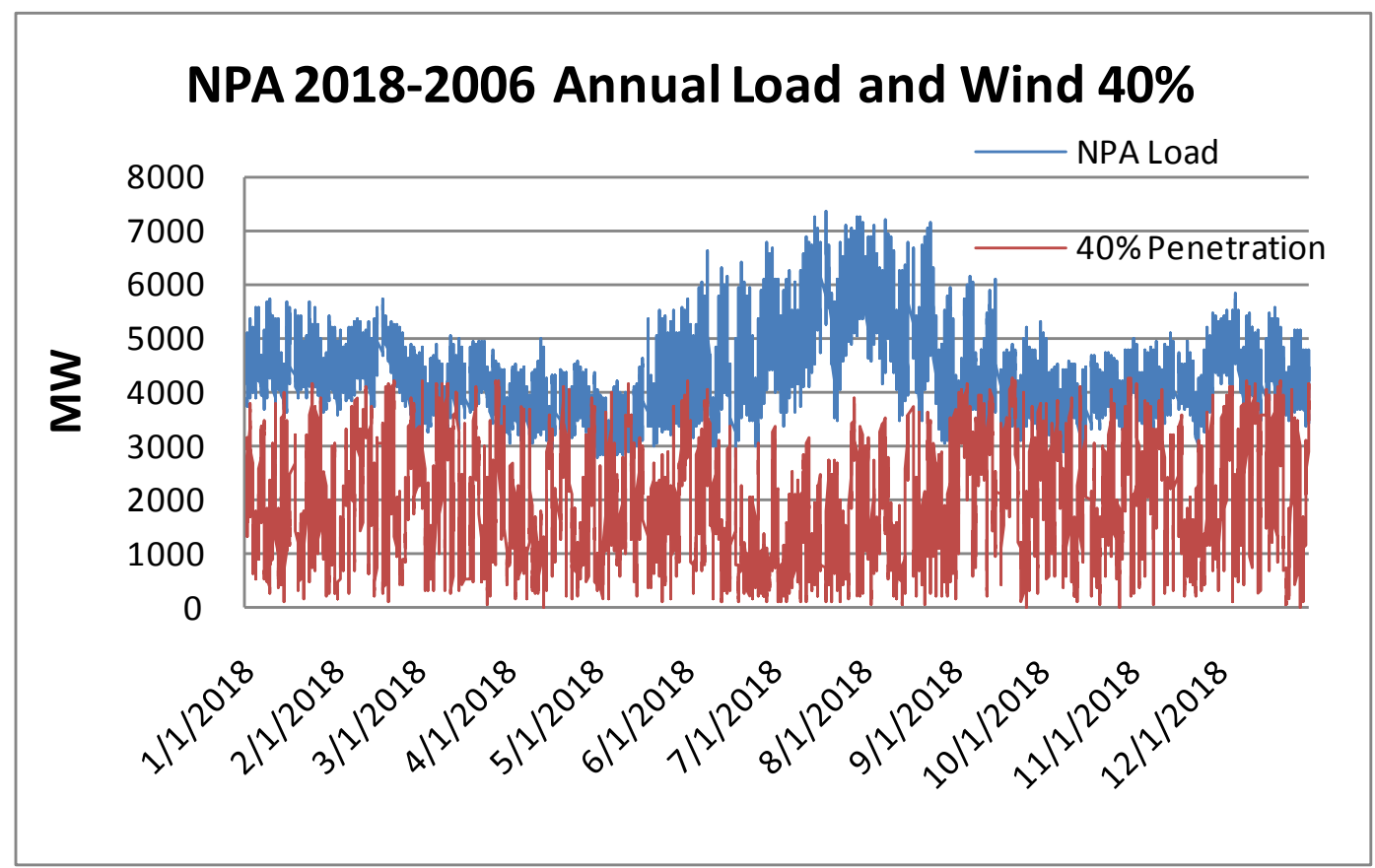

Figure 54: NPA 2006 Load and Wind Analysis - 40\% Penetration 


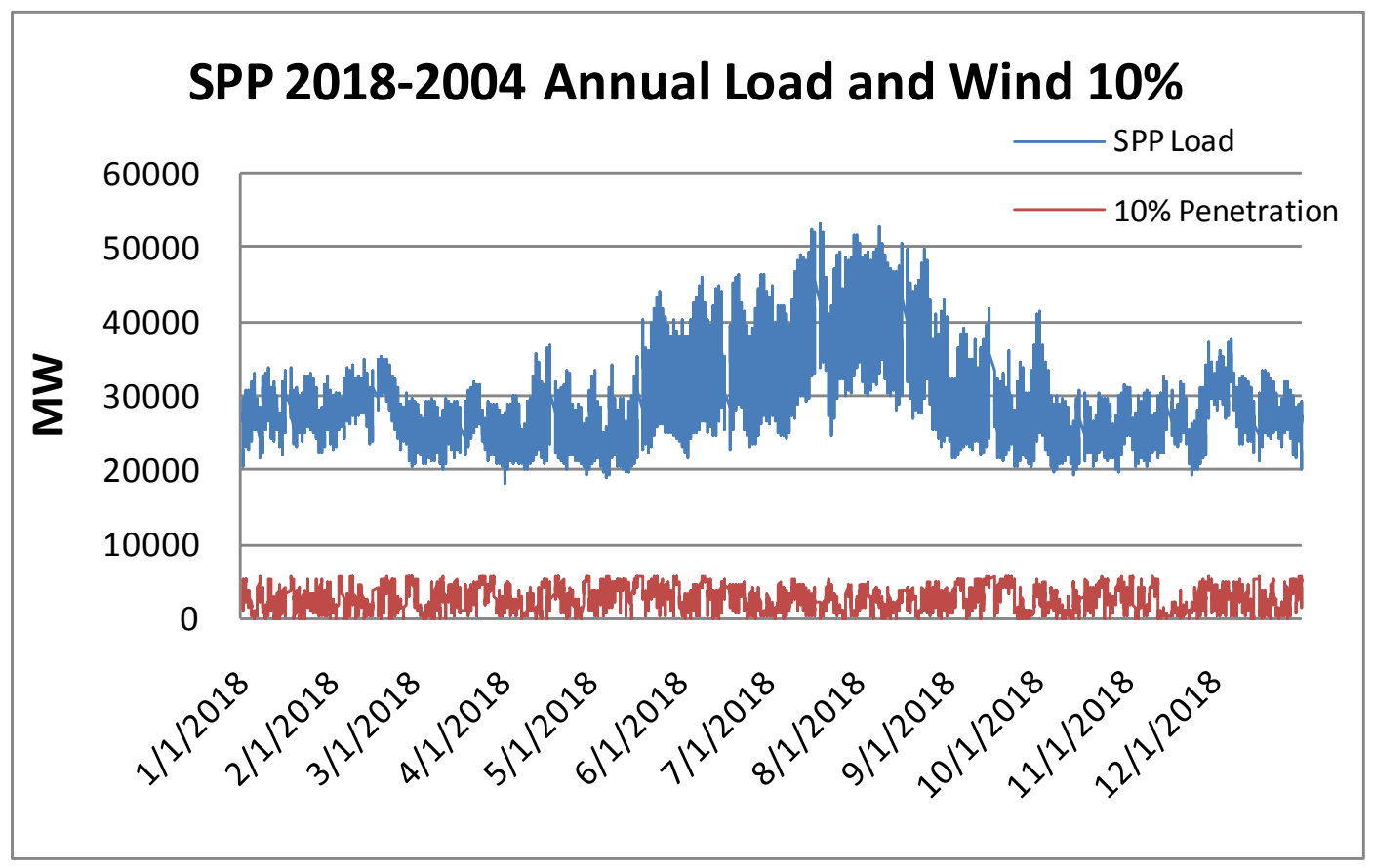

Figure 55: $\quad$ SPP 2004 Load and Wind Analysis - 10\% Penetration

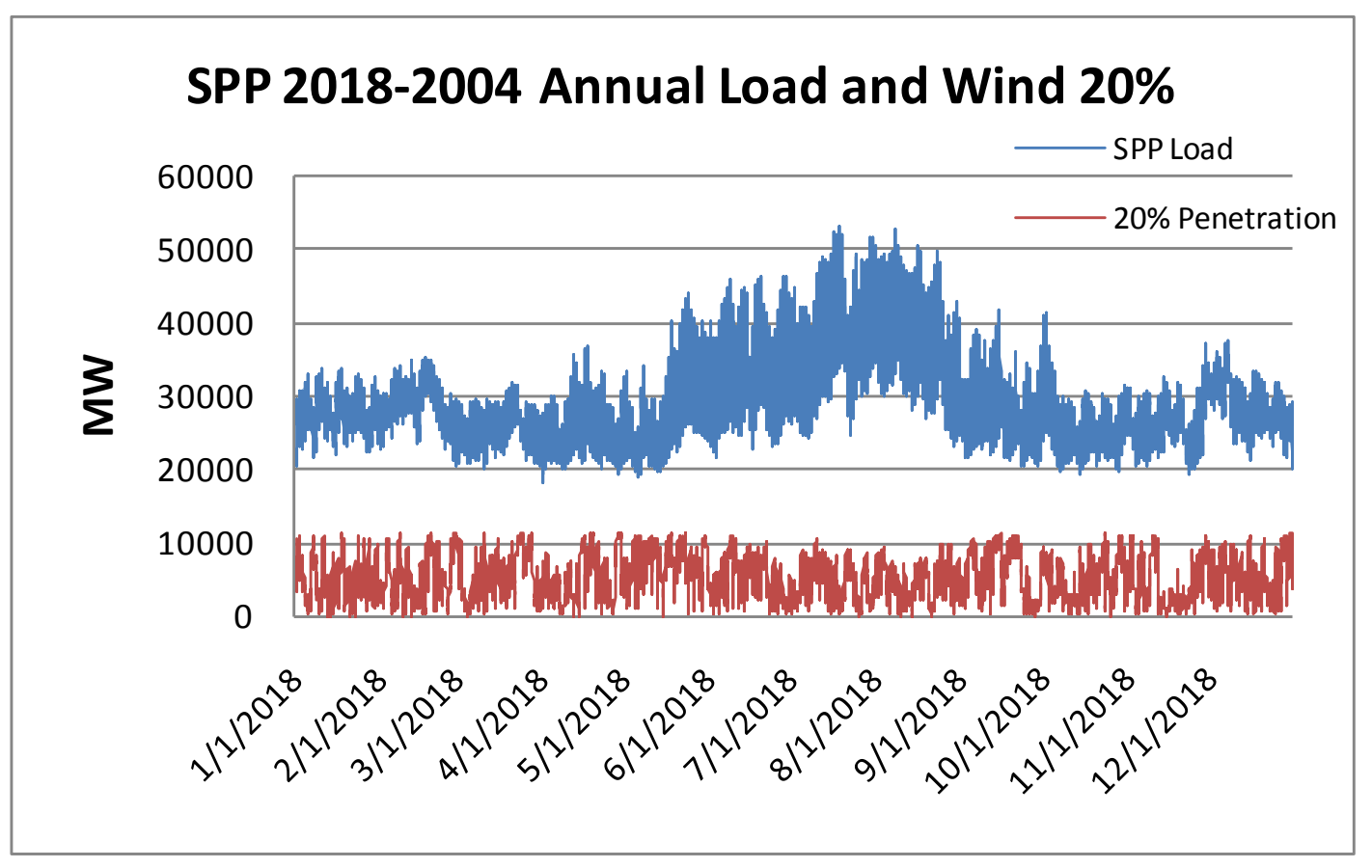

Figure 56: $\quad$ SPP 2004 Load and Wind Analysis - 20\% Penetration 


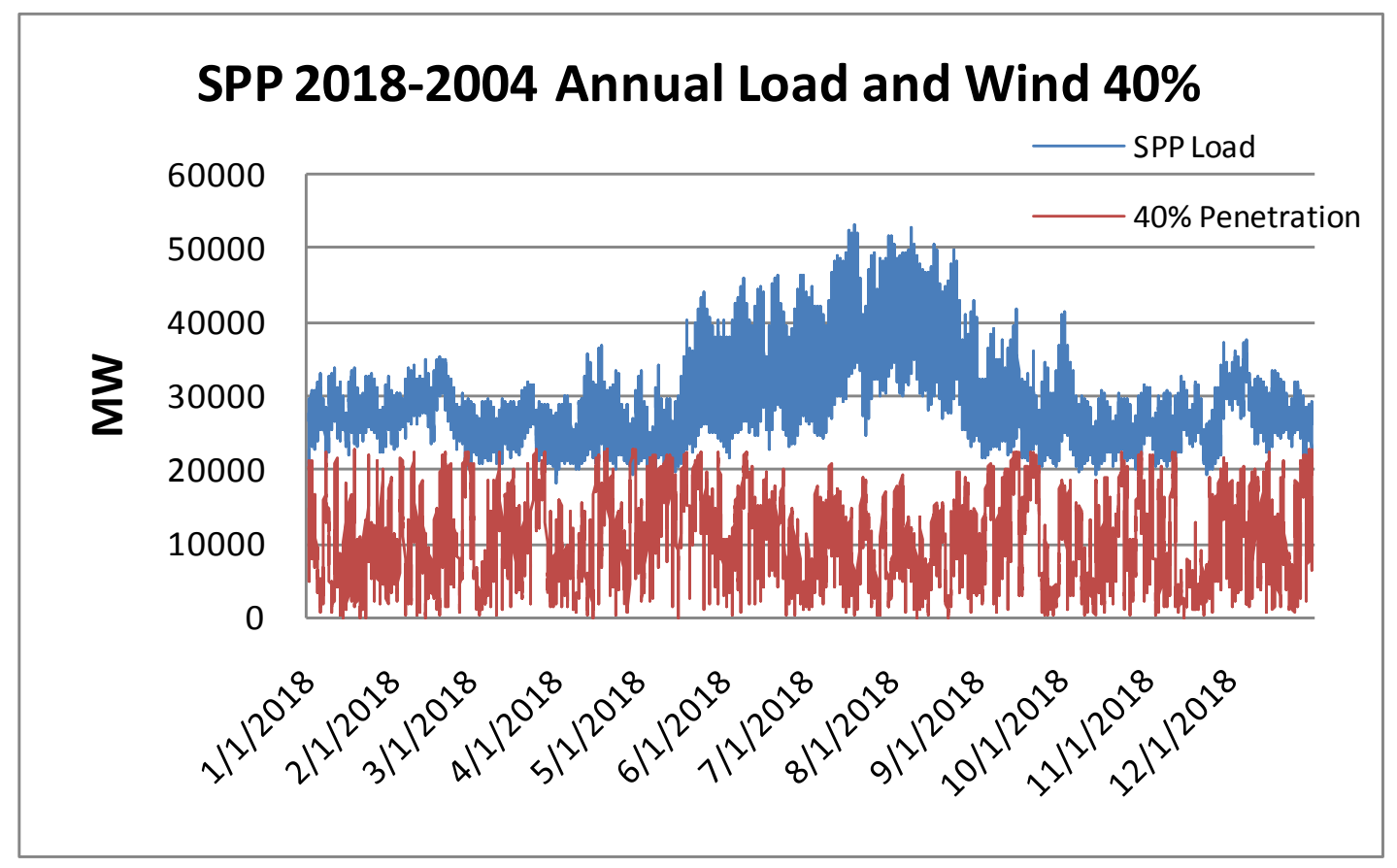

Figure 57: $\quad$ SPP 2004 Load and Wind Analysis - 40\% Penetration

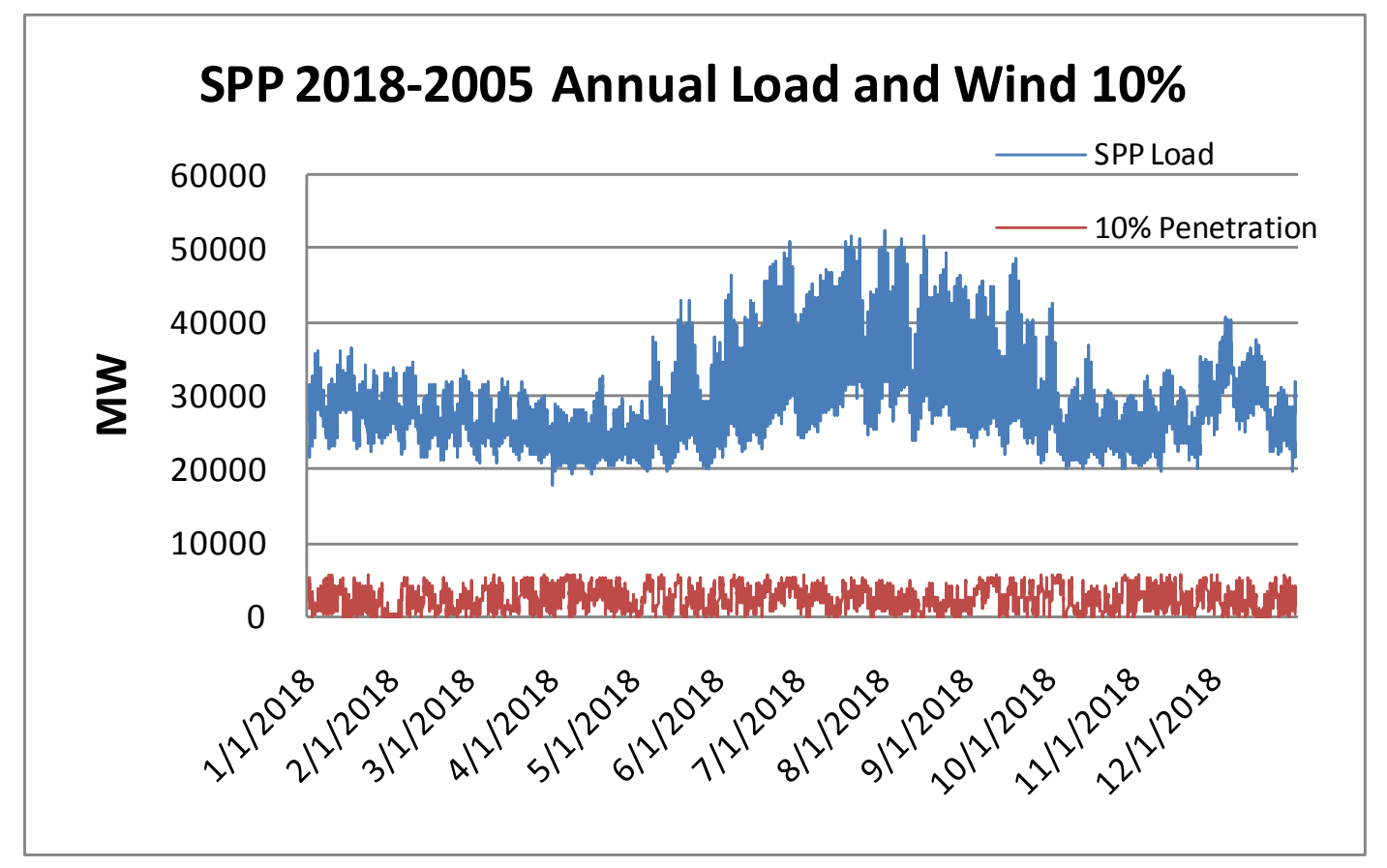

Figure 58: $\quad$ SPP 2005 Load and Wind Analysis - 10\% Penetration 


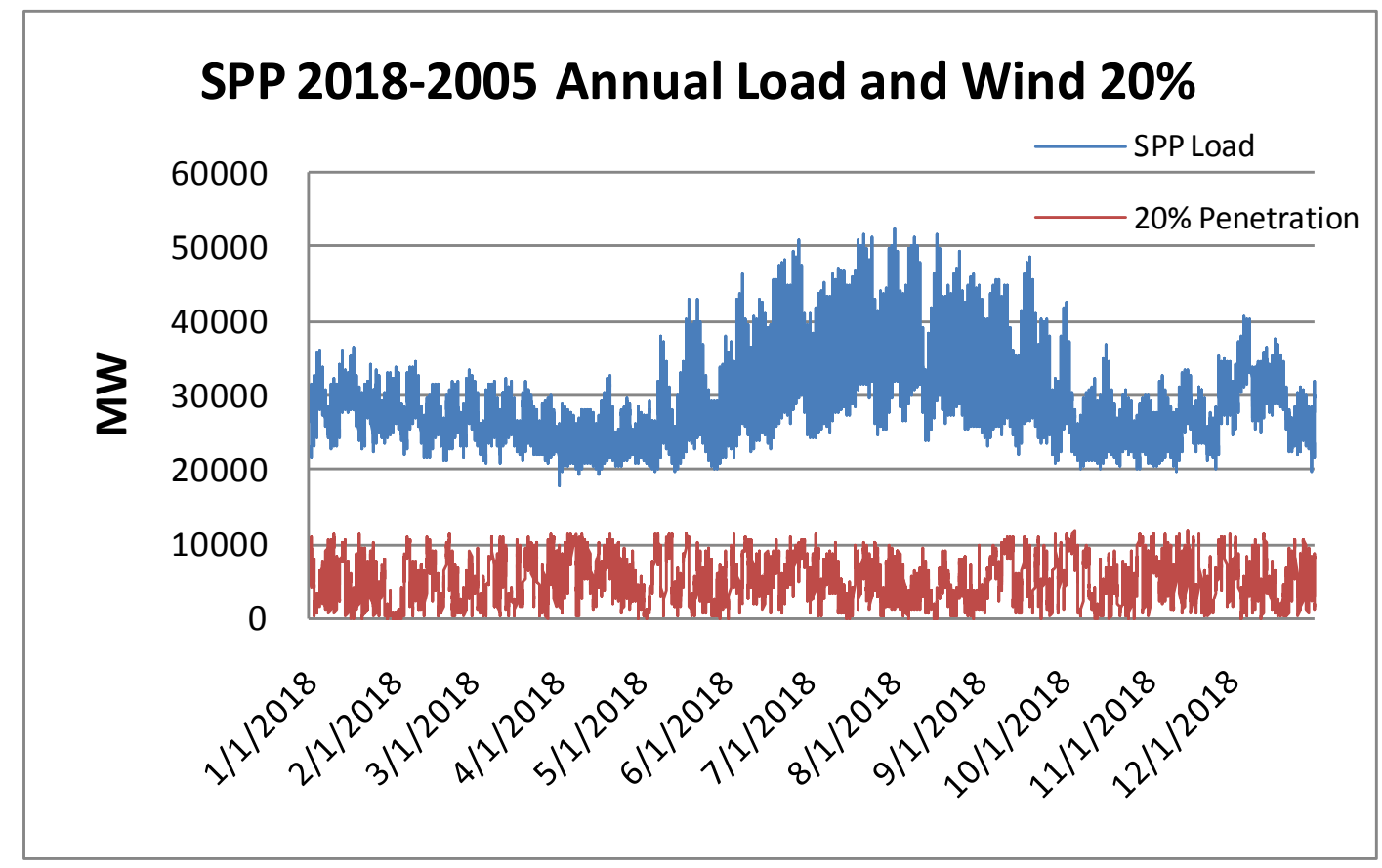

Figure 59: $\quad$ SPP 2005 Load and Wind Analysis - 20\% Penetration

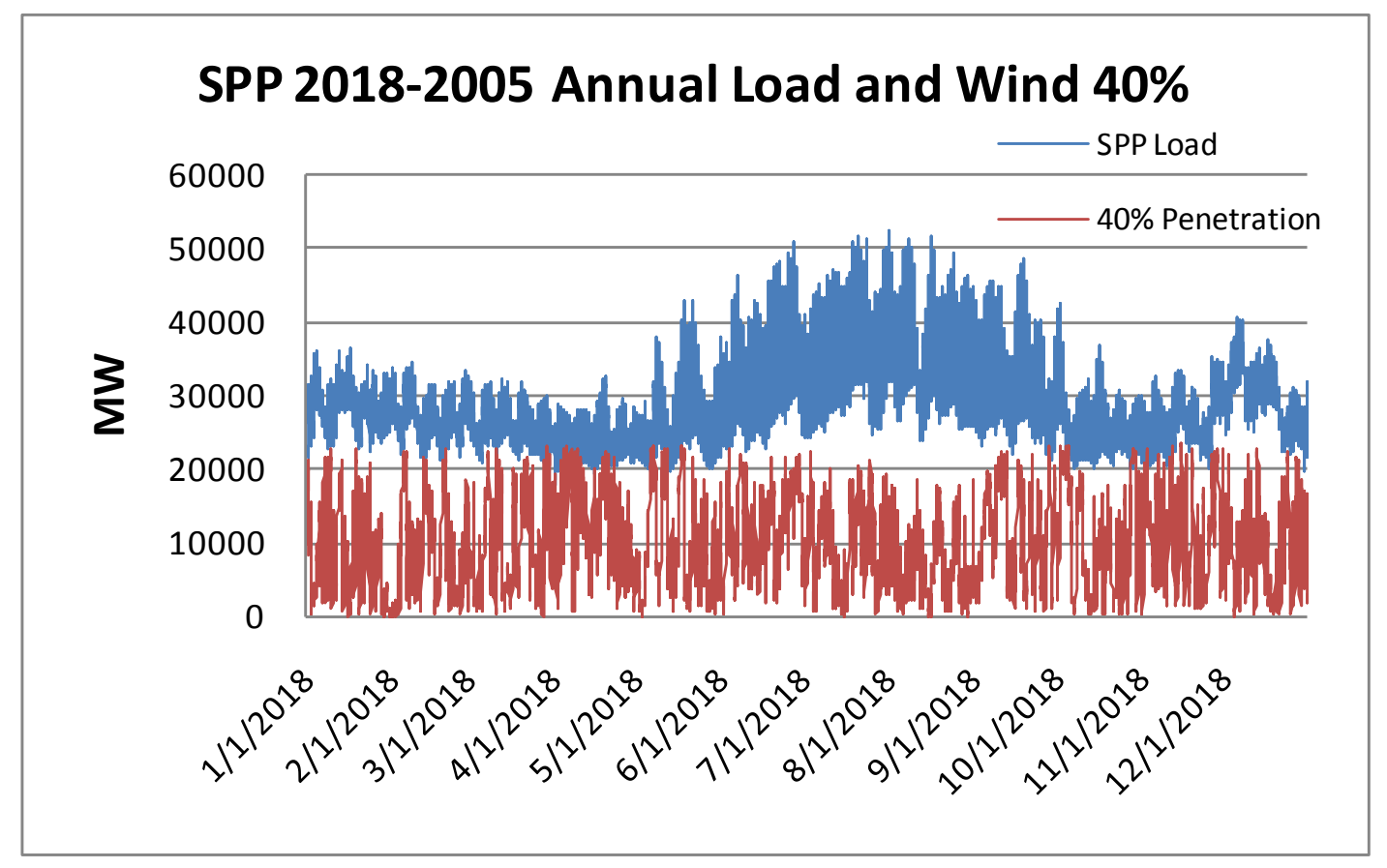

Figure 60: $\quad$ SPP 2005 Load and Wind Analysis - 40\% Penetration 


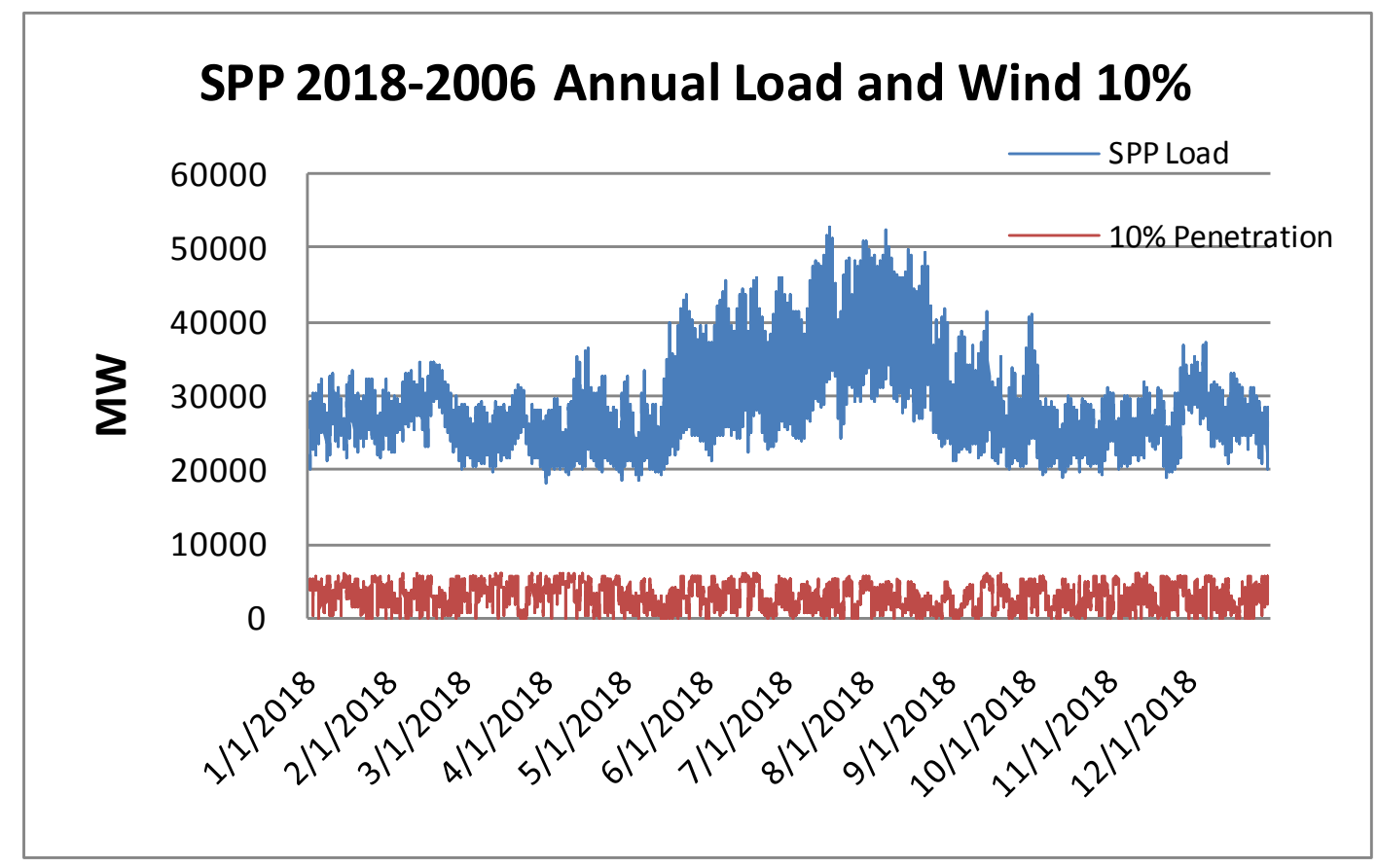

Figure 61: $\quad$ SPP 2006 Load and Wind Analysis - 10\% Penetration

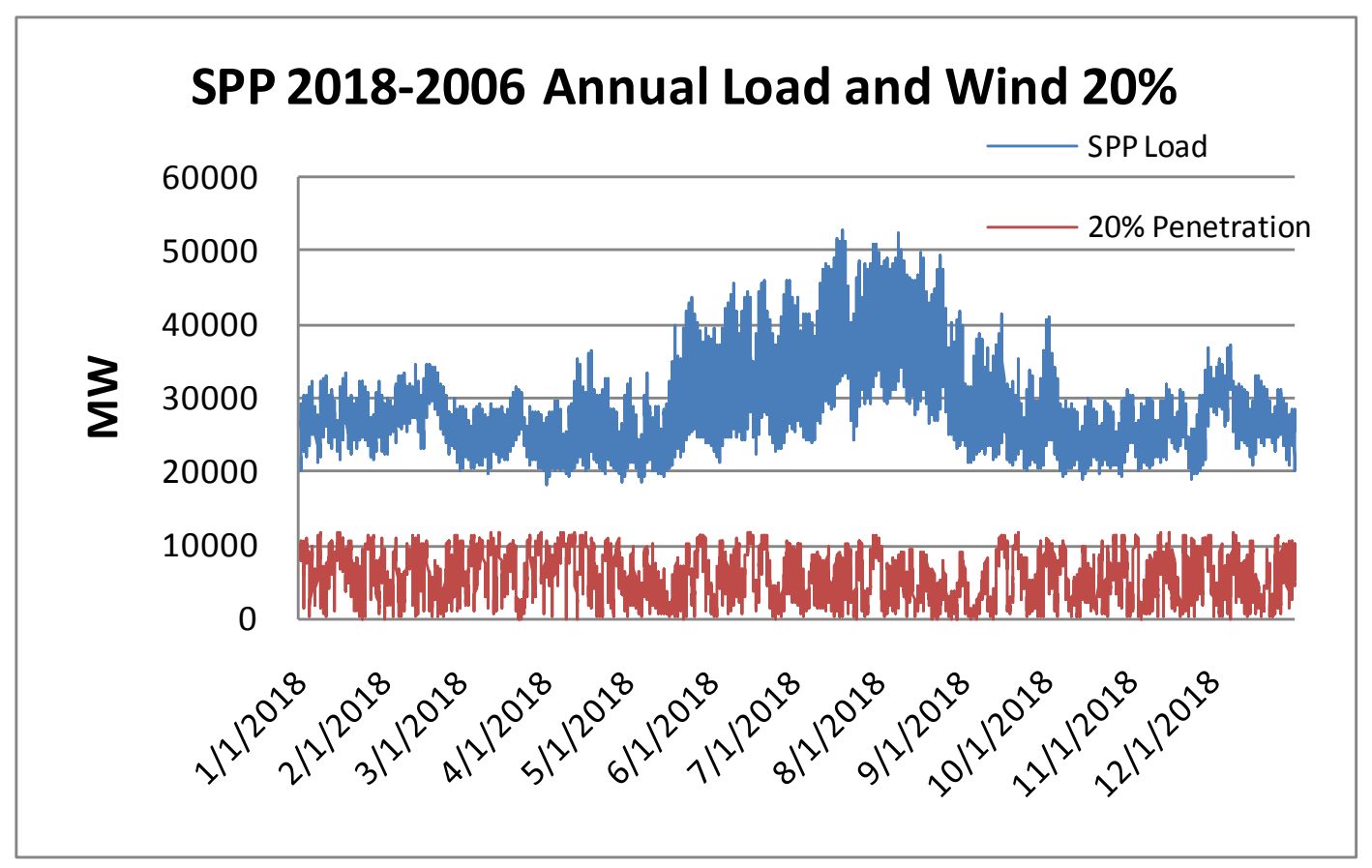

Figure 62: $\quad$ SPP 2006 Load and Wind Analysis - 20\% Penetration 


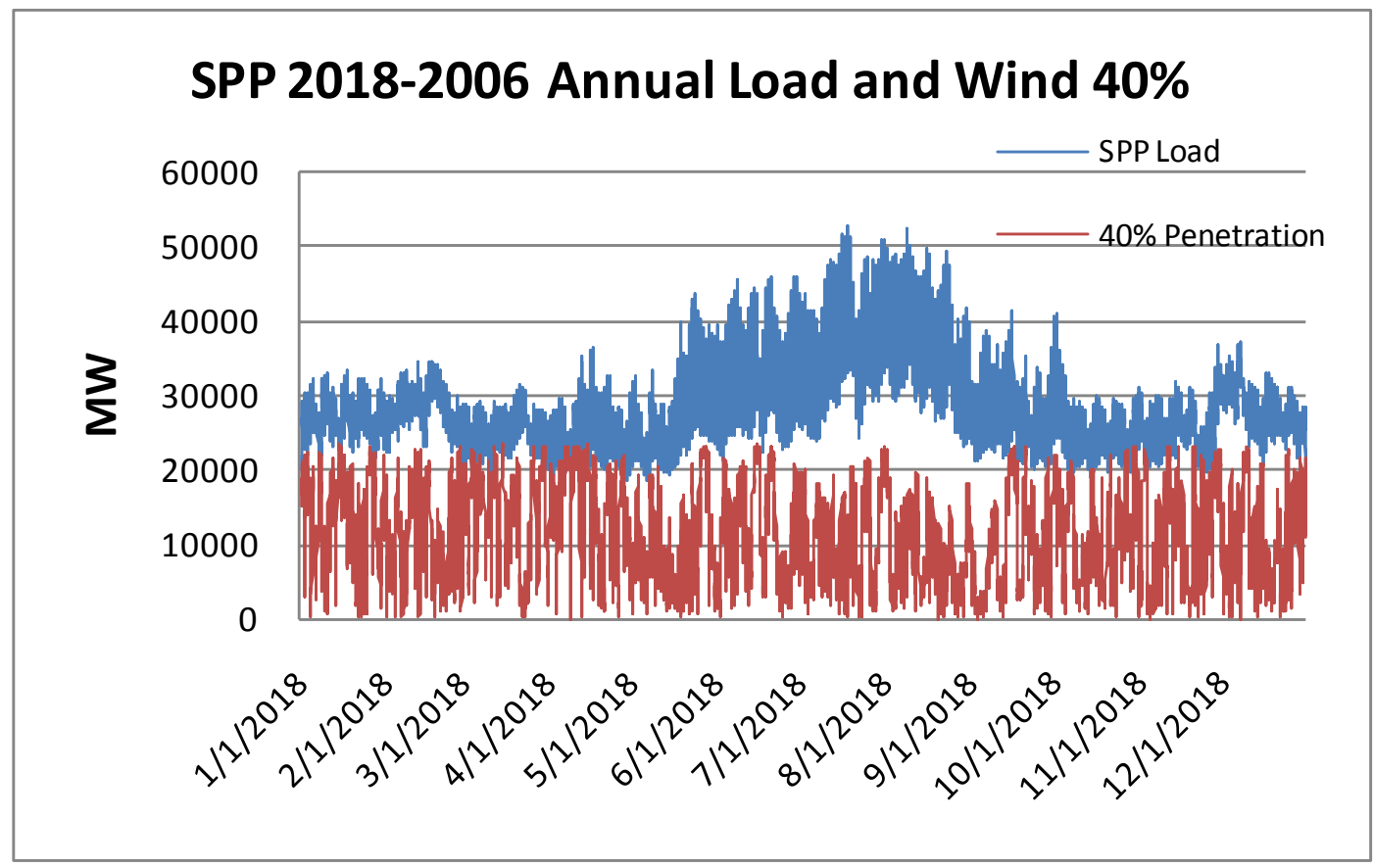

Figure 63: $\quad$ SPP 2006 Load and Wind Analysis - 40\% Penetration

\section{Section 8.2.3.2 Histogram Charts for Hourly Loads}

Three histograms, Figure 64 - Figure 66, show the distribution of average hourly loads for NPA, SPP including NPA, and SPP excluding NPA. "Average" is used here to mean the average of an hour's three values taken from 2004-2006 load data.

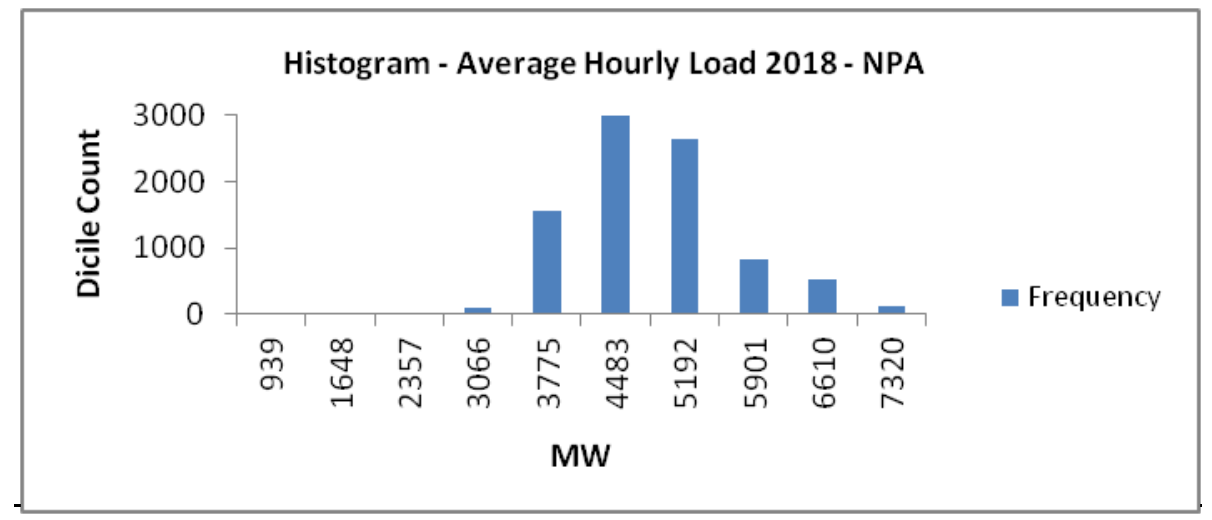

Figure 64: Histogram of Average NPA 2018 Load 


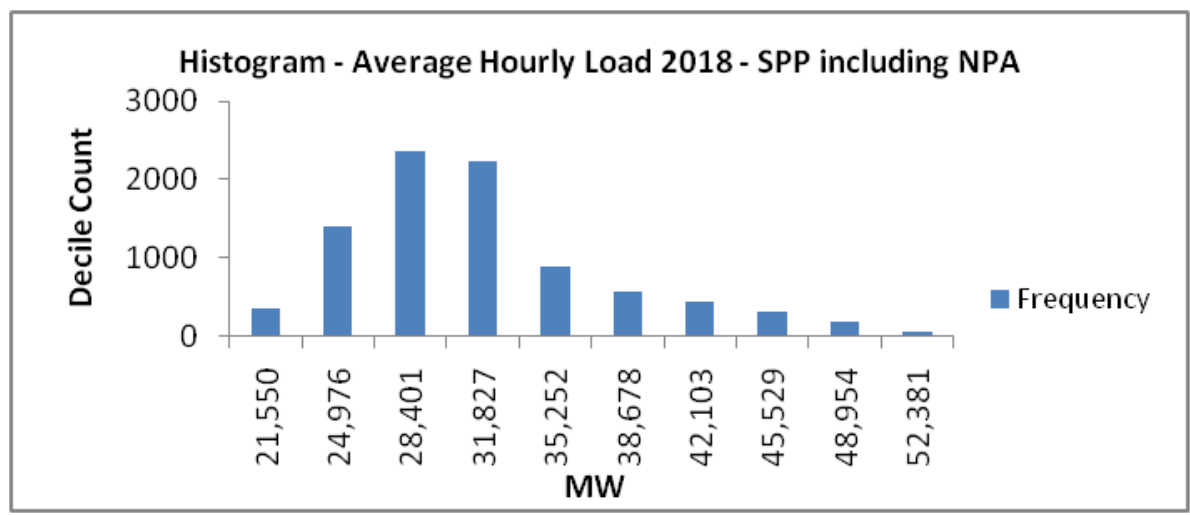

Figure 65: Histogram of Average SPP 2018 Load Including NPA

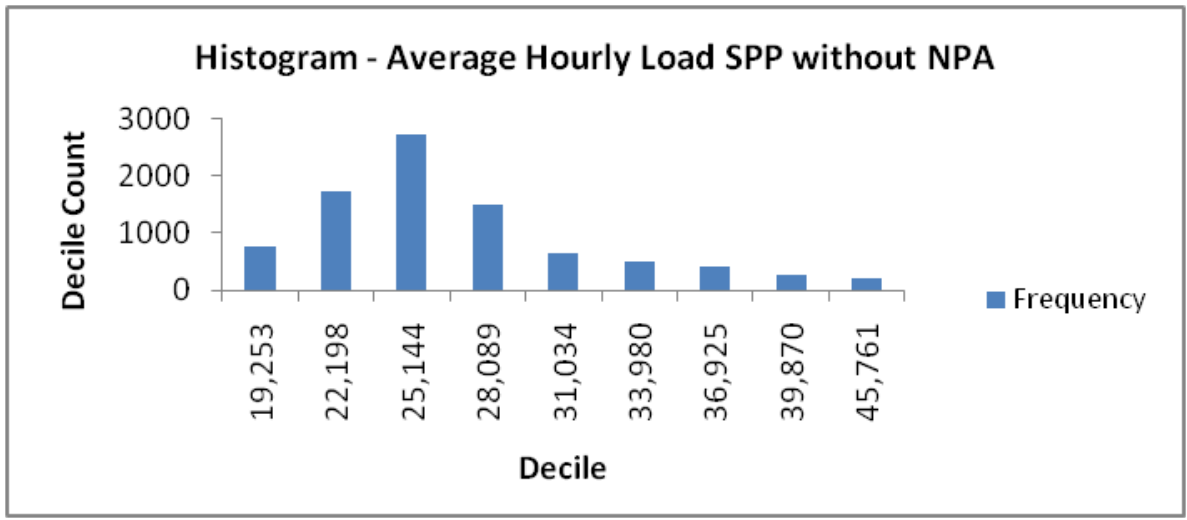

Figure 66: Histogram of Average SPP - NPA 2018 Load Excluding NPA

\section{Section 8.2.3.3 Average Seasonal Load and Wind Production}

The charts below in Figure 67 through Figure 81 show 24 hour day shapes for each region. The seasons are defined as winter: December - February; spring: March - May; summer: June August; fall: September - November. Day shapes are created by averaging each respective hour of the day for the period of interest. The relative relationship between wind and load for the $10 \%, 20 \%$ and $40 \%$ penetration scenarios can be seen as well as separate charts with wind only to enhance display of wind differences. 


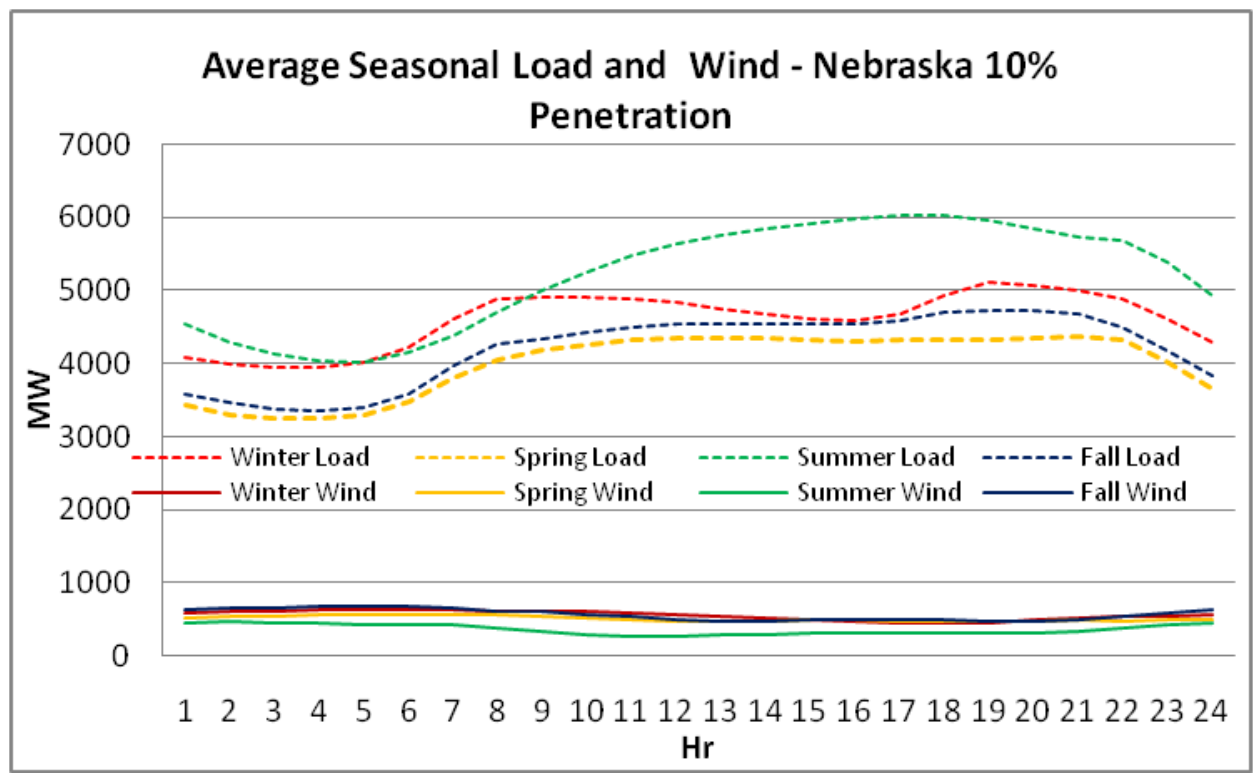

Figure 67: NPA - 24-Hour Average Seasonal Load and Wind 10\% Penetration

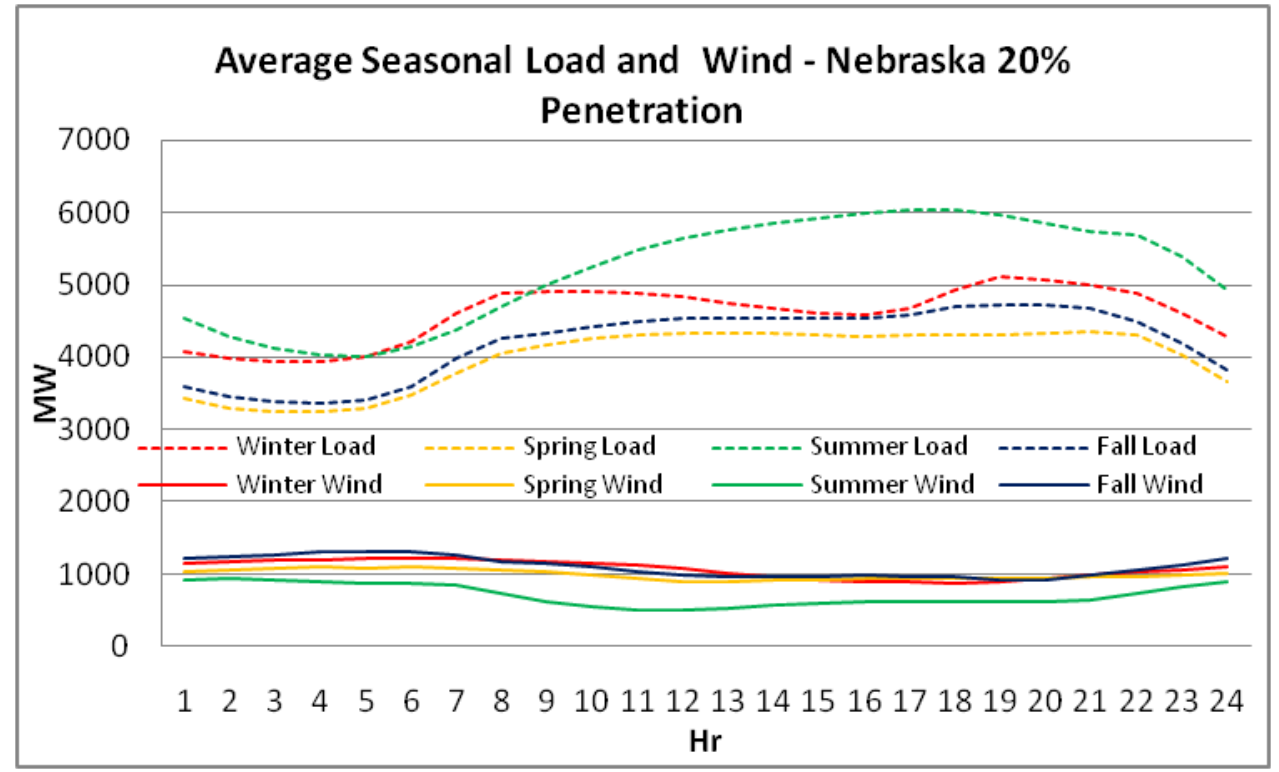

Figure 68: NPA - 24-Hour Average Seasonal Load and Wind 20\% Penetration 


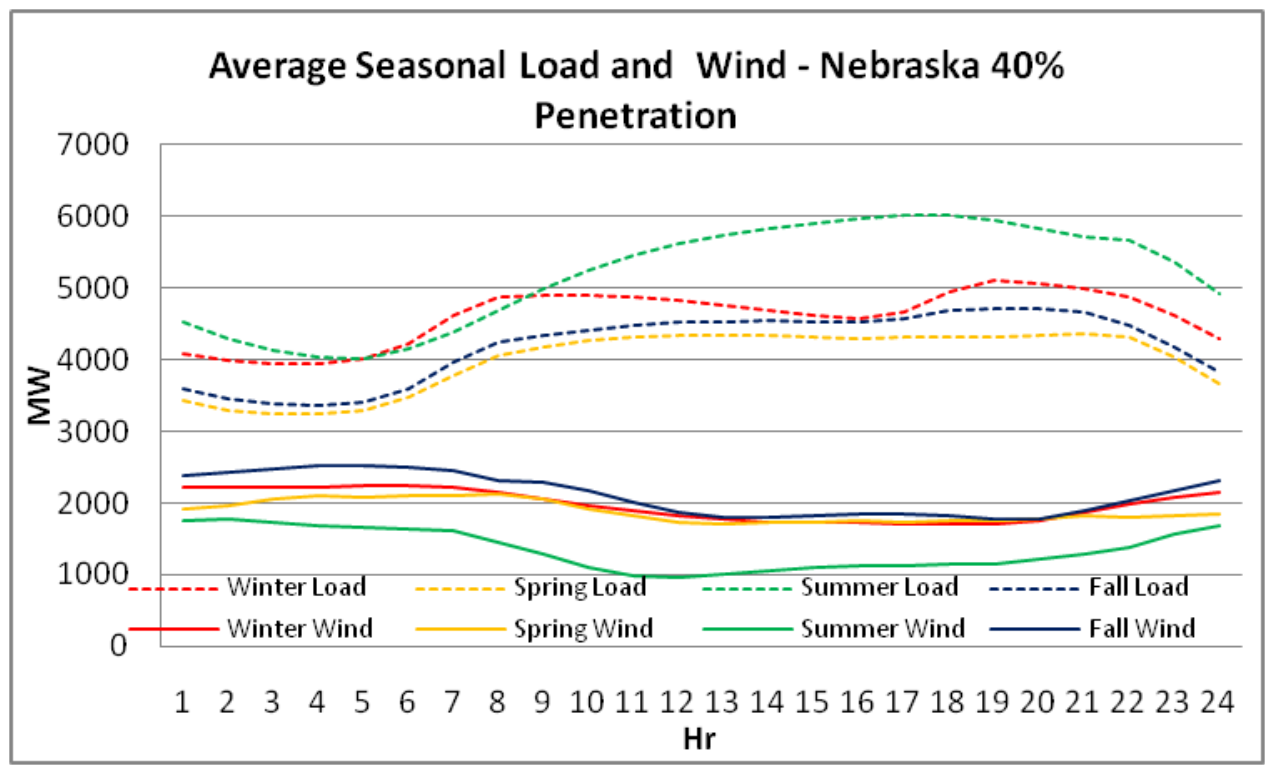

Figure 69: NPA - 24-Hour Average Seasonal Load and Wind 40\% Penetration

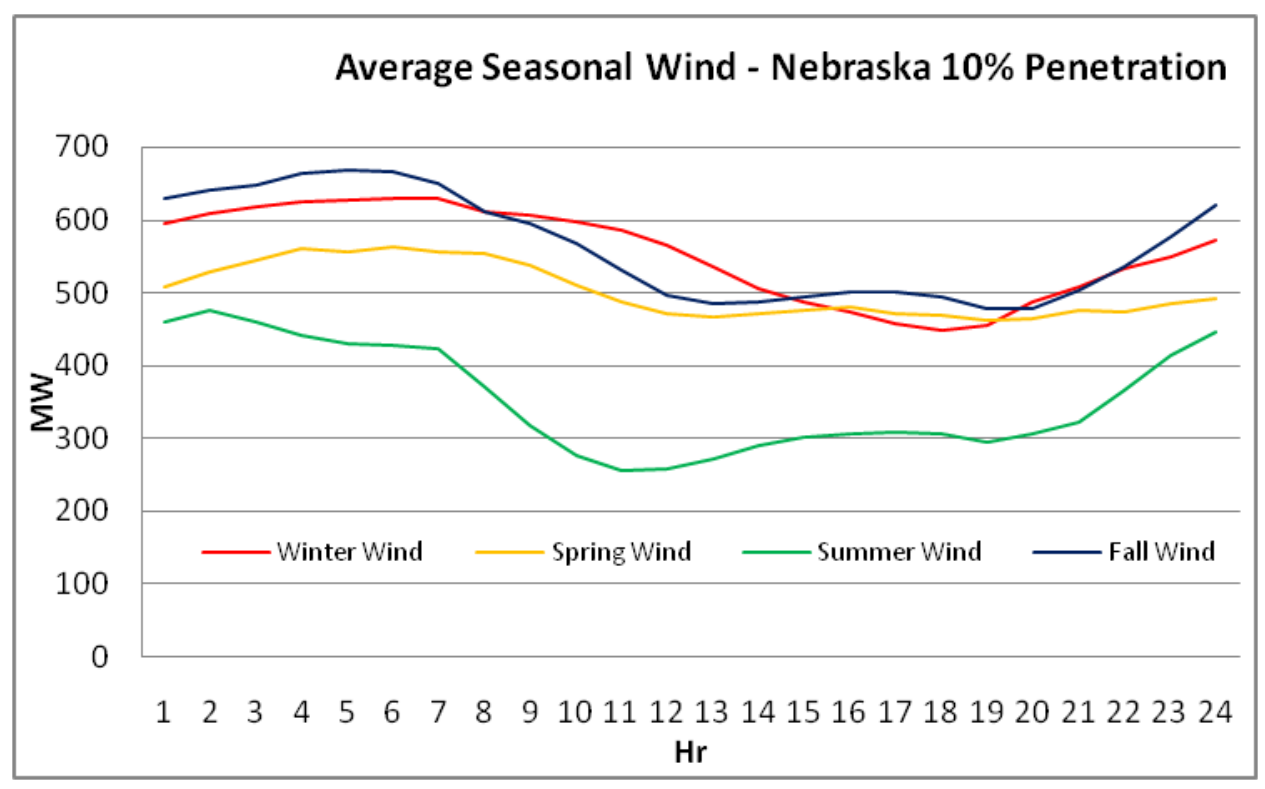

Figure 70: NPA - 24 Hour Average Seasonal Wind 10\% Penetration 


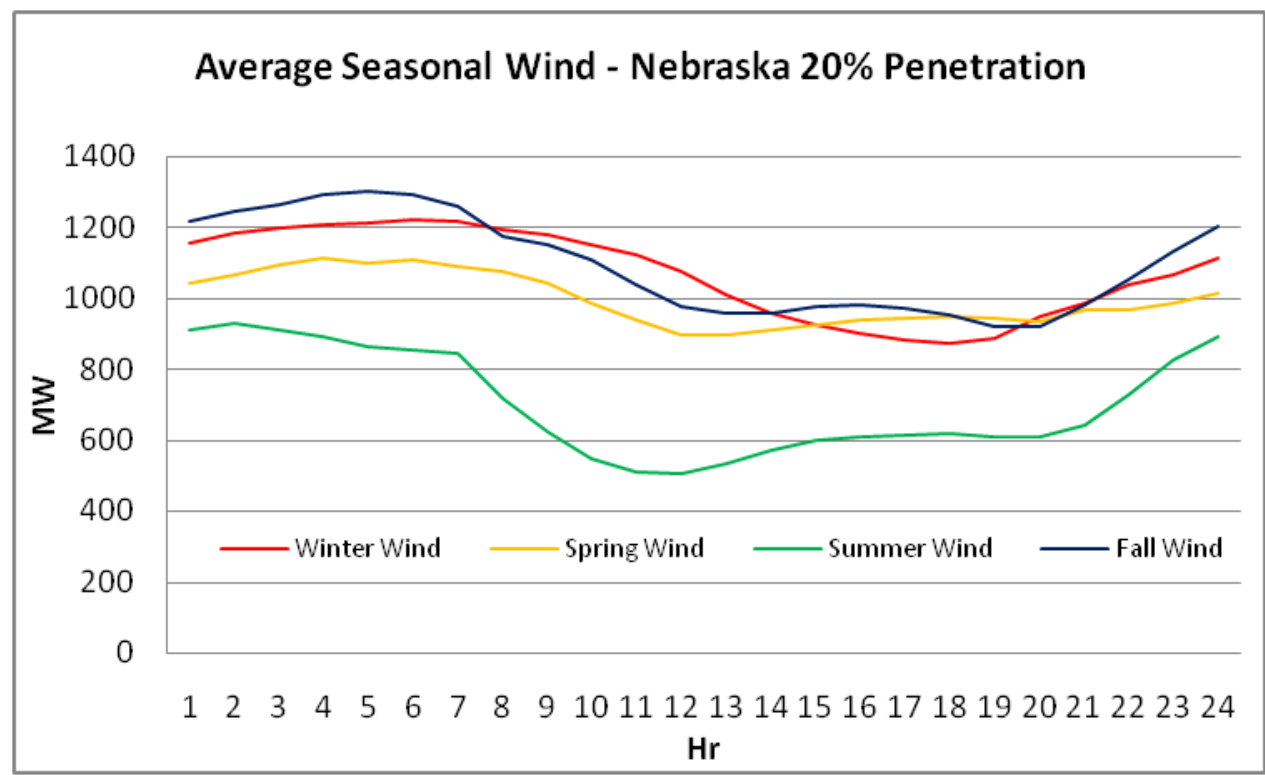

Figure 71: NPA - 24 Hour Average Seasonal Wind 20\% Penetration

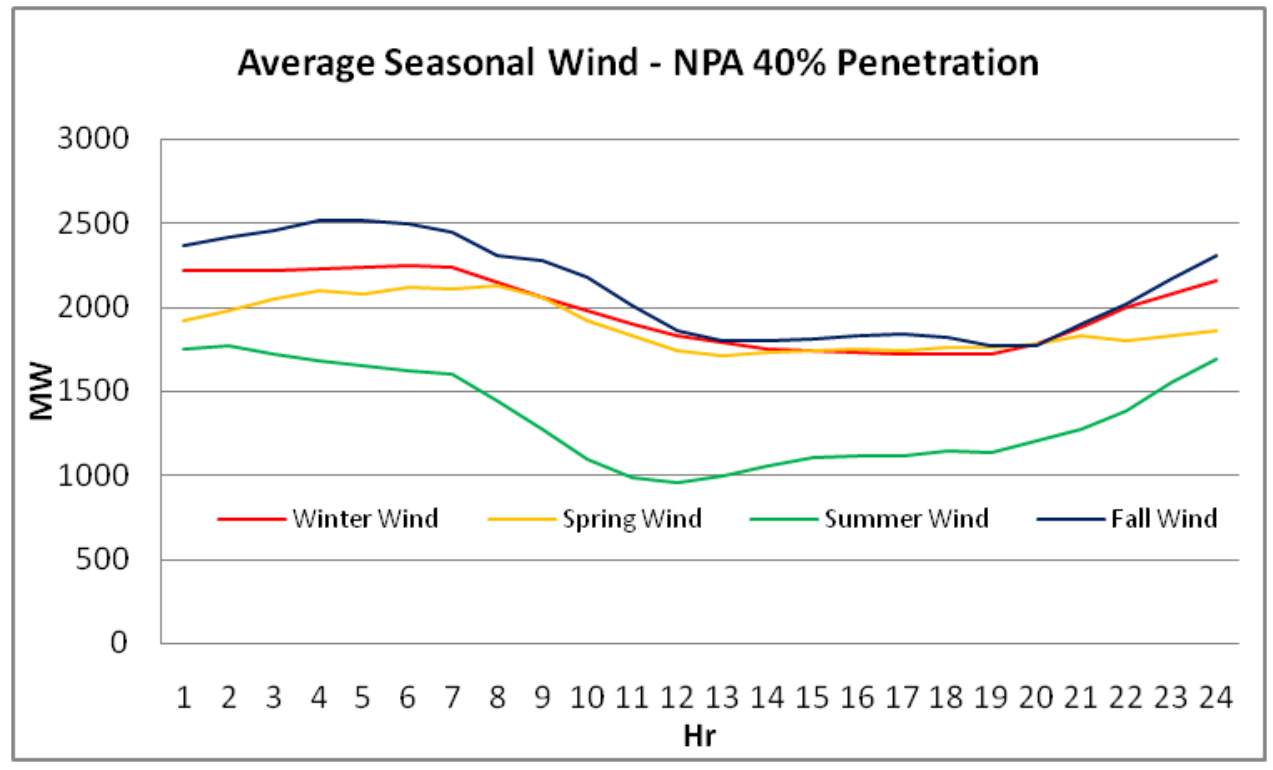

Figure 72: NPA - 24 Hour Average Seasonal Wind 40\% Penetration 


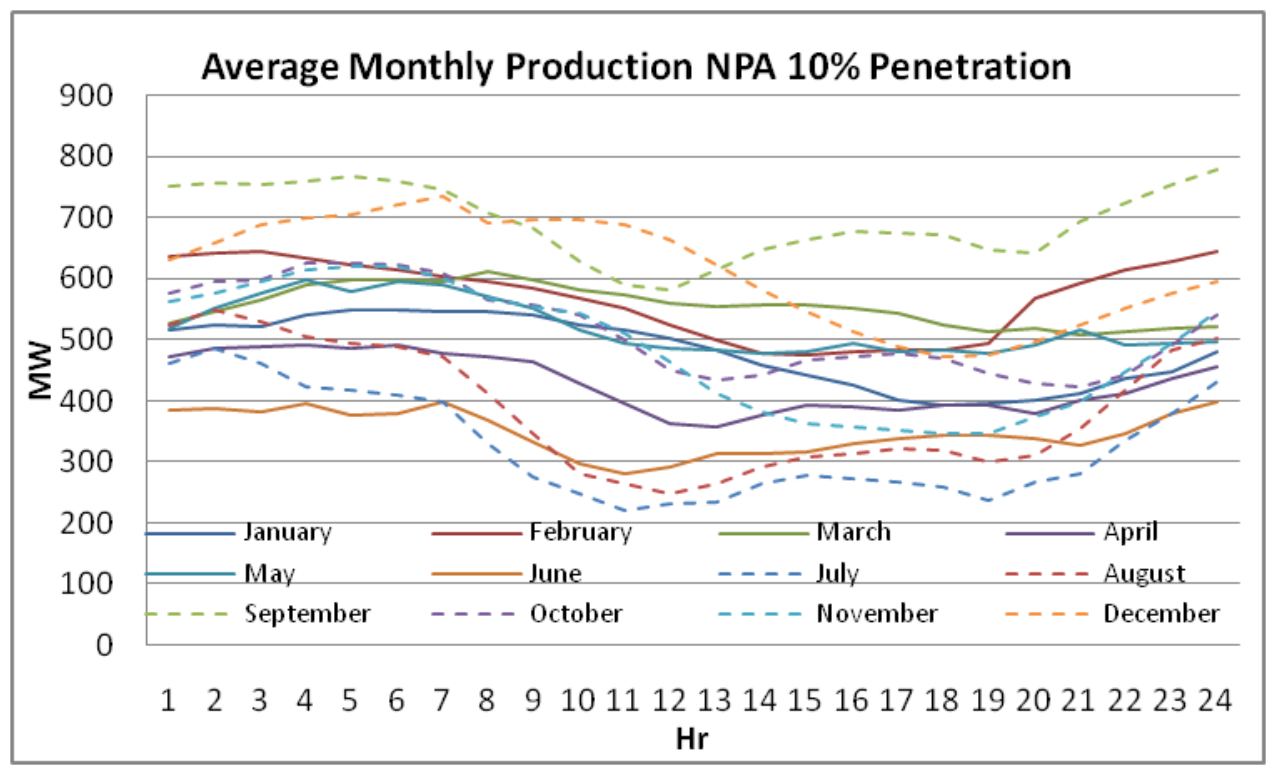

Figure 73: NPA - 24 Hour Average Monthly Wind 10\% Penetration

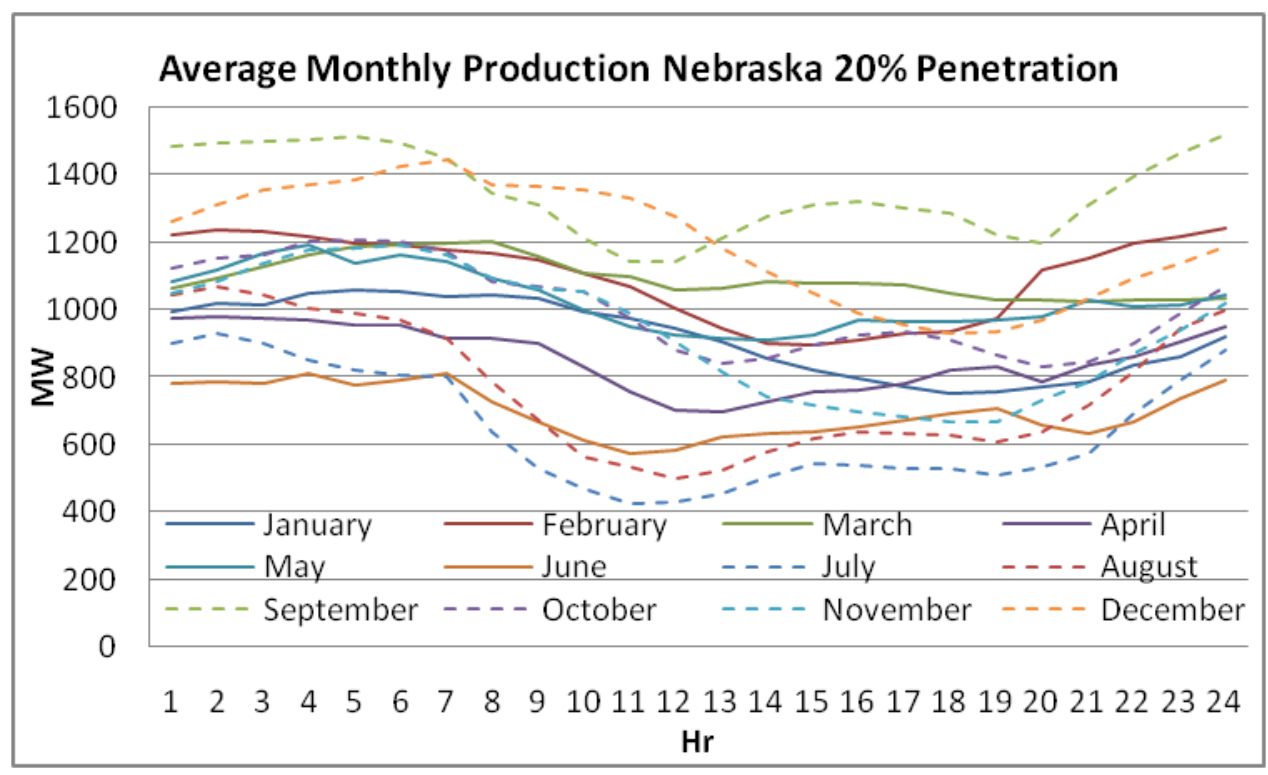

Figure 74: NPA - 24 Hour Average Monthly Wind 20\% Penetration 


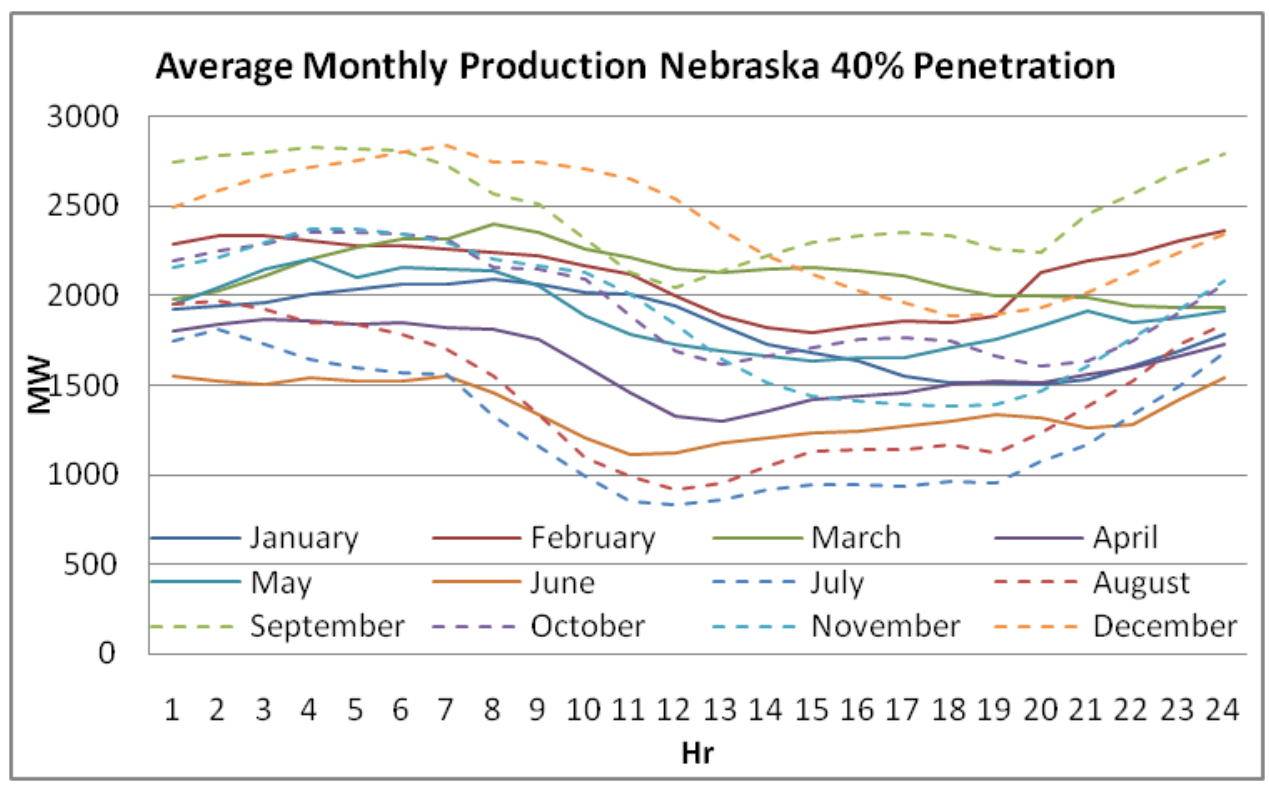

Figure 75: NPA - 24 Hour Average Monthly Wind 40\% Penetration

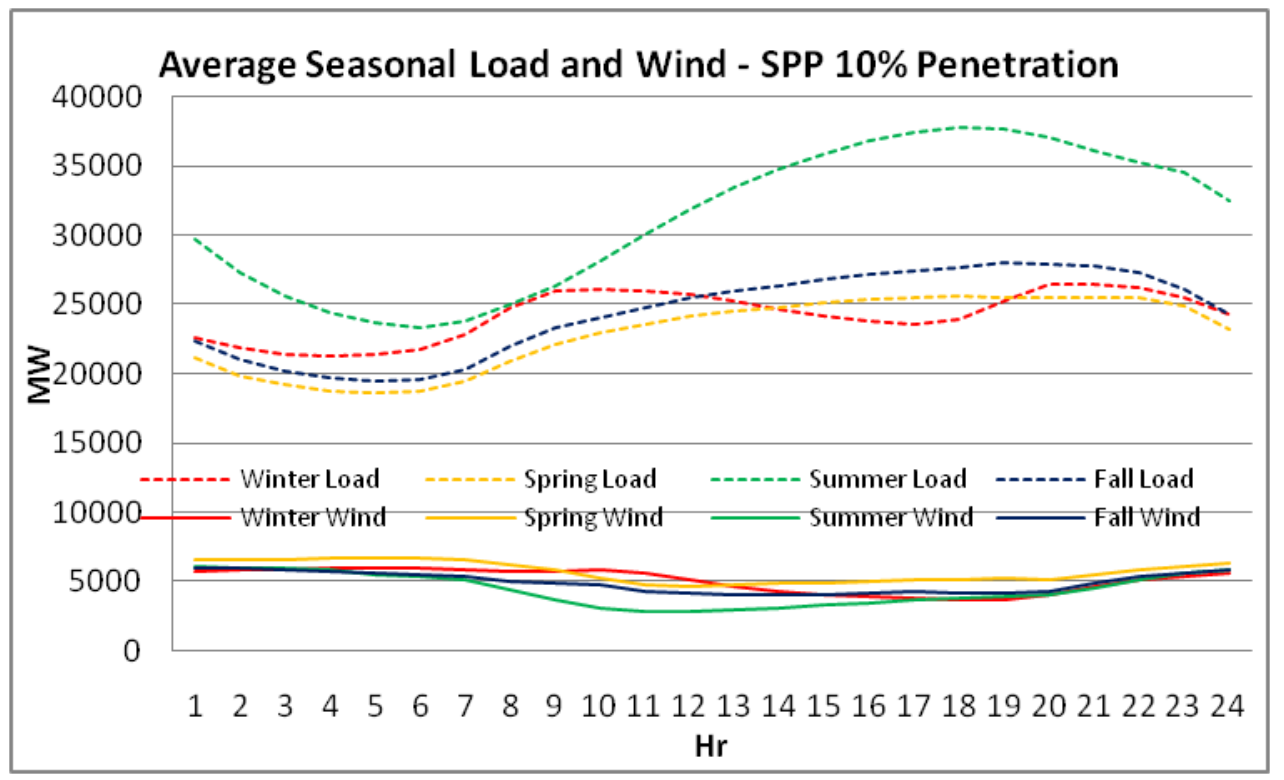

Figure 76: SPP - 24 Hour Average Seasonal Load and Wind 10\% Penetration 


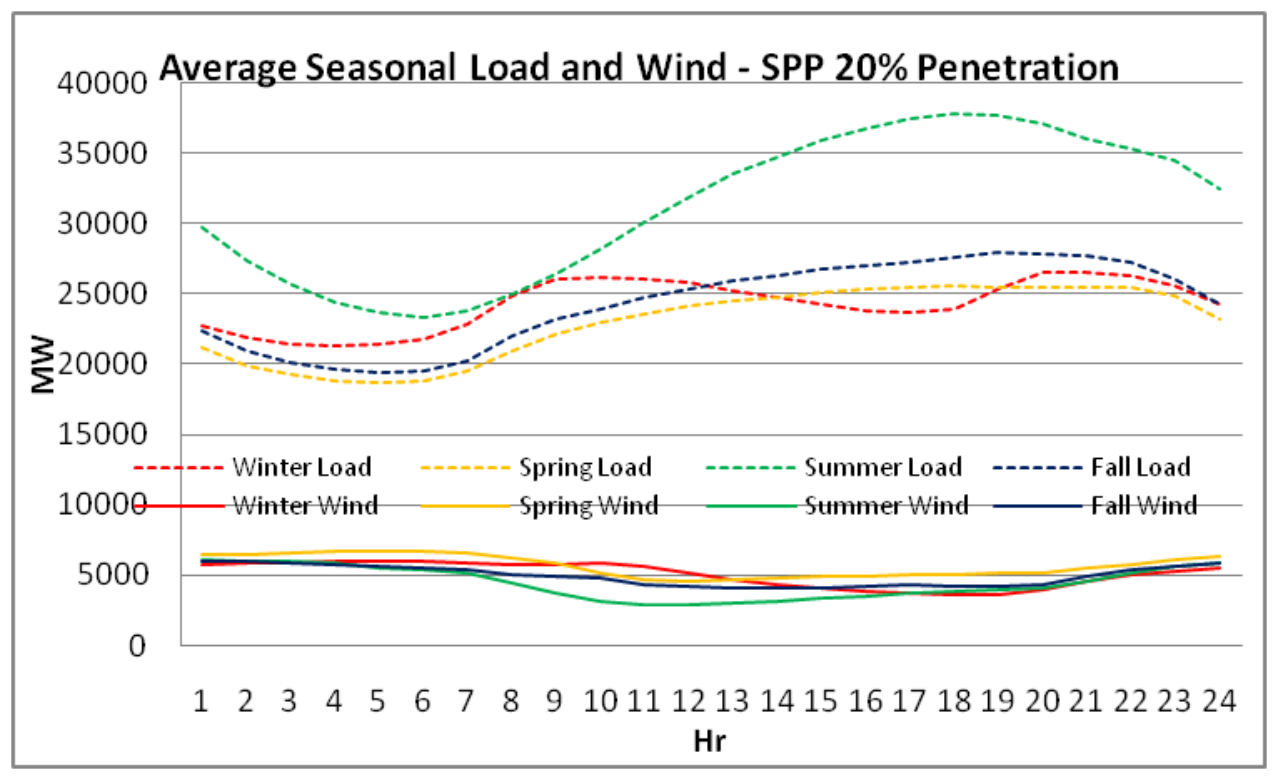

Figure 77: SPP - 24 Hour Average Seasonal Load and Wind 20\% Penetration

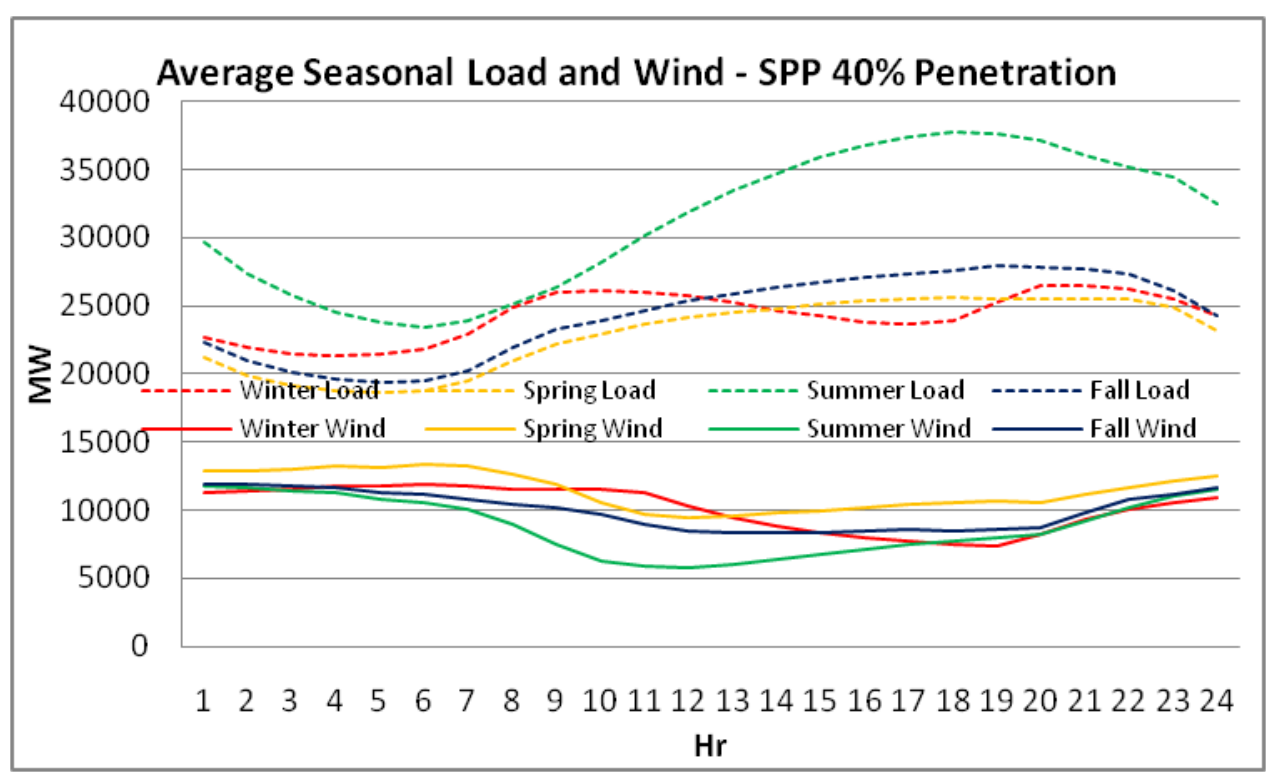

Figure 78: SPP - 24 Hour Average Seasonal Load and Wind 40\% Penetration 


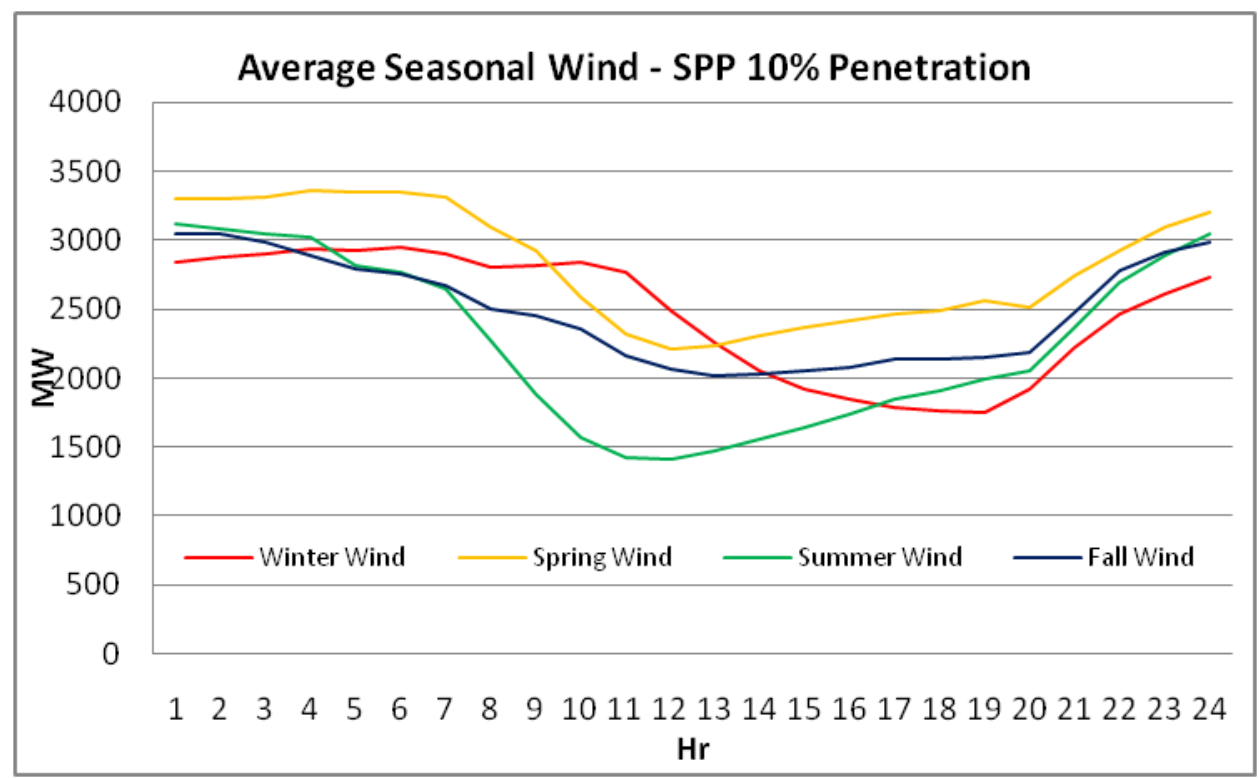

Figure 79: SPP - 24 Hour Average Seasonal Wind 10\% Penetration

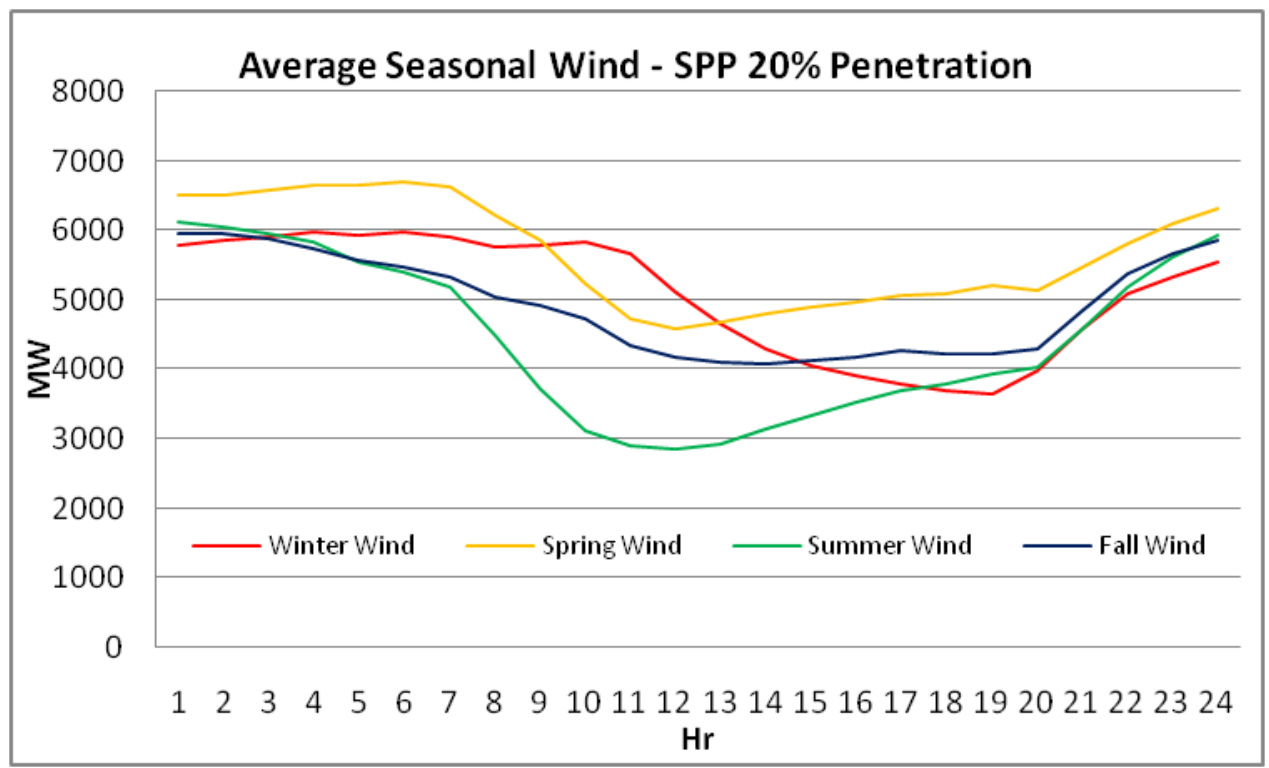

Figure 80: SPP - 24 Hour Average Seasonal Wind 20\% Penetration 


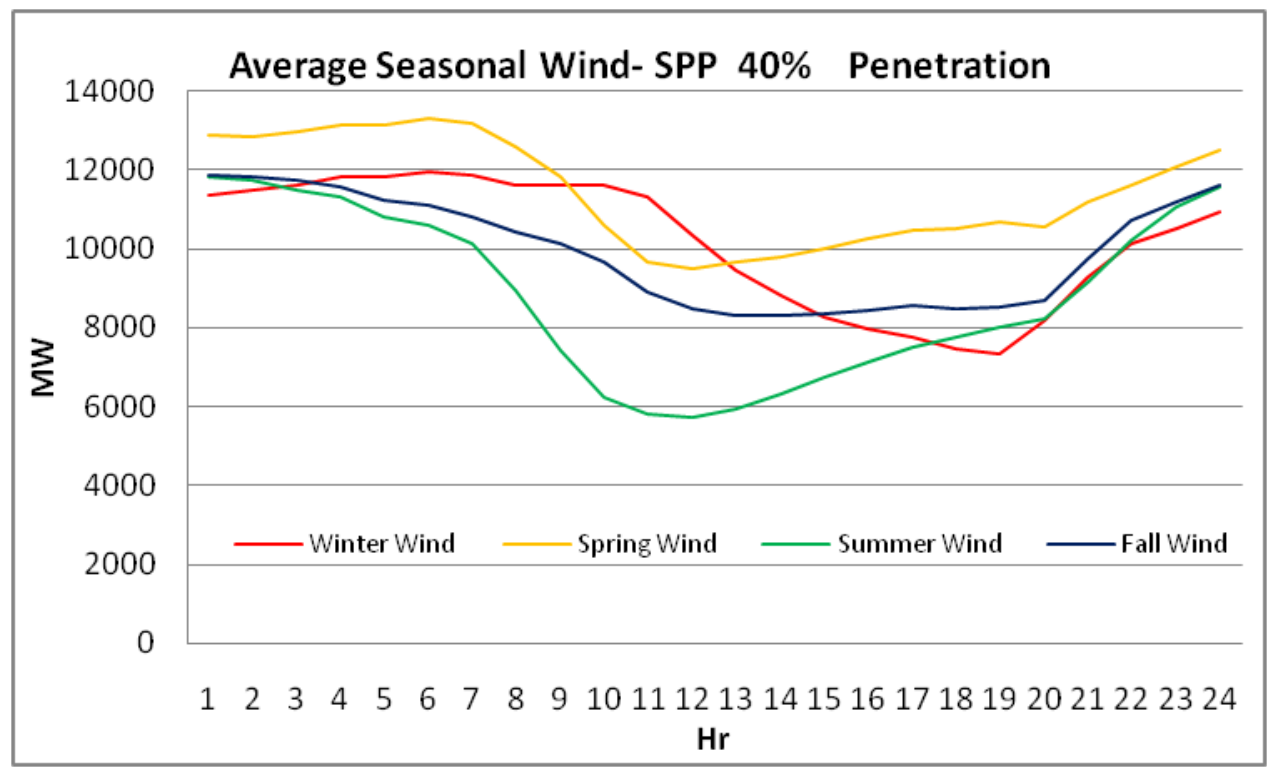

Figure 81: SPP - 24 Hour Average Seasonal Wind 40\% Penetration

\section{Section 8.2.3.4 Load, Wind, and Load net Wind}

Figure 82 and Figure 84 show time series plots for Nebraska data during a day with variable wind generation from $20 \%$ wind penetration together with low loads and then the overall week in April 2018. These figures show how the ramping requirement is increased due to the variable wind (i.e., ramping for load net wind exceeds that for the load alone).

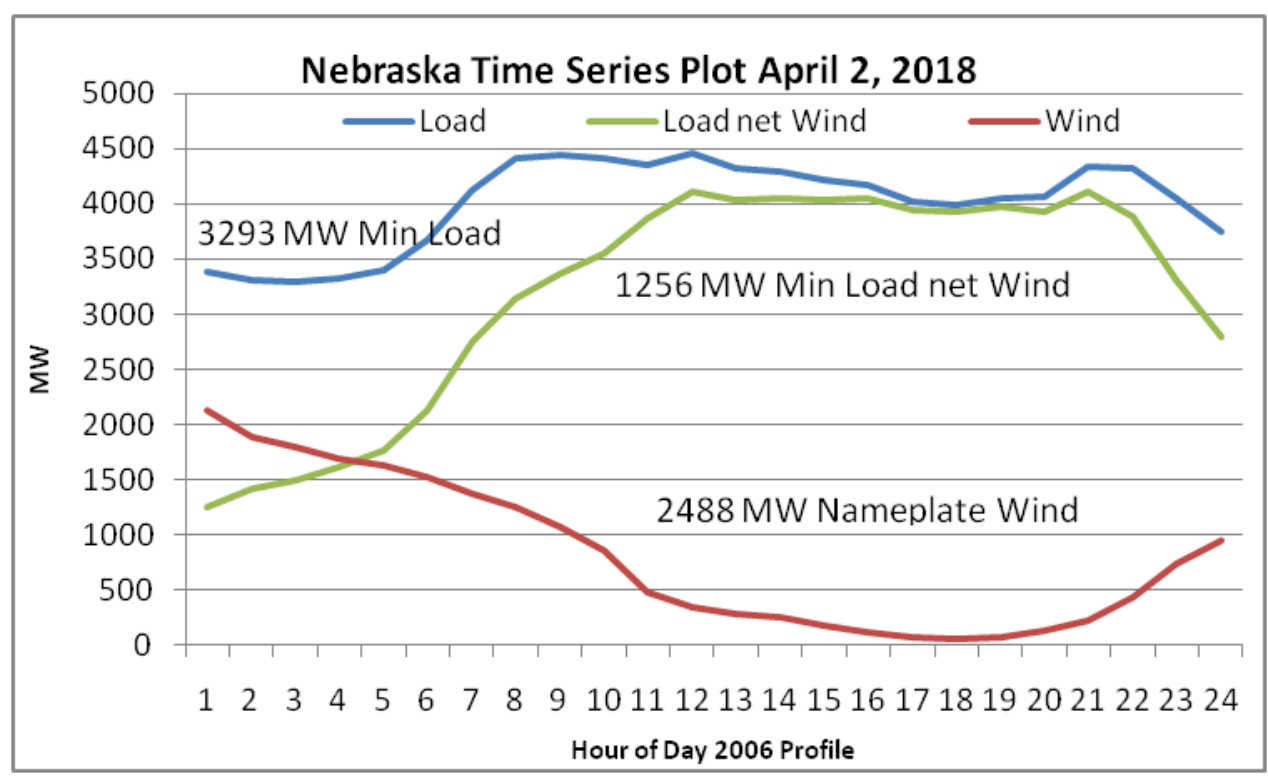

Figure 82: Time Series Plot for April Day of Load, Wind, and Load net Wind

Table 24 shows hourly data for April 2, 2018 using the 2006 profile. This data is used as an example of the effects of wind variability on ramping conditions. Note: The rule used to dispatch generation in this example is to ramp according to the ratio of the dispatch range that 
must run generation can cover (1700MW), to the min to max load net wind ramping requirement (4115MW $-1256 \mathrm{MW})$.

It is important to examine Nebraska ramping capability for a springtime day when large wind generation fluctuations may occur combined with low loads (e.g., the April 2, 2018 day being displayed). At that time, the operating range of must run generation from minimum to maximum would be approximately 1,700 MW, or from 2,500 MW to 4,210 MW. These values are applied in Table 24. This assumes some spring outages in that all coal and nuclear units are running except for four coal units. Also Nebraska hydro is running at half capacity and the WAPA purchases are scheduled at their minimum levels. Nuclear units are assumed to run flat out and contributing no range of operation. For this assumption the modeled maximum hourly ramping rates are 629MW-up per hour up and $739 \mathrm{MW}$-down per hour.

Table 24: Sample Day of Hourly Data (April 2, 2018) for Nebraska Load and Wind with 2006 profile

\begin{tabular}{|rr|rrrr|rrr|r|r|}
\hline \multicolumn{2}{|c|}{ 20\% Wind Penetration $2006,4 / 2 / 2018$} \\
\hline
\end{tabular}


Figure 83 provides a chart that shows how the must run generation ranges from min to max over the day (and with exports ramping oppositely) by plotting those data from Table 24. Also shown are the hourly ramping that will need to come from the must run units and the hourly changes to the export level by plotting that data from Table 24 .

Figure 84 shows the variations for wind generation, load, and load net wind for the full week that includes April 2, which shows similar repeated patterns throughout the week.

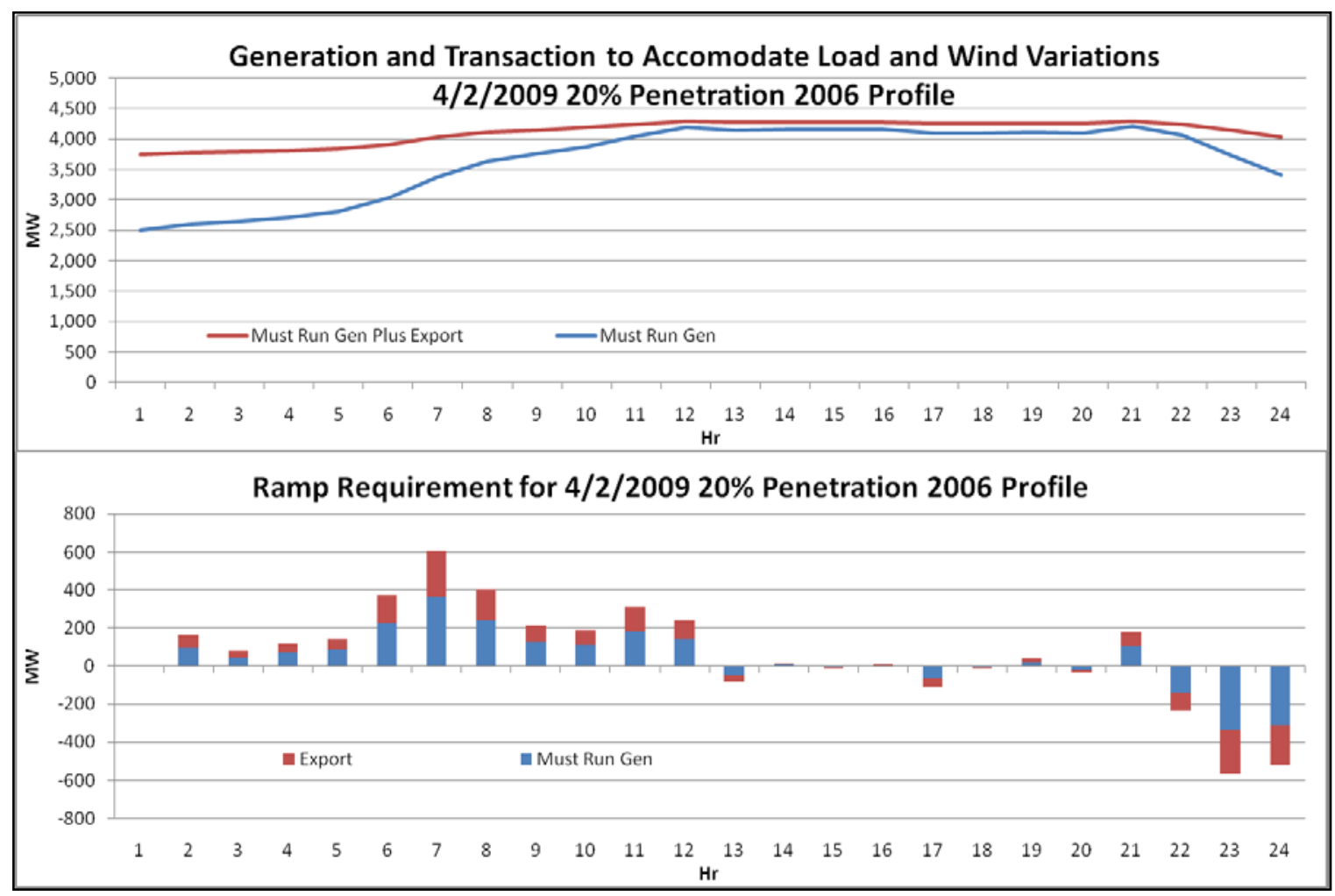

Figure 83: Generation and Transaction to accommodate load and wind variations and Ramp Requirements for April 2, 2018 for 2006 profile 


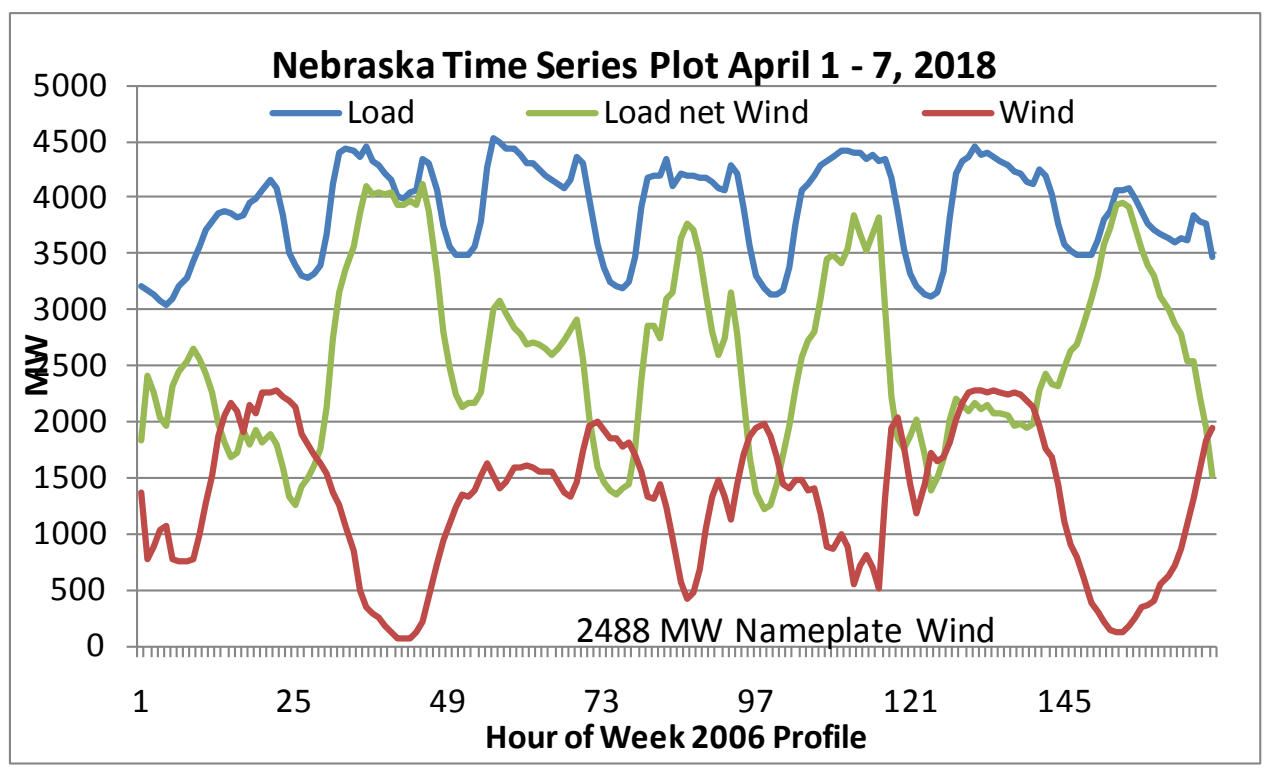

Figure 84: Time Series Plot for April Week of Load, Wind, and Load net Wind

Figure 85 provides a load duration curve of NPA 2018 load and the load net wind for $10 \%, 20 \%$ and $40 \%$ wind penetration for the 2006 wind/load pattern. This figure shows the impact of increased wind penetration on low load periods in that at $10 \%$ penetration wind generation in Nebraska exceeds the annual minimum load level only about $5 \%$ of the time but at $40 \%$ penetration the wind generation exceeds annual minimum load nearly $50 \%$ of the time. As clarification this does not mean that $40 \%$ wind penetration exceeds load $50 \%$ of the time because only the minimum load point is being referenced here. However, wind generation for $40 \%$ penetration exceeds Nebraska load approximately 600 hours of the year, or $7 \%$ of the time (time below the zero line in Figure 85).

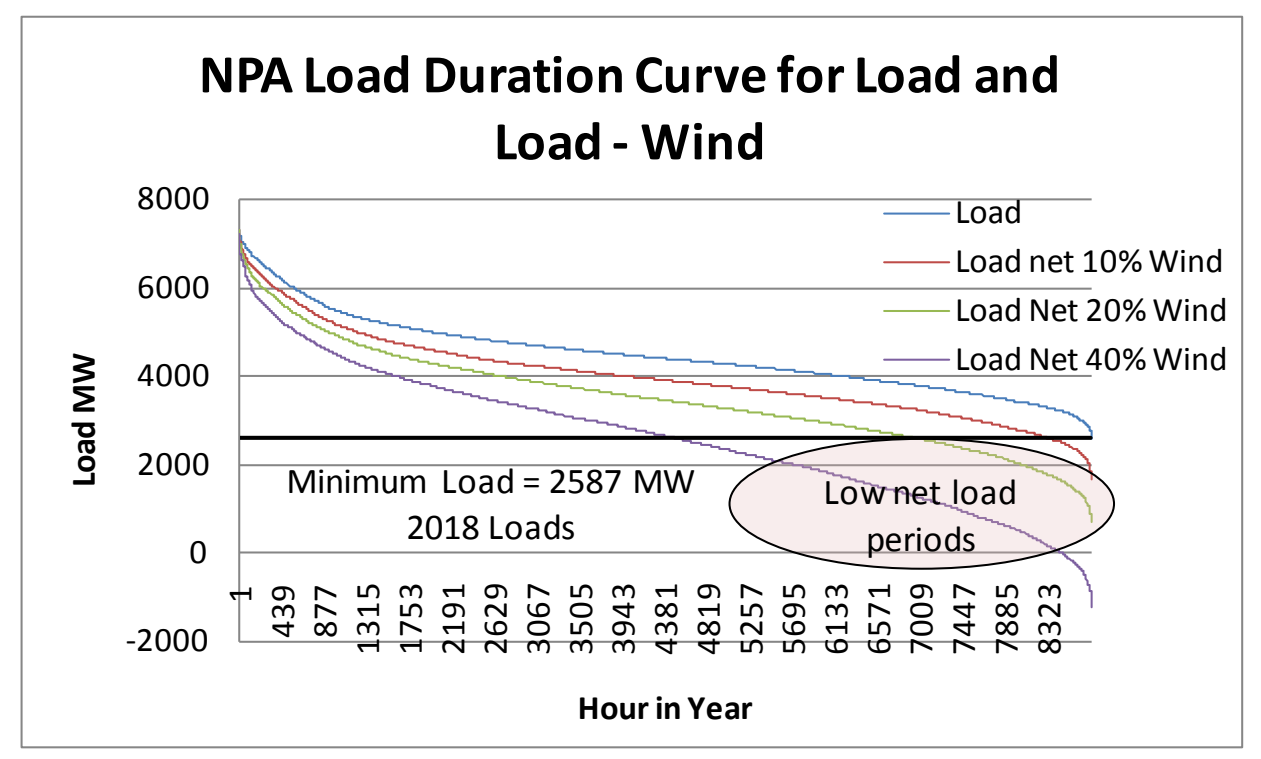

Figure 85: Load Duration for NPA load and Load net Wind 


\section{Section 8.2.3.5 Nebraska Load/Wind Hourly Correlation}

A correlation analysis was performed on NPA load and wind annually and monthly from multiple years, then on the rest of SPP, then on SPP including NPA, Figure 86 - Figure 89. A zero correlation means that on average wind generation and load change independently of one another. Positive correlation means that on average as load increases wind generation tends to increase more often than decrease and vice-versa. Negative correlation means that on average as load increases wind generation tends to decrease more often than increase and vice-versa. A perfect correlation would be $100 \%$ while a perfect inverse correlation would be $-100 \%$. Loads and wind generation are generally negatively correlated.

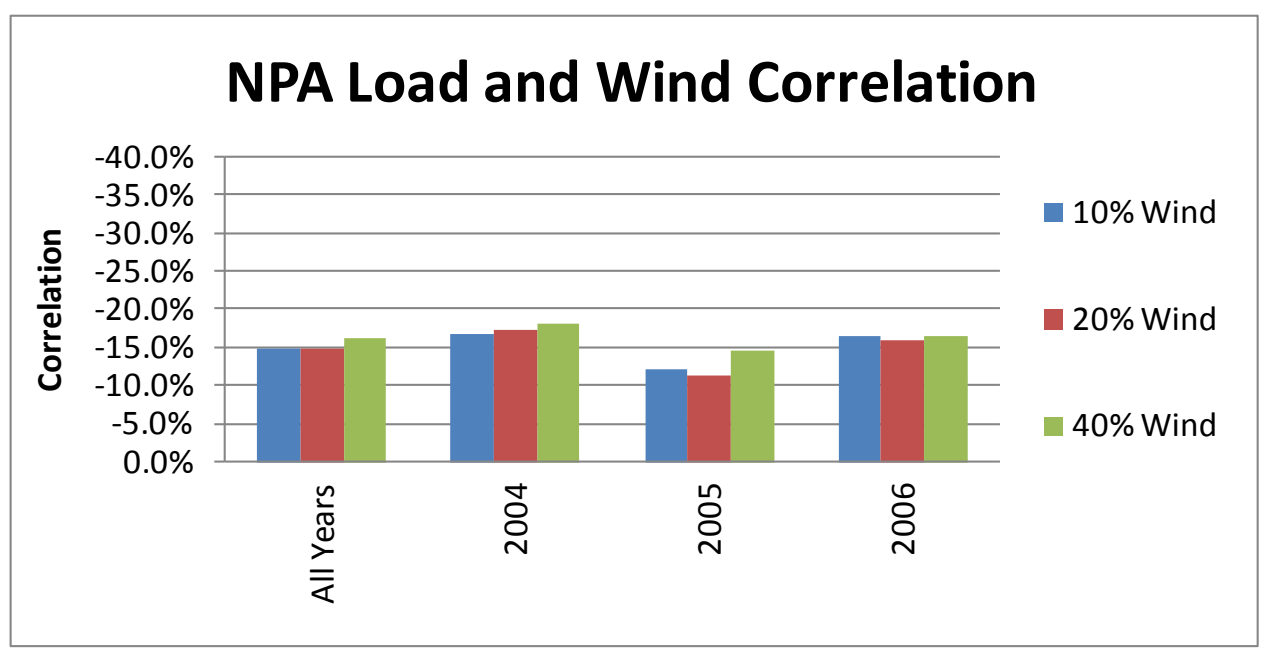

Figure 86: NPA-Annual Summary Correlation of Load and Wind

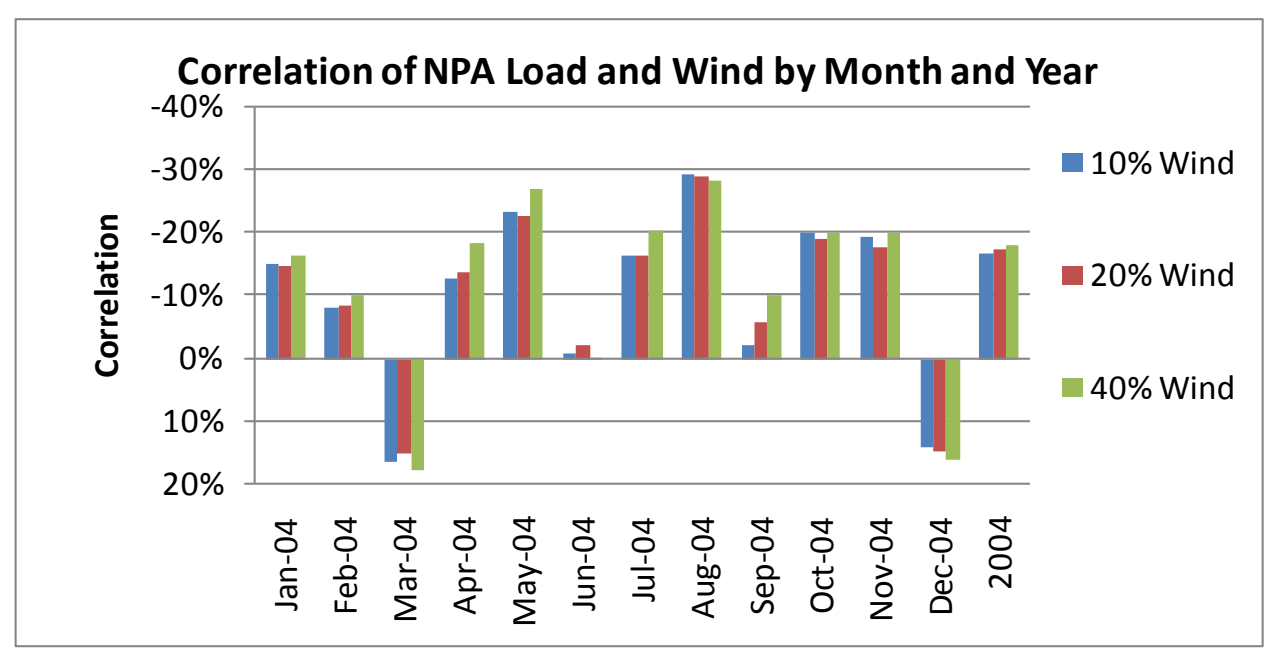

Figure 87: NPA 2004 Load and Wind Correlation by Month 


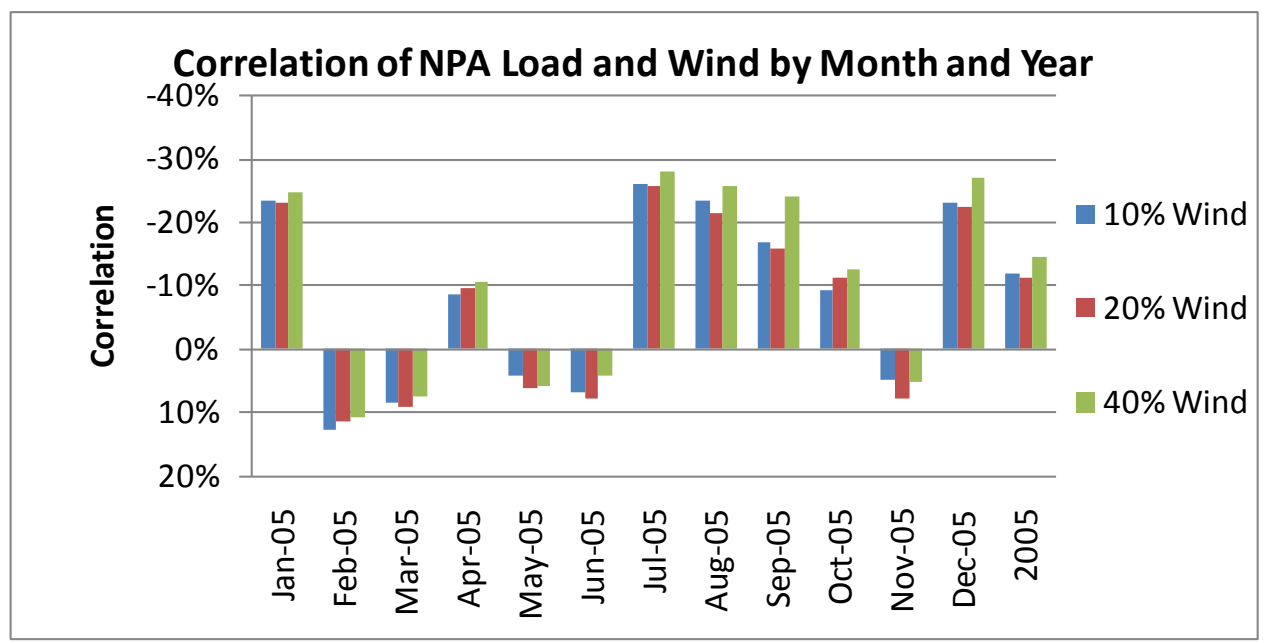

Figure 88: NPA 2005 Load and Wind Correlation by Monty

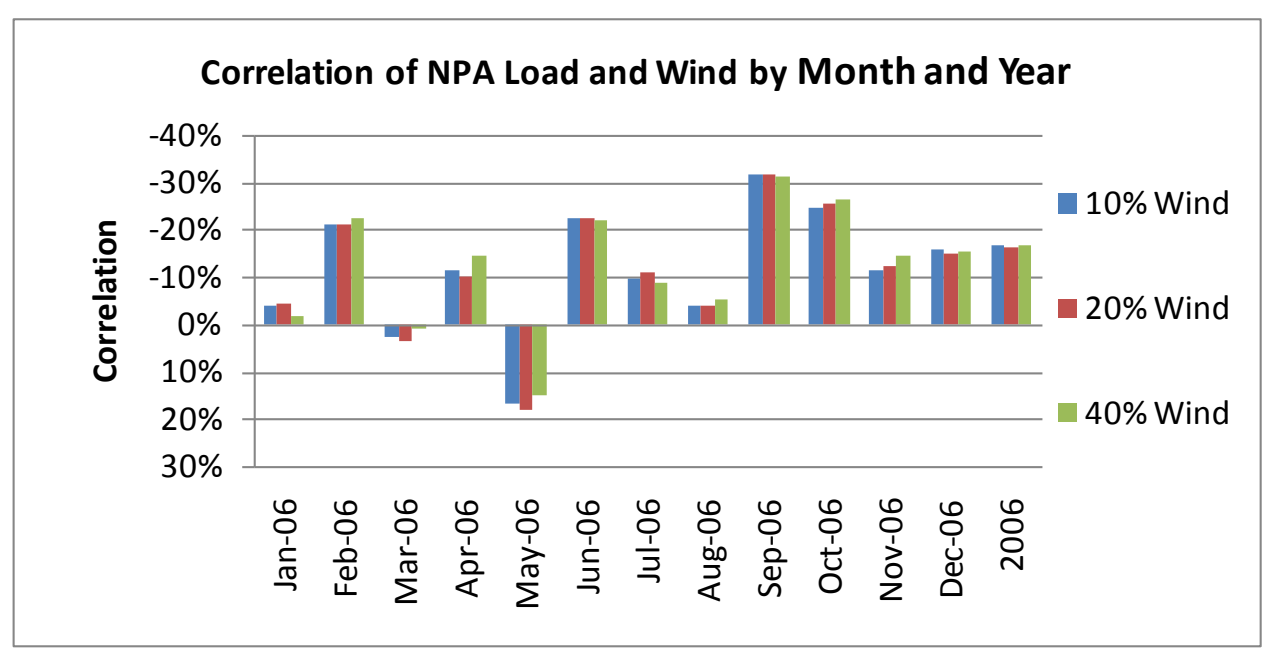

Figure 89: NPA 2006 Load and Wind Correlation by Month

Comparing correlation results between SPP and NPA:

- Overall, the rest of SPP has about 3-4\% more negative correlation than NPA, as determined by comparing Figure 86 and Figure 90.

- In the rest of SPP July is the most negatively correlated month, whereas in NPA July is down the list at $5^{\text {th }}$ most negatively correlated. In SPP the next most negatively correlated months are December, January, and April, all these monthly comparisons using $40 \%$ penetration results.

- The most positively correlated month in SPP is May, the same as in NPA. 


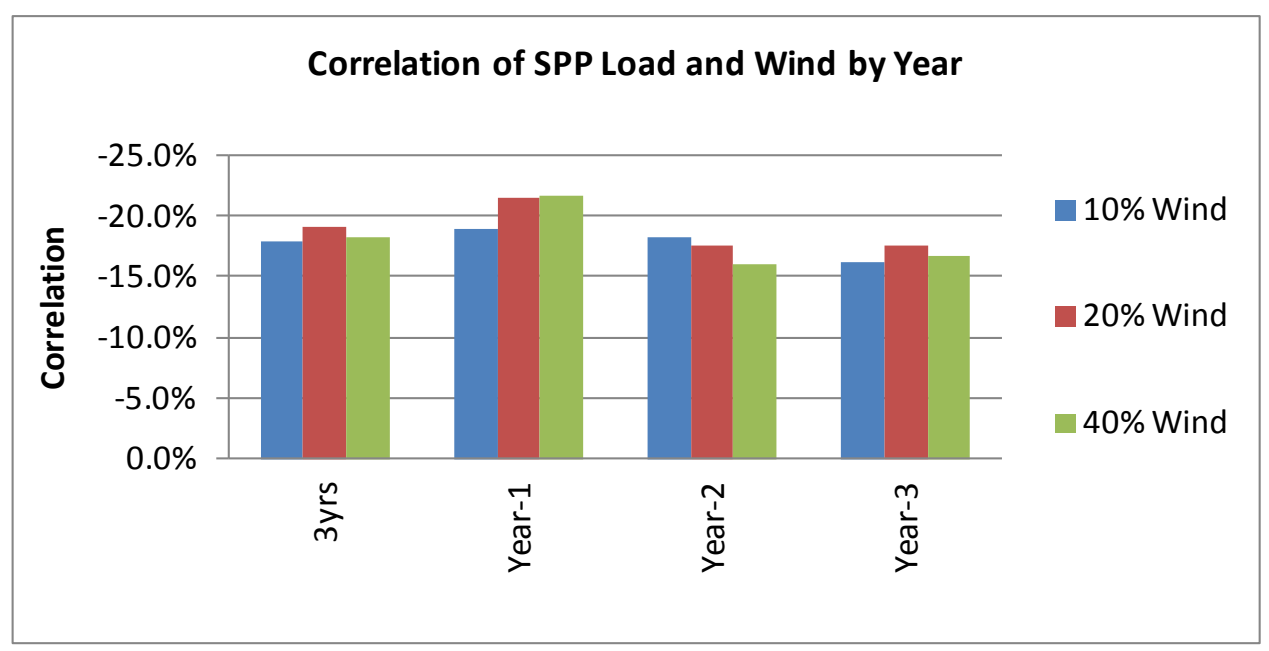

Figure 90: SPP Load and Wind Correlation by Year

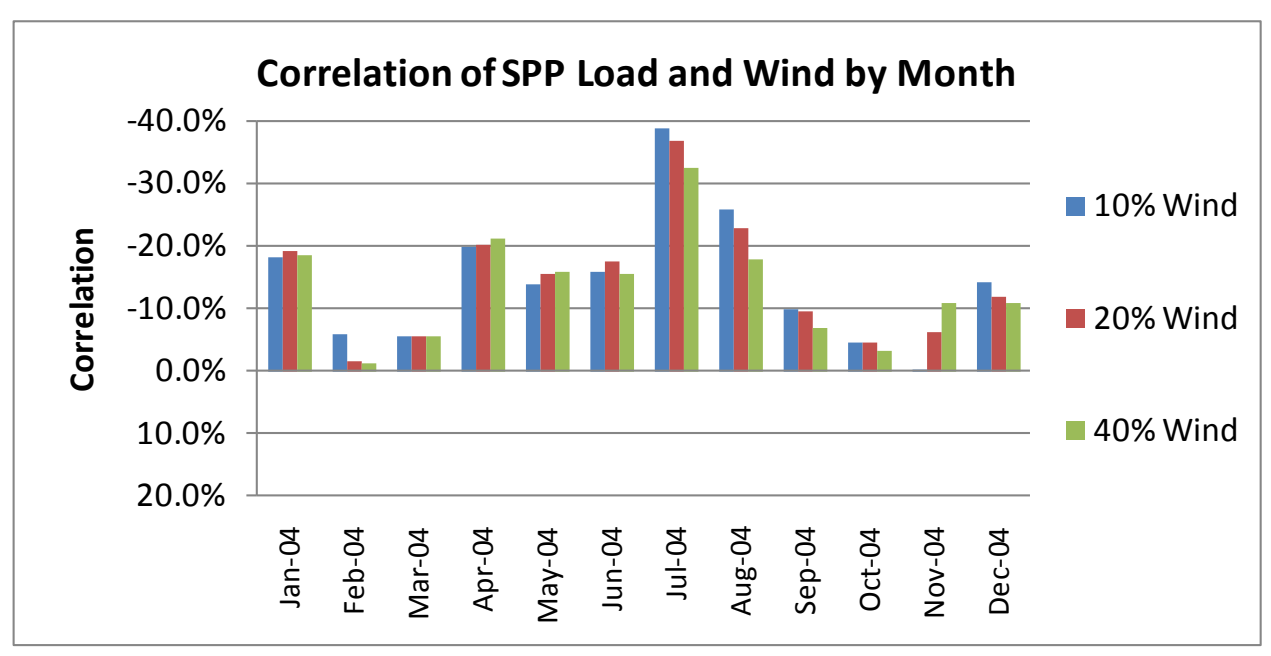

Figure 91: SPP 2004 Load and Wind Correlation by Month 


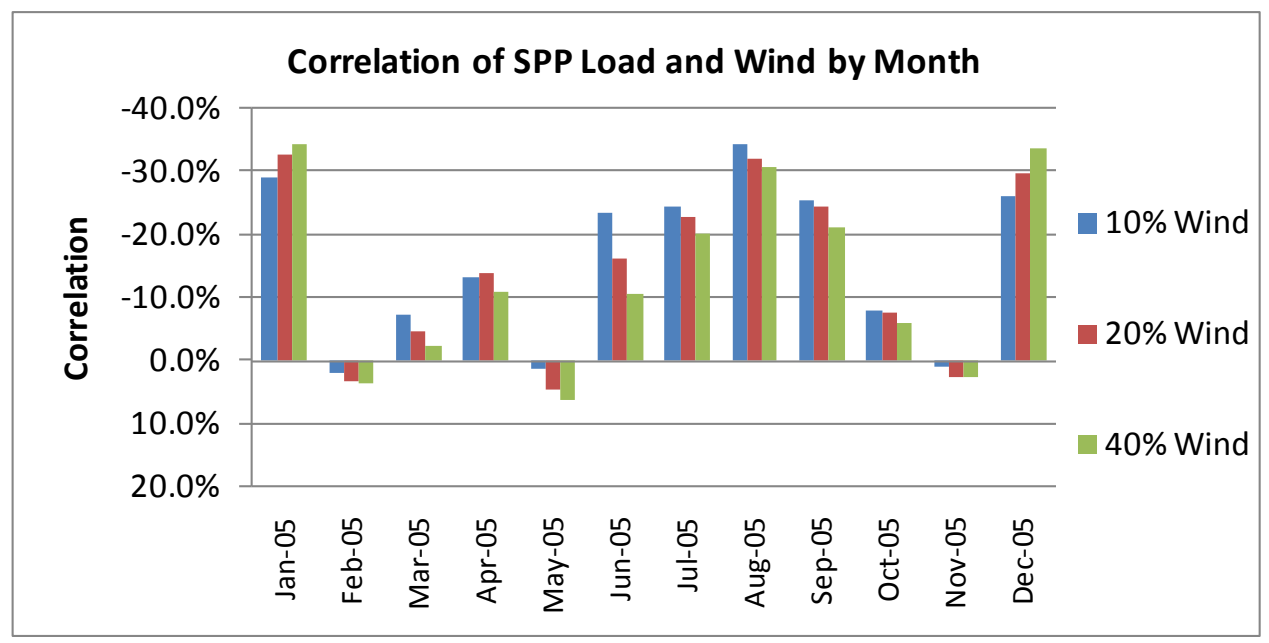

Figure 92: SPP 2005 Load and Wind Correlation by Month

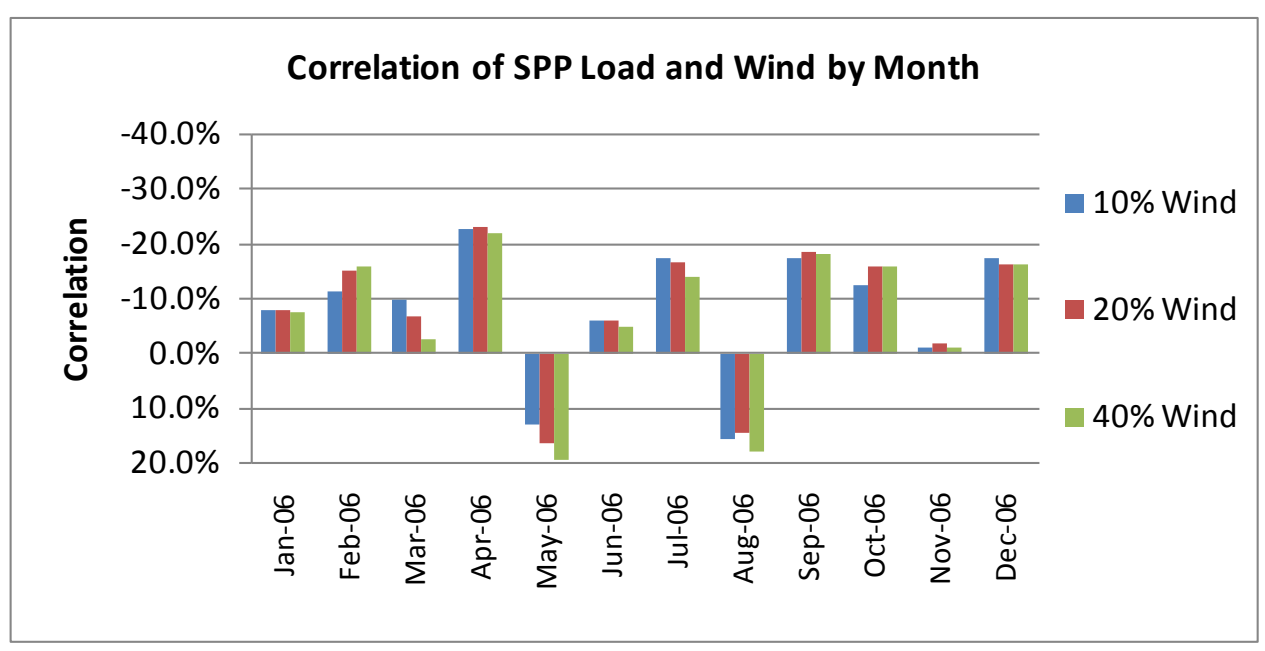

Figure 93: SPP 2006 Load and Wind Correlation by Month

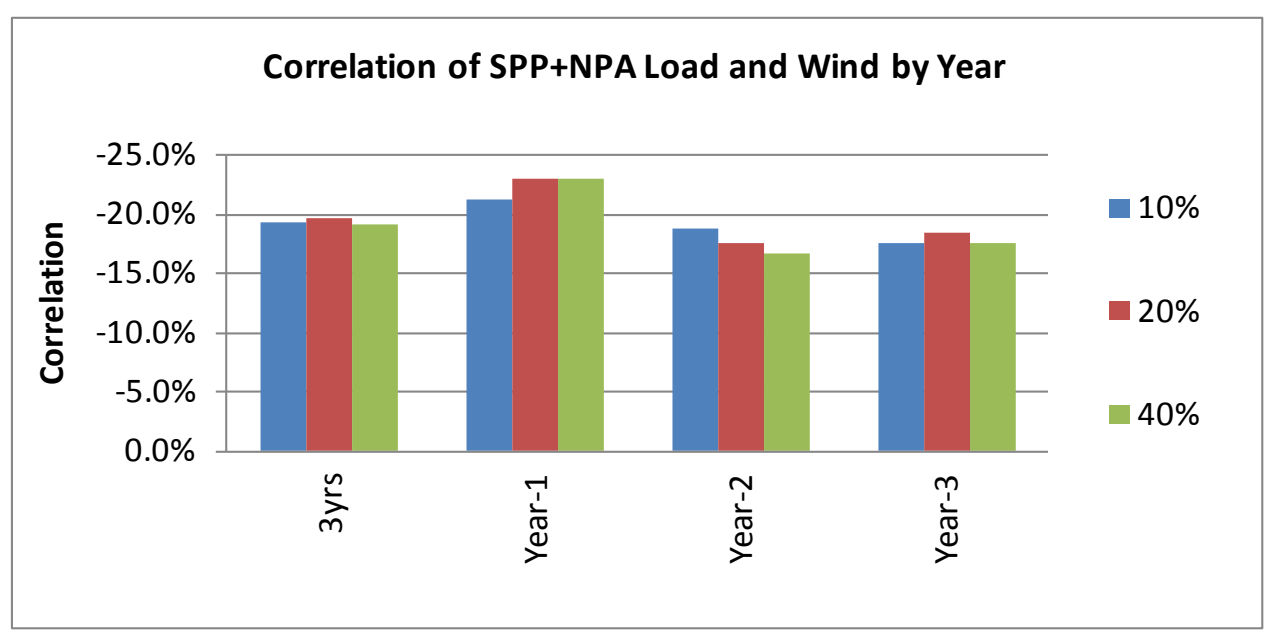

Figure 94: SPP with NPA Load and Wind Correlation by Year 


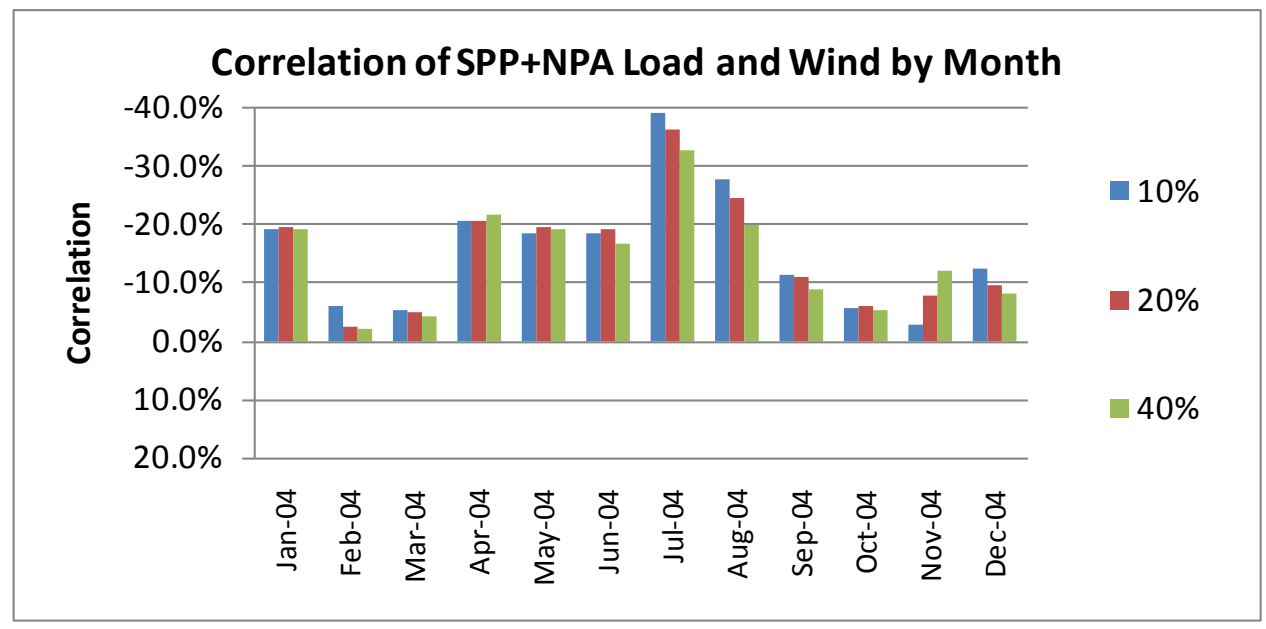

Figure 95: SPP with NPA 2004 Load and Wind Correlation by Month

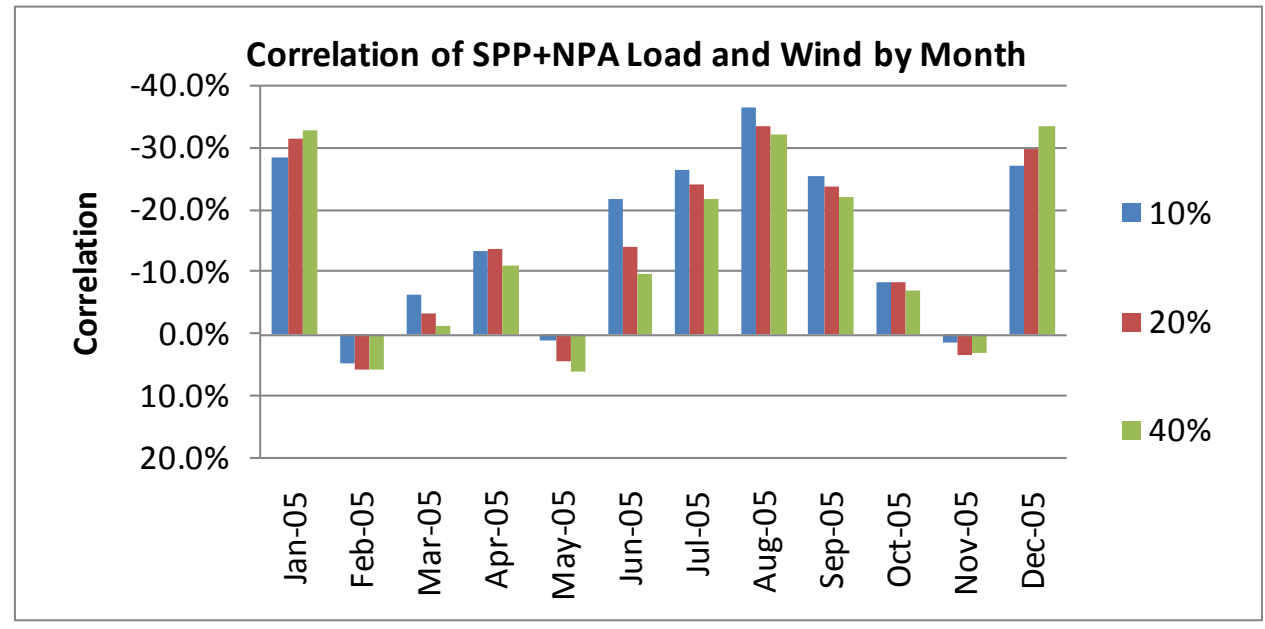

Figure 96: SPP with NPA 2005 Load and Wind Correlation by Month

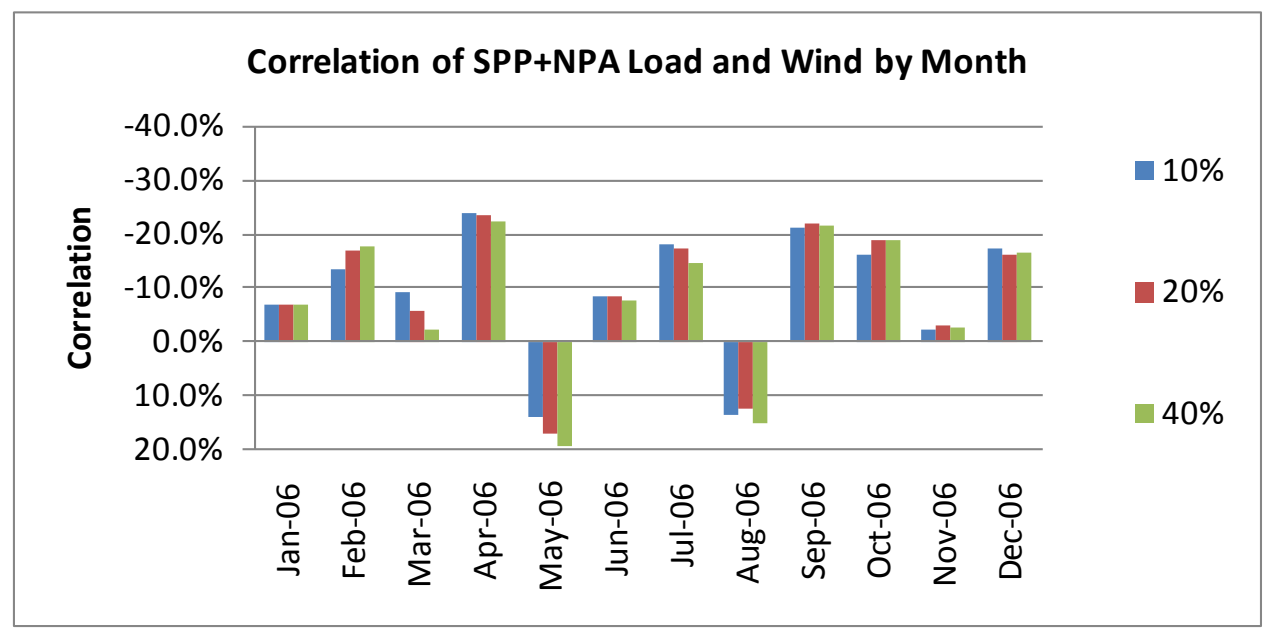

Figure 97: SPP and NPA 2006 Load and Wind Correlation by Month 


\section{Section 8.2.3.6 NPA and WAPA Load and Wind Forecast Analysis}

The Mean Absolute Error (MAE) for actual wind and day ahead wind forecast was calculated for Nebraska stand alone, WAPA stand alone and for Nebraska and WAPA with combined data. Figure 98- Figure 100 provide MAE analysis for Nebraska at wind penetrations of 10\%, $1249 \mathrm{MW}$ nameplate, 20\%, $2488 \mathrm{MW}$ nameplate, and 40\%, $4727 \mathrm{MW}$ nameplate for each of the three years. The MAE generally reduces some with penetration showing the benefit of aggregation, although much of the aggregation benefit is achieved at the $10 \%$ penetration level. Typically at single sites the MAE for the day ahead forecast is at least $15 \%$.

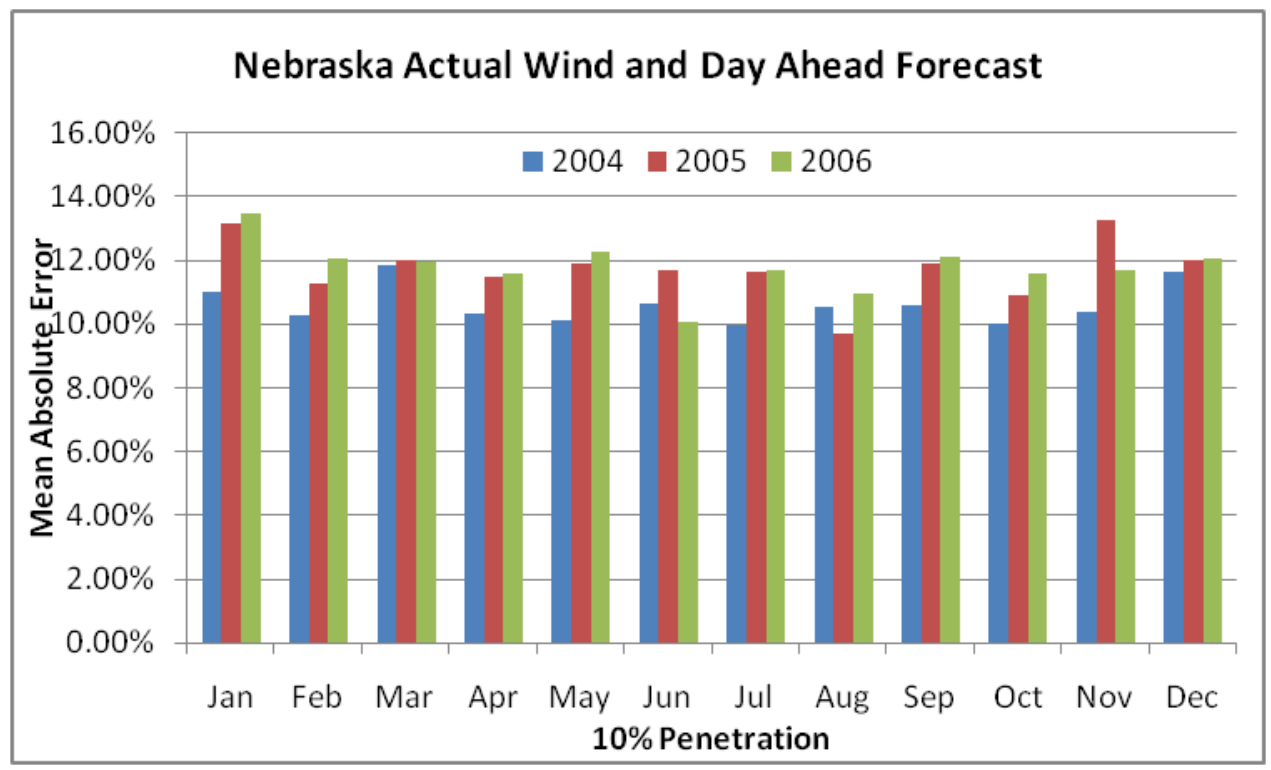

Figure 98: Nebraska Actual Wind and Day Ahead Forecast MAE for 10\% Penetration

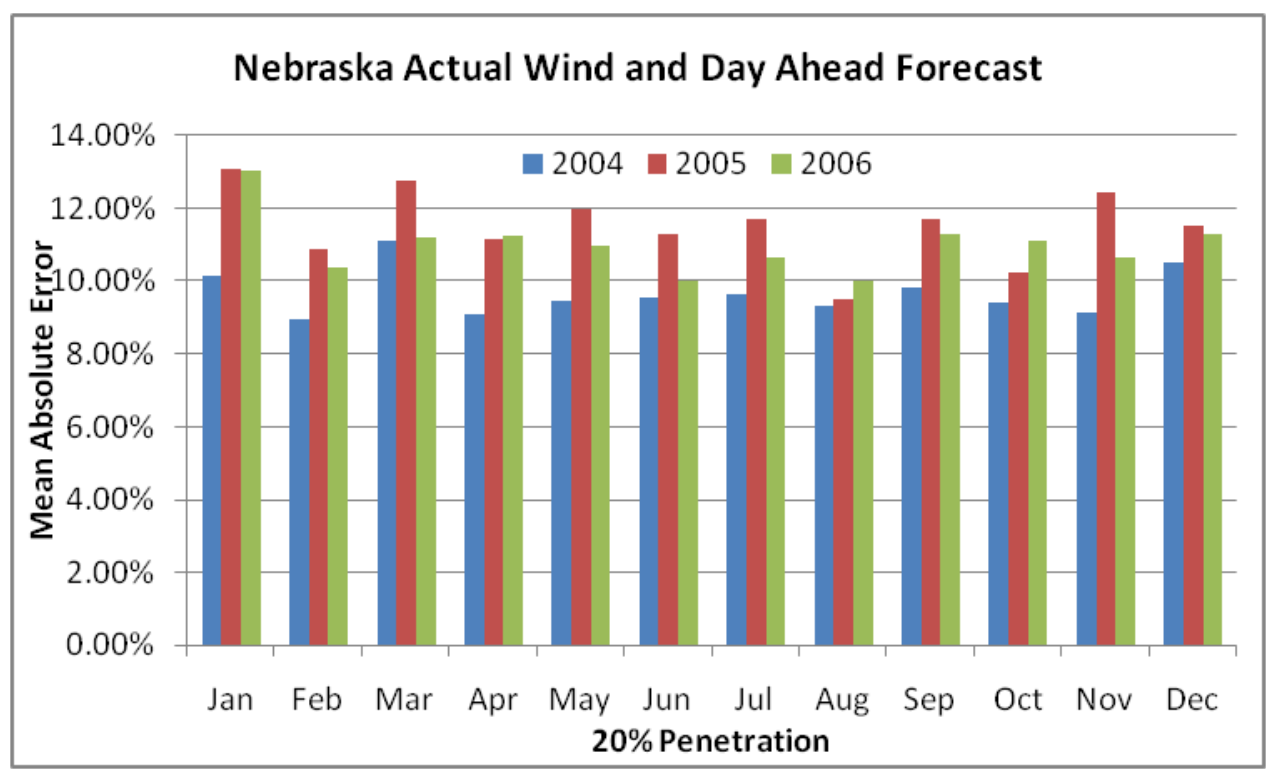

Figure 99: Nebraska Actual Wind and Day Ahead Forecast MAE for 20\% Penetration 


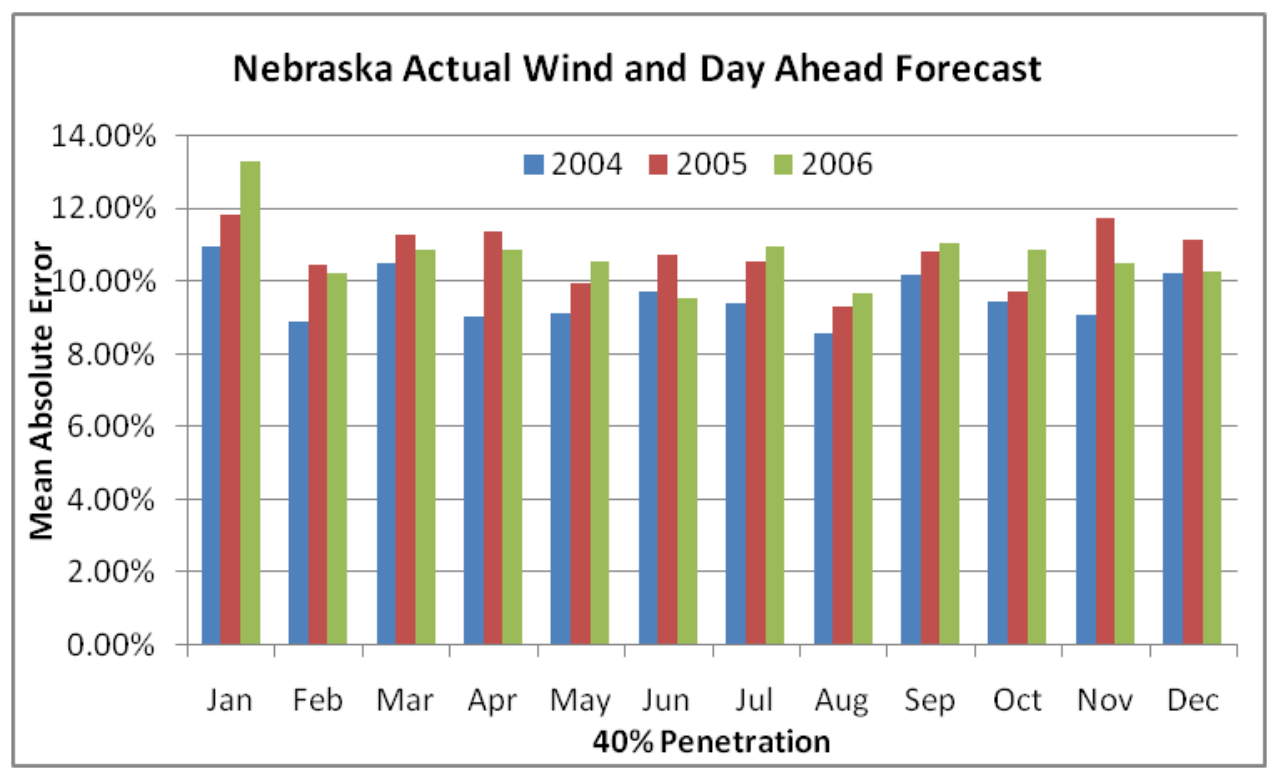

Figure 100: Nebraska Actual Wind and Day Ahead Forecast MAE for $40 \%$ Penetration

Figure 101 shows the MAE for Nebraska 2018 Load using a 1 hour persistence forecast. Study profile years for 2004, 2005 and 2006 loads were escalated to the study year, 2018.

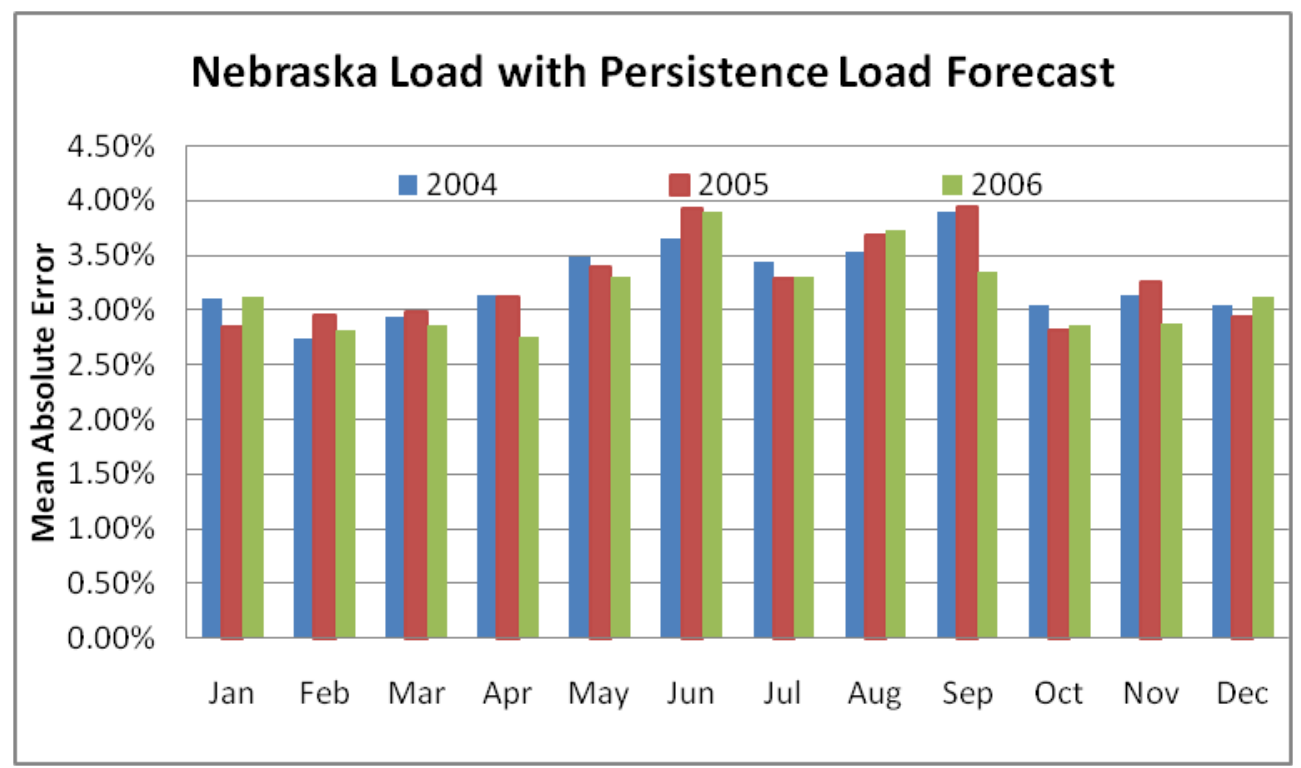

Figure 101: MAE of Nebraska Load with Persistence Forecast

For this study the WAPA wind and load remain constant. Figure 102 shows the wind MAE for each study scenario. Figure 102 shows the MAE for actual wind and day ahead forecast and Figure 103 shows the MAE of the persistence forecast for WAPA wind generation. 


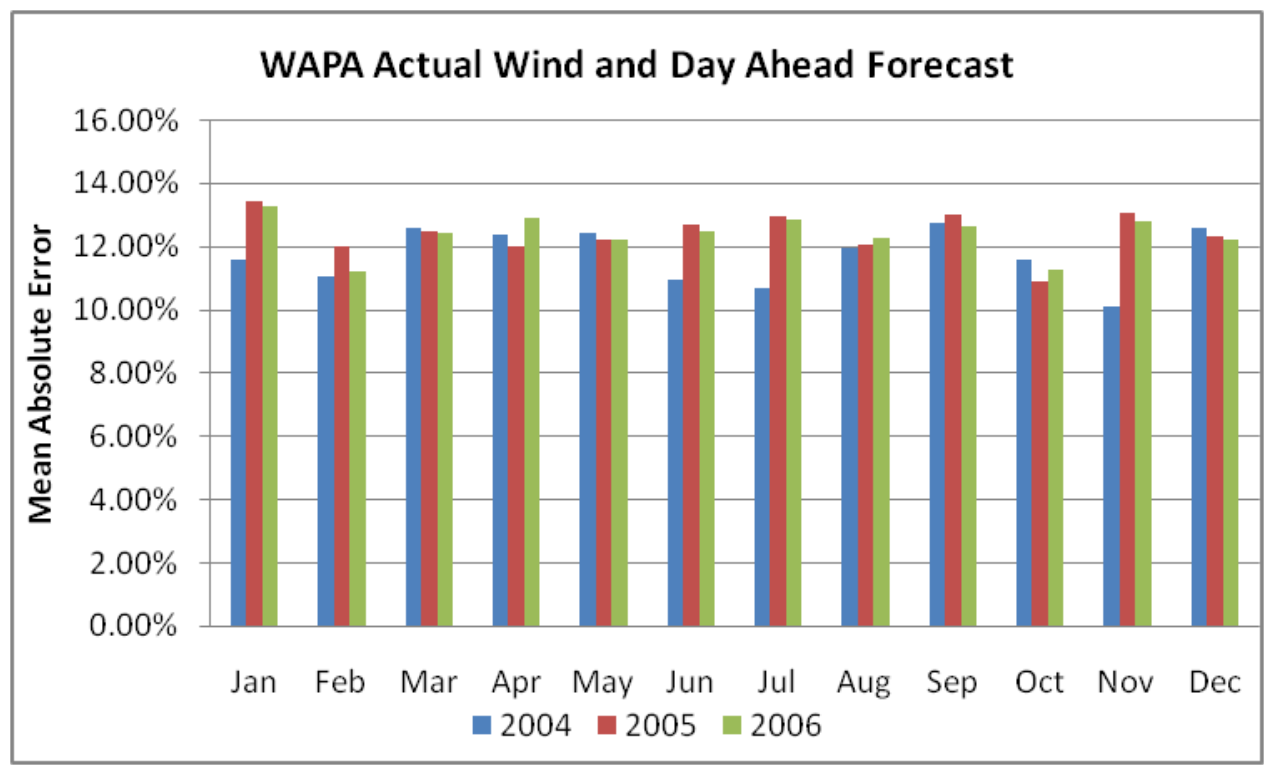

Figure 102: WAPA Actual Wind and Day Ahead Forecast MAE

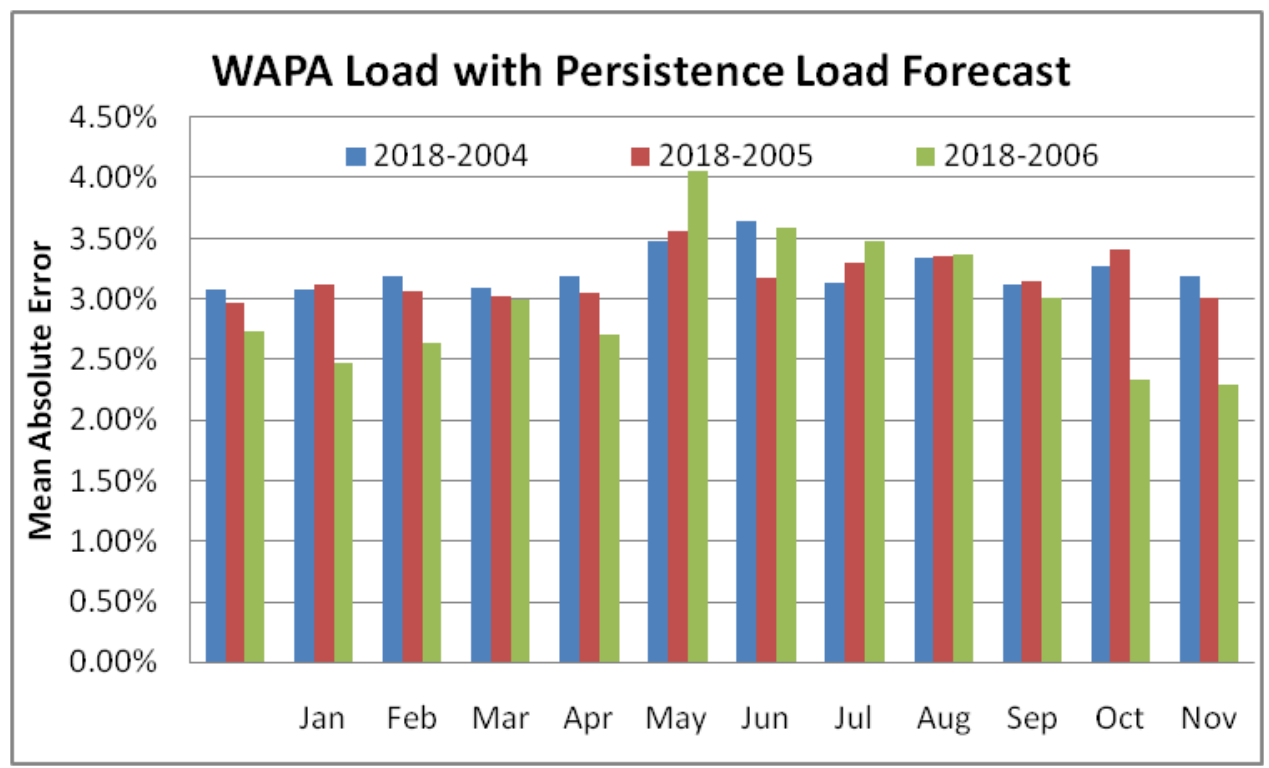

Figure 103: WAPA Load with $1 \mathrm{hr}$. Persistence Forecast MAE

Figure 104, Figure 105, and Figure 106 show the MAE for combined Nebraska and WAPA actual and day-ahead forecast wind with penetration levels and Figure 107 shows the MAE for the persistence forecast for combined Nebraska and WAPA wind generation. The purpose of this investigation of Nebraska/WAPA diversification was to see how much combining the two wind generations from different locations and associated forecasts would reduce MAE. Looking at the MAE results for the day-ahead forecasts for Nebraska 10\% (Figure 98) indicates approximately $11.35 \%$ MAE and similarly for WAPA (Figure 102) yields $12.26 \%$ MAE. In the 
combined case (Figure 104) the day-ahead MAE is reduced to $10.89 \%$ indicating that some diversity benefits would exist in coordinating Nebraska and WAPA wind generation through forecast reduction.

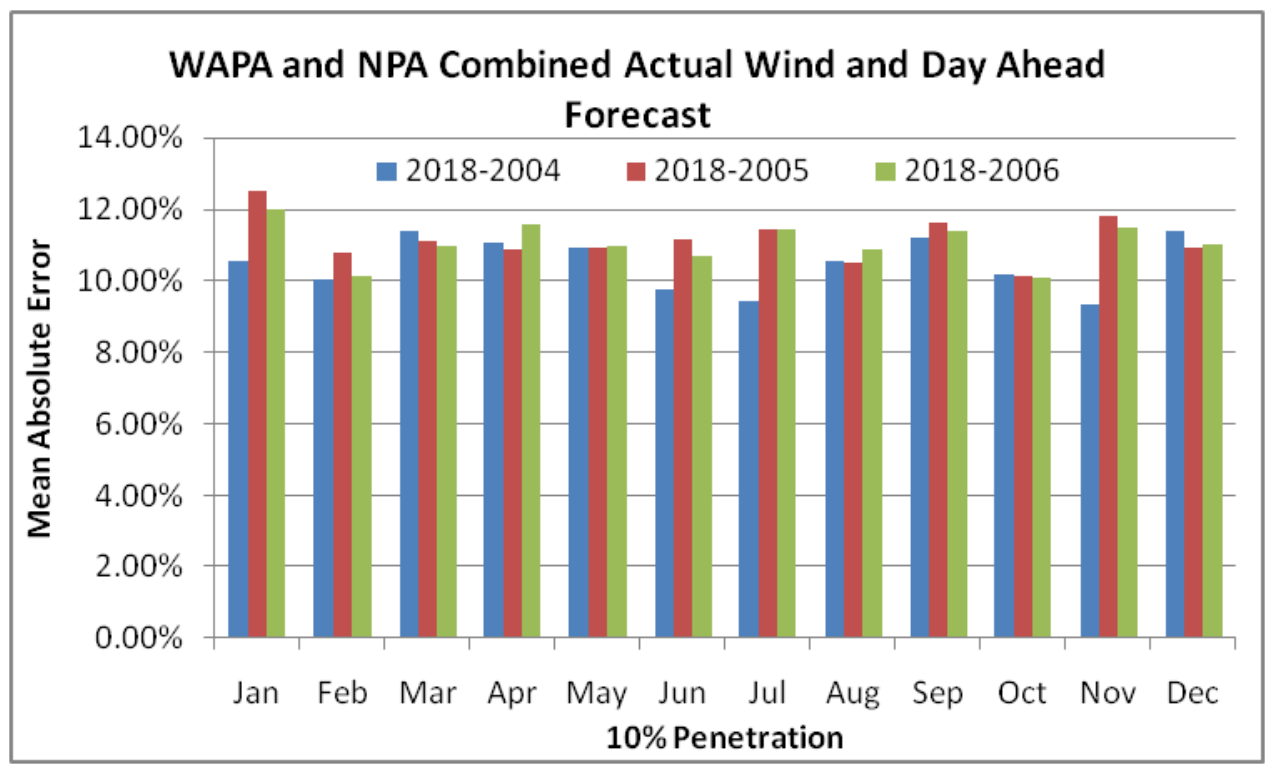

Figure 104: Nebraska and WAPA Actual Wind and Day Ahead Forecast MAE for 10\% Penetration

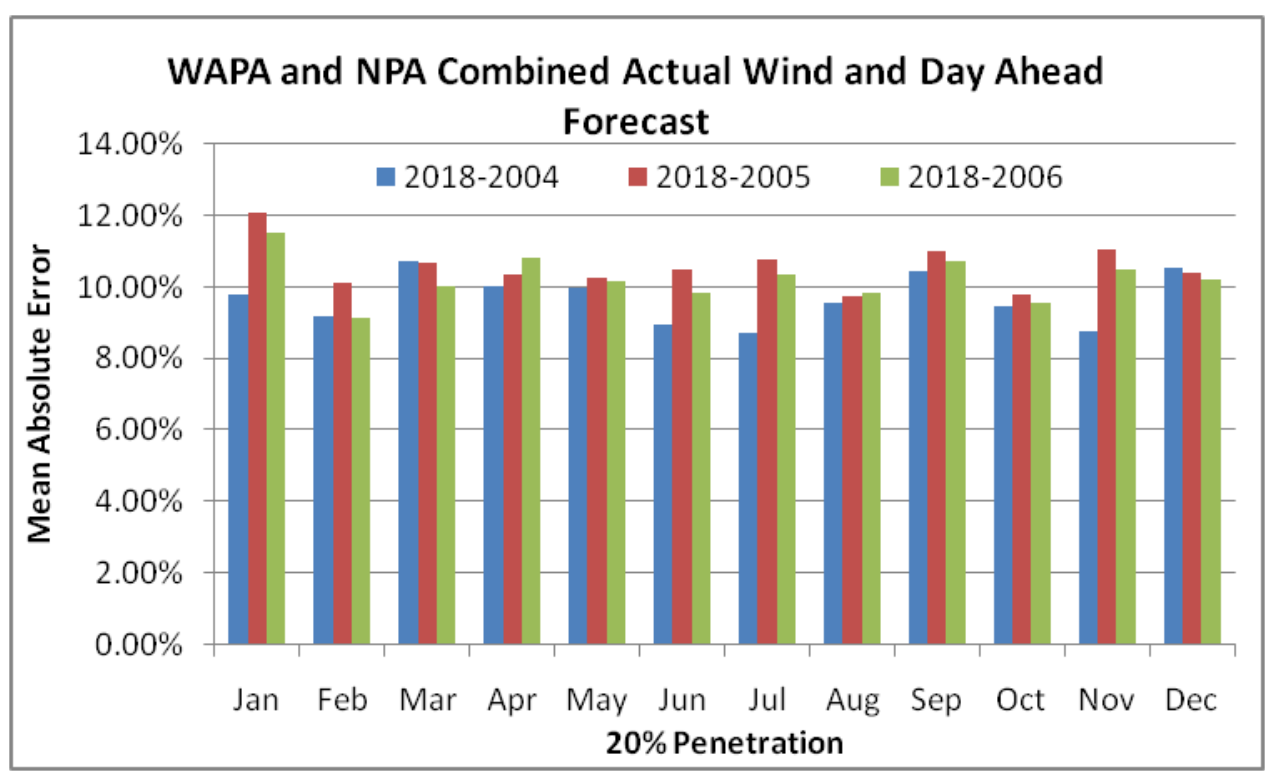

Figure 105: Nebraska and WAPA Actual Wind and Day Ahead Forecast MAE for 20\% Penetration 


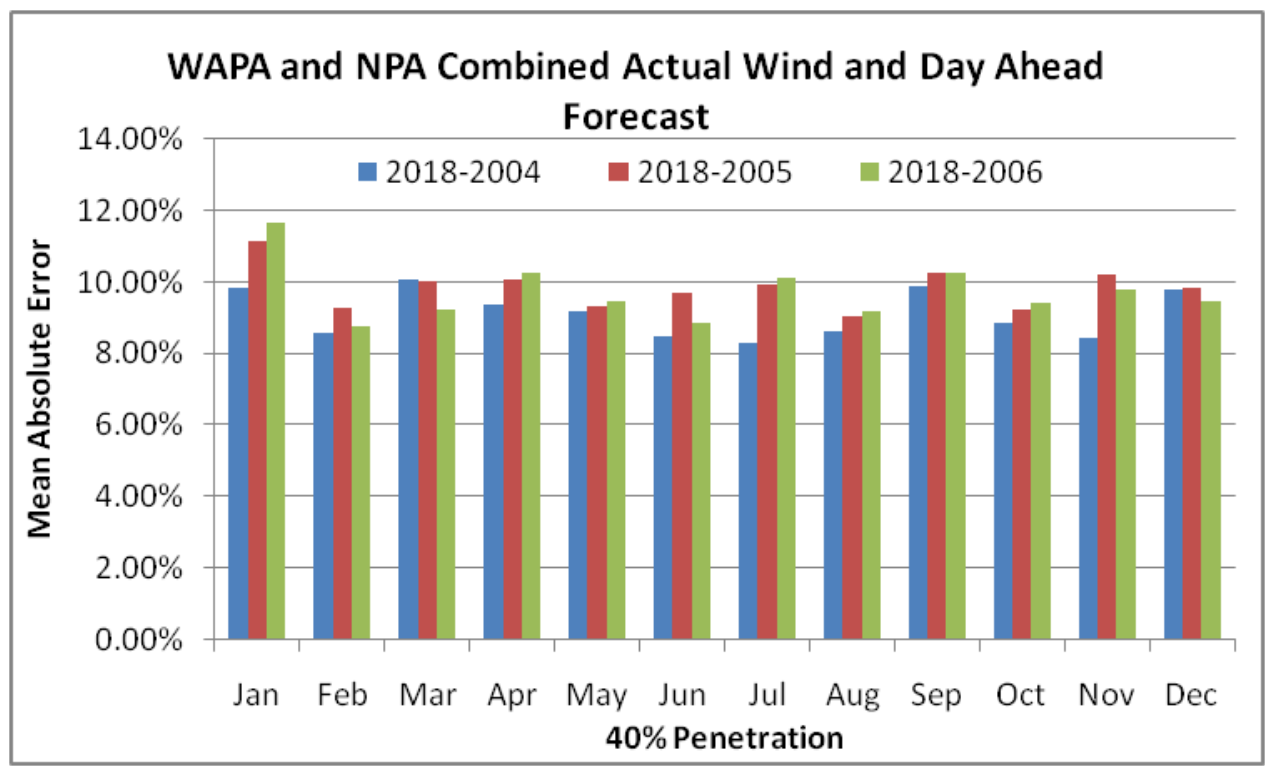

Figure 106: Nebraska and WAPA Actual Wind and Day Ahead Forecast MAE for 40\% Penetration

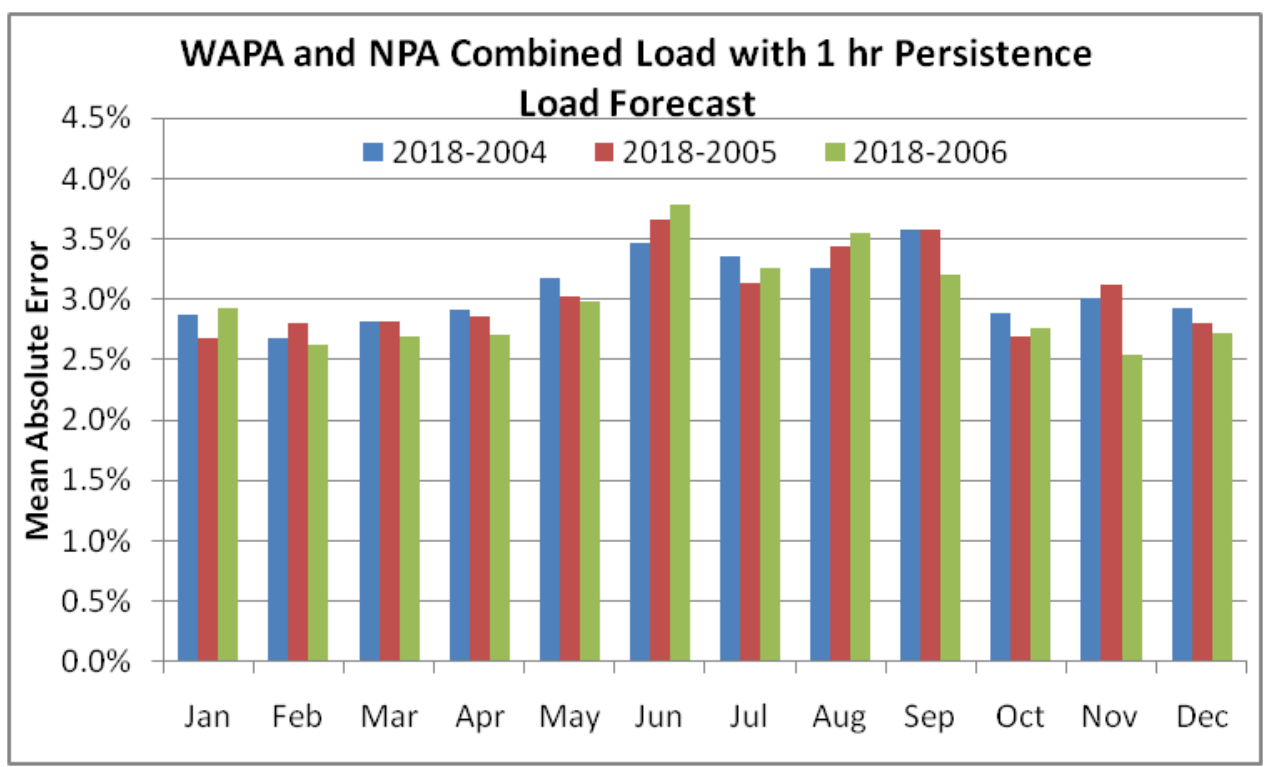

Figure 107: Nebraska and WAPA Load with $1 \mathrm{hr}$ Persistence Forecast MAE

\section{Section 8.2.4 Operational Considerations}

The wind generation's variability and uncertainty, as described in earlier tables and charts, affect unit commitment decisions and increase the amount of regulating reserves required.

Additionally, the thermal units are required to respond to the wind generation by ramping up and down to adjust to the wind generation output. In this section some of these operational considerations are described. 


\section{Section 8.2.4.1 Ramp analysis}

The morning ramp-up and night ramp-down rates were examined for Nebraska. The analysis used the Nebraska $20 \%$ wind penetration case and focused on monthly and seasonal aggregated data as follows:

Monthly NPA (average, maximum, minimum, $98^{\text {th }}$ percentile)

Figure 108 - Figure 111 morning ramp-up -- 2004

Figure 112 - Figure 115 morning ramp-up -- 2005

Figure 116 - Figure 119 morning ramp-up -- 2006

Seasonal NPA (maximum, minimum, $98^{\text {th }}$ percentile)

Figure 120 - Figure 122 morning ramp-up -- 2004

Figure 123 - Figure 125 morning ramp-up -- 2005

Figure 126 - Figure 128 morning ramp-up -- 2006

Monthly NPA (average, maximum, minimum, $98^{\text {th }}$ percentile)

Figure 129 - Figure 132 evening ramp-down -- 2004

Figure 133 - Figure 136 evening ramp-down -- 2005

Figure 137 - Figure 140 evening ramp-down -- 2006

Seasonal NPA (maximum, minimum, $98^{\text {th }}$ percentile)

Figure 141 - Figure 143 evening ramp-down -- 2004

Figure 144 - Figure 146 evening ramp-down -- 2005

Figure 147 - Figure 149 evening ramp-down -- 2006 


\begin{tabular}{|c|c|c|c|c|c|c|c|c|c|c|c|c|c|c|}
\hline & \multicolumn{14}{|c|}{ NPA Average Hourly MW Change 2018 - 2004 Profile $20 \%$ Wind } \\
\hline & \multicolumn{2}{|c|}{$\mathrm{Hr} 5$} & \multicolumn{2}{|c|}{$\mathrm{Hr} 6$} & \multicolumn{2}{|c|}{$\mathrm{Hr} 7$} & \multicolumn{2}{|c|}{$\mathrm{Hr} 8$} & \multicolumn{2}{|c|}{\begin{tabular}{|l|}
$\mathrm{Hr} 9$ \\
\end{tabular}} & \multicolumn{2}{|c|}{$\mathrm{Hr} 10$} & \multicolumn{2}{|c|}{$\operatorname{Hr} 11$} \\
\hline & Load & $\begin{array}{l}\text { Load } \\
\text { Net } \\
\text { Wind }\end{array}$ & Load & $\begin{array}{l}\text { Load } \\
\text { Net } \\
\text { Wind }\end{array}$ & Load & $\begin{array}{l}\text { Load } \\
\text { Net } \\
\text { Wind }\end{array}$ & Load & $\begin{array}{l}\text { Load } \\
\text { Net } \\
\text { Wind }\end{array}$ & Load & $\begin{array}{l}\text { Load } \\
\text { Net } \\
\text { Wind }\end{array}$ & Load & $\begin{array}{l}\text { Load } \\
\text { Net } \\
\text { Wind }\end{array}$ & Load & $\begin{array}{l}\text { Load } \\
\text { Net } \\
\text { Wind }\end{array}$ \\
\hline Jan & 85 & 73 & 237 & 241 & 410 & 426 & 294 & 290 & 10 & 20 & 11 & 49 & -2 & 19 \\
\hline Feb & 69 & 88 & 217 & 224 & 401 & 422 & 231 & 223 & 5 & 32 & -16 & 31 & -28 & 6 \\
\hline Mar & 66 & 40 & 219 & 211 & 370 & 367 & 237 & 244 & 68 & 111 & 24 & 66 & 16 & 35 \\
\hline Apr & 48 & 65 & 188 & 185 & 362 & 399 & 270 & 280 & 113 & 130 & 65 & 131 & 51 & 119 \\
\hline May & 19 & 76 & 124 & 102 & 235 & 256 & 288 & 330 & 189 & 223 & 155 & 214 & 126 & 179 \\
\hline Jun & 1 & 36 & 129 & 108 & 201 & 181 & 342 & 413 & 293 & 344 & 218 & 268 & 201 & 236 \\
\hline Jul & -38 & -3 & 112 & 134 & 194 & 200 & 333 & 500 & 347 & 452 & 289 & 347 & 250 & 298 \\
\hline Aug & -14 & -5 & 156 & 170 & 323 & 370 & 264 & 400 & 259 & 374 & 232 & 337 & 226 & 255 \\
\hline Sep & 2 & -15 & 157 & 179 & 378 & 429 & 245 & 350 & 156 & 198 & 184 & 285 & 171 & 237 \\
\hline Oct & 49 & 48 & 198 & 206 & 398 & 425 & 331 & 413 & 45 & 64 & 52 & 70 & 53 & 139 \\
\hline Nov & 70 & 64 & 222 & 217 & 389 & 419 & 268 & 338 & 27 & 53 & 8 & 18 & 4 & 74 \\
\hline Dec & 33 & 5 & 213 & 173 & 342 & 318 & 300 & 379 & 7 & 6 & 23 & 29 & -10 & 15 \\
\hline
\end{tabular}

Figure 108: 2004 Profile Monthly Average Hourly MW Change for Hours 5 to 11

\begin{tabular}{|c|c|c|c|c|c|c|c|c|c|c|c|c|c|c|}
\hline & \multicolumn{14}{|c|}{ NPA Maximum Hourly Ramp-up MW Change 2018 - 2004 Profile } \\
\hline & \multicolumn{2}{|c|}{$\mathrm{Hr} 5$} & \multicolumn{2}{|c|}{$\mathrm{Hr} 6$} & \multicolumn{2}{|c|}{$\mathrm{Hr} 7$} & \multicolumn{2}{|c|}{$\mathrm{Hr} 8$} & \multicolumn{2}{|c|}{$\mathrm{Hr} 9$} & \multicolumn{2}{|c|}{$\mathrm{Hr} 10$} & \multicolumn{2}{|c|}{ Hr 11} \\
\hline & Load & $\begin{array}{l}\text { Load } \\
\text { Net } \\
\text { Wind }\end{array}$ & Load & $\begin{array}{l}\text { Load } \\
\text { Net } \\
\text { Wind }\end{array}$ & Load & $\begin{array}{l}\text { Load } \\
\text { Net } \\
\text { Wind }\end{array}$ & Load & $\begin{array}{l}\text { Load } \\
\text { Net } \\
\text { Wind }\end{array}$ & Load & $\begin{array}{l}\text { Load } \\
\text { Net } \\
\text { Wind }\end{array}$ & Load & $\begin{array}{l}\text { Load } \\
\text { Net } \\
\text { Wind }\end{array}$ & Load & $\begin{array}{l}\text { Load } \\
\text { Net } \\
\text { Wind }\end{array}$ \\
\hline Jan & 219 & 310 & 362 & 539 & 623 & 728 & 428 & 846 & 264 & 299 & 267 & 385 & 163 & 258 \\
\hline Feb & 155 & 334 & 402 & 501 & 614 & 746 & 361 & 552 & 120 & 390 & 100 & 442 & 52 & 250 \\
\hline Mar & 148 & 277 & 314 & 471 & 584 & 663 & 340 & 749 & 281 & 429 & 237 & 404 & 116 & 256 \\
\hline Apr & 116 & 493 & 282 & 542 & 510 & 780 & 414 & 786 & 331 & 382 & 187 & 293 & 153 & 308 \\
\hline May & 101 & 532 & 208 & 389 & 426 & 507 & 407 & 746 & 344 & 507 & 323 & 675 & 265 & 600 \\
\hline Jun & 93 & 458 & 207 & 390 & 380 & 570 & 620 & 992 & 474 & 670 & 358 & 436 & 317 & 569 \\
\hline Jul & 63 & 322 & 246 & 444 & 424 & 595 & 583 & 962 & 565 & 733 & 520 & 646 & 415 & 743 \\
\hline Aug & 63 & 292 & 274 & 593 & 533 & 640 & 371 & 937 & 444 & 696 & 420 & 629 & 318 & 528 \\
\hline Sep & 132 & 198 & 276 & 450 & 599 & 693 & 421 & 845 & 352 & 613 & 449 & 509 & 356 & 497 \\
\hline Oct & 123 & 294 & 305 & 387 & 524 & 723 & 438 & 860 & 200 & 351 & 178 & 365 & 121 & 414 \\
\hline Nov & 141 & 336 & 307 & 479 & 556 & 733 & 397 & 797 & 200 & 370 & 129 & 250 & 101 & 339 \\
\hline Dec & 145 & 242 & 655 & 426 & 539 & 710 & 489 & 859 & 131 & 381 & 265 & 450 & 148 & 405 \\
\hline
\end{tabular}

Figure 109: 2004 Profile Monthly Maximum Hourly MW Change for Hours 5 to 11 


\begin{tabular}{|c|c|c|c|c|c|c|c|c|c|c|c|c|c|c|}
\hline & \multicolumn{14}{|c|}{ NPA Minimum Hourly Ramp-up MW Change 2018 - 2004 Profile 20\% Wind } \\
\hline & \multicolumn{2}{|c|}{$\mathrm{Hr} 5$} & \multicolumn{2}{|c|}{$\mathrm{Hr} 6$} & \multicolumn{2}{|c|}{$\mathrm{Hr} 7$} & \multicolumn{2}{|c|}{$\mathrm{Hr} 8$} & \multicolumn{2}{|c|}{$\mathrm{Hr} 9$} & \multicolumn{2}{|c|}{$\mathrm{Hr} 10$} & \multicolumn{2}{|c|}{$\operatorname{Hr} 11$} \\
\hline & Load & $\begin{array}{l}\text { Load } \\
\text { Net } \\
\text { Wind }\end{array}$ & Load & $\begin{array}{l}\text { Load } \\
\text { Net } \\
\text { Wind }\end{array}$ & Load & $\begin{array}{l}\text { Load } \\
\text { Net } \\
\text { Wind }\end{array}$ & Load & $\begin{array}{l}\text { Load } \\
\text { Net } \\
\text { Wind }\end{array}$ & Load & $\begin{array}{l}\text { Load } \\
\text { Net } \\
\text { Wind }\end{array}$ & Load & $\begin{array}{l}\text { Load } \\
\text { Net } \\
\text { Wind }\end{array}$ & Load & $\begin{array}{l}\text { Load } \\
\text { Net } \\
\text { Wind }\end{array}$ \\
\hline Jan & -22 & -121 & 89 & 23 & 117 & 52 & 100 & -212 & -166 & -255 & -110 & -156 & -80 & -335 \\
\hline Feb & -31 & -88 & 20 & -19 & 177 & 171 & 120 & -65 & -132 & -324 & -116 & -164 & -87 & -258 \\
\hline Mar & -79 & -304 & 55 & 23 & 27 & -104 & 71 & -221 & -94 & -192 & -69 & -325 & -83 & -406 \\
\hline Apr & -62 & -247 & 23 & -96 & 67 & 4 & 66 & -111 & -38 & -128 & -63 & -164 & -48 & -79 \\
\hline May & -75 & -226 & -17 & -228 & -36 & -205 & 68 & -174 & 31 & -66 & 20 & -180 & -21 & -208 \\
\hline Jun & -56 & -202 & 15 & -312 & -41 & -138 & 129 & 2 & 178 & -141 & 73 & 71 & 50 & 73 \\
\hline Jul & -163 & -282 & -16 & -182 & -125 & -425 & 67 & -2 & 134 & 185 & 89 & 45 & 83 & -10 \\
\hline Aug & -96 & -241 & -1 & -43 & 8 & -3 & 35 & -110 & 64 & 124 & 62 & -8 & 74 & -107 \\
\hline Sep & -129 & -335 & -11 & -133 & 16 & 20 & -13 & -46 & -26 & -202 & 11 & -235 & 31 & -197 \\
\hline Oct & -36 & -145 & -20 & -143 & 117 & 82 & 59 & 113 & -34 & -184 & -36 & -175 & -41 & -92 \\
\hline Nov & 10 & -223 & 86 & -132 & 123 & 118 & 83 & -54 & -148 & -206 & -127 & -343 & -83 & -156 \\
\hline Dec & -929 & -1101 & 51 & -127 & 131 & 29 & 153 & 43 & -343 & -327 & -143 & -295 & -86 & -307 \\
\hline
\end{tabular}

Figure 110: 2004 Profile Monthly Minimum Hourly MW Change for Hours 5 to 11

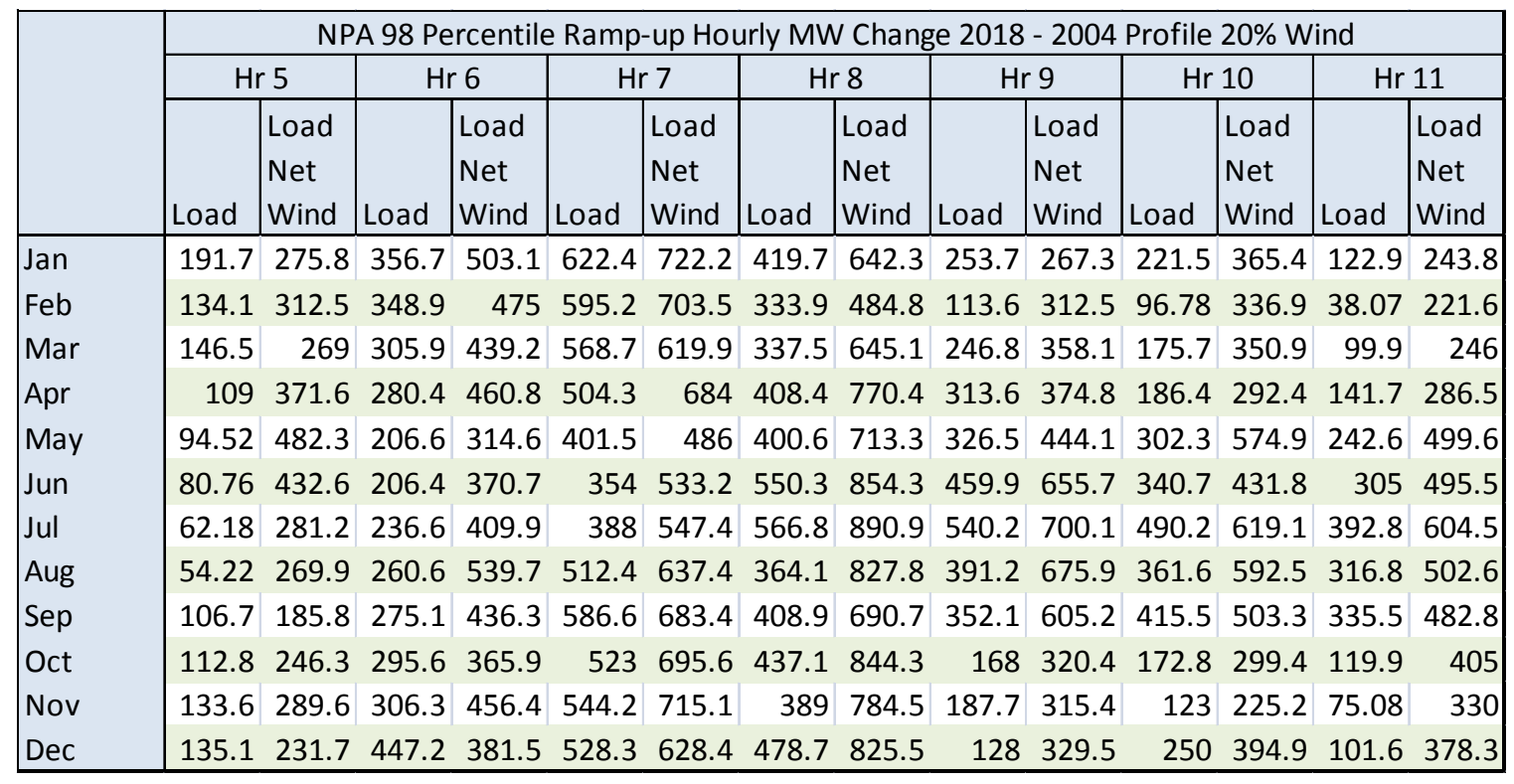

Figure 111: 2004 Profile Monthly 98 Percentile Hourly MW Change for Hours 5 to 11 


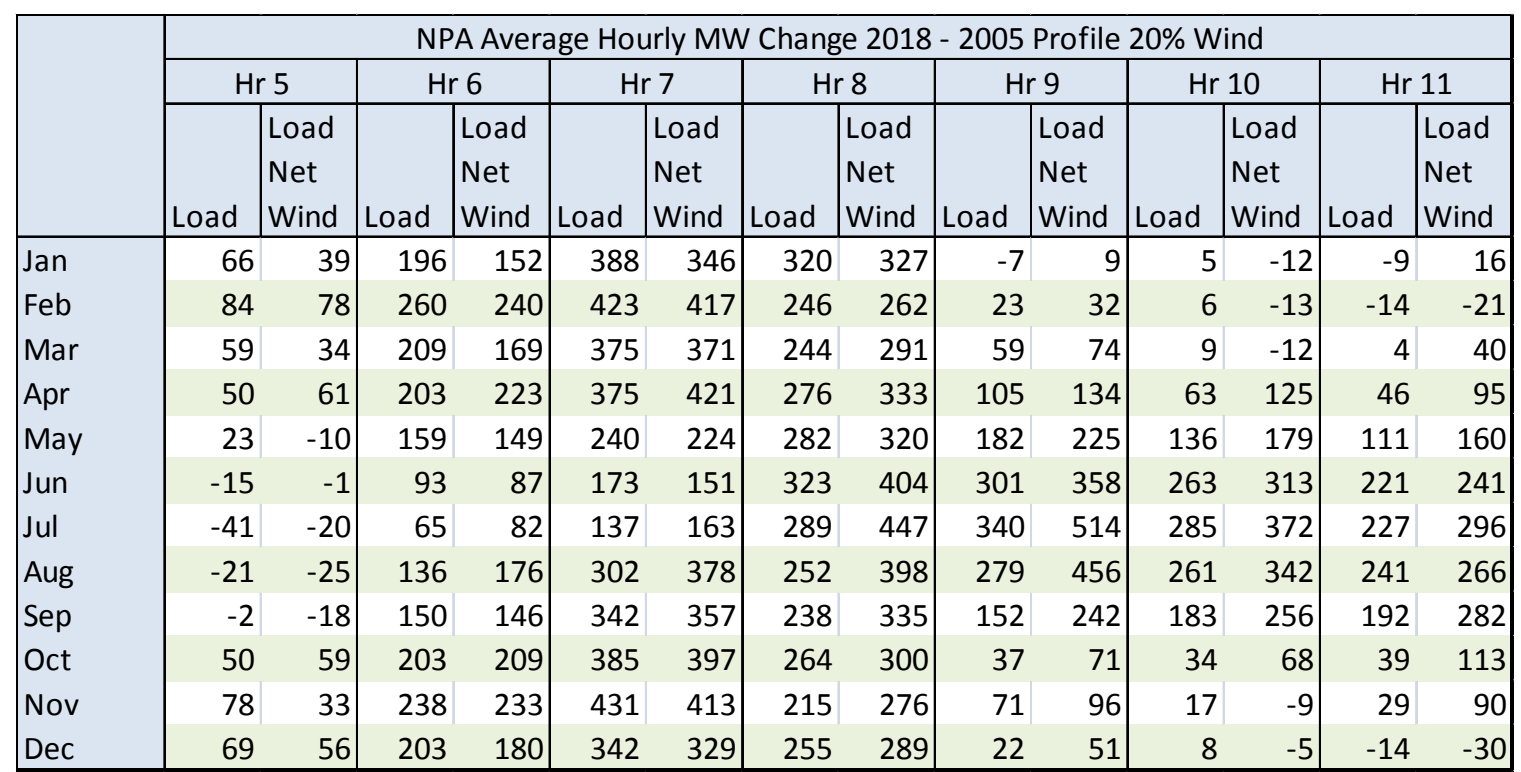

Figure 112: 2005 Profile Monthly Average Hourly MW Change for Hours 5 to 11

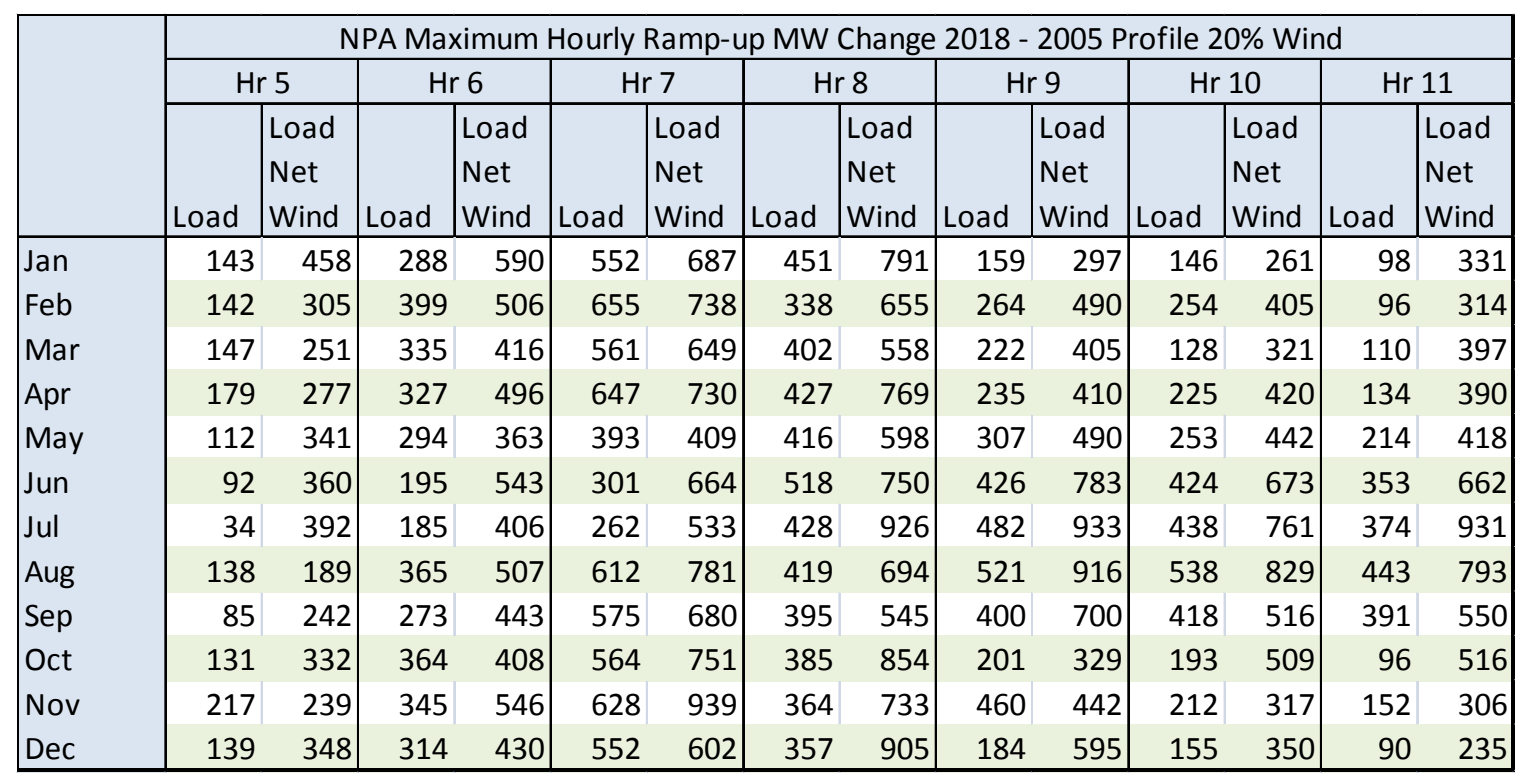

Figure 113: 2005 Profile Monthly Maximum Hourly MW Change for Hours 5 to 11 


\begin{tabular}{|c|c|c|c|c|c|c|c|c|c|c|c|c|c|c|}
\hline & \multicolumn{14}{|c|}{ NPA Minimum Hourly Ramp-up MW Change 2018 - 2005 Profile 20\% Wind } \\
\hline & \multicolumn{2}{|c|}{$\mathrm{Hr} 5$} & \multicolumn{2}{|c|}{$\operatorname{Hr} 6$} & \multicolumn{2}{|c|}{$\mathrm{Hr} 7$} & \multicolumn{2}{|c|}{$\mathrm{Hr} 8$} & \multicolumn{2}{|c|}{$\mathrm{Hr} 9$} & \multicolumn{2}{|c|}{$\mathrm{Hr} 10$} & \multicolumn{2}{|c|}{$\operatorname{Hr} 11$} \\
\hline & Load & $\begin{array}{l}\text { Load } \\
\text { Net } \\
\text { Wind }\end{array}$ & Load & $\begin{array}{l}\text { Load } \\
\text { Net } \\
\text { Wind }\end{array}$ & Load & $\begin{array}{l}\text { Load } \\
\text { Net } \\
\text { Wind }\end{array}$ & Load & $\begin{array}{l}\text { Load } \\
\text { Net } \\
\text { Wind }\end{array}$ & Load & $\begin{array}{l}\text { Load } \\
\text { Net } \\
\text { Wind } \\
\end{array}$ & Load & $\begin{array}{l}\text { Load } \\
\text { Net } \\
\text { Wind }\end{array}$ & Load & $\begin{array}{l}\text { Load } \\
\text { Net } \\
\text { Wind }\end{array}$ \\
\hline Jan & 1 & -239 & 15 & -222 & 106 & 40 & 127 & -111 & -133 & -376 & -119 & -318 & -114 & -203 \\
\hline Feb & -35 & -282 & 120 & -104 & 179 & 23 & 137 & -91 & -95 & -211 & -101 & -496 & -80 & -314 \\
\hline Mar & -11 & -326 & 55 & -254 & 76 & -54 & 54 & -111 & -131 & -148 & -128 & -374 & -58 & -389 \\
\hline Apr & -78 & -171 & 43 & -105 & 1 & -146 & 47 & -179 & -36 & -218 & -7 & -352 & -94 & -117 \\
\hline May & -91 & -533 & -4 & -116 & -67 & -100 & 78 & -37 & 1 & -299 & -39 & -243 & 19 & -111 \\
\hline Jun & -113 & -332 & -49 & -299 & -130 & -130 & 113 & -103 & 158 & -216 & 116 & 27 & 95 & -58 \\
\hline Jul & -141 & -367 & -92 & -368 & -143 & -337 & 98 & -22 & 73 & 204 & 28 & -100 & -11 & -25 \\
\hline Aug & -134 & -343 & -69 & -170 & -63 & -145 & 37 & -71 & 126 & 91 & 85 & -13 & 119 & 20 \\
\hline Sep & -103 & -299 & -54 & -348 & -10 & -247 & 17 & -50 & -39 & -124 & -12 & -120 & -32 & -62 \\
\hline Oct & -62 & -144 & 11 & -129 & 113 & 93 & 110 & -11 & -55 & -140 & -59 & -202 & -53 & -399 \\
\hline Nov & 5 & -322 & 83 & -88 & 167 & 126 & -490 & -521 & -79 & -271 & -112 & -460 & -52 & -258 \\
\hline Dec & 15 & -174 & 68 & -152 & 155 & -103 & 105 & -9 & -114 & -263 & -134 & -286 & -110 & -364 \\
\hline
\end{tabular}

Figure 114: 2005 Profile Monthly Minimum Hourly MW Change for Hours 5 to 11

\begin{tabular}{|c|c|c|c|c|c|c|c|c|c|c|c|c|c|c|}
\hline & \multicolumn{14}{|c|}{ NPA 98 Percentile Ramp-up Hourly MW Change 2018 - 2005 Profile $20 \%$ Wind } \\
\hline & \multicolumn{2}{|c|}{ Hr 5} & \multicolumn{2}{|c|}{ Hr 6} & \multicolumn{2}{|c|}{$\mathrm{Hr} 7$} & \multicolumn{2}{|c|}{$\mathrm{Hr} 8$} & \multicolumn{2}{|c|}{$\mathrm{Hr} 9$} & \multicolumn{2}{|c|}{ Hr 10} & \multicolumn{2}{|c|}{$\operatorname{Hr} 11$} \\
\hline & Load & \begin{tabular}{|l} 
Load \\
Net \\
Wind \\
\end{tabular} & Load & $\begin{array}{l}\text { Load } \\
\text { Net } \\
\text { Wind }\end{array}$ & Load & \begin{tabular}{|l|} 
Load \\
Net \\
Wind
\end{tabular} & Load & $\begin{array}{l}\text { Load } \\
\text { Net } \\
\text { Wind }\end{array}$ & Load & \begin{tabular}{|l|} 
Load \\
Net \\
Wind \\
\end{tabular} & Load & $\begin{array}{l}\text { Load } \\
\text { Net } \\
\text { Wind } \\
\end{array}$ & Load & $\begin{array}{l}\text { Load } \\
\text { Net } \\
\text { Wind }\end{array}$ \\
\hline Jan & 135.4 & 361.1 & 280.9 & 463.9 & 524.3 & 616.2 & 440 & 639.8 & 141.5 & 281.2 & 144 & 253.5 & 89.76 & 322.7 \\
\hline Feb & 138.9 & 297.4 & 387.3 & 495 & 627.2 & 715.6 & 334 & 582.1 & 257.2 & 405.2 & 238.3 & 367.9 & 93.15 & 290.1 \\
\hline Mar & 122.6 & 197.6 & 333.3 & 395.8 & 558.5 & 634.5 & 394.9 & 537.5 & 211.9 & 354.9 & 119.7 & 266.7 & 97.9 & 344.4 \\
\hline Apr & 138.2 & 265.9 & 319.5 & 465.8 & 619.6 & 724.2 & 418.9 & 690.7 & 231.2 & 402.6 & 185.1 & 362.6 & 123.8 & 315 \\
\hline May & 101 & 254 & 282.6 & 348.3 & 392.2 & 399.2 & 414.6 & 571.4 & 294.4 & 461 & 241.1 & 431.5 & 201.6 & 399.1 \\
\hline Jun & 67.03 & 276.4 & 193.8 & 478.9 & 297.1 & 570 & 509.9 & 719.6 & 420.1 & 777.9 & 423.5 & 622.9 & 344.1 & 611.4 \\
\hline Jul & 31.66 & 334.1 & 163.5 & 322 & 258.6 & 470.7 & 409 & 850.8 & 469.8 & 873.1 & 432.4 & 707.4 & 359.5 & 701.4 \\
\hline Aug & 104.5 & 179.6 & 303.8 & 495.2 & 580.7 & 754.2 & 407.3 & 684.6 & 494.1 & 782 & 497.9 & 793 & 419.6 & 612.6 \\
\hline Sep & 80.26 & 224.2 & 271.5 & 442.3 & 561.5 & 643.3 & 374 & 540.4 & 381.9 & 623.9 & 392.7 & 502.6 & 368.1 & 494.8 \\
\hline Oct & 119.8 & 264.1 & 337.3 & 368.6 & 546.1 & 663.1 & 370 & 685.5 & 199.1 & 307.3 & 162.2 & 376.9 & 95.56 & 498.8 \\
\hline Nov & 192.3 & 229.5 & 339.9 & 474.3 & 618.2 & 832.6 & 351.4 & 592.6 & 335.2 & 376.9 & 196.3 & 316.6 & 140.1 & 294.9 \\
\hline Dec & 136.8 & 315.6 & 309.4 & 391 & 538 & 586.7 & 356.4 & 682.8 & 172 & 430.3 & 142.5 & 278.7 & 82.86 & 232.9 \\
\hline
\end{tabular}

Figure 115: 2005 Profile Monthly 98 Percentile Hourly MW Change for Hours 5 to 11 


\begin{tabular}{|c|c|c|c|c|c|c|c|c|c|c|c|c|c|c|}
\hline & \multicolumn{14}{|c|}{ NPA Average Hourly MW Change 2018 - 2006 Profile 20\% Wind } \\
\hline & \multicolumn{2}{|c|}{$\operatorname{Hr} 5$} & \multicolumn{2}{|c|}{ Hr 6} & \multicolumn{2}{|c|}{$\mathrm{Hr} 7$} & \multicolumn{2}{|c|}{$\mathrm{Hr} 8$} & \multicolumn{2}{|c|}{$\mathrm{Hr} 9$} & \multicolumn{2}{|c|}{$\mathrm{Hr} 10$} & \multicolumn{2}{|c|}{$\operatorname{Hr} 11$} \\
\hline & Load & $\begin{array}{l}\text { Load } \\
\text { Net } \\
\text { Wind }\end{array}$ & Load & $\begin{array}{l}\text { Load } \\
\text { Net } \\
\text { Wind }\end{array}$ & Load & $\begin{array}{l}\text { Load } \\
\text { Net } \\
\text { Wind }\end{array}$ & Load & $\begin{array}{l}\text { Load } \\
\text { Net } \\
\text { Wind }\end{array}$ & Load & $\begin{array}{l}\text { Load } \\
\text { Net } \\
\text { Wind }\end{array}$ & Load & $\begin{array}{l}\text { Load } \\
\text { Net } \\
\text { Wind }\end{array}$ & Load & $\begin{array}{l}\text { Load } \\
\text { Net } \\
\text { Wind }\end{array}$ \\
\hline Jan & 66 & 73 & 211 & 204 & 430 & 445 & 364 & 396 & -42 & -48 & -34 & -73 & -11 & 14 \\
\hline Feb & 85 & 80 & 259 & 253 & 423 & 424 & 213 & 230 & -7 & -9 & -15 & -29 & -22 & 6 \\
\hline Mar & 71 & 43 & 219 & 204 & 348 & 344 & 208 & 214 & 63 & 75 & 14 & -1 & 6 & 27 \\
\hline Apr & 39 & 64 & 165 & 191 & 334 & 319 & 218 & 286 & 109 & 173 & 58 & 86 & 65 & 98 \\
\hline May & 22 & -24 & 120 & 80 & 212 & 218 & 272 & 272 & 181 & 216 & 127 & 168 & 123 & 187 \\
\hline Jun & -23 & -28 & 83 & 49 & 159 & 160 & 280 & 423 & 270 & 406 & 234 & 275 & 248 & 242 \\
\hline Jul & -39 & -56 & 67 & 62 & 142 & 162 & 292 & 437 & 321 & 501 & 283 & 387 & 219 & 289 \\
\hline Aug & -16 & -8 & 157 & 195 & 330 & 354 & 262 & 365 & 270 & 380 & 250 & 341 & 239 & 281 \\
\hline Sep & 28 & 36 & 153 & 166 & 373 & 394 & 231 & 311 & 136 & 199 & 135 & 300 & 136 & 202 \\
\hline Oct & 49 & 31 & 199 & 198 & 394 & 412 & 264 & 344 & 31 & 45 & 33 & 19 & 37 & 119 \\
\hline Nov & 72 & 69 & 220 & 213 & 375 & 413 & 205 & 245 & 31 & 55 & -4 & 42 & -2 & 48 \\
\hline Dec & 76 & 116 & 197 & 214 & 364 & 384 & 269 & 269 & 10 & 48 & 5 & 34 & -7 & 59 \\
\hline
\end{tabular}

Figure 116: 2006 Profile Monthly Average Hourly MW Change for Hours 5 to 11

\begin{tabular}{|c|c|c|c|c|c|c|c|c|c|c|c|c|c|c|}
\hline & \multicolumn{14}{|c|}{ NPA Maximum Hourly Ramp-up MW Change 2018 - 2006 Profile 20\% Wind } \\
\hline & \multicolumn{2}{|c|}{$\mathrm{Hr} 5$} & \multicolumn{2}{|c|}{$\mathrm{Hr} 6$} & \multicolumn{2}{|c|}{$\mathrm{Hr} 7$} & \multicolumn{2}{|c|}{$\mathrm{Hr} 8$} & \multicolumn{2}{|c|}{$\mathrm{Hr} 9$} & \multicolumn{2}{|c|}{$\operatorname{Hr} 10$} & \multicolumn{2}{|c|}{$\mathrm{Hr} 11$} \\
\hline & Load & $\begin{array}{l}\text { Load } \\
\text { Net } \\
\text { Wind }\end{array}$ & Load & $\begin{array}{l}\text { Load } \\
\text { Net } \\
\text { Wind }\end{array}$ & Load & $\begin{array}{l}\text { Load } \\
\text { Net } \\
\text { Wind }\end{array}$ & Load & $\begin{array}{l}\text { Load } \\
\text { Net } \\
\text { Wind }\end{array}$ & Load & $\begin{array}{l}\text { Load } \\
\text { Net } \\
\text { Wind } \\
\end{array}$ & Load & $\begin{array}{l}\text { Load } \\
\text { Net } \\
\text { Wind }\end{array}$ & Load & $\begin{array}{l}\text { Load } \\
\text { Net } \\
\text { Wind } \\
\end{array}$ \\
\hline Jan & 136 & 434 & 315 & 533 & 625 & 699 & 511 & 1113 & 196 & 346 & 157 & 242 & 113 & 224 \\
\hline Feb & 161 & 355 & 395 & 651 & 643 & 866 & 349 & 721 & 170 & 398 & 116 & 188 & 71 & 240 \\
\hline Mar & 134 & 272 & 314 & 610 & 592 & 676 & 358 & 673 & 247 & 388 & 165 & 356 & 81 & 308 \\
\hline Apr & 109 & 400 & 265 & 511 & 494 & 659 & 388 & 606 & 283 & 485 & 190 & 442 & 174 & 514 \\
\hline May & 81 & 335 & 226 & 489 & 374 & 582 & 397 & 674 & 367 & 728 & 329 & 395 & 275 & 522 \\
\hline Jun & 60 & 219 & 158 & 271 & 302 & 521 & 411 & 886 & 374 & 625 & 374 & 490 & 442 & 602 \\
\hline Jul & 31 & 344 & 187 & 241 & 310 & 367 & 436 & 801 & 474 & 801 & 502 & 696 & 439 & 664 \\
\hline Aug & 87 & 255 & 405 & 434 & 632 & 741 & 402 & 670 & 419 & 787 & 421 & 639 & 340 & 941 \\
\hline Sep & 99 & 238 & 267 & 450 & 586 & 659 & 398 & 661 & 352 & 523 & 393 & 674 & 328 & 583 \\
\hline Oct & 133 & 440 & 291 & 472 & 535 & 676 & 407 & 572 & 191 & 302 & 206 & 318 & 151 & 573 \\
\hline Nov & 146 & 390 & 334 & 463 & 547 & 738 & 304 & 640 & 236 & 412 & 155 & 424 & 94 & 442 \\
\hline Dec & 160 & 398 & 319 & 450 & 530 & 699 & 395 & 495 & 140 & 350 & 195 & 473 & 107 & 322 \\
\hline
\end{tabular}

Figure 117: 2006 Profile Monthly Maximum Hourly MW Change for Hours 5 to 11 


\begin{tabular}{|c|c|c|c|c|c|c|c|c|c|c|c|c|c|c|}
\hline & \multicolumn{14}{|c|}{ NPA Minimum Hourly Ramp-up MW Change 2018 - 2006 Profile 20\% Wind } \\
\hline & \multicolumn{2}{|c|}{$\operatorname{Hr} 5$} & \multicolumn{2}{|c|}{$\mathrm{Hr} 6$} & \multicolumn{2}{|c|}{$\mathrm{Hr} 7$} & \multicolumn{2}{|c|}{$\mathrm{Hr} 8$} & \multicolumn{2}{|c|}{$\operatorname{Hr} 9$} & \multicolumn{2}{|c|}{$\mathrm{Hr} 10$} & \multicolumn{2}{|c|}{$\operatorname{Hr} 11$} \\
\hline & Load & \begin{tabular}{|l|} 
Load \\
Net \\
Wind \\
\end{tabular} & Load & \begin{tabular}{|l|} 
Load \\
Net \\
Wind \\
\end{tabular} & Load & \begin{tabular}{|l|} 
Load \\
Net \\
Wind \\
\end{tabular} & Load & \begin{tabular}{|l} 
Load \\
Net \\
Wind \\
\end{tabular} & Load & \begin{tabular}{|l} 
Load \\
Net \\
Wind \\
\end{tabular} & Load & \begin{tabular}{|l|} 
Load \\
Net \\
Wind \\
\end{tabular} & Load & \begin{tabular}{|l} 
Load \\
Net \\
Wind \\
\end{tabular} \\
\hline Jan & -17 & -333 & 24 & -214 & 105 & -167 & 123 & -84 & -254 & -373 & -224 & -463 & -116 & -309 \\
\hline Feb & -21 & -73 & 55 & 32 & 119 & 16 & 73 & -410 & -116 & -420 & -112 & -307 & -94 & -182 \\
\hline Mar & -39 & -138 & 59 & -22 & 57 & -11 & 98 & -4 & -97 & -366 & -155 & -264 & -56 & -238 \\
\hline Apr & -41 & -364 & 17 & -156 & 51 & 13 & 77 & -161 & -48 & -259 & -56 & -280 & -55 & -462 \\
\hline May & -89 & -439 & -80 & -320 & -103 & -229 & 96 & -24 & 75 & -206 & 9 & -95 & 34 & -46 \\
\hline Jun & -97 & -293 & -76 & -138 & -54 & -223 & 15 & 68 & 167 & 70 & -27 & -110 & 74 & -206 \\
\hline Jul & -138 & -391 & -65 & -303 & -146 & -355 & 111 & -64 & -23 & 135 & 59 & -83 & -38 & 23 \\
\hline Aug & -111 & -308 & -76 & -206 & -43 & -163 & 58 & 110 & 108 & 120 & 78 & 108 & 93 & 12 \\
\hline Sep & -150 & -177 & -8 & -159 & 2 & 5 & -12 & -314 & -15 & -129 & 6 & -70 & 40 & -74 \\
\hline Oct & -126 & -296 & 26 & -114 & 68 & -14 & 64 & -46 & -75 & -370 & -59 & -402 & -31 & -264 \\
\hline Nov & 3 & -366 & 67 & -41 & 146 & 38 & 46 & -119 & -106 & -194 & -161 & -235 & -108 & -429 \\
\hline Dec & -65 & -172 & 31 & -54 & 120 & -66 & 147 & -242 & -139 & -262 & -141 & -541 & -106 & -235 \\
\hline
\end{tabular}

Figure 118: 2006 Profile Monthly Minimum Hourly MW Change for Hours 5 to 11

\begin{tabular}{|c|c|c|c|c|c|c|c|c|c|c|c|c|c|c|}
\hline & \multicolumn{14}{|c|}{ NPA 98 Percentile Ramp-up Hourly MW Change 2018 - 2006 Profile 20\% Wind } \\
\hline & \multicolumn{2}{|c|}{$\operatorname{Hr} 5$} & \multicolumn{2}{|c|}{$\mathrm{Hr} 6$} & \multicolumn{2}{|c|}{$\mathrm{Hr} 7$} & \multicolumn{2}{|c|}{$\mathrm{Hr} 8$} & \multicolumn{2}{|c|}{$\mathrm{Hr} 9$} & \multicolumn{2}{|c|}{$\mathrm{Hr} 10$} & \multicolumn{2}{|c|}{$\mathrm{Hr} 11$} \\
\hline & Load & \begin{tabular}{|l} 
Load \\
Net \\
Wind
\end{tabular} & Load & \begin{tabular}{|l} 
Load \\
Net \\
Wind \\
\end{tabular} & Load & \begin{tabular}{|l|} 
Load \\
Net \\
Wind \\
\end{tabular} & Load & $\mid \begin{array}{l}\text { Load } \\
\text { Net } \\
\text { Wind }\end{array}$ & Load & $\begin{array}{l}\text { Load } \\
\text { Net } \\
\text { Wind }\end{array}$ & Load & $\begin{array}{l}\text { Load } \\
\text { Net } \\
\text { Wind }\end{array}$ & Load & $\begin{array}{l}\text { Load } \\
\text { Net } \\
\text { Wind }\end{array}$ \\
\hline Jan & 127.1 & 382.5 & 307.1 & 477.6 & 607.8 & 696.8 & 502.8 & 946.1 & 177.1 & 325.4 & 155.2 & 216.4 & 92 & 221.1 \\
\hline Feb & 156.7 & 360.2 & 394.2 & 605.7 & 634.3 & 765 & 348.5 & 601.6 & 158.6 & 314.6 & 97.17 & 175.2 & 58.09 & 216.8 \\
\hline Mar & 125.6 & 231 & 311.7 & 546.6 & 553.1 & 634.3 & 322.4 & 577.4 & 241.7 & 376.6 & 156.5 & 299.1 & 75.58 & 292.1 \\
\hline Apr & 105.5 & 356.5 & 255.9 & 507.3 & 485.3 & 627.8 & 358.8 & 606 & 255.5 & 480.8 & 185.6 & 426.3 & 158.3 & 396.6 \\
\hline May & 79.54 & 292.1 & 218.3 & 400.3 & 373.9 & 544.5 & 396.3 & 636.3 & 356 & 579.8 & 283.5 & 354.4 & 250.2 & 518.4 \\
\hline Jun & 34.36 & 198.5 & 158 & 221.5 & 283 & 468.5 & 398.5 & 857 & 363.7 & 624.7 & 348.7 & 483.3 & 402.7 & 556.7 \\
\hline Jul & 22.9 & 311.2 & 180.9 & 228.4 & 309.6 & 352 & 429.4 & 768.3 & 472.6 & 775.6 & 470.8 & 675.6 & 401.2 & 561.4 \\
\hline Aug & 81.6 & 211.8 & 345 & 430.5 & 605 & 698.1 & 388.9 & 623.3 & 412.7 & 712.3 & 399.6 & 609.9 & 327 & 659.9 \\
\hline Sep & 94.53 & 223.9 & 258.3 & 418.1 & 577.5 & 642.9 & 381.6 & 626.6 & 318.9 & 476.5 & 313.2 & 658.6 & 276.9 & 527.9 \\
\hline Oct & 119.3 & 332.4 & 284.5 & 447.1 & 533.1 & 667.9 & 398.3 & 568.6 & 179.7 & 255.7 & 194.9 & 294.4 & 128.9 & 471.7 \\
\hline Nov & 137.6 & 328.5 & 323.2 & 456.9 & 534.3 & 714.1 & 301.2 & 581.1 & 210.8 & 400.4 & 123.7 & 404.6 & 72.55 & 390.8 \\
\hline Dec & 155.1 & 314.1 & 309.6 & 438.4 & 530.3 & 643.2 & 377.1 & 493.8 & 136.7 & 341.1 & 167.1 & 415.9 & 93.82 & 302.6 \\
\hline
\end{tabular}

Figure 119: 2006 Profile Monthly 98 Percentile Hourly MW Change for Hours 5 to 11 


\begin{tabular}{|c|c|c|c|c|c|c|c|c|c|c|c|c|c|c|}
\hline & \multicolumn{14}{|c|}{ NPA Maximum Hourly Ramp-up MW Change 2018 - 2004 Profile 20\% Wind } \\
\hline & \multicolumn{2}{|c|}{$\mathrm{Hr} 5$} & \multicolumn{2}{|c|}{$\mathrm{Hr} 6$} & \multicolumn{2}{|c|}{\begin{tabular}{|l|}
$\mathrm{Hr} 7$ \\
\end{tabular}} & \multicolumn{2}{|c|}{$\mathrm{Hr} 8$} & \multicolumn{2}{|c|}{$\operatorname{Hr} 9$} & \multicolumn{2}{|c|}{$\mathrm{Hr} 10$} & \multicolumn{2}{|c|}{$\mathrm{Hr} 11$} \\
\hline & Load & $\begin{array}{l}\text { Load } \\
\text { Net } \\
\text { Wind }\end{array}$ & Load & $\begin{array}{l}\text { Load } \\
\text { Net } \\
\text { Wind } \\
\end{array}$ & Load & $\begin{array}{l}\text { Load } \\
\text { Net } \\
\text { Wind } \\
\end{array}$ & Load & $\begin{array}{l}\text { Load } \\
\text { Net } \\
\text { Wind }\end{array}$ & Load & $\begin{array}{l}\text { Load } \\
\text { Net } \\
\text { Wind }\end{array}$ & Load & $\begin{array}{l}\text { Load } \\
\text { Net } \\
\text { Wind }\end{array}$ & Load & $\begin{array}{l}\text { Load } \\
\text { Net } \\
\text { Wind }\end{array}$ \\
\hline Winter & 219 & 334 & 655 & 539 & 623 & 746 & 489 & 859 & 264 & 390 & 267 & 450 & 163 & 405 \\
\hline Spring & 148 & 532 & 314 & 542 & 584 & 780 & 414 & 786 & 344 & 507 & 323 & 675 & 265 & 600 \\
\hline Summer & 93 & 458 & 274 & 593 & 533 & 640 & 620 & 992 & 565 & 733 & 520 & 646 & 415 & 743 \\
\hline Fall & 141 & 336 & 307 & 479 & 599 & 733 & 438 & 860 & 352 & 613 & 449 & 509 & 356 & 497 \\
\hline Year & 219 & 532 & 655 & 593 & 623 & 780 & 620 & 992 & 565 & 733 & 520 & 675 & 415 & 743 \\
\hline
\end{tabular}

Figure 120: 2004 Profile Seasonal Maximum Hourly MW Change for Hours 5 to 11

\begin{tabular}{|c|c|c|c|c|c|c|c|c|c|c|c|c|c|c|}
\hline & \multicolumn{14}{|c|}{ NPA Minimum Hourly Ramp-up MW Change 2018 - 2004 Profile 20\% Wind } \\
\hline & \multicolumn{2}{|c|}{$\operatorname{Hr} 5$} & \multicolumn{2}{|c|}{$\mathrm{Hr} 6$} & \multicolumn{2}{|c|}{$\mathrm{Hr} 7$} & \multicolumn{2}{|c|}{$\mathrm{Hr} 8$} & \multicolumn{2}{|c|}{$\mathrm{Hr} 9$} & \multicolumn{2}{|c|}{$\operatorname{Hr} 10$} & \multicolumn{2}{|c|}{$\operatorname{Hr} 11$} \\
\hline & Load & $\begin{array}{l}\text { Load } \\
\text { Net } \\
\text { Wind }\end{array}$ & Load & $\begin{array}{l}\text { Load } \\
\text { Net } \\
\text { Wind }\end{array}$ & Load & $\begin{array}{l}\text { Load } \\
\text { Net } \\
\text { Wind }\end{array}$ & Load & $\begin{array}{l}\text { Load } \\
\text { Net } \\
\text { Wind }\end{array}$ & Load & $\begin{array}{l}\text { Load } \\
\text { Net } \\
\text { Wind }\end{array}$ & Load & $\begin{array}{l}\text { Load } \\
\text { Net } \\
\text { Wind }\end{array}$ & Load & $\begin{array}{l}\text { Load } \\
\text { Net } \\
\text { Wind }\end{array}$ \\
\hline Winter & -929 & -1101 & 20 & -127 & 117 & 29 & 100 & -212 & -343 & -327 & -143 & -295 & -87 & -335 \\
\hline Spring & -79 & -304 & -17 & -228 & -36 & -205 & 66 & -221 & -94 & -192 & -69 & -325 & -83 & -406 \\
\hline Summer & -163 & -282 & -16 & -312 & -125 & -425 & 35 & -110 & 64 & -141 & 62 & -8 & 50 & -107 \\
\hline Fall & -129 & -335 & -20 & -143 & 16 & 20 & -13 & -54 & -148 & -206 & -127 & -343 & -83 & -197 \\
\hline Year & -929 & -1101 & -20 & -312 & -125 & -425 & -13 & -221 & -343 & -327 & -143 & -343 & -87 & -406 \\
\hline
\end{tabular}

Figure 121: 2004 Profile Seasonal Minimum Hourly MW Change for Hours 5 to 11

\begin{tabular}{|c|c|c|c|c|c|c|c|c|c|c|c|c|c|c|}
\hline & \multicolumn{14}{|c|}{ NPA 98 Percentile Ramp-up Hourly MW Change 2018 - 2004 Profile $20 \%$ Wind } \\
\hline & \multicolumn{2}{|c|}{$\mathrm{Hr} 5$} & \multicolumn{2}{|c|}{$\mathrm{Hr} 6$} & \multicolumn{2}{|c|}{\begin{tabular}{|l|}
$\mathrm{Hr} 7$ \\
\end{tabular}} & \multicolumn{2}{|c|}{\begin{tabular}{|l|}
$\mathrm{Hr} 8$ \\
\end{tabular}} & \multicolumn{2}{|c|}{\begin{tabular}{|l|}
$\mathrm{Hr} 9$ \\
\end{tabular}} & \multicolumn{2}{|c|}{$\mathrm{Hr} 10$} & \multicolumn{2}{|c|}{$\mathrm{Hr} 11$} \\
\hline & Load & $\begin{array}{l}\text { Load } \\
\text { Net } \\
\text { Wind }\end{array}$ & Load & $\begin{array}{l}\text { Load } \\
\text { Net } \\
\text { Wind }\end{array}$ & Load & $\begin{array}{l}\text { Load } \\
\text { Net } \\
\text { Wind }\end{array}$ & Load & \begin{tabular}{|l} 
Load \\
Net \\
Wind \\
\end{tabular} & Load & $\begin{array}{l}\text { Load } \\
\text { Net } \\
\text { Wind }\end{array}$ & Load & \begin{tabular}{|l|} 
Load \\
Net \\
Wind \\
\end{tabular} & Load & $\begin{array}{l}\text { Load } \\
\text { Net } \\
\text { Wind }\end{array}$ \\
\hline Winter & 159 & 298 & 371 & 484 & 616 & 721 & 437 & 813 & 167 & 317 & 246 & 398 & 108 & 280 \\
\hline Spring & 124 & 466 & 292 & 439 & 519 & 634 & 406 & 759 & 321 & 413 & 288 & 498 & 224 & 416 \\
\hline Summer & 65 & 339 & 247 & 455 & 497 & 603 & 561 & 942 & 510 & 681 & 429 & 606 & 370 & 536 \\
\hline Fall & 129 & 264 & 305 & 442 & 568 & 706 & 435 & 836 & 333 & 515 & 373 & 482 & 299 & 453 \\
\hline Year & 141 & 333 & 323 & 466 & 583 & 700 & 478 & 841 & 444 & 627 & 388 & 529 & 322 & 471 \\
\hline
\end{tabular}

Figure 122: 2004 Profile Seasonal 98 Percentile Hourly MW Change for Hours 5 to 11 


\begin{tabular}{|c|c|c|c|c|c|c|c|c|c|c|c|c|c|c|}
\hline & \multicolumn{14}{|c|}{ NPA Maximum Hourly Ramp-up MW Change 2018 - 2005 Profile 20\% Wind } \\
\hline & \multicolumn{2}{|c|}{$\mathrm{Hr} 5$} & \multicolumn{2}{|c|}{ Hr 6} & \multicolumn{2}{|c|}{$\mathrm{Hr} 7$} & \multicolumn{2}{|c|}{$\mathrm{Hr} 8$} & \multicolumn{2}{|c|}{$\mathrm{Hr} 9$} & \multicolumn{2}{|c|}{$\mathrm{Hr} 10$} & \multicolumn{2}{|c|}{$\mathrm{Hr} 11$} \\
\hline & Load & $\begin{array}{l}\text { Load } \\
\text { Net } \\
\text { Wind } \\
\end{array}$ & Load & $\begin{array}{l}\text { Load } \\
\text { Net } \\
\text { Wind }\end{array}$ & Load & $\begin{array}{l}\text { Load } \\
\text { Net } \\
\text { Wind }\end{array}$ & Load & $\begin{array}{l}\text { Load } \\
\text { Net } \\
\text { Wind } \\
\end{array}$ & Load & $\begin{array}{l}\text { Load } \\
\text { Net } \\
\text { Wind } \\
\end{array}$ & Load & $\begin{array}{l}\text { Load } \\
\text { Net } \\
\text { Wind } \\
\end{array}$ & Load & $\begin{array}{l}\text { Load } \\
\text { Net } \\
\text { Wind }\end{array}$ \\
\hline Winter & 143 & 458 & 399 & 590 & 655 & 738 & 451 & 905 & 264 & 595 & 254 & 405 & 98 & 331 \\
\hline Spring & 179 & 341 & 335 & 496 & 647 & 730 & 427 & 769 & 307 & 490 & 253 & 442 & 214 & 418 \\
\hline Summer & 138 & 392 & 365 & 543 & 612 & 781 & 518 & 926 & 521 & 933 & 538 & 829 & 443 & 931 \\
\hline Fall & 217 & 332 & 364 & 546 & 628 & 939 & 395 & 854 & 460 & 700 & 418 & 516 & 391 & 550 \\
\hline Year & 217 & 458 & 399 & 590 & 655 & 939 & 518 & 926 & 521 & 933 & 538 & 829 & 443 & 931 \\
\hline
\end{tabular}

Figure 123: 2005 Profile Seasonal Maximum Hourly MW Change for Hours 5 to 11

\begin{tabular}{|c|c|c|c|c|c|c|c|c|c|c|c|c|c|c|}
\hline & \multicolumn{14}{|c|}{ NPA Minimum Hourly Ramp-up MW Change 2018 - 2005 Profile 20\% Wind } \\
\hline & \multicolumn{2}{|c|}{$\operatorname{Hr} 5$} & \multicolumn{2}{|c|}{$\mathrm{Hr} 6$} & \multicolumn{2}{|c|}{$\mathrm{Hr} 7$} & \multicolumn{2}{|c|}{$\mathrm{Hr} 8$} & \multicolumn{2}{|c|}{$\mathrm{Hr} 9$} & \multicolumn{2}{|c|}{$\operatorname{Hr} 10$} & \multicolumn{2}{|c|}{$\operatorname{Hr} 11$} \\
\hline & Load & $\begin{array}{l}\text { Load } \\
\text { Net } \\
\text { Wind }\end{array}$ & Load & $\begin{array}{l}\text { Load } \\
\text { Net } \\
\text { Wind }\end{array}$ & Load & $\begin{array}{l}\text { Load } \\
\text { Net } \\
\text { Wind }\end{array}$ & Load & $\begin{array}{l}\text { Load } \\
\text { Net } \\
\text { Wind }\end{array}$ & Load & $\begin{array}{l}\text { Load } \\
\text { Net } \\
\text { Wind }\end{array}$ & Load & $\begin{array}{l}\text { Load } \\
\text { Net } \\
\text { Wind }\end{array}$ & Load & $\begin{array}{l}\text { Load } \\
\text { Net } \\
\text { Wind }\end{array}$ \\
\hline Winter & -35 & -282 & 15 & -222 & 106 & -103 & 105 & -111 & -133 & -376 & -134 & -496 & -114 & -364 \\
\hline Spring & -91 & -533 & -4 & -254 & -67 & -146 & 47 & -179 & -131 & -299 & -128 & -374 & -94 & -389 \\
\hline Summer & -141 & -367 & -92 & -368 & -143 & -337 & 37 & -103 & 73 & -216 & 28 & -100 & -11 & -58 \\
\hline Fall & -103 & -322 & -54 & -348 & -10 & -247 & -490 & -521 & -79 & -271 & -112 & -460 & -53 & -399 \\
\hline Year & -141 & -533 & -92 & -368 & -143 & -337 & -490 & -521 & -133 & -376 & -134 & -496 & -114 & -399 \\
\hline
\end{tabular}

Figure 124: 2005 Profile Seasonal Minimum Hourly MW Change for Hours 5 to 11

\begin{tabular}{|c|c|c|c|c|c|c|c|c|c|c|c|c|c|c|}
\hline & \multicolumn{14}{|c|}{ NPA 98 Percentile Ramp-up Hourly MW Change 2018 - 2005 Profile $20 \%$ Wind } \\
\hline & \multicolumn{2}{|c|}{$\mathrm{Hr} 5$} & \multicolumn{2}{|c|}{$\mathrm{Hr} 6$} & \multicolumn{2}{|c|}{$\mathrm{Hr} 7$} & \multicolumn{2}{|c|}{\begin{tabular}{|l|}
$\mathrm{Hr} 8$ \\
\end{tabular}} & \multicolumn{2}{|c|}{$\mathrm{Hr} 9$} & \multicolumn{2}{|c|}{$\mathrm{Hr} 10$} & \multicolumn{2}{|c|}{$\mathrm{Hr} 11$} \\
\hline & Load & $\begin{array}{l}\text { Load } \\
\text { Net } \\
\text { Wind }\end{array}$ & Load & $\begin{array}{l}\text { Load } \\
\text { Net } \\
\text { Wind }\end{array}$ & Load & $\begin{array}{l}\text { Load } \\
\text { Net } \\
\text { Wind }\end{array}$ & Load & \begin{tabular}{|l} 
Load \\
Net \\
Wind \\
\end{tabular} & Load & $\begin{array}{l}\text { Load } \\
\text { Net } \\
\text { Wind }\end{array}$ & Load & \begin{tabular}{|l|} 
Load \\
Net \\
Wind \\
\end{tabular} & Load & $\begin{array}{l}\text { Load } \\
\text { Net } \\
\text { Wind }\end{array}$ \\
\hline Winter & 140 & 315 & 368 & 490 & 582 & 689 & 425 & 685 & 234 & 367 & 170 & 339 & 92 & 315 \\
\hline Spring & 126 & 277 & 327 & 444 & 600 & 720 & 415 & 634 & 263 & 430 & 226 & 423 & 188 & 388 \\
\hline Summer & 84 & 307 & 260 & 491 & 519 & 720 & 499 & 759 & 477 & 848 & 467 & 763 & 379 & 686 \\
\hline Fall & 140 & 240 & 338 & 442 & 611 & 752 & 368 & 605 & 375 & 561 & 367 & 496 & 346 & 493 \\
\hline Year & 137 & 296 & 350 & 480 & 596 & 719 & 430 & 724 & 431 & 715 & 424 & 584 & 349 & 492 \\
\hline
\end{tabular}

Figure 125: 2005 Profile Seasonal 98 Percentile Hourly MW Change for Hours 5 to 11 


\begin{tabular}{|c|c|c|c|c|c|c|c|c|c|c|c|c|c|c|}
\hline & \multicolumn{14}{|c|}{ Nebraska Maximum Hourly Ramp-up MW Change 2018 - 2006 Profile 20\% Wind } \\
\hline & \multicolumn{2}{|c|}{$\mathrm{Hr} 5$} & \multicolumn{2}{|c|}{$\operatorname{Hr} 6$} & \multicolumn{2}{|c|}{$\mathrm{Hr} 7$} & \multicolumn{2}{|c|}{$\mathrm{Hr} 8$} & \multicolumn{2}{|c|}{$\mathrm{Hr} 9$} & \multicolumn{2}{|c|}{$\mathrm{Hr} 10$} & \multicolumn{2}{|c|}{$\operatorname{Hr} 11$} \\
\hline & Load & $\begin{array}{l}\text { Load } \\
\text { Net } \\
\text { Wind }\end{array}$ & Load & $\begin{array}{l}\text { Load } \\
\text { Net } \\
\text { Wind }\end{array}$ & Load & $\begin{array}{l}\text { Load } \\
\text { Net } \\
\text { Wind }\end{array}$ & Load & $\begin{array}{l}\text { Load } \\
\text { Net } \\
\text { Wind }\end{array}$ & Load & $\begin{array}{l}\text { Load } \\
\text { Net } \\
\text { Wind }\end{array}$ & Load & $\begin{array}{l}\text { Load } \\
\text { Net } \\
\text { Wind }\end{array}$ & Load & $\begin{array}{l}\text { Load } \\
\text { Net } \\
\text { Wind }\end{array}$ \\
\hline Winter & 161 & 434 & 395 & 651 & 643 & 866 & 511 & 1113 & 196 & 398 & 195 & 473 & 113 & 322 \\
\hline Spring & 134 & 400 & 314 & 610 & 592 & 676 & 397 & 674 & 367 & 728 & 329 & 442 & 275 & 522 \\
\hline Summer & 87 & 344 & 405 & 434 & 632 & 741 & 436 & 886 & 474 & 801 & 502 & 696 & 442 & 941 \\
\hline Fall & 146 & 440 & 334 & 472 & 586 & 738 & 407 & 661 & 352 & 523 & 393 & 674 & 328 & 583 \\
\hline Year & 161 & 440 & 405 & 651 & 643 & 866 & 511 & 1113 & 474 & 801 & 502 & 696 & 442 & 941 \\
\hline
\end{tabular}

Figure 126: 2006 Profile Seasonal Maximum Hourly MW Change for Hours 5 to 11

\begin{tabular}{|c|c|c|c|c|c|c|c|c|c|c|c|c|c|c|}
\hline & \multicolumn{14}{|c|}{ NPA Minimum Hourly Ramp-up MW Change 2018 - 2006 Profile 20\% Wind } \\
\hline & \multicolumn{2}{|c|}{$\operatorname{Hr} 5$} & \multicolumn{2}{|c|}{$\mathrm{Hr} 6$} & \multicolumn{2}{|c|}{$\mathrm{Hr} 7$} & \multicolumn{2}{|c|}{$\mathrm{Hr} 8$} & \multicolumn{2}{|c|}{$\mathrm{Hr} 9$} & \multicolumn{2}{|c|}{$\mathrm{Hr} 10$} & \multicolumn{2}{|c|}{$\operatorname{Hr} 11$} \\
\hline & Load & \begin{tabular}{|l|} 
Load \\
Net \\
Wind \\
\end{tabular} & Load & $\begin{array}{l}\text { Load } \\
\text { Net } \\
\text { Wind }\end{array}$ & Load & $\begin{array}{l}\text { Load } \\
\text { Net } \\
\text { Wind }\end{array}$ & Load & $\begin{array}{l}\text { Load } \\
\text { Net } \\
\text { Wind }\end{array}$ & Load & $\begin{array}{l}\text { Load } \\
\text { Net } \\
\text { Wind }\end{array}$ & Load & $\begin{array}{l}\text { Load } \\
\text { Net } \\
\text { Wind }\end{array}$ & Load & $\begin{array}{l}\text { Load } \\
\text { Net } \\
\text { Wind }\end{array}$ \\
\hline Winter & -65 & -333 & 24 & -214 & 105 & -167 & 73 & -410 & -254 & -420 & -224 & -541 & -116 & -309 \\
\hline Spring & -89 & -439 & -80 & -320 & -103 & -229 & 77 & -161 & -97 & -366 & -155 & -280 & -56 & -462 \\
\hline Summer & -138 & -391 & -76 & -303 & -146 & -355 & 15 & -64 & -23 & 70 & -27 & -110 & -38 & -206 \\
\hline Fall & -150 & -366 & -8 & -159 & 2 & -14 & -12 & -314 & -106 & -370 & -161 & -402 & -108 & -429 \\
\hline Year & -150 & -439 & -80 & -320 & -146 & -355 & -12 & -410 & -254 & -420 & -224 & -541 & -116 & -462 \\
\hline
\end{tabular}

Figure 127: 2006 Profile Seasonal Minimum Hourly MW Change for Hours 5 to 11

\begin{tabular}{|c|c|c|c|c|c|c|c|c|c|c|c|c|c|c|}
\hline & \multicolumn{14}{|c|}{ NPA 98 Percentile Ramp-up Hourly MW Change 2018 - 2006 Profile 20\% Wind } \\
\hline & \multicolumn{2}{|c|}{$\operatorname{Hr} 5$} & \multicolumn{2}{|c|}{$\mathrm{Hr} 6$} & \multicolumn{2}{|c|}{$\mathrm{Hr} 7$} & \multicolumn{2}{|c|}{$\mathrm{Hr} 8$} & \multicolumn{2}{|c|}{\begin{tabular}{|l|}
$\mathrm{Hr} 9$ \\
\end{tabular}} & \multicolumn{2}{|c|}{$\mathrm{Hr} 10$} & \multicolumn{2}{|c|}{$\operatorname{Hr} 11$} \\
\hline & Load & $\begin{array}{l}\text { Load } \\
\text { Net } \\
\text { Wind }\end{array}$ & Load & $\begin{array}{l}\text { Load } \\
\text { Net } \\
\text { Wind }\end{array}$ & Load & $\begin{array}{l}\text { Load } \\
\text { Net } \\
\text { Wind }\end{array}$ & Load & $\begin{array}{l}\text { Load } \\
\text { Net } \\
\text { Wind }\end{array}$ & Load & $\begin{array}{l}\text { Load } \\
\text { Net } \\
\text { Wind }\end{array}$ & Load & $\begin{array}{l}\text { Load } \\
\text { Net } \\
\text { Wind }\end{array}$ & Load & $\begin{array}{l}\text { Load } \\
\text { Net } \\
\text { Wind }\end{array}$ \\
\hline Winter & 155 & 374 & 376 & 541 & 626 & 699 & 496 & 759 & 165 & 347 & 155 & 375 & 90 & 285 \\
\hline Spring & 112 & 324 & 294 & 507 & 514 & 628 & 388 & 636 & 293 & 483 & 208 & 415 & 228 & 515 \\
\hline Summer & 72 & 267 & 299 & 390 & 550 & 633 & 413 & 807 & 464 & 764 & 427 & 654 & 387 & 613 \\
\hline Fall & 132 & 308 & 314 & 455 & 558 & 680 & 394 & 609 & 248 & 440 & 229 & 630 & 233 & 505 \\
\hline Year & 146 & 364 & 343 & 484 & 586 & 690 & 456 & 725 & 405 & 634 & 382 & 623 & 345 & 516 \\
\hline
\end{tabular}

Figure 128: 2006 Profile Seasonal 98 Percentile Hourly MW Change for Hours 5 to 11 
The following tables show the results of the down-ramp for hours 21 through hour 3 .

\begin{tabular}{|c|c|c|c|c|c|c|c|c|c|c|c|c|c|c|}
\hline & \multicolumn{14}{|c|}{ NPA Average Hourly MW Change 2018 - 2004 Profile 20\% Wind } \\
\hline & \multicolumn{2}{|c|}{$\operatorname{Hr} 21$} & \multicolumn{2}{|c|}{$\mathrm{Hr} 22$} & \multicolumn{2}{|c|}{$\mathrm{Hr} 23$} & \multicolumn{2}{|c|}{$\mathrm{Hr} 24$} & \multicolumn{2}{|c|}{$\mathrm{Hr} 1$} & \multicolumn{2}{|c|}{$\mathrm{Hr} 2$} & \multicolumn{2}{|c|}{$\mathrm{Hr} 3$} \\
\hline & Load & $\begin{array}{l}\text { Load } \\
\text { Net } \\
\text { Wind }\end{array}$ & Load & $\begin{array}{l}\text { Load } \\
\text { Net } \\
\text { Wind }\end{array}$ & Load & $\begin{array}{l}\text { Load } \\
\text { Net } \\
\text { Wind }\end{array}$ & Load & $\begin{array}{l}\text { Load } \\
\text { Net } \\
\text { Wind }\end{array}$ & Load & $\begin{array}{l}\text { Load } \\
\text { Net } \\
\text { Wind }\end{array}$ & Load & $\begin{array}{l}\text { Load } \\
\text { Net } \\
\text { Wind }\end{array}$ & Load & $\begin{array}{l}\text { Load } \\
\text { Net } \\
\text { Wind }\end{array}$ \\
\hline Jan & -64 & -78 & -139 & -184 & -260 & -286 & -337 & -396 & -204 & -236 & -98 & -121 & -36 & -32 \\
\hline Feb & -70 & -118 & -130 & -162 & -257 & -281 & -308 & -343 & -185 & -195 & -81 & -96 & -30 & -34 \\
\hline Mar & -43 & -25 & -110 & -149 & -285 & -276 & -319 & -313 & -192 & -226 & -91 & -119 & -42 & -66 \\
\hline Apr & 166 & 113 & -204 & -49 & -307 & -354 & -372 & -424 & -225 & -221 & -110 & -115 & -57 & -57 \\
\hline May & -42 & -99 & -157 & 56 & -287 & -292 & -391 & -420 & -278 & -351 & -150 & -188 & -99 & -146 \\
\hline Jun & -128 & -92 & -157 & -40 & -252 & -306 & -455 & -506 & -339 & -322 & -222 & -228 & -129 & -132 \\
\hline Jul & -136 & -170 & -104 & -161 & -267 & -372 & -441 & -533 & -378 & -400 & -281 & -306 & -194 & -151 \\
\hline Aug & -59 & -143 & -119 & -180 & -403 & -533 & -467 & -517 & -382 & -411 & -243 & -268 & -159 & -143 \\
\hline Sep & 17 & -92 & -76 & -297 & -365 & -440 & -423 & -484 & -310 & -305 & -198 & -210 & -122 & -118 \\
\hline Oct & -64 & -79 & -97 & -215 & -283 & -372 & -320 & -394 & -226 & -258 & -109 & -132 & -55 & -58 \\
\hline Nov & -91 & -135 & -192 & -259 & -287 & -355 & -312 & -393 & -201 & -259 & -94 & -126 & -33 & -89 \\
\hline Dec & -56 & -124 & -217 & -182 & -243 & -296 & -326 & -373 & -234 & -290 & -91 & -149 & -39 & -98 \\
\hline
\end{tabular}

Figure 129: 2004 Profile Monthly Average Hourly MW Change for Hours 21 to 3

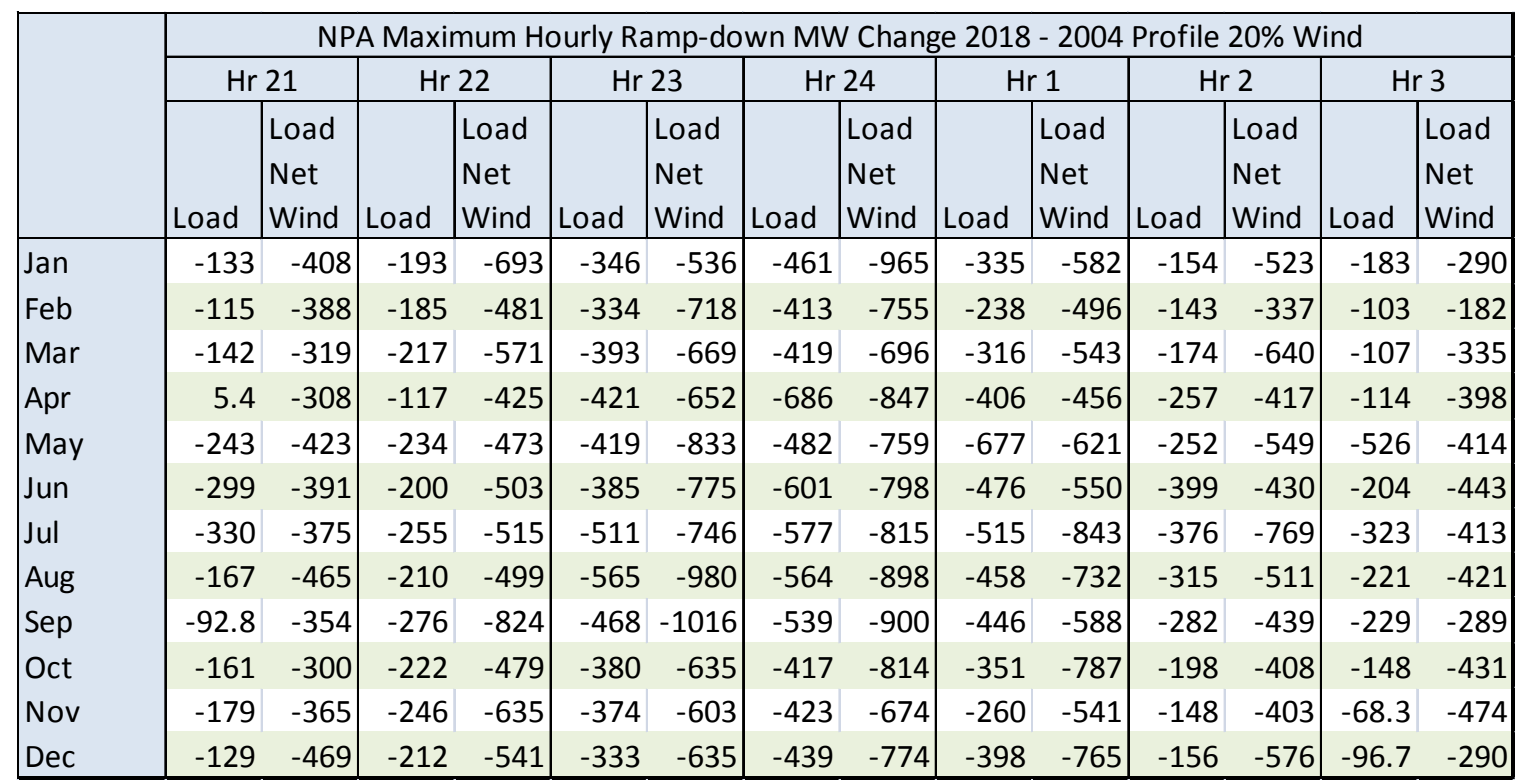

Figure 130: 2004 Profile Monthly Maximum Hourly MW Change for Hours 21 to 3 


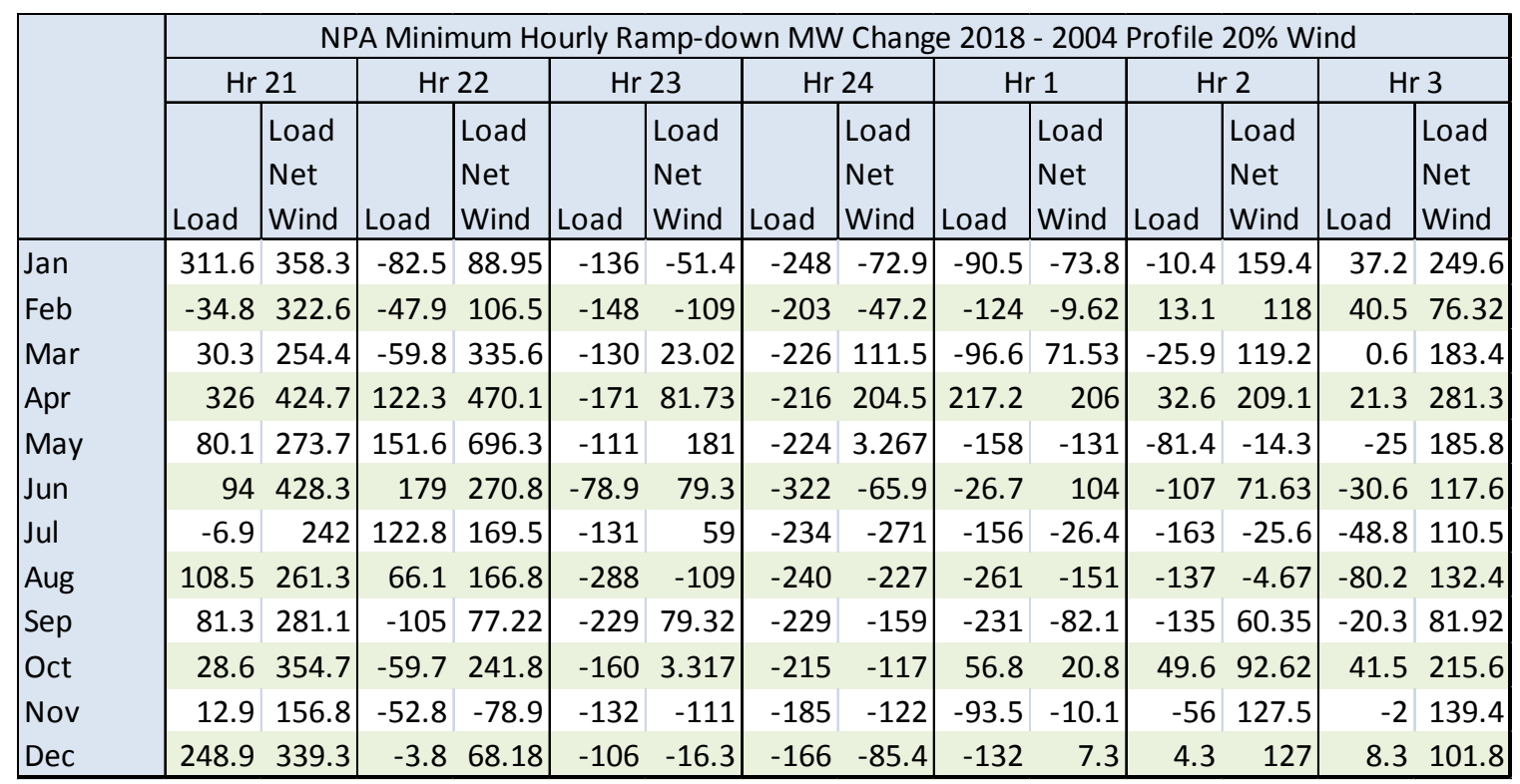

Figure 131: 2004 Profile Monthly Minimum Hourly MW Change for Hours 21 to 3

\begin{tabular}{|c|c|c|c|c|c|c|c|c|c|c|c|c|c|c|}
\hline & \multicolumn{14}{|c|}{ NPA 98 Percentile Ramp-up Hourly MW Change 2018 - 2004 Profile $20 \%$ Wind } \\
\hline & \multicolumn{2}{|c|}{$\operatorname{Hr} 21$} & \multicolumn{2}{|c|}{$\operatorname{Hr} 22$} & \multicolumn{2}{|c|}{$\mathrm{Hr} 23$} & \multicolumn{2}{|c|}{$\mathrm{Hr} 24$} & \multicolumn{2}{|c|}{$\mathrm{Hr} 1$} & \multicolumn{2}{|c|}{$\mathrm{Hr} 2$} & \multicolumn{2}{|c|}{$\mathrm{Hr} 3$} \\
\hline & Load & $\begin{array}{l}\text { Load } \\
\text { Net } \\
\text { Wind }\end{array}$ & Load & $\begin{array}{l}\text { Load } \\
\text { Net } \\
\text { Wind }\end{array}$ & Load & $\begin{array}{l}\text { Load } \\
\text { Net } \\
\text { Wind }\end{array}$ & Load & $\begin{array}{l}\text { Load } \\
\text { Net } \\
\text { Wind }\end{array}$ & Load & $\begin{array}{l}\text { Load } \\
\text { Net } \\
\text { Wind }\end{array}$ & Load & $\begin{array}{l}\text { Load } \\
\text { Net } \\
\text { Wind }\end{array}$ & Load & $\begin{array}{l}\text { Load } \\
\text { Net } \\
\text { Wind }\end{array}$ \\
\hline Jan & 129 & 214 & -83 & 51 & -142 & -57 & -249 & -80 & -54 & -44 & -21 & 157 & 25 & 237 \\
\hline Feb & -36 & 213 & -56 & 58 & -155 & -119 & -205 & -107 & -131 & -31 & -2 & 110 & 24 & 75 \\
\hline Mar & 29 & 248 & -70 & 269 & -166 & 14 & -234 & 34 & -115 & 50 & -33 & 118 & -2 & 119 \\
\hline Apr & 296 & 395 & 120 & 396 & -180 & 48 & -235 & 41 & 14 & 120 & -13 & 133 & 11 & 249 \\
\hline May & 71 & 205 & 142 & 579 & -134 & 167 & -256 & -122 & -161 & -154 & -93 & -15 & -29 & 90 \\
\hline Jun & 60 & 336 & 137 & 258 & -120 & 62 & -322 & -133 & -108 & -20 & -132 & 14 & -53 & 59 \\
\hline Jul & -17 & 143 & 113 & 121 & -147 & 8 & -247 & -278 & -241 & -103 & -188 & -33 & -78 & 88 \\
\hline Aug & 91 & 194 & 53 & 157 & -292 & -185 & -285 & -241 & -270 & -157 & -141 & -31 & -82 & 90 \\
\hline Sep & 77 & 243 & -132 & 45 & -234 & 4 & -253 & -203 & -245 & -101 & -144 & 12 & -22 & 78 \\
\hline Oct & 23 & 349 & -79 & 211 & -168 & -79 & -226 & -132 & -60 & -8 & 12 & 51 & 24 & 180 \\
\hline Nov & -6 & 106 & -72 & -85 & -141 & -164 & -196 & -158 & -117 & -47 & -58 & 118 & -2 & 128 \\
\hline Dec & 106 & 189 & -12 & 63 & -110 & -47 & -201 & -138 & -134 & -38 & -7 & 82 & 6 & 76 \\
\hline
\end{tabular}

Figure 132: 2004 Profile Monthly 98 Percentile Hourly MW Change for Hours 21 to 3 


\begin{tabular}{|c|c|c|c|c|c|c|c|c|c|c|c|c|c|c|}
\hline & \multicolumn{14}{|c|}{ NPA Average Hourly MW Change 2018 - 2005 Profile 20\% Wind } \\
\hline & \multicolumn{2}{|c|}{$\operatorname{Hr} 21$} & \multicolumn{2}{|c|}{$\mathrm{Hr} 22$} & \multicolumn{2}{|c|}{$\mathrm{Hr} 23$} & \multicolumn{2}{|c|}{$\mathrm{Hr} 24$} & \multicolumn{2}{|c|}{$\mathrm{Hr} 1$} & \multicolumn{2}{|c|}{$\mathrm{Hr} 2$} & \multicolumn{2}{|c|}{$\mathrm{Hr} 3$} \\
\hline & Load & $\begin{array}{l}\text { Load } \\
\text { Net } \\
\text { Wind } \\
\end{array}$ & Load & $\begin{array}{l}\text { Load } \\
\text { Net } \\
\text { Wind }\end{array}$ & Load & $\begin{array}{l}\text { Load } \\
\text { Net } \\
\text { Wind }\end{array}$ & Load & $\begin{array}{l}\text { Load } \\
\text { Net } \\
\text { Wind }\end{array}$ & Load & $\begin{array}{l}\text { Load } \\
\text { Net } \\
\text { Wind }\end{array}$ & Load & \begin{tabular}{|l|} 
Load \\
Net \\
Wind \\
\end{tabular} & Load & $\begin{array}{l}\text { Load } \\
\text { Net } \\
\text { Wind }\end{array}$ \\
\hline Jan & -98 & -113 & -164 & -190 & -268 & -263 & -296 & -267 & -164 & -151 & -77 & -68 & -27 & -24 \\
\hline Feb & -62 & -96 & -136 & -176 & -279 & -271 & -337 & -352 & -157 & -150 & -97 & -104 & -47 & -78 \\
\hline Mar & -66 & -111 & -178 & -217 & -299 & -270 & -286 & -239 & -182 & -146 & -71 & -68 & -17 & -20 \\
\hline Apr & 167 & 150 & -37 & -32 & -305 & -294 & -371 & -420 & -231 & -275 & -112 & -120 & -47 & -55 \\
\hline May & -24 & 15 & 51 & 88 & -262 & -263 & -385 & -370 & -293 & -296 & -156 & -178 & -99 & -110 \\
\hline Jun & -169 & -147 & -52 & -95 & -240 & -299 & -446 & -501 & -376 & -424 & -247 & -212 & -152 & -78 \\
\hline Jul & -156 & -241 & -64 & -222 & -237 & -398 & -388 & -501 & -383 & -432 & -253 & -274 & -178 & -171 \\
\hline Aug & -87 & -171 & -99 & -234 & -363 & -487 & -456 & -559 & -367 & -429 & -257 & -244 & -168 & -139 \\
\hline Sep & 1 & -129 & -202 & -343 & -356 & -448 & -425 & -499 & -327 & -384 & -199 & -214 & -131 & -89 \\
\hline Oct & -41 & -122 & -135 & -205 & -235 & -298 & -314 & -398 & -208 & -225 & -117 & -106 & -64 & -35 \\
\hline Nov & -88 & -199 & -152 & -288 & -277 & -315 & -334 & -435 & -223 & -312 & -137 & -199 & -33 & -58 \\
\hline Dec & -68 & -96 & -126 & -183 & -248 & -263 & -321 & -318 & -213 & -208 & -131 & -141 & -26 & -46 \\
\hline
\end{tabular}

Figure 133: 2005 Profile Monthly Average Hourly MW Change for Hours 21 to 3

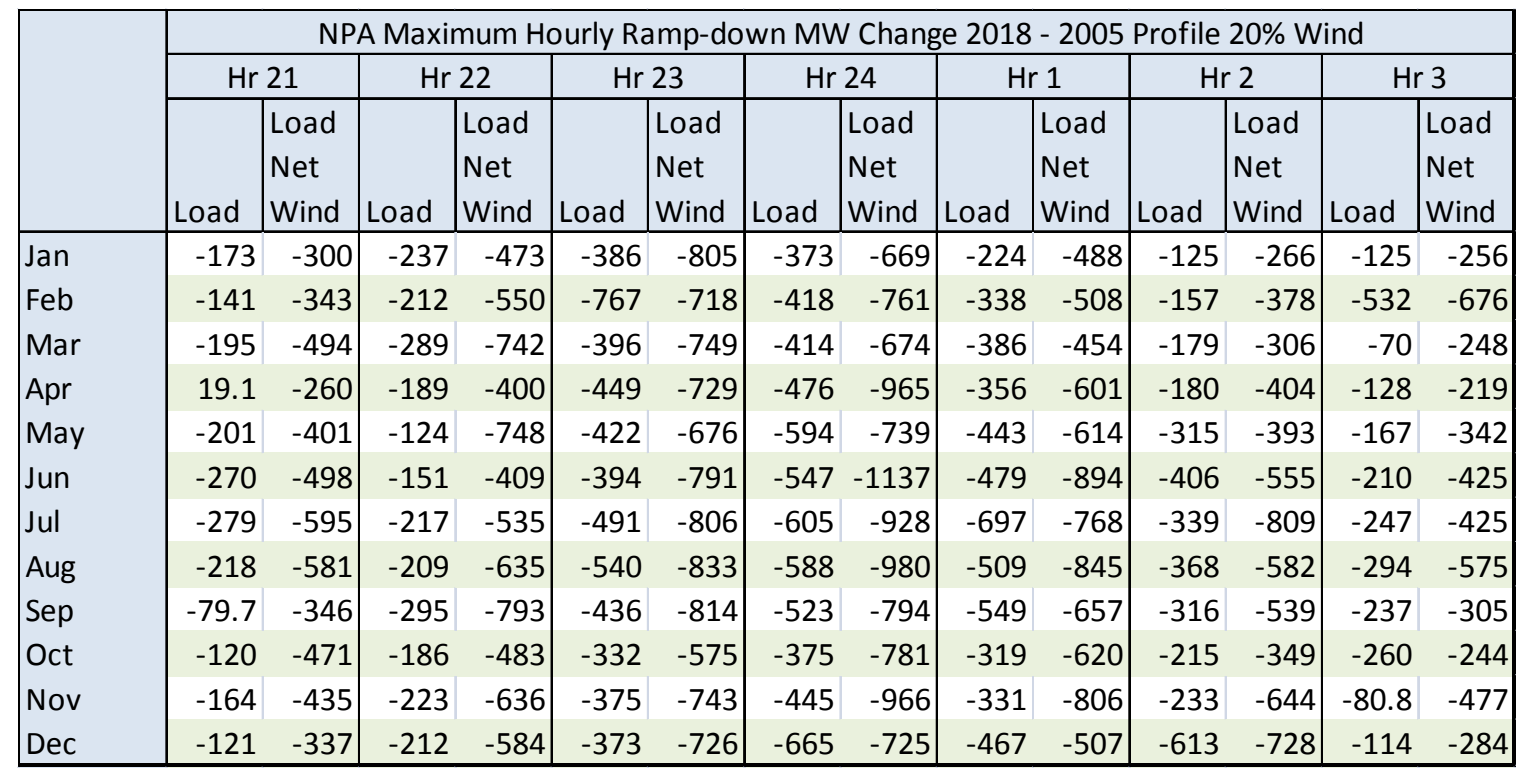

Figure 134: 2005 Profile Monthly Maximum Hourly MW Change for Hours 21 to 3 


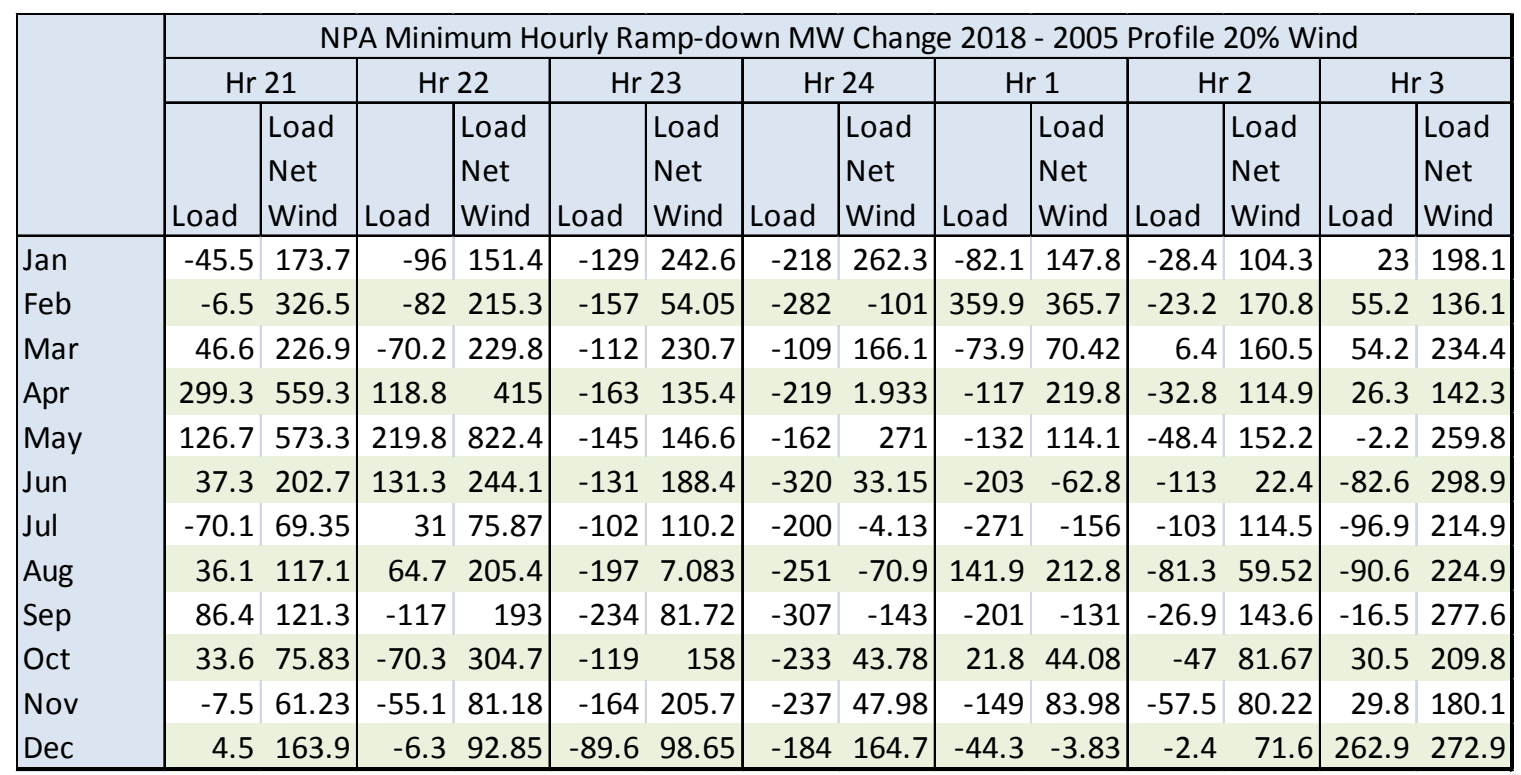

Figure 135: 2005 Profile Monthly Minimum Hourly MW Change for Hours 21 to 3

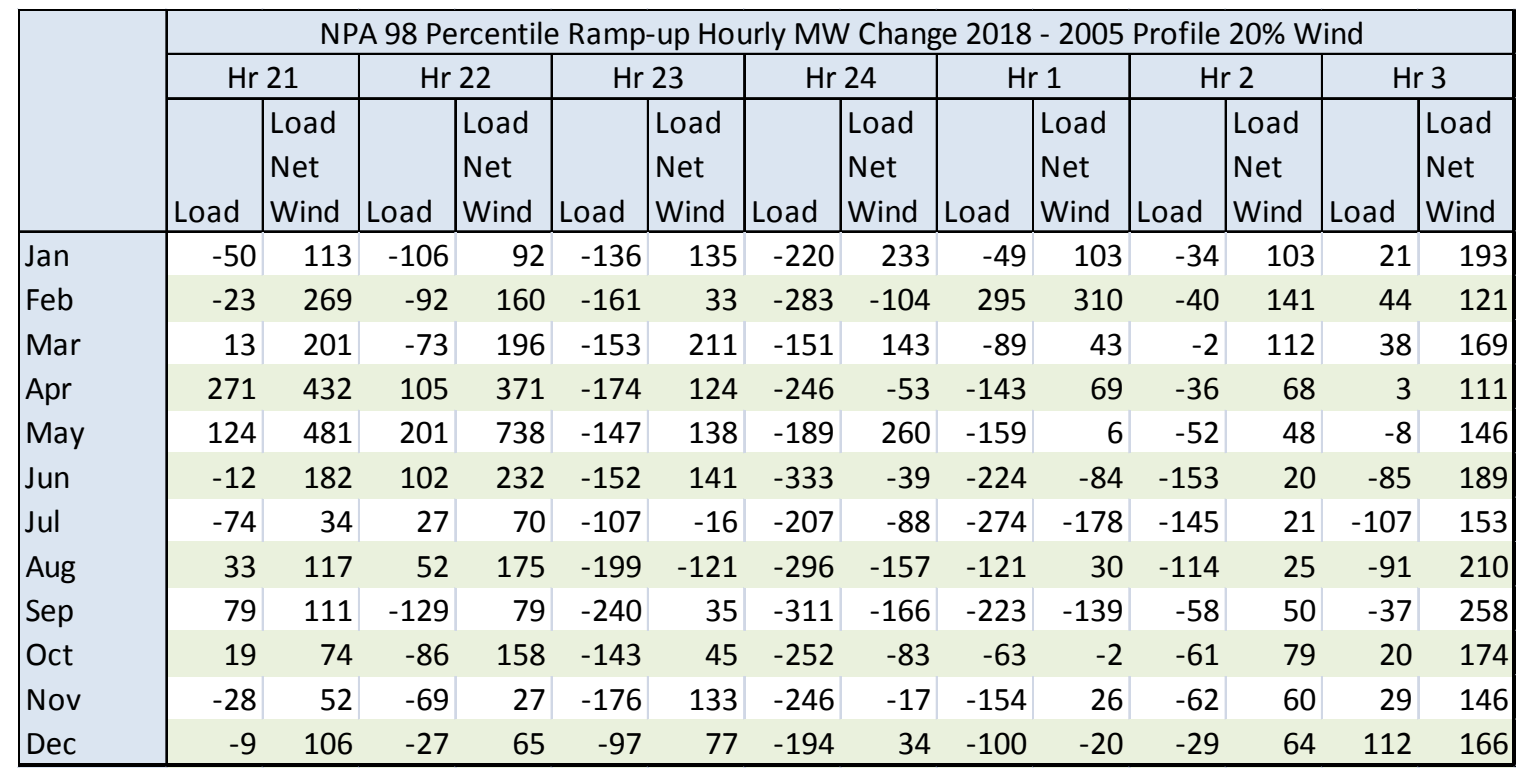

Figure 136: 2005 Profile Monthly 98 Percentile Hourly MW Change for Hours 21 to 3 


\begin{tabular}{|c|c|c|c|c|c|c|c|c|c|c|c|c|c|c|}
\hline & \multicolumn{14}{|c|}{ NPA Average Hourly MW Change 2018 - 2006 Profile 20\% Wind } \\
\hline & \multicolumn{2}{|c|}{$\operatorname{Hr} 21$} & \multicolumn{2}{|c|}{$\operatorname{Hr} 22$} & \multicolumn{2}{|c|}{$\mathrm{Hr} 23$} & \multicolumn{2}{|c|}{ Hr 24} & \multicolumn{2}{|c|}{$\mathrm{Hr} 1$} & \multicolumn{2}{|c|}{$\mathrm{Hr} 2$} & \multicolumn{2}{|c|}{$\mathrm{Hr} 3$} \\
\hline & Load & $\begin{array}{l}\text { Load } \\
\text { Net } \\
\text { Wind }\end{array}$ & Load & $\begin{array}{l}\text { Load } \\
\text { Net } \\
\text { Wind }\end{array}$ & Load & $\begin{array}{l}\text { Load } \\
\text { Net } \\
\text { Wind }\end{array}$ & Load & $\begin{array}{l}\text { Load } \\
\text { Net } \\
\text { Wind }\end{array}$ & Load & $\begin{array}{l}\text { Load } \\
\text { Net } \\
\text { Wind }\end{array}$ & Load & $\begin{array}{l}\text { Load } \\
\text { Net } \\
\text { Wind }\end{array}$ & Load & $\begin{array}{l}\text { Load } \\
\text { Net } \\
\text { Wind }\end{array}$ \\
\hline Jan & -105 & -154 & -179 & -279 & -284 & -327 & -294 & -310 & -155 & -184 & -82 & -130 & -24 & -51 \\
\hline Feb & -69 & -59 & -120 & -180 & -250 & -344 & -309 & -394 & -197 & -269 & -86 & -141 & -22 & -56 \\
\hline Mar & -65 & -76 & -157 & -197 & -255 & -298 & -287 & -317 & -173 & -222 & -79 & -99 & -27 & -50 \\
\hline Apr & 127 & 97 & -36 & -78 & -257 & -311 & -334 & -352 & -200 & -195 & -106 & -72 & -47 & -56 \\
\hline May & -35 & -31 & 15 & 60 & -278 & -208 & -365 & -303 & -276 & -266 & -148 & -138 & -84 & -99 \\
\hline Jun & -206 & -319 & -66 & -192 & -273 & -391 & -439 & -449 & -347 & -319 & -216 & -151 & -137 & -65 \\
\hline Jul & -164 & -318 & -65 & -248 & -228 & -314 & -398 & -424 & -360 & -397 & -261 & -203 & -187 & -180 \\
\hline Aug & -78 & -240 & -93 & -271 & -365 & -442 & -477 & -494 & -367 & -353 & -274 & -192 & -185 & -108 \\
\hline Sep & 69 & 0 & -191 & -280 & -353 & -441 & -384 & -406 & -246 & -255 & -159 & -147 & -72 & -70 \\
\hline Oct & -43 & -79 & -133 & -219 & -243 & -307 & -300 & -364 & -233 & -335 & -104 & -160 & -52 & -87 \\
\hline Nov & -84 & -152 & -148 & -199 & -258 & -312 & -278 & -311 & -185 & -237 & -90 & -117 & -38 & -48 \\
\hline Dec & -72 & -168 & -138 & -161 & -273 & -281 & -335 & -400 & -228 & -296 & -120 & -117 & -47 & -33 \\
\hline
\end{tabular}

Figure 137: 2006 Profile Monthly Average Hourly MW Change for Hours 21 to 3

\begin{tabular}{|c|c|c|c|c|c|c|c|c|c|c|c|c|c|c|}
\hline & \multicolumn{14}{|c|}{ NPA Maximum Hourly Ramp-down MW Change 2018 - 2006 Profile 20\% Wind } \\
\hline & \multicolumn{2}{|c|}{$\operatorname{Hr} 21$} & \multicolumn{2}{|c|}{$\mathrm{Hr} 22$} & \multicolumn{2}{|c|}{$\mathrm{Hr} 23$} & \multicolumn{2}{|c|}{$\mathrm{Hr} 24$} & \multicolumn{2}{|c|}{$\mathrm{Hr} 1$} & \multicolumn{2}{|c|}{$\mathrm{Hr} 2$} & \multicolumn{2}{|c|}{$\mathrm{Hr} 3$} \\
\hline & Load & $\begin{array}{l}\text { Load } \\
\text { Net } \\
\text { Wind }\end{array}$ & Load & $\begin{array}{l}\text { Load } \\
\text { Net } \\
\text { Wind }\end{array}$ & Load & $\begin{array}{l}\text { Load } \\
\text { Net } \\
\text { Wind }\end{array}$ & Load & $\begin{array}{l}\text { Load } \\
\text { Net } \\
\text { Wind }\end{array}$ & Load & $\begin{array}{l}\text { Load } \\
\text { Net } \\
\text { Wind }\end{array}$ & Load & $\begin{array}{l}\text { Load } \\
\text { Net } \\
\text { Wind }\end{array}$ & Load & $\begin{array}{l}\text { Load } \\
\text { Net } \\
\text { Wind }\end{array}$ \\
\hline Jan & -161 & -551 & -250 & -674 & -420 & -927 & -391 & -705 & -203 & -481 & -184 & -455 & -116 & -281 \\
\hline Feb & -460 & -451 & -214 & -599 & -354 & -758 & -471 & -900 & -400 & -652 & -186 & -393 & -115 & -291 \\
\hline Mar & -134 & -396 & -231 & -590 & -333 & -688 & -356 & -639 & -237 & -640 & -138 & -807 & -119 & -462 \\
\hline Apr & -20.7 & -604 & -161 & -671 & -350 & -612 & -422 & -622 & -306 & -516 & -186 & -429 & -125 & -241 \\
\hline May & -220 & -507 & -91.1 & -429 & -518 & -757 & -540 & -606 & -667 & -808 & -242 & -394 & -219 & -342 \\
\hline Jun & -302 & -706 & -212 & -878 & -376 & -843 & -564 & -913 & -488 & -544 & -287 & -406 & -220 & -312 \\
\hline Jul & -258 & -633 & -148 & -609 & -340 & -746 & -542 & -871 & -430 & -799 & -321 & -547 & -288 & -436 \\
\hline Aug & -224 & -582 & -197 & -757 & -502 & -807 & -620 & -902 & -481 & -779 & -383 & -437 & -284 & -370 \\
\hline Sep & -39.1 & -465 & -309 & -698 & -561 & -1008 & -508 & -914 & -397 & -777 & -308 & -464 & -177 & -304 \\
\hline Oct & -89.1 & -622 & -213 & -546 & -330 & -745 & -431 & -948 & -718 & -707 & -242 & -433 & -186 & -561 \\
\hline Nov & -147 & -488 & -232 & -555 & -337 & -777 & -383 & -611 & -303 & -467 & -181 & -430 & -87.2 & -404 \\
\hline Dec & -160 & -502 & -248 & -511 & -384 & -680 & -481 & -837 & -522 & -650 & -184 & -422 & -158 & -319 \\
\hline
\end{tabular}

Figure 138: 2006 Profile Monthly Maximum Hourly MW Change for Hours 21 to 3 


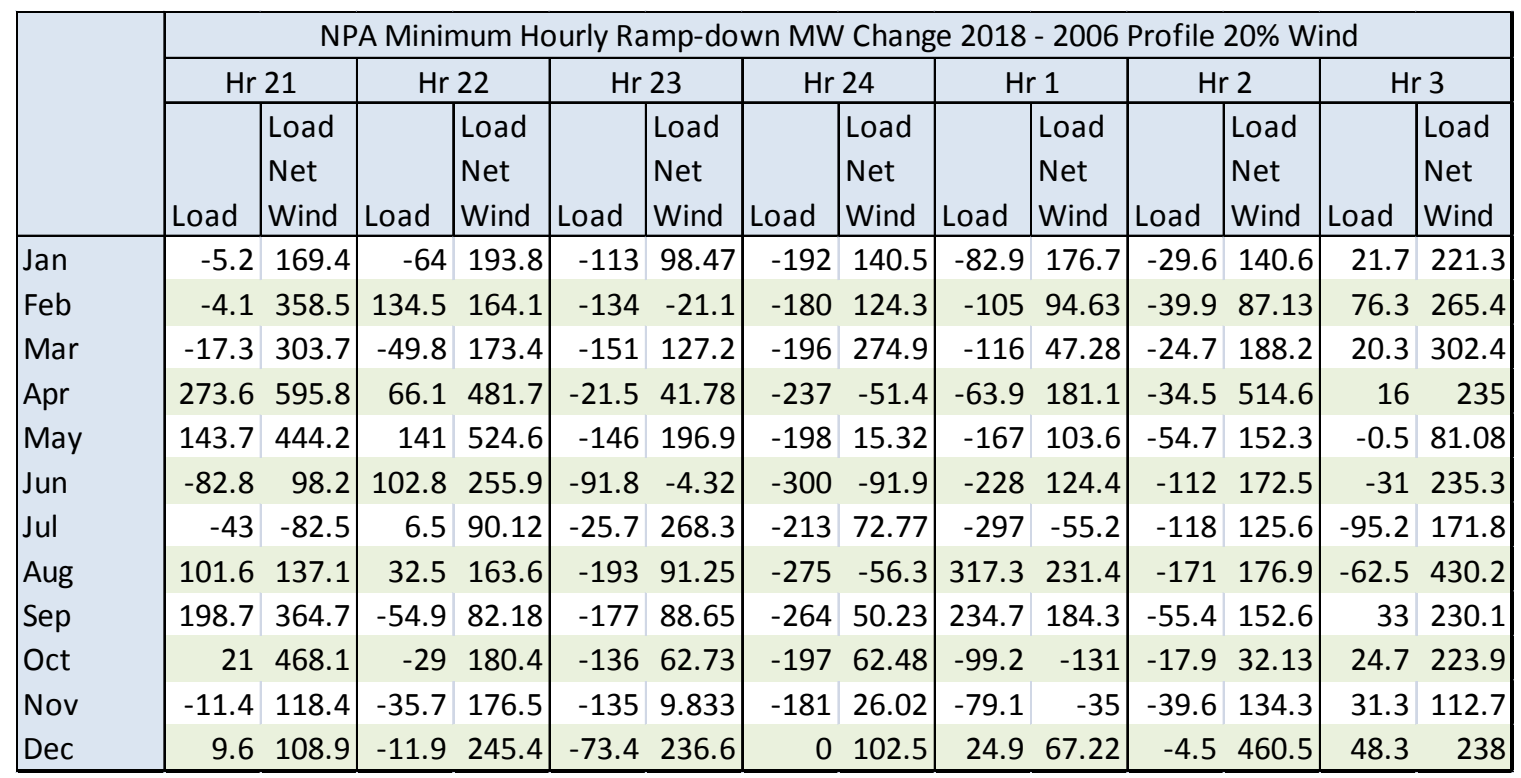

Figure 139: 2006 Profile Monthly Minimum Hourly MW Change for Hours 21 to 3

\begin{tabular}{|c|c|c|c|c|c|c|c|c|c|c|c|c|c|c|}
\hline & \multicolumn{14}{|c|}{ NPA 98 Percentile Ramp-up Hourly MW Change 2018 - 2006 Profile 20\% Wind } \\
\hline & \multicolumn{2}{|c|}{$\operatorname{Hr} 21$} & \multicolumn{2}{|c|}{$\mathrm{Hr} 22$} & \multicolumn{2}{|c|}{$\mathrm{Hr} 23$} & \multicolumn{2}{|c|}{$\mathrm{Hr} 24$} & \multicolumn{2}{|c|}{ Hr 1} & \multicolumn{2}{|c|}{$\mathrm{Hr} 2$} & \multicolumn{2}{|c|}{$\mathrm{Hr} 3$} \\
\hline & Load & \begin{tabular}{|l} 
Load \\
Net \\
Wind
\end{tabular} & Load & $\begin{array}{l}\text { Load } \\
\text { Net } \\
\text { Wind }\end{array}$ & Load & $\begin{array}{l}\text { Load } \\
\text { Net } \\
\text { Wind }\end{array}$ & Load & $\begin{array}{l}\text { Load } \\
\text { Net } \\
\text { Wind }\end{array}$ & Load & $\begin{array}{l}\text { Load } \\
\text { Net } \\
\text { Wind }\end{array}$ & Load & $\begin{array}{l}\text { Load } \\
\text { Net } \\
\text { Wind }\end{array}$ & Load & $\begin{array}{l}\text { Load } \\
\text { Net } \\
\text { Wind }\end{array}$ \\
\hline Jan & -18 & 132 & -79 & 105 & -142 & 88 & -200 & 53 & -50 & 150 & -31 & 107 & 17 & 164 \\
\hline Feb & -7 & 319 & 36 & 120 & -151 & -41 & -190 & -17 & -108 & 23 & -41 & 82 & 55 & 201 \\
\hline Mar & -18 & 278 & -72 & 116 & -170 & 104 & -203 & 88 & -119 & 25 & -30 & 183 & 20 & 222 \\
\hline Apr & 246 & 489 & 44 & 392 & -90 & -11 & -243 & -62 & -94 & 177 & -50 & 403 & 7 & 155 \\
\hline May & 123 & 377 & 113 & 523 & -148 & 120 & -221 & 9 & -176 & 80 & -71 & 126 & -18 & 81 \\
\hline Jun & -94 & 67 & 55 & 224 & -127 & -40 & -310 & -111 & -235 & 27 & -132 & 124 & -54 & 210 \\
\hline Jul & -67 & -122 & 2 & 87 & -62 & 73 & -229 & -1 & -302 & -83 & -163 & 56 & -117 & 73 \\
\hline Aug & 80 & 66 & 19 & 157 & -214 & 34 & -313 & -87 & -25 & 152 & -172 & 155 & -81 & 220 \\
\hline Sep & 193 & 314 & -56 & 27 & -182 & 64 & -271 & -8 & 2 & 121 & -63 & 152 & 6 & 213 \\
\hline Oct & 6 & 402 & -58 & 177 & -154 & 38 & -201 & -45 & -120 & -131 & -31 & 29 & 18 & 174 \\
\hline Nov & -16 & 99 & -63 & 93 & -148 & -24 & -191 & 21 & -92 & -36 & -42 & 121 & 22 & 101 \\
\hline Dec & 7 & 108 & -36 & 195 & -84 & 227 & -108 & 47 & 0 & 21 & -30 & 255 & 43 & 233 \\
\hline
\end{tabular}

Figure 140: 2006 Profile Monthly 98 Percentile Hourly MW Change for Hours 21 to 3 


\begin{tabular}{|c|c|c|c|c|c|c|c|c|c|c|c|c|c|c|}
\hline & \multicolumn{14}{|c|}{ NPA Maximum Hourly Ramp-down MW Change 2018 - 2004 Profile $20 \%$ Wind } \\
\hline & \multicolumn{2}{|c|}{$\operatorname{Hr} 21$} & \multicolumn{2}{|c|}{$\mathrm{Hr} 22$} & \multicolumn{2}{|c|}{$\mathrm{Hr} 23$} & \multicolumn{2}{|c|}{$\mathrm{Hr} 24$} & \multicolumn{2}{|c|}{$\mathrm{Hr} 1$} & \multicolumn{2}{|c|}{$\mathrm{Hr} 2$} & \multicolumn{2}{|c|}{$\mathrm{Hr} 3$} \\
\hline & Load & $\begin{array}{l}\text { Load } \\
\text { Net } \\
\text { Wind }\end{array}$ & Load & $\begin{array}{l}\text { Load } \\
\text { Net } \\
\text { Wind }\end{array}$ & Load & $\begin{array}{l}\text { Load } \\
\text { Net } \\
\text { Wind }\end{array}$ & Load & $\begin{array}{l}\text { Load } \\
\text { Net } \\
\text { Wind } \\
\end{array}$ & Load & $\begin{array}{l}\text { Load } \\
\text { Net } \\
\text { Wind }\end{array}$ & Load & $\begin{array}{l}\text { Load } \\
\text { Net } \\
\text { Wind }\end{array}$ & Load & $\begin{array}{l}\text { Load } \\
\text { Net } \\
\text { Wind }\end{array}$ \\
\hline Winter & -133 & -469 & -212 & -693 & -346 & -718 & -461 & -965 & -398 & -765 & -156 & -576 & -183 & -290 \\
\hline Spring & -243 & -423 & -234 & -571 & -421 & -833 & -686 & -847 & -677 & -621 & -257 & -640 & -526 & -414 \\
\hline Summer & -330 & -465 & -255 & -515 & -565 & -980 & -601 & -898 & -515 & -843 & -399 & -769 & -323 & -443 \\
\hline Fall & -179 & -365 & -276 & -824 & -468 & -1016 & -539 & -900 & -446 & -787 & -282 & -439 & -229 & -474 \\
\hline Year & -330 & -469 & -276 & -824 & -565 & -1016 & -686 & -965 & -677 & -843 & -399 & -769 & -526 & -474 \\
\hline
\end{tabular}

Figure 141: 2004 Profile Season Maximum Hourly MW Change for Hours 21 to 3

\begin{tabular}{|c|c|c|c|c|c|c|c|c|c|c|c|c|c|c|}
\hline & \multicolumn{14}{|c|}{ NPA Minimum Hourly Ramp-down MW Change 2018 - 2004 Profile 20\% Wind } \\
\hline & \multicolumn{2}{|c|}{ Hr 21} & \multicolumn{2}{|c|}{$\mathrm{Hr} 22$} & \multicolumn{2}{|c|}{$\mathrm{Hr} 23$} & \multicolumn{2}{|c|}{$\mathrm{Hr} 24$} & \multicolumn{2}{|c|}{$\mathrm{Hr} 1$} & \multicolumn{2}{|c|}{$\mathrm{Hr} 2$} & \multicolumn{2}{|c|}{$\mathrm{Hr} 3$} \\
\hline & Load & $\begin{array}{l}\text { Load } \\
\text { Net } \\
\text { Wind }\end{array}$ & Load & $\begin{array}{l}\text { Load } \\
\text { Net } \\
\text { Wind }\end{array}$ & Load & $\begin{array}{l}\text { Load } \\
\text { Net } \\
\text { Wind }\end{array}$ & Load & $\begin{array}{l}\text { Load } \\
\text { Net } \\
\text { Wind }\end{array}$ & Load & $\begin{array}{l}\text { Load } \\
\text { Net } \\
\text { Wind }\end{array}$ & Load & $\begin{array}{l}\text { Load } \\
\text { Net } \\
\text { Wind }\end{array}$ & Load & $\begin{array}{l}\text { Load } \\
\text { Net } \\
\text { Wind }\end{array}$ \\
\hline Winter & 312 & 358 & -4 & 107 & -106 & -16 & -166 & -47 & -91 & 7 & 13 & 159 & 41 & 250 \\
\hline Spring & 326 & 425 & 152 & 696 & -111 & 181 & -216 & 204 & 217 & 206 & 33 & 209 & 21 & 281 \\
\hline Summer & 109 & 428 & 179 & 271 & -79 & 79 & -234 & -66 & -27 & 104 & -107 & 72 & -31 & 132 \\
\hline Fall & 81 & 355 & -53 & 242 & -132 & 79 & -185 & -117 & 57 & 21 & 50 & 128 & 42 & 216 \\
\hline Year & 326 & 428 & 179 & 696 & -79 & 181 & -166 & 204 & 217 & 206 & 50 & 209 & 42 & 281 \\
\hline
\end{tabular}

Figure 142: 2004 Profile Season Minimum Hourly MW Change for Hours 21 to 3

\begin{tabular}{|c|c|c|c|c|c|c|c|c|c|c|c|c|c|c|}
\hline & \multicolumn{14}{|c|}{ NPA 98 Percentile Ramp-down Hourly MW Change 2018 - 2004 Profile 20\% Wind } \\
\hline & \multicolumn{2}{|c|}{$\operatorname{Hr} 21$} & \multicolumn{2}{|c|}{$\mathrm{Hr} 22$} & \multicolumn{2}{|c|}{$\mathrm{Hr} 23$} & \multicolumn{2}{|c|}{$\mathrm{Hr} 24$} & \multicolumn{2}{|c|}{$\mathrm{Hr} 1$} & \multicolumn{2}{|c|}{$\mathrm{Hr} 2$} & \multicolumn{2}{|c|}{$\mathrm{Hr} 3$} \\
\hline & Load & $\begin{array}{l}\text { Load } \\
\text { Net } \\
\text { Wind }\end{array}$ & Load & $\begin{array}{l}\text { Load } \\
\text { Net } \\
\text { Wind }\end{array}$ & Load & $\begin{array}{l}\text { Load } \\
\text { Net } \\
\text { Wind }\end{array}$ & Load & $\begin{array}{l}\text { Load } \\
\text { Net } \\
\text { Wind } \\
\end{array}$ & Load & $\begin{array}{l}\text { Load } \\
\text { Net } \\
\text { Wind } \\
\end{array}$ & Load & $\begin{array}{l}\text { Load } \\
\text { Net } \\
\text { Wind }\end{array}$ & Load & $\begin{array}{l}\text { Load } \\
\text { Net } \\
\text { Wind }\end{array}$ \\
\hline Winter & -120 & -392 & -189 & -503 & -333 & -620 & -432 & -759 & -315 & -527 & -152 & -401 & -129 & -280 \\
\hline Spring & -171 & -398 & -197 & -473 & -419 & -659 & -476 & -786 & -406 & -605 & -228 & -435 & -144 & -398 \\
\hline Summer & -268 & -390 & -209 & -500 & -512 & -838 & -578 & -801 & -482 & -719 & -366 & -599 & -295 & -414 \\
\hline Fall & -145 & -323 & -248 & -638 & -434 & -705 & -537 & -825 & -385 & -574 & -247 & -405 & -193 & -389 \\
\hline Year & -213 & -389 & -240 & -513 & -481 & -751 & -564 & -798 & -454 & -617 & -328 & -507 & -234 & -396 \\
\hline
\end{tabular}

Figure 143: 2004 Profile Season 98 Percentile Hourly MW Change for Hours 21 to 3 


\begin{tabular}{|c|c|c|c|c|c|c|c|c|c|c|c|c|c|c|}
\hline & \multicolumn{14}{|c|}{ NPA Maximum Hourly Ramp-down MW Change 2018 - 2005 Profile 20\% Wind } \\
\hline & \multicolumn{2}{|c|}{$\operatorname{Hr} 21$} & \multicolumn{2}{|c|}{$\mathrm{Hr} 22$} & \multicolumn{2}{|c|}{$\mathrm{Hr} 23$} & \multicolumn{2}{|c|}{$\mathrm{Hr} 24$} & \multicolumn{2}{|c|}{$\mathrm{Hr} 1$} & \multicolumn{2}{|c|}{$\mathrm{Hr} 2$} & \multicolumn{2}{|c|}{$\mathrm{Hr} 3$} \\
\hline & Load & $\begin{array}{l}\text { Load } \\
\text { Net } \\
\text { Wind }\end{array}$ & Load & $\begin{array}{l}\text { Load } \\
\text { Net } \\
\text { Wind }\end{array}$ & Load & $\begin{array}{l}\text { Load } \\
\text { Net } \\
\text { Wind }\end{array}$ & Load & $\begin{array}{l}\text { Load } \\
\text { Net } \\
\text { Wind }\end{array}$ & Load & $\begin{array}{l}\text { Load } \\
\text { Net } \\
\text { Wind }\end{array}$ & Load & $\begin{array}{l}\text { Load } \\
\text { Net } \\
\text { Wind }\end{array}$ & Load & $\begin{array}{l}\text { Load } \\
\text { Net } \\
\text { Wind }\end{array}$ \\
\hline Winter & -173 & -343 & -237 & -584 & -767 & -805 & -665 & -761 & -467 & -508 & -613 & -728 & -532 & -676 \\
\hline Spring & -201 & -494 & -289 & -748 & -449 & -749 & -594 & -965 & -443 & -614 & -315 & -404 & -167 & -342 \\
\hline Summer & -279 & -595 & -217 & -635 & -540 & -833 & -605 & -1137 & -697 & -894 & -406 & -809 & -294 & -575 \\
\hline Fall & -164 & -471 & -295 & -793 & -436 & -814 & -523 & -966 & -549 & -806 & -316 & -644 & -260 & -477 \\
\hline Year & -279 & -595 & -295 & -793 & -767 & -833 & -665 & -1137 & -697 & -894 & -613 & -809 & -532 & -676 \\
\hline
\end{tabular}

Figure 144: 2005 Profile Season Maximum Hourly MW Change for Hours 21 to 3

\begin{tabular}{|c|c|c|c|c|c|c|c|c|c|c|c|c|c|c|}
\hline & \multicolumn{14}{|c|}{ NPA Minimum Hourly Ramp-down MW Change 2018 - 2005 Profile $20 \%$ Wind } \\
\hline & \multicolumn{2}{|c|}{$\operatorname{Hr} 21$} & \multicolumn{2}{|c|}{$\operatorname{Hr} 22$} & \multicolumn{2}{|c|}{$\mathrm{Hr} 23$} & \multicolumn{2}{|c|}{$\mathrm{Hr} 24$} & \multicolumn{2}{|c|}{$\mathrm{Hr} 1$} & \multicolumn{2}{|c|}{$\mathrm{Hr} 2$} & \multicolumn{2}{|c|}{$\mathrm{Hr} 3$} \\
\hline & Load & $\begin{array}{l}\text { Load } \\
\text { Net } \\
\text { Wind } \\
\end{array}$ & Load & $\begin{array}{l}\text { Load } \\
\text { Net } \\
\text { Wind }\end{array}$ & Load & $\begin{array}{l}\text { Load } \\
\text { Net } \\
\text { Wind }\end{array}$ & Load & $\begin{array}{l}\text { Load } \\
\text { Net } \\
\text { Wind }\end{array}$ & Load & \begin{tabular}{|l|} 
Load \\
Net \\
Wind \\
\end{tabular} & Load & $\begin{array}{l}\text { Load } \\
\text { Net } \\
\text { Wind }\end{array}$ & Load & $\begin{array}{l}\text { Load } \\
\text { Net } \\
\text { Wind }\end{array}$ \\
\hline Winter & 5 & 326 & -6 & 215 & -90 & 243 & -184 & 262 & 360 & 366 & -2 & 171 & 263 & 273 \\
\hline Spring & 299 & 573 & 220 & 822 & -112 & 231 & -109 & 271 & -74 & 220 & 6 & 161 & 54 & 260 \\
\hline Summer & 37 & 203 & 131 & 244 & -102 & 188 & -200 & 33 & 142 & 213 & -81 & 115 & -83 & 299 \\
\hline Fall & 86 & 121 & -55 & 305 & -119 & 206 & -233 & 48 & 22 & 84 & -27 & 144 & 31 & 278 \\
\hline Year & 299 & 573 & 220 & 822 & -90 & 243 & -109 & 271 & 360 & 366 & 6 & 171 & 263 & 299 \\
\hline
\end{tabular}

Figure 145: 2005 Profile Season Minimum Hourly MW Change for Hours 21 to 3

\begin{tabular}{|c|c|c|c|c|c|c|c|c|c|c|c|c|c|c|}
\hline & \multicolumn{14}{|c|}{ NPA 98 Percentile Ramp-down Hourly MW Change 2018 - 2005 Profile 20\% Wind } \\
\hline & \multicolumn{2}{|c|}{$\operatorname{Hr} 21$} & \multicolumn{2}{|c|}{$\mathrm{Hr} 22$} & \multicolumn{2}{|c|}{$\mathrm{Hr} 23$} & \multicolumn{2}{|c|}{$\mathrm{Hr} 24$} & \multicolumn{2}{|c|}{$\mathrm{Hr} 1$} & \multicolumn{2}{|c|}{$\mathrm{Hr} 2$} & \multicolumn{2}{|c|}{$\mathrm{Hr} 3$} \\
\hline & Load & $\begin{array}{l}\text { Load } \\
\text { Net } \\
\text { Wind }\end{array}$ & Load & $\begin{array}{l}\text { Load } \\
\text { Net } \\
\text { Wind }\end{array}$ & Load & \begin{tabular}{|l|} 
Load \\
Net \\
Wind \\
\end{tabular} & Load & \begin{tabular}{|l|} 
Load \\
Net \\
Wind \\
\end{tabular} & Load & \begin{tabular}{|l|} 
Load \\
Net \\
Wind \\
\end{tabular} & Load & $\begin{array}{l}\text { Load } \\
\text { Net } \\
\text { Wind }\end{array}$ & Load & $\begin{array}{l}\text { Load } \\
\text { Net } \\
\text { Wind }\end{array}$ \\
\hline Winter & -145 & -338 & -222 & -551 & -376 & -734 & -402 & -719 & \begin{tabular}{|l|}
-292 \\
\end{tabular} & -495 & -178 & -398 & -123 & -293 \\
\hline Spring & -165 & -369 & -264 & -651 & -431 & -729 & -487 & -828 & -437 & -601 & -228 & -391 & -164 & -306 \\
\hline Summer & -266 & -513 & -209 & -565 & -497 & -798 & -578 & -937 & -484 & -807 & -344 & -596 & -241 & -425 \\
\hline Fall & -135 & -434 & -277 & -647 & -427 & -782 & -508 & -807 & -400 & -674 & -284 & -515 & -222 & -303 \\
\hline Year & -223 & -435 & -259 & -614 & -463 & -778 & -545 & -864 & -461 & -718 & -323 & -505 & -229 & -355 \\
\hline
\end{tabular}

Figure 146: 2005 Profile Season 98 Percentile Hourly MW Change for Hours 21 to 3 


\begin{tabular}{|c|c|c|c|c|c|c|c|c|c|c|c|c|c|c|}
\hline & \multicolumn{14}{|c|}{ NPA Maximum Hourly Ramp-down MW Change 2018 - 2006 Profile $20 \%$ Wind } \\
\hline & \multicolumn{2}{|c|}{$\operatorname{Hr} 21$} & \multicolumn{2}{|c|}{$\mathrm{Hr} 22$} & \multicolumn{2}{|c|}{$\mathrm{Hr} 23$} & \multicolumn{2}{|c|}{$\mathrm{Hr} 24$} & \multicolumn{2}{|c|}{$\mathrm{Hr} 1$} & \multicolumn{2}{|c|}{$\mathrm{Hr} 2$} & \multicolumn{2}{|c|}{$\mathrm{Hr} 3$} \\
\hline & Load & $\begin{array}{l}\text { Load } \\
\text { Net } \\
\text { Wind }\end{array}$ & Load & $\begin{array}{l}\text { Load } \\
\text { Net } \\
\text { Wind }\end{array}$ & Load & $\begin{array}{l}\text { Load } \\
\text { Net } \\
\text { Wind }\end{array}$ & Load & $\begin{array}{l}\text { Load } \\
\text { Net } \\
\text { Wind }\end{array}$ & Load & $\begin{array}{l}\text { Load } \\
\text { Net } \\
\text { Wind }\end{array}$ & Load & $\begin{array}{l}\text { Load } \\
\text { Net } \\
\text { Wind }\end{array}$ & Load & $\begin{array}{l}\text { Load } \\
\text { Net } \\
\text { Wind }\end{array}$ \\
\hline Winter & -460 & -551 & -250 & -674 & -420 & -927 & -481 & -900 & -522 & -652 & -186 & -455 & -158 & -319 \\
\hline Spring & -220 & -604 & -231 & -671 & -518 & -757 & -540 & -639 & -667 & -808 & -242 & -807 & -219 & -462 \\
\hline Summer & -302 & -706 & -212 & -878 & -502 & -843 & -620 & -913 & -488 & -799 & -383 & -547 & -288 & -436 \\
\hline Fall & -147 & -622 & -309 & -698 & -561 & -1008 & -508 & -948 & -718 & -777 & -308 & -464 & -186 & -561 \\
\hline Year & -460 & -706 & -309 & -878 & -561 & -1008 & -620 & -948 & -718 & -808 & -383 & -807 & -288 & -561 \\
\hline
\end{tabular}

Figure 147: 2006 Profile Season Maximum Hourly MW Change for Hours 21 to 3

\begin{tabular}{|c|c|c|c|c|c|c|c|c|c|c|c|c|c|c|}
\hline & \multicolumn{14}{|c|}{ NPA Minimum Hourly Ramp-down MW Change 2018 - 2006 Profile 20\% Wind } \\
\hline & \multicolumn{2}{|c|}{$\operatorname{Hr} 21$} & \multicolumn{2}{|c|}{$\mathrm{Hr} 22$} & \multicolumn{2}{|c|}{\begin{tabular}{|l|}
$\mathrm{Hr} 23$ \\
\end{tabular}} & \multicolumn{2}{|c|}{$\mathrm{Hr} 24$} & \multicolumn{2}{|c|}{$\mathrm{Hr} 1$} & \multicolumn{2}{|c|}{$\mathrm{Hr} 2$} & \multicolumn{2}{|c|}{$\mathrm{Hr} 3$} \\
\hline & Load & $\begin{array}{l}\text { Load } \\
\text { Net } \\
\text { Wind }\end{array}$ & Load & $\begin{array}{l}\text { Load } \\
\text { Net } \\
\text { Wind }\end{array}$ & Load & $\begin{array}{l}\text { Load } \\
\text { Net } \\
\text { Wind }\end{array}$ & Load & $\begin{array}{l}\text { Load } \\
\text { Net } \\
\text { Wind }\end{array}$ & Load & $\begin{array}{l}\text { Load } \\
\text { Net } \\
\text { Wind }\end{array}$ & Load & $\begin{array}{l}\text { Load } \\
\text { Net } \\
\text { Wind } \\
\end{array}$ & Load & $\begin{array}{l}\text { Load } \\
\text { Net } \\
\text { Wind }\end{array}$ \\
\hline Winter & 10 & 358 & 135 & 245 & -73 & 237 & 0 & 141 & 25 & 177 & -5 & 460 & 76 & 265 \\
\hline Spring & 274 & 596 & 141 & 525 & -22 & 197 & -196 & 275 & -64 & 181 & -25 & 515 & 20 & 302 \\
\hline Summer & 102 & 137 & 103 & 256 & -26 & 268 & -213 & 73 & 317 & 231 & -112 & 177 & -31 & 430 \\
\hline Fall & 199 & 468 & -29 & 180 & -135 & 89 & -181 & 62 & 235 & 184 & -18 & 153 & 33 & 230 \\
\hline Year & 274 & 596 & 141 & 525 & -22 & 268 & 0 & 275 & 317 & 231 & -5 & 515 & 76 & 430 \\
\hline
\end{tabular}

Figure 148: 2006 Profile Season Minimum Hourly MW Change for Hours 21 to 3

\begin{tabular}{|c|c|c|c|c|c|c|c|c|c|c|c|c|c|c|}
\hline & \multicolumn{14}{|c|}{ NPA 98 Percentile Ramp-down Hourly MW Change 2018 - 2006 Profile 20\% Wind } \\
\hline & \multicolumn{2}{|c|}{$\operatorname{Hr} 21$} & \multicolumn{2}{|c|}{$\mathrm{Hr} 22$} & \multicolumn{2}{|c|}{\begin{tabular}{|l|}
$\mathrm{Hr} 23$ \\
\end{tabular}} & \multicolumn{2}{|c|}{\begin{tabular}{|l|}
$\mathrm{Hr} 24$ \\
\end{tabular}} & \multicolumn{2}{|c|}{$\mathrm{Hr} 1$} & \multicolumn{2}{|c|}{$\mathrm{Hr} 2$} & \multicolumn{2}{|c|}{$\mathrm{Hr} 3$} \\
\hline & Load & $\begin{array}{l}\text { Load } \\
\text { Net } \\
\text { Wind }\end{array}$ & Load & $\begin{array}{l}\text { Load } \\
\text { Net } \\
\text { Wind }\end{array}$ & Load & $\begin{array}{l}\text { Load } \\
\text { Net } \\
\text { Wind }\end{array}$ & Load & $\begin{array}{l}\text { Load } \\
\text { Net } \\
\text { Wind }\end{array}$ & Load & $\begin{array}{l}\text { Load } \\
\text { Net } \\
\text { Wind } \\
\end{array}$ & Load & $\begin{array}{l}\text { Load } \\
\text { Net } \\
\text { Wind }\end{array}$ & Load & $\begin{array}{l}\text { Load } \\
\text { Net } \\
\text { Wind }\end{array}$ \\
\hline Winter & -160 & -463 & -248 & -596 & -386 & -735 & -451 & -835 & -417 & -601 & -184 & -399 & -116 & -296 \\
\hline Spring & -188 & -451 & -210 & -543 & -355 & -664 & -485 & -622 & -370 & -586 & -219 & -408 & -169 & -310 \\
\hline Summer & -280 & -641 & -183 & -712 & -490 & -800 & -581 & -904 & -469 & -787 & -324 & -443 & -259 & -378 \\
\hline Fall & -134 & -470 & -291 & -618 & -483 & -901 & -491 & -798 & -377 & -671 & -243 & -435 & -158 & -420 \\
\hline Year & -251 & -578 & -250 & -647 & -475 & -773 & -555 & -854 & -443 & -704 & -310 & -433 & -228 & -376 \\
\hline
\end{tabular}

Figure 149: 2006 Profile Season 98 Percentile Hourly MW Change for Hours 21 to 3

The contour plot in Figure 150 shows the changes in load net wind from one hour to the next. Along the vertical axis plot displays the change in load net wind for each hour of the day while the horizontal axis is each day of the year. Increase in load net wind is represented by yellow, orange and red colors with red being the largest increase in hourly load net wind change. Colors green, light blue and dark blue indicate decrease in load net wind from one hour to the next with dark blue being the largest decrease. On examination of the plot it can be seen that increases in hourly load net wind change tend to occur during early morning hours between 6 
and 7 am on days 1 through 120 and days 240 through 365 (winter, spring and fall). On days 120 through 240 (summer) the increase in load net wind is later in the morning between hours 7 and 10. The largest decrease in load net wind tends to be between the hours of 21 and 1 . This plot also shows the spread of hourly load net wind increases (hours 5-17) during the summer time period (days 140 and 250).

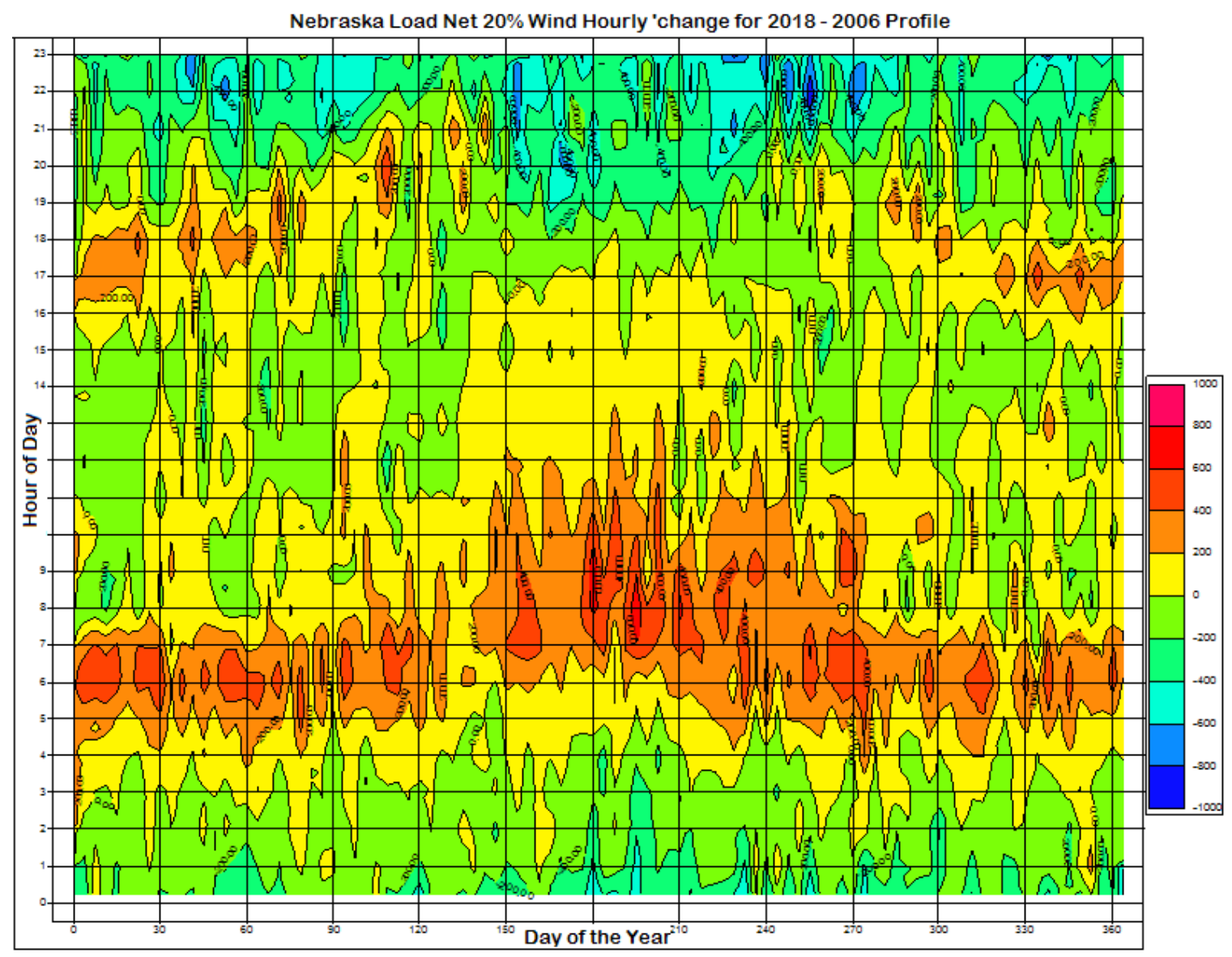

Figure 150: Contour plot of Nebraska Load Net Wind Hourly Changes with 20\% Penetration for 2006 profile. 


\section{Nebraska Statewide Wind Integration Study}

\section{Part 2 of Report Section $\mathbf{8 . 2}$}




\section{CONTENTS}

Section $8 \quad$ Appendix continued

88

Section 8.2 Additional Wind and System Generation Characteristics and Scenario Information Part 288

Section 8.2.4.2 Weekly Load, Resource and Wind Charts: 


\section{FIGURES}

Figure 151: Nebraska 10\% wind Penetration, 2006 Profile week of 1/1/2018 .................. 89

Figure 152: Nebraska 10\% wind Penetration, 2006 Profile week of 1/8/2018 .................. 90

Figure 153: Nebraska 10\% wind Penetration, 2006 Profile week of 1/15/2018 ................. 91

Figure 154: Nebraska 10\% wind Penetration, 2006 Profile week of 1/22/2018 ................. 92

Figure 155: Nebraska 10\% wind Penetration, 2006 Profile week of 1/29/2018 ................. 93

Figure 156: Nebraska 10\% wind Penetration, 2006 Profile week of 2/5/2018 .................. 94

Figure 157: Nebraska 10\% wind Penetration, 2006 Profile week of 2/12/2018 ................. 95

Figure 158: Nebraska 10\% wind Penetration, 2006 Profile week of 2/19/2018 ................. 96

Figure 159: Nebraska 10\% wind Penetration, 2006 Profile week of 2/26/2018 ................. 97

Figure 160: Nebraska 10\% wind Penetration, 2006 Profile week of 3/5/2018 ................... 98

Figure 161: Nebraska 10\% wind Penetration, 2006 Profile week of 3/12/2018 ................. 99

Figure 162: Nebraska 10\% wind Penetration, 2006 Profile week of 3/19/2018 ............... 100

Figure 163: Nebraska 10\% wind Penetration, 2006 Profile week of 3/26/2018 ................ 101

Figure 164: Nebraska 10\% wind Penetration, 2006 Profile week of 4/2/2018 ................. 102

Figure 165: Nebraska 10\% wind Penetration, 2006 Profile week of 4/9/2018 ................ 103

Figure 166: Nebraska 10\% wind Penetration, 2006 Profile week of 4/16/2018 ............... 104

Figure 167: Nebraska 10\% wind Penetration, 2006 Profile week of 4/23/2018 ............... 105

Figure 168: Nebraska 10\% wind Penetration, 2006 Profile week of 4/30/2018 ............... 106

Figure 169: Nebraska 10\% wind Penetration, 2006 Profile week of 5/7/2018 ................. 107

Figure 170: Nebraska 10\% wind Penetration, 2006 Profile week of 5/14/2018 ............... 108

Figure 171: Nebraska 10\% wind Penetration, 2006 Profile week of 5/21/2018 ............... 109

Figure 172: Nebraska 10\% wind Penetration, 2006 Profile week of 5/28/2018 ............... 110

Figure 173: Nebraska 10\% wind Penetration, 2006 Profile week of 6/4/2018 ................. 111

Figure 174: Nebraska 10\% wind Penetration, 2006 Profile week of 6/11/2018 ............... 112

Figure 175: Nebraska 10\% wind Penetration, 2006 Profile week of 6/18/2018 ............... 113

Figure 176: Nebraska 10\% wind Penetration, 2006 Profile week of 6/25/2018 ............... 114

Figure 177: Nebraska 10\% wind Penetration, 2006 Profile week of 7/2/2018 ................ 115 
Figure 178: Nebraska 10\% wind Penetration, 2006 Profile week of 7/9/2018 ................ 116

Figure 179: Nebraska 10\% wind Penetration, 2006 Profile week of 7/16/2018 ............... 117

Figure 180: Nebraska 10\% wind Penetration, 2006 Profile week of 7/23/2018 ............... 118

Figure 181: Nebraska 10\% wind Penetration, 2006 Profile week of 7/30/2018 .............. 119

Figure 182: Nebraska 10\% wind Penetration, 2006 Profile week of 8/6/2018 ................ 120

Figure 183: Nebraska 10\% wind Penetration, 2006 Profile week of 8/13/2018 ............... 121

Figure 184: Nebraska 10\% wind Penetration, 2006 Profile week of 8/20/2018 ............... 122

Figure 185: Nebraska 10\% wind Penetration, 2006 Profile week of 8/27/2018 ............... 123

Figure 186: Nebraska 10\% wind Penetration, 2006 Profile week of 9/3/2018 ................. 124

Figure 187: Nebraska 10\% wind Penetration, 2006 Profile week of 9/10/2018 ............... 125

Figure 188: Nebraska 10\% wind Penetration, 2006 Profile week of 9/17/2018 ............... 126

Figure 189: Nebraska 10\% wind Penetration, 2006 Profile week of 9/24/2018 ............... 127

Figure 190: Nebraska 10\% wind Penetration, 2006 Profile week of 10/1/2018 ............... 128

Figure 191: Nebraska 10\% wind Penetration, 2006 Profile week of 10/8/2018 ............... 129

Figure 192: Nebraska 10\% wind Penetration, 2006 Profile week of 10/15/2018 ............. 130

Figure 193 Nebraska 10\% wind Penetration, 2006 Profile week of 10/22/2018 ............. 131

Figure 194: Nebraska 10\% wind Penetration, 2006 Profile week of 10/29/2018 ............. 132

Figure $195 \quad$ Nebraska 10\% wind Penetration, 2006 Profile week of 11/5/2018 ................ 133

Figure 196: Nebraska 10\% wind Penetration, 2006 Profile week of 11/12/2018 ............. 134

Figure 197: Nebraska 10\% wind Penetration, 2006 Profile week of 11/19/2018 ............. 135

Figure 198: Nebraska 10\% wind Penetration, 2006 Profile week of 11/26/2018 ............. 136

Figure 199: Nebraska 10\% wind Penetration, 2006 Profile week of 12/3/2018 ............... 137

Figure 200: Nebraska 10\% wind Penetration, 2006 Profile week of 12/10/2018 ............. 138

Figure 201: Nebraska 10\% wind Penetration, 2006 Profile week of 12/17/2018 ............. 139

Figure 202: Nebraska 10\% wind Penetration, 2006 Profile week of 12/24/2018 ............. 140

Figure 203: $\quad$ SPP 10\% wind Penetration, 2006 Profile week of 1/1/2018 .......................... 142

Figure 204: $\quad$ SPP 10\% wind Penetration, 2006 Profile week of 1/8/2018 ......................... 143

Figure 205: $\quad$ SPP 10\% wind Penetration, 2006 Profile week of 1/15/2018 ........................ 144

Figure 206: $\quad$ SPP 10\% wind Penetration, 2006 Profile week of 1/22/2018 ...................... 145

Figure 207: $\quad$ SPP 10\% wind Penetration, 2006 Profile week of 1/29/2018 ....................... 146

Figure 208: $\quad$ SPP 10\% wind Penetration, 2006 Profile week of 2/5/2018 ......................... 147

Figure 209: $\quad$ SPP 10\% wind Penetration, 2006 Profile week of 2/12/2018 ....................... 148 
Figure 210: $\quad$ SPP 10\% wind Penetration, 2006 Profile week of 2/19/2018 ...................... 149

Figure 211: SPP 10\% wind Penetration, 2006 Profile week of 2/26/2018 ........................ 150

Figure 212: $\quad$ SPP 10\% wind Penetration, 2006 Profile week of 3/5/2018 .......................... 151

Figure 213: $\quad$ SPP 10\% wind Penetration, 2006 Profile week of 3/12/2018 ...................... 152

Figure 214: $\quad$ SPP 10\% wind Penetration, 2006 Profile week of 3/19/2018 ....................... 153

Figure 215: $\quad$ SPP 10\% wind Penetration, 2006 Profile week of 3/26/2018 ......................... 154

Figure 216: $\quad$ SPP 10\% wind Penetration, 2006 Profile week of 4/2/2018 .......................... 155

Figure 217: $\quad$ SPP 10\% wind Penetration, 2006 Profile week of 4/9/2018 .......................... 156

Figure 218: $\quad$ SPP 10\% wind Penetration, 2006 Profile week of 4/16/2018 ....................... 157

Figure 219: $\quad$ SPP 10\% wind Penetration, 2006 Profile week of $4 / 23 / 2018 \ldots \ldots \ldots \ldots . . . . . . . . . . . . .158$

Figure 220: $\quad$ SPP 10\% wind Penetration, 2006 Profile week of $4 / 30 / 2018$........................ 159

Figure 221: $\quad$ SPP 10\% wind Penetration, 2006 Profile week of 5/7/2018 .......................... 160

Figure 222: $\quad$ SPP 10\% wind Penetration, 2006 Profile week of 5/14/2018 ........................ 161

Figure 223: $\quad$ SPP 10\% wind Penetration, 2006 Profile week of 5/21/2018 ....................... 162

Figure 224: SPP 10\% wind Penetration, 2006 Profile week of 5/28/2018 ........................ 163

Figure 225: $\quad$ SPP 10\% wind Penetration, 2006 Profile week of 6/4/2018 ........................... 164

Figure 226: $\quad$ SPP 10\% wind Penetration, 2006 Profile week of 6/11/2018 ......................... 165

Figure 227: $\quad$ SPP 10\% wind Penetration, 2006 Profile week of 6/18/2018 ....................... 166

Figure 228: $\quad$ SPP 10\% wind Penetration, 2006 Profile week of 6/25/2018 ........................ 167

Figure 229: $\quad$ SPP 10\% wind Penetration, 2006 Profile week of 7/2/2018 …...................... 168

Figure 230: $\quad$ SPP 10\% wind Penetration, 2006 Profile week of 7/9/2018 ......................... 169

Figure 231: SPP 10\% wind Penetration, 2006 Profile week of 7/16/2018 ....................... 170

Figure 232: $\quad$ SPP 10\% wind Penetration, 2006 Profile week of 7/23/2018 …................... 171

Figure 233: $\quad$ SPP 10\% wind Penetration, 2006 Profile week of $7 / 30 / 2018$......................... 172

Figure 234: $\quad$ SPP 10\% wind Penetration, 2006 Profile week of 8/6/2018 .......................... 173

Figure 235: $\quad$ SPP 10\% wind Penetration, 2006 Profile week of 8/13/2018 ......................... 174

Figure 236: $\quad$ SPP 10\% wind Penetration, 2006 Profile week of 8/20/2018 ....................... 175

Figure 237: $\quad$ SPP 10\% wind Penetration, 2006 Profile week of 8/27/2018 ........................ 176

Figure 238: $\quad$ SPP 10\% wind Penetration, 2006 Profile week of 9/3/2018 ........................ 177

Figure 239: $\quad$ SPP 10\% wind Penetration, 2006 Profile week of 9/10/2018 ....................... 178

Figure 240: $\quad$ SPP 10\% wind Penetration, 2006 Profile week of 9/17/2018 ...................... 179

Figure 241: $\quad$ SPP 10\% wind Penetration, 2006 Profile week of 9/24/2018 ....................... 180 
Figure 242: $\quad$ SPP 10\% wind Penetration, 2006 Profile week of 10/1/2018 ...................... 181

Figure 243: $\quad$ SPP 10\% wind Penetration, 2006 Profile week of 10/8/2018 ….................... 182

Figure 244: SPP 10\% wind Penetration, 2006 Profile week of 10/15/2018 ...................... 183

Figure 245: $\quad$ SPP 10\% wind Penetration, 2006 Profile week of 10/22/2018 ....................... 184

Figure 246: $\quad$ SPP 10\% wind Penetration, 2006 Profile week of 10/29/2018 ..................... 185

Figure 247: SPP 10\% wind Penetration, 2006 Profile week of 11/5/2018 ......................... 186

Figure 248: $\quad$ SPP 10\% wind Penetration, 2006 Profile week of 11/12/2018 ...................... 187

Figure 249: $\quad$ SPP 10\% wind Penetration, 2006 Profile week of 11/19/2018 ...................... 188

Figure 250: $\quad$ SPP 10\% wind Penetration, 2006 Profile week of 11/26/2018 ...................... 189

Figure 251: $\quad$ SPP 10\% wind Penetration, 2006 Profile week of 12/3/2018 ...................... 190

Figure 252: $\quad$ SPP 10\% wind Penetration, 2006 Profile week of 12/10/2018 ...................... 191

Figure 253: SPP 10\% wind Penetration, 2006 Profile week of 12/17/2018 ..................... 192

Figure 254: SPP 10\% wind Penetration, 2006 Profile week of 12/24/2018 ..................... 193 


\section{TABLES}

Table 25: Nebraska Seasonal Peak and Minimum Energy

88

Table 26: $\quad$ SPP with Nebraska Seasonal Peak and Minimum Energy

141 


\section{Section $8 \quad$ APPENDIX CONTINUED}

\section{Section 8.2 Additional Wind and System Generation Characteristics and Scenario Information Part 2}

\section{Section 8.2.4.2 Weekly Load, Resource and Wind Charts:}

The following charts (Figure 151-Figure 254) provide a weekly analysis of PROMOD output results.

On each page the top chart displays Load, Transactions, and Load net Transaction for the week, dates at the bottom of the chart, the first day of the week is Monday. Transactions that are shown as negative are exports, which is the typical situation for Nebraska. Then, load net transactions are a larger generation obligation than load alone. It is this load net transaction that needs to be met by the generation in the center chart. The center chart shows the resource stack aggregated into 5 categories

- Nuclear (Bottom of chart)

- Base Fossil ( $2^{\text {nd }}$ on stack): ST Coal, Fixed Energy Resources, Steam Turbine

- Midrange ( $3^{\text {rd }}$ on stack): Combined Cycle, Combustion Turbine, Existing Hydro, St Gas, ST Oil, St Other

- Peak ( $4^{\text {th }}$ on stack): CT Gas, CT Oil, CT Other, Internal Combustion, Interruptible Load, and Pumped Storage

- Wind (Top of stack)

Bottom chart plots the actual wind and the dispatched wind. Red color on the chart represents periods of curtailment. Note there are no wind curtailments for Nebraska.

Nebraska seasonal peak and minimum wind energies are shown in Table 25.

Table 25: Nebraska Seasonal Peak and Minimum Energy

\begin{tabular}{|c|c|c|c|c|c|c|}
\hline $\begin{array}{l}\text { Weekly } \\
\text { Energy }\end{array}$ & $\begin{array}{l}\text { Peak } \\
\text { MWH }\end{array}$ & $\begin{array}{c}\text { Week of } \\
\text { Year }\end{array}$ & $\begin{array}{l}\text { Start } \\
\text { Date }\end{array}$ & $\begin{array}{c}\text { Min } \\
\text { MWH }\end{array}$ & $\begin{array}{c}\text { Weak of } \\
\text { Year }\end{array}$ & $\begin{array}{l}\text { Start } \\
\text { Date }\end{array}$ \\
\hline Season1 & 126771 & 49 & $12 / 3 / 2018$ & 82076 & 7 & $2 / 12 / 2018$ \\
\hline Season2 & 138559 & 21 & $5 / 21 / 2018$ & 61961 & 20 & $5 / 14 / 2018$ \\
\hline Season3 & 90803 & 24 & $6 / 11 / 2018$ & 47022 & 26 & $6 / 25 / 2018$ \\
\hline Season4 & 113732 & 37 & $9 / 10 / 2018$ & 55662 & 36 & $9 / 3 / 2018$ \\
\hline
\end{tabular}

In general these 104 charts are organized as:

- NPA profiles for the 52 weeks of the 2006 pattern applied in $2018,10 \%$ penetration

- SPP with NPA profiles for the 52 weeks of the 2006 pattern applied in $2018,10 \%$ penetration. 


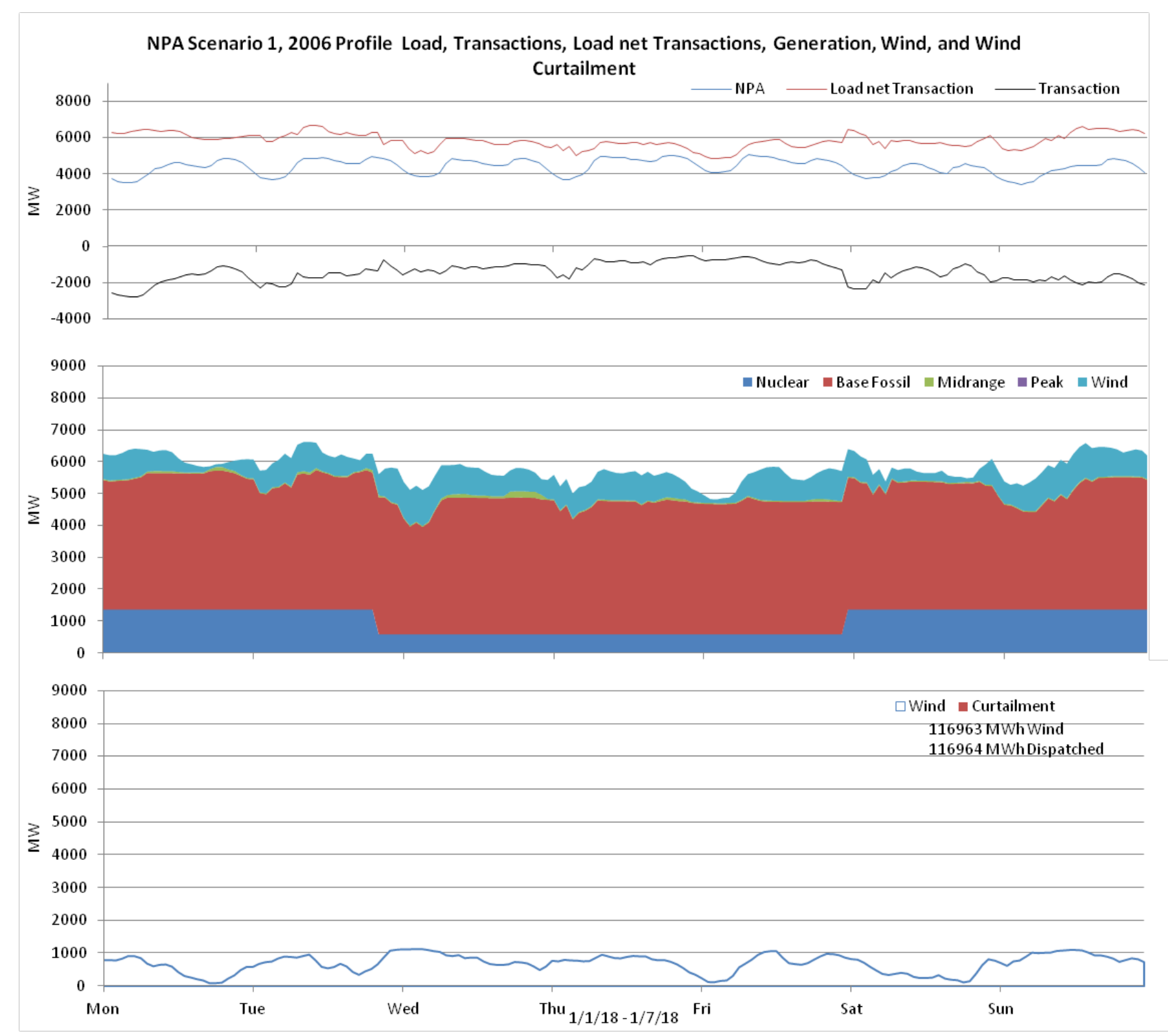

Figure 151: Nebraska 10\% wind Penetration, 2006 Profile week of 1/1/2018 


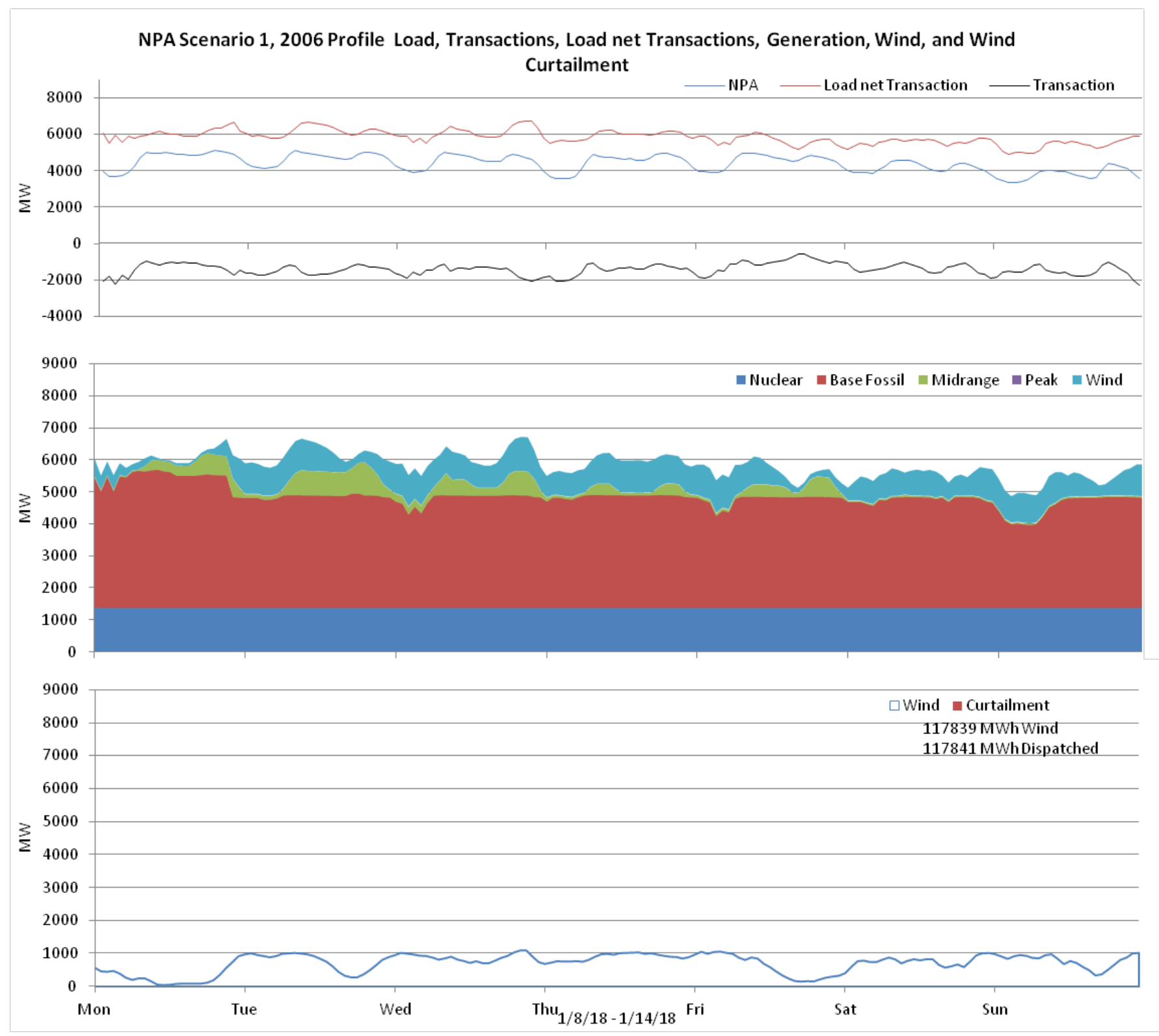

Figure 152: Nebraska 10\% wind Penetration, 2006 Profile week of 1/8/2018 


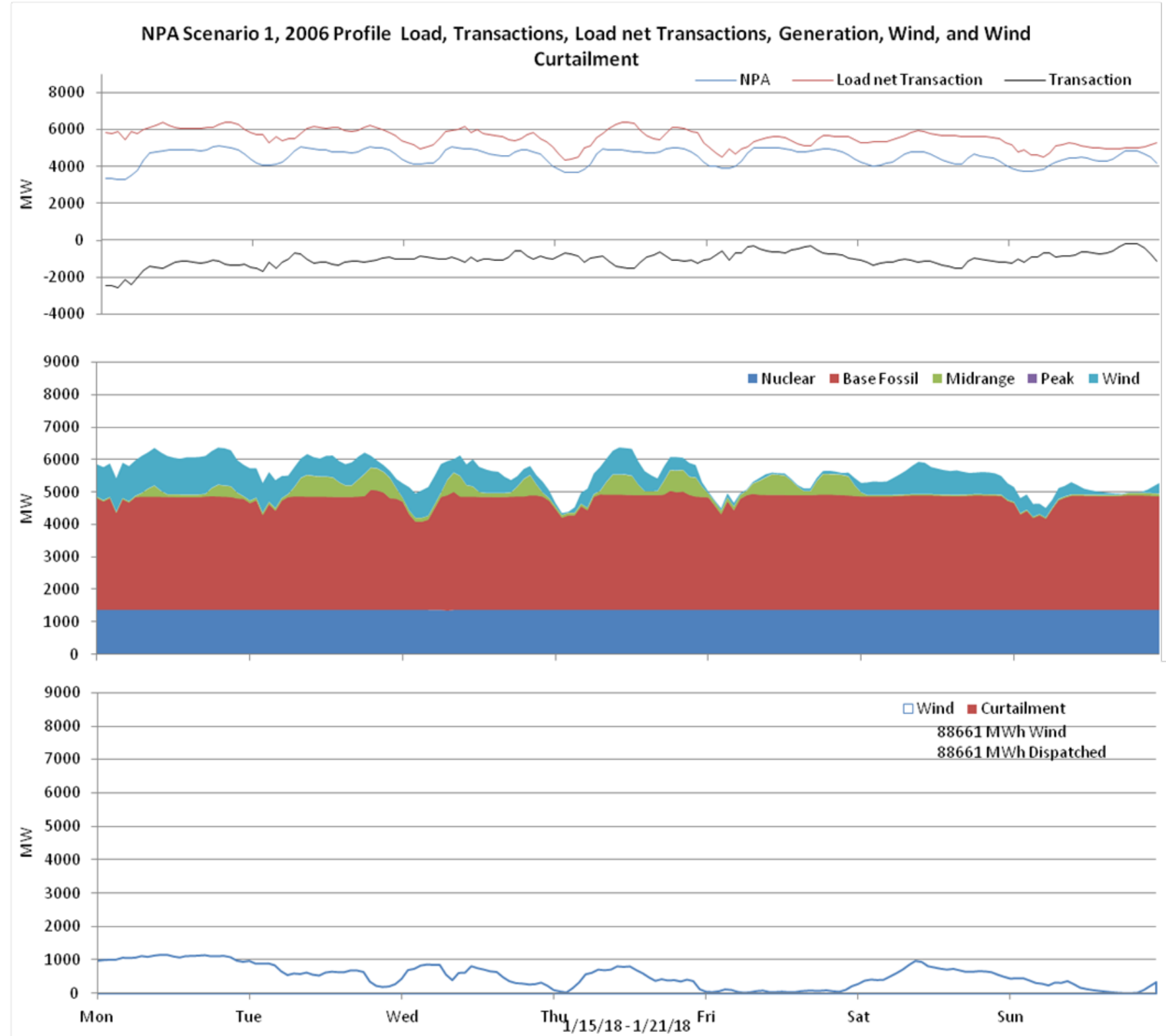

Figure 153: Nebraska 10\% wind Penetration, 2006 Profile week of $1 / 15 / 2018$ 


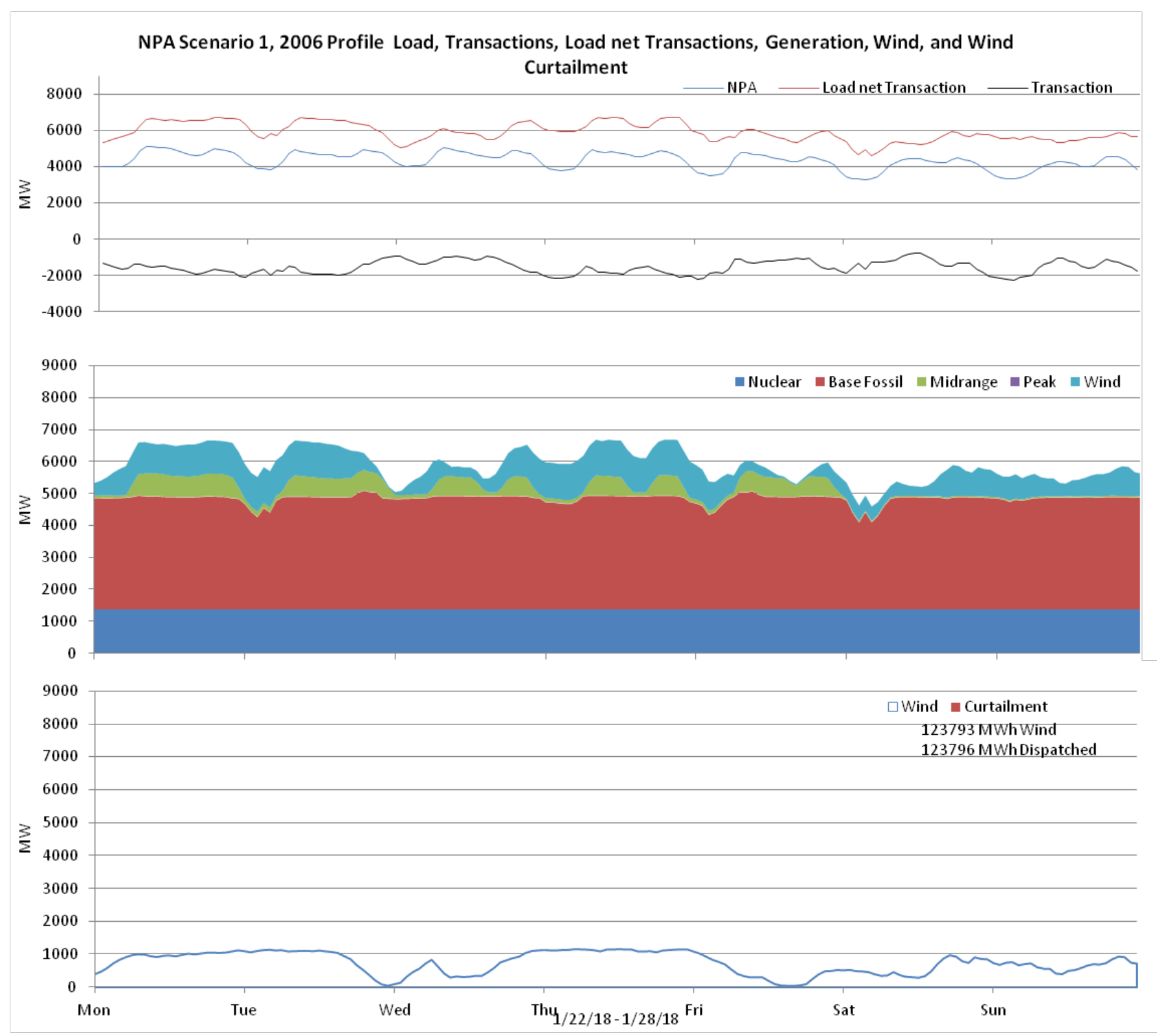

Figure 154: Nebraska 10\% wind Penetration, 2006 Profile week of 1/22/2018 


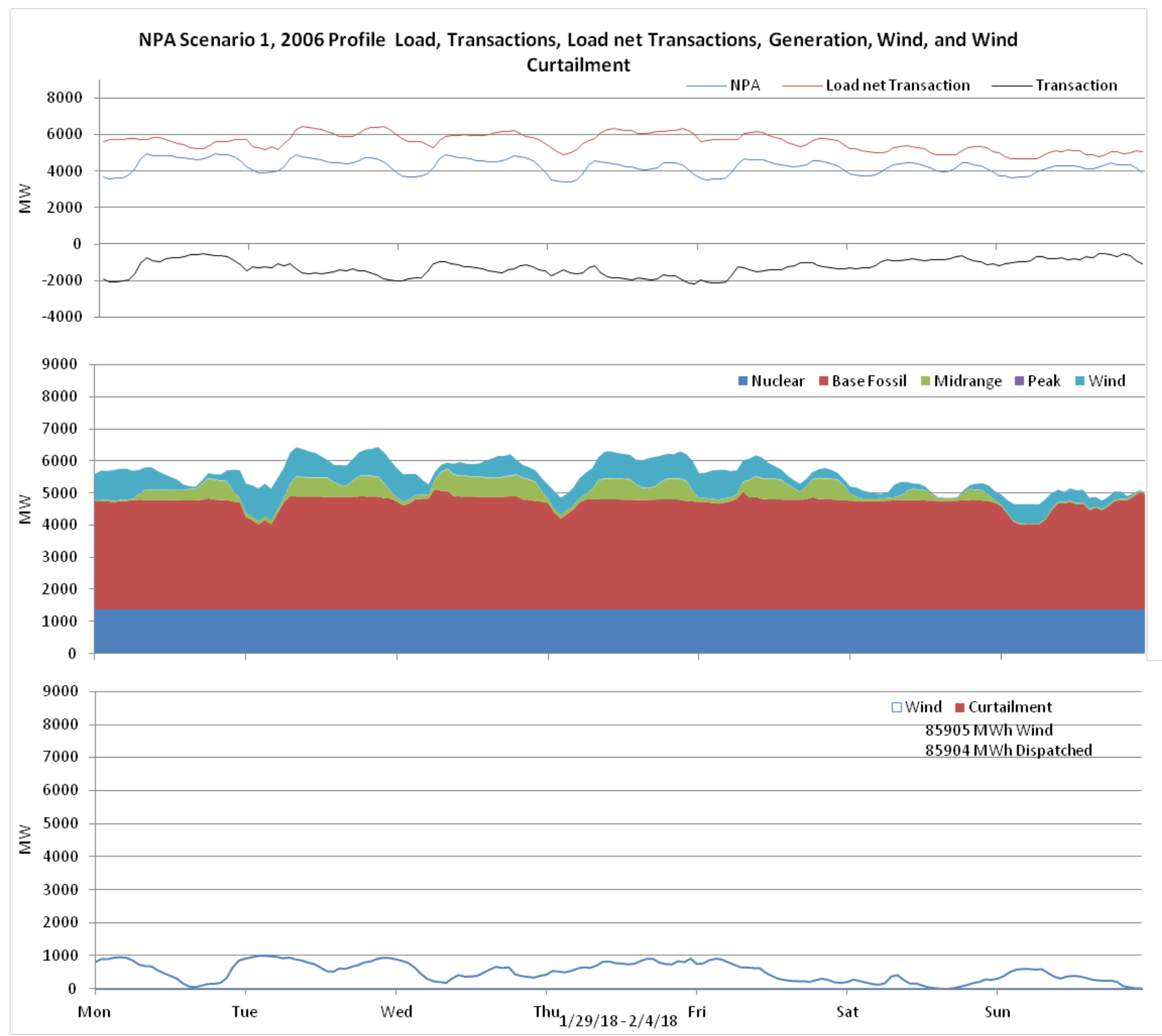

Figure 155: Nebraska 10\% wind Penetration, 2006 Profile week of 1/29/2018 


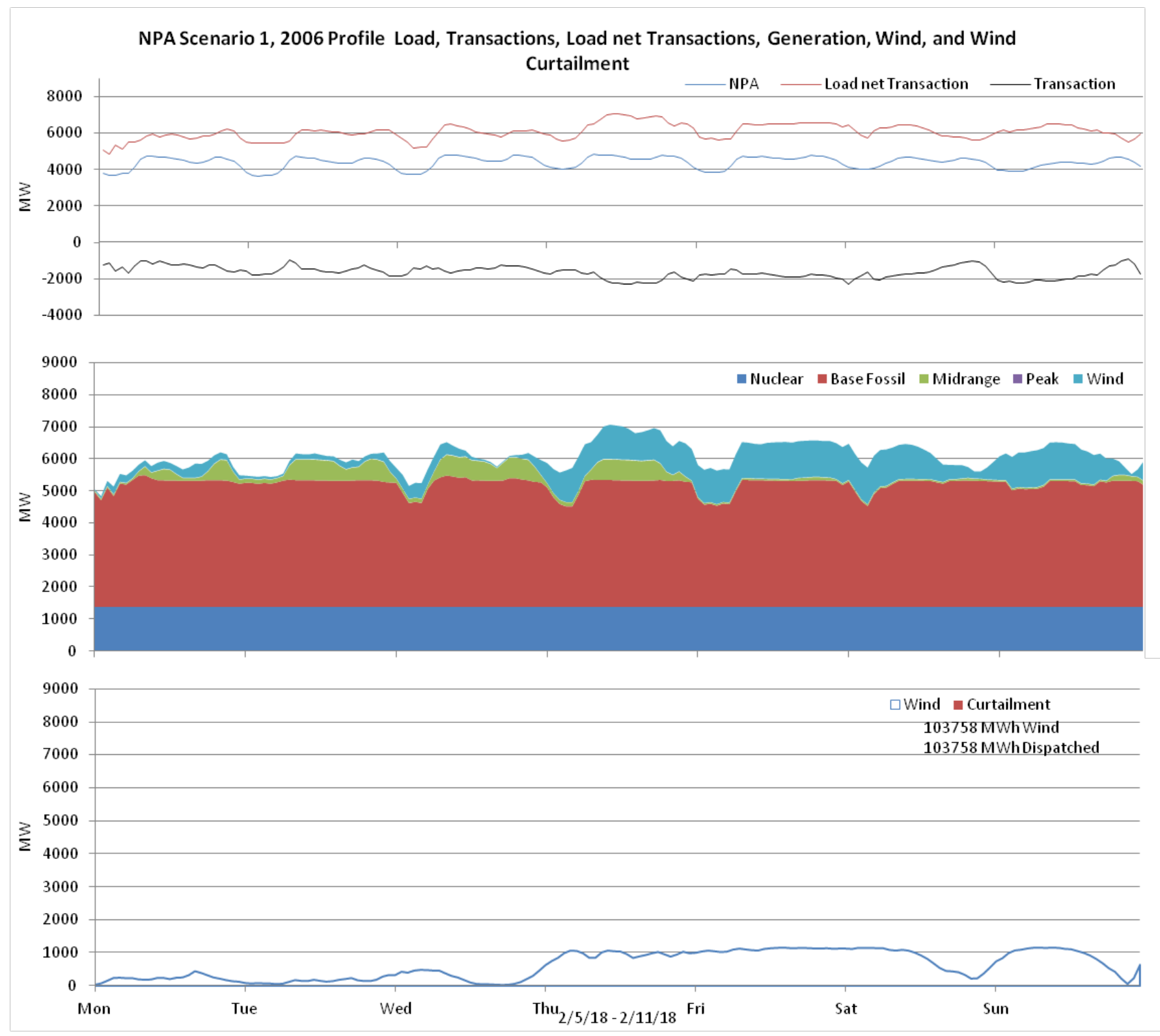

Figure 156: Nebraska 10\% wind Penetration, 2006 Profile week of 2/5/2018 


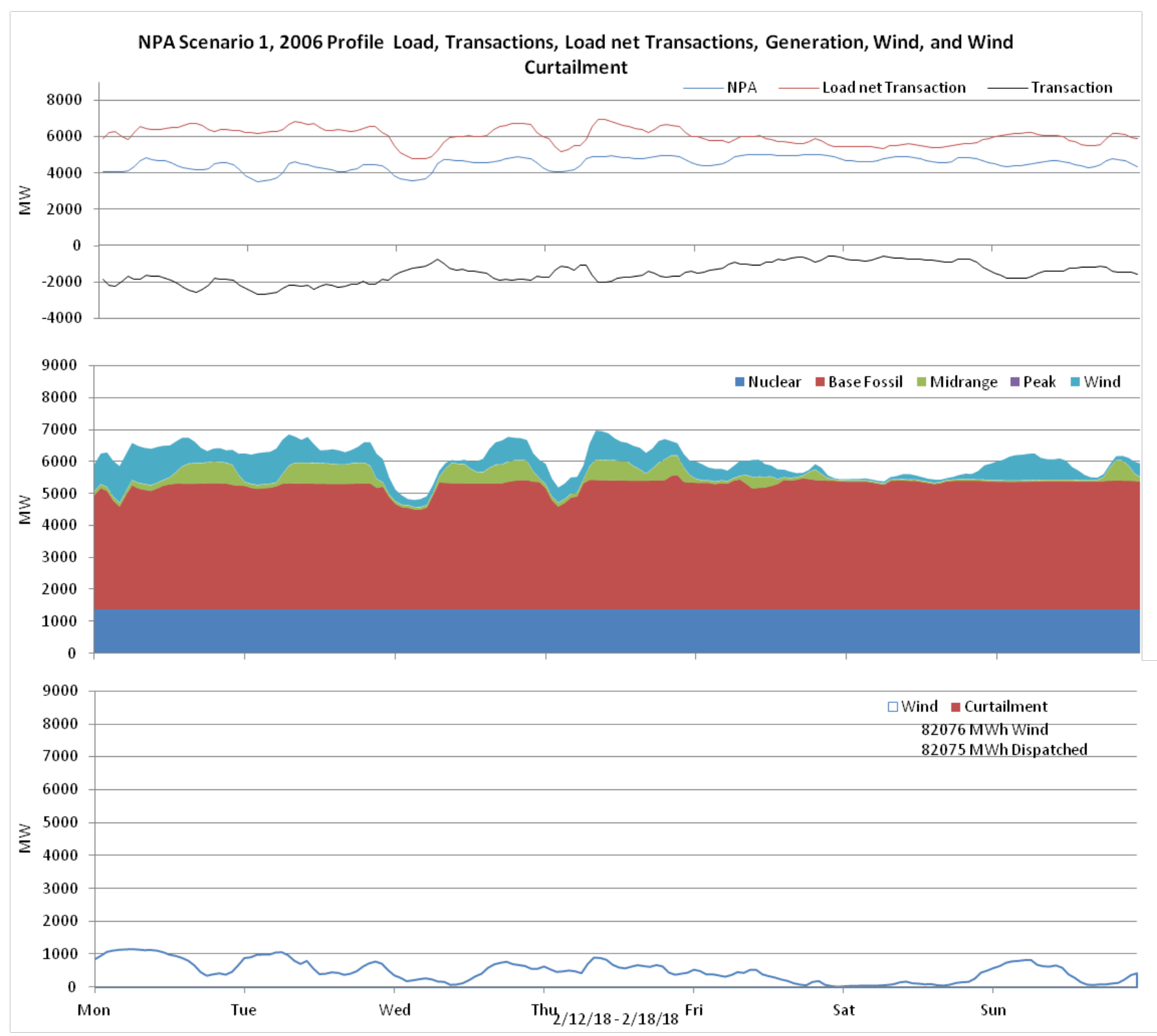

Figure 157: Nebraska 10\% wind Penetration, 2006 Profile week of 2/12/2018 


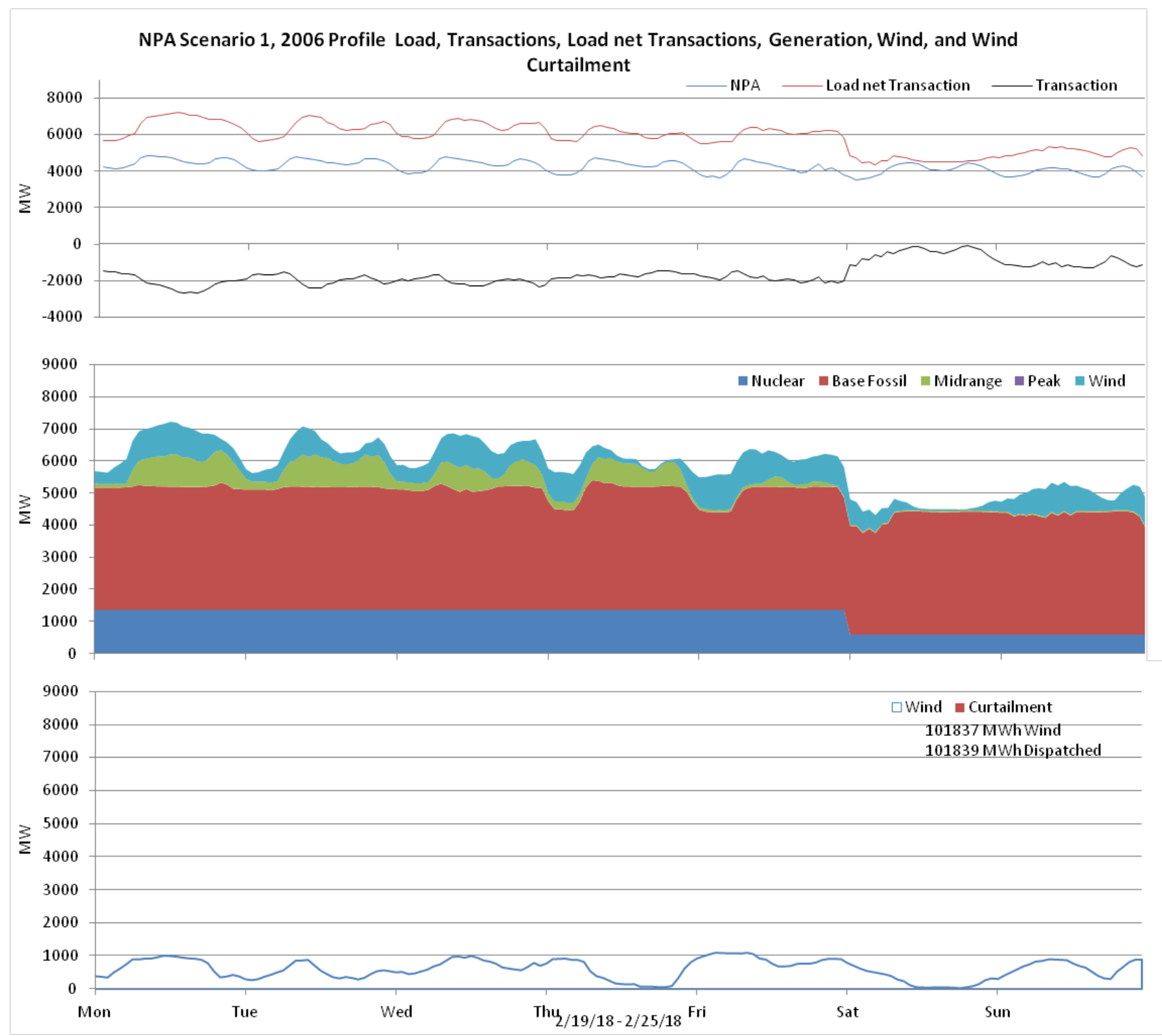

Figure 158: Nebraska 10\% wind Penetration, 2006 Profile week of 2/19/2018 


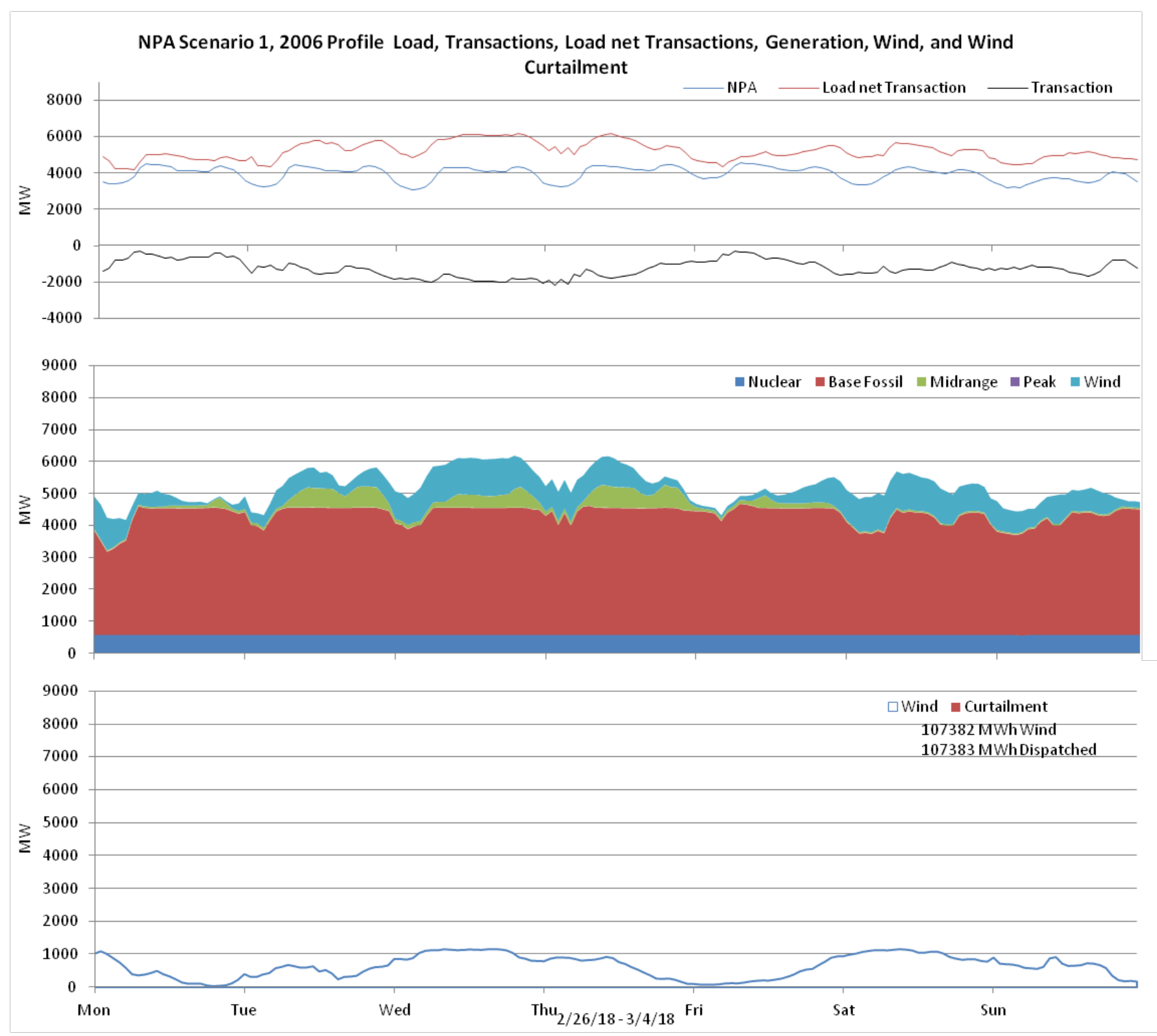

Figure 159: Nebraska 10\% wind Penetration, 2006 Profile week of 2/26/2018 


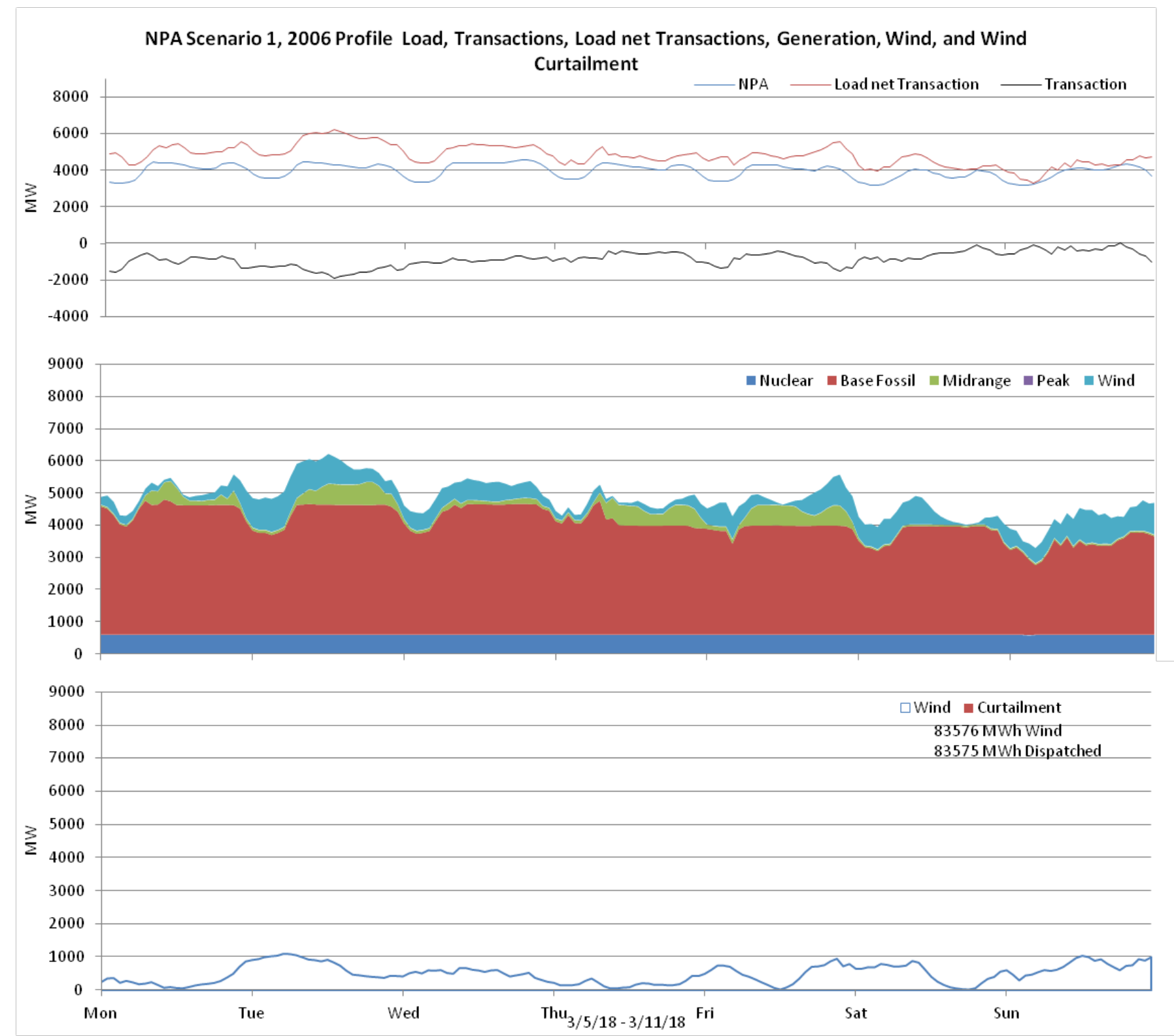

Figure 160: Nebraska 10\% wind Penetration, 2006 Profile week of 3/5/2018 


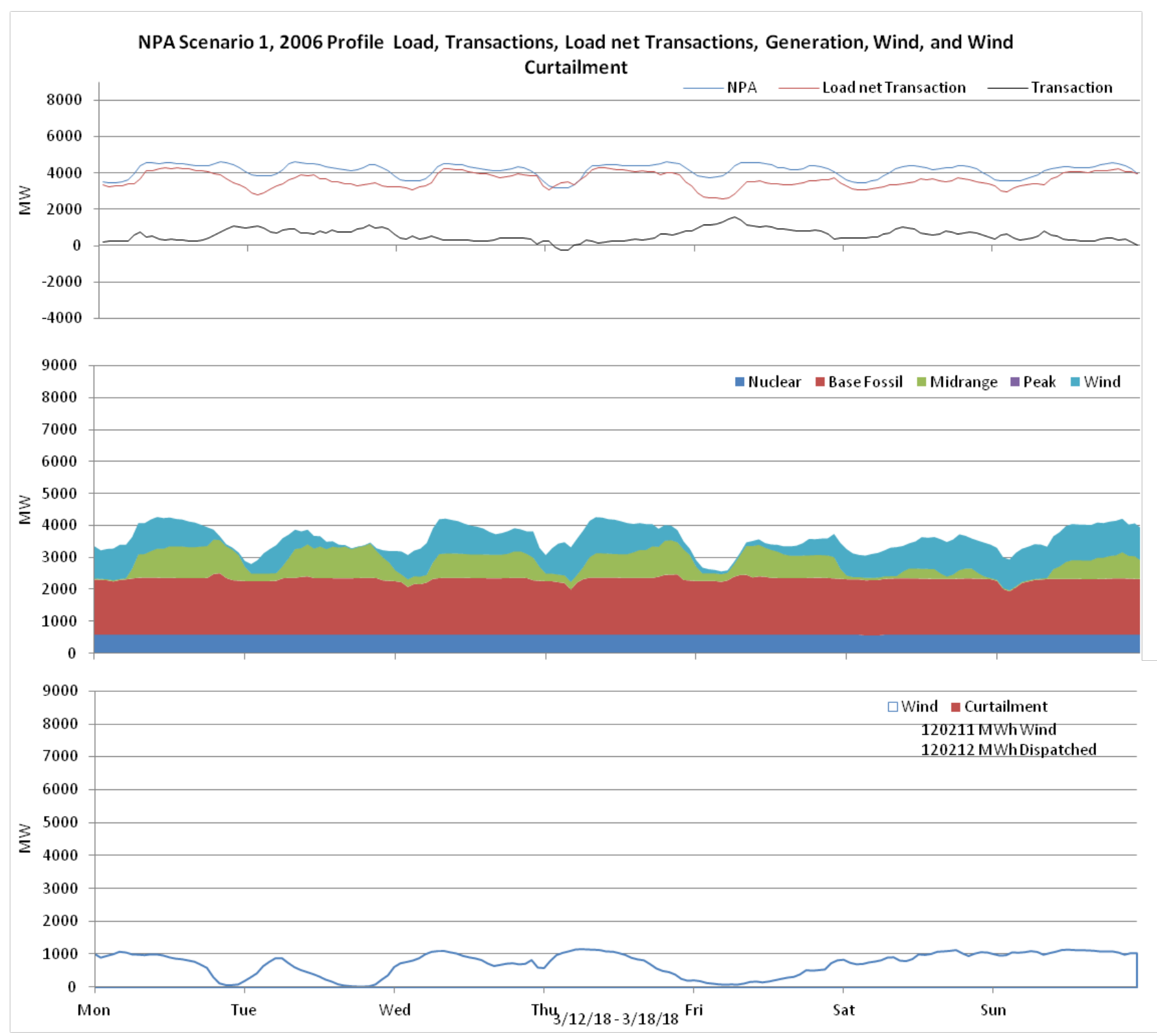

Figure 161: Nebraska 10\% wind Penetration, 2006 Profile week of 3/12/2018 


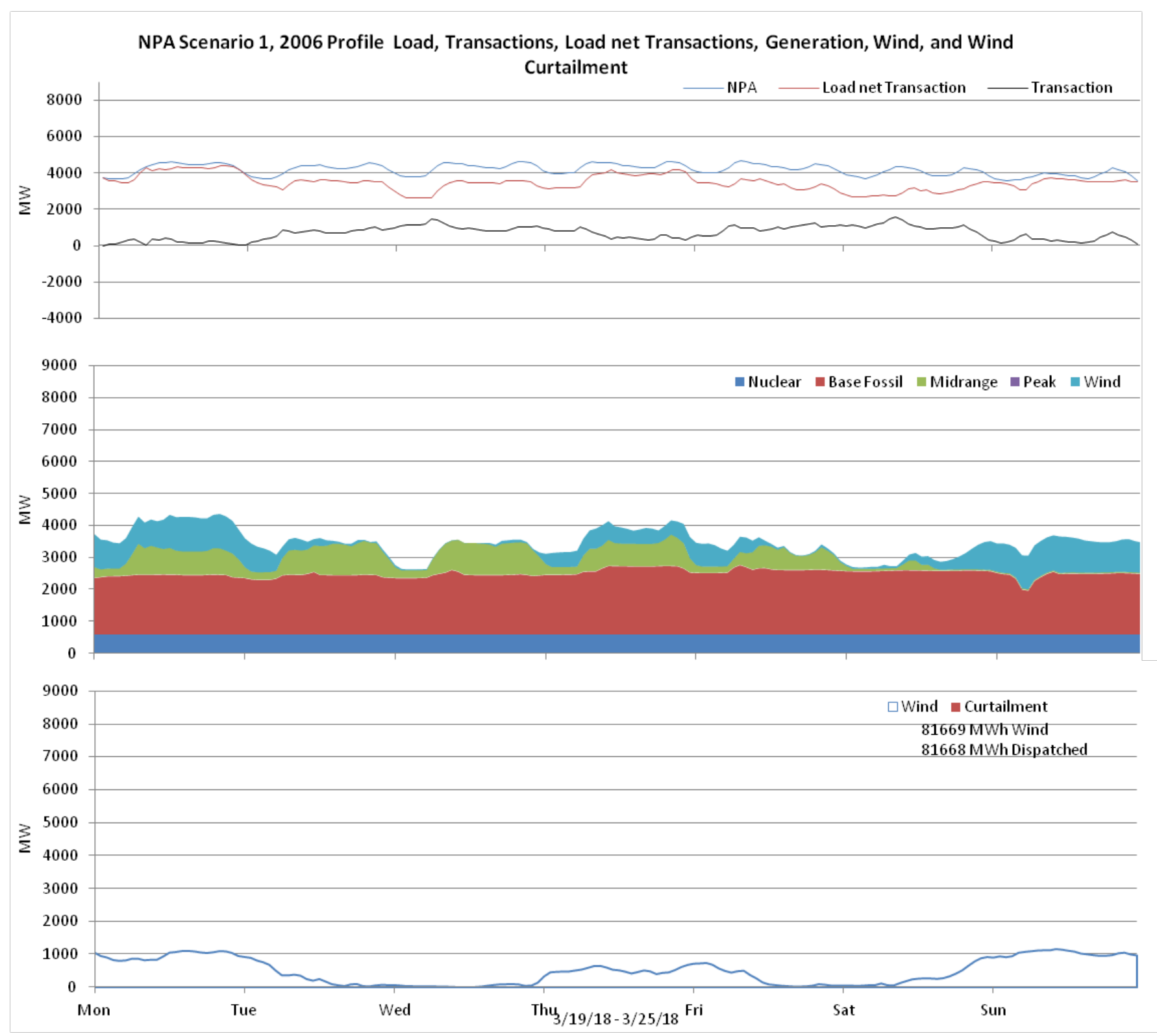

Figure 162: Nebraska 10\% wind Penetration, 2006 Profile week of 3/19/2018 


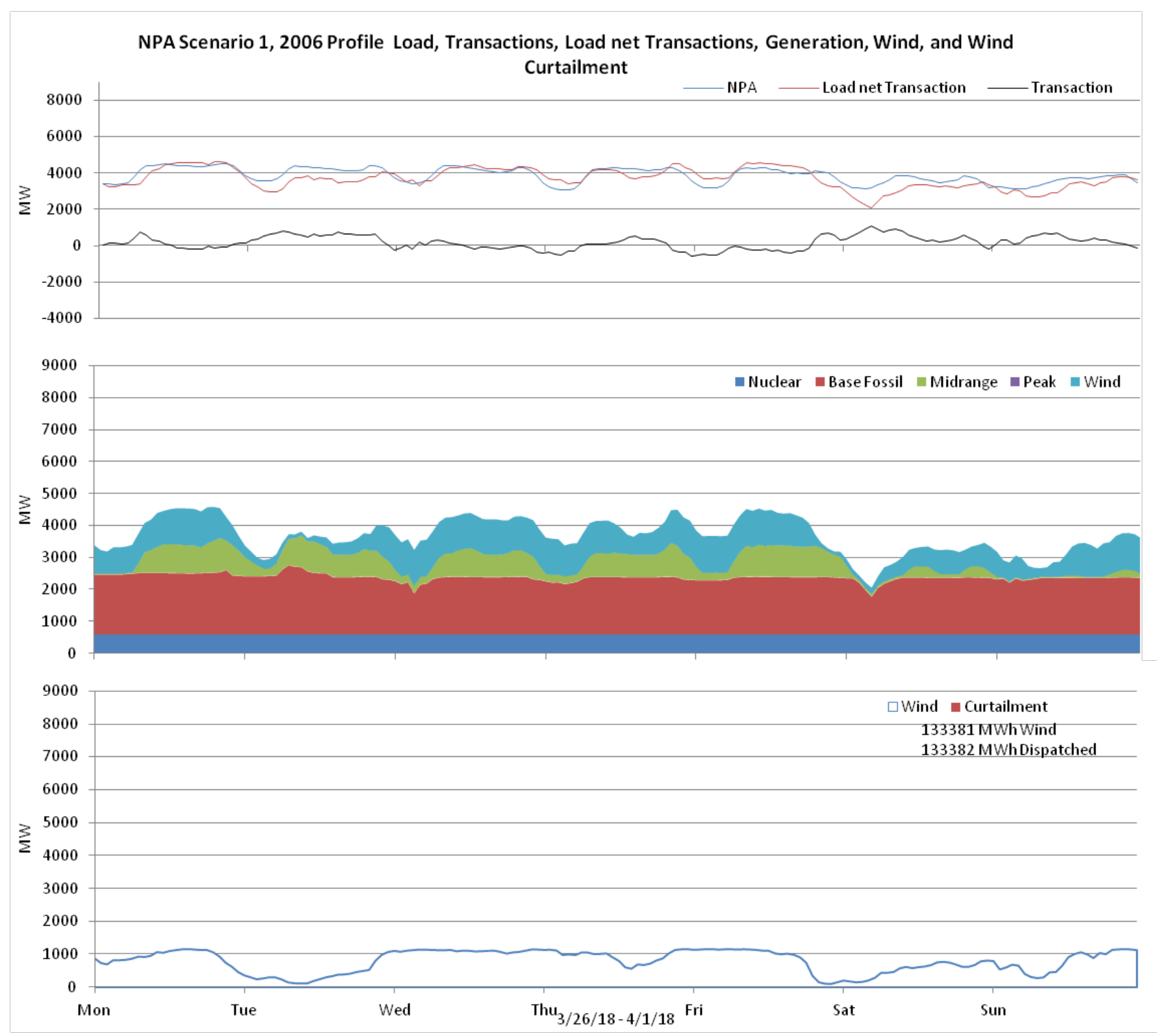

Figure 163: Nebraska 10\% wind Penetration, 2006 Profile week of 3/26/2018 


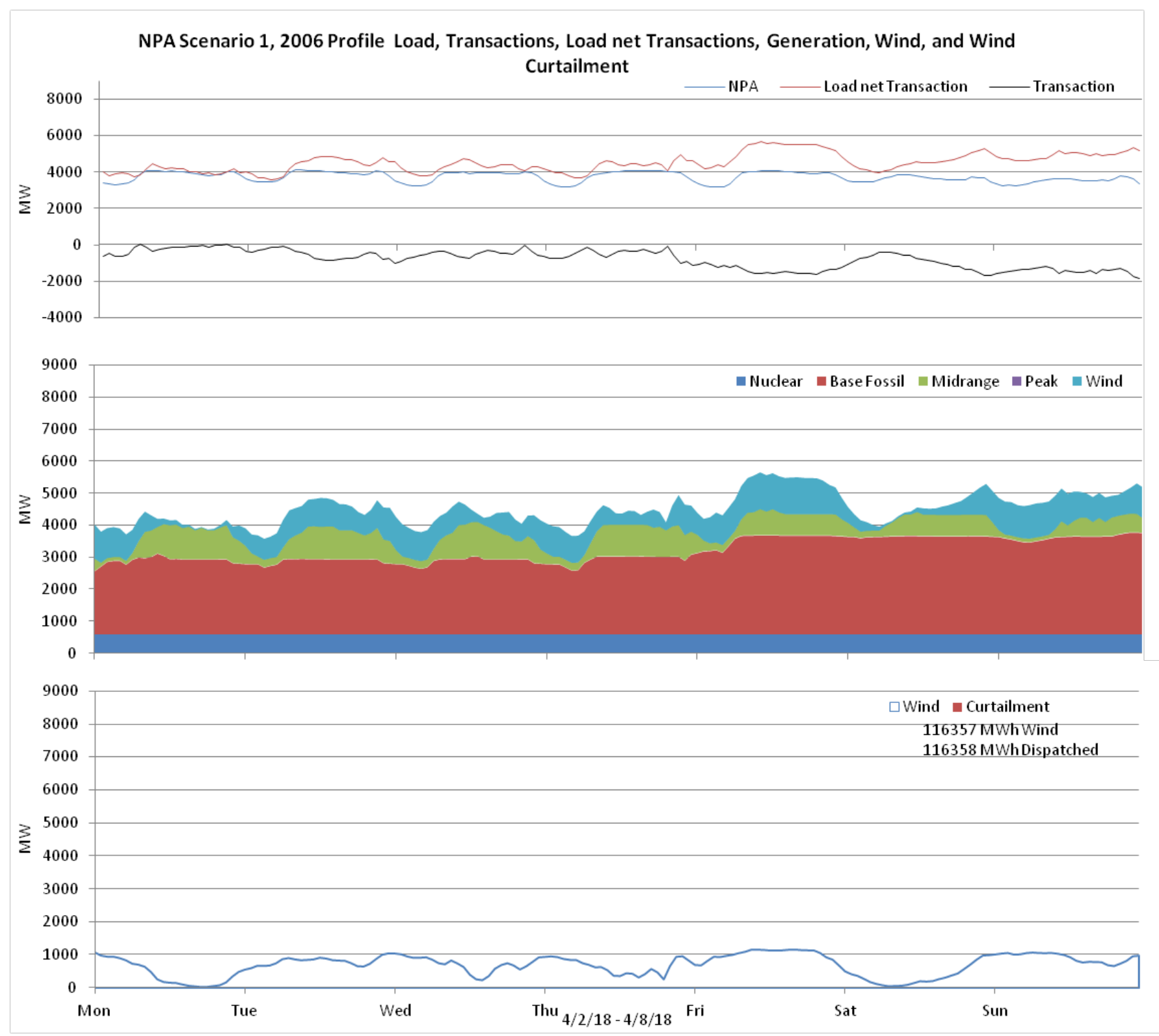

Figure 164: Nebraska 10\% wind Penetration, 2006 Profile week of 4/2/2018 


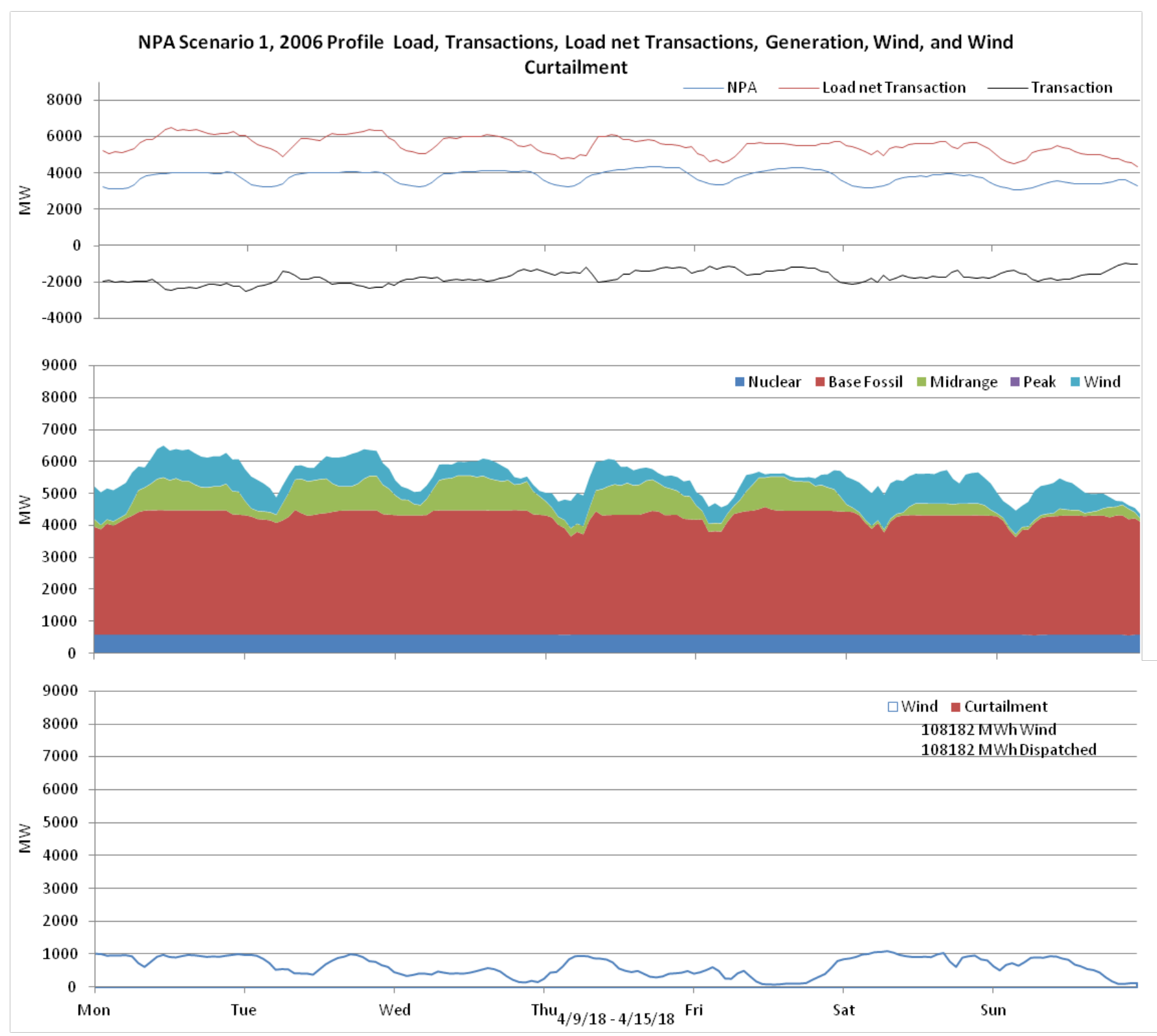

Figure 165: Nebraska 10\% wind Penetration, 2006 Profile week of 4/9/2018 


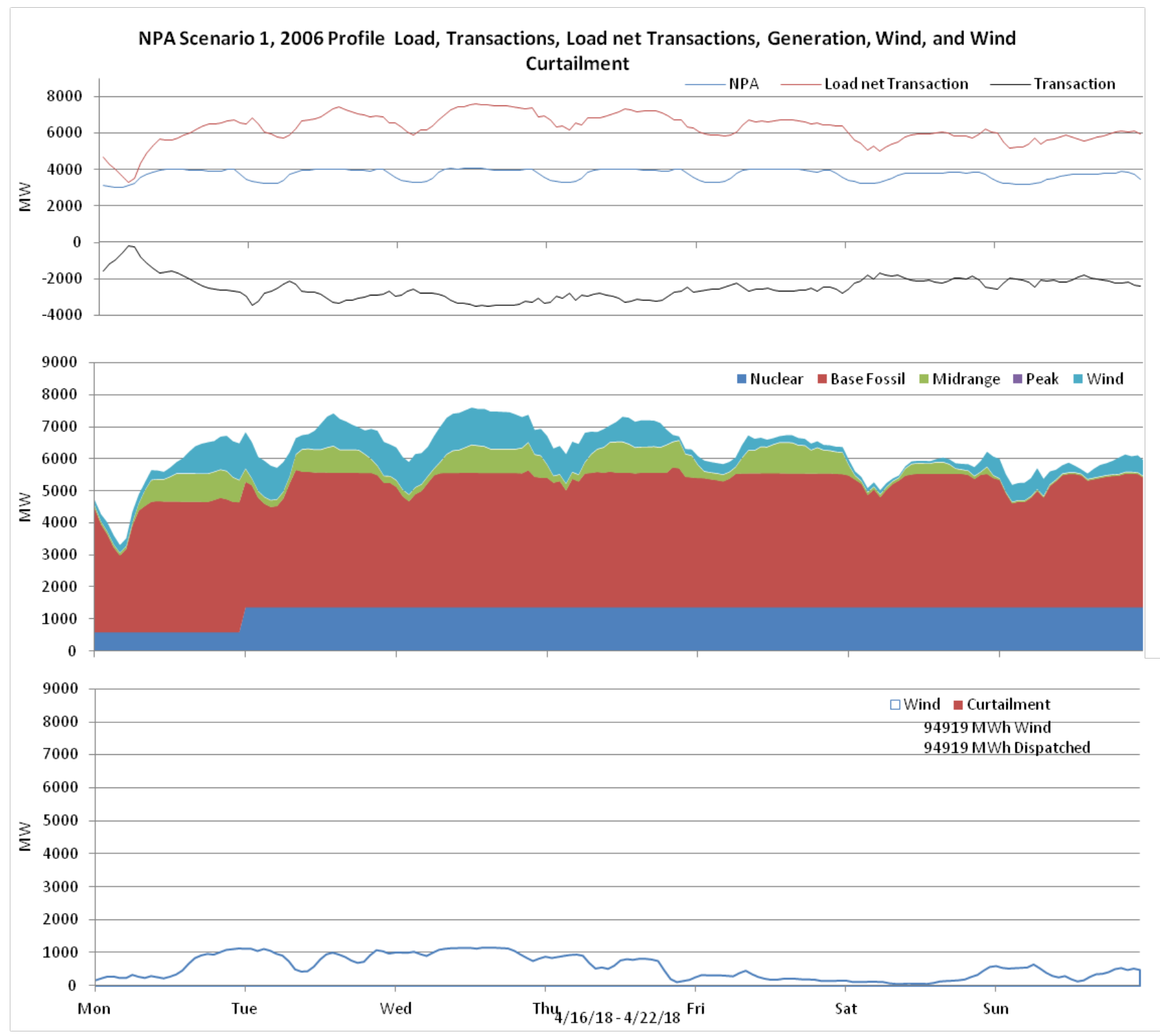

Figure 166: Nebraska 10\% wind Penetration, 2006 Profile week of 4/16/2018 


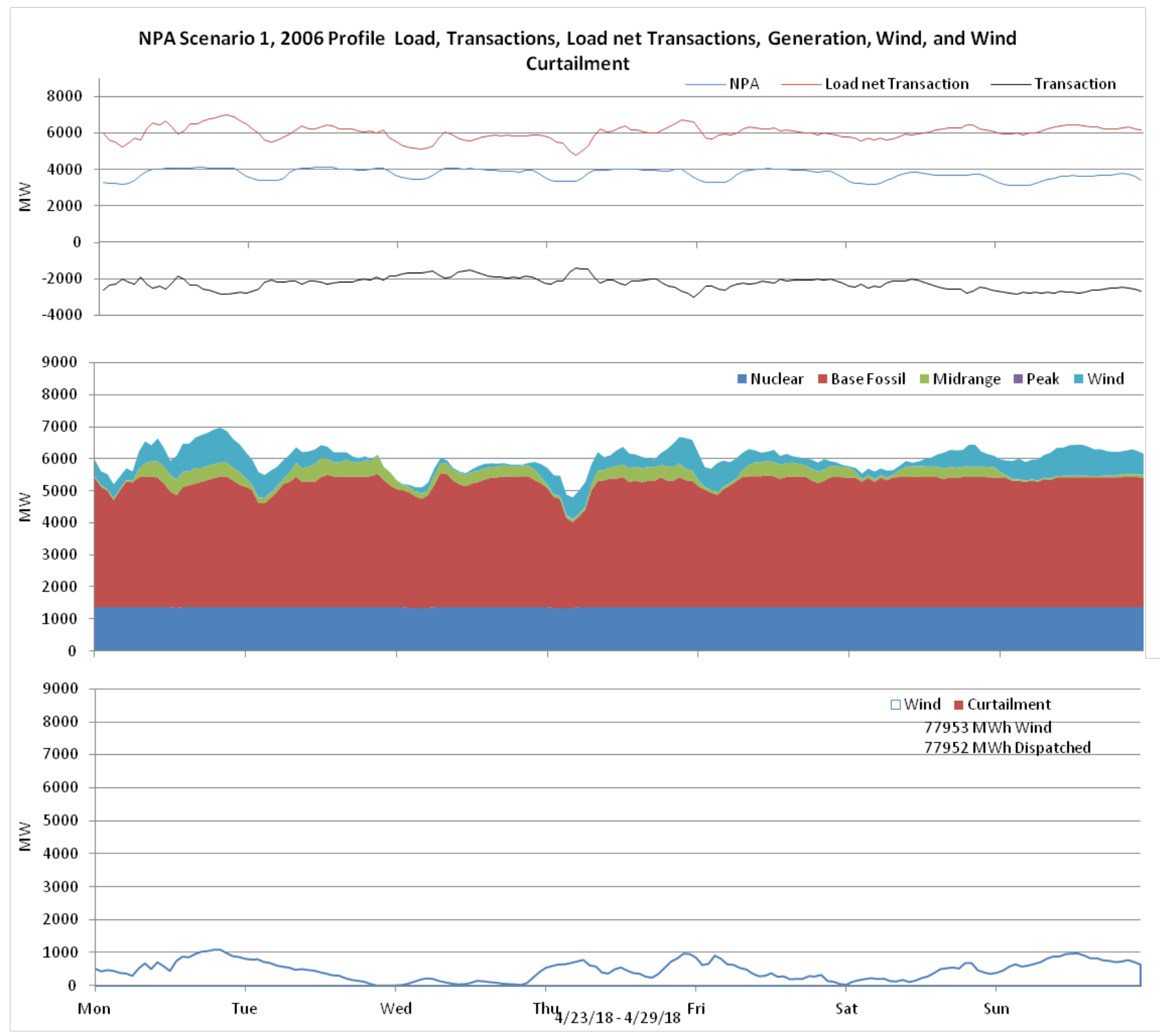

Figure 167: Nebraska 10\% wind Penetration, 2006 Profile week of 4/23/2018 


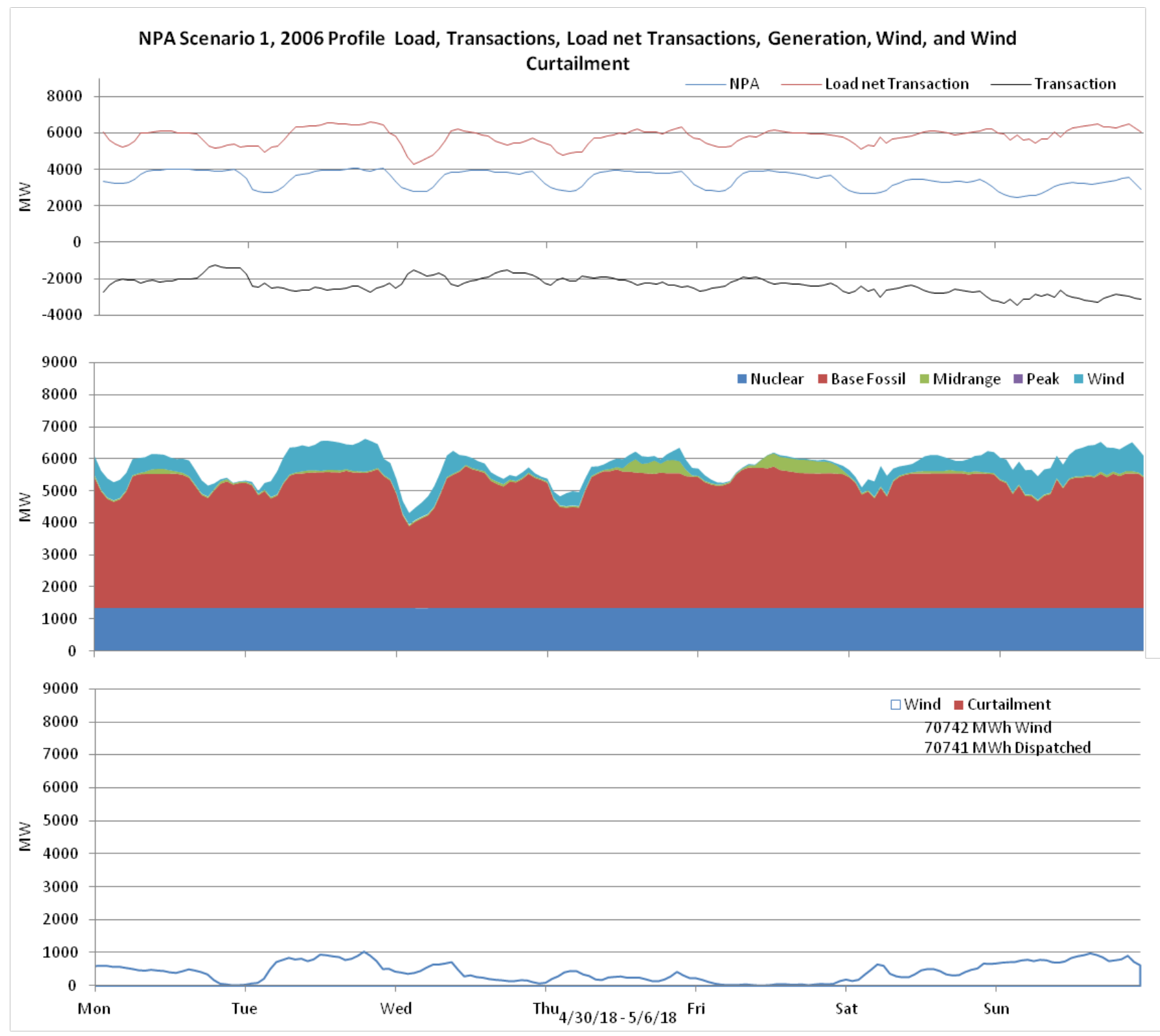

Figure 168: Nebraska 10\% wind Penetration, 2006 Profile week of 4/30/2018 


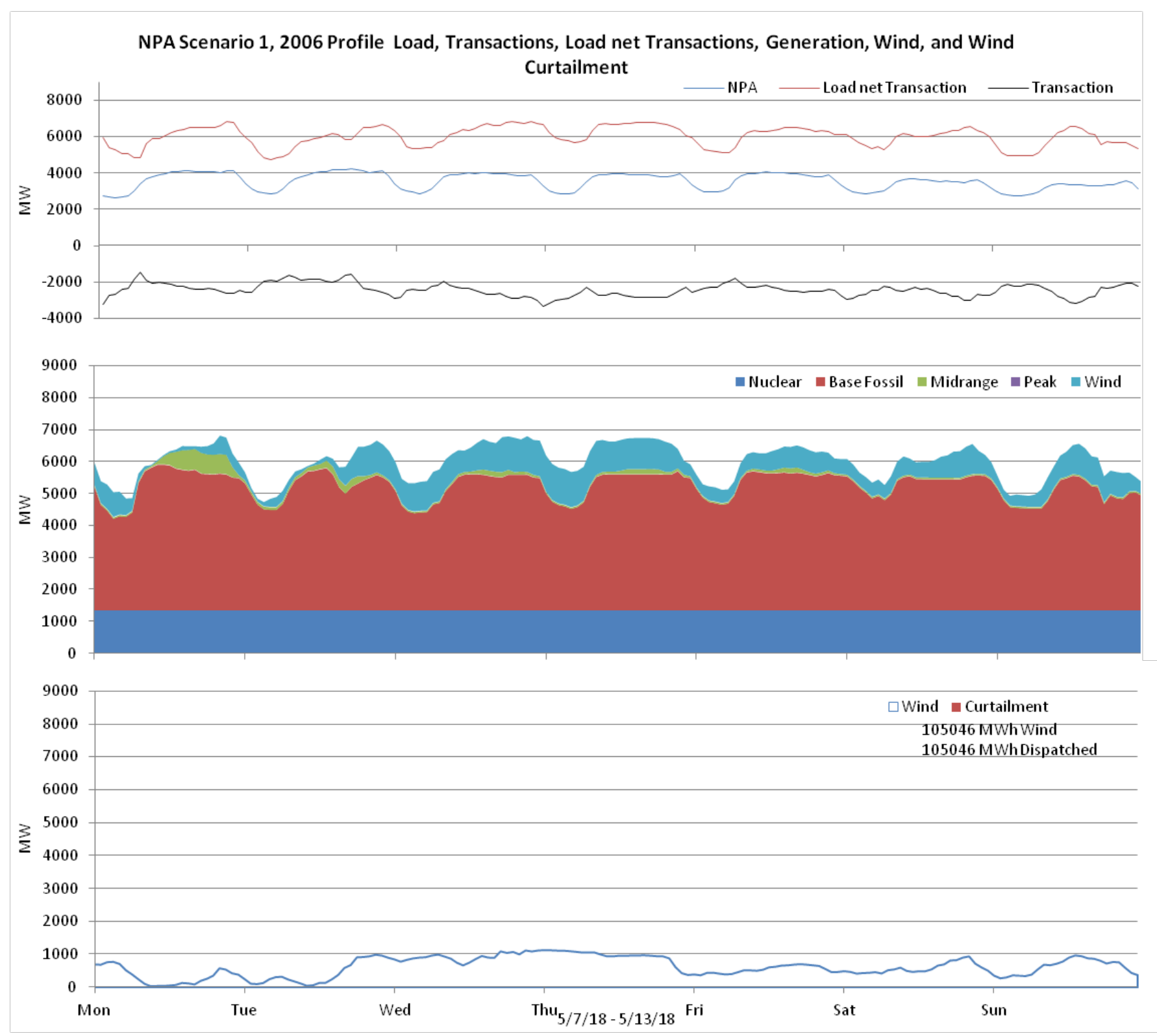

Figure 169: Nebraska 10\% wind Penetration, 2006 Profile week of 5/7/2018 


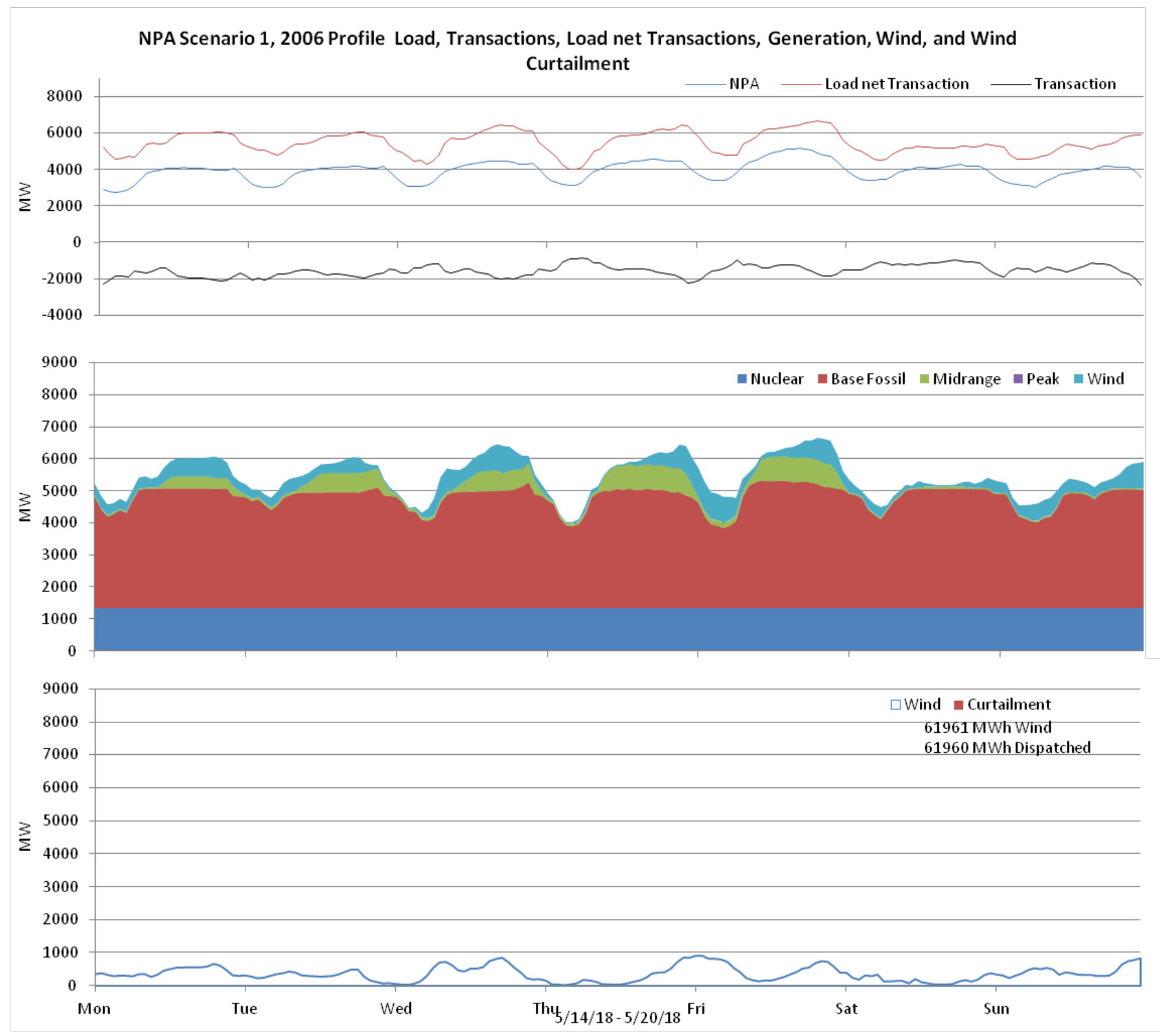

Figure 170: Nebraska 10\% wind Penetration, 2006 Profile week of 5/14/2018 


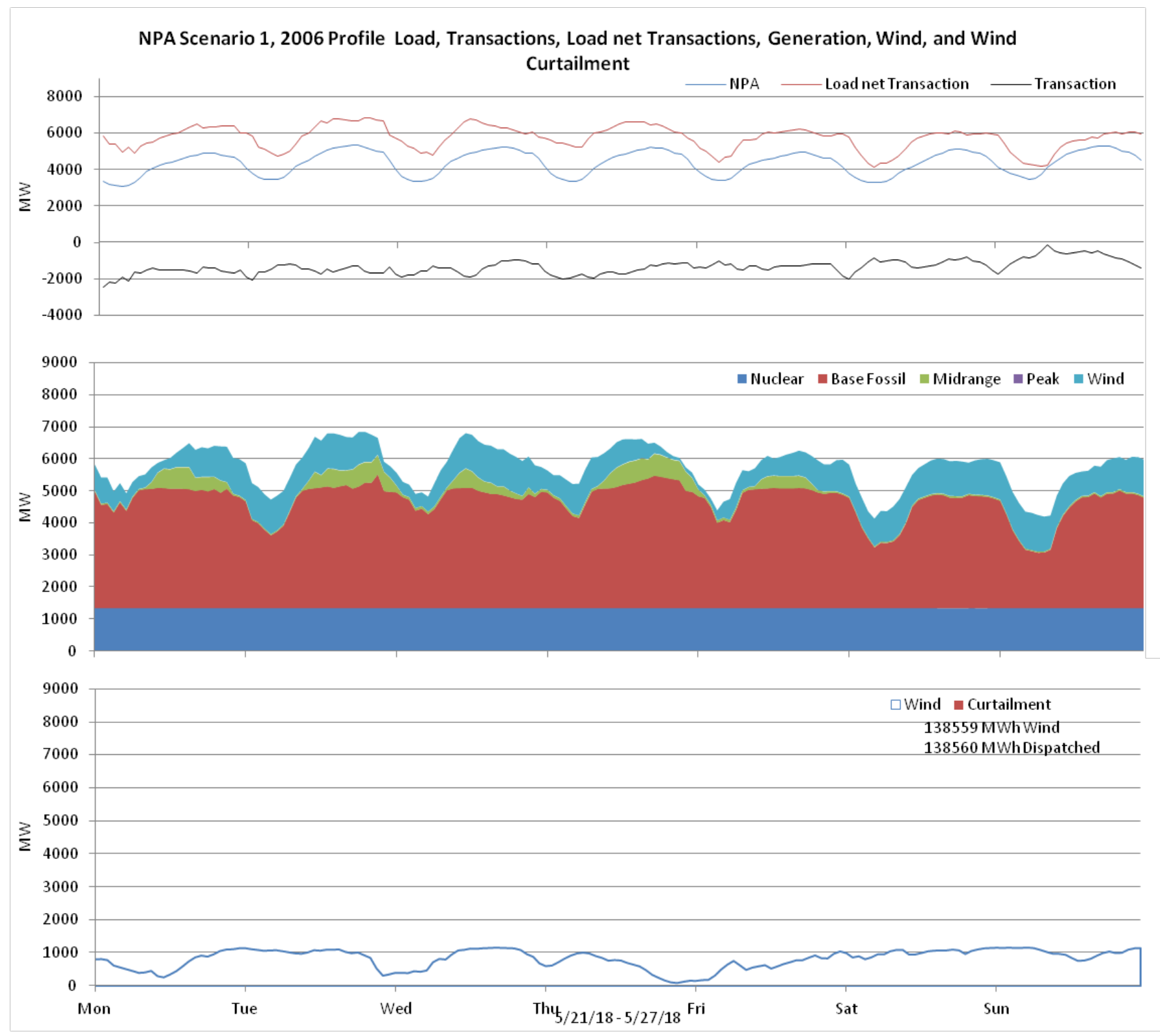

Figure 171: Nebraska 10\% wind Penetration, 2006 Profile week of 5/21/2018 


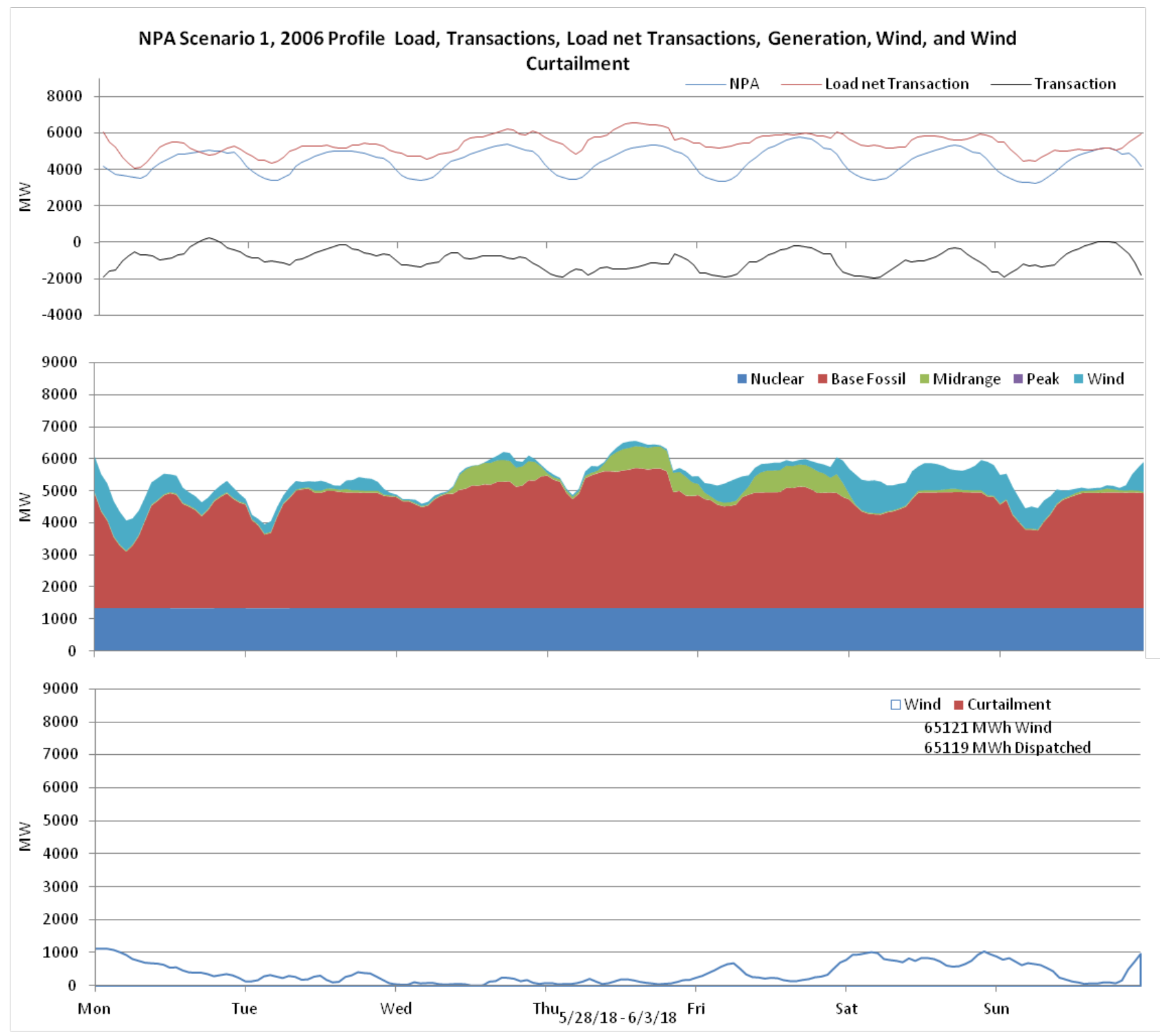

Figure 172: Nebraska 10\% wind Penetration, 2006 Profile week of 5/28/2018 


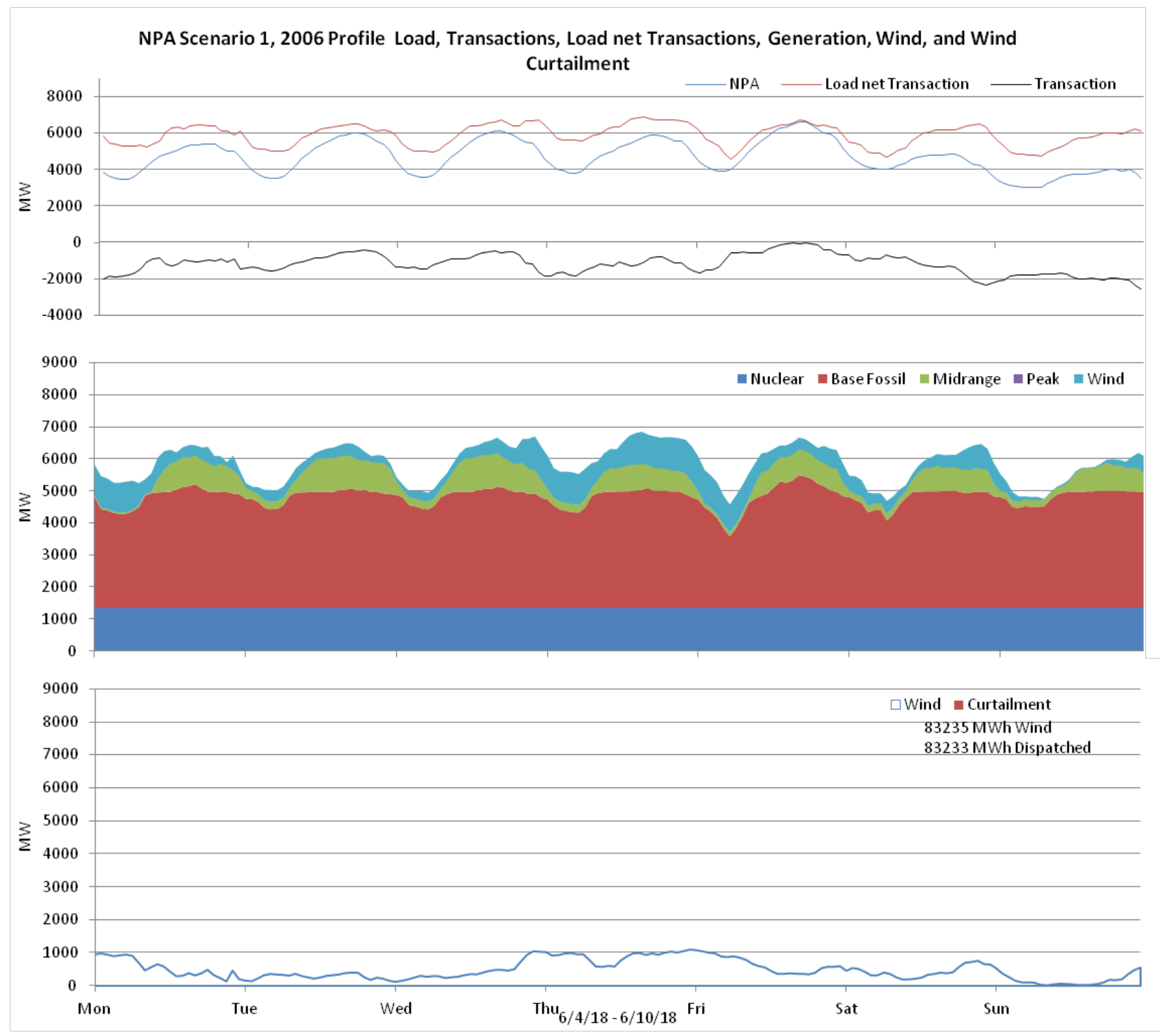

Figure 173: Nebraska 10\% wind Penetration, 2006 Profile week of 6/4/2018 


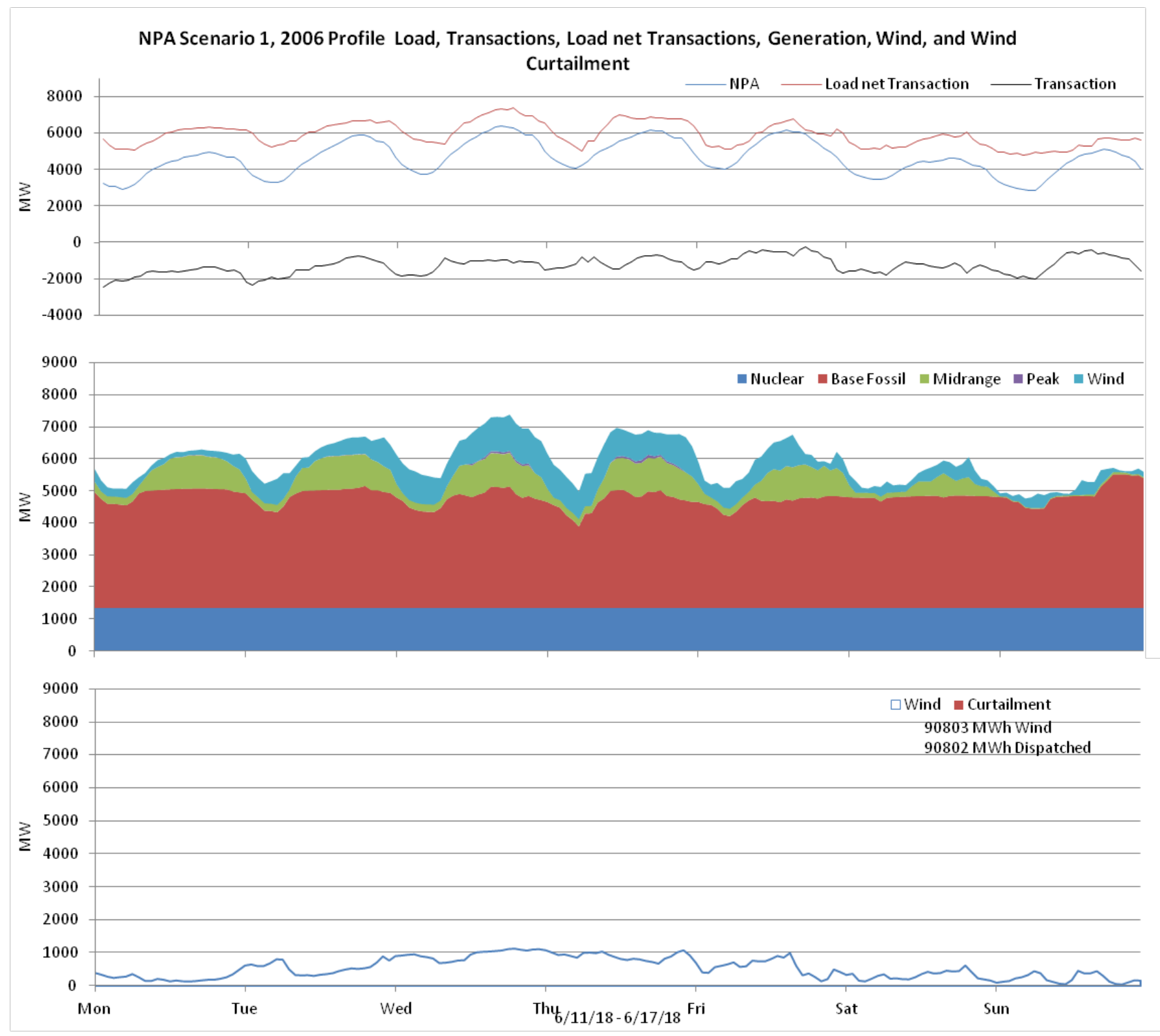

Figure 174: Nebraska 10\% wind Penetration, 2006 Profile week of 6/11/2018 


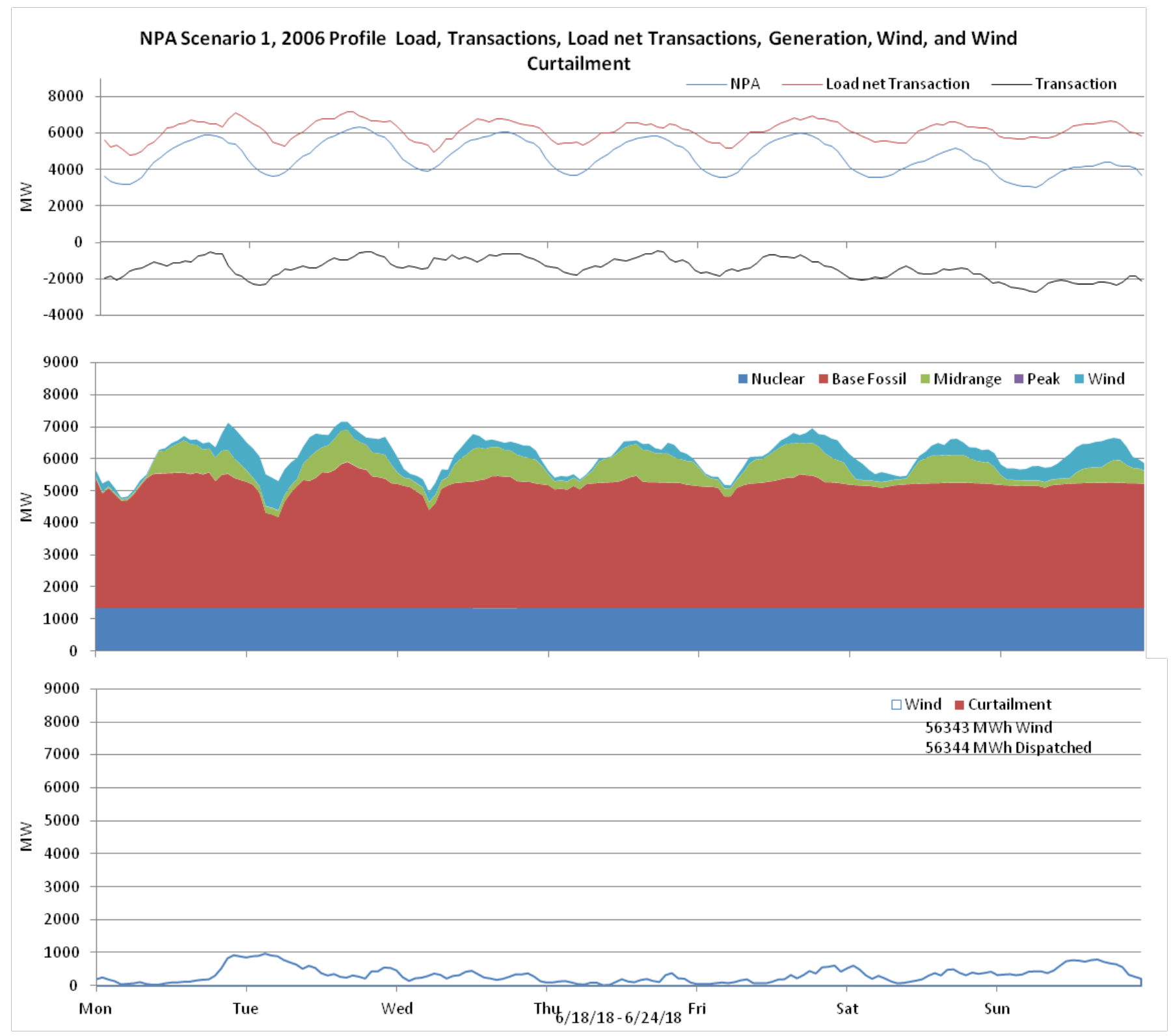

Figure 175: Nebraska 10\% wind Penetration, 2006 Profile week of 6/18/2018 


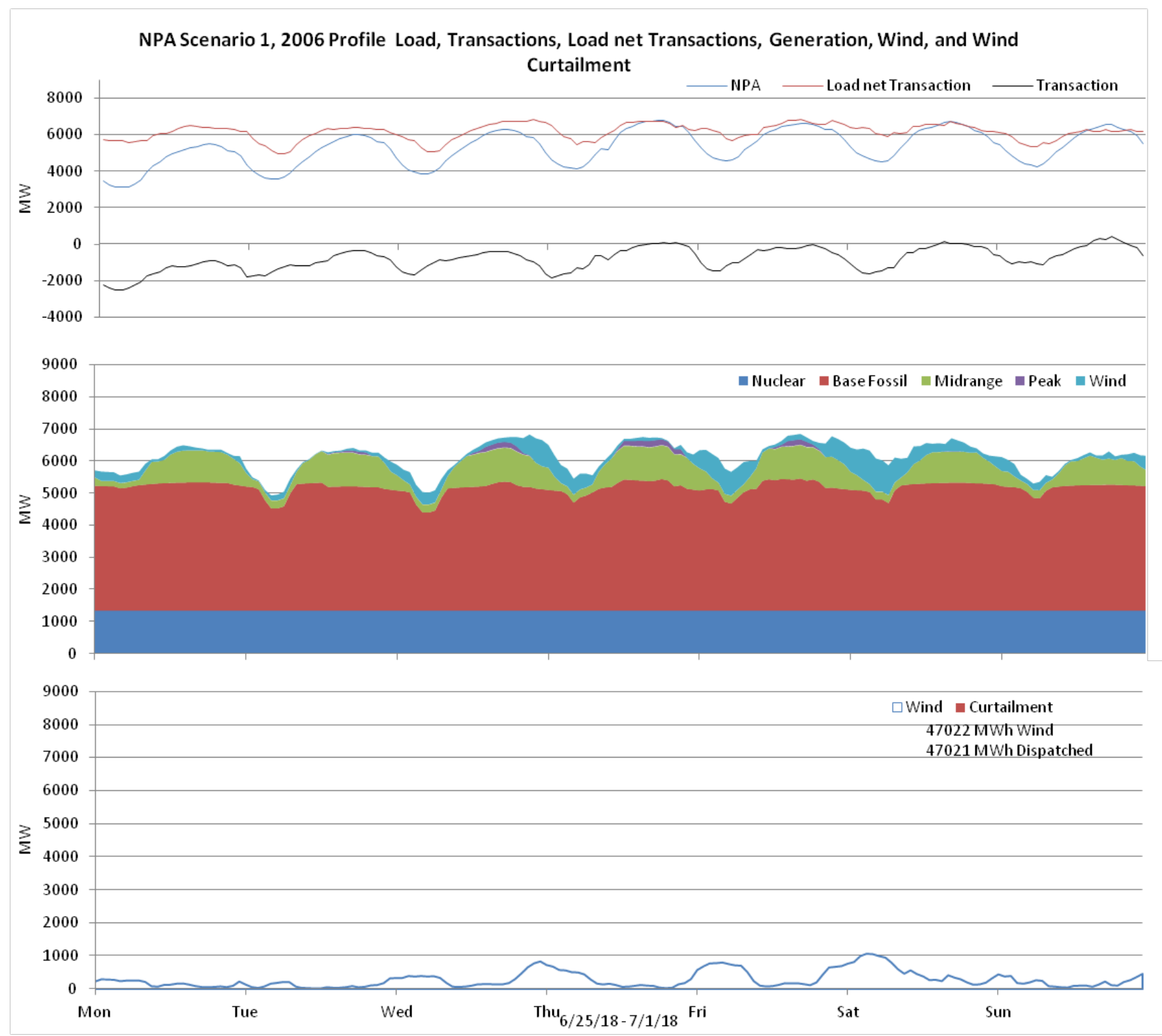

Figure 176: Nebraska 10\% wind Penetration, 2006 Profile week of 6/25/2018 


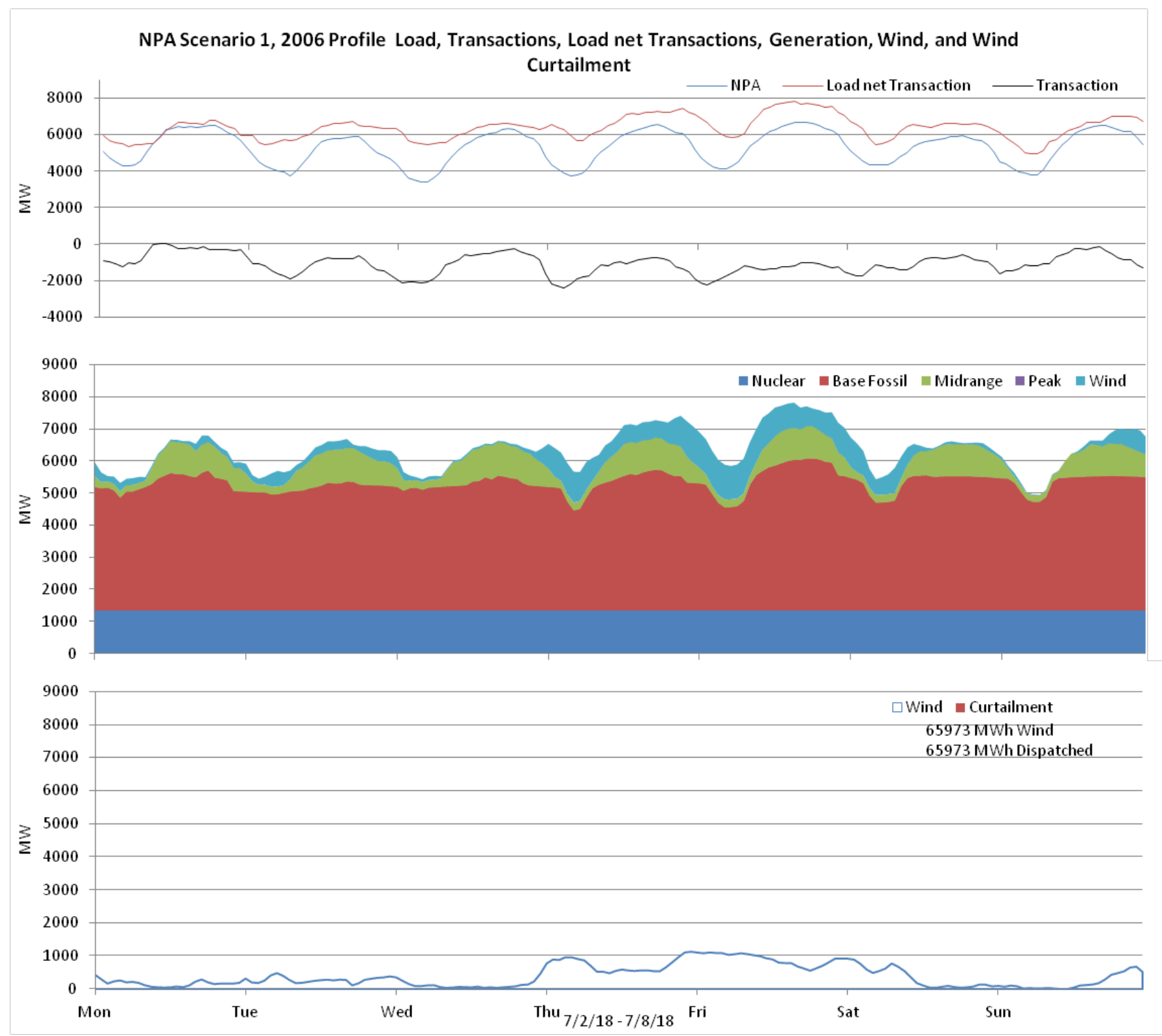

Figure 177: Nebraska 10\% wind Penetration, 2006 Profile week of 7/2/2018 


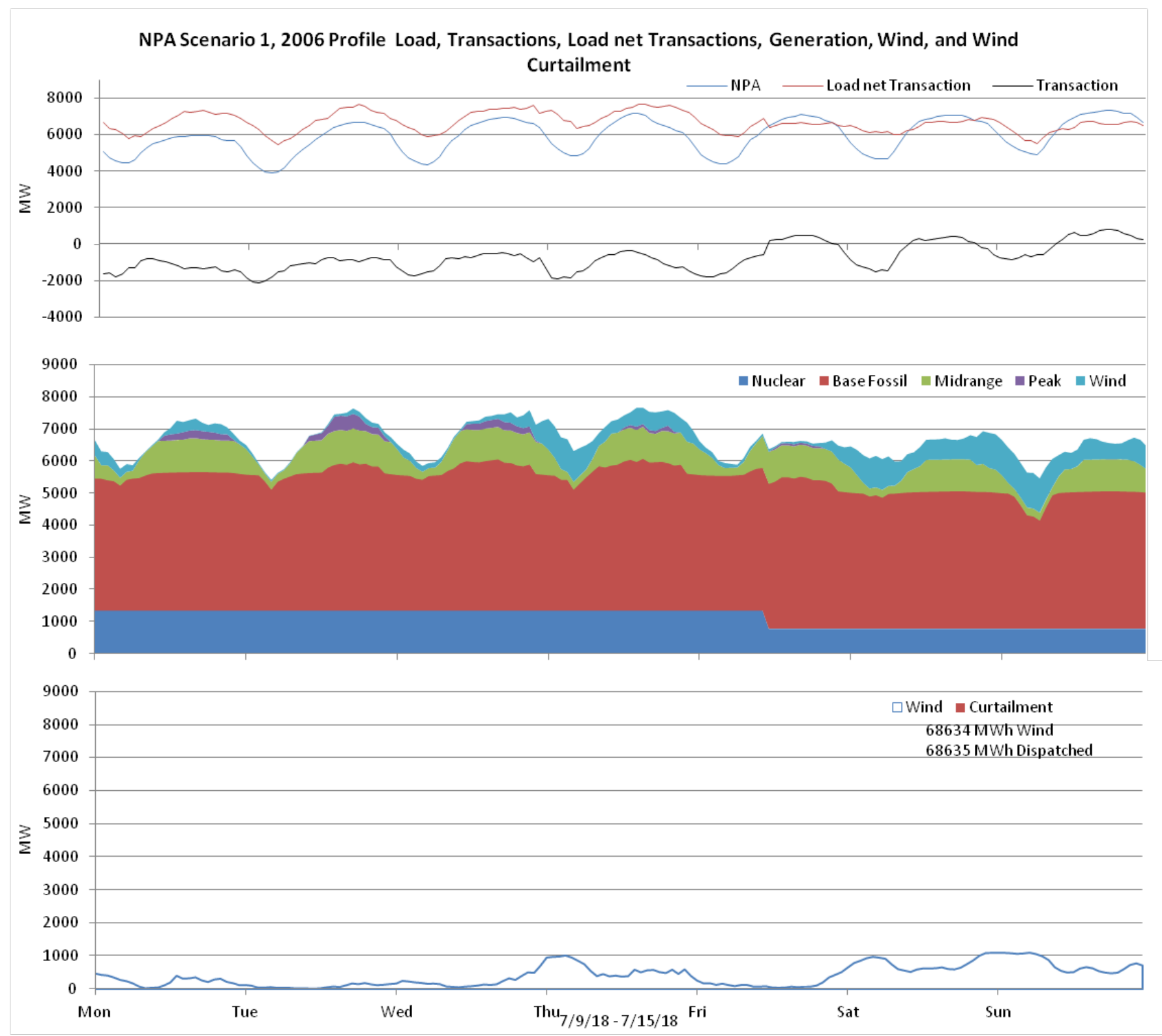

Figure 178: Nebraska 10\% wind Penetration, 2006 Profile week of 7/9/2018 


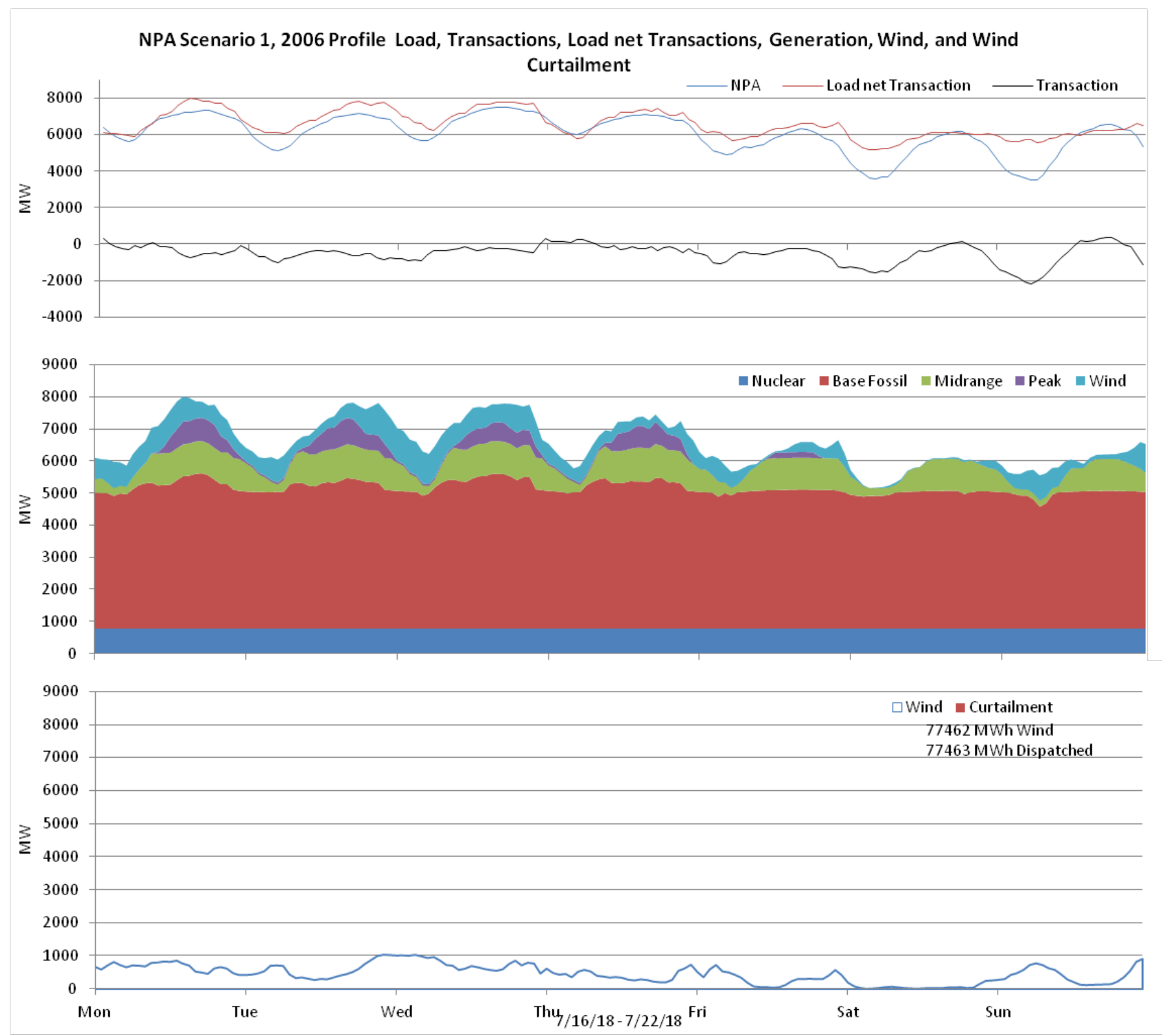

Figure 179: Nebraska 10\% wind Penetration, 2006 Profile week of 7/16/2018 


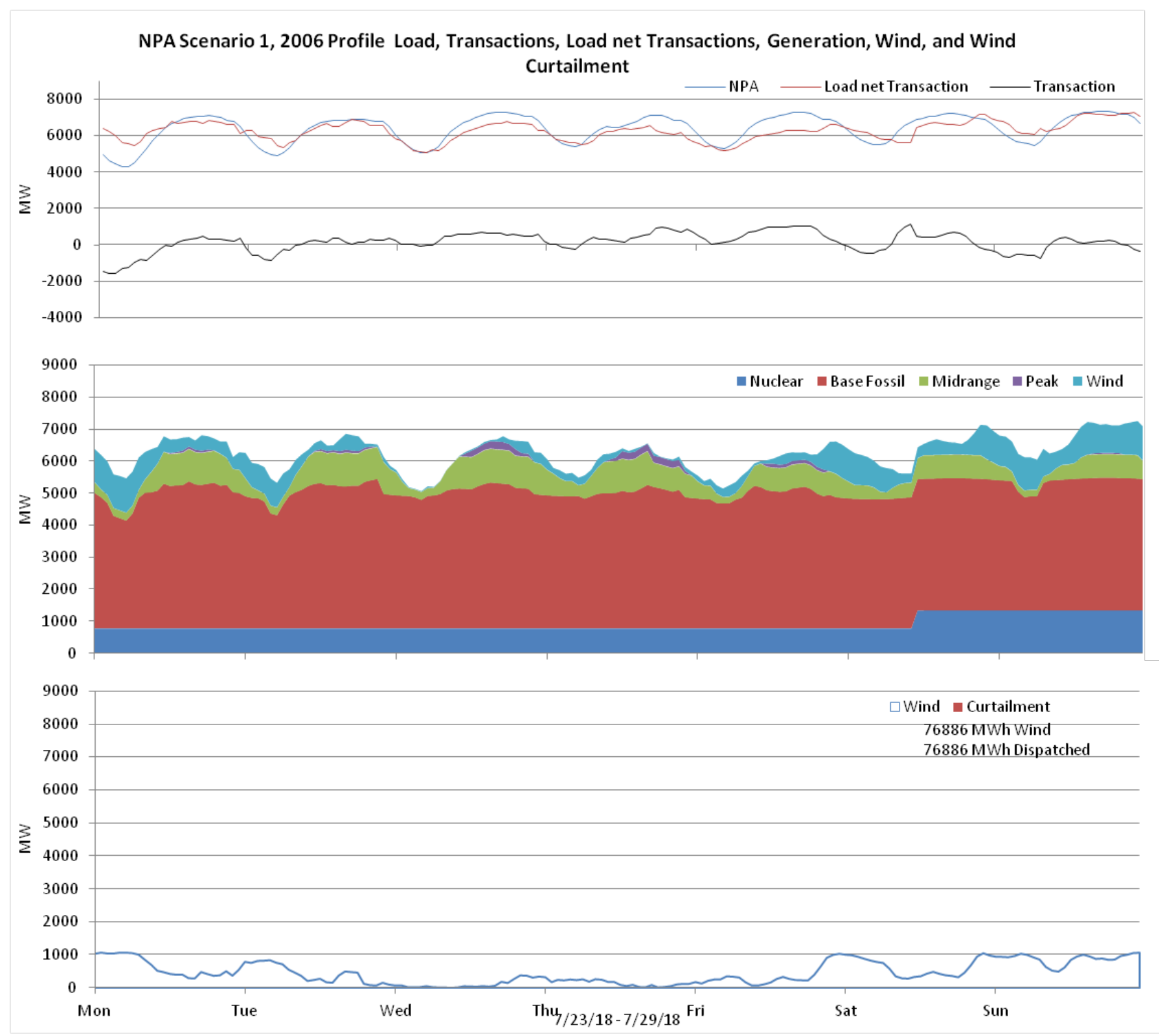

Figure 180: Nebraska 10\% wind Penetration, 2006 Profile week of 7/23/2018 


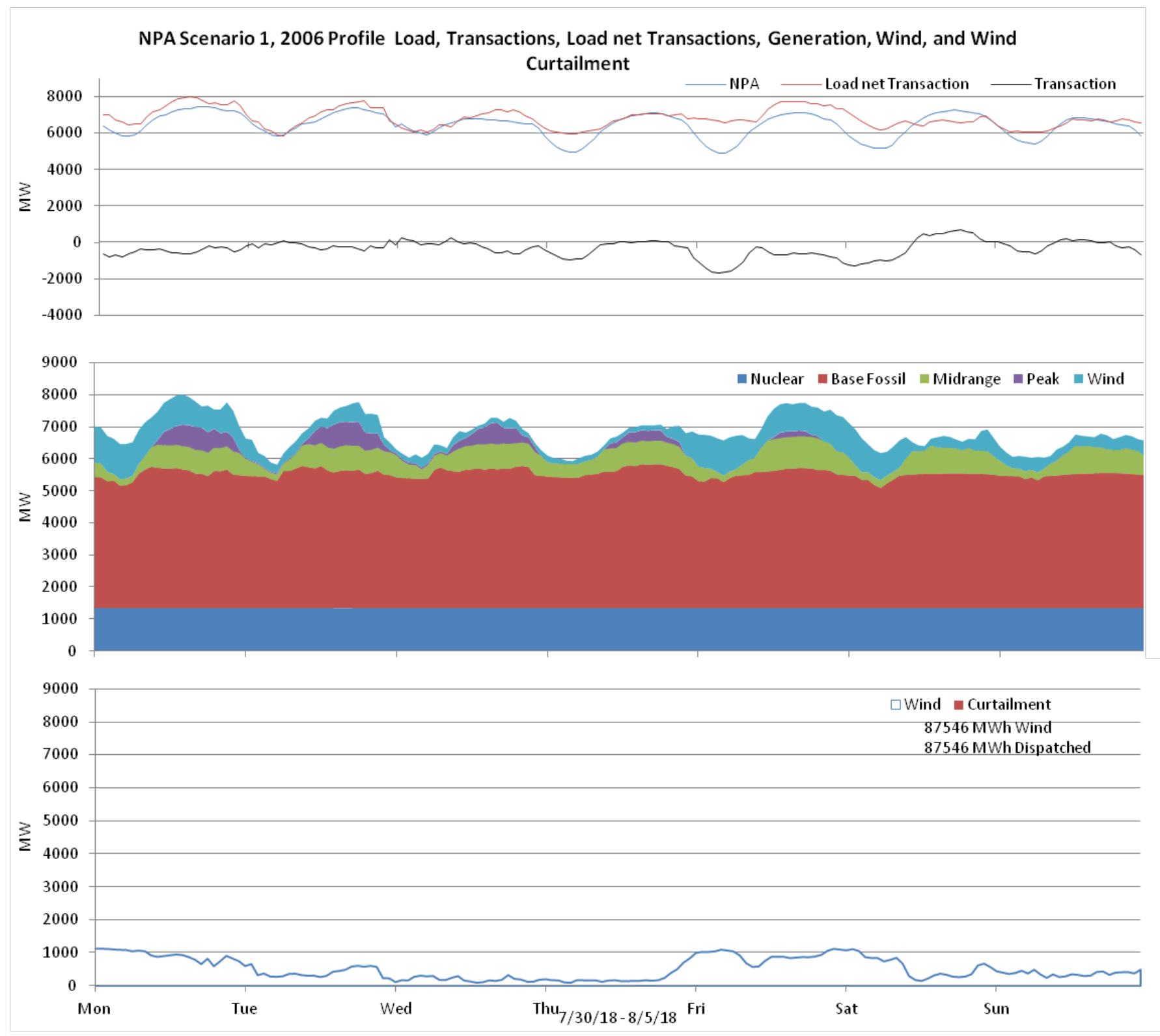

Figure 181: Nebraska 10\% wind Penetration, 2006 Profile week of 7/30/2018 


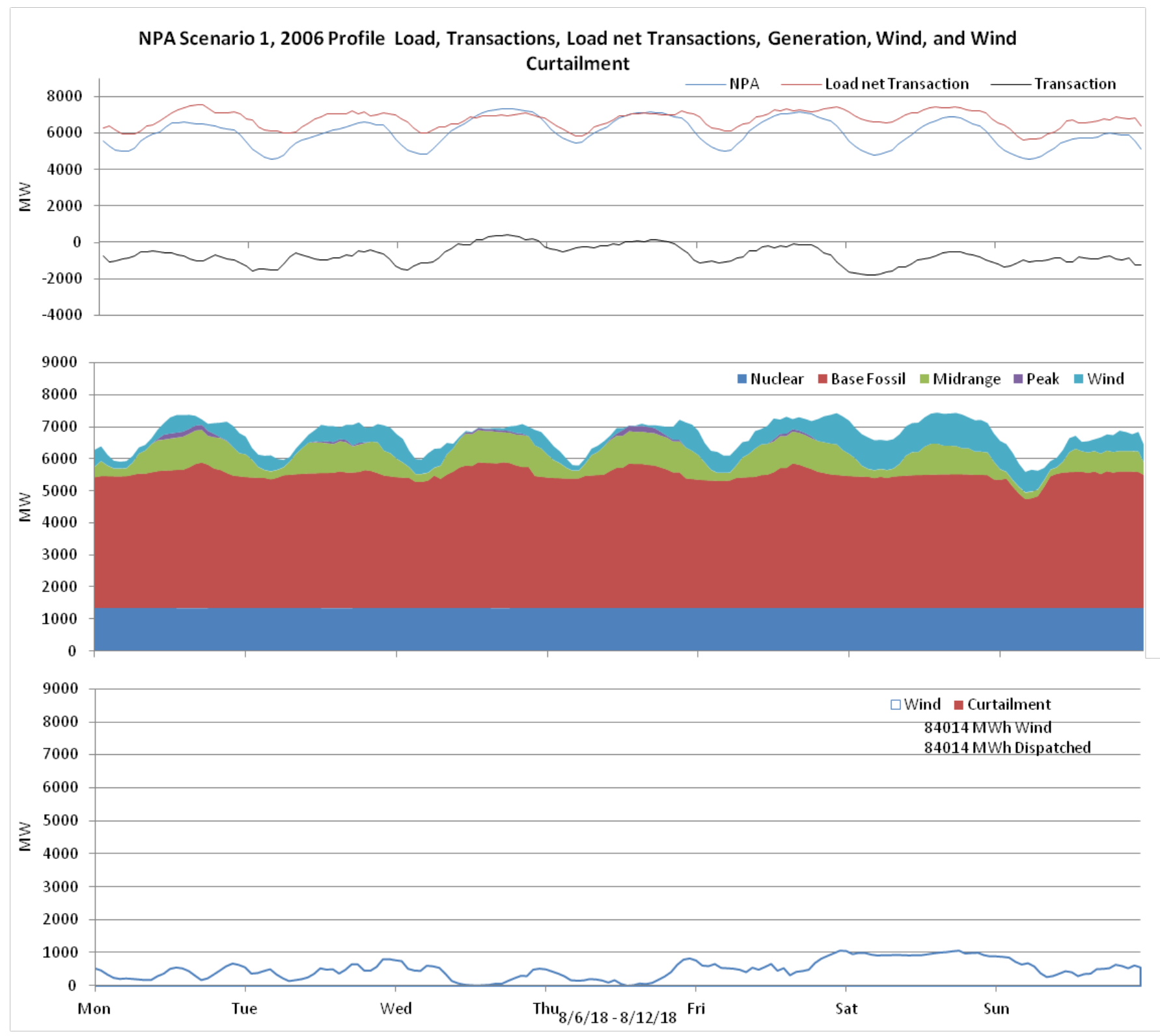

Figure 182: Nebraska 10\% wind Penetration, 2006 Profile week of $8 / 6 / 2018$ 


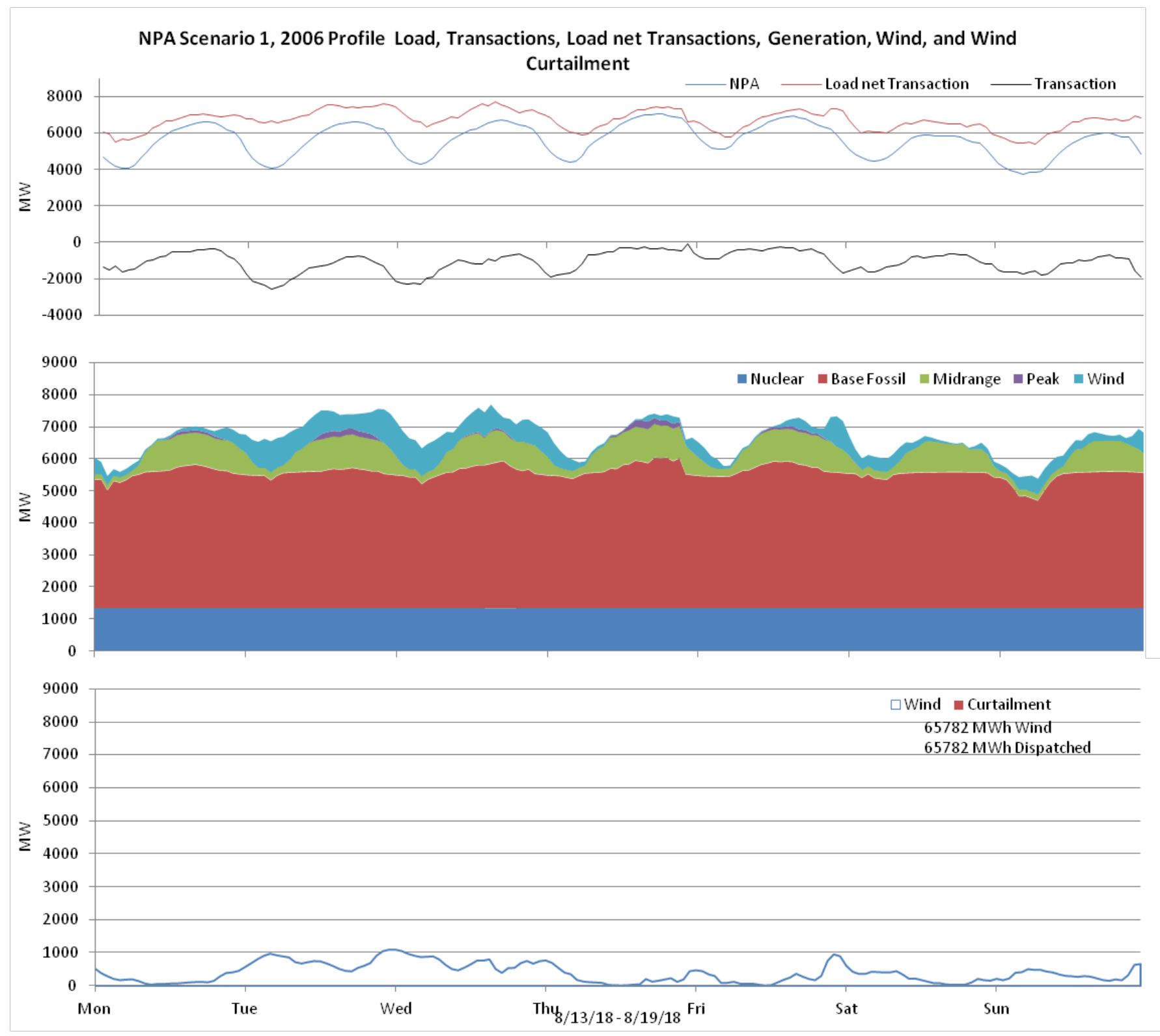

Figure 183: Nebraska 10\% wind Penetration, 2006 Profile week of 8/13/2018 


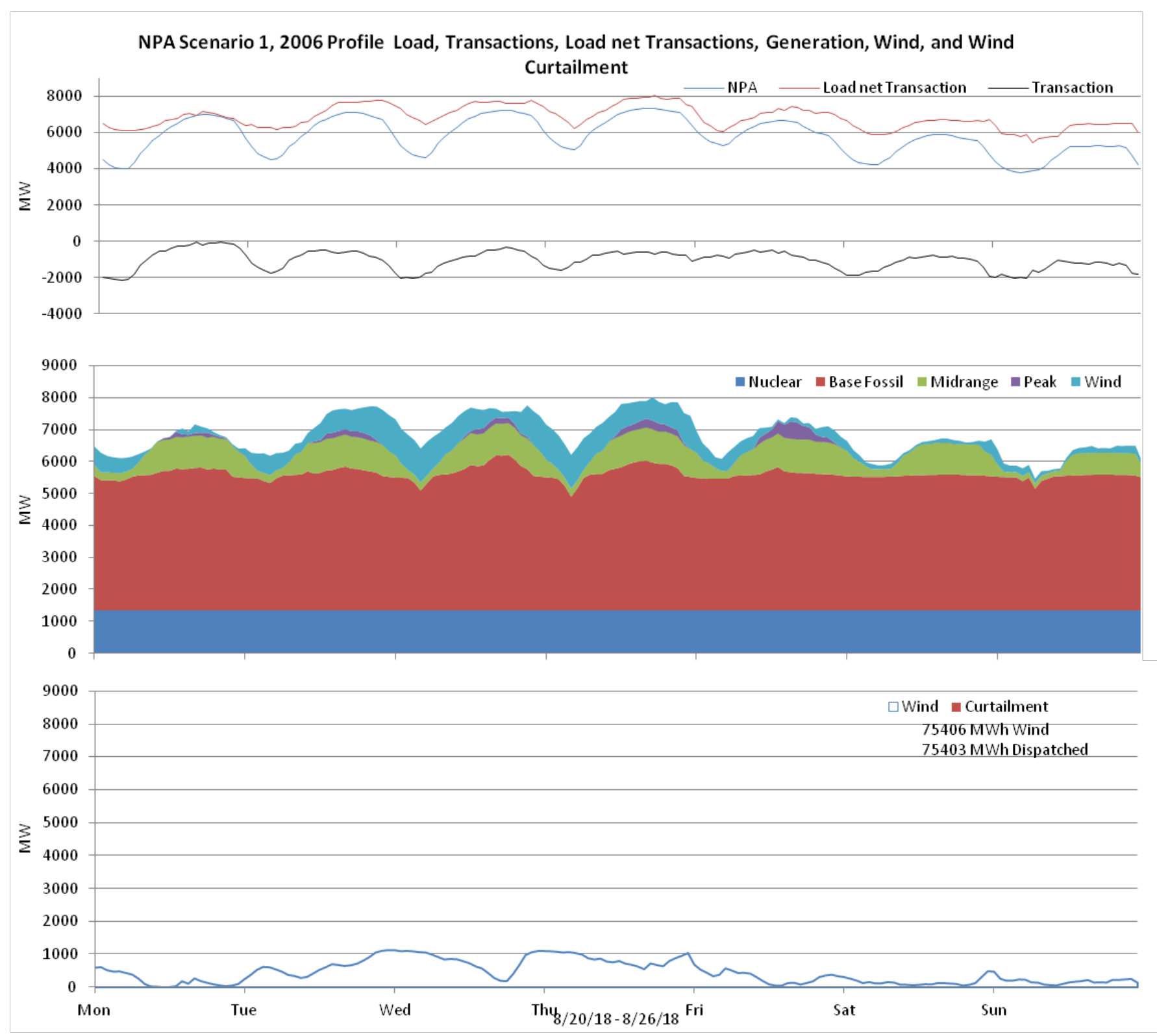

Figure 184: Nebraska 10\% wind Penetration, 2006 Profile week of 8/20/2018 


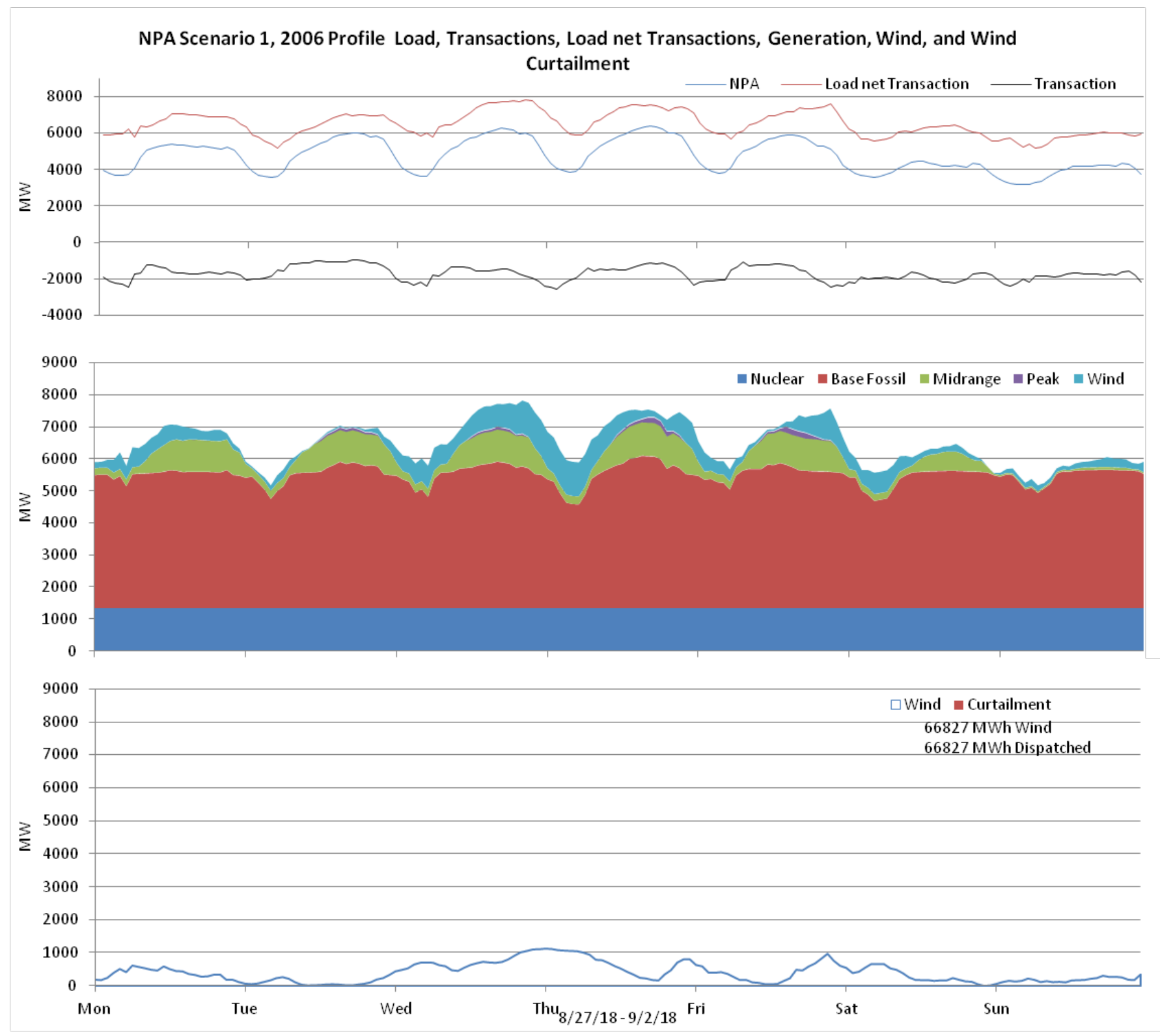

Figure 185: Nebraska 10\% wind Penetration, 2006 Profile week of $8 / 27 / 2018$ 


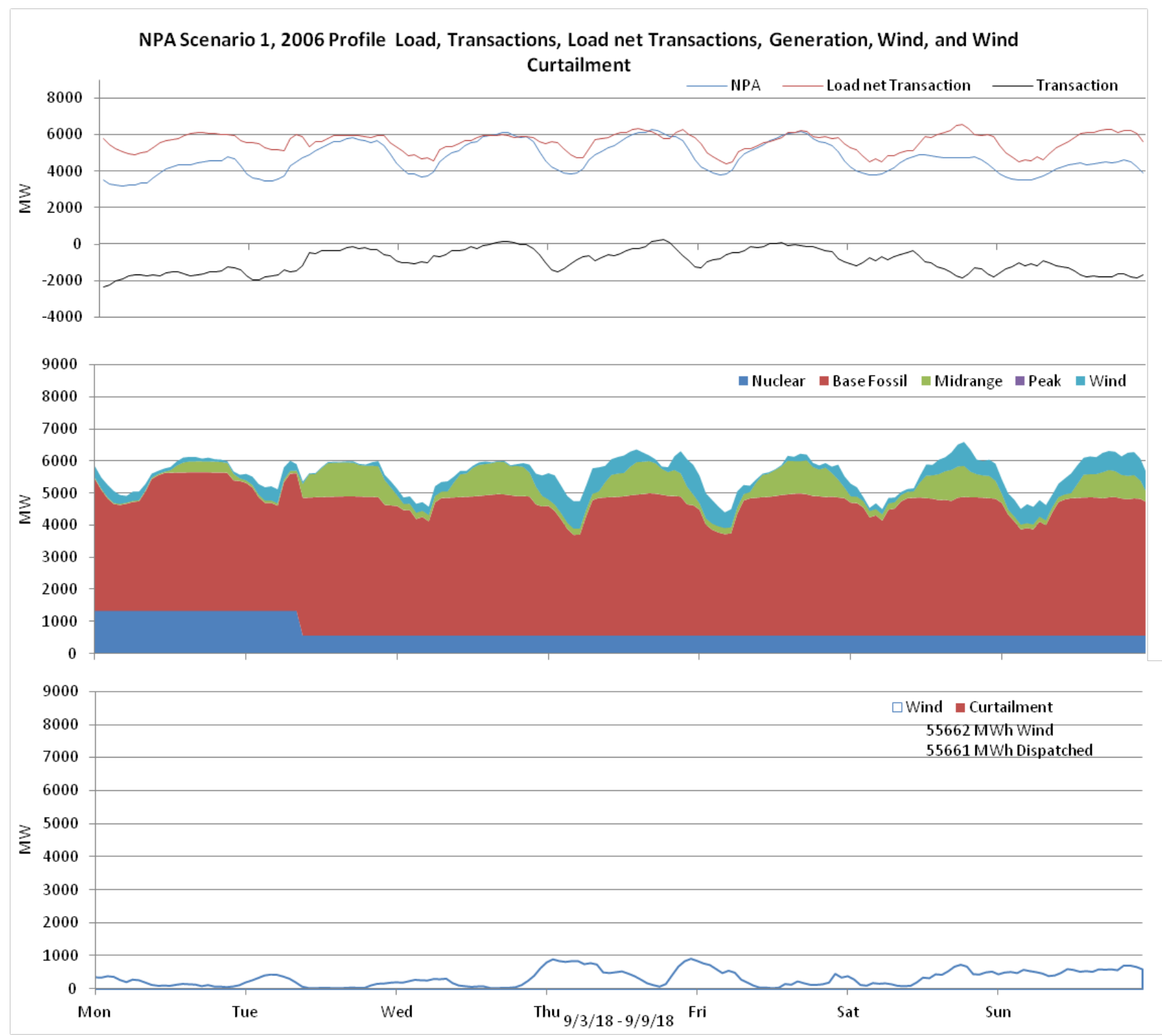

Figure 186: Nebraska 10\% wind Penetration, 2006 Profile week of 9/3/2018 


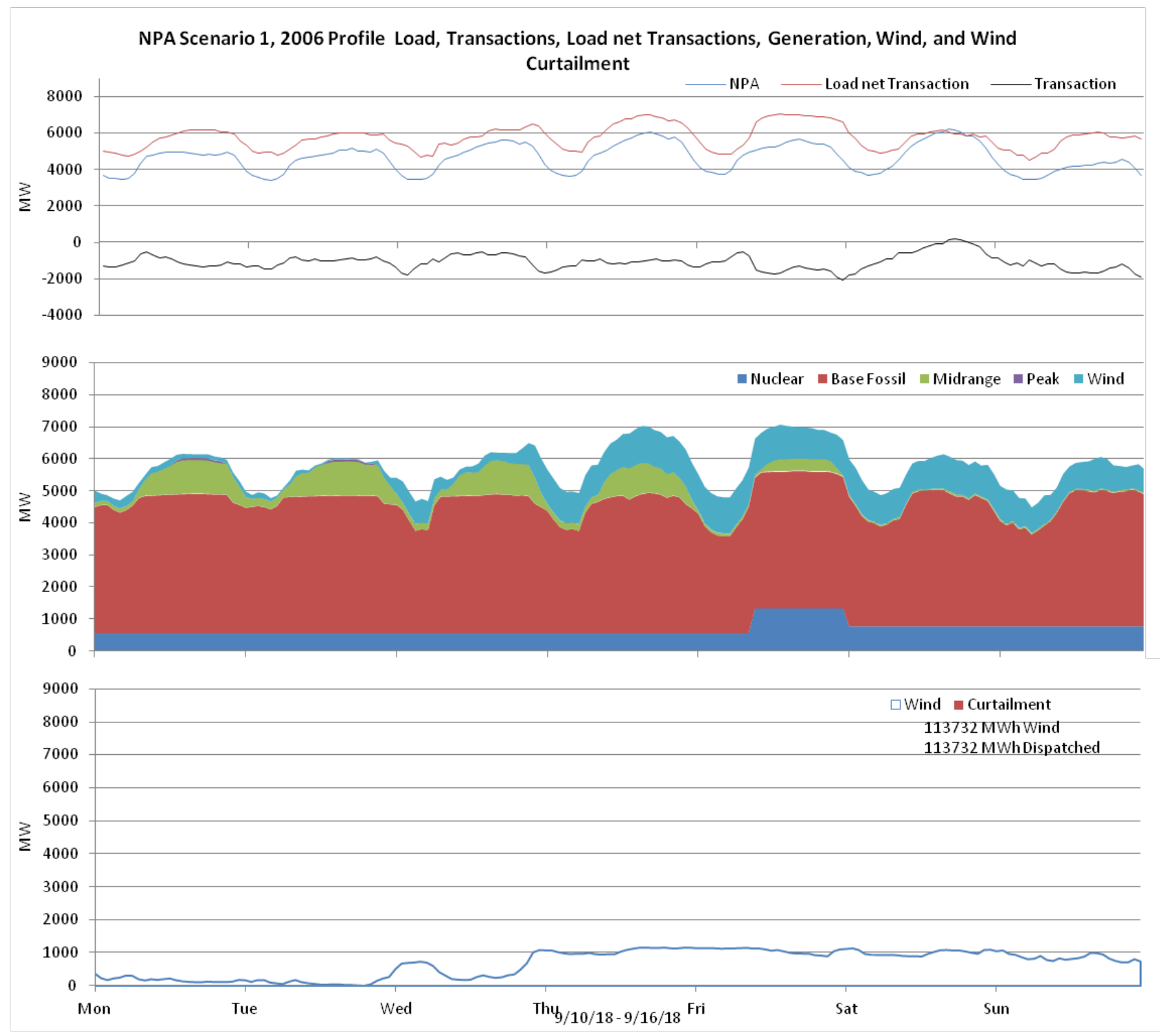

Figure 187: Nebraska 10\% wind Penetration, 2006 Profile week of 9/10/2018 


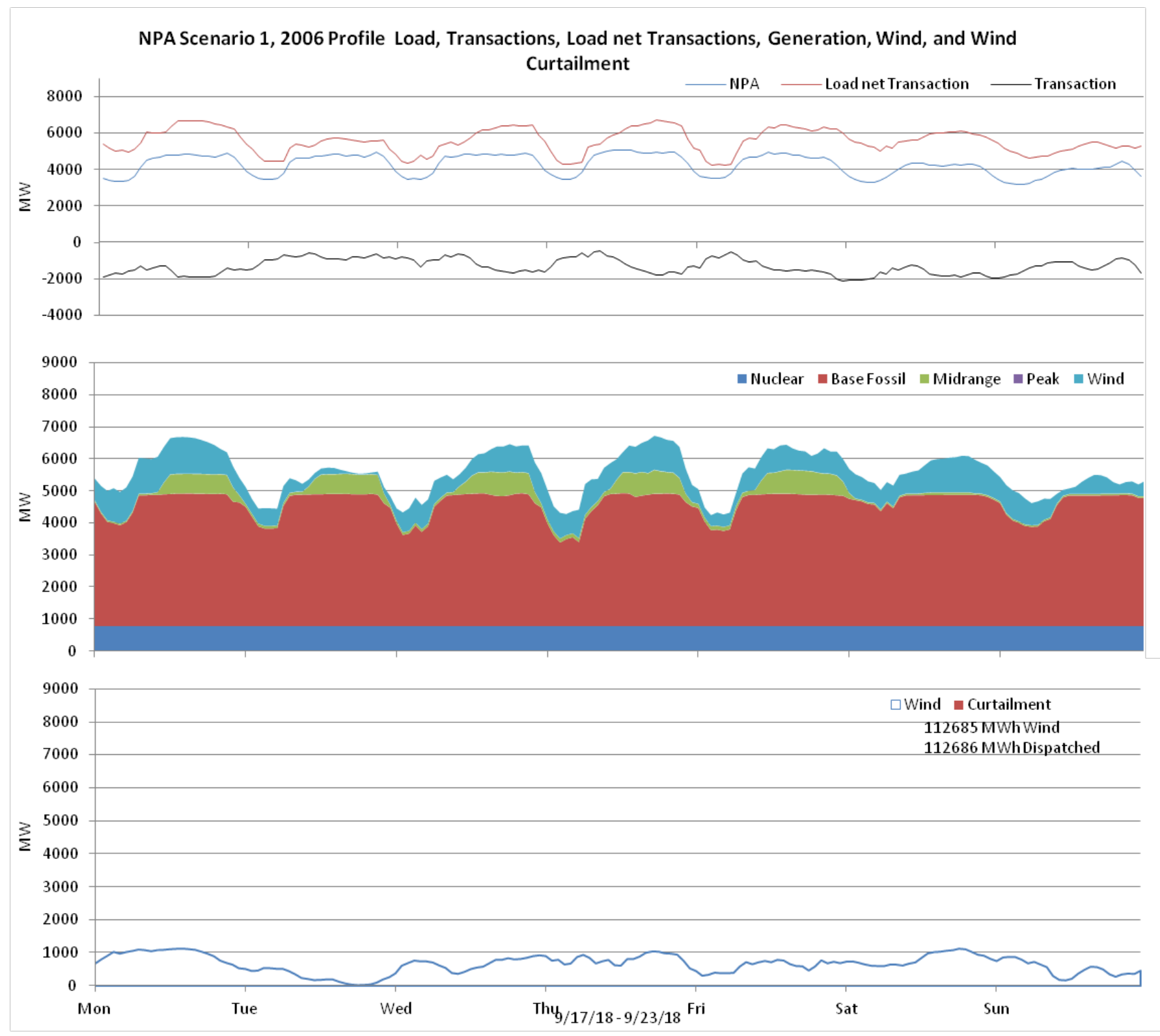

Figure 188: Nebraska 10\% wind Penetration, 2006 Profile week of 9/17/2018 


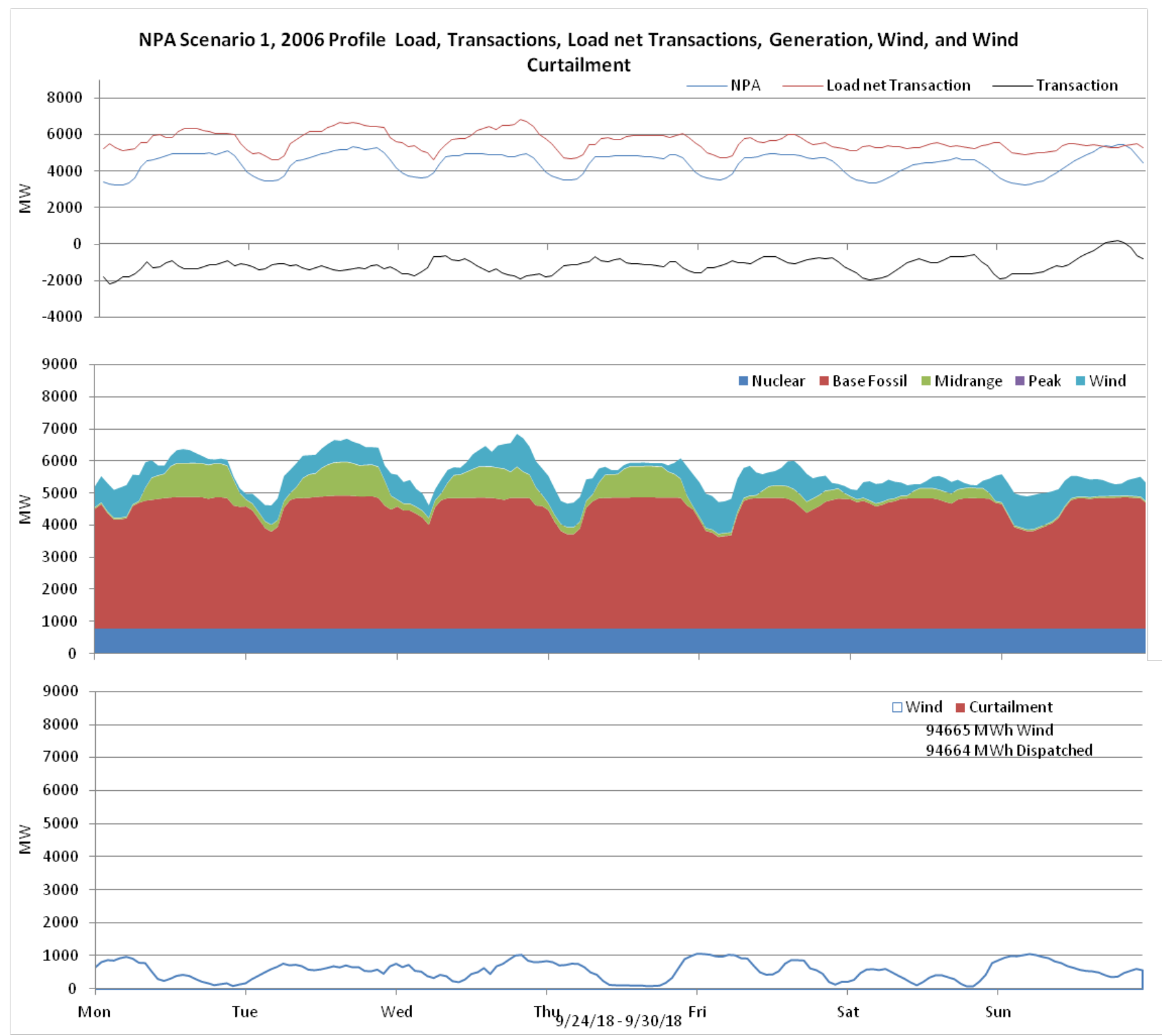

Figure 189: Nebraska 10\% wind Penetration, 2006 Profile week of 9/24/2018 


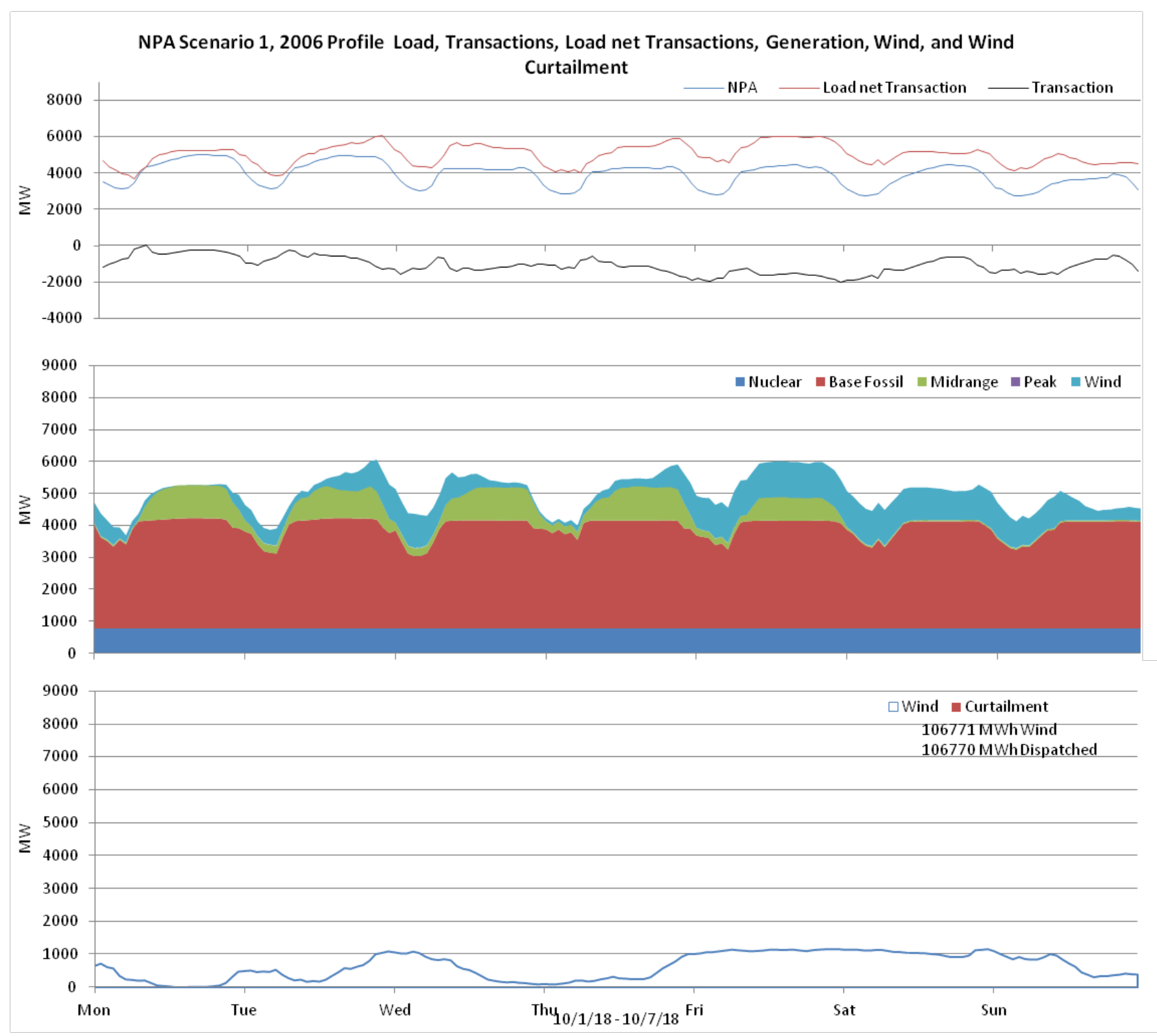

Figure 190: Nebraska 10\% wind Penetration, 2006 Profile week of 10/1/2018 


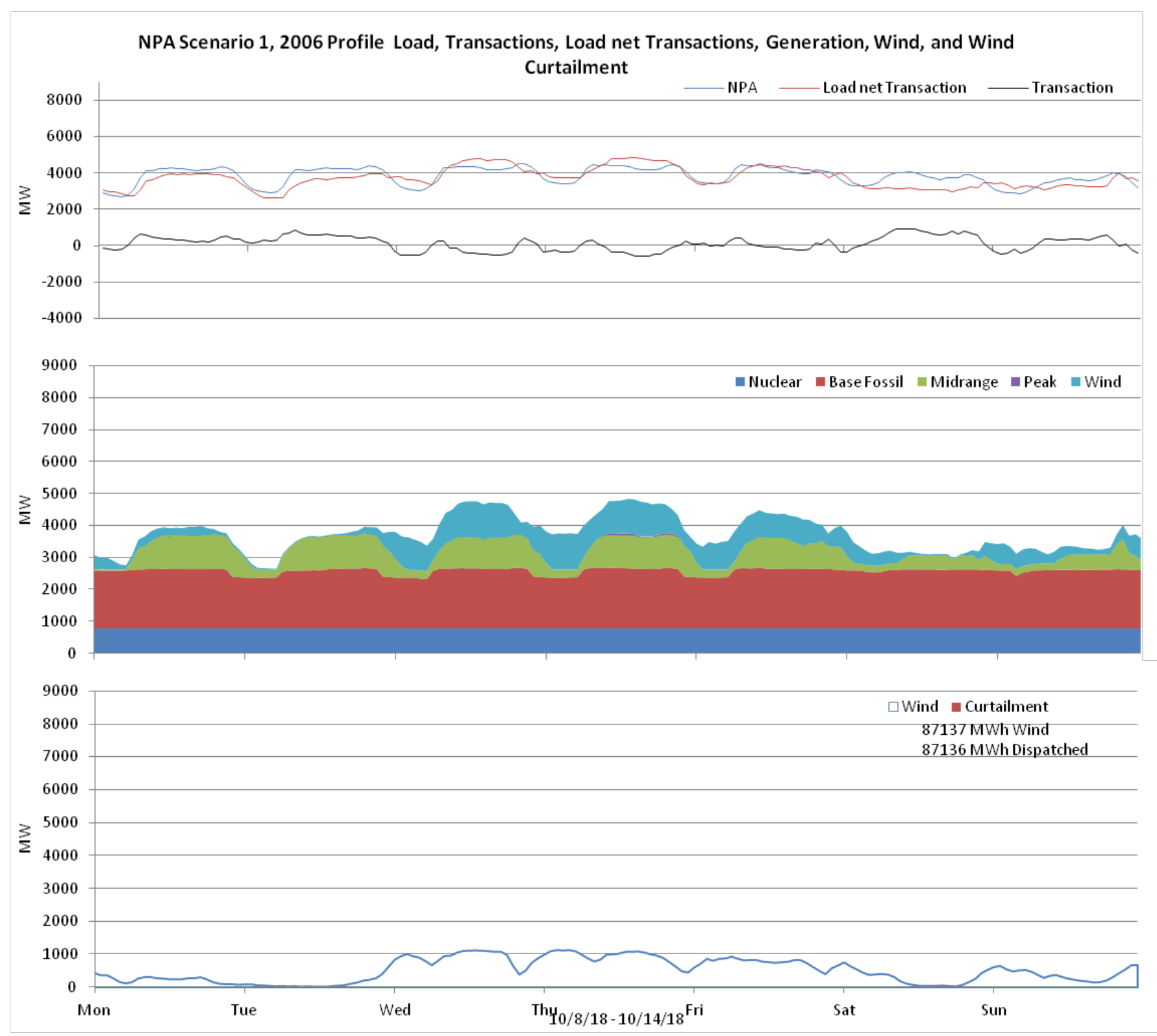

Figure 191: Nebraska 10\% wind Penetration, 2006 Profile week of 10/8/2018 


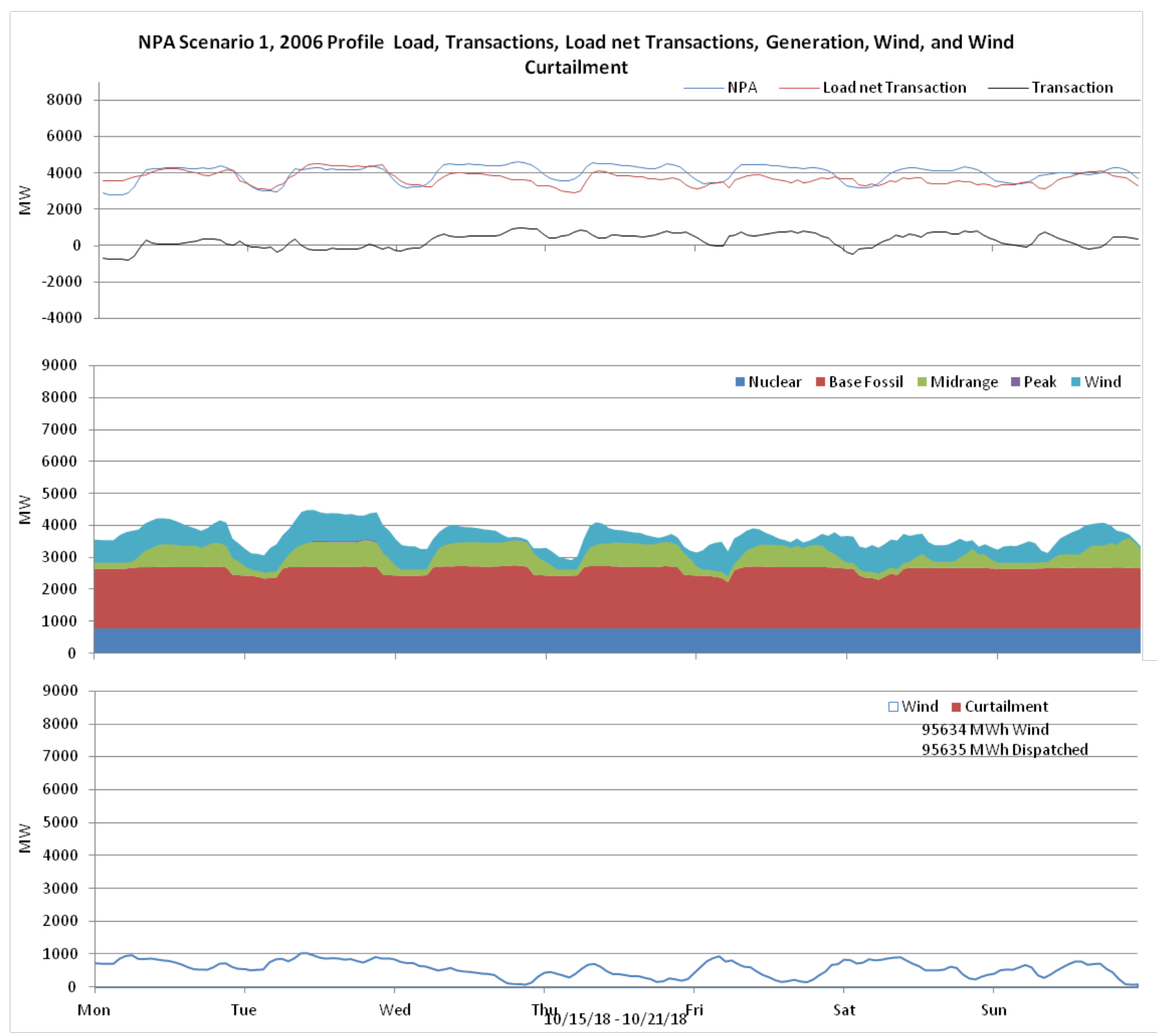

Figure 192: Nebraska 10\% wind Penetration, 2006 Profile week of 10/15/2018 


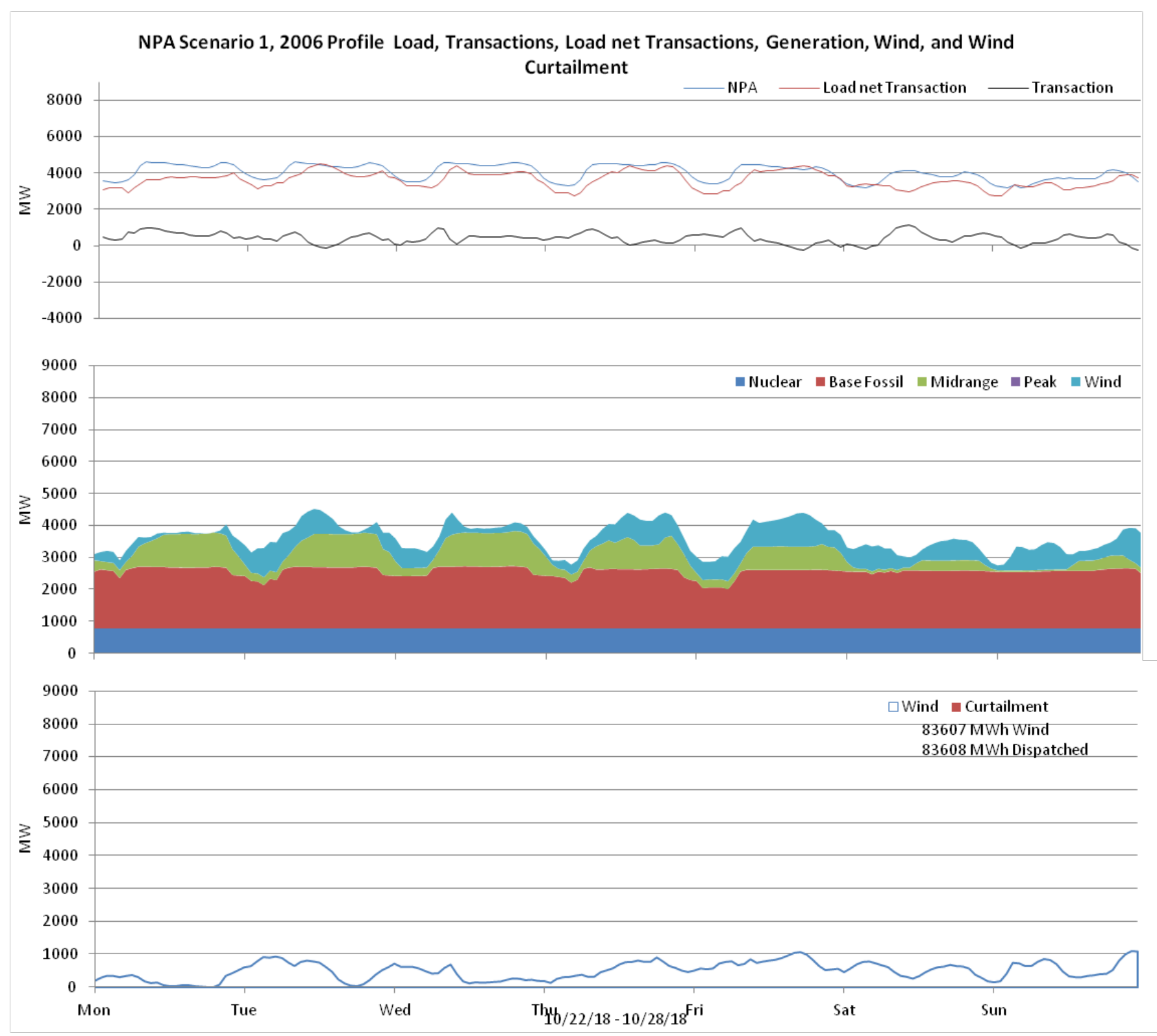

Figure 193 Nebraska 10\% wind Penetration, 2006 Profile week of 10/22/2018 


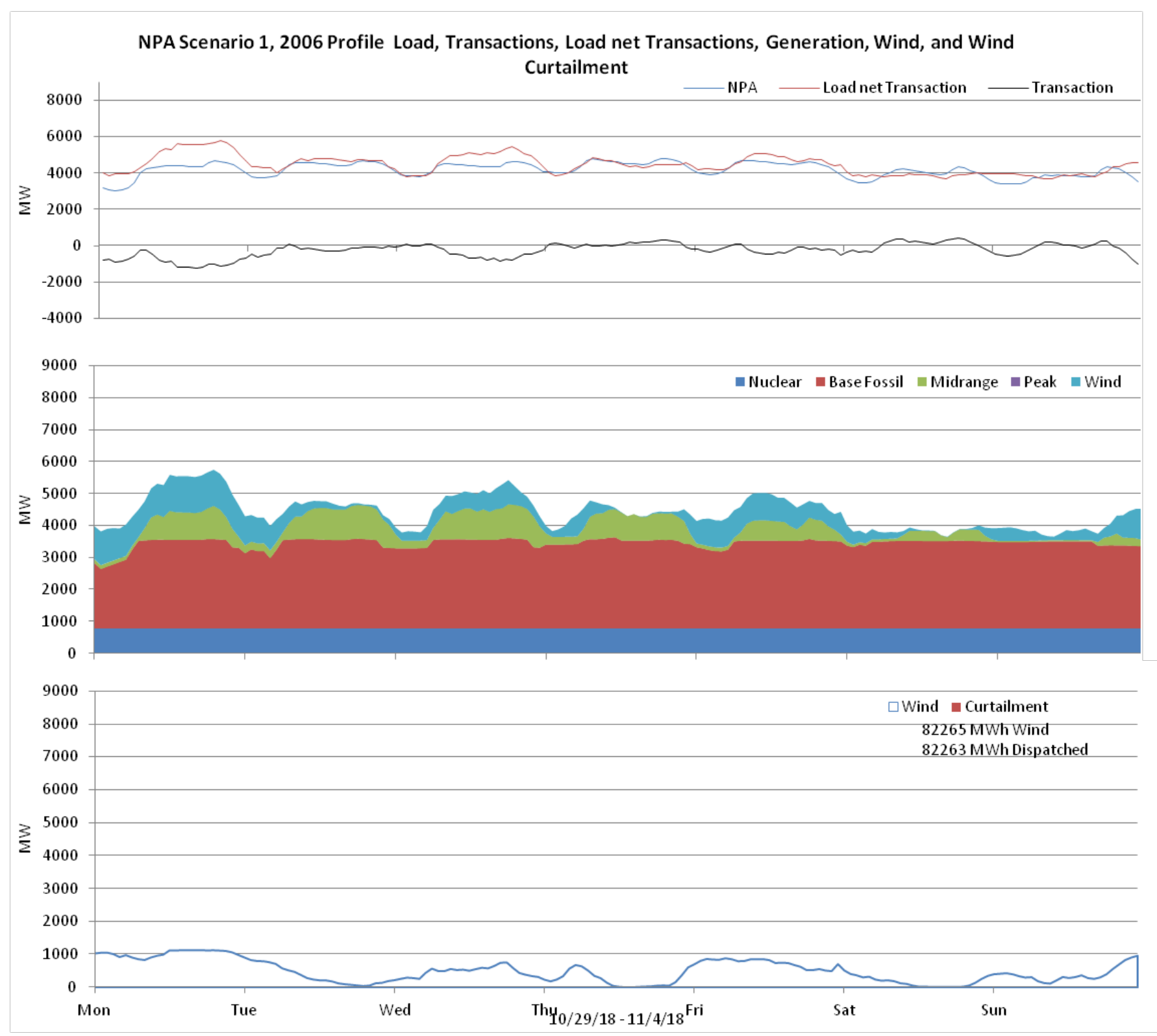

Figure 194: Nebraska 10\% wind Penetration, 2006 Profile week of 10/29/2018 


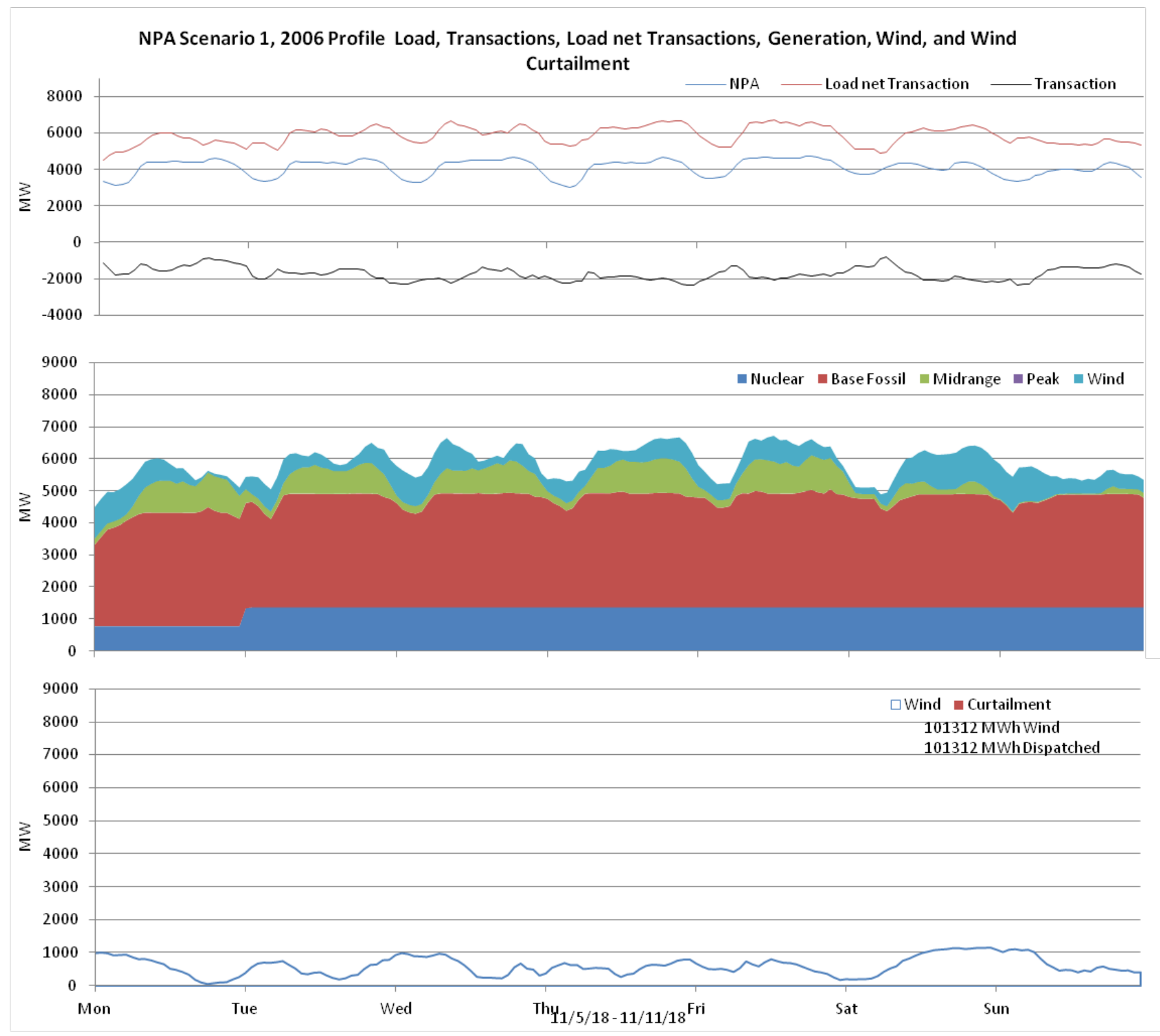

Figure 195 Nebraska 10\% wind Penetration, 2006 Profile week of 11/5/2018 


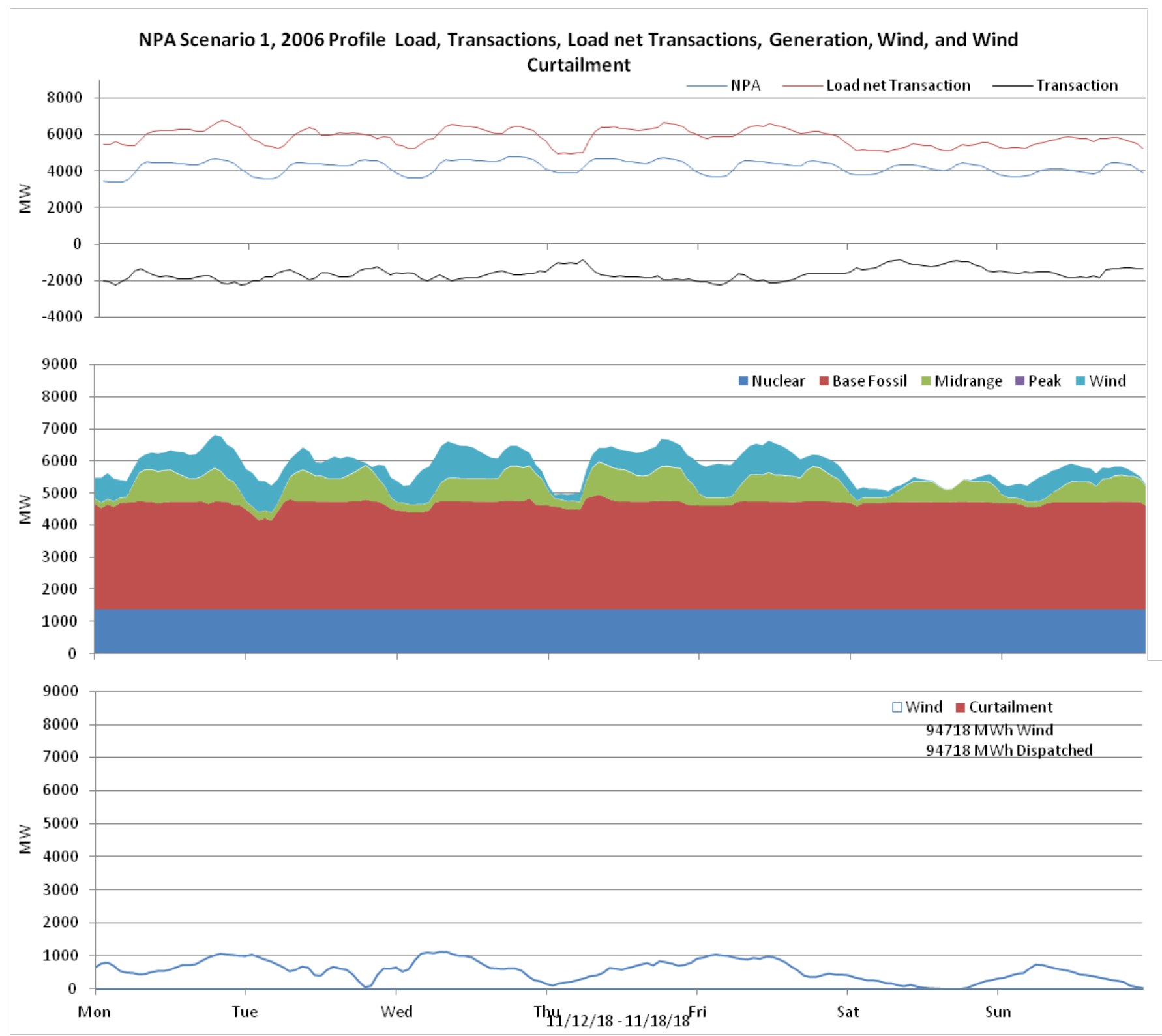

Figure 196: Nebraska 10\% wind Penetration, 2006 Profile week of 11/12/2018 


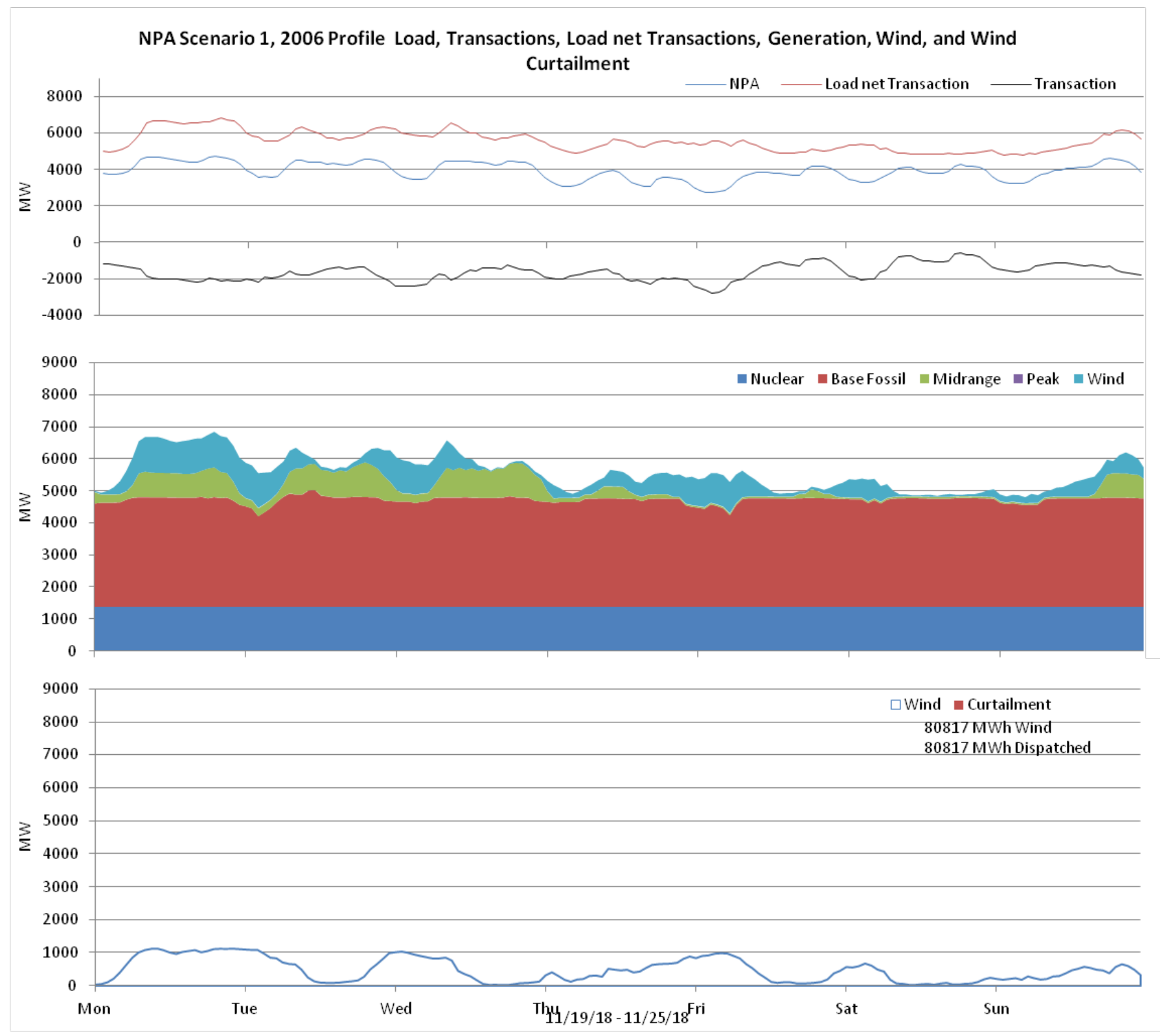

Figure 197: Nebraska 10\% wind Penetration, 2006 Profile week of 11/19/2018 


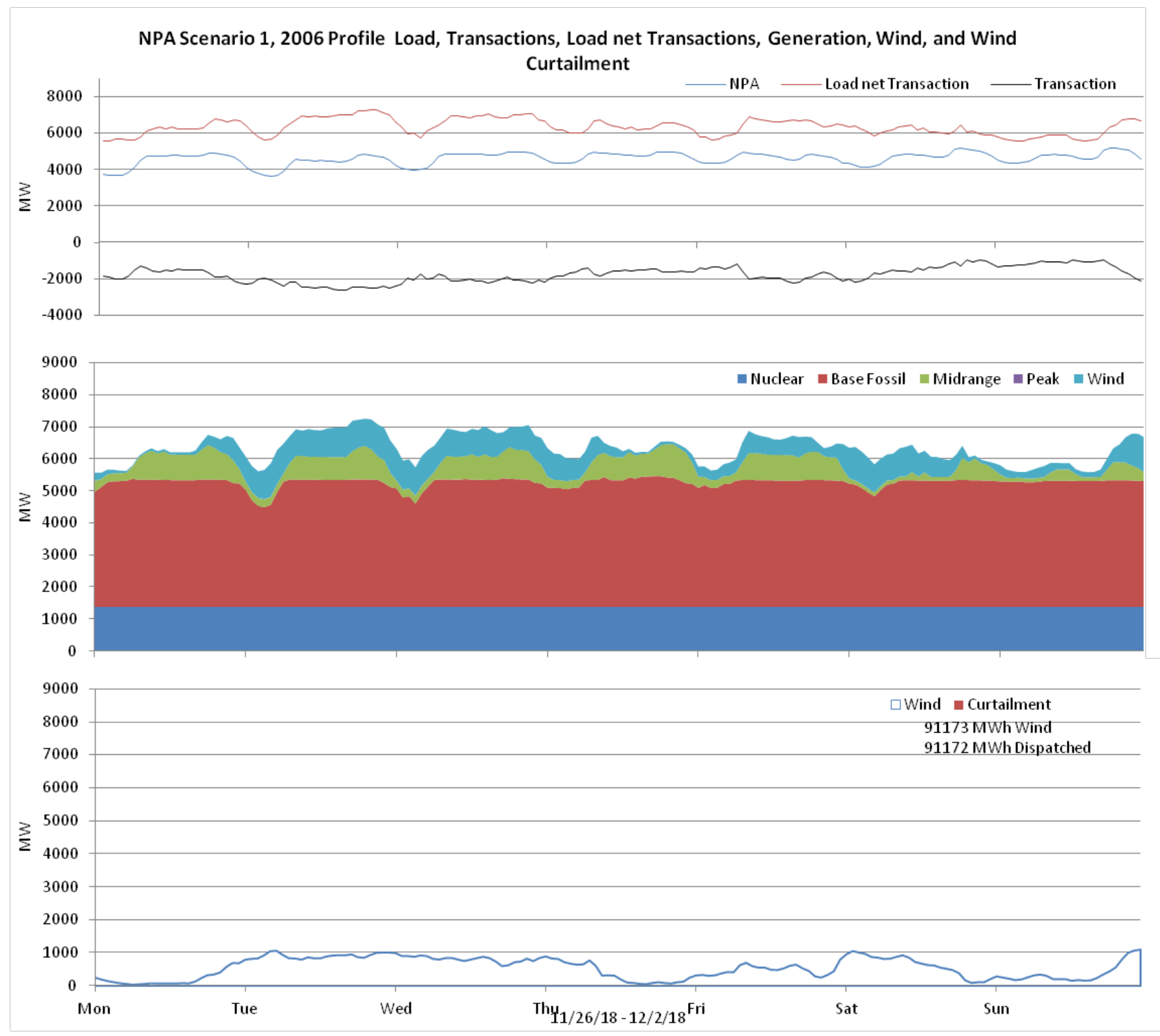

Figure 198: Nebraska 10\% wind Penetration, 2006 Profile week of 11/26/2018 


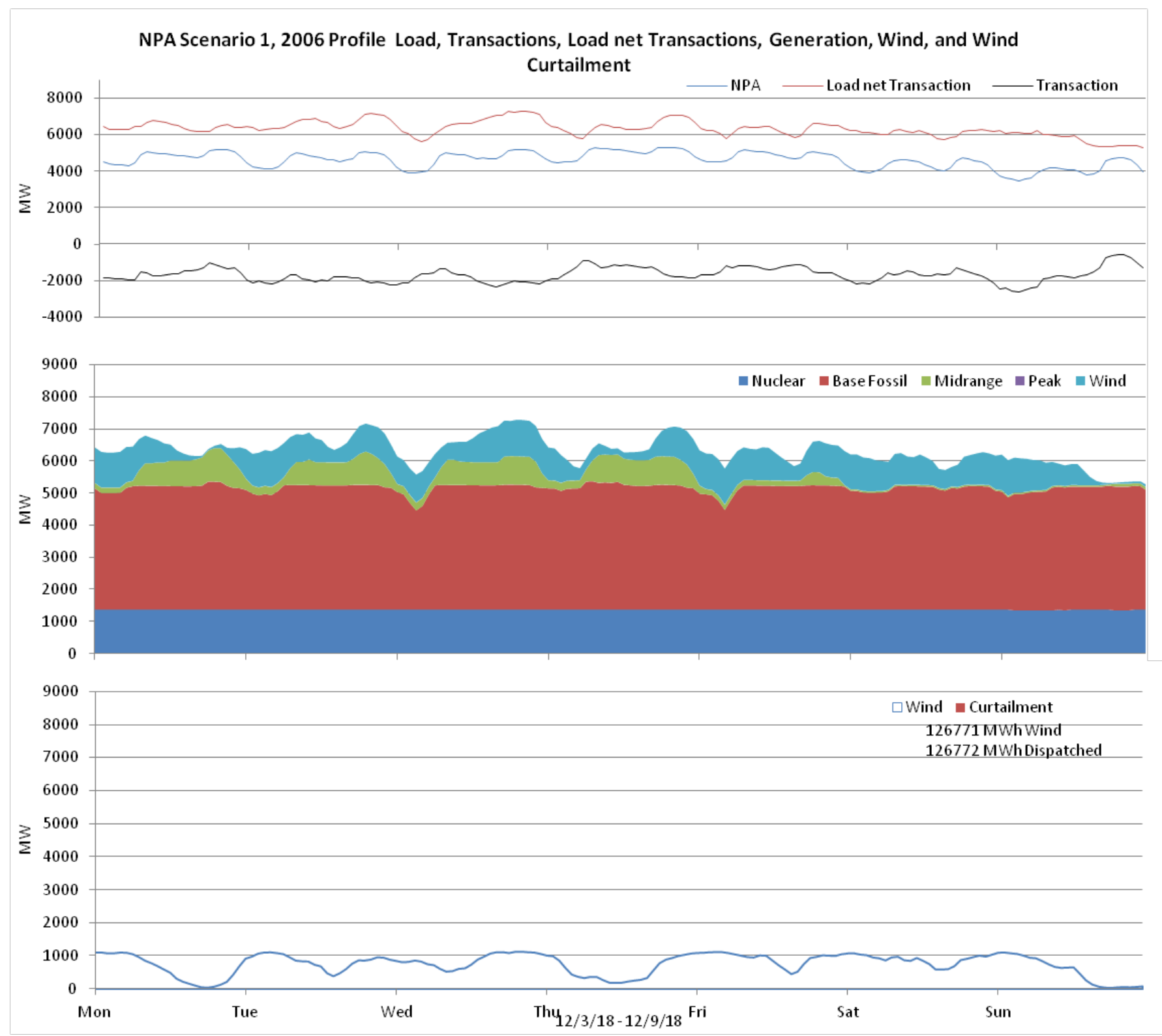

Figure 199: Nebraska 10\% wind Penetration, 2006 Profile week of 12/3/2018 


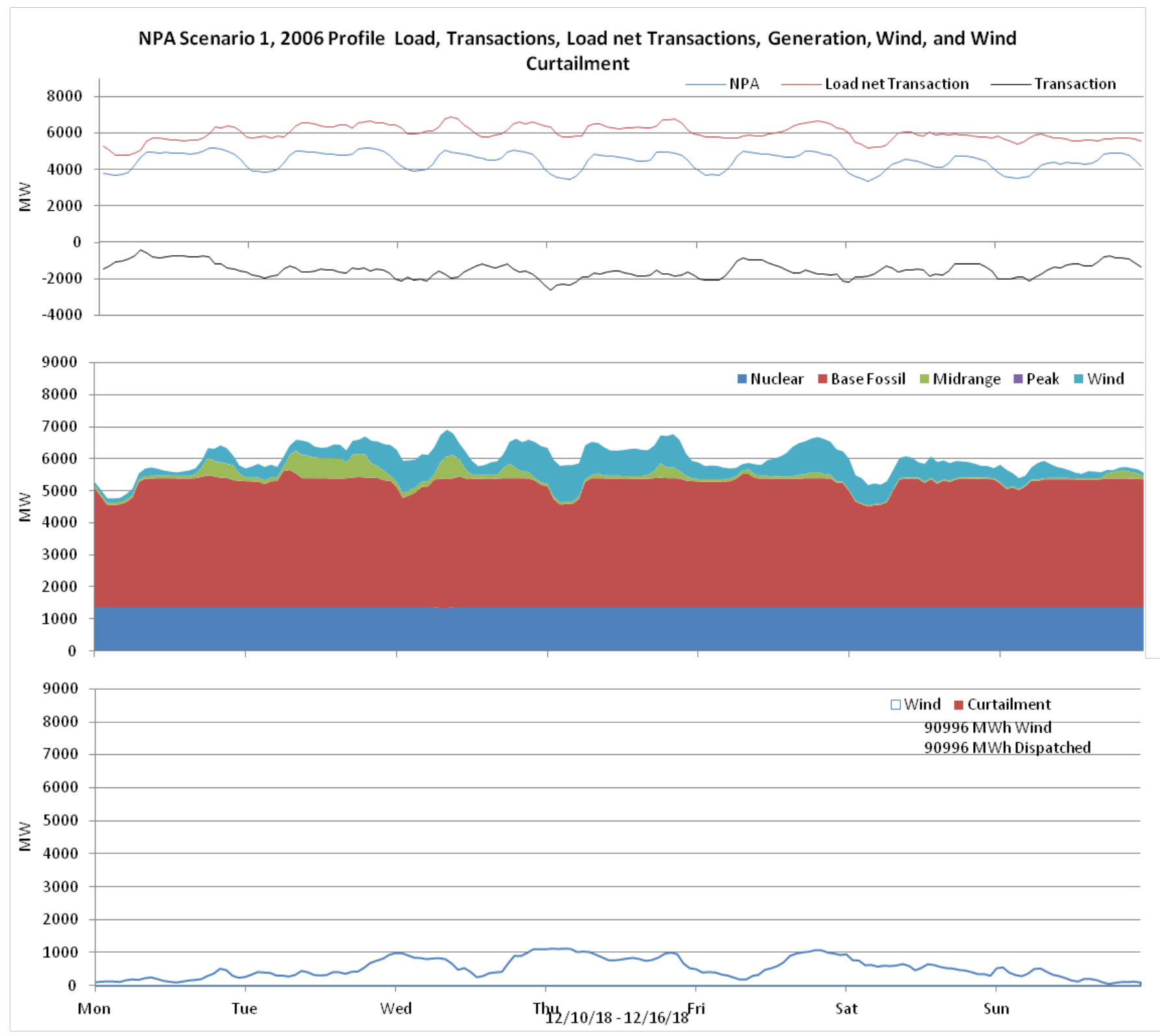

Figure 200: Nebraska 10\% wind Penetration, 2006 Profile week of 12/10/2018 


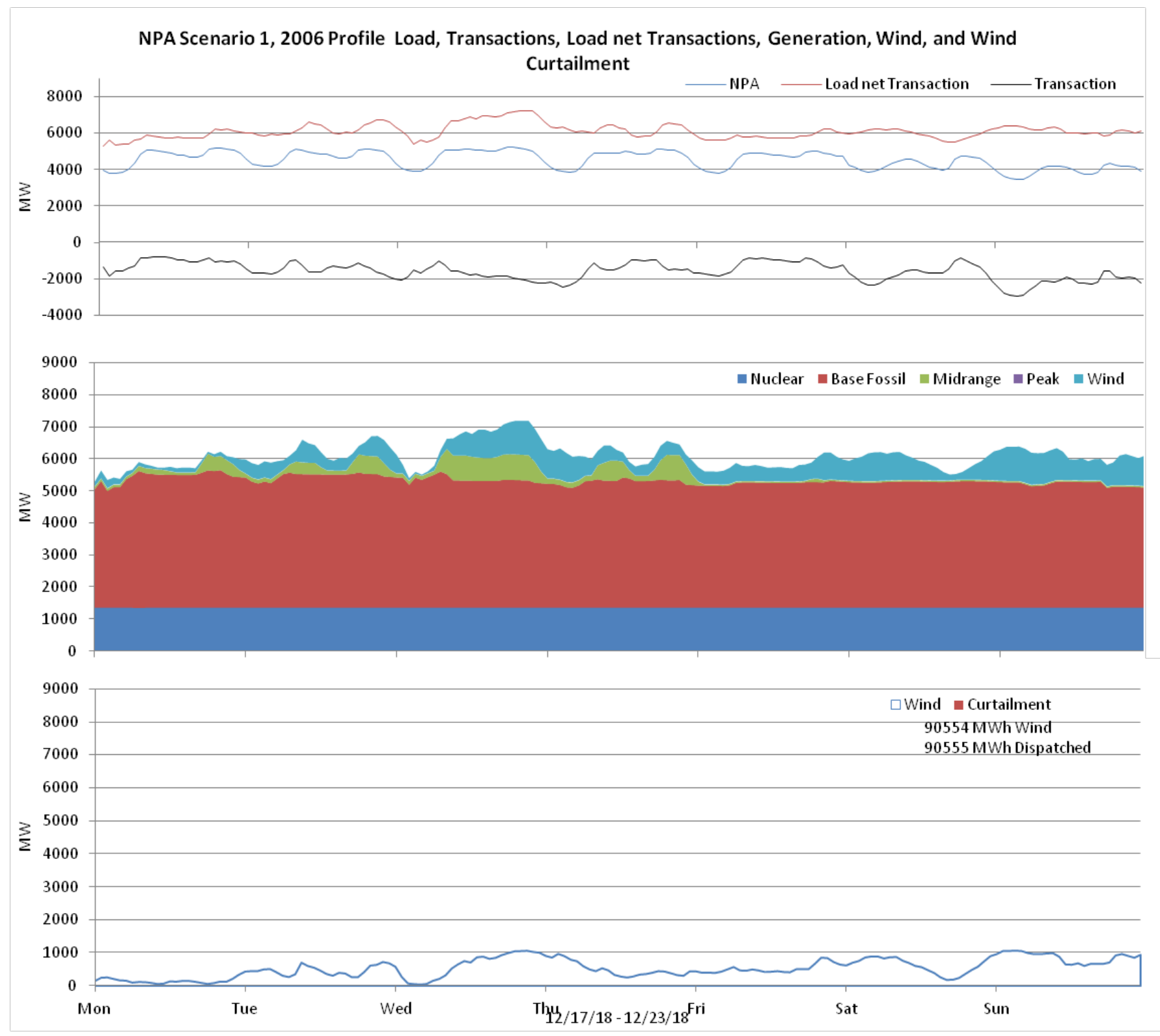

Figure 201: Nebraska 10\% wind Penetration, 2006 Profile week of 12/17/2018 


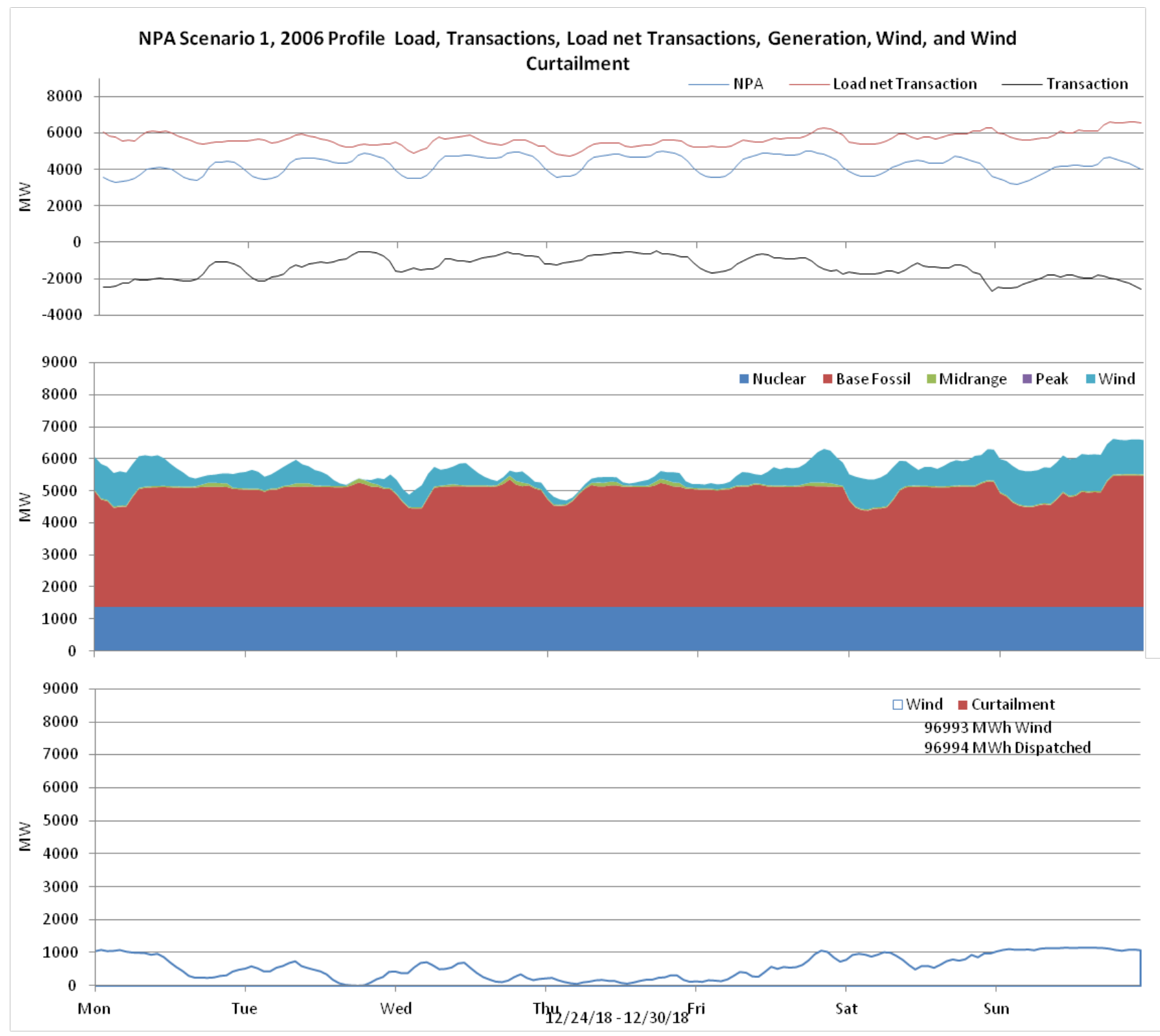

Figure 202: Nebraska 10\% wind Penetration, 2006 Profile week of 12/24/2018 
For SPP with Nebraska seasonal peak and minimum wind energies are shown in Table 26.

Table 26: SPP with Nebraska Seasonal Peak and Minimum Energy

\begin{tabular}{|c|c|c|c|c|c|c|}
\hline $\begin{array}{l}\text { Weekly } \\
\text { Energy }\end{array}$ & $\begin{array}{l}\text { Peak } \\
\text { MWH }\end{array}$ & $\begin{array}{c}\text { Week of } \\
\text { Year }\end{array}$ & $\begin{array}{l}\text { Start } \\
\text { Date }\end{array}$ & $\begin{array}{c}\text { Min } \\
\text { MWH }\end{array}$ & $\begin{array}{c}\text { Weak of } \\
\text { Year }\end{array}$ & $\begin{array}{l}\text { Start } \\
\text { Date }\end{array}$ \\
\hline Season1 & 776093 & 2 & $1 / 8 / 2018$ & 464849 & 51 & $12 / 17 / 2018$ \\
\hline Season2 & 922523 & 21 & $5 / 21 / 2018$ & 359033 & 20 & $5 / 14 / 2018$ \\
\hline Season3 & 785389 & 24 & $6 / 11 / 2018$ & 359675 & 34 & $8 / 20 / 2018$ \\
\hline Season4 & 780341 & 40 & $10 / 1 / 2018$ & 403535 & 36 & $9 / 3 / 2018$ \\
\hline
\end{tabular}



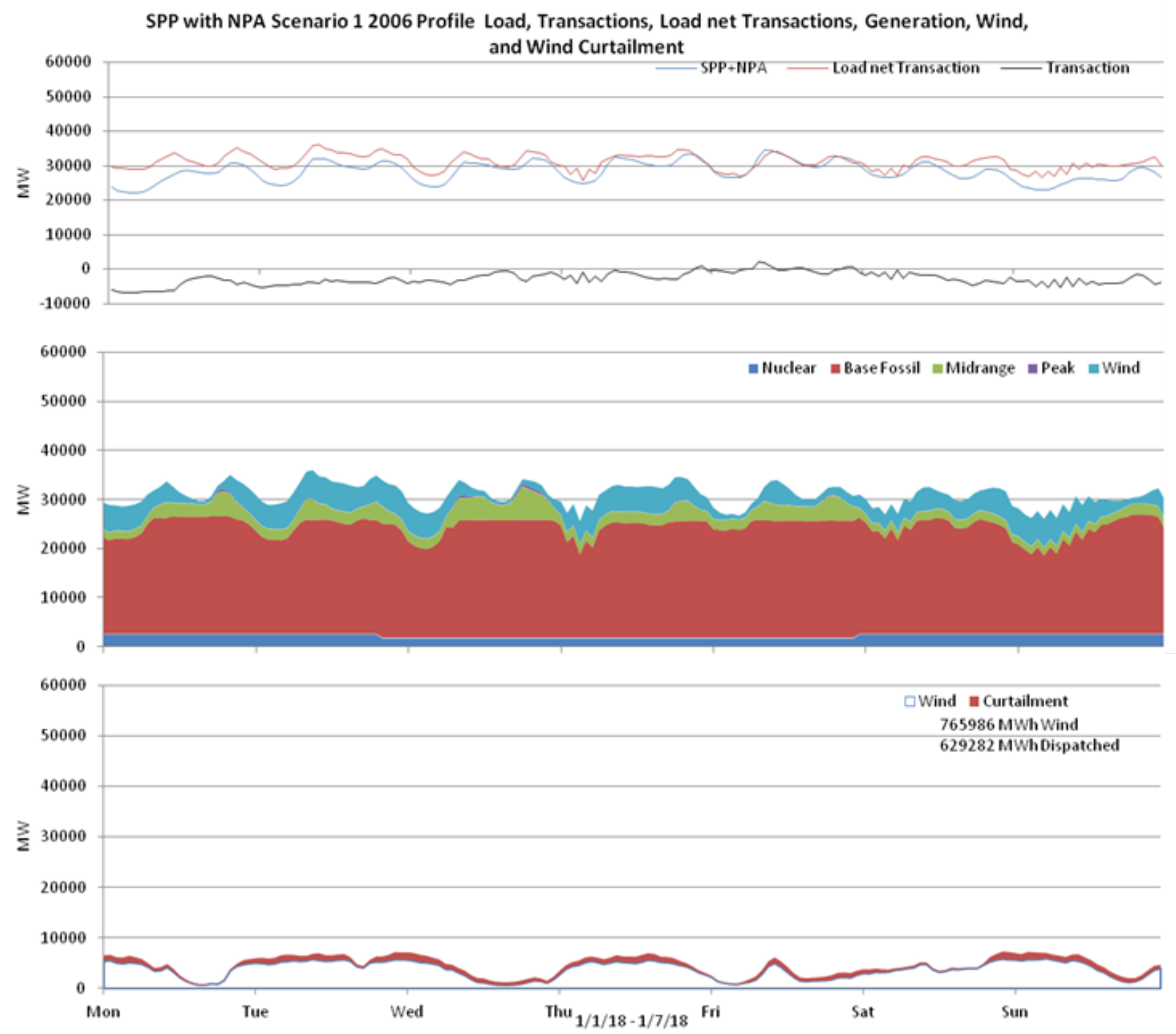

Figure 203: SPP 10\% wind Penetration, 2006 Profile week of $1 / 1 / 2018$ 


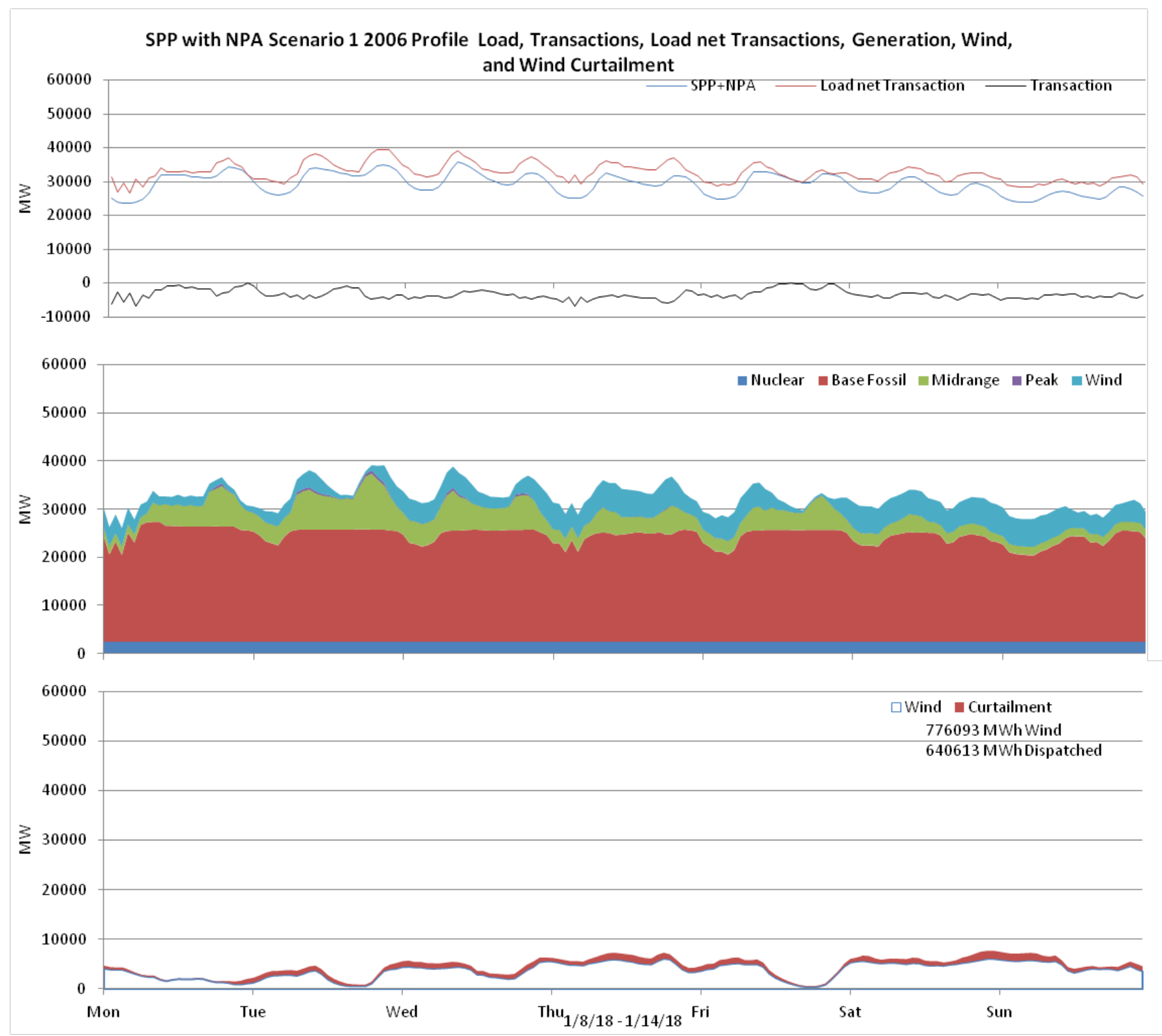

Figure 204: SPP 10\% wind Penetration, 2006 Profile week of $1 / 8 / 2018$ 


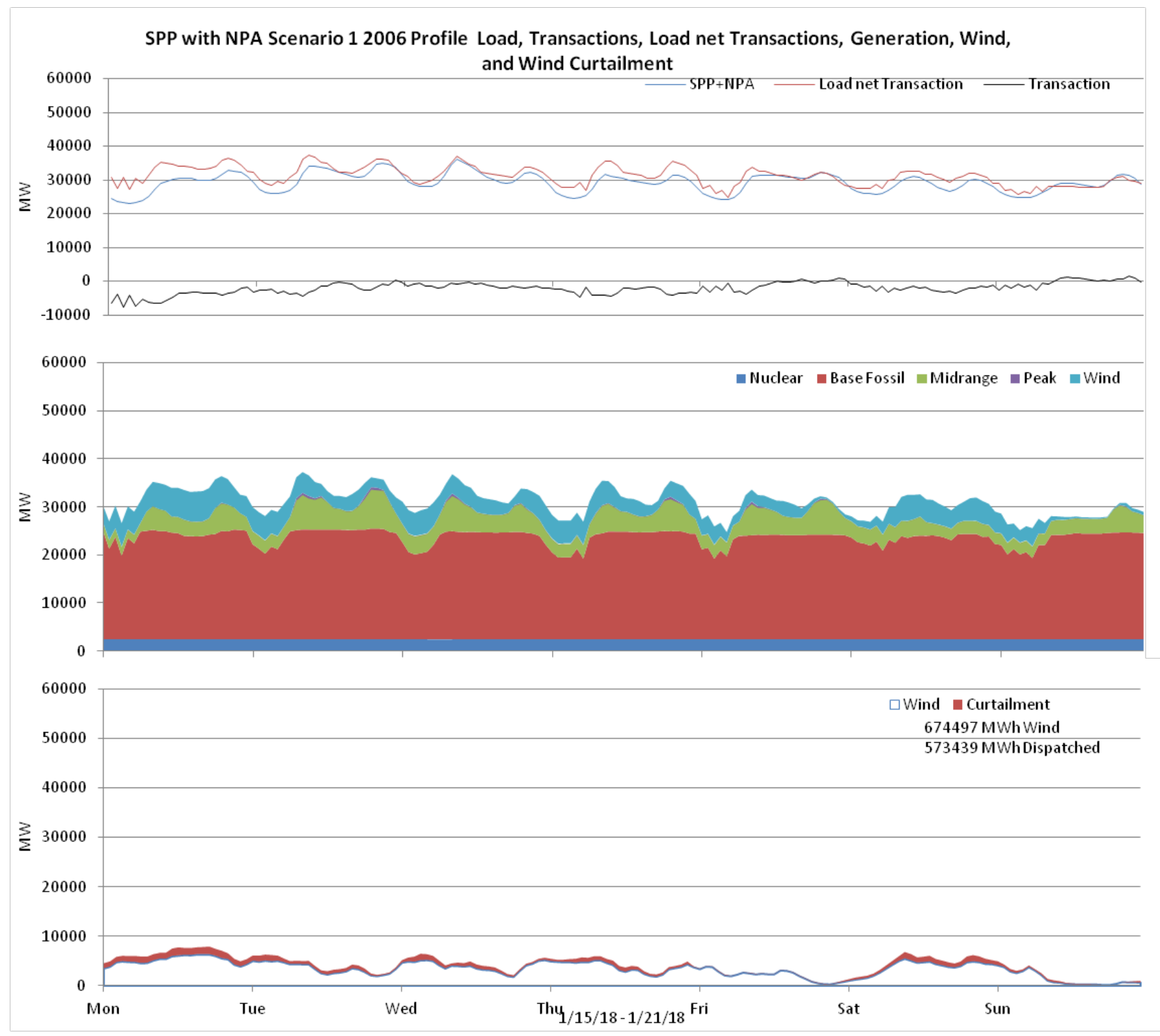

Figure 205: SPP 10\% wind Penetration, 2006 Profile week of 1/15/2018 


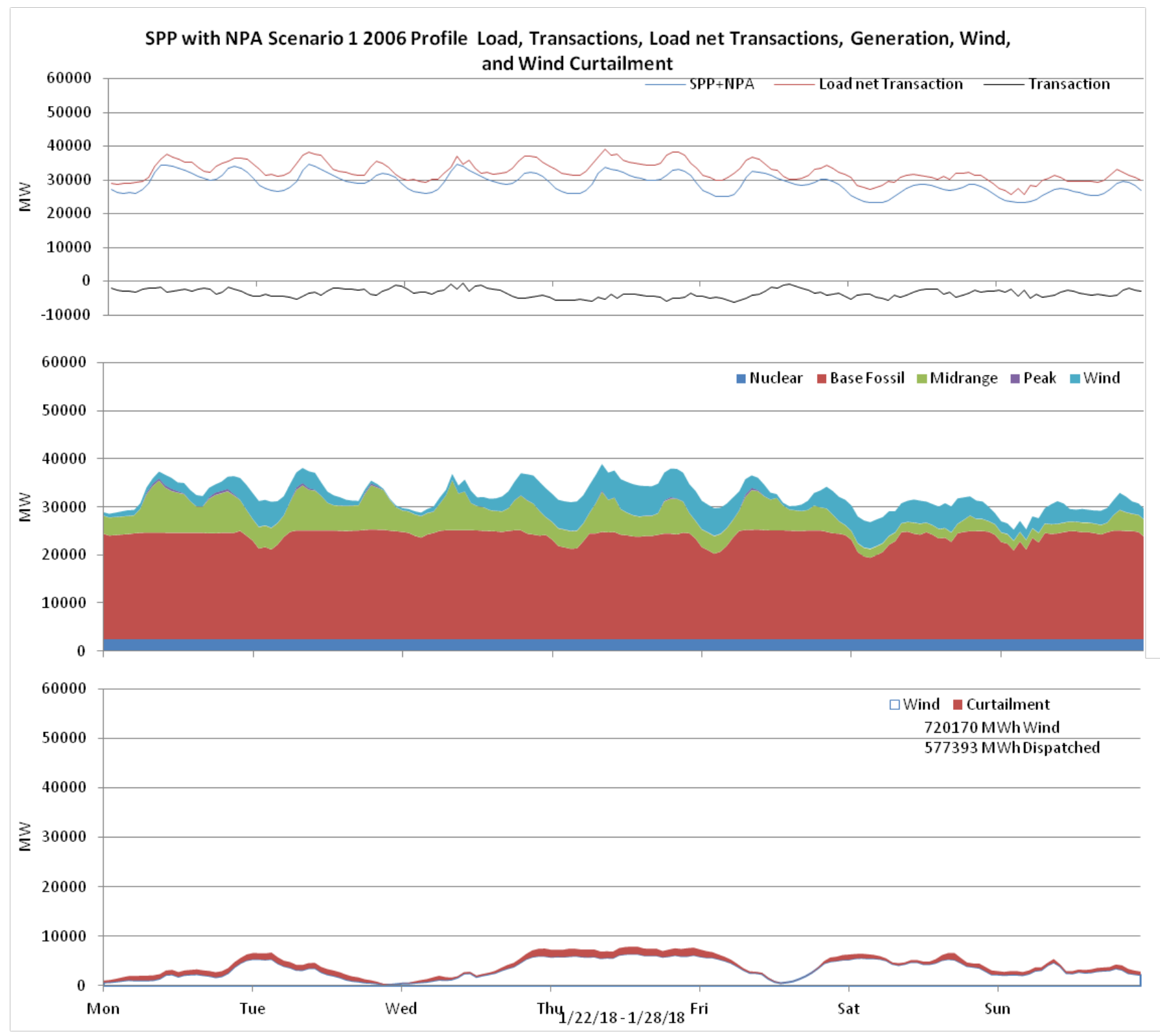

Figure 206: SPP 10\% wind Penetration, 2006 Profile week of 1/22/2018 


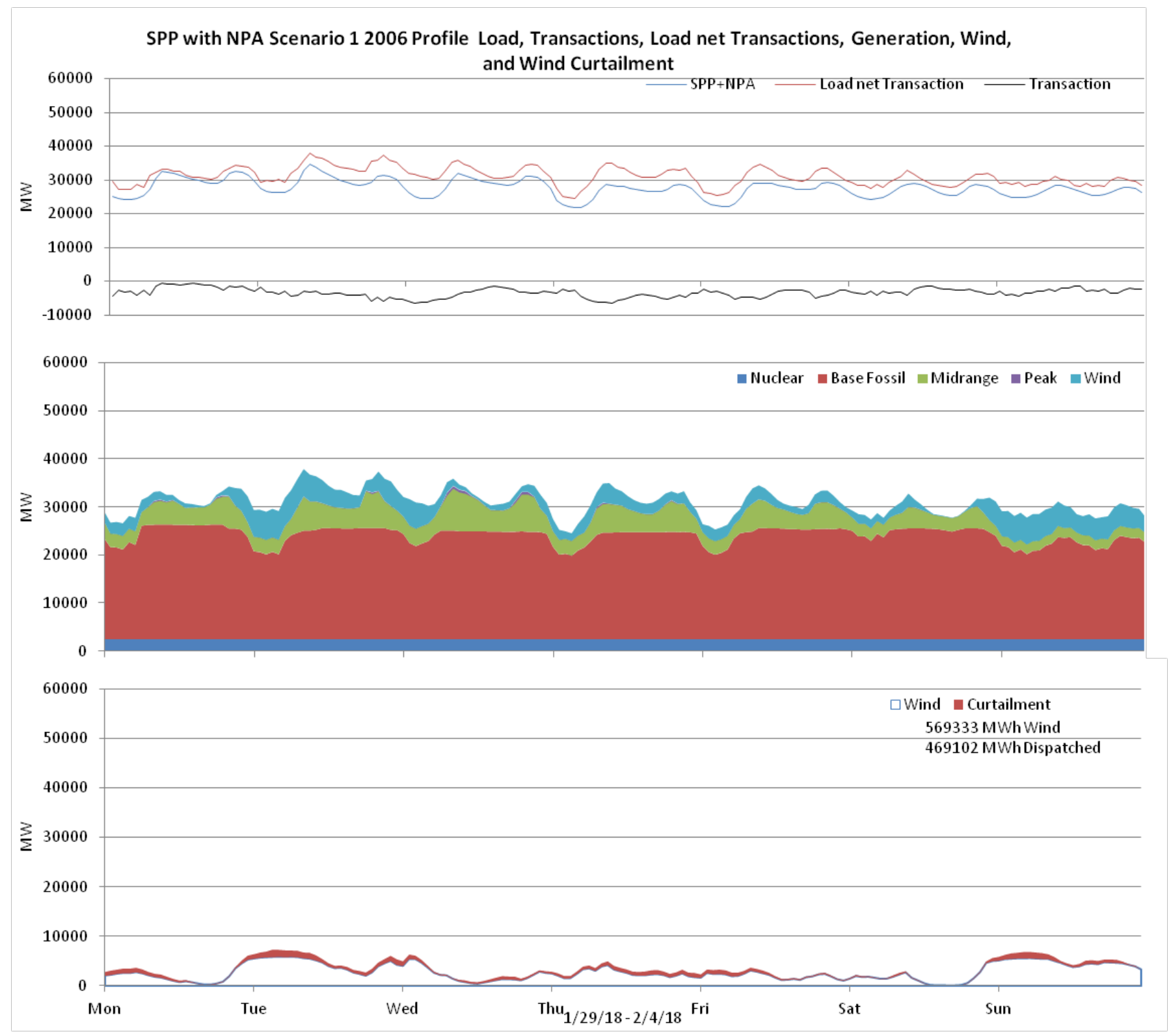

Figure 207: SPP 10\% wind Penetration, 2006 Profile week of 1/29/2018 


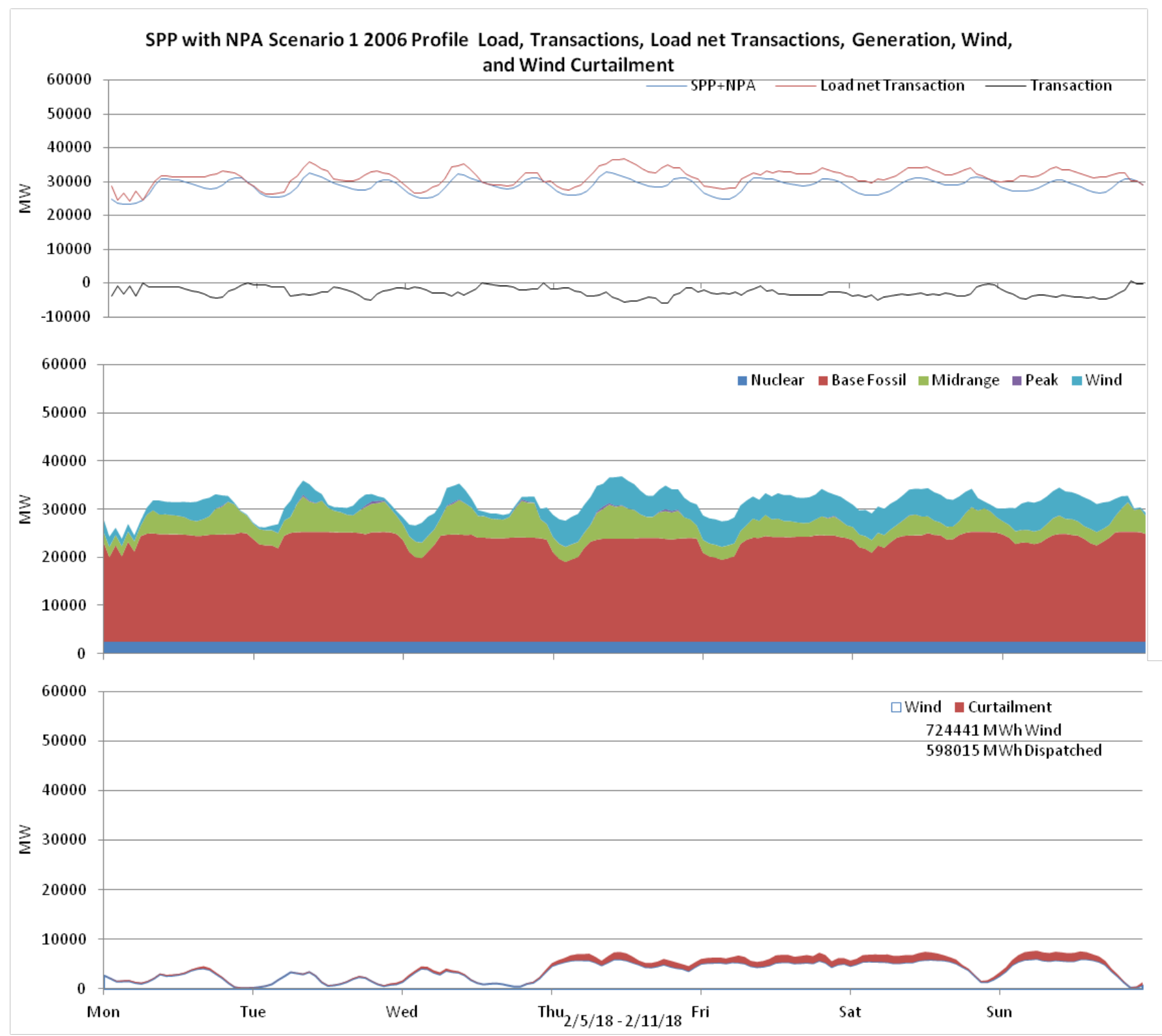

Figure 208: SPP 10\% wind Penetration, 2006 Profile week of 2/5/2018 


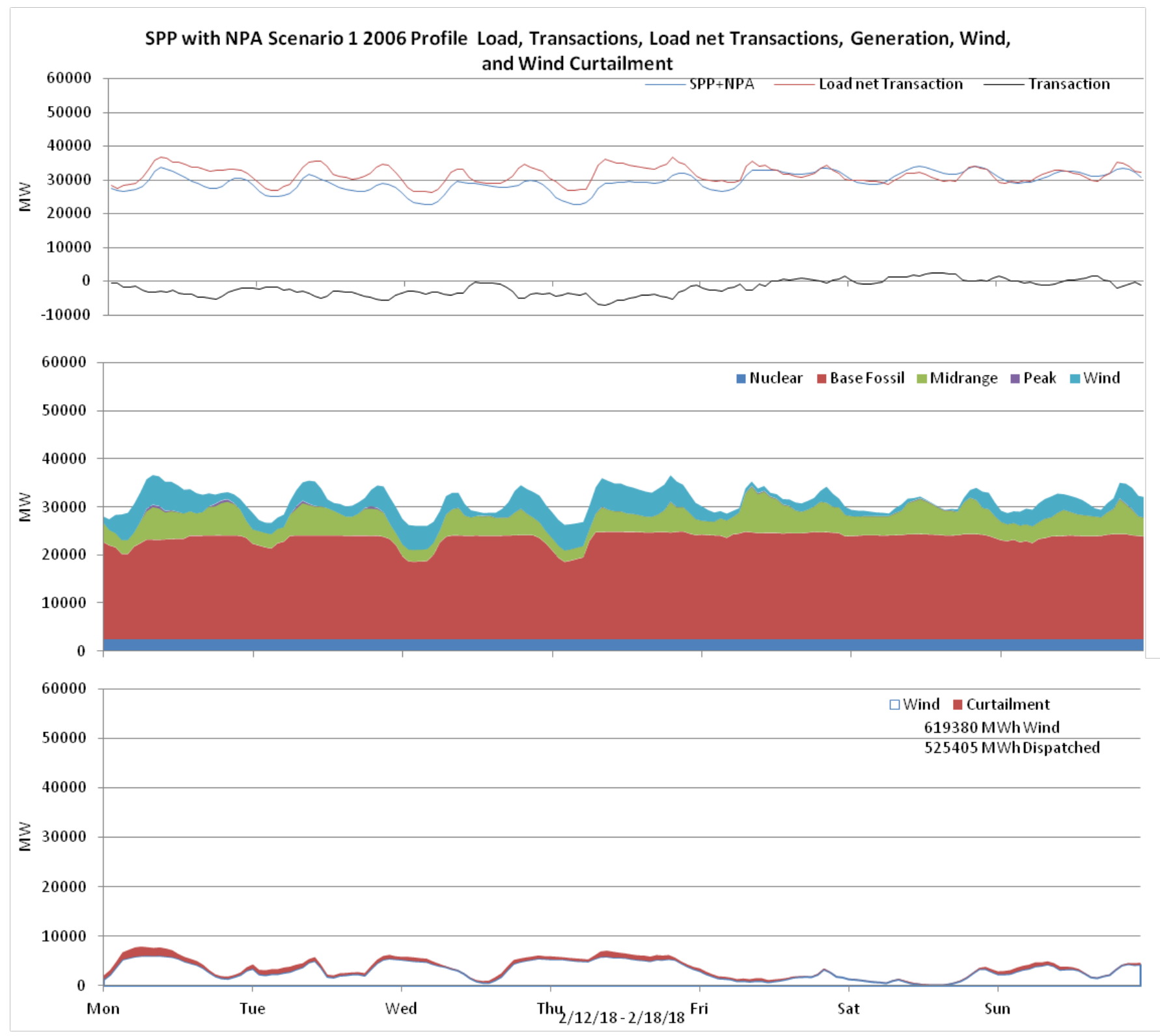

Figure 209: SPP 10\% wind Penetration, 2006 Profile week of 2/12/2018 


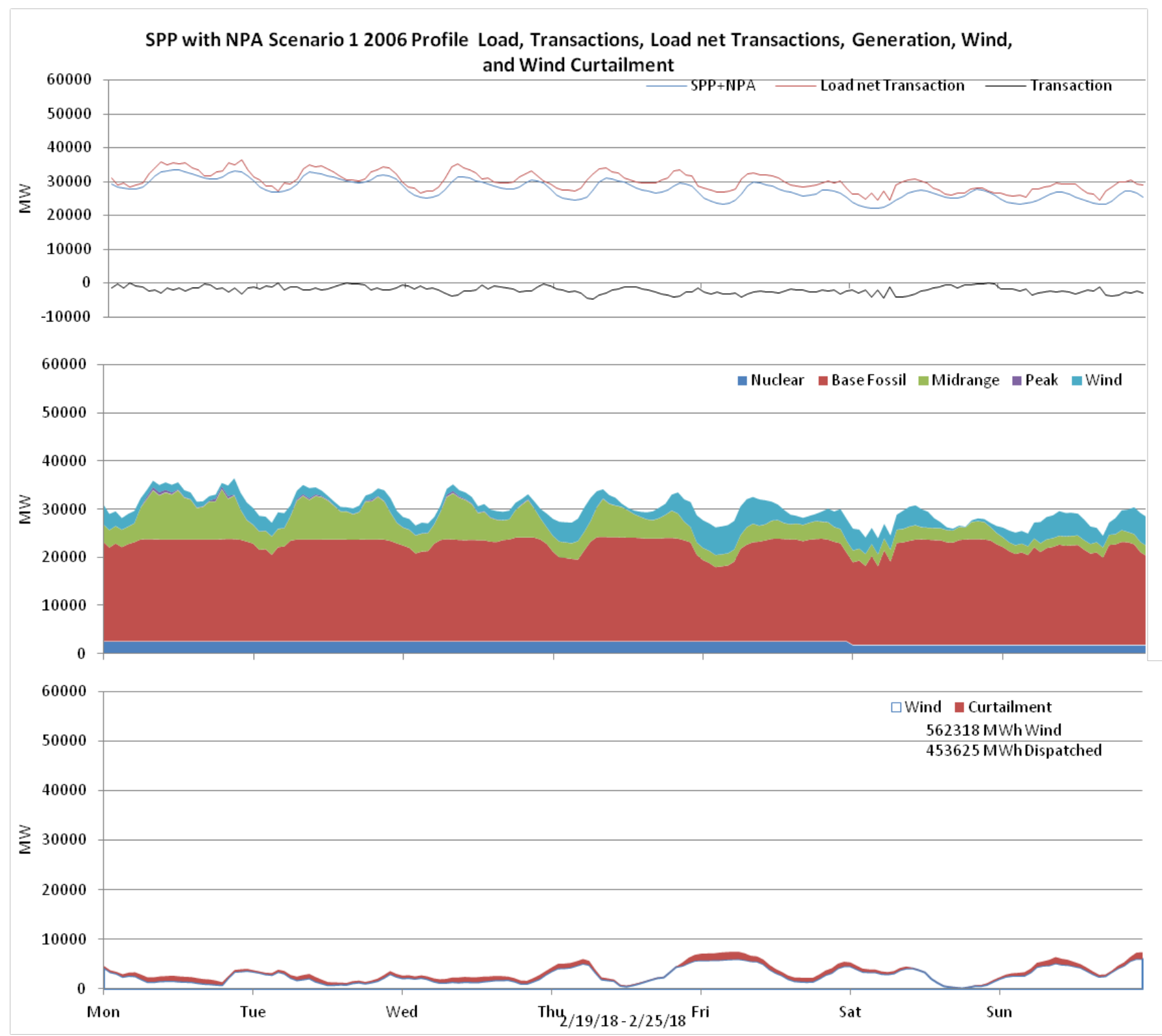

Figure 210: SPP 10\% wind Penetration, 2006 Profile week of 2/19/2018 


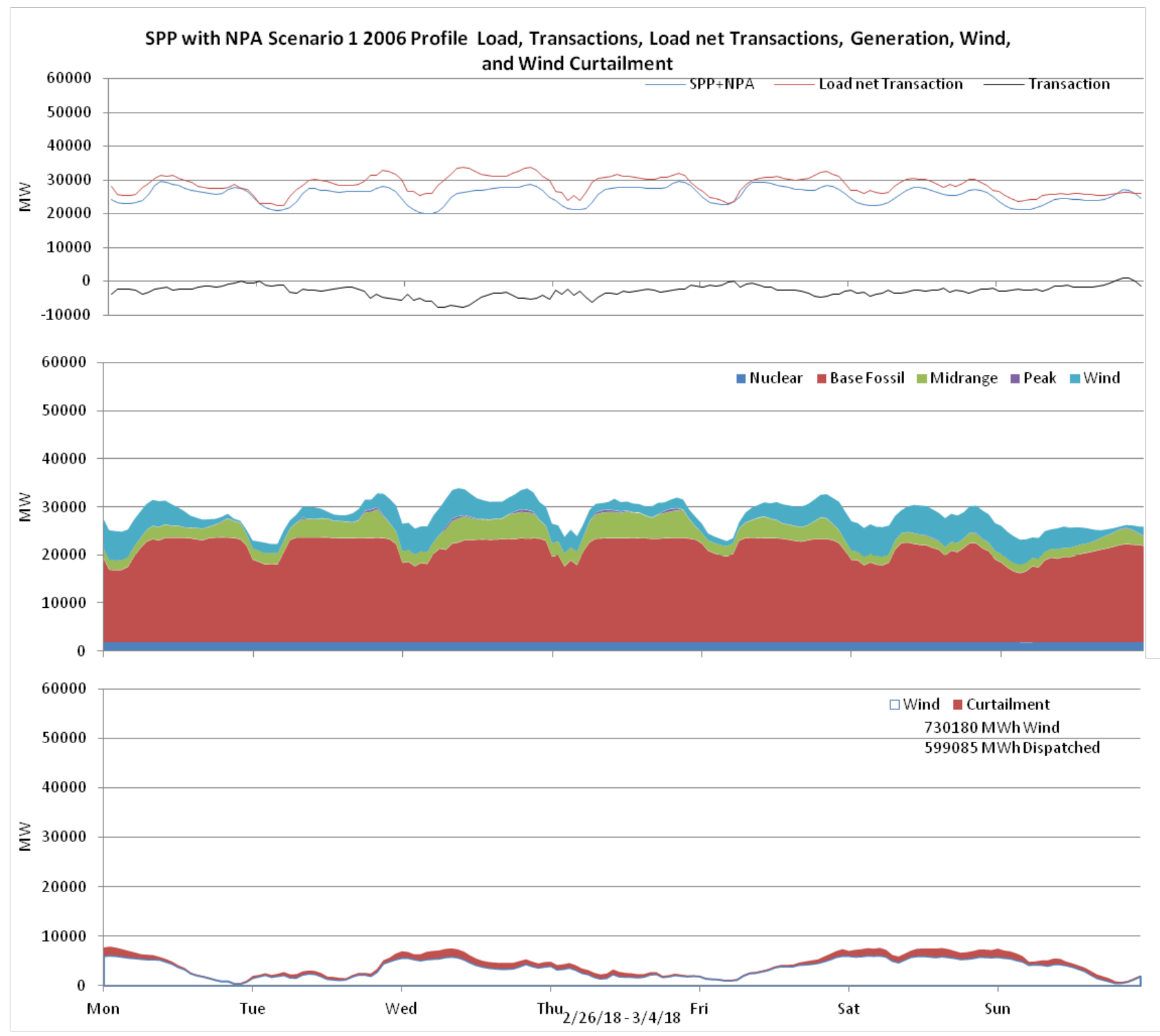

Figure 211: SPP 10\% wind Penetration, 2006 Profile week of 2/26/2018 


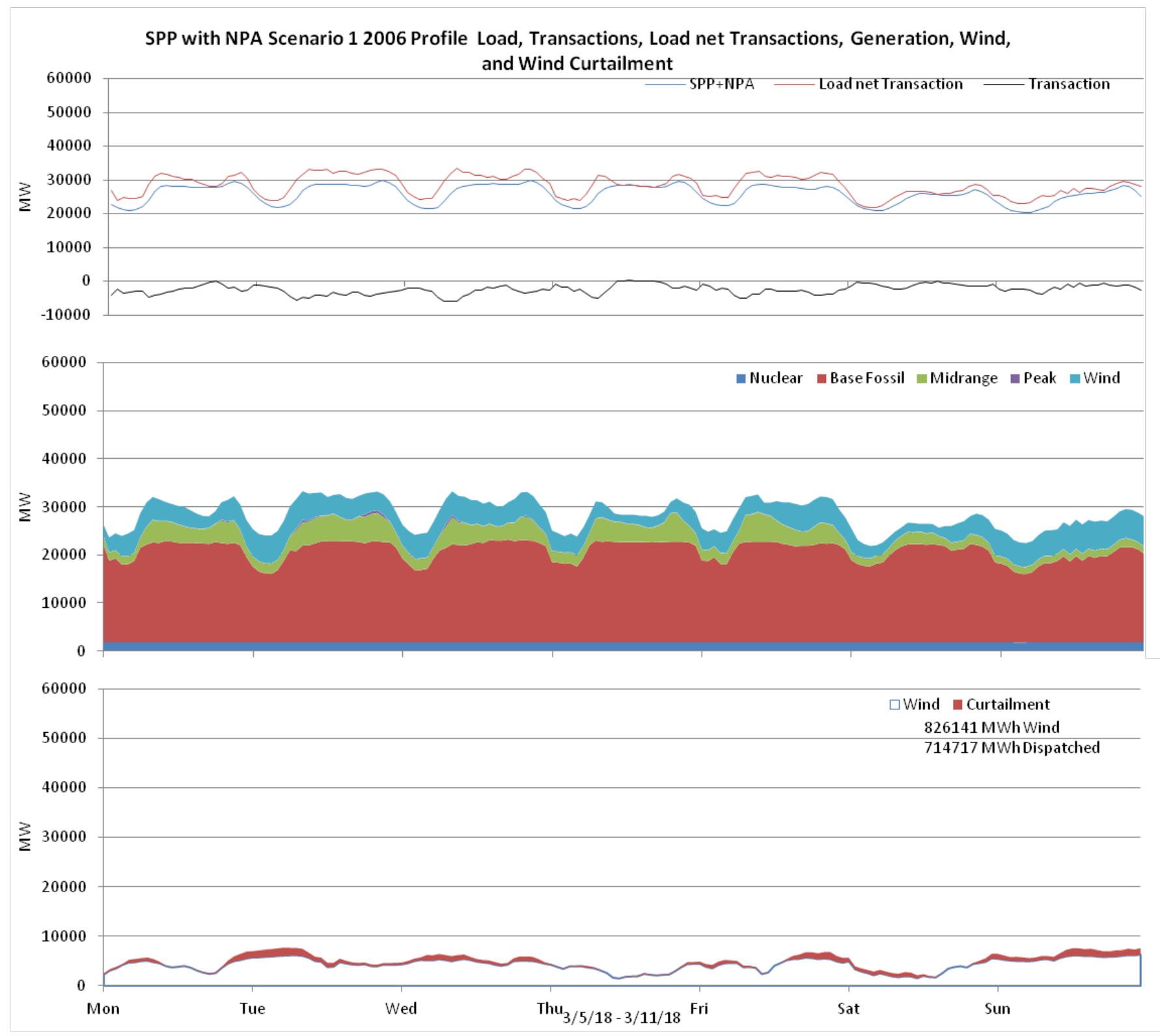

Figure 212: SPP 10\% wind Penetration, 2006 Profile week of 3/5/2018 


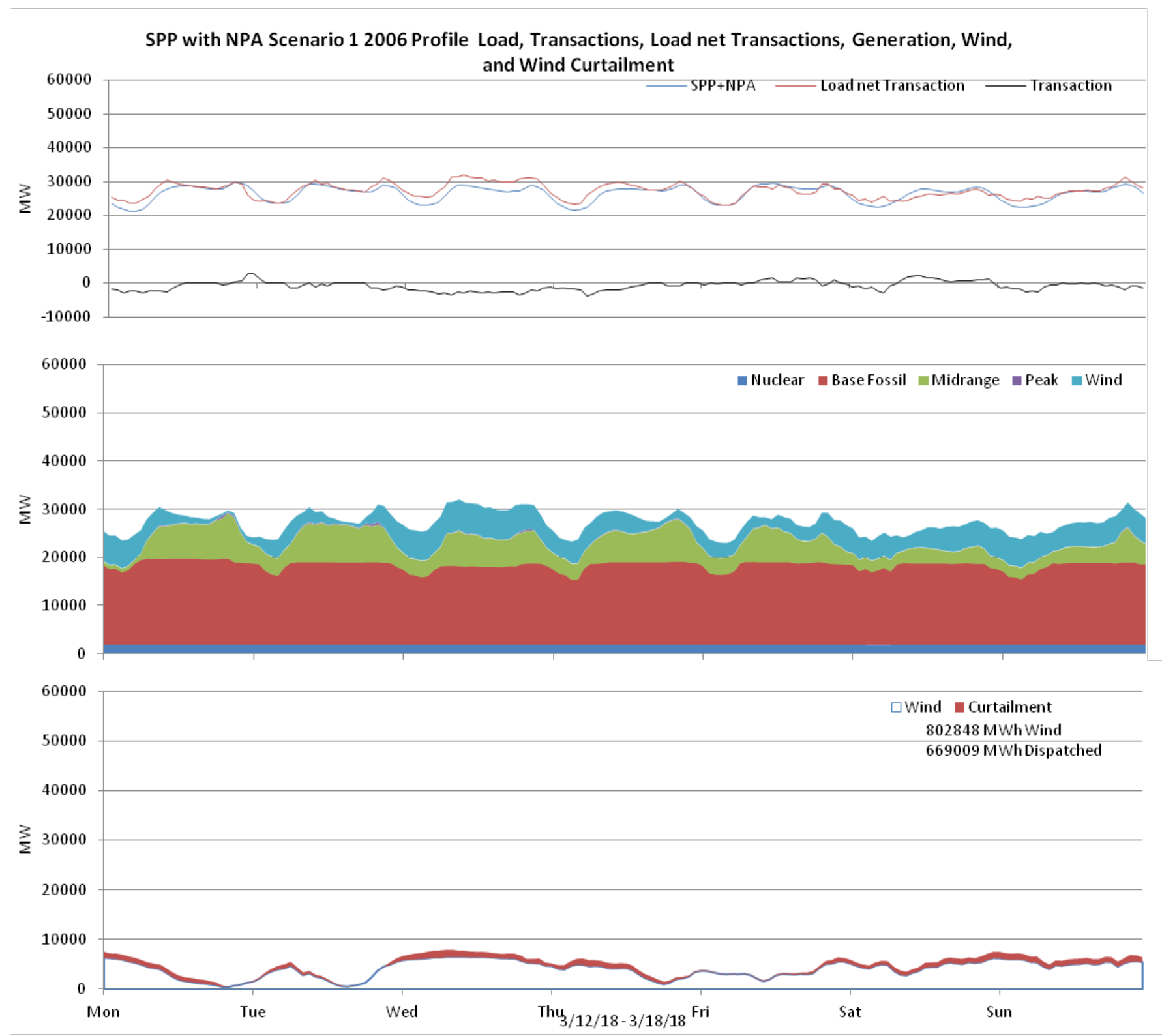

Figure 213: SPP $10 \%$ wind Penetration, 2006 Profile week of $3 / 12 / 2018$ 


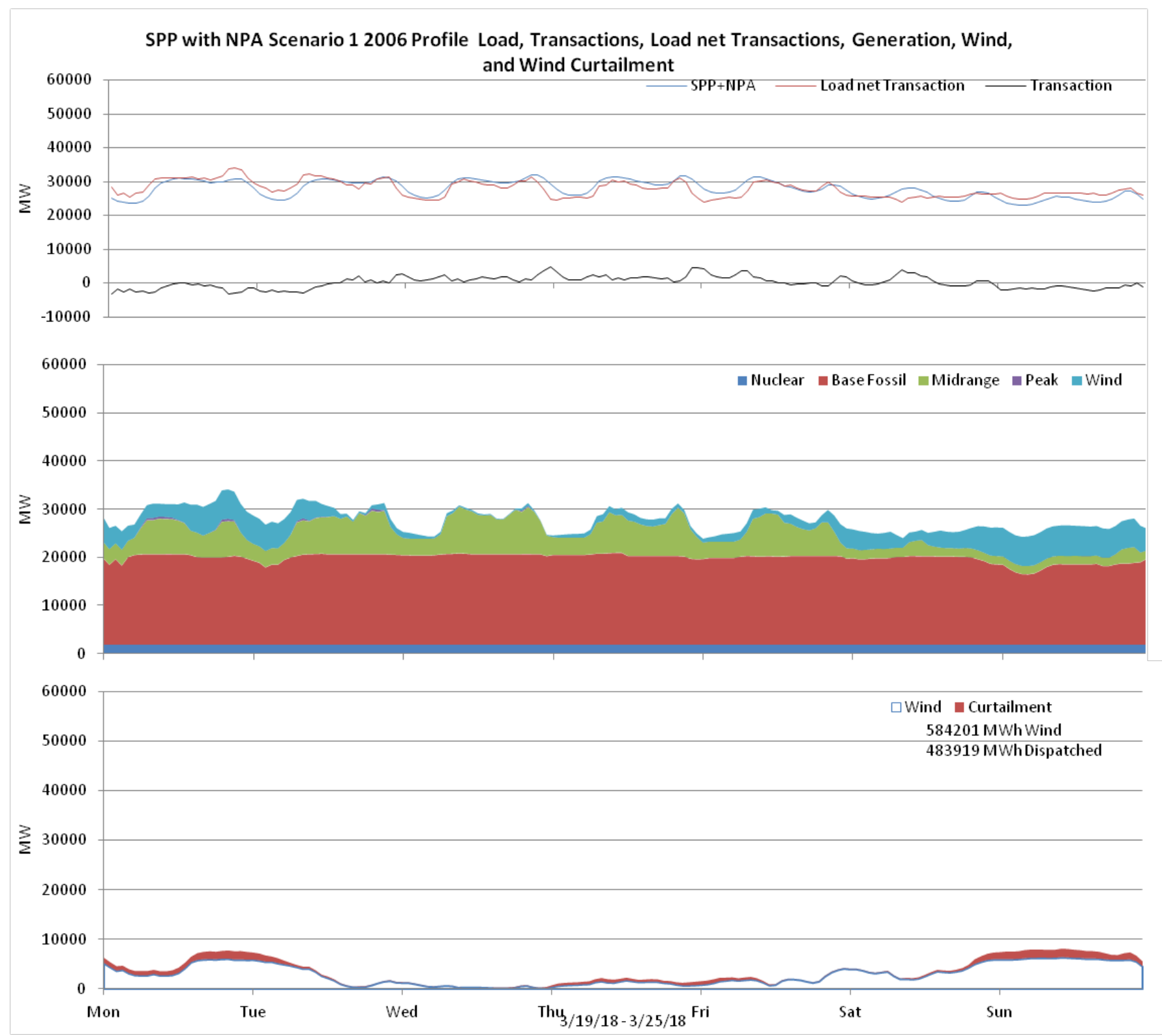

Figure 214: SPP 10\% wind Penetration, 2006 Profile week of 3/19/2018 


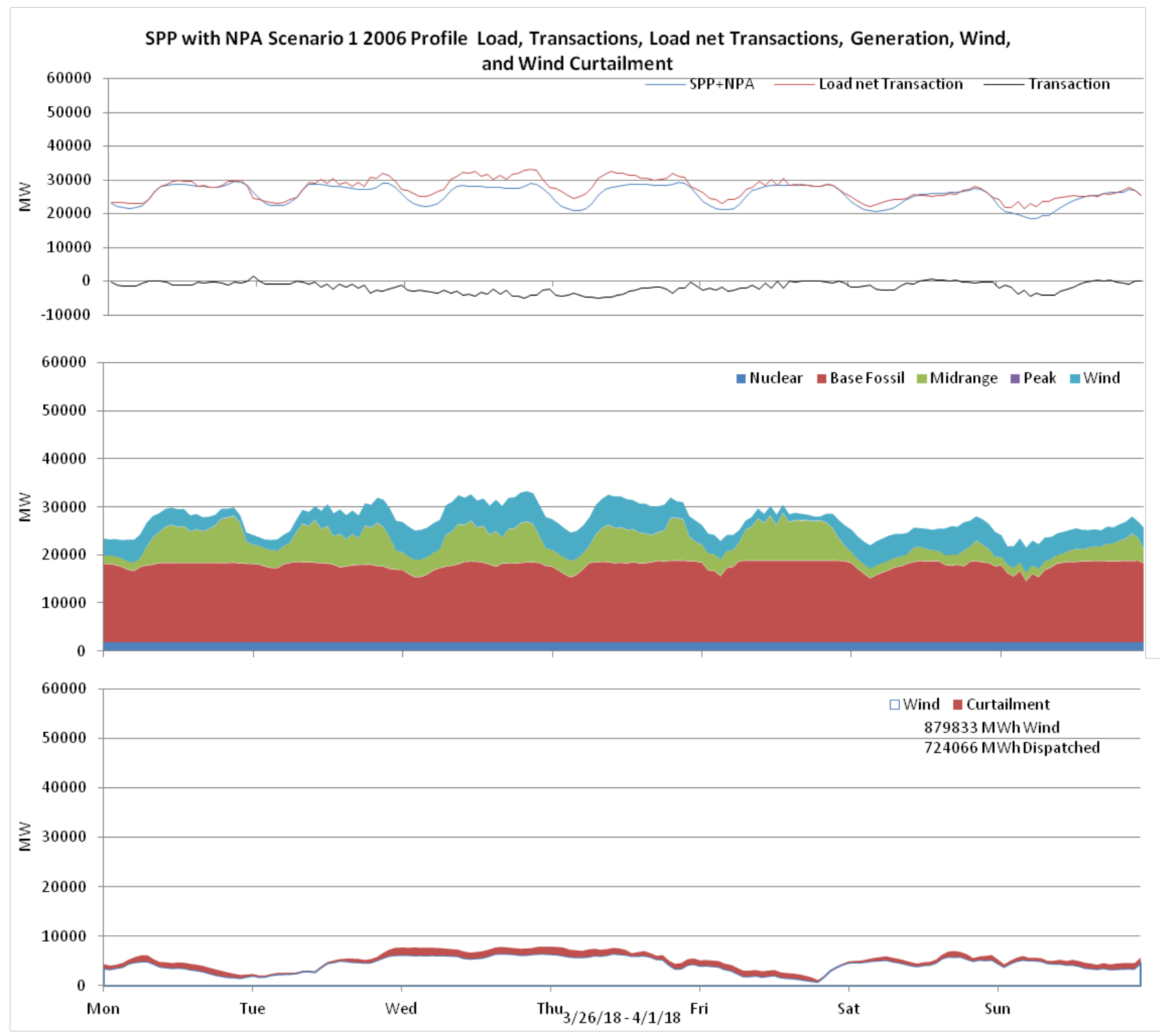

Figure 215: SPP 10\% wind Penetration, 2006 Profile week of 3/26/2018 


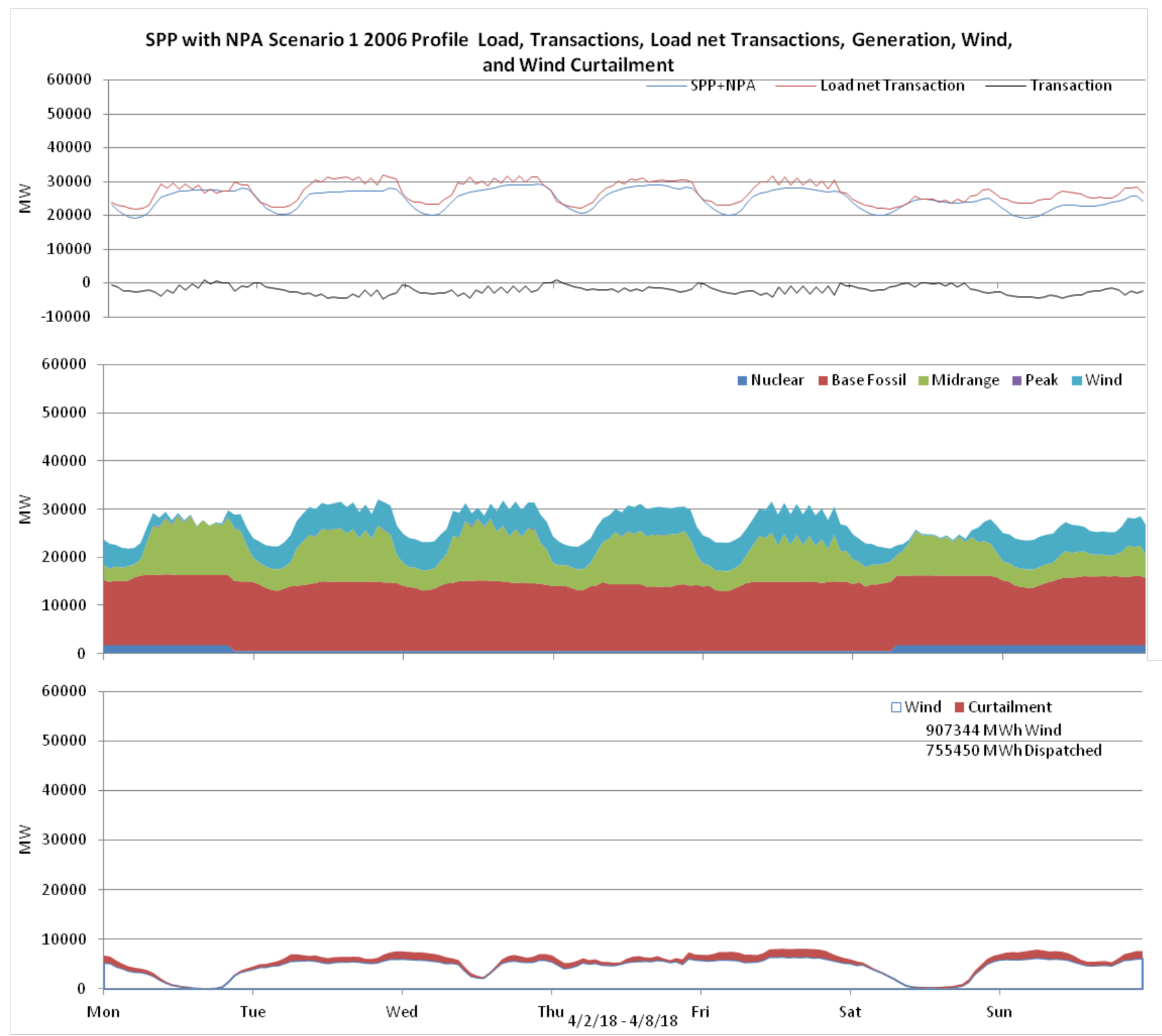

Figure 216: SPP 10\% wind Penetration, 2006 Profile week of 4/2/2018 


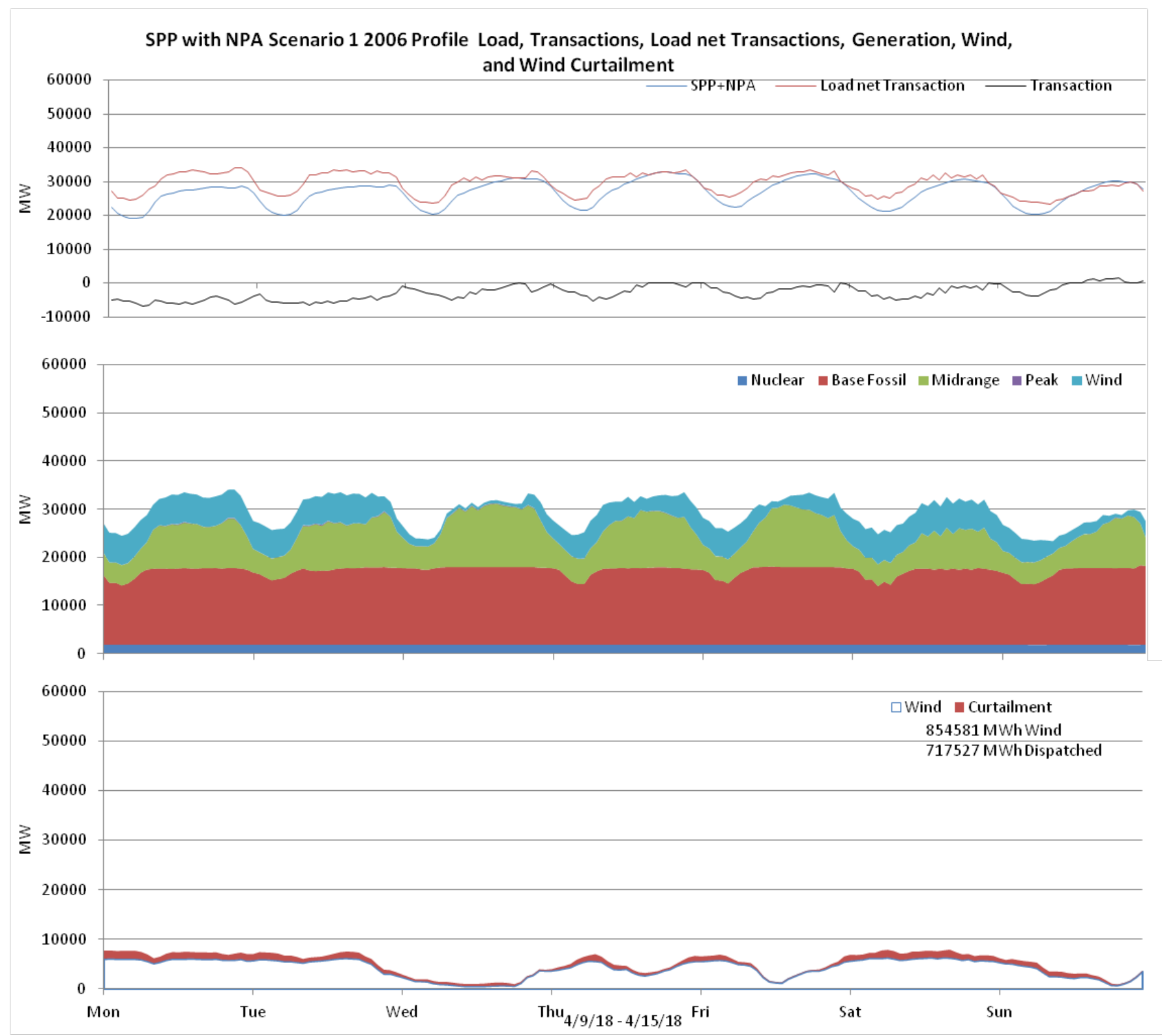

Figure 217: SPP 10\% wind Penetration, 2006 Profile week of 4/9/2018 


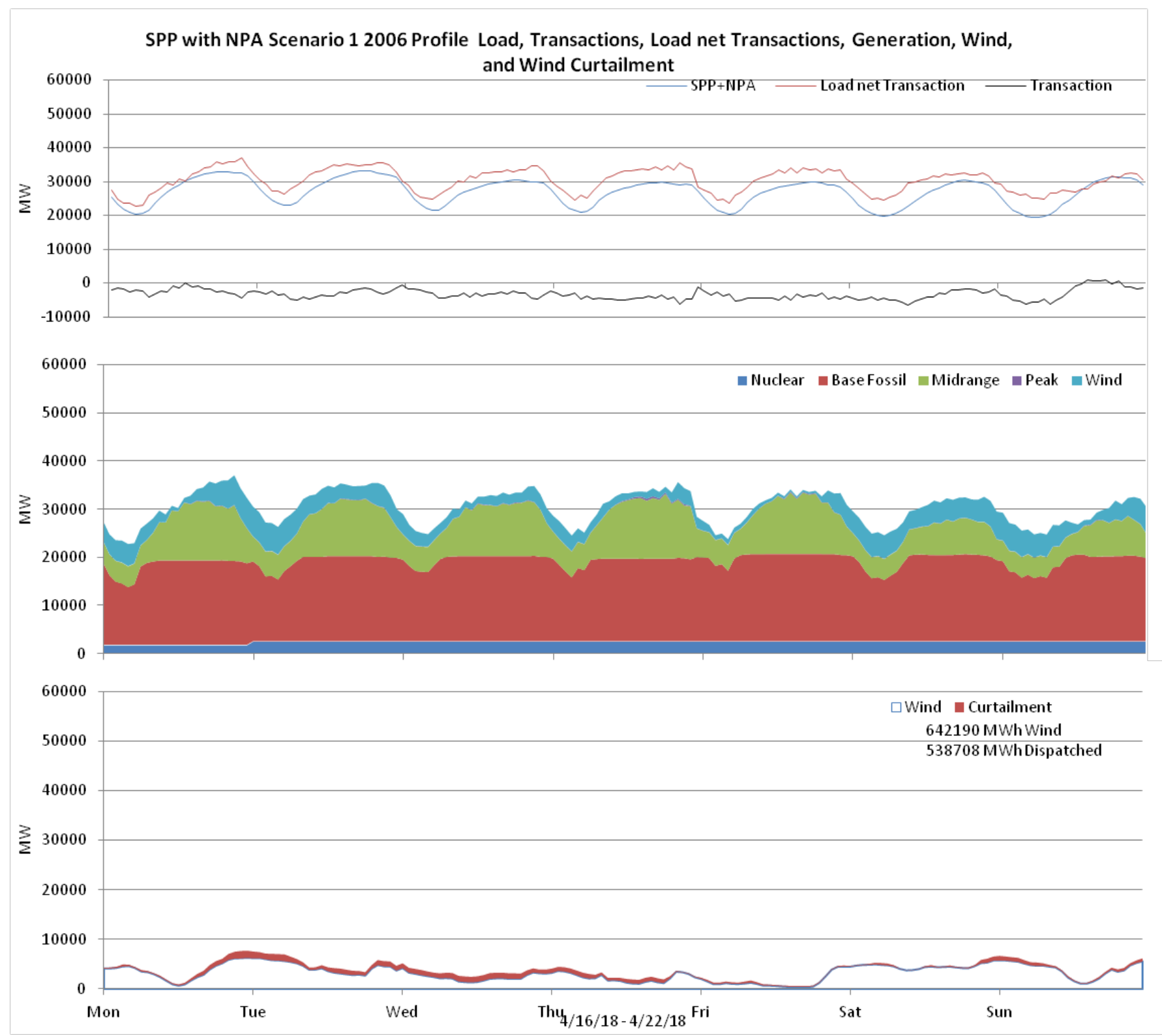

Figure 218: SPP 10\% wind Penetration, 2006 Profile week of 4/16/2018 


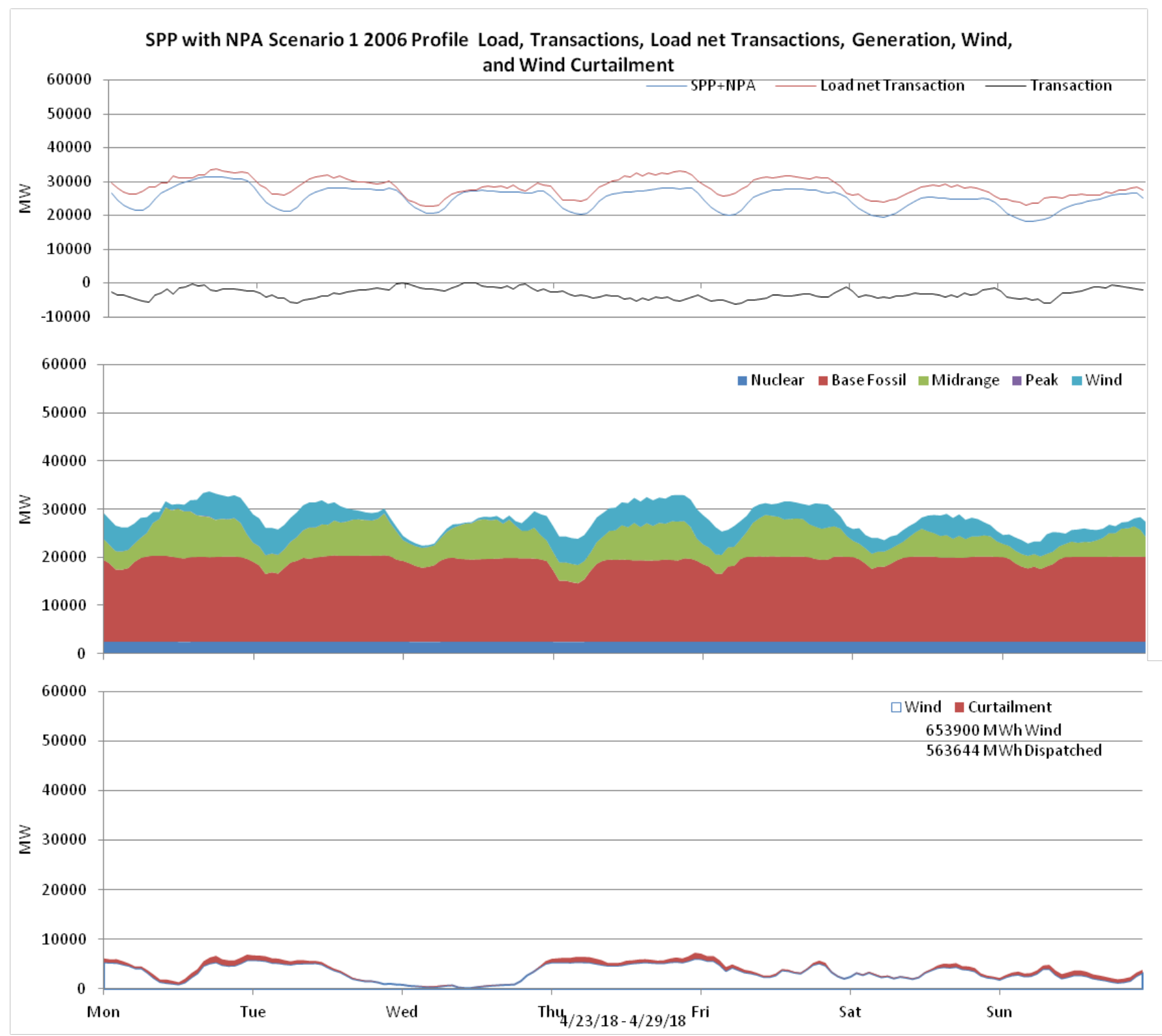

Figure 219: SPP 10\% wind Penetration, 2006 Profile week of 4/23/2018 


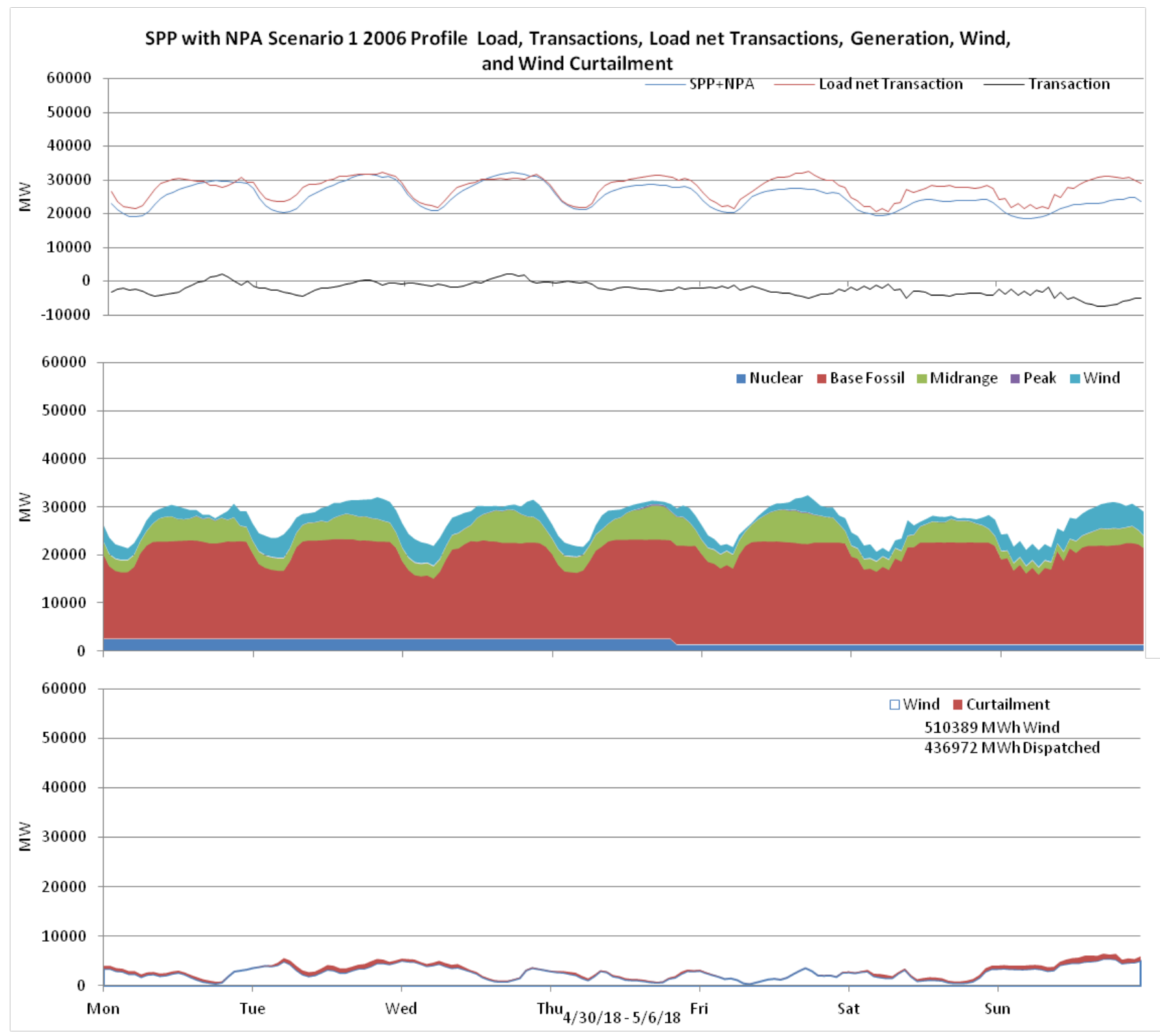

Figure 220: SPP 10\% wind Penetration, 2006 Profile week of 4/30/2018 


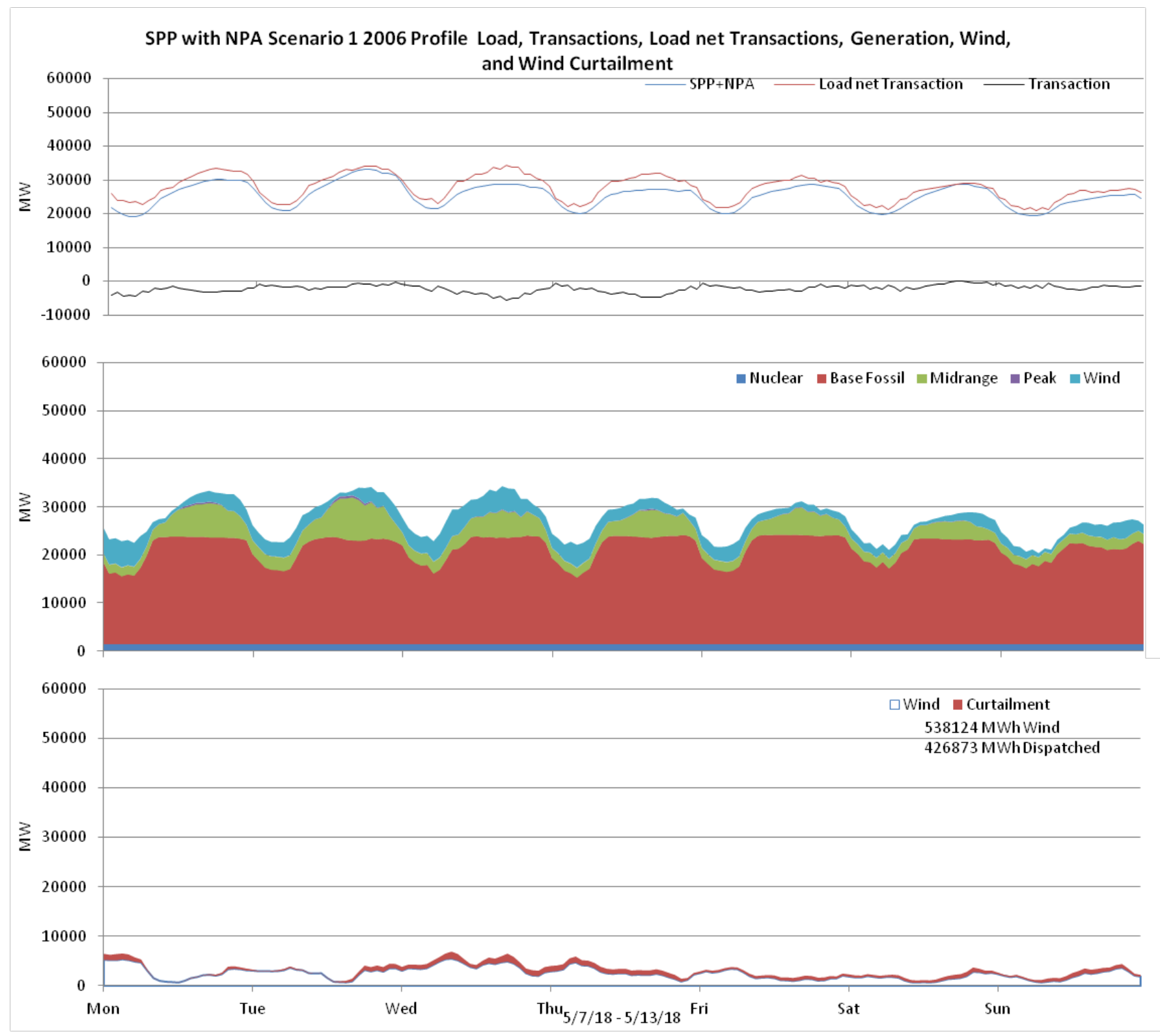

Figure 221: SPP 10\% wind Penetration, 2006 Profile week of 5/7/2018 


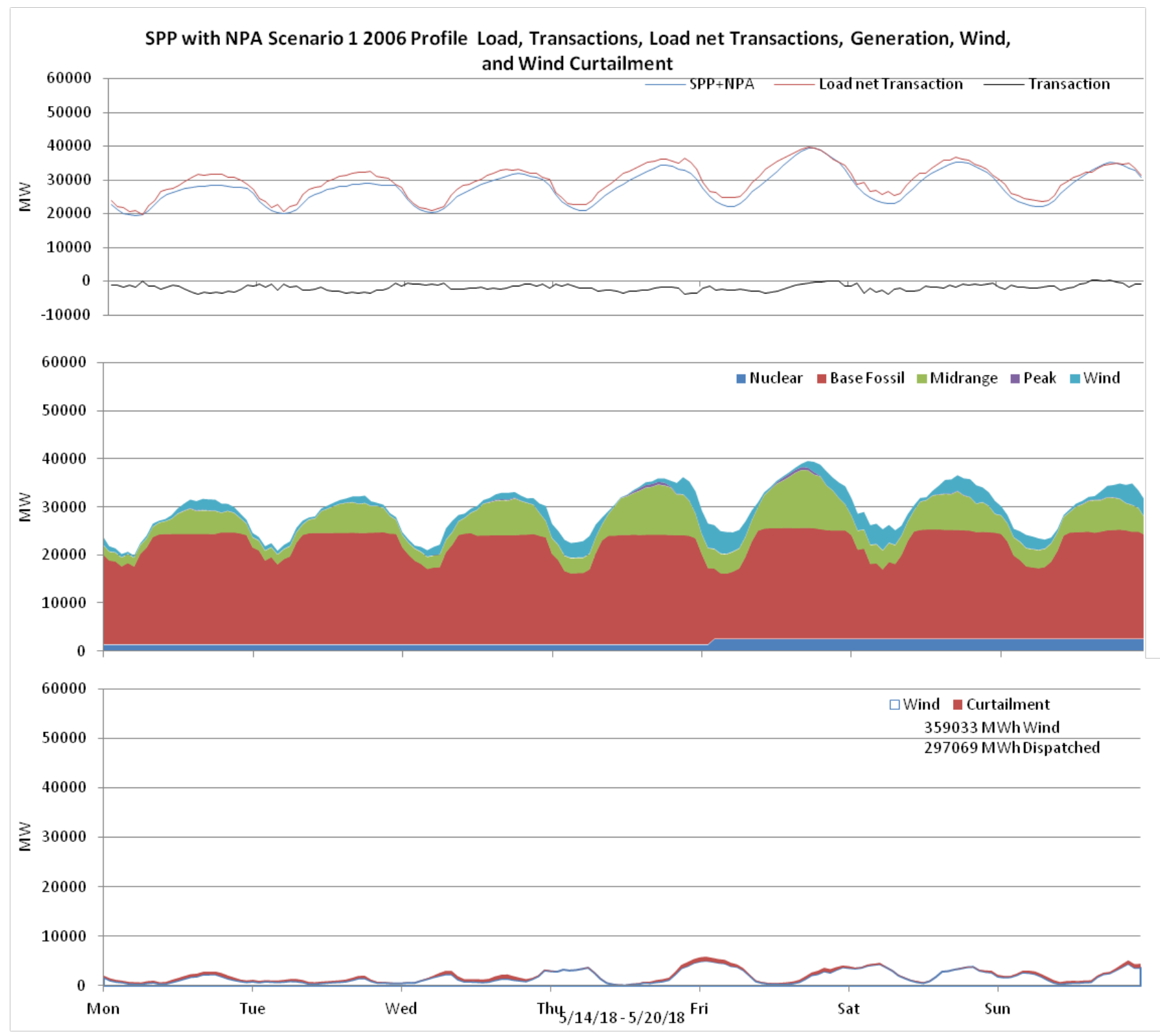

Figure 222: SPP 10\% wind Penetration, 2006 Profile week of 5/14/2018 


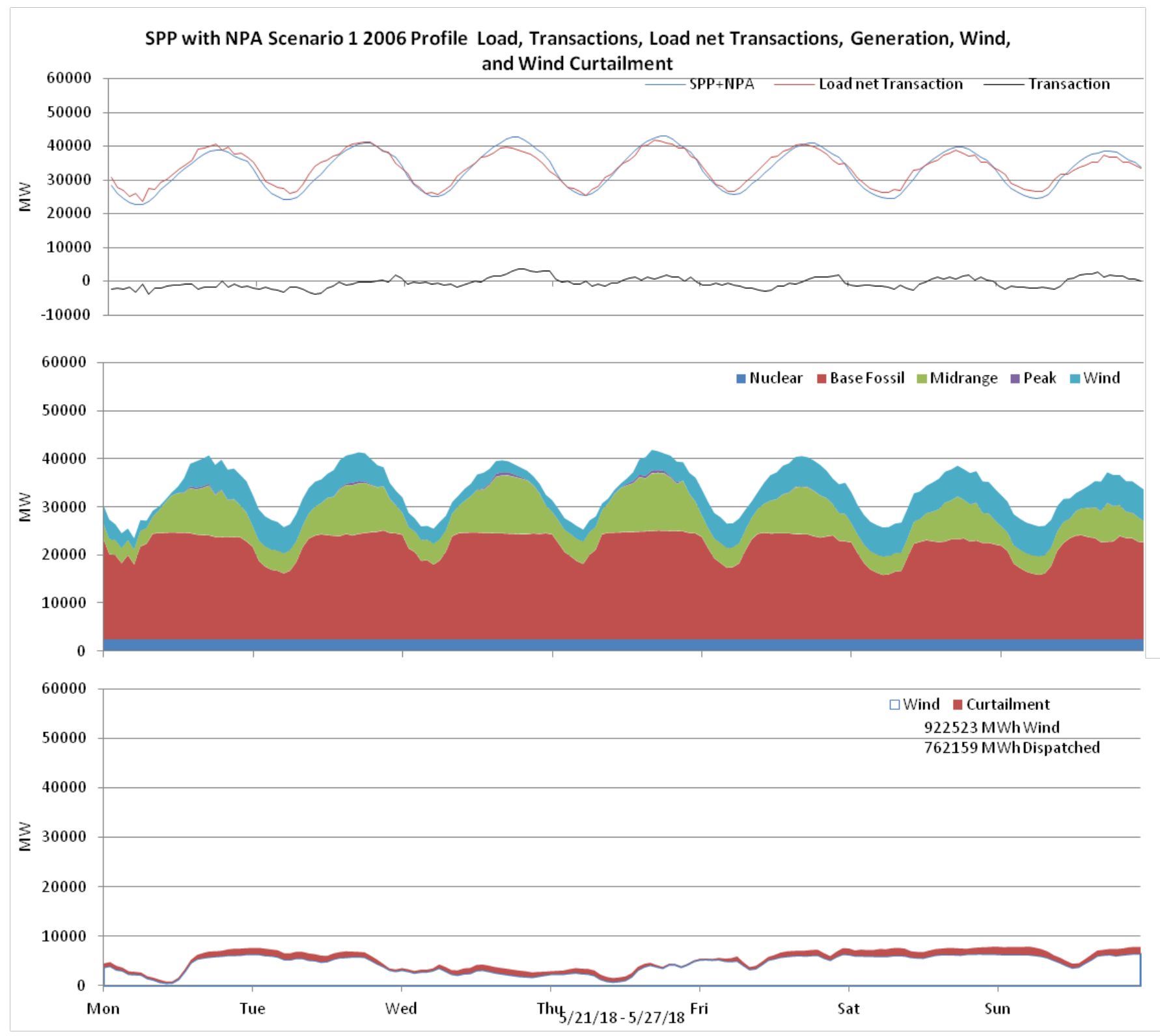

Figure 223: SPP 10\% wind Penetration, 2006 Profile week of 5/21/2018 


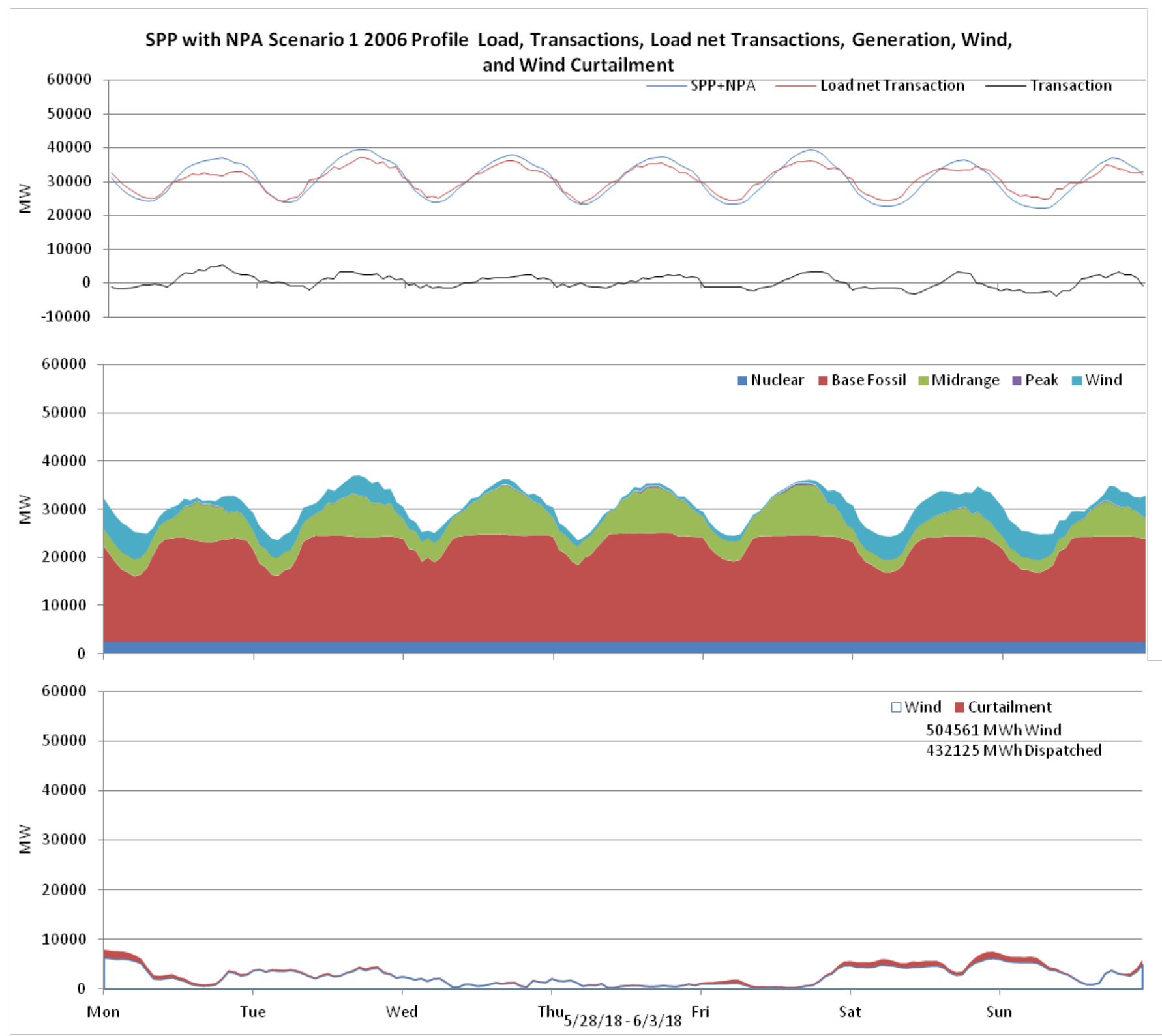

Figure 224: SPP 10\% wind Penetration, 2006 Profile week of 5/28/2018 


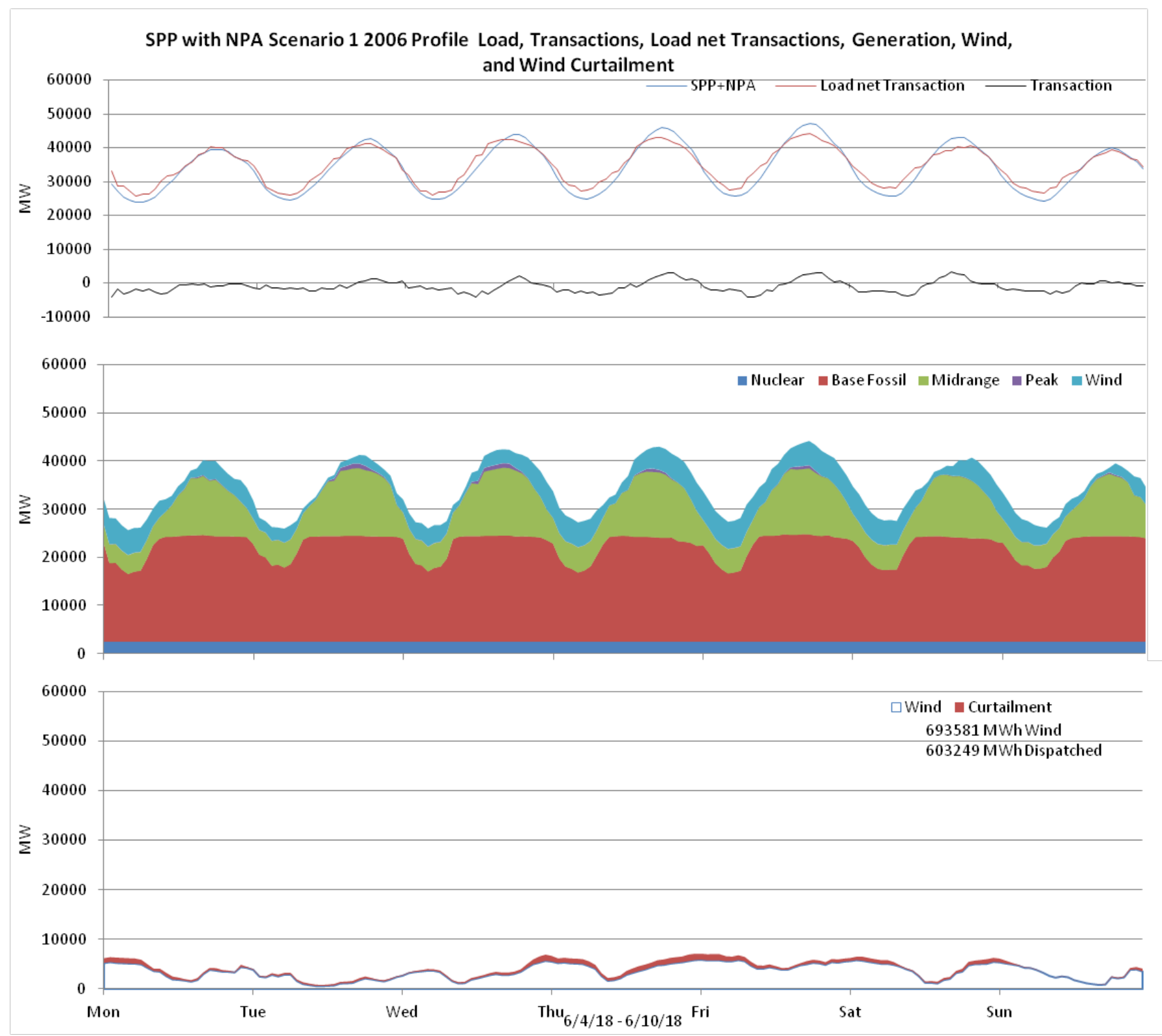

Figure 225: SPP 10\% wind Penetration, 2006 Profile week of 6/4/2018 


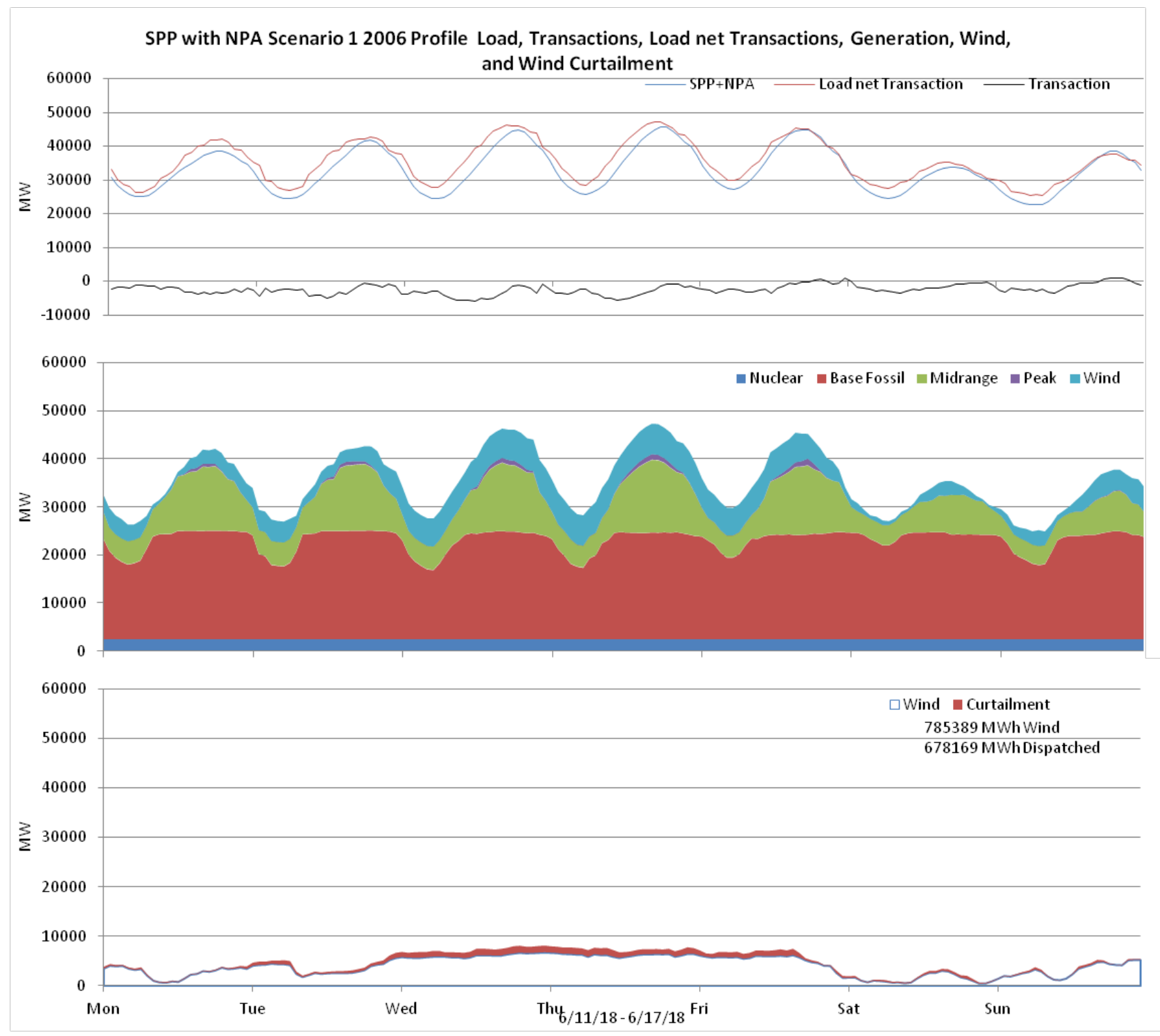

Figure 226: SPP 10\% wind Penetration, 2006 Profile week of $6 / 11 / 2018$ 


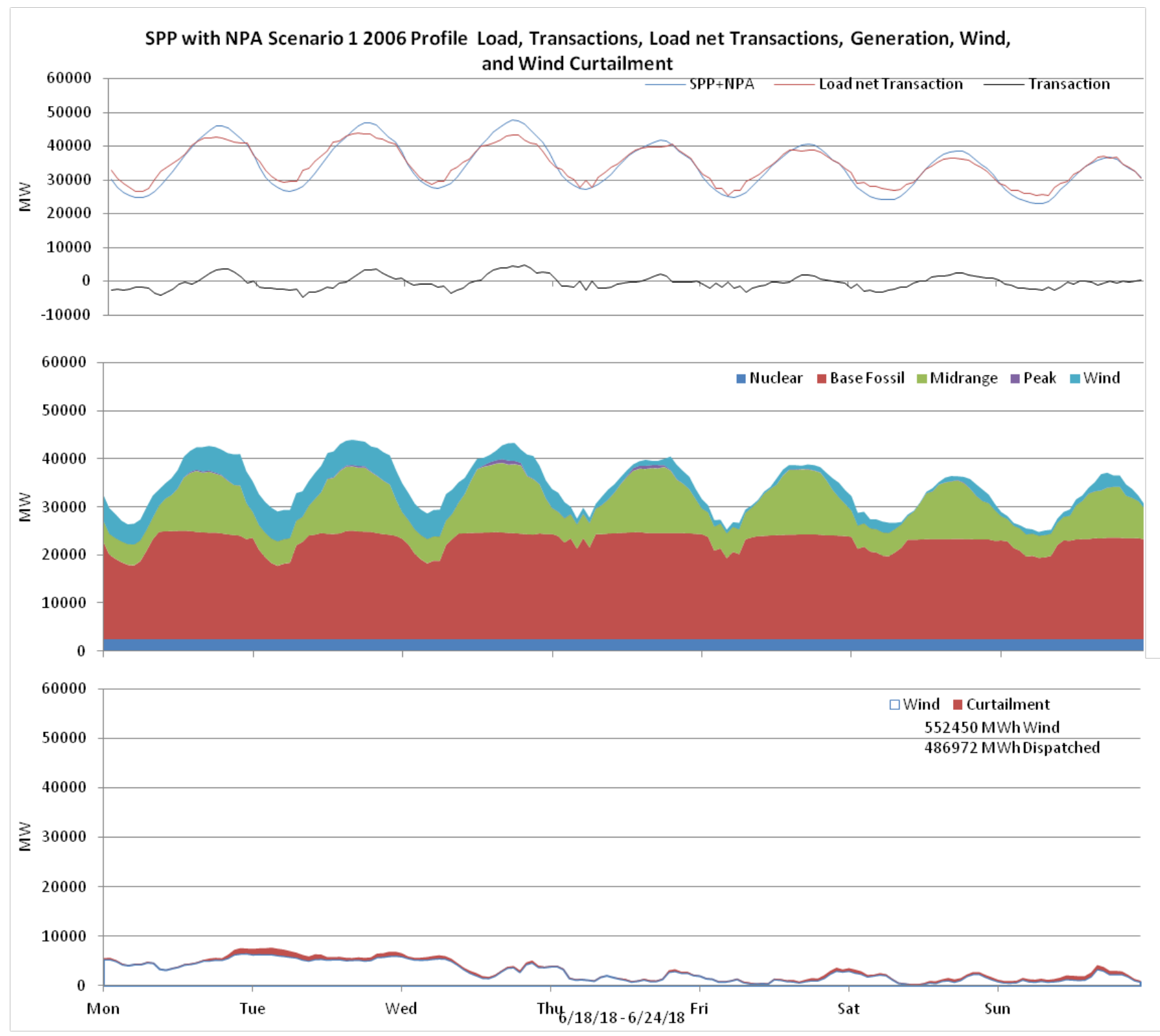

Figure 227: SPP 10\% wind Penetration, 2006 Profile week of 6/18/2018 


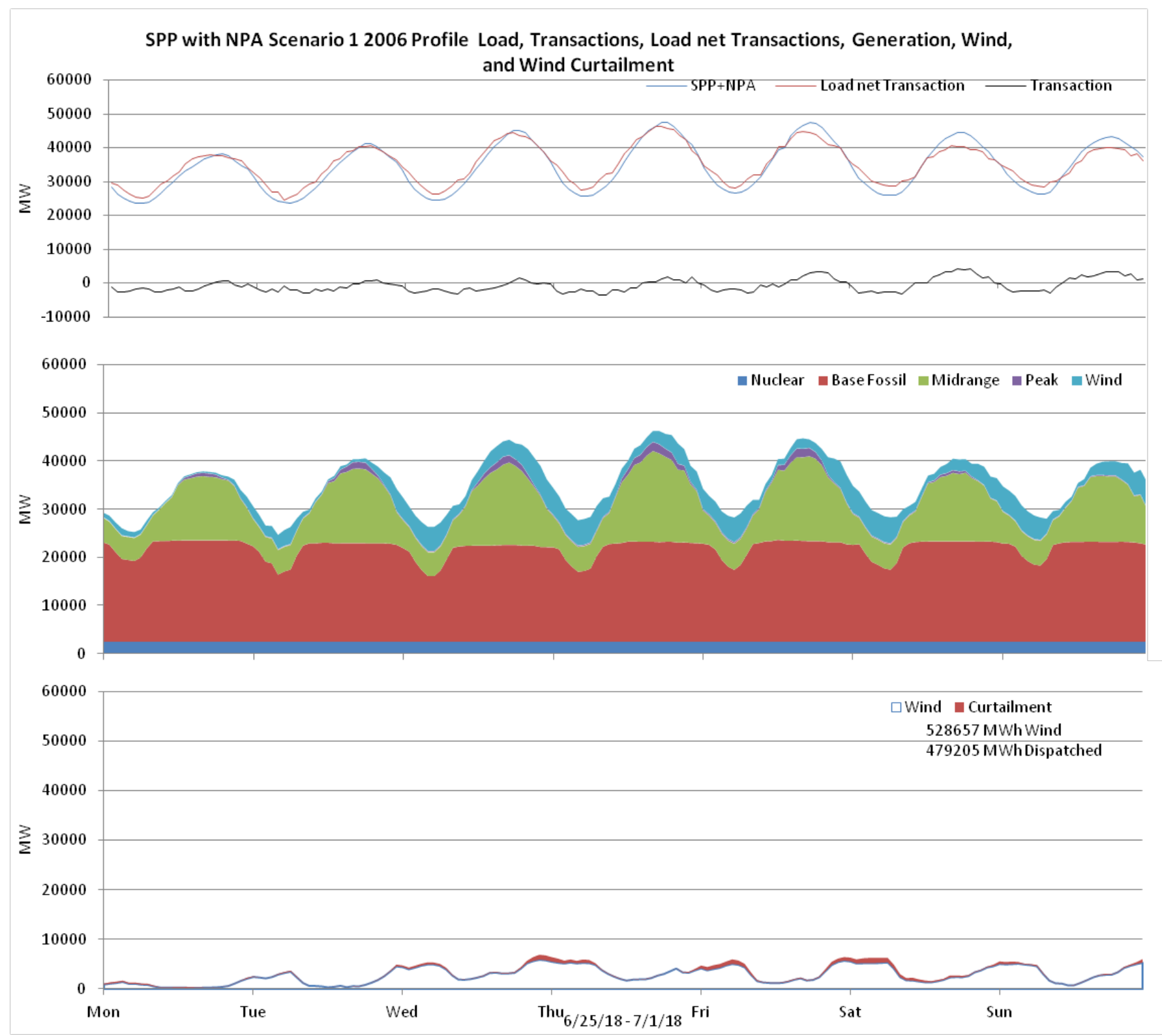

Figure 228: SPP 10\% wind Penetration, 2006 Profile week of 6/25/2018 


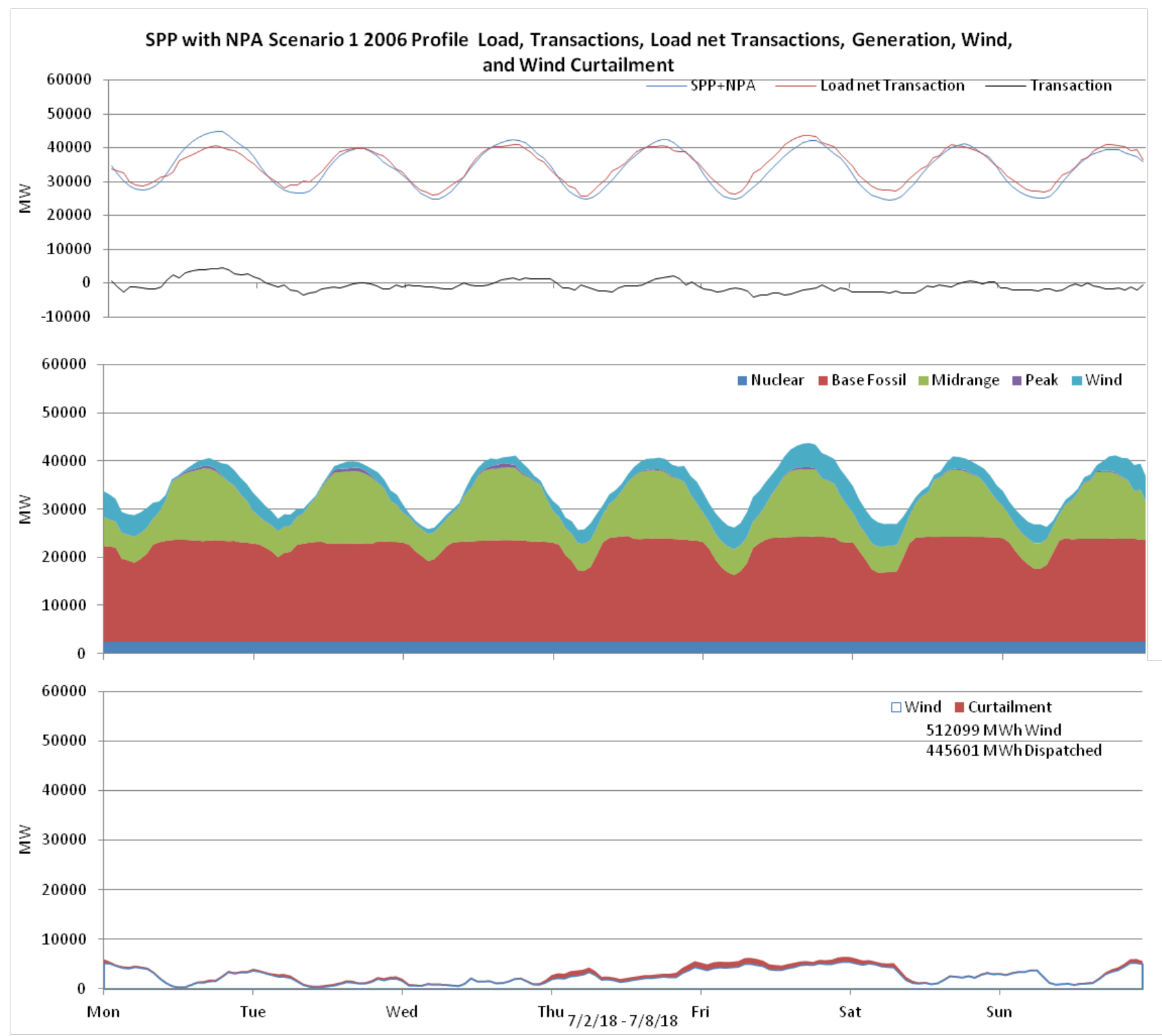

Figure 229: SPP 10\% wind Penetration, 2006 Profile week of 7/2/2018 


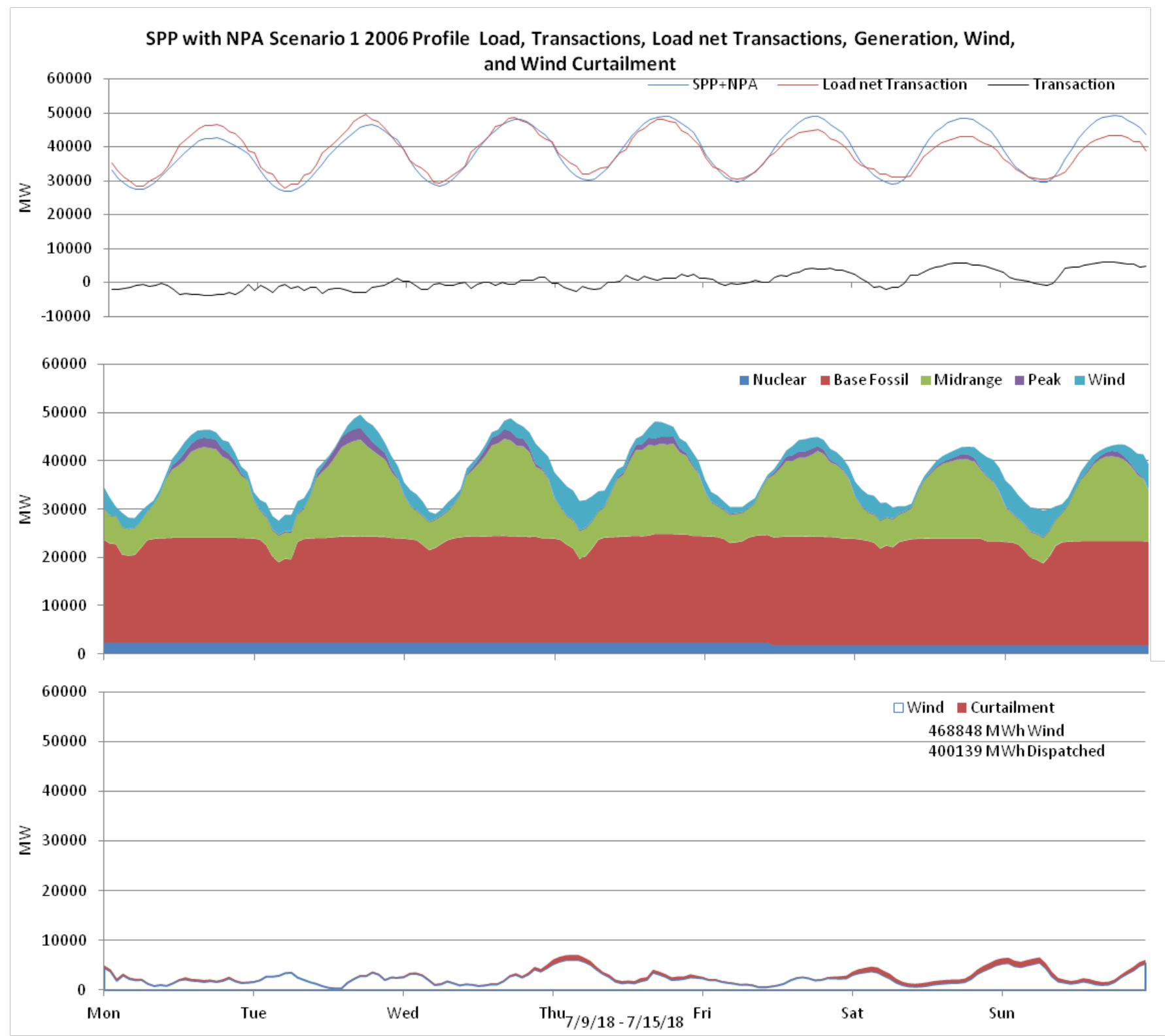

Figure 230: SPP 10\% wind Penetration, 2006 Profile week of 7/9/2018 


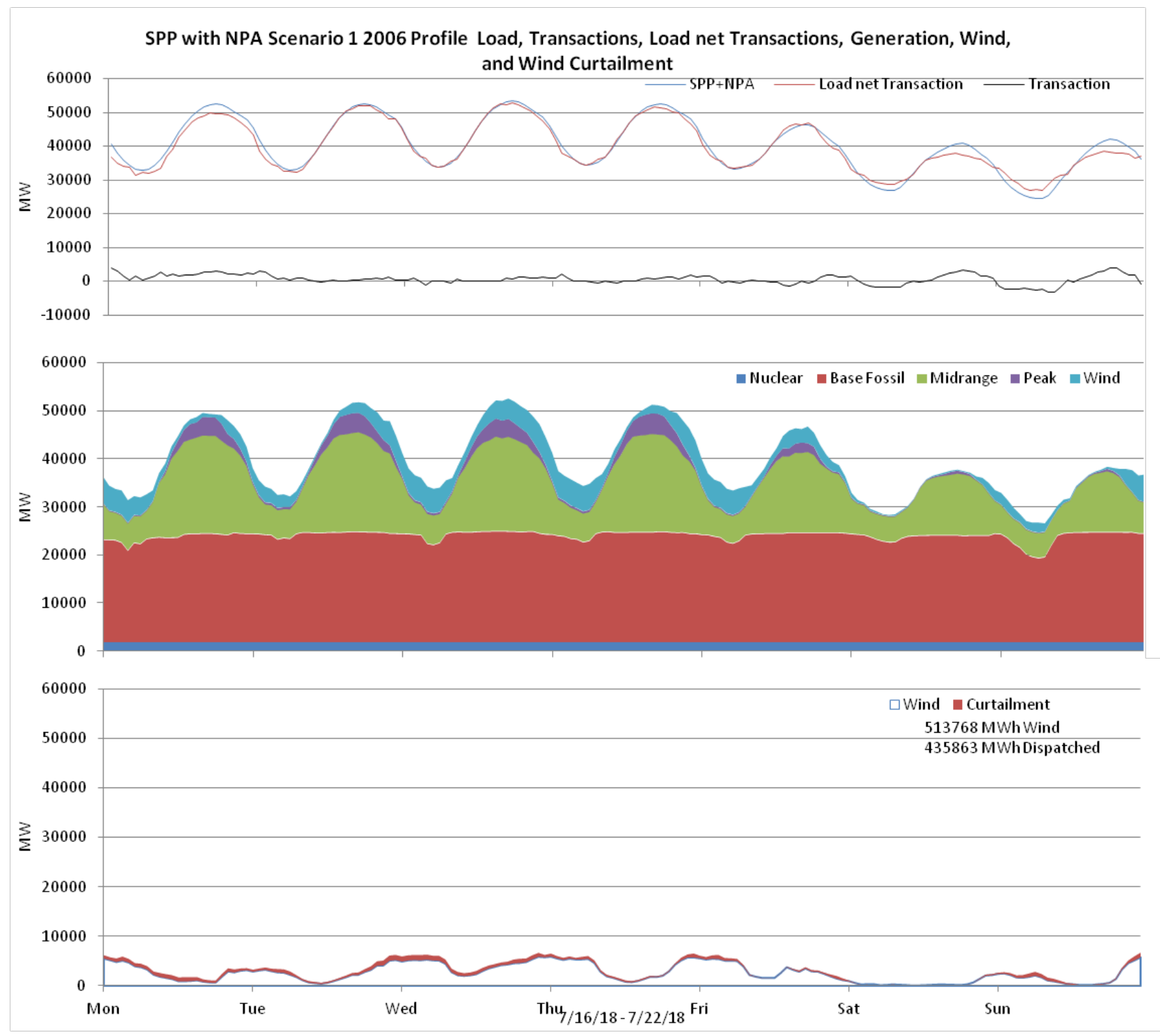

Figure 231: SPP 10\% wind Penetration, 2006 Profile week of 7/16/2018 


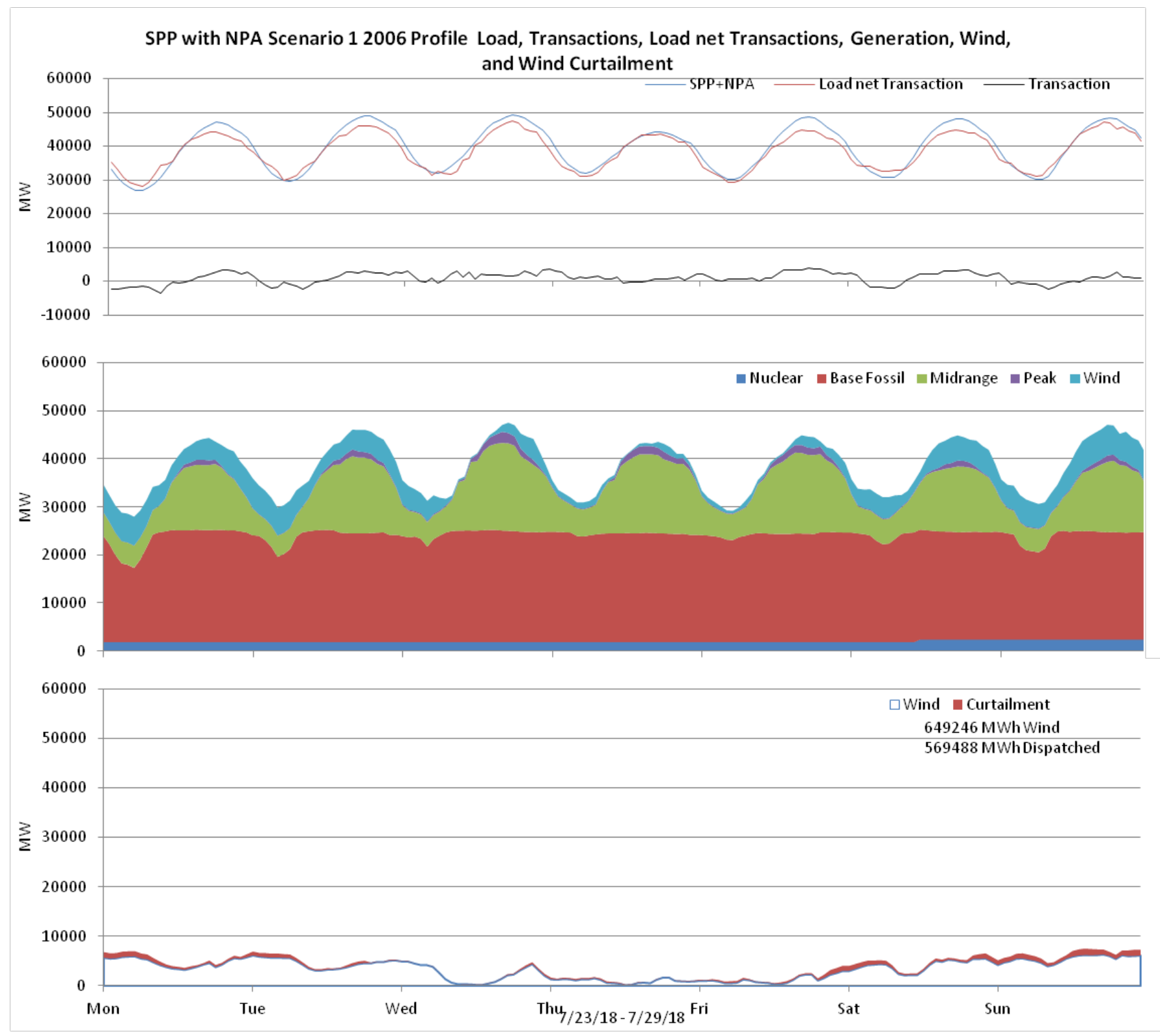

Figure 232: SPP 10\% wind Penetration, 2006 Profile week of 7/23/2018 


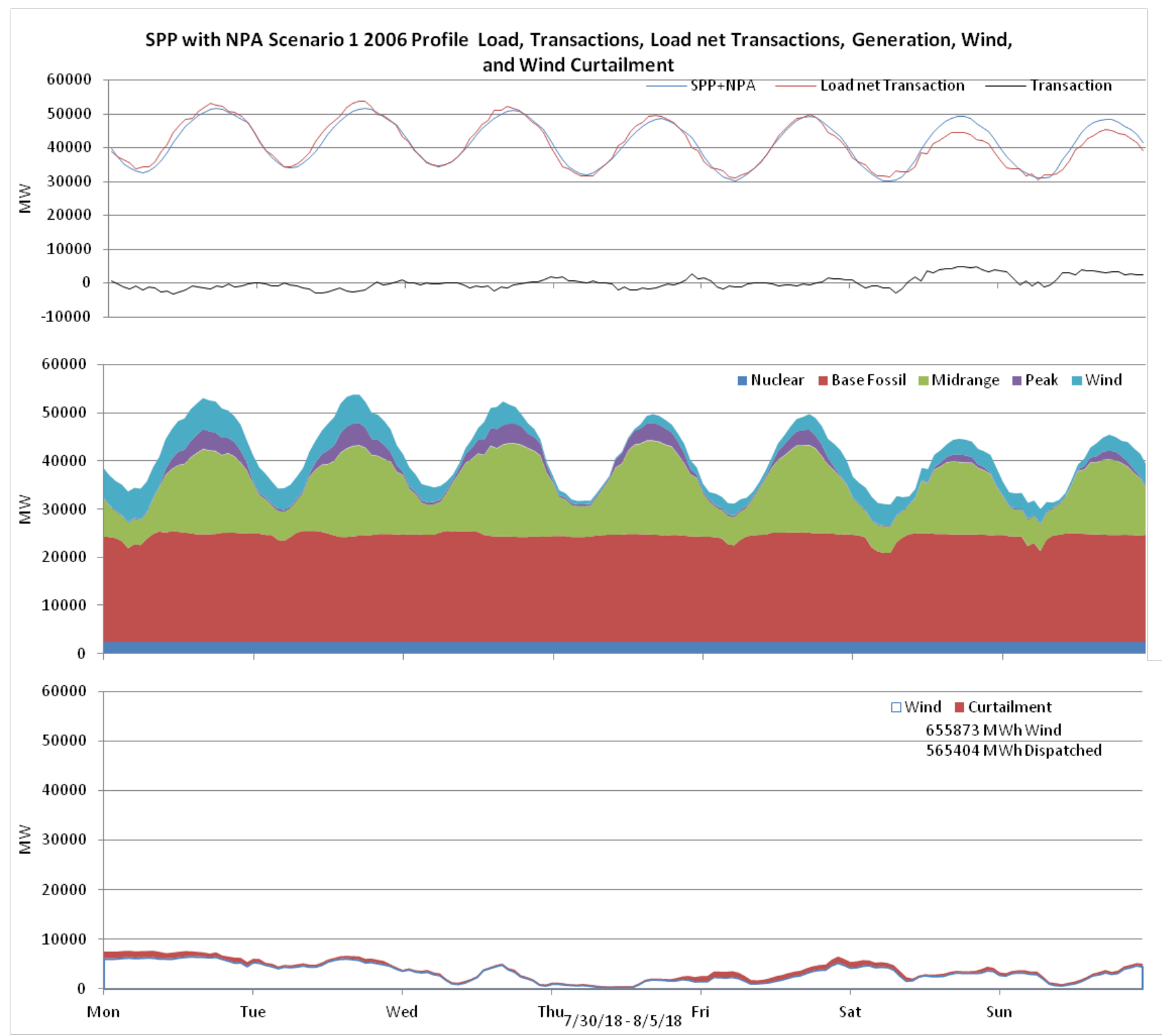

Figure 233: SPP 10\% wind Penetration, 2006 Profile week of 7/30/2018 


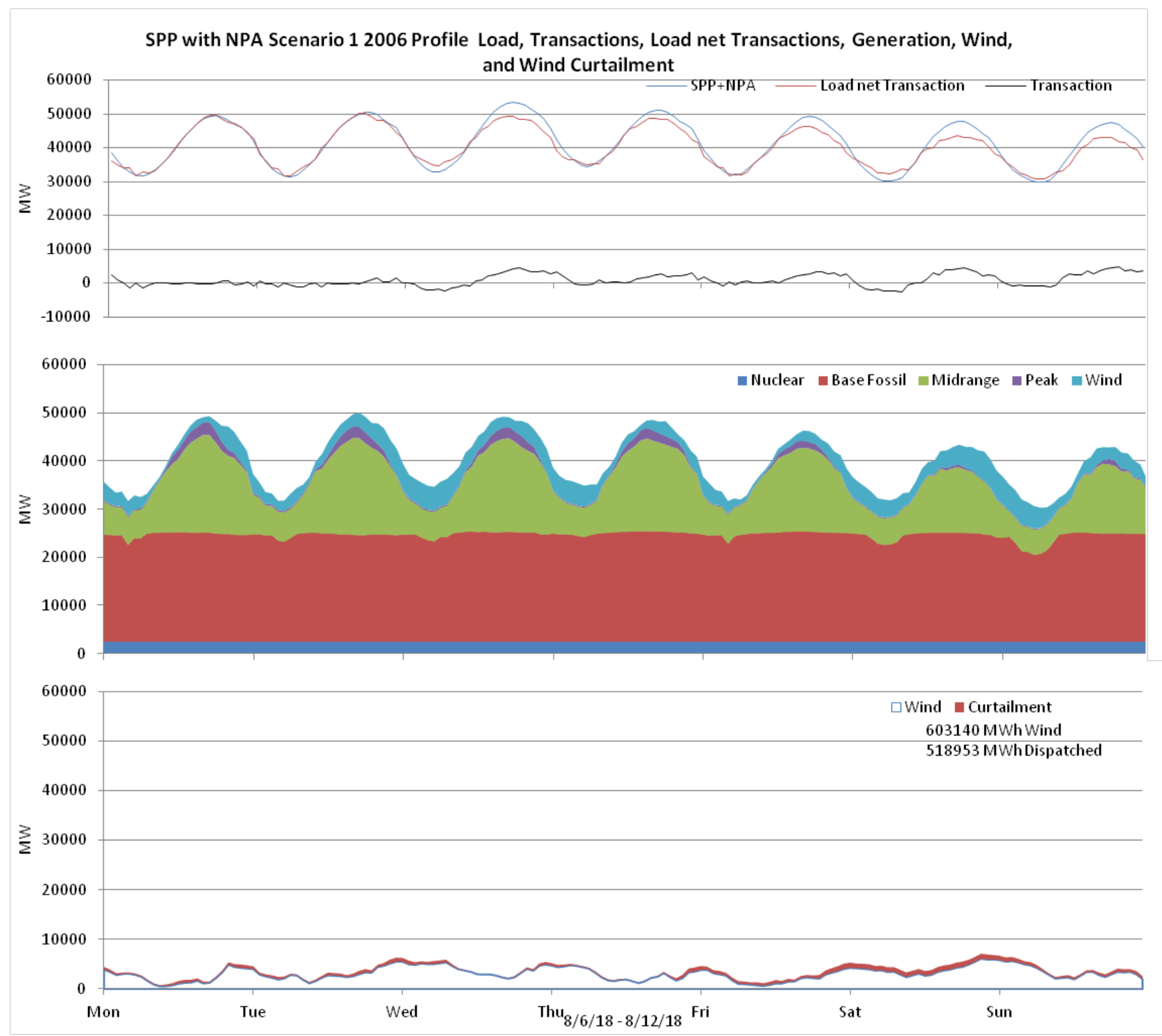

Figure 234: SPP 10\% wind Penetration, 2006 Profile week of 8/6/2018 


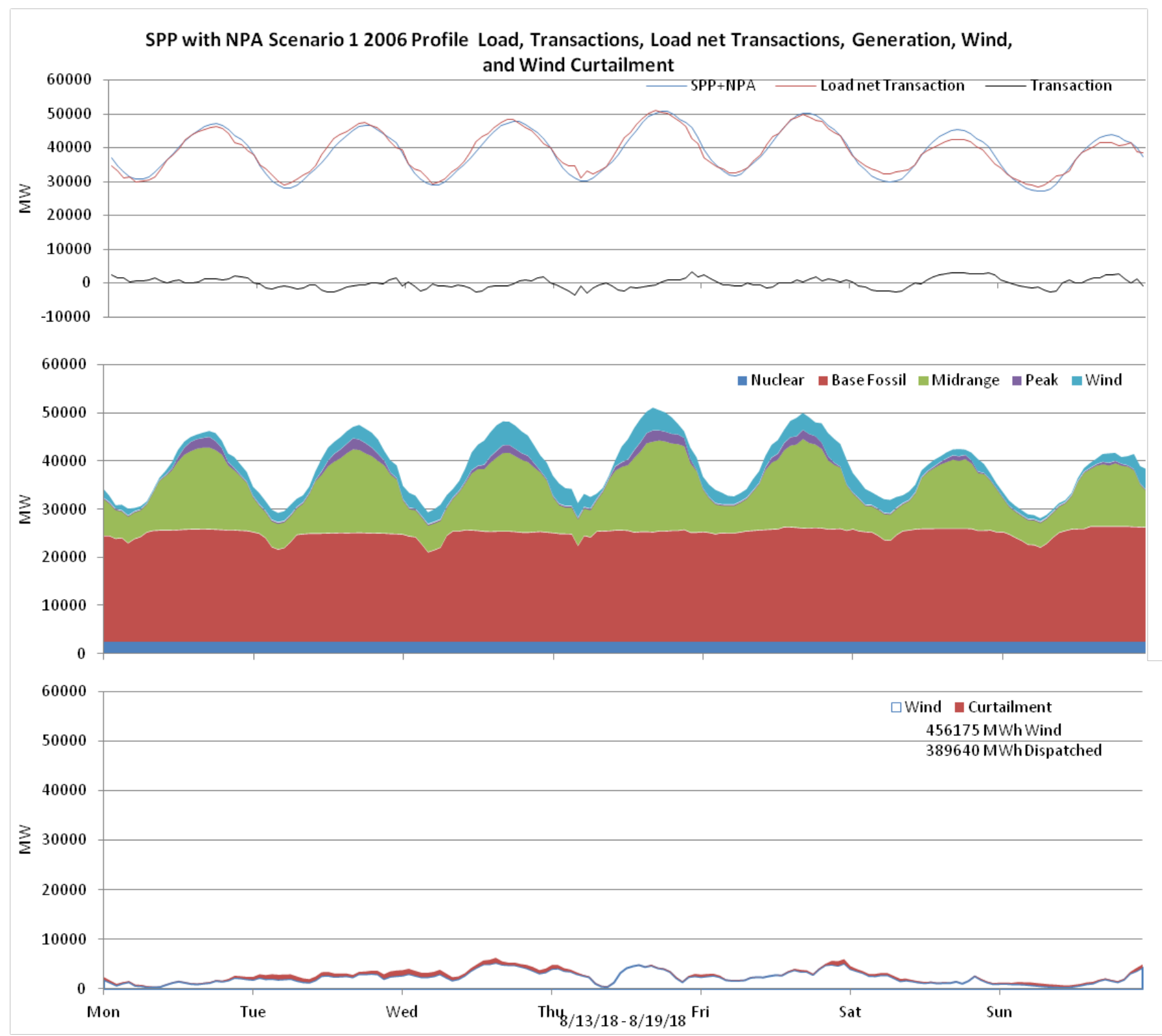

Figure 235: SPP 10\% wind Penetration, 2006 Profile week of 8/13/2018 


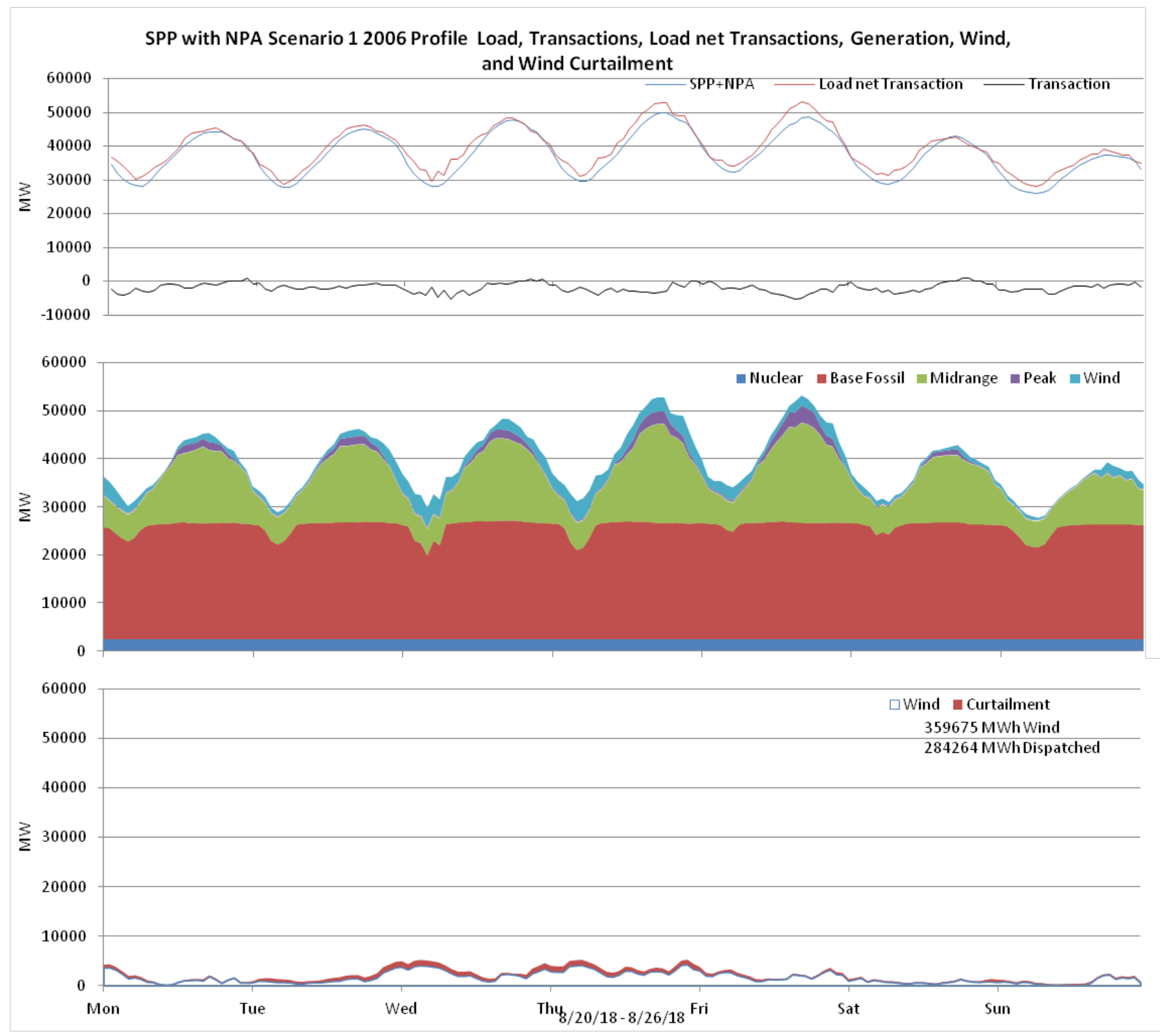

Figure 236: SPP 10\% wind Penetration, 2006 Profile week of $8 / 20 / 2018$ 


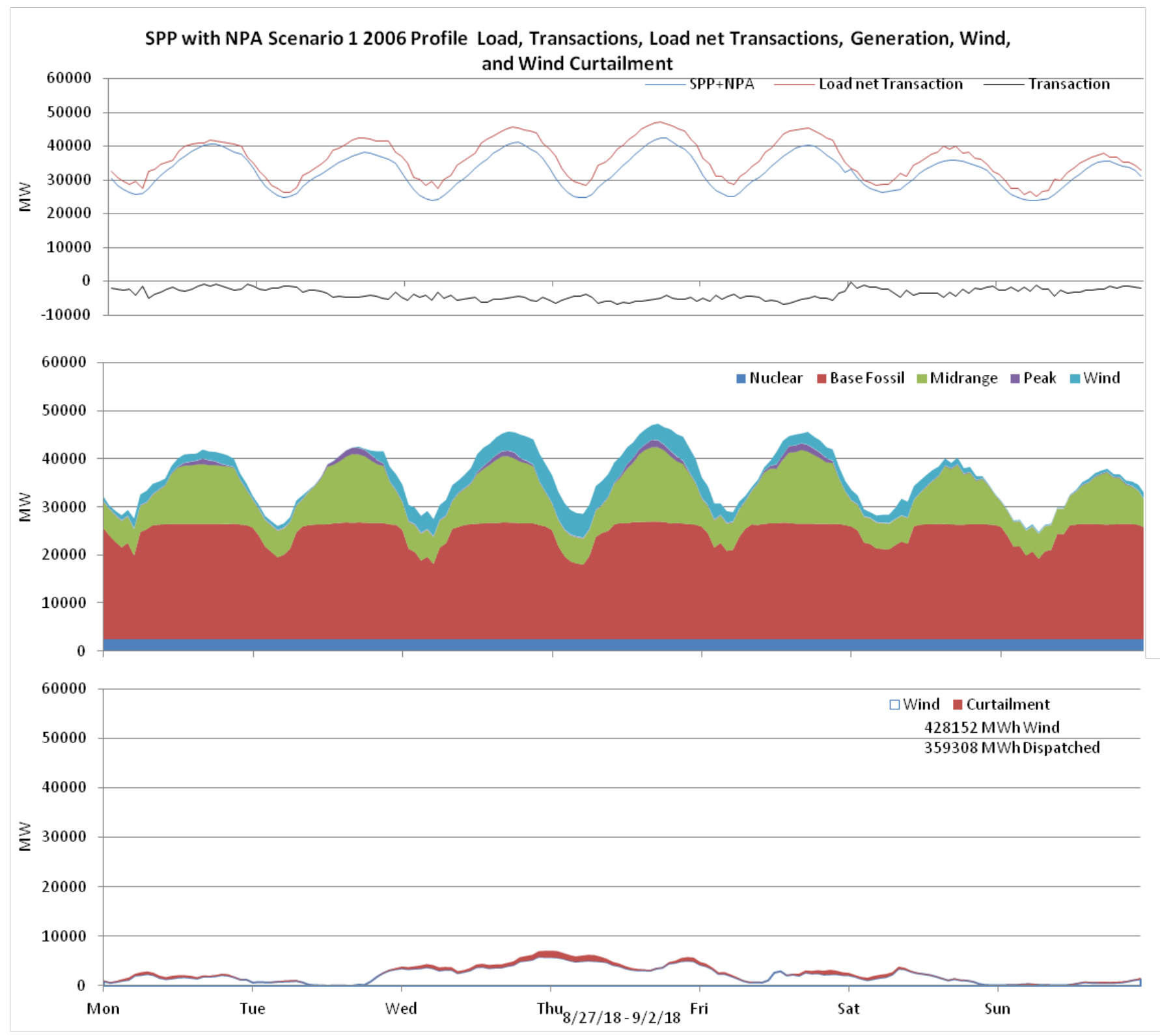

Figure 237: SPP 10\% wind Penetration, 2006 Profile week of $8 / 27 / 2018$ 


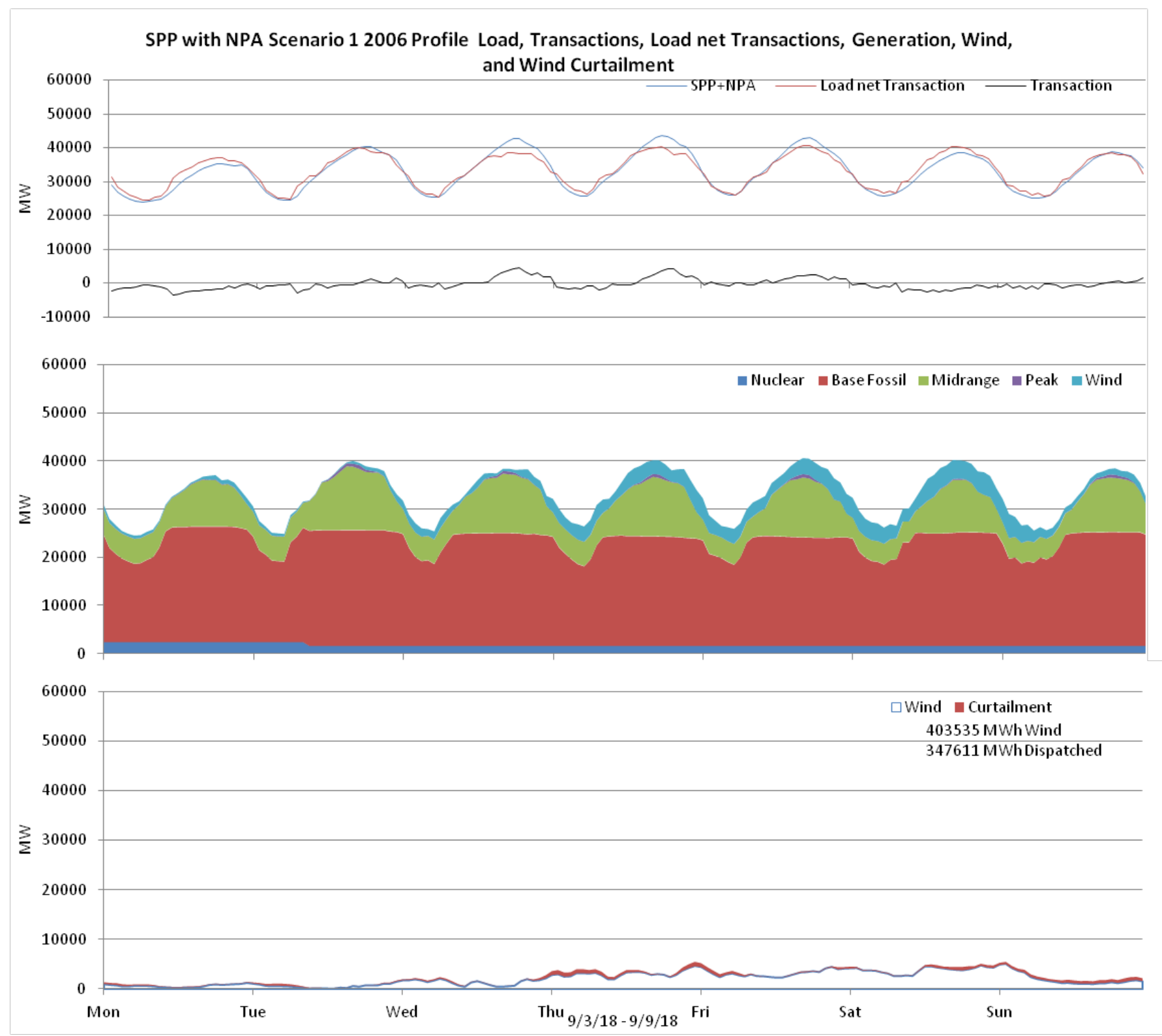

Figure 238: SPP 10\% wind Penetration, 2006 Profile week of $9 / 3 / 2018$ 


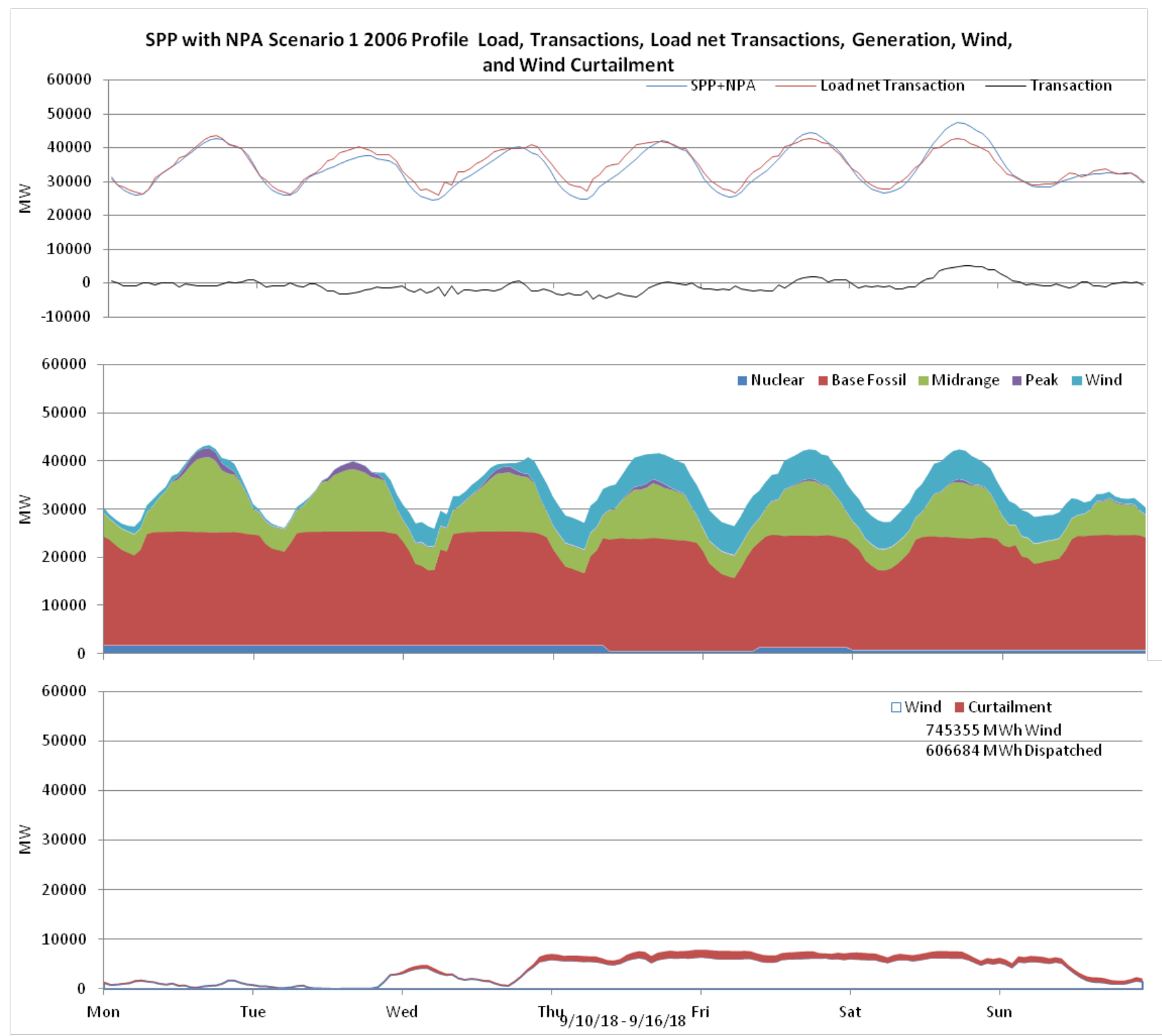

Figure 239: SPP 10\% wind Penetration, 2006 Profile week of $9 / 10 / 2018$ 


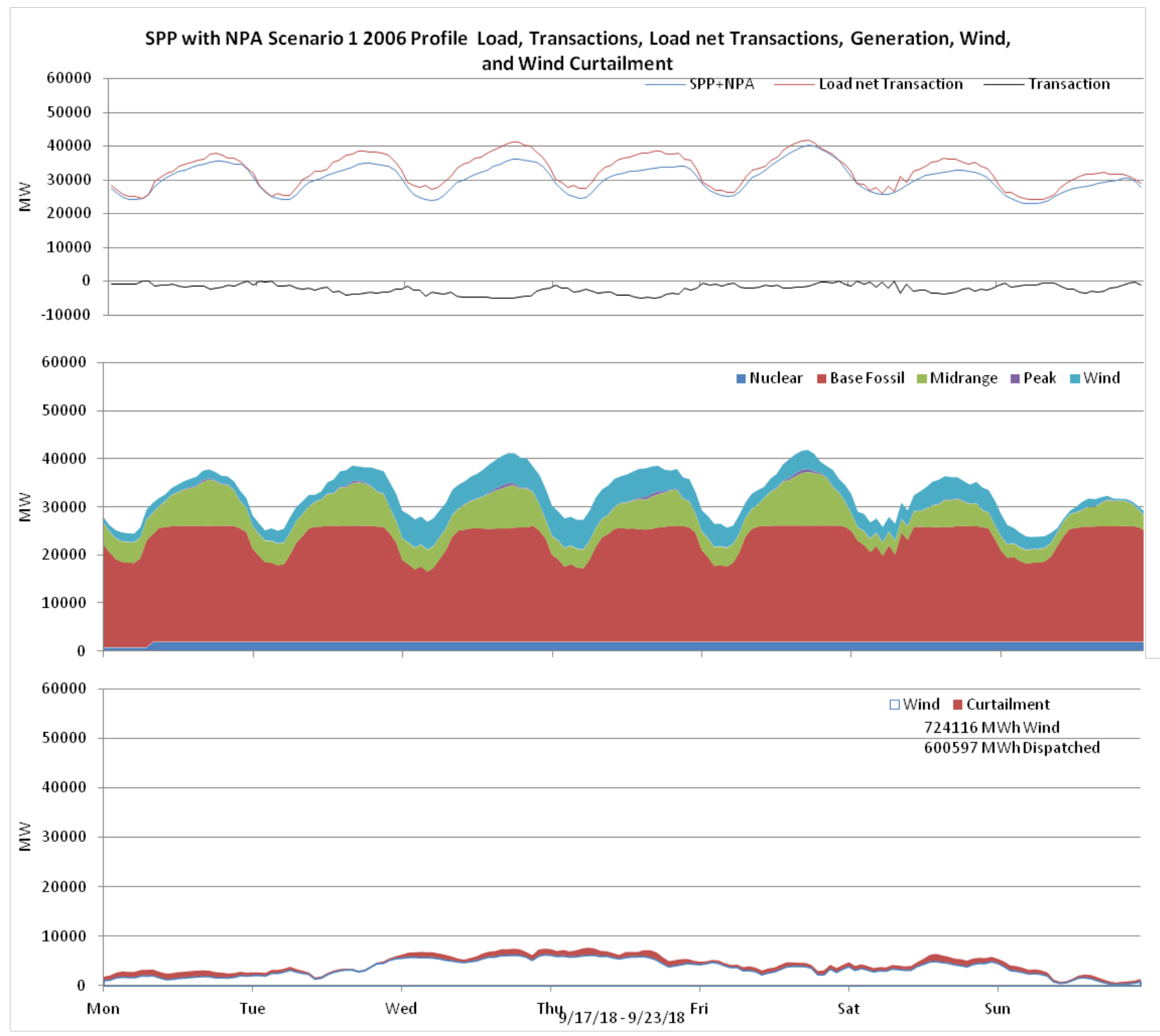

Figure 240: SPP 10\% wind Penetration, 2006 Profile week of 9/17/2018 


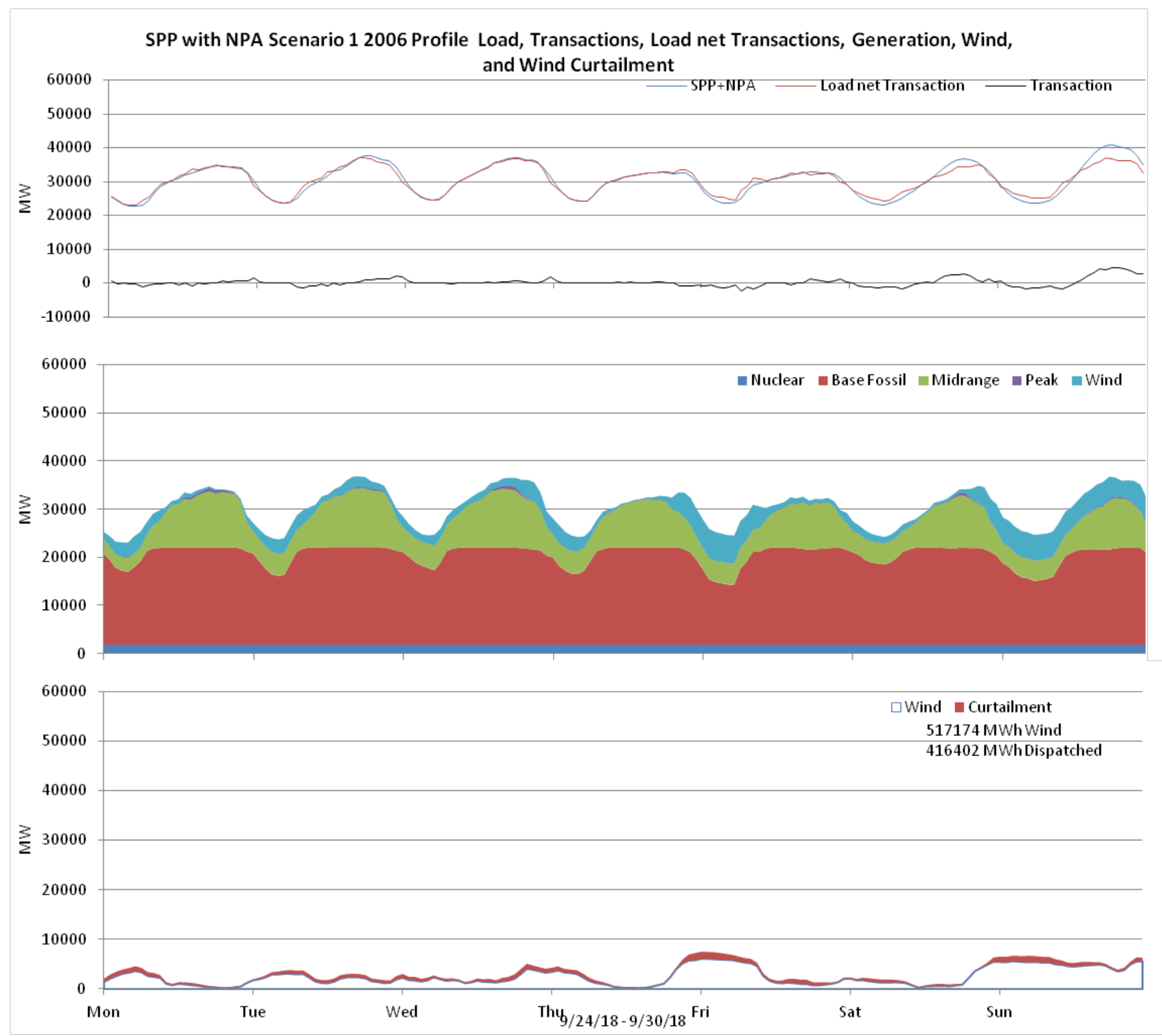

Figure 241: SPP 10\% wind Penetration, 2006 Profile week of 9/24/2018 


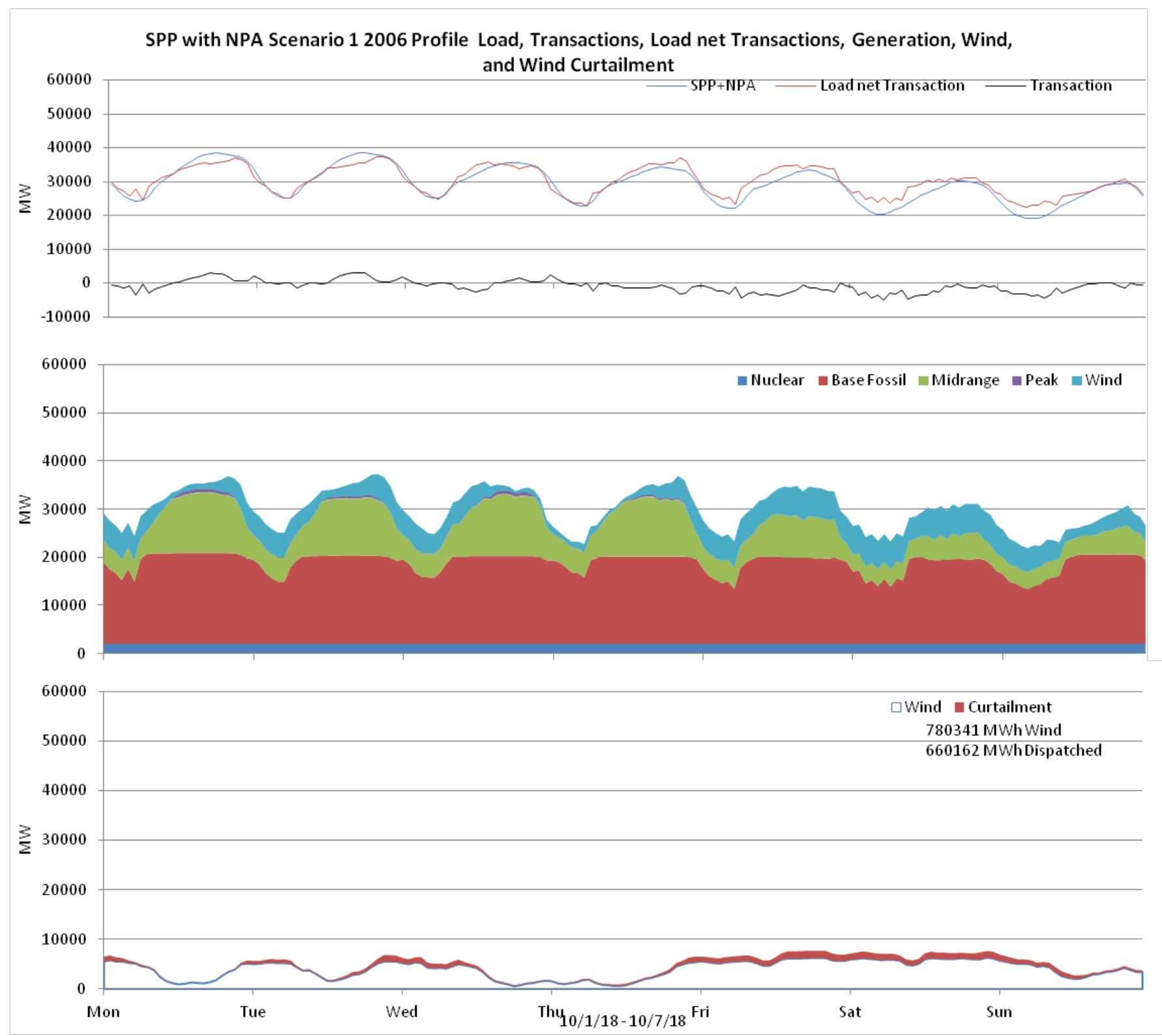

Figure 242: SPP 10\% wind Penetration, 2006 Profile week of 10/1/2018 


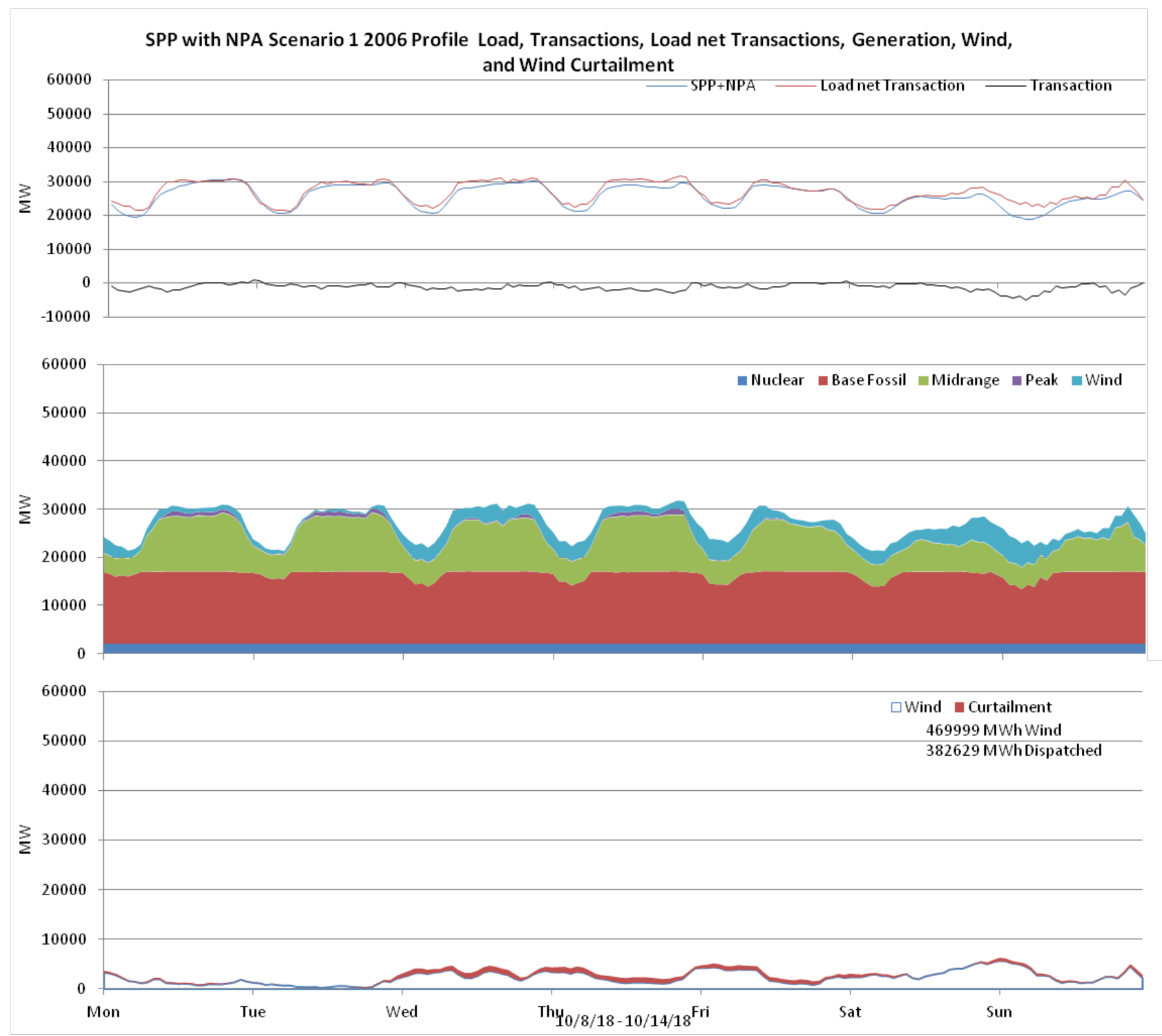

Figure 243: SPP 10\% wind Penetration, 2006 Profile week of 10/8/2018 


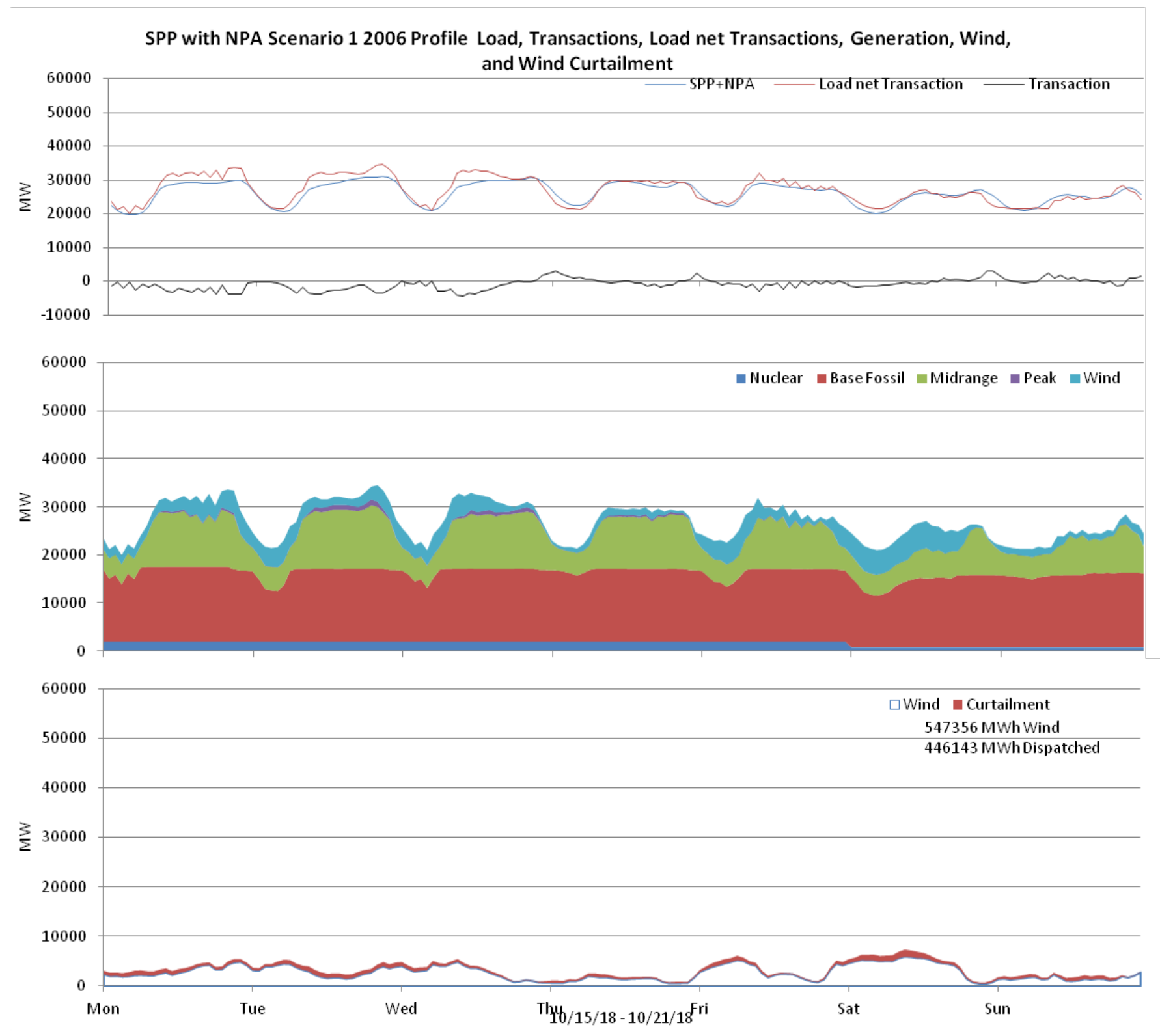

Figure 244: SPP 10\% wind Penetration, 2006 Profile week of 10/15/2018 


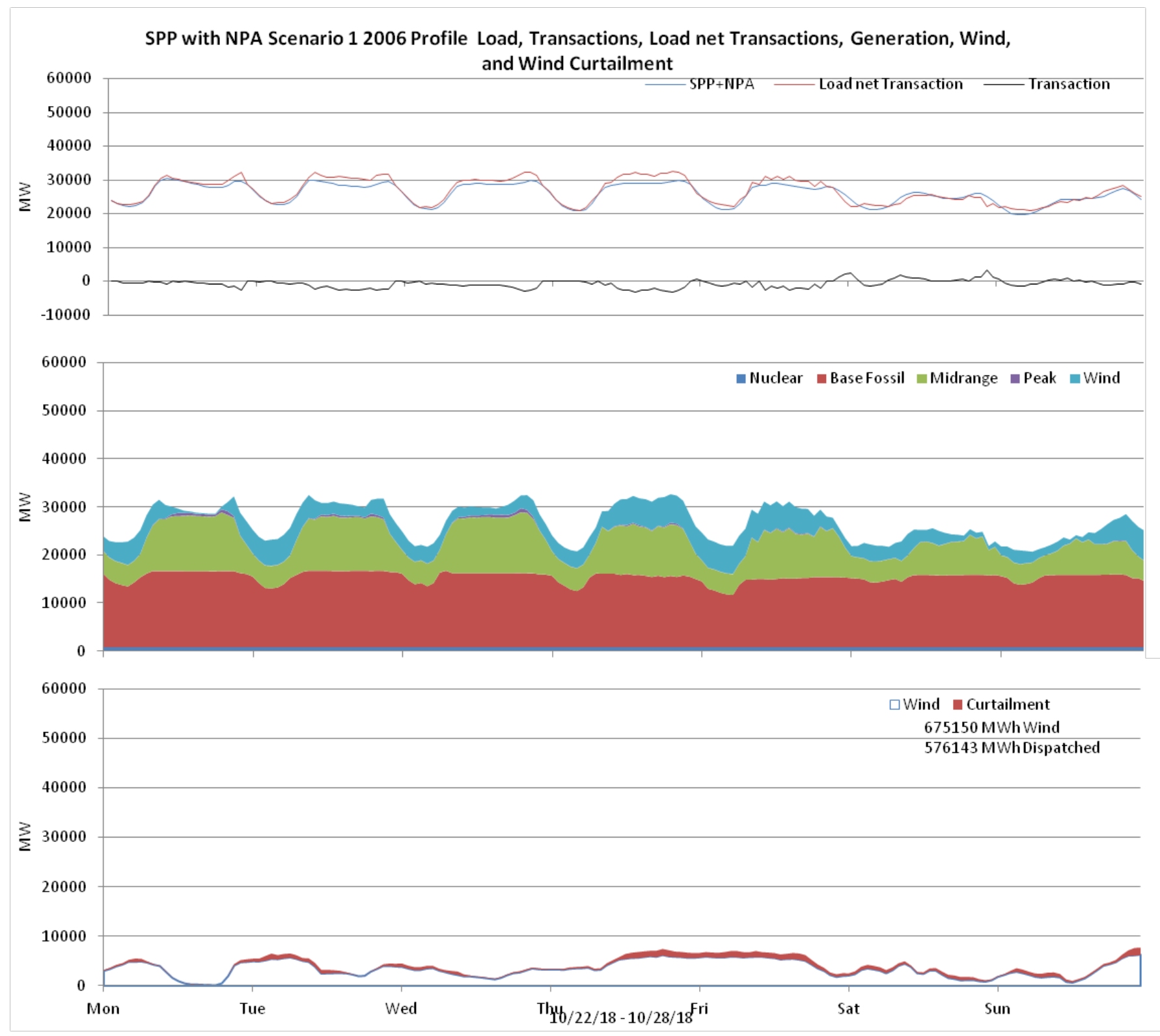

Figure 245: SPP 10\% wind Penetration, 2006 Profile week of 10/22/2018 


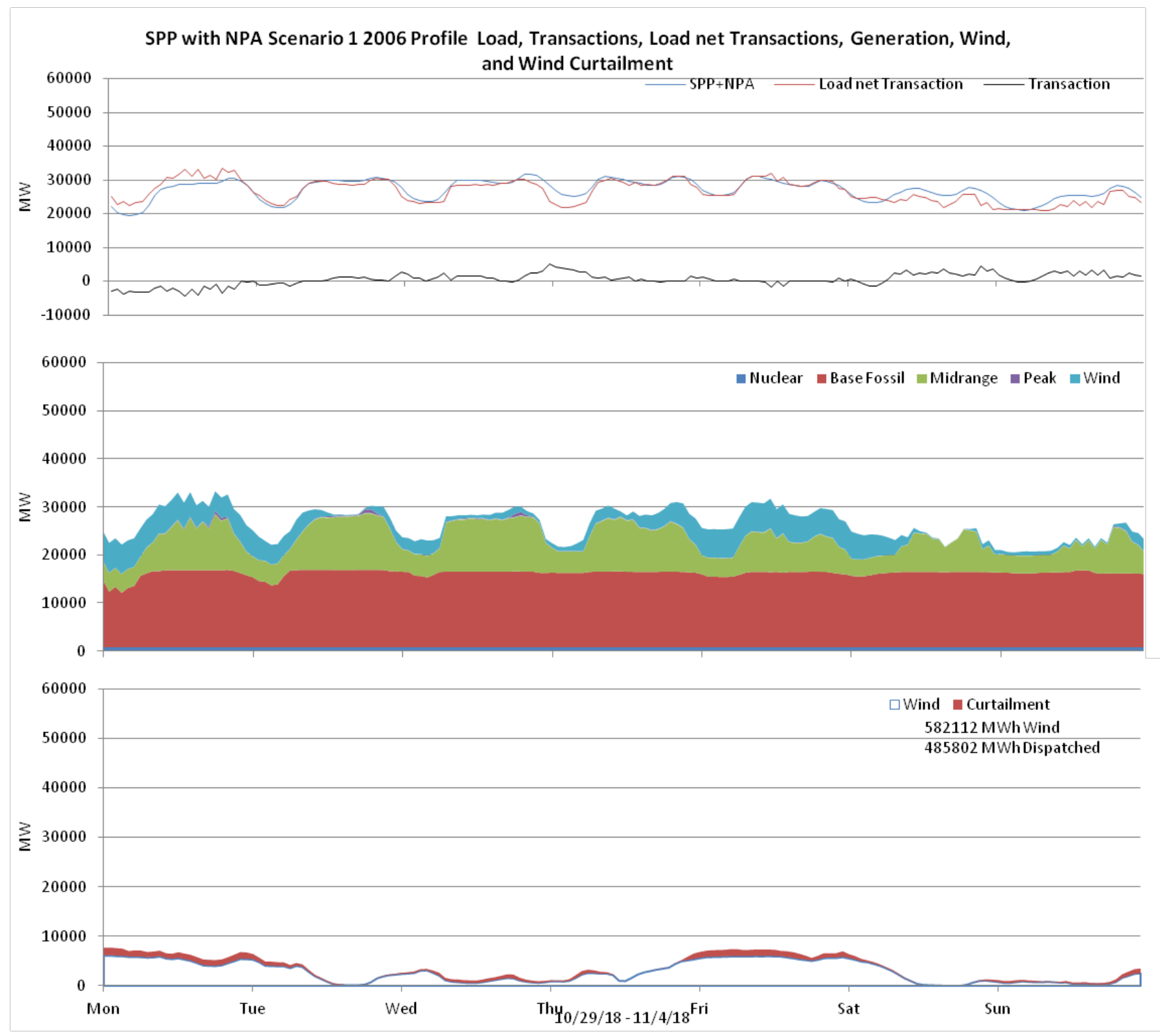

Figure 246: SPP 10\% wind Penetration, 2006 Profile week of 10/29/2018 


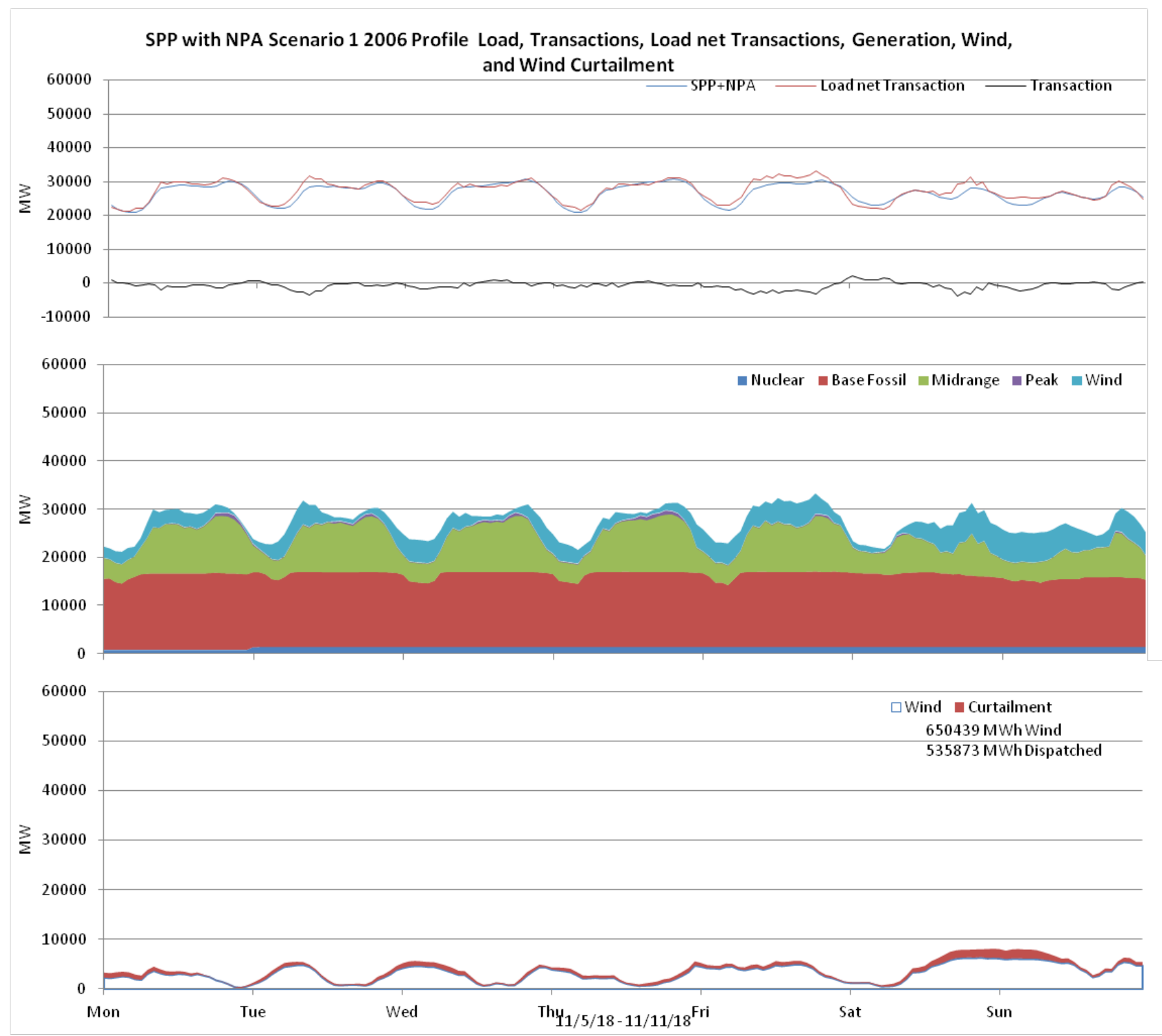

Figure 247: SPP 10\% wind Penetration, 2006 Profile week of 11/5/2018 


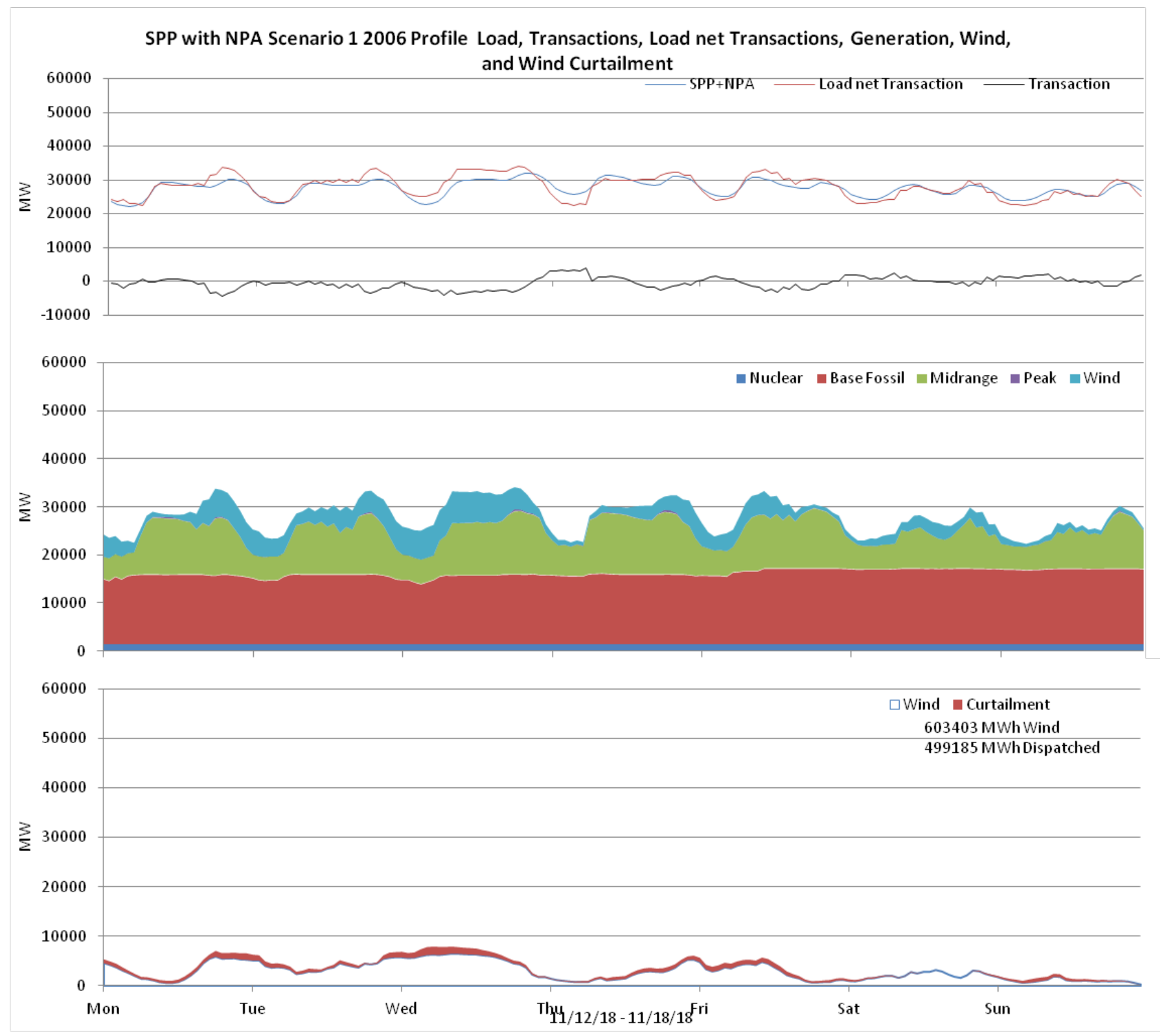

Figure 248: SPP 10\% wind Penetration, 2006 Profile week of 11/12/2018 


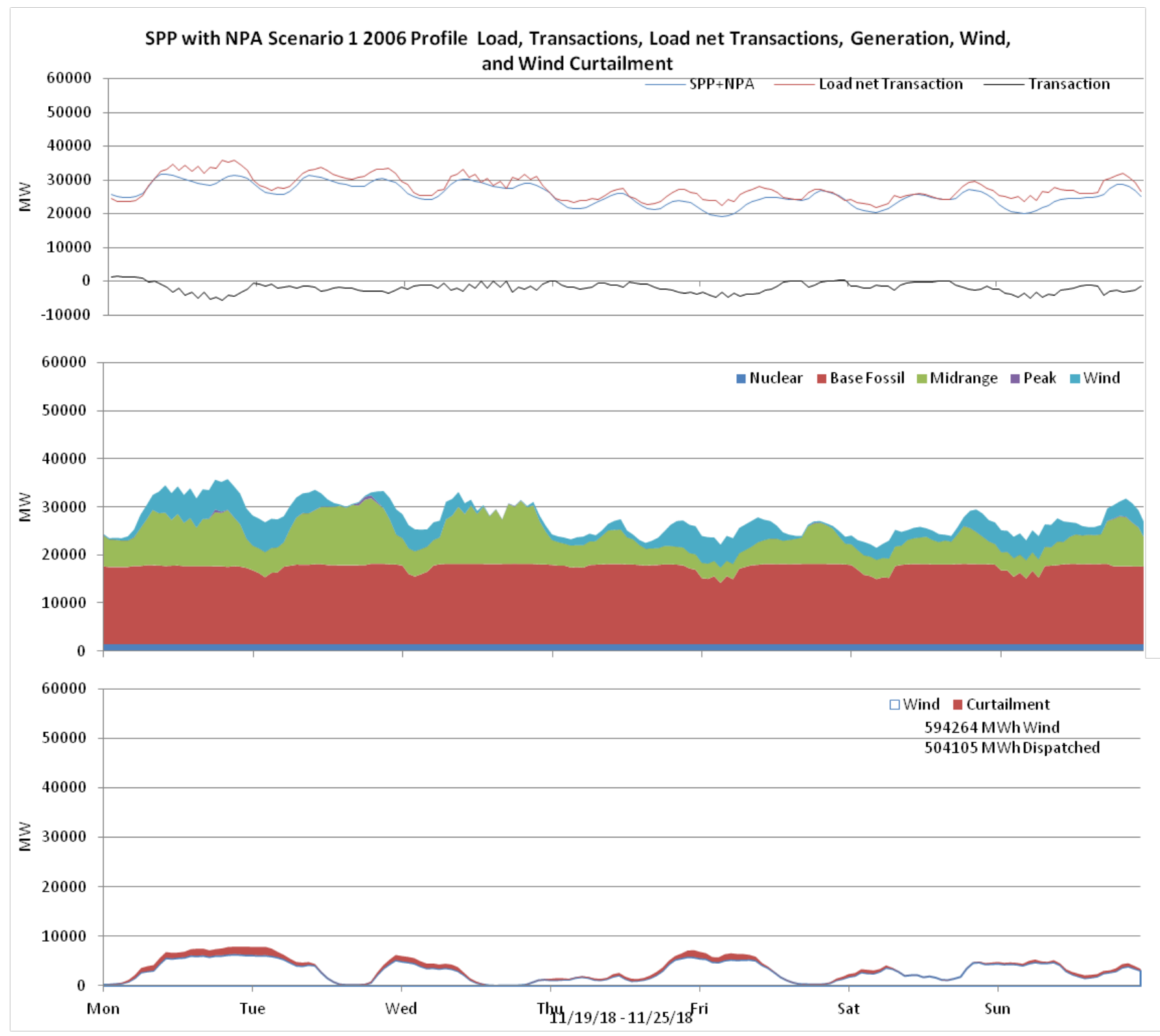

Figure 249: SPP 10\% wind Penetration, 2006 Profile week of 11/19/2018 


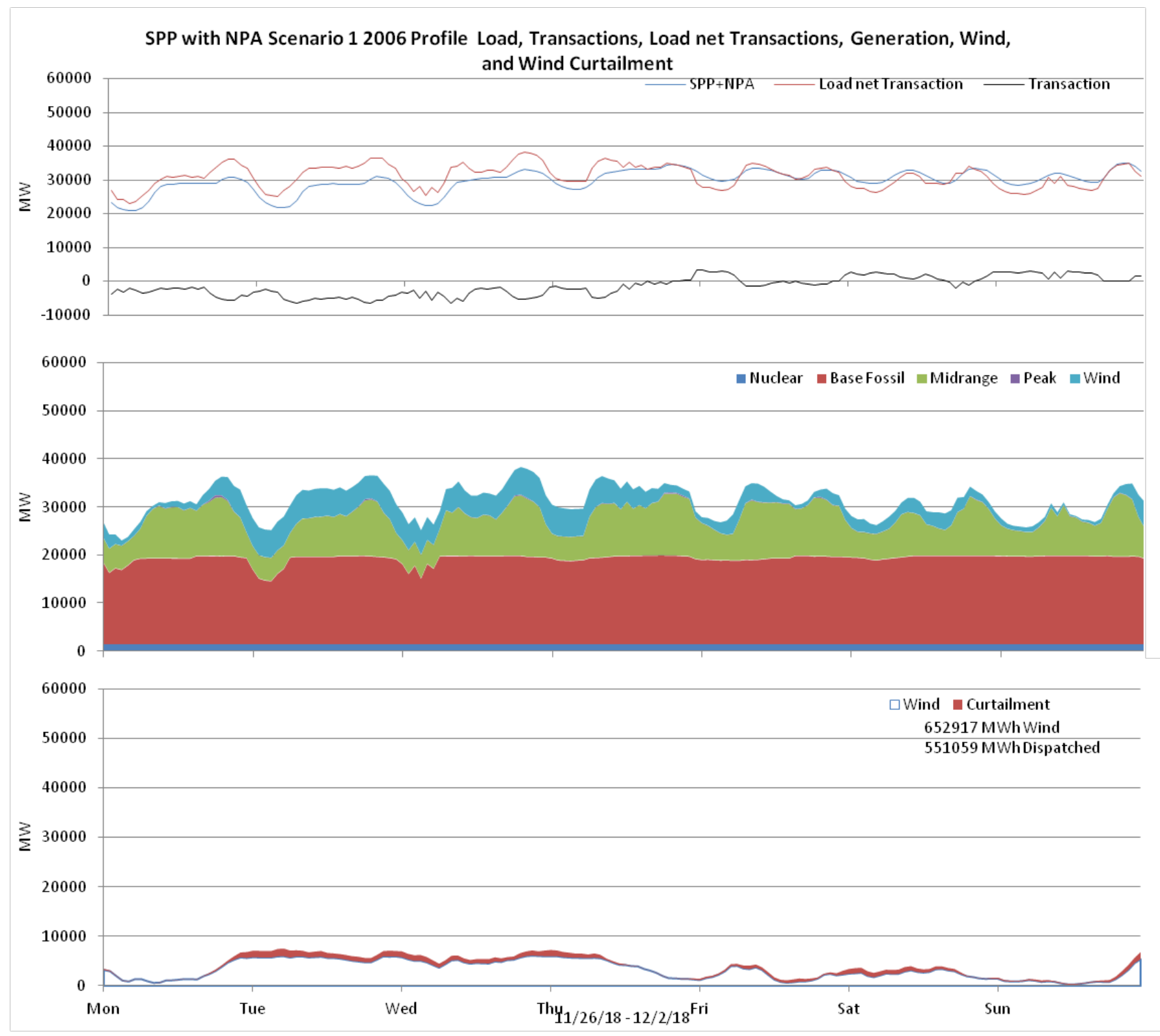

Figure 250: SPP 10\% wind Penetration, 2006 Profile week of 11/26/2018 


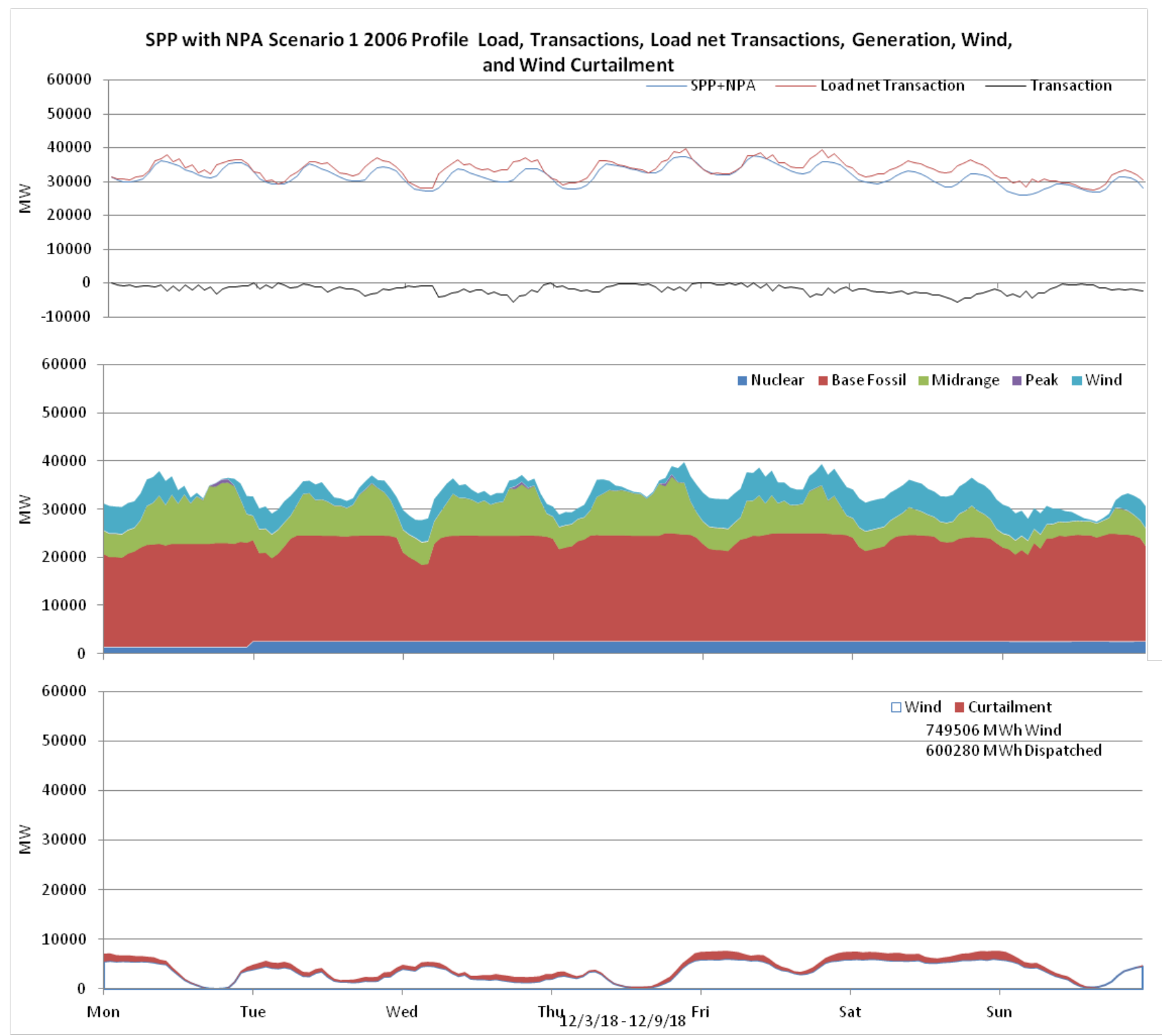

Figure 251: SPP 10\% wind Penetration, 2006 Profile week of 12/3/2018 


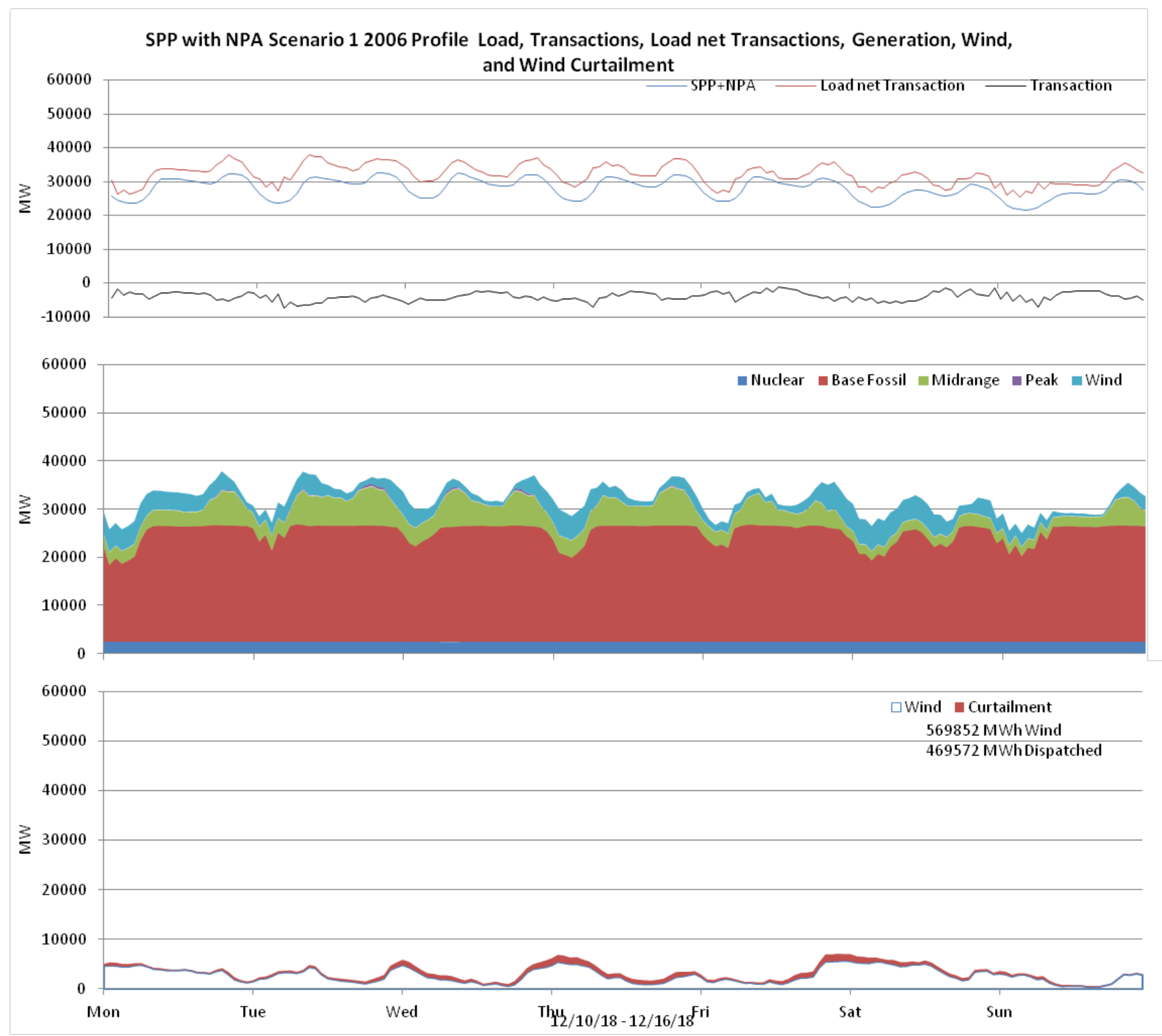

Figure 252: SPP 10\% wind Penetration, 2006 Profile week of 12/10/2018 


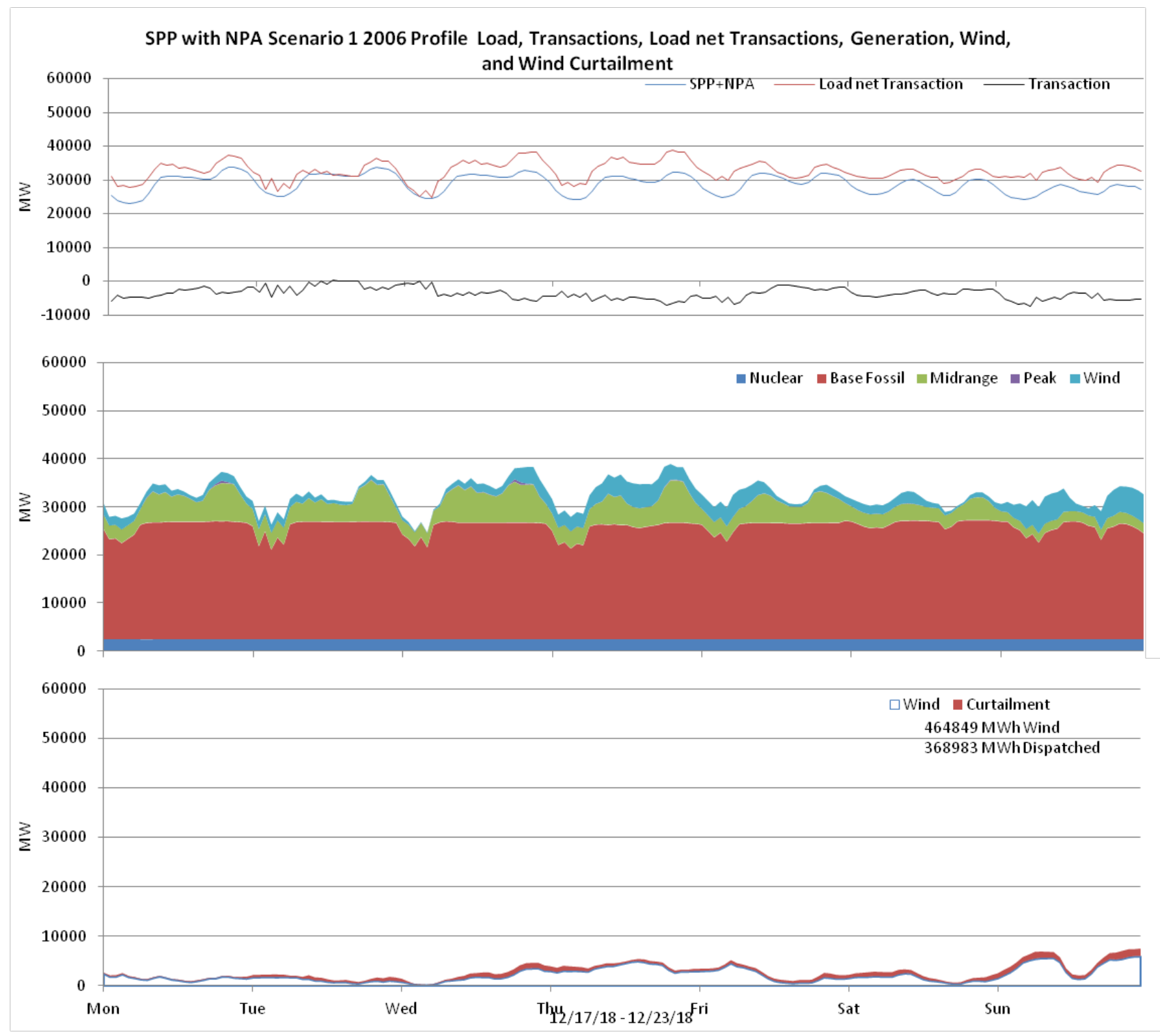

Figure 253: SPP 10\% wind Penetration, 2006 Profile week of 12/17/2018 


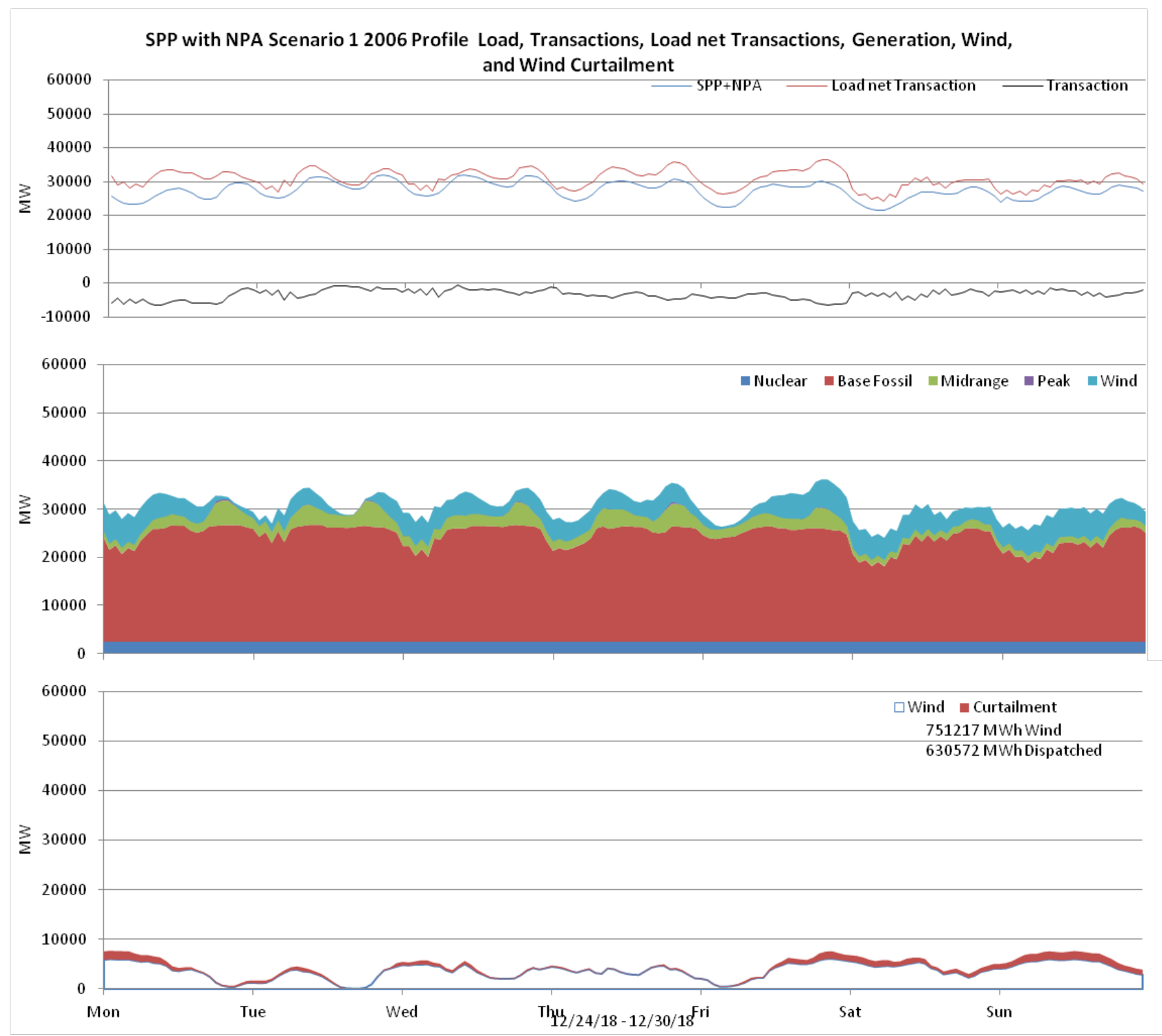

Figure 254: SPP 10\% wind Penetration, 2006 Profile week of 12/24/2018 


\subsection{Production Simulation Details and Analysis}

\subsubsection{Production Simulation Key Inputs}

Table 64: Modeled Nebraska Generation (non-Wind)

\begin{tabular}{|c|c|c|}
\hline UnitDescription & UnitCategory & Max Capacity* \\
\hline ADM Cogen Coal & ST Coal & 50.0 \\
\hline Ansley 1-2 & Internal Combustion & 1.2 \\
\hline Arnold 1-3 & Internal Combustion & 0.9 \\
\hline Auburn 1 & Internal Combustion & 16.3 \\
\hline Beatrice: CC & Combined Cycle & 250.0 \\
\hline Beaver City 1 & Internal Combustion & 1.2 \\
\hline Belleville 4-8 & Internal Combustion & 10.0 \\
\hline Benkleman & Internal Combustion & 0.8 \\
\hline Blue Hill 1-2 & Internal Combustion & 1.2 \\
\hline Broken Bow 1-6 & Internal Combustion & 8.3 \\
\hline Burwell 1-4 & Internal Combustion & 3.0 \\
\hline C W Burdick: 1 & ST Gas & 17.0 \\
\hline C W Burdick: 2 & ST Gas & 22.0 \\
\hline C W Burdick: 3 & ST Gas & 54.0 \\
\hline C W Burdick: GT1 & CT Gas & 15.0 \\
\hline C W Burdick: GT2 & CT Gas & 40.0 \\
\hline C W Burdick: GT3 & CT Gas & 40.0 \\
\hline Callaway 1-3 & Internal Combustion & 0.9 \\
\hline Cambridge & Internal Combustion & 3.0 \\
\hline Canaday: 1 & ST Gas & 118.0 \\
\hline Cass County: GT1 & Combined Cycle & 275.0 \\
\hline Cass County: GT2 & Combined Cycle & 275.0 \\
\hline Chappell 2-3 & Internal Combustion & 1.2 \\
\hline Columbus (NE) & Hydro & 45.0 \\
\hline Cooper: 1 & Nuclear & 773.0 \\
\hline Council Bluffs: ST3 & ST Coal & 49.7 \\
\hline Council Bluffs: ST4 & ST Coal & 107.4 \\
\hline Crete 1-7 & Internal Combustion & 15.7 \\
\hline Curtis 1-3 & Internal Combustion & 3.0 \\
\hline David City 1-7 & Internal Combustion & 8.8 \\
\hline Deshler 1-4 & Internal Combustion & 2.3 \\
\hline Don Henry: 1 & CT Gas & 18.0 \\
\hline Elk City (NE): IC 1 & Internal Combustion & 6.0 \\
\hline Emerson 2-4 & Internal Combustion & 1.7 \\
\hline Fairbury: 1 & ST Gas & 4.0 \\
\hline Fairbury: 2 & ST Gas & 3.0 \\
\hline Fairbury: 4 & ST Gas & 13.0 \\
\hline Falls City 1-2 & Internal Combustion & 1.7 \\
\hline Falls City 3-8 & Internal Combustion & 18.7 \\
\hline Fort Calhoun: 1 & Nuclear & 588.0 \\
\hline Franklin 1-4 & Internal Combustion & 3.8 \\
\hline Fremont CT & CT Gas & 36.0 \\
\hline Fremont: 6 & ST Coal & 17.5 \\
\hline Fremont: 7 & ST Coal & 22.4 \\
\hline Fremont: 8 & ST Coal & 84.4 \\
\hline Gerald Gentleman: 1 & ST Coal & 630.0 \\
\hline Gerald Gentleman: 2 & ST Coal & 665.0 \\
\hline Hallam: 1 & CT Gas & 56.0 \\
\hline Hebron: 1 & CT Oil & 55.0 \\
\hline Inter-LES & Interruptible & 4.0 \\
\hline J Street: 1 & CT Gas & 30.0 \\
\hline Jeffrey & Hydro & 18.0 \\
\hline Johnson 1 & Hydro & 18.0 \\
\hline Johnson 2 & Hydro & 18.0 \\
\hline Jones St: 1 & CT Oil & 65.0 \\
\hline Jones St: 2 & CT Oil & 65.0 \\
\hline Kearney Hydro & Hydro & 1.0 \\
\hline Kingsley & Hydro & 38.0 \\
\hline Laramie River: 1 & ST Coal & 188.1 \\
\hline
\end{tabular}




\begin{tabular}{|c|c|c|}
\hline UnitDescription & UnitCategory & Max Capacity ${ }^{*}$ \\
\hline Lyons 2-3 & Internal Combustion & $\begin{array}{r}1.1 \\
\end{array}$ \\
\hline Madison 1-4 & Internal Combustion & 4.0 \\
\hline McCook: 1 & CT Oil & 50.0 \\
\hline Monroe (NE) & Hydro & 4.0 \\
\hline Mullen 1-2 & Internal Combustion & 1.0 \\
\hline Nebraska City 2-10 & Internal Combustion & 25.0 \\
\hline Nebraska City No 2: 11 & Internal Combustion & 5.0 \\
\hline Nebraska City No 2: 12 & Internal Combustion & 5.0 \\
\hline Nebraska City No 2: 13 & Internal Combustion & 5.0 \\
\hline Nebraska City Unit 2: ST1 & ST Coal & 538.2 \\
\hline Nebraska City: 1 & ST Coal & 653.0 \\
\hline North Denver: 4 & ST Gas & 16.0 \\
\hline North Denver: 5 & ST Gas & 25.0 \\
\hline North Omaha: 1 & ST Coal & 55.8 \\
\hline North Omaha: 2 & ST Coal & 95.1 \\
\hline North Omaha: 3 & ST Coal & 95.1 \\
\hline North Omaha: 4 & ST Coal & 115.0 \\
\hline North Omaha: 5 & ST Coal & 173.2 \\
\hline North Omaha:1_GASTOPPING & ST Coal & 22.8 \\
\hline North Omaha:2_GASTOPPING & ST Coal & 15.9 \\
\hline North Omaha:3_GASTOPPING & ST Coal & 15.9 \\
\hline North Omaha:4 GASTOPPING & ST Coal & 23.2 \\
\hline North Omaha:5_GASTOPPING & ST Coal & 50.8 \\
\hline North Platte & Hydro & 24.0 \\
\hline Ord 1-5 & Internal Combustion & 10.8 \\
\hline Oxford 1-5 & Internal Combustion & 3.3 \\
\hline Pender 1-4 & Internal Combustion & 4.0 \\
\hline Platte: 1 & ST Coal & 100.0 \\
\hline Red Cloud 2-5 & Internal Combustion & 4.0 \\
\hline Rokeby Black Start & Internal Combustion & 3.1 \\
\hline Rokeby: GT1 & CT Gas & 74.0 \\
\hline Rokeby: GT2 & CT Gas & 86.0 \\
\hline Rokeby: GT3 & CT Gas & 97.0 \\
\hline Salt Valley (CC): CC & Combined Cycle & 120.0 \\
\hline Salt Valley (CC): GT3 & CT Gas & 47.0 \\
\hline Salt Valley Black Start & Internal Combustion & 1.6 \\
\hline Sargent 1-3 & Internal Combustion & 1.0 \\
\hline Sarpy County: 1 & CT Gas & 62.0 \\
\hline Sarpy County: 2 & CT Gas & 62.0 \\
\hline Sarpy County: 3 & CT Gas & 120.0 \\
\hline Sarpy County: 4 & CT Gas & 48.0 \\
\hline Sarpy County: 5 & CT Gas & 48.0 \\
\hline Sarpy County: BSD & Internal Combustion & 3.0 \\
\hline Sheldon (NE): 1 & ST Coal & 105.0 \\
\hline Sheldon (NE): 2 & ST Coal & 120.0 \\
\hline Spalding 2-5 & Internal Combustion & 2.3 \\
\hline Spencer 1-2 & Hydro & 1.8 \\
\hline Stuart 1-4 & Internal Combustion & 2.1 \\
\hline Sutherland 1-4 & Internal Combustion & 2.7 \\
\hline Tecumseh 1-5 & Internal Combustion & 6.6 \\
\hline Wahoo 1 & Internal Combustion & 10.0 \\
\hline Wakefield 2 & Internal Combustion & 3.4 \\
\hline Wayne 1 & Internal Combustion & 19.0 \\
\hline West Point 1 & Internal Combustion & 7.4 \\
\hline Whelan Energy Center: 1 & ST Coal & 77.0 \\
\hline Whelan Energy Center: ST & ST Coal & 140.8 \\
\hline Wilber 4-6 & Internal Combustion & 2.9 \\
\hline York 1-2 & Internal Combustion & 2.6 \\
\hline
\end{tabular}

Total (excluding Wind and Purchases from WAPA) 82 or Capacity Sales assumed for 2018 
NOTE for the above table: Section 1.1.2 states that there is today approximately 9,000 MW of generation capacity (including capacity associated with out-of-state purchases and sales). For comparison, the above list for 2018 uses winter (not summer) capacity for thermal units; includes future capacity at Whelan, ADM, and Cass County; excludes wind generation, WAPA purchases, and out-of-state sales of Nebraska unit shares.

Table 65: Nebraska Wind Site Mapping to Bus Number

\begin{tabular}{|r|r|r|l|}
\hline SitelD & NamePlate & Bus Number & Bus Name \\
\hline 22 & 260.8 & 640226 & HOSKINS3 \\
\hline 47 & 284.5 & 640226 & HOSKINS3 \\
\hline 70 & 1100 & 652509 & FTRANDL4 \\
\hline 76 & 479.2 & 640183 & GENTLMN3 \\
\hline 143 & 268 & 652509 & FTRANDL4 \\
\hline 160 & 239.8 & 659247 & ARTHUR 7 \\
\hline 205 & 234.6 & 640227 & HOSKINS4 \\
\hline 245 & 453.1 & 640392 & VALENTN7 \\
\hline 208 & 245.4 & 640271 & MCCOOL 3 \\
\hline 695 & 660 & 659133 & SIDNEY 3 \\
\hline 1149 & 501.4 & 640271 & MCCOOL 3 \\
\hline
\end{tabular}

Table 66: Modeled Flow Gates

\begin{tabular}{|l|l|}
\hline NERC Flowgate ID & Description \\
\hline
\end{tabular}

5221 "RedWillMingo" - Single Monitored Branch (SPP Flowgate)

6006 "GGS" - Interface

6007 "GENTLMN3 345 REDWILO3 345 1" - Single Monitored Branch

6008 "GRIS LNC" - Interface

6009 "COOPER_S" - Interface

6030 "Nebraska City-Cooper 345kV" - Single Monitored Branch (OPPD Flowgate)

5272 "GILCLRREDMIN" - Contingency, Gill to Clearwater flo Red Willow to Mingo (SPP Flowgate)

6170 "Sheldon-20th \& Pioneer 115kV flo Wagener-Mark Moore 345kV" - Contingency (LES Flowgate)

6152 "St. Joe-Midway 161kV flo Fairport-Cooper/St. Joe 345kV" - Contingency (AECl Flowgate)

6127 "Sub 1214-70th \& Bluff 161kV flo Cooper-Nebraska City 345kV" - Contingency (OPPD Flowgate)

6014 "FTCAL S" - Interface (OPPD Flowgate)

6147 "Sub 3451-Raun 345kV" - Single Monitored Branch (OPPD Flowgate)

6122 "Council Bluffs-Avoca 161kV flo Council Bluffs-Madison County 345kV" - Contingency (MEC Flowgate)

6161 "Sub 701-Sub 1211 161kV flo Council Bluffs-Madison County 345kV" - Contingency (OPPD/MEC Flowgate)

6162 "Council Bluffs-Manawa 161kV flo Council Bluffs-Madison County 345kV" - Contingency (MEC Flowgate)

6126 "S1226-Tekamah 161kV flo S3451-Raun 345kV" - Contingency (OPPD Flowgate)

6127 "Sub 1214-70th \& Bluff 161kV flo Cooper-Nebraska City 345kV" - Contingency (OPPD Flowgate)

6163 "70th \& Bluff Xfmr flo Sub 3454-Wagener 345kV" - Contingency (LES Flowgate)

6199 BRVRBCOPSTJ" (Council Bluffs - River Bend $161 \mathrm{kV}$ FLO Cooper - St.Joe $345 \mathrm{kV}$ )

5345 ELTECCOOSTL" (Kelly-Tecumseh 161 kV FLO Cooper - St.Joe 345 kV) 
8.3.2. Production Simulation Generation Results

Table 67: Base Case Generation 2004 Scenario 1

\begin{tabular}{|l|r|r|r|r|r|r|}
\multicolumn{1}{c}{2004} & \multicolumn{2}{c|}{ Ideal } & \multicolumn{2}{c|}{ Actual } & \multicolumn{2}{c|}{ Delta } \\
\hline Scenario 1 - 10\% & Nebraska & Rest of SPP & Nebraska & Rest of SPP & Nebraska & Rest of SPP \\
\hline Combined Cycle & $3,052,657$ & $40,619,627$ & $3,244,397$ & $41,193,793$ & $6 \%$ & $1 \%$ \\
\hline CT Gas & 81,301 & $5,426,470$ & 97,267 & $5,441,857$ & $20 \%$ & $0 \%$ \\
\hline CT Other & - & - & - & - & & \\
\hline Hydro & 577,219 & $5,100,234$ & 577,219 & $5,100,234$ & $0 \%$ & $0 \%$ \\
\hline Internal Combustion & 17,576 & 96,961 & 18,430 & 97,346 & $5 \%$ & $0 \%$ \\
\hline Interruptible & - & - & - & - & & \\
\hline Nuclear & $9,682,811$ & $8,415,466$ & $9,682,565$ & $8,415,466$ & $0 \%$ & $0 \%$ \\
\hline Pumped Storage & - & 343,519 & - & 343,530 & & $0 \%$ \\
\hline ST Coal & $29,023,704$ & $138,031,190$ & $28,865,605$ & $137,544,699$ & $-1 \%$ & $0 \%$ \\
\hline ST Gas & 20,758 & $3,011,647$ & 23,463 & $3,082,796$ & $13 \%$ & $2 \%$ \\
\hline ST Other & 369,315 & $2,176,637$ & 369,310 & $2,177,282$ & $0 \%$ & $0 \%$ \\
\hline Wind & $4,303,032$ & $21,380,592$ & $4,303,032$ & $21,449,797$ & $0 \%$ & $0 \%$ \\
\hline Other (Fixed Energy) & $1,675,421$ & $2,769,624$ & $1,675,421$ & $2,769,624$ & $0 \%$ & $0 \%$ \\
\hline Total & $48,803,794$ & $227,371,967$ & $48,856,709$ & $227,616,422$ & $0 \%$ & $0 \%$ \\
\hline
\end{tabular}

Table 68: $\quad$ Base Case Generation 2004 Scenario 2

\begin{tabular}{|c|c|c|c|c|c|c|}
\hline 2004 & \multicolumn{2}{|c|}{ Ideal } & \multicolumn{2}{|c|}{ Actual } & \multicolumn{2}{|c|}{ Delta } \\
\hline Scenario $2-20 \%$ & Nebraska & Rest of SPP & Nebraska & Rest of SPP & Nebraska & Rest of SPP \\
\hline Combined Cycle & $2,641,355$ & $34,548,168$ & $2,842,542$ & $35,721,180$ & $8 \%$ & $3 \%$ \\
\hline CT Gas & 64,935 & $5,176,038$ & 85,512 & $5,180,558$ & $32 \%$ & $0 \%$ \\
\hline CT Other & - & - & - & - & & \\
\hline Hydro & 577,217 & $5,100,243$ & 577,217 & $5,100,243$ & $0 \%$ & $0 \%$ \\
\hline Internal Combustion & 14,474 & 77,081 & 15,837 & 75,277 & $9 \%$ & $-2 \%$ \\
\hline Interruptible & - & - & - & - & & \\
\hline Nuclear & $9,682,606$ & $8,415,466$ & $9,682,370$ & $8,415,466$ & $0 \%$ & $0 \%$ \\
\hline Pumped Storage & - & 350,703 & - & 350,706 & & $0 \%$ \\
\hline ST Coal & $28,439,206$ & $132,208,257$ & $28,131,937$ & $131,730,367$ & $-1 \%$ & $0 \%$ \\
\hline ST Gas & 12,700 & $2,340,312$ & 17,585 & $2,467,862$ & $38 \%$ & $5 \%$ \\
\hline ST Other & 369,230 & $2,122,185$ & 369,145 & $2,130,354$ & $0 \%$ & $0 \%$ \\
\hline Wind & $8,436,517$ & $41,170,639$ & $8,436,517$ & $41,258,298$ & $0 \%$ & $0 \%$ \\
\hline Other (Fixed Energy) & $1,675,421$ & $2,769,624$ & $1,675,421$ & $2,769,624$ & $0 \%$ & $0 \%$ \\
\hline Total & $51,913,662$ & $234,278,717$ & $51,834,082$ & $235,199,935$ & $0 \%$ & $0 \%$ \\
\hline
\end{tabular}

Table 69: Base Case Generation 2004 Scenario 3

\begin{tabular}{|l|r|r|r|r|r|r|}
\multicolumn{1}{c}{2004} & \multicolumn{2}{c|}{ Ideal } & \multicolumn{2}{c|}{ Actual } \\
\cline { 2 - 7 } \multicolumn{1}{c|}{ Scenario 3 - 20\% } & Nebraska & Rest of SPP & Nebraska & \multicolumn{1}{c|}{ Rest of SPP } & Nebraska & Rest of SPP \\
\hline Combined Cycle & $2,901,217$ & $31,393,787$ & $3,081,791$ & $32,829,682$ & $6 \%$ & $5 \%$ \\
\hline CT Gas & 40,872 & $5,184,676$ & 62,689 & $5,187,832$ & $53 \%$ & $0 \%$ \\
\hline CT Other & - & - & - & - & & \\
\hline Hydro & 577,217 & $5,100,243$ & 577,217 & $5,100,243$ & $0 \%$ & $0 \%$ \\
\hline Internal Combustion & 11,454 & 69,638 & 12,756 & 65,047 & $11 \%$ & $-7 \%$ \\
\hline Interruptible & - & - & - & - & & $0 \%$ \\
\hline Nuclear & $9,683,424$ & $8,415,466$ & $9,683,250$ & $8,415,466$ & & $0 \%$ \\
\hline Pumped Storage & - & 350,703 & - & 350,706 & & $0 \%$ \\
\hline ST Coal & $29,296,327$ & $134,189,599$ & $29,239,169$ & $133,409,829$ & $0 \%$ & $-1 \%$ \\
\hline ST Gas & 13,205 & $2,059,899$ & 14,595 & $2,167,160$ & $11 \%$ & $5 \%$ \\
\hline ST Other & 369,355 & $2,160,509$ & 369,355 & $2,162,329$ & $0 \%$ & $0 \%$ \\
\hline Wind & $8,436,517$ & $43,947,627$ & $8,436,517$ & $43,948,633$ & $0 \%$ & $0 \%$ \\
\hline Other (Fixed Energy) & $1,675,421$ & $2,769,624$ & $1,675,421$ & $2,769,624$ & $0 \%$ & $0 \%$ \\
\hline Total & $53,005,009$ & $235,641,770$ & $53,152,759$ & $236,406,550$ & $0 \%$ & 0 \\
\hline
\end{tabular}


Table 70: $\quad$ Base Case Generation 2004 Scenario 4

\begin{tabular}{|l|r|r|r|r|r|r|}
\multicolumn{1}{c}{2004} & \multicolumn{2}{c|}{ Ideal } & \multicolumn{2}{c|}{ Actual } \\
\cline { 2 - 7 } \multicolumn{1}{c|}{ Scenario 4 - 40\% } & Nebraska & Rest of SPP & Nebraska & \multicolumn{1}{c|}{ Rest of SPP } & Nebraska & Rest of SPP \\
\hline Combined Cycle & $1,872,788$ & $24,046,125$ & $2,176,054$ & $26,576,392$ & $16 \%$ & $11 \%$ \\
\hline CT Gas & 19,391 & $5,017,144$ & 42,231 & $5,033,977$ & $118 \%$ & $0 \%$ \\
\hline CT Other & - & - & - & - & & \\
\hline Hydro & 577,220 & $5,100,240$ & 577,220 & $5,100,240$ & $0 \%$ & $0 \%$ \\
\hline Internal Combustion & 6,040 & 44,579 & 9,422 & 38,924 & $56 \%$ & $-13 \%$ \\
\hline Interruptible & - & - & - & - & & $0 \%$ \\
\hline Nuclear & $9,682,153$ & $8,415,466$ & $9,681,657$ & $8,415,466$ & & $0 \%$ \\
\hline Pumped Storage & - & 378,127 & - & 378,128 & & $0 \%$ \\
\hline ST Coal & $27,371,438$ & $122,671,983$ & $26,981,916$ & $121,003,842$ & $-1 \%$ & $-1 \%$ \\
\hline ST Gas & 3,664 & $1,518,326$ & 8,594 & $1,733,432$ & $135 \%$ & $14 \%$ \\
\hline ST Other & 366,649 & $1,851,544$ & 366,060 & $1,846,155$ & $0 \%$ & $0 \%$ \\
\hline Wind & $16,233,240$ & $84,037,575$ & $16,232,551$ & $84,075,578$ & $0 \%$ & $0 \%$ \\
\hline Other (Fixed Energy) & $1,675,421$ & $2,769,624$ & $1,675,421$ & $2,769,624$ & $0 \%$ & $0 \%$ \\
\hline Total & $57,808,005$ & $255,850,732$ & $57,751,126$ & $256,971,757$ & $0 \%$ & $0 \%$ \\
\hline
\end{tabular}

Table 71: Base Case Generation 2005 Scenario 1

\begin{tabular}{|c|c|c|c|c|c|c|}
\hline 2005 & \multicolumn{2}{|c|}{ Ideal } & \multicolumn{2}{|c|}{ Actual } & \multicolumn{2}{|c|}{ Delta } \\
\hline Scenario $1-10 \%$ & Nebraska & Rest of SPP & Nebraska & Rest of SPP & Nebraska & Rest of SPP \\
\hline Combined Cycle & $2,942,068$ & $40,031,098$ & $3,154,628$ & $40,561,113$ & $7 \%$ & $1 \%$ \\
\hline CT Gas & 65,983 & $5,357,280$ & 79,199 & $5,375,645$ & $20 \%$ & $0 \%$ \\
\hline CT Other & - & - & - & - & & \\
\hline Hydro & 577,215 & $5,100,230$ & 577,215 & $5,100,230$ & $0 \%$ & $0 \%$ \\
\hline Internal Combustion & 15,496 & 91,001 & 15,744 & 90,969 & $2 \%$ & $0 \%$ \\
\hline Interruptible & - & - & - & - & & \\
\hline Nuclear & $9,683,573$ & $8,415,466$ & $9,683,573$ & $8,415,466$ & $0 \%$ & $0 \%$ \\
\hline Pumped Storage & - & 292,467 & - & 292,475 & & $0 \%$ \\
\hline ST Coal & $29,164,560$ & $137,929,102$ & $29,003,310$ & $137,493,613$ & $-1 \%$ & $0 \%$ \\
\hline ST Gas & 19,476 & $3,026,711$ & 22,452 & $3,078,503$ & $15 \%$ & $2 \%$ \\
\hline ST Other & 369,355 & $2,173,993$ & 369,355 & $2,174,530$ & $0 \%$ & $0 \%$ \\
\hline Wind & $4,559,266$ & $22,587,373$ & $4,559,266$ & $22,672,479$ & $0 \%$ & $0 \%$ \\
\hline Other (Fixed Energy) & $1,675,421$ & $2,769,624$ & $1,675,421$ & $2,769,624$ & $0 \%$ & $0 \%$ \\
\hline Total & $49,072,414$ & $227,774,343$ & $49,140,162$ & $228,024,646$ & $0 \%$ & $0 \%$ \\
\hline
\end{tabular}

Table 72: Base Case Generation 2005 Scenario 2

\begin{tabular}{|c|c|c|c|c|c|c|}
\hline 2005 & \multicolumn{2}{|c|}{ Ideal } & \multicolumn{2}{|c|}{ Actual } & \multicolumn{2}{|c|}{ Delta } \\
\hline Scenario $2-20 \%$ & Nebraska & Rest of SPP & Nebraska & Rest of SPP & Nebraska & Rest of SPP \\
\hline Combined Cycle & $2,545,111$ & $33,831,336$ & $2,732,906$ & $35,102,760$ & $7 \%$ & $4 \%$ \\
\hline CT Gas & 53,456 & $5,120,541$ & 69,419 & $5,136,111$ & $30 \%$ & $0 \%$ \\
\hline CT Other & - & - & - & - & & \\
\hline Hydro & 577,214 & $5,100,233$ & 577,214 & $5,100,233$ & $0 \%$ & $0 \%$ \\
\hline Internal Combustion & 13,177 & 71,668 & 14,072 & 70,611 & $7 \%$ & $-1 \%$ \\
\hline Interruptible & - & - & - & - & & \\
\hline Nuclear & $9,683,574$ & $8,415,466$ & $9,683,574$ & $8,415,466$ & $0 \%$ & $0 \%$ \\
\hline Pumped Storage & - & 304,971 & - & 304,968 & & $0 \%$ \\
\hline ST Coal & $28,682,778$ & $131,991,777$ & $28,351,970$ & $131,554,348$ & $-1 \%$ & $0 \%$ \\
\hline ST Gas & 10,555 & $2,337,505$ & 14,785 & $2,484,990$ & $40 \%$ & $6 \%$ \\
\hline ST Other & 369,355 & $2,115,437$ & 369,355 & $2,125,883$ & $0 \%$ & $0 \%$ \\
\hline Wind & $8,963,788$ & $42,549,838$ & $8,963,787$ & $42,662,609$ & $0 \%$ & $0 \%$ \\
\hline Other (Fixed Energy) & $1,675,421$ & $2,769,624$ & $1,675,421$ & $2,769,624$ & $0 \%$ & $0 \%$ \\
\hline Total & $52,574,429$ & $234,608,395$ & $52,452,503$ & $235,727,604$ & $0 \%$ & $0 \%$ \\
\hline
\end{tabular}


Table 73: Base Case Generation 2005 Scenario 3

\begin{tabular}{|l|r|r|r|r|r|r|}
\multicolumn{1}{c|}{2005} & \multicolumn{2}{c|}{ Ideal } & \multicolumn{2}{c|}{ Actual } & \multicolumn{2}{c|}{ Delta } \\
\cline { 2 - 7 } \multicolumn{1}{c|}{ Scenario 3 - 20\% } & \multicolumn{1}{c|}{ Nebraska } & \multicolumn{1}{c|}{ Rest of SPP } & \multicolumn{1}{c|}{ Nebraska } & \multicolumn{1}{c|}{ Rest of SPP } & Nebraska & Rest of SPP \\
\hline Combined Cycle & $2,713,596$ & $30,531,563$ & $2,954,850$ & $32,096,860$ & $9 \%$ & $5 \%$ \\
\hline CT Gas & 40,819 & $5,140,987$ & 59,905 & $5,150,027$ & $47 \%$ & $0 \%$ \\
\hline CT Other & - & - & - & - & & \\
\hline Hydro & 577,214 & $5,100,233$ & 577,214 & $5,100,233$ & $0 \%$ & $0 \%$ \\
\hline Internal Combustion & 11,379 & 64,046 & 12,634 & 59,032 & $11 \%$ & $-8 \%$ \\
\hline Interruptible & - & - & - & - & & \\
\hline Nuclear & $9,683,575$ & $8,415,466$ & $9,683,575$ & $8,415,466$ & $0 \%$ & $0 \%$ \\
\hline Pumped Storage & - & 304,971 & - & 304,968 & & $0 \%$ \\
\hline ST Coal & $29,395,842$ & $134,337,162$ & $29,342,286$ & $133,398,058$ & $0 \%$ & $-1 \%$ \\
\hline ST Gas & 13,538 & $2,036,437$ & 14,556 & $2,143,402$ & $8 \%$ & $5 \%$ \\
\hline ST Other & 369,355 & $2,160,273$ & 369,355 & $2,160,447$ & $0 \%$ & $0 \%$ \\
\hline Wind & $8,963,788$ & $45,819,950$ & $8,963,787$ & $45,822,339$ & $0 \%$ & $0 \%$ \\
\hline Other (Fixed Energy) & $1,675,421$ & $2,769,624$ & $1,675,421$ & $2,769,624$ & $0 \%$ & $0 \%$ \\
\hline Total & $53,444,526$ & $236,680,712$ & $53,653,583$ & $237,420,457$ & $0 \%$ & $0 \%$ \\
\hline
\end{tabular}

Table 74: $\quad$ Base Case Generation 2005 Scenario 4

\begin{tabular}{|lr|r|r|r|r|r|}
\multicolumn{1}{c|}{2005} & \multicolumn{2}{c|}{ Ideal } & \multicolumn{2}{c|}{ Actual } & \multicolumn{2}{c|}{ Delta } \\
\cline { 2 - 7 } \multicolumn{1}{c|}{ Scenario 4 - 40\% } & \multicolumn{1}{c|}{ Nebraska } & \multicolumn{1}{c|}{ Rest of SPP } & \multicolumn{1}{c|}{ Nebraska } & \multicolumn{1}{c|}{ Rest of SPP } & Nebraska & Rest of SPP \\
\hline Combined Cycle & $1,710,373$ & $23,342,695$ & $2,061,443$ & $25,831,327$ & $21 \%$ & $11 \%$ \\
\hline CT Gas & 22,425 & $5,031,827$ & 42,245 & $5,034,029$ & $88 \%$ & $0 \%$ \\
\hline CT Other & - & - & - & - & & \\
\hline Hydro & 577,218 & $5,100,226$ & 577,218 & $5,100,226$ & $0 \%$ & $0 \%$ \\
\hline Internal Combustion & 7,786 & 43,771 & 9,937 & 39,344 & $28 \%$ & $-10 \%$ \\
\hline Interruptible & - & - & - & - & & \\
\hline Nuclear & $9,683,086$ & $8,415,466$ & $9,682,965$ & $8,415,466$ & $0 \%$ & $0 \%$ \\
\hline Pumped Storage & - & 374,053 & - & 374,051 & & $0 \%$ \\
\hline ST Coal & $27,367,397$ & $121,554,470$ & $26,916,420$ & $119,947,004$ & $-2 \%$ & $-1 \%$ \\
\hline ST Gas & 4,244 & $1,570,039$ & 8,632 & $1,769,812$ & $103 \%$ & $13 \%$ \\
\hline ST Other & 367,397 & $1,823,596$ & 366,478 & $1,830,375$ & $0 \%$ & $0 \%$ \\
\hline Wind & $17,027,217$ & $87,115,685$ & $17,027,849$ & $87,168,829$ & $0 \%$ & $0 \%$ \\
\hline Other (Fixed Energy) & $1,675,421$ & $2,769,624$ & $1,675,421$ & $2,769,624$ & $0 \%$ & $0 \%$ \\
\hline Total & $58,442,563$ & $257,141,452$ & $58,368,608$ & $258,280,087$ & $0 \%$ & $0 \%$ \\
\hline
\end{tabular}

Table 75: Base Case Generation 2006 Scenario 1

\begin{tabular}{|c|c|c|c|c|c|c|}
\hline 2006 & \multicolumn{2}{|c|}{ Ideal } & \multicolumn{2}{|c|}{ Actual } & \multicolumn{2}{|c|}{ Delta } \\
\hline Scenario 1 - 10\% & Nebraska & Rest of SPP & Nebraska & Rest of SPP & Nebraska & Rest of SPP \\
\hline Combined Cycle & $2,935,336$ & $40,169,138$ & $3,162,431$ & $40,718,123$ & $8 \%$ & $1 \%$ \\
\hline CT Gas & 71,248 & $5,373,608$ & 87,127 & $5,380,117$ & $22 \%$ & $0 \%$ \\
\hline CT Other & & & & & & \\
\hline Hydro & 577,218 & $5,100,230$ & 577,218 & $5,100,230$ & $0 \%$ & $0 \%$ \\
\hline Internal Combustion & 16,501 & 97,310 & 16,965 & 97,332 & $3 \%$ & $0 \%$ \\
\hline Interruptible & 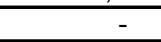 & - & - & & & \\
\hline \begin{tabular}{|l|} 
Nuclear \\
\end{tabular} & $9,682,925$ & $8,415,466$ & $9,682,711$ & $8,415,466$ & $0 \%$ & $0 \%$ \\
\hline Pumped Storage & - & 331,349 & - & 331,378 & & $0 \%$ \\
\hline ST Coal & $29,063,883$ & $137,974,717$ & $28,913,624$ & $137,451,394$ & $-1 \%$ & $0 \%$ \\
\hline ST Gas & 15,221 & $3,011,926$ & 19,068 & $3,106,484$ & $25 \%$ & $3 \%$ \\
\hline ST Other & 369,335 & $2,174,567$ & 369,335 & $2,174,857$ & $0 \%$ & $0 \%$ \\
\hline Wind & $4,749,670$ & $22,696,709$ & $4,749,670$ & $22,774,389$ & $0 \%$ & $0 \%$ \\
\hline Other (Fixed Energy) & $1,675,421$ & $2,769,624$ & $1,675,421$ & $2,769,624$ & $0 \%$ & $0 \%$ \\
\hline Total & $49,156,758$ & $228,114,644$ & $49,253,568$ & $228,319,394$ & $0 \%$ & $0 \%$ \\
\hline
\end{tabular}


Table 76: $\quad$ Base Case Generation 2006 Scenario 2

\begin{tabular}{|l|r|r|r|r|r|r|}
\multicolumn{1}{c}{2006} & \multicolumn{2}{c}{ Ideal } & \multicolumn{2}{c}{ Actual } & \multicolumn{2}{c}{ Delta } \\
\cline { 2 - 7 } & Nebraska & Rest of SPP & Nebraska & Rest of SPP & Nebraska & Rest of SPP \\
\hline Combined Cycle & $2,507,868$ & $33,960,953$ & $2,707,139$ & $35,177,239$ & $8 \%$ & $4 \%$ \\
\hline CT Gas & 55,108 & $5,106,417$ & 70,068 & $5,124,149$ & $27 \%$ & $0 \%$ \\
\hline CT Other & - & - & - & - & & \\
\hline Hydro & 577,217 & $5,100,231$ & 577,217 & $5,100,231$ & $0 \%$ & $0 \%$ \\
\hline Internal Combustion & 14,049 & 72,585 & 14,499 & 72,359 & $3 \%$ & $0 \%$ \\
\hline Interruptible & - & - & - & - & & \\
\hline Nuclear & $9,682,878$ & $8,415,466$ & $9,682,648$ & $8,415,466$ & $0 \%$ & $0 \%$ \\
\hline Pumped Storage & - & 340,387 & - & 340,386 & & $0 \%$ \\
\hline ST Coal & $28,514,679$ & $131,736,640$ & $28,197,962$ & $131,231,646$ & $-1 \%$ & $0 \%$ \\
\hline ST Gas & 8,744 & $2,241,940$ & 13,933 & $2,424,364$ & $59 \%$ & $8 \%$ \\
\hline ST Other & 369,321 & $2,116,160$ & 369,290 & $2,124,901$ & $0 \%$ & $0 \%$ \\
\hline Wind & $9,263,808$ & $43,597,804$ & $9,263,808$ & $43,684,117$ & $0 \%$ & $0 \%$ \\
\hline Other (Fixed Energy) & $1,675,421$ & $2,769,624$ & $1,675,421$ & $2,769,624$ & $0 \%$ & $0 \%$ \\
\hline Total & $52,669,093$ & $235,458,208$ & $52,571,985$ & $236,464,482$ & $0 \%$ & $0 \%$ \\
\hline
\end{tabular}

Table 77: $\quad$ Base Case Generation 2006 Scenario 3

\begin{tabular}{|c|c|c|c|c|c|c|}
\hline 2006 & \multicolumn{2}{|c|}{ Ideal } & \multicolumn{2}{|c|}{ Actual } & \multicolumn{2}{|c|}{ Delta } \\
\hline Scenario 3 - 20\% & Nebraska & Rest of SPP & Nebraska & Rest of SPP & Nebraska & Rest of SPP \\
\hline Combined Cycle & $2,695,369$ & $30,636,111$ & $2,974,523$ & $32,385,091$ & $10 \%$ & $6 \%$ \\
\hline CT Gas & 31,609 & $5,102,804$ & 55,129 & $5,130,240$ & $74 \%$ & $1 \%$ \\
\hline CT Other & - & 3,864 & - & 1,407 & & $-64 \%$ \\
\hline Hydro & 577,217 & $5,100,231$ & 577,217 & $5,100,231$ & $0 \%$ & $0 \%$ \\
\hline Internal Combustion & 9,716 & 66,874 & 11,622 & 61,329 & $20 \%$ & $-8 \%$ \\
\hline Interruptible & - & - & - & - & & \\
\hline Nuclear & $9,683,459$ & $8,415,466$ & $9,683,452$ & $8,415,466$ & $0 \%$ & $0 \%$ \\
\hline Pumped Storage & - & 340,387 & - & 340,386 & & $0 \%$ \\
\hline ST Coal & $29,354,885$ & $133,737,626$ & $29,283,695$ & $132,803,967$ & $0 \%$ & $-1 \%$ \\
\hline ST Gas & 8,921 & $1,904,263$ & 12,171 & $2,022,132$ & $36 \%$ & $6 \%$ \\
\hline ST Other & 369,355 & $2,151,946$ & 369,355 & $2,154,734$ & $0 \%$ & $0 \%$ \\
\hline Wind & $9,263,808$ & $47,048,666$ & $9,263,808$ & $47,052,104$ & $0 \%$ & $0 \%$ \\
\hline Other (Fixed Energy) & $1,675,421$ & $2,769,624$ & $1,675,421$ & $2,769,624$ & $0 \%$ & $0 \%$ \\
\hline Total & $53,669,760$ & $237,277,863$ & $53,906,392$ & $238,236,711$ & $0 \%$ & $0 \%$ \\
\hline
\end{tabular}

Table 78: $\quad$ Base Case Generation 2006 Scenario 4

\begin{tabular}{|l|r|r|r|r|r|r|}
\multicolumn{1}{c}{2006} & \multicolumn{2}{c|}{ Ideal } & \multicolumn{2}{c|}{ Actual } & \multicolumn{2}{c|}{ Delta } \\
\hline Scenario 4 - 40\% & Nebraska & Rest of SPP & Nebraska & \multicolumn{1}{c|}{ Rest of SPP } & Nebraska & Rest of SPP \\
\hline Combined Cycle & $1,662,261$ & $23,518,913$ & $1,965,732$ & $26,150,652$ & $18 \%$ & $11 \%$ \\
\hline CT Gas & 14,957 & $4,963,829$ & 32,554 & $4,982,664$ & $118 \%$ & $0 \%$ \\
\hline CT Other & - & 8,660 & - & 5,089 & & $-41 \%$ \\
\hline Hydro & 577,217 & $5,100,233$ & 577,217 & $5,100,233$ & $0 \%$ & $0 \%$ \\
\hline Internal Combustion & 4,597 & 41,582 & 8,927 & 37,825 & $94 \%$ & $-9 \%$ \\
\hline Interruptible & - & - & - & - & & $0 \%$ \\
\hline Nuclear & $9,682,591$ & $8,415,466$ & $9,681,925$ & $8,415,466$ & $0 \%$ & $0 \%$ \\
\hline Pumped Storage & - & 364,527 & - & 364,526 & & $0 \%$ \\
\hline ST Coal & $27,201,789$ & $120,545,524$ & $26,744,936$ & $118,771,567$ & $-2 \%$ & $-1 \%$ \\
\hline ST Gas & 3,024 & $1,334,302$ & 7,615 & $1,552,100$ & $152 \%$ & $16 \%$ \\
\hline ST Other & 366,600 & $1,778,973$ & 365,760 & $1,788,143$ & $0 \%$ & $1 \%$ \\
\hline Wind & $17,746,435$ & $89,432,319$ & $17,746,456$ & $89,482,765$ & $0 \%$ & $0 \%$ \\
\hline Other (Fixed Energy) & $1,675,421$ & $2,769,624$ & $1,675,421$ & $2,769,624$ & $0 \%$ & $0 \%$ \\
\hline Total & $58,934,892$ & $258,273,951$ & $58,806,542$ & $259,420,654$ & $0 \%$ & $0 \%$ \\
\hline
\end{tabular}


Table 79: Sensitivity Case Generation 2006 Nebraska 345 kV 20\% Penetration

\begin{tabular}{|c|c|c|c|c|c|c|}
\hline 2006 & \multicolumn{2}{|c|}{ Ideal } & \multicolumn{2}{|c|}{ Actual } & \multicolumn{2}{|c|}{ Delta } \\
\hline Nebraska 345 kV 20\% & Nebraska & Rest of SPP & Nebraska & Rest of SPP & Nebraska & Rest of SPP \\
\hline Combined Cycle & $2,674,888$ & $31,584,162$ & $2,946,498$ & $33,138,334$ & $10 \%$ & $5 \%$ \\
\hline CT Gas & 56,333 & $5,049,235$ & 77,337 & $5,079,619$ & $37 \%$ & $1 \%$ \\
\hline CT Other & - & 3,537 & - & 706 & & $-80 \%$ \\
\hline Hydro & 577,217 & $5,100,231$ & 577,217 & $5,100,231$ & $0 \%$ & $0 \%$ \\
\hline Internal Combustion & 13,827 & 97,476 & 14,747 & 88,603 & $7 \%$ & $-9 \%$ \\
\hline Interruptible & - & - & - & - & & \\
\hline Nuclear & $9,681,807$ & $8,415,466$ & $9,681,252$ & $8,415,466$ & $0 \%$ & $0 \%$ \\
\hline Pumped Storage & - & 340,387 & - & 340,386 & & $0 \%$ \\
\hline ST Coal & $28,986,062$ & $134,775,777$ & $28,894,418$ & $133,910,870$ & $0 \%$ & $-1 \%$ \\
\hline ST Gas & 15,354 & $1,881,406$ & 17,968 & $2,017,238$ & $17 \%$ & $7 \%$ \\
\hline ST Other & 369,285 & $2,152,349$ & 369,275 & $2,158,010$ & $0 \%$ & $0 \%$ \\
\hline Wind & $9,263,808$ & $47,037,944$ & $9,263,808$ & $47,042,970$ & $0 \%$ & $0 \%$ \\
\hline Other (Fixed Energy) & $1,675,421$ & $2,769,624$ & $\overline{1,675,421}$ & $2,769,624$ & $0 \%$ & $0 \%$ \\
\hline Total & $53,314,002$ & $239,207,594$ & $53,517,940$ & $240,062,058$ & $0 \%$ & $0 \%$ \\
\hline
\end{tabular}

Table 80: Sensitivity Case Generation 2006 Nebraska 345 kV 40\% Penetration

\begin{tabular}{|l|r|r|r|r|r|r|}
\multicolumn{1}{r|}{2006} & \multicolumn{2}{c|}{ Ideal } & \multicolumn{2}{c|}{ Actual } \\
\hline Nebraska 345 kV 40\% & Nebraska & Rest of SPP & Nebraska & \multicolumn{1}{c|}{ Rest of SPP } & Nebraska & Rest of SPP \\
\hline Combined Cycle & $1,880,406$ & $24,154,008$ & $2,128,137$ & $26,645,147$ & $13 \%$ & $10 \%$ \\
\hline CT Gas & 31,597 & $4,896,086$ & 52,148 & $4,935,710$ & $65 \%$ & $1 \%$ \\
\hline CT Other & - & 8,906 & - & 6,306 & & $-29 \%$ \\
\hline Hydro & 577,217 & $5,100,233$ & 577,217 & $5,100,233$ & $0 \%$ & $0 \%$ \\
\hline Internal Combustion & 8,253 & 78,350 & 10,707 & 66,504 & $30 \%$ & $-15 \%$ \\
\hline Interruptible & - & - & - & - & & \\
\hline Nuclear & $9,678,882$ & $8,415,466$ & $9,678,169$ & $8,415,466$ & $0 \%$ & $0 \%$ \\
\hline Pumped Storage & - & 364,527 & - & 364,526 & & $0 \%$ \\
\hline ST Coal & $26,900,850$ & $120,536,625$ & $26,453,437$ & $118,844,631$ & $-2 \%$ & $-1 \%$ \\
\hline ST Gas & 7,873 & $1,314,496$ & 11,487 & $1,541,494$ & $46 \%$ & $17 \%$ \\
\hline ST Other & 367,037 & $1,755,994$ & 366,159 & $1,749,344$ & $0 \%$ & $0 \%$ \\
\hline Wind & $17,746,713$ & $89,126,779$ & $17,746,793$ & $89,171,138$ & $0 \%$ & $0 \%$ \\
\hline Other (Fixed Energy) & $1,675,421$ & $2,769,624$ & $1,675,421$ & $2,769,624$ & $0 \%$ & $0 \%$ \\
\hline Total & $56,993,842$ & $234,367,085$ & $56,571,537$ & $232,964,976$ & $-1 \%$ & $-1 \%$ \\
\hline
\end{tabular}

Table 81: Sensitivity Case Generation 2006 Tariff $=\$ 20 /$ MWh 20\% Penetration 2006 Ideal Actual Delta

\begin{tabular}{|c|c|c|c|c|c|c|}
\hline 2000 & \multicolumn{2}{|c|}{ Jeal } & \multicolumn{2}{|c|}{ Rutual } & \multicolumn{2}{|c|}{ Della } \\
\hline Tariff $=\$ 20 /$ MWh $20 \%$ & Nebraska & Rest of SPP & Nebraska & Rest of SPP & Nebraska & Rest of SPP \\
\hline Combined Cycle & $2,074,444$ & $24,832,020$ & $2,260,242$ & $26,470,490$ & $9 \%$ & $7 \%$ \\
\hline CT Gas & 31,609 & $5,086,801$ & 55,129 & $5,111,397$ & $74 \%$ & $0 \%$ \\
\hline CT Other & - & 3,414 & - & 480 & & $-86 \%$ \\
\hline Hydro & 577,217 & $5,100,231$ & 577,217 & $5,100,231$ & $0 \%$ & $0 \%$ \\
\hline Internal Combustion & 10,647 & 70,813 & 12,422 & 63,554 & $17 \%$ & $-10 \%$ \\
\hline Interruptible & - & - & - & - & & \\
\hline Nuclear & $9,683,459$ & $8,415,466$ & $9,683,452$ & $8,415,466$ & $0 \%$ & $0 \%$ \\
\hline Pumped Storage & - & 340,387 & - & 340,386 & & $0 \%$ \\
\hline ST Coal & $28,304,518$ & $125,141,241$ & $28,159,297$ & $123,988,737$ & $-1 \%$ & $-1 \%$ \\
\hline ST Gas & 8,732 & $1,834,242$ & 12,542 & $2,001,330$ & $44 \%$ & $9 \%$ \\
\hline ST Other & 369,210 & $2,143,215$ & 369,130 & $2,142,141$ & $0 \%$ & $0 \%$ \\
\hline Wind & $9,263,808$ & $47,039,273$ & $9,263,808$ & $47,053,027$ & $0 \%$ & $0 \%$ \\
\hline Other (Fixed Energy) & $1,675,421$ & $2,769,624$ & $1,675,421$ & $2,769,624$ & $0 \%$ & $0 \%$ \\
\hline Total & $51,999,064$ & $222,776,727$ & $52,068,660$ & $223,456,863$ & $0 \%$ & $0 \%$ \\
\hline
\end{tabular}


Table 82: Sensitivity Case Generation 2006 CO2 = \$0/MWh 20\% Penetration

\begin{tabular}{|l|r|r|r|r|r|r|}
\multicolumn{1}{c|}{2006} & \multicolumn{2}{c|}{ Ideal } & \multicolumn{2}{c|}{ Actual } \\
\hline CO2 = \$0/MWh 20\% & Nebraska & Rest of SPP & \multicolumn{1}{c|}{ Debraska } & \multicolumn{1}{c|}{ Rest of SPP } & Nebraska & Rest of SPP \\
\hline Combined Cycle & $2,674,888$ & $31,584,162$ & $2,555,361$ & $28,330,025$ & $-4 \%$ & $-10 \%$ \\
\hline CT Gas & 56,333 & $5,049,235$ & 55,590 & $5,058,634$ & $-1 \%$ & $0 \%$ \\
\hline CT Other & - & 3,537 & - & 1,967 & & $-44 \%$ \\
\hline Hydro & 577,217 & $5,100,231$ & 577,217 & $5,100,231$ & $0 \%$ & $0 \%$ \\
\hline Internal Combustion & 13,827 & 97,476 & 13,231 & 70,215 & $-4 \%$ & $-28 \%$ \\
\hline Interruptible & - & - & - & - & & $0 \%$ \\
\hline Nuclear & $9,681,807$ & $8,415,466$ & $9,682,588$ & $8,415,466$ & $0 \%$ & $0 \%$ \\
\hline Pumped Storage & - & 340,387 & - & 685,005 & & $101 \%$ \\
\hline ST Coal & $28,986,062$ & $134,775,777$ & $29,721,105$ & $137,231,859$ & $3 \%$ & $2 \%$ \\
\hline ST Gas & 15,354 & $1,881,406$ & 12,896 & $2,110,687$ & $-16 \%$ & $12 \%$ \\
\hline ST Other & 369,285 & $2,152,349$ & 369,355 & $2,057,728$ & $0 \%$ & $-4 \%$ \\
\hline Wind & $9,263,808$ & $47,037,944$ & $9,263,808$ & $47,045,054$ & $0 \%$ & $0 \%$ \\
\hline Other (Fixed Energy) & $1,675,421$ & $2,769,624$ & $1,675,421$ & $2,769,624$ & $0 \%$ & $0 \%$ \\
\hline Total & $53,314,002$ & $239,207,594$ & $53,926,573$ & $238,876,496$ & $1 \%$ & $0 \%$ \\
\hline
\end{tabular}

Table 83: Sensitivity Case Generation 2006 CO2 $=\$ 5020 \%$ Penetration

\begin{tabular}{|c|c|c|c|c|c|c|}
\hline 2006 & \multicolumn{2}{|c|}{ Ideal } & \multicolumn{2}{|c|}{ Actual } & \multicolumn{2}{|c|}{ Delta } \\
\hline CO2 $=\$ 5020 \%$ & Nebraska & Rest of SPP & Nebraska & Rest of SPP & Nebraska & Rest of SPP \\
\hline Combined Cycle & $4,125,176$ & $38,665,973$ & $4,428,162$ & $40,170,982$ & $7 \%$ & $4 \%$ \\
\hline CT Gas & 33,955 & $5,465,177$ & 58,138 & $5,477,077$ & $71 \%$ & $0 \%$ \\
\hline CT Other & - & 3,781 & - & 1,764 & & $-53 \%$ \\
\hline \begin{tabular}{|l} 
Hydro \\
\end{tabular} & 577,217 & $5,100,231$ & 577,217 & $5,100,231$ & $0 \%$ & $0 \%$ \\
\hline Internal Combustion & 9,942 & 78,736 & 11,658 & 72,573 & $17 \%$ & $-8 \%$ \\
\hline Interruptible & - & - & - & - & & \\
\hline Nuclear & $9,683,574$ & $8,415,466$ & $9,683,574$ & $8,415,466$ & $0 \%$ & $0 \%$ \\
\hline Pumped Storage & - & 156,554 & - & 156,560 & & $0 \%$ \\
\hline ST Coal & $28,458,938$ & $125,520,584$ & $28,375,701$ & $124,616,022$ & $0 \%$ & $-1 \%$ \\
\hline ST Gas & 8,651 & $1,979,051$ & 12,822 & $2,114,567$ & $48 \%$ & $7 \%$ \\
\hline ST Other & 369,355 & $2,177,734$ & 369,355 & $2,176,814$ & $0 \%$ & $0 \%$ \\
\hline Wind & $9,263,808$ & $47,051,605$ & $9,263,808$ & $47,053,822$ & $0 \%$ & $0 \%$ \\
\hline Other (Fixed Energy) & $1,675,421$ & $2,769,624$ & $1,675,421$ & $2,769,624$ & $0 \%$ & $0 \%$ \\
\hline Total & $54,206,038$ & $237,384,515$ & $54,455,856$ & $238,125,502$ & $0 \%$ & $0 \%$ \\
\hline
\end{tabular}

Table 84: Sensitivity Case Generation 2006 CO2 $=\$ 12020 \%$ Penetration

\begin{tabular}{|c|c|c|c|c|c|c|}
\hline 2006 & \multicolumn{2}{|c|}{ Ideal } & \multicolumn{2}{|c|}{ Actual } & \multicolumn{2}{|c|}{ Delta } \\
\hline CO2= \$120 20\% & Nebraska & Rest of SPP & Nebraska & Rest of SPP & Nebraska & Rest of SPP \\
\hline Combined Cycle & $7,094,636$ & $73,733,780$ & $7,073,233$ & $73,878,476$ & $0 \%$ & $0 \%$ \\
\hline CT Gas & 93,536 & $7,836,575$ & 133,244 & $7,942,305$ & $42 \%$ & $1 \%$ \\
\hline CT Other & - & 2,858 & - & 1,758 & & $-39 \%$ \\
\hline Hydro & 577,217 & $5,100,231$ & 577,217 & $5,100,231$ & $0 \%$ & $0 \%$ \\
\hline Internal Combustion & 23,377 & 358,958 & 23,704 & 363,179 & $1 \%$ & $1 \%$ \\
\hline Interruptible & - & - & - & - & & \\
\hline Nuclear & $9,683,574$ & $8,415,466$ & $9,683,574$ & $8,415,466$ & $0 \%$ & $0 \%$ \\
\hline Pumped Storage & - & 61,983 & - & 61,981 & & $0 \%$ \\
\hline ST Coal & $22,894,548$ & $90,532,911$ & $22,744,709$ & $90,036,628$ & $-1 \%$ & $-1 \%$ \\
\hline ST Gas & 30,639 & $3,319,713$ & 36,456 & $3,784,108$ & $19 \%$ & $14 \%$ \\
\hline ST Other & 369,075 & $2,069,047$ & 369,050 & $2,074,945$ & $0 \%$ & $0 \%$ \\
\hline Wind & $9,263,808$ & $47,055,522$ & $9,263,808$ & $47,058,055$ & $0 \%$ & $0 \%$ \\
\hline Other (Fixed Energy) & $1,675,421$ & $2,769,624$ & $1,675,421$ & $2,769,624$ & $0 \%$ & $0 \%$ \\
\hline Total & $51,705,830$ & $241,256,669$ & $51,580,416$ & $241,486,757$ & $0 \%$ & $0 \%$ \\
\hline
\end{tabular}


Table 85: Sensitivity Case Generation 2006 Nebraska Market

\begin{tabular}{|l|r|r|r|r|r|r|}
\multicolumn{1}{c|}{2006} & \multicolumn{2}{c|}{ Ideal } & \multicolumn{2}{c|}{ Actual } & \multicolumn{1}{c|}{ Delta } \\
\hline Nebraska Market & Nebraska & Rest of SPP & Nebraska & Rest of SPP & Nebraska & Rest of SPP \\
\hline Combined Cycle & $2,579,440$ & $39,821,782$ & $2,289,261$ & $41,272,213$ & $-11 \%$ & $4 \%$ \\
\hline CT Gas & 60,421 & $5,320,016$ & 65,547 & $5,398,316$ & $8 \%$ & $1 \%$ \\
\hline CT Other & - & - & - & - & & \\
\hline Hydro & 577,216 & $5,100,231$ & 577,218 & $5,100,230$ & $0 \%$ & $0 \%$ \\
\hline Internal Combustion & 12,882 & 81,955 & 14,444 & 97,784 & $12 \%$ & $19 \%$ \\
\hline Interruptible & - & - & - & & \\
\hline Nuclear & $9,681,973$ & $8,415,466$ & $9,681,530$ & $8,415,466$ & $0 \%$ & $0 \%$ \\
\hline Pumped Storage & - & 325,141 & - & 331,342 & & $2 \%$ \\
\hline ST Coal & $28,382,660$ & $138,853,086$ & $27,766,974$ & $137,959,780$ & $-2 \%$ & $-1 \%$ \\
\hline ST Gas & 23,206 & $2,919,195$ & 38,957 & $3,146,680$ & $68 \%$ & $8 \%$ \\
\hline ST Other & 369,325 & $2,181,680$ & 369,300 & $2,175,191$ & $0 \%$ & $0 \%$ \\
\hline Wind & $4,749,939$ & $23,123,822$ & $4,749,670$ & $22,791,600$ & $0 \%$ & $-1 \%$ \\
\hline Other (Fixed Energy) & $1,675,421$ & $2,769,624$ & $1,675,421$ & $2,769,624$ & $0 \%$ & $0 \%$ \\
\hline Total & $48,112,482$ & $228,911,998$ & $47,228,323$ & $229,458,226$ & $-2 \%$ & \\
\hline
\end{tabular}

Table 86: Sensitivity Case Generation 2006 Daily block Proxy 10\% Penetration

\begin{tabular}{|c|c|c|c|c|c|c|}
\hline 2006 & \multicolumn{2}{|c|}{ Ideal } & \multicolumn{2}{|c|}{ Actual } & \multicolumn{2}{|c|}{ Delta } \\
\hline Daily Block Proxy & Nebraska & Rest of SPP & Nebraska & Rest of SPP & Nebraska & Rest of SPP \\
\hline Combined Cycle & $3,033,513$ & $39,520,874$ & $3,162,431$ & $40,718,123$ & $4 \%$ & $3 \%$ \\
\hline CT Gas & 74,230 & $5,303,758$ & 87,127 & $5,380,117$ & $17 \%$ & $1 \%$ \\
\hline CT Other & - & - & - & - & & \\
\hline Hydro & 577,216 & $5,100,232$ & 577,218 & $5,100,230$ & $0 \%$ & $0 \%$ \\
\hline Internal Combustion & 15,286 & 79,658 & 16,965 & 97,332 & $11 \%$ & $22 \%$ \\
\hline Interruptible & - & - & - & - & & \\
\hline Nuclear & $9,682,717$ & $8,415,466$ & $9,682,711$ & $8,415,466$ & $0 \%$ & $0 \%$ \\
\hline Pumped Storage & - & 325,139 & - & 331,378 & & $2 \%$ \\
\hline ST Coal & $29,151,412$ & $138,346,993$ & $28,913,624$ & $137,451,394$ & $-1 \%$ & $-1 \%$ \\
\hline ST Gas & 15,371 & $2,902,651$ & 19,068 & $3,106,484$ & $24 \%$ & $7 \%$ \\
\hline ST Other & 369,335 & $2,181,184$ & 369,335 & $2,174,857$ & $0 \%$ & $0 \%$ \\
\hline Wind & $4,749,939$ & $23,116,395$ & $4,749,670$ & $22,774,389$ & $0 \%$ & $-1 \%$ \\
\hline Other (Fixed Energy) & $1,675,421$ & $2,769,624$ & $1,675,421$ & $2,769,624$ & $0 \%$ & $0 \%$ \\
\hline Total & $49,344,438$ & $228,061,975$ & $49,253,568$ & $228,319,394$ & $0 \%$ & $0 \%$ \\
\hline
\end{tabular}

Table 87: Sensitivity Case Generation 2006 5-Hr MA 10\% Penetration $2006 \quad$ Ideal Actual Delta

\begin{tabular}{|c|c|c|c|c|c|c|}
\hline & & & \\
\hline $5 \mathrm{Hr}$ MA $10 \%$ & Nebraska & Rest of SPP & Nebraska & Rest of SPP & Nebraska & Rest of SPP \\
\hline Combined Cycle & $3,046,882$ & $40,132,014$ & $3,162,431$ & $40,718,123$ & $4 \%$ & $1 \%$ \\
\hline CT Gas & 78,703 & $5,341,915$ & 87,127 & $5,380,117$ & $11 \%$ & $1 \%$ \\
\hline CT Other & & & & & & \\
\hline Hydro & 577,214 & $5,100,231$ & 577,218 & $5,100,230$ & $0 \%$ & $0 \%$ \\
\hline Internal Combustion & 16,496 & 92,753 & 16,965 & 97,332 & $3 \%$ & $5 \%$ \\
\hline Interruptible & & & & & & \\
\hline Nuclear & $9,682,674$ & $8,415,466$ & $9,682,711$ & $8,415,466$ & $0 \%$ & $0 \%$ \\
\hline Pumped Storage & & 330,958 & & 331,378 & & $0 \%$ \\
\hline ST Coal & $29,038,469$ & $137,762,732$ & $28,913,624$ & $137,451,394$ & $0 \%$ & $0 \%$ \\
\hline ST Gas & 15,966 & $2,983,472$ & 19,068 & $3,106,484$ & $19 \%$ & $4 \%$ \\
\hline ST Other & 369,335 & $2,171,063$ & 369,335 & $2,174,857$ & $0 \%$ & $0 \%$ \\
\hline Wind & $4,749,268$ & $22,867,628$ & $4,749,670$ & $22,774,389$ & $0 \%$ & $0 \%$ \\
\hline Other (Fixed Energy) & $1,675,421$ & $2,769,624$ & $1,675,421$ & $2,769,624$ & $0 \%$ & $0 \%$ \\
\hline Total & $49,250,428$ & $227,967,856$ & $49,253,568$ & $228,319,394$ & $0 \%$ & $0 \%$ \\
\hline
\end{tabular}


Table 88: Sensitivity Case Generation 2006 Sub-Period Block 10\% Penetration

\begin{tabular}{|c|c|c|c|c|c|c|}
\hline 2006 & \multicolumn{2}{|c|}{ Ideal } & \multicolumn{2}{|c|}{ Actual } & \multicolumn{2}{|c|}{ Delta } \\
\hline Sub-Period Block & Nebraska & Rest of SPP & Nebraska & Rest of SPP & Nebraska & Rest of SPP \\
\hline Combined Cycle & $3,067,549$ & $39,856,553$ & $3,162,431$ & $40,718,123$ & $3 \%$ & $2 \%$ \\
\hline CT Gas & 84,820 & $5,359,432$ & 87,127 & $5,380,117$ & $3 \%$ & $0 \%$ \\
\hline CT Other & - & - & - & - & & \\
\hline Hydro & 577,218 & $5,100,236$ & 577,218 & $5,100,230$ & $0 \%$ & $0 \%$ \\
\hline Internal Combustion & 16,755 & 88,178 & 16,965 & 97,332 & $1 \%$ & $10 \%$ \\
\hline Interruptible & - & - & - & - & & \\
\hline Nuclear & $9,682,572$ & $8,415,466$ & $9,682,711$ & $8,415,466$ & $0 \%$ & $0 \%$ \\
\hline Pumped Storage & - & 339,647 & - & 331,378 & & $-2 \%$ \\
\hline ST Coal & $29,021,770$ & $137,863,566$ & $28,913,624$ & $137,451,394$ & $0 \%$ & $0 \%$ \\
\hline ST Gas & 21,104 & $2,953,646$ & 19,068 & $3,106,484$ & $-10 \%$ & $5 \%$ \\
\hline ST Other & 369,315 & $2,173,068$ & 369,335 & $2,174,857$ & $0 \%$ & $0 \%$ \\
\hline Wind & $4,780,584$ & $23,145,530$ & $4,749,670$ & $22,774,389$ & $-1 \%$ & $-2 \%$ \\
\hline Other (Fixed Energy) & $1,675,421$ & $2,769,624$ & $1,675,421$ & $2,769,624$ & $0 \%$ & $0 \%$ \\
\hline Total & $47,621,687$ & $225,295,322$ & $47,578,148$ & $225,549,770$ & $0 \%$ & $0 \%$ \\
\hline
\end{tabular}

Table 89: Sensitivity Case Generation 2006 13-Hr MA 10\% Penetration

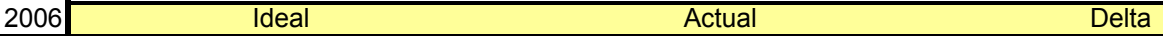

\begin{tabular}{|c|c|c|c|c|c|c|}
\hline 2006 & & & & & & elta \\
\hline 13-Hr MA & Nebraska & Rest of SPP & Nebraska & Rest of SPP & Nebraska & Rest of SPP \\
\hline Combined Cycle & $3,045,211$ & $39,820,544$ & $3,162,431$ & $40,718,123$ & $4 \%$ & $2 \%$ \\
\hline CT Gas & 77,106 & $5,330,636$ & 87,127 & $5,380,117$ & $13 \%$ & $1 \%$ \\
\hline CT Other & - & - & - & - & & \\
\hline Hydro & 577,219 & $5,100,229$ & 577,218 & $5,100,230$ & $0 \%$ & $0 \%$ \\
\hline Internal Combustion & 16,010 & 86,753 & 16,965 & 97,332 & $6 \%$ & $12 \%$ \\
\hline Interruptible & - & - & - & - & & \\
\hline Nuclear & $9,682,693$ & $8,415,466$ & $9,682,711$ & $8,415,466$ & $0 \%$ & $0 \%$ \\
\hline Pumped Storage & - & 328,304 & - & 331,378 & & $1 \%$ \\
\hline ST Coal & $29,092,420$ & $137,966,692$ & $28,913,624$ & $137,451,394$ & $-1 \%$ & $0 \%$ \\
\hline ST Gas & 17,555 & $2,938,038$ & 19,068 & $3,106,484$ & $9 \%$ & $6 \%$ \\
\hline ST Other & 369,335 & $2,175,435$ & 369,335 & $2,174,857$ & $0 \%$ & $0 \%$ \\
\hline Wind & $4,749,399$ & $23,001,848$ & $4,749,670$ & $22,774,389$ & $0 \%$ & $-1 \%$ \\
\hline Other (Fixed Energy) & $1,675,421$ & $2,769,624$ & $1,675,421$ & $2,769,624$ & $0 \%$ & $0 \%$ \\
\hline Total & $47,626,948$ & $225,163,945$ & $47,578,148$ & $225,549,770$ & $0 \%$ & $0 \%$ \\
\hline
\end{tabular}

Table 90: Sensitivity Case Generation 2006 WAPA Sensitivity

\begin{tabular}{|l|r|r|r|r|r|r|}
\multicolumn{1}{c}{2006} & \multicolumn{1}{c|}{ Schedule to Load Net Wind } & \multicolumn{2}{c|}{ Schedule to Load } & \multicolumn{2}{c|}{ Delta } \\
\hline WAPA Sensitivity & Nebraska & Rest of SPP & Nebraska & Rest of SPP & Nebraska & Rest of SPP \\
\hline Combined Cycle & $2,155,950$ & $40,853,889$ & $2,162,184$ & $40,880,954$ & $0 \%$ & $0 \%$ \\
\hline CT Gas & 59,886 & $5,354,559$ & 59,564 & $5,354,515$ & $-1 \%$ & $0 \%$ \\
\hline CT Other & - & - & - & - & & \\
\hline Hydro & 577,219 & $5,100,227$ & 577,219 & $5,100,227$ & $0 \%$ & $0 \%$ \\
\hline Internal Combustion & 12,967 & 95,343 & 13,094 & 95,495 & $1 \%$ & $0 \%$ \\
\hline Interruptible & 0 & - & - & $0 \%$ & $0 \%$ \\
\hline Nuclear & $9,683,541$ & $8,415,466$ & $9,683,540$ & $8,415,466$ & $0 \%$ & $0 \%$ \\
\hline Pumped Storage & - & 291,469 & - & 291,477 & & $0 \%$ \\
\hline ST Coal & $27,737,885$ & $138,020,652$ & $27,725,483$ & $138,019,735$ & $0 \%$ & $0 \%$ \\
\hline ST Gas & 36,172 & $3,034,708$ & 36,356 & $3,033,867$ & $1 \%$ & $0 \%$ \\
\hline ST Other & 369,355 & $2,174,539$ & 369,355 & $2,174,606$ & $0 \%$ & $0 \%$ \\
\hline Wind & $4,749,670$ & $22,775,640$ & $4,749,670$ & $22,775,705$ & $0 \%$ & $0 \%$ \\
\hline Other (Fixed Energy) & $1,675,421$ & $2,769,624$ & $1,675,421$ & $2,769,624$ & $0 \%$ & $0 \%$ \\
\hline Total & $47,058,066$ & $228,886,115$ & $47,051,887$ & $228,911,670$ & $0 \%$ & $0 \%$ \\
\hline
\end{tabular}


Table 91: Sensitivity Case Generation 2006 WAPA II - Pure

\begin{tabular}{|l|r|r|r|r|r|r|}
\multicolumn{1}{c|}{2006} & \multicolumn{1}{c|}{ Schedule to Load Net Wind } & \multicolumn{2}{c|}{ Schedule to Load } & \multicolumn{3}{c|}{ Delta } \\
\hline WAPA II - Pure & Nebraska & Rest of SPP & Nebraska & Rest of SPP & Nebraska & Rest of SPP \\
\hline Combined Cycle & $2,167,250$ & $40,846,469$ & $2,170,011$ & $40,840,421$ & $0 \%$ & $0 \%$ \\
\hline CT Gas & 59,732 & $5,358,591$ & 60,083 & $5,351,775$ & $1 \%$ & $0 \%$ \\
\hline CT Other & - & - & - & - & & \\
\hline Hydro & 577,219 & $5,100,226$ & 577,219 & $5,100,226$ & $0 \%$ & $0 \%$ \\
\hline Internal Combustion & 12,951 & 95,030 & 13,049 & 95,077 & $1 \%$ & $0 \%$ \\
\hline Interruptible & 0 & - & 0 & - & $-22 \%$ & $0 \%$ \\
\hline Nuclear & $9,683,541$ & $8,415,466$ & $9,683,541$ & $8,415,466$ & $0 \%$ & $0 \%$ \\
\hline Pumped Storage & - & 291,279 & - & 291,457 & & $0 \%$ \\
\hline ST Coal & $27,750,359$ & $138,028,017$ & $27,741,381$ & $138,039,545$ & $0 \%$ & $0 \%$ \\
\hline ST Gas & 35,646 & $3,035,066$ & 36,721 & $3,045,426$ & $3 \%$ & $0 \%$ \\
\hline ST Other & 369,355 & $2,174,574$ & 369,355 & $2,174,685$ & $0 \%$ & $0 \%$ \\
\hline Wind & $4,749,670$ & $22,773,324$ & $4,749,670$ & $22,775,335$ & $0 \%$ & $0 \%$ \\
\hline Other (Fixed Energy) & $1,675,421$ & $2,769,624$ & $1,675,421$ & $2,769,624$ & $0 \%$ & $0 \%$ \\
\hline Total & $47,081,143$ & $228,887,667$ & $47,076,451$ & $228,899,038$ & $0 \%$ & $0 \%$ \\
\hline
\end{tabular}

Table 92: Sensitivity Case Generation 2006 Existing Wind

\begin{tabular}{|l|r|r|r|r|r|r|}
\multicolumn{1}{c|}{2006} & \multicolumn{3}{c|}{ Ideal } & \multicolumn{2}{c|}{ Actual } \\
\cline { 2 - 7 } & Eebraska & Rest of SPP & Nebraska & \multicolumn{1}{c|}{ Rest of SPP } & Nebraska & Rest of SPP \\
\hline Combinting Wind & $3,029,551$ & $43,869,680$ & $2,953,246$ & $44,439,736$ & $-3 \%$ & $1 \%$ \\
\hline CT Gas & 80,648 & $5,563,957$ & 80,530 & $5,621,081$ & $0 \%$ & $1 \%$ \\
\hline CT Other & - & - & - & - & & \\
\hline Hydro & 577,217 & $5,100,232$ & 577,216 & $5,100,228$ & $0 \%$ & $0 \%$ \\
\hline Internal Combustion & 16,377 & 125,996 & 16,651 & 129,876 & $2 \%$ & $3 \%$ \\
\hline Interruptible & 0 & - & 0 & - & $29 \%$ & 0 \\
\hline Nuclear & $9,682,224$ & $8,415,466$ & $9,682,040$ & $8,415,466$ & $0 \%$ & $0 \%$ \\
\hline Pumped Storage & - & 322,389 & - & 325,967 & & $1 \%$ \\
\hline ST Coal & $28,832,469$ & $141,121,083$ & $28,703,107$ & $140,817,697$ & $0 \%$ & $0 \%$ \\
\hline ST Gas & 34,467 & $3,543,997$ & 38,812 & $3,651,080$ & $13 \%$ & $3 \%$ \\
\hline ST Other & 369,335 & $2,207,726$ & 369,350 & $2,207,220$ & $0 \%$ & $0 \%$ \\
\hline Wind & 492,115 & $11,203,623$ & 492,118 & $11,203,631$ & $0 \%$ & $0 \%$ \\
\hline Other (Fixed Energy) & $1,675,421$ & $2,769,624$ & $1,675,421$ & $2,769,624$ & $0 \%$ & $0 \%$ \\
\hline Total & $44,789,824$ & $224,243,772$ & $44,588,492$ & $224,681,607$ & $0 \%$ & $0 \%$ \\
\hline
\end{tabular}

Table 93: Sensitivity Case Generation 20065 Hour MA 20\%

\begin{tabular}{|c|c|c|c|c|c|c|}
\hline 2006 & \multicolumn{2}{|c|}{ Ideal } & \multicolumn{2}{|c|}{ Actual } & \multicolumn{2}{|c|}{ Delta } \\
\hline $5 \mathrm{Hr}$ MA $20 \%$ & Nebraska & Rest of SPP & Nebraska & Rest of SPP & Nebraska & Rest of SPP \\
\hline Combined Cycle & $2,564,981$ & $33,758,759$ & $2,707,139$ & $35,177,239$ & $6 \%$ & $4 \%$ \\
\hline CT Gas & 62,491 & $5,078,530$ & 70,068 & $5,124,149$ & $12 \%$ & $1 \%$ \\
\hline CT Other & - & - & - & - & & \\
\hline Hydro & 577,218 & $5,100,232$ & 577,217 & $5,100,231$ & $0 \%$ & $0 \%$ \\
\hline Internal Combustion & 13,783 & 68,139 & 14,499 & 72,359 & $5 \%$ & $6 \%$ \\
\hline Interruptible & - & - & - & - & & \\
\hline Nuclear & $9,682,776$ & $8,415,466$ & $9,682,648$ & $8,415,466$ & $0 \%$ & $0 \%$ \\
\hline Pumped Storage & - & 339,179 & - & 340,386 & & $0 \%$ \\
\hline ST Coal & $28,477,992$ & $131,649,015$ & $28,197,962$ & $131,231,646$ & $-1 \%$ & $0 \%$ \\
\hline ST Gas & 9,449 & $2,221,254$ & 13,933 & $2,424,364$ & $47 \%$ & $9 \%$ \\
\hline ST Other & 369,315 & $2,114,746$ & 369,290 & $2,124,901$ & $0 \%$ & $0 \%$ \\
\hline Wind & $9,264,080$ & $44,076,704$ & $9,263,808$ & $43,684,117$ & $0 \%$ & $-1 \%$ \\
\hline Other (Fixed Energy) & $1,675,421$ & $2,769,624$ & $1,675,421$ & $2,769,624$ & $0 \%$ & $0 \%$ \\
\hline Total & $52,697,505$ & $235,591,648$ & $52,571,985$ & $236,464,482$ & $0 \%$ & $0 \%$ \\
\hline
\end{tabular}


Table 94: Sensitivity Case Generation 2006 Daily Block Wind 20\%

\begin{tabular}{|c|c|c|c|c|c|c|}
\hline 200 & \multicolumn{2}{|c|}{ Ideal } & \multicolumn{2}{|c|}{ Actual } & \multicolumn{2}{|c|}{ Delta } \\
\hline Daily Block Proxy $20 \%$ & Nebraska & Rest of SPP & Nebraska & Rest of SPP & Nebraska & Rest of SPP \\
\hline Combined Cycle & $2,490,948$ & $32,626,919$ & $2,707,139$ & $35,177,239$ & $9 \%$ & $8 \%$ \\
\hline CT Gas & 60,162 & $5,020,357$ & 70,068 & $5,124,149$ & $16 \%$ & $2 \%$ \\
\hline CT Other & - & - & - & - & & \\
\hline Hydro & 577,218 & $5,100,232$ & 577,217 & $5,100,231$ & $0 \%$ & $0 \%$ \\
\hline Internal Combustion & 12,960 & 54,420 & 14,499 & 72,359 & $12 \%$ & $33 \%$ \\
\hline Interruptible & - & - & - & - & & \\
\hline Nuclear & $9,682,890$ & $8,415,466$ & $9,682,648$ & $8,415,466$ & $0 \%$ & $0 \%$ \\
\hline Pumped Storage & - & 327,845 & - & 340,386 & & $4 \%$ \\
\hline ST Coal & $28,616,091$ & $132,456,830$ & $28,197,962$ & $131,231,646$ & $-1 \%$ & $-1 \%$ \\
\hline ST Gas & 8,075 & $2,060,790$ & 13,933 & $2,424,364$ & $73 \%$ & $18 \%$ \\
\hline ST Other & 369,320 & $2,116,682$ & 369,290 & $2,124,901$ & $0 \%$ & $0 \%$ \\
\hline Wind & $9,264,080$ & $44,076,704$ & $9,263,808$ & $43,684,117$ & $0 \%$ & $-1 \%$ \\
\hline Other (Fixed Energy) & $1,675,421$ & $2,769,624$ & $1,675,421$ & $2,769,624$ & $0 \%$ & $0 \%$ \\
\hline Total & $52,757,165$ & $235,025,867$ & $52,571,985$ & $236,464,482$ & $0 \%$ & $1 \%$ \\
\hline
\end{tabular}

Table 95: Sensitivity Case Generation 2006 Sub-period Block Wind 20\% $2 0 0 6 \longdiv { \text { Ideal } }$ Actual Delta

\begin{tabular}{|c|c|c|c|c|c|c|}
\hline & \multicolumn{2}{|c|}{ Tueal } & \multicolumn{2}{|c|}{ Actual } & \multicolumn{2}{|c|}{ Eetia } \\
\hline Sub-Period Block 20\% & Nebraska & Rest of SPP & Nebraska & Rest of SPP & Nebraska & Rest of SPP \\
\hline Combined Cycle & $2,519,885$ & $33,270,991$ & $2,707,139$ & $35,177,239$ & $7 \%$ & $6 \%$ \\
\hline CT Gas & 59,134 & $5,068,367$ & 70,068 & $5,124,149$ & $18 \%$ & $1 \%$ \\
\hline CT Other & - & - & - & - & & \\
\hline Hydro & 577,218 & $5,100,230$ & 577,217 & $5,100,231$ & $0 \%$ & $0 \%$ \\
\hline Internal Combustion & 13,390 & 61,143 & 14,499 & 72,359 & $8 \%$ & $18 \%$ \\
\hline Interruptible & - & - & - & - & & \\
\hline Nuclear & $9,682,793$ & $8,415,466$ & $9,682,648$ & $8,415,466$ & $0 \%$ & $0 \%$ \\
\hline Pumped Storage & - & 336,685 & - & 340,386 & & $1 \%$ \\
\hline ST Coal & $28,513,619$ & $131,815,223$ & $28,197,962$ & $131,231,646$ & $-1 \%$ & $0 \%$ \\
\hline ST Gas & 11,047 & $2,170,937$ & 13,933 & $2,424,364$ & $26 \%$ & $12 \%$ \\
\hline ST Other & 369,325 & $2,115,576$ & 369,290 & $2,124,901$ & $0 \%$ & $0 \%$ \\
\hline Wind & $9,264,080$ & $44,076,704$ & $9,263,808$ & $43,684,117$ & $0 \%$ & $-1 \%$ \\
\hline Other (Fixed Energy) & $1,675,421$ & $2,769,624$ & $1,675,421$ & $2,769,624$ & $0 \%$ & $0 \%$ \\
\hline Total & $51,010,490$ & $232,431,321$ & $50,896,564$ & $233,694,858$ & $0 \%$ & $1 \%$ \\
\hline
\end{tabular}

Table 96: $\quad$ Sensitivity Case Generation 200613 Hour Moving Average 20\%

\begin{tabular}{|c|c|c|c|c|c|c|}
\hline 2006 & \multicolumn{2}{|c|}{ Ideal } & \multicolumn{2}{|c|}{ Actual } & \multicolumn{2}{|c|}{ Delta } \\
\hline 13-Hr MA $20 \%$ & Nebraska & Rest of SPP & Nebraska & Rest of SPP & Nebraska & Rest of SPP \\
\hline Combined Cycle & $2,535,217$ & $33,233,200$ & $2,707,139$ & $35,177,239$ & $7 \%$ & $6 \%$ \\
\hline CT Gas & 61,421 & $5,056,705$ & 70,068 & $5,124,149$ & $14 \%$ & $1 \%$ \\
\hline CT Other & - & - & - & - & & \\
\hline Hydro & 577,219 & $5,100,233$ & 577,217 & $5,100,231$ & $0 \%$ & $0 \%$ \\
\hline Internal Combustion & 13,542 & 58,910 & 14,499 & 72,359 & $7 \%$ & $23 \%$ \\
\hline Interruptible & - & - & - & - & & \\
\hline Nuclear & $9,682,843$ & $8,415,466$ & $9,682,648$ & $8,415,466$ & $0 \%$ & $0 \%$ \\
\hline Pumped Storage & - & 335,897 & - & 340,386 & & $1 \%$ \\
\hline ST Coal & $28,549,948$ & $132,022,105$ & $28,197,962$ & $131,231,646$ & $-1 \%$ & $-1 \%$ \\
\hline ST Gas & 10,412 & $2,150,908$ & 13,933 & $2,424,364$ & $34 \%$ & $13 \%$ \\
\hline ST Other & 369,310 & $2,114,844$ & 369,290 & $2,124,901$ & $0 \%$ & $0 \%$ \\
\hline Wind & $9,264,080$ & $44,076,704$ & $9,263,808$ & $43,684,117$ & $0 \%$ & $-1 \%$ \\
\hline Other (Fixed Energy) & $1,675,421$ & $2,769,624$ & $1,675,421$ & $2,769,624$ & $0 \%$ & $0 \%$ \\
\hline Total & $51,063,992$ & $232,564,972$ & $50,896,564$ & $233,694,858$ & $0 \%$ & $0 \%$ \\
\hline
\end{tabular}


Table 97: Nebraska Non-monitored Branches with number of hours of potential overloads (2006 load and wind profiles)

\begin{tabular}{|c|c|c|c|c|c|c|c|c|c|c|c|c|}
\hline & \multicolumn{3}{|c|}{ Scenario 1} & \multicolumn{3}{|c|}{ Scenario 2} & \multicolumn{3}{|c|}{ Scenario 3} & \multicolumn{3}{|c|}{ Scenario 4} \\
\hline Nebraska Branch Name & VIOLATION & $90 \%$ & $80 \%$ & VIOLATION & $90 \%$ & $80 \%$ & VIOLATION & $90 \%$ & $80 \%$ & VIOLATION & $90 \%$ & $80 \%$ \\
\hline 640050AINSWND7 640096 CALAMS 7 & 0 & 0 & 0 & 0 & 0 & 3 & 0 & 0 & 0 & 0 & 0 & 0 \\
\hline 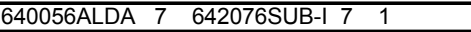 & 0 & 2 & 23 & 0 & 1 & 14 & 0 & 0 & 10 & 0 & 0 & 2 \\
\hline 640058ATKINSN7 640165EMMET 7 & 0 & 0 & 0 & 568 & 1242 & 2050 & 0 & 0 & 0 & 0 & 0 & 0 \\
\hline 640058ATKINSN7 $\quad$ 640367STUART 7 1 & 0 & 0 & 0 & 710 & 1408 & 2194 & 0 & 0 & 0 & 0 & 0 & 0 \\
\hline 640065AXTELL $3 \quad$ 640312PAULINE3 1 & 0 & 0 & 0 & 0 & 0 & 0 & 0 & 0 & 0 & 0 & 0 & 3 \\
\hline 640066AXTELL 7 640250KEARNEY7 1 & 0 & 0 & 0 & 0 & 0 & 0 & 0 & 0 & 0 & 2 & 10 & 83 \\
\hline 640076BEATRCE7 640208HARBINE7 1 & 317 & 652 & 1232 & 129 & 326 & 612 & 89 & 238 & 622 & 3 & 22 & 186 \\
\hline 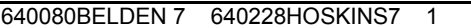 & 0 & 2 & 42 & 2 & 46 & 172 & 0 & 16 & 118 & 0 & 0 & 35 \\
\hline 640096CALAMS 7 640381THEDFRD7 1 & 0 & 0 & 0 & 0 & 0 & 3 & 0 & 0 & 0 & 0 & 0 & 0 \\
\hline 640098CALAWAY7 $\quad$ 640267MAXWELS7 1 & 0 & 0 & 0 & 0 & 0 & 36 & 0 & 0 & 0 & 0 & 0 & 0 \\
\hline 640100CAMBRIG7 $\quad$ 640269MCCOOK 71 & 0 & 0 & 0 & 0 & 0 & 5 & 0 & 0 & 0 & 0 & 0 & 0 \\
\hline 640113CLRWATR7 $\quad$ 640305ONEILL 7 1 & 0 & 0 & 0 & 0 & 0 & 15 & 0 & 0 & 0 & 0 & 0 & 0 \\
\hline 640131COLMB.W4 640133COLMBUS4 1 & 0 & 0 & 0 & 0 & 0 & 16 & 0 & 0 & 0 & 0 & 0 & 0 \\
\hline 640200GR ISLD4 1 & 0 & 0 & 0 & 0 & 5 & 67 & 0 & 0 & 0 & 0 & 0 & 0 \\
\hline 652509FTRANDL4 1 & 0 & 0 & 1 & 0 & 0 & 0 & 0 & 0 & 0 & 21 & 109 & 369 \\
\hline $\begin{array}{lll}\text { 640136COLMBUS7 } & \text { 640336SCHUYLR7 } & 1\end{array}$ & 0 & 0 & 9 & 0 & 28 & 203 & 0 & 0 & 7 & 21 & 122 & 442 \\
\hline 640165EMMET 7 640305ONEILL 71 & 0 & 0 & 0 & 386 & 1045 & 1825 & 0 & 0 & 0 & 0 & 0 & 0 \\
\hline 640171FIRTH 7 640278SHELDON7 1 & 0 & 0 & 51 & 0 & 11 & 137 & 0 & 0 & 18 & 0 & 0 & 31 \\
\hline 640183GENTLMN3 640252KEYSTON3 1 & 0 & 0 & 0 & 0 & 0 & 0 & 0 & 0 & 0 & 0 & 0 & 4 \\
\hline 640196GOTHNBG7 640238JEFFREY7 & 0 & 62 & 709 & 0 & 223 & 1003 & 0 & 0 & 2 & 0 & 0 & 140 \\
\hline $\begin{array}{lll}640210 H A R M O N Y 7 & 640351 S T . F R A N C & 1 \\
\end{array}$ & 0 & 0 & 0 & 4426 & 4681 & 4927 & 6 & 48 & 186 & 27 & 133 & 491 \\
\hline 640392VALENTN7 1 & 0 & 0 & 0 & 4610 & 4861 & 5097 & 34 & 141 & 355 & 83 & 365 & 819 \\
\hline 640227HOSKINS4 $640226 \mathrm{HOSKINS3} 1$ & 0 & 0 & 11 & 3 & 14 & 31 & 2 & 13 & 30 & 18 & 35 & 72 \\
\hline 640227HOSKINS4 640386TWIN CH4 1 & 0 & 0 & 0 & 0 & 0 & 1 & 0 & 0 & 0 & 0 & 0 & 2 \\
\hline 640228HOSKINS7 640227 HOSKINS4 1 & 0 & 1 & 7 & 2 & 5 & 17 & 2 & 7 & 28 & 18 & 33 & 52 \\
\hline 640228HOSKINS7 640363STNTN.N7 1 & 0 & 0 & 0 & 0 & 0 & 4 & 0 & 0 & 1 & 0 & 2 & 7 \\
\hline 640252KEYSTON3 659133SIDNEY 3 & 0 & 0 & 0 & 0 & 0 & 0 & 0 & 0 & 0 & 0 & 0 & 8 \\
\hline 640259LOUPCTY7 $\quad$ 640284N.LOUP 71 & 0 & 0 & 1 & 0 & 1 & 78 & 0 & 0 & 0 & 7603 & 7725 & 7853 \\
\hline 640267MAXWELS7 $\quad$ 640287N.PLATT7 1 & 6 & 121 & 652 & 5 & 65 & 316 & 0 & 0 & 0 & 0 & 0 & 0 \\
\hline 640271MCCOOL $3 \quad$ 640277MOORE 3 & 0 & 0 & 0 & 0 & 22 & 324 & 0 & 0 & 0 & 0 & 0 & 0 \\
\hline 640277MOORE 3 650114NW68HOL3 & 0 & 0 & 0 & 0 & 0 & 2 & 0 & 0 & 0 & 1440 & 2002 & 2610 \\
\hline 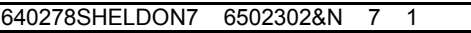 & 0 & 0 & 1 & 0 & 0 & 2 & 0 & 0 & 3 & 4140 & 4757 & 5409 \\
\hline 640278SHELDON7 $65023820 \&$ PIO $7 \quad 1$ & 0 & 0 & 17 & 0 & 1 & 49 & 0 & 4 & 30 & 0 & 3 & 11 \\
\hline 640281N.BEND $7 \quad$ 640336SCHUYLR7 1 & 0 & 0 & 0 & 0 & 0 & 0 & 0 & 0 & 0 & 2016 & 3361 & 5081 \\
\hline 640305ONEILL 7 640349SPENCER7 1 & 0 & 45 & 274 & 0 & 8 & 49 & 9 & 55 & 272 & 0 & 0 & 0 \\
\hline $\begin{array}{lll}640349 S P E N C E R 7 & 652510 F T R A N D L 7 & 1 \\
\end{array}$ & 8 & 94 & 380 & 1 & 10 & 76 & 20 & 88 & 363 & 117 & 359 & 902 \\
\hline 640351ST.FRANC $\quad$ 652482MISSION7 1 & 0 & 0 & 0 & 4295 & 4524 & 4776 & 0 & 17 & 82 & 2473 & 3421 & 4433 \\
\hline $\begin{array}{lll}\text { 640357STANTON7 } & \text { 640363STNTN.N7 } & 1 \\
\end{array}$ & 0 & 0 & 0 & 0 & 0 & 0 & 0 & 0 & 0 & 7 & 43 & 230 \\
\hline 640374SWEET W3 652571GR ISLD3 1 & 0 & 0 & 5 & 0 & 1 & 81 & 0 & 0 & 0 & 0 & 0 & 0 \\
\hline 640386TWIN CH4 652565SIOUXCY4 & 0 & 0 & 2 & 0 & 0 & 0 & 0 & 0 & 2 & 3485 & 3945 & 4455 \\
\hline $645455 S 345533645456 S 34563 \quad 1$ & 0 & 0 & 0 & 0 & 0 & 0 & 0 & 0 & 0 & 0 & 0 & 0 \\
\hline $645458 S 34583 \quad 1$ & 0 & 0 & 1 & 0 & 0 & 3 & 0 & 0 & 7 & 0 & 0 & 1 \\
\hline 648056S3456T4T 1 & 0 & 0 & 4 & 0 & 0 & 4 & 0 & 0 & 0 & 1524 & 2399 & 3578 \\
\hline 648056S3456T4T & 0 & 0 & 4 & 0 & 0 & 4 & 0 & 0 & 0 & 0 & 0 & 0 \\
\hline 648009S1209T1T 1 & 0 & 0 & 13 & 0 & 0 & 8 & 0 & 0 & 10 & 8716 & 8752 & 8759 \\
\hline $646221 S 122155 \quad 646255 S 1255551$ & 0 & 2 & 158 & 0 & 23 & 214 & 0 & 13 & 173 & 0 & $\begin{array}{ll}0 \\
\end{array}$ & 0 \\
\hline 650208WLINC $7 \quad 6502302 \& N \quad 7 \quad 1$ & 3 & 9 & 24 & 3 & 11 & 24 & 3 & 8 & 21 & 836 & 1387 & 2482 \\
\hline 65021519\&ALVO7 $\quad 65026970 \& B L U F 7 \quad 1$ & 0 & 0 & 1 & 0 & 0 & 0 & 0 & 0 & 1 & 6 & 16 & 35 \\
\hline 65026257\&GAR $7 \quad$ 65026784\&LEIG7 1 & 0 & 0 & 3 & 0 & 0 & 2 & 0 & 0 & 3 & 0 & 0 & 1 \\
\hline 659132OGALALA7 $\quad$ 659187ROSCOE 71 & 0 & 0 & 0 & 0 & 0 & 0 & 0 & 0 & 0 & 18 & 121 & 514 \\
\hline 659132OGALALA7 $\quad$ 659246MCONGHY7 1 & 4546 & 4779 & 5027 & 4546 & 4779 & 5027 & 4546 & 4779 & 5027 & 240 & 896 & 2278 \\
\hline 659246MCONGHY7 $\quad$ 659247ARTHUR 7 1 & 4580 & 4814 & 5059 & 4580 & 4814 & 5059 & 4580 & 4814 & 5059 & 4546 & 4779 & 5027 \\
\hline
\end{tabular}




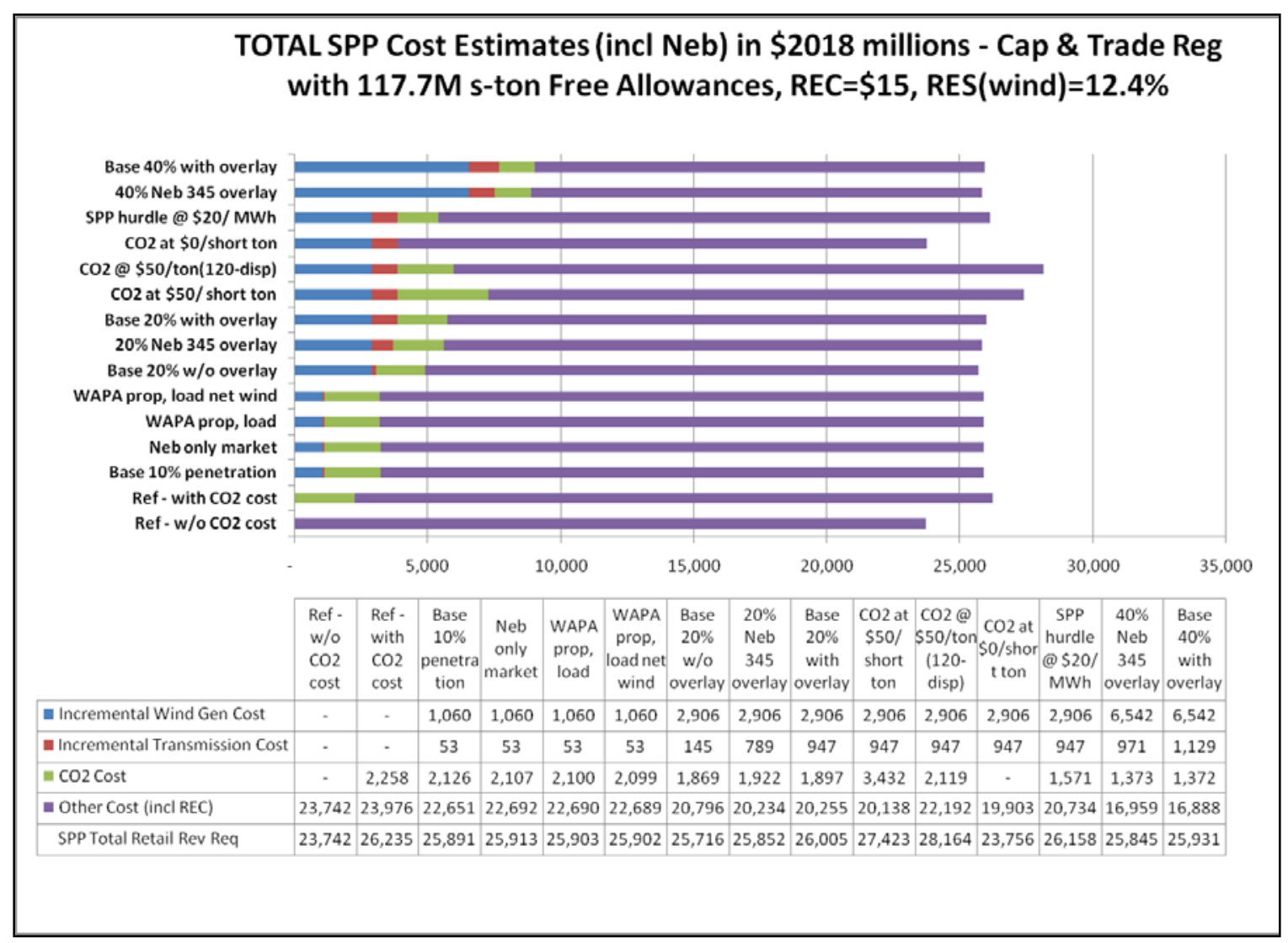

Figure 98: Total SPP Cost Estimates (inc Neb) in $\$ 2018$ millions - Cap \& Trade Reg with 117.7M s-ton Free Allowances, REC $=\$ 15$, RES (wind) $=12.4 \%$ 


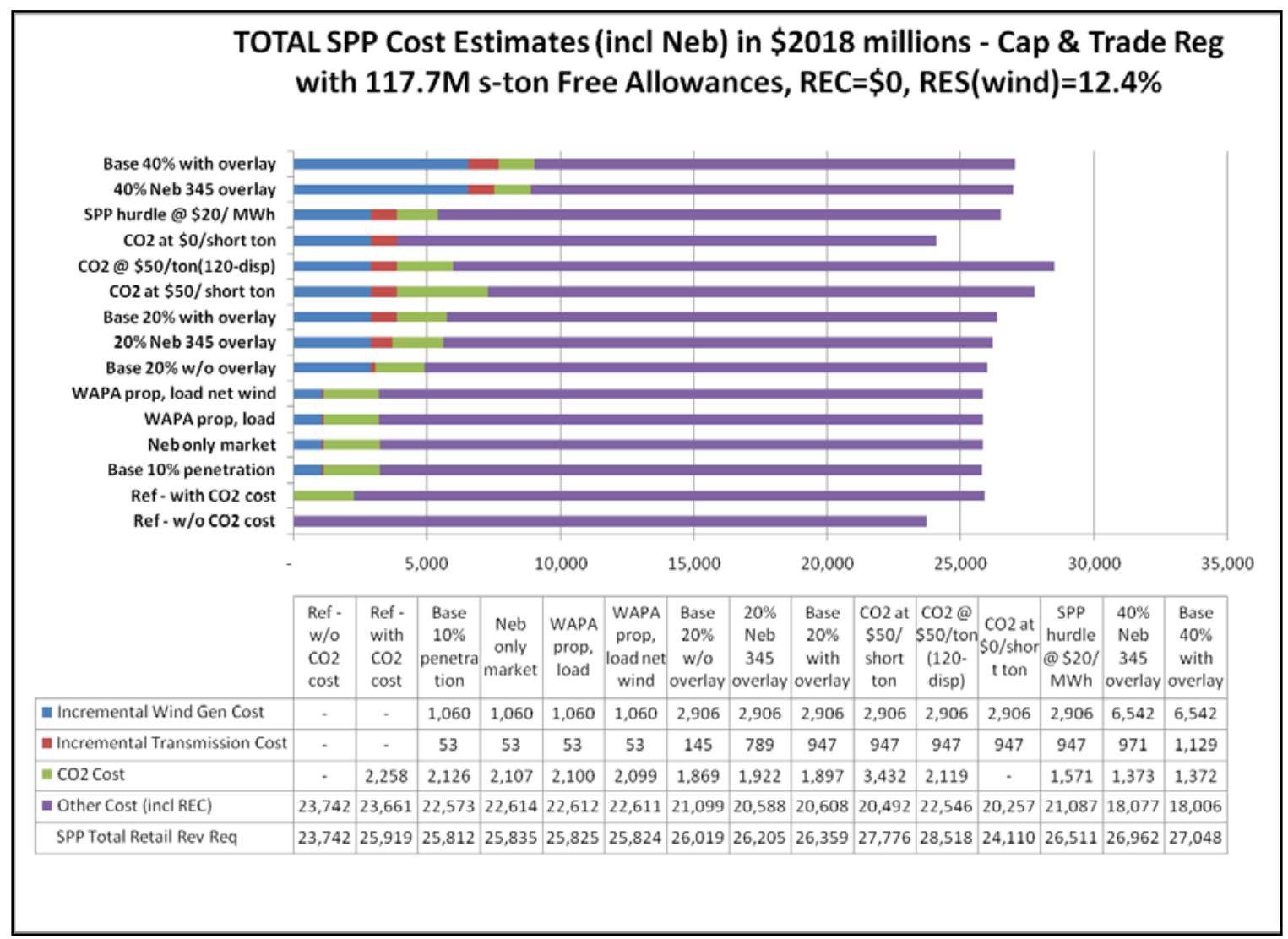

Figure 99: Total SPP Cost Estimates (inc Neb) in $\$ 2018$ millions - Cap \& Trade Reg with 117.7M s-ton Free Allowances, REC= $\$ 0, R E S$ (wind)=12.4\% 


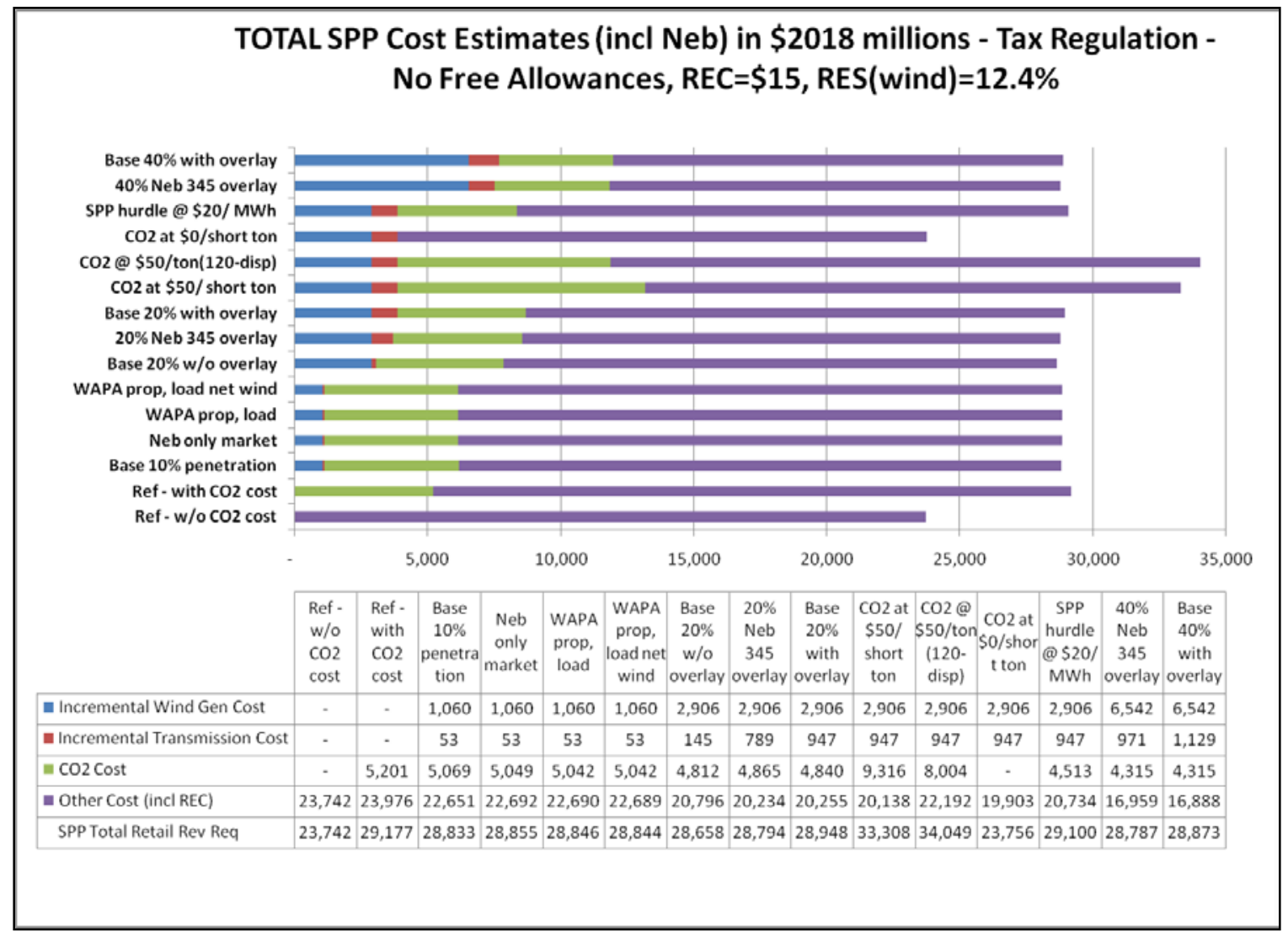

Figure 100: Total SPP Cost Estimates (inc Neb) in $\$ 2018$ millions - Tax Regulation - No Free Allowances, REC $=\$ 15, \operatorname{RES}($ wind) $=12.4 \%$ 


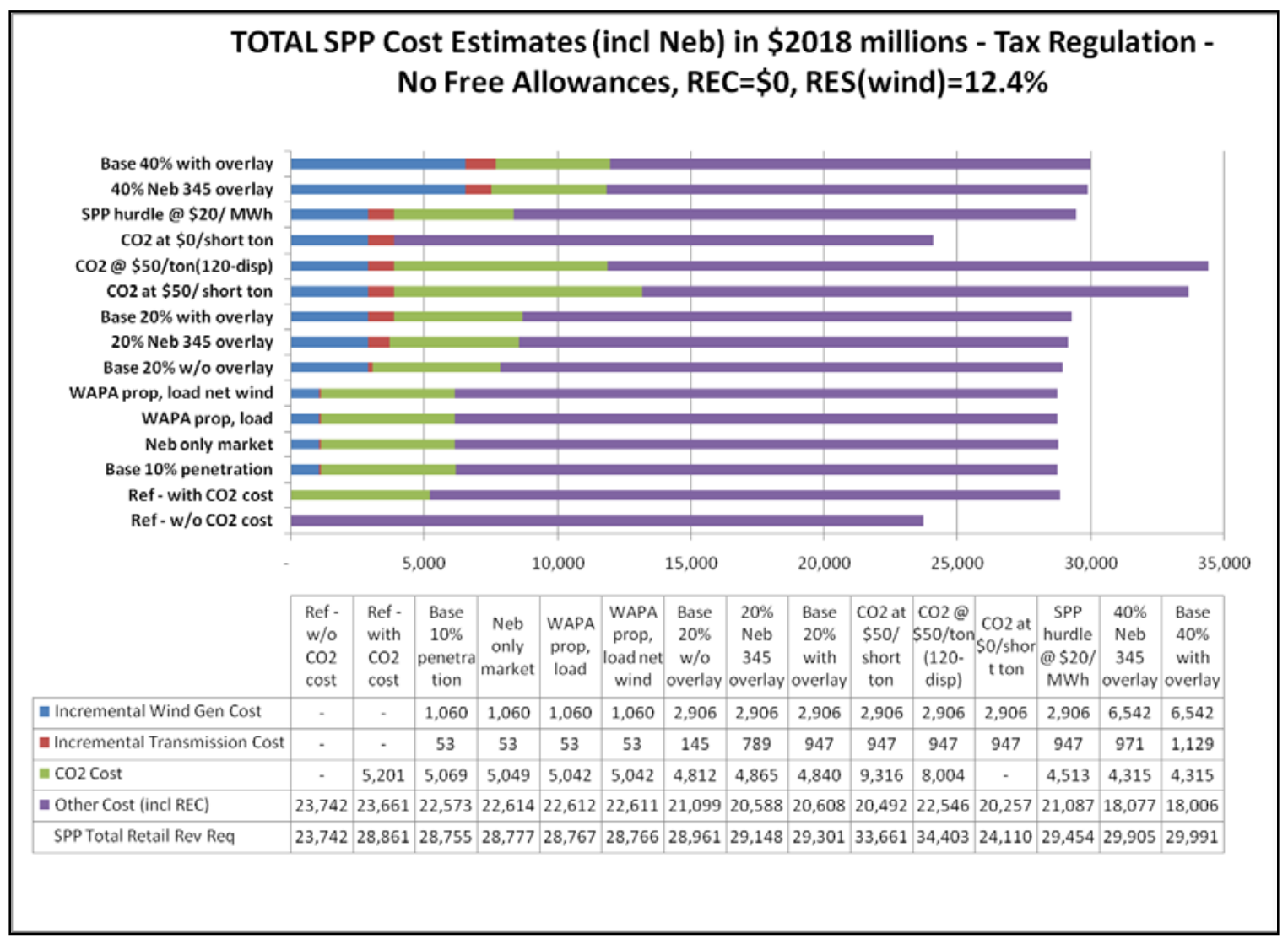

Figure 101: Total SPP Cost Estimates (inc Neb) in $\$ 2018$ millions - Tax Regulation - No Free Allowances, REC $=\$ 0, \operatorname{RES}($ wind) $=12.4 \%$ 
Table 98: Additional Revenue Requirements (CO2 Cap \& Trade Reg, REC= $\$ 15$

NPA-NREL Wind Integration Study - Addn'I Revenue Requirements (CO2 Cap \& Trade Reg, REC=\$15)

\begin{tabular}{|c|c|c|c|c|c|c|c|c|}
\hline 2006 Case & $\begin{array}{l}\text { SPP Pen- } \\
\text { etration } \\
(\%)\end{array}$ & $\begin{array}{c}\text { REC cost in } \\
\text { add'l RR } \\
\left({ }^{*} 2018 \mathrm{M} \$\right)\end{array}$ & $\begin{array}{c}\text { CO2 Price } \\
\text { (2018\$/short } \\
\text { ton) }\end{array}$ & $\begin{array}{c}\text { Reference } \\
\text { Rev Req } \\
\text { (2018 M\$) }\end{array}$ & $\begin{array}{c}\text { Additonal } \\
\text { Rev Req } \\
(2018 \text { M\$) }\end{array}$ & $\begin{array}{c}\text { Additional } \\
\text { Rev Req } \\
(\%)\end{array}$ & $\begin{array}{c}\text { CO2 } \\
\text { Emissions } \\
\text { millions st }\end{array}$ & $\begin{array}{c}\text { Exports } \\
\text { (million } \\
\text { MWh) }\end{array}$ \\
\hline Ref - w/o CO2 cost & $4.3 \%$ & 0 & 0 & 23,742 & 0 & $0.0 \%$ & NA & NA \\
\hline Ref - with CO2 cost & $4.3 \%$ & 316 & 25 & 23,742 & 2,493 & $10.5 \%$ & 208.0 & 4.3 \\
\hline Base $10 \%$ penetration & $10 \%$ & 78 & 25 & 23,742 & 2,149 & $9.1 \%$ & 202.8 & 12.6 \\
\hline Neb only market & $10 \%$ & 78 & 25 & 23,742 & 2,171 & $9.1 \%$ & 202.0 & 11.7 \\
\hline WAPA prop, load & $10 \%$ & 78 & 25 & 23,742 & 2,162 & $9.1 \%$ & 201.7 & 11.1 \\
\hline WAPA prop, load net wind & $10 \%$ & 78 & 25 & 23,742 & 2,160 & $9.1 \%$ & 201.7 & 11.1 \\
\hline Base $20 \%$ w/o overlay & $20 \%$ & (303) & 25 & 23,742 & 1,974 & $8.3 \%$ & 192.5 & 23.1 \\
\hline $20 \%$ Neb 345 overlay & $20 \%$ & (354) & 25 & 23,742 & 2,110 & $8.9 \%$ & 194.6 & 28.6 \\
\hline Base $20 \%$ with overlay & $20 \%$ & (354) & 25 & 23,742 & 2,264 & $9.5 \%$ & 193.6 & 27.1 \\
\hline $\mathrm{CO} 2$ at $\$ 50 /$ short ton & $20 \%$ & (354) & 50 & 23,742 & 3,681 & $15.5 \%$ & 186.3 & 27.8 \\
\hline CO2 @ \$50/ton(120-disp) & $20 \%$ & (354) & $' 120 ' / 50$ & 23,742 & 4,423 & $18.6 \%$ & 160.1 & 28.5 \\
\hline $\mathrm{CO} 2$ at $\$ 0 /$ short ton & $20 \%$ & (354) & 0 & 23,742 & 15 & $0.1 \%$ & 197.9 & 40.6 \\
\hline SPP hurdle @ \$20/MWh & $20 \%$ & $(354)$ & 25 & 23,742 & 2,416 & $10.2 \%$ & 180.5 & 10.5 \\
\hline $40 \%$ Neb 345 overlay & $40 \%$ & $(1,117)$ & 25 & 23,742 & 2,103 & $8.9 \%$ & 172.6 & 52.7 \\
\hline Base $40 \%$ with overlay & $40 \%$ & $(1,117)$ & 25 & 23,742 & 2,189 & $9.2 \%$ & 172.6 & 52.9 \\
\hline
\end{tabular}

*REC cost is positive/negative of RECs bought/sold in market

FREE Allowances Assumed in s-ton millions $=$

Renewable (wind) portion of Renewable Energy Standard $=12.4 \%$ 
Table 99: $\quad$ Additional Revenue Requirements (CO2 Cap \& Trade Reg, REC $=\$ 0$

NPA-NREL Wind Integration Study - Addn'I Revenue Requirements (CO2 Cap \& Trade Reg, REC=\$0)

\begin{tabular}{|c|c|c|c|c|c|c|c|c|}
\hline 2006 Case & $\begin{array}{l}\text { SPP Pen- } \\
\text { etration } \\
(\%)\end{array}$ & $\begin{array}{c}\text { REC cost in } \\
\text { add'I RR } \\
\left({ }^{*} 2018 \mathrm{M} \$\right)\end{array}$ & $\begin{array}{c}\text { CO2 Price } \\
\text { (2018\$/short } \\
\text { ton) }\end{array}$ & $\begin{array}{l}\text { Reference } \\
\text { Rev Req } \\
(2018 \text { M\$) }\end{array}$ & $\begin{array}{c}\text { Additonal } \\
\text { Rev Req } \\
(2018 \mathrm{M} \$)\end{array}$ & $\begin{array}{c}\text { Additional } \\
\text { Rev Req } \\
(\%)\end{array}$ & $\begin{array}{c}\mathrm{CO} 2 \\
\text { Emissions } \\
\text { millions st }\end{array}$ & $\begin{array}{l}\text { Exports } \\
\text { (million } \\
\text { MWh) }\end{array}$ \\
\hline Ref - w/o CO2 cost & $4.3 \%$ & 0 & 0 & 23,742 & 0 & $0.0 \%$ & NA & NA \\
\hline Ref - with CO2 cost & $4.3 \%$ & 0 & 25 & 23,742 & 2,177 & $9.2 \%$ & 208.0 & 4.3 \\
\hline Base $10 \%$ penetration & $10 \%$ & 0 & 25 & 23,742 & 2,071 & $8.7 \%$ & 202.8 & 12.6 \\
\hline Neb only market & $10 \%$ & 0 & 25 & 23,742 & 2,093 & $8.8 \%$ & 202.0 & 11.7 \\
\hline WAPA prop, load & $10 \%$ & 0 & 25 & 23,742 & 2,084 & $8.8 \%$ & 201.7 & 11.1 \\
\hline WAPA prop, load net wind & $10 \%$ & 0 & 25 & 23,742 & 2,082 & $8.8 \%$ & 201.7 & 11.1 \\
\hline Base $20 \%$ w/o overlay & $20 \%$ & 0 & 25 & 23,742 & 2,278 & $9.6 \%$ & 192.5 & 23.1 \\
\hline $20 \%$ Neb 345 overlay & $20 \%$ & 0 & 25 & 23,742 & 2,464 & $10.4 \%$ & 194.6 & 28.6 \\
\hline Base $20 \%$ with overlay & $20 \%$ & 0 & 25 & 23,742 & 2,617 & $11.0 \%$ & 193.6 & 27.1 \\
\hline $\mathrm{CO} 2$ at $\$ 50 /$ short ton & $20 \%$ & 0 & 50 & 23,742 & 4,035 & $17.0 \%$ & 186.3 & 27.8 \\
\hline CO2 @ \$50/ton(120-disp) & $20 \%$ & 0 & '120'/50 & 23,742 & 4,777 & $20.1 \%$ & 160.1 & 28.5 \\
\hline $\mathrm{CO} 2$ at $\$ 0 /$ short ton & $20 \%$ & 0 & 0 & 23,742 & 368 & $1.6 \%$ & 197.9 & 40.6 \\
\hline SPP hurdle @ \$20/ MWh & $20 \%$ & 0 & 25 & 23,742 & 2,770 & $11.7 \%$ & 180.5 & 10.5 \\
\hline $40 \%$ Neb 345 overlay & $40 \%$ & 0 & 25 & 23,742 & 3,221 & $13.6 \%$ & 172.6 & 52.7 \\
\hline Base $40 \%$ with overlay & $40 \%$ & 0 & 25 & 23,742 & 3,307 & $13.9 \%$ & 172.6 & 52.9 \\
\hline
\end{tabular}

2018 cap at $13.5 \%$ reduction from ROUGH Estimate of 2005 emissions-->156.6

$\begin{array}{ll}* \text { REC cost is positive/negative of RECs bought/sold in market } & \text { FREE Allowances Assumed in s-ton millions }=117.7\end{array}$

Renewable (wind) portion of Renewable Energy Standard $=12.4 \%$ 
Table 100: Additional Revenue Requirements (CO2 TAX Reg, REC $=\$ 15$ )

NPA-NREL Wind Integration Study - Additional Revenue Requirements (CO2 TAX Reg, REC=\$15)

\begin{tabular}{|c|c|c|c|c|c|c|c|c|}
\hline 2006 Case & $\begin{array}{c}\text { SPP Pen- } \\
\text { etration } \\
(\%)\end{array}$ & $\begin{array}{c}\text { REC cost in } \\
\text { add'I RR } \\
\left({ }^{*} 2018 \mathrm{M} \$\right)\end{array}$ & $\begin{array}{c}\text { CO2 Price } \\
\text { (2018\$/short } \\
\text { ton) }\end{array}$ & $\begin{array}{c}\text { Reference } \\
\text { Rev Req } \\
(2018 \mathrm{M} \$)\end{array}$ & $\begin{array}{c}\text { Additonal } \\
\text { Rev Req } \\
(2018 \text { M\$) } \\
\end{array}$ & $\begin{array}{c}\text { Additional } \\
\text { Rev Req } \\
(\%)\end{array}$ & $\begin{array}{c}\mathrm{CO} 2 \\
\text { Emissions } \\
\text { millions st }\end{array}$ & $\begin{array}{c}\text { Exports } \\
\text { (million } \\
\text { MWh) }\end{array}$ \\
\hline Ref - w/o CO2 cost & $4.3 \%$ & 0 & 0 & 23,742 & 0 & $0.0 \%$ & NA & NA \\
\hline Ref - with CO2 cost & $4.3 \%$ & 316 & 25 & 23,742 & 5,435 & $22.9 \%$ & 208.0 & 4.3 \\
\hline Base $10 \%$ penetration & $10 \%$ & 78 & 25 & 23,742 & 5,091 & $21.4 \%$ & 202.8 & 12.6 \\
\hline Neb only market & $10 \%$ & 78 & 25 & 23,742 & 5,113 & $21.5 \%$ & 202.0 & 11.7 \\
\hline WAPA prop, load & $10 \%$ & 78 & 25 & 23,742 & 5,104 & $21.5 \%$ & 201.7 & 11.1 \\
\hline WAPA prop, load net wind & $10 \%$ & 78 & 25 & 23,742 & 5,103 & $21.5 \%$ & 201.7 & 11.1 \\
\hline Base $20 \%$ w/o overlay & $20 \%$ & (303) & 25 & 23,742 & 4,917 & $20.7 \%$ & 192.5 & 23.1 \\
\hline $20 \%$ Neb 345 overlay & $20 \%$ & (354) & 25 & 23,742 & 5,053 & $21.3 \%$ & 194.6 & 28.6 \\
\hline Base $20 \%$ with overlay & $20 \%$ & (354) & 25 & 23,742 & 5,206 & $21.9 \%$ & 193.6 & 27.1 \\
\hline $\mathrm{CO} 2$ at $\$ 50 /$ short ton & $20 \%$ & (354) & 50 & 23,742 & 9,566 & $40.3 \%$ & 186.3 & 27.8 \\
\hline CO2 @ \$50/ton(120-disp) & $20 \%$ & (354) & $' 120 ' / 50$ & 23,742 & 10,308 & $43.4 \%$ & 160.1 & 28.5 \\
\hline $\mathrm{CO} 2$ at $\$ 0 /$ short ton & $20 \%$ & (354) & 0 & 23,742 & 15 & $0.1 \%$ & 197.9 & 40.6 \\
\hline SPP hurdle @ \$20/ MWh & $20 \%$ & $(354)$ & 25 & 23,742 & 5,358 & $22.6 \%$ & 180.5 & 10.5 \\
\hline $40 \%$ Neb 345 overlay & $40 \%$ & $(1,117)$ & 25 & 23,742 & 5,046 & $21.3 \%$ & 172.6 & 52.7 \\
\hline Base $40 \%$ with overlay & $40 \%$ & $(1,117)$ & 25 & 23,742 & 5,132 & $21.6 \%$ & 172.6 & 52.9 \\
\hline
\end{tabular}

${ }^{*} \mathrm{REC}$ cost is positive/negative of RECs bought/sold in market

FREE Allowances Assumed in s-ton millions $=0.0$

Renewable (wind) portion of Renewable Energy Standard $=12.4 \%$ 
Table 101: $\quad$ Additional Revenue Requirements (CO2 TAX Reg, REC $=\$ 0$ )

NPA-NREL Wind Integration Study - Additional Revenue Requirements (CO2 TAX Reg, REC $=\$ 0$ )

\begin{tabular}{|c|c|c|c|c|c|c|c|c|}
\hline 2006 Case & $\begin{array}{c}\text { SPP Pen- } \\
\text { etration } \\
(\%) \\
\end{array}$ & $\begin{array}{c}\text { REC cost in } \\
\text { add'I RR } \\
\left({ }^{\star} 2018 \mathrm{M} \$\right)\end{array}$ & $\begin{array}{c}\text { CO2 Price } \\
\text { (2018\$/short } \\
\text { ton) }\end{array}$ & $\begin{array}{c}\text { Reference } \\
\text { Rev Req } \\
(2018 \mathrm{M} \$) \\
\end{array}$ & $\begin{array}{c}\text { Additonal } \\
\text { Rev Req } \\
(2018 \mathrm{M} \$) \\
\end{array}$ & $\begin{array}{c}\text { Additional } \\
\text { Rev Req } \\
(\%) \\
\end{array}$ & $\begin{array}{c}\mathrm{CO} 2 \\
\text { Emissions } \\
\text { millions st } \\
\end{array}$ & $\begin{array}{l}\text { Exports } \\
\text { (million } \\
\text { MWh) } \\
\end{array}$ \\
\hline Ref - w/o CO2 cost & $4.3 \%$ & 0 & 0 & 23,742 & 0 & $0.0 \%$ & NA & NA \\
\hline Ref - with $\mathrm{CO} 2$ cost & $4.3 \%$ & 0 & 25 & 23,742 & 5,120 & $21.6 \%$ & 208.0 & 4.3 \\
\hline Base $10 \%$ penetration & $10 \%$ & 0 & 25 & 23,742 & 5,013 & $21.1 \%$ & 202.8 & 12.6 \\
\hline Neb only market & $10 \%$ & 0 & 25 & 23,742 & 5,035 & $21.2 \%$ & 202.0 & 11.7 \\
\hline WAPA prop, load & $10 \%$ & 0 & 25 & 23,742 & 5,026 & $21.2 \%$ & 201.7 & 11.1 \\
\hline WAPA prop, load net wind & $10 \%$ & 0 & 25 & 23,742 & 5,024 & $21.2 \%$ & 201.7 & 11.1 \\
\hline Base $20 \%$ w/o overlay & $20 \%$ & 0 & 25 & 23,742 & 5,220 & $22.0 \%$ & 192.5 & 23.1 \\
\hline $20 \%$ Neb 345 overlay & $20 \%$ & 0 & 25 & 23,742 & 5,406 & $22.8 \%$ & 194.6 & 28.6 \\
\hline Base $20 \%$ with overlay & $20 \%$ & 0 & 25 & 23,742 & 5,560 & $23.4 \%$ & 193.6 & 27.1 \\
\hline $\mathrm{CO} 2$ at $\$ 50 /$ short ton & $20 \%$ & 0 & 50 & 23,742 & 9,920 & $41.8 \%$ & 186.3 & 27.8 \\
\hline CO2 @ \$50/ton(120-disp) & $20 \%$ & 0 & $' 120 ' / 50$ & 23,742 & 10,661 & $44.9 \%$ & 160.1 & 28.5 \\
\hline $\mathrm{CO} 2$ at $\$ 0 /$ short ton & $20 \%$ & 0 & 0 & 23,742 & 368 & $1.6 \%$ & 197.9 & 40.6 \\
\hline SPP hurdle @ \$20/ MWh & $20 \%$ & 0 & 25 & 23,742 & 5,712 & $24.1 \%$ & 180.5 & 10.5 \\
\hline $40 \%$ Neb 345 overlay & $40 \%$ & 0 & 25 & 23,742 & 6,163 & $26.0 \%$ & 172.6 & 52.7 \\
\hline Base $40 \%$ with overlay & $40 \%$ & 0 & 25 & 23,742 & 6,249 & $26.3 \%$ & 172.6 & 52.9 \\
\hline
\end{tabular}

${ }^{*} \mathrm{REC}$ cost is positive/negative of RECs bought/sold in market

FREE Allowances Assumed in s-ton millions $=0.0$

Renewable (wind) portion of Renewable Energy Standard $=12.4 \%$ 


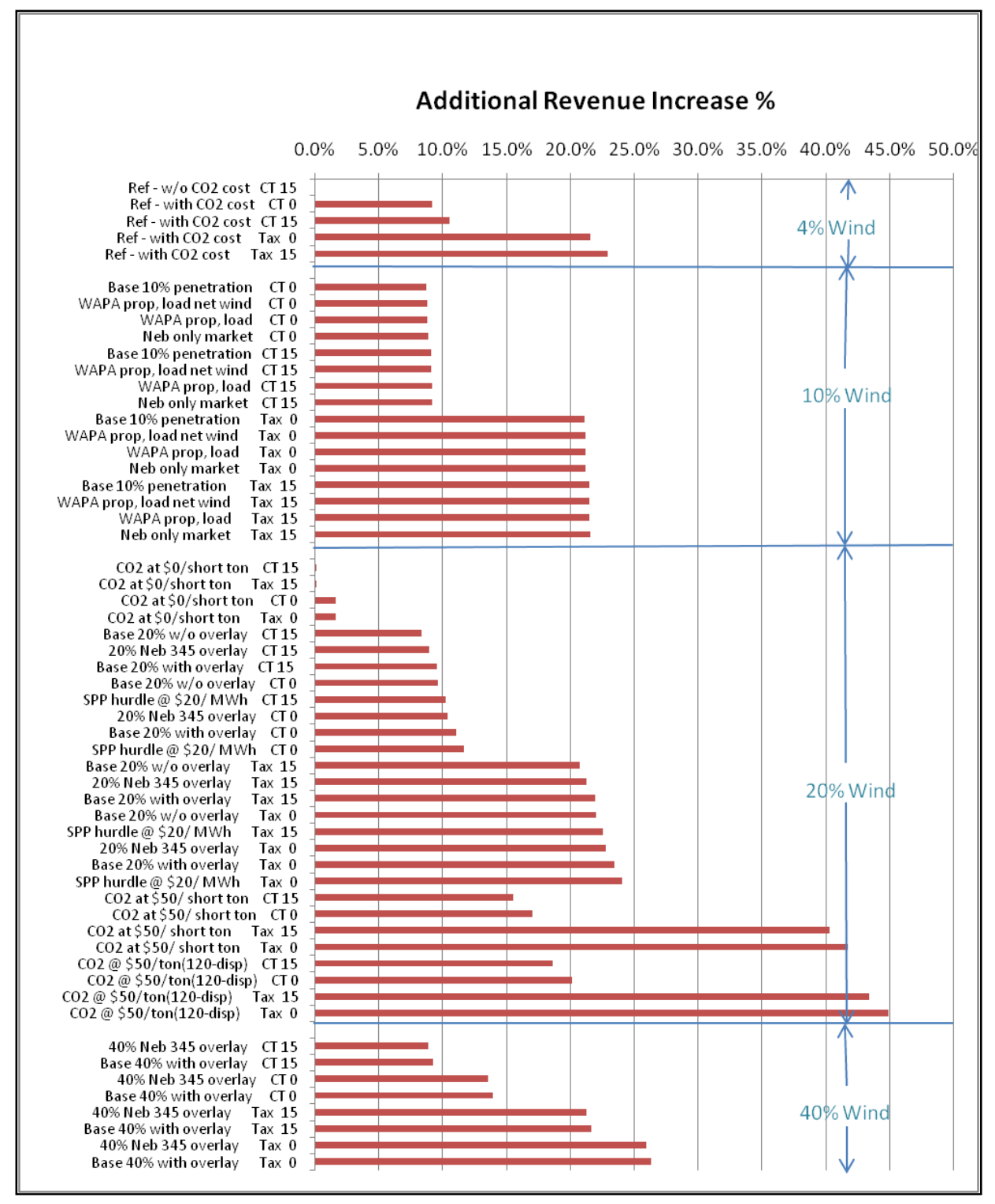

Figure 102: Additional Revenue Increase \% 


\subsection{GLOSSARY (MEANINGS AS USED IN THIS REPORT)}

Actual Wind Case - Simulation case where wind is dispatched up to the actual wind profile excluding curtailment.

Adjusted Production Cost (as modeled in this study in PROMOD) - Area or regional production costs adjusted to account for purchase and sale energy.

Annual Carrying Charge Rate - the percentage that is applied to a lump-sum dollar investment amount that yields the annual cost for capital and operating and maintenance related to the investment.

Area Control Error (ACE) - see Section 3.1.3.

Automatic Generation Control (AGC) - see Section 3.1.2.

Balancing Area (Control Area) - a modeled region within the PROMOD data set with a defined topology sUch as SPP, MISO, SERC, etc.

Basecase - a PROMOD run used as reference case for comparison.

Cap-and-Trade - an administrative approach used to control pollution by providing economic incentives for achieving reductions in the emissions of pollutants.

Case - a PROMOD runs with a set of defined parameters; may be interchanged with scenario.

CO2 Emission - a power plant's emission of carbon dioxide from fossil-fuel burning and measured in short tons (as used in this study).

CO2 Emission Price - the dollars per short ton associated with a CO2 emission in a cap-and-trade regulation environment.

CO2 Emission Tax - the dollars per short ton associated with a CO2 emission in a tax regulation environment.

Commitment (Unit) - The identification of a generating resource to be on or off line

Congestion - in the production simulation when the transmission system reaches a thermal or other operating limit that is monitored as a flowgate.

Constraint (in PROMOD) - a characteristic of the modeled system (generation or transmission) that is required to be met in the solution.

Contingency Reserve - see Table 15

Contingency Reserve (Spinning) - see Section 3.1

Contingency Reserve (Supplemental) - see Section 3.1

Control Performance Standard (CPS) - see Section 3.1.3.

CPS1 - see Section 3.1.3 
CPS2 - see Section 3.1.3

Daily Block Proxy - a proxy wind resource that is a flat average of delivered energy for a given day. Like other proxy resources it requires no additional regulating reserves and is known perfectly.

Day-Ahead Forecast (NREL Wind) -Wind generation data within the NREL database that represents a 24 hour forecast created 18 hours before to the first hour of the forecast.

Day-Ahead Market - a formal organized market for energy transactions occurring in the next day.

Dispatch (Unit) - the generation level setting of a unit committed to be on line.

DOE - U.S. Department of Energy (see energy.gov)

Dump Energy - the total amount of violations of unit minimums required to create a net zero load and energy production balance.

Duration Curve - a curve that shows the relationship between a value and its utilization by plotting values in descending order instead of chronologically.

ECAR - East Central Area Reliability Council, which is now a part of Reliability First Corporation (see reliabilityfirst.com)

EHV - Extra High Voltage (voltages above 345kV)

Energy Management System (EMS) - see Section 3.1.2.

Energy - Electrical energy typically referenced in kWh, MWh, GWh or TWh.

ERCOT - Electric Reliability Council of Texas (see ercot.com)

ERO - Electric Reliability Organization (presently NERC)

EWITS - Eastern Wind Integration and Transmission Study (see wind.nrel.gov/public/EWITS)

Export/Import - energy exchanged with other regions.

Flowgate - $a$ line or set of lines for which the line flows need to be constrained not to exceed a certain level due to operational reasons.

FRCC - Florida Reliability Council (see frcc.com)

Free Allowances - a permit to emit carbon dioxide, as evaluated in this study, which is provided to the utility free of charge by the regulating entity, rather than an allowance having been purchased on the market or at auction.

Generation - Output of a generator measured in MW.

Generation Unit Cycling - process of ramping up (turning on) or ramping down (turning off) a generating unit to meet load fluctuations.

Heat Rate (as modeled in this study in PROMOD) - amount of heat input required per unit of generator output. Typically expressed in btu / kWh or some variation thereof.

Hourly Production Simulation Analysis - computer simulation of generation and transmission system using security constrained economic dispatch in PROMOD. 
Hurdle Rate - Amount (in \$/MWh) of LMP differential required for interchange between pools to occur in PROMOD simulations.

Ideal Wind Case - production simulation case where wind is perfectly known and requires no incremental operating reserves. Typically compared to Actual Wind Case to determine wind integration costs.

Incremental - an additional amount beyond some other reference level.

Interconnection (Eastern, Western) - synchronized frequency power grid. The state of Nebraska includes portions of the both the Eastern and Western Interconnections of the United States. The bulk of the study focuses on portions of the Eastern Interconnection, with Section 6 focused on those portions of Nebraska in the Western Interconnection.

ISO - Independent System Operator

JCSP - Joint Coordinated System Plan (see jcspstudy.org)

kV - kilovolts (or 1,000 Volts)

kW, MW, GW, TW - units of power: kilowatt $(\mathrm{kW})=$ one thousand watts; Megawatt $(\mathrm{MW})=$ one million watts; Gigawatt $(G W)=$ one billion watts; Terawatt $(T W)=$ one trillion watts.

kWh, MWh, GWh, TWh - units of energy: kilowatt hour $(\mathrm{kWh})=$ one thousand watt hours; Megawatt hour $(\mathrm{MWh})=$ one million watt hours; Gigawatt hour (GWh) = one billion watt hours; Terawatt hour $(\mathrm{TWh})=$ one trillion watt hours.

Load Following - adjusting generator output coincident with movements in load.

Load Net Wind - the result of subtracting the MW value of wind generation in a given hour from the $M W$ value of Load in the same hour.

Locational Marginal Price (LMP) - the marginal cost of energy at a specific bus (or node) in the transmission system. Often expressed as Generation or Load Weighted LMP which is a regional average determined by summing the product of bus generation (or load) and bus LMP and dividing that sum by the total bus generation (or load).

Losses - in context of energy, losses refer to the amount of energy lost from point of generation to point of receipt.

MAAC - Mid-Atlantic Area Council, which is now a part of Reliability First Corporation (see reliabilityfirst.com)

MAIN - Mid-America Interconnected Network, which is now a part of Reliability First Corporation (see reliabilityfirst.com)

MAPP - Mid-Continent Area Power Pool (see mapp.org)

Market - see Section 4.4.2 for SPP market and Section 4.4.1 for Nebraska only market.

Max Capacity - The maximum possible output of a generating unit.

Mean Absolute Error - is a quantity used to measure how close forecasts or predictions are to the eventual outcomes. In this study the MAE is calculated using predicted values, 10 minute, 1hour or day-ahead forecasts, and comparing these values to actual value. The smaller the MAE result the better the forecast or lower the forecast error. The MAE does not take 
into account if the forecast is high or low it is an absolute that provides limits around the actual value. Take for example the error of the wind hour ahead forecast is determined by subtracting the forecast by the actual wind value. Calculating the absolute value for each error for every hourly value in the study period and finding the average of these values results in the MAE for the wind during this period.

Meso-Scale - a meteorological phenomenon approximately 10 to 1000 kilometers having horizontal scales such as wind

Min Capacity - The minimum possible output of an online generating unit.

MISO - Midwest Independent Transmission System Operator (see midwestiso.org)

Mitigation - a resource or methodology that provides a helpful solution to the integration requirements imposed by wind generation due to its variability and uncertainty.

Moving Average Proxy - a proxy wind resource that is a moving average of delivered energy for a given hour and a certain number of hours surrounding it. Like other proxy resources it requires no additional regulating reserves and is known perfectly.

MRO - Midwest Reliability Organization (see midwestreliability.org)

Must-Run Generator - a generator forced online in PROMOD rather than being economically committed.

NERC - North-American Electric Reliability Corporation (see nerc.com)

Net Position - the net of all resources minus loads for an area or region.

Non-monitored Branches - in PROMOD, unlike in a powerflow model like PSS/E, not every branch is required to adhere to its modeled limit. Branches or groups of branches required to honor their limits (whether in basecase or $\mathrm{N}-1$ conditions) are referred to as flowgates; other branches are called non-monitored branches and can theoretically have unlimited flow but are usually limited by the flowgates around them. Flows can still be reported on theses non-monitored branches.

NPA - Nebraska Power Association. (see nepower.org)

NPCC - Northeast Power Coordinating Council (see npcc.org)

NREL - National Renewable Energy Laboratory. (see nrel.gov)

NREL Wind Generation Site - See Section 2.3.1.

NYISO - New York Independent System Operator (see nyiso.com)

Operating Reserve - See Table 15

Operating Reserve (Spinning) - See Table 15

Operating Reserve (Supplemental) - See Table 15

Penetration (Wind) - the percentage calculated by dividing annual wind generation in GWh of a utility by the utility's annual load in GWh, both measured at the generator busbar (as it is used in this study). Other usages calculate penetration on a MW basis, or sometimes divide by retail load. The usage in this study results in the lowest (most conservative) penetration value, in that $7.41 \%$ losses are included in the denominator, and that the energy basis is usually lower than the capacity basis. 
Persistence Forecast (Wind) - choosing the value of a wind forecast in hour $n+1$ by selecting the metered or actual value of the wind in hour $n$.

PJM - a regional transmission organization (see pjm.com)

Production Cost (as modeled in this study in PROMOD) - the calculated operating cost for an area, region or footprint in PROMOD. Include generator fuel costs and operations and maintenance.

PROMOD-Security constrained economic dispatch production simulation model produced and maintained by Ventyx.

Proxy Resource - a fictitious wind resources that is perfectly known, requires no additional regulating reserves and has limited variability. Used for comparison against actual wind resource to determined integration costs. See also Daily Block Proxy, Sub-period Block Proxy, Moving Average Proxy and Shaped Proxy.

Ramp - MW movement in generation either up or down.

Ramp Rate (as modeled in this study in PROMOD) -maximum generator output change from hour to hour.

Regulation - see Section 3.1.1.

Reliability-Related Services - see Section 3.1

Renewable Energy Credit (REC) - an official, tradable certificate that a MWh of qualifying renewable energy has been produced.

Renewable Energy Standard (RES) - in this report, refers to a federal requirement for utilities to include renewable resources in its supply mix and a portion may contain energy efficiency projects. In this study, the RES described in the Waxman-Markey bill is used as a proxy.

Regulating Reserve - See Table 15

Revenue Requirement - In this study, refers to the total revenue that the SPP utilities need to or are authorized to recover, which for Nebraska's public utilities and other non-profits amounts to their operating costs, and for private non-profits includes a reasonable return on rate base.

RTO - Regional Transmission Organization

Scenario - PROMOD runs with a set of defined parameters; may be interchanged with case.

Schedules (energy) - In this study a agreed-upon amount of electric energy transacted and delivered between a buyer and seller for a set time period, typically specified as MW amounts in an hour and may include ramping specifications between varying hourly levels. The schedules are coordinated through the electric system operator.

Security-Constrained Unit Commitment (SCUC) - see Section 3.1.1

Sensitivity case - a (PROMOD) simulation used to test the effect on results of varying a specific input assumption.

SERC - South East Reliability Corporation (see sercl.org) 
Shaped Proxy - a proxy wind resource that uses the actual delivered shape of the wind but is perfectly known (no forecast error) and requires no additional regulating reserves.

Short ton - two thousand pounds.

Simulation - computer imitation of real world generation, transmission and load system operations.

Spinning Reserve - See Table 15

Sub-hourly Statistical Analysis - analysis of chronological data sampled at 10 minute intervals.

Sub-period Block Proxy - a proxy wind resource that is a flat average of delivered energy for each sub-period (on-peak and off-peak) of a given day. Like other proxy resources it requires no additional regulating reserves and is known perfectly.

Supplemental (non-Spinning) Reserves - see Section 3.1.1

SPP - Southwest Power Pool. (see spp.org)

System Control and Data Acquisition (SCADA) - see Section 3.1.2.

Thermal Limit - physical limit of energy carrying capability of a transmission line.

Total Cost (as used in this study) - all the revenue requirements that are recovered in the end-use retail rates for the utilities in the Southwest Power Pool.

Transaction - energy interchange between utility entities.

Transmission Overlay - transmission system upgrades, typically extra high voltage with large interregional carrying capacity.

TRC - Technical Review Committee (see Appendix Section 8.1)

TVA - Tennessee Valley Authority (see tva.gov)

Unserved Energy - known as emergency energy in PROMOD, this is the amount of energy beyond modeled generation resources required to meet load.

WAPA - Western Area Power Administration. (see wapa.gov)

WECC - Western Electricity Coordinating Council (see wecc.biz)

Wind Generation Curtailment - a reduction in requested output of a wind generator due to an excess of energy determined by LMP at the wind injection site being below a certain threshold.

Wind Integration Cost - production cost increase due to wind forecast error, wind regulating reserves and wind variability. Typically normalized to wind energy by dividing production cost delta by total wind energy.

Wind Intermittency - this refers to wind generation characteristic of stopping for a period of time and then restarting for a period of time.

Wind Reserves - used in Figure 45 and Figure 54

Wind Uncertainty - In this report this term refers to the unpredictability of wind generation output. 
Wind Variability - The fact that wind generation output can increase or decrease dependent upon weather. 


\section{REPORT DOCUMENTATION PAGE}

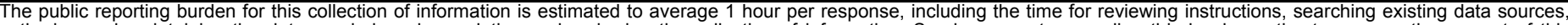

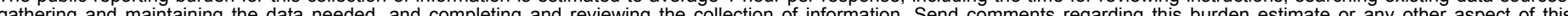

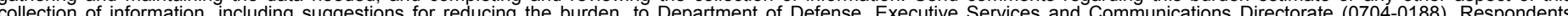

should be aware that nothithstanding any other provision of law, no person shall be subject to any penalty for faili

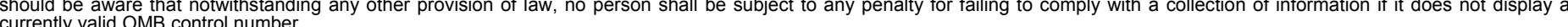

PLEASE DO NOT RETURN YOUR FORM TO THE ABOVE ORGANIZATION.
1. REPORT DATE (DD-MM-YYYY)
2. REPORT TYPE
March 2010
Subcontract Report
3. DATES COVERED (From - To)
$4 / 2008$ to $1 / 2010$

4. TITLE AND SUBTITLE

Nebraska Statewide Wind Integration Study:

April 2008 - January 2010

6. AUTHOR(S)

C. Johannes and D. Kallesen 5a. CONTRACT NUMBER

DE-AC36-08-GO28308

5b. GRANT NUMBER

5c. PROGRAM ELEMENT NUMBER

5d. PROJECT NUMBER

NREL/SR-550-47519

5e. TASK NUMBER

WE10.4311

5f. WORK UNIT NUMBER
7. PERFORMING ORGANIZATION NAME(S) AND ADDRESS(ES)

Nebraska Power Association

P.O. Box 49

Columbus, NE 68602-0499
8. PERFORMING ORGANIZATION REPORT NUMBER

AAM-9-89030-01

9. SPONSORING/MONITORING AGENCY NAME(S) AND ADDRESS(ES)

National Renewable Energy Laboratory

1617 Cole Blvd.

Golden, CO 80401-3393

10. SPONSOR/MONITOR'S ACRONYM(S)

NREL

11. SPONSORING/MONITORING AGENCY REPORT NUMBER NREL/SR-550-47519

12. DISTRIBUTION AVAILABILITY STATEMENT

National Technical Information Service

U.S. Department of Commerce

5285 Port Royal Road

Springfield, VA 22161

13. SUPPLEMENTARY NOTES

NREL Technical Monitor: Brian Parsons

14. ABSTRACT (Maximum 200 Words)

Wind generation resources in Nebraska will play an increasingly important role in the environmental and energy security solutions for the state and the nation. In this context, the Nebraska Power Association conducted a statewide wind integration study.

\section{SUBJECT TERMS}

Nebraska; wind; integration study; Nebraska Power Association.

\begin{tabular}{|c|c|c|}
\hline $\begin{array}{l}\text { a. REPORT } \\
\text { Unclassified }\end{array}$ & $\begin{array}{l}\text { b. ABSTRACT } \\
\text { Unclassified }\end{array}$ & $\begin{array}{l}\text { c. THIS PAGE } \\
\text { Unclassified }\end{array}$ \\
\hline
\end{tabular}

\begin{tabular}{|c|c|}
\hline $\begin{array}{l}\text { 17. LIMITATION } \\
\text { OF ABSTRACT }\end{array}$ & $\begin{array}{l}\text { 18. } \\
\text { NUMBER } \\
\text { OF PAGES }\end{array}$ \\
\hline UL & \\
\hline
\end{tabular}

19a. NAME OF RESPONSIBLE PERSON

19b. TELEPHONE NUMBER (Include area code) 